\title{
Breast Reconstruction After Mastectomy: A Systematic Review and Meta-Analysis
}




\section{Breast Reconstruction After Mastectomy: A Systematic Review and Meta-Analysis}

Prepared for:

Agency for Healthcare Research and Quality

U.S. Department of Health and Human Services

5600 Fishers Lane

Rockville, MD 20857

www.ahrq.gov

Contract No. 75Q80120D00001

Prepared by:

Brown Evidence-based Practice Center

Providence, RI

Investigators:

Ian J. Saldanha, M.B.B.S., M.P.H., Ph.D.

Wangnan Cao, Ph.D.

Justin M. Broyles, M.D.

Gaelen P. Adam, M.L.I.S., M.P.H.

Monika Reddy Bhuma, B.D.S., M.P.H.

Shivani Mehta, M.P.H.

Laura S. Dominici, M.D.

Andrea L. Pusic, M.D.

Ethan M. Balk, M.D., M.P.H.

AHRQ Publication No. 21-EHC027

July 2021

Revised October 2021 


\section{Errata}

The original systematic review reported that autologous reconstruction (AR) with deep inferior epigastric perforator (DIEP) compared with latissimus dorsi (LD) flaps may result in comparable patient satisfaction with breasts (low strength of evidence [SoE]), based on two studies. Upon rereview of the studies, we found that only a single study reported relevant data for this comparison. The SoE was downgraded from low to insufficient based on a single study with high risk of bias and imprecise effect size. Based on this, a conclusion cannot be made for this comparison. This change is reflected in the Abstract, Main Points, Key Points for Key Question 6, and in Tables 8 and 9 and Appendix Tables E-6.4 and G-6. 
This report is based on research conducted by the Brown Evidence-based Practice Center (EPC) under contract to the Agency for Healthcare Research and Quality (AHRQ), Rockville, MD (Contract No. 75Q80120D00001). The findings and conclusions in this document are those of the authors, who are responsible for its contents; the findings and conclusions do not necessarily represent the views of AHRQ. Therefore, no statement in this report should be construed as an official position of AHRQ or of the U.S. Department of Health and Human Services.

\section{None of the investigators have any affiliations or financial involvement that conflicts with the material presented in this report.}

The information in this report is intended to help healthcare decision makers - patients and clinicians, health system leaders, and policymakers, among others-make well-informed decisions and thereby improve the quality of healthcare services. This report is not intended to be a substitute for the application of clinical judgment. Anyone who makes decisions concerning the provision of clinical care should consider this report in the same way as any medical reference and in conjunction with all other pertinent information, i.e., in the context of available resources and circumstances presented by individual patients.

This report is made available to the public under the terms of a licensing agreement between the author and the Agency for Healthcare Research and Quality. This report may be used and reprinted without permission except those copyrighted materials that are clearly noted in the report. Further reproduction of those copyrighted materials is prohibited without the express permission of copyright holders.

AHRQ or U.S. Department of Health and Human Services endorsement of any derivative products that may be developed from this report, such as clinical practice guidelines, other quality enhancement tools, or reimbursement or coverage policies, may not be stated or implied.

AHRQ appreciates appropriate acknowledgment and citation of its work. Suggested language for acknowledgment: This work was based on an evidence report, Breast Reconstruction After Mastectomy: A Systematic Review and Meta-Analysis, by the Evidence-based Practice Center Program at the Agency for Healthcare Research and Quality (AHRQ).

Suggested citation: Saldanha IJ, Cao W, Broyles JM, Adam GP, Bhuma MR, Mehta S, Dominici LS, Pusic AL, Balk EM. Breast Reconstruction After Mastectomy: A Systematic Review and Meta-Analysis. Comparative Effectiveness Review No. 245. (Prepared by the Brown Evidencebased Practice Center under Contract No. 75Q80120D00001.) AHRQ Publication No. 21EHC027. Rockville, MD: Agency for Healthcare Research and Quality; July 2021. Revised October 2021. DOI: https://doi.org/10.23970/AHRQEPCCER245. Posted final reports are located on the Effective Health Care Program search page. 


\section{Preface}

The Agency for Healthcare Research and Quality (AHRQ), through its Evidence-based Practice Centers (EPCs), sponsors the development of systematic reviews to assist public- and private-sector organizations in their efforts to improve the quality of healthcare in the United States. These reviews provide comprehensive, science-based information on common, costly medical conditions, and new healthcare technologies and strategies.

Systematic reviews are the building blocks underlying evidence-based practice; they focus attention on the strength and limits of evidence from research studies about the effectiveness and safety of a clinical intervention. In the context of developing recommendations for practice, systematic reviews can help clarify whether assertions about the value of the intervention are based on strong evidence from clinical studies. For more information about AHRQ EPC systematic reviews, see https://effectivehealthcare.ahrq.gov/about/epc/evidence-synthesis.

AHRQ expects that these systematic reviews will be helpful to health plans, providers, purchasers, government programs, and the healthcare system as a whole. Transparency and stakeholder input are essential to the Effective Health Care Program. Please visit the website (www.effectivehealthcare.ahrq.gov) to see draft research questions and reports or to join an email list to learn about new program products and opportunities for input.

If you have comments on this systematic review, they may be sent by mail to the Task Order Officer named below at: Agency for Healthcare Research and Quality, 5600 Fishers Lane, Rockville, MD 20857, or by email to epc@ahrq.hhs.gov.

David Meyers, M.D. Acting Director Agency for Healthcare Research and Quality

Christine Chang, M.D., M.P.H.

Acting Director

Center for Evidence and Practice Improvement

Agency for Healthcare Research and Quality
Arlene S. Bierman, M.D., M.S.

Director

Center for Evidence and Practice

Improvement

Agency for Healthcare Research and Quality

Jill Huppert, M.D., M.P.H.

Task Order Officer

Center for Evidence and Practice Improvement

Agency for Healthcare Research and Quality 


\section{Acknowledgments}

The authors gratefully acknowledge the following individuals for their contributions to this project: Associate Editor Timothy Wilt, M.D., M.P.H., from the Minnesota Evidence-based Practice Center, Minneapolis, MN; Task Order Officer Jill Huppert, M.D., M.P.H., from AHRQ; and Acting EPC Program Director Christine Chang, M.D., M.P.H., from AHRQ.

\section{Key Informants}

In designing the study questions, the EPC consulted several Key Informants who represent the end-users of research. The EPC sought the Key Informant input on the priority areas for research and synthesis. Key Informants are not involved in the analysis of the evidence or the writing of the report. Therefore, in the end, study questions, design, methodological approaches, and/or conclusions do not necessarily represent the views of individual Key Informants.

Key Informants must disclose any financial conflicts of interest greater than $\$ 5,000$ and any other relevant business or professional conflicts of interest. Because of their role as end-users, individuals with potential conflicts may be retained. The TOO and the EPC work to balance, manage, or mitigate any conflicts of interest.

The list of Key Informants who provided input to this report follows:

Binita Ashar, M.D.

Center for Devices and Radiological Health

Food and Drug Administration

Silver Spring, MD

Katelyn Donnelly, M.P.H. ${ }^{\dagger}$

American Society of Plastic Surgeons

The Plastic Surgery Foundation

Arlington Heights, IL

Phyllis Greenberger, M.S.W.

HealthyWomen

Middletown, NJ

Priscilla McAuliffe, M.D., Ph.D.

Department of Surgery

University of Pittsburgh School of Medicine

Pittsburgh, PA
Terence Myckatyn, M.D.

Department of Surgery

Washington University School of Medicine

in St. Louis

St. Louis, MO

Anne Taylor, M.D.

Aesthetica Surgery \& Spa

Worthington, $\mathrm{OH}$

Myelin Torres, M.D. ${ }^{* \dagger}$

Department of Radiation Oncology

Emory University School of Medicine

Atlanta, GA

*Also provided input on Draft Report.

${ }^{\dagger}$ Also a Technical Expert Panel member. 


\section{Technical Expert Panel}

In designing the study questions and methodology at the outset of this report, the EPC consulted several technical and content experts. Broad expertise and perspectives were sought. Divergent and conflicted opinions are common and perceived as healthy scientific discourse that results in a thoughtful, relevant systematic review. Therefore, in the end, study questions, design, methodologic approaches, and/or conclusions do not necessarily represent the views of individual technical and content experts.

Technical Experts must disclose any financial conflicts of interest greater than $\$ 5,000$ and any other relevant business or professional conflicts of interest. Because of their unique clinical or content expertise, individuals with potential conflicts may be retained. The TOO and the EPC work to balance, manage, or mitigate any potential conflicts of interest identified.

The list of Technical Experts who provided input to this report follows:

Michele Manahan, M.D.

Department of Plastic and Reconstructive Surgery

Johns Hopkins University School of

Medicine

Baltimore, MD

Steven Nagel, M.D.*

Center for Devices and Radiological Health

Food and Drug Administration

Silver Spring, MD

William Sikov, M.D.*

Department of Medicine

Warren Alpert Medical School of Brown

University

Providence, RI

*Also provided input on Draft Report.
Edwin Wilkins, M.D.

Department of Plastic Surgery

University of Michigan School of Medicine

Ann Arbor, MI

Sung Yoon, M.D.*

Center for Devices and Radiological Health

Food and Drug Administration

Silver Spring, MD

\section{Peer Reviewers}

Prior to publication of the final evidence report, EPCs sought input from independent Peer Reviewers without financial conflicts of interest. However, the conclusions and synthesis of the scientific literature presented in this report do not necessarily represent the views of individual reviewers.

Peer Reviewers must disclose any financial conflicts of interest greater than $\$ 5,000$ and any other relevant business or professional conflicts of interest. Because of their unique clinical or content expertise, individuals with potential nonfinancial conflicts may be retained. The TOO 
and the EPC work to balance, manage, or mitigate any potential nonfinancial conflicts of interest identified.

The list of Peer Reviewers follows:

Melissa Camp, M.D., M.P.H.

Department of Surgery

Johns Hopkins University School of

Medicine

Baltimore, MD

Olivia Ho, M.D., M.S.

Department of Plastic and Reconstructive

Surgery

Mayo Clinic

Rochester, MN

Sameer Nath, M.D.

Department of Radiation Oncology

University of Colorado Anschutz Medical

Campus

Aurora, CO
Kilian Salerno, M.D.

Radiation Oncology Branch, Center for

Cancer Research

National Cancer Institute

Bethesda, MD

Jean Wright, M.D.

Department of Radiation Oncology and Molecular Radiation Sciences

Johns Hopkins University School of

Medicine

Baltimore, MD 


\section{Breast Reconstruction After Mastectomy: A Systematic Review and Meta-Analysis}

\section{Structured Abstract}

Objectives. This systematic review evaluates breast reconstruction options for women after mastectomy for breast cancer (or breast cancer prophylaxis). We addressed six Key Questions (KQs): (1) implant-based reconstruction (IBR) versus autologous reconstruction (AR), (2) timing of IBR and AR in relation to chemotherapy and radiation therapy, (3) comparisons of implant materials, (4) comparisons of anatomic planes for IBR, (5) use versus nonuse of human acellular dermal matrices (ADMs) during IBR, and (6) comparisons of AR flap types.

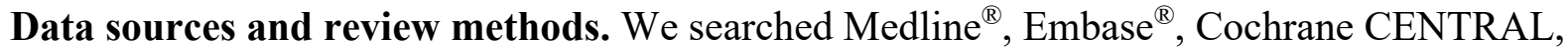
CINAHL $^{\circledR}$, and ClinicalTrials.gov from inception to March 23, 2021, to identify comparative and single group studies. We extracted study data into the Systematic Review Data Repository Plus (SRDR + ). We assessed the risk of bias and evaluated the strength of evidence (SoE) using standard methods. The protocol was registered in PROSPERO (registration number CRD42020193183).

Results. We found 8 randomized controlled trials, 83 nonrandomized comparative studies, and 69 single group studies. Risk of bias was moderate to high for most studies. KQ1: Compared with IBR, AR is probably associated with clinically better patient satisfaction with breasts and sexual well-being but comparable general quality of life and psychosocial well-being (moderate SoE, all outcomes). AR probably poses a greater risk of deep vein thrombosis or pulmonary embolism (moderate SoE), but IBR probably poses a greater risk of reconstructive failure in the long term (1.5 to 4 years) (moderate SoE) and may pose a greater risk of breast seroma (low SoE). KQ 2: Conducting IBR either before or after radiation therapy may result in comparable physical well-being, psychosocial well-being, sexual well-being, and patient satisfaction with breasts (all low SoE), and probably results in comparable risks of implant failure/loss or need for explant surgery (moderate SoE). We found no evidence addressing timing of IBR or AR in relation to chemotherapy or timing of $A R$ in relation to radiation therapy. KQ 3: Silicone and saline implants may result in clinically comparable patient satisfaction with breasts (low SoE). There is insufficient evidence regarding double lumen implants. KQ 4: Whether the implant is placed in the prepectoral or total submuscular plane may not be associated with risk of infections that are not explicitly implant related (low SoE). There is insufficient evidence addressing the comparisons between prepectoral and partial submuscular and between partial and total submuscular planes. KQ 5: The evidence is inconsistent regarding whether human ADM use during IBR impacts physical well-being, psychosocial well-being, or satisfaction with breasts. However, ADM use probably increases the risk of implant failure/loss or need for explant surgery (moderate SoE) and may increase the risk of infections not explicitly implant related (low SoE). Whether or not ADM is used probably is associated with comparable risks of seroma and unplanned repeat surgeries for revision (moderate SoE for both), and possibly necrosis (low SoE). KQ 6: AR with either transverse rectus abdominis (TRAM) or deep inferior epigastric perforator (DIEP) flaps may result in comparable patient satisfaction with breasts (low SoE), but TRAM flaps probably increase the risk of harms to the area of flap harvest (moderate SoE). 
Conclusion. Evidence regarding surgical breast reconstruction options is largely insufficient or of only low or moderate SoE. New high-quality research is needed, especially for timing of IBR and $\mathrm{AR}$ in relation to chemotherapy and radiation therapy, for comparisons of implant materials, and for comparisons of anatomic planes of implant placement. 


\section{Contents}

Evidence Summary ........................................................................................................................... ES-1

Introduction ............................................................................................................................... 1

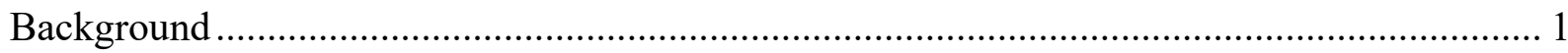

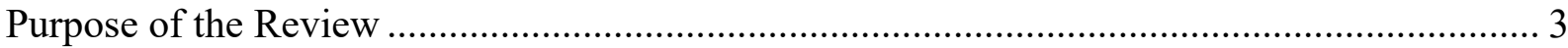

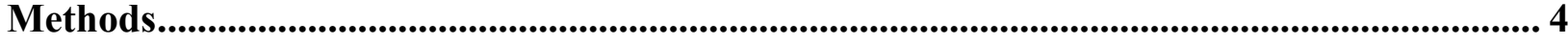

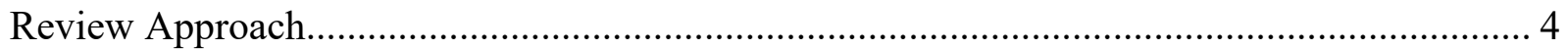

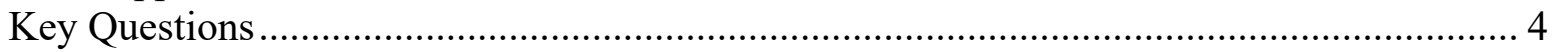

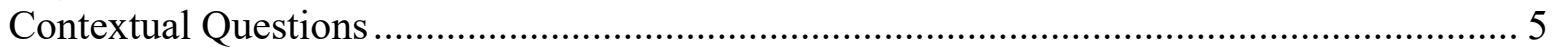

Analytic Framework and Criteria for Inclusion and Exclusion .......................................... 5

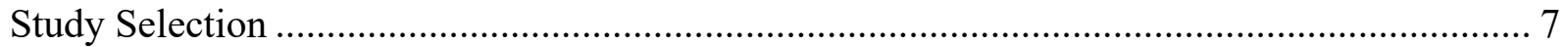

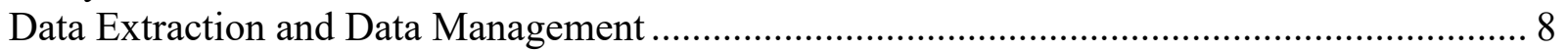

Assessment of Risk of Bias in Individual Studies ............................................................. 9

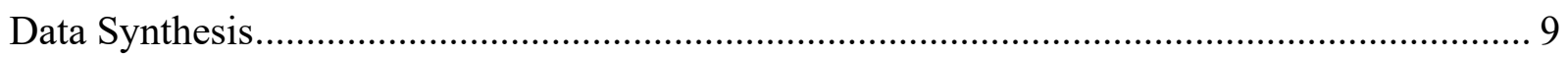

Grading the Strength of Evidence for Major Comparisons and Outcomes ………….............. 10

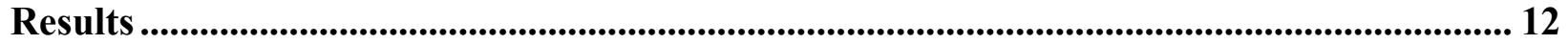

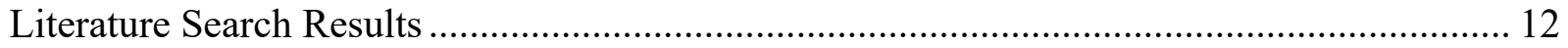

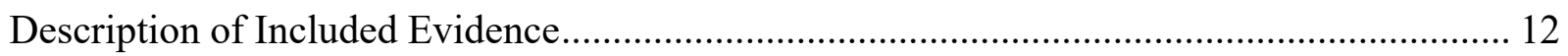

Key Question 1: Implant-Based Reconstruction Versus Autologous Reconstruction ............... 14

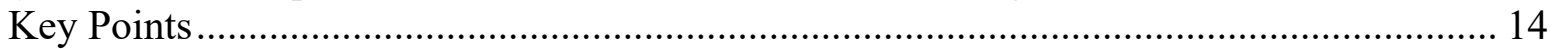

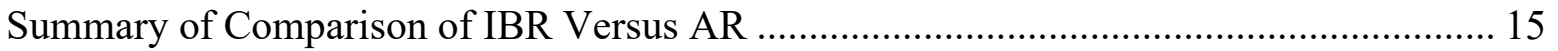

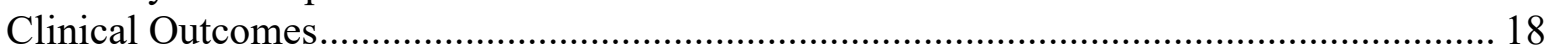

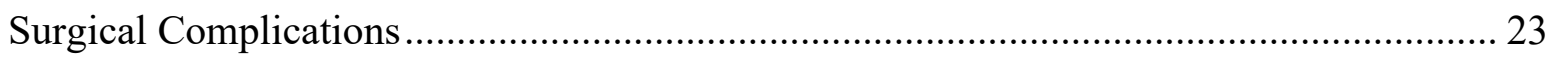

Heterogeneity of Treatment Effects (Subgroup Differences) ……………………………..... 31

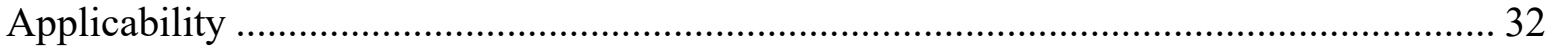

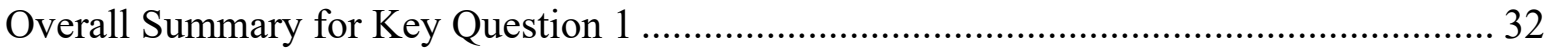

Key Question 2: Timing of Implant-Based Reconstruction or Autologous Reconstruction in

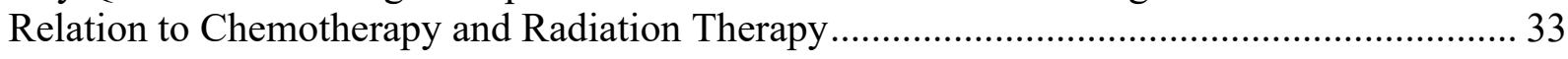

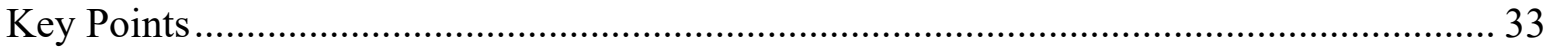

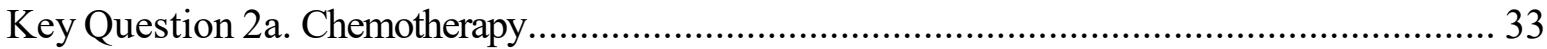

Key Question 2b. Radiation Therapy .................................................................................. 33

Summary of Comparison of Timing of Chemotherapy and Radiation Therapy Relative to

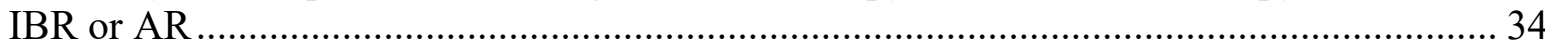

Heterogeneity of Treatment Effects (Subgroup Differences) ................................................. 38

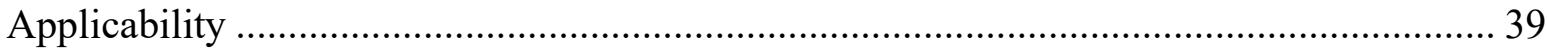

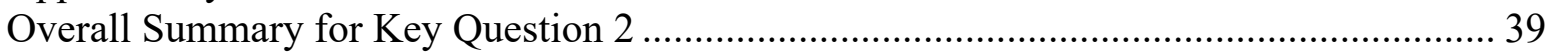

Key Question 3: Comparisons of Implant Materials for Implant-Based Reconstruction.......... 40

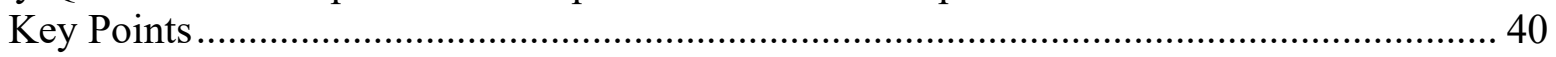

Summary of Comparison of Implant Materials .............................................................. 40

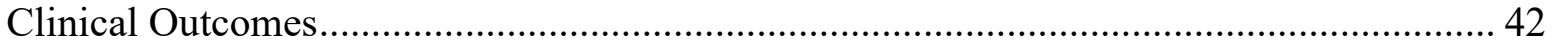

Surgical Complications .................................................................................................. 42

Heterogeneity of Treatment Effects (Subgroup Differences) ................................................ 43

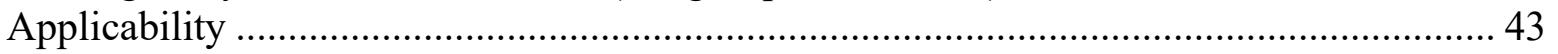

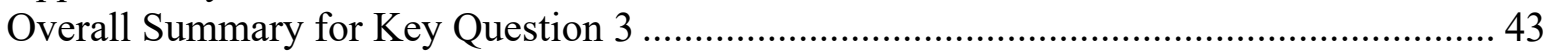

Key Question 4: Comparisons of Anatomic Planes of Implant Placement for Implant-Based

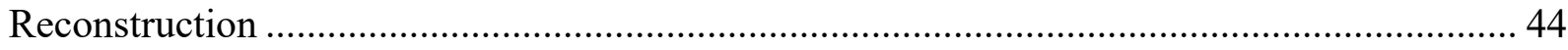




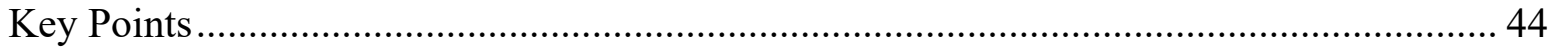

Summary of Comparisons of Anatomic Planes of Implant Placement............................. 44

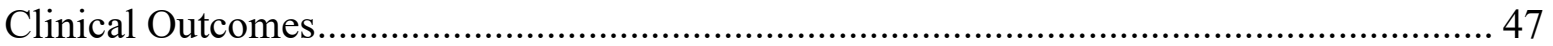

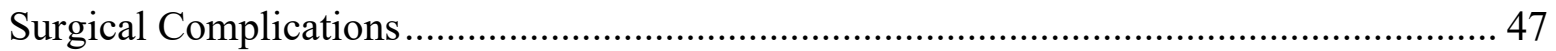

Heterogeneity of Treatment Effects (Subgroup Differences) ....................................... 49

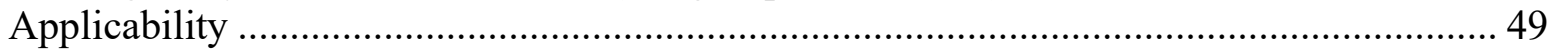

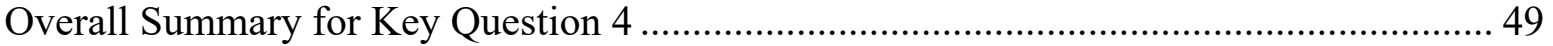

Key Question 5: Use of Human Acellular Dermal Matrices for Implant-Based Reconstruction

50

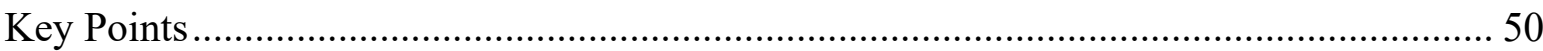

Summary of Comparison of Use Versus Nonuse of Human ADMs ................................ 51

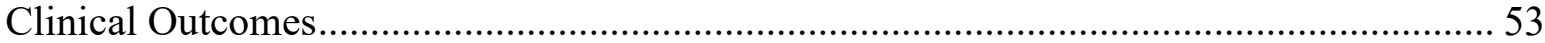

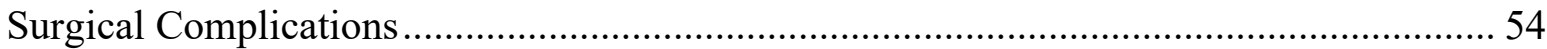

Heterogeneity of Treatment Effects (Subgroup Differences) ......................................... 60

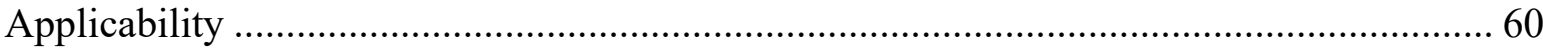

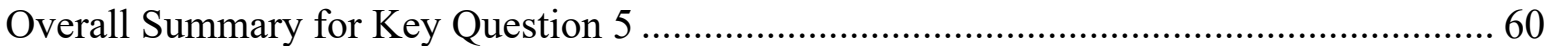

Key Question 6. Comparisons of Flap Types for Autologous Reconstruction....................... 61

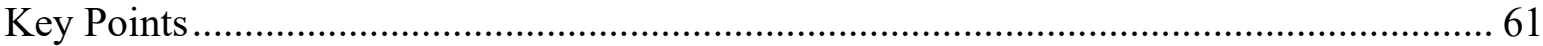

Summary of Comparisons of Flap Types for Autologous Reconstruction....................... 62

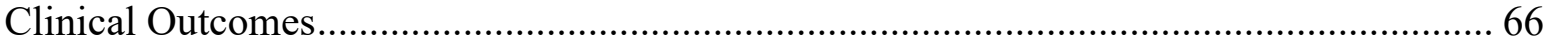

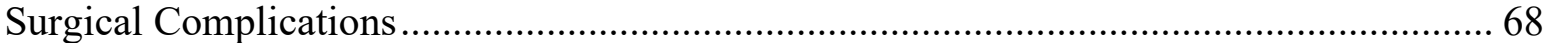

Heterogeneity of Treatment Effects (Subgroup Differences) ........................................... 70

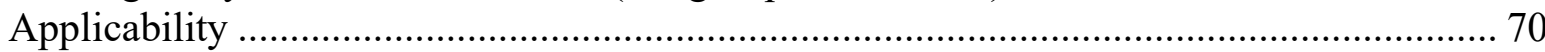

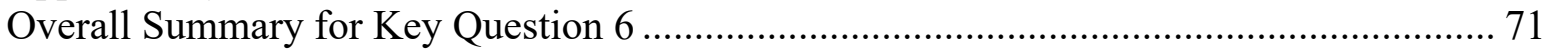

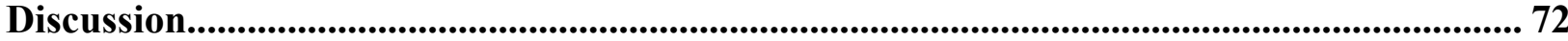

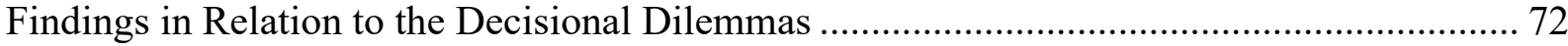

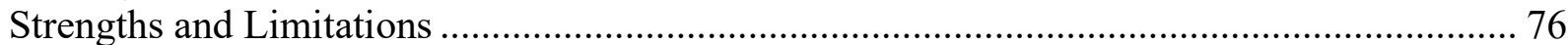

Strengths and Limitations of the Evidence Base .......................................................... 76

Strengths and Limitations of the Systematic Review Process ...................................... 77

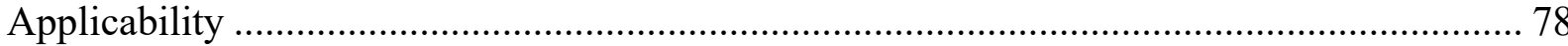

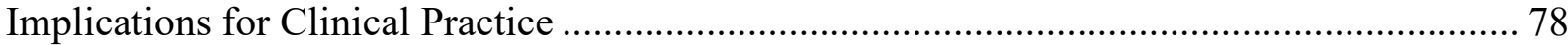

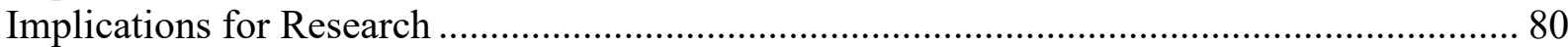

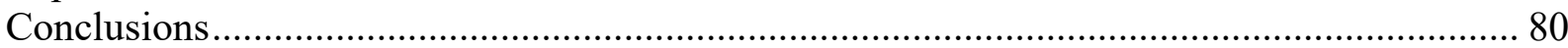

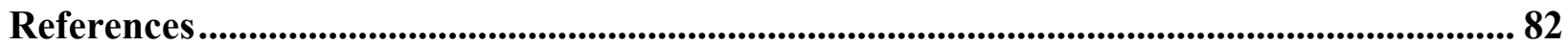

Tables

Table A. Summary of evidence identified in this systematic review (KQ 2 data only refers to IBR

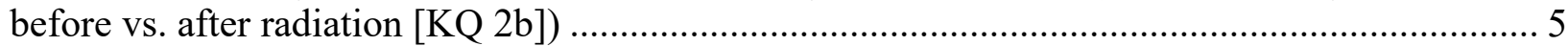

Table 1. Eligibility criteria for each Key Question............................................................. 7

Table 2. Number of studies addressing each Key Question, by study design ........................... 12

Table 3. Evidence profile for Key Question 1: IBR vs. AR ............................................... 16

Table 4. Evidence profile for Key Question 2: Timing of IBR and AR in relation to

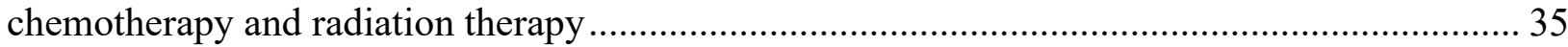

Table 5. Evidence profile for Key Question 3: Comparisons of implant materials for IBR ....... 41 Table 6. Evidence profile for Key Question 4: Comparisons of anatomic planes of implant

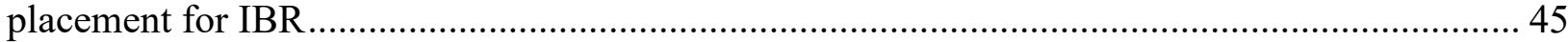

Table 7. Evidence profile for Key Question 5: Use versus nonuse of human ADMs during IBR52 
Table 8. Evidence profile for Key Question 6: Comparisons of flap types for AR.................... 63

Table 9. Summary of evidence identified in this systematic review ......................................... 74

\section{Figures}

Figure 1. Analytic framework for Key Question 1: Implant-based versus autologous breast reconstruction

Figure 2. Analytic framework for Key Questions focusing on timing of reconstruction (Key

Questions 2a and 2b) and use of implants (Key Questions 3, 4, and 5) ..

Figure 3. Analytic framework for Key Question 6: Comparisons of flap types for autologous reconstruction.

Figure 4. Meta-analysis for Key Question 1: IBR versus AR - psychosocial well-being........... 20

Figure 5. Meta-analysis for Key Question 1: IBR versus AR - sexual well-being .................... 21

Figure 6. Meta-analysis for Key Question 1: IBR versus AR - patient satisfaction with breasts 22 Figure 7. Meta-analysis for Key Question 2b: Timing of IBR in relation to radiation therapy implant failure/loss or need for explant surgery ............................................................... 38

Figure 8. Meta-analysis for Key Question 5: Use versus nonuse of human ADMs during IBR -

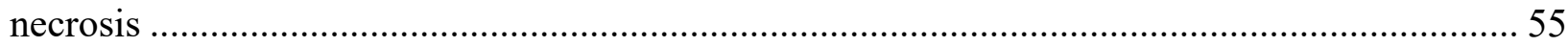

Figure 9. Meta-analysis for Key Question 5: Use versus nonuse of human ADMs during IBR -

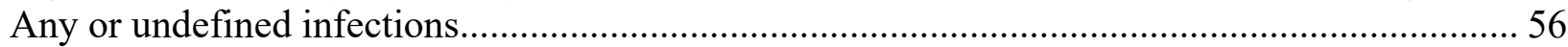
Figure 10. Meta-analysis for Key Question 5: Use versus nonuse of human ADMs during IBR -

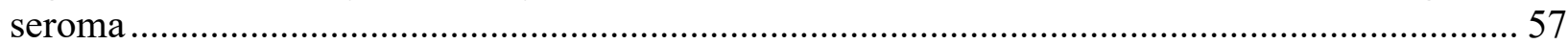
Figure 11. Meta-analysis for Key Question 5: Use versus nonuse of human ADMs during IBR implant failure/loss or need for explant surgery ............................................................ 58 Figure 12. Meta-analysis for Key Question 5: Use versus nonuse of human ADMs during IBR hematoma

\section{Appendixes}

Appendix A. Methods

Appendix B. List of Excluded Studies

Appendix C. Results: Design, Arm, and Sample Details

Appendix D. Results: Risk of Bias

Appendix E. Results: Summary Tables for Outcomes

Appendix F. Results: Full Evidence Tables for Outcomes

Appendix G. Results: Evidence Profiles

Appendix H. Appendix References 


\section{Evidence Summary}

\section{Main Points}

- Implant-Based Reconstruction (IBR) Versus Autologous Reconstruction (AR)

- Compared with IBR, AR is probably associated with clinically better sexual well-being and patient satisfaction with breasts, but comparable general quality of life and psychosocial well-being (Moderate strength of evidence [SoE], all outcomes).

- Compared with IBR, AR probably poses a greater risk of deep vein thrombosis or pulmonary embolism but comparable risk of unplanned repeat hospitalization (both Moderate SoE).

- Compared with AR, although results in the short term (1 to 1.3 months) are inconsistent, IBR probably poses greater risk of reconstructive failure in the long term (1.5 to 4 years) (Moderate SoE). IBR may also pose a greater risk of breast seroma (Low SoE).

- Timing of IBR and AR in Relation to Chemotherapy and Radiation Therapy

- Conducting IBR either before or after radiation therapy may result in comparable physical well-being, psychosocial well-being, sexual well-being, and patient satisfaction with breasts (Low SoE for all).

- Conducting IBR either before or after radiation therapy probably results in comparable risk of implant failure/loss or need for explant surgery (Moderate SoE).

- We found no evidence comparing timing of IBR or AR before or after chemotherapy or timing of AR before or after radiation therapy.

- Comparisons of Implant Materials for IBR

- Silicone or saline implants may result in clinically comparable patient satisfaction with breasts (Low SoE).

- There is insufficient evidence to make conclusions about surgical complications when comparing silicone and saline implants.

O There is insufficient evidence regarding double lumen implants.

- Comparisons of Anatomic Planes of Implant Placement During IBR

$\circ$ Whether the implant is placed in the prepectoral or total submuscular plane may not impact the risk of infections that are not explicitly implant-related (Low SoE).

- There is insufficient evidence for all outcomes comparing prepectoral versus partial submuscular planes and partial versus total submuscular planes.

- Use Versus Nonuse of Acellular Dermal Matrices (ADMs) During IBR

- The evidence is inconsistent regarding whether human ADM use during IBR impacts patient physical well-being, psychosocial well-being, or satisfaction with breasts.

$\circ$ ADM use probably increases the risk of implant failure/loss or need for explant surgery (Moderate SoE) and may increase the risk of infections not explicitly related to the implants or ADM (Low SoE). The risks of seroma or of unplanned repeat surgery for revision probably are comparable with or without ADM use (Moderate SoE); the risk of necrosis may be comparable (Low SoE).

- Comparisons of Flap Types for AR

- AR with either transverse rectus abdominis (TRAM) or deep inferior epigastric perforator (DIEP) flaps may result in comparable patient satisfaction with breasts (Low SoE); however, TRAM flaps probably increase the risk of harms to the area of flap harvest (Moderate SoE).

- There is insufficient evidence regarding other flap types. 


\section{Background and Purpose}

Breast cancer is the most common new cancer diagnosis among women in the United States and the second most common cause of cancer death. For women who choose to undergo breast reconstruction surgery (more than $40 \%$ of women in the United States who undergo mastectomy), various decisions must be made related to the timing and type of reconstruction. Based on the type of procedure and composition of the newly reconstructed breast, reconstruction is categorized into IBR and AR. Implants are prosthetic devices that replace the surgically removed breast tissue. With AR, breast reconstruction is done with the patient's own tissue, thereby obviating the need for implants (except for latissimus dorsi [LD] flaps, which usually require an implant).

This systematic review (SR) aims to inform plastic surgeons, breast surgical oncologists, medical oncologists, radiation oncologists, other care providers, patients, policymakers, and developers of clinical guidance about surgical breast reconstruction options after mastectomy for breast cancer (or breast cancer prophylaxis). The SR addresses six Key Questions (KQs): (1) IBR versus AR, (2) timing of IBR and AR in relation to chemotherapy and radiation therapy, (3) comparisons of implant materials for IBR, (4) comparisons of anatomic planes of implant placement during IBR, (5) use versus nonuse of human ADMs during IBR, and (6) comparisons of flap types for AR.

\section{Methods}

We used methods consistent with those outlined in the Agency for Healthcare Research and Quality Evidence-based Practice Center Program Methods Guidance (https://effectivehealthcare.ahrq.gov/topics/cer-methods-guide/overview). Our searches targeted comparative studies and single group studies (i.e., studies without a comparison group) from database inception to March 23, 2021. We extracted study data into the Systematic Review Data Repository Plus (SRDR+). Our conclusions about comparative effectiveness and harms are based solely on the comparative studies. Single group studies provided additional information about incidence of surgical complications. Where there was sufficient evidence with an acceptable amount of heterogeneity, we conducted pairwise meta-analyses. In the Results section of this Evidence Summary, we provide numeric estimates of summary treatment effects only where meta-analyses were feasible for prioritized outcomes. We assessed the risk of bias and evaluated the SoE using standard methods. The PROSPERO protocol registration number is CRD42020193183. This Evidence Summary incorporates SoE ratings into the Main Points through qualifying language to communicate SoE of conclusions: "probably" for Moderate SoE and "may" for Low SoE.

\section{Results}

We found 160 primary studies comprising 478,650 patients in total. These included 8 randomized controlled trials $(\mathrm{N}=570$ patients), 83 nonrandomized comparative studies (i.e., observational studies that compared 2 or more interventions; $\mathrm{N}=202,862$ ), and 69 single group studies $(\mathrm{N}=275,218)$.

IBR versus AR: Compared with $I B R, A R$ is probably associated with clinically significant better sexual well-being (summary adjusted mean difference [adjMD] 5.8, 95\% confidence interval [CI] 3.4 to $8.2 ; 3$ studies) and satisfaction with breasts (summary adjMD 8.1,95\% CI 6.1 to $10.1 ; 3$ 
studies) but comparable psychosocial well-being (summary adjMD 3.1, 95\% CI 1.3 to 5.0; 3 studies) and general quality of life (Moderate SoE, all outcomes) (Table A). Compared with IBR, AR may be associated with greater risks of deep vein thrombosis or pulmonary embolism (Moderate SoE) but comparable risk of unplanned repeat hospitalizations (Moderate SoE). On the other hand, IBR may be associated with greater risk of seroma (Low SoE). Results were inconsistent regarding whether the choice of IBR versus AR impacts physical well-being, satisfaction with surgical outcome, or risks of reconstructive failure, infections that are not explicitly implant-related, pain, analgesic use, or unplanned surgeries for revision or for complications.

Timing of IBR and AR in relation to chemotherapy and radiation therapy: Whether IBR is conducted before or after radiation therapy may result in comparable physical well-being, psychosocial well-being, sexual well-being, and patient satisfaction with breasts (Low SoE for each). We found that IBR probably results in comparable risk of implant failure/loss or need for explant surgery whether conducted before or after radiation therapy (summary adjusted odds ratio [adjOR] $0.87,95 \%$ CI 0.62 to $1.24 ; 3$ studies) (Moderate SoE). We found no evidence addressing timing of $\mathrm{AR}$ in relation to radiation therapy. We did not find any studies comparing timing of IBR or AR before or after chemotherapy.

Comparisons of implant materials for IBR: Silicone and saline implants may result in clinically comparable assessments of satisfaction with breasts (Low SoE). We found insufficient evidence addressing surgical complications when comparing silicone and saline implants. We found insufficient evidence addressing double lumen implants.

Comparisons of anatomic planes of implant placement for IBR: Prepectoral and total submuscular placements of implants may result in comparable risks of infections that are not explicitly implant-related (Low SoE). We found insufficient evidence for clinical outcomes for this comparison. We found insufficient evidence for all outcomes when comparing prepectoral versus partial submuscular placements and partial versus total submuscular placements.

Use versus nonuse of human ADMs during IBR: ADM use probably increases the risk of implant failure/loss or need for explant surgery (summary adjOR 1.28, 95\% CI 0.97 to 1.70; 6 studies) (Moderate SoE) and may increase the risk of infections not explicitly related to the implants or ADM (summary adjOR $1.56,95 \%$ CI 0.96 to 2.53; 7 studies) (Low SoE). However, ADM use and nonuse groups probably experience comparable risks of seroma (summary adjOR $1.52,95 \%$ CI 0.62 to $3.71 ; 4$ studies) (Moderate $\mathrm{SoE}$ ) and unplanned repeat surgeries for revision (Moderate SoE). ADM use and nonuse groups may experience comparable risks of necrosis (summary adjOR $0.89,95 \%$ CI 0.63 to $1.25 ; 4$ studies) (Low SoE). The results are inconsistent regarding whether ADM use impacts physical well-being, psychosocial well-being, satisfaction with breasts, pain, or risks of wound dehiscence or capsular contracture.

Comparisons of flap types for AR: TRAM versus DIEP flaps: These two flap types may result in clinically comparable patient satisfaction with breasts (Low SoE) and risk of necrosis (Low SoE), but TRAM probably poses greater risk of harms to the area of flap harvest (abdominal bulge/hernia and need for abdominal hernia surgery) (Moderate SoE). Other flaps: We found insufficient evidence addressing LD, lateral thoracodorsal (LTD), superficial inferior epigastric artery (SIEA), and thoracodorsal artery perforator (TAP) flaps. 


\section{Limitations}

Although we found a large body of evidence, it included many single group studies and relatively few studies reported the same outcomes pertaining to similar comparisons. Thus, evidence regarding surgical breast reconstruction options is largely insufficient or of only low or moderate SoE. Nonrandomized comparative studies often did not report adjusted effect sizes or omitted confidence intervals and $\mathrm{P}$ values. When subgroup data were reported, statistical analyses evaluating heterogeneity of treatment effects were not reported. The included studies were mostly at moderate to high risk of bias. Several prioritized outcomes, including general quality of life and risk of animation deformity, were infrequently reported.

\section{Implications and Conclusions}

Our analysis of all surgical choices examined as KQs in this review finds no clear winners when all outcomes are considered. We encourage clinicians to inform patients about the limitations of existing research and to help patients make decisions regarding options for breast reconstruction based on their values and preferences, together with the clinician's expertise and experience. Research is needed to address various questions related to breast reconstruction, particularly the timing of IBR and AR in relation to chemotherapy and radiation therapy, and the choices of implant materials, anatomic planes of implant placement during IBR, and flaps used for AR. Future studies should either randomize patients or adequately account for important confounders and evaluate key outcomes, especially those in the existing core outcome set for breast reconstruction after mastectomy. 
Table A. Summary of evidence identified in this systematic review

\begin{tabular}{|c|c|c|c|c|c|c|c|}
\hline Category & Outcomes & KQ 1 & KQ 2* & $\begin{array}{ll}K Q \\
3\end{array}$ & $\begin{array}{l}\mathrm{KQ} \\
4\end{array}$ & KQ 5 & KQ 6 \\
\hline \multirow{9}{*}{$\begin{array}{l}\text { Clinical } \\
\text { outcomes }\end{array}$} & General quality of life & $\sim \sim$ & nd & $?$ & nd & nd & nd \\
\hline & Physical well-being & $\uparrow \downarrow$ & $\sim$ & $?$ & $?$ & $\uparrow \downarrow$ & $?$ \\
\hline & Psychosocial well-being & $\sim \sim$ & $\sim$ & $?$ & ? & $\uparrow \downarrow$ & $?$ \\
\hline & Sexual well-being & $\Delta \mathbf{\Delta}$ AR clinically better & $\sim$ & $?$ & nd & $?$ & $?$ \\
\hline & Patient satisfaction with breasts & $\Delta \mathbf{\Delta}$ AR clinically better & $\sim$ & $\sim$ & $?$ & $\uparrow \downarrow$ & $\sim$ \\
\hline & Patient satisfaction with outcome & $\uparrow \downarrow$ & $?$ & $?$ & nd & nd & $?$ \\
\hline & Planned surgeries for reconstruction & $\mathrm{N} / \mathrm{P}$ & $\mathrm{N} / \mathrm{P}$ & nd & nd & nd & nd \\
\hline & Duration of initial hospitalization & & & & & & $?$ \\
\hline & Mortality & $?$ & nd & $?$ & nd & $?$ & $?$ \\
\hline \multirow{24}{*}{$\begin{array}{l}\text { Surgical } \\
\text { complications }\end{array}$} & Unplanned repeat hospitalization & $\sim \sim$ & nd & nd & nd & nd & nd \\
\hline & Duration of unplanned repeat hospitalization & nd & nd & nd & nd & nd & nd \\
\hline & Unplanned repeat surgery for revision & $\uparrow \downarrow$ & $?$ & nd & $?$ & $\sim \sim$ & $?$ \\
\hline & Unplanned repeat surgery for complications & $\uparrow \downarrow$ & nd & nd & nd & $?$ & nd \\
\hline & Pain & $\uparrow \downarrow$ & $?$ & nd & $\uparrow \downarrow$ & $\uparrow \downarrow$ & $?$ \\
\hline & Analgesic use & $?$ & nd & nd & $?$ & $?$ & nd \\
\hline & Necrosis & $?$ & $?$ & nd & $?$ & $\sim$ & $\sim$ \\
\hline & Harms to area of flap harvest & $\cdot$ & . & & . & . & $\begin{array}{l}\text { Increased abdominal bulge/hernia, } \\
\text { hernia repair surgery with TRAM than } \\
\text { DIEP }\end{array}$ \\
\hline & Animation deformity & nd & nd & nd & nd & nd & . \\
\hline & Implant-related infections & . & nd & nd & nd & nd & . \\
\hline & Implant rupture & & nd & nd & nd & $?$ & . \\
\hline & Implant deflation & . & nd & nd & nd & nd & . \\
\hline & Implant malposition & . & nd & nd & nd & $?$ &. \\
\hline & Implant failure/loss or needing explant & . & $\sim \sim$ & $?$ & $?$ & $\begin{array}{l}\bullet \text { with } \\
\text { ADM }\end{array}$ & . \\
\hline & Capsular contracture & . & $\mathrm{N} / \mathrm{P}$ & $?$ & $?$ & $\uparrow \downarrow$ &. \\
\hline & New neoplasms & - & 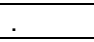 & nd & nd & nd & . \\
\hline & $\begin{array}{l}\text { Complications delaying other cancer } \\
\text { treatments }\end{array}$ & nd & nd & nd & nd & nd & nd \\
\hline & Thromboembolic events & $\diamond \triangleleft$ DVT or PE with AR & nd & nd & nd & $?$ & $?$ \\
\hline & Infections not explicitly implant-related & $\uparrow \downarrow$ & $\mathrm{N} / \mathrm{P}$ &. & $\sim$ & $\diamond$ with ADM & $?$ \\
\hline & Wound dehiscence & $\mathrm{N} / \mathrm{P}$ & $\mathrm{N} / \mathrm{P}$ & $\mathrm{N} / \mathrm{P}$ & $\mathrm{N} / \mathrm{P}$ & $\uparrow \downarrow$ & $?$ \\
\hline & Delayed healing & $\mathrm{N} / \mathrm{P}$ & N/P & $\mathrm{N} / \mathrm{P}$ & $\mathrm{N} / \mathrm{P}$ & $?$ & nd \\
\hline & Seroma & $\checkmark$ with IBR & $?$ & nd & $?$ & $\sim \sim$ & nd \\
\hline & Chronic conditions & $\mathrm{N} / \mathrm{P}$ & $\mathrm{N} / \mathrm{P}$ & nd & nd & $\mathrm{N} / \mathrm{P}$ &. \\
\hline & Reconstructive failure & $\diamond$ with IBR in the long term & . & . & . &. &. \\
\hline
\end{tabular}

$* \mathrm{KQ} 2$ data refer only to IBR before versus after radiation $[\mathrm{KQ} 2 \mathrm{~b}]$

Abbreviations: $\mathrm{ADM}=$ acellular dermal matrix, $\mathrm{AR}=$ autologous reconstruction, $\mathrm{DIEP}=$ deep inferior epigastric perforator, $\mathrm{DVT}=$ deep vein thrombosis, $\mathrm{IBR}=$ implant-based reconstruction, $\mathrm{KQ}=$ Key Question, $\mathrm{LD}=$ latissimus dorsi, $\mathrm{N} / \mathrm{P}=$ not prioritized (for strength of evidence assessment), nd $=$ no data (no evidence identified), $\mathrm{PE}=$ pulmonary embolism, $\mathrm{SoE}=$ strength of evidence, $\mathrm{TRAM}=$ transverse rectus abdominis myocutaneous. $\boldsymbol{\Lambda}=$ Low SoE of better clinical outcomes, $\boldsymbol{\Lambda} \boldsymbol{\Lambda}=$ Moderate SoE of better clinical 
outcomes, $\boldsymbol{\Delta} \boldsymbol{\Delta} \boldsymbol{\Delta}=$ High SoE of better clinical outcomes (no instances in this table)

$\bullet=$ Low SoE of increased complications, $\bullet=$ Moderate SoE of increased complications, $\bullet \bullet=$ High SoE of increased complications (no instances in this table)

$\sim$ = Low SoE of comparable outcomes, $\sim=$ Moderate SoE of comparable outcomes, $\sim \sim=$ High SoE of comparable outcomes (no instances in this table)

$?=$ Insufficient SoE due to sparse evidence, $\uparrow \downarrow=$ Insufficient SoE due to inconsistent or conflicting results, . = not applicable (i.e., outcome not applicable to KQ)

Colors: Insufficient SoE, Low SoE, Moderate SoE, High SoE (no instances). The colors do not add unique information. 


\section{Introduction}

\section{Background}

Breast cancer is the most common new cancer diagnosis among women in the United States and the second most common cause of cancer death. ${ }^{1}$ Approximately 268,600 new breast cancer diagnoses and 41,760 cancer-related deaths in the U.S. were estimated for $2019 .^{2}$ Surgery is a standard component of the treatment strategy for most patients with breast cancer. Surgical options include mastectomy (where the entire breast is removed) and lumpectomy or segmental mastectomy (where a portion of the breast is removed). Radiation is generally recommended following lumpectomy, but less frequently following mastectomy. The indications for post mastectomy radiation therapy (PMRT) include four or more positive axillary lymph nodes, axillary nodal involvement that persists after systemic therapy, and stage T3 breast tumors. ${ }^{3}$ The evidence to support PMRT in patients with one to three positive lymph nodes, younger age, tumor margins less than $1 \mathrm{~mm}$, lymphovascular tumor invasion, and high nuclear grade or negative nodal disease is less clear. Mastectomy is chosen or recommended for approximately 50 percent of women in the U.S. with breast cancer. ${ }^{4}$ Nonsurgical treatments that are used in conjunction with surgery include radiation therapy as well as chemotherapy and endocrine (hormonal) therapy with a range of pharmacologic agents.

Breast reconstruction is commonly offered to women receiving mastectomy for breast cancer. (In this report, we use the term "women" to refer to phenotypic females, regardless of gender.) Women are increasingly choosing to undergo breast reconstruction, although some women are not considered candidates and some choose to avoid reconstruction. ${ }^{5,6}$ As of 2016, more than 40 percent of women in the U.S. who underwent mastectomy for breast cancer had reconstruction. ${ }^{4}$ According to the American Society of Plastic Surgeons/Plastic Surgery Foundation, approximately 107,200 women in the U.S. underwent breast reconstruction in $2019 .{ }^{7}$ Federal regulations require that health insurance policies that cover mastectomy also cover breast reconstruction. ${ }^{8}$ Breast reconstruction is also offered to women who undergo mastectomy for prophylaxis against breast cancer, such as women with high-risk gene mutations such as BRCA1 and BRCA2. ${ }^{9-11}$

For women who choose breast reconstruction surgery, two main considerations must be made: timing and type of reconstruction. Breast reconstruction can be initiated either at the time of mastectomy (immediate reconstruction) or at a later date (delayed reconstruction). Immediate reconstruction is the most common practice in the U.S., selected for approximately 75 percent of patients. ${ }^{7}$ Immediate reconstruction is believed to be associated with better aesthetic results, lower overall costs, and better patient psychological well-being than delayed reconstruction. ${ }^{12}$ This is at least in part related to the fact that both mastectomy and reconstruction are done during the same surgery, thus reducing the number of surgeries and exposures to anesthesia. Although immediate reconstruction has traditionally been thought to be associated with more postoperative complications than delayed reconstruction in the setting of PMRT, a recent systematic review suggested found that complication rates were comparable. ${ }^{13}$ Immediate reconstruction may impact the planning and delivery of radiation therapy in a negative fashion, particularly in regard to chest wall and/or nodal coverage and heart/lung minimization. ${ }^{14,15}$

Based on the type of procedure and composition of the newly reconstructed breast, reconstruction can be categorized into either implant-based reconstruction (IBR) or autologous reconstruction (AR). Most reconstruction procedures in the U.S. (81\%) are implant-based. Implants are prosthetic devices that replace the surgically removed breast tissue or, in the case of 
breast augmentation, are intended to increase the size of the intact breast. IBR can occur in either one or two stages. In planned single-stage implant placement, also known as direct-to-implant placement, IBR is accomplished through a single implantation procedure. In planned two-stage implant placement, a tissue expander is placed as a first procedure, followed by permanent implant placement at a later date. Direct-to-implant placements comprise 16 percent and tissue expander-based reconstructions 84 percent of IBR procedures. ${ }^{7}$ IBR can be further divided based on the physical design of the implant (silicone, saline, or double lumen [e.g., may contain both silicone and saline $\left.{ }^{16}\right]$ ), the anatomic plane in which the device is placed (prepectoral, partial submuscular, or total submuscular), and whether or not an adjunctive human acellular dermal matrix $(\mathrm{ADM})$ is incorporated into the reconstruction. Regardless of these factors, IBR poses potential risks, such as infection, rupture, deflation, and malposition. ${ }^{17}$

Approximately 95 percent of implants for breast reconstruction used in the U.S. are siliconefilled because of the more natural feel and appearance and greater patient satisfaction than with saline implants. ${ }^{71}{ }^{18} 2015$ systematic review concluded that the evidence remained inconclusive about any association between silicone implants and long-term cancer or rheumatologic health outcomes. ${ }^{19}$ However, there have been continued reports of systemic symptoms, such as joint pains, muscle aches, and chronic fatigue. Additionally, breast implant-associated anaplastic large cell lymphoma (BIA-ALCL), a rare type of non-Hodgkin's lymphoma, may occur, especially with textured implants (regardless of fill type). The risk of BIA-ALCL led the U.S. Food and Drug Administration to request a recall of one manufacturer's textured implant and tissue expander in $2019^{20}$ and to recommend a boxed warning for all breast implants in $2020 .^{21}$

The anatomic plane in which the implant is placed during breast reconstruction can have implications on complications, aesthetics, and cost. The most common anatomic plane has traditionally been total submuscular placement, where the implant is placed beneath the pectoralis major muscle. It provides vascularized soft tissue coverage of the implant. However, total submuscular placement has challenges, such as limits to the possible size of the breast reconstruction and incidence of "animation deformity" (i.e., distortion of the reconstructed breast during contraction of the pectoralis major muscle). Animation deformity is experienced by as many as 80 percent of patients who receive total submuscular placement of the implant. ${ }^{22}$ To overcome these challenges, another option is partial submuscular placement with ADM use (which creates a musculofascial pocket to leave the rib cage covered by a portion of the muscle $^{23}$ ) or prepectoral placement with ADM (in front of, or superficial to, the muscle). Prepectoral placement also obviates the need for pectoralis muscle dissection and may cause less pain. ${ }^{24}$ However, the prepectoral technique currently used is relatively novel, and evidence regarding comparative effectiveness, aesthetics, and harms of the various anatomic planes of implant placement is lacking.

ADMs can be derived from human (allografts), animal (xenografts), or synthetic sources. They represent a heterogeneous group of biologic scaffolds that are used in reconstructive surgery to hold the implant in place. ADMs allow for repopulation, revascularization, and integration of the host's cells into the implanted tissue. ${ }^{25}$ Use of ADMs may reduce the incidence of capsular contracture and may improve the aesthetic definition of the inframammary fold (where the lower breast meets the chest wall) and the medial (i.e., midline) border of the breast. However, ADMs may lead to postoperative complications, such as infection and seroma. ${ }^{26-29}$

In AR, breast reconstruction is done with the patient's own tissue, thereby generally obviating the need for implants. In 2018, AR represented approximately 19 percent of breast reconstruction procedures performed in the U.S. ${ }^{7}$ AR is generally described by the anatomic 
region from which the tissue flap is sourced. These include deep inferior epigastric perforator (DIEP; $52 \%$ of ARs), latissimus dorsi (LD; 22\%), transverse rectus abdominis myocutaneous (TRAM; $21 \%$ ), and others (5\%). ${ }^{7}$ DIEP flaps use fat and skin from the patient's abdomen. LD flaps use muscle, fat, and/or skin from the patient's back and are often accompanied by implant placement ("hybrid" reconstruction ${ }^{30}$ ). TRAM flaps include muscle, fat, and skin from the patient's abdomen. The options regarding source of the AR flap are limited by the patient's body habitus, prior surgery, medical comorbidities, and preference. Different flap types vary in their associated types and frequencies of complications. In contrast to IBR, AR can have several advantages, including: (1) AR is intended to be completed in a single, albeit multi-site, surgery (as opposed to most IBRs, which require two-stage implant placements) and (2) AR is intended to be life-long although some patients require small revision surgeries of the breast and/or the donor site (implants, even when single-stage, are recommended to be replaced every 10 years). However, AR requires a larger operation and may have more major complications, such as deep vein thrombosis, abdominal bulge or hernias, wound dehiscence, delayed healing, and scarring. The long-term sequelae of AR, especially patient-reported clinical outcomes, such as satisfaction, psychosocial well-being, and sexual well-being, as well as long-term harms, e.g., harms to the area of flap harvest, remain unclear.

\section{Purpose of the Review}

This systematic review assesses the surgical breast reconstruction treatments for women who are undergoing (or have undergone) mastectomy for breast cancer (or breast cancer prophylaxis). The review does not address the choice of whether patients who have undergone mastectomy should undergo breast reconstruction.

Specifically, the review addresses the (comparative) benefits and harms of:

- IBR versus AR (Key Question [KQ] 1)

- Timing of IBR and AR in relation to chemotherapy and radiation therapy (KQ 2)

- Various options for IBR, including implant materials (KQ 3), implant placement planes (KQ 4), and use of human ADMs (KQ 5)

- Various flap types for AR (KQ 6).

The intended audience for this systematic review includes plastic surgeons, breast surgical oncologists, medical oncologists, radiation oncologists, other care providers for women undergoing mastectomy for breast cancer, guideline developers, healthcare policy makers, and patients. It is expected that the findings will inform clinical guidance for breast reconstruction after mastectomy. 


\section{Methods}

\section{Review Approach}

For all Key Questions (KQs), the systematic review followed Evidence-based Practice Center (EPC) Program methodology, as laid out in its Methods Guide, particularly as it pertains to reviews of comparative effectiveness, diagnostic tests, and complex meta-analyses. ${ }^{31}$ As described below, the Contextual Questions were addressed using a nonsystematic approach. We registered the protocol for this systematic review in PROSPERO (registration number CRD42020193183).

\section{Key Questions}

KQ 1: For adult women who are undergoing (or have undergone) mastectomy for breast cancer, what are the comparative benefits and harms of implant-based (IBR) versus autologous (AR) breast reconstruction?

$K Q$ 2: For adult women undergoing IBR or $A R$ after mastectomy for breast cancer that requires chemotherapy or radiation therapy,

KQ 2a: What is the optimal time for IBR or AR with respect to chemotherapy?

KQ 2b: What is the optimal time for IBR or AR with respect to radiation therapy?

KQ 3: For adult women undergoing IBR after mastectomy for breast cancer, what are the comparative benefits and harms of different types of implants (e.g., silicone, saline)?

KQ 4: For adult women undergoing IBR after mastectomy for breast cancer, what are the comparative benefits and harms of different anatomic planes of implant placement (prepectoral, partial submuscular, and total submuscular)?

KQ 5: For adult women undergoing IBR after mastectomy for breast cancer, what are the comparative benefits and harms of IBR with versus without the use of a human acellular dermal matrix (ADM) in the reconstruction procedure? 
KQ 6: For adult women undergoing $A R$ after mastectomy for breast cancer, what are the comparative benefits and harms of different flap types for AR?

\section{Contextual Questions}

Contextual Question 1: What patient preferences and values inform decision making about breast reconstruction after mastectomy for breast cancer? This includes the initial choice to undergo reconstruction, as well as the type and timing of surgery.

Contextual Question 2: What strategies or tools (including shared decision making) are available to help women make informed choices about breast reconstruction after mastectomy for breast cancer?

\section{Analytic Framework and Criteria for Inclusion and Exclusion}

Based on discussions with Key Informants and Technical Expert Panel members, we developed three analytic frameworks for the six KQs (Figures 1 to 3).

Figure 1. Analytic framework for Key Question 1: Implant-based versus autologous breast reconstruction

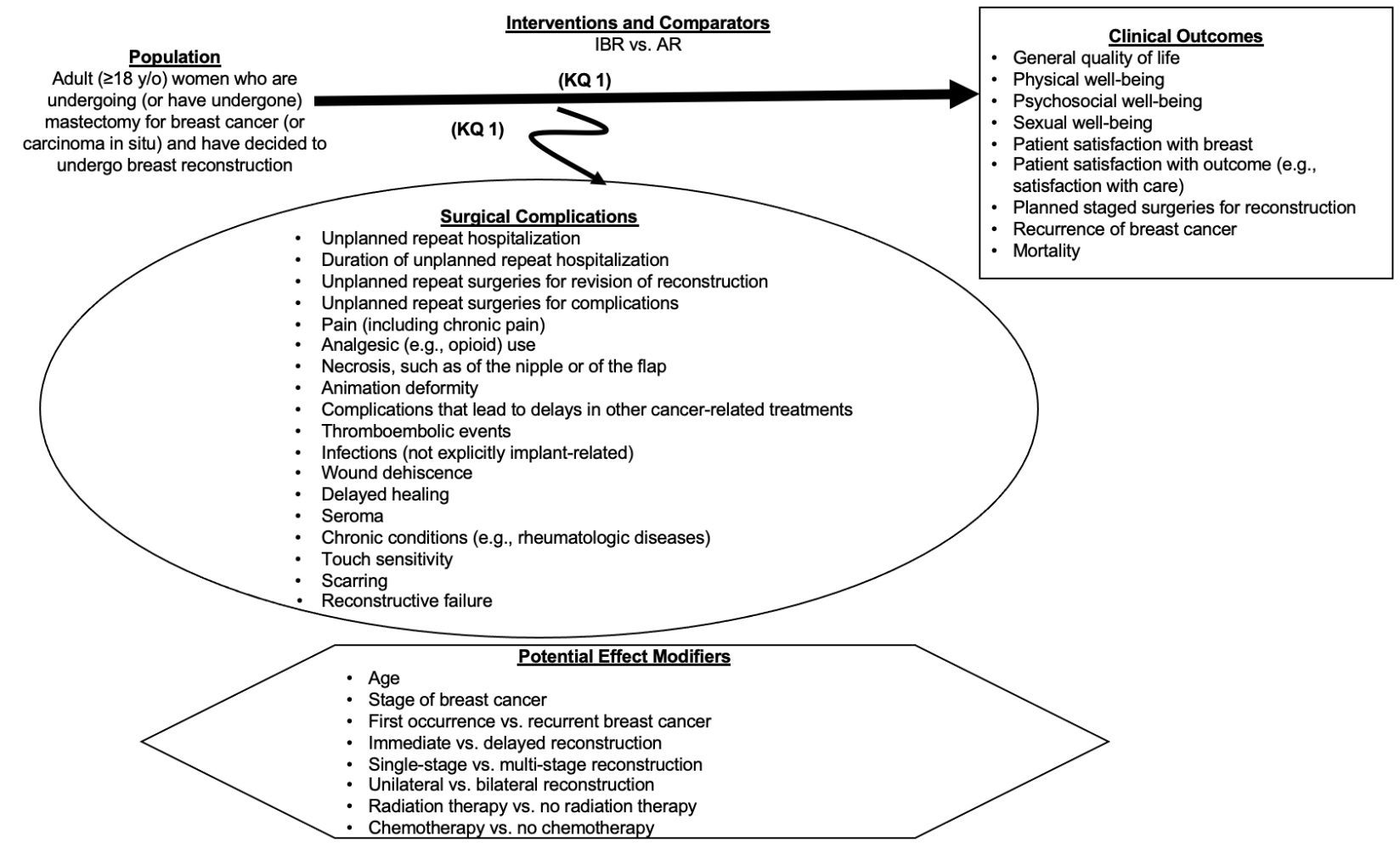

Abbreviations: $\mathrm{AR}=$ autologous reconstruction, $\mathrm{IBR}=$ implant-based reconstruction, $\mathrm{KQ}=$ Key Question. 


\section{Figure 2. Analytic framework for Key Questions focusing on timing of reconstruction (Key Questions 2a and 2b) and use of implants (Key Questions 3, 4, and 5)}

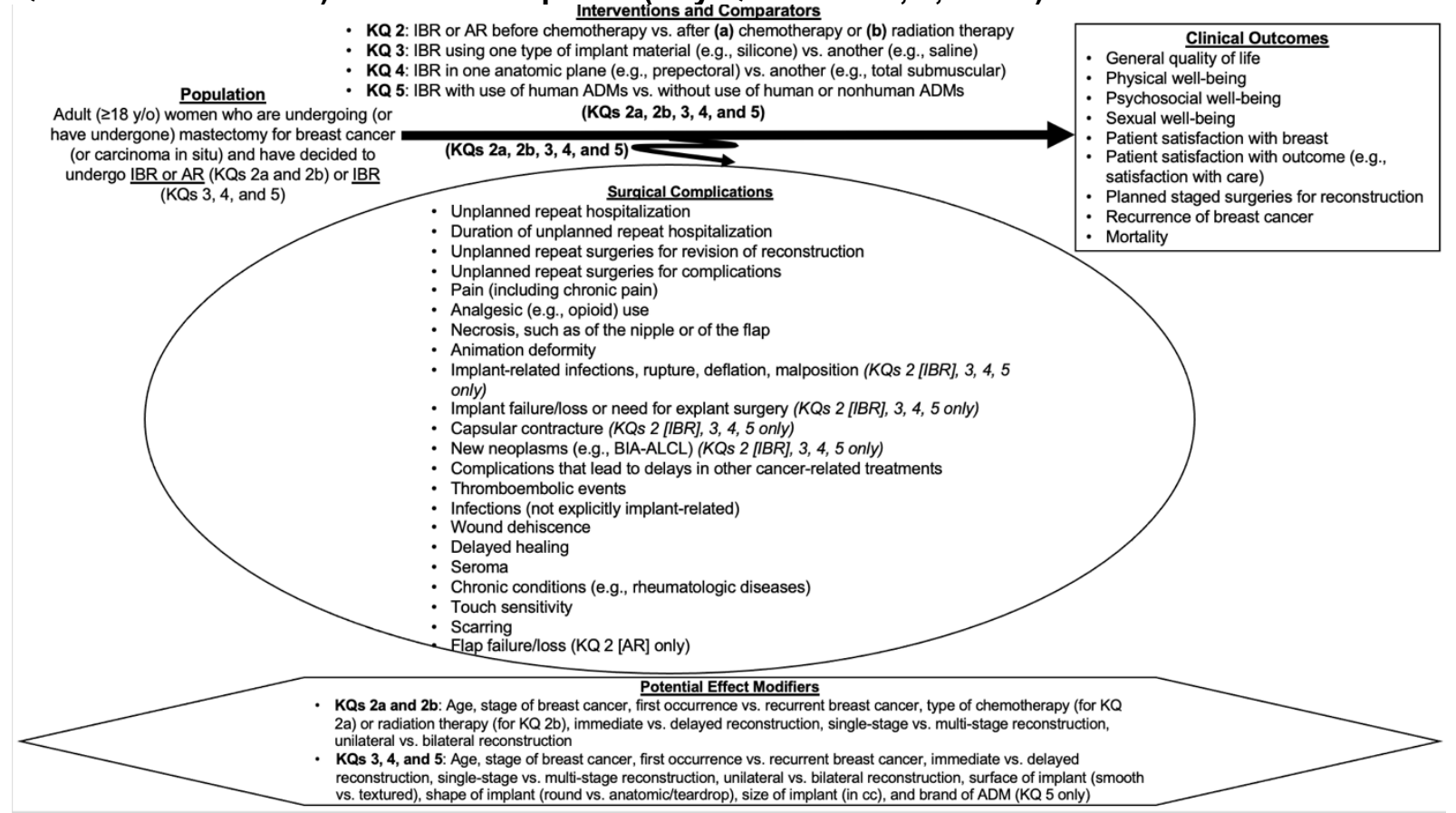

Abbreviations: $\mathrm{ADM}=$ acellular dermal matrix, $\mathrm{AR}=$ autologous reconstruction, $\mathrm{BIA}-\mathrm{ALCL}=$ breast implant-associated anaplastic large cell lymphoma, IBR = implant-based reconstruction, KQ = Key Question.

\section{Figure 3. Analytic framework for Key Question 6: Comparisons of flap types for autologous reconstruction}

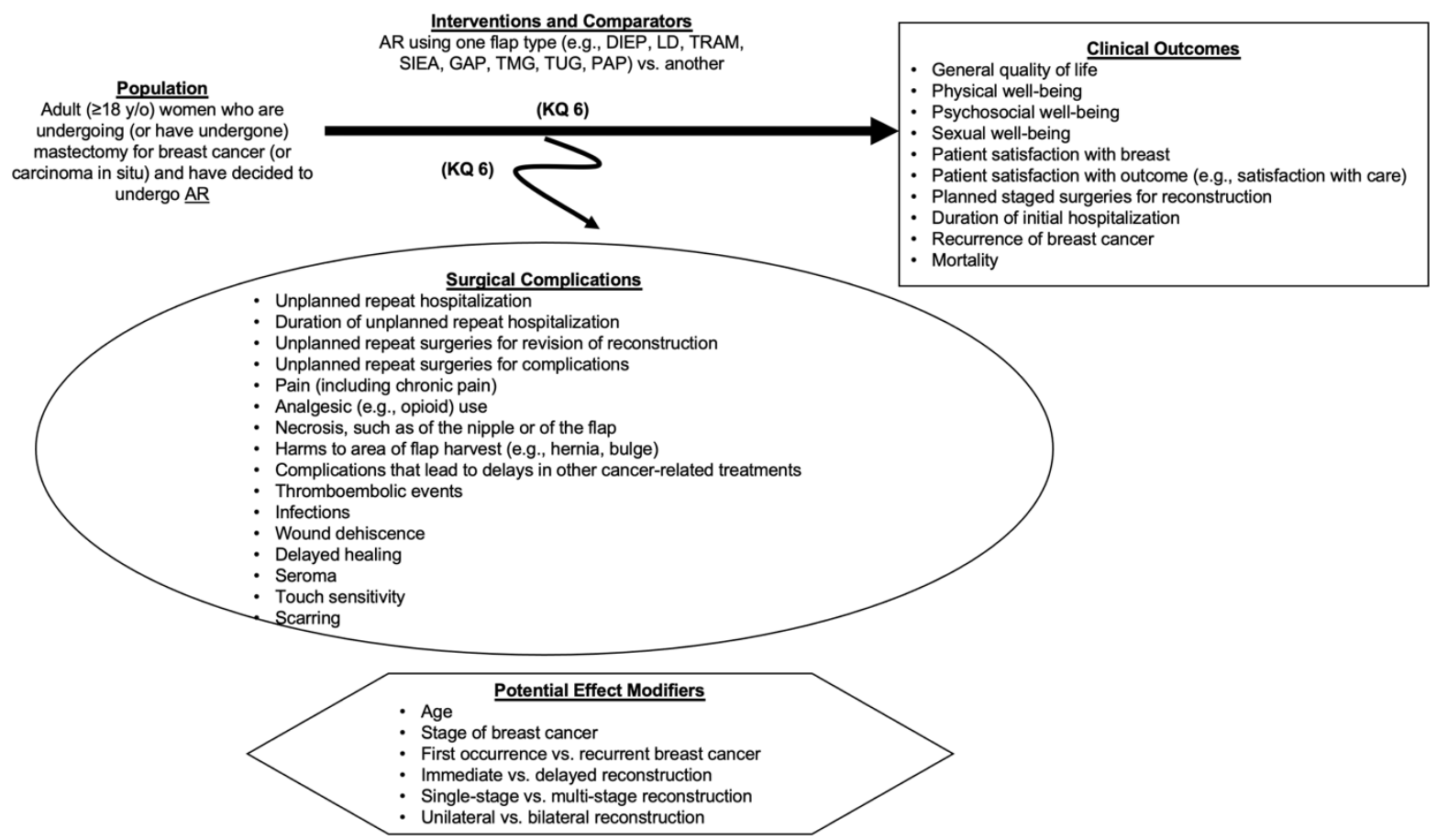

Abbreviations: $\mathrm{AR}=$ autologous reconstruction, $\mathrm{DIEP}=$ deep inferior epigastric perforator, $\mathrm{GAP}=$ gluteal artery perforator, $\mathrm{KQ}=$ Key Question, $\mathrm{LD}=$ latissimus dorsi, $\mathrm{PAP}=$ profundal artery perforator, SIEA $=$ superficial inferior epigastric artery 
perforator, $\mathrm{TMG}=$ transverse musculocutaneous gracilis, $\mathrm{TRAM}=$ transverse rectus abdominis myocutaneous, $\mathrm{TUG}=$ transverse upper gracilis.

\section{Study Selection}

Appendix A provides full details on all search strategies, inclusion and exclusion criteria, and screening processes. Briefly, we searched for published studies for all KQs in Medline ${ }^{\circledR}$ (via PubMed $^{\circledR}$ ), Embase ${ }^{\circledR}$, the Cochrane Central Register of Clinical Trials, and CINAHL ${ }^{\circledR}$, and for unpublished studies in ClinicalTrials.gov from database inception through March 23, 2021. We included controlled vocabulary terms, along with free-text words, related to breast, cancer, mastectomy, implants/implantation, and autologous reconstruction. We did not employ any date or language restrictions to the search but included filters to remove nonhuman studies and articles not describing primary studies.

Table 1 summarizes the eligibility criteria for all KQs (Appendix A provides detailed inclusion and exclusion criteria). For KQ 1 (IBR versus AR) and KQ 2 (IBR or AR before versus the same type of reconstruction after [a] chemotherapy or [b] radiation therapy), the population of interest was all women who had decided to undergo breast reconstruction after mastectomy. For KQs 3, 4, and 5, we were specifically interested in women undergoing IBR: KQ 3 compared different types of implant materials (of any kind), KQ 4 compared different anatomic planes of implant placement (of any kind), and KQ 5 compared use versus nonuse of human ADMs. For KQ 6, we were specifically interested in women undergoing AR; the comparisons of interest were different flap types (of any kind).

For all KQs, we examined various clinical outcomes (such as psychosocial well-being, sexual well-being, and general quality of life) and surgical complications (such as necrosis, seroma, and reconstructive failure) at any followup time-point.

We included randomized controlled trials (RCTs), nonrandomized comparative studies (NRCSs; prospective or retrospective cohort studies comparing two or more treatments), casecontrol studies, and single group studies (prospective or retrospective, without a comparison group).

Table 1. Eligibility criteria for each Key Question

\begin{tabular}{|c|c|c|c|c|c|c|c|}
\hline Element & Eligibility Criteria & $\begin{array}{l}\mathrm{KQ} \\
1\end{array}$ & $\begin{array}{l}\mathrm{KQ} \\
2\end{array}$ & $\begin{array}{l}\mathrm{KQ} \\
3\end{array}$ & $\begin{array}{l}\mathrm{KQ} \\
4\end{array}$ & $\begin{array}{l}\mathrm{KQ} \\
5\end{array}$ & $\begin{array}{l}\mathrm{KQ} \\
6\end{array}$ \\
\hline \multirow[t]{3}{*}{ Population } & $\begin{array}{l}\text { Adult ( } \geq 18 \text { years old) women who are undergoing } \\
\text { (or have undergone) therapeutic or prophylactic } \\
\text { mastectomy for breast cancer (or carcinoma in situ) } \\
\text { and have decided to undergo } \\
\text { Any breast reconstruction }\end{array}$ & $X$ & $X$ & 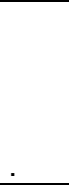 & & & \\
\hline & Implant-based breast reconstruction & $\mathrm{X}$ & $\mathrm{X}$ & $\mathrm{X}$ & $\mathrm{X}$ & $\mathrm{X}$ & \\
\hline & Autologous breast reconstruction & $\mathrm{X}$ & $\mathrm{X}$ & . & 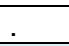 & . & $\mathrm{X}$ \\
\hline \multirow{7}{*}{$\begin{array}{l}\text { Interventions } \\
\text { and } \\
\text { Comparators }\end{array}$} & Implant-based reconstruction (any) & $\mathrm{X}$ & $\mathrm{X}$ & . & . & . & . \\
\hline & $\begin{array}{l}\text { Implant-based reconstruction before or after } \\
\text { chemotherapy }\end{array}$ & . & $\mathrm{X}$ & . & . & . & $\cdot$ \\
\hline & $\begin{array}{l}\text { Implant-based reconstruction before or after } \\
\text { radiation therapy }\end{array}$ & . & $\mathrm{X}$ & . & . & . & . \\
\hline & $\begin{array}{l}\text { Implant-based reconstruction with specific } \\
\text { materials }\end{array}$ & & . & $\mathrm{X}$ & & & \\
\hline & $\begin{array}{l}\text { Implant-based reconstruction with specific } \\
\text { anatomic placements }\end{array}$ & . & . & & $\mathrm{X}$ & & \\
\hline & $\begin{array}{l}\text { Implant-based reconstruction with vs without } \\
\text { human ADMs }\end{array}$ & $\cdot$ & . & . & . & $\mathrm{X}$ & $\cdot$ \\
\hline & Autologous reconstruction (any) & $\mathrm{X}$ & $\mathrm{X}$ & & & & $\mathrm{X}$ \\
\hline
\end{tabular}




\begin{tabular}{|c|c|c|c|c|c|c|c|}
\hline Element & Eligibility Criteria & $\begin{array}{l}\mathrm{KQ} \\
1\end{array}$ & $\begin{array}{l}\mathrm{KQ} \\
2\end{array}$ & $\begin{array}{l}\mathrm{KQ} \\
3\end{array}$ & $\begin{array}{l}\mathrm{KQ} \\
4\end{array}$ & $\begin{array}{l}\mathrm{KQ} \\
5\end{array}$ & $\begin{array}{l}\mathrm{KQ} \\
6\end{array}$ \\
\hline & $\begin{array}{l}\text { Autologous reconstruction before or after } \\
\text { chemotherapy }\end{array}$ & . & $\mathrm{X}$ & . & . & . & . \\
\hline & $\begin{array}{l}\text { Autologous reconstruction before or after radiation } \\
\text { therapy }\end{array}$ & . & $\mathrm{X}$ & . & . & . & . \\
\hline & Autologous reconstruction with specific flap types & $\mathrm{X}$ & $\mathrm{X}$ &. & . &. & $\mathrm{X}$ \\
\hline \multirow{7}{*}{$\begin{array}{l}\text { Outcomes - } \\
\text { Clinical }\end{array}$} & General quality of life & $\mathrm{X}$ & $\mathrm{X}$ & $\mathrm{X}$ & $\mathrm{X}$ & $\mathrm{X}$ & $\mathrm{X}$ \\
\hline & Physical, psychosocial, or sexual well-being & $\mathrm{X}$ & $\mathrm{X}$ & $\mathrm{X}$ & $\mathrm{X}$ & $\mathrm{X}$ & $\mathrm{X}$ \\
\hline & Patient satisfaction with breasts or with outcome & $\mathrm{X}$ & $\mathrm{X}$ & $\mathrm{X}$ & $\mathrm{X}$ & $\mathrm{X}$ & $\mathrm{X}$ \\
\hline & Planned surgeries for reconstruction & $\mathrm{X}$ & $\mathrm{X}$ & $\mathrm{X}$ & $\mathrm{X}$ & $\mathrm{X}$ & $\mathrm{X}$ \\
\hline & Recurrence of breast cancer & $\mathrm{X}$ & $\mathrm{X}$ & $\mathrm{X}$ & $\mathrm{X}$ & $\mathrm{X}$ & $\mathrm{X}$ \\
\hline & Duration of initial hospitalization & . & . & . & . & . & $\mathrm{X}$ \\
\hline & Mortality & $\mathrm{X}$ & $\mathrm{X}$ & $\mathrm{X}$ & $\mathrm{X}$ & $\mathrm{X}$ & $\mathrm{X}$ \\
\hline \multirow{25}{*}{$\begin{array}{l}\text { Outcomes - } \\
\text { Surgical } \\
\text { Complications }\end{array}$} & Unplanned repeat hospitalization & $\mathrm{X}$ & $\mathrm{X}$ & $\mathrm{X}$ & $\mathrm{X}$ & $\mathrm{X}$ & $\mathrm{X}$ \\
\hline & Duration of unplanned repeat hospitalization & $\mathrm{X}$ & $\mathrm{X}$ & $\mathrm{X}$ & $\mathrm{X}$ & $\mathrm{X}$ & $\mathrm{X}$ \\
\hline & $\begin{array}{l}\text { Unplanned repeat surgery for revision of } \\
\text { reconstruction }\end{array}$ & $\mathrm{X}$ & $\mathrm{X}$ & $\mathrm{X}$ & $\mathrm{X}$ & $\mathrm{X}$ & $\mathrm{X}$ \\
\hline & Unplanned repeat surgery for complications & $\mathrm{X}$ & $\mathrm{X}$ & $\mathrm{X}$ & $\mathrm{X}$ & $\mathrm{X}$ & $\mathrm{X}$ \\
\hline & Pain & $\mathrm{X}$ & $\mathrm{X}$ & $\mathrm{X}$ & $\mathrm{X}$ & $\mathrm{X}$ & $\mathrm{X}$ \\
\hline & Analgesic use & $\mathrm{X}$ & $\mathrm{X}$ & $\mathrm{X}$ & $\mathrm{X}$ & $\mathrm{X}$ & $\mathrm{X}$ \\
\hline & Necrosis & $\mathrm{X}$ & $\mathrm{X}$ & $\mathrm{X}$ & $\mathrm{X}$ & $\mathrm{X}$ & $\mathrm{X}$ \\
\hline & Harms to area of flap harvest & & . & 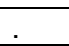 & & & $\mathrm{X}$ \\
\hline & Animation deformity & $\mathrm{X}$ & $\mathrm{X}$ & $\mathrm{X}$ & $\mathrm{X}$ & $\mathrm{X}$ & . \\
\hline & $\begin{array}{l}\text { Implant-related infection, rupture, deflation, or } \\
\text { malposition }\end{array}$ & . & $\mathrm{X}$ & $\mathrm{X}$ & $\mathrm{X}$ & $\mathrm{X}$ & . \\
\hline & Implant failure/loss or need for explant surgery & . & $\mathrm{X}$ & $\mathrm{X}$ & $\mathrm{X}$ & $\mathrm{X}$ & . \\
\hline & Capsular contracture & . & $\mathrm{X}$ & $\mathrm{X}$ & $\mathrm{X}$ & $\mathrm{X}$ & . \\
\hline & New neoplasms & . & . & $\mathrm{X}$ & $\mathrm{X}$ & $\mathrm{X}$ & . \\
\hline & $\begin{array}{l}\text { Complications that lead to delay in cancer-related } \\
\text { treatment }\end{array}$ & $\mathrm{X}$ & $\mathrm{X}$ & $\mathrm{X}$ & $\mathrm{X}$ & $\mathrm{X}$ & $\mathrm{X}$ \\
\hline & Thromboembolic events & $\mathrm{X}$ & $\mathrm{X}$ & $\mathrm{X}$ & $\mathrm{X}$ & $\mathrm{X}$ & $\mathrm{X}$ \\
\hline & Infections & $\mathrm{X}$ & $\mathrm{X}$ & . & $\mathrm{X}$ & $\mathrm{X}$ & $\mathrm{X}$ \\
\hline & Wound dehiscence & $\mathrm{X}$ & $\mathrm{X}$ & $\mathrm{X}$ & $\mathrm{X}$ & $\mathrm{X}$ & $\mathrm{X}$ \\
\hline & Delayed healing & $\mathrm{X}$ & $\mathrm{X}$ & $\mathrm{X}$ & $\mathrm{X}$ & $\mathrm{X}$ & $\mathrm{X}$ \\
\hline & Seroma & $\mathrm{X}$ & $\mathrm{X}$ & $\mathrm{X}$ & $\mathrm{X}$ & $\mathrm{X}$ & $\mathrm{X}$ \\
\hline & Chronic conditions & $\mathrm{X}$ & $\mathrm{X}$ & $\mathrm{X}$ & $\mathrm{X}$ & $\mathrm{X}$ & \\
\hline & Touch sensitivity & $\mathrm{X}$ & $\mathrm{X}$ & $\mathrm{X}$ & $\mathrm{X}$ & $\mathrm{X}$ & $\mathrm{X}$ \\
\hline & Scarring & $\mathrm{X}$ & $\mathrm{X}$ & $\mathrm{X}$ & $\mathrm{X}$ & $\mathrm{X}$ & $\mathrm{X}$ \\
\hline & Red breast syndrome & . & . & $\mathrm{X}$ & $\mathrm{X}$ & $\mathrm{X}$ & 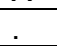 \\
\hline & Flap failure/loss & & $\mathrm{X}$ & . & . & . & $\mathrm{X}$ \\
\hline & Reconstructive failure & $\mathrm{X}$ & . & . & . & . & . \\
\hline \multirow[t]{5}{*}{ Study Designs } & $\begin{array}{l}\text { Randomized controlled trials, } \mathrm{N} \geq 10 \text { patients per } \\
\text { group }\end{array}$ & $\mathrm{X}$ & $\mathrm{X}$ & $\mathrm{X}$ & $\mathrm{X}$ & $\mathrm{X}$ & $\mathrm{X}$ \\
\hline & $\begin{array}{l}\text { Nonrandomized comparative studies, } \mathrm{N} \geq 30 \\
\text { patients per group, provided adjusted analyses }\end{array}$ & $\mathrm{X}$ & $\mathrm{X}$ & $\mathrm{X}$ & $\mathrm{X}$ & $\mathrm{X}$ & $\mathrm{X}$ \\
\hline & Case-control studies, $\mathrm{N} \geq 100$ patients per group & $\mathrm{X}$ & $\mathrm{X}$ & $\mathrm{X}$ & $\mathrm{X}$ & $\mathrm{X}$ & $\mathrm{X}$ \\
\hline & $\begin{array}{l}\text { Single group studies, } \mathrm{N} \geq 500 \text { patients (for } \\
\text { complications only) }\end{array}$ & $\mathrm{X}$ & $\mathrm{X}$ & $\mathrm{X}$ & $\mathrm{X}$ & $\mathrm{X}$ & $\mathrm{X}$ \\
\hline & Prospective or retrospective & $\mathrm{X}$ & $\mathrm{X}$ & $\mathrm{X}$ & $\mathrm{X}$ & $\mathrm{X}$ & $\mathrm{X}$ \\
\hline Timing & Any & $\mathrm{X}$ & $\mathrm{X}$ & $\mathrm{X}$ & $\mathrm{X}$ & $\mathrm{X}$ & $\mathrm{X}$ \\
\hline Setting & Any, including single- and multi-center studies & $\mathrm{X}$ & $\mathrm{X}$ & $\mathrm{X}$ & $\mathrm{X}$ & $\mathrm{X}$ & $\mathrm{X}$ \\
\hline
\end{tabular}

Abbreviations: $\mathrm{ADM}=$ acellular dermal matrix, $\mathrm{KQ}=$ Key Question, $\mathrm{X}=$ relevant to $\mathrm{KQ}$, . = not relevant to KQ.

\section{Data Extraction and Data Management}

We extracted data into the Systematic Review Data Repository Plus (SRDR+) software (https://srdrplus.ahrq.gov). Each eligible study was extracted and assessed for risk of bias/quality by one researcher, and extracted data were confirmed by a second, independent researcher. 


\section{Assessment of Risk of Bias in Individual Studies}

We evaluated each study for risk of bias and methodological quality.

Because we included a variety of study designs, we incorporated items from three different existing commonly used tools and tailored the set of items for each study design. The three tools include the Cochrane Risk of Bias Tool, ${ }^{32}$ the Risk of Bias in Nonrandomized Studies (ROBINSI) Tool, ${ }^{33}$ and the National Heart, Lung, and Blood Institute (NHLBI) Quality Assessment Tool. ${ }^{34}$

For RCTs, we used all the items from the Cochrane Risk of Bias Tool, ${ }^{32}$ which addresses issues related to randomization and allocation concealment methodology; blinding of patients, study personnel/care providers, objective outcome assessors, and subjective outcome assessors; completeness of outcome data; selective outcome reporting; and other issues that could be related to bias. We also used items from the NHLBI Tool focusing on the adequacy of descriptions of study eligibility criteria, interventions, and outcomes. ${ }^{34}$

For NRCSs, we used the specific sections of the ROBINS-I Tool ${ }^{33}$ that pertain to confounding and selection bias. ROBINS-I requires the identification of specific confounders of interest for the systematic review. To assess the presence of potential confounding in studies, we considered age, body mass index, and stage of breast cancer as potential confounders for all KQs. In addition, we considered history of abdominal surgeries as a potential confounder for KQ 6. Because NRCSs, like RCTs, can be impacted by the lack of blinding and by participant loss to followup, we also used the items from the Cochrane Risk of Bias Tool ${ }^{32}$ that focus on issues related to blinding of patients, study personnel or care providers, objective outcome assessors, and subjective outcome assessors; incomplete outcome data; selective outcome reporting; and other issues that could be related to bias. We also used items from the NHLBI Tool that pertain to the adequacy of descriptions of study eligibility criteria, interventions, and outcomes. ${ }^{34}$

No case-control studies were identified.

For single group studies, we used items from the Cochrane Risk of Bias Tool ${ }^{32}$ that pertain to issues of participant loss to followup, specifically, incomplete outcome data, selective outcome reporting, and other issues that could relate to bias. We also used items from the NHLBI Tool focusing on the adequacy of descriptions of eligibility criteria, interventions, and outcomes. ${ }^{34}$

\section{Data Synthesis}

We summarized the evidence both qualitatively and, when feasible and appropriate, quantitatively. Each study included in the systematic review is described in summary and evidence tables presenting study design features, study participant characteristics, descriptions of interventions, outcome results, and risk of bias/methodological quality. Summary tables briefly describe the studies and their findings.

For all KQs, we compared interventions with their comparators for their effects, preferentially with odds ratios for dichotomous outcomes (e.g., recurrence of breast cancer), net mean differences (between-intervention comparison of within-intervention changes) for continuous outcomes with both pre- and post-intervention data (e.g., pain or general quality of life scales), and mean differences (between interventions) in continuous outcome data evaluated only postintervention (e.g., patient satisfaction with breasts). Other effect sizes were included (e.g., hazard ratio) when the preferred effect sizes could not be elicited. For continuous outcomes, we used published estimates of minimal clinically important differences as a guide for interpreting whether differences between groups were clinically significant. Adjusted analyses 
were preferentially included over unadjusted (crude) comparisons. Unadjusted analyses from observational studies (NRCSs) were extracted but are not included in our findings.

Where there were at least three studies reporting results from sufficiently similar analyses, we conducted meta-analyses using random-effects models. In the key points sections of the text and the evidence profile tables for each KQ, we provide numeric estimates of summary treatment effects only where meta-analyses were feasible for prioritized outcomes; these are denoted as "summary" estimates. The data did not allow for network meta-analyses.

\section{Grading the Strength of Evidence for Major Comparisons and Outcomes}

We graded the strength of the body of evidence (SoE) as per the Agency for Healthcare Research and Quality (AHRQ) Methods Guide on assessing SoE. ${ }^{31,35}$ We evaluated SoE for each major comparison or evaluation within each KQ.

We assessed SoE for each outcome that was deemed to be important prior to compiling the evidence. We determined the relative importance of the outcomes with input from the Technical Expert Panel, which included experts in plastic surgery, medical oncology, radiation oncology, breast reconstructive device regulation, and clinical practice guideline development. Examples of prioritized clinical outcomes include:

- General quality of life

- Physical, psychosocial, and sexual well-being

- Patient satisfaction with breasts

- Mortality.

Examples of prioritized surgical complications include:

- Repeat hospitalization

- Duration of repeat hospitalization

- Unplanned repeat surgeries

- Pain.

For specific KQs, we also prioritized certain additional surgical complications, for example:

- Animation deformity

- Implant-related infections (KQ 3, types of implants for IBR)

- Harms to area of flap harvest (KQ 6, flap types for AR).

The prioritized outcomes are consistent with the outcomes in a "core outcome set" published in 2015 for research on breast reconstructive surgery. ${ }^{36}$ Core outcome sets are agreed minimum sets of outcomes that should be reported in research in a given topic area. ${ }^{37}$

For each SoE assessment, we considered the number of studies, their study designs, the study limitations (i.e., risk of bias and overall methodological quality), the directness of the evidence to the KQs, the consistency of study results, the precision of any estimates of effect, the likelihood of reporting bias, other limitations, and the overall findings across studies. Based on these assessments, we assigned a SoE rating as being either high, moderate, low, or insufficient evidence to estimate an effect.

Outcomes with highly imprecise estimates (with a 95\% confidence interval that extends beyond both 0.50 and 2.0 for categorical outcomes), highly inconsistent findings across studies, or with data from only one study were deemed to have insufficient evidence to allow for a conclusion (with the exception that a particularly large and generalizable single study could provide at least low SoE). This approach is consistent with the concept that for imprecise 
evidence "any estimate of effect is very uncertain," the definition of Very Low quality evidence per the Grading of Recommendations, Assessment, Development, and Evaluations (GRADE) approach. ${ }^{38}$

We summarize the data sources, study characteristics, and each SoE dimensional rating in an evidence profile table. This table details our reasoning for arriving at the overall SoE rating.

In accordance with AHRQ guidance for describing treatment effects, ${ }^{39,} 40$ we have incorporated qualifying language regarding $\mathrm{SoE}$ when communicating conclusions (e.g., in Key Points sections of the text) as follows: "probably" for conclusion statements with Moderate SoE and "may" for conclusion statements with Low SoE. 


\section{Results}

\section{Literature Search Results}

The electronic literature search, combined with a hand search of existing systematic reviews, yielded 15,936 unique citations. A total of 160 primary studies met criteria. Appendix B provides a list of excluded studies. Appendix Figure C-1 summarizes the results of the search and screening processes.

The 160 included studies were reported in 202 articles that were published between 1989 and 2021. Across studies, patients were enrolled and followed between 1977 and 2020. The 160 studies comprised eight randomized controlled trials (RCTs), 83 nonrandomized comparative studies (NRCSs), and 69 single group studies. The 160 included studies enrolled a total of 478,650 patients. The 160 studies comprised eight RCTs with 570 patients (ranging from 34 to 150 patients each), 83 NRCSs with 202,862 patients (ranging from 70 to 32,897 patients), and 69 single group studies with 275,218 patients (ranging from 501 to 56,522 patients).

Table 2 summarizes the number of studies that addressed each Key Question (KQ), by study design. Most (69\%) of the studies (111/160) addressed KQ 1 (implant-based reconstruction [IBR] versus autologous reconstruction [AR]); in some of these studies, IBR was further categorized into direct-to-implant IBR- and tissue expander IBR-specific groups, and AR was further categorized into specific flap types. KQ 2 was addressed by five NRCSs, each of which addressed the timing of reconstruction before or after radiation therapy (none before or after chemotherapy). All five NRCSs addressing KQ 3 (comparisons of implant materials for IBR) compared silicone versus saline implants, and one NRCS also included a third group of women who received double lumen silicone implants. The RCT and seven NRCSs addressing KQ 4 compared prepectoral, total submuscular, and partial submuscular planes of implant placement. KQ 5 (human acellular dermal matrix [ADM] use versus nonuse during IBR) was addressed by 22 studies and KQ 6 (comparisons of flap types for AR) was addressed by 20 studies. KQ 6 studies compared six flap types.

Table 2. Number of studies addressing each Key Question, by study design

\begin{tabular}{|l|c|c|c|c|c|c|c|}
\hline Design & KQ 1 & KQ 2 & KQ 3 & KQ 4 & KQ 5 & KQ 6 & Total \\
\hline Randomized controlled trials & 2 & 0 & 0 & 1 & 2 & 3 & $\mathbf{8}$ \\
\hline Nonrandomized comparative studies & 40 & 5 & 5 & 7 & 20 & 16 & $\mathbf{8 3}^{*}$ \\
\hline Single group studies & 69 & 0 & 0 & 0 & 0 & 0 & $\mathbf{6 9}$ \\
\hline Total & $\mathbf{1 1 1}$ & $\mathbf{5}$ & $\mathbf{5}$ & $\mathbf{8}$ & $\mathbf{2 2}$ & $\mathbf{1 9}$ & $\mathbf{1 6 0}$ \\
\hline
\end{tabular}

* Some nonrandomized comparative studies addressed multiple Key Questions (KQs).

For all 160 included studies, Appendix Tables C-1 to C-7 summarize the design, arm, and patient characteristics (separate subtables for each KQ, with two tables for KQ 1: one for comparative studies [RCTs and NRCSs] and another for single group studies) and Appendix Tables D-1 to D-4 summarize the risk of bias assessments (separate subtables by study design, with two tables for NRCSs: one for selection bias and confounding and the other for other types of bias). Further details about the literature search, included studies, and excluded studies (with reasons for their exclusion) are in Appendixes A and B.

\section{Description of Included Evidence}

Detailed findings are in the appendixes. These include tables describing study designs, groups, and sample characteristics (Appendix C); risk of bias (Appendix D); all outcomes 
(Appendixes E and F); and evidence profiles (Appendix G). Appendix H includes the references cited in the rest of the appendixes. Where relevant, we call attention to specific appendix table numbers in the relevant subsections of this main report. 


\section{Key Question 1: Implant-Based Reconstruction Versus Autologous Reconstruction}

\section{Key Points}

- Compared with patients who undergo IBR, those who undergo AR probably experience clinically significant better sexual well-being (summary adjusted mean difference [adjMD] 5.8, 95\% confidence interval [CI] 3.4 to 8.2; 3 studies) and satisfaction with breasts (summary adjMD 8.1, 95\% CI 6.1 to 10.1; 3 studies) (Moderate strength of evidence [Moderate SoE], both outcomes). However, IBR and AR are probably associated with clinically comparable psychosocial well-being (summary adjMD 3.1, 95\% CI 1.3 to 5.0; 3 studies) and general quality of life (Moderate SoE, both outcomes).

- Because of inconsistent results, evidence is insufficient regarding whether the choice of IBR versus AR impacts physical well-being, satisfaction with nipples, satisfaction with surgical outcome, or risks of unplanned repeat surgeries for revision, unplanned repeat surgeries for complications, pain, analgesic use, or infections that are not explicitly implant-related.

- Compared with patients who undergo IBR, those who undergo AR probably are at a greater risk of deep vein thrombosis or pulmonary embolism (Moderate SoE).

- Compared with patients who undergo AR, although results were inconsistent in the shortterm (1 to 1.3 months), those who undergo IBR probably are at greater risk of reconstructive failure in the long-term (1.5 to 4 years) (Moderate SoE). Those who under IBR may also be at greater risk of breast seroma (Low SoE).

- IBR and AR are probably associated with comparable risks of unplanned repeat hospitalizations (Moderate SoE).

- Because of sparse data, there is insufficient evidence to compare IBR and AR in terms of risks of mortality or necrosis.

We found 109 eligible studies. We found two RCTs and 38 NRCSs (with adjusted analyses) for the comparison between IBR and AR. An additional 69 single group studies of either IBR or AR (with 500 or more patients) provided data on surgical complications. An additional two NRCSs did not report adjusted effect sizes or P values. ${ }^{41,42}$ The IBR and AR groups in these two NRCSs did not meet the sample size threshold of 500 patients, so we could not consider them as single group studies. They are thus not discussed further in this section. Appendix Tables C-1, D2, D-3, and F-1.1 to F-1.26 include full data for all RCTs and NRCSs, irrespective of whether they reported adjusted effect sizes. Details of the 69 single group studies are in Appendix Tables C-2, D-4, and F-1.13 to F-1.26.

\section{Randomized Controlled Trials}

The two RCTs ${ }^{43,44}$ compared IBR and AR in a total of 223 patients in Sweden. We rated one $\mathrm{RCT}$ to be at overall high risk of bias and the other at overall moderate risk of bias. Average ages of patients were similar in the RCTs, with mean ages ranging from 52 to 56 years. Average body mass indices (BMIs) ranged from 25 to $26 \mathrm{~kg} / \mathrm{m}^{2}$. Neither RCT reported the racial distribution of patients.

The RCT result summaries are in Appendix Tables E-1.1 to E-1.7 and Appendix Table F1.26 . 


\section{Nonrandomized Comparative Studies}

The 38 adjusted NRCSs, reported in 53 articles, ${ }^{45-97}$ compared IBR and AR in a total of 121,302 patients. Among the 38 NRCSs, 10 (26\%) were prospective and 28 (74\%) were retrospective. We rated 25 of the $38 \mathrm{NRCSs}$ to be at overall high risk of bias, mostly related to serious risk of confounding and the lack of blinding of participants, study personnel, and/or outcome assessors. We rated the remaining 13 NRCSs to be at overall moderate risk of bias.

The 38 NRCSs enrolled between 70 and 32,897 women each. More than half $(n=22 ; 58 \%)$ were conducted in North America (19 in the U.S., 2 in Canada, and 1 in both). The remaining NRCSs were conducted in China $(n=5)$, the Netherlands $(n=3)$, South Korea $(n=3)$, and Finland, France, Italy, Japan, and Portugal (1 each). Average ages of patients were similar across NRCSs, ranging from 43 to 53 years. Average BMIs ranged from 22 to $35 \mathrm{~kg} / \mathrm{m}^{2}$. In the only eight NRCSs that reported patient races, between 63 and 89 percent were White and between 4 and 7 percent were Black.

The NRCS result summaries are in Appendix Tables E-1.1 to E-1.7 and Appendix Table F1.26 .

\section{Single Group Studies}

The 69 single group studies, reported in 86 articles, ${ }^{98-183}$ evaluated IBR $(n=30)$ or AR $(n=39)$ in a total of 275,245 patients. We rated one single group study to be at overall high risk of bias, 14 at moderate risk of bias, and 54 at low risk of bias. Note that because these studies only involved a single group, issues of confounding are not relevant. High and moderate risks of bias were mostly related to eligibility criteria not being clearly described, interventions not being clearly described or consistently delivered, and/or selective outcome reporting.

The 69 single group studies included between 501 and 56,522 women each. Most ( $\mathrm{n}=44$; 64\%) were conducted in North America (43 in the U.S. and 1 in Canada). Other studies were conducted in South Korea $(n=5)$, the U.K. $(n=4)$, Germany $(n=4)$, Belgium $(n=3)$, Sweden $(n=2)$, and in seven other countries $(\mathrm{n}=1 \mathrm{each})$. Average ages of patients were similar across studies, ranging from 46 to 54 years. Average BMIs ranged from 22 to $29 \mathrm{~kg} / \mathrm{m}^{2}$. Only 17 of the 69 studies reported patient races: between 42 and 98 percent were White and between 2 and 15 percent were Black.

The single group study results are in Appendix Tables F-1.13 to F-1.26.

\section{Summary of Comparison of IBR Versus AR}

Table 3 summarizes the evidence for the comparison of IBR versus AR. There is low to moderate SoE for all conclusions. (We did not make any conclusions based on insufficient evidence.) AR is probably associated with clinically better experiences for some patient-reported clinical outcomes (e.g., sexual well-being, satisfaction with breasts) but not others (e.g., general quality of life). In terms of surgical complications, patients who undergo AR may be at greater risks of deep vein thrombosis or pulmonary embolism. However, although results are inconsistent in the short-term ( 1 to 1.3 months), patients who undergo IBR probably are at greater risk of reconstructive failure in the long-term (1.5 to 4 years). Additionally, patients who undergo IBR may be at greater risk of breast seroma. Risks of some other surgical complications (e.g., unplanned repeat hospitalizations) may be comparable between IBR and AR. For others, the evidence is sparse (e.g., necrosis) and/or the results are inconsistent (e.g., infections, pain). 
Table 3. Evidence profile for Key Question 1: IBR versus AR

\begin{tabular}{|c|c|c|c|c|c|c|c|c|c|}
\hline $\begin{array}{l}\text { Outcome } \\
\text { Category }\end{array}$ & Outcome & $\begin{array}{l}\text { N Studies } \\
\text { (Patients) }\end{array}$ & RoB & Consistency & Precision & Directness & Other & SoE & Conclusions (Reason, if None) \\
\hline \multirow{7}{*}{$\begin{array}{l}\text { Clinical } \\
\text { outcomes }\end{array}$} & General quality of life & $3(709)$ & Moderate & Consistent & Precise & Direct & None & Moderate & Comparable in both groups \\
\hline & Physical well-being & $6(5717)$ & Moderate & Inconsistent & Precise & Direct & None & Insufficient & None (Inconsistent results) \\
\hline & Psychosocial well-being & $5(2760)$ & Moderate & Consistent & Precise & Direct & None & Moderate & $\begin{array}{l}\text { Clinically comparable in both } \\
\text { groups: summary adjMD } 3.14 \\
(95 \% \text { Cl } 1.26,5.02) ; 3 \text { studies }\end{array}$ \\
\hline & Sexual well-being & $4(3307)$ & Moderate & Consistent & Precise & Direct & None & Moderate & $\begin{array}{l}\text { Clinically significant better with AR: } \\
\text { summary adjMD } 5.83(95 \% \mathrm{Cl} \\
3.44,8.23) ; 3 \text { studies }\end{array}$ \\
\hline & $\begin{array}{l}\text { Patient satisfaction with } \\
\text { breasts }\end{array}$ & $7(4557)$ & Moderate & Consistent & Precise & Direct & None & Moderate & $\begin{array}{l}\text { Clinically significant better } \\
\text { satisfaction with breast with AR: } \\
\text { summary adjMD } 8.08(95 \% \mathrm{Cl} \\
6.11,10.1) ; 3 \text { studies. }\end{array}$ \\
\hline & $\begin{array}{l}\text { Patient satisfaction with } \\
\text { surgical outcome }\end{array}$ & $5(1432)$ & Moderate & Inconsistent & Precise & Direct & None & Insufficient & None (Inconsistent results) \\
\hline & Mortality & $1(4061)$ & High & $\mathrm{N} / \mathrm{A}$ & Precise & Direct & Sparse & Insufficient & None (Sparse evidence) \\
\hline \multirow[t]{10}{*}{$\begin{array}{l}\text { Surgical } \\
\text { complications }\end{array}$} & $\begin{array}{l}\text { Unplanned repeat } \\
\text { hospitalization }\end{array}$ & $3(50675)$ & High & Consistent & Precise & Direct & None & Moderate & Comparable in both groups \\
\hline & $\begin{array}{l}\text { Unplanned repeat } \\
\text { surgeries for revision }\end{array}$ & $3(3138)$ & High & Inconsistent & Precise & Direct & None & Insufficient & None (Inconsistent results) \\
\hline & $\begin{array}{l}\text { Unplanned repeat } \\
\text { surgeries for } \\
\text { complications }\end{array}$ & $3(14313)$ & High & Inconsistent & Precise & Direct & None & Insufficient & None (Inconsistent results) \\
\hline & Pain & $5(3173)$ & Moderate & Inconsistent & Precise & Direct & None & Low & None (Inconsistent results) \\
\hline & Analgesic use & $1(90)$ & High & $\mathrm{N} / \mathrm{A}$ & Imprecise & Direct & Sparse & Insufficient & None (Sparse evidence) \\
\hline & Necrosis & $4(2176)$ & High & Inconsistent & Imprecise & Direct & None & Insufficient & None (Inconsistent results) \\
\hline & Thromboembolic events & $4(34742)$ & High & Consistent & Precise & Direct & None & Moderate & $\begin{array}{l}\text { Increased risk of deep vein } \\
\text { thrombosis or pulmonary } \\
\text { embolism with AR }\end{array}$ \\
\hline & Infections & $4(17246)$ & Moderate & Inconsistent & Imprecise & Direct & None & Insufficient & None (Inconsistent results) \\
\hline & Seroma & $2(1300)$ & Moderate & Consistent & Unclear & Direct & None & Low & $\begin{array}{l}\text { Increased risk of breast seroma } \\
\text { with IBR }\end{array}$ \\
\hline & Reconstructive Failure & $5(21090)$ & Moderate & Consistent & Precise & Direct & None & Moderate & $\begin{array}{l}\text { Increased risk with IBR in the long- } \\
\text { term ( } 1.5 \text { to } 4 \text { years of followup) }\end{array}$ \\
\hline
\end{tabular}

Abbreviations: adj = adjusted, $\mathrm{AR}=$ autologous reconstruction, $\mathrm{CI}=$ confidence interval, $\mathrm{DIEP}=$ deep inferior epigastric perforator, IBR $=$ implant-based reconstruction, $\mathrm{MD}=$

mean difference, $\mathrm{N} / \mathrm{A}=$ not applicable, $\mathrm{NR}=$ not reported, RoB $=$ risk of bias, SIEA = superficial inferior epigastric artery, SoE $=$ strength of evidence, TRAM = transverse rectus

abdominis myocutaneous.

For continuous outcomes, clinical significance is based on published estimates of minimal clinically important differences (MCIDs), where available.

Colors: Header rows are shaded orange. The color does not add unique information. 
Appendix Table G-1 provides the complete version of this evidence profile, including displaying all outcomes for which no evidence was identified. 


\section{Clinical Outcomes}

One RCT and 13 NRCSs reported on clinical outcomes comparing IBR and AR (Appendix Tables E-1.1 to E-1.5). Note that we did not evaluate single group studies for clinical outcomes.

\section{General Quality of Life}

Three NRCSs (Kouwenberg 2019, Kouwenberg 2020, and Roth 2007) reported on general quality of life (Appendix Table E-1.1). Kouwenberg 2019 and Kouwenberg 2020 used the EuroQoL Group 5-dimension 5-level scale (EQ-5D-5L). The EQ-5D-5L includes two components: (1) a visual analog scale (VAS) that ranges from 0 to 100 , with higher scores indicating better quality of life and (2) a utilities score that ranges from 0 to 1 , with higher scores indicating better preference-weighted quality of life. The minimum clinically important differences (MCIDs) have been estimated to be 7 points for the VAS component and 0.06 points for the utilities component. ${ }^{184}$ Kouwenberg 2019 reported that patients who underwent IBR and AR had comparable utilities scores $(\mathrm{P}=0.7$; adjusted effect size not reported). Kouwenberg 2020 also reported that patients who underwent IBR and AR had comparable utilities scores as well as VAS scores ( $\mathrm{P}=\mathrm{NS}$; adjusted effect size not reported).

Kouwenberg 2020 also used the global health status component of the European Organization for Research and Treatment of Cancer Quality of Life Questionnaire C30 (EORTC QLQ-C30), which was designed to measure changes (or differences) in health-related quality of life in patients with cancer. The score ranges from 0 to 100 , with higher scores indicating better quality of life. The MCID has been estimated to be 4 to 10 points. ${ }^{185}$ Patients who underwent IBR and $\mathrm{AR}$ had comparable scores ( $\mathrm{P}=$ not significant $[\mathrm{NS}]$; adjusted effect size not reported).

Roth 2007 reported on quality of life using five measurements: the functional well-being component of the Functional Assessment of Cancer Therapy (FACT-B); the role emotional, vitality, and general mental health components of the Short Form-36 (SF-36); and a score evaluating body image ( 9 to 45 ; higher is better). The functional well-being component of the FACT-B ranges from 0 to 28 (higher is better; MCID 2 points ${ }^{186}$ ). Each of the SF-36 component scores ranges from 0 to 100 (higher is better; MCID not available for component scores). Although adjusted effect sizes were not reported, Roth 2007 reported that, at 2 years of followup, scores for each of the five measurements were comparable between patients who underwent IBR and $\mathrm{AR}(\mathrm{P} \geq 0.05)$.

\section{Physical Well-Being}

One RCT (Tallroth 2020) and five NRCSs (Eltahir 2015, Kouwenberg 2020, Kulkarni 2017, McCarthy 2014, and Nelson 2019) reported on physical well-being (Appendix Tables E-1.2 and E-1.3).

The RCT (Tallroth 2020) and four NRCSs (Eltahir 2015, Kouwenberg 2020, Kulkarni 2017, and McCarthy 2014) reported physical well-being as continuous data using seven different measurement instruments. Data were inconsistent across studies. All five studies used the BREAST-Q, an instrument designed to evaluate changes (or differences) in patient-reported outcomes in patients who have undergone breast surgery. Scores range from 0 to 100, with higher scores indicating better well-being. The MCID for physical well-being has been estimated to be 3 points. ${ }^{187}$

Tallroth 2020 (the RCT) reported that patients randomized to AR had clinically significant higher BREAST-Q physical well-being: chest and upper body scores at 5.3 years of followup 
(mean difference [MD] 7.6, 95\% CI 0.30 to 14.9). Among the NRCSs, Eltahir 2015 reported comparable BREAST-Q physical well-being: overall scores between IBR and AR groups, at 2.2 years of followup (adjMD -2.60, 95\% CI -9.77 to 4.57). However, McCarthy 2014 reported that, at 1 to 5 years of followup, patients who underwent AR had higher physical well-being scores $(\mathrm{P}<0.05$; adjusted effect size not reported). Similarly, Kouwenberg 2020 reported that patients who underwent AR had higher chest scores beyond 6 months of followup $(\mathrm{P}<0.05$; adjusted effect size not reported). Kulkarni 2017 reported data only for the chest- and upper body-specific BREAST-Q scores. For the chest, scores at 1 and 2 years of followup were comparable between IBR and AR overall as well as within subgroups of women who had unilateral or bilateral reconstructions. For the chest and upper body scores, however, the group of women who specifically underwent AR with pedicled transverse rectus abdominis myocutaneous (TRAM) flaps had clinically important poorer well-being than patients who underwent IBR.

One NRCS (Kulkarni 2017) also used the pain interference and physical function components of the Patient-Reported Outcomes Measurement Information System (PROMIS) (scores 0 to 100; higher is worse; MCID 3 to 4.5 points $^{188}$ ) and reported comparable scores between IBR and AR groups at 1 year of followup. Eltahir 2015 also used the physical functioning component of the SF-36 (scores 0 to 100; higher is better) and reported comparable scores between IBR and AR groups (adjMD 2.13, 95\% CI -4.20 to 8.46). Kouwenberg also used the EORTC QLQ-C30 (0-100; higher is better; MCID 3 points $\left.{ }^{187}\right)$ and reported comparable scores between IBR and AR beyond 6 months of followup.

One NRCS (Nelson 2019) reported categorical data for the likelihood of patients having higher BREAST-Q physical well-being: chest scores (Appendix Table E-1.3). Although the likelihoods at 1 and 3 years of followup were comparable between IBR and AR groups, patients who underwent AR were more likely to have higher (versus unchanged) scores at 5 years (adjusted odds ratio [adjOR] 4.52, 95\% CI 2.03 to 10.1) and at 7 years (adjOR 3.08, 95\% 1.03 to 9.15).

\section{Psychosocial Well-Being}

One RCT (Tallroth 2020) and four NRCSs (Eltahir 2015, Kouwenberg 2020, Kulkarni 2017, and Roth 2007) reported on psychosocial well-being using six different measurement instruments (Appendix Table E-1.1). Psychosocial well-being was generally comparable between IBR and AR groups.

The RCT (Tallroth 2020) and three NRCSs (Eltahir 2015, Kouwenberg 2020, and Kulkarni 2017) reported data using the BREAST-Q questionnaire (0-100; higher is better; MCID 4 points ${ }^{187}$ ) (Figure 4 and Appendix Table E-1.1). Only two of the NRCSs (Eltahir 2015 and Kulkarni 2017) reported adjusted effect sizes, and therefore we combined their estimates and the RCT's estimate at average followup durations ranging from 2 to 5.3 years (Figure 4). Effect sizes ranged from 0.30 to 4.60 across these studies. The meta-analysis provided evidence that patients who underwent IBR or AR experienced clinically comparable psychosocial well-being (adjMD $3.14,95 \%$ CI 1.26 to $\left.5.02 ; I^{2}=0 \%\right)$. 


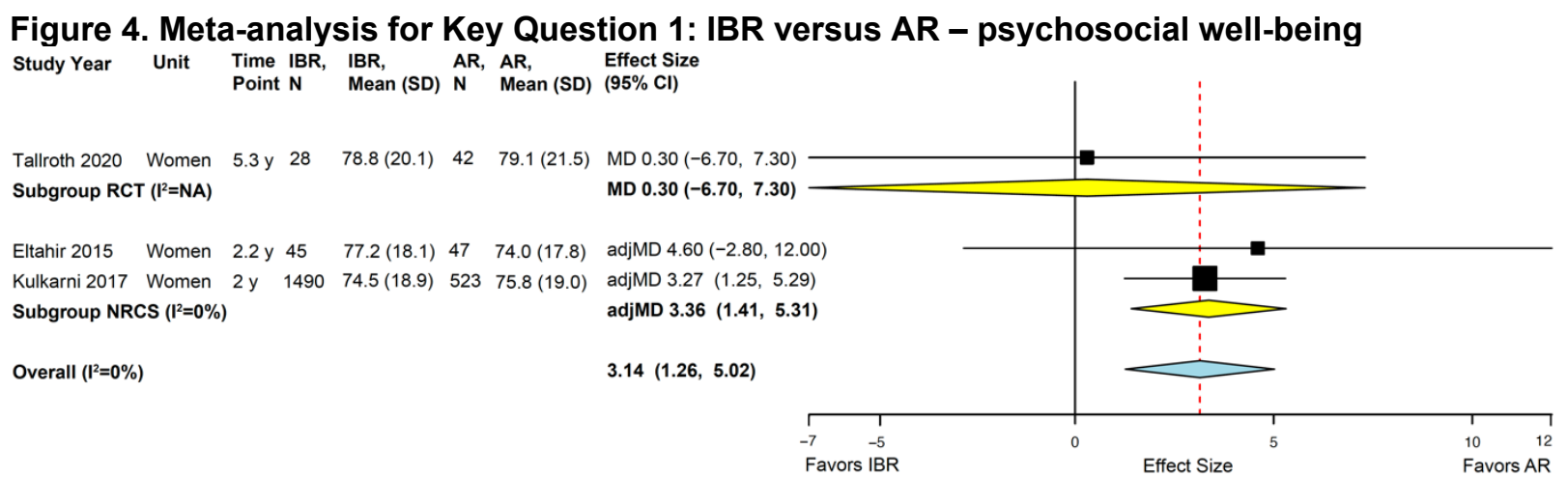

Abbreviations: adj $=$ adjusted, $\mathrm{AR}=$ autologous reconstruction, $\mathrm{CI}=$ confidence interval, $\mathrm{IBR}=$ implant-based reconstruction, $\mathrm{I}^{2}=$ measure of statistical heterogeneity ( $\%$ of total variability that is due to between-study variability), $\mathrm{MD}=$ mean difference, $\mathrm{NA}=$ not applicable, $\mathrm{NRCS}=$ nonrandomized comparative study, $\mathrm{RCT}=$ randomized controlled trial, $\mathrm{SD}=$ standard deviation, $\mathrm{y}=$ years.

Kulkarni 2017, which was included in the meta-analysis, reported additional data regarding psychosocial well-being. At 2 years, scores were comparable even within the subgroups of women who had unilateral reconstruction or bilateral reconstruction.

Psychosocial well-being data were also comparable between IBR and AR groups in studies that used other measurement instruments. Kulkarni 2017 also used the PROMIS anxiety and depression components (scores 0 to 100; higher is worse; MCID 3 to 4.5 points for each ${ }^{188}$ ) and reported comparable scores between IBR and AR groups at 1 year of followup.

Two NRCSs (Eltahir 2015 and Roth 2007) reported on the social functioning component of SF-36 (scores 0 to 100; higher is better; MCID not available). Both NRCSs reported that IBR and AR groups had comparable scores. Eltahir 2015 reported an adjMD of -1.21 (95\% CI, -8.44 to 6.02), while Roth 2007 reported that the $P$ value was $\geq 0.05$ (adjusted effect size not reported). Roth 2007 also reported on the social/family well-being component of the FACT-B (0-28; higher is better; MCID 2 points $\left.{ }^{186}\right)$. Although an adjusted effect size was not reported, at 2 years of followup, patients who underwent IBR and AR had comparable FACT-B scores ( $\mathrm{P}=0.24)$.

One NRCS (Kouwenberg 2020) reported on the social, emotional, cognitive, and role function components of the EORTC QLQC30 (each scored from 0 to 100; higher is better; MCIDs not available). Only the role function component scores were statistically significantly different between IBR and AR groups; patients who underwent IBR had higher scores $(\mathrm{P}<0.05$; adjusted effect size not reported).

\section{Sexual Well-Being}

One RCT (Tallroth 2020) and three NRCSs (Eltahir 2015, Kouwenberg 2020, and Kulkarni 2017) reported on sexual well-being using the BREAST-Q questionnaire (0-100; higher is better; MCID $5^{187}$ ) (Figure 5 and Appendix Table E-1.1). Only two of the NRCSs (Eltahir 2015 and Kulkarni 2017) reported adjusted effect sizes, and therefore we combined their estimates and the RCT's estimate at average followup durations ranging from 2 to 5.3 years (Figure 5). Effect sizes ranged from 5.53 to 8.70 across these studies. The meta-analysis provided evidence that patients who underwent AR experienced clinically better sexual well-being than patients who underwent IBR (adjMD 5.83, 95\% CI 3.44 to $8.23 ; \mathrm{I}^{2}=0 \%$ ). 


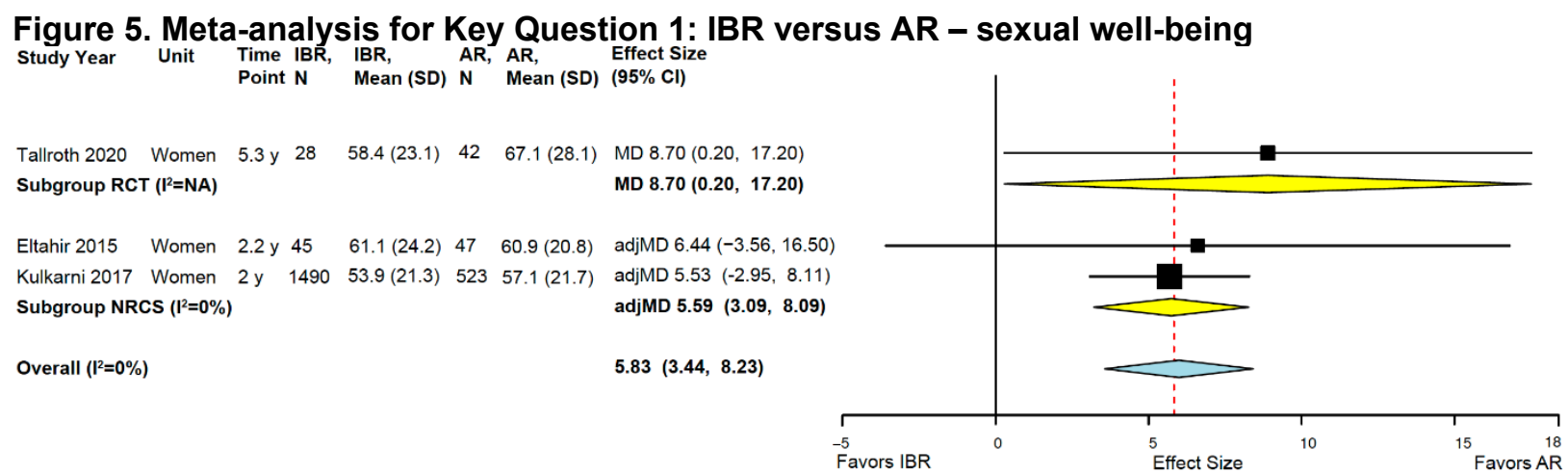

Abbreviations: adj $=$ adjusted, $\mathrm{AR}=$ autologous reconstruction, $\mathrm{CI}=$ confidence interval, $\mathrm{IBR}=$ implant-based reconstruction, $\mathrm{I}^{2}=$ measure of statistical heterogeneity ( $\%$ of total variability that is due to between-study variability), $\mathrm{MD}=$ mean difference, $\mathrm{NA}=$ not applicable, $\mathrm{NRCS}=$ nonrandomized comparative study, $\mathrm{RCT}=$ randomized controlled trial, $\mathrm{SD}=$ standard deviation, $\mathrm{y}=$ years.

Kulkarni 2017, which was included in the meta-analysis, reported additional data regarding sexual well-being. At 2 years, the clinically better sexual well-being advantage of AR over IBR existed within the subgroup of women who had unilateral reconstruction (adjMD 11.4; $\mathrm{P}<0.001$ ) but not bilateral reconstruction (adjMD 4.2; $\mathrm{P}<0.001)$.

The one NRCS reporting sexual well-being data that were not included in the meta-analysis (Kouwenberg 2020) reported that, compared with patients who underwent IBR, patients who underwent AR experienced better sexual well-being beyond 6 months $(\mathrm{P}<0.05$; adjusted effect size not reported). Kouwenberg 2020 also reported data on sexual functioning and sexual enjoyment using the EORTC QLQBR23 (0 to 100; higher is better; MCID 5 ${ }^{189}$ ). Beyond 6 months of followup, compared with patients who underwent IBR, those who underwent AR experienced better sexual functioning $(\mathrm{P}<0.05)$ but not better sexual enjoyment $(\mathrm{P}=\mathrm{NS})$; adjusted effect sizes were not reported.

\section{Satisfaction With Breasts}

One RCT and six NRCS reported data on satisfaction with breasts or nipples.

The RCT (Tallroth 2020) and four NRCSs (Brito 2020, Eltahir 2015, Kouwenberg 2020, and Kulkarni 2017) reported on satisfaction with breasts using the BREAST-Q (0-100; higher is better; MCID 5 ${ }^{187}$ ) (Figure 6 and Appendix Table E-1.1). Only two of the NRCSs (Eltahir 2015 and Kulkarni 2017) reported adjusted effect sizes, and therefore we combined their estimates and the RCT's estimate at average followup durations ranging from 2 to 5.3 years (Figure 6). Effect sizes ranged from 7.94 to 8.70 across these studies. The meta-analysis provided evidence that patients who underwent AR experienced clinically better satisfaction with breasts than patients who underwent IBR (adjMD $8.08,95 \%$ CI 6.11 to $10.1 ; \mathrm{I}^{2}=0 \%$ ). 


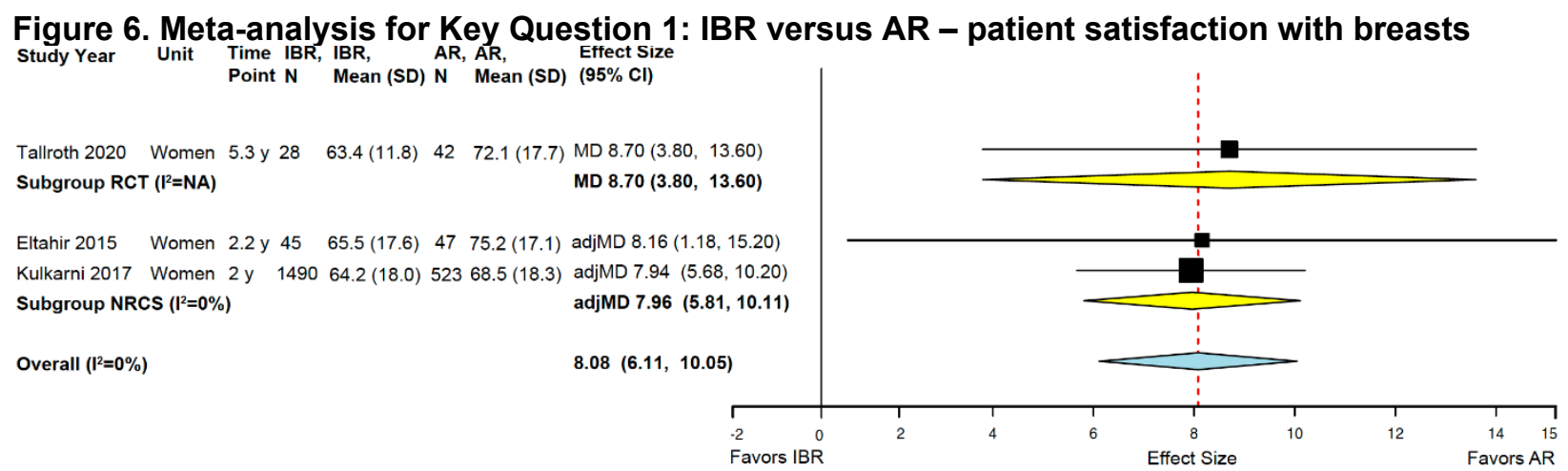

Abbreviations: adj $=$ adjusted, $\mathrm{AR}=$ autologous reconstruction, $\mathrm{CI}=$ confidence interval, $\mathrm{IBR}=$ implant-based reconstruction, $\mathrm{I}^{2}=$ measure of statistical heterogeneity ( $\%$ of total variability that is due to between-study variability), $\mathrm{MD}=$ mean difference, $\mathrm{NA}=$ not applicable, NRCS $=$ nonrandomized comparative study, RCT $=$ randomized controlled trial, $\mathrm{SD}=$ standard deviation, $\mathrm{y}=$ years.

Kulkarni 2017, which was included in the meta-analysis, reported additional data regarding satisfaction with breasts. At 2 years, the satisfaction advantage of AR over IBR existed even within the subgroups of women who had unilateral reconstruction (adjMD 9.85; $\mathrm{P}=0.001$ ) or bilateral reconstruction (adjMD 5.13; $\mathrm{P}=0.001$ ).

The two NRCSs reporting sexual satisfaction with breasts data that were not included in the meta-analysis (Brito 2020 and Kouwenberg 2020) also reported that, compared with patients who underwent IBR, patients who underwent AR experienced better satisfaction beyond 6 months (Kouwenberg 2020: $\mathrm{P}<0.05$; adjusted effect size not reported) and at an unreported timepoint (Brito 2020: $\mathrm{P}=0.004$; adjusted effect size not reported).

Two NRCSs (Lei 2020 and Yueh 2009) reported categorical data regarding satisfaction with breasts (Appendix Table E-1.4). Lei 2020 reported that a comparable proportion of patients who underwent IBR or AR were satisfied (adjOR 0.85, 95\% CI 0.36 to 1.63). However, Yueh 2009 reported that patients who underwent AR 2 were more likely to be satisfied than were patients who underwent IBR (adjOR 1.43, 95\% 1.18 to 1.73). These findings were also observed when specifically comparing women who underwent AR with TRAM flaps versus women who underwent IBR (adjOR 3.49, 95\% CI 1.91 to 6.40) and when comparing women who underwent AR with latissimus dorsi (LD) flaps versus women who underwent IBR (adjOR 1.99, 95\% CI 1.09 to 3.65). However, the odds of being satisfied were comparable between the AR with deep inferior epigastric perforator (DIEP) flap and IBR groups.

The RCT (Tallroth 2020) and two NRCSs (Eltahir 2015 and Kouwenberg 2020) reported on satisfaction with nipples using the BREAST-Q questionnaire (0-100; higher is better; MCID

$\left.5^{187}\right)$. The results were inconsistent. Two studies reported comparable satisfaction scores between IBR and AR groups: Tallroth 2020 at 5.3 years of followup (MD 2.3, 95\% CI -5.5 to 10.1) and Eltahir 2015 at 2.2 years of followup (adjMD 1.70, 95\% CI -14.2 to 17.6). However, Kouwenberg 2020 reported that patients who underwent AR had higher scores beyond 6 months of followup ( $\mathrm{P}<0.05$; adjusted effect size not reported).

\section{Satisfaction With Surgical Outcome}

One RCT (Tallroth 2020) and four NRCSs (Eltahir 2015, Kouwenberg 2020, Yueh 2009, and Lei 2020) reported on satisfaction with the surgical outcome. The results were inconsistent. Three of these studies reported continuous data (using the BREAST-Q; 0-100; higher is better; MCID 5) (Appendix Table E-1.1). Two studies reported comparable satisfaction scores between 
IBR and AR groups: Tallroth at 5.3 years of followup (MD 2.9, 95\% CI -3.1 to 8.9) and Eltahir 2015 at 2.2 years of followup (adjMD 4.9, 95\% CI -3.1 to 12.9). However, Kouwenberg 2020 reported that patients who underwent AR had higher scores beyond 6 months of followup ( $\mathrm{P}<0.05$; adjusted effect size not reported).

Two NRCSs (Lei 2020 and Yueh 2009) reported categorical data on whether patients reported being satisfied with the surgical outcome (Appendix Table E-1.4). Lei 2020 reported comparable satisfaction in the IBR and AR groups (adjOR 0.69, 95\% CI 0.45 to 1.67). On the other hand, Yueh 2009 reported that, compared with women who underwent IBR, there was greater likelihood of satisfaction in the overall AR group (adjOR 1.83, 95\% CI 1.11 to 3.03) and in the AR with TRAM flap group (adjOR 2.05, 95\% CI 1.13 to 3.72). However, the odds of being satisfied with the surgical outcome was comparable between the IBR and AR with DIEP flap group and between the IBR and AR with LD flap group.

\section{Recurrence of Breast Cancer}

Three NRCSs (Ha 2020, Kouwenberg 2020, and Wu 2021) reported inconsistent data for recurrence of breast cancer (Appendix Table E-1.3). Ha 2020 reported that at 4.8 years of followup, compared with women who underwent IBR, women who underwent AR experienced a greater risk of recurrence of high histologic grade (Grade III) breast cancer (adjOR 3.39, $95 \%$ CI 1.23 to 9.32). However, risks of locoregional recurrence were comparable $(\mathrm{P}=0.70$; adjusted effect size not reported). The two other NRCSs (Kouwenberg 2020 and Wu 2021) also reported that local and distant recurrence risks were comparable between IBR and AR groups.

Note that recurrence of breast cancer was not a prioritized outcome for this review because its linkage to breast reconstruction surgery is not clear, and so does not appear in the Evidence Profiles.

\section{Mortality}

One NRCS (Jiang 2013) reported mortality data (Appendix Table E-1.5). At 8.9 years of followup, patients in the IBR and AR groups experienced comparable risks of overall mortality (adjOR $0.96,95 \%$ CI 0.89 to 1.04 ) and breast cancer-specific mortality (adjOR $0.95,95 \%$ CI 0.87 to 1.04$)$.

\section{Surgical Complications}

Both RCTs, 35 of the 38 NRCSs, and all 69 single group studies reported on surgical complications for this KQ. For each outcome, we first summarize results from the comparative studies (i.e., RCTs and NRCSs; Appendix Tables E-1.5 to E-1.7 and Appendix Tables F-1.11 to F-1.26). Additional information on surgical complications from the single group studies is presented in Appendix Tables F-1.13 to F-1.26 and is summarized briefly here. Because this KQ is about the comparison between IBR and AR, conclusions (including SoE assessments) are based on results from the comparative studies only.

\section{Unplanned Repeat Hospitalizations}

Three NRCSs and four single group studies reported data for this outcome.

Two NRCSs (Merchant 2015 and Mioton 2013) reported that risks of unplanned repeat hospitalizations within the first month of followup were comparable between patients in the IBR and AR groups (Appendix Table E-1.5). Merchant 2015 reported an adjOR of 1.07 (95\% CI 0.95 to 1.20 ) and Mioton 2013 reported that the comparison was not statistically significant (an 
adjusted effect size was not reported). The third NRCS (Nasser 2018) reported that IBR and AR groups had comparable 1-month risks of unplanned emergency department (ED) visits (adjOR 1.11, 95\% CI 0.91 to 1.25) and unplanned ED visits with pain-related diagnoses (adjOR 1.11, 95\% CI 0.83 to 1.67 ).

Among the four single group studies, three assessed IBR and one AR (Appendix Table F1.14). Among the three IBR studies, two studies reported 3-month risks of unplanned repeat hospitalizations of 18 and 16.5 percent, while the third study, without reporting the time point, reported a risk of 2.8 percent. In the AR study, the risk at 1 month was 6.7 percent.

\section{Unplanned Repeat Surgeries for Revision}

Three NRCSs and nine single group studies reported data for this outcome.

Findings in the three NRCSs (Fischer 2014, Zhang 2019, and Kulkarni 2017) were inconsistent (Appendix Table E-1.6). Two NRCSs (Fischer 2014 and Zhang 2019) reported that risks of unplanned repeat surgeries for revision were lower in the AR group than the IBR group, while Kulkarni 2017 reported the reverse. Without reporting adjusted effect sizes, Fischer 2014 reported lower risks in the AR group within the first 1 year $(\mathrm{P}=0.017)$ and at 2 years of followup ( $\mathrm{P}=0.003)$. Zhang 2019 reported an adjOR of 0.72 (95\% CI 0.50 to 1.06) at 4.9 years of followup. Kulkarni 2017, on the other hand, reported higher risks in most of the individual flap groups (DIEP, free TRAM, LD, and superficial inferior epigastric artery perforator [SIEA]) than in the IBR with tissue expanders group, with adjORs ranging from 1.83 to 2.66. The pedicled TRAM group, however, had a risk that was comparable to the IBR with tissue expanders group (adjOR 1.33, 95\% CI 0.75 to 2.40).

Among the nine single group studies, three assessed IBR and six AR (Appendix Table F1.14). Among the IBR studies, Park 2019 reported a 6-month risk of unplanned surgeries for revision of 8.7 percent, Coroneos 2019 reported a 3-year risk of 20.4 percent, and Rogoff 2020 reported a risk of 0.6 percent at an unreported time-point. The six AR studies reported that at various followup time-points between 5.6 months and 10 years, risks ranged from 1.0 to 19.1 percent.

\section{Unplanned Repeat Surgeries for Complications}

Three NRCSs and eight single group studies reported data for this outcome.

Findings in the three NRCSs (Hangge 2013, Mioton 2013, and Zhang 2011) were inconsistent (Appendix Table E-1.6). One NRCS (Hangge 2013) reported that, compared with the AR group, risks of unplanned repeat surgeries for unspecified complications were higher in the IBR direct-to-implant group (adjOR 2.03, 95\% CI 1.03 to 3.98) and the IBR with tissue expanders group (adjOR 1.81, 95\% CI 0.90 to 3.64). On the other hand, Mioton 2013 reported comparable risks of the above outcome between IBR and AR groups (adjOR 1.08, 95\% CI 0.88 to 1.32) and Zhang 2011 reported comparable risks of unplanned repeat surgeries for compromised implants or flaps (adjOR $0.63,95 \%$ CI 0.29 to 1.37 ).

Among the eight single group studies, three studies assessed IBR and five AR (Appendix Table F-1.15). Among the IBR studies, Sewart 2021 reported that, at 3 months of followup, the risk of undergoing unplanned repeat surgeries for infections was 16.8 percent; Hamdi 2011 reported that, at 2.6 years of followup, the risk of undergoing AR after failure of IBR was 7.8 percent; and Acosta 2011 reported that, at 9 years of followup, the risk of undergoing wound revision was 11.4 percent. The five AR studies reported risks of reoperation for complications at up to 3.1 years of followup ranging from 2.0 to 18.0 percent. 


\section{Pain}

Five NRCSs and three single group studies reported inconsistent data regarding pain.

Three NRCSs (Kulkarni 2017, Shiraishi 2020, and Roth 2007) reported that, compared with IBR, AR, particularly when conducted using abdominal-based flaps (TRAM, SIEA, and DIEP), was generally associated with more chronic pain beyond 3 months after surgery (Appendix Table E-1.7). Kulkarni 2017 reported pain data using three different measurements: the sensory and affective components of the McGill Pain Questionnaire Short Form (MPQ-SF) (for each component: 0 to 10 ; higher is worse; MCIDs not available ${ }^{190}$ ) and a VAS (0 to 10; higher is worse; MCID 2 points ${ }^{191}$ ). At 1 week after surgery, compared with patients who underwent IBR, patients who underwent AR with SIEA flaps had higher MPQ-SF sensory pain scores (adjMD $2.41,95 \%$ CI 0.38 to 4.44 ). At 3 months after surgery, compared with patients who underwent IBR with tissue expanders, patients who underwent AR with free TRAM flaps had higher scores (adjMD 2.48; $\mathrm{P}<0.001$ ). At 1 week after surgery, MPQ-SF affective pain scores were comparable between various AR flap and IBR groups. However, at 2 years after surgery, compared with patients who underwent IBR with tissue expanders, scores were higher (i.e., worse) in patients who underwent AR with abdominally sourced flaps: DIEP (adjMD 0.33, 95\% CI 0.07 to 0.59), free TRAM (adjMD 0.84; $\mathrm{P}<0.001$ ), and SIEA (adjMD 1.24; $\mathrm{P}<0.0001$ ). Using the VAS, however, Kulkarni 2017 reported comparable pain across study groups at 1 week after surgery whether analyzed as a continuous score (Appendix Table E-1.7) or as a dichotomous outcome of moderate to severe pain versus not (Appendix Table E-1.6). Shiraishi 2020 also reported data using the MPQ-SF. At 1 year of followup, patients who underwent AR (all with DIEP flaps) had higher mean MPQ-SF scores for overall (adjMD 1.08) as well as for the sensory (adjMD 0.80) and affective components (adjMD 0.28); no estimates of uncertainty or P values were reported (MCID also not available). Roth 2007 reported data using a different VAS (1 to 5; higher is better; MCID not available). At 2 years of followup, patients who underwent AR experienced lower (i.e., worse) abdominal pain scores $(\mathrm{P}<0.0001$; adjusted effect size not reported). Scores for the other scores (i.e., bodily pain, breast pain, and back pain) were comparable between IBR and AR groups.

On the other hand, Eltahir 2015 reported comparable pain scores between IBR and AR groups (adjMD 2.40, 95\% CI -5.37 to 10.2). This was based on the pain component of the SF36 (scores 0 to 100; higher is better; MCID unavailable). Similarly, Kouwenberg 2020 reported comparable pain scores between IBR and AR groups ( $\mathrm{P}=\mathrm{NS}$; adjusted effect size not reported). This was based on the pain component of the EORTC QLQC30 (scores 0 to 100; higher is worse; MCID unavailable).

Each of the three single group studies assessed IBR (Appendix Table F-1.16). Risks of breast pain or tightness were 5.1 percent at 1 year ( 1 study), 1.8 percent at 2 years ( 1 study), and 4.7 percent at an unreported time-point (1 study). Although a statistical test evaluating subgroup effects was not reported, obese and nonobese patients had comparable risks of breast pain at 1 year $(5.8 \%$ and $4.8 \%)$ (1 study).

\section{Analgesic Use}

One NRCS (Shiraishi 2020) reported data using a score from 0 to 5 where 0 implies no analgesic use and 5 implies daily analgesic use (no MCID is available). At 1 year of followup, patients who underwent AR had higher mean analgesic use scores (adjMD 0.37), but no estimates of uncertainty or $\mathrm{P}$ values were reported. 


\section{Necrosis}

Four NRCSs and 41 single group studies reported necrosis data.

Findings in the four NRCSs (Woo 2018, Naoum 2020a, Abedi 2016, and de Araujo 2016) were inconsistent (Appendix Table E-1.5). Locations of necrosis reported included the mastectomy flap and fat at followup time points ranging from 1.6 to 10 years. One NRCS (Woo 2018) reported that patients who underwent AR had a lower risk of mastectomy flap necrosis (adjOR 0.31, 95\% CI 0.11 to 0.86), while two other NRCSs reported that IBR and AR groups had comparable risks (Naoum 2020a: adjOR 0.83, 95\% CI 0.19 to 3.5 and Abedi 2016: adjOR $0.66,95 \%$ CI 0.38 to 1.16). However, another NRCS (de Araujo 2016) reported a higher risk in the AR group, but the estimate was very imprecise (adjOR 17.9, 95\% CI 0.52 to 610.5 ).

Naoum 2020a also reported that patients who underwent AR had a higher risk of fat necrosis at 4 to 10 years of followup, but the estimate was imprecise (adjOR 21.2, 95\% CI 2.5 to 174.5).

Among the 41 single group studies, 16 studies assessed IBR and 25 AR (Appendix Table F1.17). Among the 16 IBR studies, risk of breast fat necrosis was 5.0 percent in the first 12 months (1 study). Risk of mastectomy flap necrosis was 1.8 percent in the first month (1 study), between 0.1 and 8.5 percent between 1 month and 1 year ( 5 studies), between 1.6 and 3.1 percent after 1 year ( 2 studies), and between 2.0 and 12.3 percent at unreported time-points ( 4 studies). Risk of necrosis at unspecified locations was 1.5 percent in the first month ( 1 study), between 2.0 and 12.4 percent between 1 month and 1 year ( 3 studies), 9.7 percent after 1 year ( 1 study), and 3.2 percent at an unreported time-point (1 study). Although statistical tests evaluating subgroup effects were not reported, differences in risks of necrosis were reported among subgroups: immediate IBR had higher risk than delayed IBR (1 study); two-stage IBR had higher risk than single-stage IBR (2 studies), and patients who underwent radiation therapy before IBR had higher risk than who did not (2 studies). Obese patients had a higher risk of necrosis of the breast fat but not of the skin flap (1 study).

Among the 25 AR studies, risk of breast fat necrosis was between 10.0 and 11.2 percent in the first month ( 2 studies), between 1.3 and 11.1 percent between 1 month and 1 year ( 3 studies), between 0.3 and 13.0 percent after 1 year ( 5 studies), and between 0.9 percent and 19.4 percent at unreported time-points (10 studies). Risk of mastectomy flap necrosis was 0.4 percent in the first year ( 1 study), between 1.9 percent and 14.3 percent after 1 year ( 5 studies), and between 0.5 percent and 18.2 percent at unreported time-points ( 5 studies). Risk of AR flap necrosis was 5.5 percent in the first year ( 1 study), 2.5 percent at 2 years ( 1 study), and 5.5 percent at an unreported time-point (1 study). Risk of umbilical necrosis was 3.5 percent after 1 year (1 study) and 3.3 percent at an unreported time-point ( 1 study).

Although a statistical test evaluating subgroup effects was not reported, patients in the oldest age group ( $>70$ years) had a higher risk of fat necrosis $(26.3 \%)$ than younger categories of women (approximately 15\% to 17\%) (1 study).

\section{Thromboembolic Events}

Both RCTs, two NRCSs, and 26 single group studies reported thromboembolic event data.

One NRCS (Mioton 2013) reported that risks of deep vein thrombosis (DVT) in IBR and AR groups were comparable (adjOR $0.99,95 \%$ CI 0.41 to 2.41 ), but patients who underwent AR had a statistically nonsignificant higher risk of pulmonary embolism (PE) (adjOR 1.84, 95\% CI 0.71 to 4.77) (Appendix Table E-1.5). The other NRCS (Momeni 2018) reported that patients who underwent AR had a statistically significant higher risk of the composite outcome of DVT or PE (adjOR 2.27, 95\% CI 1.79 to 2.86). Both RCTs (Brorson 2020a or Tallroth 2020) 
provided unusable data for DVT, PE, arterial stop, and venous stasis because for each outcome one or both study groups (IBR and AR) experienced no events in the short (1-month) followup, so no effect sizes were calculable.

Among the 26 single group studies, one study assessed IBR and 25 AR (Appendix Table F1.18). In the IBR study (Chen 2018a), risk of DVT was 0.04 percent and PE 0.09 percent (time points not reported).

Among the 25 AR studies, risk of DVT was 0.1 percent in the first month ( 1 study) and between 0.1 and 2.5 percent at unreported time-points ( 4 studies). Risk of PE was 0.2 percent in the first year ( 1 study), between 0.1 percent and 1.4 percent after 1 year ( 2 studies), and between 0.1 percent and 0.8 percent at unreported time-points (4 studies). Risk of the composite outcome of DVT or PE was 0.5 percent in the first month ( 1 study) and between 0 percent and 1.1 percent at unreported time-points (4 studies).

Risk of venous congestion of the AR flap was 1.2 percent in the first month (1 study), 4.4 percent between 1 month and 1 year ( 1 study), and 0.6 percent after 1 year ( 1 study). Risk of venous thrombosis of the AR flap was 2.7 percent between 1 month and 1 year ( 1 study). Risk of venous thrombosis or occlusion of the AR flap was between 0.5 percent and 5.6 percent in the first month (4 studies), 3.8 percent after 1 year (1 study), and between 1.2 percent and 3.9 percent at unreported time-points ( 3 studies). Risk of insufficient venous drainage of the AR flap was 0.8 percent after 1 year ( 1 study). Risk of arterial thrombosis of the AR flap was 1.6 percent between 1 month and 1 year ( 1 study). Risk of arterial thrombosis or occlusion of the AR flap was between 0.8 percent and 4.0 percent in the first month (4 studies), 0.5 percent after 1 year ( 1 study), and between 1.4 percent and 3.8 percent at unreported time-points ( 2 studies). Risk of insufficient arterial supply of the AR flap was 0.1 percent after 1 year ( 1 study). Risk of the composite outcome of arterial or venous thrombosis or occlusion of the AR flap was 0.1 percent in the first year ( 1 study) and between 0.8 percent and 3.5 percent at unreported timepoints ( 2 studies). Although a statistical test evaluating subgroup effects was not reported, patients in the oldest age group (>70 years) had a higher risk of this composite outcome (5.3\%) than younger categories of women (between $2.9 \%$ and $3.9 \%$ risk) (1 study).

\section{Infections Not Explicitly Implant Related}

Six NRCSs and 47 single group studies reported data on infections that were not explicitly implant-related.

Findings in the six NRCSs were inconsistent (Appendix Table E-1.6). One NRCS (Naoum $2020 \mathrm{~b}$ ) reported that patients who underwent AR had higher risks of infections (location not specified) than patients who underwent single-staged IBR (adjOR 3.2, 95\% CI 0.6 to 16) and patients who underwent two-staged IBR (adjOR 8.1, 95\% CI 1.7 to 39). Another NRCS (Mioton 2013) reported that, compared with patients who underwent IBR, patients who underwent AR had higher risks of wound infections (adjOR 1.40, 95\% CI 1.01 to 1.96). Mioton 2013 also reported that patients who underwent AR had higher risks of deep surgical site infections (adjOR 1.81, 95\% CI 1.12 to 2.94) but not superficial surgical site infections (adjOR 1.20, 95\% CI 0.81 to 1.76). Two NRCSs reported imprecise comparisons of risks of infections between AR and IBR groups (de Araujo 2016: adjOR 0.86, 95\% CI 0.18 to 4.11 and Naoum 2020a: adjOR $0.77,95 \%$ CI 0.20 to 2.50). On the other hand, two NRCSs reported that AR may be associated with a lower risk of infections. Although Kulkarni 2017 reported comparable risks of breast wound infections between the AR flap types and IBR, patients in the DIEP flap group had a 
lower risk than the IBR group (adjOR 0.44, 95\% CI 0.25 to 0.78 ). Garvey 2012 reported a lower risk in the AR group than the IBR group $(\mathrm{P}<0.001)$, but an adjusted effect size was not reported.

Among the 47 single group studies, 25 studies assessed IBR and 22 AR (Appendix Table F1.19). In the 25 IBR studies, risk of infections ranged from 1.7 to 3.0 percent in the first month ( 3 studies), from 2.1 to 25.7 percent between 1 month and 1 year (11 studies), from 1.7 to 10.8 percent after 1 year ( 7 studies), and from 0.3 to 24.7 percent at unreported time-points ( 7 studies).

In the $22 \mathrm{AR}$ studies, risk of breast infections ranged from 2.3 to 10.6 percent in the first month ( 2 studies), from 0.4 to 6.9 percent between 1 month and 1 year ( 4 studies), from 1.1 to 10.3 percent after 1 year ( 5 studies), and from 0.4 to 7.8 percent at unreported time-points ( 8 studies). The risk of donor site or flap infections ranged from 0.5 to 7.4 percent between 1 month and 1 year ( 2 studies) and ranged from 1.0 to 1.9 percent at unreported time-points ( 3 studies). The risk of major or systemic infections was 3.8 percent after 1 year (1 study) and 0 percent (i.e., no major or systemic infections) at an unreported time-point (1 study).

\section{Wound Dehiscence}

Two NRCSs and 21 single group studies reported would dehiscence data.

One NRCS (Mioton 2013) reported that, compared with patients who underwent IBR, those who underwent AR had a near-significant higher risk of wound dehiscence at 1 month of followup (adjOR 1.79, 95\% CI 0.83 to 3.84), but the other NRCS (Garvey 2012) reported that risks in IBR and AR groups were comparable at 1.5 years of followup $(\mathrm{P}=0.25$; adjusted effect size not reported), (Appendix Table E-1.5).

Among the 21 single group studies, 10 studies assessed IBR and 11 assessed AR (Appendix Table F-1.20). Among the 10 IBR studies, risk of breast wound dehiscence was 1.9 percent in the first month ( 1 study), between 1.6 and 4.9 percent between 1 month and 1 year ( 4 studies), between 0.3 and 2.1 percent after 1 year ( 4 studies), and between 0.7 and 11.0 percent at unreported time-points ( 2 studies). Although a statistical test evaluating subgroup effects was not reported, compared with patients without prior radiation therapy, patients with prior radiation had comparable 1 -year risk of wound dehiscence in one study $(0.8 \%$ vs. $0.3 \%)$ but higher 3.4year risk in another study (11.9\% vs. 1.1\%). In another study, obese and nonobese patients had comparable risks at 1 year of followup (3.6\% vs. 2.8\%). In yet another study, patients in singleand two-stage reconstruction had comparable risks at 3 months (2.5\% vs. $4.2 \%)$.

Among the 11 AR studies, risk of breast wound dehiscence was 1.2 percent in the first month ( 1 study), between 0.3 and 3.9 percent between 1 month and 1 year ( 4 studies), 11.4 percent after 1 year ( 1 study), and between 0.1 and 1.0 percent at unreported time-points ( 6 studies). Risk of donor site wound dehiscence was 1.7 percent between 1 month and 1 year (1 study) and 1.4 percent at an unreported time-point (1 study). Although a statistical test evaluating subgroup effects was not reported, patients with and without prior radiation therapy had comparable 1-year risk of wound dehiscence in one study $(0.8 \%$ vs. $0.3 \%)$ but higher 3.4 -year risk in another study (11.9\% vs. 1.1\%).

\section{Delayed Healing}

Two NRCSs and 11 single group studies reported data for the outcome of delayed healing.

Both NRCSs (Fischer 2013 and Garvey 2012) reported higher risks of delayed healing among women who underwent AR than IBR (Appendix Table E-1.5). Fischer 2013 reported a higher risk of delayed breast wound healing in the AR group at 4 years of followup (adjOR 
2.2, 95\% CI 1.0 to 5.2) and Garvey 2012 reported a higher risk of delayed healing at an unspecified location in the AR group at 1.5 years of followup $(\mathrm{P}=0.01$; adjusted effect size not reported).

Among the 11 single group studies, two assessed IBR and nine AR (Appendix Table F-1.21). In the two IBR studies, 1-year risk of a nonhealing wound was 4.8 percent ( 1 study) and 0.4 percent at an unreported time-point (1 study). Although statistical tests evaluating subgroup effects were not reported, risks were comparable between patients with or without prior radiation therapy ( 1 study), between patients undergoing immediate or delayed IBR (1 study), and between patients undergoing direct-to-implant or tissue expander-based IBR (1 study). In another study, obese patients had a higher risk than nonobese patients at 1 year of followup (7.6\% vs. $3.7 \%$ ).

Among the nine AR studies, the risk of delayed breast wound healing was between 1.5 and 7.6 percent between 1 month and 1 year ( 2 studies), 2.7 percent beyond 1 year (1 study), and 25.0 percent at an unreported time-point ( 1 study). The risk of delayed donor site wound healing was between 1.7 and 3.4 percent between 1 month and 1 year ( 2 studies), 0.5 percent beyond 1 year, and 11.5 percent at an unreported time-point ( 1 study). The other AR studies reported risks of delayed healing at unspecified locations. Risks were 9.2 percent at 1 month (1 study), 19.7 percent between 4.3 and 5.4 years ( 1 study), and between 1.2 and 18.7 percent at unreported time-points (2 studies).

\section{Seroma}

Two NRCSs and 33 single group studies reported seroma data.

Although they did not report adjusted effect sizes, both NRCSs (Fischer 2014 and Garvey 2012) reported higher risks of breast seroma among women who underwent IBR than AR. Fischer 2014 reported a higher risk of seroma $(\mathrm{P}=0.009)$, and Garvey 2012 reported a higher risk of the composite outcome of seroma or hematoma $(\mathrm{P}<0.001)$.

Among the 33 single group studies, 14 studies assessed IBR and 19 studies AR (Appendix Table F-1.22). Among the 14 IBR studies, breast seroma risks ranged from 1.6 to 4.6 percent in the first month ( 2 studies), from 1.1 to 12.6 percent between 1 month and 1 year ( 7 studies), from 0.3 to 5.6 percent after 1 year ( 3 studies), and from 0.2 to 4.5 percent at unreported time-points (3 studies). Four of the IBR studies also reported subgroup-specific data for seroma. Although statistical tests evaluating subgroup effects were not reported, comparable risks of seroma were reported among subgroups defined by history of prior radiation therapy ( 2 studies), use of immediate versus delayed IBR (1 study), and use of single- versus two-stage IBR ( 2 studies). However, in one study, obese patients had a higher risk of seroma than nonobese patients $(11.4 \%$ vs. $4.7 \%$ ).

Among the 19 AR studies, breast seroma risk was 3.1 percent in the first year (1 study), 3.9 percent beyond 1 year ( 1 study), and 2.7 percent at an unreported time-point (1 study). Risk of donor site or flap seroma was between 0.5 and 5.5 percent after the first year ( 2 studies) and between 0.4 and 4.1 percent at unreported time-points ( 3 studies). Although a statistical test evaluating subgroup effects was not reported, one study (Chang 2011) reported comparable risks of donor site seroma among patients aged $<50$ years, 50 to 59 years, 60 to 69 years, and $>70$ years. Risks of seroma at unspecified locations were 1.9 percent in the first month (1 study), between 1.6 and 2.9 percent between 1 month and 1 year ( 3 studies), between 0.3 and 4.6 percent after 1 year (4 studies), and between 0.6 and 2.1 percent at unreported time-points ( 5 studies). 


\section{Scarring}

No NRCS reported on scarring.

Two single group studies (Cordeiro 2015a and Yoo 2014) reported scarring data (Appendix Table F-1.23). Cordeiro 2015a, which assessed IBR, reported that at 2 years of followup, 1.4 percent of patients experienced hypertrophic or other scarring. Yoo 2014, which assessed AR, reported that 2.6 percent of patients experienced breast hypertrophic scarring and 5.2 percent experienced donor site hypertrophic scarring. Yoo 2014 did not report time-points for either outcome.

\section{Reconstructive Failure}

Five NRCSs reported data for reconstructive failure (Appendix Table E-1.6).

Two NRCSs (Chetta 2017 and Mioton 2013) reported inconsistent data in the short-term (1 to 1.3 months of followup). Chetta 2017, without defining reconstructive failure, reported that, compared with women who underwent IBR, women who underwent AR had a considerably lower risk of reconstructive failure (adjOR $0.09,95 \%$ CI 0.07 to 0.13 ). On the other hand, Mioton 2013, defining reconstructive failure as implant or flap failure, reported a higher risk among women who underwent AR than IBR (adjOR 1.69, 95\% CI 1.08 to 2.62).

Three NRCSs (Fischer 2013, Garvey 2012, and Kulkarni 2017) reported considerably lower risks among women who underwent AR than IBR in the long-term (1.5 to 4 years of followup). Fischer 2013, defining reconstructive failure as unplanned nonaesthetic tissue expander or implant removal related to a complication in patients with IBR or flap loss in patients with AR, reported an adjOR of 0.19 (95\% CI 0.04 to 0.80$)$ at 4 years. Garvey 2012, without defining reconstructive failure, reported $\mathrm{P}<0.001$ (adjusted effect size not reported) at 1.5 years. The findings of Kulkarni 2017 agreed with these two NRCSs, but Kulkarni 2017 reported data (without defining reconstructive failure) separately for women who underwent unilateral reconstructions (adjOR $0.12,95 \%$ CI 0.04 to 0.36 ) and for women who underwent bilateral reconstructions (adjOR $0.14,95 \%$ CI 0.05 to 0.45 ). We do not report a meta-analysis for reconstructive failure because of substantial heterogeneity across studies $\left(\mathrm{I}^{2}=98 \%\right)$.

\section{Hematoma or Hemorrhage}

One NRCS and 37 single group studies reported hematoma or hemorrhage data.

The NRCS (Fischer 2014) reported comparable risks of breast hematoma at 1.8 to 2.1 years between women who underwent IBR and $\mathrm{AR}(\mathrm{P}=1.0$; adjusted effect size not reported).

Among the 37 single group studies, 13 studies assessed IBR and 24 studies AR (Appendix Table F-1.25). Among the 13 IBR studies, breast hematoma risks ranged from 2.7 to 6.3 percent in the first month ( 2 studies), from 0 to 12.6 percent between 1 month and 1 year ( 6 studies), from 2.0 to 2.5 percent after 1 year ( 2 studies), and from 0.4 to 4.1 percent at unreported time-points ( 2 studies). One study reported that the risk of breast hematoma or hemorrhage was 2.1 percent at an unreported time-point. In two other studies, the risk of needing blood transfusion were 3.2 and 8.6 percent, but the time-points were not reported in either study.

Among the 24 AR studies, breast (or unspecified location) hematoma risks were between 1.6 and 4.7 percent in the first month (4 studies), between 1.3 and 3.2 percent between 1 month and 1 year ( 3 studies), between 1.3 and 5.8 percent after 1 year ( 4 studies), and between 0.9 and 6.4 percent at unreported time-points ( 8 studies). Breast (or unspecified location) hematoma or hemorrhage risks were between 3.1 and 3.5 percent at unreported time-points ( 2 studies). Risks of breast (or unspecified location) hematoma necessitating transfusion or operation 
were 8.0 percent in the first month ( 1 study), 5.7 percent after 1 year ( 1 study), and between 0.9 and 8.0 percent at unreported time-points ( 2 studies). Donor site or flap hematoma risks were 0.8 percent between 1 month and 1 year ( 2 studies), 0.4 percent beyond 1 year ( 1 study), and 0.1 to 1.7 percent at unreported time-points ( 2 studies).

\section{Composite or Unspecified Harms}

One RCT, 13 NRCSs, and five single group studies reported on various composite or unspecified harms (Appendix Table F-1.26). There was considerable inconsistency across studies in how harms were defined, which precluded meta-analysis.

The RCT (Brorson 2020a) used the Clavien-Dindo classification system and reported that, at 1 month of followup, women who underwent IBR or AR experienced comparable risks of Grades I, II, IIIa, and IIIb complications. No patients experienced Grades IV or V complications.

Among the 13 NRCSs, patients who underwent AR generally experienced higher risks of composite or unspecified harms. Six NRCSs reported risks of overall (or "any") complications. Seven of these NRCSs (Chetta 2017, Kouwenberg 2020, Kulkarni 2017, Mak 2020, Palve 2020, Qin 2018, and Simon 2020), reported that, between 1 month and 3.7 years of followup, risks were higher among patients who underwent AR than those who underwent IBR (adjORs ranged from 1.36 to 8.28). Kulkarni 2017 reported separate comparisons between various AR flap types and IBR; risks of overall complications were higher for each flap group (DIEP, free TRAM, pedicled TRAM, LD, and SIEA) than the IBR group at both 1 and 2 years. On the other hand, two NRCSs (Laporta 2017 and Xu 2018) reported comparable risks of overall complications between IBR and AR groups.

Six NRCSs reported on risks of major complications or complications requiring hospitalization. Four of these NRCSs (Dauplat 2021, Fischer 2015, Kulkarni 2017, and Liu 2014) reported that, between 1 month and 1 year of followup, risks were higher among patients who underwent AR than those who underwent IBR (adjORs ranged from 1.36 to 5.36). Kulkarni 2017 reported that, compared with the IBR group, 1-year risks of major complications were higher for each flap group, except for the LD flap group, for which the risk was comparable to the IBR group (adjOR 0.98, 95\% CI 0.47 to 2.00). Kulkarni 2017 reported a similar pattern of data for the outcome re-operative complications at 2 years of followup. However, the other two NRCSs (Mak 2020 and Fischer 2014) reported conflicting results. Mak 2020 reported that risks of re-operative complications were comparable in the IBR and AR groups at 1 month $(\mathrm{P}=0.99$; adjusted effect size not reported), while Fischer 2014 reported that the risk of complications requiring hospitalization was near-significantly higher in the IBR group at 1.8-2.1 years ( $\mathrm{P}=0.08$; adjusted effect size not reported).

Among the five single group studies, one assessed IBR and four AR (Appendix Table F1.26). In the IBR study (Salibian 2019), major ischemic complications occurred in 6.7 percent of patients at 3.3 years of followup. In the four AR studies, the risk of overall complications at 9 years of followup was 19.3 percent ( 1 study), the risk of wound problems at 9 to 10 months of followup was 12.6 percent ( 1 study), the risk of breast seroma, hematoma, or wound infection at 5 years of followup was 3.4 percent ( 1 study), and the risk of deep vein thrombosis, pulmonary embolism, myocardial infarction and others was 6.4 percent (1 study).

\section{Heterogeneity of Treatment Effects (Subgroup Differences)}

No NRCS formally analyzed possible heterogeneity of treatment effects, i.e., statistical tests for whether the comparative effect of AR versus IBR (e.g., the odds ratio [OR]) differs in one 
subgroup of patients versus another. One NRCS (Kulkarni 2017) report data separately for the subgroups of women who underwent unilateral and bilateral reconstructions, but it is unclear whether relative outcomes differed between the subgroups. In Kulkarni 2017, the inferences regarding the relative effects of AR versus IBR on the clinical outcomes of psychosocial wellbeing, sexual well-being, and satisfaction with breasts as well as the surgical complication of reconstructive failure were similar between the unilateral and bilateral reconstruction subgroups.

\section{Applicability}

A majority of the studies were conducted in North America (55\% of NRCSs and $64 \%$ of single group studies). From limited reported data, it appears that the large majority of women in the North American studies were White. However, because about 80 to 90 percent of women who undergo breast reconstruction in the U.S are White, ${ }^{19}$ it is likely that the studies are applicable to the U.S. population, despite the implied large disparity.

\section{Overall Summary for Key Question 1}

Compared with IBR, AR is probably associated with clinically better patient satisfaction with breasts and sexual well-being but comparable general quality of life and psychosocial well-being (Moderate SoE, all outcomes). AR probably poses a greater risk of deep vein thrombosis or pulmonary embolism (Moderate SoE), but IBR probably poses a greater risk of reconstructive failure in the long term (1.5 to 4 years) (Moderate SoE) and may pose a greater risk of breast seroma (Low SoE). 


\section{Key Question 2: Timing of Implant-Based Reconstruction or Autologous Reconstruction in Relation to Chemotherapy and Radiation Therapy}

\section{Key Points}

- We did not find any studies addressing timing of IBR or AR in relation to chemotherapy.

- Conducting IBR either before or after radiation therapy may result in comparable physical well-being, psychosocial well-being, sexual well-being, and patient satisfaction with breasts (Low SoE for all). Because of sparse data, there is insufficient evidence for patient satisfaction with outcome regarding timing of IBR in relation to radiation therapy.

- Conducting IBR either before or after radiation therapy probably results in comparable risk of implant failure/loss or need for explant surgery (summary adjOR $0.87,95 \% \mathrm{CI}$ 0.62 to $1.24 ; 3$ studies) (Moderate SoE).

- Because of sparse data, there is insufficient evidence regarding timing of IBR and risks of unplanned repeat surgeries for revision of reconstruction, pain, necrosis, or seroma.

- We did not find any studies addressing timing of AR in relation to radiation therapy.

\section{Key Question 2a. Chemotherapy}

None of the studies compared timing of chemotherapy relative to IBR or to AR.

\section{Key Question 2b. Radiation Therapy}

Five NRCSs, reported in 10 articles, ${ }^{192-201}$ evaluated the timing of radiation therapy relative to IBR in a total of 2,834 patients. The studies are detailed in Appendix Tables C-3, D-2, and D3. Appendix Tables C-1, D-2, D-3, and F-2.1 to F-2.15 include full results data for all NRCSs, irrespective of whether they reported adjusted effect sizes.

In Eriksson 2013, all patients received partial submuscular implants; other studies did not report the anatomic plane of implant placement. In Stein 2020, the mean size of the implants ranged from 406 to $444 \mathrm{cc}$; other studies did not report implant sizes. None of the studies reported additional information about the implants, such as surface (smooth versus textured) or shape (round versus tear drop). We rated four NRCSs to be at overall high risk of bias, mostly related to serious risk of confounding and the lack of blinding of participants, study personnel, and/or outcome assessors. We rated one NRCS (Yoon 2020) to be at overall moderate risk of bias.

The five studies included between 130 and 1,143 women each. The studies were conducted in the U.S. $(n=2)$, U.S. and Canada $(n=1)$, Canada $(n=1)$, and Sweden $(n=1)$. Average ages of patients were similar across studies, ranging from 45 to 55 years. Average BMIs were also similar across studies, ranging from 23.7 to $26.5 \mathrm{~kg} / \mathrm{m}^{2}$. In one of the North American studies (Yoon 2020) 94 percent of patients were White and 3 percent were Black; the other studies did not report on race. Studies followed women for between 2 and 3.6 years.

The study result summaries are in Figure 7 and Appendix Tables E-2.1 and E-2.2. Full results are in Appendix Tables F-2.1 to F-2.15. 


\section{Summary of Comparison of Timing of Chemotherapy and Radiation Therapy Relative to IBR or AR}

Table 4 summarizes the evidence for the comparison of timing of chemotherapy and radiation therapy relative to IBR or AR. There is low SoE that physical well-being, psychosocial well-being, sexual well-being, and patient satisfaction with breasts may be comparable whether IBR is conducted before or after radiation therapy There is moderate SoE that the risk of implant failure/loss or need for explant surgery may be comparable whether IBR is conducted before or after radiation therapy (adjOR $0.87,95 \%$ CI 0.62 to 1.24 ). There is no evidence to support whether the effect of IBR timing differs based on patient, surgeon, implant, or other characteristics. There is insufficient (or no) comparative evidence regarding other outcomes related to timing of radiation therapy with IBR. There is no comparative evidence regarding timing of chemotherapy relative to IBR or AR or regarding timing of radiation therapy relative to AR. 
Table 4. Evidence profile for Key Question 2: Timing of IBR and AR in relation to chemotherapy and radiation therapy

\begin{tabular}{|c|c|c|c|c|c|c|c|c|c|c|}
\hline Comparison & $\begin{array}{l}\text { Outcome } \\
\text { Category }\end{array}$ & Outcome & $\begin{array}{l}\text { N } \\
\text { Studies } \\
\text { (Patients) }\end{array}$ & RoB & Consistency & Precision & Directness & Other & SoE & $\begin{array}{l}\text { Conclusions (Reason, if } \\
\text { None) }\end{array}$ \\
\hline \multirow{2}{*}{$\begin{array}{l}\text { IBR before vs. } \\
\text { after } \\
\text { chemotherapy }\end{array}$} & $\begin{array}{l}\text { Clinical } \\
\text { outcomes }\end{array}$ & All & $0(0)$ & $\mathrm{N} / \mathrm{A}$ & $\mathrm{N} / \mathrm{A}$ & $\mathrm{N} / \mathrm{A}$ & $\mathrm{N} / \mathrm{A}$ & $\mathrm{N} / \mathrm{A}$ & $\mathrm{N} / \mathrm{A}$ & $\mathrm{N} / \mathrm{A}$ \\
\hline & $\begin{array}{l}\text { Surgical } \\
\text { complications }\end{array}$ & All & $0(0)$ & N/A & $\mathrm{N} / \mathrm{A}$ & $\mathrm{N} / \mathrm{A}$ & $\mathrm{N} / \mathrm{A}$ & $\mathrm{N} / \mathrm{A}$ & $\mathrm{N} / \mathrm{A}$ & $\mathrm{N} / \mathrm{A}$ \\
\hline \multirow{10}{*}{$\begin{array}{l}\text { IBR before vs. } \\
\text { after radiation } \\
\text { therapy }\end{array}$} & $\begin{array}{l}\text { Clinical } \\
\text { outcomes }\end{array}$ & $\begin{array}{l}\text { Physical well- } \\
\text { being }\end{array}$ & $2(423)$ & High & Consistent & Precise & Direct & Sparse & Low & $\begin{array}{l}\text { Comparable in both } \\
\text { groups }\end{array}$ \\
\hline & $\begin{array}{l}\text { Clinical } \\
\text { outcomes }\end{array}$ & $\begin{array}{l}\text { Psychosocial } \\
\text { well-being }\end{array}$ & $2(423)$ & High & Consistent & Precise & Direct & Sparse & Low & $\begin{array}{l}\text { Comparable in both } \\
\text { groups }\end{array}$ \\
\hline & $\begin{array}{l}\text { Clinical } \\
\text { outcomes }\end{array}$ & $\begin{array}{l}\text { Sexual well- } \\
\text { being }\end{array}$ & $2(423)$ & High & Consistent & Precise & Direct & Sparse & Low & $\begin{array}{l}\text { Comparable in both } \\
\text { groups }\end{array}$ \\
\hline & $\begin{array}{l}\text { Clinical } \\
\text { outcomes }\end{array}$ & $\begin{array}{l}\text { Satisfaction with } \\
\text { breasts }\end{array}$ & $2(423)$ & High & Consistent & Precise & Direct & Sparse & Low & $\begin{array}{l}\text { Comparable in both } \\
\text { groups }\end{array}$ \\
\hline & $\begin{array}{l}\text { Clinical } \\
\text { outcomes }\end{array}$ & $\begin{array}{l}\text { Satisfaction with } \\
\text { outcome }\end{array}$ & $1(106)$ & High & Unclear & Precise & Direct & Sparse & Insufficient & None (Sparse evidence) \\
\hline & $\begin{array}{l}\text { Surgical } \\
\text { complications }\end{array}$ & $\begin{array}{l}\text { Unplanned } \\
\text { repeat surgeries } \\
\text { for revision }\end{array}$ & $1(368)$ & High & Unclear & Precise & Direct & Sparse & Insufficient & None (Sparse evidence) \\
\hline & $\begin{array}{l}\text { Surgical } \\
\text { complications }\end{array}$ & Pain & $1(317)$ & Moderate & Unclear & Precise & Direct & Sparse & Insufficient & None (Sparse evidence) \\
\hline & $\begin{array}{l}\text { Surgical } \\
\text { complications }\end{array}$ & Necrosis & $1(876)$ & High & Unclear & Precise & Direct & Sparse & Insufficient & None (Sparse evidence) \\
\hline & $\begin{array}{l}\text { Surgical } \\
\text { complications }\end{array}$ & $\begin{array}{l}\text { Implant } \\
\text { failure/loss or } \\
\text { need for explant }\end{array}$ & $4(2537)$ & High & Consistent & Precise & Direct & None & Moderate & $\begin{array}{l}\text { Comparable in both } \\
\text { groups: summary adjOR } \\
0.87 \text { ( } 95 \% \mathrm{Cl} 0.62,1.24) \text {; } \\
3 \text { studies }\end{array}$ \\
\hline & $\begin{array}{l}\text { Surgical } \\
\text { complications }\end{array}$ & Seroma & $1(150)$ & Moderate & Unclear & Unclear & Direct & Sparse & Insufficient & None (Sparse evidence) \\
\hline \multirow{2}{*}{$\begin{array}{l}\text { AR before vs. } \\
\text { after } \\
\text { chemotherapy }\end{array}$} & $\begin{array}{l}\text { Clinical } \\
\text { outcomes }\end{array}$ & All & $0(0)$ & $\mathrm{N} / \mathrm{A}$ & $\mathrm{N} / \mathrm{A}$ & $\mathrm{N} / \mathrm{A}$ & $\mathrm{N} / \mathrm{A}$ & $\mathrm{N} / \mathrm{A}$ & $\mathrm{N} / \mathrm{A}$ & $\mathrm{N} / \mathrm{A}$ \\
\hline & $\begin{array}{l}\text { Surgical } \\
\text { complications }\end{array}$ & All & $0(0)$ & $\mathrm{N} / \mathrm{A}$ & $\mathrm{N} / \mathrm{A}$ & $\mathrm{N} / \mathrm{A}$ & $\mathrm{N} / \mathrm{A}$ & $\mathrm{N} / \mathrm{A}$ & $\mathrm{N} / \mathrm{A}$ & $\mathrm{N} / \mathrm{A}$ \\
\hline \multirow{2}{*}{$\begin{array}{l}\text { AR before vs. } \\
\text { after radiation } \\
\text { therapy }\end{array}$} & $\begin{array}{l}\text { Clinical } \\
\text { outcomes }\end{array}$ & All & $0(0)$ & $\mathrm{N} / \mathrm{A}$ & $\mathrm{N} / \mathrm{A}$ & $\mathrm{N} / \mathrm{A}$ & $\mathrm{N} / \mathrm{A}$ & $\mathrm{N} / \mathrm{A}$ & $\mathrm{N} / \mathrm{A}$ & $\mathrm{N} / \mathrm{A}$ \\
\hline & $\begin{array}{l}\text { Surgical } \\
\text { complications }\end{array}$ & All & $0(0)$ & $\mathrm{N} / \mathrm{A}$ & $\mathrm{N} / \mathrm{A}$ & $\mathrm{N} / \mathrm{A}$ & $\mathrm{N} / \mathrm{A}$ & $\mathrm{N} / \mathrm{A}$ & N/A & N/A \\
\hline
\end{tabular}

Abbreviations: adj $=$ adjusted, $\mathrm{AR}=$ autologous reconstruction, $\mathrm{CI}=$ confidence interval, $\mathrm{IBR}=$ implant-based reconstruction, $\mathrm{N} / \mathrm{A}=$ not applicable, $\mathrm{OR}=$ odds ratio, $\mathrm{RoB}=$ risk

of bias, SoE $=$ strength of evidence. 
Colors: Header rows are shaded orange. Rows for every alternate comparison are shaded blue. The colors do not add unique information.

Appendix Table G-2 provides the complete version of this evidence profile, including displaying all outcomes for which no evidence was identified 


\section{Clinical Outcomes}

Two NRCSs (Cordeiro 2015 and Yoon 2020) compared clinical outcomes in women receiving IBR before versus or after radiation therapy (Appendix Table E-2.1).

Both NRCSs evaluated subscales of the BREAST-Q at a mean of 2 years (Yoon 2020) and 3.3 years (Cordeiro 2015) of followup. Each subscale score ranges from 0 to 100, with higher scores indicating better well-being or satisfaction. For patients receiving implants, the MCID for physical well-being has been estimated to be 3 points, psychosocial well-being 4 points, sexual well-being 5 points, and satisfaction with breasts 5 points. ${ }^{187}$ We considered 5 points as the MCID for satisfaction with outcome. Yoon 2020 also reported data for physical function using PROMIS (scores 0 to 100; higher is worse; MCID 3 to 4.5 points ${ }^{188}$ ) and sexual function using the EORTC (scores 0 to 100; higher is worse; MCID 4 to 10 points $^{185}$ ).

For physical well-being, Yoon 2020 reported comparable BREAST-Q scores before versus after radiation therapy (adjMD $-0.64,95 \% \mathrm{CI}-7.19$ to 5.90) and so did Cordeiro 2015 (mean 73.4 vs. 72.5; $\mathrm{P}=\mathrm{NS}$ ). Yoon 2020 also reported comparable PROMIS physical function scores (adjMD -0.04, 95\% CI -2.40 to 2.32). For psychosocial well-being, Yoon 2020 reported comparable BREAST-Q scores before versus after radiation therapy (adjMD 0.48, 95\% CI -7.72 to 8.68 ), and Cordeiro 2015 reported a difference that was statistically but not clinically significant (mean 72.3 vs. 71.1; $\mathrm{P}<0.01$ ). For sexual well-being, Yoon 2020 reported comparable BREAST-Q scores before versus after radiation therapy (adjMD $-1.00,95 \% \mathrm{CI}$ -8.41 to 6.40 ), and Cordeiro 2015 reported a difference that was statistically but not clinically significant (mean 54.0 vs. 55.4; $\mathrm{P}<0.01$ ). Yoon 2020 also reported comparable EORTC sexual function scores (adjMD $-1.40,95 \% \mathrm{CI}-8.58$ to 5.77). For satisfaction with breasts, Yoon 2020 reported comparable BREAST-Q scores before versus after radiation therapy (adjMD -3.89, 95\% CI -11.0 to 3.23) and so did Cordeiro 2015 (mean 56.2 vs. 57.2; P=NS). For satisfaction with surgical outcome, Cordeiro 2015 reported a difference in BREAST-Q scores that was statistically but not clinically significant (mean 68.4 vs. $70.2 ; \mathrm{P}=0.02$ ).

\section{Surgical Complications}

All five NRCSs reported on surgical complications comparing IBR before versus after radiation therapy (Figure 7 and Appendix Tables E-2.1 and E-2.2).

One NRCS (Yoon 2020) reported continuous data for pain using the pain interference component of PROMIS (scores 0 to 100; higher is worse; MCID 3 to 4.5 points ${ }^{188}$ ). At 2 years of followup, PROMIS scores were comparable before versus after radiation therapy (adjMD 2.86, $95 \% \mathrm{CI}-1.05$ to 6.77$)$.

One NRCS (Eriksson 2013) reported that, at 3.6 years of followup, rates of unplanned repeat surgeries for revision of reconstruction were comparable between women who underwent IBR before radiation and those who underwent IBR after radiation (adjusted hazard ratio [adjHR] 0.94, 95\% CI 0.63 to 1.40). Similarly, one NRCS (Hirsch 2014) reported that, at 3.1 years of followup, risks of necrosis were comparable between women who underwent IBR before radiation and after radiation (adjHR 0.96, 95\% CI 0.68 to 1.35 ).

One NRCS (Yoon 2020) reported on major and minor infections at 2 years of followup after IBR. Major infections were defined as those requiring treatment with intravenous antibiotics with or without return to surgery, while minor infections were defined as those treated with oral antibiotics. Although adjusted effect sizes were not reported, the authors reported that there were no significant differences in risks of either major $(\mathrm{P}=0.40)$ or minor infections 
$(\mathrm{P}=0.96)$ when comparing IBR before versus after radiation therapy. Yoon 2020 additionally reported that, at the same followup time-point (2 years), the incidence of three other surgical complications were also similar between the treatment groups: wound dehiscence $(\mathrm{P}=0.32)$, seroma $(P=0.46)$, and capsular contracture $(P=0.80)$.

Two NRCSs (Hirsch 2014 and Yoon 2020) reported that hematoma rates were similar between women receiving IBR before or after radiation therapy (Appendix Table E-2.1). Hirsch 2014 reported data at 3.1 years of followup (adjOR 0.56, 95\% CI 0.22 to 1.45), while Yoon 2020 reported data at 2 years of followup $(\mathrm{P}=0.632)$.

Three NRCSs reported on implant failure/loss or need for explant surgery at average followup durations ranging from 2 to 3.5 years (Figure 7). Two studies reported adjORs, while one (Eriksson 2013) reported adjHRs. Because the events were rare $(<2 \%)$ in both groups of the Eriksson 2013 study, we considered the adjHR to be a reliable estimate of the adjOR and therefore combined estimates from all four studies. Effect sizes ranged from 0.62 to 1.12 across studies. The meta-analysis did not provide evidence for a between-group difference for the outcome of implant failure/loss or need for explant surgery (adjOR 0.87, 95\% CI 0.62 to 1.24; $\left.\mathrm{I}^{2}=54 \%\right)$.

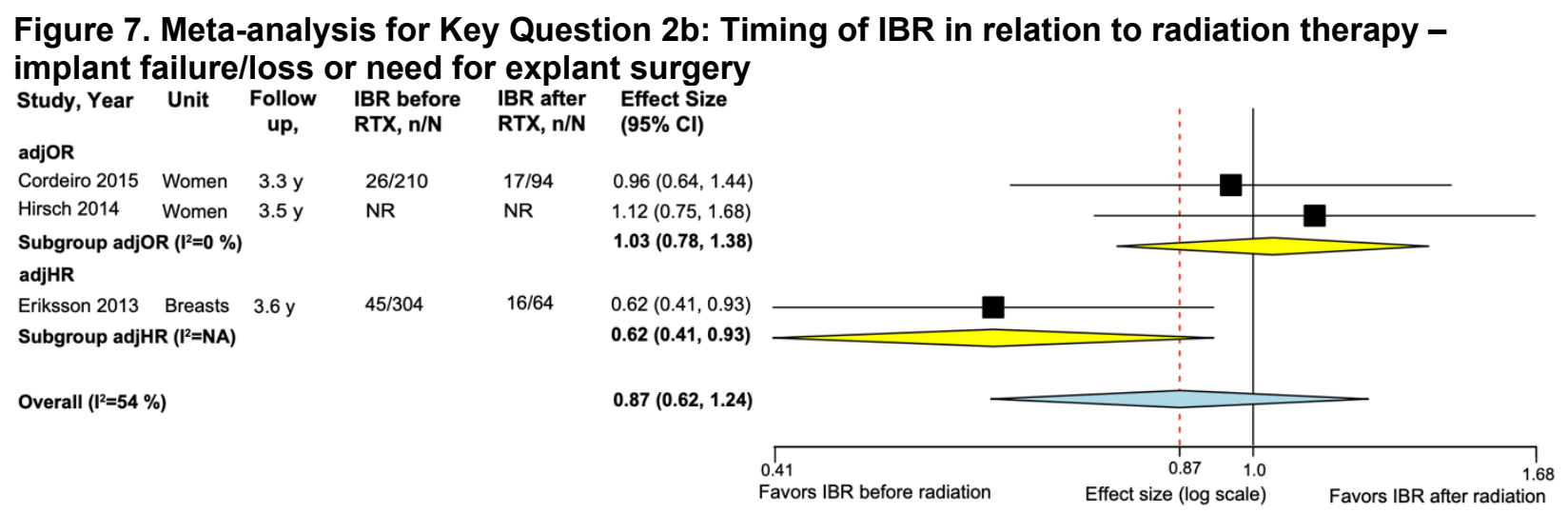

Abbreviations: adj $=$ adjusted, $\mathrm{CI}=$ confidence interval, $\mathrm{HR}=$ hazard ratio $=\mathrm{IBR}=$ implant-based reconstruction, $\mathrm{I}^{2}=$ measure of statistical heterogeneity ( $\%$ of total variability that is due to between-study variability), NR = not reported, OR $=$ odds ratio, $\mathrm{RTX}=$ radiation therapy, $\mathrm{y}=$ years.

One NRCS reporting implant failure data that was not included in the meta-analysis due to the lack of a reported adjusted effect size (Yoon 2020) reported that implant failure was more common in patients who underwent IBR after radiation therapy $(\mathrm{P}=0.04)$.

Three NRCSs (Hirsch 2014, Stein 2020, and Yoon 2020) reported data for a heterogeneous group of composite or unspecified harms, each of which was comparable between groups of women receiving IBR before or after radiation therapy. These included any complication, major complications, major complications needing hospitalization or surgery, operative complications, nonoperative complications, and minor complications.

\section{Heterogeneity of Treatment Effects (Subgroup Differences)}

None of the studies reported subgroup results or other analyses of possible heterogeneity of treatment effects. The studies were too sparse to allow exploration of possible differences based on patient or other characteristics. 


\section{Applicability}

Four of the five studies addressing this KQ were conducted in North America. All studies were published in the last 8 years. Generally, these studies, including the Swedish study (Eriksson 2013), contribute evidence that is directly applicable to the population of women receiving implants in the U.S. that has been shown to be between 80 and 90 percent White. ${ }^{19}$

\section{Overall Summary for Key Question 2}

Conducting IBR either before or after radiation therapy may result in comparable physical well-being, psychosocial well-being, sexual well-being, and patient satisfaction with breasts (all Low SoE) and probably results in comparable risks of implant failure/loss or need for explant surgery (Moderate SoE). We found no evidence addressing timing of IBR or AR in relation to chemotherapy or timing of AR in relation to radiation therapy. 


\section{Key Question 3: Comparisons of Implant Materials for Implant-Based Reconstruction}

\section{Key Points}

- Silicone and saline implants may result in clinically comparable assessments of patient satisfaction with breasts (Low SoE).

- Because of sparse data, there is insufficient evidence to make conclusions about other clinical outcomes or about surgical complications when comparing materials used for IBR (silicone, saline, or double lumen [i.e., silicone and saline]).

Five retrospective NRCSs, reported in five articles, ${ }^{18,192,202-204}$ compared different implant materials in a total of 2,929 patients undergoing IBR. The studies are detailed in Appendix Tables C-4, D-2, and D-3. None of the studies reported additional information about the implants, such as surface (smooth versus textured), shape (round versus tear drop), size, or anatomic plane of placement. We rated all five NRCSs to be at overall high risk of bias, mostly related to serious risk of confounding and the lack of blinding of participants, study personnel, and/or outcome assessors. Not all the NRCSs reported adjusted effect sizes with confidence intervals; some reported adjusted $P$ values without effect sizes.

All five NRCSs compared silicone and saline implants. One study (Le 2005) also included a third group of women with double lumen (i.e., silicone and saline) implants. The studies included between 143 and 1,143 women each and were conducted in the U.S. $(n=3)$, Canada $(n=1)$, or both $(n=1)$. Among the studies reporting data, the women's mean ages ranged from 47 to 56 years and their mean BMIs ranged from 24.3 to $27.2 \mathrm{~kg} / \mathrm{m}^{2}$. Most studies did not report patient race. In Le 2005, the large majority (94\%) were White; in Macadam 2010, a study conducted in Vancouver, Canada, the majority (66\%) were Asian. Studies mostly followed women for about 3 to 5 years, with the exception of Le 2005, which included a median of 12.4 years of followup.

The study result summaries are in Appendix Tables E-3.1 and E-3.2. Full results are in Appendix Tables F-3.1 to F-3.9.

\section{Summary of Comparison of Implant Materials}

Table 5 summarizes the evidence comparing implant materials for IBR. The only conclusion we are able to make is that, in comparison with saline implants, silicone implants are associated with clinically comparable satisfaction with breasts. Due to sparse evidence, no conclusions regarding other clinical outcomes or regarding surgical complications are feasible for this comparison or for any comparison involving double lumen implants. 
Table 5. Evidence profile for Key Question 3: Comparisons of implant materials for IBR

\begin{tabular}{|c|c|c|c|c|c|c|c|c|c|c|}
\hline $\begin{array}{l}\text { Comparis } \\
\text { on }\end{array}$ & $\begin{array}{l}\text { Outcome } \\
\text { Category }\end{array}$ & Outcome & $\begin{array}{l}\text { N } \\
\text { Studies } \\
\text { (Patients) }\end{array}$ & RoB & Consistency & Precision & Directness & Other & SoE & $\begin{array}{l}\text { Conclusions } \\
\text { (Reason, if } \\
\text { None) }\end{array}$ \\
\hline \multirow[t]{9}{*}{$\begin{array}{l}\text { Silicone } \\
\text { vs. saline }\end{array}$} & $\begin{array}{l}\text { Clinical } \\
\text { outcomes }\end{array}$ & General quality of life & $1(139)$ & High & $\mathrm{N} / \mathrm{A}$ & Unclear & Direct & Sparse & Insufficient & $\begin{array}{l}\text { None (Sparse } \\
\text { evidence) }\end{array}$ \\
\hline & $\begin{array}{l}\text { Clinical } \\
\text { outcomes }\end{array}$ & Physical well-being & $1(142)$ & High & $\mathrm{N} / \mathrm{A}$ & Unclear & Direct & Sparse & Insufficient & $\begin{array}{l}\text { None (Sparse } \\
\text { evidence) }\end{array}$ \\
\hline & $\begin{array}{l}\text { Clinical } \\
\text { outcomes }\end{array}$ & Psychosocial well-being & $1(142)$ & High & $\mathrm{N} / \mathrm{A}$ & Unclear & Direct & Sparse & Insufficient & $\begin{array}{l}\text { None (Sparse } \\
\text { evidence) }\end{array}$ \\
\hline & $\begin{array}{l}\text { Clinical } \\
\text { outcomes }\end{array}$ & Sexual well-being & $1(137)$ & High & $\mathrm{N} / \mathrm{A}$ & Unclear & Direct & Sparse & Insufficient & $\begin{array}{l}\text { None (Sparse } \\
\text { evidence) }\end{array}$ \\
\hline & $\begin{array}{l}\text { Clinical } \\
\text { outcomes }\end{array}$ & $\begin{array}{l}\text { Patient satisfaction with } \\
\text { breasts }\end{array}$ & $2(624)$ & High & Consistent & Unclear & Direct & None & Low & $\begin{array}{l}\text { Comparable ir } \\
\text { both groups }\end{array}$ \\
\hline & $\begin{array}{l}\text { Clinical } \\
\text { outcomes }\end{array}$ & $\begin{array}{l}\text { Patient satisfaction with } \\
\text { outcome }\end{array}$ & $1(143)$ & High & $\mathrm{N} / \mathrm{A}$ & Unclear & Direct & Sparse & Insufficient & $\begin{array}{l}\text { None (Sparse } \\
\text { evidence) }\end{array}$ \\
\hline & $\begin{array}{l}\text { Clinical } \\
\text { outcomes }\end{array}$ & Mortality & 1 (NR) & High & $\mathrm{N} / \mathrm{A}$ & Imprecise & Direct & Sparse & Insufficient & $\begin{array}{l}\text { None (Sparse } \\
\text { evidence) }\end{array}$ \\
\hline & $\begin{array}{l}\text { Surgical } \\
\text { complications }\end{array}$ & $\begin{array}{l}\text { Implant failure/loss or } \\
\text { need for explant } \\
\text { surgery }\end{array}$ & $1(288)$ & High & $\mathrm{N} / \mathrm{A}$ & Precise & Direct & Sparse & Insufficient & $\begin{array}{l}\text { None (Sparse } \\
\text { evidence) }\end{array}$ \\
\hline & $\begin{array}{l}\text { Surgical } \\
\text { complications }\end{array}$ & Capsular contracture & $1(345)$ & High & $\mathrm{N} / \mathrm{A}$ & Unclear & Direct & Sparse & Insufficient & $\begin{array}{l}\text { None (Sparse } \\
\text { evidence) }\end{array}$ \\
\hline \multirow{2}{*}{$\begin{array}{l}\text { Silicone } \\
\text { vs. } \\
\text { double } \\
\text { lumen }\end{array}$} & $\begin{array}{l}\text { Clinical } \\
\text { outcomes }\end{array}$ & Mortality & 1 (NR) & High & $\mathrm{N} / \mathrm{A}$ & Imprecise & Direct & Sparse & Insufficient & $\begin{array}{l}\text { None (Sparse } \\
\text { evidence) }\end{array}$ \\
\hline & $\begin{array}{l}\text { Surgical } \\
\text { complications }\end{array}$ & All & $0(0)$ & $\mathrm{N} / \mathrm{A}$ & $\mathrm{N} / \mathrm{A}$ & $\mathrm{N} / \mathrm{A}$ & $\mathrm{N} / \mathrm{A}$ & $\mathrm{N} / \mathrm{A}$ & $\mathrm{N} / \mathrm{A}$ & $\mathrm{N} / \mathrm{A}$ \\
\hline \multirow{2}{*}{$\begin{array}{l}\text { Saline vs. } \\
\text { double } \\
\text { lumen }\end{array}$} & $\begin{array}{l}\text { Clinical } \\
\text { outcomes }\end{array}$ & All & $0(0)$ & $\mathrm{N} / \mathrm{A}$ & $\mathrm{N} / \mathrm{A}$ & $\mathrm{N} / \mathrm{A}$ & $\mathrm{N} / \mathrm{A}$ & $\mathrm{N} / \mathrm{A}$ & $\mathrm{N} / \mathrm{A}$ & $\mathrm{N} / \mathrm{A}$ \\
\hline & $\begin{array}{l}\text { Surgical } \\
\text { complications }\end{array}$ & All & $0(0)$ & $\mathrm{N} / \mathrm{A}$ & $\mathrm{N} / \mathrm{A}$ & $\mathrm{N} / \mathrm{A}$ & $\mathrm{N} / \mathrm{A}$ & $\mathrm{N} / \mathrm{A}$ & $\mathrm{N} / \mathrm{A}$ & $\mathrm{N} / \mathrm{A}$ \\
\hline
\end{tabular}

Abbreviations: $\mathrm{IBR}=$ implant-based reconstruction, $\mathrm{N} / \mathrm{A}=$ not applicable, $\mathrm{NR}=$ not reported, $\mathrm{RoB}=$ risk of bias, $\mathrm{SoE}=$ strength of evidence.

For continuous outcomes, clinical significance is based on published estimates of minimal clinically important differences (MCIDs), where available.

Colors: Header rows are shaded orange. Rows for every alternate comparison are shaded blue. The colors do not add unique information.

Appendix Table G-3 provides the complete version of this evidence profile, including displaying all outcomes for which no evidence was identified. 


\section{Clinical Outcomes}

Three NRCSs (Macadam 2010, McCarthy 2010, and Le 2005) assessed clinical outcomes in women receiving either silicone or saline implants (Appendix Tables E-3.1 and E-3.2).

One NRCS (Macadam 2010) reported on general quality of life using the EORTC QLQC30 (0 to 100; higher is better; MCID 5 to 10 points $^{205}$ ) (Appendix Table E-3.1). Patients with silicone implants had higher mean global health status scores than those with saline implants. The difference was marginally clinically important, favoring silicone, but was not statistically significant (79.9 vs. $74.9 ; \mathrm{P}=0.13$ ).

Macadam 2010 also evaluated five subscales of the BREAST-Q at a mean of 3.6 years (range 2.6 to 4.5 ). Each subscale score ranges from 0 to 100, with higher scores indicating better wellbeing or satisfaction. For patients receiving implants, the MCID for physical well-being has been estimated to be 3 points, psychosocial well-being 4 points, sexual well-being 5 points, and satisfaction with breasts 5 points. ${ }^{187}$ We considered 5 points as the MCID for satisfaction with outcome. Physical well-being (per the physical function score) was similar between women receiving silicone and saline implants (mean 76.2 vs. $73.4 ; \mathrm{P}=0.29$ ). Psychosocial well-being was both clinically and statistically significantly better among women receiving silicone implants (77.6 vs. 70.8; $\mathrm{P}=0.03$ ). Sexual well-being was clinically but not statistically significantly better among women receiving silicone implants (54.4 vs. 47.6; $\mathrm{P}=0.06$ ). Satisfaction with outcome (defined as overall satisfaction) was also clinically but not statistically significantly better among women receiving silicone implants (75.4 vs. 69.5; $\mathrm{P}=0.08$ ).

Satisfaction with breasts on the BREAST-Q was evaluated by two NRCSs (Macadam 2010 and McCarthy 2010) at similar time-points (ranging from 2.4 to 4.5 years). McCarthy 2010 reported that patients in the silicone group scored higher by 4.1 points (95\% CI 1.31 to 6.89), but this difference was not clinically significant. Macadam 2010 reported that patients with silicone implants had statistically significantly higher scores $(\mathrm{P}=0.008)$, but no effect size was reported.

One NRCS (Le 2005) reported mortality data at a median of 12.4 years comparing groups of patients who underwent IBR with silicone, saline, or double lumen implants (Appendix Table E3.2). Breast cancer mortality was similar in the silicone and saline groups (adjHR 1.01, 95\% CI 0.44 to 2.34 ). Near-significantly higher breast cancer mortality was reported in the double lumen implant group than in the silicone group (adjHR 1.49, 95\% CI 0.83 to 2.70). Similar results were observed for non-breast cancer mortality, although the estimates were highly imprecise.

\section{Surgical Complications}

Two NRCSs (Cordeiro 2015a and Antony 2014) reported surgical complications (Appendix Table E-3.2).

Cordeiro 2015a reported that, at 3.3 years of followup, implant failure/loss occurred less frequently among patients with silicone implants than patients with saline implants, but this was not statistically significant (adjOR $0.61,95 \%$ CI 0.36 to 1.07 ).

Antony 2014 reported that, at 3 to 5 years of followup, patients who received silicone or saline implants experienced capsular contractures (of Baker Classification Grades 3 or 4) at rates that were not statistically significantly different. No further data were reported. 


\section{Heterogeneity of Treatment Effects (Subgroup Differences)}

No studies reported subgroup results or other analyses of heterogeneity of treatment effects. The studies were too sparse to explore differences based on patient or other characteristics.

\section{Applicability}

All five studies addressing this KQ were conducted in North America. All studies were published in the last 10 years, except for Le 2005, which was published 16 years ago. In one U.S. study (Le 2005), the large majority of women were White, while in another study in Vancouver, Canada (Macadam 2010), a substantial proportion of women (66\%) were Asian.

Notwithstanding the one Canadian study, these studies generally contribute evidence that is directly applicable to the population of women receiving implants in the U.S. who have been shown to be between 80 and 90 percent White. ${ }^{19}$

\section{Overall Summary for Key Question 3}

Silicone and saline implants may result in clinically comparable patient satisfaction with breasts (Low SoE). There is insufficient evidence regarding double lumen implants. 


\section{Key Question 4: Comparisons of Anatomic Planes of Implant Placement for Implant-Based Reconstruction}

\section{Key Points}

- Whether the implant is placed in prepectoral or total submuscular planes may not impact the risk of infections that are not explicitly implant-related (Low SoE). Because of sparse data, there is insufficient evidence addressing clinical outcomes for this comparison of planes.

- Because of sparse data, there is insufficient evidence addressing clinical outcomes and surgical complications for comparisons of prepectoral versus partial submuscular planes.

- There is no evidence for partial versus total submuscular planes of implant placement.

One RCT (Lee 2021b ${ }^{206}$ ) and seven NRCSs (Avila 2020, Cattelani 2018, Gabriel 2020, Kim

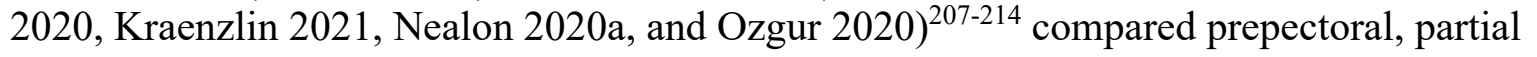
submuscular, and total submuscular planes of implant placement in a total of 1,555 patients undergoing IBR. Cattelani 2018 and Kim 2021b, conducted in Italy and South Korea, respectively, used textured implants, while Nealon 2020a, conducted in the U.S., used smooth implants; the other five studies did not report on the surface of the implants. In seven of the eight studies, reconstruction was immediate (i.e., implanted during the mastectomy) and single-staged (i.e., without use of tissue expanders).

We rated the RCT to be at overall moderate risk of bias, mostly related to lack of blinding of participants and study personnel and unclear risk of selective outcome reporting. We rated six of the seven NRCSs to be at overall high risk of bias and one at overall moderate risk of bias. Ratings were mostly related to critical or serious risk of confounding and the lack of blinding of participants, study personnel, and/or outcome assessors. The women's mean ages in the eight studies ranged from 43 to 53 years and mean BMIs 24.9 and $27.4 \mathrm{~kg} / \mathrm{m}^{2}$. None of the studies reported information on patient race. Participant followup ranged from 6 months to 6.1 years.

The studies are detailed in Appendix Tables C-5, D-2, D-3, E-4.1, and E-4.2. Full results data are in Appendix Tables F-4.1 to F-4.13.

\section{Summary of Comparisons of Anatomic Planes of Implant Placement}

Table 6 summarizes the evidence for the comparison of anatomic planes of implant placement for IBR. Due to sparse evidence, the only conclusion that is feasible regarding any comparison of anatomic planes is that prepectoral and total submuscular placement of implants may be associated with comparable risks of infections that are not explicitly implant-related. No conclusions regarding clinical outcomes are feasible. 
Table 6. Evidence profile for Key Question 4: Comparisons of anatomic planes of implant placement for IBR

\begin{tabular}{|c|c|c|c|c|c|c|c|c|c|c|}
\hline Comparison & $\begin{array}{l}\text { Outcome } \\
\text { Category }\end{array}$ & Outcome & $\begin{array}{l}\text { N } \\
\text { Studies } \\
\text { (Patients) }\end{array}$ & RoB & Consistency & Precision & Directness & Other & SoE & Conclusions \\
\hline \multirow[t]{11}{*}{$\begin{array}{l}\text { Prepectoral } \\
\text { vs. total } \\
\text { submuscular }\end{array}$} & $\begin{array}{l}\text { Clinical } \\
\text { outcomes }\end{array}$ & Physical well-being & $1(84)$ & High & $\mathrm{N} / \mathrm{A}$ & Unclear & Direct & Sparse & Insufficient & $\begin{array}{l}\text { None } \\
\text { (Sparse } \\
\text { evidence) }\end{array}$ \\
\hline & $\begin{array}{l}\text { Clinical } \\
\text { outcomes }\end{array}$ & $\begin{array}{l}\text { Psychosocial well- } \\
\text { being }\end{array}$ & $1(84)$ & High & $\mathrm{N} / \mathrm{A}$ & Unclear & Direct & Sparse & Insufficient & $\begin{array}{l}\text { None } \\
\text { (Sparse } \\
\text { evidence) }\end{array}$ \\
\hline & $\begin{array}{l}\text { Clinical } \\
\text { outcomes }\end{array}$ & $\begin{array}{l}\text { Satisfaction with } \\
\text { breasts }\end{array}$ & $1(84)$ & High & $\mathrm{N} / \mathrm{A}$ & Unclear & Direct & Sparse & Insufficient & $\begin{array}{l}\text { None } \\
\text { (Sparse } \\
\text { evidence) }\end{array}$ \\
\hline & $\begin{array}{l}\text { Surgical } \\
\text { complications }\end{array}$ & $\begin{array}{l}\text { Unplanned repeat } \\
\text { surgeries for revision }\end{array}$ & $1(405)$ & High & $\mathrm{N} / \mathrm{A}$ & Precise & Direct & Sparse & Insufficient & $\begin{array}{l}\text { None } \\
\text { (Sparse } \\
\text { evidence) }\end{array}$ \\
\hline & $\begin{array}{l}\text { Surgical } \\
\text { complications }\end{array}$ & Pain & $2(230)$ & High & Inconsistent & Precise & Direct & $\mathrm{N} / \mathrm{A}$ & Insufficient & $\begin{array}{l}\text { None } \\
\text { (Inconsistent } \\
\text { results) }\end{array}$ \\
\hline & $\begin{array}{l}\text { Surgical } \\
\text { complications }\end{array}$ & Analgesic use & $1(146)$ & High & $\mathrm{N} / \mathrm{A}$ & Precise & Direct & Sparse & Insufficient & $\begin{array}{l}\text { None } \\
\text { (Sparse } \\
\text { evidence) }\end{array}$ \\
\hline & $\begin{array}{l}\text { Surgical } \\
\text { complications }\end{array}$ & Necrosis & $1(256)$ & High & $\mathrm{N} / \mathrm{A}$ & Imprecise & Direct & Sparse & Insufficient & $\begin{array}{l}\text { None } \\
\text { (Sparse } \\
\text { evidence) } \\
\end{array}$ \\
\hline & $\begin{array}{l}\text { Surgical } \\
\text { complications }\end{array}$ & $\begin{array}{l}\text { Implant failure/loss or } \\
\text { need for explant } \\
\text { surgery }\end{array}$ & $1(256)$ & High & $\mathrm{N} / \mathrm{A}$ & Imprecise & Direct & Sparse & Insufficient & $\begin{array}{l}\text { None } \\
\text { (Sparse } \\
\text { evidence) }\end{array}$ \\
\hline & $\begin{array}{l}\text { Surgical } \\
\text { complications }\end{array}$ & $\begin{array}{l}\text { Infections (not explicitly } \\
\text { implant-related) }\end{array}$ & $2(542)$ & High & Direct & Precise & Direct & $\mathrm{N} / \mathrm{A}$ & Low & $\begin{array}{l}\text { Comparable } \\
\text { risk }\end{array}$ \\
\hline & $\begin{array}{l}\text { Surgical } \\
\text { complications }\end{array}$ & Capsular contracture & $1(256)$ & High & $\mathrm{N} / \mathrm{A}$ & Imprecise & Direct & Sparse & Insufficient & $\begin{array}{l}\text { None } \\
\text { (Sparse } \\
\text { evidence) } \\
\end{array}$ \\
\hline & $\begin{array}{l}\text { Surgical } \\
\text { complications }\end{array}$ & Seroma & $1(256)$ & High & $\mathrm{N} / \mathrm{A}$ & Imprecise & Direct & Sparse & Insufficient & $\begin{array}{l}\text { None } \\
\text { (Sparse } \\
\text { evidence) }\end{array}$ \\
\hline
\end{tabular}




\begin{tabular}{|c|c|c|c|c|c|c|c|c|c|c|}
\hline Comparison & $\begin{array}{l}\text { Outcome } \\
\text { Category }\end{array}$ & Outcome & $\begin{array}{l}\mathbf{N} \\
\text { Studies } \\
\text { (Patients) }\end{array}$ & RoB & Consistency & Precision & Directness & Other & SoE & Conclusions \\
\hline \multirow[t]{5}{*}{$\begin{array}{l}\text { Prepectoral } \\
\text { vs. partial } \\
\text { submuscular }\end{array}$} & $\begin{array}{l}\text { Clinical } \\
\text { outcomes }\end{array}$ & Physical well-being & $1(34)$ & Moderate & $\mathrm{N} / \mathrm{A}$ & Precise & Direct & Sparse & Insufficient & $\begin{array}{l}\text { None } \\
\text { (Sparse } \\
\text { evidence) }\end{array}$ \\
\hline & $\begin{array}{l}\text { Clinical } \\
\text { outcomes }\end{array}$ & $\begin{array}{l}\text { Psychosocial well- } \\
\text { being }\end{array}$ & $1(34)$ & Moderate & $\mathrm{N} / \mathrm{A}$ & Precise & Direct & Sparse & Insufficient & $\begin{array}{l}\text { None } \\
\text { (Sparse } \\
\text { evidence) }\end{array}$ \\
\hline & $\begin{array}{l}\text { Surgical } \\
\text { complications }\end{array}$ & Pain & $1(167)$ & Moderate & $\mathrm{N} / \mathrm{A}$ & Precise & Direct & Sparse & Insufficient & $\begin{array}{l}\text { None } \\
\text { (Sparse } \\
\text { evidence) } \\
\end{array}$ \\
\hline & $\begin{array}{l}\text { Surgical } \\
\text { complications }\end{array}$ & Capsular contracture & $1(34)$ & Moderate & $\mathrm{N} / \mathrm{A}$ & Imprecise & Direct & Sparse & Insufficient & $\begin{array}{l}\text { None } \\
\text { (Sparse } \\
\text { evidence) }\end{array}$ \\
\hline & $\begin{array}{l}\text { Surgical } \\
\text { complications }\end{array}$ & Seroma & $1(34)$ & Moderate & $\mathrm{N} / \mathrm{A}$ & Imprecise & Direct & Sparse & Insufficient & $\begin{array}{l}\text { None } \\
\text { (Sparse } \\
\text { evidence) }\end{array}$ \\
\hline \multirow{2}{*}{$\begin{array}{l}\text { Partial vs. } \\
\text { total } \\
\text { submuscular }\end{array}$} & $\begin{array}{l}\text { Clinical } \\
\text { outcomes }\end{array}$ & All & $0(0)$ & $\mathrm{N} / \mathrm{A}$ & $\mathrm{N} / \mathrm{A}$ & $\mathrm{N} / \mathrm{A}$ & $\mathrm{N} / \mathrm{A}$ & $\mathrm{N} / \mathrm{A}$ & $\mathrm{N} / \mathrm{A}$ & $\mathrm{N} / \mathrm{A}$ \\
\hline & $\begin{array}{l}\text { Surgical } \\
\text { complications }\end{array}$ & All & $0(0)$ & $\mathrm{N} / \mathrm{A}$ & $\mathrm{N} / \mathrm{A}$ & $\mathrm{N} / \mathrm{A}$ & $\mathrm{N} / \mathrm{A}$ & $\mathrm{N} / \mathrm{A}$ & $\mathrm{N} / \mathrm{A}$ & $\mathrm{N} / \mathrm{A}$ \\
\hline
\end{tabular}

Abbreviations: IBR = implant-based reconstruction, $\mathrm{N} / \mathrm{A}=$ not applicable, RoB $=$ risk of bias, $\mathrm{SoE}=$ strength of evidence.

Colors: Header rows are shaded orange. Rows for every alternate comparison are shaded blue. The colors do not add unique information.

Appendix Table G-4 provides the complete version of this evidence profile, including displaying all outcomes for which no evidence was identified. 


\section{Clinical Outcomes}

The RCT (Lee 2021b) and one NRCS (Cattelani 2018) assessed patient-reported clinical outcomes (Appendix Table E-4.1).

Both studies reported on physical well-being. The RCT (Lee 2021b) used the physical component summary of the SF-36 (scores 0 to 100; higher is better; MCID not available) and reported comparable scores between patients who underwent IBR with prepectoral and partial submuscular implants $(\mathrm{P}=0.689)$. The NRCS (Cattelani 2018) used two different measurements: the Constant Murley score and the Disabilities of the Arm, Shoulder, and Hand (DASH) score. The Constant Murley score was designed to measure changes (or differences) in physical function in patients who have undergone shoulder surgery. The score ranges from 0 to 100 , with higher scores indicating better function. The MCID has been estimated to be 10 points. $^{215}$ Compared with patients who underwent IBR with total submuscular placement, patients who underwent prepectoral placement had both clinically and statistically significantly higher mean Constant Murley scores at 1 day ( 71.6 vs. $60.4 ; \mathrm{P}<0.001)$ as well as at 7 days after surgery $(65.7$ vs. 52.4; $\mathrm{P}<0.001)$. The DASH was designed to measure the ability of patients with upper extremity musculoskeletal disorders to perform certain upper extremity activities. The score ranges from 0 to 100, with higher scores indicating less ability. The MCID has been estimated to be 10 to 15 points. ${ }^{216}$ Cattelani 2018 reported that at 1 year of followup, patients with prepectoral placement had both clinically and statistically significantly lower (i.e., better) mean DASH scores than patients with total submuscular placement (9.9 vs. 29.2; $\mathrm{P}<0.001$ ).

Both studies reported on psychosocial well-being. The RCT (Lee 2021b) used the mental component summary of the SF-36 (scores 0 to 100; higher is better; MCID not available) and reported comparable scores between patients who underwent IBR with prepectoral and partial submuscular implants at 6 months $(\mathrm{P}=0.904)$. Lee $2021 \mathrm{~b}$ also reported on anxiety and depression using the Hospital Anxiety and Depression Scale (scores 0 to 21 for each; higher is worse; MCID not available). At 6 months of followup, patients who underwent IBR with prepectoral and partial submuscular implants had comparable levels of anxiety $(\mathrm{P}=0.959)$ and depression $(\mathrm{P}=0.924)$. The NRCS (Cattelani 2018) reported that patients who underwent prepectoral placement experienced better psychosocial well-being (measured by number of days until return to usual work). Patients with prepectoral placement returned to their usual work sooner than did patients who underwent total submuscular placement (mean 34.6 vs. 57.3 days; $\mathrm{P}<0.001$ ).

Cattelani 2018 reported on patient satisfaction with breasts using the BREAST-Q ( 0 to 100 , higher is better, MCID 5 points ${ }^{187}$ ). At 1 year of followup, patients with prepectoral placement had both clinically and statistically significantly greater satisfaction with breasts than patients with total submuscular placement (92.2 vs. $76.1 ; \mathrm{P}<0.001)$.

\section{Surgical Complications}

All eight studies reported on surgical complications.

One NRCS (Cattelani 2018) reported data on unplanned repeat surgeries for revision of reconstruction (Appendix Table E-4.2). Patients who underwent prepectoral or total submuscular placements of implants had comparable risks ( $\mathrm{P}=\mathrm{NS}$; adjusted effect size not reported).

Three NRCSs reported inconsistent data on pain. Two NRCS used the VAS (0 to 10; higher is worse; MCID 2 points $\left.{ }^{191}\right)$. Avila 2020 reported that patients who underwent IBR with prepectoral and total submuscular placement had clinically comparable albeit statistically 
significantly different pain levels (3.94 vs. 5.25; $\mathrm{P}<0.001$ ). Kim 2020 reported that patients who underwent prepectoral and partial submuscular placement had comparable pain levels at 1 day (adjMD $-0.08 ; \mathrm{P}=0.33)$ at 7 days after surgery (adjMD $-0.12 ; \mathrm{P}=0.12)$. Cattelani 2018 reported pain data using the Brief Pain Inventory-Short Form (BPI-SF) (Appendix Table E-4.1). The score ranges from 0 to 100 , with higher scores indicating greater pain. The MCID has been estimated to be 2 points. ${ }^{217}$ Compared with patients who underwent IBR with total submuscular placement, patients with prepectoral placement had both clinically and statistically significantly lower pain at 1 day (17.6 vs. $44.1 ; \mathrm{P}<0.001)$ as well as at 7 days after surgery (8.2 vs. 22.0; $\mathrm{P}<0.001)$.

One NRCS (Avila 2020) reported on analgesic use expressed in terms of oral morphine equivalents. Patients who underwent IBR with total submuscular placement had higher levels of analgesic use at 1 month (63.0 vs. $17.4 ; \mathrm{P}=0.03)$.

One NRCS (Nealon 2020a) reported that risks of necrosis (of the skin) at 1.7 to 2.4 years were comparable between women who underwent prepectoral or total submuscular implants (adjOR 1.01, 95\% CI 0.74 to 5.95).

Two NRCSs (Nealon 2020a and Kraenzlin 2021) reported that risks of infections that were not explicitly implant related were comparable between groups of women who underwent prepectoral or total submuscular implants. Nealon 2020a reported an imprecise adjOR of 0.31 $(95 \% \mathrm{CI}<0.01$ to 8.65$)$ at 1.7 to 2.4 years and Kraenzlin 2021 reported a P value of 0.21 (adjusted effect size not reported) at an unreported time-point.

One NRCS (Nealon 2020a) reported that risks of needing explant surgery by 1.7 to 2 years were comparable between women who underwent prepectoral or total submuscular implants, although the estimate was highly imprecise (adjOR 1.01, 95\% CI 0.07 to 14.1).

Two studies reported on capsular contracture. No events occurred in the partial submuscular in the RCT by 6 months (Lee 2021b), so an effect size was not calculable. One NRCS (Nealon 2020a) reported that patients who underwent IBR with prepectoral and total submuscular implants had comparable risks at 1.7 to 2.4 years, but the estimate was imprecise (adjOR $0.30,95 \%$ CI 0.03 to 1.55 ).

Two studies reported on seroma although the estimates were highly imprecise. The RCT (Lee 2021b) reported that patients who underwent IBR with prepectoral and partial submuscular implants had comparable risks at 6 months (OR 1.06, 95\% CI 0.15 to 7.34). One NRCS (Nealon 2020a) reported that patients who underwent IBR with prepectoral and total submuscular implants had comparable risks at 1.7 to 2.4 years (adjOR $1.49,95 \%$ CI 0.37 to 6.11 ).

One NRCS (Nealon 2020a) reported that risks of hematoma at 1.7 to 2.4 years were comparable between women who underwent prepectoral or total submuscular implants, although the estimate was highly imprecise (adjOR 5.18, 95\% CI 0.39 to 7.05 ).

Three NRCSs reported a variety of composite/unspecified harms. Avila 2020 reported that patients with prepectoral and total submuscular implants had comparable risks of a composite of necrosis, infection, wound dehiscence, hematoma, and seroma at 1 month $(\mathrm{P}=\mathrm{NS}$; adjusted effect size not reported). Gabriel 2020 reported that at 2 years, compared with patients with prepectoral implants, patients with partial submuscular implants had a higher risk of any complication (adjOR 3.04, 95\% CI 1.34 to 7.61). Ozgur 2020 reported that at 5.3 to 6.1 years, compared with patients with partial submuscular implants, patients with total submuscular implants had a higher risk of a composite of capsular contracture, inframammary fold problems, bottoming out, rippling, mechanical shift, and animation deformity (adjOR 3.28, 95\% CI 1.39 to 7.76). 


\section{Heterogeneity of Treatment Effects (Subgroup Differences)}

None of the eight studies reported subgroup results or other analyses of possible heterogeneity of treatment effects. The studies were too sparse to allow exploration of possible differences based on patient or other characteristics.

\section{Applicability}

None of the eight studies addressing this KQ reported information about patient race. The Italian study (Cattelani 2018) and one of the South Korean studies (Kim 2021b) used textured implants, which are not commonly used in the U.S.

\section{Overall Summary for Key Question 4}

Whether the implant is placed in the prepectoral or total submuscular plane may not be associated with risk of infections that are not explicitly implant-related (Low SoE). There is insufficient evidence addressing the comparisons between prepectoral and partial submuscular and between partial and total submuscular planes. 


\section{Key Question 5: Use of Human Acellular Dermal Matrices for Implant-Based Reconstruction}

\section{Key Points}

- In patients undergoing IBR, because of inconsistent results, the evidence is insufficient regarding whether human ADM use impacts the patient-reported outcomes of physical well-being, psychosocial well-being, or satisfaction with breasts. Because of sparse data, there is insufficient evidence regarding whether human ADM use improves sexual wellbeing.

- Patients undergoing IBR with human ADMs probably are at greater risk than those not receiving human ADMs of implant failure/loss or need for explant surgery (summary adjOR 1.28, 95\% CI 0.97 to 1.70; 6 studies) (Moderate SoE) and may be at greater risk of infections not explicitly implant- or ADM-related (summary adjOR $1.56,95 \%$ CI 0.96 to 2.53; 7 studies) (Low SoE). Compared with nonuse, use of human ADMs probably does not impact the risks of unplanned repeat surgeries for revision (Moderate SoE) and seroma (summary adjOR $1.52,95 \%$ CI 0.62 to 3.71 ; 4 studies) (Moderate SoE). Compared with nonuse, use of human ADMs may not impact the risk of necrosis (summary adjOR $0.89,95 \%$ CI 0.63 to $1.25 ; 4$ studies) (Low SoE).

- Because of inconsistent results, there is insufficient evidence regarding whether use of human ADMs impacts pain or risks of wound dehiscence, implant malposition, or capsular contracture.

- Because of sparse data, there is insufficient evidence regarding whether human ADM use is associated with risks of mortality, unplanned repeat surgeries for complications, analgesic use, implant rupture, thromboembolic events, or delayed healing.

Twenty-two studies, including two RCTs reported in two articles ${ }^{218,219}$ and 20 NRCSs reported in 29 articles $^{65,66,194,196-198,200,209,218-238}$ evaluated the comparison of use versus nonuse of human ADMs in a total of 43,334 patients undergoing IBR. The studies are detailed in Figures 8 to 12 and Appendix Tables C-6, D-1 to D-3, and E-5.1 to E-5.4.

Most studies did not report any additional information about the implants, such as surface (smooth versus textured), shape (round versus tear drop), size, or anatomic plane of placement. Among the two RCTs, we rated Wendel 2013 to be at high risk of bias because of the lack of blinding of participants and study personnel and because of incomplete outcome data and selective outcome reporting. We rated McCarthy 2012 to be at moderate risk of bias because of the lack of blinding of participants and study personnel and because of selective outcome reporting. Among the 20 NRCSs, we rated 15 to be at high risk of bias, four at moderate risk, and one at low risk. High risk of bias ratings were mostly related to critical or serious risk of confounding and the lack of blinding of study personnel and/or outcome assessors. Not all the NRCSs reported complete results data: some reported adjusted $\mathrm{P}$ values without effect sizes or effect sizes without CIs or P values.

The 22 studies included between 36 and 18,977 women and were conducted mostly in North America (16 in the U.S., 2 in Canada, 1 in both). Two studies were conducted in South Korea and one in Italy. Among the studies reporting data, the women's mean ages ranged from 47 to 51 years and their mean BMIs ranged from 22.3 to $27.0 \mathrm{~kg} / \mathrm{m}^{2}$. Only the NRCS by Ibrahim 2013 
reported on patient race. The majority of women (79\%) were White, 6.4 percent were Black, and 2.8 percent were Asian. Studies mostly followed women for about 2 to 5 years.

The study result summaries are in Figures 4 to 9 and Appendix Tables E-5.1 to E-5.4. Full results are in Appendix Tables F-5.1 to F-5.21.

\section{Summary of Comparison of Use Versus Nonuse of Human ADMs}

There is moderate SoE that, when compared with ADM nonuse during IBR, ADM use is associated with comparable risks of seroma and unplanned repeat surgeries for revision of reconstruction, but increased risk of implant failure/loss or need for explant surgery (Table 7). There is low SoE that ADM use may not impact the clinical outcomes of physical well-being, psychosocial well-being, and patient satisfaction with breasts, and the surgical complications of pain and capsular contracture. There is also low SoE of comparable risks between ADM use and nonuse groups in necrosis and seroma, but of increased risk of infections that are not explicitly related to implants or ADMs. 
Table 7. Evidence profile for Key Question 5: Use versus nonuse of human ADMs during IBR

\begin{tabular}{|c|c|c|c|c|c|c|c|c|c|}
\hline $\begin{array}{l}\text { Outcome } \\
\text { Category }\end{array}$ & Outcome & $\begin{array}{l}\text { N Studies } \\
\text { (Patients) }\end{array}$ & RoB & Consistency & Precision & Directness & Other & SoE & $\begin{array}{l}\text { Conclusions (Reason, if } \\
\text { None) }\end{array}$ \\
\hline \multirow{5}{*}{$\begin{array}{l}\text { Clinical } \\
\text { outcomes }\end{array}$} & Physical well-being & $3(1604)$ & Moderate & Inconsistent & Precise & Direct & None & Insufficient & None (Inconsistent results) \\
\hline & Psychosocial well-being & $2(1535)$ & Moderate & Inconsistent & Precise & Direct & None & Insufficient & None (Inconsistent results) \\
\hline & Sexual well-being & $1(1451)$ & Moderate & $\mathrm{N} / \mathrm{A}$ & Precise & Direct & Sparse & Insufficient & None (Sparse evidence) \\
\hline & $\begin{array}{l}\text { Patient satisfaction with } \\
\text { breasts }\end{array}$ & $2(1535)$ & Moderate & Inconsistent & Precise & Direct & None & Insufficient & None (Inconsistent results) \\
\hline & Mortality & $1(36)$ & High & $\mathrm{N} / \mathrm{A}$ & $\mathrm{N} / \mathrm{A}$ & Direct & Sparse & Insufficient & None (Sparse evidence) \\
\hline \multirow[t]{14}{*}{$\begin{array}{l}\text { Surgical } \\
\text { complications }\end{array}$} & $\begin{array}{l}\text { Unplanned repeat } \\
\text { surgeries for revision }\end{array}$ & $3(20808)$ & High & Consistent & Precise & Direct & None & Moderate & Comparable in both groups \\
\hline & $\begin{array}{l}\text { Unplanned repeat } \\
\text { surgeries for complications }\end{array}$ & $1(128)$ & High & $\mathrm{N} / \mathrm{A}$ & Unclear & Direct & Sparse & Insufficient & None (Sparse evidence) \\
\hline & Pain & $2(153)$ & Moderate & Inconsistent & Unclear & Direct & None & Insufficient & None (Inconsistent results) \\
\hline & Analgesic use & $1(68)$ & Moderate & $\mathrm{N} / \mathrm{A}$ & Precise & Direct & Sparse & Insufficient & None (Sparse evidence) \\
\hline & Necrosis & $7(2101)$ & High & Consistent & Precise & Direct & None & Low & $\begin{array}{l}\text { Comparable in both groups: } \\
\text { summary adjOR } 0.89 \text { ( } 95 \% \\
\text { Cl } 0.63,1.25) ; 4 \text { studies }\end{array}$ \\
\hline & Implant rupture & $1(1451)$ & Moderate & $\mathrm{N} / \mathrm{A}$ & Unclear & Direct & Sparse & Insufficient & None (Sparse evidence) \\
\hline & Implant malposition & $2(1654)$ & Moderate & Inconsistent & Unclear & Direct & None & Insufficient & None (Inconsistent results) \\
\hline & $\begin{array}{l}\text { Implant failure/loss or need } \\
\text { for explant surgery }\end{array}$ & $10(38983)$ & Moderate & Consistent & Precise & Direct & None & Moderate & $\begin{array}{l}\text { Higher risk with ADM: } \\
\text { summary adjOR } 1.28 \text { ( } 95 \% \\
\mathrm{Cl} 0.97,1.70) ; 6 \text { studies }\end{array}$ \\
\hline & Capsular contracture & $4(3485)$ & High & Inconsistent & Precise & Direct & None & Insufficient & None (Inconsistent results) \\
\hline & Thromboembolic events & $1(18997)$ & Moderate & $\mathrm{N} / \mathrm{A}$ & Unclear & Direct & Sparse & Insufficient & None (Sparse evidence) \\
\hline & $\begin{array}{l}\text { Infections (not explicitly } \\
\text { implant- or ADM-related) }\end{array}$ & $13(25228)$ & Moderate & Inconsistent & Precise & Direct & None & Low & $\begin{array}{l}\text { Higher risk with ADM: } \\
\text { summary adjOR } 1.56(95 \% \\
\mathrm{Cl} 0.96,2.53) ; 7 \text { studies }\end{array}$ \\
\hline & Wound dehiscence & $4(21798)$ & Moderate & Inconsistent & Unclear & Direct & None & Insufficient & None (Inconsistent results) \\
\hline & Delayed healing & $1(398)$ & High & $\mathrm{N} / \mathrm{A}$ & Imprecise & Direct & Sparse & Insufficient & None (Sparse evidence) \\
\hline & Seroma & $6(3575)$ & Moderate & Consistent & Precise & Direct & None & Moderate & $\begin{array}{l}\text { Comparable in both groups: } \\
\text { summary adjOR } 1.52 \text { ( } 95 \% \\
\text { Cl } 0.62,3.71) ; 4 \text { studies }\end{array}$ \\
\hline
\end{tabular}

Abbreviations: adj = adjusted, $\mathrm{ADM}=$ acellular dermal matrix, $\mathrm{CI}=$ confidence interval, $\mathrm{IBR}=$ implant-based reconstruction, $\mathrm{N} / \mathrm{A}=$ not applicable, $\mathrm{OR}=\mathrm{odds}$ ratio, $\mathrm{RoB}=$ risk of

bias, $\mathrm{SoE}=$ strength of evidence.

Colors: Header rows are shaded orange. The color does not add unique information.

Appendix Table G-5 provides the complete version of this evidence profile, including displaying all outcomes for which no evidence was identified 


\section{Clinical Outcomes}

One RCT (McCarthy 2017) and two NRCSs (Cattelani 2018 and Ganesh Kumar 2021) reported on clinical outcomes comparing women who underwent IBR with or without the use of ADMs (Appendix Tables E-5.1 and E-5.2).

All three studies reported on physical well-being. The RCT (McCarthy 2017) used the BREAST-Q chest and upper body scores (0-100; higher is better; MCID $3^{187}$ ) (Appendix Table E-5.1). Scores were comparable whether or not ADM was used during IBR, when measured during the expansion phase of the tissue expander (net mean difference [NMD] 0.60, 95\% CI -4.87 to 6.07 ), or after expansion (NMD 0.50, 95\% CI -5.93 to 6.93). One NRCS (Ganesh Kumar 2021) used the overall physical well-being score of the BREAST-Q (0-100; higher is better; MCID $3^{187}$ ) and similarly reported that scores were comparable whether or not ADM was used (adjMD -0.82, 95\% CI -3.01 to 1.37). The other NRCS (Cattelani 2018) reported on physical well-being using two different measurements: the Constant Murley score, which measures shoulder function (0 to 100; higher is better; MCID 10) and the DASH score, which measures function of the arm, shoulder, and hand (0 to 100; higher is worse; MCID 10 to $\left.15^{216}\right)$. Compared with patients who underwent IBR with ADM, patients without ADM had both clinically and statistically significantly higher mean Constant Murley scores at 1 day (71.6 vs. 60.4; $\mathrm{P}<0.001)$ and 7 days after surgery (65.7 vs. 52.4; $\mathrm{P}<0.001)$. At 1 year of followup, patients who underwent IBR with ADMs had both clinically and statistically significantly lower (i.e., better) mean DASH scores than patients who underwent IBR without ADMs (9.9 vs. 29.2; $\mathrm{P}<0.001)$.

Two NRCSs (Ganesh Kumar 2021 and Cattelani 2018) reported on psychosocial well-being. Ganesh Kumar 2021 used the BREAST-Q: psychosocial well-being scores ( 0 to 100 ; higher is better; MCID $4^{187}$ ) at 2 years of followup. Psychosocial well-being scores were comparable whether or not ADMs were used (adjMD -0.26, 95\% CI -2.97 to 2.45). Cattelani 2018 reported that patients who underwent IBR with ADMs returned to their usual work sooner than did patients without ADMs (mean 34.6 vs. 57.3 days; $\mathrm{P}<0.001$ ).

Ganesh Kumar 2021 also reported on sexual well-being using the BREAST-Q: sexual wellbeing scores ( 0 to 100 ; higher is better; MCID $5^{187}$ ) at 2 years of followup. Sexual well-being scores were comparable whether or not ADMs were used during IBR (adjMD $-2.28,95 \%$ CI -5.63 to 1.06 ).

Both NRCSs (Cattelani 2018 and Ganesh Kumar 2021) reported on patient satisfaction with breasts using the BREAST-Q: satisfaction with breast scores (0 to 100; higher is better; MCID $5^{187}$ ). Cattelani 2018, using data at 1 year of followup, reported that patients who had received ADMs were more satisfied with their breasts $(\mathrm{P}<0.001)$, although an adjusted effect size was not reported. Ganesh Kumar 2021, however, reported that, at 2 years of followup, satisfaction scores were comparable whether or not ADMs were used (adjMD -1.95, 95\% CI -4.96 to 1.06).

\section{Mortality}

One RCT (Wendel 2013) reported on 1-month mortality, but no deaths occurred (Appendix Table E-5.2). 


\section{Surgical Complications}

All 22 studies (2 RCTs and 20 NRCSs) reported on surgical complications comparing women who underwent IBR with or without use of ADMs (Figures 5 to 9 and Appendix Tables E-5.2 to E-5.4).

\section{Unplanned Repeat Surgeries}

Three NRCSs (Ibrahim 2013, Nealon 2020b, and Sobti 2018) reported on unplanned repeat surgeries for revision of reconstruction (Appendix Table E-5.2). Ibrahim 2013 reported that, at 6 months of followup, risks were comparable whether or not ADMs were used $(\mathrm{P}=0.14)$. An adjusted effect size was not reported. At approximately 5 years of followup, no significant between-group differences in risks of unplanned surgeries were reported by both Nealon 2020b (adjOR 0.86, 95\% CI 0.69 to 1.08 ) and Sobti 2018 (adjOR 1.10, 95\% CI 0.63 to 1.92 ).

One NRCS (Peled 2012) reported on unplanned repeat surgeries for complications, specifically wound-healing or infectious complications. At 2.6 to 3.3 years of followup, patients receiving ADMs underwent fewer unplanned surgeries for complications $(\mathrm{P}<0.05)$. However, an adjusted effect size was not reported.

\section{Pain and Analgesic Use}

One RCT (McCarthy 2012) and one NRCS (Cattelani 2018) reported on the outcome of pain (Appendix Table E-5.3). McCarthy 2012 reported data using a VAS at 24 hours, during the expansion phase, and upon completion of the expansion phase. The VAS score used ranges from 0 to 100 , with higher scores indicating greater pain. The MCID has been estimated to be 5 points. ${ }^{239}$ McCarthy 2012 reported that patients randomized to receive ADMs experienced clinically significant greater increases in pain in the first 24 hours (NMD 6.2, 95\% CI -4.9 to 17.3) and during the expansion phase (NMD 6.8, 95\% CI 1.1 to 12.5); although, the earlier time point was not statistically significant. After the expansion phase, changes in pain were comparable between ADM use and nonuse groups (NMD -4.6, 95\% CI -9.8 to 0.6).

Cattelani 2018 reported on pain using the BPI-SF ( 0 to 100; higher implies greater pain; MCID has been estimated to be 2 points ${ }^{217}$ ). Compared with patients who did not receive ADMs (with total submuscular implant placement), patients who received ADMs (with prepectoral implant placement) experienced both clinically and statistically significantly lower pain at 1 day (17.6 vs. 44.1; $\mathrm{P}<0.001)$ and 7 days after surgery (8.2 vs. 22.0; $\mathrm{P}<0.001)$.

One RCT (McCarthy 2012) reported on analgesic use within the first 24 hours after surgery. McCarthy 2012 reported that analgesic use overall in the first 24 hours was statistically similar whether or not ADMs were used during IBR (MD -134 mg, 95\% CI -440 to 172).

\section{Necrosis}

Seven NRCSs reported data on necrosis (Appendix Table F-5.10). Among them, four NRCSs (Hirsch 2014, Nealon 2020b, Seth 2012, and Sobti 2018) reported adjusted effect sizes and thus were included in a meta-analysis (Figure 8). Effect sizes ranged from 0.53 to 1.32 . The meta-analysis suggested comparable risks of necrosis comparing patients who received or did not receive ADMs during their IBR (adjOR 0.83, 95\% CI 0.63 to $1.25 ; \mathrm{I}^{2}=25 \%$ ). 
Figure 8. Meta-analysis for Key Question 5: Use versus nonuse of human ADMs during IBR necrosis

$\begin{array}{llll}\text { Study Year Unit Time Point } & \begin{array}{l}\text { Use of } \\ \text { ADM, }\end{array} & \begin{array}{l}\text { Nonuse of } \\ \text { ADM, }\end{array} & \begin{array}{l}\text { Adjusted Odds } \\ \text { Ratio }\end{array}\end{array}$ $\begin{array}{lll}\text { ADM, } & \text { ADM, } & \text { Ratio } \\ \mathrm{n} / \mathrm{N} & \mathrm{n} / \mathrm{N} & (95 \% \mathrm{Cl})\end{array}$

Hirsch 2014 Women 3.1 y NR NR $0.98(0.58,1.66)$

Nealon 2020b Women 5.3 y NR NR $\quad 0.87(0.50,1.52)$

Seth $2012 \quad$ Breasts 2.0 y $\quad 17 / 199 \quad 26 / 393 \quad 1.32(0.70,2.49)$

$\begin{array}{lllll}\text { Sobti } 2018 \text { Women } & 5.0 \mathrm{y} & 14 / 338 & 30 / 376 & 0.53(0.28,1.02)\end{array}$

Overall $\left(I^{2}=25 \%\right)$

NR

NR

$0.89(0.63,1.25)$

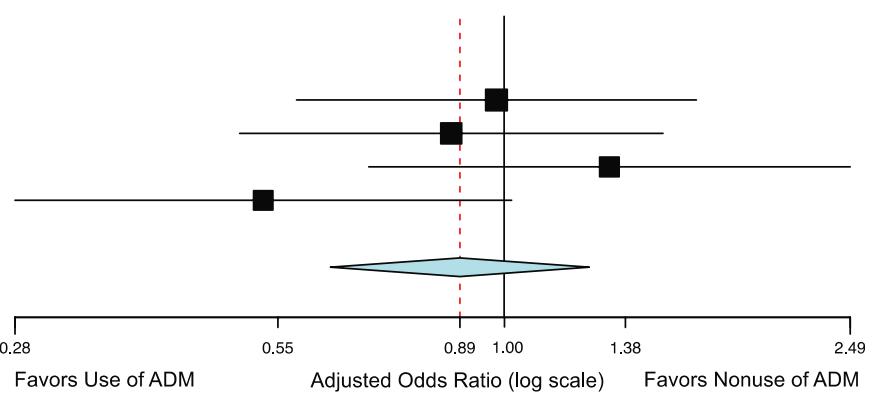

Abbreviations: $\mathrm{ADM}=$ acellular dermal matrix, $\mathrm{CI}=$ confidence interval, $\mathrm{IBR}=$ implant-based reconstruction, $\mathrm{I}^{2}=$ measure of statistical heterogeneity ( $\%$ of total variability that is due to between-study variability), $\mathrm{NR}=$ not reported, $\mathrm{OR}=$ odds ratio.

Among the three studies reporting necrosis data that were not included in the meta-analysis, Craig 2019 reported an adjusted effect size for only the subgroup of patients who did not receive postoperative radiation therapy; it was therefore excluded from the meta-analysis. In that subgroup, patients who received ADMs had a higher risk of necrosis at 7 months of followup (adjOR 4.99, 95\% CI 3.28 to 8.03). Qureshi 2016 was not included in the meta-analysis because only an adjOR (3.1) with no CI was reported. Ganesh Kumar 2021 reported that, at 2 years of followup, risks of necrosis were comparable whether or not $\mathrm{ADMs}$ were used $(\mathrm{P}=0.28$; adjusted effect size not reported).

Thus, among the three NRCSs not included in the meta-analysis, two studies (Craig 2019 and Qureshi 2016) suggested that ADM use may be associated with increased risk of necrosis, while one study (Ganesh Kumar 2021) was in agreement with the meta-analysis findings of comparable risk of necrosis.

\section{Thromboembolic Events}

One NRCS (Ibrahim 2013) reported on thromboembolic events (Appendix Table E-5.2). No differences were found between IBR with or without ADMs in terms of risks of deep vein thrombosis $(\mathrm{P}=0.47)$ or pulmonary embolism $(\mathrm{P}=0.11)$. No adjusted effect sizes were reported.

\section{Infections Not Explicitly Related to Implants or ADMs}

Thirteen studies (2 RCTs and 11 NRCSs) reported data on infections that were not explicitly related to implants or ADMs (Appendix Table F-5.15). Various types and extents of infections were described, and we summarize them separately below.

\section{Any or Undefined Infections}

Nine studies (both RCTs and seven NRCSs) reported on any or undefined infections. Seven studies, comprising both RCTs (McCarthy 2012 and Wendel 2013) and five NRCSs (Chun 2010, Nealon 2020b, Seth 2012, Sobti 2018, and Woo 2017), reported effect sizes that adequately accounted for confounders and were thus included in a meta-analysis (Figure 9). Effect sizes ranged from 0.88 to 5.37 . The meta-analysis suggested a near-significant increased risk of infections in patients who received ADMs during their IBR (adjOR 1.56, 95\% CI 0.96 to 2.53; $\left.\mathrm{I}^{2}=46 \%\right)$. The findings in the RCTs were not significantly different from the NRCSs $(\mathrm{P}=0.44$ from a meta-regression, and as suggested by the lack of heterogeneity across studies). 
Figure 9. Meta-analysis for Key Question 5: Use versus nonuse of human ADMs during IBR - Any or undefined infections

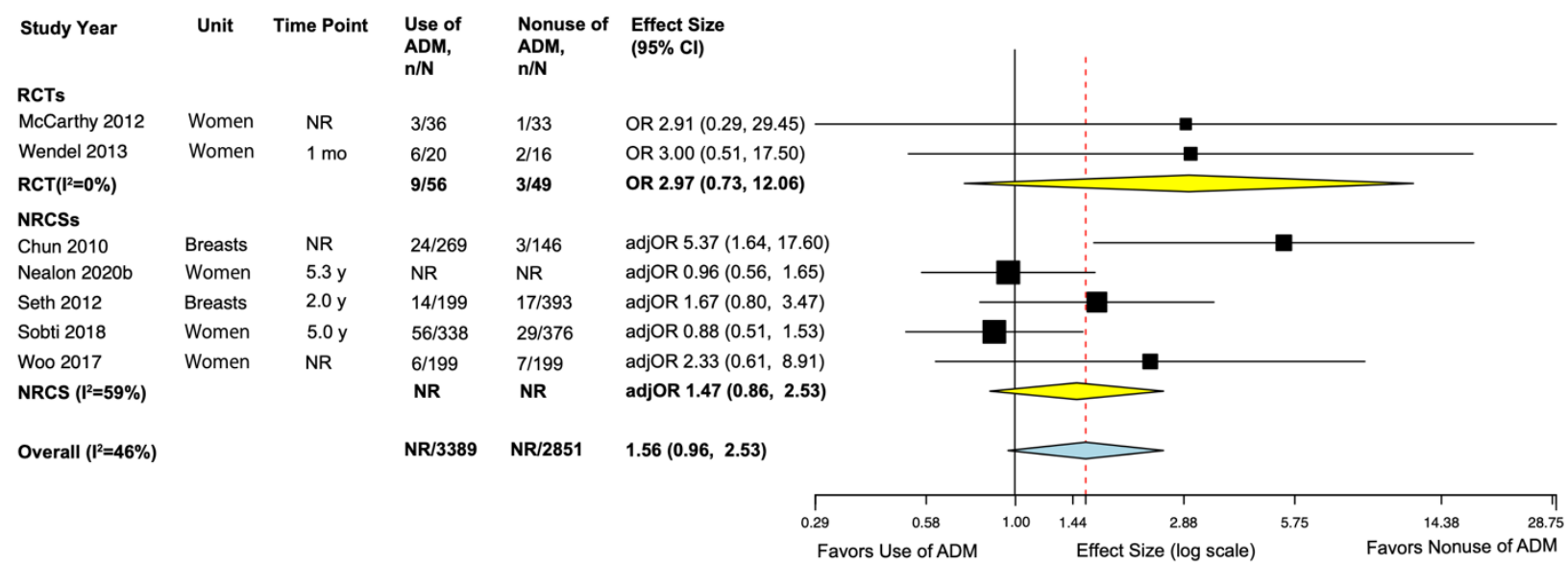

Abbreviations: $\mathrm{ADM}=$ acellular dermal matrix, $\mathrm{CI}=$ confidence interval, $\mathrm{IBR}=$ implant-based reconstruction, $\mathrm{I}^{2}=$ measure of statistical heterogeneity ( $\%$ of total variability that is due to between-study variability), mo $=$ months, $\mathrm{NR}=$ not reported, $\mathrm{NRCS}=$ nonrandomized comparative study, $\mathrm{OR}=$ odds ratio, $\mathrm{RCT}=$ randomized controlled trial, $\mathrm{y}=$ years.

The two NRCSs that were not included in the meta-analysis reported inconsistent findings. Brooke 2012 reported comparable risks of infections whether or not ADMs were used $(\mathrm{P}=0.09$; no adjusted effect size was reported), while Craig 2019 reported an adjusted effect size for only the subgroup of patients who did not receive postoperative radiation therapy (adjOR 2.68, 95\% CI 1.54 to 5.06 ).

Thus, among the two NRCSs not included in the meta-analysis, one NRCS (Brooke 2012) suggested that ADM use or nonuse may be associated with comparable risks of infections, while subgroup data from another NRCS (Craig 2019) were in agreement with the meta-analysis findings of increased risk with ADM use.

\section{Defined Infections}

Five NRCSs (Liu 2011, Ganesh Kumar 2021, Ibrahim 2013, Chun 2010, and Peled 2012) reported data for defined infections. Their findings were inconsistent.

Three NRCSs (Liu 2011, Ganesh Kumar 2021, and Ibrahim 2013) reported on wound infections. Liu 2011 reported that ADM use was associated with greater risk of major or minor wound infections (adjOR 3.25, 95\% CI 0.80 to 13.1). Ganesh Kumar 2021 reported that ADM use and nonuse groups had comparable overall risks of wound infections $(\mathrm{P}=0.138)$ and infections requiring intravenous antibiotics or reoperation $(\mathrm{P}=0.045)$, but $\mathrm{ADM}$ use was associated with greater risks of wound infections requiring oral antibiotics (adjOR 1.49, 95\% CI 0.90 to 2.44). Ibrahim 2013 reported that ADM use was associated with greater risk of superficial surgical site infection $(\mathrm{P}=0.021)$ but not deep incisional surgical site infection $(\mathrm{P}=0.366)$. Ibrahim 2013 also reported that $\mathrm{ADM}$ use and nonuse groups experienced comparable risks of organ space infection $(\mathrm{P}=0.290)$ and sepsis $(\mathrm{P}=0.516)$.

Chun 2010 reported that ADM use was associated with a greater risk of major infection requiring admission for intravenous antibiotics and/or surgery $(\mathrm{P}=0.0016)$. However, Peled 2012 reported that ADM use was associated with a lower risk of localized or systemic infections that were treated with oral antibiotics or admission for intravenous antibiotics $(\mathrm{P}<0.05)$. Neither Chun 2010 nor Peled 2012 reported adjusted effect sizes. 


\section{Wound Dehiscence and Delayed Healing}

Four NRCSs (Ganesh Kumar 2021, Qureshi 2016, Ibrahim 2013, and Craig 2019) reported on wound dehiscence, but their findings were inconsistent (Appendix Table E-5.2). Ganesh Kumar 2021 reported that risk of wound dehiscence was higher among patients receiving ADMs $(\mathrm{P}=0.009)$ but did not report an adjusted effect size. Ibrahim 2013 reported that risks of wound dehiscence were comparable in the two groups $(\mathrm{P}=0.26)$ but did not report an adjusted effect size or CIs. However, Qureshi 2016 reported that the risk of wound dehiscence was lower among patients receiving ADMs (adjOR 0.4; $\mathrm{P}<0.05$ ). Craig 2019 reported adjusted data only for the subgroup of patients who did not receive postoperative radiation therapy; ADM use was associated with higher risk of wound dehiscence at 7 months of followup (adjOR $2.46,95 \% \mathrm{CI}$ 1.23 to 4.93$)$.

One NRCS (Woo 2017) reported on delayed healing, defined as a composite outcome of delayed wound healing or skin flap necrosis. Comparable risks were observed in women receiving or not receiving ADMs during IBR (adjOR 1.41, 95\% CI 0.67 to 2.96).

\section{Seroma}

Six studies (one RCT and five NRCSs) reported data on seroma (Appendix Table F-5.14). Among them, four studies, comprising one RCT (McCarthy 2012) and three NRCSs (Chun 2010, Seth 2012, and Woo 2017), reported effect sizes that adequately accounted for confounders and were thus included in a meta-analysis (Figure 10). Effect sizes ranged from 0.29 to 4.24 across all studies. The meta-analysis suggests that seroma risks in patients who received or did not receive ADMs during their IBR were comparable (adjOR $1.52,95 \%$ CI 0.62 to $3.71 ; \mathrm{I}^{2}=52 \%$ ). The single RCT (McCarthy 2012) provided a highly imprecise estimate, but it was not significantly different from the NRCSs' estimates $(\mathrm{P}=0.30$ from a metaregression).

Figure 10. Meta-analysis for Key Question 5: Use versus nonuse of human ADMs during IBR seroma

\begin{tabular}{|c|c|c|c|c|c|}
\hline Study Year & Unit & Time Point & $\begin{array}{l}\text { Use of } \\
\text { ADM, } \\
\mathrm{n} / \mathrm{N}\end{array}$ & $\begin{array}{l}\text { Nonuse of } \\
\text { ADM, } \\
\mathrm{n} / \mathrm{N}\end{array}$ & $\begin{array}{l}\text { Effect Size } \\
(95 \% \mathrm{Cl})\end{array}$ \\
\hline \multicolumn{6}{|l|}{ RCTs } \\
\hline McCarthy 2012 & Women & NR & $1 / 36$ & $3 / 33$ & OR $0.29(0.03,2.89)$ \\
\hline RCT $\left(I^{2}=N A\right)$ & & & $1 / 36$ & $3 / 33$ & OR $0.29(0.03,2.89)$ \\
\hline \multicolumn{6}{|l|}{ NRCSs } \\
\hline Chun 2010 & Breasts & NR & $38 / 269$ & $4 / 146$ & adjOR $4.24(1.28,14.00)$ \\
\hline Seth 2012 & Breasts & $2.0 \mathrm{y}$ & 8/199 & $8 / 393$ & adjOR $2.02(0.75,5.45)$ \\
\hline Woo 2017 & Women & NR & $8 / 199$ & 17/199 & $\operatorname{adjOR} 0.89(0.33,2.39)$ \\
\hline \multicolumn{3}{|l|}{ NRCS $\left(I^{2}=50 \%\right)$} & $54 / 667$ & $29 / 738$ & adjOR $1.87(0.79,4.42)$ \\
\hline \multicolumn{3}{|l|}{ Overall $\left(I^{2}=52 \%\right)$} & $55 / 703$ & $58 / 771$ & $1.52(0.62,3.71)$ \\
\hline
\end{tabular}

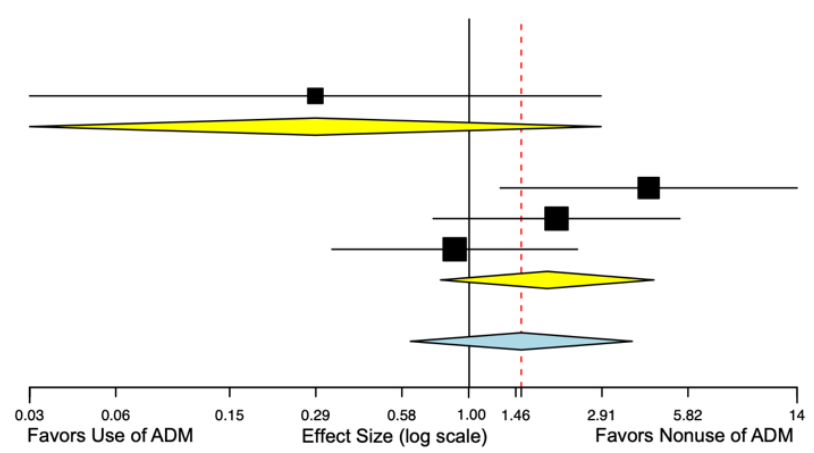

Abbreviations: $\mathrm{ADM}=$ acellular dermal matrix, $\mathrm{CI}=$ confidence interval, $\mathrm{IBR}=$ implant-based reconstruction, $\mathrm{I}^{2}=$ measure of statistical heterogeneity ( $\%$ of total variability that is due to between-study variability), $\mathrm{NR}=$ not reported, OR $=$ odds ratio.

The two NRCSs that were not included in the meta-analysis (Craig 2019 and Ganesh Kumar 2021) reported inconsistent findings. Craig 2019, excluded because it reported an adjusted effect size for only the subgroup of patients who did not receive postoperative radiation therapy, reported that patients who received ADMs had a higher risk of seroma at 7 months of followup (adjOR 3.19, 95\% CI 1.84 to 5.52). However, Ganesh Kumar 2021, excluded because of the lack 
of a reported adjusted effect size, reported that risks of seroma at 2 years of followup in ADM use and nonuse groups were comparable $(\mathrm{P}=0.72)$.

\section{Implant Rupture, Malposition, and Extrusion}

One NRCS (Ganesh Kumar 2021) reported that risks of implant rupture (defined as implant rupture, leakage, or deflation) were comparable in ADM use and nonuse groups $(\mathrm{P}=0.665)$ (Appendix Table E-5.2). An adjusted effect size was not reported. Two NRCSs (Ganesh Kumar 2021 and Vardanian 2011) reported on implant malposition, but the data were conflicting. Ganesh Kumar 2021, without reporting an adjusted effect size, reported that risks of implant malposition were comparable between ADM use and nonuse groups $(\mathrm{P}=0.83)$, but Vardanian 2011 reported that ADM use was associated with a lower risk of implant malposition (adjOR $0.23,95 \%$ CI 0.06 to 0.78 ). One NRCS (Seth 2012) reported a highly imprecise estimate comparing risks of implant extrusion between ADM use and nonuse groups.

\section{Implant Failure/Loss or Need for Explant Surgery}

Ten NRCSs reported data on implant failure/loss or need for explant surgery (Appendix Table F-5.16). Among them, six NRCSs (Ganesh Kumar 2021, Hirsch 2014, Nealon 2020b, Pannucci 2013, Seth 2012, and Woo 2017) reported effect sizes that adequately accounted for confounders and were thus included in a meta-analysis (Figure 11). AdjORs ranged from 0.41 to 1.92 across these studies. The meta-analysis suggested a near-significant increased risk in patients who received ADMs during their IBR (adjOR 1.28, 95\% CI 0.97 to $1.70 ; \mathrm{I}^{2}=16 \%$ ).

Figure 11. Meta-analysis for Key Question 5: Use versus nonuse of human ADMs during IBR implant failure/loss or need for explant surgery

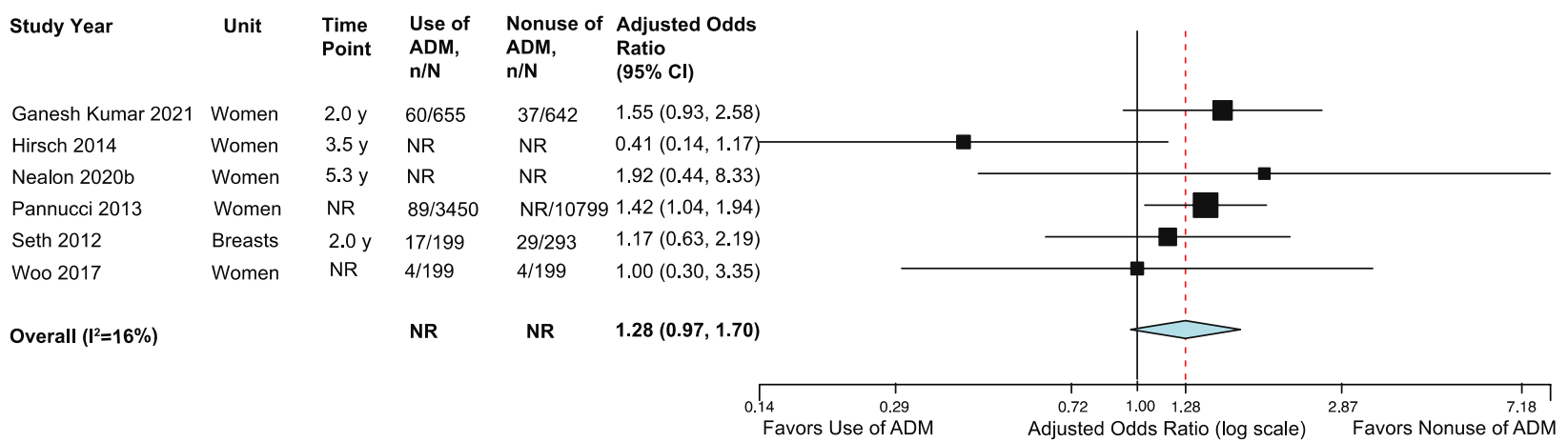

Abbreviations: $\mathrm{ADM}=$ acellular dermal matrix, mo $=$ months, $\mathrm{CI}=$ confidence interval, $\mathrm{IBR}=$ implant-based reconstruction, $\mathrm{I}^{2}=$ measure of statistical heterogeneity ( $\%$ of total variability that is due to between-study variability), NR $=$ not reported.

The four NRCSs that were not included in the meta-analysis (Craig 2019, Ibrahim 2013, Qureshi 2016, and Peled 2012) reported inconsistent findings. Craig 2019, excluded because it reported an adjusted effect size for only the subgroup of patients who did not receive postoperative radiation therapy, reported that patients who received ADMs had a higher risk of implant failure/loss at 7 months of followup (adjOR 1.90, 95\% CI 1.03 to 3.51). The other three NRCSs were excluded because they did not report an adjusted effect size. Ibrahim 2013 reported that risks of implant failure were comparable whether or not ADMs were used $(\mathrm{P}=0.9)$. However, Qureshi 2016 reported an adjOR for explant surgery of 0.2 (without CIs or a $\mathrm{P}$ value) and Peled 2012 reported that ADM use was associated with a lower risk of implant failure/loss $(\mathrm{P}<0.05)$. 


\section{Capsular Contracture and Harms to the Inframammary Fold}

Four NRCSs (Ganesh Kumar 2021, Nealon 2020b, Sobti 2018, and Vardanian 2011) reported on risk of capsular contracture, but their findings were inconsistent (Appendix Table E-5.2). We do not report a meta-analysis for this outcome due to substantial statistical heterogeneity (i.e., marked between-study variability in results, as suggested by an $\mathrm{I}^{2}$ of $85 \%$ ). Three of these NRCSs reported that rates of capsular contracture were comparable, irrespective of ADM use. These included one NRCS at 2 years of followup (Ganesh Kumar 2021) and two at approximately 5 years of followup (Nealon 2020b and Sobti 2018). Vardanian 2011, however, reported that ADM use was associated with a lower risk of capsular contracture at 2.4 years of followup (adjOR 0.18, 95\% CI 0.08 to 0.43 ).

One NRCS (Vardanian 2011) reported on harms to the inframammary fold, defined as issues related to the integrity of the fold but not bottoming out or shifting of the fold. ADM use was associated with a lower risk of this outcome (adjOR $0.49,95 \%$ CI 0.23 to 1.01 ).

\section{Hematoma}

Six NRCSs reported data on hematoma (Appendix Table F-5.19). Five of these NRCSs (Hirsch 2014, Lee 2020, Nealon 2020b, Seth 2012, and Sobti 2018) reported effect sizes that adequately accounted for confounders and were thus included in a meta-analysis (Figure 12). AdjORs ranged from 0.50 to 1.47 across these studies. The meta-analysis suggested that risks of hematoma were comparable whether or not ADMs were used during IBR (adjOR 0.88, 95\% CI 0.58 to $1.34 ; \mathrm{I}^{2}=0 \%$ ).

Figure 12. Meta-analysis for Key Question 5: Use versus nonuse of human ADMs during IBR hematoma

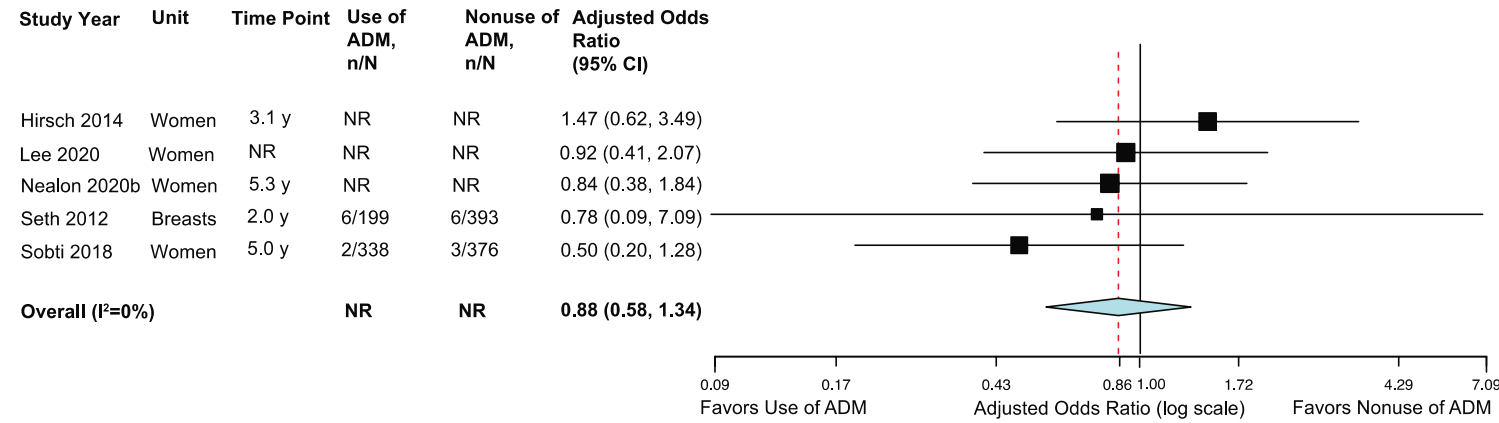

Abbreviations: $\mathrm{ADM}=$ acellular dermal matrix, $\mathrm{CI}=$ confidence interval, $\mathrm{IBR}=$ implant-based reconstruction, $\mathrm{I}^{2}=$ measure of statistical heterogeneity ( $\%$ of total variability that is due to between-study variability), OR = odds ratio.

Ganesh Kumar 2021, excluded from the meta-analysis because of the lack of a reported adjusted effect size, also reported that risks of hematoma were comparable whether or not ADMs were used $(\mathrm{P}=0.12)$.

\section{Composite or Unspecified Harms}

One RCT (Wendel 2013) and eight NRCSs (Brooke, 2012, Ganesh Kumar 2021, Hirsch 2014, Liu 2011, Safran 2020, Stein 2020, Weichman 2012, and Woo 2017) reported on various composite or unspecified harms (Appendix Table E-5.4). We did not conduct a meta-analysis of these studies because of great variability in how these outcomes were defined.

The RCT (Wendel 2013) reported that no serious adverse events occurred in either the ADM use or nonuse groups. Among the NRCSs, Liu 2011 reported a higher risk of surgical complications among patients who received ADMs than those who did not (adjOR 1.76, 95\% CI 
1.03 to 3.01) and Ganesh Kumar 2021 reported a higher risk of major complications among patients who received ADMs than those who did not (adjOR 1.43, 95\% CI 1.00 to 2.05). The other six NRCSs reported comparable risks in ADM and non-ADM groups for major complications and various other composite or unspecified harms, such as any complication, minor complications, and operative complications except explant surgery.

\section{Heterogeneity of Treatment Effects (Subgroup Differences)}

No study evaluated whether the relative effect of ADM use varied in different subgroups of patients who underwent IBR. Craig 2019 reported wound dehiscence data among the subgroup of women who had not received postoperative radiation therapy but did not compare these results with those who had. Across studies, the studies were too sparse to allow exploration of possible differences based on patient or other characteristics.

\section{Applicability}

Only one eligible study, which was conducted in the U.S., reported on race, with the large majority of women being White. However, most studies (20 of 22) were conducted in North America. Generally, the studies addressing this KQ contribute evidence that is directly applicable to the population of women receiving implants in the U.S. who have been shown to be between 80 and 90 percent White. ${ }^{19}$ All 22 studies addressing this KQ were published in the last 10 years.

\section{Overall Summary for Key Question 5}

The evidence is inconsistent regarding whether human ADM use during IBR impacts physical well-being, psychosocial well-being, or satisfaction with breasts. However, ADM use probably increases the risk of implant failure/loss or need for explant surgery (Moderate SoE) and may increase the risk of infections not explicitly implant-related (Low SoE). Whether or not $\mathrm{ADM}$ is used probably is associated with comparable risks of seroma and unplanned repeat surgeries for revision (Moderate SoE for both) and possibly necrosis (Low SoE). 


\section{Key Question 6. Comparisons of Flap Types for Autologous Reconstruction}

\section{Key Points}

- TRAM versus DIEP flaps

- AR with TRAM and DIEP flaps may result in comparable levels of patient satisfaction with breasts (Low SoE).

- Although risks of necrosis may be comparable (Low SoE), AR with TRAM flaps probably poses a greater risk of harms to the area of flap harvest (abdominal bulge/hernia and need for abdominal hernia repair surgery) (Moderate SoE).

- Because of sparse data, there is insufficient evidence to make conclusions about other clinical outcomes (physical, psychosocial, or social well-being, patient satisfaction with breasts, and duration of initial hospitalization) or about surgical complications (infections and wound dehiscence).

- Other comparisons of flaps

- Because of sparse data, there is insufficient evidence regarding various clinical outcomes or surgical complications.

We found 13 studies (reported in 15 articles $^{43,45,51,96,240-250}$ ) that conducted randomized or adjusted analyses for the comparison of different flap types among women undergoing AR. An additional six studies (reported in 15 articles $^{41,42,47,50,63,78,80,83,84,87,90,91,251-253}$ ) did not report any adjusted effect sizes or $\mathrm{P}$ values and are thus not discussed further in this section. Appendix Tables C-7, D-1, D-2, and D-3 describe all 19 studies, irrespective of whether they reported adjusted effect sizes. They reported data for six different flap types: TRAM, DIEP, LD, SIEA, lateral thoracodorsal (LTD), and thoracodorsal artery perforator (TAP).

The 13 studies included three RCTs and 10 NRCSs. Among the three RCTs, we rated Brandberg 2000 and Brorson 2020b to be at high risk of bias because of the lack of blinding of participants, study personnel, and outcome assessors and because of incomplete outcome data and selective outcome reporting. We rated Rindom 2019 to be at moderate risk of bias because of the lack of blinding of participants, study personnel, and outcome assessors. Among the 10 NRCSs, we rated eight to be at high risk of bias and two at moderate risk. High risk of bias ratings for NRCSs were mostly related to critical or serious risk of confounding and the lack of blinding of participants, study personnel, and/or outcome assessors. Not all NRCSs reported adjusted effect sizes with confidence intervals; some reported adjusted $\mathrm{P}$ values without effect sizes or effect sizes without confidence intervals or $P$ values.

The 13 studies included between 50 and 15,836 women and were conducted mostly in North America (four in the U.S., three in Canada, and one in both). The remaining five studies were conducted in Sweden $(n=2)$ and Denmark, France, and the U.K. ( $\mathrm{n}=1$ each). Among the studies reporting data, the women's mean ages ranged from 49 to 52 years and their mean BMIs ranged from 25.9 to $28.6 \mathrm{~kg} / \mathrm{m} 2$. Two studies, both from the U.S., reported on patient race; the majority $(80 \%$ and $70 \%)$ of patients were White. Studies followed women for about up to 2 to 3 years. The study result summaries are in Appendix Tables E-6.1 to E-6.6. Full results are in Appendix Tables F-6.1 to F-6.25. 


\section{Summary of Comparisons of Flap Types for Autologous Reconstruction}

Table 8 summarizes the evidence for the comparisons of various flap types for AR. Women who undergo AR with TRAM and DIEP flaps may experience similar levels of satisfaction with their breasts. However, when compared with women with DIEP flaps, women with TRAM flaps may be at greater risks of abdominal bulge/hernia and of needing abdominal hernia repair surgery but not at greater risk of necrosis.

While six different flaps have been compared, the evidence for comparisons of specific pairs of flaps is sparse. The evidence identified for this KQ was too sparse to allow pairwise metaanalysis, let alone network meta-analysis. It is unclear whether any specific flap type is clearly associated with better patient-reported (clinical) outcomes than the others. 
Table 8. Evidence profile for Key Question 6: Comparisons of flap types for AR 


\begin{tabular}{|c|c|c|c|c|c|c|c|c|c|}
\hline Comparison & $\begin{array}{l}\text { Outcome } \\
\text { Category }\end{array}$ & Outcome & $\begin{array}{l}\text { N Studies } \\
\text { (Patients) }\end{array}$ & RoB & Consistency & Precision & Directness & SoE & $\begin{array}{l}\text { Conclusions (Reason, if } \\
\text { None) }\end{array}$ \\
\hline \multirow[t]{10}{*}{$\begin{array}{l}\text { TRAM vs. } \\
\text { DIEP }\end{array}$} & $\begin{array}{l}\text { Clinical } \\
\text { outcomes }\end{array}$ & Physical well-being & $1(\mathrm{NR})$ & Moderate & $\mathrm{N} / \mathrm{A}$ & Precise & Direct & Insufficient & None (Sparse evidence) \\
\hline & $\begin{array}{l}\text { Clinical } \\
\text { outcomes }\end{array}$ & $\begin{array}{l}\text { Psychosocial well- } \\
\text { being }\end{array}$ & 1 (NR) & Moderate & $\mathrm{N} / \mathrm{A}$ & Precise & Direct & Insufficient & None (Sparse evidence) \\
\hline & $\begin{array}{l}\text { Clinical } \\
\text { outcomes }\end{array}$ & Sexual well-being & $1(\mathrm{NR})$ & Moderate & $\mathrm{N} / \mathrm{A}$ & Precise & Direct & Insufficient & None (Sparse evidence) \\
\hline & $\begin{array}{l}\text { Clinical } \\
\text { outcomes }\end{array}$ & $\begin{array}{l}\text { Patient satisfaction } \\
\text { with breasts }\end{array}$ & $2(\mathrm{NR})$ & Moderate & Consistent & Precise & Direct & Low & Comparable in both groups \\
\hline & $\begin{array}{l}\text { Clinical } \\
\text { outcomes }\end{array}$ & $\begin{array}{l}\text { Patient satisfaction } \\
\text { with outcome }\end{array}$ & $1(260)$ & High & $\mathrm{N} / \mathrm{A}$ & Imprecise & Direct & Insufficient & None (Sparse evidence) \\
\hline & $\begin{array}{l}\text { Clinical } \\
\text { outcomes }\end{array}$ & $\begin{array}{l}\text { Duration of initial } \\
\text { hospitalization }\end{array}$ & $1(15836)$ & High & $\mathrm{N} / \mathrm{A}$ & Unclear & Direct & Insufficient & None (Sparse evidence) \\
\hline & $\begin{array}{l}\text { Surgical } \\
\text { complications }\end{array}$ & Necrosis & 2 (959) & High & Consistent & Unclear & Direct & Low & Comparable in both groups \\
\hline & $\begin{array}{l}\text { Surgical } \\
\text { complications }\end{array}$ & $\begin{array}{l}\text { Harms to area of } \\
\text { flap harvest }\end{array}$ & $4(9253)$ & High & Consistent & Precise & Direct & Moderate & $\begin{array}{l}\text { TRAM had increased risk } \\
\text { of abdominal bulge/hernia } \\
\text { and abdominal hernia } \\
\text { repair surgery }\end{array}$ \\
\hline & $\begin{array}{l}\text { Surgical } \\
\text { complications }\end{array}$ & Infections & $1(15836)$ & High & $\mathrm{N} / \mathrm{A}$ & Unclear & Direct & Insufficient & None (Sparse evidence) \\
\hline & $\begin{array}{l}\text { Surgical } \\
\text { complications }\end{array}$ & Wound dehiscence & $1(15836)$ & High & $\mathrm{N} / \mathrm{A}$ & Unclear & Direct & Insufficient & None (Sparse evidence) \\
\hline \multirow[t]{3}{*}{ DIEP vs. LD } & $\begin{array}{l}\text { Clinical } \\
\text { outcomes }\end{array}$ & $\begin{array}{l}\text { Patient satisfaction } \\
\text { with breasts }\end{array}$ & $1(229)$ & HIgh & $\mathrm{N} / \mathrm{A}$ & Imprecise & Direct & Insufficient & None (Sparse evidence) \\
\hline & $\begin{array}{l}\text { Clinical } \\
\text { outcomes }\end{array}$ & $\begin{array}{l}\text { Patient satisfaction } \\
\text { with outcome }\end{array}$ & $1(229)$ & High & $\mathrm{N} / \mathrm{A}$ & Imprecise & Direct & Insufficient & None (Sparse evidence) \\
\hline & $\begin{array}{l}\text { Surgical } \\
\text { complications }\end{array}$ & $\begin{array}{l}\text { Thromboembolic } \\
\text { events }\end{array}$ & $1(56)$ & High & $\mathrm{N} / \mathrm{A}$ & Imprecise & Direct & Insufficient & None (Sparse evidence) \\
\hline \multirow[t]{5}{*}{$\begin{array}{l}\text { SIEA vs. } \\
\text { DIEP }\end{array}$} & $\begin{array}{l}\text { Clinical } \\
\text { outcomes }\end{array}$ & Physical well-being & $1(\mathrm{NR})$ & Moderate & $\mathrm{N} / \mathrm{A}$ & Precise & Direct & Insufficient & None (Sparse evidence) \\
\hline & $\begin{array}{l}\text { Clinical } \\
\text { outcomes }\end{array}$ & $\begin{array}{l}\text { Psychosocial well- } \\
\text { being }\end{array}$ & 1 (NR) & Moderate & $\mathrm{N} / \mathrm{A}$ & Precise & Direct & Insufficient & None (Sparse evidence) \\
\hline & $\begin{array}{l}\text { Clinical } \\
\text { outcomes }\end{array}$ & Sexual well-being & 1 (NR) & Moderate & $\mathrm{N} / \mathrm{A}$ & Precise & Direct & Insufficient & None (Sparse evidence) \\
\hline & $\begin{array}{l}\text { Clinical } \\
\text { outcomes }\end{array}$ & $\begin{array}{l}\text { Patient satisfaction } \\
\text { with breasts }\end{array}$ & 1 (NR) & Moderate & $\mathrm{N} / \mathrm{A}$ & Precise & Direct & Insufficient & None (Sparse evidence) \\
\hline & $\begin{array}{l}\text { Surgical } \\
\text { complications }\end{array}$ & $\begin{array}{l}\text { Harms to area of } \\
\text { flap harvest }\end{array}$ & $1(417)$ & Moderate & $\mathrm{N} / \mathrm{A}$ & Precise & Direct & Insufficient & None (Sparse evidence) \\
\hline \multirow[t]{2}{*}{ TAP vs. LD } & $\begin{array}{l}\text { Clinical } \\
\text { outcomes }\end{array}$ & Physical well-being & $1(40)$ & Moderate & $\mathrm{N} / \mathrm{A}$ & Imprecise & Direct & Insufficient & None (Sparse evidence) \\
\hline & $\begin{array}{l}\text { Clinical } \\
\text { outcomes }\end{array}$ & $\begin{array}{l}\text { Duration of initial } \\
\text { hospitalization }\end{array}$ & $1(40)$ & Moderate & $\mathrm{N} / \mathrm{A}$ & Precise & Direct & Insufficient & None (Sparse evidence) \\
\hline
\end{tabular}




\begin{tabular}{|c|c|c|c|c|c|c|c|c|c|}
\hline & $\begin{array}{l}\text { Surgical } \\
\text { complications }\end{array}$ & Pain & $1(40)$ & Moderate & $\mathrm{N} / \mathrm{A}$ & Imprecise & Direct & Insufficient & None (Sparse evidence) \\
\hline & $\begin{array}{l}\text { Surgical } \\
\text { complications }\end{array}$ & Necrosis & $1(40)$ & Moderate & $\mathrm{N} / \mathrm{A}$ & Imprecise & Direct & Insufficient & None (Sparse evidence) \\
\hline & $\begin{array}{l}\text { Surgical } \\
\text { complications }\end{array}$ & Infections & $1(40)$ & Moderate & $\mathrm{N} / \mathrm{A}$ & Imprecise & Direct & Insufficient & None (Sparse evidence) \\
\hline & $\begin{array}{l}\text { Surgical } \\
\text { complications }\end{array}$ & Seroma & $1(40)$ & Moderate & $\mathrm{N} / \mathrm{A}$ & Imprecise & Direct & Insufficient & None (Sparse evidence) \\
\hline \multirow[t]{4}{*}{$\begin{array}{l}\text { TRAM vs. } \\
\text { LD }\end{array}$} & $\begin{array}{l}\text { Clinical } \\
\text { outcomes }\end{array}$ & $\begin{array}{l}\text { Patient satisfaction } \\
\text { with breasts }\end{array}$ & $1(49)$ & High & $\mathrm{N} / \mathrm{A}$ & Unclear & Direct & Insufficient & None (Sparse evidence) \\
\hline & $\begin{array}{l}\text { Clinical } \\
\text { outcomes }\end{array}$ & $\begin{array}{l}\text { Patient satisfaction } \\
\text { with outcome }\end{array}$ & $1(255)$ & High & $\mathrm{N} / \mathrm{A}$ & Imprecise & Direct & Insufficient & None (Sparse evidence) \\
\hline & $\begin{array}{l}\text { Clinical } \\
\text { outcomes }\end{array}$ & Mortality & $1(59)$ & High & $\mathrm{N} / \mathrm{A}$ & Imprecise & Direct & Insufficient & None (Sparse evidence) \\
\hline & $\begin{array}{l}\text { Surgical } \\
\text { complications }\end{array}$ & $\begin{array}{l}\text { Unplanned repeat } \\
\text { surgeries for } \\
\text { revision }\end{array}$ & $1(3296)$ & High & $\mathrm{N} / \mathrm{A}$ & Precise & Direct & Insufficient & None (Sparse evidence) \\
\hline \multirow[t]{3}{*}{$\begin{array}{l}\text { TRAM vs. } \\
\text { LTD }\end{array}$} & $\begin{array}{l}\text { Clinical } \\
\text { outcomes }\end{array}$ & $\begin{array}{l}\text { Patient satisfaction } \\
\text { with breasts }\end{array}$ & $1(38)$ & High & $\mathrm{N} / \mathrm{A}$ & Unclear & Direct & Insufficient & None (Sparse evidence) \\
\hline & $\begin{array}{l}\text { Clinical } \\
\text { outcomes }\end{array}$ & Mortality & $1(45)$ & High & $\mathrm{N} / \mathrm{A}$ & Imprecise & Direct & Insufficient & None (Sparse evidence) \\
\hline & $\begin{array}{l}\text { Surgical } \\
\text { complications }\end{array}$ & All & $0(0)$ & N/A & $\mathrm{N} / \mathrm{A}$ & N/A & $\mathrm{N} / \mathrm{A}$ & N/A & N/A \\
\hline \multirow[t]{3}{*}{ LD vs. LTD } & $\begin{array}{l}\text { Clinical } \\
\text { outcomes }\end{array}$ & $\begin{array}{l}\text { Patient satisfaction } \\
\text { with breasts }\end{array}$ & $1(35)$ & High & $\mathrm{N} / \mathrm{A}$ & Unclear & Direct & Insufficient & None (Sparse evidence) \\
\hline & $\begin{array}{l}\text { Clinical } \\
\text { outcomes }\end{array}$ & Mortality & $1(46)$ & High & $\mathrm{N} / \mathrm{A}$ & Imprecise & Direct & Insufficient & None (Sparse evidence) \\
\hline & $\begin{array}{l}\text { Surgical } \\
\text { complications }\end{array}$ & All & $0(0)$ & $\mathrm{N} / \mathrm{A}$ & $\mathrm{N} / \mathrm{A}$ & $\mathrm{N} / \mathrm{A}$ & $\mathrm{N} / \mathrm{A}$ & $\mathrm{N} / \mathrm{A}$ & N/A \\
\hline
\end{tabular}

Abbreviations: adj = adjusted, $\mathrm{AR}=$ autologous reconstruction, $\mathrm{CI}=$ confidence interval, $\mathrm{DIEP}=$ deep inferior epigastric perforator, $\mathrm{LD}=$ latissimus dorsi, $\mathrm{LTD}=$ lateral

thoracodorsal, $\mathrm{OR}=$ odds ratio, $\mathrm{N} / \mathrm{A}=$ not applicable, $\mathrm{RoB}=$ risk of bias, $\mathrm{SIEA}=$ superficial inferior epigastric artery, $\mathrm{SoE}=$ strength of evidence, $\mathrm{TAP}=$ thoracodorsal artery perforator, TRAM $=$ transverse rectus abdominis myocutaneous.

Colors: Header rows are shaded orange. Rows for every alternate comparison are shaded blue. The colors do not add unique information.

Appendix Table G-6 provides the complete version of this evidence profile, including displaying all outcomes for which no evidence was identified. 


\section{Clinical Outcomes}

Two RCTs (Brandberg 2000 and Rindom 2019) and three NRCSs (Erdmann-Sager 2018, Yueh 2009, and Zoghbi 2017) reported on clinical outcomes (Appendix Tables E-6.1 to E-6.5).

\section{Physical Well-Being}

One RCT (Rindom 2019) and one NRCS (Erdmann-Sager 2018) reported on physical wellbeing (Appendix Table E-6.1).

Rindom 2019, which randomized women undergoing AR to receive either the LD or TAP flap, reported overall and subscale data for the Constant Murley Score evaluating physical function of the shoulder at 1 year of followup. The overall score ranges from 0 to 100 , with subscale scores ranging from 0 to 15 (pain), 0 to 20 (activity in daily life), 0 to 40 (range of motion), and 0 to 25 (strength). Higher scores indicate better physical function of the shoulder. The MCID for the overall Constant Murley Score has been estimated to be 10 points. ${ }^{215}$ To our knowledge, MCIDs have not been estimated for the subscale scores. Rindom 2019 reported that, overall and across the subscales, women with TAP and LD flaps had clinically similar scores (Appendix Table E-6.1). However, women who received TAP flaps had statistically significantly higher scores overall and for the pain and activity in daily life subscales, but not for the range of motion and strength subscales.

Erdmann-Sager 2018 reported on physical well-being at 1 and 2 years of followup for patients undergoing AR with DIEP, free TRAM, pedicled TRAM, or SIEA flaps. Data were reported for the BREAST-Q physical function: abdomen- and chest/upper body-specific score (0 to 100; higher is better; MCID 3 for patients undergoing $\mathrm{AR}^{187}$ ). At 1 year of followup, patients undergoing AR with DIEP flaps reported clinically better abdominal physical function than patients undergoing AR with TRAM flaps, whether free (adjMD 4.16, 95\% CI 0.02 to 8.33) or pedicled (adjMD 4.01, 95\% CI 0.45 to 8.48). Similar results were observed at 2 years of followup. At 1 year, patients undergoing AR with SIEA flaps experienced abdominal physical function that was clinically better than patients who received DIEP flaps (adjMD 4.72, 95\% CI -0.07 to 9.52 ). However, at 2 years, abdominal physical function scores were similar between SIEA and DIEP flap groups (adjMD 0.58, 95\% CI -4.79 to 5.95).

Erdmann-Sager 2018 reported that, at 1 year of followup, patients undergoing AR with DIEP flaps experienced clinically similar chest and upper body physical function as patients undergoing AR with TRAM flaps, whether free or pedicled. However, patients undergoing AR with SIEA flaps experienced clinically better chest and upper body physical function than patients who received DIEP flaps (adjMD 3.42, 95\% CI -0.22 to 7.05). At 2 years of followup, though, chest and upper body physical function scores were comparable between SIEA and DIEP groups and between DIEP and both TRAM groups.

Erdmann-Sager 2018 also reported data for the physical functioning and pain interference components of the PROMIS instrument. Both component scores range from 0 to 100 , with higher scores indicating poorer outcomes. For both, the MCID has been estimated to be 4 to 6 points. ${ }^{188}$ At both 1 and 2 years of followup, Erdmann-Sager 2018 reported that physical functioning as well as pain interference were clinically comparable between SIEA and DIEP groups and between DIEP and both TRAM groups. 


\section{Psychosocial and Sexual Well-Being}

One NRCS (Erdmann-Sager 2018) reported on psychosocial well-being using the BREAST$\mathrm{Q}$ (0 to 100; higher is better; MCID 4 for patients undergoing $\mathrm{AR}^{187}$ ) (Appendix Table E-6.2). At both 1 and 2 years of followup, psychosocial well-being was clinically comparable between patients undergoing AR with DIEP flaps, free TRAM flaps, and pedicled TRAM flaps, and between patients undergoing AR with DIEP and SIEA flaps.

Erdmann-Sager 2018 also reported on sexual well-being using the BREAST-Q (0 to 100; higher is better; MCID 5 for patients undergoing $\mathrm{AR}^{187}$ ) (Appendix Table E-6.2). At both 1 and 2 years of followup, sexual well-being was clinically comparable between patients undergoing AR with DIEP flaps, free TRAM flaps, and pedicled TRAM flaps, and between patients undergoing AR with DIEP and SIEA flaps.

\section{Patient Satisfaction With Breasts}

One RCT (Brandberg 2000) and two NRCSs (Erdmann-Sager 2018 and Yueh 2009) reported on patient satisfaction with breasts (Appendix Tables E-6.3 and E-6.4). Brandberg 2000 randomized women undergoing AR to receive either TRAM, LD, or LTD flaps (Appendix Table E-6.3). The authors constructed their own questionnaire that included six satisfaction-related items: cosmetic, shape, size, scars on the breast, donor site scars, and similarity with contralateral breast. Each item was measured on a scale of 1 to 6 and reported separately (without a total score), with higher scores representing greater satisfaction. To our knowledge, an MCID has not been estimated for this questionnaire. At 1 year of followup, scores for each of the six items were reported to be similar between the TRAM, LD, and LTD groups. All between-flap differences for each item were within 1 point.

One NRCS (Erdmann-Sager 2018) reported on patient satisfaction with breasts at 1 and 2 years of followup for patients undergoing AR with DIEP, free TRAM, pedicled TRAM, or SIEA flaps. Data were reported using the BREAST-Q satisfaction with breasts ( 0 to 100 ; higher is better; MCID 5 for patients undergoing $\mathrm{AR}^{187}$ ). At both 1 and 2 years of followup, satisfaction levels with breasts were clinically similar between patients undergoing AR with DIEP flaps, free TRAM flaps, and pedicled TRAM flaps, and between patients undergoing AR with DIEP and SIEA flaps.

The other NRCS (Yueh 2009), which evaluated patients who underwent AR with DIEP, TRAM, or LD flaps, reported data on whether or not patients were satisfied with their breasts (Appendix Table E-6.4). The proportions of patients reporting satisfaction were comparable between patients with DIEP and TRAM flaps (adjOR 0.67, 95\% CI 0.37 to 1.23 ), between patients with LD and TRAM flaps (adjOR 0.78, 95\% CI 0.54, 1.14), and between patients with DIEP and LD flaps (adjOR 0.90, 95\% CI 0.60, 1.34).

\section{Patient Satisfaction With Surgical Outcome}

One NRCS (Yueh 2009) reported that proportions of patients who reported satisfaction with their surgical outcome (defined as general satisfaction with surgery) were comparable between patients who underwent AR with DIEP, TRAM, and LD flaps (Appendix Table E-6.4).

\section{Recurrence of Breast Cancer}

One RCT (Brandberg 2000) reported low risks of breast cancer recurrence at 1 year of followup across flap types in patients who underwent AR with TRAM, LD, or LTD flaps (Appendix Table E-6.4). Data for between-flap comparisons were highly imprecise. 


\section{Duration of Initial Hospitalization}

One RCT (Rindom 2019) and one NRCS (Zoghbi 2017) reported data for duration of initial hospitalization (Appendix Tables E-6.3 and E-6.4). Rindom 2019 reported that patients randomized to AR with LD or TAP flaps had similar durations of initial hospitalization (adjMD 0.9 days, 95\% CI -1.4 to 3.2) (Appendix Table E-6.3). Zoghbi 2017, which was a large NRCS $(15,836$ women), reported that women with TRAM flaps had statistically significant longer hospital stays than women with DIEP flaps ( $\mathrm{P}<0.001$; an adjusted effect size was not reported). Zoghbi 2017 also reported that women with TRAM flaps had higher odds of having an increased length of stay (adjOR 1.59, 95\% CI 1.45 to 1.72) (Appendix Table E-6.4).

\section{Mortality}

One RCT (Brandberg 2000) reported low rates of mortality at 1 year of followup across flap types in patients who underwent AR with TRAM, LD, or LTD flaps (Appendix Table E-6.5). Data for between-flap comparisons were highly imprecise.

\section{Surgical Complications}

Both RCTs (Brandberg 2000 and Rindom 2019) and eight NRCSs (Abedi 2016, ErdmannSager 2018, Knox 2016, Kroll 2000, Massenburg 2015, Mennie 2015, Zhong 2014, and Zoghbi 2017) reported on surgical complications of various flap types.

\section{Unplanned Repeat Surgeries for Revision of Reconstruction}

One NRCS (Massenburg 2015) reported on unplanned repeat surgeries for revision of reconstruction in women who underwent AR with pedicled TRAM or LD flaps (Appendix Table E-6.4). Compared with women who underwent AR with LD flaps, risks of unplanned repeat surgery at 1 month of followup were higher in women who underwent AR with pedicled TRAM flaps (adjOR 1.71, 95\% CI 1.25 to 2.33 ).

\section{Pain and Necrosis}

One RCT (Rindom 2019) reported that patients randomized to undergo AR with TAP flaps were considerably less likely than patients randomized to undergo AR with LD flaps to experience shoulder-related pain at 1 year of followup (OR $0.05,95 \%$ CI $0.005,0.51$ ) (Appendix Table E-6.5).

One RCT (Rindom 2019) and two NRCSs (Abedi 2016 and Kroll 2000) reported on the outcome of necrosis (Appendix Table E-6.5). Rindom 2019 randomized patients to undergo AR with LD or TAP flaps (both flaps required the use of implants). Data were reported for major necrosis (defined as necrosis necessitating removal of the implant) and minor necrosis (defined as epidermolysis or small necrosis of the most distal part of the flap) at 1 year of followup. For both outcomes, data for between-flap comparisons were highly imprecise. Abedi 2016 reported that risks of mastectomy flap necrosis at 1.6 to 1.9 years of followup were comparable between patients who underwent AR with DIEP and TRAM flaps $(\mathrm{P}=0.610$; adjusted effect size not reported). Kroll 2000 reported, however, that the risk of fat necrosis at 3 months of followup was higher among patients undergoing AR with DIEP flaps than TRAM flaps (adjOR 2.10, 95\% CI $0.87,5.10)$, but this was not statistically significant. 


\section{Harms to Area of Flap Harvest}

Four NRCSs (Erdmann-Sager 2018, Knox 2016, Mennie 2015, and Zhong 2014) reported on harms to the area of flap harvest (Appendix Table E-6.5). Erdmann-Sager 2018 reported that, at 2 years of followup, compared with patients who underwent AR with DIEP flaps, patients who underwent AR with free TRAM flaps had a lower risk of donor site complications (adjOR 0.52, 95\% CI 0.27 to 1.02), while women who underwent AR with SIEA flaps had a higher risk (adjOR 2.73, 95\% CI 1.51 to 4.96). Patients who underwent AR with DIEP or pedicled TRAM flaps had comparable risks.

The other three NRCSs (Knox 2016, Mennie 2015, and Zhong 2014) reported that AR with TRAM flaps were associated with greater risks of harms to the area of flap harvest than was AR with DIEP flaps. Higher risks of abdominal bulge/hernia were reported for AR with TRAM flaps by Knox 2016 (adjOR 5.2, 95\% CI 1.3 to 20.9) and by Zhong 2014 (adjOR 2.73, 95\% CI 1.01 to 7.07). Mennie 2015 reported higher risks of needing hernia repair surgery in both the free TRAM flap group (adjOR 1.81, 95\% CI 1.24 to 2.64) and the pedicled TRAM flap group (adjOR 2.89, 95\% CI 1.91 to 4.37) when compared with the DIEP flap group.

\section{Thromboembolic Events, Infections, Wound Dehiscence, Seroma, and Hematoma}

One RCT (Brorson 2020b) reported on the outcome of thromboembolic events in patients who underwent AR with DIEP and LD flaps. (Appendix Table E-6.5). Neither group experienced any DVT or PE events at 1 month of followup.

One RCT (Rindom 2019) and one NRCS (Zoghbi 2017) reported on the outcome of infections (Appendix Table E-6.5). Rindom 2019 reported low risks (one case of infection each) in the LD and TAP groups. Data for between-flap comparisons were highly imprecise. Zoghbi 2017 reported that wound infections were more common in women who underwent AR with TRAM than DIEP flaps (adjOR 1.67, 95\% CI 1.23 to 2.27 ).

One NRCS (Zoghbi 2017) reported that the risk of wound dehiscence was higher among patients undergoing AR with TRAM than DIEP flaps (adjOR 4.3; CIs not reported; $\mathrm{P}<0.001$ ) (Appendix Table E-6.5).

One RCT (Rindom 2019) reported on the outcome of seroma in patients randomized to AR with either LD or TAP flaps (Appendix Table E-6.5). Only one case of seroma occurred in the LD group and none in the TAP group. Data for the between-flap comparison were highly imprecise.

One RCT (Rindom 2019) reported on the outcome of hematoma at 1 year of followup in patients randomized to AR with either LD or TAP flaps (Appendix Table E-6.5). Only one case of hematoma occurred in the TAP group and none in the LD group. Data for the between-flap comparison were highly imprecise.

\section{Flap Failure/Loss}

Two NRCSs (Kroll 2000 and Massenburg 2015) reported on flap failure or loss (Appendix Table E-6.5). Kroll 2000 reported a considerably higher risk of partial flap loss at 3 months of followup among patients undergoing AR with DIEP than TRAM flaps (adjOR 6.74, 95\% CI 1.83 to 24.7). Without specifying whether flap failure/loss was defined as partial or total, Massenburg 2015 reported that, compared with patients who underwent AR with LD flaps, higher risks of flap failure/loss were observed at 1 month of followup in patients who underwent AR with pedicled TRAM flaps (adjOR 2.28, 95\% CI 1.38 to 3.77). 


\section{Composite or Unspecified Harms}

Two RCTs (Brorson 2020b and Rindom 2019) and four NRCSs (Dauplat 2021, ErdmannSager 2018, Massenburg 2015, and Zhong 2014) reported on various composite or unspecified harms (Appendix Table E-6.6). There was considerable inconsistency across studies in how harms were defined.

Brorson 2020b reported on complications using the Clavien-Dindo Grading system in patients randomized to undergo AR with either DIEP or LD flaps. Risks of complications were similar between groups at 1 month of followup, except that patients who underwent AR with LD flaps had a lower incidence of Grade IIIb complications (OR 0.22, 95\% CI 0.05 to 0.87; $\mathrm{P}=0.031$ )

Rindom 2019 reported on both major and minor complications at 1 year of followup in patients randomized to undergo AR with either LD or TAP flaps. For both outcomes, data for the between-flap comparisons were highly imprecise.

Dauplat 2021 reported on major breast complications requiring surgical intervention or readmission at 1 year of followup. Patients who underwent AR with LD without implant had a higher risk than patients who underwent AR with TRAM (adjOR 1.69, 95\% CI 1.19 to 2.41) or AR with LD without implant (adjOR 4.85, 95\% CI 1.67 to 14.1).

Zhong 2014 reported on major breast complications, defined as the composite of total or partial flap loss, fat necrosis, and breast hematoma (time-point not reported). Patients who underwent AR with DIEP or LD flaps experienced similar risks for this composite outcome.

Erdmann-Sager 2018 reported that, at 2 years of followup, compared with patients who underwent AR with DIEP flaps, patients who underwent AR with free TRAM flaps had a lower risk of any complication (adjOR $0.51,95 \%$ CI 0.25 to 1.02). Women who underwent AR with pedicled TRAM flaps or SIEA flaps had risks that were comparable with women who underwent AR with DIEP flaps.

Massenburg 2015 reported that, compared with patients who underwent AR with LD flaps, higher risks of any complication were observed at 1 month of followup in patients who underwent AR with pedicled TRAM flaps (adjOR 1.92, 95\% CI 1.45 to 2.55). Similarly, higher risks of the composite outcome of superficial or deep surgical site infection, organ space infection, or wound disruption/dehiscence were also observed at 1 month of followup in patients who underwent AR with free TRAM flaps (adjOR 1.46, 95\% CI 1.00 to 2.12) or pedicled TRAM flaps (adjOR 1.80, 95\% CI 1.29 to 2.51 ).

\section{Heterogeneity of Treatment Effects (Subgroup Differences)}

None of the studies reported subgroup results or other analyses of possible heterogeneity of treatment effects. The studies were too sparse to allow exploration of possible differences based on patient or other characteristics.

\section{Applicability}

Although only 2 (of 13) studies addressing this KQ reported information about patient race, more than half of the studies (8 of 13) were conducted in North America. Generally, these studies contribute evidence that is directly applicable to the population of women undergoing AR in the U.S. However, in addition to the paucity of the evidence for the flap types addressed, we did not find any studies of other flap types that are used in the U.S. These include the profunda artery perforator (PAP), superior gluteal artery perforator (SGAP), transverse musculocutaneous 
gracilis (TMG), and transverse upper gracilis (TUG) flaps. Eleven of the 13 studies were published in the last 12 years. Two studies (Brandberg 2000 and Kroll 2000) were published 20 years ago with data from mostly the 1990s, and so their applicability to today's clinical practice should be interpreted with caution.

\section{Overall Summary for Key Question 6}

AR with either TRAM or DIEP flaps may result in comparable patient satisfaction with breasts (Low SoE), but TRAM flaps probably increase the risk of harms to the area of flap harvest (Moderate SoE). AR with either DIEP or LD flaps may result in comparable patient satisfaction with breasts (Low SoE), but there is insufficient evidence regarding thromboembolic events and no evidence regarding other surgical complications. 


\section{Discussion}

\section{Findings in Relation to the Decisional Dilemmas}

Despite a large overall body of evidence (160 studies), the evidence was sparse for most specific questions of interest in this systematic review (SR). Notably, we found only eight randomized controlled trials (RCTs). Table 9 summarizes the identified evidence addressing the six Key Questions (KQs).

Although the largest number of studies addressed the overall choice between implant-based reconstruction (IBR) and autologous reconstruction (AR), the evidence does not clearly establish which is more likely to be preferred. AR is probably associated with better sexual wellbeing and satisfaction with breasts, but the evidence suggests that it is associated with comparable general quality of life and psychosocial well-being. In terms of surgical complications, AR probably poses a greater risk of deep vein thrombosis or pulmonary embolism, but IBR may pose a greater risk of breast seroma. Risks of other adverse events are largely comparable, or the evidence is insufficient and/or inconsistent across studies, precluding conclusions. Although the findings were inconsistent in the short term ( 1 to 1.3 months), compared with AR, IBR probably poses greater risk of reconstructive failure in the long term (1.5 to 4 years).

Among women who decide to undergo IBR, we found little evidence to address the best timing of the IBR in relation to the two main categories of nonsurgical treatments for cancer: chemotherapy and radiation therapy. We found no evidence regarding whether the IBR should be conducted before or after chemotherapy. We found that timing of IBR in relation to radiation therapy may not affect the patient-reported clinical outcomes of physical well-being, psychosocial well-being, sexual well-being, and satisfaction with breasts. The evidence, though, suggests that the risks of implant failure or loss is probably comparable whether the IBR is conducted before or after radiation therapy. The evidence was insufficient to make conclusions about other harms, such as pain, necrosis, and seroma.

For women choosing between silicone and saline implants, the evidence suggests that the two types of implant materials may be associated with clinically comparable levels of patient satisfaction with breasts. The evidence was insufficient to make conclusions about surgical complications, such as implant failure or loss and capsular contracture. There was also insufficient evidence comparing double lumen implants and other implant types. Studies eligible for this SR did not address the risk of new neoplasms, in particular implant-associated anaplastic large cell lymphoma (BIA-ALCL).

Evidence was largely insufficient regarding the choice of anatomic plane of implant placement for IBR. Prepectoral and total submuscular placements may be associated with comparable risks of infections that are not explicitly implant-related. Evidence for the comparisons involving partial submuscular placement was insufficient. The eligible studies provided insufficient or no evidence regarding two of the principal concerns when determining which plane to use, animation deformity and pain.

Studies examining use versus nonuse of human acellular dermal matrices (ADMs) during IBR report inconsistent results addressing the clinical outcomes of physical well-being, psychosocial well-being, and satisfaction with breasts. However, ADM use probably increases the risk of implant failure or loss and may increase the risk of infections that are not explicitly implant- or ADM-related. Some surgical complications, such as necrosis and seroma may be comparable whether or not ADMs are used, although results for others, such as pain and capsular 
contracture, are inconsistent. The inconsistent (and thus insufficient) evidence regarding whether ADM use impacts capsular contracture risk is a surprising finding because this risk has traditionally been thought to be lower when ADMs are used. ${ }^{28}$

Among women who decide to undergo AR, we found no evidence to address the best timing of the AR before or after chemotherapy or radiation therapy. Finally, regarding choice of flap type to use for AR, we found evidence for six different flap types, but conclusions could only be made for the comparison between deep inferior epigastric perforator (DIEP) and transverse rectus abdominis myocutaneous (TRAM) flaps. The only patient-reported clinical outcome for which a conclusion is feasible is patient satisfaction with breasts, which was comparable. The only surgical complications for which conclusions were feasible are risks of necrosis and of harms to the area of flap harvest. Necrosis risks were comparable between DIEP and TRAM flaps, but TRAM flaps had a higher risk of harms to the area of flap harvest (abdominal bulge/ hernia and needing abdominal hernia repair surgery). 
Table 9. Full summary of evidence identified in this systematic review

\begin{tabular}{|c|c|c|c|c|c|c|c|}
\hline $\begin{array}{l}\text { Outcome } \\
\text { Categories }\end{array}$ & Outcomes & $\begin{array}{l}\text { KQ 1: IBR Versus } \\
\text { AR }\end{array}$ & $\begin{array}{l}\text { KQ 2b:* IBR } \\
\text { Before Versus } \\
\text { After Radiation }\end{array}$ & $\begin{array}{l}\text { KQ 3: IBR } \\
\text { Materials: } \\
\text { Silicone Versus } \\
\text { Saline }\end{array}$ & $\begin{array}{l}\text { KQ 4: Prepectoral } \\
\text { Versus Total } \\
\text { Submuscular } \\
\text { Placement for IBR }\end{array}$ & $\begin{array}{l}\text { KQ 5: ADM Use } \\
\text { Versus Nonuse } \\
\text { During IBR }\end{array}$ & KQ 6: AR Flap Types \\
\hline \multirow[t]{10}{*}{$\begin{array}{l}\text { Clinical } \\
\text { outcomes }\end{array}$} & $\begin{array}{l}\text { General quality of } \\
\text { life }\end{array}$ & $\sim \sim$ Comparable & nd & ? No conclusion & nd & nd & nd \\
\hline & Physical well-being & $\uparrow \downarrow$ No conclusion & $\sim$ Comparable & ? No conclusion & ? No conclusion & $\uparrow \downarrow$ No conclusion & ? No conclusion \\
\hline & $\begin{array}{l}\text { Psychosocial well- } \\
\text { being }\end{array}$ & $\sim$ Comparable & $\sim$ Comparable & ? No conclusion & ? No conclusion & $\uparrow \downarrow$ No conclusion & ? No conclusion \\
\hline & Sexual well-being & $\begin{array}{l}\Delta \boldsymbol{\Delta} \text { AR clinically } \\
\text { better }\end{array}$ & $\sim$ Comparable & ? No conclusion & nd & ? No conclusion & ? No conclusion \\
\hline & $\begin{array}{l}\text { Patient satisfaction } \\
\text { with breasts }\end{array}$ & $\begin{array}{l}\boldsymbol{\Delta} \boldsymbol{\Delta} \text { AR clinically } \\
\text { better }\end{array}$ & $\sim$ Comparable & $\sim$ Comparable & ? No conclusion & $\uparrow \downarrow$ No conclusion & Comparable for TRAM vs. DIEP \\
\hline & $\begin{array}{l}\text { Patient satisfaction } \\
\text { with outcome }\end{array}$ & $\uparrow \downarrow$ No conclusion & ? No conclusion & ? No conclusion & nd & nd & ? No conclusion \\
\hline & $\begin{array}{l}\text { Planned surgeries } \\
\text { for reconstruction }\end{array}$ & $\mathrm{N} / \mathrm{P}$ & $\mathrm{N} / \mathrm{P}$ & nd & nd & nd & nd \\
\hline & $\begin{array}{l}\text { Recurrence of } \\
\text { breast cancer }\end{array}$ & $\mathrm{N} / \mathrm{P}$ & $\mathrm{N} / \mathrm{P}$ & $\mathrm{N} / \mathrm{P}$ & $\mathrm{N} / \mathrm{P}$ & $\mathrm{N} / \mathrm{P}$ & $\mathrm{N} / \mathrm{P}$ \\
\hline & $\begin{array}{l}\text { Duration of initial } \\
\text { hospitalization }\end{array}$ & . & . & . & . & . & ? No conclusion \\
\hline & Mortality & ? No conclusion & nd & $\begin{array}{l}\text { ? No conclusion: } \\
\text { silicone vs. } \\
\text { saline } \\
\text { ? No conclusion: } \\
\text { silicone vs. } \\
\text { double lumen } \\
\end{array}$ & nd & ? No conclusion & ? No conclusion \\
\hline \multirow[t]{10}{*}{$\begin{array}{l}\text { Surgical } \\
\text { complications }\end{array}$} & $\begin{array}{l}\text { Unplanned repeat } \\
\text { hospitalization }\end{array}$ & $\sim \sim$ Comparable & nd & nd & nd & nd & nd \\
\hline & $\begin{array}{l}\text { Duration of } \\
\text { unplanned repeat } \\
\text { hospitalization }\end{array}$ & nd & nd & nd & nd & nd & nd \\
\hline & $\begin{array}{l}\text { Unplanned repeat } \\
\text { surgery for revision }\end{array}$ & $\uparrow \downarrow$ No conclusion & ? No conclusion & nd & ? No conclusion & $\sim \sim$ Comparable & ? No conclusion \\
\hline & $\begin{array}{l}\text { Unplanned repeat } \\
\text { surgery for } \\
\text { complications }\end{array}$ & $\uparrow \downarrow$ No conclusion & nd & nd & nd & ? No conclusion & nd \\
\hline & Pain & $\uparrow \downarrow$ No conclusion & ? No conclusion & nd & $\uparrow \downarrow$ No conclusion & $\uparrow \downarrow$ No conclusion & ? No conclusion \\
\hline & Analgesic use & ? No conclusion & nd & nd & ? No conclusion & ? No conclusion & nd \\
\hline & Necrosis & ? No conclusion & ? No conclusion & nd & ? No conclusion & $\sim$ Comparable & $\sim$ Comparable for TRAM vs. DIEP \\
\hline & $\begin{array}{l}\text { Harms to area of } \\
\text { flap harvest }\end{array}$ & . & . & . & . & . & $\begin{array}{l}\text { Abdominal bulge/ hernia, } \\
\text { hernia repair surgery with TRAM } \\
\text { than DIEP }\end{array}$ \\
\hline & Animation deformity & nd & nd & nd & nd & nd & \\
\hline & $\begin{array}{l}\text { Implant-related } \\
\text { infections }\end{array}$ & & nd & nd & nd & nd & . \\
\hline
\end{tabular}




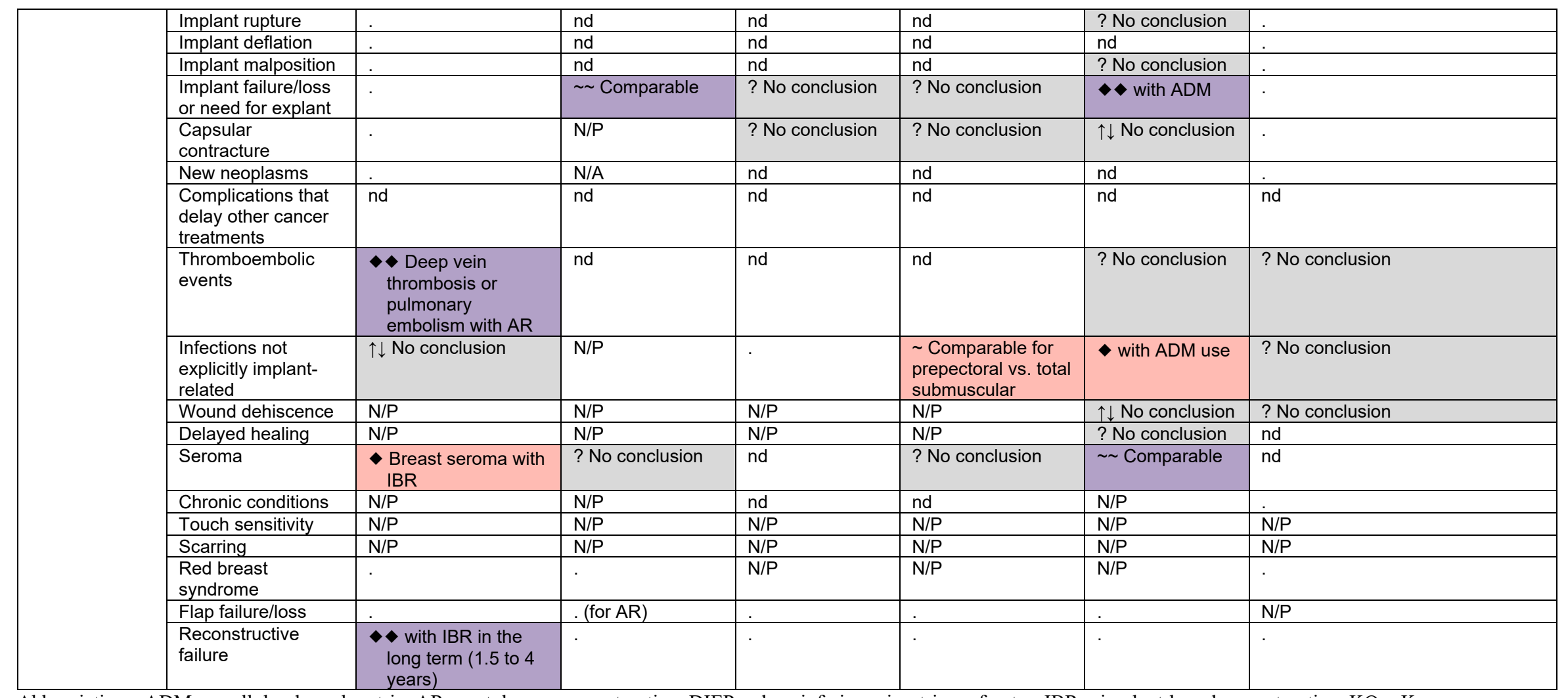

Abbreviations: $\mathrm{ADM}=$ acellular dermal matrix, $\mathrm{AR}=$ autologous reconstruction, $\mathrm{DIEP}=$ deep inferior epigastric perforator, IBR $=$ implant-based reconstruction, $\mathrm{KQ}=\mathrm{Key}$

Question, $\mathrm{LD}=$ latissimus dorsi, $\mathrm{N} / \mathrm{P}=$ not prioritized (for strength of evidence assessment), nd = no data (no evidence identified), TRAM = transverse rectus abdominis myocutaneous.

* No evidence addressed timing of IBR or AR in relation to chemotherapy (KQ 2a) or timing of AR in relation to radiation therapy (KQ 2b).

$\boldsymbol{\Delta}=$ Low SoE of better clinical outcomes, $\boldsymbol{\Delta} \mathbf{\Delta}=$ Moderate SoE of better clinical outcomes, $\boldsymbol{\Delta} \boldsymbol{\Delta} \boldsymbol{\Delta}=$ High SoE of better clinical outcomes (no instances in this table)

$\bullet=$ Low SoE of increased complications, $\bullet=$ Moderate SoE of increased complications, $\bullet \bullet=$ High SoE of increased complications (no instances in this table)

$\sim=$ Low SoE of comparable outcomes, $\sim$ = Moderate SoE of comparable outcomes, $\sim \sim=$ High SoE of comparable outcomes (no instances in this table)

$?=$ Insufficient strength of evidence due to sparse evidence, $\uparrow \downarrow=$ Insufficient strength of evidence due to inconsistent or conflicting results, . $=$ not applicable (i.e., outcome not applicable to KQ)

Color legend: Insufficient strength of evidence (gray), Low strength of evidence (pink), Moderate strength of evidence (purple), High strength of evidence (tan) (no instances in this table). The colors do not provide unique information compared with the text and symbols. 


\section{Strengths and Limitations}

\section{Strengths and Limitations of the Evidence Base}

The main strength of the evidence base is its applicability to the decisionmaking context in the U.S. The evidence is relevant to various decisional dilemmas underpinning surgical options for women undergoing breast reconstruction after mastectomy for breast cancer (see section Applicability).

However, despite numerous being published studies on the topic of breast reconstruction after mastectomy, the evidence is still sparse for many topics. A sizeable proportion of the studies included in this SR $(69 / 160 ; 43 \%)$ were single group studies. Although these single group studies provided estimates of risks of various surgical complications in women who underwent either IBR or AR, their noncomparative nature precluded their use in informing conclusions regarding choices between surgical options posed by the six KQs. Among the 91 comparative studies in this SR, there were only eight RCTs, each of which was small, with sample sizes ranging from 36 to 150 patients. We identified RCTs only on the choice of IBR versus AR (KQ 1), anatomic planes of implant placement for IBR (KQ 4), ADM use during IBR (KQ 5), and the choice of flap types for AR (KQ 6). Few studies of any design addressed timing of reconstruction (KQ 2; 5 nonrandomized comparative studies [NRCSs]), implant materials for IBR (KQ 3; five NRCSs), or anatomic planes of implant placement for IBR (KQ 4; one RCT and seven NRCSs). All five NRCSs that addressed timing of reconstruction (KQ 2) addressed timing of IBR in relation to radiation therapy; none addressed timing of IBR in relation to chemotherapy or timing of AR in relation to chemotherapy or radiation therapy.

Two limitations are specific to the NRCSs identified. First, NRCSs commonly did not report adjusted between-group effect sizes for all outcomes of clinical interest. Without access to the individual patient data, we were unable to calculate adjusted effect sizes. Given the personal decision making involved regarding choice of breast reconstruction surgery, use of implanted materials, and timing in relation to cancer therapy (both by the patient and the surgeons), it is very likely that the women in each treatment group within a study would be fundamentally dissimilar on one or more important confounders. Thus, we determined a priori that unadjusted effect sizes would likely be highly biased and of limited value. We also decided a priori on a list of confounders that would be necessary for studies to have adjusted for in order for us to include their results. Second, some NRCSs reported adjusted effect sizes without confidence intervals or $\mathrm{P}$ values or they reported $\mathrm{P}$ values without adjusted effect sizes. These forms of inadequate reporting limited our ability to make conclusions because we could comment only on directionality (i.e., only adjusted effect sizes) or statistical significance (i.e., only P values). Both of these limitations to the NRCSs compromised our ability to make conclusions for some outcomes.

Across study designs, data were often reported within subgroups based on factors such as age, obesity status, unilateral versus bilateral reconstruction, and (for IBR) number of stages. However, none of the studies reported statistical analyses that evaluated either differences between subgroups or, preferable, evidence of heterogeneity of treatment effects (different relative effects in different subgroups of patients). Thus, although in this report we commented wherever results for a certain outcome appeared to be different in one subgroup versus another, we refrained from concluding that there was (or was not) heterogeneity of treatment effects.

The evidence base identified in this SR is also limited by the lack of very long term followup. The longest followup time-point at which studies reported data was 10 years. Breast 
reconstruction is intended to last for decades, and so the very long-term benefit and harm outcomes of the various reconstruction options is largely unknown. Another limitation is that most studies reporting data for the patient satisfaction outcomes did not report information regarding who collected the data. Thus, there is the potential for social desirability bias if patients did not accurately report satisfaction data. However, most studies reporting data for this patient-reported outcome used the BREAST-Q, a validated and standard instrument.

We assessed most of the comparative studies (RCTs and NRCSs) to be at overall moderate or high risk of bias, primarily because participants, care providers, and/or outcome assessors were not blinded and because of incomplete outcome data. While blinding of participants (i.e., patients) and care providers (i.e., surgeons) will almost always be impossible in studies addressing the surgical KQs in this SR, lack of blinding can still lead to bias. Moreover, although for subjective patient-reported outcomes, such as sexual well-being, it may be impossible to blind the outcome assessors (i.e., patients), it is possible to blind the outcome assessors (e.g., nurses) for objective outcomes, such as most harms. The NRCSs were also often considered to be at serious or critical risk of confounding. Furthermore, the participant eligibility criteria, interventions, and outcomes were often inadequately described.

\section{Strengths and Limitations of the Systematic Review Process}

We followed contemporary standards for SRs, including multiple stakeholder engagement in KQ development and refinement and careful adherence to recommended methods for literature searching, screening, data extraction, risk of bias assessment, qualitative synthesis, quantitative synthesis, and SoE assessment. We were very inclusive in our eligibility criteria for studies, especially in terms of study designs, including RCTs, NRCSs, and (large) single group studies of reconstruction surgeries for women who had undergone or were undergoing mastectomy for breast cancer.

Despite our comprehensive search for studies and our inclusion of a total of 160 studies, all conclusions made in this SR are either based on low or moderate strength of evidence. For all interventions examined, our conclusions were predominantly about surgical complications rather than patient-reported clinical outcomes. Perhaps our most definitive conclusions were for the overall comparison of IBR versus AR (KQ 1). Regarding IBR, we were able to make only a few conclusions about timing in relation to radiation therapy (KQ 2b), about implant materials (KQ 3), about anatomic planes of implant placement (KQ 4), and about use of ADMs (KQ 5), but no conclusions about timing in relation to chemotherapy (KQ 2a; lack of evidence). Regarding AR, we were able to make only a few conclusions about comparisons between different flap types (KQ 6), but no conclusions about timing in relation to chemotherapy (KQ 2a) or radiation therapy (KQ 2b).

During protocol development, we prioritized outcomes in consultation with panels of key informants and technical experts and in keeping with a published core outcome set for breast reconstruction surgery. ${ }^{36}$ However, many of the prioritized outcomes were either not reported in any included study or were reported in an insufficient number of studies to merit conclusions. Unreported or rarely reported clinical outcomes included general quality of life and number of planned surgeries for reconstruction. Unreported or rarely reported surgical complications included duration of unplanned repeat hospitalizations, analgesic use, animation deformity, and complications that delay other cancer-related treatments. 


\section{Applicability}

Most studies in this SR were conducted in North America (U.S. or Canada), Europe, or highor middle-income East Asian countries (South Korea and China, respectively). Among the North American studies, the racial makeup of study participants largely mirrored the population of women who undergo breast reconstruction in the U.S., who have been shown to be overwhelmingly White. ${ }^{19}$ Average ages of patients ranged from the early to late $50 \mathrm{~s}$ and their average body mass indices (BMIs) ranged from 22 to $29 \mathrm{~kg} / \mathrm{m}^{2}$. Large proportions of patients in most studies had undergone mastectomy for therapeutic purposes, with few undergoing prophylactic mastectomies (for high risk such as due to BRCA1 or BRCA2 gene mutations). As such, the conclusions in this SR apply generally to mostly White, middle-aged, nonobese women in high-income countries who are being treated for breast cancer. It is unclear to what extent the findings of this SR are broadly applicable beyond these populations.

The applicability of the findings may also be limited to the specific interventions that have been studied. Specifically, the evidence reflects the implant materials that are in use in the U.S. (silicone and saline implants), and we restricted our review to human ADMs, which are commonly used in the U.S. In terms of anatomic planes, the sparse evidence we identified addresses prepectoral and total submuscular implants, but we found insufficient evidence for partial submuscular implants, the other anatomic plane commonly used in the U.S. The evidence identified addressing flap types for AR considered some but not all the commonly used flap types in the U.S.

\section{Implications for Clinical Practice}

The findings in this SR summarize what is known about the comparative effectiveness and harms of various treatment options for patients who have undergone or are undergoing mastectomy and have made the decision to undergo breast reconstruction.

Briefly, our analysis of all surgical choices examined as KQs in this SR finds no clear winners. The strongest evidentiary basis is for the KQ addressing the broad choice of IBR versus AR. When making this choice, clinicians and patients should note that although some patientreported outcomes may be better with AR than IBR (e.g., sexual well-being), this is not true for other patient-reported outcomes (e.g., physical well-being). In terms of harms, some serious harms, such as pulmonary embolism, are probably more likely with AR, but other harms, such as breast seroma, may be more likely with IBR. The choice of IBR versus AR also needs to consider that IBR typically involves multiple surgeries and that implants may require monitoring and replacement, but AR usually involves a single surgery (revision surgeries may at times be needed), and the reconstruction is intended to be lifelong. However, AR surgery is usually more extensive (involving both breast and donor site incisions) and, in the case of abdominal donor sites, may lead to greater chronic abdominal pain than IBR. Moreover, based on such factors as body habitus and history of previous surgery, some women may not be candidates for AR.

For women who choose to undergo IBR, issues of timing of reconstruction relative to other cancer therapies (chemotherapy and radiation therapy), type of implant materials, anatomic plane of implant placement, and use of ADMs need consideration. Unfortunately, the evidence supporting these choices is weaker. There is no evidence to inform the issue of timing of IBR in relation to chemotherapy; this lack of evidence may be related to the preference of practitioners to base decisions regarding timing of chemotherapy on the severity of the underlying cancer for patients with more aggressive cancers, chemotherapy is usually administered before surgery. 
There is only limited evidence suggesting that conducting IBR before or after radiation therapy may not affect physical well-being, psychosocial well-being, sexual well-being, and patient satisfaction with breasts, and probably does not affect the risk of implant failure or loss. The evidence is also weak for whether silicone or saline should be used as implant materials and whether the implant should be placed in the prepectoral, total submuscular, or partial submuscular planes. Implant placement in prepectoral or total submuscular planes may result in comparable risk of infections that are not explicitly implant-related. Regarding ADMs, there is insufficient evidence whether their use impact patient-reported clinical outcomes. However, ADM use may be associated with some surgical complications, such as infections that are not explicitly implant-related and implant failure, but not others, such as necrosis and seroma. It is worth noting that ADM use may be used more frequently when the implant is placed in prepectoral or partial submuscular planes. Although studies of ADM use did not frequently report the anatomic plane of implant placement, this factor is a possible confounder of the observed treatment effect of ADM use. Nevertheless, our findings that ADM use may be associated with a higher incidence of infections and implant failure are consistent with a recent U.S. Food and Drug Administration Safety Communication regarding ADM use during IBR. ${ }^{254}$

If the decision is made to undergo AR, the choices of timing of reconstruction relative to other cancer therapies (chemotherapy and radiation therapy) and of which flap types should be used need consideration. Unfortunately, there is no evidence to inform the choice of timing of AR in relation to either chemotherapy or radiation therapy. As in the case of IBR, for patients with more aggressive cancers, practitioners may more often choose to administer chemotherapy before AR. We hypothesize a possible reason why the issue of timing of AR in relation to radiation therapy has not been researched. Practitioners may generally prefer to deliver the radiation therapy before AR to avoid radiating tissue from another location in the body, which in some cases involves very delicate microvascular anastomoses by the plastic surgeon. In terms of flap types, DIEP flaps may be associated with comparable satisfaction with breasts as TRAM flaps. However, compared with DIEP flaps, TRAM flaps may be associated with a greater risk of harms to the area of flap harvest, such as abdominal bulge/hernia and needing abdominal hernia repair surgery. Decisions regarding flap types should consider the location of the source tissue, patient body habitus, and availability of plastic surgeons with advanced training in microvascular techniques (for free flaps, such as DIEP and free TRAM flaps).

Given the relatively weak evidence addressing some breast reconstruction-related key decisions that need to be made in clinical practice and the highly patient preference-sensitive nature of the decisions, ${ }^{255,256}$ we encourage clinicians to inform patients about the limitations of existing research. The patient's values and preferences and the clinician's expertise and experience are highly important.

Various clinical decision support tools have been developed to facilitate the decision making process. ${ }^{256-259}$ These tools range from those that provide standard information about breast reconstruction options and their risks ${ }^{256,257,259}$ to tools that provide personalized risk assessments tailored to individual patients. ${ }^{260}$ The BREASTChoice tool is one example of a personalized tool. It was developed by incorporating the perspectives of breast cancer patients who had undergone mastectomy, plastic surgeons performing reconstructions, and nurses caring for patients who undergo reconstructions. ${ }^{260}$ Although we are not aware of (and did not systematically search for) SRs comparing clinical decision support tools for breast reconstruction, one tool, BREASTChoice, has been compared with usual care in an RCT of patients undergoing breast reconstruction after mastectomy in the U.S. ${ }^{258}$ Use of the tool was associated with patients 
having better knowledge about reconstruction options and their risks, but there were no differences in decision process quality, patient quality of life, or patient decisions made. ${ }^{258}$

Clinicians should also consider and emphasize to patients that much of the research that has been done addressing breast reconstruction has focused largely on patients whose mastectomy was performed for therapeutic (and not prophylactic) purposes. In addition, patients in existing studies have been mostly White, middle-aged, and nonobese women living in high-income countries. For patients in clinical practice who do not belong to these categories, clinicians and patients will need to consider the appropriateness of extrapolating information about benefits and harms of breast reconstruction options from the evidence to the decision making context.

\section{Implications for Research}

Research is needed to address various questions related to breast reconstruction, particularly the timing of IBR and AR in relation to chemotherapy and radiation therapy, implant materials (for IBR), anatomic planes of implant placement (for IBR), and choice of flaps (for AR). Because of the absence of studies that predominantly enrolled women undergoing mastectomy for prophylactic purposes, researchers should also design studies that, either entirely or in part, enroll these patients. The recent decade has witnessed a steady and sizeable increase in the number of prophylactic mastectomies. ${ }^{261-265}$ The risk-benefit assessments for reconstruction choices among these women may be different than for women who undergo therapeutic mastectomies. Perceived (subjective) benefits and harms may also differ. When enrolled as part of a larger study, subgroup-specific data for patients undergoing mastectomy for prophylactic purposes, should be reported. In addition, studies should also enroll more diverse groups of women, such as by age group, race, ethnicity, and socioeconomic status.

It is important that when possible future studies conduct randomization (to avoid selection bias). We recognize that studies may not always be possible; in that context, studies should report between-group estimates of treatment effect that adequately account for important confounders, such as age, BMI, and stage of breast cancer. Ideally, propensity score analyses (or similar rigorous techniques) should be used to adequately adjust for potential confounders. A propensity score analysis, for example, estimates the likelihood that each patient had one or the other intervention (conditional on her measured characteristics) and controls for this likelihood. These analyses generally require relatively large numbers of patients for whom there are granular data about risk factors for outcomes. In terms of performance and detection biases, while blinding of participants and care providers will rarely be feasible (if at all), studies should blind the assessors of outcomes that are not patient reported. In addition, there is a need for long-term followup of large, prospective studies to assess long term risks of surgical complications.

Future studies should also evaluate important outcomes that were not adequately reported in the identified evidence, such as quality of life, number of planned surgeries for reconstruction, incidence and duration of unplanned repeat hospitalizations and surgeries, analgesic use, animation deformity, and complications that may delay other cancer-related treatments.

\section{Conclusions}

Although we found a large body of evidence, we were able to make only a few specific conclusions in this SR, all of which were based on low or moderate strength of evidence. Future research, ideally comprising large RCTs and/or well-designed and well-analyzed long-term observational studies, is needed to compare timing of reconstruction relative to chemotherapy 
and radiation therapy, different implant materials, different anatomic planes of implant placement, and use of ADMs in patients undergoing reconstruction. 


\section{References}

1. U.S. Department of Health and Human Services, Centers for Disease Control and Prevention and National Cancer Institute. U.S. Cancer Statistics Working Group. U.S. Cancer Statistics Data Visualizations Tool, based on November 2018 submission data (19992016). 2019. www.cdc.gov/cancer/dataviz. Accessed on December 28, 2019.

2. American Cancer Society. Breast Cancer Facts \& Figures 2019-2020. . 2019. https://www.cancer.org/content/dam/cancerorg/research/cancer-facts-andstatistics/breast-cancer-facts-andfigures/breast-cancer-facts-and-figures2019-2020.pdf. Accessed on December 28, 2019.

3. Recht A, Comen EA, Fine RE, et al. Postmastectomy Radiotherapy: An American Society of Clinical Oncology, American Society for Radiation Oncology, and Society of Surgical Oncology Focused Guideline Update. Pract Radiat Oncol. 2016 Nov-Dec;6(6):e219-e34. doi: 10.1016/j.prro.2016.08.009. PMID: 27659727.

4. Jonczyk MM, Jean J, Graham R, et al. Surgical trends in breast cancer: a rise in novel operative treatment options over a 12 year analysis. Breast Cancer Res Treat. 2019 Jan;173(2):267-74. doi: 10.1007/s10549018-5018-1. PMID: 30361873.

5. Baker JL, Dizon DS, Wenziger CM, et al. "Going Flat" After Mastectomy: Patient-Reported Outcomes by Online Survey. Ann Surg Oncol. 2021 May;28(5):2493-505. doi: 10.1245/s10434-020-09448-9. PMID: 33393025 .

6. Wakeley ME, Bare CF, Pine R, et al. A social media survey of women who do not pursue reconstruction after mastectomy for breast cancer: Characterizing the "Going Flat" movement. Breast J. 2020 Jul;26(7):1455-7. doi: 10.1111/tbj.13781. PMID: 32067326.

7. American Society of Plastic Surgeons. 2019 Plastic Surgery Statistics Report. 2019. https://www.plasticsurgery.org/documents/ $\mathrm{N}$ ews/Statistics/2019/plastic-surgerystatistics-full-report-2019.pdf. Accessed on February 13, 2021.
8. Employee Benefits Security Administration. Your Rights After a Mastectomy.; 2018. https://www.dol.gov/sites/default/files/ebsa/ about-ebsa/our-activities/resourcecenter/publications/your-rights-after-amastectomy.pdf. Accessed on December 28, 2019.

9. Haffty BG, Harrold E, Khan AJ, et al. Outcome of conservatively managed early-onset breast cancer by BRCA $1 / 2$ status. Lancet. 2002 Apr 27;359(9316):1471-7. doi: 10.1016/s0140-6736(02)08434-9. PMID: 11988246.

10. Shahedi K, Emanuelsson M, Wiklund F, et al. High risk of contralateral breast carcinoma in women with hereditary/familial nonBRCA1/BRCA2 breast carcinoma. Cancer. 2006 Mar 15;106(6):1237-42. doi: 10.1002/cncr.21753. PMID: 16475207.

11. van den Broek AJ, van 't Veer LJ, Hooning MJ, et al. Impact of Age at Primary Breast Cancer on Contralateral Breast Cancer Risk in BRCA1/2 Mutation Carriers. J Clin Oncol. 2016 Feb 10;34(5):409-18. doi: 10.1200/jco.2015.62.3942. PMID: 26700119.

12. Al-Ghazal SK, Sully L, Fallowfield L, et al. The psychological impact of immediate rather than delayed breast reconstruction. Eur J Surg Oncol. 2000 Feb;26(1):17-9. doi: 10.1053/ejso.1999.0733. PMID: 10718173.

13. Hershenhouse KS, Bick K, Shauly O, et al. "Systematic review and meta-analysis of immediate versus delayed autologous breast reconstruction in the setting of postmastectomy adjuvant radiation therapy". J Plast Reconstr Aesthet Surg. 2020 Dec 5. doi: 10.1016/j.bjps.2020.11.027. PMID: 33423976.

14. Motwani SB, Strom EA, Schechter NR, et al. The impact of immediate breast reconstruction on the technical delivery of postmastectomy radiotherapy. Int J Radiat Oncol Biol Phys. 2006 Sep 1;66(1):76-82. doi: 10.1016/j.jijrobp.2006.03.040. PMID: 16765534 . 
15. Schechter NR, Strom EA, Perkins GH, et al. Immediate breast reconstruction can impact postmastectomy irradiation. Am J Clin Oncol. 2005 Oct;28(5):485-94. doi: 10.1097/01.coc.0000170582.38634.b6. PMID: 16199989.

16. Middleton MS, McNamara MP, Jr. Breast implant classification with MR imaging correlation: (CME available on RSNA link). Radiographics. 2000 May;20(3):E1. doi: 10.1148/radiographics.20.3.g00mae11. PMID: 10835136.

17. U.S. Food and Drug Administration Center for Devices and Radiological Health. FDA Update on the Safety of Silicone Gel-Filled Breast Implants. 2011. https://www.fda.gov/media/80685/download . Accessed on April 9, 2020.

18. McCarthy CM, Klassen AF, Cano SJ, et al. Patient satisfaction with postmastectomy breast reconstruction: a comparison of saline and silicone implants. Cancer. 2010 Dec 15;116(24):5584-91. doi: 10.1002/cncr.25552. PMID: 21136577.

19. Balk EM, Earley A, Avendano EA, et al. LongTerm Health Outcomes in Women With Silicone Gel Breast Implants: A Systematic Review. Ann Intern Med. $2016 \mathrm{Feb}$ 2;164(3):164-75. doi: 10.7326/m15-1169. PMID: 26550776.

20. U.S. Food and Drug Administration. The FDA Requests Allergan Voluntarily Recall Natrelle BIOCELL Textured Breast Implants and Tissue Expanders from the Market to Protect Patients: FDA Safety Communication.; 2019. https://www.fda.gov/medical-devices/safetycommunications/fda-requests-allerganvoluntarily-recall-natrelle-biocell-texturedbreast-implants-and-tissue. Accessed on April 9, 2020.

21. U.S. Food and Drug Administration. Breast Implants - Certain Labeling Recommendations to Improve Patient Communication. Guidance for Industry and Food and Drug Administration Staff. 2020. https://www.fda.gov/media/131885/downloa d. Accessed on February 13, 2021.
22. Hanna KR, DeGeorge BR, Jr., Mericli AF, et al. Comparison study of two types of expanderbased breast reconstruction: acellular dermal matrix-assisted versus total submuscular placement. Ann Plast Surg. 2013 Jan;70(1):10-5. doi: 10.1097/SAP.0b013e31822f6765. PMID: 21862915.

23. Cordeiro PG, Jazayeri L. Two-Stage ImplantBased Breast Reconstruction: An Evolution of the Conceptual and Technical Approach over a Two-Decade Period. Plast Reconstr Surg. 2016 Jul;138(1):1-11. doi: 10.1097/prs.0000000000002243. PMID: 27348635.

24. Walia GS, Aston J, Bello R, et al. Prepectoral Versus Subpectoral Tissue Expander Placement: A Clinical and Quality of Life Outcomes Study. Plast Reconstr Surg Glob Open. 2018 Apr;6(4):e1731. doi: 10.1097/gox.0000000000001731. PMID: 29876176.

25. Wainwright DJ. Use of an acellular allograft dermal matrix (AlloDerm) in the management of full-thickness burns. Burns. 1995 Jun;21(4):243-8. doi: 10.1016/03054179(95)93866-i. PMID: 7662122.

26. Dikmans RE, Negenborn VL, Bouman MB, et al. Two-stage implant-based breast reconstruction compared with immediate one-stage implant-based breast reconstruction augmented with an acellular dermal matrix: an open-label, phase 4, multicentre, randomised, controlled trial. Lancet Oncol. 2017 Feb;18(2):251-8. doi: 10.1016/s1470-2045(16)30668-4. PMID: 28012977.

27. Negenborn VL, Young-Afat DA, Dikmans REG, et al. Quality of life and patient satisfaction after one-stage implant-based breast reconstruction with an acellular dermal matrix versus two-stage breast reconstruction (BRIOS): primary outcome of a randomised, controlled trial. Lancet Oncol. 2018 Sep;19(9):1205-14. doi: 10.1016/s1470-2045(18)30378-4. PMID: 30104147. 
28. Salzberg CA, Ashikari AY, Berry C, et al. Acellular Dermal Matrix-Assisted Direct-toImplant Breast Reconstruction and Capsular Contracture: A 13-Year Experience. Plast Reconstr Surg. 2016 Aug;138(2):329-37. doi: $10.1097 /$ prs. 0000000000002331 . PMID: 27064232.

29. Smith JM, Broyles JM, Guo Y, et al. Human acellular dermis increases surgical site infection and overall complication profile when compared with submuscular breast reconstruction: An updated meta-analysis incorporating new products(). J Plast Reconstr Aesthet Surg. 2018 Nov;71(11):1547-56. doi: 10.1016/j.bjps.2018.06.012. PMID: 30093286.

30. Feng J, Pardoe CI, Mota AM, et al. Two-Stage Latissimus Dorsi Flap with Implant for Unilateral Breast Reconstruction: Getting the Size Right. Arch Plast Surg. 2016 Mar;43(2):197-203. doi: 10.5999/aps.2016.43.2.197. PMID: 27018318.

31. Berkman ND, Lohr KN, Ansari M, et al. AHRQ Methods for Effective Health Care: Grading the Strength of a Body of Evidence When Assessing Health Care Interventions for the Effective Health Care Program of the Agency for Healthcare Research and Quality: An Update. Methods Guide for Effectiveness and Comparative Effectiveness Reviews. Rockville (MD): Agency for Healthcare Research and Quality (US); 2008.

32. Higgins JP, Altman DG, Gotzsche PC, et al. The Cochrane Collaboration's tool for assessing risk of bias in randomised trials. Bmj. 2011 Oct 18;343:d5928. doi: 10.1136/bmj.d5928. PMID: 22008217.

33. Sterne JA, Hernan MA, Reeves BC, et al. ROBINS-I: a tool for assessing risk of bias in non-randomised studies of interventions. Bmj. 2016 Oct 12;355:i4919. doi: 10.1136/bmj.i4919. PMID: 27733354.

34. National Heart, Lung, and Blood Institute. Study Quality Assessment Tools.; 2019. https://www.nhlbi.nih.gov/healthtopics/study-quality-assessment-tools. Accessed on January 23, 2020.
35. Berkman ND, Lohr KN, Ansari MT, et al. Grading the strength of a body of evidence when assessing health care interventions: an EPC update. J Clin Epidemiol. 2015 Nov;68(11):1312-24. doi: 10.1016/j.jclinepi.2014.11.023. PMID: 25721570.

36. Potter S, Holcombe C, Ward JA, et al. Development of a core outcome set for research and audit studies in reconstructive breast surgery. Br J Surg. 2015 Oct;102(11):1360-71. doi: 10.1002/bjs.9883. PMID: 26179938.

37. Williamson PR, Altman DG, Blazeby JM, et al. Developing core outcome sets for clinical trials: issues to consider. Trials. 2012 Aug 6;13:132. doi: 10.1186/1745-6215-13-132. PMID: 22867278.

38. Guyatt GH, Oxman AD, Kunz R, et al. Going from evidence to recommendations. Bmj. 2008 May 10;336(7652):1049-51. doi: 10.1136/bmj.39493.646875.AE. PMID: 18467413.

39. Gerrity M, Fiordalisi C, Pillay J, et al. AHRQ Methods for Effective Health Care. Roadmap for Narratively Describing Effects of Interventions in Systematic Reviews. Rockville (MD): Agency for Healthcare Research and Quality (US); 2020.

40. Murad MH, Fiordalisi C, Pillay J, et al. Making Narrative Statements to Describe Treatment Effects. J Gen Intern Med. 2020 Oct 27. doi: 10.1007/s11606-020-06330-y. PMID: 33111244 .

41. Carramaschi F, Bricout N, Servant JM, et al. [Breast reconstruction after mastectomy. Critical analysis of 240 cases]. AMB Rev Assoc Med Bras. 1989 Jan-Feb;35(1):15-9. PMID: 2602589.

42. Garbay JR, Rietjens M, Petit JY. [Esthetic results of breast reconstruction after amputation for cancer. 323 cases]. J Gynecol Obstet Biol Reprod (Paris). 1992;21(4):405-12. PMID: 1624727. 
43. Brorson F, Thorarinsson A, Kölby L, et al. Early complications in delayed breast reconstruction: A prospective, randomized study comparing different reconstructive methods in radiated and non-radiated patients. Eur J Surg Oncol. 2020 Dec;46(12):2208-17. doi: 10.1016/j.ejso.2020.07.010. PMID: 32807615 .

44. Tallroth L, Velander P, Klasson S. A short-term comparison of expander prosthesis and DIEP flap in breast reconstructions: A prospective randomized study. J Plast Reconstr Aesthet Surg. 2020 Nov 12. doi: 10.1016/j.bjps.2020.10.104. PMID: 33436336 .

45. Abedi N, Ho AL, Knox A, et al. Predictors of Mastectomy Flap Necrosis in Patients Undergoing Immediate Breast Reconstruction: A Review of 718 Patients. Ann Plast Surg. 2016 Jun;76(6):629-34. doi: 10.1097/sap.0000000000000262. PMID: 25003437.

46. Atisha D, Alderman AK, Lowery JC, et al. Prospective analysis of long-term psychosocial outcomes in breast reconstruction: two-year postoperative results from the Michigan Breast Reconstruction Outcomes Study. Ann Surg. 2008 Jun;247(6):1019-28. doi: 10.1097/SLA.0b013e3181728a5c. PMID: 18520230 .

47. Bennett KG, Qi J, Kim HM, et al. Comparison of 2-Year Complication Rates Among Common Techniques for Postmastectomy Breast Reconstruction. JAMA Surg. 2018 Oct 1;153(10):901-8. doi: 10.1001/jamasurg.2018.1687. PMID: 29926077.

48. Brito IM, Fernandes A, Andresen C, et al. Patient satisfaction with breast reconstruction: how much do timing and surgical technique matter? Eur J Plast Surg. 2020;43:809-18.

49. Chetta MD, Aliu O, Zhong L, et al. Reconstruction of the Irradiated Breast: A National Claims-Based Assessment of Postoperative Morbidity. Plast Reconstr Surg. 2017 Apr;139(4):783-92. doi: 10.1097/prs.0000000000003168. PMID: 28002254 .
50. Cohen WA, Ballard TN, Hamill JB, et al. Understanding and Optimizing the Patient Experience in Breast Reconstruction. Ann Plast Surg. 2016 Aug;77(2):237-41. doi: 10.1097/sap.0000000000000550. PMID: 26101986.

51. Dauplat J, Thivat E, Rouanet P, et al. Risk Factors Associated With Complications After Unilateral Immediate Breast Reconstruction: A French Prospective Multicenter Study. In Vivo. 2021 MarApr;35(2):937-45. doi: 10.21873/invivo.12334. PMID: 33622886.

52. de Araujo TB, Jue Xu M, Susarla SM, et al. Impact of Prior Unilateral Chest Wall Radiotherapy on Outcomes in Bilateral Breast Reconstruction. Plast Reconstr Surg. 2016 Oct;138(4):575e-80e. doi: 10.1097/prs.0000000000002532. PMID: 27673527.

53. Eltahir Y, Werners LL, Dreise MM, et al. Which breast is the best? Successful autologous or alloplastic breast reconstruction: patientreported quality-of-life outcomes. Plast Reconstr Surg. 2015 Jan;135(1):43-50. doi: 10.1097/prs.0000000000000804. PMID: 25539295.

54. Fischer JP, Fox JP, Nelson JA, et al. A Longitudinal Assessment of Outcomes and Healthcare Resource Utilization After Immediate Breast ReconstructionComparing Implant- and Autologous-based Breast Reconstruction. Ann Surg. 2015 Oct;262(4):692-9. doi: 10.1097/sla.0000000000001457. PMID: 26366550 .

55. Fischer JP, Nelson JA, Cleveland E, et al. Breast reconstruction modality outcome study: a comparison of expander/implants and free flaps in select patients. Plast Reconstr Surg. 2013 May;131(5):928-34. doi: 10.1097/PRS.0b013e3182865977. PMID: 23629074.

56. Fischer JP, Wes AM, Nelson JA, et al. Propensity-matched, longitudinal outcomes analysis of complications and cost: comparing abdominal free flaps and implant-based breast reconstruction. J Am Coll Surg. 2014 Aug;219(2):303-12. doi: 10.1016/j.jamcollsurg.2014.02.028. PMID: 24916480. 
57. Garvey PB, Villa MT, Rozanski AT, et al. The advantages of free abdominal-based flaps over implants for breast reconstruction in obese patients. Plast Reconstr Surg. 2012 Nov;130(5):991-1000. doi: 10.1097/PRS.0b013e318267efc5. PMID: 23096600.

58. Ha JH, Hong KY, Lee HB, et al. Oncologic outcomes after immediate breast reconstruction following mastectomy: comparison of implant and flap using propensity score matching. BMC Cancer. 2020 Jan 30;20(1):78. doi: 10.1186/s12885020-6568-2. PMID: 32000718.

59. Hangge PT, Jogerst K, Mohsen A, et al. Making an informed choice: Which breast reconstruction type has the lowest complication rate? Am J Surg. 2019 Dec;218(6):1040-5. doi: 10.1016/j.amjsurg.2019.09.033. PMID: 31606126 .

60. Jiang YZ, Liu YR, Yu KD, et al. Immediate postmastectomy breast reconstruction showed limited advantage in patient survival after stratifying by family income. PLoS One. 2013;8(12):e82807. doi: 10.1371/journal.pone.0082807. PMID: 24349366.

61. Kouwenberg CAE, de Ligt KM, Kranenburg LW, et al. Long-Term Health-Related Quality of Life after Four Common Surgical Treatment Options for Breast Cancer and the Effect of Complications: A Retrospective PatientReported Survey among 1871 Patients. Plast Reconstr Surg. 2020 Jul;146(1):1-13. doi: 10.1097/prs.0000000000006887. PMID: 32590633.

62. Kouwenberg CAE, Kranenburg LW, Visser MS, et al. "The validity of the EQ-5D-5L in measuring quality of life benefits of breast reconstruction". J Plast Reconstr Aesthet Surg. 2019 Jan;72(1):52-61. doi: 10.1016/j.bjps.2018.08.023. PMID: 30270015 .

63. Kulkarni AR, Pusic AL, Hamill JB, et al. Factors Associated with Acute Postoperative Pain Following Breast Reconstruction. JPRAS Open. 2017 Mar;11:1-13. doi: 10.1016/j.jpra.2016.08.005. PMID: 28713853.
64. Laporta R, Sorotos M, Longo B, et al. Breast Reconstruction in Elderly Patients: Risk Factors, Clinical Outcomes, and Aesthetic Results. J Reconstr Microsurg. 2017 May;33(4):257-67. doi: 10.1055/s-00361597822. PMID: 28061518.

65. Lee KT, Bang SI, Pyon JK, et al. Method of breast reconstruction and the development of lymphoedema. Br J Surg. 2017 Feb;104(3):230-7. doi: 10.1002/bjs.10397. PMID: 27861758.

66. Lee KT, Pyon JK, Bang SI, et al. Does the reconstruction method influence development of mastectomy flap complications in nipple-sparing mastectomy? J Plast Reconstr Aesthet Surg. 2013 Nov;66(11):1543-50. doi: 10.1016/j.bjps.2013.06.032. PMID: 23834909.

67. Lei $\mathrm{C}, \mathrm{Xu} \mathrm{L}, \mathrm{Xu} F$, et al. Patient satisfaction in one-stage immediate breast reconstruction after mastectomy: A multi-center comparative patient evaluation of prosthesis, LDMF, and TRAM techniques. Medicine (Baltimore). 2020 May 29;99(22):e19991. doi: 10.1097/md.0000000000019991. PMID: 32481367.

68. Liu C, Momeni A, Zhuang Y, et al. Outcome analysis of expander/implant versus microsurgical abdominal flap breast reconstruction: a critical study of 254 cases. Ann Surg Oncol. 2014 Jun;21(6):2074-82. doi: 10.1245/s10434-014-3521-0. PMID: 24558063.

69. Mak JC, Kwong A. Complications in Postmastectomy Immediate Breast Reconstruction: A Ten-year Analysis of Outcomes. Clin Breast Cancer. 2020 Oct;20(5):402-7. doi: 10.1016/j.clbc.2019.12.002. PMID: 32665188.

70. McCarthy CM, Mehrara BJ, Long T, et al. Chest and upper body morbidity following immediate postmastectomy breast reconstruction. Ann Surg Oncol. 2014 Jan;21(1):107-12. doi: 10.1245/s10434-0133231-z. PMID: 24201740. 
71. Merchant SJ, Goldstein L, Kruper LL. Patterns and Trends in Immediate Postmastectomy Reconstruction in California: Complications and Unscheduled Readmissions. Plast Reconstr Surg. 2015 Jul;136(1):10e-9e. doi: 10.1097/prs.0000000000001326. PMID: 26111325.

72. Mioton LM, Smetona JT, Hanwright PJ, et al. Comparing thirty-day outcomes in prosthetic and autologous breast reconstruction: a multivariate analysis of 13,082 patients? J

Plast Reconstr Aesthet Surg. 2013 Jul;66(7):917-25. doi: 10.1016/j.bjps.2013.03.009. PMID: 23562485 .

73. Mlodinow AS, Ver Halen JP, Lim S, et al. Predictors of readmission after breast reconstruction: a multi-institutional analysis of 5012 patients. Ann Plast Surg. 2013 Oct;71(4):335-41. doi: 10.1097/SAP.0b013e3182a0df25. PMID: 24025652 .

74. Momeni A, Fox JP. Venous Thromboembolism After Surgical Treatment of Breast Cancer. Ann Plast Surg. 2018 Feb;80(2):188-92. doi: 10.1097/sap.0000000000001249. PMID: 29095189.

75. Naoum GE, Oladeru OT, Niemierko A, et al. Optimal breast reconstruction type for patients treated with neoadjuvant chemotherapy, mastectomy followed by radiation therapy. Breast Cancer Res Treat. 2020 Aug;183(1):127-36. doi: 10.1007/s10549-020-05747-7. PMID: 32607638 .

76. Naoum GE, Salama L, Niemierko A, et al. Single Stage Direct-to-Implant Breast Reconstruction Has Lower Complication Rates Than Tissue Expander and Implant and Comparable Rates to Autologous Reconstruction in Patients Receiving Postmastectomy Radiation. Int J Radiat Oncol Biol Phys. 2020 Mar 1;106(3):51424. doi: 10.1016/j.ijrobp.2019.11.008. PMID: 31756414.

77. Nasser JS, Huetteman HE, Chung TT, et al. Unplanned Emergency Department Visits within 30 Days of Mastectomy and Breast Reconstruction. Plast Reconstr Surg. 2018 Dec;142(6):1411-20. doi: 10.1097/prs.0000000000004970. PMID: 30204678 .
78. Nelson JA, Voineskos SH, Qi J, et al. Elective Revisions after Breast Reconstruction: Results from the Mastectomy Reconstruction Outcomes Consortium. Plast Reconstr Surg. 2019 Dec;144(6):1280-90. doi: $10.1097 /$ prs.0000000000006225. PMID: 31764633.

79. Palve JS, Luukkaala TH, Kääriäinen MT. Predictive risk factors of complications in different breast reconstruction methods. Breast Cancer Res Treat. 2020 Jul;182(2):345-54. doi: 10.1007/s10549020-05705-3. PMID: 32468337.

80. Pusic AL, Matros E, Fine N, et al. PatientReported Outcomes 1 Year After Immediate Breast Reconstruction: Results of the Mastectomy Reconstruction Outcomes Consortium Study. J Clin Oncol. 2017 Aug 1;35(22):2499-506. doi: 10.1200/jco.2016.69.9561. PMID: 28346808 .

81. Qin Q, Tan Q, Lian B, et al. Postoperative outcomes of breast reconstruction after mastectomy: A retrospective study. Medicine (Baltimore). 2018 Feb;97(5):e9766. doi: 10.1097/md.0000000000009766. PMID: 29384865.

82. Roth RS, Lowery JC, Davis J, et al. Persistent pain following postmastectomy breast reconstruction: long-term effects of type and timing of surgery. Ann Plast Surg. 2007 Apr;58(4):371-6. doi: 10.1097/01.sap.0000239810.38137.84. PMID: 17413877.

83. Roth RS, Qi J, Hamill JB, et al. Is chronic postsurgical pain surgery-induced? A study of persistent postoperative pain following breast reconstruction. Breast. 2018 Feb;37:119-25. doi: 10.1016/j.breast.2017.11.001. PMID: 29145033.

84. Santosa KB, Qi J, Kim HM, et al. Long-term Patient-Reported Outcomes in Postmastectomy Breast Reconstruction. JAMA Surg. 2018 Oct 1;153(10):891-9. doi: 10.1001/jamasurg.2018.1677. PMID: 29926096. 
85. Shiraishi M, Sowa Y, Fujikawa K, et al. Factors associated with chronic pain following breast reconstruction in Japanese women. J Plast Surg Hand Surg. 2020 Oct;54(5):31722. doi: 10.1080/2000656x.2020.1780246. PMID: 32589082.

86. Simon P, Barrou J, Cohen M, et al. Types of Mastectomies and Immediate Reconstructions for Ipsilateral Breast Local Recurrences. Front Oncol. 2020;10:567298. doi: 10.3389/fonc.2020.567298. PMID: 33363007 .

87. Taylor EM, Wilkins EG, Pusic AL, et al. Impact of Unilateral versus Bilateral Breast Reconstruction on Procedure Choices and Outcomes. Plast Reconstr Surg. 2019 Jun;143(6):1159e-68e. doi: 10.1097/prs.0000000000005602. PMID: 31136472 .

88. Tong WM, Baumann DP, Villa MT, et al. Obese Women Experience Fewer Complications after Oncoplastic Breast Repair following Partial Mastectomy Than after Immediate Total Breast Reconstruction. Plast Reconstr Surg. 2016 Mar;137(3):777-91. doi: 10.1097/01.prs.0000479939.69211.19. PMID: 26910658.

89. Weichman KE, Clavin NW, Miller HC, et al. Does the use of biopatch devices at drain sites reduce perioperative infectious complications in patients undergoing immediate tissue expander breast reconstruction? Plast Reconstr Surg. 2015 Jan;135(1):9e-17e. doi: 10.1097/prs.0000000000000810. PMID: 25539357.

90. Weichman KE, Hamill JB, Kim HM, et al. Understanding the recovery phase of breast reconstructions: Patient-reported outcomes correlated to the type and timing of reconstruction. J Plast Reconstr Aesthet Surg. 2015 Oct;68(10):1370-8. doi: 10.1016/j.bjps.2015.05.039. PMID: 26165633.

91. Wilkins EG, Hamill JB, Kim HM, et al. Complications in Postmastectomy Breast Reconstruction: One-year Outcomes of the Mastectomy Reconstruction Outcomes Consortium (MROC) Study. Ann Surg. 2018 Jan;267(1):164-70. doi: 10.1097/sla.0000000000002033. PMID: 27906762 .
92. Woo KJ, Lee KT, Mun GH, et al. Effect of breast reconstruction modality on the development of postmastectomy shoulder morbidity. J Plast Reconstr Aesthet Surg. 2018 Dec;71(12):1761-7. doi: 10.1016/j.bjps.2018.07.033. PMID: 30360958.

93. Wu ZY, Han HH, Kim HJ, et al. A propensity score-matched comparison of recurrence outcomes after immediate implant vs autologous flap reconstruction in patients receiving neoadjuvant chemotherapy for breast cancer. Breast Cancer Res Treat. 2021 Mar 19. doi: 10.1007/s10549-021-06114-w. PMID: 33740204.

94. Xu F, Sun H, Zhang C, et al. Comparison of surgical complication between immediate implant and autologous breast reconstruction after mastectomy: A multicenter study of 426 cases. J Surg Oncol. 2018

Nov;118(6):953-8. doi: 10.1002/jso.25238. PMID: 30261115.

95. Yueh JH, Slavin SA, Adesiyun T, et al. Patient satisfaction in postmastectomy breast reconstruction: a comparative evaluation of DIEP, TRAM, latissimus flap, and implant techniques. Plast Reconstr Surg. 2010 Jun;125(6):1585-95. doi: 10.1097/PRS.0b013e3181cb6351. PMID: 20517080 .

96. Yueh JH, Slavin SA, Bar-Meir ED, et al. Impact of regional referral centers for microsurgical breast reconstruction: the New England perforator flap program experience. J Am Coll Surg. 2009 Feb;208(2):246-54. doi: 10.1016/j.jamcollsurg.2008.10.033. PMID: 19228537.

97. Zhang L, Jin K, Wang X, et al. The Impact of Radiotherapy on Reoperation Rates in Patients Undergoing Mastectomy and Breast Reconstruction. Ann Surg Oncol. 2019 Apr;26(4):961-8. doi: 10.1245/s10434-01807135-4. PMID: 30675702.

98. Acosta R, Smit JM, Audolfsson T, et al. A clinical review of 9 years of free perforator flap breast reconstructions: an analysis of 675 flaps and the influence of new techniques on clinical practice. J Reconstr Microsurg. 2011 Feb;27(2):91-8. doi: 10.1055/s-0030-1267835. PMID: 21046538. 
99. Albornoz CR, Cordeiro PG, Hishon L, et al. A nationwide analysis of the relationship between hospital volume and outcome for autologous breast reconstruction. Plast Reconstr Surg. 2013 Aug;132(2):192e-200e. doi: 10.1097/PRS.0b013e31829586c1. PMID: 23897346.

100. Andree C, Munder BI, Seidenstuecker K, et al. Skin-sparing mastectomy and immediate reconstruction with DIEP flap after breastconserving therapy. Med Sci Monit. 2012 Dec;18(12):Cr716-20. doi: 10.12659/msm.883598. PMID: 23197233.

101. Banuelos J, Taslakian EN, Nguyen MT, et al. The American College of Surgeons National Quality Improvement Program Incompletely Captures Implant-Based Breast Reconstruction Complications. Ann Plast Surg. 2020 Mar;84(3):271-5. doi: 10.1097/sap.0000000000002051. PMID: 31663932.

102. Beugels J, Bod L, van Kuijk SMJ, et al. Complications following immediate compared to delayed deep inferior epigastric artery perforator flap breast reconstructions. Breast Cancer Res Treat. 2018 Jun;169(2):349-57. doi: 10.1007/s10549018-4695-0. PMID: 29399731.

103. Beugels J, Kool M, Hoekstra LT, et al. Quality of Life of Patients After Immediate or Delayed Autologous Breast Reconstruction: A Multicenter Study. Ann Plast Surg. 2018 Nov;81(5):523-7. doi: 10.1097/sap.0000000000001618. PMID: 30247195.

104. Brooks S, Djohan R, Tendulkar R, et al. Risk factors for complications of radiation therapy on tissue expander breast reconstructions. Breast J. 2012 JanFeb;18(1):28-34. doi: 10.1111/j.15244741.2011.01182.x. PMID: 22098451.

105. Chang DW, Wang B, Robb GL, et al. Effect of obesity on flap and donor-site complications in free transverse rectus abdominis myocutaneous flap breast reconstruction. Plast Reconstr Surg. 2000 Apr;105(5):16408. doi: 10.1097/00006534-20000405000007. PMID: 10809092.
106. Chang EI, Chang EI, Soto-Miranda MA, et al. Demystifying the use of internal mammary vessels as recipient vessels in free flap breast reconstruction. Plast Reconstr Surg. 2013 Oct;132(4):763-8. doi: 10.1097/PRS.0b013e31829fe321. PMID: 24076668.

107. Chang EI, Chang EI, Soto-Miranda MA, et al. Comprehensive Evaluation of Risk Factors and Management of Impending Flap Loss in 2138 Breast Free Flaps. Ann Plast Surg. 2016 Jan;77(1):67-71. doi: 10.1097/sap.0000000000000263. PMID: 25003429.

108. Chang EI, Chang EI, Soto-Miranda MA, et al. Comprehensive analysis of donor-site morbidity in abdominally based free flap breast reconstruction. Plast Reconstr Surg. 2013 Dec;132(6):1383-91. doi: 10.1097/PRS.0b013e3182a805a3. PMID: 24005365.

109. Chang EI, Vaca L, DaLio AL, et al. Assessment of advanced age as a risk factor in microvascular breast reconstruction. Ann Plast Surg. 2011 Sep;67(3):255-9. doi: 10.1097/SAP.0b013e3181f9b20c. PMID: 21407063.

110. Chen AD, Chi D, Wu WW, et al. The Influence of Connective Tissue Disease in Breast Reconstruction: A National Database Analysis. Ann Plast Surg. 2018 Apr;80(4 Suppl 4):S182-s8. doi: 10.1097/sap.0000000000001387. PMID: 29596085.

111. Chen CF, Lin SF, Hung CF, et al. Risk of infection is associated more with drain duration than daily drainage volume in prosthesis-based breast reconstruction: A cohort study. Medicine (Baltimore). 2016 Dec;95(49):e5605. doi: 10.1097/md.0000000000005605. PMID: 27930584.

112. Chen Y, Chen J, Chen J, et al. [Current trends of breast reconstruction after mastectomy for breast cancer patients in China: a survey report]. Zhonghua Zhong Liu Za Zhi. 2014 Nov;36(11):851-7. PMID: 25620484. 
113. Cleveland EC, Fischer JP, Nelson JA, et al. Optimizing the fascial closure: an analysis of 1261 abdominally based free flap reconstructions. Ann Plast Surg. 2013 Sep;71(3):255-60. doi: 10.1097/SAP.0b013e318286380e. PMID: 23945529.

114. Collier W, Scheefer Van Boerum M, Kim J, et al. Are 30-Day Outcomes Enough? Late Infectious Readmissions following Prosthetic-Based Breast Reconstruction. Plast Reconstr Surg. 2019 Sep;144(3):360e8e. doi: $10.1097 /$ prs.0000000000005903. PMID: 31461001.

115. Cordeiro PG, Albornoz CR, McCormick B, et al. The impact of postmastectomy radiotherapy on two-stage implant breast reconstruction: an analysis of long-term surgical outcomes, aesthetic results, and satisfaction over 13 years. Plast Reconstr Surg. 2014 Oct;134(4):588-95. doi: 10.1097/prs.0000000000000523. PMID: 25357021 .

116. Cordeiro PG, McCarthy CM. A single surgeon's 12-year experience with tissue expander/implant breast reconstruction: part I. A prospective analysis of early complications. Plast Reconstr Surg. 2006 Sep 15;118(4):825-31. doi: 10.1097/01.prs.0000232362.82402.e8. PMID: 16980842.

117. Cordeiro PG, McGuire P, Murphy DK. Natrelle 410 Extra-Full Projection Silicone Breast Implants: 2-Year Results from Two Prospective Studies. Plast Reconstr Surg. 2015 Oct;136(4):638-46. doi: 10.1097/prs.0000000000001636. PMID: 26090764.

118. Cordeiro PG, Snell L, Heerdt A, et al. Immediate tissue expander/implast breast reconstruction after salvage mastectomy for cancer recurrence following lumpectomy/irradiation. Plast Reconstr Surg. 2012 Feb;129(2):341-50. doi: 10.1097/PRS.0b013e318205f203. PMID: 22286416.

119. Coroneos CJ, Selber JC, Offodile AC, 2nd, et al. US FDA Breast Implant Postapproval Studies: Long-term Outcomes in 99,993 Patients. Ann Surg. 2019 Jan;269(1):30-6. doi: 10.1097/sla.0000000000002990. PMID: 30222598 .
120. Daly LT, Doval AF, Lin SJ, et al. Role of CTA in Women with Abdominal Scars Undergoing DIEP Breast Reconstruction: Review of 1,187 Flaps. J Reconstr Microsurg. 2020 May;36(4):294-300. doi: 10.1055/s-0039-1701040. PMID: 31994156.

121. Enajat M, Rozen WM, Whitaker IS, et al. A single center comparison of one versus two venous anastomoses in 564 consecutive DIEP flaps: investigating the effect on venous congestion and flap survival. Microsurgery. 2010;30(3):185-91. doi: 10.1002/micr.20712. PMID: 19790180.

122. Fitzgerald O'Connor E, Rozen WM, Chowdhry $\mathrm{M}$, et al. The microvascular anastomotic coupler for venous anastomoses in free flap breast reconstruction improves outcomes. Gland Surg. 2016 Apr;5(2):88-92. doi: 10.3978/j.issn.2227-684X.2015.05.14. PMID: 27047776.

123. Gfrerer L, Mattos D, Mastroianni M, et al. Assessment of patient factors, surgeons, and surgeon teams in immediate implant-based breast reconstruction outcomes. Plast Reconstr Surg. 2015 Feb;135(2):245e-52e. doi: $10.1097 /$ prs.0000000000000912. PMID: 25626807.

124. Gill PS, Hunt JP, Guerra AB, et al. A 10-year retrospective review of 758 DIEP flaps for breast reconstruction. Plast Reconstr Surg. 2004 Apr 1;113(4):1153-60. doi: 10.1097/01.prs.0000110328.47206.50. PMID: 15083015.

125. Goodwin SJ, McCarthy CM, Pusic AL, et al. Complications in smokers after postmastectomy tissue expander/implant breast reconstruction. Ann Plast Surg. 2005 Jul;55(1):16-9; discussion 9-20. doi: 10.1097/01.sap.0000168282.81348.b3. PMID: 15985785.

126. Haddock NT, Cho MJ, Teotia SS. Comparative Analysis of Single versus Stacked Free Flap Breast Reconstruction: A Single-Center Experience. Plast Reconstr Surg. 2019 Sep;144(3):369e-77e. doi: 10.1097/prs.0000000000005906. PMID: 31461004. 
127. Haddock NT, Culver AJ, Teotia SS. Abdominal weakness, bulge, or hernia after DIEP flaps: An algorithm of management, prevention, and surgical repair with classification. $\mathrm{J}$ Plast Reconstr Aesthet Surg. 2020 Dec 26. doi: 10.1016/j.bjps.2020.12.044. PMID: 33487570 .

128. Hamdi M, Andrades P, Thiessen F, et al. Is a second free flap still an option in a failed free flap breast reconstruction? Plast Reconstr Surg. 2010 Aug;126(2):375-84. doi: 10.1097/PRS.0b013e3181de22f3. PMID: 20679823.

129. Hamdi M, Casaer B, Andrades P, et al. Salvage (tertiary) breast reconstruction after implant failure. J Plast Reconstr Aesthet Surg. 2011 Mar;64(3):353-9. doi: 10.1016/j.bjps.2010.05.019. PMID: 20576480 .

130. Hansen N, Espino S, Blough JT, et al. Evaluating Mastectomy Skin Flap Necrosis in the Extended Breast Reconstruction Risk Assessment Score for 1-Year Prediction of Prosthetic Reconstruction Outcomes. J Am Coll Surg. 2018 Jul;227(1):96-104. doi: 10.1016/j.jamcollsurg.2018.05.003. PMID: 29778821.

131. Hembd AS, Yan J, Zhu H, et al. Intraoperative Assessment of DIEP Flap Breast Reconstruction Using Indocyanine Green Angiography: Reduction of Fat Necrosis, Resection Volumes, and Postoperative Surveillance. Plast Reconstr Surg. 2020 Jul;146(1):1e-10e. doi: 10.1097/prs.0000000000006888. PMID: 32590635.

132. Heo JW, Park SO, Jin US. Donor-site morbidities in 615 patients after breast reconstruction using a free muscle-sparing type I transverse rectus abdominis myocutaneous flap: a single surgeon experience. J Plast Surg Hand Surg. 2018 Dec;52(6):325-32. doi: 10.1080/2000656x.2018.1493389. PMID: 30039735 .

133. Hunsicker LM, Ashikari AY, Berry C, et al. Short-Term Complications Associated With Acellular Dermal Matrix-Assisted Direct-toImplant Breast Reconstruction. Ann Plast Surg. 2017 Jan;78(1):35-40. doi: 10.1097/sap.0000000000000742. PMID: 26849284.
134. Huo J, Smith BD, Giordano SH, et al. A comparison of patient-centered economic and clinical outcomes of post-mastectomy breast reconstruction between obese and non-obese patients. Breast. 2016 Dec;30:118-24. doi: 10.1016/j.breast.2016.09.004. PMID: 27697676.

135. Jo T, Hur J, Min K, et al. Immediate breast reconstruction after salvage mastectomy: Case control outcome comparisons of DIEP flap and DTI reconstruction. J Plast Reconstr Aesthet Surg. 2020 Dec 9. doi: 10.1016/j.bjps.2020.11.034. PMID: 33386262 .

136. Kanuri A, Liu AS, Guo L. Whom should we SPY? A cost analysis of laser-assisted indocyanine green angiography in prevention of mastectomy skin flap necrosis during prosthesis-based breast reconstruction. Plast Reconstr Surg. 2014 Apr;133(4):448e-54e. doi: 10.1097/prs.0000000000000025. PMID: 24675199.

137. Kato H, Nakagami G, Iwahira Y, et al. Risk factors and risk scoring tool for infection during tissue expansion in tissue expander and implant breast reconstruction. Breast $\mathrm{J}$. 2013 Nov-Dec;19(6):618-26. doi: 10.1111/tbj.12175. PMID: 24011080.

138. Kim EK, Eom JS, Ahn SH, et al. Evolution of the pedicled TRAM flap: a prospective study of 500 consecutive cases by a single surgeon in Asian patients. Ann Plast Surg. 2009 Oct;63(4):378-82. doi: 10.1097/SAP.0b013e3181951708. PMID: 19745709.

139. Kim EK, Eom JS, Ahn SH, et al. The efficacy of prophylactic low-molecular-weight heparin to prevent pulmonary thromboembolism in immediate breast reconstruction using the TRAM flap. Plast Reconstr Surg. 2009 Jan;123(1):9-12. doi: 10.1097/PRS.0b013e3181904be7. PMID: 19116509.

140. Kim HJ, Park EH, Lim WS, et al. Nipple areola skin-sparing mastectomy with immediate transverse rectus abdominis musculocutaneous flap reconstruction is an oncologically safe procedure: a single center study. Ann Surg. 2010 Mar;251(3):493-8. doi: 10.1097/SLA.0b013e3181c5dc4e. PMID: 20134317. 
141. Langer S, Munder B, Seidenstuecker K, et al. Development of a surgical algorithm and optimized management of complications based on a review of 706 abdominal free flaps for breast reconstruction. Med Sci Monit. 2010 Nov;16(11):Cr518-22. PMID: 20980954.

142. Lantieri L, Hivelin M, Benjoar MD, et al. [Setting of a breast autologous microsurgical reconstructive surgery evolution in 20 years and review of 1138 cases]. Ann Chir Plast Esthet. 2015 Dec;60(6):484-9. doi: 10.1016/j.anplas.2015.06.009. PMID: 26238173.

143. Law TY, Moeller E, Hubbard ZS, et al. Preoperative Hypoglycemia and Hyperglycemia Are Related to Postoperative Infection Rates in Implant-Based Breast Reconstruction. J Surg Res. 2018 Dec;232:437-41. doi: 10.1016/j.jss.2018.06.068. PMID: 30463754 .

144. Lee KT, Bae J, Jeon BJ, et al. Adjuvant Chemotherapy in Two-Stage Tissue Expander/Implant Breast Reconstruction: Does it Affect Final Outcomes? Ann Surg Oncol. 2021 Apr;28(4):2191-8. doi: 10.1245/s10434-020-09177-z. PMID: 32974692 .

145. Lee KT, Kim S, Jeon BJ, et al. Association of the Implant Surface Texture Used in Reconstruction With Breast Cancer Recurrence. JAMA Surg. 2020 Dec 1;155(12):1132-40. doi: 10.1001/jamasurg.2020.4124. PMID: 33026424.

146. Liao EC, Taghinia AH, Nguyen LP, et al. Incidence of hematoma complication with heparin venous thrombosis prophylaxis after TRAM flap breast reconstruction. Plast Reconstr Surg. 2008 Apr;121(4):1101-7. doi: 10.1097/01.prs.0000302454.43201.83. PMID: 18349626.

147. Lovecchio F, Jordan SW, Lim S, et al. Risk Factors for Complications Differ Between Stages of Tissue-Expander Breast Reconstruction. Ann Plast Surg. 2015 Sep;75(3):275-80. doi: 10.1097/sap.0000000000000109. PMID: 24691330.
148. Masoomi H, Blumenauer BJ, Blakkolb CL, et al. Predictors of blood transfusion in autologous breast reconstruction surgery: A retrospective study using the nationwide inpatient sample database. J Plast Reconstr Aesthet Surg. 2019 Oct;72(10):1616-22. doi: 10.1016/j.bjps.2019.06.012. PMID: 31331721 .

149. Masoomi H, Paydar KZ, Wirth GA, et al. Predictive risk factors of venous thromboembolism in autologous breast reconstruction surgery. Ann Plast Surg. 2014 Jan;72(1):30-3. doi: 10.1097/sap.0000000000000003. PMID: 24317244.

150. McCarthy CM, Mehrara BJ, Riedel E, et al. Predicting complications following expander/implant breast reconstruction: an outcomes analysis based on preoperative clinical risk. Plast Reconstr Surg. 2008 Jun;121(6):1886-92. doi: 10.1097/PRS.0b013e31817151c4. PMID: 18520873.

151. Mehrara BJ, Santoro TD, Arcilla E, et al. Complications after microvascular breast reconstruction: experience with 1195 flaps. Plast Reconstr Surg. 2006 Oct;118(5):11009; discussion 10-1. doi: 10.1097/01.prs.0000236898.87398.d6. PMID: 17016173.

152. Mirzabeigi MN, Nelson JA, Fischer JP, et al. Tamoxifen (selective estrogen-receptor modulators) and aromatase inhibitors as potential perioperative thrombotic risk factors in free flap breast reconstruction. Plast Reconstr Surg. 2015 Apr;135(4):670e9e. doi: 10.1097/prs.0000000000001127. PMID: 25811579.

153. Munder B, Andree C, Witzel C, et al. The DIEP Flap as Well-established Method of Choice for Autologous Breast Reconstruction with a Low Complication Rate - Retrospective Single-centre 10-Year Experience. Geburtshilfe Frauenheilkd. 2020 Jun;80(6):628-38. doi: 10.1055/a-11162102. PMID: 32565553.

154. Nelson JA, Fischer JP, Grover R, et al. The impact of anemia on microsurgical breast reconstruction complications and outcomes. Microsurgery. 2014 May;34(4):261-70. doi: 10.1002/micr.22202. PMID: 24436188. 
155. Nelson JA, Stransky CA, Fischer JP, et al. Reexamining free flap breast reconstruction in the community and university setting: is there a difference? Ann Plast Surg. 2014 Dec;73 Suppl 2:S171-4. doi: 10.1097/sap.0000000000000204. PMID: 25046665.

156. O'Neill AC, Barandun M, Cha J, et al. Restrictive use of perioperative blood transfusion does not increase complication rates in microvascular breast reconstruction. J Plast Reconstr Aesthet Surg. 2016 Aug;69(8):1092-6. doi: 10.1016/j.bjps.2016.04.021. PMID: 27236504.

157. O'Neill AC, Sebastiampillai S, Zhong T, et al. Increasing body mass index increases complications but not failure rates in microvascular breast reconstruction: A retrospective cohort study. J Plast Reconstr Aesthet Surg. 2019 Sep;72(9):1518-24. doi: 10.1016/j.bjps.2019.05.013. PMID: 31196805 .

158. Parikh RP, Brown GM, Sharma K, et al. Immediate Implant-Based Breast Reconstruction with Acellular Dermal Matrix: A Comparison of Sterile and Aseptic AlloDerm in 2039 Consecutive Cases. Plast Reconstr Surg. 2018 Dec;142(6):1401-9. doi: 10.1097/prs.0000000000004968. PMID: 30204676.

159. Park JW, Lee KT, Jeon BJ, et al. Effects of Antihypertensive Drugs on Outcomes of Breast Reconstruction. Ann Surg Oncol. 2019 Jun;26(6):1712-9. doi: 10.1245/s10434-019-07293-z. PMID: 30863940 .

160. Phan R, Rozen WM, Chowdhry M, et al. Risk factors and timing of postoperative hematomas following microvascular breast reconstruction: A prospective cohort study. Microsurgery. 2020 Feb;40(2):99-103. doi: 10.1002/micr.30473. PMID: 31124177.

161. Polanco TO, Shamsunder MG, Hicks MEV, et al. Goal-directed fluid therapy in autologous breast reconstruction results in less fluid and more vasopressor administration without outcome compromise. J Plast Reconstr Aesthet Surg. 2021 Feb 4. doi: 10.1016/j.bjps.2021.01.017. PMID: 33745850 .
162. Potter S, Conroy EJ, Cutress RI, et al. Shortterm safety outcomes of mastectomy and immediate implant-based breast reconstruction with and without mesh (iBRA): a multicentre, prospective cohort study. Lancet Oncol. 2019 Feb;20(2):25466. doi: 10.1016/s1470-2045(18)30781-2. PMID: 30639093.

163. Prantl L, Moellhoff N, von Fritschen U, et al. Immediate versus secondary DIEP flap breast reconstruction: a multicenter outcome study. Arch Gynecol Obstet. 2020 Dec;302(6):1451-9. doi: 10.1007/s00404020-05779-w. PMID: 32895743.

164. Ricci JA, Kamali P, Becherer BE, et al. Umbilical necrosis rates after abdominalbased microsurgical breast reconstruction. J Surg Res. 2017 Jul;215:257-63. doi: 10.1016/j.jss.2017.04.010. PMID: 28688657.

165. Rogoff H, Marquez JE, Ghosh K, et al. Clinical Outcomes After Hematoma Development: A Study of 627 Tissue Expander Breast Reconstructions. Ann Plast Surg. 2020 Jul;85(S1 Suppl 1):S63-s7. doi: 10.1097/sap.0000000000002343. PMID: 32243320.

166. Roy M, Sebastiampillai S, Zhong T, et al. Synergistic Interaction Increases Complication Rates following Microvascular Breast Reconstruction. Plast Reconstr Surg. 2019 Jul;144(1):1e-8e. doi: 10.1097/prs.0000000000005695. PMID: 31246793.

167. Rubio GA, McGee CS, Thaller SR. Autologous breast reconstruction surgery outcomes in patients with autoimmune connective tissue disease. J Plast Reconstr Aesthet Surg. 2019 May;72(5):848-62. doi: 10.1016/j.bjps.2018.12.038. PMID: 30665841 .

168. Salibian AA, Frey JD, Bekisz JM, et al. Ischemic Complications after Nipplesparing Mastectomy: Predictors of Reconstructive Failure in Implant-based Reconstruction and Implications for Decision-making. Plast Reconstr Surg Glob Open. 2019 May;7(5):e2280. doi: 10.1097/gox.0000000000002280. PMID: 31333984. 
169. Seidenstuecker K, van Waes C, Munder BI, et al. DIEAP flap for safe definitive autologous breast reconstruction. Breast. 2016 Apr;26:59-66. doi: 10.1016/j.breast.2015.12.005. PMID: 27017243.

170. Selber JC, Bergey M, Sonnad SS, et al. Free flap breast reconstruction in advanced age: is it safe? Plast Reconstr Surg. 2009 Oct;124(4):1015-22. doi: 10.1097/PRS.0b013e3181b453fd. PMID: 19935283.

171. Seth AK, Silver HR, Hirsch EM, et al. Comparison of Delayed and Immediate Tissue Expander Breast Reconstruction in the Setting of Postmastectomy Radiation Therapy. Ann Plast Surg. 2015

Nov;75(5):503-7. doi: 10.1097/sap.0000000000000191. PMID: 25180955.

172. Sewart E, Turner NL, Conroy EJ, et al. Patientreported outcomes of immediate implantbased breast reconstruction with and without biological or synthetic mesh. BJS Open. 2021 Jan 8;5(1). doi: 10.1093/bjsopen/zraa063. PMID: 33609398.

173. Shaikh IA, Thomas H, Vidyadharan R, et al. Post mastectomy immediate breast reconstruction 13 years experience in a single centre. Indian J Surg Oncol. 2010 Sep;1(3):250-5. doi: 10.1007/s13193-0110051-0. PMID: 22693373.

174. Shammas RL, Ren Y, Thomas SM, et al. The Association of Overall Annual Hospital Volume and Perioperative Outcomes following Free Flap Breast Reconstruction. Plast Reconstr Surg. 2021 Feb 1;147(2):196e-206e. doi: 10.1097/prs.0000000000007549. PMID: 33565821 .

175. Singh D, Slavin BR, Holton T. Comparing Surgical Site Occurrences in 1 versus 2stage Breast Reconstruction via Federated EMR Network. Plast Reconstr Surg Glob Open. 2021 Jan;9(1):e3385. doi: 10.1097/gox.0000000000003385. PMID: 33564597.
176. Singh N, Reaven NL, Funk SE. Immediate 1stage vs. tissue expander postmastectomy implant breast reconstructions: a retrospective real-world comparison over 18 months. J Plast Reconstr Aesthet Surg. 2012 Jul;65(7):917-23. doi: 10.1016/j.bjps.2011.12.040. PMID: 22342636.

177. Song D, Slater K, Papsdorf M, et al. Autologous Breast Reconstruction in Women Older Than 65 Years Versus Women Younger Than 65 Years: A Multi-Center Analysis. Ann Plast Surg. 2016 Feb;76(2):155-63. doi: 10.1097/sap.0000000000000527. PMID: 26637165.

178. Tran BNN, Ruan QZ, Cohen JB, et al. Does Hormone Therapy Use Increase Perioperative Complications in Abdominally Based Microsurgical Breast Reconstruction? Plast Reconstr Surg. 2018 Jun;141(6):805e13e. doi: 10.1097/prs.0000000000004359. PMID: 29794694.

179. Vargas CR, Koolen PG, Ho OA, et al. Preoperative CT-angiography in autologous breast reconstruction. Microsurgery. 2016 Nov;36(8):623-7. doi: 10.1002/micr.30065. PMID: 27159778.

180. Warren DK, Nickel KB, Hostler CJ, et al. Surgeon choice in the use of postdischarge antibiotics for prophylaxis following mastectomy with and without breast reconstruction. Infect Control Hosp Epidemiol. 2021 Apr;42(4):467-70. doi: 10.1017/ice.2020.462. PMID: 33040748.

181. Watterson PA, Bostwick J, 3rd, Hester TR, Jr., et al. TRAM flap anatomy correlated with a 10-year clinical experience with 556 patients. Plast Reconstr Surg. 1995 Jun;95(7):1185-94. doi: 10.1097/00006534199506000-00007. PMID: 7761505.

182. Williams JK, Bostwick J, 3rd, Bried JT, et al. TRAM flap breast reconstruction after radiation treatment. Ann Surg. 1995 Jun;221(6):756-64; discussion 64-6. doi: 10.1097/00000658-199506000-00014. PMID: 7794079. 
183. Yoo H, Kim BH, Kim HH, et al. Local recurrence of breast cancer in reconstructed breasts using TRAM flap after skin-sparing mastectomy: clinical and imaging features. Eur Radiol. 2014 Sep;24(9):2220-6. doi: 10.1007/s00330-014-3214-x. PMID: 24852813.

184. Pickard AS, Neary MP, Cella D. Estimation of minimally important differences in EQ-5D utility and VAS scores in cancer. Health Qual Life Outcomes. 2007 Dec 21;5:70. doi: 10.1186/1477-7525-5-70. PMID: 18154669.

185. Musoro JZ, Coens C, Fiteni F, et al. Minimally Important Differences for Interpreting EORTC QLQ-C30 Scores in Patients With Advanced Breast Cancer. JNCI Cancer Spectr. 2019 Sep;3(3):pkz037. doi: 10.1093/jncics/pkz037. PMID: 32328553.

186. Eton DT, Cella D, Yost KJ, et al. A combination of distribution- and anchor-based approaches determined minimally important differences (MIDs) for four endpoints in a breast cancer scale. J Clin Epidemiol. 2004 Sep;57(9):898-910. doi: 10.1016/j.jclinepi.2004.01.012. PMID: 15504633 .

187. Voineskos SH, Klassen AF, Cano SJ, et al. Giving Meaning to Differences in BREASTQ Scores: Minimal Important Difference for Breast Reconstruction Patients. Plast Reconstr Surg. 2020 Jan;145(1):11e-20e. doi: 10.1097/prs.0000000000006317. PMID: 31577663.

188. Yost KJ, Eton DT, Garcia SF, et al. Minimally important differences were estimated for six Patient-Reported Outcomes Measurement Information System-Cancer scales in advanced-stage cancer patients. J Clin Epidemiol. 2011 May;64(5):507-16. doi: 10.1016/j.jclinepi.2010.11.018. PMID: 21447427.

189. Hamidou Z, Dabakuyo-Yonli TS, Guillemin F, et al. Impact of response shift on time to deterioration in quality of life scores in breast cancer patients. PLoS One. 2014;9(5):e96848. doi: 10.1371/journal.pone.0096848. PMID: 24828426.

190. Harrington S, Gilchrist L, Sander A. Breast Cancer EDGE Task Force Outcomes: Clinical Measures of Pain. Rehabil Oncol. 2014;32(1):13-21. PMID: 25346950.
191. Farrar JT, Young JP, Jr., LaMoreaux L, et al. Clinical importance of changes in chronic pain intensity measured on an 11-point numerical pain rating scale. Pain. 2001 Nov;94(2):149-58. doi: 10.1016/s03043959(01)00349-9. PMID: 11690728.

192. Cordeiro PG, Albornoz CR, McCormick B, et al. What Is the Optimum Timing of Postmastectomy Radiotherapy in Two-Stage Prosthetic Reconstruction: Radiation to the Tissue Expander or Permanent Implant? Plast Reconstr Surg. 2015 Jun;135(6):150917. doi: $10.1097 /$ prs. 0000000000001278 . PMID: 25742523.

193. Eriksson M, Anveden L, Celebioglu F, et al. Radiotherapy in implant-based immediate breast reconstruction: risk factors, surgical outcomes, and patient-reported outcome measures in a large Swedish multicenter cohort. Breast Cancer Res Treat. 2013 Dec;142(3):591-601. doi: 10.1007/s10549013-2770-0. PMID: 24258257.

194. Hirsch EM, Seth AK, Kim JY, et al. Analysis of risk factors for complications in expander/implant breast reconstruction by stage of reconstruction. Plast Reconstr Surg. 2014 Nov;134(5):692e-9e. doi: 10.1097/prs.0000000000000607. PMID: 25347643.

195. Santosa KB, Chen X, Qi J, et al. Postmastectomy Radiation Therapy and Two-Stage Implant-Based Breast Reconstruction: Is There a Better Time to Irradiate? Plast Reconstr Surg. 2016 Oct;138(4):761-9. doi: 10.1097/prs.0000000000002534. PMID: 27673513.

196. Seth AK, Hirsch EM, Fine NA, et al. Additive risk of tumescent technique in patients undergoing mastectomy with immediate reconstruction. Ann Surg Oncol. 2011 Oct;18(11):3041-6. doi: 10.1245/s10434011-1913-y. PMID: 21947584.

197. Seth AK, Hirsch EM, Kim JY, et al. Two surgeons, one patient: the impact of surgeon-surgeon familiarity on patient outcomes following mastectomy with immediate reconstruction. Breast. 2013 Oct;22(5):914-8. doi: 10.1016/j.breast.2013.04.013. PMID: 23673077 . 
198. Seth AK, Hirsch EM, Kim JY, et al. Hematoma after mastectomy with immediate reconstruction: an analysis of risk factors in 883 patients. Ann Plast Surg. 2013 Jul;71(1):20-3. doi: 10.1097/SAP.0b013e318243355f. PMID: 22791066.

199. Seth AK, Hirsch EM, Kim JY, et al. Long-term outcomes following fat grafting in prosthetic breast reconstruction: a comparative analysis. Plast Reconstr Surg. 2012 Nov;130(5):984-90. doi: 10.1097/PRS.0b013e318267d34d. PMID: 22777039.

200. Stein MJ, Chung A, Arnaout A, et al. Complication rates of acellular dermal matrix in immediate breast reconstruction with radiation: A single-institution retrospective comparison study. J Plast Reconstr Aesthet Surg. 2020 Dec;73(12):2156-63. doi: 10.1016/j.bjps.2020.05.065. PMID: 32561384.

201. Yoon AP, Qi J, Kim HM, et al. Patient-Reported Outcomes after Irradiation of Tissue Expander versus Permanent Implant in Breast Reconstruction: A Multicenter Prospective Study. Plast Reconstr Surg. 2020 May;145(5):917e-26e. doi: 10.1097/prs.0000000000006724. PMID: 32332528 .

202. Antony AK, McCarthy C, Disa JJ, et al. Bilateral implant breast reconstruction: outcomes, predictors, and matched cohort analysis in 730 2-stage breast reconstructions over 10 years. Ann Plast Surg. 2014;72(6):625-30. doi: 10.1097/SAP.0b013e31826af03e. PMID: 24135689.

203. Le GM, O'Malley CD, Glaser SL, et al. Breast implants following mastectomy in women with early-stage breast cancer: prevalence and impact on survival. Breast Cancer Res. 2005;7(2):R184-93. doi: 10.1186/bcr974. PMID: 15743498.
204. Macadam SA, Ho AL, Cook EF, Jr., et al. Patient satisfaction and health-related quality of life following breast reconstruction: patient-reported outcomes among saline and silicone implant recipients. Plast Reconstr Surg. 2010 Mar;125(3):761-71. doi: 10.1097/PRS.0b013e3181cb5cf8. PMID: 20009795.

205. Osoba D, Rodrigues G, Myles J, et al. Interpreting the significance of changes in health-related quality-of-life scores. J Clin Oncol. 1998 Jan;16(1):139-44. doi: 10.1200/jco.1998.16.1.139. PMID: 9440735 .

206. Lee JS, Park E, Lee JH, et al. A prospective comparison study of early functional outcomes after implant-based breast reconstruction: subpectoral versus prepectoral technique. Ann Palliat Med. 2021 Mar;10(3):2520-9. doi: 10.21037/apm20-1550. PMID: 33691448.

207. Avila A, Bartholomew AJ, Sosin M, et al. Acute Postoperative Complications in Prepectoral versus Subpectoral Reconstruction following Nipple-Sparing Mastectomy. Plast Reconstr Surg. 2020 Dec;146(6):715e-20e. doi: 10.1097/prs.0000000000007326. PMID: 33234947.

208. Bozzuto LM, Bartholomew AJ, Tung S, et al. Decreased postoperative pain and opioid use following prepectoral versus subpectoral breast reconstruction after mastectomy: A retrospective cohort study: Pain after preversus subpectoral reconstruction. J Plast Reconstr Aesthet Surg. 2020 Dec 13. doi: 10.1016/j.bjps.2020.12.009. PMID: 33451949.

209. Cattelani L, Polotto S, Arcuri MF, et al. OneStep Prepectoral Breast Reconstruction With Dermal Matrix-Covered Implant Compared to Submuscular Implantation: Functional and Cost Evaluation. Clin Breast Cancer. 2018 Aug;18(4):e703-e11. doi: 10.1016/j.clbc.2017.11.015. PMID: 29275104.

210. Gabriel A, Sigalove S, Storm-Dickerson TL, et al. Dual-Plane versus Prepectoral Breast Reconstruction in High-Body Mass Index Patients. Plast Reconstr Surg. 2020 Jun;145(6):1357-65. doi: $10.1097 /$ prs.0000000000006840. PMID: 32195862. 
211. Kim JH, Hong SE. A Comparative Analysis between Subpectoral versus Prepectoral Single Stage Direct-to-Implant Breast Reconstruction. Medicina (Kaunas). 2020 Oct $13 ; 56(10)$. doi: 10.3390/medicina56100537. PMID: 33066236.

212. Kraenzlin F, Darrach H, Khavanin N, et al. Tissue Expander-Based Breast Reconstruction in the Prepectoral Versus Subpectoral Plane: An Analysis of ShortTerm Outcomes. Ann Plast Surg. 2021 Jan;86(1):19-23. doi: 10.1097/sap.0000000000002415. PMID: 32568752.

213. Nealon KP, Weitzman RE, Sobti N, et al. Prepectoral Direct-to-Implant Breast Reconstruction: Safety Outcome Endpoints and Delineation of Risk Factors. Plast Reconstr Surg. 2020 Feb 5. doi: 10.1097/prs.0000000000006721. PMID: 32032345 .

214. Ozgur I, Kurul S, Bademler S, et al. Comparison of subpectoral versus dual-plane implant based immediate breast reconstruction after nipple-areola sparing mastectomy. Ann Chir Plast Esthet. 2020 Nov 19. doi: 10.1016/j.anplas.2020.10.002. PMID: 33223365 .

215. Kukkonen J, Kauko T, Vahlberg T, et al. Investigating minimal clinically important difference for Constant score in patients undergoing rotator cuff surgery. J Shoulder Elbow Surg. 2013 Dec;22(12):1650-5. doi: 10.1016/j.jse.2013.05.002. PMID: 23850308 .

216. Franchignoni F, Vercelli S, Giordano A, et al. Minimal clinically important difference of the disabilities of the arm, shoulder and hand outcome measure (DASH) and its shortened version (QuickDASH). J Orthop Sports Phys Ther. 2014 Jan;44(1):30-9. doi: 10.2519/jospt.2014.4893. PMID: 24175606.

217. Mathias SD, Crosby RD, Qian Y, et al. Estimating minimally important differences for the worst pain rating of the Brief Pain Inventory-Short Form. J Support Oncol. 2011 Mar-Apr;9(2):72-8. doi: 10.1016/j.suponc.2010.12.004. PMID: 21542414.
218. McCarthy CM, Lee CN, Halvorson EG, et al. The use of acellular dermal matrices in twostage expander/implant reconstruction: a multicenter, blinded, randomized controlled trial. Plast Reconstr Surg. 2012 Nov;130(5 Suppl 2):57s-66s. doi: 10.1097/PRS.0b013e31825f05b4. PMID: 23096987.

219. Wendel J. Use of Dermal Matrix in Breast Reconstruction. ClinicalTrials.gov; 2013.

220. Brooke S, Mesa J, Uluer M, et al. Complications in tissue expander breast reconstruction: a comparison of AlloDerm, DermaMatrix, and FlexHD acellular inferior pole dermal slings. Ann Plast Surg. 2012 Oct;69(4):347-9. doi: 10.1097/SAP.0b013e31824b3d97. PMID: 22868313.

221. Chun YS, Verma K, Rosen H, et al. Implantbased breast reconstruction using acellular dermal matrix and the risk of postoperative complications. Plast Reconstr Surg. 2010 Feb;125(2):429-36. doi: 10.1097/PRS.0b013e3181c82d90. PMID: 20124828.

222. Clarke-Pearson EM, Lin AM, Hertl C, et al. Revisions in Implant-Based Breast Reconstruction: How Does Direct-toImplant Measure Up? Plast Reconstr Surg. 2016 Jun;137(6):1690-9. doi: 10.1097/prs.0000000000002173. PMID: 27219225.

223. Craig ES, Clemens MW, Koshy JC, et al. Outcomes of Acellular Dermal Matrix for Immediate Tissue Expander Reconstruction with Radiotherapy: A Retrospective Cohort Study. Aesthet Surg J. 2019 Feb 15;39(3):279-88. doi: 10.1093/asj/sjy127. PMID: 29800083.

224. Ganesh Kumar N, Berlin NL, Kim HM, et al. Development of an evidence-based approach to the use of acellular dermal matrix in immediate expander-implant-based breast reconstruction. J Plast Reconstr Aesthet Surg. 2021 Jan;74(1):30-40. doi: 10.1016/j.bjps.2020.10.005. PMID: 33172826. 
225. Ibrahim AM, Shuster M, Koolen PG, et al. Analysis of the National Surgical Quality Improvement Program database in 19,100 patients undergoing implant-based breast reconstruction: complication rates with acellular dermal matrix. Plast Reconstr Surg. 2013 Nov;132(5):1057-66. doi: 10.1097/PRS.0b013e3182a3beec. PMID: 24165587.

226. Lee KT, Lee H, Jeon BJ, et al. Impact of overweight/obesity on the development of hematoma following tissue expander-based breast reconstruction. J Plast Reconstr Aesthet Surg. 2020;74(2):307-15.

227. Liu AS, Kao HK, Reish RG, et al. Postoperative complications in prosthesis-based breast reconstruction using acellular dermal matrix. Plast Reconstr Surg. 2011 May;127(5):1755-62. doi: 10.1097/PRS.0b013e31820cf233. PMID: 21228744.

228. Nealon KP, Sobti N, Gadd M, et al. Assessing the additional surgical risk of contralateral prophylactic mastectomy and immediate breast implant reconstruction. Breast Cancer Res Treat. 2020 Jan;179(2):255-65. doi: 10.1007/s10549-019-05460-0. PMID: 31605310 .

229. Pannucci CJ, Antony AK, Wilkins EG. The impact of acellular dermal matrix on tissue expander/implant loss in breast reconstruction: an analysis of the tracking outcomes and operations in plastic surgery database. Plast Reconstr Surg. 2013 Jul;132(1):1-10. doi: 10.1097/PRS.0b013e318290f917. PMID: 23508050.

230. Peled AW, Foster RD, Garwood ER, et al. The effects of acellular dermal matrix in expander-implant breast reconstruction after total skin-sparing mastectomy: results of a prospective practice improvement study. Plast Reconstr Surg. 2012 Jun;129(6):901e8e. doi: 10.1097/PRS.0b013e31824ec447. PMID: 22634688.

231. Qureshi AA, Broderick KP, Belz J, et al. Uneventful versus Successful Reconstruction and Outcome Pathways in Implant-Based Breast Reconstruction with Acellular Dermal Matrices. Plast Reconstr Surg. 2016 Aug;138(2):173e-83e. doi: 10.1097/prs.0000000000002402. PMID: 27465177.
232. Safran T, Al-Halabi B, Viezel-Mathieu A, et al. Direct-to-Implant, Prepectoral Breast Reconstruction: A Single-Surgeon Experience with 201 Consecutive Patients. Plast Reconstr Surg. 2020 Apr;145(4):686e96e. doi: $10.1097 /$ prs. 0000000000006654 . PMID: 32221195.

233. Seth AK, Hirsch EM, Fine NA, et al. Utility of acellular dermis-assisted breast reconstruction in the setting of radiation: a comparative analysis. Plast Reconstr Surg. 2012 Oct;130(4):750-8. doi: 10.1097/PRS.0b013e318262f009. PMID: 23018687.

234. Sobti N, Ji E, Brown RL, et al. Evaluation of Acellular Dermal Matrix Efficacy in Prosthesis-Based Breast Reconstruction. Plast Reconstr Surg. 2018 Mar;141(3):5419. doi: $10.1097 /$ prs.0000000000004109. PMID: 29481386.

235. Sorkin M, Qi J, Kim HM, et al. Acellular Dermal Matrix in Immediate Expander/Implant Breast Reconstruction: A Multicenter Assessment of Risks and Benefits. Plast Reconstr Surg. 2017 Dec;140(6):1091-100. doi: 10.1097/prs.0000000000003842. PMID: 28806288.

236. Vardanian AJ, Clayton JL, Roostaeian J, et al. Comparison of implant-based immediate breast reconstruction with and without acellular dermal matrix. Plast Reconstr Surg. 2011 Nov;128(5):403e-10e. doi: 10.1097/PRS.0b013e31822b6637. PMID: 22030500.

237. Weichman KE, Wilson SC, Weinstein AL, et al. The use of acellular dermal matrix in immediate two-stage tissue expander breast reconstruction. Plast Reconstr Surg. 2012 May;129(5):1049-58. doi: 10.1097/PRS.0b013e31824a2acb. PMID: 22544088.

238. Woo KJ, Park JW, Mun GH, et al. Does the Use of Acellular Dermal Matrix Increase Postoperative Complications of the FirstStage Reconstruction of Immediate Expander-Implant Breast Reconstruction: A Matched Cohort Study. Ann Plast Surg. 2017 Oct; 79(4):341-5. doi: 10.1097/sap.0000000000001067. PMID: 28509694. 
239. Flaherty SA. Pain measurement tools for clinical practice and research. Aana j. 1996 Apr;64(2):133-40. PMID: 9095685.

240. Brandberg Y, Malm M, Blomqvist L. A prospective and randomized study, "SVEA," comparing effects of three methods for delayed breast reconstruction on quality of life, patient-defined problem areas of life, and cosmetic result. Plast Reconstr Surg. 2000 Jan;105(1):66-74; discussion 5-6. doi: 10.1097/00006534-200001000-00011. PMID: 10626972.

241. Brandberg Y, Malm M, Rutqvist LE, et al. A prospective randomised study (named SVEA) of three methods of delayed breast reconstruction. Study design, patients' preoperative problems and expectations. Scand J Plast Reconstr Surg Hand Surg. 1999 Jun;33(2):209-16. doi: 10.1080/02844319950159479. PMID: 10450579 .

242. Erdmann-Sager J, Wilkins EG, Pusic AL, et al. Complications and Patient-Reported Outcomes after Abdominally Based Breast Reconstruction: Results of the Mastectomy Reconstruction Outcomes Consortium Study. Plast Reconstr Surg. 2018 Feb;141(2):271-81. doi: 10.1097/prs.0000000000004016. PMID: 29019862.

243. Gart MS, Smetona JT, Hanwright PJ, et al. Autologous options for postmastectomy breast reconstruction: a comparison of outcomes based on the American College of Surgeons National Surgical Quality Improvement Program. J Am Coll Surg. 2013 Feb;216(2):229-38. doi: 10.1016/j.jamcollsurg.2012.11.003. PMID: 23211118 .

244. Knox AD, Ho AL, Leung L, et al. Comparison of Outcomes following Autologous Breast Reconstruction Using the DIEP and Pedicled TRAM Flaps: A 12-Year Clinical Retrospective Study and Literature Review. Plast Reconstr Surg. 2016 Jul;138(1):16-28. doi: $10.1097 /$ prs.0000000000001747. PMID: 26267400.

245. Kroll SS. Fat necrosis in free transverse rectus abdominis myocutaneous and deep inferior epigastric perforator flaps. Plast Reconstr Surg. 2000 Sep;106(3):576-83. doi: 10.1097/00006534-200009030-00008. PMID: 10987463.
246. Massenburg BB, Sanati-Mehrizy P, Ingargiola MJ, et al. Flap Failure and Wound Complications in Autologous Breast Reconstruction: A National Perspective. Aesthetic Plast Surg. 2015 Dec;39(6):902-9. doi: 10.1007/s00266-015-0575-8. PMID: 26487657.

247. Mennie JC, Mohanna PN, O'Donoghue JM, et al. Donor-Site Hernia Repair in Abdominal Flap Breast Reconstruction: A PopulationBased Cohort Study of 7929 Patients. Plast Reconstr Surg. 2015 Jul;136(1):1-9. doi: 10.1097/prs.0000000000001398. PMID: 25839173.

248. Rindom MB, Gunnarsson GL, Lautrup MD, et al. Shoulder-related donor site morbidity after delayed breast reconstruction with pedicled flaps from the back: An open label randomized controlled clinical trial. J Plast Reconstr Aesthet Surg. 2019

Dec;72(12):1942-9. doi: 10.1016/j.bjps.2019.07.027. PMID: 31515191 .

249. Zhong T, Novak CB, Bagher S, et al. Using propensity score analysis to compare major complications between DIEP and free muscle-sparing TRAM flap breast reconstructions. Plast Reconstr Surg. 2014 Apr;133(4):774-82. doi: 10.1097/prs.0000000000000024. PMID: 24675183.

250. Zoghbi Y, Gerth DJ, Tashiro J, et al. Deep Inferior Epigastric Perforator Versus Free Transverse Rectus Abdominis Myocutaneous Flap: Complications and Resource Utilization. Ann Plast Surg. 2017 May;78(5):516-20. doi: 10.1097/sap.0000000000000936. PMID: 28052051.

251. Baumann DP, Lin HY, Chevray PM. Perforator number predicts fat necrosis in a prospective analysis of breast reconstruction with free TRAM, DIEP, and SIEA flaps. Plast Reconstr Surg. 2010 May;125(5):1335-41. doi: 10.1097/PRS.0b013e3181d4fb4a. PMID: 20440154.

252. Israeli R, Funk S, Reaven NL. Comparative analysis of 18-month outcomes and costs of breast reconstruction flap procedures. Plast Reconstr Surg. 2014 Mar;133(3):471-9. doi: 10.1097/prs.0000000000000064. PMID: 24572840. 
253. Macadam SA, Zhong T, Weichman K, et al. Quality of Life and Patient-Reported Outcomes in Breast Cancer Survivors: A Multicenter Comparison of Four Abdominally Based Autologous Reconstruction Methods. Plast Reconstr Surg. 2016 Mar;137(3):758-71. doi: 10.1097/01.prs.0000479932.11170.8f. PMID: 26910656.

254. U.S. Food and Drug Administration. Acellular Dermal Matrix (ADM) Products Used in Implant-Based Breast Reconstruction Differ in Complication Rates: FDA Safety Communication.; 2021. https://www.fda.gov/medical-devices/safetycommunications/acellular-dermal-matrixadm-products-used-implant-based-breastreconstruction-differ-complication. Accessed on May 18, 2021.

255. Keirns CC, Goold SD. Patient-centered care and preference-sensitive decision making. Jama. 2009 Oct 28;302(16):1805-6. doi: 10.1001/jama.2009.1550. PMID: 19861674.

256. Lee CN, Ubel PA, Deal AM, et al. How Informed Is the Decision About Breast Reconstruction After Mastectomy?: A Prospective, Cross-sectional Study. Ann Surg. 2016 Dec;264(6):1103-9. doi: 10.1097/sla.0000000000001561. PMID: 26727092 .

257. Lee CN, Belkora J, Chang Y, et al. Are patients making high-quality decisions about breast reconstruction after mastectomy? [outcomes article]. Plast Reconstr Surg. 2011

Jan;127(1):18-26. doi: 10.1097/PRS.0b013e3181f958de. PMID: 21200195.

258. Politi MC, Lee CN, Philpott-Streiff SE, et al. A Randomized Controlled Trial Evaluating the BREASTChoice Tool for Personalized Decision Support About Breast Reconstruction After Mastectomy. Ann Surg. 2020 Feb;271(2):230-7. doi: 10.1097/sla.0000000000003444. PMID: 31305282 .
259. Sun CS, Reece GP, Crosby MA, et al. Plastic Surgeon Expertise in Predicting Breast Reconstruction Outcomes for Patient Decision Analysis. Plast Reconstr Surg Glob Open. 2013 Nov 1;1(6):e78. doi: 10.1097/gox.0000000000000010. PMID: 24910814.

260. Hasak JM, Myckatyn TM, Grabinski VF, et al. Stakeholders' Perspectives on Postmastectomy Breast Reconstruction: Recognizing Ways to Improve Shared Decision Making. Plast Reconstr Surg Glob Open. 2017 Nov;5(11):e1569. doi: 10.1097/gox.0000000000001569. PMID: 29263969.

261. Alaofi RK, Nassif MO, Al-Hajeili MR. Prophylactic mastectomy for the prevention of breast cancer: Review of the literature. Avicenna J Med. 2018 Jul-Sep;8(3):67-77. doi: 10.4103/ajm.AJM_21_18. PMID: 30090744.

262. Euhus DM, Diaz J. Breast cancer prevention. Breast J. 2015 Jan-Feb;21(1):76-81. doi: 10.1111/tbj.12352. PMID: 25413630.

263. Morrow M, Mehrara B. Prophylactic mastectomy and the timing of breast reconstruction. Br J Surg. 2009 Jan;96(1):12. doi: 10.1002/bjs.6463. PMID: 19109821.

264. Tuttle TM, Habermann EB, Grund EH, et al. Increasing use of contralateral prophylactic mastectomy for breast cancer patients: a trend toward more aggressive surgical treatment. J Clin Oncol. 2007 Nov 20;25(33):5203-9. doi: 10.1200/jco.2007.12.3141. PMID: 17954711.

265. Wong SM, Freedman RA, Sagara Y, et al. Growing Use of Contralateral Prophylactic Mastectomy Despite no Improvement in Long-term Survival for Invasive Breast Cancer. Ann Surg. 2017 Mar;265(3):581-9. doi: 10.1097/sla.0000000000001698. PMID: 28169929. 


\section{Appendix Contents}

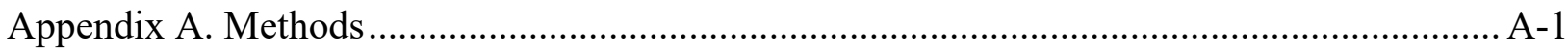

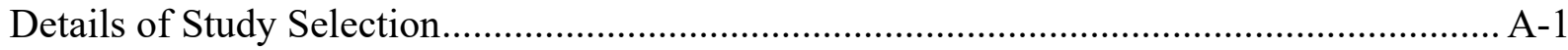

Search Strategy (Details) ........................................................................................... A-

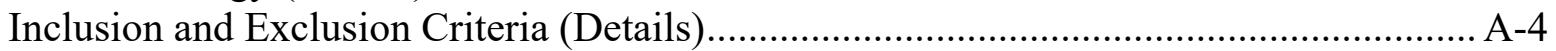

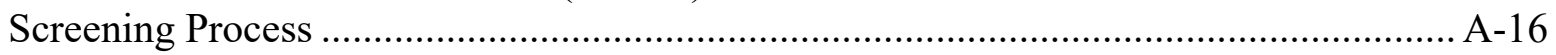

Data Extraction and Data Management (Details) ……………..................................... A-17

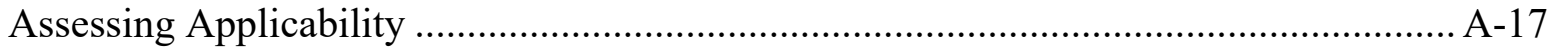

Addressing the Contextual Questions ........................................................................ A-17

Peer Review and Public Commentary .......................................................................... A-17

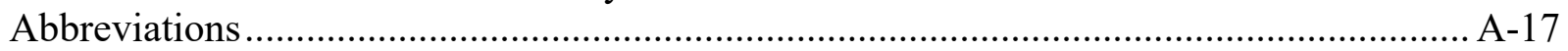

Appendix B. List of Excluded Studies.............................................................................. B-1

Appendix C. Results: Design, Arm, and Sample Details …………………………………..... C-1

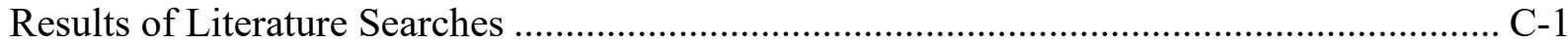

Description of Included Studies .................................................................................. C-1

Overall Summary of Study Characteristics................................................................ C-1

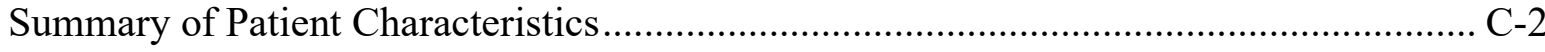

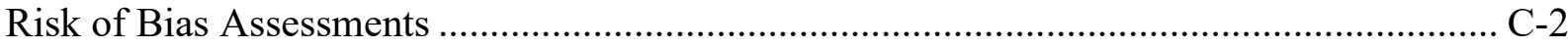

Appendix D. Results: Risk of Bias .................................................................................. D-1

Appendix E. Results: Summary Tables for Outcomes …………………………………….....

Appendix F. Results: Full Evidence Tables for Outcomes ........................................................F-1

Appendix G. Results: Evidence Profiles.............................................................................. G-1

Appendix H. Appendix References ............................................................................... H-1

\section{Tables}

Table B-1. Excluded primary studies with reasons for exclusion ................................................. 1

Table C-1. Key Question 1: Implant-based versus autologous reconstruction - comparative studies, summary of design, arm, and sample details ................................................................ 1

Table C-2. Key Question 1: Implant-based versus autologous reconstruction - single group studies, summary of design, arm, and sample details ............................................................ 34 Table C-3. Key Question 2: Timing of chemotherapy and radiation therapy relative to IBR or AR - summary of design, arm, and sample details

Table C-4. Key Question 3: Comparison of materials for IBR - summary of design, arm, and sample details.

Table C-5. Key Question 4: Comparison of anatomic planes for IBR - summary of design, arm, and sample details

Table C-6. Key Question 5: Use versus nonuse of human ADM for IBR - summary of design, arm, and sample details.

Table C-7. Key Question 6: Comparison of flap types for autologous reconstruction - summary

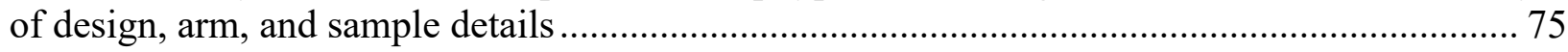

Table D-1. Risk of bias assessment for all Key Questions - randomized controlled trials ............ 1 Table D-2. Risk of bias assessment for all Key Questions - nonrandomized comparative studies, confounding and selection bias....

Table D-3. Risk of bias assessment for all Key Questions - nonrandomized comparative studies, assessment of remaining biases, quality, and overall risk of bias............................................... 6 Table D-4. Risk of bias and quality assessment for all Key Questions - single group studies .... 12 
Table E-1.1. Summary Table - Key Question 1: IBR versus AR - continuous outcomes (general quality of life, psychosocial well-being, sexual well-being, patient satisfaction with breasts, and patient satisfaction with outcome)

Table E-1.2. Summary Table - Key Question 1: IBR versus AR - continuous outcomes (physical well-being).....

Table E-1.3. Summary Table - Key Question 1: IBR versus AR - NRCSs, categorical outcomes (physical well-being and recurrence of breast cancer) 7 Table E-1.4. Summary Table - Key Question 1: IBR versus AR - NRCSs, categorical outcomes (patient satisfaction with breasts and with outcome). 7 Table E-1.5. Summary Table - Key Question 1: IBR versus AR - categorical outcomes, mortality, unplanned repeat hospitalizations, necrosis, thromboembolic events, wound dehiscence, delayed healing, seroma, and hematoma. 8 Table E-1.6. Summary Table - Key Question 1: IBR versus AR - NRCSs, categorical outcomes (unplanned repeat surgeries for revision, unplanned repeat surgeries for complications, pain, infections not explicitly implant related, and reconstructive failure) ....................................... 10 Table E-1.7. Summary Table - Key Question 1: IBR versus AR - NRCSs, continuous outcomes (pain and analgesic use)

Table E-2.1. Summary Table - Key Question 2b: Timing of IBR in relation to radiation therapy - NRCSs, continuous outcomes (various) .......................................................................... 17 Table E-2.2. Summary Table - Key Question 2b: Timing of IBR in relation to radiation therapy - NRCSs, categorical outcomes (various)

Table E-3.1. Summary Table - Key Question 3: Comparisons of implant materials for IBR NRCSs, continuous outcomes (general quality of life, physical well-being, psychosocial wellbeing, sexual well-being, satisfaction with outcome, and satisfaction with breasts).................. 19 Table E-3.2. Summary Table - Key Question 3: Comparisons of implant materials for IBR NRCSs, categorical outcomes (mortality, implant failure/loss, and capsular contracture) ......... 20 Table E-4.1. Summary Table - Key Question 4: Comparisons of anatomic planes of implant placement for IBR - continuous outcomes (various) .

Table E-4.2. Summary Table - Key Question 4: Comparisons of anatomic planes of implant placement for IBR - categorical outcomes (various)

Table E-5.1. Summary Table - Key Question 5: Use versus nonuse of human ADMs during IBR - continuous outcomes (physical well-being, psychosocial well-being, sexual well-being, and satisfaction with breasts).....

Table E-5.2. Summary Table - Key Question 5: Use versus nonuse of human ADMs during IBR

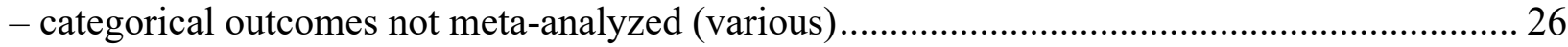
Table E-5.3. Summary Table - Key Question 5: Use versus nonuse of human ADMs during IBR - continuous outcomes (pain and analgesic use) .......................................................... 28 Table E-5.4. Summary Table - Key Question 5: Use versus nonuse of human ADMs during IBR - categorical outcomes (composite or unspecified harms) ................................................... 29 Table E-6.1. Summary Table - Key Question 6: Comparisons of flap types for AR - continuous outcomes (physical well-being)....

Table E-6.2. Summary Table - Key Question 6: Comparisons of flap types for AR - NRCS, continuous outcomes (psychosocial and sexual well-being) .............................................. 33 Table E-6.3. Summary Table - Key Question 6: Comparisons of flap types for AR - continuous outcomes (patient satisfaction with breasts and duration of initial hospitalization). 
Table E-6.4. Summary Table - Key Question 6: Comparisons of flap types for AR - categorical outcomes (patient satisfaction with breasts, patient satisfaction with outcome, recurrence of breast cancer, and duration of initial hospitalization) .......................................................... 36 Table E-6.5. Summary Table - Key Question 6: Comparisons of flap types for AR - categorical

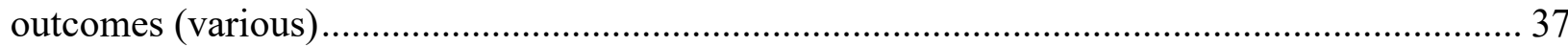
Table E-6.6. Summary Table - Key Question 6: Comparisons of flap types for AR - categorical outcomes (composite or unspecified harms)....................................................................... 40 Table F-1.1. Full Evidence Table - Key Question 1: IBR versus AR - continuous outcomes (general quality of life) .......................................................................................... 1

Table F-1.2. Full Evidence Table - Key Question 1: IBR versus AR - continuous outcomes

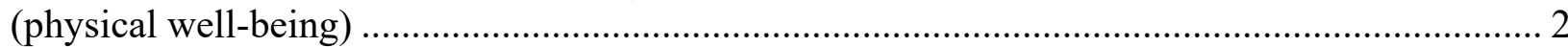

Table F-1.3. Full Evidence Table - Key Question 1: IBR versus AR - continuous outcomes (psychosocial well-being)

Table F-1.4. Full Evidence Table - Key Question 1: IBR versus AR - continuous outcomes (sexual well-being). . .11

Table F-1.5. Full Evidence Table - Key Question 1: IBR versus AR - continuous outcomes (patient satisfaction with breast) .................................................................................. 18

Table F-1.6. Full Evidence Table - Key Question 1: IBR versus AR - categorical outcomes (physical well-being) 22

Table F-1.7. Full Evidence Table - Key Question 1: IBR versus AR - categorical outcomes (patient satisfaction with breast).

Table F-1.8. Full Evidence Table - Key Question 1: IBR versus AR - continuous outcomes (patient satisfaction with outcome).

Table F-1.9. Full Evidence Table - Key Question 1: IBR versus AR - categorical outcomes (patient satisfaction with outcome).

Table F-1.10. Full Evidence Table - Key Question 1: IBR versus AR - categorical outcomes (recurrence of breast cancer)...

Table F-1.11. Full Evidence Table - Key Question 1: IBR versus AR - continuous outcomes (harms, pain, and analgesic use) .

Table F-1.12. Full Evidence Table - Key Question 1: IBR versus AR - categorical outcomes, mortality.

Table F-1.13. Full Evidence Table - Key Question 1: IBR versus AR - categorical outcomes (harms - unplanned repeat hospitalizations)......

Table F-1.14. Full Evidence Table - Key Question 1: IBR versus AR - categorical outcomes (harms - unplanned repeat surgery for revision of reconstruction).

Table F-1.15. Full Evidence Table - Key Question 1: IBR versus AR - categorical outcomes (harms - unplanned repeat surgery for complications).....

Table F-1.16. Full Evidence Table - Key Question 1: IBR versus AR - categorical outcomes (harms - pain, including chronic pain)

Table F-1.17. Full Evidence Table - Key Question 1: IBR versus AR - categorical outcomes (harms - necrosis)

Table F-1.18. Full Evidence Table - Key Question 1: IBR versus AR - categorical outcomes (harms - thromboembolic events)

Table F-1.19. Full Evidence Table - Key Question 1: IBR versus AR - categorical outcomes (harms - infections not explicitly implant related)..... 
Table F-1.20. Full Evidence Table - Key Question 1: IBR versus AR - categorical outcomes (harms - wound dehiscence)...

Table F-1.21. Full Evidence Table - Key Question 1: IBR versus AR - categorical outcomes (harms - delayed healing)....

Table F-1.22. Full Evidence Table - Key Question 1: IBR versus AR - categorical outcomes (harms - seroma).

Table F-1.23. Full Evidence Table - Key Question 1: IBR versus AR - categorical outcomes (harms - scarring)

Table F-1.24. Full Evidence Table - Key Question 1: IBR versus AR - categorical outcomes (harms - reconstructive failure)

Table F-1.25. Full Evidence Table - Key Question 1: IBR versus AR - categorical outcomes (harms - hematoma or hemorrhage)

Table F-1.26. Full Evidence Table - Key Question 1: IBR versus AR - categorical outcomes (composite/unspecified harms).

Table F-2.1. Full Evidence Table - Key Question 2: Timing of radiation therapy relative to IBR

- continuous outcomes (physical well-being)

Table F-2.2. Full Evidence Table - Key Question 2: Timing of radiation therapy relative to IBR

- continuous outcomes (psychosocial well-being)

Table F-2.3. Full Evidence Table - Key Question 2: Timing of radiation therapy relative to IBR

- continuous outcomes (sexual well-being)....

Table F-2.4. Full Evidence Table - Key Question 2: Timing of radiation therapy relative to IBR

- continuous outcomes (patient satisfaction with breast)

Table F-2.5. Full Evidence Table - Key Question 2: Timing of radiation therapy relative to IBR

- continuous outcomes (patient satisfaction with outcome)

Table F-2.6. Full Evidence Table - Key Question 2: Timing of radiation therapy relative to IBR

- continuous outcomes (pain).

Table F-2.7. Full Evidence Table - Key Question 2: Timing of radiation therapy relative to IBR

- categorical outcomes (unplanned repeat surgeries for revision of reconstruction).....

Table F-2.8. Full Evidence Table - Key Question 2: Timing of radiation therapy relative to IBR

- categorical outcomes (necrosis).

Table F-2.9. Full Evidence Table - Key Question 2: Timing of radiation therapy relative to IBR

- categorical outcomes (infections not explicitly implant related)....

Table F-2.10. Full Evidence Table - Key Question 2: Timing of radiation therapy relative to IBR - categorical outcomes (wound dehiscence).

Table F-2.11. Full Evidence Table - Key Question 2: Timing of radiation therapy relative to IBR - categorical outcomes (seroma) 72

Table F-2.12. Full Evidence Table - Key Question 2: Timing of radiation therapy relative to IBR - categorical outcomes (implant failure/loss) 72

Table F-2.13. Full Evidence Table - Key Question 2: Timing of radiation therapy relative to IBR - categorical outcomes (capsular contracture). 72

Table F-2.14. Full Evidence Table - Key Question 2: Timing of radiation therapy relative to IBR - categorical outcomes (hematoma)

Table F-2.15. Full Evidence Table - Key Question 2: Timing of radiation therapy relative to IBR - categorical outcomes (combined/unspecified harms). 73

Table F-3.1. Full Evidence Table - Key Question 3: Comparison of materials for IBR continuous outcomes (general quality of life) 
Table F-3.2. Full Evidence Table - Key Question 3: Comparison of materials for IBR continuous outcomes (physical well-being)

Table F-3.3. Full Evidence Table - Key Question 3: Comparison of materials for IBR continuous outcomes (psychosocial well-being) ............................................................... 74

Table F-3.4. Full Evidence Table - Key Question 3: Comparison of materials for IBR continuous outcomes (sexual well-being).....

Table F-3.5. Full Evidence Table - Key Question 3: Comparison of materials for IBR continuous outcomes (patient satisfaction with breast) ..... 75

Table F-3.6. Full Evidence Table - Key Question 3: Comparison of materials for IBR continuous outcomes (patient satisfaction with outcome) .................................................... 75

Table F-3.7. Full Evidence Table - Key Question 3: Comparison of materials for IBR -

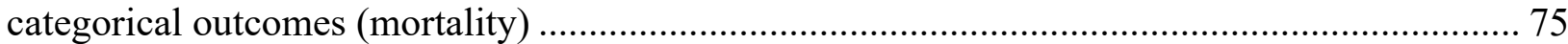

Table F-3.8. Full Evidence Table - Key Question 3: Comparison of materials for IBR categorical outcomes (capsular contracture) .................................................................. 76

Table F-3.9. Full Evidence Table - Key Question 3: Comparison of materials for IBR categorical outcomes (implant failure/loss). 76

Table F-4.1. Full Evidence Table - Key Question 4: Comparison of anatomic planes for IBR continuous outcomes (physical well-being). 76 Table F-4.2. Full Evidence Table - Key Question 4: Comparison of anatomic planes for IBR continuous outcomes (psychosocial well-being) .... 76 Table F-4.3. Full Evidence Table - Key Question 4: Comparison of anatomic planes for IBR continuous outcomes (patient satisfaction with breast) ... 77 Table F-4.4. Full Evidence Table - Key Question 4: Comparison of anatomic planes for IBR continuous outcomes (pain, including chronic pain and analgesic use) .................................. 77 Table F-4.5. Full Evidence Table - Key Question 4: Comparison of anatomic planes for IBR categorical outcomes (analgesic use). 78

Table F-4.6. Key Question 4: Comparison of anatomic planes for IBR - categorical outcomes (unplanned repeat surgeries for revision) ..... 78 Table F-4.7. Full Evidence Table - Key Question 4: Comparison of anatomic planes for IBR -

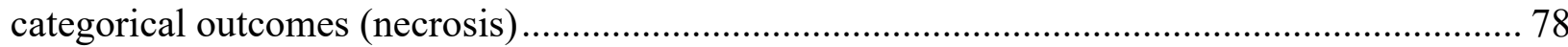
Table F-4.8. Full Evidence Table - Key Question 4: Comparison of anatomic planes for IBR categorical outcomes (infections) 79 Table F-4.9. Full Evidence Table - Key Question 4: Comparison of anatomic planes for IBR categorical outcomes (seroma) 79 Table F-4.10. Full Evidence Table - Key Question 4: Comparison of anatomic planes for IBR categorical outcomes (need for explant surgery) ............................................................. 79 Table F-4.11. Full Evidence Table - Key Question 4: Comparison of anatomic planes for IBR categorical outcomes (capsular contracture) ................................................................... 80 Table F-4.12. Full Evidence Table - Key Question 4: Comparison of anatomic planes for IBR -

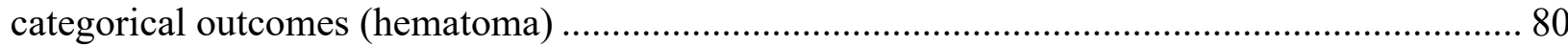
Table F-4.13. Full Evidence Table - Key Question 4: Comparison of anatomic planes for IBR categorical outcomes (combined/unspecified harms) 80 Table F-5.1. Full Evidence Table - Key Question 5: Use versus nonuse of human ADM during IBR - continuous outcomes (physical well-being). 81 Table F-5.2. Full Evidence Table - Key Question 5: Use versus nonuse of human ADM during IBR - continuous outcomes (psychosocial well-being). 
Table F-5.3. Full Evidence Table - Key Question 5: Use versus nonuse of human ADM during IBR - continuous outcomes (sexual well-being).

Table F-5.4. Full Evidence Table - Key Question 5: Use versus nonuse of human ADM during IBR - continuous outcomes (patient satisfaction with breast)............................................. 82 Table F-5.5. Full Evidence Table - Key Question 5: Use versus nonuse of human ADM during IBR - continuous outcomes (analgesic use)

Table F-5.6. Full Evidence Table - Key Question 5: Use versus nonuse of human ADM during IBR - categorical outcomes (analgesic use)

Table F-5.7. Full Evidence Table - Key Question 5: Use versus nonuse of human ADM during IBR - categorical outcomes (mortality).

Table F-5.8. Full Evidence Table - Key Question 5: Use versus nonuse of human ADM during IBR - categorical outcomes (unplanned repeat surgeries) ................................................ 83 Table F-5.9. Full Evidence Table - Key Question 5: Use versus nonuse of human ADM during IBR - continuous outcomes (pain, including chronic pain) 84 Table F-5.10. Full Evidence Table - Key Question 5: Use versus nonuse of human ADM during IBR - categorical outcomes (necrosis) 84

Table F-5.11. Full Evidence Table - Key Question 5: Use versus nonuse of human ADM during IBR - categorical outcomes (thromboembolic events). 85 Table F-5.12. Full Evidence Table - Key Question 5: Use versus nonuse of human ADM during IBR - categorical outcomes (wound dehiscence)..

Table F-5.13. Full Evidence Table - Key Question 5: Use versus nonuse of human ADM during IBR - categorical outcomes (delayed healing). 85 Table F-5.14. Full Evidence Table - Key Question 5: Use versus nonuse of human ADM during IBR - categorical outcomes (seroma). 86 Table F-5.15. Full Evidence Table - Key Question 5: Use versus nonuse of human ADM during IBR - categorical outcomes (infections not explicitly implant related) .................................. 86 Table F-5.16. Full Evidence Table - Key Question 5: Use versus nonuse of human ADM during IBR - categorical outcomes (implant failure/loss or need for explant surgery) ........................ 88 Table F-5.17. Full Evidence Table - Key Question 5: Use versus nonuse of human ADM during IBR - categorical outcomes (implant complications except for implant failure) ...................... 88 Table F-5.18. Full Evidence Table - Key Question 5: Use versus nonuse of human ADM during IBR - categorical outcomes (capsular contracture).

Table F-5.19. Full Evidence Table - Key Question 5: Use versus nonuse of human ADM during IBR - categorical outcomes (hematoma).

Table F-5.20. Full Evidence Table - Key Question 5: Use versus nonuse of human ADM during IBR - categorical outcomes (combined/unspecified harms) ............................................... 90 Table F-5.21: Full Evidence Table - Key Question 5: Use versus nonuse of human ADM during IBR - categorical outcomes (harms to the inframammary fold) 91 Table F-6.1. Full Evidence Table - Key Question 6: Comparison of flap types for AR continuous outcomes (physical well-being).

Table F-6.2. Full Evidence Table - Key Question 6: Comparison of flap types for AR continuous outcomes (psychosocial well-being) .... 94 Table F-6.3. Full Evidence Table - Key Question 6: Comparison of flap types for AR continuous outcomes (sexual well-being)..... 95 Table F-6.4. Full Evidence Table - Key Question 6: Comparison of flap types for AR continuous outcomes (patient satisfaction with breast)..... 
Table F-6.5. Full Evidence Table - Key Question 6: Comparison of flap types for AR categorical outcomes (patient satisfaction with breast)

Table F-6.6. Full Evidence Table - Key Question 6: Comparison of flap types for AR continuous outcomes (patient satisfaction with outcome) 98

Table F-6.7. Full Evidence Table - Key Question 1: IBR versus AR - categorical outcomes (patient satisfaction with outcome). 100

Table F-6.8. Full Evidence Table - Key Question 6: Comparison of flap types for AR categorical outcomes (recurrence of breast cancer).

Table F-6.9. Full Evidence Table - Key Question 6: Comparison of flap types for AR continuous outcomes (duration of initial hospitalization) 100 Table F-6.10. Full Evidence Table - Key Question 6: Comparison of flap types for AR categorical outcomes (duration of initial hospitalization)

Table F-6.11. Full Evidence Table - Question 6: Comparison of flap types for AR - categorical outcomes (mortality)

Table F-6.12. Full Evidence Table - Question 6: Comparison of flap types for AR - categorical outcomes (unplanned repeat surgeries for revision of reconstruction).

Table F-6.13. Full Evidence Table - Key Question 6: IBR versus AR - comparison of flap types

for AR - continuous outcomes (pain, including chronic pain). 102 Table F-6.14. Full Evidence Table - Key Question 6: IBR versus AR - comparison of flap types for AR - categorical outcomes (pain, including chronic pain). 102 Table F-6.15. Full Evidence Table - Key Question 6: Comparison of flap types for AR categorical outcomes (necrosis). 102

Table F-6.16. Full Evidence Table - Key Question 6: Comparison of flap types for AR categorical outcomes (thromboembolic events) 105 Table F-6.17. Full Evidence Table - Key Question 6: Comparison of flap types for AR categorical outcomes (infections not explicitly implant related) 106 Table F-6.18. Full Evidence Table - Key Question 6: Comparison of flap types for AR categorical outcomes (wound dehiscence). 108

Table F-6.19. Full Evidence Table - Key Question 6: Comparison of flap types for AR categorical outcomes (delayed healing).

Table F-6.20. Full Evidence Table - Key Question 6: Comparison of flap types for AR categorical outcomes (seroma) .....

Table F-6.21. Full Evidence Table - Key Question 6: Comparison of flap types for AR categorical outcomes (scarring) 110

Table F-6.22. Full Evidence Table - Key Question 6: Comparison of flap types for AR categorical outcomes (harms to area of flap harvest)

Table F-6.23. Full Evidence Table - Key Question 6: Comparison of flap types for AR categorical outcomes (flap failure/loss)....

Table F-6.24. Full Evidence Table - Key Question 6: Comparison of flap types for AR categorical outcomes (hematoma/hemorrhage)

Table F-6.25. Full Evidence Table - Key Question 6: Comparison of flap types for AR categorical outcomes (composite/unspecified harms) 114

Table G-1. Key Question 1: IBR versus AR - full evidence profile . 1 Table G-2. Key Question 2: Timing of chemotherapy and radiation therapy relative to IBR and AR - full evidence profile..................................................................................... 3

Table G-3. Key Question 3: Comparison of materials for IBR - full evidence profile................ 5 
Table G-4. Key Question 4: Comparison of anatomic planes of implant placement for IBR - full

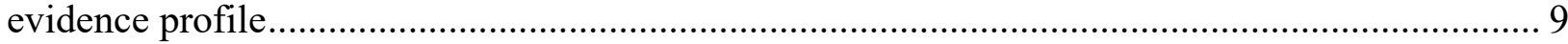

Table G-5. Key Question 5: Use versus nonuse of human ADMs during IBR - full evidence profile.................................................................................................................. 13

Table G-6. Key Question 6: Comparison of flap types for AR - full evidence profile............... 15

\section{Figures}

Figure C-1. Flow diagram for studies ........................................................................ C-1 


\section{Appendix A. Methods}

\section{Details of Study Selection}

\section{Search Strategy (Details)}

We searched for studies for all Key Questions in MEDLINE (via PubMed), Embase, The Cochrane Central Register of Clinical Trials, and CINAHL. Duplicate citations were removed prior to screening. We did not employ any date or language restrictions to the search but included filters to remove nonhuman studies and articles that are not primary studies. We included MeSH or Emtree terms, along with free-text words, related to breast, cancer, mastectomy, implants/implantation, and autologous reconstruction. The searches were independently peer reviewed. The exact search terms used for identifying studies in each database are listed below. To identify additional eligible studies, we also reviewed the reference lists of relevant existing systematic reviews (SRs).

We also ran a search of the ClinicalTrials.gov registry for ongoing studies, unpublished study protocols, and unpublished study results.

\section{Medline (via PubMed) \\ Last run on March 23, 2021 \\ Search 1}

("Mastectomy"[Mesh] OR Mastectomy[Title/Abstract] OR Mammectomies[Title/Abstract] OR Mastectomies[Title/Abstract] OR Mammectomy[Title/Abstract])

AND

(“Mammaplasty”[MeSH Terms] OR “Breast implants”[MeSH Terms] OR "Breast Implantation"[Mesh] OR mammaplasty OR mammoplasty OR Mammaplasties OR Mammoplasties OR ((breast* OR mammar*) AND (implant OR implants OR Reconstruction* OR prosthe* OR "Prostheses and Implants"[MeSH Terms] OR "Transplantation, Autologous”[Mesh]) OR “Autografts"[Mesh] OR Autotransplantation OR Autotransplantations OR Autografting OR Autograftings OR Autologous OR Autograft OR Autotransplants OR Autotransplant OR "Silicones"[Mesh] OR "Saline Solution”[Mesh] OR "Prosthesis Design"[Mesh] OR Silicone OR silicones OR saline OR "gummy bear" OR nanomaterials OR ((“Acellular Dermis"[Mesh] OR “Tissue Expansion Devices”[Mesh] OR acellular OR dermal OR peritoneal) AND (matrices OR matrix)) OR "Surgical Flaps”[Mesh] OR “Adipose Tissue/transplantation"[Mesh] OR “Tissue Transplantation”[Mesh] OR ((flap OR flaps) AND (surgical OR surgery))))

Search 2 to get AE studies not captured by search 1 . Searches 1 and 2 were subsequently to identify unique records.

("Mammaplasty"[MeSH Terms] OR "Breast implants"[MeSH Terms] OR "Breast Implantation"[Mesh] OR mammaplasty OR mammoplasty OR Mammaplasties OR 
Mammoplasties OR ((breast* OR mammar*) AND (implant OR implants OR Reconstruction* OR prosthe* OR "Prostheses and Implants"[MeSH Terms])))

\title{
AND
}

(“Transplantation, Autologous"[Mesh] OR “Autografts"[Mesh] OR Autotransplantation OR Autotransplantations OR Autografting OR Autograftings OR Autologous OR Autograft OR Autotransplants OR Autotransplant OR "Silicones"[Mesh] OR "Saline Solution"[Mesh] OR "Prosthesis Design"[Mesh] OR Silicone OR silicones OR saline OR "gummy bear" OR nanomaterials OR ((“Acellular Dermis”[Mesh] OR "Tissue Expansion Devices”[Mesh] OR acellular OR dermal OR peritoneal) AND (matrices OR matrix)) OR "Surgical Flaps"[Mesh] OR "Adipose Tissue/transplantation"[Mesh] OR "Tissue Transplantation"[Mesh] OR ((flap OR flaps) AND (surgical OR surgery)))

\begin{abstract}
AND
(safe* or adverse* or undesirable or harm or harms or injurious or risk or risks or reaction* or complication* or poison* OR side effect* or safety or unsafe OR ((adverse or undesirable or harm or harms or toxic or injurious or serious or fatal) AND (effect* or reaction* or event* or outcome* or incident*)) OR death or deaths or fatal or fatality or fatalities OR Rupture)
\end{abstract}

NOT

("address"[pt] or "autobiography"[pt] or "bibliography"[pt] or "biography" [pt] or "case reports" [pt] or "comment" [pt] or "congress"[pt] or "dictionary"[pt] or "directory" [pt] or "festschrift"[pt] or "government publication"[pt] or "historical article" [pt] or "interview"[pt] or "lecture"[pt] or "legal case"[pt] or "legislation" [pt] or "news"[pt] or "newspaper article"[pt] or "patient education handout"[pt] or "periodical index"[pt] or "comment on" or ("Animals"[Mesh] NOT "Humans"[Mesh]))

\section{EMBASE}

Last run on March 23, 2021

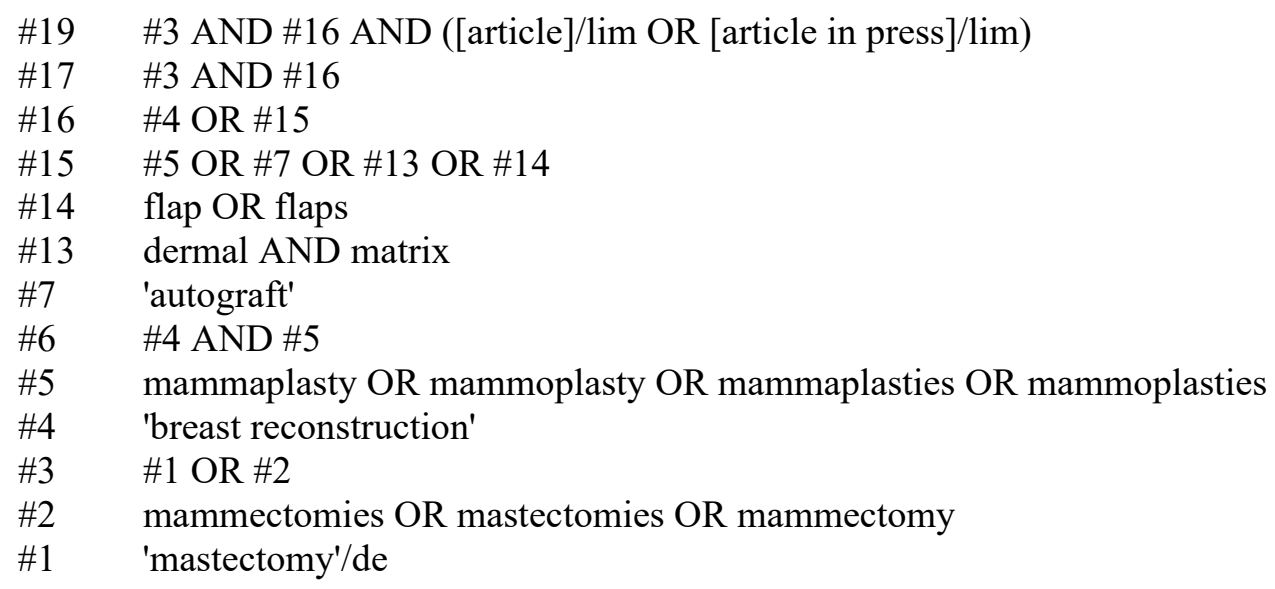




\title{
Cochrane CENTRAL
}

\author{
Last run on March 23, 2021
}

\#1 MeSH descriptor: [Mastectomy] explode all trees

\#2 Mastectomy OR Mammectomies OR Mastectomies OR Mammectomy

\#3 \#1 OR \#2

\#4 MeSH descriptor: [Mammaplasty] explode all trees

\#5 MeSH descriptor: [Breast Implants] explode all trees

\#6 MeSH descriptor: [Breast Implantation] explode all trees

\#7 mammaplasty OR mammoplasty OR Mammaplasties OR Mammoplasties

\#8 \#4 OR \#5 OR \#6 OR \#7

\#9 breast* OR mammar*

\#10 MeSH descriptor: [Prostheses and Implants] explode all trees

\#11 MeSH descriptor: [Transplantation, Autologous] explode all trees

\#12 implant OR implants OR Reconstruction* OR prosthe*

\#13 \#10 OR \#11 OR \#12

\#14 \#9 AND \#13

\#15 \#8 OR \#14

\#16 MeSH descriptor: [Autografts] explode all trees

\#17 MeSH descriptor: [Silicones] explode all trees

\#18 MeSH descriptor: [Saline Solution] explode all trees

\#19 MeSH descriptor: [Prosthesis Design] explode all trees

\#20 Silicone OR silicones OR saline OR "gummy bear" OR nanomaterials OR Autotransplantation OR Autotransplantations OR Autografting OR Autograftings OR Autologous OR Autograft OR Autotransplants OR Autotransplant

\#21 \#15 OR \#16 OR \#17 OR \#18 OR \#19 OR \#20

\#22 MeSH descriptor: [Acellular Dermis] explode all trees

\#23 MeSH descriptor: [Tissue Expansion Devices] explode all trees

\#24 acellular OR dermal OR peritoneal

\#25 \#22 OR \#23 OR \#24

\#26 matrices OR matrix

\#27 \#25 AND \#26

\#28 \#27 OR \#21

\#29 MeSH descriptor: [Surgical Flaps] explode all trees

\#30 MeSH descriptor: [Tissue Transplantation] explode all trees

\#31 ((flap OR flaps) AND (surgical OR surgery))

\#32 \#28 OR \#29 OR \#30 OR \#31

\#33 \#32 AND \#3

\section{CINAHL}

Last run on March 23, 2021

(Mastectomy OR Mammectomies OR Mastectomies OR Mammectomy)

\section{AND}

(mammaplasty OR mammoplasty OR Mammaplasties OR Mammoplasties OR ((breast* OR mammar*) AND (implant OR implants OR Reconstruction* OR prosthe*)) OR Silicone OR silicones OR saline OR "gummy bear" OR nanomaterials OR Autotransplantation OR 
Autotransplantations OR Autografting OR Autograftings OR Autologous OR Autograft OR Autotransplants OR Autotransplant OR ((acellular OR dermal OR peritoneal) AND (matrices OR matrix)) OR ((flap OR flaps) AND (surgical OR surgery)))

We also asked all members of the Technical Expert Panel (TEP) and the American Society for Plastic Surgeons (ASPS) to review our list of included studies and suggest any additional studies that might be relevant, which we checked against our list of citations and, where applicable, added to our list. Non-English language articles were screened by readers of the relevant languages or after translation via Google Translate (https://translate.google.com/), where possible. Additional articles suggested to us in any language from any source, during peer and public review, will be screened applying identical eligibility criteria.

\section{Inclusion and Exclusion Criteria (Details)}

\section{Key Question 1 (IBR Versus AR)}

\section{Population}

- Adult ( $\geq 18$ years old) women who are undergoing (or have undergone) mastectomy for any type of breast cancer (or carcinoma in situ) and have decided to undergo breast reconstruction

- Either therapeutic or prophylactic mastectomy

- Exclude: Studies where $\geq 10 \%$ of women underwent breast reconstruction (combined across reasons):

○ for solely cosmetic purposes (i.e., augmentation)

- for revision reconstruction (i.e., after a previous reconstruction for breast cancer)

\section{Interventions}

- IBR

- Either single- or multi-stage

- Any type of implant material, either smooth or textured, silicone or saline

- Any anatomic plane of implant placement

- With or without use of human ADM

- With or without mastectomy and reconstruction of the contralateral breast (i.e., unilateral or bilateral)

- With or without symmetry procedure (e.g., mastopexy) in the contralateral breast

\section{Comparators}

- AR using any flap (either free flap or pedicled), for example:

- Deep inferior epigastric perforator (DIEP)

- Latissimus dorsi (LD)

- Transverse rectus abdominis myocutaneous (TRAM)

- Superficial inferior epigastric artery perforator (SIEA)

- Gluteal artery perforator (GAP)

- Transverse musculocutaneous gracilis (TMG)

- Transverse upper gracilis (TUG) 
- Profundal artery perforator (PAP)

- Combination of IBR and AR

- Exclude: Non-autologous flap transplants (i.e., cadaveric or xenotransplant)

- Exclude: Exclusive lipofilling/autologous fat reconstruction

\section{Outcomes (* denotes important outcomes that were used when developing Strength of Evidence tables)}

- Clinical outcomes

- General quality of life*

○ Physical well-being (e.g., pain, discomfort)*

○ Psychosocial well-being (e.g., self-esteem, emotionality, normality)*

○ Sexual well-being*

- Patient satisfaction with breast*

- Patient satisfaction with outcome (e.g., satisfaction with care)*

- Planned surgeries for reconstruction

- Recurrence of breast cancer

○ Mortality*

- Surgical complications

○ Unplanned repeat hospitalization*

- Duration of unplanned repeat hospitalization*

- Unplanned repeat surgeries - for revision of reconstruction (e.g., for asymmetry)*

- Unplanned repeat surgeries - for complications (e.g., for infection, bleeding)*

- Pain, including chronic pain*

○ Analgesic (e.g., opioid) use*

- Necrosis, such as of the nipple or of the flap*

- Animation deformity*

- Complications that lead to delays in other cancer-related treatments (e.g., chemotherapy, radiation therapy)*

- Thromboembolic events*

- Infections not explicitly implant-related*

○ Wound dehiscence

- Delayed healing

- Seroma*

- Chronic conditions (e.g., rheumatologic diseases)

- Touch sensitivity

- Scarring

- Reconstructive failure/loss

\section{Potential Effect Modifiers}

- Age

- Stage of breast cancer

- First occurrence versus recurrent breast cancer

- Immediate versus delayed reconstruction

- Single-stage (direct to reconstruction) versus multi-stage (with tissue expander) reconstruction 
- Unilateral versus bilateral reconstruction

- Radiation therapy versus no radiation therapy

- Chemotherapy versus no chemotherapy

\section{Timing}

- Any

\section{Setting}

- Any, including single- and multicenter

\section{Design}

- Randomized controlled trials (RCTs), $\mathrm{N} \geq 10$ per group

- Nonrandomized comparative studies (NRCSs), $\mathrm{N} \geq 30$ per group, provided adjusted analyses

- Case-control studies, $\mathrm{N} \geq 100$ per group

- Single group studies, $\mathrm{N} \geq 500$

- Studies may be prospective or retrospective

- Exclude: case reports and series of individually reported case reports

\section{Key Question 2 (Optimal Time for IBR or AR)}

\section{Population(s)}

- Adult ( $\geq 18$ years old) women who are undergoing IBR or AR after a mastectomy for breast cancer (or carcinoma in situ) that requires either chemotherapy or radiation therapy

- Therapeutic mastectomy

- Exclude: Studies where $\geq 10 \%$ of women underwent breast reconstruction (combined across reasons):

$\bigcirc$ for solely cosmetic purposes (i.e., augmentation)

○ for solely prophylactic purposes (i.e., without diagnosed breast cancer)

- for revision reconstruction (i.e., after a previous reconstruction for breast cancer)

\section{Interventions}

- IBR or AR before chemotherapy

- IBR (whether tissue expander or implant itself) or AR before radiation therapy

$\circ$ Either single- or multistage

- With or without mastectomy and reconstruction of the contralateral breast (i.e., unilateral or bilateral)

- With or without symmetry procedure (e.g., mastopexy) in the contralateral breast

- With or without use of human ADM

- For IBR - Any type of implant material, either smooth or textured

- For IBR - Any anatomic plane of implant placement

○ For AR - Any flap type

\section{Comparators}

- IBR or AR after chemotherapy (when used for the current treatment of breast cancer) 
- IBR (whether tissue expander or implant itself) or AR after radiation therapy (when used for the current treatment of breast cancer)

\section{Outcomes (* denotes important outcomes that were used when developing Strength of Evidence tables)}

- Clinical outcomes

- General quality of life*

○ Physical well-being (e.g., pain, discomfort)*

○ Psychosocial well-being (e.g., self-esteem, emotionality, normality)*

○ Sexual well-being*

- Patient satisfaction with breast*

- Patient satisfaction with outcome (e.g., satisfaction with care)*

- Planned surgeries for reconstruction

- Recurrence of breast cancer

- Mortality*

- Surgical complications

○ Unplanned repeat hospitalization*

- Duration of unplanned repeat hospitalization*

- Unplanned repeat surgeries - for revision of reconstruction (e.g., for asymmetry)*

- Unplanned repeat surgeries - for complications (e.g., for infection, bleeding)*

- Pain, including chronic pain*

- Analgesic (e.g., opioid) use*

- Necrosis, such as of the nipple or of the flap*

- Animation deformity*

- Implant-related infections (for IBR)*

- Implant rupture, including asymptomatic rupture (for IBR)*

○ Implant deflation (for IBR)*

- Implant malposition (for IBR)*

- Implant failure/loss or need for explant surgery (for IBR)*

- Capsular contracture (for IBR)

- Complications that cause delays in other cancer-related treatments (e.g., chemotherapy, radiation therapy)*

- Thromboembolic events*

- Infections

- Wound dehiscence

○ Delayed healing

- Seroma*

- Chronic conditions (e.g., rheumatologic diseases)

- Touch sensitivity

○ Scarring

- Flap failure/loss (for AR)

\section{Potential Effect Modifiers}

- Age

- Stage of breast cancer

- First occurrence versus recurrent breast cancer 
- Type of chemotherapy (for KQ 2a) or radiation therapy (for KQ 2b)

- Immediate versus delayed reconstruction

- Single-stage (direct to reconstruction) versus multi-stage (with tissue expander) reconstruction

- Unilateral versus bilateral reconstruction

\section{Timing}

- Any

\section{Setting}

- Any, including single- and multicenter

\section{Design}

- $\quad$ RCTs, $N \geq 10$ per group

- NRCSs, $\mathrm{N} \geq 30$ per group, provided adjusted analyses

- Case-control studies, $\mathrm{N} \geq 100$ per group

- Single group studies, $\mathrm{N} \geq 500$

- Studies may be prospective or retrospective

- Exclude: case reports and series of individually reported case reports

\section{Key Question 3 (Type of Implant Material)}

\section{Population(s)}

- Adult ( $\geq 18$ years old) women who are undergoing (or have undergone) mastectomy for any type of breast cancer (or carcinoma in situ) and have decided to undergo IBR

- Either therapeutic or prophylactic mastectomy

- Exclude: Studies where $\geq 10 \%$ of women underwent breast reconstruction (combined across reasons):

○ for solely cosmetic purposes (i.e., augmentation)

- for revision reconstruction (i.e., after a previous reconstruction for breast cancer)

\section{Interventions}

- IBR using one type of implant material

○ Saline

○ Silicone

○ Other materials

○ Either smooth or textured

- Either single- or multistage

- Any anatomic plane of implant placement

- With or without use of human ADM

- With or without mastectomy and reconstruction of the contralateral breast (i.e., unilateral or bilateral)

- With or without symmetry procedure (e.g., mastopexy) in the contralateral breast 


\section{Comparators}

- IBR using another type of implant material

Outcomes $(*$ denotes important outcomes [i.e., unilateral or bilateral] that were used when developing Strength of Evidence tables)

- Clinical outcomes

$\circ$ General quality of life*

○ Physical well-being (e.g., pain, discomfort)*

○ Psychosocial well-being (e.g., self-esteem, emotionality, normality)*

- Sexual well-being*

○ Patient satisfaction with breast*

- Patient satisfaction with outcome (e.g., satisfaction with care)*

- Planned surgeries for reconstruction*

- Recurrence of breast cancer

- Mortality*

- Surgical complications

○ Unplanned repeat hospitalization*

- Duration of unplanned repeat hospitalization*

- Unplanned repeat surgeries - for revision of reconstruction (e.g., for asymmetry)*

- Unplanned repeat surgeries - for complications (e.g., for infection, bleeding)*

○ Pain, including chronic pain*

○ Analgesic (e.g., opioid) use*

- Necrosis, such as of the nipple*

- Animation deformity*

- Implant-related infections*

- Implant rupture, including asymptomatic rupture*

○ Implant deflation*

- Implant malposition*

- Implant failure/loss or need for explant surgery*

- Capsular contracture*

○ New neoplasms (e.g., BIA-ALCL)*

- Complications that cause delays in other cancer-related treatments (e.g., chemotherapy, radiation therapy)*

- Thromboembolic events*

$\circ$ Wound dehiscence

$\circ$ Delayed healing

- Seroma*

- Chronic conditions (e.g., rheumatologic diseases)*

- Touch sensitivity

○ Scarring

- Red breast syndrome

\section{Potential Effect Modifiers}

- Age

- Stage of breast cancer

- First occurrence versus recurrent breast cancer 
- Immediate versus delayed reconstruction

- Single-stage (direct to reconstruction) versus multistage (with tissue expander) reconstruction

- Unilateral versus bilateral reconstruction

- Surface of implant (smooth versus textured)

- Shape of implant (round versus anatomic/teardrop)

- Size of implant (volume)

\section{Timing}

- Any

\section{Setting}

- Any, including single- and multicenter

\section{Design}

- $\mathrm{RCTs}, \mathrm{N} \geq 10$ per group

- $\mathrm{NRCSs}, \mathrm{N} \geq 30$ per group, provided adjusted analyses

- Case-control studies, $\mathrm{N} \geq 100$ per group

- Single group studies, $\mathrm{N} \geq 500$

- Studies may be prospective or retrospective

- Exclude: case reports and series of individually reported case reports

\section{Key Question 4 (Anatomic Plane of Implant Placement)}

\section{Population(s)}

- Adult ( $\geq 18$ years old) women who are undergoing (or have undergone) mastectomy for any type of breast cancer (or carcinoma in situ) and have decided to undergo IBR

- Either therapeutic or prophylactic mastectomy

- Exclude: Studies where $\geq 10 \%$ of women underwent breast reconstruction (combined across reasons):

○ for solely cosmetic purposes (i.e., augmentation)

- for revision reconstruction (i.e., after a previous reconstruction for breast cancer)

\section{Interventions}

- IBR with implant placement in one anatomic plane

○ Prepectoral placement

- Partial submuscular placement

- Total submuscular placement

- Either single- or multi-stage

- Any type of implant material, either smooth or textured

- With or without use of human ADM

- With or without mastectomy and reconstruction of the contralateral breast (i.e., unilateral or bilateral)

- With or without symmetry procedure (e.g., mastopexy) in the contralateral breast 


\section{Comparators}

- IBR with implant placement in a different anatomic plane

Outcomes $(*$ denotes important outcomes that were used when developing Strength of Evidence tables)

- Clinical outcomes

$\circ$ General quality of life*

○ Physical well-being (e.g., pain, discomfort)*

○ Psychosocial well-being (e.g., self-esteem, emotionality, normality)*

○ Sexual well-being*

- Patient satisfaction with breast*

○ Patient satisfaction with outcome (e.g., satisfaction with care)*

- Planned surgeries for reconstruction*

- Recurrence of breast cancer

- Mortality*

- Surgical complications

○ Unplanned repeat hospitalization*

- Duration of unplanned repeat hospitalization*

- Unplanned repeat surgeries - for revision of reconstruction (e.g., for asymmetry)*

- Unplanned repeat surgeries - for complications (e.g., for infection, bleeding)*

- Pain, including chronic pain*

○ Analgesic (e.g., opioid) use*

- Necrosis, such as of the nipple*

- Animation deformity*

- Implant-related infections*

- Implant rupture, including asymptomatic rupture*

○ Implant deflation*

- Implant malposition*

- Implant failure/loss or need for explant surgery*

- Capsular contracture*

○ New neoplasms (e.g., BIA-ALCL)*

- Complications that cause delays in other cancer-related treatments (e.g., chemotherapy, radiation therapy)*

○ Thromboembolic events*

- Infections not explicitly implant-related*

○ Wound dehiscence

○ Delayed healing

- Seroma*

○ Chronic conditions (e.g., rheumatologic diseases)*

- Touch sensitivity

- Scarring

- Red breast syndrome

\section{Potential Effect Modifiers}

- Age

- Stage of breast cancer 
- First occurrence versus recurrent breast cancer

- Immediate versus delayed reconstruction

- Single-stage (direct to reconstruction) versus multistage (with tissue expander) reconstruction

- Unilateral versus bilateral reconstruction

- Surface of implant (smooth versus textured)

- Shape of implant (round versus anatomic/teardrop)

- Size of implant (volume)

\section{Timing}

- Any

\section{Setting}

- Any, including single- and multicenter

\section{Design}

- $\quad$ RCTs, $\mathrm{N} \geq 10$ per group

- $\mathrm{NRCSs}, \mathrm{N} \geq 30$ per group, provided adjusted analyses

- Case-control studies, $\mathrm{N} \geq 100$ per group

- Single group studies, $\mathrm{N} \geq 500$

- Studies may be prospective or retrospective

- Exclude: case reports and series of individually reported case reports

\section{Key Question 5 (Use of Human ADM)}

\section{Population(s)}

- Adult ( $\geq 18$ years old) women who are undergoing (or have undergone mastectomy) for any type of breast cancer (or carcinoma in situ) and have decided to undergo IBR

- Either therapeutic or prophylactic mastectomy

- Exclude: Studies where $\geq 10 \%$ of women underwent breast reconstruction (combined across reasons):

○ for solely cosmetic purposes (i.e., augmentation)

- for revision reconstruction (i.e., after a previous reconstruction for breast cancer)

\section{Interventions}

- IBR with use of human ADM

- Either single- or multistage

- Any anatomic plane of implant placement

- Any type of implant material, either smooth or textured

- With or without mastectomy and reconstruction of the contralateral breast (i.e., unilateral or bilateral)

- With or without symmetry procedure (e.g., mastopexy) in the contralateral breast

\section{Comparators}

- IBR without use of human or nonhuman ADM 
Outcomes $(*$ denotes important outcomes that were used when developing Strength of Evidence tables)

- Clinical outcomes

○ General quality of life*

- Physical well-being (e.g., pain, discomfort)*

○ Psychosocial well-being (e.g., self-esteem, emotionality, normality)*

○ Sexual well-being*

- Patient satisfaction with breast*

- Patient satisfaction with outcome (e.g., satisfaction with care)*

- Planned surgeries for reconstruction*

- Recurrence of breast cancer

○ Mortality*

- Surgical complications

- Unplanned repeat hospitalization*

- Duration of unplanned repeat hospitalization*

- Unplanned repeat surgeries - for revision of reconstruction (e.g., for asymmetry)*

- Unplanned repeat surgeries - for complications (e.g., for infection, bleeding)*

- Pain, including chronic pain*

- Analgesic (e.g., opioid) use*

- Necrosis, such as of the nipple*

- Animation deformity*

- Implant-related infections*

- Implant rupture, including asymptomatic rupture*

○ Implant deflation*

- Implant malposition*

- Implant failure/loss or need for explant surgery*

- Capsular contracture*

○ New neoplasms (e.g., BIA-ALCL)*

- Complications that cause delays in other cancer-related treatments (e.g., chemotherapy, radiation therapy)*

- Thromboembolic events*

○ Infections*

○ Wound dehiscence*

- Delayed healing*

- Seroma*

- Chronic conditions (e.g., rheumatologic diseases)

- Touch sensitivity

- Scarring

- Red breast syndrome

\section{Potential Effect Modifiers}

- Age

- Stage of breast cancer

- First occurrence versus recurrent breast cancer

- Immediate versus delayed reconstruction 
- Single-stage (direct to reconstruction) versus multi-stage (with tissue expander) reconstruction

- Unilateral versus bilateral reconstruction

- Anatomic plane of implant placement (prepectoral versus partial submuscular versus total submusclar)

- Surface of implant (smooth versus textured)

- Shape of implant (round versus anatomic/teardrop)

- Size of implant (volume)

- Brand of human ADM (e.g., Alloderm ${ }^{\circledR}$, FlexHD $^{\circledR}$, BellaDerm $^{\circledR}$, AlloMax $^{\circledR}$, Cortiva $^{\circledR}$, DermACELL ${ }^{\circledR}$ )

\section{Timing}

- Any

\section{Setting}

- Any, including single- and multicenter

\section{Design}

- $\quad$ RCTs, $\mathrm{N} \geq 10$ per group

- NRCSs, $\mathrm{N} \geq 30$ per group, provided adjusted analyses

- Case-control studies, $\mathrm{N} \geq 100$ per group

- Single group studies, $\mathrm{N} \geq 500$

- Studies may be prospective or retrospective

- Exclude: case reports and series of individually reported case reports

\section{Key Question 6 (Different Flap Types for AR)}

\section{Population(s)}

- Adult ( $\geq 18$ years old) women who are undergoing (or have undergone mastectomy) for any type of breast cancer (or carcinoma in situ) and have decided to undergo AR

- Either therapeutic or prophylactic mastectomy

- Exclude: Studies where $\geq 10 \%$ of women underwent breast reconstruction (combined across reasons):

○ for solely cosmetic purposes (i.e., augmentation)

- for revision reconstruction (i.e., after a previous reconstruction for breast cancer)

\section{Interventions}

- AR using one flap (either free flap or pedicled), for example:

$\circ$ Deep inferior epigastric perforator (DIEP)

- Latissimus dorsi (LD)

- Transverse rectus abdominis myocutaneous (TRAM)

- Superficial inferior epigastric artery perforator (SIEA)

- Gluteal artery perforator (GAP)

- Transverse musculocutaneous gracilis (TMG)

- Transverse upper gracilis (TUG) 
- Profunda artery perforator (PAP)

- Superior gluteal artery perforator (SGAP)

- With or without mastectomy and reconstruction of the contralateral breast (i.e., unilateral or bilateral)

- With or without symmetry procedure (e.g., mastopexy) in the contralateral breast

- Exclude: Non-autologous flap transplants (i.e., cadaveric or xenotransplant)

- Exclude: Exclusive lipofilling/autologous fat reconstruction

\section{Comparators}

- AR using a different flap (either free flap or pedicled)

- Combination of IBR and AR

- Exclude: Non-autologous flap transplants (i.e., cadaveric or xenotransplant)

- Exclude: Exclusive lipofilling/autologous fat reconstruction

\section{Outcomes (* denotes important outcomes that were used when developing Strength of Evidence tables)}

- Clinical outcomes

$\circ$ General quality of life*

- Physical well-being (e.g., pain, discomfort)*

○ Psychosocial well-being (e.g., self-esteem, emotionality, normality)*

- Sexual well-being*

- Patient satisfaction with breast*

- Patient satisfaction with outcome (e.g., satisfaction with care)*

- Planned surgeries for reconstruction*

- Recurrence of breast cancer

○ Duration of initial hospitalization*

○ Mortality*

- Surgical complications

○ Unplanned repeat hospitalization*

- Duration of unplanned repeat hospitalization*

- Unplanned repeat surgeries - for revision of reconstruction (e.g., for asymmetry)*

- Unplanned repeat surgeries - for complications (e.g., for infection, bleeding)*

○ Pain, including chronic pain*

- Analgesic (e.g., opioid) use*

- Necrosis, such as of the nipple or of the flap*

- Harms to area of flap harvest (e.g., hernia, bulge formation)*

- Complications that lead to delays in other cancer-related treatments (e.g., chemotherapy, radiation therapy)*

- Thromboembolic events*

- Infections*

○ Wound dehiscence*

- Delayed healing*

- Seroma*

- Touch sensitivity

- Scarring 
○ Flap failure/loss

\section{Potential Effect Modifiers}

- Age

- Stage of breast cancer

- First occurrence versus recurrent breast cancer

- Immediate versus delayed reconstruction

- Single-stage (direct to reconstruction) versus multi-stage (with tissue expander) reconstruction

- Unilateral versus bilateral reconstruction

\section{Timing}

- Any

\section{Setting}

- Any, including single- and multicenter

\section{Design}

- $\quad$ RCTs, $\mathrm{N} \geq 10$ per group

- NRCSs, $\mathrm{N} \geq 30$ per group, provided adjusted analyses

- Case-control studies, $\mathrm{N} \geq 100$ per group

- Single group studies, $\mathrm{N} \geq 500$

- Studies may be prospective or retrospective

Exclude: case reports and series of individually reported case reports

\section{Screening Process}

Citations from all searches were deduplicated and then entered into abstrackr software (http://abstrackr.cebm.brown.edu/) to enable title and abstract screening. The team conducted three rounds of pilot screening. During each pilot round, we all screened the same 100 abstracts and discuss conflicts, with the goal of training the team in the nuances of the eligibility criteria and refining them as needed. After the pilot rounds, we screened all remaining abstracts in duplicate. The abstrackr software has machine learning capabilities that predict the likelihood of relevance of each citation. Daily, the list of unscreened abstracts was sorted so that the most potentially relevant articles were presented first. This process made screening more efficient and enabled us to capture the large majority of relevant articles relatively early in the abstract screening process.

Based on empirical research on abstrackr (that is soon to be submitted for publication), we switched to single screening of remaining abstracts once both of the following criteria were fulfilled: (1) all remaining unscreened abstracts had a prediction value less than 0.40 (on a scale of 0 to 1), and (2) no eligible citations were identified in a consecutive sample of 400 abstracts (this threshold for number of abstracts was chosen because it comfortably exceeds 370 abstracts, which is the threshold above which the upper $97.5 \%$ confidence interval bound for a proportion of irrelevant abstracts [i.e., 0/370] is less than $1 \%$ ). The empirical research suggests that at this threshold, all remaining abstracts would have been rejected. Potentially relevant citations were retrieved in full text. These articles were rescreened in duplicate. 


\section{Data Extraction and Data Management (Details)}

We extracted data from eligible primary studies into the Systematic Review Data RepositoryPlus (https://srdrplus.ahrq.gov). For each study, one researcher extracted and entered data, which were confirmed by a second, independent researcher. Each individual study that was reported in multiple articles was extracted as a single record. In the instance where two studies were reported within a single article, each study was extracted separately.

For each study, we extracted article-identifying information, study design features, funding source, population characteristics and sample sizes, intervention and comparator names and descriptions, and relevant clinical outcomes and surgical complication outcomes and their definitions.

\section{Assessing Applicability}

For each KQ (or specific subquestion), we assessed the applicability of the included studies primarily based on the studies' eligibility criteria and their included participants, specifically related to such factors as age, type of breast cancer, and first occurrence versus recurrent breast cancer. These were qualitatively compared with typical distributions of these factors among patients undergoing breast reconstruction in the U.S.

\section{Addressing the Contextual Questions}

Based on data and input garnered during our systematic review of the KQs, we answered the Contextual Questions in a narrative format. We did not systematically extract or review eligible studies, create summary tables, or assess the strength of evidence for the Contextual Questions.

\section{Peer Review and Public Commentary}

Experts in plastic surgery, breast surgical oncology, medical oncology, radiation oncology, national policy, clinical practice guidelines, and individuals representing stakeholder and user communities are being invited to provide external peer review of this SR. AHRQ and an Associate Editor from a fellow Evidence-based Practice Center were invited to provide comments. The draft report was posted on the AHRQ Website to elicit public comment for a period of 4 weeks. We addressed all reviewer and public comments, revising the text as appropriate. A disposition of comments table of peer and public comments is posted on the EHC Website.

\section{Abbreviations}

ADM
AHRQ
AR
ASPS
BIA-ALCL
BMI
BPI-SF
CI
CINAHL
COI

acellular dermal matrix Agency for Healthcare Research and Quality autologous reconstruction American Society of Plastic Surgeons breast implant-associated anaplastic large cell lymphoma body mass index Brief Pain Inventory-Short Form confidence interval Cumulative Index to Nursing and Allied Health Literature conflicts of interest 


$\begin{array}{ll}\text { DASH } & \text { Disabilities of the Arm, Should, and Hand } \\ \text { DIEP } & \text { deep inferior epigastric perforator } \\ \text { DVT } & \text { deep vein thrombosis } \\ \text { EHC } & \text { Effective Health Care Program } \\ \text { EORTC QLQ-C30 } & \text { European Organization for Research and Treatment of Cancer } \\ & \text { Quality of Life Questionnaire C30 } \\ \text { EPC } & \text { Evidence-based Practice Center } \\ \text { FACT-B } & \text { Functional Assessment of Cancer Therapy } \\ \text { FDA } & \text { Food and Drug Administration } \\ \text { GAP } & \text { gluteal artery perforator } \\ \text { HR } & \text { hazard ratio } \\ \text { IBR } & \text { implant-based reconstruction } \\ \text { KI } & \text { Key Informant } \\ \text { KQ } & \text { Key Question } \\ \text { LD } & \text { latissimus dorsi } \\ \text { LTD } & \text { lateral thoracodorsal } \\ \text { MCID } & \text { minimal clinically important difference } \\ \text { MD } & \text { mean difference } \\ \text { MeSH } & \text { medical subject heading } \\ \text { MPQ-SF } & \text { McGill Pain Questionnaire Short Form } \\ \text { NHLBI } & \text { National Heart, Lung, and Blood Institute } \\ \text { NLM } & \text { National Library of Medicine } \\ \text { NMD } & \text { net mean difference } \\ \text { NRCS } & \text { nonrandomized comparative study } \\ \text { OR } & \text { odds ratio } \\ \text { PAP } & \text { profundal artery perforator } \\ \text { PE } & \text { pulmonary embolism } \\ \text { PMID } & \text { PubMed identifier } \\ \text { PMRT } & \text { postmastectomy radiation therapy } \\ \text { PROMIS } & \text { Patient-Reported Outcomes Measurement Information System } \\ \text { RCT } & \text { randomized controlled trial } \\ \text { RoB } & \text { risk of bias } \\ \text { ROBINS-I } & \text { Risk of Bias in Nonrandomized Studies of Interventions } \\ \text { RR } & \text { Telative risk } \\ \text { SD } & \text { standard deviation } \\ \text { SE } & \text { standard error } \\ \text { SF-36 } & \text { Short Form-36 } \\ \text { SGAP } & \text { superior gluteal artery perforator } \\ \text { SIEA } & \text { superficial inferior epigastric artery perforator } \\ \text { SoE } & \text { strength of evidence } \\ \text { SR } & \text { systematic review } \\ \text { SRDR+ } & \text { Systematic Review Data Repository Plus } \\ \text { TAP } & \text { thoracodorsal artery perforator } \\ \text { TEP } & \text { technical Expert Panel } \\ \text { TMG } & \text { Tanserse musculocutaneous gracilis } \\ \text { TOO } & \end{array}$


TRAM

TUG

U.K.

U.S.

VAS transverse rectus abdominis myocutaneous transverse upper gracilis

United Kingdom

United States

Visual Analog Scale 


\section{Appendix B. List of Excluded Studies}

The 1,050 excluded articles, along with reasons for exclusion, are summarized in Appendix Table B-1. The most common reasons for exclusion were that the articles did not address any Key Question ( $\mathrm{n}=313$ articles), described nonrandomized comparative studies without adequate adjustment ( $\mathrm{n}=234$ articles), or described Single group studies with fewer than 500 participants ( $\mathrm{n}=187$ articles).

\begin{tabular}{|c|c|c|c|c|c|}
\hline No. & $\begin{array}{l}\text { PMID or } \\
\text { Other } \\
\text { Identifier }\end{array}$ & $\begin{array}{l}\text { First Author } \\
\text { Last Name }\end{array}$ & Title & Journal & Reason for Exclusion \\
\hline 1 & 31772899 & Abdelrahman & $\begin{array}{l}\text { Oncoplastic Volume Replacement for Breast Cancer: Latissimus Dorsi Flap versus } \\
\text { Thoracodorsal Artery Perforator Flap }\end{array}$ & $\begin{array}{l}\text { Plast Reconstr } \\
\text { Surg Glob Open }\end{array}$ & $\begin{array}{l}\text { Not mastectomy for } \\
\text { breast cancer }\end{array}$ \\
\hline 2 & 25133469 & Abt & $\begin{array}{l}\text { Neoadjuvant chemotherapy and short-term morbidity in patients undergoing } \\
\text { mastectomy with and without breast reconstruction }\end{array}$ & JAMA Surg & $\begin{array}{l}\text { Does not address KQ1- } \\
\text { KQ6 }\end{array}$ \\
\hline 3 & 32162180 & Adachi & $\begin{array}{l}\text { Effects of neoadjuvant chemotherapy on operative adverse events and } \\
\text { chemotherapy and radiotherapy in patients undergoing immediate breast } \\
\text { reconstruction }\end{array}$ & Breast Cancer & $\begin{array}{l}\text { Does not address KQ1- } \\
\text { KQ6 }\end{array}$ \\
\hline 4 & 20584583 & Adesiyun & $\begin{array}{l}\text { Impact of sequencing of postmastectomy radiotherapy and breast reconstruction } \\
\text { on timing and rate of complications and patient satisfaction }\end{array}$ & $\begin{array}{l}\text { Int J Radiat Oncol } \\
\text { Biol Phys }\end{array}$ & NRCS not adjusted \\
\hline 5 & 22156884 & Adetayo & $\begin{array}{l}\text { A Meta-analysis of Outcomes Using Acellular Dermal Matrix in Breast and } \\
\text { Abdominal Wall Reconstructions: Event Rates and Risk Factors Predictive of } \\
\text { Complications }\end{array}$ & Ann Plast Surg & $\begin{array}{l}\text { Narrative review/ } \\
\text { Commentary }\end{array}$ \\
\hline 6 & 22487264 & Agarwal & $\begin{array}{l}\text { A population-based study of breast cancer-specific survival following mastectomy } \\
\text { and immediate or early-delayed breast reconstruction }\end{array}$ & Breast J & $\begin{array}{l}\text { Single group }>500, \text { but } \\
\text { no complications data }\end{array}$ \\
\hline 7 & 20604794 & Agarwal & Survival in breast cancer patients undergoing immediate breast reconstruction & Breast J & $\begin{array}{l}\text { Does not address KQ1- } \\
\text { KQ6 }\end{array}$ \\
\hline 8 & 24119787 & Agrawal & $\begin{array}{l}\text { Surgical and oncological outcome after skin-sparing mastectomy and immediate } \\
\text { breast reconstruction }\end{array}$ & Clin Breast Cancer & $\begin{array}{l}\text { Single group N enrolled } \\
<500\end{array}$ \\
\hline 9 & 107920554 & Agrawal & $\begin{array}{l}\text { Surgical and oncological outcome after skin-sparing mastectomy and immediate } \\
\text { breast reconstruction }\end{array}$ & $\begin{array}{l}\text { Clinical Breast } \\
\text { Cancer }\end{array}$ & $\begin{array}{l}\text { Single group } \mathrm{N} \text { enrolled } \\
<500\end{array}$ \\
\hline 10 & 28582783 & Akita & $\begin{array}{l}\text { Contribution of Simultaneous Breast Reconstruction by Deep Inferior Epigastric } \\
\text { Artery Perforator Flap to the Efficacy of Vascularized Lymph Node Transfer in } \\
\text { Patients with Breast Cancer-Related Lymphedema }\end{array}$ & $\begin{array}{l}\text { J Reconstr } \\
\text { Microsurg }\end{array}$ & NRCS $<30$ per arm \\
\hline 11 & 31810892 & Akyurek & $\begin{array}{l}\text { Two-stage prosthetic breast reconstruction with latissimus flap: Prepectoral versus } \\
\text { subpectoral approach }\end{array}$ & $\begin{array}{l}\text { J Plast Reconstr } \\
\text { Aesthet Surg }\end{array}$ & NRCS $<30$ per arm \\
\hline 12 & 26208580 & Al-Hilli & $\begin{array}{l}\text { Reoperation for Complications after Lumpectomy and Mastectomy for Breast } \\
\text { Cancer from the } 2012 \text { National Surgical Quality Improvement Program (ACS- } \\
\text { NSQIP) }\end{array}$ & Ann Surg Oncol & $\begin{array}{l}\text { Does not address KQ1- } \\
\text { KQ6 }\end{array}$ \\
\hline 13 & $\begin{array}{l}\text { CN- } \\
01984885\end{array}$ & Alamouti & $\begin{array}{l}\text { Multidisciplinary management of risk-reducing mastectomy and immediate } \\
\text { reconstruction: treatment algorithm and patient satisfaction }\end{array}$ & $\begin{array}{l}\text { European journal of } \\
\text { plastic surgery }\end{array}$ & NRCS $<30$ per arm \\
\hline 14 & 31645075 & Alba & $\begin{array}{l}\text { Postoperative Upper Extremity Function in Implant and Autologous Breast } \\
\text { Reconstruction }\end{array}$ & $\begin{array}{l}J \text { Reconstr } \\
\text { Microsurg }\end{array}$ & NRCS not adjusted \\
\hline
\end{tabular}




\begin{tabular}{|c|c|c|c|c|c|}
\hline No. & $\begin{array}{l}\text { PMID or } \\
\text { Other } \\
\text { Identifier }\end{array}$ & $\begin{array}{l}\text { First Author } \\
\text { Last Name }\end{array}$ & Title & Journal & Reason for Exclusion \\
\hline 15 & 23271515 & Albornoz & A paradigm shift in U.S. Breast reconstruction: increasing implant rates & $\begin{array}{l}\text { Plast Reconstr } \\
\text { Surg }\end{array}$ & $\begin{array}{l}\text { Not breast } \\
\text { reconstruction }\end{array}$ \\
\hline 16 & 25158715 & Albornoz & Diminishing relative contraindications for immediate breast reconstruction & $\begin{array}{l}\text { Plast Reconstr } \\
\text { Surg }\end{array}$ & $\begin{array}{l}\text { Does not address KQ1- } \\
\text { KQ6 }\end{array}$ \\
\hline 17 & 25159019 & Albornoz & $\begin{array}{l}\text { Diminishing relative contraindications for immediate breast reconstruction: a } \\
\text { multicenter study }\end{array}$ & J Am Coll Surg & $\begin{array}{l}\text { Does not address KQ1- } \\
\text { KQ6 }\end{array}$ \\
\hline 18 & 17189107 & Alderman & $\begin{array}{l}\text { Does patient satisfaction with breast reconstruction change over time? Two-year } \\
\text { results of the Michigan Breast Reconstruction Outcomes Study }\end{array}$ & J Am Coll Surg & $\begin{array}{l}\text { Single group } \mathrm{N} \text { enrolled } \\
<500\end{array}$ \\
\hline 19 & 16772906 & Alderman & A two-year prospective analysis of trunk function in TRAM breast reconstructions & $\begin{array}{l}\text { Plastic and } \\
\text { Reconstructive } \\
\text { Surgery }\end{array}$ & No outcome of interest \\
\hline 20 & 12045548 & Alderman & $\begin{array}{l}\text { Complications in postmastectomy breast reconstruction: two-year results of the } \\
\text { Michigan Breast Reconstruction Outcome Study }\end{array}$ & $\begin{array}{l}\text { Plast Reconstr } \\
\text { Surg }\end{array}$ & NRCS not adjusted \\
\hline 21 & 33526361 & Aliotta & $\begin{array}{l}\text { A controlled cost and outcomes analysis of acellular dermal matrix and implant- } \\
\text { based reconstruction }\end{array}$ & $\begin{array}{l}\text { J Plast Reconstr } \\
\text { Aesthet Surg }\end{array}$ & NRCS not adjusted \\
\hline 22 & 32201323 & Allan & $\begin{array}{l}\text { The effect of operative time on complication profile and length of hospital stay in } \\
\text { autologous and implant-based breast reconstruction patients: An analysis of the } \\
\text { 2007-2012 ACS-NSQIP database }\end{array}$ & $\begin{array}{l}J \text { Plast Reconstr } \\
\text { Aesthet Surg }\end{array}$ & $\begin{array}{l}\text { Does not address KQ1- } \\
\text { KQ6 }\end{array}$ \\
\hline 23 & 30545644 & Allue Cabanuz & $\begin{array}{l}\text { Influence of radiotherapy on immediate breast reconstruction after skin-sparing } \\
\text { mastectomy. Before or after: Does it matter? }\end{array}$ & Cir Esp & NRCS not adjusted \\
\hline 24 & 19121986 & Alonso-Burgos & $\begin{array}{l}\text { Preoperative planning of DIEP and SGAP flaps: preliminary experience with } \\
\text { magnetic resonance angiography using 3-tesla equipment and blood-pool contrast } \\
\text { medium }\end{array}$ & $\begin{array}{l}J \text { Plast Reconstr } \\
\text { Aesthet Surg }\end{array}$ & $\begin{array}{l}\text { Single group } \mathrm{N} \text { enrolled } \\
<500\end{array}$ \\
\hline 25 & 30899456 & Alshammari & $\begin{array}{l}\text { Patient-reported outcomes after breast reconstructive surgery: A prospective } \\
\text { cross-sectional study }\end{array}$ & $\begin{array}{l}\text { Ann Med Surg } \\
\text { (Lond) }\end{array}$ & NRCS $<30$ per arm \\
\hline 26 & 104823788 & Anavekar & $\begin{array}{l}\text { Achieving autologous breast reconstruction for breast cancer patients in the setting } \\
\text { of post-mastectomy radiotherapy }\end{array}$ & $\begin{array}{l}\text { Journal of Cancer } \\
\text { Survivorship }\end{array}$ & $\begin{array}{l}\text { Narrative review/ } \\
\text { Commentary }\end{array}$ \\
\hline 27 & 32190584 & Anbiyaiee & $\begin{array}{l}\text { Breast Reconstruction after Mastectomy in Women with Breast Cancer: A } \\
\text { Systematic and Meta-Analysis Review }\end{array}$ & World J Plast Surg & $\begin{array}{l}\text { Does not address KQ1- } \\
\text { KQ6 }\end{array}$ \\
\hline 28 & 15234042 & Anderson & $\begin{array}{l}\text { Low complication rates are achievable after postmastectomy breast reconstruction } \\
\text { and radiation therapy }\end{array}$ & $\begin{array}{l}\text { Int J Radiat Oncol } \\
\text { Biol Phys }\end{array}$ & NRCS not adjusted \\
\hline 29 & 15234042 & Anderson & $\begin{array}{l}\text { Low complication rates are achievable after postmastectomy breast reconstruction } \\
\text { and radiation therapy }\end{array}$ & $\begin{array}{l}\text { International } \\
\text { Journal of } \\
\text { Radiation Oncology } \\
\text { Biology Physics }\end{array}$ & NRCS not adjusted \\
\hline 30 & 23770544 & Andree & $\begin{array}{l}\text { A single center prospective study of bilateral breast reconstruction with free } \\
\text { abdominal flaps: A critical analyses of } 144 \text { patients }\end{array}$ & $\begin{array}{l}\text { Medical Science } \\
\text { Monitor }\end{array}$ & $\begin{array}{l}>=10 \% \text { revision } \\
\text { reconstruction only }\end{array}$ \\
\hline 31 & 23197233 & Andree & $\begin{array}{l}\text { Skin-sparing mastectomy and immediate reconstruction with DIEP flap after } \\
\text { breast-conserving therapy }\end{array}$ & $\begin{array}{l}\text { Medical Science } \\
\text { Monitor }\end{array}$ & $\begin{array}{l}\text { Duplicate of another } \\
\text { publication }\end{array}$ \\
\hline
\end{tabular}




\begin{tabular}{|c|c|c|c|c|c|}
\hline No. & $\begin{array}{l}\text { PMID or } \\
\text { Other } \\
\text { Identifier }\end{array}$ & $\begin{array}{l}\text { First Author } \\
\text { Last Name }\end{array}$ & Title & Journal & Reason for Exclusion \\
\hline 32 & 31526955 & Angarita & $\begin{array}{l}\text { Does timing of alloplastic breast reconstruction in older women impact immediate } \\
\text { postoperative complications? An analysis of the American College of Surgeons } \\
\text { National Surgical Quality Improvement Program (ACS NSQIP) database }\end{array}$ & Breast & $\begin{array}{l}\text { Does not address KQ1- } \\
\text { KQ6 }\end{array}$ \\
\hline 33 & 31154580 & Angarita & $\begin{array}{l}\text { Is immediate breast reconstruction safe in women over 70? An analysis of the } \\
\text { National Surgical Quality Improvement Program (NSQIP) database }\end{array}$ & $\begin{array}{l}\text { Breast Cancer Res } \\
\text { Treat }\end{array}$ & $\begin{array}{l}\text { Copublication of } \\
\text { included study with no } \\
\text { new data }\end{array}$ \\
\hline 34 & 32449079 & Angarita & $\begin{array}{l}\text { Does oncoplastic surgery increase immediate (30- } \\
\text { day) postoperative complications? An analysis of the American College of } \\
\text { Surgeons National Surgical Quality Improvement Program (ACS NSQIP) database }\end{array}$ & $\begin{array}{l}\text { Breast Cancer Res } \\
\text { Treat }\end{array}$ & $\begin{array}{l}\text { Does not address KQ1- } \\
\text { KQ6 }\end{array}$ \\
\hline 35 & 29660924 & Anker & $\begin{array}{l}\text { Vasopressor support vs. liberal fluid administration in deep inferior epigastric } \\
\text { perforator (DIEP) free flap breast reconstruction - a randomized controlled trial }\end{array}$ & $\begin{array}{l}\text { Clin Hemorheol } \\
\text { Microcirc }\end{array}$ & $\begin{array}{l}\text { Does not address KQ1- } \\
\text { KQ6 }\end{array}$ \\
\hline 36 & 31468214 & Anker & $\begin{array}{l}\text { Assessment of DIEP Flap Perfusion with Intraoperative Indocyanine Green } \\
\text { Fluorescence Imaging in Vasopressor-Dominated Hemodynamic Support Versus } \\
\text { Liberal Fluid Administration: A Randomized Controlled Trial With Breast Cancer } \\
\text { Patients }\end{array}$ & Ann Surg Oncol & $\begin{array}{l}\text { Single group } \mathrm{N} \text { enrolled } \\
<500\end{array}$ \\
\hline 37 & 31738381 & $\begin{array}{l}\text { Anna Loch- } \\
\text { Wilkinson }\end{array}$ & $\begin{array}{l}\text { Breast Implant-Associated Anaplastic Large Cell Lymphoma in Australia: A } \\
\text { Longitudinal Study of Implant and Other Related Risk Factors }\end{array}$ & Aesthet Surg J & $\begin{array}{l}\text { Single group } \mathrm{N} \text { enrolled } \\
<500\end{array}$ \\
\hline 38 & 28628501 & Arikawa & $\begin{array}{l}\text { Comparison of Donor Site Drainage Duration and Seroma Rate Between } \\
\text { Latissimus Dorsi Musculocutaneous Flaps and Thoracodorsal Artery Perforator } \\
\text { Flaps }\end{array}$ & Ann Plast Surg & NRCS not adjusted \\
\hline 39 & 27229369 & Armstrong & $\begin{array}{l}\text { Determinants of increased acute postoperative pain after autologous breast } \\
\text { reconstruction within an enhanced recovery after surgery protocol: A prospective } \\
\text { cohort study }\end{array}$ & $\begin{array}{l}\text { J Plast Reconstr } \\
\text { Aesthet Surg }\end{array}$ & $\begin{array}{l}\text { Single group } \mathrm{N} \text { enrolled } \\
<500\end{array}$ \\
\hline 40 & 23582505 & Arya & $\begin{array}{l}\text { Post-operative assessment of perfusion of Deep Inferior Epigastric Perforator } \\
\text { (DIEP) free flaps via Pulsatility Index (PI) using a portable colour Doppler } \\
\text { sonogram device }\end{array}$ & $\begin{array}{l}\text { J Plast Reconstr } \\
\text { Aesthet Surg }\end{array}$ & NRCS $<30$ per arm \\
\hline 41 & 32803638 & Asaad & $\begin{array}{l}\text { The impact of co-surgeons on complication rates and healthcare cost in patients } \\
\text { undergoing microsurgical breast reconstruction: analysis of } 8680 \text { patients }\end{array}$ & $\begin{array}{l}\text { Breast Cancer } \\
\text { Research and } \\
\text { Treatment }\end{array}$ & $\begin{array}{l}\text { Does not address KQ1- } \\
\text { KQ6 }\end{array}$ \\
\hline 42 & 24756811 & Ashfaq & $\begin{array}{l}\text { Impact of breast reconstruction on the decision to undergo contralateral } \\
\text { prophylactic mastectomy }\end{array}$ & Ann Surg Oncol & $\begin{array}{l}\text { Single group }>500 \text {, but } \\
\text { no complications data }\end{array}$ \\
\hline 43 & 23706394 & Ashraf & $\begin{array}{l}\text { Patient involvement in the decision-making process improves satisfaction and } \\
\text { quality of life in postmastectomy breast reconstruction }\end{array}$ & $\begin{array}{l}\text { Journal of Surgical } \\
\text { Research }\end{array}$ & $\begin{array}{l}\text { Does not address KQ1- } \\
\text { KQ6 }\end{array}$ \\
\hline 44 & 19593108 & Atisha & $\begin{array}{l}\text { A systematic review of abdominal wall function following abdominal flaps for } \\
\text { postmastectomy breast reconstruction }\end{array}$ & Ann Plast Surg & $\begin{array}{l}\text { Does not address KQ1- } \\
\text { KQ6 }\end{array}$ \\
\hline 45 & 18520874 & Atisha & The impact of obesity on patient satisfaction with breast reconstruction & $\begin{array}{l}\text { Plast Reconstr } \\
\text { Surg }\end{array}$ & NRCS not adjusted \\
\hline 46 & 25465378 & Atisha & A national snapshot of satisfaction with breast cancer procedures & Ann Surg Oncol & $\begin{array}{l}\text { Does not address KQ1- } \\
\text { KQ6 }\end{array}$ \\
\hline 47 & 30589826 & Atisha & $\begin{array}{l}\text { A National Snapshot of Patient-Reported Outcomes Comparing Types of } \\
\text { Abdominal Flaps for Breast Reconstruction }\end{array}$ & $\begin{array}{l}\text { Plast Reconstr } \\
\text { Surg }\end{array}$ & $\begin{array}{l}\text { Single group }>500, \text { but } \\
\text { no complications data }\end{array}$ \\
\hline
\end{tabular}




\begin{tabular}{|c|c|c|c|c|c|}
\hline No. & $\begin{array}{l}\text { PMID or } \\
\text { Other } \\
\text { Identifier }\end{array}$ & $\begin{array}{l}\text { First Author } \\
\text { Last Name }\end{array}$ & Title & Journal & Reason for Exclusion \\
\hline 48 & 8197555 & August & Breast reconstruction in older women & Surgery & Unable to retrieve article \\
\hline 49 & 30892486 & Augustinho & $\begin{array}{l}\text { Patient satisfaction with breast reconstruction using musculocutaneous flap from } \\
\text { latissimus dorsi versus from rectus abdominis: A cross-sectional study }\end{array}$ & $\begin{array}{l}\text { Sao Paulo Medical } \\
\text { Journal }\end{array}$ & $\begin{array}{l}\text { Duplicate of another } \\
\text { publication }\end{array}$ \\
\hline 50 & None & Augustinho & $\begin{array}{l}\text { Patient satisfaction with breast reconstructionusing musculocutaneous flap from } \\
\text { latissimus dorsiversus from rectus abdominis: a cross-sectional study }\end{array}$ & Sao Paulo Med J & NRCS not adjusted \\
\hline 51 & 26132336 & Avraham & $\begin{array}{l}\text { Postoperative Expansion is not a Primary Cause of Infection in Immediate Breast } \\
\text { Reconstruction with Tissue Expanders }\end{array}$ & Breast $J$ & $\begin{array}{l}\text { Does not address KQ1- } \\
\text { KQ6 }\end{array}$ \\
\hline 52 & 32718111 & Azizi & $\begin{array}{l}\text { Does surgical procedure type impact postoperative pain and recovery in deep } \\
\text { inferior epigastric artery perforator flap breast reconstruction? }\end{array}$ & Arch Plast Surg & $\begin{array}{l}\text { Single group } \mathrm{N} \text { enrolled } \\
<500\end{array}$ \\
\hline 53 & 28522026 & Babin & $\begin{array}{l}\text { [Breast reconstruction in elderly patients: Studies of the practices at institut } \\
\text { Bergonie during 2005-2015] }\end{array}$ & Bull Cancer & $\begin{array}{l}\text { Single group } \mathrm{N} \text { enrolled } \\
<500\end{array}$ \\
\hline 54 & 25396188 & Baek & A retrospective analysis of ruptured breast implants & Arch Plast Surg & NRCS <30 per arm \\
\hline 55 & 16525258 & Bajaj & $\begin{array}{l}\text { Comparison of donor-site complications and functional outcomes in free muscle- } \\
\text { sparing TRAM flap and free DIEP flap breast reconstruction }\end{array}$ & $\begin{array}{l}\text { Plast Reconstr } \\
\text { Surg }\end{array}$ & NRCS not adjusted \\
\hline 56 & 29697602 & Baker & $\begin{array}{l}\text { A Prospective Comparison of Short-Term Outcomes of Subpectoral and } \\
\text { Prepectoral Strattice-Based Immediate Breast Reconstruction }\end{array}$ & $\begin{array}{l}\text { Plast Reconstr } \\
\text { Surg }\end{array}$ & NRCS $<30$ per arm \\
\hline 57 & 33051937 & Baker & $\begin{array}{l}\text { The impact of axillary node surgery on outcomes following immediate breast } \\
\text { reconstruction }\end{array}$ & Breast J & NRCS not adjusted \\
\hline 58 & 27219260 & Baldelli & $\begin{array}{l}\text { Implant-Based Breast Reconstruction Using a Polyester Mesh (Surgimesh-PET): A } \\
\text { Retrospective Single-Center Study }\end{array}$ & $\begin{array}{l}\text { Plast Reconstr } \\
\text { Surg }\end{array}$ & $\begin{array}{l}\text { Does not address KQ1- } \\
\text { KQ6 }\end{array}$ \\
\hline 59 & 26550776 & Balk & $\begin{array}{l}\text { Long-Term Health Outcomes in Women With Silicone Gel Breast Implants: A } \\
\text { Systematic Review }\end{array}$ & Ann Intern Med & $\begin{array}{l}\text { Not mastectomy for } \\
\text { breast cancer }\end{array}$ \\
\hline 60 & 28458959 & Baltodano & $\begin{array}{l}\text { Preoperative Radiotherapy Is Not Associated with Increased Post-mastectomy } \\
\text { Short-term Morbidity: Analysis of } 77,902 \text { Patients }\end{array}$ & $\begin{array}{l}\text { Plast Reconstr } \\
\text { Surg Glob Open }\end{array}$ & $\begin{array}{l}\text { Does not address KQ1- } \\
\text { KQ6 }\end{array}$ \\
\hline 61 & 32842096 & Baltodano & $\begin{array}{l}\text { Early Discontinuation of Breast Free Flap Monitoring: A Strategy Driven by } \\
\text { National Data }\end{array}$ & $\begin{array}{l}\text { Plast Reconstr } \\
\text { Surg }\end{array}$ & $\begin{array}{l}\text { Does not address KQ1- } \\
\text { KQ6 }\end{array}$ \\
\hline 62 & 24091489 & Bank & $\begin{array}{l}\text { Economic analysis and review of the literature on implant-based breast } \\
\text { reconstruction with and without the use of the acellular dermal matrix }\end{array}$ & $\begin{array}{l}\text { Aesthetic Plast } \\
\text { Surg }\end{array}$ & NRCS $<30$ per arm \\
\hline 63 & 31513083 & Banuelos & $\begin{array}{l}\text { Microbiology of Implant-Based Breast Reconstruction Infections: A Systematic } \\
\text { Review }\end{array}$ & Ann Plast Surg & $\begin{array}{l}\text { Does not address KQ1- } \\
\text { KQ6 }\end{array}$ \\
\hline 64 & 31663932 & Banuelos & $\begin{array}{l}\text { The American College of Surgeons National Quality Improvement Program } \\
\text { Incompletely Captures Implant-Based Breast Reconstruction Complications }\end{array}$ & $\begin{array}{l}\text { Annals of plastic } \\
\text { surgery }\end{array}$ & $\begin{array}{l}\text { Duplicate of another } \\
\text { publication }\end{array}$ \\
\hline 65 & 21336948 & Barry & Radiotherapy and breast reconstruction: a meta-analysis & $\begin{array}{l}\text { Breast Cancer Res } \\
\text { Treat }\end{array}$ & $\begin{array}{l}\text { Narrative review/ } \\
\text { Commentary }\end{array}$ \\
\hline 66 & 29452438 & Bartlett & $\begin{array}{l}\text { Algorithmic Approach for Intraoperative Salvage of Venous Congestion in DIEP } \\
\text { Flaps }\end{array}$ & $\begin{array}{l}J \text { Reconstr } \\
\text { Microsurg }\end{array}$ & $\begin{array}{l}\text { Single group } \mathrm{N} \text { enrolled } \\
<500\end{array}$ \\
\hline 67 & 26218386 & Basta & $\begin{array}{l}\text { A Propensity-Matched Analysis of the Influence of Breast Reconstruction on } \\
\text { Subsequent Development of Lymphedema }\end{array}$ & $\begin{array}{l}\text { Plast Reconstr } \\
\text { Surg }\end{array}$ & No outcome of interest \\
\hline 68 & 26595013 & Basta & $\begin{array}{l}\text { A Systematic Review and Head-to-Head Meta-Analysis of Outcomes following } \\
\text { Direct-to-Implant versus Conventional Two-Stage Implant Reconstruction }\end{array}$ & $\begin{array}{l}\text { Plast Reconstr } \\
\text { Surg }\end{array}$ & $\begin{array}{l}\text { Does not address KQ1- } \\
\text { KQ6 }\end{array}$ \\
\hline
\end{tabular}




\begin{tabular}{|c|c|c|c|c|c|}
\hline No. & $\begin{array}{l}\text { PMID or } \\
\text { Other } \\
\text { Identifier }\end{array}$ & $\begin{array}{l}\text { First Author } \\
\text { Last Name }\end{array}$ & Title & Journal & Reason for Exclusion \\
\hline 69 & 31650347 & Batenburg & $\begin{array}{l}\text { Patient-reported cosmetic satisfaction and the long-term association with quality of } \\
\text { life in irradiated breast cancer patients }\end{array}$ & $\begin{array}{l}\text { Breast Cancer Res } \\
\text { Treat }\end{array}$ & $\begin{array}{l}\text { Single group } \mathrm{N} \text { enrolled } \\
<500\end{array}$ \\
\hline 70 & 28743179 & Becherer & $\begin{array}{l}\text { Prevalence of psychiatric comorbidities among women undergoing free tissue } \\
\text { autologous breast reconstruction }\end{array}$ & J Surg Oncol & $\begin{array}{l}\text { Does not address KQ1- } \\
\text { KQ6 }\end{array}$ \\
\hline 71 & 18843123 & Beier & $\begin{array}{l}\text { Breast reconstruction after breast-cancer surgery...N Engl J Med. } 2008 \text { Oct } \\
\text { 9;359(15):1590-601 }\end{array}$ & $\begin{array}{l}\text { New England } \\
\text { Journal of Medicine }\end{array}$ & $\begin{array}{l}\text { Narrative review/ } \\
\text { Commentary }\end{array}$ \\
\hline 72 & None & Beier & $\begin{array}{l}\text { Human Acellular Dermal Matrix (Epiflex®) in Immediate Implant-Based Breast } \\
\text { Reconstruction after Skin- A nd Nipple-Sparing Mastectomy and Treatment of } \\
\text { Capsular Fibrosis: Results of a Multicenter, Prospective, Observational NOGGO- } \\
\text { AWOGyn Study }\end{array}$ & Breast Care & $\begin{array}{l}\text { Single group } \mathrm{N} \text { enrolled } \\
<500\end{array}$ \\
\hline 73 & 10783510 & Benadiba & [Survivorship of breast implants used in breast reconstruction. 949 implants] & $\begin{array}{l}\text { Ann Chir Plast } \\
\text { Esthet }\end{array}$ & Unable to retrieve article \\
\hline 74 & None & Bence & $\begin{array}{l}\text { Assessing the needs of Hungarian breast cancer patients for modern oncoplastic } \\
\text { breast surgical treatment: Questionnaire study of } 500 \text { patients }\end{array}$ & Orvosi Hetilap & Unable to retrieve article \\
\hline 75 & 30003763 & Benderli Cihan & The role of radiotherapy following mastectomy and reconstruction & J buon & $\begin{array}{l}\text { Narrative review/ } \\
\text { Commentary }\end{array}$ \\
\hline 76 & 24737845 & $\begin{array}{l}\text { Benditte- } \\
\text { Klepetko }\end{array}$ & $\begin{array}{l}\text { Analysis of patient satisfaction and donor-site morbidity after different types of } \\
\text { breast reconstruction }\end{array}$ & Scand J Surg & NRCS $<30$ per arm \\
\hline 77 & 18090813 & Bengtson & Style 410 highly cohesive silicone breast implant core study results at 3 years & $\begin{array}{l}\text { Plast Reconstr } \\
\text { Surg }\end{array}$ & NRCS not adjusted \\
\hline 78 & 28658472 & Bennett & $\begin{array}{l}\text { Association of Fat Grafting With Patient-Reported Outcomes in Postmastectomy } \\
\text { Breast Reconstruction }\end{array}$ & JAMA Surg & $\begin{array}{l}\text { Does not address KQ1- } \\
\text { KQ6 }\end{array}$ \\
\hline 79 & 29506826 & Berlin & $\begin{array}{l}\text { Nonresponse bias in survey research: lessons from a prospective study of breast } \\
\text { reconstruction }\end{array}$ & J Surg Res & $\begin{array}{l}\text { Does not address KQ1- } \\
\text { KQ6 }\end{array}$ \\
\hline 80 & 28215963 & Berlin & $\begin{array}{l}\text { Racial and ethnic variations in one-year clinical and patient-reported outcomes } \\
\text { following breast reconstruction }\end{array}$ & Am J Surg & $\begin{array}{l}\text { Does not address KQ1- } \\
\text { KQ6 }\end{array}$ \\
\hline 81 & 29489482 & Berlin & $\begin{array}{l}\text { Hospital Variations in Clinical Complications and Patient-reported Outcomes at } 2 \\
\text { Years After Immediate Breast Reconstruction }\end{array}$ & Ann Surg & $\begin{array}{l}\text { Does not address KQ1- } \\
\text { KQ6 }\end{array}$ \\
\hline 82 & 20853034 & Berry & $\begin{array}{l}\text { Complication rates of radiation on tissue expander and autologous tissue breast } \\
\text { reconstruction }\end{array}$ & Ann Surg Oncol & No outcome of interest \\
\hline 83 & 28918997 & Berthet & $\begin{array}{l}\text { Tolerance of latissimus dorsi in immediate breast reconstruction without implant to } \\
\text { radiotherapy }\end{array}$ & $\begin{array}{l}\text { J Plast Reconstr } \\
\text { Aesthet Surg }\end{array}$ & $\begin{array}{l}\text { Single group } \mathrm{N} \text { enrolled } \\
<500\end{array}$ \\
\hline 84 & 28831365 & Bettinger & $\begin{array}{l}\text { Comparative Study of Prepectoral and Subpectoral Expander-Based Breast } \\
\text { Reconstruction and Clavien IIlb Score Outcomes }\end{array}$ & $\begin{array}{l}\text { Plast Reconstr } \\
\text { Surg Glob Open }\end{array}$ & $\begin{array}{l}\text { Single group } \mathrm{N} \text { enrolled } \\
<500\end{array}$ \\
\hline 85 & 29399731 & Beugels & $\begin{array}{l}\text { Complications following immediate compared to delayed deep inferior epigastric } \\
\text { artery perforator flap breast reconstructions }\end{array}$ & $\begin{array}{l}\text { Breast Cancer } \\
\text { Research and } \\
\text { Treatment }\end{array}$ & $\begin{array}{l}\text { Duplicate of another } \\
\text { publication }\end{array}$ \\
\hline 86 & 30247195 & Beugels & $\begin{array}{l}\text { Quality of Life of Patients After Immediate or Delayed Autologous Breast } \\
\text { Reconstruction: A Multicenter Study }\end{array}$ & $\begin{array}{l}\text { Annals of plastic } \\
\text { surgery }\end{array}$ & $\begin{array}{l}\text { Duplicate of another } \\
\text { publication }\end{array}$ \\
\hline 87 & 31030303 & Beugels & $\begin{array}{l}\text { The influence of neoadjuvant chemotherapy on complications of immediate DIEP } \\
\text { flap breast reconstructions }\end{array}$ & $\begin{array}{l}\text { Breast Cancer Res } \\
\text { Treat }\end{array}$ & $\begin{array}{l}\text { Single group } \mathrm{N} \text { enrolled } \\
<500\end{array}$ \\
\hline
\end{tabular}




\begin{tabular}{|c|c|c|c|c|c|}
\hline No. & $\begin{array}{l}\text { PMID or } \\
\text { Other } \\
\text { Identifier }\end{array}$ & $\begin{array}{l}\text { First Author } \\
\text { Last Name }\end{array}$ & Title & Journal & Reason for Exclusion \\
\hline 88 & 27449746 & Beugels & $\begin{array}{l}\text { Reply to the Letter to the Editor by Wade et al. 'The importance of the Unit of } \\
\text { Analysis'. Commentary on: Beugels } \mathrm{J} \text { et al. Complications in unilateral versus } \\
\text { bilateral deep inferior epigastric artery perforator flap breast reconstructions: A } \\
\text { multicentre study }\end{array}$ & $\begin{array}{l}J \text { Plast Reconstr } \\
\text { Aesthet Surg }\end{array}$ & $\begin{array}{l}\text { Single group } \mathrm{N} \text { enrolled } \\
<500\end{array}$ \\
\hline 89 & 19634163 & Bezuhly & $\begin{array}{l}\text { Immediate postmastectomy reconstruction is associated with improved breast } \\
\text { cancer-specific survival: Evidence and new challenges from the surveillance, } \\
\text { epidemiology, and end results database }\end{array}$ & Cancer & $\begin{array}{l}\text { Duplicate of another } \\
\text { publication }\end{array}$ \\
\hline 90 & 19634163 & Bezuhly & $\begin{array}{l}\text { Immediate postmastectomy reconstruction is associated with improved breast } \\
\text { cancer-specific survival: evidence and new challenges from the Surveillance, } \\
\text { Epidemiology, and End Results database }\end{array}$ & Cancer & $\begin{array}{l}\text { Does not address KQ1- } \\
\text { KQ6 }\end{array}$ \\
\hline 91 & 28198770 & Billig & $\begin{array}{l}\text { Should Immediate Autologous Breast Reconstruction Be Considered in Women } \\
\text { Who Require Postmastectomy Radiation Therapy? A Prospective Analysis of } \\
\text { Outcomes }\end{array}$ & $\begin{array}{l}\text { Plast Reconstr } \\
\text { Surg }\end{array}$ & $\begin{array}{l}\text { Single group } \mathrm{N} \text { enrolled } \\
<500\end{array}$ \\
\hline 92 & 28724133 & Billig & $\begin{array}{l}\text { A Nationwide Analysis of Cost Variation for Autologous Free Flap Breast } \\
\text { Reconstruction }\end{array}$ & JAMA Surg & $\begin{array}{l}\text { Single group }>500 \text {, but } \\
\text { no complications data }\end{array}$ \\
\hline 93 & 27286854 & Billner & $\begin{array}{l}\text { Poly Implant Prothese and Rofil Substandard Breast Implant Explantations from a } \\
\text { Large German Single Centre from } 2011 \text { to 2014: A Comparative Study }\end{array}$ & $\begin{array}{l}\text { Aesthetic Plast } \\
\text { Surg }\end{array}$ & $\begin{array}{l}\text { Single group } \mathrm{N} \text { enrolled } \\
<500\end{array}$ \\
\hline 94 & 28676319 & Billon & $\begin{array}{l}\text { Impact of adjuvant anti-estrogen therapies (tamoxifen and aromatase inhibitors) on } \\
\text { perioperative outcomes of breast reconstruction }\end{array}$ & $\begin{array}{l}J \text { Plast Reconstr } \\
\text { Aesthet Surg }\end{array}$ & $\begin{array}{l}\text { Does not address KQ1- } \\
\text { KQ6 }\end{array}$ \\
\hline 95 & None & Bjelic-Radisic & $\begin{array}{l}\text { Short-term outcome and complications rate after immediate breast reconstruction } \\
\text { with implants and acellular dermis }\end{array}$ & $\begin{array}{l}\text { European Journal } \\
\text { of Cancer }\end{array}$ & $\begin{array}{l}\text { Single group } \mathrm{N} \text { enrolled } \\
<500\end{array}$ \\
\hline 96 & 28185324 & Blackburn & $\begin{array}{l}\text { The musculoskeletal consequences of breast reconstruction using the latissimus } \\
\text { dorsi muscle for women following mastectomy for breast cancer: A critical review }\end{array}$ & $\begin{array}{l}\text { Eur J Cancer Care } \\
\text { (Engl) }\end{array}$ & $\begin{array}{l}\text { Does not address KQ1- } \\
\text { KQ6 }\end{array}$ \\
\hline 97 & 32557451 & Blankensteijn & $\begin{array}{l}\text { Racial Disparities in Outcomes of Reconstructive Breast Surgery: An Analysis of } \\
51,362 \text { Patients from the ACS-NSQIP }\end{array}$ & $\begin{array}{l}\text { J Reconstr } \\
\text { Microsurg }\end{array}$ & NRCS not adjusted \\
\hline 98 & 9583482 & Blomqvist & The inflammatory reaction in elective flap surgery & $\begin{array}{l}\text { Plast Reconstr } \\
\text { Surg }\end{array}$ & NRCS $<30$ per arm \\
\hline 99 & 9245865 & Blondeel & $\begin{array}{l}\text { The donor site morbidity of free DIEP flaps and free TRAM flaps for breast } \\
\text { reconstruction }\end{array}$ & Br J Plast Surg & NRCS not adjusted \\
\hline 100 & 10343589 & Blondeel & $\begin{array}{l}\text { Sensory nerve repair in perforator flaps for autologous breast reconstruction: } \\
\text { sensational or senseless? }\end{array}$ & Br J Plast Surg & NRCS not adjusted \\
\hline 101 & 25382588 & Bodin & $\begin{array}{l}\text { Venous coupler use for free-flap breast reconstructions: specific analyses of TMG } \\
\text { and DIEP flaps }\end{array}$ & Microsurgery & NRCS $<30$ per arm \\
\hline 102 & 32434696 & Boehm & $\begin{array}{l}\text { Increasing abdominal wall thickness predicts complications in abdominally based } \\
\text { breast reconstruction: A review of } 106 \text { consecutive patients }\end{array}$ & $\begin{array}{l}\text { J Plast Reconstr } \\
\text { Aesthet Surg }\end{array}$ & $\begin{array}{l}\text { Single group } \mathrm{N} \text { enrolled } \\
<500\end{array}$ \\
\hline 103 & 10826338 & Boikov & [Reconstructive surgery after mastectomy--the anatomical prerequisites] & $\begin{array}{l}\text { Akush Ginekol } \\
\text { (Sofiia) }\end{array}$ & Unable to retrieve article \\
\hline 104 & 22395320 & Bonomi & $\begin{array}{l}\text { Current indications for and comparative analysis of three different types of } \\
\text { latissimus dorsi flaps }\end{array}$ & Aesthet Surg J & $\begin{array}{l}\text { Single group N enrolled } \\
<500\end{array}$ \\
\hline 105 & 28766231 & Boughey & $\begin{array}{l}\text { Contralateral Prophylactic Mastectomy with Immediate Breast Reconstruction } \\
\text { Increases Healthcare Utilization and Cost }\end{array}$ & Ann Surg Oncol & No outcome of interest \\
\hline
\end{tabular}




\begin{tabular}{|c|c|c|c|c|c|}
\hline No. & $\begin{array}{l}\text { PMID or } \\
\text { Other } \\
\text { Identifier }\end{array}$ & $\begin{array}{l}\text { First Author } \\
\text { Last Name }\end{array}$ & Title & Journal & Reason for Exclusion \\
\hline 106 & 19483561 & Boyd & $\begin{array}{l}\text { Comparison of superior gluteal artery musculocutaneous and superior gluteal } \\
\text { artery perforator flaps for microvascular breast reconstruction }\end{array}$ & $\begin{array}{l}\text { Plast Reconstr } \\
\text { Surg }\end{array}$ & NRCS not adjusted \\
\hline 107 & 31083604 & Brandon & $\begin{array}{l}\text { New Evaluation Procedure for Multi-Dimensional Mechanical Strains and Tangent } \\
\text { Moduli of Breast Implants: IDEAL IMPLANT((R)) Structured Breast Implant } \\
\text { Compared to Silicone Gel Implants }\end{array}$ & $\begin{array}{l}\text { Bioengineering } \\
\text { (Basel) }\end{array}$ & $\begin{array}{l}\text { Not breast } \\
\text { reconstruction }\end{array}$ \\
\hline 108 & 32699924 & Braun & $\begin{array}{l}\text { Do Nipple Necrosis Rates Differ in Prepectoral Versus Submuscular Implant-Based } \\
\text { Reconstruction After Nipple-Sparing Mastectomy? }\end{array}$ & Ann Surg Oncol & NRCS not adjusted \\
\hline 109 & 28513834 & Browne & $\begin{array}{l}\text { The association between complications and quality of life after mastectomy and } \\
\text { breast reconstruction for breast cancer }\end{array}$ & Cancer & $\begin{array}{l}\text { Does not address KQ1- } \\
\text { KQ6 }\end{array}$ \\
\hline 110 & 28958570 & Browne & $\begin{array}{l}\text { Measuring the patient perspective on latissimus dorsi donor site outcomes } \\
\text { following breast reconstruction }\end{array}$ & $\begin{array}{l}J \text { Plast Reconstr } \\
\text { Aesthet Surg }\end{array}$ & $\begin{array}{l}\text { Does not address KQ1- } \\
\text { KQ6 }\end{array}$ \\
\hline 111 & 125841979 & Browne & $\begin{array}{l}\text { The association between complications and quality of life after mastectomy and } \\
\text { breast reconstruction for breast cancer }\end{array}$ & Cancer (0008543X) & $\begin{array}{l}\text { Duplicate of another } \\
\text { publication }\end{array}$ \\
\hline 112 & 28701264 & Buckley & $\begin{array}{l}\text { Impact of rural-urban status on survival after mastectomy without reconstruction } \\
\text { versus mastectomy with reconstruction }\end{array}$ & Am J Surg & $\begin{array}{l}\text { Does not address KQ1- } \\
\text { KQ6 }\end{array}$ \\
\hline 113 & 28634720 & Bucknor & $\begin{array}{l}\text { The financial impact and drivers of hospital charges in contralateral prophylactic } \\
\text { mastectomy and reconstruction: a Nationwide Inpatient Sample hospital analysis }\end{array}$ & $\begin{array}{l}\text { Breast Cancer Res } \\
\text { Treat }\end{array}$ & $\begin{array}{l}\text { Single group }>500 \text {, but } \\
\text { no complications data }\end{array}$ \\
\hline 114 & 23542856 & Buseman & $\begin{array}{l}\text { Comparison of sterile versus nonsterile acellular dermal matrices for breast } \\
\text { reconstruction }\end{array}$ & Ann Plast Surg & NRCS $<30$ per arm \\
\hline 115 & 25576165 & Butler & $\begin{array}{l}\text { African-American women have equivalent outcomes following autologous free flap } \\
\text { breast reconstruction despite greater preoperative risk factors }\end{array}$ & Am J Surg & $\begin{array}{l}\text { Copublication of } \\
\text { included study with no } \\
\text { new data }\end{array}$ \\
\hline 116 & 26545345 & Butler & $\begin{array}{l}\text { Racial and age disparities persist in immediate breast reconstruction: an updated } \\
\text { analysis of } 48,564 \text { patients from the } 2005 \text { to } 2011 \text { American College of Surgeons } \\
\text { National Surgery Quality Improvement Program data sets }\end{array}$ & Am J Surg & $\begin{array}{l}\text { Does not address KQ1- } \\
\text { KQ6 }\end{array}$ \\
\hline 117 & 25626808 & Butz & $\begin{array}{l}\text { Advanced age is a predictor of } 30 \text {-day complications after autologous but not } \\
\text { implant-based postmastectomy breast reconstruction }\end{array}$ & $\begin{array}{l}\text { Plast Reconstr } \\
\text { Surg }\end{array}$ & $\begin{array}{l}\text { Does not address KQ1- } \\
\text { KQ6 }\end{array}$ \\
\hline 118 & 16490892 & Byung & $\begin{array}{l}\text { Changing patterns in the clinical characteristics of Korean patients with breast } \\
\text { cancer during the last } 15 \text { years }\end{array}$ & Archives of Surgery & $\begin{array}{l}\text { Single group } \mathrm{N} \text { enrolled } \\
<500\end{array}$ \\
\hline 119 & 27047784 & Cabalag & Alloplastic adjuncts in breast reconstruction & Gland Surg & $\begin{array}{l}\text { Does not address KQ1- } \\
\text { KQ6 }\end{array}$ \\
\hline 120 & 31079136 & Cai & $\begin{array}{l}\text { Autologous Breast Reconstruction with Transverse Rectus Abdominis } \\
\text { Musculocutaneous (TRAM) or Deep Inferior Epigastric Perforator (DIEP) Flaps: An } \\
\text { Analysis of the } 100 \text { Most Cited Articles }\end{array}$ & Med Sci Monit & $\begin{array}{l}\text { Narrative review/ } \\
\text { Commentary }\end{array}$ \\
\hline 121 & 32149849 & Cai & $\begin{array}{l}\text { National Trends in Hospitalization Charges for Autologous Free Flap Breast } \\
\text { Reconstruction }\end{array}$ & Ann Plast Surg & $\begin{array}{l}\text { Single group }>500 \text {, but } \\
\text { no complications data }\end{array}$ \\
\hline 122 & 29595713 & Calobrace & $\begin{array}{l}\text { Introduction to 'Sientra Shaped and Round Cohesive Gel Implants: Long-Term } \\
\text { Safety Outcomes' }\end{array}$ & $\begin{array}{l}\text { Plast Reconstr } \\
\text { Surg }\end{array}$ & $\begin{array}{l}\text { Narrative review/ } \\
\text { Commentary }\end{array}$ \\
\hline 123 & 25158767 & Caplin & $\begin{array}{l}\text { Indications for the use of MemoryShape breast implants in aesthetic and } \\
\text { reconstructive breast surgery: long-term clinical outcomes of shaped versus round } \\
\text { silicone breast implants }\end{array}$ & $\begin{array}{l}\text { Plast Reconstr } \\
\text { Surg }\end{array}$ & $\begin{array}{l}\text { Not breast } \\
\text { reconstruction }\end{array}$ \\
\hline
\end{tabular}




\begin{tabular}{|c|c|c|c|c|c|}
\hline No. & $\begin{array}{l}\text { PMID or } \\
\text { Other } \\
\text { Identifier }\end{array}$ & $\begin{array}{l}\text { First Author } \\
\text { Last Name }\end{array}$ & Title & Journal & Reason for Exclusion \\
\hline 124 & 33308993 & Caputo & $\begin{array}{l}\text { Quality of Life and Early Functional Evaluation in Direct-to-Implant Breast } \\
\text { Reconstruction After Mastectomy: A Comparative Study Between Prepectoral } \\
\text { Versus Dual-Plane Reconstruction }\end{array}$ & Clin Breast Cancer & NRCS not adjusted \\
\hline 125 & 11293510 & Carlson & Results of immediate breast reconstruction after skin-sparing mastectomy & Ann Plast Surg & NRCS not adjusted \\
\hline 126 & 18434833 & Carlson & $\begin{array}{l}\text { Effects of radiation therapy on pedicled transverse rectus abdominis } \\
\text { myocutaneous flap breast reconstruction }\end{array}$ & Ann Plast Surg & NRCS $<30$ per arm \\
\hline 127 & 12620903 & Carlson & $\begin{array}{l}\text { Local recurrence after skin-sparing mastectomy: Tumor biology or surgical } \\
\text { conservatism? }\end{array}$ & $\begin{array}{l}\text { Annals of Surgical } \\
\text { Oncology }\end{array}$ & $\begin{array}{l}\text { Single group }>500 \text {, but } \\
\text { no complications data }\end{array}$ \\
\hline 128 & 14998566 & Carlson & $\begin{array}{l}\text { The use of skin sparing mastectomy in the treatment of breast cancer: The Emory } \\
\text { experience }\end{array}$ & Surg Oncol & $\begin{array}{l}\text { Not breast } \\
\text { reconstruction }\end{array}$ \\
\hline 129 & 30656095 & Carminati & $\begin{array}{l}\text { Immediate Implant-based Breast Reconstruction with Acellular Dermal Matrix } \\
\text { Compared with Tissue-expander Breast Reconstruction: Rate of Infection }\end{array}$ & $\begin{array}{l}\text { Plast Reconstr } \\
\text { Surg Glob Open }\end{array}$ & $\begin{array}{l}\text { Does not address KQ1- } \\
\text { KQ6 }\end{array}$ \\
\hline 130 & 27406093 & Carter & $\begin{array}{l}\text { Operative and Oncologic Outcomes in } 9861 \text { Patients with Operable Breast Cancer: } \\
\text { Single-Institution Analysis of Breast Conservation with Oncoplastic Reconstruction }\end{array}$ & Ann Surg Oncol & $\begin{array}{l}\text { Does not address KQ1- } \\
\text { KQ6 }\end{array}$ \\
\hline 131 & 27600636 & Casella & $\begin{array}{l}\text { Current trends and outcomes of breast reconstruction following nipple-sparing } \\
\text { mastectomy: results from a national multicentric registry with } 1006 \text { cases over a 6- } \\
\text { year period }\end{array}$ & Breast Cancer & $\begin{array}{l}\text { Single group }>500 \text {, but } \\
\text { no complications data }\end{array}$ \\
\hline 132 & 25339795 & Casella & $\begin{array}{l}\text { TiLoop® Bra mesh used for immediate breast reconstruction: comparison } \\
\text { of retropectoral and subcutaneous implant placement in a prospective } \\
\text { single-institution series }\end{array}$ & $\begin{array}{l}\text { European journal of } \\
\text { plastic surgery }\end{array}$ & NRCS $<30$ per arm \\
\hline 133 & 25339795 & Casella & $\begin{array}{l}\text { TiLoop® Bra mesh used for immediate breast reconstruction: comparison of } \\
\text { retropectoral and subcutaneous implant placement in a prospective single- } \\
\text { institution series }\end{array}$ & $\begin{array}{l}\text { European Journal } \\
\text { of Plastic Surgery }\end{array}$ & $\begin{array}{l}\text { Duplicate of another } \\
\text { publication }\end{array}$ \\
\hline 134 & 22941163 & Casey & Etiology of breast masses after autologous breast reconstruction & Ann Surg Oncol & $\begin{array}{l}\text { Single group N enrolled } \\
<500\end{array}$ \\
\hline 135 & 29316048 & Cassidy & Does response to neo-adjuvant chemotherapy impact breast reconstruction? & Breast J & $\begin{array}{l}\text { Does not address KQ1- } \\
\text { KQ6 }\end{array}$ \\
\hline 136 & 30507481 & Catanuto & Quality of life after breast reconstruction-the BRIOS study & Lancet Oncology & $\begin{array}{l}\text { Duplicate of another } \\
\text { publication }\end{array}$ \\
\hline 137 & 29275104 & Cattelani & $\begin{array}{l}\text { One-Step Prepectoral Breast Reconstruction With Dermal Matrix-Covered Implant } \\
\text { Compared to Submuscular Implantation: Functional and Cost Evaluation }\end{array}$ & Clin Breast Cancer & $\begin{array}{l}\text { Duplicate of another } \\
\text { publication }\end{array}$ \\
\hline 138 & 8579262 & Cederna & $\begin{array}{l}\text { Postmastectomy reconstruction: comparative analysis of the psychosocial, } \\
\text { functional, and cosmetic effects of transverse rectus abdominis musculocutaneous } \\
\text { flap versus breast implant reconstruction }\end{array}$ & Ann Plast Surg & NRCS $<30$ per arm \\
\hline 139 & 31356269 & Cerullo & $\begin{array}{l}\text { Is Bigger Better?: The Effect of Hospital Consolidation on Index Hospitalization } \\
\text { Costs and Outcomes Among Privately Insured Recipients of Immediate Breast } \\
\text { Reconstruction }\end{array}$ & Ann Surg & $\begin{array}{l}\text { Single group }>500 \text {, but } \\
\text { no complications data }\end{array}$ \\
\hline 140 & 24316992 & Cha & $\begin{array}{l}\text { Patient-reported outcomes following breast reconstruction surgery in a public } \\
\text { hospital: use of the Breast-Q questionnaire }\end{array}$ & N Z Med J & NRCS not adjusted \\
\hline 141 & 32309084 & Chan & $\begin{array}{l}\text { No-drain Technique in Abdominal Closure for Breast Reconstruction: Lower } \\
\text { Complication Rate, Shorter Hospitalization Stay }\end{array}$ & $\begin{array}{l}\text { Plast Reconstr } \\
\text { Surg Glob Open }\end{array}$ & NRCS $<30$ per arm \\
\hline
\end{tabular}




\begin{tabular}{|c|c|c|c|c|c|}
\hline No. & $\begin{array}{l}\text { PMID or } \\
\text { Other } \\
\text { Identifier }\end{array}$ & $\begin{array}{l}\text { First Author } \\
\text { Last Name }\end{array}$ & Title & Journal & Reason for Exclusion \\
\hline 142 & 18626350 & Chang & $\begin{array}{l}\text { Effects of an autologous flap combined with an implant for breast reconstruction: } \\
\text { an evaluation of } 1000 \text { consecutive reconstructions of previously irradiated breasts }\end{array}$ & $\begin{array}{l}\text { Plast Reconstr } \\
\text { Surg }\end{array}$ & NRCS not adjusted \\
\hline 143 & 20048582 & Chang & Breast reconstruction and lymphedema & $\begin{array}{l}\text { Plast Reconstr } \\
\text { Surg }\end{array}$ & NRCS not adjusted \\
\hline 144 & 10845289 & Chang & $\begin{array}{l}\text { Effect of smoking on complications in patients undergoing free TRAM flap breast } \\
\text { reconstruction }\end{array}$ & $\begin{array}{l}\text { Plast Reconstr } \\
\text { Surg }\end{array}$ & $\begin{array}{l}\text { Copublication of } \\
\text { included study with no } \\
\text { new data }\end{array}$ \\
\hline 145 & 10845289 & Chang & $\begin{array}{l}\text { Effect of smoking on complications in patients undergoing free TRAM flap breast } \\
\text { reconstruction }\end{array}$ & $\begin{array}{l}\text { Plastic and } \\
\text { Reconstructive } \\
\text { Surgery }\end{array}$ & $\begin{array}{l}\text { Duplicate of another } \\
\text { publication }\end{array}$ \\
\hline 146 & 10809092 & Chang & $\begin{array}{l}\text { Effect of obesity on flap and donor-site complications in free transverse rectus } \\
\text { abdominis myocutaneous flap breast reconstruction }\end{array}$ & $\begin{array}{l}\text { Plastic and } \\
\text { Reconstructive } \\
\text { Surgery }\end{array}$ & $\begin{array}{l}\text { Duplicate of another } \\
\text { publication }\end{array}$ \\
\hline 147 & 25626809 & Chang & $\begin{array}{l}\text { Challenging a traditional paradigm: 12-year experience with autologous free flap } \\
\text { breast reconstruction for inflammatory breast cancer }\end{array}$ & $\begin{array}{l}\text { Plast Reconstr } \\
\text { Surg }\end{array}$ & $\begin{array}{l}\text { Single group } \mathrm{N} \text { enrolled } \\
<500\end{array}$ \\
\hline 148 & 23123613 & Chang & $\begin{array}{l}\text { Simultaneous contralateral reduction mammoplasty or mastopexy during unilateral } \\
\text { free flap breast reconstruction }\end{array}$ & Ann Plast Surg & $\begin{array}{l}\text { Single group } \mathrm{N} \text { enrolled } \\
<500\end{array}$ \\
\hline 149 & 27866726 & Chang & $\begin{array}{l}\text { Trends in mastectomy and reconstruction for breast cancer; a twelve year } \\
\text { experience from a tertiary care center }\end{array}$ & Am J Surg & $\begin{array}{l}\text { Single group } \mathrm{N} \text { enrolled } \\
<500\end{array}$ \\
\hline 150 & 132784872 & Chang & $\begin{array}{l}\text { Influence of Hypofractionated Radiation Therapy Following Mastectomy on } \\
\text { Complication in Breast Cancer Patients Undergoing Two-Stage Prosthetic Breast } \\
\text { Reconstruction }\end{array}$ & $\begin{array}{l}\text { International } \\
\text { Journal of } \\
\text { Radiation } \\
\text { Oncology, Biology, } \\
\text { Physics }\end{array}$ & $\begin{array}{l}\text { Single group } \mathrm{N} \text { enrolled } \\
<500\end{array}$ \\
\hline 151 & 15096930 & Chang & Reconstruction of complex oncologic chest wall defects: a 10-year experience & Ann Plast Surg & $\begin{array}{l}\text { Single group } \mathrm{N} \text { enrolled } \\
<500\end{array}$ \\
\hline 152 & 26289806 & Chao & $\begin{array}{l}\text { Processes of Care in Breast Reconstruction and the Long-Term Impact of a } \\
\text { Comprehensive Breast Center }\end{array}$ & Ann Surg Oncol & $\begin{array}{l}\text { Does not address KQ1- } \\
\text { KQ6 }\end{array}$ \\
\hline 153 & $\begin{array}{l}\text { CN- } \\
01421299\end{array}$ & Charton & $\begin{array}{l}\text { Time to health-related quality of life score deterioration at 1-year follow-up after } \\
\text { immediate latissimus dorsi breast reconstructions: a prospective study in breast } \\
\text { cancer }\end{array}$ & $\begin{array}{l}\text { Quality of life } \\
\text { research }\end{array}$ & $\begin{array}{l}\text { Single group } \mathrm{N} \text { enrolled } \\
<500\end{array}$ \\
\hline 154 & 25719690 & Chatterjee & $\begin{array}{l}\text { The use of mesh versus primary fascial closure of the abdominal donor site when } \\
\text { using a transverse rectus abdominis myocutaneous flap for breast reconstruction: } \\
\text { a cost-utility analysis }\end{array}$ & $\begin{array}{l}\text { Plast Reconstr } \\
\text { Surg }\end{array}$ & $\begin{array}{l}\text { Does not address KQ1- } \\
\text { KQ6 }\end{array}$ \\
\hline 155 & 28833196 & Chattha & $\begin{array}{l}\text { Comparison of risk factors and complications in patients by stratified mastectomy } \\
\text { weight: An institutional review of } 1041 \text { consecutive cases }\end{array}$ & J Surg Oncol & $\begin{array}{l}\text { Does not address KQ1- } \\
\text { KQ6 }\end{array}$ \\
\hline 156 & 32102003 & Chattha & $\begin{array}{l}\text { Revisiting the Relationship Between Hospital Case Volume and Outcomes in } \\
\text { Abdominally Based Free Flap Breast Reconstruction }\end{array}$ & Ann Plast Surg & $\begin{array}{l}\text { Does not address KQ1- } \\
\text { KQ6 }\end{array}$ \\
\hline 157 & 12243831 & Chawla & $\begin{array}{l}\text { Radiotherapy and breast reconstruction: complications and cosmesis with TRAM } \\
\text { versus tissue expander/implant }\end{array}$ & $\begin{array}{l}\text { Int J Radiat Oncol } \\
\text { Biol Phys }\end{array}$ & NRCS $<30$ per arm \\
\hline
\end{tabular}




\begin{tabular}{|c|c|c|c|c|c|}
\hline No. & $\begin{array}{l}\text { PMID or } \\
\text { Other } \\
\text { Identifier }\end{array}$ & $\begin{array}{l}\text { First Author } \\
\text { Last Name }\end{array}$ & Title & Journal & Reason for Exclusion \\
\hline 158 & 20548236 & Chen & $\begin{array}{l}\text { Effects of vasopressor administration on the outcomes of microsurgical breast } \\
\text { reconstruction }\end{array}$ & Ann Plast Surg & NRCS not adjusted \\
\hline 159 & 18040176 & Chen & $\begin{array}{l}\text { Immediate postoperative complications in DIEP versus free/muscle-sparing TRAM } \\
\text { flaps }\end{array}$ & $\begin{array}{l}\text { Plast Reconstr } \\
\text { Surg }\end{array}$ & NRCS not adjusted \\
\hline 160 & 30497928 & Chen & $\begin{array}{l}\text { Immediate Breast Reconstruction in De Novo Metastatic Breast Cancer: An } \\
\text { Analysis of } 563 \text { Cases Based on the SEER Database }\end{array}$ & Clin Breast Cancer & $\begin{array}{l}\text { Does not address KQ1- } \\
\text { KQ6 }\end{array}$ \\
\hline 161 & 29442215 & Chen & $\begin{array}{l}\text { Meta-analysis for psychological impact of breast reconstruction in patients with } \\
\text { breast cancer }\end{array}$ & Breast Cancer & $\begin{array}{l}\text { Does not address KQ1- } \\
\text { KQ6 }\end{array}$ \\
\hline 162 & 16128096 & Chen & [The clinic analysis of complications of varied breast implant] & $\begin{array}{l}\text { Zhonghua Zheng } \\
\text { Xing Wai Ke Za Zhi }\end{array}$ & NRCS not adjusted \\
\hline 163 & 31417055 & Chen & $\begin{array}{l}\text { A short follow-up of prosthesis-based breast reconstruction using TiLOOP((R)) Bra } \\
\text { surgical mesh }\end{array}$ & Niger J Clin Pract & $\begin{array}{l}\text { Single group } \mathrm{N} \text { enrolled } \\
<500\end{array}$ \\
\hline 164 & 16772907 & Cheng & $\begin{array}{l}\text { Comparisons of resource costs and success rates between immediate and delayed } \\
\text { breast reconstruction using DIEP or SIEA flaps under a well-controlled clinical trial }\end{array}$ & $\begin{array}{l}\text { Plast Reconstr } \\
\text { Surg }\end{array}$ & NRCS $<30$ per arm \\
\hline 165 & 32895461 & Cheng & $\begin{array}{l}\text { A retrospective study to compare the clinical effects of individualized anatomic } \\
\text { single- and double-bundle anterior cruciate ligament reconstruction surgery }\end{array}$ & Sci Rep & $\begin{array}{l}\text { Not breast } \\
\text { reconstruction }\end{array}$ \\
\hline 166 & 32901309 & Cheng & $\begin{array}{l}\text { Comparisons Between Normal Body Mass Index and Overweight Patients Who } \\
\text { Underwent Unilateral Microsurgical Breast Reconstructions }\end{array}$ & Ann Surg Oncol & $\begin{array}{l}\text { Single group } \mathrm{N} \text { enrolled } \\
<500\end{array}$ \\
\hline 167 & 15457015 & Chevray & $\begin{array}{l}\text { Breast reconstruction with superficial inferior epigastric artery flaps: a prospective } \\
\text { comparison with TRAM and DIEP flaps }\end{array}$ & $\begin{array}{l}\text { Plast Reconstr } \\
\text { Surg }\end{array}$ & NRCS $<30$ per arm \\
\hline 168 & 32670569 & Chirappapha & $\begin{array}{l}\text { Comparisons of complications between extended latissimus dorsi flap and } \\
\text { latissimus dorsi flap in total breast reconstruction: A prospective cohort study }\end{array}$ & $\begin{array}{l}\text { Ann Med Surg } \\
\text { (Lond) }\end{array}$ & NRCS $<30$ per arm \\
\hline 169 & 31801159 & Cho & $\begin{array}{l}\text { Clinical Decision Making Using CTA in Conjoined, Bipedicled DIEP and SIEA for } \\
\text { Unilateral Breast Reconstruction }\end{array}$ & $\begin{array}{l}J \text { Reconstr } \\
\text { Microsurg }\end{array}$ & $\begin{array}{l}\text { Single group } \mathrm{N} \text { enrolled } \\
<500\end{array}$ \\
\hline 170 & 27465178 & Choi & $\begin{array}{l}\text { Breast in a Day': Examining Single-Stage Immediate, Permanent Implant } \\
\text { Reconstruction in Nipple-Sparing Mastectomy }\end{array}$ & $\begin{array}{l}\text { Plast Reconstr } \\
\text { Surg }\end{array}$ & $\begin{array}{l}\text { Single group } \mathrm{N} \text { enrolled } \\
<500\end{array}$ \\
\hline 171 & 1410349 & Chu & Radiation therapy of cancer in prosthetically augmented or reconstructed breasts & Radiology & NRCS $<30$ per arm \\
\hline 172 & 20555301 & Chun & $\begin{array}{l}\text { Comparison of morbidity, functional outcome, and satisfaction following bilateral } \\
\text { TRAM versus bilateral DIEP flap breast reconstruction }\end{array}$ & $\begin{array}{l}\text { Plast Reconstr } \\
\text { Surg }\end{array}$ & NRCS not adjusted \\
\hline 173 & 30791946 & Chung & $\begin{array}{l}\text { The effect of post mastectomy radiation therapy on breast reconstruction with and } \\
\text { without acellular dermal matrix: a systematic review and meta-analysis protocol }\end{array}$ & Syst Rev & $\begin{array}{l}\text { Protocol/methods with } \\
\text { no results }\end{array}$ \\
\hline 174 & 25910179 & Chung & $\begin{array}{l}\text { Surgical Site Infections after Free Flap Breast Reconstruction: An Analysis of 2,899 } \\
\text { Patients from the ACS-NSQIP Datasets }\end{array}$ & $\begin{array}{l}J \text { Reconstr } \\
\text { Microsurg }\end{array}$ & $\begin{array}{l}\text { Does not address KQ1- } \\
\text { KQ6 }\end{array}$ \\
\hline 175 & 23096982 & Clemens & $\begin{array}{l}\text { Acellular dermal matrix in irradiated tissue expander/implant-based breast } \\
\text { reconstruction: evidence-based review }\end{array}$ & $\begin{array}{l}\text { Plast Reconstr } \\
\text { Surg }\end{array}$ & Systematic review \\
\hline 176 & 26161307 & Clemens & $\begin{array}{l}\text { Current perspectives on radiation therapy in autologous and prosthetic breast } \\
\text { reconstruction }\end{array}$ & Gland Surg & $\begin{array}{l}\text { Narrative review/ } \\
\text { Commentary }\end{array}$ \\
\hline 177 & 12890459 & Cocquyt & $\begin{array}{l}\text { Better cosmetic results and comparable quality of life after skin-sparing } \\
\text { mastectomy and immediate autologous breast reconstruction compared to breast } \\
\text { conservative treatment }\end{array}$ & Br J Plast Surg & $\begin{array}{l}\text { Single group } \mathrm{N} \text { enrolled } \\
<500\end{array}$ \\
\hline
\end{tabular}




\begin{tabular}{|c|c|c|c|c|c|}
\hline No. & $\begin{array}{l}\text { PMID or } \\
\text { Other } \\
\text { Identifier }\end{array}$ & $\begin{array}{l}\text { First Author } \\
\text { Last Name }\end{array}$ & Title & Journal & Reason for Exclusion \\
\hline 178 & 12890459 & Cocquyt & $\begin{array}{l}\text { Better cosmetic results and comparable quality of life after skin-sparing } \\
\text { mastectomy and immediate autologous breast reconstruction compared to breast } \\
\text { conservative treatment }\end{array}$ & $\begin{array}{l}\text { British Journal of } \\
\text { Plastic Surgery }\end{array}$ & $\begin{array}{l}\text { Single group } \mathrm{N} \text { enrolled } \\
<500\end{array}$ \\
\hline 179 & 26505698 & Cohen & $\begin{array}{l}\text { Breast Implant-Associated Infections: The Role of the National Surgical Quality } \\
\text { Improvement Program and the Local Microbiome }\end{array}$ & $\begin{array}{l}\text { Plast Reconstr } \\
\text { Surg }\end{array}$ & $\begin{array}{l}>=10 \% \text { augmentation } \\
\text { reconstruction only }\end{array}$ \\
\hline 180 & 28336245 & Cohen & $\begin{array}{l}\text { Does the Timing of Chemotherapy Affect Post-Mastectomy Breast Reconstruction } \\
\text { Complications? }\end{array}$ & Clin Breast Cancer & $\begin{array}{l}\text { Single group N enrolled } \\
<500\end{array}$ \\
\hline 181 & 28841600 & Cohen & $\begin{array}{l}\text { Determining the Oncologic Safety of Autologous Fat Grafting as a Reconstructive } \\
\text { Modality: An Institutional Review of Breast Cancer Recurrence Rates and Surgical } \\
\text { Outcomes }\end{array}$ & $\begin{array}{l}\text { Plast Reconstr } \\
\text { Surg }\end{array}$ & NRCS not adjusted \\
\hline 182 & 26534828 & Cohen & $\begin{array}{l}\text { Is Unilateral Implant or Autologous Breast Reconstruction Better in Obtaining } \\
\text { Breast Symmetry? }\end{array}$ & Breast J & NRCS not adjusted \\
\hline 183 & 21460651 & Colakoglu & Impact of complications on patient satisfaction in breast reconstruction & $\begin{array}{l}\text { Plast Reconstr } \\
\text { Surg }\end{array}$ & $\begin{array}{l}\text { Single group N enrolled } \\
<500\end{array}$ \\
\hline 184 & 24581765 & Collier & $\begin{array}{l}\text { The effect of timing of postmastectomy radiation on implant-based breast } \\
\text { reconstruction: a retrospective comparison of complication outcomes }\end{array}$ & Am J Surg & NRCS not adjusted \\
\hline 185 & 21825969 & Collis & $\begin{array}{l}\text { Acellular dermal matrix slings in tissue expander breast reconstruction: are there } \\
\text { substantial benefits? }\end{array}$ & Ann Plast Surg & NRCS not adjusted \\
\hline 186 & 10873353 & Contant & $\begin{array}{l}\text { Morbidity of immediate breast reconstruction (IBR) after mastectomy by a } \\
\text { subpectorally placed silicone prosthesis: the adverse effect of radiotherapy }\end{array}$ & Eur J Surg Oncol & NRCS $<30$ per arm \\
\hline 187 & 106511232 & Contant & $\begin{array}{l}\text { Satisfaction and prosthesis related complaints in women with immediate breast } \\
\text { reconstruction following prophylactic and oncological mastectomy }\end{array}$ & $\begin{array}{l}\text { Psychology, Health } \\
\text { and Medicine }\end{array}$ & $\begin{array}{l}\text { Duplicate of another } \\
\text { publication }\end{array}$ \\
\hline 188 & 106511232 & Contant & $\begin{array}{l}\text { Satisfaction and prosthesis related complaints in women with immediate breast } \\
\text { reconstruction following prophylactic and oncological mastectomy }\end{array}$ & $\begin{array}{l}\text { Psychology, Health } \\
\text { \& Medicine }\end{array}$ & $\begin{array}{l}\text { Single group } \mathrm{N} \text { enrolled } \\
<500\end{array}$ \\
\hline 189 & 27879581 & Cooney & $\begin{array}{l}\text { Matching Procedures at the Time of Immediate Breast Reconstruction: An } \\
\text { American College of Surgeons National Surgical Quality Improvement Program } \\
\text { Study of } 24,191 \text { Patients }\end{array}$ & $\begin{array}{l}\text { Plast Reconstr } \\
\text { Surg }\end{array}$ & $\begin{array}{l}\text { Does not address KQ1- } \\
\text { KQ6 }\end{array}$ \\
\hline 190 & 28410984 & Corban & $\begin{array}{l}\text { A systematic review of complications associated with direct implants vs. tissue } \\
\text { expanders following Wise pattern skin-sparing mastectomy }\end{array}$ & $\begin{array}{l}J \text { Plast Reconstr } \\
\text { Aesthet Surg }\end{array}$ & $\begin{array}{l}\text { Does not address KQ1- } \\
\text { KQ6 }\end{array}$ \\
\hline 191 & 27890331 & Cordeiro & $\begin{array}{l}\text { The safety of same-day breast reconstructive surgery: An analysis of short-term } \\
\text { outcomes }\end{array}$ & Am J Surg & $\begin{array}{l}\text { Does not address KQ1- } \\
\text { KQ6 }\end{array}$ \\
\hline 192 & 32008941 & Cordeiro & $\begin{array}{l}\text { Risk of breast implant associated anaplastic large cell lymphoma (BIA-ALCL) in a } \\
\text { cohort of } 3546 \text { women prospectively followed long term after reconstruction with } \\
\text { textured breast implants }\end{array}$ & $\begin{array}{l}J \text { Plast Reconstr } \\
\text { Aesthet Surg }\end{array}$ & $\begin{array}{l}\text { Does not address KQ1- } \\
\text { KQ6 }\end{array}$ \\
\hline 193 & 25919262 & Coroneos & SIEA versus DIEP Arterial Complications: A Cohort Study & $\begin{array}{l}\text { Plast Reconstr } \\
\text { Surg }\end{array}$ & NRCS not adjusted \\
\hline 194 & 23806951 & Costa & $\begin{array}{l}\text { Incidence of surgical-site infection is not affected by method of immediate breast } \\
\text { reconstruction }\end{array}$ & $\begin{array}{l}\text { Plast Reconstr } \\
\text { Surg }\end{array}$ & $\begin{array}{l}\text { Copublication of } \\
\text { included study with no } \\
\text { new data }\end{array}$ \\
\hline
\end{tabular}

B-11 


\begin{tabular}{|c|c|c|c|c|c|}
\hline No. & $\begin{array}{l}\text { PMID or } \\
\text { Other } \\
\text { Identifier }\end{array}$ & $\begin{array}{l}\text { First Author } \\
\text { Last Name }\end{array}$ & Title & Journal & Reason for Exclusion \\
\hline 195 & 21460649 & Craft & $\begin{array}{l}\text { Patient satisfaction in unilateral and bilateral breast reconstruction [outcomes } \\
\text { article] }\end{array}$ & $\begin{array}{l}\text { Plast Reconstr } \\
\text { Surg }\end{array}$ & $\begin{array}{l}\text { Copublication of } \\
\text { included study with no } \\
\text { new data }\end{array}$ \\
\hline 196 & 22437263 & Craig & $\begin{array}{l}\text { Venous thromboembolism risk factors in breast cancer patients undergoing deep } \\
\text { inferior epigastric perforator flap reconstruction }\end{array}$ & Microsurgery & $\begin{array}{l}\text { Narrative review/ } \\
\text { Commentary }\end{array}$ \\
\hline 197 & 8060066 & Crespo & Postmastectomy complications in breast reconstruction & Ann Plast Surg & NRCS not adjusted \\
\hline 198 & 22544109 & Crosby & Immediate breast reconstruction and lymphedema incidence & $\begin{array}{l}\text { Plast Reconstr } \\
\text { Surg }\end{array}$ & No outcome of interest \\
\hline 199 & 31738641 & Cuccolo & $\begin{array}{l}\text { Does age or frailty have more predictive effect on outcomes following pedicled flap } \\
\text { reconstruction? An analysis of } 44,986 \text { cases(dagger) }\end{array}$ & $\begin{array}{l}\text { J Plast Surg Hand } \\
\text { Surg }\end{array}$ & $\begin{array}{l}\text { Does not address KQ1- } \\
\text { KQ6 }\end{array}$ \\
\hline 200 & 19437068 & Cunningham & Safety and effectiveness of Mentor's MemoryGel implants at 6 years & $\begin{array}{l}\text { Aesthetic Plast } \\
\text { Surg }\end{array}$ & $\begin{array}{l}\text { Single group N enrolled } \\
<500\end{array}$ \\
\hline 201 & None & Cuomo & Optimization of Prepectoral Breast Reconstruction & Breast Care & NRCS $<30$ per arm \\
\hline 202 & 21717397 & Curtis & $\begin{array}{l}\text { Immediate microsurgical breast reconstruction and simultaneous sentinel lymph } \\
\text { node dissection: issues with node positivity and recipient vessel selection }\end{array}$ & $\begin{array}{l}\text { J Reconstr } \\
\text { Microsurg }\end{array}$ & $\begin{array}{l}\text { Does not address KQ1- } \\
\text { KQ6 }\end{array}$ \\
\hline 203 & 21735435 & D'Souza & Immediate versus delayed reconstruction following surgery for breast cancer & $\begin{array}{l}\text { Cochrane } \\
\text { Database Syst Rev }\end{array}$ & $\begin{array}{l}\text { Does not address KQ1- } \\
\text { KQ6 }\end{array}$ \\
\hline 204 & 23642795 & Damen & $\begin{array}{l}\text { Improving outcomes in microsurgical breast reconstruction: lessons learnt from } 406 \\
\text { consecutive DIEP/TRAM flaps performed by a single surgeon }\end{array}$ & $\begin{array}{l}J \text { Plast Reconstr } \\
\text { Aesthet Surg }\end{array}$ & NRCS not adjusted \\
\hline 205 & 21317054 & Damen & $\begin{array}{l}\text { Medium-term cost analysis of breast reconstructions in a single Dutch centre: a } \\
\text { comparison of implants, implants preceded by tissue expansion, LD transpositions } \\
\text { and DIEP flaps }\end{array}$ & $\begin{array}{l}J \text { Plast Reconstr } \\
\text { Aesthet Surg }\end{array}$ & NRCS not adjusted \\
\hline 206 & None & Darrach & Pectoral placement of tissue expanders affects inpatient opioid use & Breast Journal & NRCS not adjusted \\
\hline 207 & 28401542 & Dauplat & Quality of life after mastectomy with or without immediate breast reconstruction & Br J Surg & $\begin{array}{l}\text { Single group }>500 \text {, but } \\
\text { no complications data }\end{array}$ \\
\hline 208 & 8901297 & Dauplat & $\begin{array}{l}\text { Mastectomy with immediate reconstruction for invasive breast cancer. Comments } \\
\text { on indications and techniques. A series of } 112 \text { cases }\end{array}$ & $\begin{array}{l}\text { Journal de } \\
\text { Gynecologie } \\
\text { Obstetrique et } \\
\text { Biologie de la } \\
\text { Reproduction }\end{array}$ & Unable to retrieve article \\
\hline 209 & 8901297 & Dauplat & $\begin{array}{l}\text { [Mastectomy with immediate reconstruction for invasive breast cancer. Comments } \\
\text { on indications and technique. A series of } 112 \text { cases] }\end{array}$ & $\begin{array}{l}\text { J Gynecol Obstet } \\
\text { Biol Reprod (Paris) }\end{array}$ & Unable to retrieve article \\
\hline 210 & 27855106 & Dave & $\begin{array}{l}\text { The iBRA-2 (immediate breast reconstruction and adjuvant therapy audit) study: } \\
\text { protocol for a prospective national multicentre cohort study to evaluate the impact } \\
\text { of immediate breast reconstruction on the delivery of adjuvant therapy }\end{array}$ & BMJ Open & $\begin{array}{l}\text { Protocol/methods with } \\
\text { no results }\end{array}$ \\
\hline 211 & 32990360 & Dave & $\begin{array}{l}\text { Risk factors for complications and implant loss after prepectoral implant-based } \\
\text { immediate breast reconstruction: medium-term outcomes in a prospective cohort }\end{array}$ & Br J Surg & $\begin{array}{l}\text { Single group N enrolled } \\
<500\end{array}$ \\
\hline 212 & 23547540 & Davila & $\begin{array}{l}\text { Immediate two-stage tissue expander breast reconstruction compared with one- } \\
\text { stage permanent implant breast reconstruction: a multi-institutional comparison of } \\
\text { short-term complications }\end{array}$ & $\begin{array}{l}J \text { Plast Surg Hand } \\
\text { Surg }\end{array}$ & $\begin{array}{l}\text { Does not address KQ1- } \\
\text { KQ6 }\end{array}$ \\
\hline
\end{tabular}

B-12 


\begin{tabular}{|c|c|c|c|c|c|}
\hline No. & $\begin{array}{l}\text { PMID or } \\
\text { Other } \\
\text { Identifier }\end{array}$ & $\begin{array}{l}\text { First Author } \\
\text { Last Name }\end{array}$ & Title & Journal & Reason for Exclusion \\
\hline 213 & 23547540 & Davila & $\begin{array}{l}\text { Immediate two-stage tissue expander breast reconstruction compared with one- } \\
\text { stage permanent implant breast reconstruction: A multi-institutional comparison of } \\
\text { short-term complications }\end{array}$ & $\begin{array}{l}\text { Journal of Plastic } \\
\text { Surgery and Hand } \\
\text { Surgery }\end{array}$ & $\begin{array}{l}\text { Duplicate of another } \\
\text { publication }\end{array}$ \\
\hline 214 & 23362476 & Davila & $\begin{array}{l}\text { Human acellular dermis versus submuscular tissue expander breast } \\
\text { reconstruction: A multivariate analysis of short-term complications }\end{array}$ & $\begin{array}{l}\text { Archives of Plastic } \\
\text { Surgery }\end{array}$ & $\begin{array}{l}\text { Duplicate of another } \\
\text { publication }\end{array}$ \\
\hline 215 & 29064925 & Dayan & Lower Extremity Free Flaps for Breast Reconstruction & $\begin{array}{l}\text { Plast Reconstr } \\
\text { Surg }\end{array}$ & $\begin{array}{l}\text { Narrative review/ } \\
\text { Commentary }\end{array}$ \\
\hline 216 & 21587037 & de Blacam & Cost analysis of implant-based breast reconstruction with acellular dermal matrix & Ann Plast Surg & $\begin{array}{l}\text { Does not address KQ1- } \\
\text { KQ6 }\end{array}$ \\
\hline 217 & 22179850 & $\begin{array}{l}\text { de la Pena- } \\
\text { Salcedo }\end{array}$ & $\begin{array}{l}\text { Back to the future: a } 15 \text {-year experience with polyurethane foam-covered breast } \\
\text { implants using the partial-subfascial technique }\end{array}$ & $\begin{array}{l}\text { Aesthetic Plast } \\
\text { Surg }\end{array}$ & $\begin{array}{l}\text { Single group } \mathrm{N} \text { enrolled } \\
<500\end{array}$ \\
\hline 218 & 25539293 & De Lorenzi & $\begin{array}{l}\text { Poly implant prothese asymmetrical anatomical breast implants: a product recall } \\
\text { study }\end{array}$ & $\begin{array}{l}\text { Plast Reconstr } \\
\text { Surg }\end{array}$ & $\begin{array}{l}\text { Does not address KQ1- } \\
\text { KQ6 }\end{array}$ \\
\hline 219 & 28121852 & De Vita & Outcome Evaluation after 2023 Nipple-Sparing Mastectomies: Our Experience & $\begin{array}{l}\text { Plast Reconstr } \\
\text { Surg }\end{array}$ & $\begin{array}{l}\text { Single group } \mathrm{N} \text { enrolled } \\
<500\end{array}$ \\
\hline 220 & 22739071 & Decker & Impact of neoadjuvant chemotherapy on wound complications after breast surgery & Surgery & $\begin{array}{l}\text { Does not address KQ1- } \\
\text { KQ6 }\end{array}$ \\
\hline 221 & 25096386 & Degnim & $\begin{array}{l}\text { Randomized trial of drain antisepsis after mastectomy and immediate prosthetic } \\
\text { breast reconstruction }\end{array}$ & Ann Surg Oncol & $\begin{array}{l}\text { Single group } \mathrm{N} \text { enrolled } \\
<500\end{array}$ \\
\hline 222 & 31042802 & DelMauro & $\begin{array}{l}\text { Reducing Length of Stay after Microsurgical Breast Reconstruction with a } \\
\text { Standardized Postoperative Protocol }\end{array}$ & $\begin{array}{l}J \text { Reconstr } \\
\text { Microsurg }\end{array}$ & $\begin{array}{l}\text { Single group } \mathrm{N} \text { enrolled } \\
<500\end{array}$ \\
\hline 223 & 31764630 & DeLong & $\begin{array}{l}\text { Systematic Review of the Impact of Acellular Dermal Matrix on Aesthetics and } \\
\text { Patient Satisfaction in Tissue Expander-to-Implant Breast Reconstructions }\end{array}$ & $\begin{array}{l}\text { Plast Reconstr } \\
\text { Surg }\end{array}$ & $\begin{array}{l}\text { Narrative review/ } \\
\text { Commentary }\end{array}$ \\
\hline 224 & 28346311 & DeLong & $\begin{array}{l}\text { Latissimus Dorsi Flap Breast Reconstruction-A Nationwide Inpatient Sample } \\
\text { Review }\end{array}$ & Ann Plast Surg & $\begin{array}{l}\text { Single group }>500 \text {, but } \\
\text { no complications data }\end{array}$ \\
\hline 225 & 29372268 & Demiri & $\begin{array}{l}\text { Outcomes of Fat-Augmented Latissimus Dorsi (FALD) Flap Versus Implant-Based } \\
\text { Latissimus Dorsi Flap for Delayed Post-radiation Breast Reconstruction }\end{array}$ & $\begin{array}{l}\text { Aesthetic Plast } \\
\text { Surg }\end{array}$ & $\begin{array}{l}\text { Single group } \mathrm{N} \text { enrolled } \\
<500\end{array}$ \\
\hline 226 & 32892331 & Demiri & $\begin{array}{l}\text { Fat-Augmented Latissimus Dorsi versus Deep Inferior Epigastric Perforator Flap: } \\
\text { Comparative Study in Delayed Autologous Breast Reconstruction }\end{array}$ & $\begin{array}{l}\text { J Reconstr } \\
\text { Microsurg }\end{array}$ & NRCS not adjusted \\
\hline 227 & 25480591 & Deng & Two modified surgical procedures for treating early stage breast cancer in China & $\begin{array}{l}\text { J Huazhong Univ } \\
\text { Sci Technolog Med } \\
\text { Sci }\end{array}$ & $\begin{array}{l}\text { Single group N enrolled } \\
<500\end{array}$ \\
\hline 228 & 29481390 & Devulapalli & $\begin{array}{l}\text { The Effect of Radiation on Quality of Life throughout the Breast Reconstruction } \\
\text { Process: A Prospective, Longitudinal Pilot Study of } 200 \text { Patients with Long-Term } \\
\text { Follow-Up }\end{array}$ & $\begin{array}{l}\text { Plast Reconstr } \\
\text { Surg }\end{array}$ & $\begin{array}{l}\text { Does not address KQ1- } \\
\text { KQ6 }\end{array}$ \\
\hline 229 & 31235319 & Dewael & $\begin{array}{l}\text { Immediate versus delayed autologous breast reconstruction: A retrospective } \\
\text { matched cohort study of irradiated patients }\end{array}$ & $\begin{array}{l}\text { J Plast Reconstr } \\
\text { Aesthet Surg }\end{array}$ & NRCS $<30$ per arm \\
\hline 230 & $\begin{array}{l}\text { CN- } \\
01139314\end{array}$ & Dikmans & $\begin{array}{l}\text { Two-stage implant-based breast reconstruction is safer than immediate one-stage } \\
\text { implant-based breast reconstruction augmented with an a cellular dermal matrix: a } \\
\text { multicentre randomized controlled trial }\end{array}$ & $\begin{array}{l}\text { European journal of } \\
\text { cancer }\end{array}$ & $\begin{array}{l}\text { Does not address KQ1- } \\
\text { KQ6 }\end{array}$ \\
\hline
\end{tabular}

B-13 


\begin{tabular}{|c|c|c|c|c|c|}
\hline No. & $\begin{array}{l}\text { PMID or } \\
\text { Other } \\
\text { Identifier }\end{array}$ & $\begin{array}{l}\text { First Author } \\
\text { Last Name }\end{array}$ & Title & Journal & Reason for Exclusion \\
\hline 231 & $\begin{array}{l}\text { CN- } \\
01340949\end{array}$ & Dikmans & $\begin{array}{l}\text { Two-stage implant-based breast reconstruction compared with immediate one- } \\
\text { stage implant-based breast reconstruction augmented with an acellular dermal } \\
\text { matrix: an open-label, phase } 4 \text {, multicentre, randomised, controlled trial }\end{array}$ & $\begin{array}{l}\text { The lancet. } \\
\text { Oncology }\end{array}$ & $\begin{array}{l}\text { Duplicate of another } \\
\text { publication }\end{array}$ \\
\hline 232 & 28012977 & Dikmans & $\begin{array}{l}\text { Two-stage implant-based breast reconstruction compared with immediate one- } \\
\text { stage implant-based breast reconstruction augmented with an acellular dermal } \\
\text { matrix: an open-label, phase } 4 \text {, multicentre, randomised, controlled trial }\end{array}$ & Lancet Oncol & $\begin{array}{l}\text { Duplicate of another } \\
\text { publication }\end{array}$ \\
\hline 233 & 28012977 & Dikmans & $\begin{array}{l}\text { Two-stage implant-based breast reconstruction compared with immediate one- } \\
\text { stage implant-based breast reconstruction augmented with an acellular dermal } \\
\text { matrix: an open-label, phase } 4 \text {, multicentre, randomised, controlled trial }\end{array}$ & $\begin{array}{l}\text { The Lancet } \\
\text { Oncology }\end{array}$ & $\begin{array}{l}\text { Does not address KQ1- } \\
\text { KQ6 }\end{array}$ \\
\hline 234 & 121069020 & Dikmans & $\begin{array}{l}\text { Two-stage implant-based breast reconstruction compared with immediate one- } \\
\text { stage implant-based breast reconstruction augmented with an acellular dermal } \\
\text { matrix: an open-label, phase } 4 \text {, multicentre, randomised, controlled trial }\end{array}$ & Lancet Oncology & $\begin{array}{l}\text { Duplicate of another } \\
\text { publication }\end{array}$ \\
\hline 235 & 10541166 & Disa & The premature removal of tissue expanders in breast reconstruction & $\begin{array}{l}\text { Plast Reconstr } \\
\text { Surg }\end{array}$ & No outcome of interest \\
\hline 236 & 10597680 & Disa & $\begin{array}{l}\text { Efficacy of conventional monitoring techniques in free tissue transfer: an 11-year } \\
\text { experience in } 750 \text { consecutive cases }\end{array}$ & $\begin{array}{l}\text { Plast Reconstr } \\
\text { Surg }\end{array}$ & $\begin{array}{l}\text { Does not address KQ1- } \\
\text { KQ6 }\end{array}$ \\
\hline 237 & 31531928 & Doherty & $\begin{array}{l}\text { Trends in immediate breast reconstruction and radiation after mastectomy: A } \\
\text { population study }\end{array}$ & Breast J & No outcome of interest \\
\hline 238 & 26942453 & Dolen & $\begin{array}{l}\text { Impact of Neoadjuvant and Adjuvant Chemotherapy on Immediate Tissue } \\
\text { Expander Breast Reconstruction }\end{array}$ & Ann Surg Oncol & No outcome of interest \\
\hline 239 & 24409778 & Dong & $\begin{array}{l}\text { [The impact of acellular dermal matrix on complications of breast reconstruction } \\
\text { using tissue expander/implant: a meta-analysis] }\end{array}$ & $\begin{array}{l}\text { Zhonghua Zheng } \\
\text { Xing Wai Ke Za Zhi }\end{array}$ & $\begin{array}{l}\text { Does not address KQ1- } \\
\text { KQ6 }\end{array}$ \\
\hline 240 & 21963981 & Donker & $\begin{array}{l}\text { Surgical complications of skin sparing mastectomy and immediate prosthetic } \\
\text { reconstruction after neoadjuvant chemotherapy for invasive breast cancer }\end{array}$ & $\begin{array}{l}\text { European Journal } \\
\text { of Surgical } \\
\text { Oncology }\end{array}$ & $\begin{array}{l}\text { Single group } \mathrm{N} \text { enrolled } \\
<500\end{array}$ \\
\hline 241 & 26505700 & Doren & $\begin{array}{l}\text { Comparison of Allergan, Mentor, and Sientra Contoured Cohesive Gel Breast } \\
\text { Implants: A Single Surgeon's 10-Year Experience }\end{array}$ & $\begin{array}{l}\text { Plast Reconstr } \\
\text { Surg }\end{array}$ & $\begin{array}{l}\text { Single group N enrolled } \\
<500\end{array}$ \\
\hline 242 & 29267860 & Dorfman & $\begin{array}{l}\text { The Effect of Implant Type on Nipple Position Geometry and Aesthetics Following } \\
\text { Tissue Expander Reconstruction After Nipple Sparing Mastectomy }\end{array}$ & Aesthet Surg J & NRCS not adjusted \\
\hline 243 & 30059382 & Doval & $\begin{array}{l}\text { Deep Inferior Epigastric Artery Perforator Flap Breast Reconstruction in Women } \\
\text { With Previous Abdominal Incisions: A Comparison of Complication Rates }\end{array}$ & Ann Plast Surg & $\begin{array}{l}\text { Single group } \mathrm{N} \text { enrolled } \\
<500\end{array}$ \\
\hline 244 & 31056434 & Drinane & $\begin{array}{l}\text { Depression is associated with worse outcomes among women undergoing breast } \\
\text { reconstruction following mastectomy }\end{array}$ & $\begin{array}{l}\text { J Plast Reconstr } \\
\text { Aesthet Surg }\end{array}$ & $\begin{array}{l}\text { Does not address KQ1- } \\
\text { KQ6 }\end{array}$ \\
\hline 245 & 8041820 & Duffy & Health risks of failed silicone gel breast implants: a 30-year clinical experience & $\begin{array}{l}\text { Plast Reconstr } \\
\text { Surg }\end{array}$ & $\begin{array}{l}\text { Single group } \mathrm{N} \text { enrolled } \\
<500\end{array}$ \\
\hline 246 & 110864376 & Duraes & $\begin{array}{l}\text { Aesthetics and patient-reported outcomes following microsurgical breast } \\
\text { reconstruction after nipple-sparing mastectomy }\end{array}$ & $\begin{array}{l}\text { Journal of the } \\
\text { American College } \\
\text { of Surgeons }\end{array}$ & $\begin{array}{l}\text { Single group N enrolled } \\
<500\end{array}$ \\
\hline 247 & 23025955 & Durkan & $\begin{array}{l}\text { Postmastectomy radiation of latissimus dorsi myocutaneous flap reconstruction is } \\
\text { well tolerated in women with breast cancer }\end{array}$ & Am Surg & NRCS not adjusted \\
\hline
\end{tabular}

B-14 


\begin{tabular}{|c|c|c|c|c|c|}
\hline No. & $\begin{array}{l}\text { PMID or } \\
\text { Other } \\
\text { Identifier }\end{array}$ & $\begin{array}{l}\text { First Author } \\
\text { Last Name }\end{array}$ & Title & Journal & Reason for Exclusion \\
\hline 248 & 30595378 & Durry & $\begin{array}{l}\text { [Patients' satisfaction after immediate breast reconstruction: Comparison between } \\
\text { five surgical techniques] }\end{array}$ & $\begin{array}{l}\text { Ann Chir Plast } \\
\text { Esthet }\end{array}$ & NRCS not adjusted \\
\hline 249 & 30611562 & Durry & $\begin{array}{l}\text { [Postoperative course after immediate breast reconstruction: Comparison between } \\
\text { five surgical techniques] }\end{array}$ & $\begin{array}{l}\text { Ann Chir Plast } \\
\text { Esthet }\end{array}$ & NRCS not adjusted \\
\hline 250 & 11505709 & Duskova & $\begin{array}{l}\text { Breast reconstruction as an integral part of breast carcinoma therapy (a self- } \\
\text { present final report of a research project IGA MZ CR) }\end{array}$ & $\begin{array}{l}\text { Acta Chirurgiae } \\
\text { Plasticae }\end{array}$ & Unable to retrieve article \\
\hline 251 & 11505709 & Duskova & $\begin{array}{l}\text { Breast reconstruction as an integral part of breast carcinoma therapy (a self- } \\
\text { present final report of a research project IGA MZ CR) }\end{array}$ & Acta Chir Plast & $\begin{array}{l}\text { Single group } \mathrm{N} \text { enrolled } \\
<500\end{array}$ \\
\hline 252 & 29040345 & Duteille & Eight-Year Safety Data for Round and Anatomical Silicone Gel Breast Implants & Aesthet Surg J & $\begin{array}{l}>=10 \% \text { augmentation } \\
\text { reconstruction only }\end{array}$ \\
\hline 253 & 25289331 & Duteille & $\begin{array}{l}\text { Five-year Safety Data for Eurosilicone's Round and Anatomical Silicone Gel Breast } \\
\text { Implants }\end{array}$ & $\begin{array}{l}\text { Plast Reconstr } \\
\text { Surg Glob Open }\end{array}$ & NRCS $<30$ per arm \\
\hline 254 & 26887685 & Duxbury & $\begin{array}{l}\text { Systematic review of the effectiveness of polyurethane-coated compared with } \\
\text { textured silicone implants in breast surgery }\end{array}$ & $\begin{array}{l}J \text { Plast Reconstr } \\
\text { Aesthet Surg }\end{array}$ & $\begin{array}{l}\text { Does not address KQ1- } \\
\text { KQ6 }\end{array}$ \\
\hline 255 & 32041661 & Dyrberg & $\begin{array}{l}\text { Direct-to-Implant Extracellular Matrix Hammock-based Breast Reconstruction; } \\
\text { Prepectoral or Subpectoral' }\end{array}$ & Trials & $\begin{array}{l}\text { Duplicate of another } \\
\text { publication }\end{array}$ \\
\hline 256 & 32041661 & Dyrberg & $\begin{array}{l}\text { Direct-to-Implant Extracellular Matrix Hammock-based Breast Reconstruction; } \\
\text { Prepectoral or Subpectoral? }\end{array}$ & Trials & $\begin{array}{l}\text { Protocol/methods with } \\
\text { no results }\end{array}$ \\
\hline 257 & 32041661 & Dyrberg & $\begin{array}{l}\text { Direct-to-Implant Extracellular Matrix Hammock-based Breast Reconstruction; } \\
\text { Prepectoral or Subpectoral' }\end{array}$ & Trials & $\begin{array}{l}\text { Protocol/methods with } \\
\text { no results }\end{array}$ \\
\hline 258 & 32041661 & Dyrberg & $\begin{array}{l}\text { Direct-to-Implant Extracellular Matrix Hammock-based Breast Reconstruction; } \\
\text { Prepectoral or Subpectoral? }\end{array}$ & Trials & $\begin{array}{l}\text { Protocol/methods with } \\
\text { no results }\end{array}$ \\
\hline 259 & 32855081 & Early & $\begin{array}{l}\text { Breast Cancer and Secondary Cancer Recurrences After Autologous Tissue } \\
\text { Reconstruction }\end{array}$ & $\begin{array}{l}\text { Clinical Breast } \\
\text { Cancer }\end{array}$ & $\begin{array}{l}\text { Single group } \mathrm{N} \text { enrolled } \\
<500\end{array}$ \\
\hline 260 & 22841854 & Egeberg & $\begin{array}{l}\text { Comparing the donor-site morbidity using DIEP, SIEA or MS-TRAM flaps for breast } \\
\text { reconstructive surgery: a meta-analysis }\end{array}$ & $\begin{array}{l}J \text { Plast Reconstr } \\
\text { Aesthet Surg }\end{array}$ & $\begin{array}{l}\text { Narrative review/ } \\
\text { Commentary }\end{array}$ \\
\hline 261 & 29487671 & El-Haddad & $\begin{array}{l}\text { A 10-Year Prospective Study of Implant-Based Breast Augmentation and } \\
\text { Reconstruction }\end{array}$ & Eplasty & $\begin{array}{l}\text { Single group } \mathrm{N} \text { enrolled } \\
<500\end{array}$ \\
\hline 262 & 28416138 & El-Sabawi & $\begin{array}{l}\text { Patient-centered outcomes of breast reconstruction in the setting of post- } \\
\text { mastectomy radiotherapy: A comprehensive review of the literature }\end{array}$ & $\begin{array}{l}J \text { Plast Reconstr } \\
\text { Aesthet Surg }\end{array}$ & Systematic review \\
\hline 263 & 26345465 & El-Sabawi & $\begin{array}{l}\text { Breast reconstruction and adjuvant therapy: A systematic review of surgical } \\
\text { outcomes }\end{array}$ & J Surg Oncol & Systematic review \\
\hline 264 & 32420440 & Eltahir & $\begin{array}{l}\text { Satisfaction with cosmetic outcomes of breast reconstruction: Investigations into } \\
\text { the correlation between the patients' Breast-Q outcome and the judgment of panels }\end{array}$ & JPRAS Open & NRCS not adjusted \\
\hline 265 & 24022602 & Enajat & $\begin{array}{l}\text { Effect of acetylsalicylic acid on microvascular thrombosis in autologous breast } \\
\text { reconstruction }\end{array}$ & $\begin{array}{l}\text { J Reconstr } \\
\text { Microsurg }\end{array}$ & $\begin{array}{l}\text { Single group } \mathrm{N} \text { enrolled } \\
<500\end{array}$ \\
\hline 266 & 23924650 & Endara & $\begin{array}{l}\text { Breast reconstruction following nipple-sparing mastectomy: a systematic review of } \\
\text { the literature with pooled analysis }\end{array}$ & $\begin{array}{l}\text { Plast Reconstr } \\
\text { Surg }\end{array}$ & $\begin{array}{l}\text { Does not address KQ1- } \\
\text { KQ6 }\end{array}$ \\
\hline 267 & 33268290 & Erlichman & $\begin{array}{l}\text { Comparing outcomes of post-mastectomy breast reconstruction between United } \\
\text { States and Western Europe }\end{array}$ & $\begin{array}{l}\text { J Plast Reconstr } \\
\text { Aesthet Surg }\end{array}$ & Systematic review \\
\hline
\end{tabular}




\begin{tabular}{|c|c|c|c|c|c|}
\hline No. & $\begin{array}{l}\text { PMID or } \\
\text { Other } \\
\text { Identifier }\end{array}$ & $\begin{array}{l}\text { First Author } \\
\text { Last Name }\end{array}$ & Title & Journal & Reason for Exclusion \\
\hline 268 & 8273633 & Fajardo & $\begin{array}{l}\text { Mammographic surveillance of breast cancer patients: should the mastectomy site } \\
\text { be imaged? }\end{array}$ & $\begin{array}{l}\text { AJR Am J } \\
\text { Roentgenol }\end{array}$ & $\begin{array}{l}\text { Single group } \mathrm{N} \text { enrolled } \\
<500\end{array}$ \\
\hline 269 & 18952229 & Fancher & A woman's influence to choose mastectomy as treatment for breast cancer & J Surg Res & $\begin{array}{l}\text { Single group N enrolled } \\
<500\end{array}$ \\
\hline 270 & 28489683 & Fang & $\begin{array}{l}\text { Intraoperative Use of Vasopressors Does Not Increase the Risk of Free Flap } \\
\text { Compromise and Failure in Cancer Patients }\end{array}$ & Ann Surg & $\begin{array}{l}\text { Does not address KQ1- } \\
\text { KQ6 }\end{array}$ \\
\hline 271 & 10654733 & Feng & Analysis of risk factors associated with rupture of silicone gel breast implants & $\begin{array}{l}\text { Plast Reconstr } \\
\text { Surg }\end{array}$ & $\begin{array}{l}\text { Not breast } \\
\text { reconstruction }\end{array}$ \\
\hline 272 & 18390839 & $\begin{array}{l}\text { Fernandez- } \\
\text { Delgado }\end{array}$ & $\begin{array}{l}\text { Satisfaction with and psychological impact of immediate and deferred breast } \\
\text { reconstruction }\end{array}$ & Ann Oncol & $\begin{array}{l}\text { Single group } \mathrm{N} \text { enrolled } \\
<500\end{array}$ \\
\hline 273 & 19228514 & $\begin{array}{l}\text { Fernandez- } \\
\text { Frias }\end{array}$ & $\begin{array}{l}\text { Immediate reconstruction after mastectomy for breast cancer: which factors affect } \\
\text { its course and final outcome? }\end{array}$ & J Am Coll Surg & $\begin{array}{l}\text { Narrative review/ } \\
\text { Commentary }\end{array}$ \\
\hline 274 & 25536206 & Fischer & $\begin{array}{l}\text { A Systematic Meta-analysis of Prosthetic-Based Breast Reconstruction in } \\
\text { Irradiated Fields With or Without Autologous Muscle Flap Coverage }\end{array}$ & Ann Plast Surg & $\begin{array}{l}\text { Does not address KQ1- } \\
\text { KQ6 }\end{array}$ \\
\hline 275 & 24076667 & Fischer & $\begin{array}{l}\text { Breast reconstruction in the morbidly obese patient: assessment of 30-day } \\
\text { complications using the } 2005 \text { to } 2010 \text { National Surgical Quality Improvement } \\
\text { Program data sets }\end{array}$ & $\begin{array}{l}\text { Plast Reconstr } \\
\text { Surg }\end{array}$ & $\begin{array}{l}\text { Does not address KQ1- } \\
\text { KQ6 }\end{array}$ \\
\hline 276 & 23865900 & Fischer & $\begin{array}{l}\text { Complications and morbidity following breast reconstruction--a review of 16,063 } \\
\text { cases from the } 2005-2010 \text { NSQIP datasets }\end{array}$ & $\begin{array}{l}\text { J Plast Surg Hand } \\
\text { Surg }\end{array}$ & $\begin{array}{l}\text { Copublication of } \\
\text { included study with no } \\
\text { new data }\end{array}$ \\
\hline 277 & 23891077 & Fischer & $\begin{array}{l}\text { Impact of obesity on outcomes in breast reconstruction: analysis of } 15,937 \text { patients } \\
\text { from the ACS-NSQIP datasets }\end{array}$ & J Am Coll Surg & NRCS not adjusted \\
\hline 278 & 23845908 & Fischer & $\begin{array}{l}\text { Peri-operative risk factors associated with early tissue expander (TE) loss following } \\
\text { immediate breast reconstruction (IBR): a review of } 9305 \text { patients from the 2005- } \\
2010 \text { ACS-NSQIP datasets }\end{array}$ & $\begin{array}{l}\text { J Plast Reconstr } \\
\text { Aesthet Surg }\end{array}$ & No outcome of interest \\
\hline 279 & 23629107 & Fischer & $\begin{array}{l}\text { Free tissue transfer in the obese patient: an outcome and cost analysis in } 1258 \\
\text { consecutive abdominally based reconstructions }\end{array}$ & $\begin{array}{l}\text { Plast Reconstr } \\
\text { Surg }\end{array}$ & NRCS not adjusted \\
\hline 280 & 23357982 & Fischer & $\begin{array}{l}\text { Comprehensive outcome and cost analysis of free tissue transfer for breast } \\
\text { reconstruction: an experience with } 1303 \text { flaps }\end{array}$ & $\begin{array}{l}\text { Plast Reconstr } \\
\text { Surg }\end{array}$ & $\begin{array}{l}\text { Does not address KQ1- } \\
\text { KQ6 }\end{array}$ \\
\hline 281 & 24328902 & Fischer & $\begin{array}{l}\text { A 30-day risk assessment of mastectomy alone compared to immediate breast } \\
\text { reconstruction (IBR) }\end{array}$ & $\begin{array}{l}\text { J Plast Surg Hand } \\
\text { Surg }\end{array}$ & $\begin{array}{l}\text { Does not address KQ1- } \\
\text { KQ6 }\end{array}$ \\
\hline 282 & 24443774 & Fischer & $\begin{array}{l}\text { Effect of BMI on modality-specific outcomes in immediate breast reconstruction } \\
\text { (IBR)--a propensity-matched analysis using the 2005-2011 ACS-NSQIP datasets }\end{array}$ & $\begin{array}{l}\text { J Plast Surg Hand } \\
\text { Surg }\end{array}$ & $\begin{array}{l}\text { Does not address KQ1- } \\
\text { KQ6 }\end{array}$ \\
\hline 283 & 25175274 & Fischer & $\begin{array}{l}\text { Mastectomy with or without immediate implant reconstruction has similar 30-day } \\
\text { perioperative outcomes }\end{array}$ & $\begin{array}{l}\text { J Plast Reconstr } \\
\text { Aesthet Surg }\end{array}$ & $\begin{array}{l}\text { Does not address KQ1- } \\
\text { KQ6 }\end{array}$ \\
\hline 284 & 24074811 & Fischer & $\begin{array}{l}\text { Risk analysis and stratification of surgical morbidity after immediate breast } \\
\text { reconstruction }\end{array}$ & J Am Coll Surg & $\begin{array}{l}\text { Does not address KQ1- } \\
\text { KQ6 }\end{array}$ \\
\hline 285 & 24572870 & Fischer & $\begin{array}{l}\text { Venous thromboembolism risk in mastectomy and immediate breast } \\
\text { reconstruction: analysis of the } 2005 \text { to } 2011 \text { American College of Surgeons } \\
\text { National Surgical Quality Improvement Program data sets }\end{array}$ & $\begin{array}{l}\text { Plast Reconstr } \\
\text { Surg }\end{array}$ & $\begin{array}{l}\text { Does not address KQ1- } \\
\text { KQ6 }\end{array}$ \\
\hline
\end{tabular}




\begin{tabular}{|c|c|c|c|c|c|}
\hline No. & $\begin{array}{l}\text { PMID or } \\
\text { Other } \\
\text { Identifier }\end{array}$ & $\begin{array}{l}\text { First Author } \\
\text { Last Name }\end{array}$ & Title & Journal & Reason for Exclusion \\
\hline 286 & 23973103 & Fischer & $\begin{array}{l}\text { Risk analysis of early implant loss after immediate breast reconstruction: a review } \\
\text { of } 14,585 \text { patients }\end{array}$ & J Am Coll Surg & $\begin{array}{l}\text { Copublication of } \\
\text { included study with no } \\
\text { new data }\end{array}$ \\
\hline 287 & 27047777 & $\begin{array}{l}\text { Fitzgerald } \\
\text { O'Connor }\end{array}$ & $\begin{array}{l}\text { Preoperative computed tomography angiography for planning DIEP flap breast } \\
\text { reconstruction reduces operative time and overall complications }\end{array}$ & Gland Surg & NRCS not adjusted \\
\hline 288 & 23407253 & Fitzpatrick & Cost and outcome analysis of breast reconstruction paradigm shift & Ann Plast Surg & $\begin{array}{l}\text { Does not address KQ1- } \\
\text { KQ6 }\end{array}$ \\
\hline 289 & 23975313 & Fitzsullivan & $\begin{array}{l}\text { Incidence and consequence of close margins in patients with ductal carcinoma-in } \\
\text { situ treated with mastectomy: is further therapy warranted? }\end{array}$ & Ann Surg Oncol & $\begin{array}{l}\text { Does not address KQ1- } \\
\text { KQ6 }\end{array}$ \\
\hline 290 & 31342397 & Flanagan & $\begin{array}{l}\text { A Comparison of Patient-Reported Outcomes After Breast-Conserving Surgery and } \\
\text { Mastectomy with Implant Breast Reconstruction }\end{array}$ & Ann Surg Oncol & $\begin{array}{l}\text { Single group }>500 \text {, but } \\
\text { no complications data }\end{array}$ \\
\hline 291 & 110864110 & Flanagan & $\begin{array}{l}\text { Adverse outcomes and quality of life associated with immediate breast } \\
\text { reconstruction after mastectomy in Washington state, 2011-2013 }\end{array}$ & $\begin{array}{l}\text { Journal of the } \\
\text { American College } \\
\text { of Surgeons }\end{array}$ & No outcome of interest \\
\hline 292 & 24374398 & Forsberg & $\begin{array}{l}\text { Aesthetic outcomes of acellular dermal matrix in tissue expander/implant-based } \\
\text { breast reconstruction }\end{array}$ & Ann Plast Surg & NRCS not adjusted \\
\hline 293 & 21681129 & Fosnot & $\begin{array}{l}\text { Closer to an understanding of fate: the role of vascular complications in free flap } \\
\text { breast reconstruction }\end{array}$ & $\begin{array}{l}\text { Plastic and } \\
\text { reconstructive } \\
\text { surgery }\end{array}$ & No outcome of interest \\
\hline 294 & 21681129 & Fosnot & $\begin{array}{l}\text { Closer to an understanding of fate: the role of vascular complications in free flap } \\
\text { breast reconstruction }\end{array}$ & $\begin{array}{l}\text { Plast Reconstr } \\
\text { Surg }\end{array}$ & $\begin{array}{l}\text { Duplicate of another } \\
\text { publication }\end{array}$ \\
\hline 295 & 12052757 & Foster & $\begin{array}{l}\text { Skin-sparing mastectomy and immediate breast reconstruction: a prospective } \\
\text { cohort study for the treatment of advanced stages of breast carcinoma }\end{array}$ & Ann Surg Oncol & $\begin{array}{l}\text { Single group } \mathrm{N} \text { enrolled } \\
<500\end{array}$ \\
\hline 296 & 26545214 & Fracol & $\begin{array}{l}\text { Bilateral Free Flap Breast Reconstruction After Unilateral Radiation: Comparing } \\
\text { Intraoperative Vascular Complications and Postoperative Outcomes in Radiated } \\
\text { Versus Nonradiated Breasts }\end{array}$ & Ann Plast Surg & $\begin{array}{l}\text { Single group } \mathrm{N} \text { enrolled } \\
<500\end{array}$ \\
\hline 297 & 33133885 & Fracol & $\begin{array}{l}\text { Lateral and Inferior Implant Malposition in Prosthetic Breast Reconstruction: } \\
\text { Incidence and Risk Factors }\end{array}$ & $\begin{array}{l}\text { Plast Reconstr } \\
\text { Surg Glob Open }\end{array}$ & $\begin{array}{l}\text { Single group }>500 \text {, but } \\
\text { no harms data }\end{array}$ \\
\hline 298 & 29651851 & Fracon & $\begin{array}{l}\text { PATIENT SATISFACTION AFTER BREAST RECONSTRUCTION: IMPLANTS VS. } \\
\text { AUTOLOGOUS TISSUES }\end{array}$ & $\begin{array}{l}\text { Acta chirurgiae } \\
\text { plasticae }\end{array}$ & NRCS not adjusted \\
\hline 299 & 29651851 & Fracon & $\begin{array}{l}\text { PATIENT SATISFACTION AFTER BREAST RECONSTRUCTION: IMPLANTS VS. } \\
\text { AUTOLOGOUS TISSUES }\end{array}$ & Acta Chir Plast & NRCS not adjusted \\
\hline 300 & 32875463 & Franceschini & $\begin{array}{l}\text { Compliance with Specific Recommendations and Tasks Reduces Nipple Necrosis } \\
\text { Rates in Prepectoral Implant-Based Reconstruction After Nipple-Sparing } \\
\text { Mastectomy }\end{array}$ & Ann Surg Oncol & $\begin{array}{l}\text { Narrative } \\
\text { review/Commentary }\end{array}$ \\
\hline 301 & 33671712 & Franceschini & $\begin{array}{l}\text { Immediate Prosthetic Breast Reconstruction after Nipple-Sparing Mastectomy: } \\
\text { Traditional Subpectoral Technique versus Direct-to-Implant Prepectoral } \\
\text { Reconstruction without Acellular Dermal Matrix }\end{array}$ & J Pers Med & NRCS not adjusted \\
\hline 302 & 7761508 & Franchelli & $\begin{array}{l}\text { Psychological evaluation of patients undergoing breast reconstruction using two } \\
\text { different methods: autologous tissues versus prostheses }\end{array}$ & $\begin{array}{l}\text { Plast Reconstr } \\
\text { Surg }\end{array}$ & NRCS not adjusted \\
\hline
\end{tabular}




\begin{tabular}{|c|c|c|c|c|c|}
\hline No. & $\begin{array}{l}\text { PMID or } \\
\text { Other } \\
\text { Identifier }\end{array}$ & $\begin{array}{l}\text { First Author } \\
\text { Last Name }\end{array}$ & Title & Journal & Reason for Exclusion \\
\hline 303 & 7761508 & Franchelli & $\begin{array}{l}\text { Psychological evaluation of patients undergoing breast reconstruction using two } \\
\text { different methods: Autologous tissues versus prostheses }\end{array}$ & $\begin{array}{l}\text { Plastic and } \\
\text { Reconstructive } \\
\text { Surgery }\end{array}$ & NRCS not adjusted \\
\hline 304 & 9678622 & Franchelli & $\begin{array}{l}\text { Can the cost affect the choice of various methods of postmastectomy breast } \\
\text { reconstruction? }\end{array}$ & Tumori & $\begin{array}{l}\text { Single group N enrolled } \\
<500\end{array}$ \\
\hline 305 & 7478060 & Franchelli & $\begin{array}{l}\text { [Psychological assessment of patients who have ++undergone breast } \\
\text { reconstruction using } 2 \text { different technics: autologous tissue versus prosthesis] }\end{array}$ & Minerva Chir & NRCS not adjusted \\
\hline 306 & 7761508 & Franchelli & $\begin{array}{l}\text { Psychological evaluation of patients undergoing breast reconstruction using two } \\
\text { different methods: Autologous tissues versus prostheses }\end{array}$ & $\begin{array}{l}\text { Plast Reconstr } \\
\text { Surg }\end{array}$ & NRCS not adjusted \\
\hline 307 & 29660396 & Franchelli & $\begin{array}{l}\text { Analysis of clinical management of infected breast implants and of factors } \\
\text { associated to successful breast pocket salvage in infections occurring after breast } \\
\text { reconstruction }\end{array}$ & $\begin{array}{l}\text { International } \\
\text { Journal of } \\
\text { Infectious Diseases }\end{array}$ & $\begin{array}{l}\text { Single group N enrolled } \\
<500\end{array}$ \\
\hline 308 & 104164872 & Fraser & $\begin{array}{l}\text { Lumbar herniation following extended autologous latissimus dorsi breast } \\
\text { reconstruction }\end{array}$ & BMC Surgery & $\begin{array}{l}\text { Case report or series of } \\
\text { case reports }\end{array}$ \\
\hline 309 & 28066037 & Fraser & THE EPIDEMIOLOGY AND OUTCOMES OF BREAST CANCER SURGERY & $\begin{array}{l}\text { Trans Am Clin } \\
\text { Climatol Assoc }\end{array}$ & NRCS not adjusted \\
\hline 310 & 31663939 & Freniere & $\begin{array}{l}\text { Outcomes Following Breast Reconstruction in Patients With Prior Mantle Radiation } \\
\text { for Treatment of Hodgkin's Lymphoma }\end{array}$ & Ann Plast Surg & NRCS $<30$ per arm \\
\hline 311 & 26495218 & Frey & $\begin{array}{l}\text { Breast Reconstruction Using Contour Fenestrated AlloDerm: Does Improvement in } \\
\text { Design Translate to Improved Outcomes? }\end{array}$ & $\begin{array}{l}\text { Plast Reconstr } \\
\text { Surg Glob Open }\end{array}$ & NRCS not adjusted \\
\hline 312 & 28538548 & Frey & $\begin{array}{l}\text { Comparison of Outcomes with Tissue Expander, Immediate Implant, and } \\
\text { Autologous Breast Reconstruction in Greater Than } 1000 \text { Nipple-Sparing } \\
\text { Mastectomies }\end{array}$ & $\begin{array}{l}\text { Plast Reconstr } \\
\text { Surg }\end{array}$ & NRCS not adjusted \\
\hline 313 & 29794639 & Frey & $\begin{array}{l}\text { Comparing Therapeutic versus Prophylactic Nipple-Sparing Mastectomy: Does } \\
\text { Indication Inform Oncologic and Reconstructive Outcomes? }\end{array}$ & $\begin{array}{l}\text { Plast Reconstr } \\
\text { Surg }\end{array}$ & NRCS not adjusted \\
\hline 314 & 25059787 & Freyvogel & $\begin{array}{l}\text { Screening mammography following autologous breast reconstruction: an } \\
\text { unnecessary effort }\end{array}$ & Ann Surg Oncol & $\begin{array}{l}\text { Does not address KQ1- } \\
\text { KQ6 }\end{array}$ \\
\hline 315 & 25059787 & Freyvogel & $\begin{array}{l}\text { Screening Mammography Following Autologous Breast Reconstruction: An } \\
\text { Unnecessary Effort }\end{array}$ & $\begin{array}{l}\text { Annals of Surgical } \\
\text { Oncology }\end{array}$ & $\begin{array}{l}\text { Single group }>500 \text {, but } \\
\text { no complications data }\end{array}$ \\
\hline 316 & 9229085 & Friis & $\begin{array}{l}\text { Connective tissue disease and other rheumatic conditions following breast implants } \\
\text { in Denmark }\end{array}$ & Ann Plast Surg & $\begin{array}{l}\text { Single group }>500 \text {, but } \\
\text { no complications data }\end{array}$ \\
\hline 317 & 11000074 & Futter & $\begin{array}{l}\text { A retrospective comparison of abdominal muscle strength following breast } \\
\text { reconstruction with a free TRAM or DIEP flap }\end{array}$ & Br J Plast Surg & NRCS $<30$ per arm \\
\hline 318 & 28279888 & Fuzesi & Validation of the electronic version of the BREAST-Q in the army of women study & Breast & $\begin{array}{l}\text { Single group }>500 \text {, but } \\
\text { no complications data }\end{array}$ \\
\hline 319 & 31788914 & Gabrick & $\begin{array}{l}\text { Breast reconstruction patterns and outcomes in academic and community } \\
\text { practices within a single institution }\end{array}$ & Breast J & $\begin{array}{l}\text { Does not address KQ1- } \\
\text { KQ6 }\end{array}$ \\
\hline 320 & 8190133 & Gabriel & Risk of connective-tissue diseases and other disorders after breast implantation & N Engl J Med & $\begin{array}{l}\text { Single group } \mathrm{N} \text { enrolled } \\
<500\end{array}$ \\
\hline 321 & 9041097 & Gabriel & Complications leading to surgery after breast implantation & $\begin{array}{l}\text { New England } \\
\text { Journal of Medicine }\end{array}$ & $\begin{array}{l}\text { Single group } \mathrm{N} \text { enrolled } \\
<500\end{array}$ \\
\hline
\end{tabular}

B-18 


\begin{tabular}{|c|c|c|c|c|c|}
\hline No. & $\begin{array}{l}\text { PMID or } \\
\text { Other } \\
\text { Identifier }\end{array}$ & $\begin{array}{l}\text { First Author } \\
\text { Last Name }\end{array}$ & Title & Journal & Reason for Exclusion \\
\hline 322 & 23211118 & Gart & $\begin{array}{l}\text { Autologous options for postmastectomy breast reconstruction: A comparison of } \\
\text { outcomes based on the american college of surgeons national surgical quality } \\
\text { improvement program }\end{array}$ & $\begin{array}{l}\text { Journal of the } \\
\text { American College } \\
\text { of Surgeons }\end{array}$ & $\begin{array}{l}\text { Duplicate of another } \\
\text { publication }\end{array}$ \\
\hline 323 & 16651940 & Garvey & DIEP and pedicled TRAM flaps: a comparison of outcomes & $\begin{array}{l}\text { Plast Reconstr } \\
\text { Surg }\end{array}$ & NRCS not adjusted \\
\hline 324 & 24469158 & Garvey & $\begin{array}{l}\text { Muscle-sparing TRAM flap does not protect breast reconstruction from } \\
\text { postmastectomy radiation damage compared with the DIEP flap }\end{array}$ & $\begin{array}{l}\text { Plast Reconstr } \\
\text { Surg }\end{array}$ & $\begin{array}{l}\text { Does not address KQ1- } \\
\text { KQ6 }\end{array}$ \\
\hline 325 & 21617453 & Garvey & $\begin{array}{l}\text { Abdominal donor-site outcomes for medial versus lateral deep inferior epigastric } \\
\text { artery branch perforator harvest }\end{array}$ & $\begin{array}{l}\text { Plast Reconstr } \\
\text { Surg }\end{array}$ & $\begin{array}{l}\text { Does not address KQ1- } \\
\text { KQ6 }\end{array}$ \\
\hline 326 & 25626783 & Gassman & $\begin{array}{l}\text { Comparison of postoperative pain control in autologous abdominal free flap versus } \\
\text { implant-based breast reconstructions }\end{array}$ & $\begin{array}{l}\text { Plast Reconstr } \\
\text { Surg }\end{array}$ & NRCS not adjusted \\
\hline 327 & 26761517 & Gdalevitch & $\begin{array}{l}\text { Reply to: Comments on Effects of Nitroglycerin Ointment on Mastectomy Flap } \\
\text { Necrosis in Immediate Breast Reconstruction: A Randomized Controlled Trial }\end{array}$ & $\begin{array}{l}\text { Plast Reconstr } \\
\text { Surg }\end{array}$ & $\begin{array}{l}\text { Not breast } \\
\text { reconstruction }\end{array}$ \\
\hline 328 & 24867734 & Gdalevitch & $\begin{array}{l}\text { Direct-to-implant single-stage immediate breast reconstruction with acellular } \\
\text { dermal matrix: predictors of failure }\end{array}$ & $\begin{array}{l}\text { Plast Reconstr } \\
\text { Surg }\end{array}$ & $\begin{array}{l}\text { Not mastectomy for } \\
\text { breast cancer }\end{array}$ \\
\hline 329 & 9588052 & Germain & [Breast reconstruction using free rectus abdominis myocutaneous flap] & Chirurgie & Unable to retrieve article \\
\hline 330 & 9588052 & Germain & $\begin{array}{l}\text { Breast reconstruction for cancer with free transverse rectus abdominis } \\
\text { myocutaneous flap }\end{array}$ & $\begin{array}{l}\text { Chirurgie - } \\
\text { Memoires de } \\
\text { l'Academie de } \\
\text { Chirurgie }\end{array}$ & NRCS $<30$ per arm \\
\hline 331 & 20502959 & Giacalone & $\begin{array}{l}\text { New concept for immediate breast reconstruction for invasive cancers: feasibility, } \\
\text { oncological safety and esthetic outcome of post-neoadjuvant therapy immediate } \\
\text { breast reconstruction versus delayed breast reconstruction: a prospective pilot } \\
\text { study }\end{array}$ & $\begin{array}{l}\text { Breast Cancer Res } \\
\text { Treat }\end{array}$ & NRCS not adjusted \\
\hline 332 & 122477529 & Gibreel & $\begin{array}{l}\text { Mastectomy and Immediate Breast Reconstruction for Cancer in the Elderly: } \\
\text { A National Cancer Data Base Study }\end{array}$ & $\begin{array}{l}\text { Journal of the } \\
\text { American College } \\
\text { of Surgeons }\end{array}$ & $\begin{array}{l}\text { Does not address KQ1- } \\
\text { KQ6 }\end{array}$ \\
\hline 333 & 105522143 & Gill & $\begin{array}{l}\text { Quality of life, abdominal muscle strength and endurance of women following } \\
\text { breast reconstruction }\end{array}$ & $\begin{array}{l}\text { Journal of } \\
\text { Women's Health } \\
\text { Physical Therapy }\end{array}$ & $\begin{array}{l}\text { Single group } \mathrm{N} \text { enrolled } \\
<500\end{array}$ \\
\hline 334 & 22327891 & Glasberg & $\begin{array}{l}\text { AlloDerm and Strattice in breast reconstruction: a comparison and techniques for } \\
\text { optimizing outcomes }\end{array}$ & $\begin{array}{l}\text { Plast Reconstr } \\
\text { Surg }\end{array}$ & $\begin{array}{l}\text { Does not address KQ1- } \\
\text { KQ6 }\end{array}$ \\
\hline 335 & 27743083 & Golpanian & Free Versus Pedicled TRAM Flaps: Cost Utilization and Complications & $\begin{array}{l}\text { Aesthetic Plast } \\
\text { Surg }\end{array}$ & $\begin{array}{l}\text { Does not address KQ1- } \\
\text { KQ6 }\end{array}$ \\
\hline 336 & 29146071 & $\begin{array}{l}\text { Gomez- } \\
\text { Escolar } \\
\text { Larranaga }\end{array}$ & $\begin{array}{l}\text { Comparison among the levels of patients' satisfaction according to the surgical } \\
\text { technique used in breast reconstruction after mastectomy }\end{array}$ & Cir Esp & NRCS not adjusted \\
\hline 337 & 23983109 & Gopie & $\begin{array}{l}\text { Impact of delayed implant and DIEP flap breast reconstruction on body image and } \\
\text { sexual satisfaction: a prospective follow-up study }\end{array}$ & Psychooncology & NRCS $<30$ per arm \\
\hline 338 & 22033976 & Gopie & The short-term psychological impact of complications after breast reconstruction & Psychooncology & NRCS not adjusted \\
\hline
\end{tabular}




\begin{tabular}{|c|c|c|c|c|c|}
\hline No. & $\begin{array}{l}\text { PMID or } \\
\text { Other } \\
\text { Identifier }\end{array}$ & $\begin{array}{l}\text { First Author } \\
\text { Last Name }\end{array}$ & Title & Journal & Reason for Exclusion \\
\hline 339 & 21514911 & Gopie & $\begin{array}{l}\text { Information-seeking behaviour and coping style of women opting for either implant } \\
\text { or DIEP-flap breast reconstruction }\end{array}$ & $\begin{array}{l}\text { J Plast Reconstr } \\
\text { Aesthet Surg }\end{array}$ & $\begin{array}{l}\text { Does not address KQ1- } \\
\text { KQ6 }\end{array}$ \\
\hline 340 & 25910024 & Grinsell & The Deep Inferior Epigastric Perforator Learning Curve in the Current Era & Ann Plast Surg & NRCS not adjusted \\
\hline 341 & 33062963 & Groth & $\begin{array}{l}\text { A Picture of Breast Reconstruction in a Public Oncology Hospital in Latin America: } \\
\text { A Ten-Year Experience }\end{array}$ & Eur J Breast Health & $\begin{array}{l}\text { Does not address KQ1- } \\
\text { KQ6 }\end{array}$ \\
\hline 342 & 2523544 & Grotting & $\begin{array}{l}\text { Conventional TRAM flap versus free microsurgical TRAM flap for immediate breast } \\
\text { reconstruction }\end{array}$ & $\begin{array}{l}\text { Plast Reconstr } \\
\text { Surg }\end{array}$ & $\begin{array}{l}\text { Single group } \mathrm{N} \text { enrolled } \\
<500\end{array}$ \\
\hline 343 & 7232564 & Gruber & $\begin{array}{l}\text { Breast reconstruction following mastectomy: A comparison of submuscular and } \\
\text { subcutaneous techniques }\end{array}$ & $\begin{array}{l}\text { Plastic and } \\
\text { Reconstructive } \\
\text { Surgery }\end{array}$ & NRCS not adjusted \\
\hline 344 & 7232564 & Gruber & $\begin{array}{l}\text { Breast reconstruction following mastectomy: a comparison of submuscular and } \\
\text { subcutaneous techniques }\end{array}$ & $\begin{array}{l}\text { Plast Reconstr } \\
\text { Surg }\end{array}$ & NRCS not adjusted \\
\hline 345 & 24792480 & Gryskiewicz & $\begin{array}{l}\text { Transaxillary Nonendoscopic Subpectoral Augmentation Mammaplasty: A 10-Year } \\
\text { Experience With Gel vs Saline in } 2000 \text { Patients-With Long-Term Patient } \\
\text { Satisfaction Measured by the BREAST-Q }\end{array}$ & Aesthet Surg J & $\begin{array}{l}>=10 \% \text { augmentation } \\
\text { reconstruction only }\end{array}$ \\
\hline 346 & 26947961 & $\begin{array}{l}\text { Gschwantler- } \\
\text { Kaulich }\end{array}$ & $\begin{array}{l}\text { Mesh versus acellular dermal matrix in immediate implant-based breast } \\
\text { reconstruction - A prospective randomized trial }\end{array}$ & Eur J Surg Oncol & $\begin{array}{l}\text { Does not address KQ1- } \\
\text { KQ6 }\end{array}$ \\
\hline 347 & 16932175 & Gusenoff & $\begin{array}{l}\text { Free tissue transfer: comparison of outcomes between university hospitals and } \\
\text { community hospitals }\end{array}$ & $\begin{array}{l}\text { Plast Reconstr } \\
\text { Surg }\end{array}$ & $\begin{array}{l}\text { Does not address KQ1- } \\
\text { KQ6 }\end{array}$ \\
\hline 348 & 141474299 & $\mathrm{Ha}$ & $\begin{array}{l}\text { Oncologic outcomes after immediate breast reconstruction following mastectomy: } \\
\text { comparison of implant and flap using propensity score matching }\end{array}$ & BMC Cancer & $\begin{array}{l}\text { Duplicate of another } \\
\text { publication }\end{array}$ \\
\hline 349 & 30616906 & Hadad & $\begin{array}{l}\text { Sub-muscular plane for augmentation mammoplasty patients increases silicone gel } \\
\text { implant rupture rate }\end{array}$ & $\begin{array}{l}\text { J Plast Reconstr } \\
\text { Aesthet Surg }\end{array}$ & $\begin{array}{l}>=10 \% \text { augmentation } \\
\text { reconstruction only }\end{array}$ \\
\hline 350 & 29068920 & Haddock & Five Steps to Internal Mammary Vessel Preparation in Less than 15 Minutes & $\begin{array}{l}\text { Plast Reconstr } \\
\text { Surg }\end{array}$ & $\begin{array}{l}\text { Single group } \mathrm{N} \text { enrolled } \\
<500\end{array}$ \\
\hline 351 & 32440397 & Haddock & $\begin{array}{l}\text { Consecutive } 265 \text { Profunda Artery Perforator Flaps: Refinements, Satisfaction, and } \\
\text { Functional Outcomes }\end{array}$ & $\begin{array}{l}\text { Plast Reconstr } \\
\text { Surg Glob Open }\end{array}$ & $\begin{array}{l}\text { Single group N enrolled } \\
<500\end{array}$ \\
\hline 352 & 32221196 & Hagarty & $\begin{array}{l}\text { Decreased Length of Postoperative Drain Use, Parenteral Opioids, Length of Stay, } \\
\text { and Complication Rates in Patients Receiving Meshed versus Unmeshed Acellular } \\
\text { Dermal Matrix in } 194 \text { Submuscular Tissue Expander-Based Breast } \\
\text { Reconstructions: A Single-Surgeon Cohort Study }\end{array}$ & $\begin{array}{l}\text { Plast Reconstr } \\
\text { Surg }\end{array}$ & $\begin{array}{l}\text { Does not address KQ1- } \\
\text { KQ6 }\end{array}$ \\
\hline 353 & 21200201 & Hall-Findlay & Breast implant complication review: double capsules and late seromas & $\begin{array}{l}\text { Plast Reconstr } \\
\text { Surg }\end{array}$ & $\begin{array}{l}\text { Not breast } \\
\text { reconstruction }\end{array}$ \\
\hline 354 & 29320921 & Hallberg & $\begin{array}{l}\text { Benefits and risks with acellular dermal matrix (ADM) and mesh support in } \\
\text { immediate breast reconstruction: a systematic review and meta-analysis }\end{array}$ & $\begin{array}{l}\text { J Plast Surg Hand } \\
\text { Surg }\end{array}$ & Systematic review \\
\hline 355 & 31144025 & Hamann & $\begin{array}{l}\text { Quality of life in breast cancer patients and surgical results of immediate tissue } \\
\text { expander/implant-based breast reconstruction after mastectomy }\end{array}$ & $\begin{array}{l}\text { Arch Gynecol } \\
\text { Obstet }\end{array}$ & NRCS $<30$ per arm \\
\hline 356 & 33480982 & Hamdi & $\begin{array}{l}\text { The 'Hug Flap': Surgical Technique to Enhance the Aesthetic Breast Projection in } \\
\text { Autologous Breast Reconstruction }\end{array}$ & Aesthet Surg J & $\begin{array}{l}\text { Single group } \mathrm{N} \text { enrolled } \\
<500\end{array}$ \\
\hline 357 & 32988607 & Hammond & $\begin{array}{l}\text { Capsular contracture in the modern era: A multidisciplinary look at the incidence } \\
\text { and risk factors after mastectomy and implant-based breast reconstruction }\end{array}$ & Am J Surg & NRCS not adjusted \\
\hline
\end{tabular}




\begin{tabular}{|c|c|c|c|c|c|}
\hline No. & $\begin{array}{l}\text { PMID or } \\
\text { Other } \\
\text { Identifier }\end{array}$ & $\begin{array}{l}\text { First Author } \\
\text { Last Name }\end{array}$ & Title & Journal & Reason for Exclusion \\
\hline 358 & 19338905 & Handel & Long-term safety and efficacy of polyurethane foam-covered breast implants & Aesthet Surg J & $\begin{array}{l}\text { Single group } \mathrm{N} \text { enrolled } \\
<500\end{array}$ \\
\hline 359 & 1871226 & Handel & $\begin{array}{l}\text { Comparative experience with smooth and polyurethane breast implants using the } \\
\text { Kaplan-Meier method of survival analysis }\end{array}$ & $\begin{array}{l}\text { Plast Reconstr } \\
\text { Surg }\end{array}$ & $\begin{array}{l}\text { Single group } \mathrm{N} \text { enrolled } \\
<500\end{array}$ \\
\hline 360 & 31606126 & Hangge & $\begin{array}{l}\text { Making an informed choice: Which breast reconstruction type has the lowest } \\
\text { complication rate? }\end{array}$ & $\begin{array}{l}\text { American Journal } \\
\text { of Surgery }\end{array}$ & $\begin{array}{l}\text { Duplicate of another } \\
\text { publication }\end{array}$ \\
\hline 361 & 21862915 & Hanna & $\begin{array}{l}\text { Comparison study of two types of expander-based breast reconstruction: acellular } \\
\text { dermal matrix-assisted versus total submuscular placement }\end{array}$ & Ann Plast Surg & NRCS not adjusted \\
\hline 362 & 130221949 & Hansen & $\begin{array}{l}\text { Evaluating Mastectomy Skin Flap Necrosis in the Extended Breast Reconstruction } \\
\text { Risk Assessment Score for } 1 \text {-Year Prediction of Prosthetic Reconstruction } \\
\text { Outcomes }\end{array}$ & $\begin{array}{l}\text { Journal of the } \\
\text { American College } \\
\text { of Surgeons }\end{array}$ & $\begin{array}{l}\text { Duplicate of another } \\
\text { publication }\end{array}$ \\
\hline 363 & 27757331 & Hanson & $\begin{array}{l}\text { Fewer Revisions in Abdominal-based Free Flaps than Latissimus Dorsi Breast } \\
\text { Reconstruction after Radiation }\end{array}$ & $\begin{array}{l}\text { Plast Reconstr } \\
\text { Surg Glob Open }\end{array}$ & $\begin{array}{l}\text { Does not address KQ1- } \\
\text { KQ6 }\end{array}$ \\
\hline 364 & 33051871 & Hansson & $\begin{array}{l}\text { First-year complications after immediate breast reconstruction with a biological and } \\
\text { a synthetic mesh in the same patient: A randomized controlled study }\end{array}$ & J Surg Oncol & $\begin{array}{l}\text { Does not address KQ1- } \\
\text { KQ6 }\end{array}$ \\
\hline 365 & 23769660 & Hanwright & $\begin{array}{l}\text { The differential effect of BMI on prosthetic versus autogenous breast } \\
\text { reconstruction: a multivariate analysis of } 12,986 \text { patients }\end{array}$ & Breast & $\begin{array}{l}\text { Does not address KQ1- } \\
\text { KQ6 }\end{array}$ \\
\hline 366 & 27505449 & Hart & The Impact of Diabetes Mellitus on Wound Healing in Breast Reconstruction & Ann Plast Surg & $\begin{array}{l}\text { Does not address KQ1- } \\
\text { KQ6 }\end{array}$ \\
\hline 367 & 32405724 & Hartmann & $\begin{array}{l}\text { A Novel Method of Outcome Assessment in Breast Reconstruction Surgery: } \\
\text { Comparison of Autologous and Alloplastic Techniques Using Three-Dimensional } \\
\text { Surface Imaging }\end{array}$ & $\begin{array}{l}\text { Aesthetic Plast } \\
\text { Surg }\end{array}$ & NRCS $<30$ per arm \\
\hline 368 & 30217311 & Hauck & Secondary breast reconstruction after mastectomy using the DIEP flap & Surg Oncol & $\begin{array}{l}\text { Single group } \mathrm{N} \text { enrolled } \\
<500\end{array}$ \\
\hline 369 & 19546667 & Haykal & $\begin{array}{l}\text { One hundred forty-one consecutive attempts at autologous tissue single-stage } \\
\text { breast cancer reconstruction }\end{array}$ & Ann Plast Surg & $\begin{array}{l}\text { Single group N enrolled } \\
<500\end{array}$ \\
\hline 370 & 27798424 & $\mathrm{He}$ & $\begin{array}{l}\text { Considering the Optimal Timing of Breast Reconstruction With Abdominal Flaps } \\
\text { With Adjuvant Irradiation in } 370 \text { Consecutive Pedicled Transverse Rectus } \\
\text { Abdominis Myocutaneous Flap and Free Deep Inferior Epigastric Perforator Flap } \\
\text { Performed in a Chinese Oncology Center: Is There a Significant Difference } \\
\text { Between Immediate and Delayed? }\end{array}$ & Ann Plast Surg & NRCS not adjusted \\
\hline 371 & 31386193 & Heeg & $\begin{array}{l}\text { Nationwide population-based study of the impact of immediate breast } \\
\text { reconstruction after mastectomy on the timing of adjuvant chemotherapy }\end{array}$ & $\begin{array}{l}\text { British Journal of } \\
\text { Surgery }\end{array}$ & No outcome of interest \\
\hline 372 & 33241589 & Heidekrueger & $\begin{array}{l}\text { Comparison of venous couplers versus hand-sewn technique in } 4577 \text { cases of } \\
\text { DIEP-flap breast reconstructions - A multicenter study }\end{array}$ & Microsurgery & $\begin{array}{l}\text { Copublication of } \\
\text { included study with no } \\
\text { new data }\end{array}$ \\
\hline 373 & 33461890 & Heidekrueger & $\begin{array}{l}\text { Impact of body mass index on free DIEP flap breast reconstruction: A multicenter } \\
\text { cohort study }\end{array}$ & $\begin{array}{l}\text { J Plast Reconstr } \\
\text { Aesthet Surg }\end{array}$ & $\begin{array}{l}\text { Copublication of } \\
\text { included study with no } \\
\text { new data }\end{array}$ \\
\hline
\end{tabular}




\begin{tabular}{|c|c|c|c|c|c|}
\hline No. & $\begin{array}{l}\text { PMID or } \\
\text { Other } \\
\text { Identifier }\end{array}$ & $\begin{array}{l}\text { First Author } \\
\text { Last Name }\end{array}$ & Title & Journal & Reason for Exclusion \\
\hline 374 & 29464161 & Heidemann & $\begin{array}{l}\text { Complications following Nipple-Sparing Mastectomy and Immediate Acellular } \\
\text { Dermal Matrix Implant-based Breast Reconstruction-A Systematic Review and } \\
\text { Meta-analysis }\end{array}$ & $\begin{array}{l}\text { Plast Reconstr } \\
\text { Surg Glob Open }\end{array}$ & $\begin{array}{l}\text { Does not address KQ1- } \\
\text { KQ6 }\end{array}$ \\
\hline 375 & 16365235 & Henriksen & $\begin{array}{l}\text { Reconstructive breast implantation after mastectomy for breast cancer: clinical } \\
\text { outcomes in a nationwide prospective cohort study }\end{array}$ & Arch Surg & $\begin{array}{l}\text { Single group } \mathrm{N} \text { enrolled } \\
<500\end{array}$ \\
\hline 376 & 31913883 & Henton & $\begin{array}{l}\text { Microsurgical Training Opportunities at the Queen Victoria Hospital: A } \\
\text { Retrospective Review of } 848 \text { Free Flaps for Breast Reconstruction }\end{array}$ & Ann Plast Surg & $\begin{array}{l}\text { Single group }>500 \text {, but } \\
\text { no complications data }\end{array}$ \\
\hline 377 & 33625028 & Hermiz & $\begin{array}{l}\text { Use of a 5-Item Modified Frailty Index for Risk Stratification in Patients Undergoing } \\
\text { Breast Reconstruction }\end{array}$ & Ann Plast Surg & NRCS not adjusted \\
\hline 378 & 22531395 & Hill & $\begin{array}{l}\text { Infectious complications associated with the use of acellular dermal matrix in } \\
\text { implant-based bilateral breast reconstruction }\end{array}$ & Ann Plast Surg & NRCS $<30$ per arm \\
\hline 379 & 30100676 & Hillberg & $\begin{array}{l}\text { Is single-stage implant-based breast reconstruction (SSBR) with an acellular matrix } \\
\text { safe?: Strattice or Meso Biomatrix(R) in SSBR }\end{array}$ & Eur J Plast Surg & NRCS $<30$ per arm \\
\hline 380 & 22286418 & Hirsch & $\begin{array}{l}\text { Outcomes of tissue expander/implant breast reconstruction in the setting of } \\
\text { prereconstruction radiation }\end{array}$ & $\begin{array}{l}\text { Plast Reconstr } \\
\text { Surg }\end{array}$ & $\begin{array}{l}\text { Single group N enrolled } \\
<500\end{array}$ \\
\hline 381 & 21918963 & Ho & $\begin{array}{l}\text { Long-term outcomes in breast cancer patients undergoing immediate 2-stage } \\
\text { expander/implant reconstruction and postmastectomy radiation }\end{array}$ & Cancer & $\begin{array}{l}\text { Does not address KQ1- } \\
\text { KQ6 }\end{array}$ \\
\hline 382 & 22421476 & $\mathrm{Ho}$ & $\begin{array}{l}\text { A systematic review and meta-analysis of complications associated with acellular } \\
\text { dermal matrix-assisted breast reconstruction }\end{array}$ & Ann Plast Surg & Systematic review \\
\hline 383 & 31350217 & Hoejvig & Delayed two-stage breast reconstruction: The impact of radiotherapy & $\begin{array}{l}J \text { Plast Reconstr } \\
\text { Aesthet Surg }\end{array}$ & $\begin{array}{l}\text { Single group N enrolled } \\
<500\end{array}$ \\
\hline 384 & 7622124 & Hoflehner & $\begin{array}{l}\text { [Mammography of the reconstructed breast--a comparison of different methods of } \\
\text { reconstruction] }\end{array}$ & $\begin{array}{l}\text { Handchir Mikrochir } \\
\text { Plast Chir }\end{array}$ & NRCS $<30$ per arm \\
\hline 385 & 30930124 & Holmes & $\begin{array}{l}\text { Salvage of the failed implant-based breast reconstruction using the Deep Inferior } \\
\text { Epigastric Perforator Flap: A single centre experience with tertiary breast } \\
\text { reconstruction }\end{array}$ & $\begin{array}{l}J \text { Plast Reconstr } \\
\text { Aesthet Surg }\end{array}$ & NRCS $<30$ per arm \\
\hline 386 & 18580143 & Holmich & $\begin{array}{l}\text { Delayed breast reconstruction with implants after invasive breast cancer does not } \\
\text { impair prognosis }\end{array}$ & Ann Plast Surg & $\begin{array}{l}\text { Single group }>500 \text {, but } \\
\text { no complications data }\end{array}$ \\
\hline 387 & 31075801 & Holoyda & $\begin{array}{l}\text { Immediate Bilateral Breast Reconstruction Using Abdominally Based Flaps: An } \\
\text { Analysis of the Nationwide Inpatient Sample Database }\end{array}$ & $\begin{array}{l}J \text { Reconstr } \\
\text { Microsurg }\end{array}$ & NRCS not adjusted \\
\hline 388 & 30694847 & Homsy & $\begin{array}{l}\text { Regional Anesthetic Blocks in Plastic Surgery Using Portable Ultrasound: A } \\
\text { Simplified Approach }\end{array}$ & Ann Plast Surg & $\begin{array}{l}\text { Single group N enrolled } \\
<500\end{array}$ \\
\hline 389 & 22084645 & Hoppe & $\begin{array}{l}\text { Complications following expander/implant breast reconstruction utilizing acellular } \\
\text { dermal matrix: a systematic review and meta-analysis }\end{array}$ & Eplasty & $\begin{array}{l}\text { Narrative review/ } \\
\text { Commentary }\end{array}$ \\
\hline 390 & 112727680 & Hsueh-Hsing & $\begin{array}{l}\text { Predictors for Reconstruction and Mood Disorder Associated With Reconstruction } \\
\text { in Patients With Breast Cancer and Mastectomy: A Retrospective Cohort Study }\end{array}$ & Medicine & $\begin{array}{l}\text { Duplicate of another } \\
\text { publication }\end{array}$ \\
\hline 391 & 21264833 & $\mathrm{Hu}$ & Impact of neoadjuvant chemotherapy on breast reconstruction & Cancer & $\begin{array}{l}\text { Does not address KQ1- } \\
\text { KQ6 }\end{array}$ \\
\hline 392 & 104650234 & $\mathrm{Hu}$ & Impact of neoadjuvant chemotherapy on breast reconstruction & Cancer (0008543X) & $\begin{array}{l}\text { Duplicate of another } \\
\text { publication }\end{array}$ \\
\hline
\end{tabular}




\begin{tabular}{|c|c|c|c|c|c|}
\hline No. & $\begin{array}{l}\text { PMID or } \\
\text { Other } \\
\text { Identifier }\end{array}$ & $\begin{array}{l}\text { First Author } \\
\text { Last Name }\end{array}$ & Title & Journal & Reason for Exclusion \\
\hline 393 & 32167044 & Huang & Factors Associated with Reconstruction in Patients Undergoing Mastectomy & Am Surg & $\begin{array}{l}\text { Does not address KQ1- } \\
\text { KQ6 }\end{array}$ \\
\hline 394 & 27198885 & Huang & $\begin{array}{l}\text { Routine sampling of internal mammary lymph nodes during microsurgical breast } \\
\text { reconstruction-Experience based on } 524 \text { microsurgical breast reconstructions }\end{array}$ & $\begin{array}{l}\text { Journal of Surgical } \\
\text { Oncology }\end{array}$ & $\begin{array}{l}\text { Does not address KQ1- } \\
\text { KQ6 }\end{array}$ \\
\hline 395 & 27294034 & Huang & $\begin{array}{l}\text { Current status of breast reconstruction in China: an experience of } 951 \text { breast } \\
\text { reconstructions from a single institute }\end{array}$ & Gland Surg & $\begin{array}{l}\text { Single group } \mathrm{N} \text { enrolled } \\
<500\end{array}$ \\
\hline 396 & 12800900 & Hultman & $\begin{array}{l}\text { Skin-sparing mastectomy flap complications after breast reconstruction: review of } \\
\text { incidence, management, and outcome }\end{array}$ & Ann Plast Surg & NRCS $<30$ per arm \\
\hline 397 & 12800900 & Hultman & $\begin{array}{l}\text { Skin-sparing mastectomy flap complications after breast reconstruction: Review of } \\
\text { incidence, management, and outcome }\end{array}$ & $\begin{array}{l}\text { Annals of Plastic } \\
\text { Surgery }\end{array}$ & NRCS $<30$ per arm \\
\hline 398 & 26796375 & Hunsinger & Long-Term Follow-Up of Quality of Life following DIEP Flap Breast Reconstruction & $\begin{array}{l}\text { Plast Reconstr } \\
\text { Surg }\end{array}$ & $\begin{array}{l}\text { Single group } \mathrm{N} \text { enrolled } \\
<500\end{array}$ \\
\hline 399 & 26808742 & Hunter & Superior Gluteal Artery Perforator Flap: The Beauty of the Buttock & Ann Plast Surg & NRCS $<30$ per arm \\
\hline 400 & 27178333 & Huo & $\begin{array}{l}\text { Post-mastectomy breast reconstruction and its subsequent complications: a } \\
\text { comparison between obese and non-obese women with breast cancer }\end{array}$ & $\begin{array}{l}\text { Breast Cancer } \\
\text { Research and } \\
\text { Treatment }\end{array}$ & $\begin{array}{l}\text { Does not address KQ1- } \\
\text { KQ6 }\end{array}$ \\
\hline 401 & 27178333 & Huo & $\begin{array}{l}\text { Post-mastectomy breast reconstruction and its subsequent complications: a } \\
\text { comparison between obese and non-obese women with breast cancer }\end{array}$ & $\begin{array}{l}\text { Breast Cancer Res } \\
\text { Treat }\end{array}$ & $\begin{array}{l}\text { Does not address KQ1- } \\
\text { KQ6 }\end{array}$ \\
\hline 402 & 25894022 & Ibrahim & $\begin{array}{l}\text { Does acellular dermal matrix really improve aesthetic outcome in tissue } \\
\text { expander/implant-based breast reconstruction? }\end{array}$ & $\begin{array}{l}\text { Aesthetic Plast } \\
\text { Surg }\end{array}$ & NRCS $<30$ per arm \\
\hline 403 & 27988412 & Ilonzo & $\begin{array}{l}\text { Breast reconstruction after mastectomy: A ten-year analysis of trends and } \\
\text { immediate postoperative outcomes }\end{array}$ & Breast & $\begin{array}{l}\text { Does not address KQ1- } \\
\text { KQ6 }\end{array}$ \\
\hline 404 & 28032163 & Imahiyerobo & $\begin{array}{l}\text { Transition from Round to Shaped Implants in Immediate Breast Reconstruction: } \\
\text { Our Preferred Approach and Clinical Outcomes }\end{array}$ & $\begin{array}{l}\text { Aesthetic Plast } \\
\text { Surg }\end{array}$ & NRCS not adjusted \\
\hline 405 & 31119358 & Isaksson & $\begin{array}{l}\text { Bilateral Risk-Reducing Mastectomies with Implant-Based Reconstructions } \\
\text { Followed Long Term: A Consecutive Series of } 185 \text { Patients }\end{array}$ & World J Surg & $\begin{array}{l}\text { Does not address KQ1- } \\
\text { KQ6 }\end{array}$ \\
\hline 406 & 27475116 & Islam & $\begin{array}{l}\text { The largest and neglected giant phyllodes tumor of the breast - A case report and } \\
\text { literature review }\end{array}$ & $\begin{array}{l}\text { International } \\
\text { Journal of Surgery } \\
\text { Case Reports }\end{array}$ & $\begin{array}{l}\text { Case report or series of } \\
\text { case reports }\end{array}$ \\
\hline 407 & 23710783 & $\begin{array}{l}\text { Israeli Ben- } \\
\text { Noon }\end{array}$ & $\begin{array}{l}\text { The effect of acellular dermal matrix on drain secretions after immediate prosthetic } \\
\text { breast reconstruction }\end{array}$ & $\begin{array}{l}\text { J Plast Surg Hand } \\
\text { Surg }\end{array}$ & NRCS $<30$ per arm \\
\hline 408 & $\begin{array}{l}\text { CN- } \\
01848602\end{array}$ & Isrctn & The iBRA (implant breast reconstruction evaluation) study & $\begin{array}{l}\text { http://www.who.int/t } \\
\text { rialsearch/Trial2.as } \\
\text { px?TriallD=ISRCT } \\
\text { N37664281 }\end{array}$ & $\begin{array}{l}\text { Protocol/methods with } \\
\text { no results }\end{array}$ \\
\hline 409 & $\begin{array}{l}\text { CN- } \\
01832640\end{array}$ & Isrctn & QUEST Trial B - Quality of life following mastectomy and breast reconstruction & $\begin{array}{l}\text { http://www.who.int/t } \\
\text { rialsearch/Trial2.as } \\
\text { px?TriallD=ISRCT } \\
\text { N92581226 }\end{array}$ & NRCS $<30$ per arm \\
\hline 410 & $\begin{array}{l}\text { CN- } \\
01843414\end{array}$ & Isrctn & $\begin{array}{l}\text { A trial evaluating outcomes of immediate implant-based breast reconstruction } \\
\text { using an acellular dermal matrix (ADM) (POBRAD trial) }\end{array}$ & $\begin{array}{l}\text { http://www.who.int/t } \\
\text { rialsearch/Trial2.as }\end{array}$ & $\begin{array}{l}\text { Protocol/methods with } \\
\text { no results }\end{array}$ \\
\hline
\end{tabular}




\begin{tabular}{|c|c|c|c|c|c|}
\hline No. & $\begin{array}{l}\text { PMID or } \\
\text { Other } \\
\text { Identifier }\end{array}$ & $\begin{array}{l}\text { First Author } \\
\text { Last Name }\end{array}$ & Title & Journal & Reason for Exclusion \\
\hline & & & & $\begin{array}{l}\text { px?TrialID }=I S R C T \\
\text { N67956295 }\end{array}$ & \\
\hline 411 & $\begin{array}{l}\text { CN- } \\
01821781\end{array}$ & Isrctn & QUEST Trial A - Quality of life following mastectomy and breast reconstruction & $\begin{array}{l}\text { http://www. who.int/t } \\
\text { rialsearch/Trial2.as } \\
\text { px?TriallD=ISRCT } \\
\text { N38846532 }\end{array}$ & $\begin{array}{l}\text { Protocol/methods with } \\
\text { no results }\end{array}$ \\
\hline 412 & 30589768 & Ivey & $\begin{array}{l}\text { Total Muscle Coverage versus AlloDerm Human Dermal Matrix for Implant-Based } \\
\text { Breast Reconstruction }\end{array}$ & $\begin{array}{l}\text { Plast Reconstr } \\
\text { Surg }\end{array}$ & NRCS not adjusted \\
\hline 413 & 26380109 & Iwahira & $\begin{array}{l}\text { Nummular Eczema of Breast: A Potential Dermatologic Complication after } \\
\text { Mastectomy and Subsequent Breast Reconstruction }\end{array}$ & Plast Surg Int & $\begin{array}{l}\text { Single group N enrolled } \\
<500\end{array}$ \\
\hline 414 & 30014454 & Jabo & $\begin{array}{l}\text { Impact of Breast Reconstruction on Time to Definitive Surgical Treatment, Adjuvant } \\
\text { Therapy, and Breast Cancer Outcomes }\end{array}$ & Ann Surg Oncol & $\begin{array}{l}\text { Does not address KQ1- } \\
\text { KQ6 }\end{array}$ \\
\hline 415 & 124774782 & Jacobson & $\begin{array}{l}\text { Risk Factors for Implant-Based Reconstruction Failure after Mastectomy with or } \\
\text { without Radiation in Patients Treated for Breast Cancer }\end{array}$ & $\begin{array}{l}\text { International } \\
\text { Journal of } \\
\text { Radiation } \\
\text { Oncology, Biology, } \\
\text { Physics }\end{array}$ & NRCS not adjusted \\
\hline 416 & 25876011 & Jagsi & $\begin{array}{l}\text { Complications After Mastectomy and Immediate Breast Reconstruction for Breast } \\
\text { Cancer: A Claims-Based Analysis }\end{array}$ & Ann Surg & $\begin{array}{l}\text { Copublication of } \\
\text { included study with no } \\
\text { new data }\end{array}$ \\
\hline 417 & 28954300 & Jagsi & $\begin{array}{l}\text { Impact of radiotherapy on complications and patient-reported satisfaction with } \\
\text { breast reconstruction: findings from the prospective multicenter MROC study }\end{array}$ & $\begin{array}{l}\text { Cancer research. } \\
\text { Conference: 39th } \\
\text { annual CTRC- } \\
\text { AACR san antonio } \\
\text { breast cancer } \\
\text { symposium. United } \\
\text { states }\end{array}$ & $\begin{array}{l}\text { Does not address KQ1- } \\
\text { KQ6 }\end{array}$ \\
\hline 418 & 28954300 & Jagsi & $\begin{array}{l}\text { Impact of Radiotherapy on Complications and Patient-Reported Outcomes After } \\
\text { Breast Reconstruction }\end{array}$ & J Natl Cancer Inst & $\begin{array}{l}\text { Duplicate of another } \\
\text { publication }\end{array}$ \\
\hline 419 & 21617454 & Jandali & $\begin{array}{l}\text { Breast reconstruction with free tissue transfer from the abdomen in the morbidly } \\
\text { obese }\end{array}$ & $\begin{array}{l}\text { Plast Reconstr } \\
\text { Surg }\end{array}$ & $\begin{array}{l}\text { Single group } \mathrm{N} \text { enrolled } \\
<500\end{array}$ \\
\hline 420 & 20195107 & Jandali & $\begin{array}{l}1000 \text { consecutive venous anastomoses using the microvascular anastomotic } \\
\text { coupler in breast reconstruction }\end{array}$ & $\begin{array}{l}\text { Plast Reconstr } \\
\text { Surg }\end{array}$ & NRCS not adjusted \\
\hline 421 & 10717013 & Janowsky & $\begin{array}{l}\text { Meta-analyses of the relation between silicone breast implants and the risk of } \\
\text { connective-tissue diseases }\end{array}$ & N Engl J Med & Systematic review \\
\hline 422 & 2333815 & Jarrett & Aesthetic refinements in prophylactic mastectomy with immediate reconstruction & $\begin{array}{l}\text { Aesthetic Plast } \\
\text { Surg }\end{array}$ & $\begin{array}{l}\text { Duplicate of another } \\
\text { publication }\end{array}$ \\
\hline 423 & 2333815 & Jarrett & Aesthetic refinements in prophylatic mastectomy with immediate reconstruction & $\begin{array}{l}\text { Aesthetic Plastic } \\
\text { Surgery }\end{array}$ & $\begin{array}{l}\text { Does not address KQ1- } \\
\text { KQ6 }\end{array}$ \\
\hline 424 & 16482786 & Javaid & $\begin{array}{l}\text { Radiation effects on the cosmetic outcomes of immediate and delayed autologous } \\
\text { breast reconstruction: an argument about timing }\end{array}$ & $\begin{array}{l}J \text { Plast Reconstr } \\
\text { Aesthet Surg }\end{array}$ & $\begin{array}{l}\text { Does not address KQ1- } \\
\text { KQ6 }\end{array}$ \\
\hline
\end{tabular}




\begin{tabular}{|c|c|c|c|c|c|}
\hline No. & $\begin{array}{l}\text { PMID or } \\
\text { Other } \\
\text { Identifier }\end{array}$ & $\begin{array}{l}\text { First Author } \\
\text { Last Name }\end{array}$ & Title & Journal & Reason for Exclusion \\
\hline 425 & 32596186 & Jayaraman & $\begin{array}{l}\text { The Impact of Combined Risk-Reducing Gynecological Surgeries on Outcomes in } \\
\text { DIEP Flap and Tissue-Expander Breast Reconstruction }\end{array}$ & Plast Surg (Oakv) & NRCS $<30$ per arm \\
\hline 426 & 31418290 & Jeevan & $\begin{array}{l}\text { Reconstructive utilisation and outcomes following mastectomy surgery in women } \\
\text { with breast cancer treated in England }\end{array}$ & $\begin{array}{l}\text { Ann R Coll Surg } \\
\text { Engl }\end{array}$ & No outcome of interest \\
\hline 427 & 28445349 & Jeevan & $\begin{array}{l}\text { Surgical Determinants of Patient-Reported Outcomes following Postmastectomy } \\
\text { Reconstruction in Women with Breast Cancer }\end{array}$ & $\begin{array}{l}\text { Plast Reconstr } \\
\text { Surg }\end{array}$ & $\begin{array}{l}\text { Does not address KQ1- } \\
\text { KQ6 }\end{array}$ \\
\hline 428 & 26075654 & Jeevan & $\begin{array}{l}\text { Socioeconomic deprivation and inpatient complication rates following mastectomy } \\
\text { and breast reconstruction surgery }\end{array}$ & Br J Surg & $\begin{array}{l}\text { Does not address KQ1- } \\
\text { KQ6 }\end{array}$ \\
\hline 429 & 24908545 & Jeevan & $\begin{array}{l}\text { Findings of a national comparative audit of mastectomy and breast reconstruction } \\
\text { surgery in England }\end{array}$ & $\begin{array}{l}J \text { Plast Reconstr } \\
\text { Aesthet Surg }\end{array}$ & $\begin{array}{l}\text { Does not address KQ1- } \\
\text { KQ6 }\end{array}$ \\
\hline 430 & 24908545 & Jeevan & $\begin{array}{l}\text { Findings of a national comparative audit of mastectomy and breast reconstruction } \\
\text { surgery in England }\end{array}$ & $\begin{array}{l}\text { Journal of Plastic, } \\
\text { Reconstructive and } \\
\text { Aesthetic Surgery }\end{array}$ & $\begin{array}{l}\text { Duplicate of another } \\
\text { publication }\end{array}$ \\
\hline 431 & 21174155 & Jensen & Nipple-sparing mastectomy in 99 patients with a mean follow-up of 5 years & Ann Surg Oncol & NRCS not adjusted \\
\hline 432 & 29227815 & Jeong & $\begin{array}{l}\text { Meta-analysis of flap perfusion and donor site complications for breast } \\
\text { reconstruction using pedicled versus free TRAM and DIEP flaps }\end{array}$ & Breast & $\begin{array}{l}\text { Does not address KQ1- } \\
\text { KQ6 }\end{array}$ \\
\hline 433 & 30665838 & Jepsen & $\begin{array}{l}\text { Complications, patient-reported outcomes, and aesthetic results in immediate } \\
\text { breast reconstruction with a dermal sling: A systematic review and meta-analysis }\end{array}$ & $\begin{array}{l}\text { J Plast Reconstr } \\
\text { Aesthet Surg }\end{array}$ & $\begin{array}{l}\text { Does not address KQ1- } \\
\text { KQ6 }\end{array}$ \\
\hline 434 & 18472362 & Jhaveri & $\begin{array}{l}\text { Clinical outcomes of postmastectomy radiation therapy after immediate breast } \\
\text { reconstruction }\end{array}$ & $\begin{array}{l}\text { Int J Radiat Oncol } \\
\text { Biol Phys }\end{array}$ & NRCS not adjusted \\
\hline 435 & 26313786 & Jia-jian & $\begin{array}{l}\text { Current Status of Breast Reconstruction in Southern China: A } 15 \text { Year, Single } \\
\text { Institutional Experience of } 20,551 \text { Breast Cancer Patients }\end{array}$ & $\begin{array}{l}\text { Medicine } \\
\text { (Baltimore) }\end{array}$ & $\begin{array}{l}\text { Does not address KQ1- } \\
\text { KQ6 }\end{array}$ \\
\hline 436 & 27159061 & $\begin{array}{l}\text { Jimenez- } \\
\text { Puente }\end{array}$ & $\begin{array}{l}\text { [Breast Reconstruction Post-Mastectomy in the Public Health System of Andalusia, } \\
\text { Spain] }\end{array}$ & $\begin{array}{l}\text { Rev Esp Salud } \\
\text { Publica }\end{array}$ & NRCS not adjusted \\
\hline 437 & 21388901 & $\begin{array}{l}\text { JoAnna } \\
\text { Nguyen }\end{array}$ & $\begin{array}{l}\text { Use of human acellular dermal matrix in implant- based breast reconstruction: } \\
\text { evaluating the evidence }\end{array}$ & $\begin{array}{l}\text { J Plast Reconstr } \\
\text { Aesthet Surg }\end{array}$ & $\begin{array}{l}\text { Narrative review/ } \\
\text { Commentary }\end{array}$ \\
\hline 438 & 25954837 & Johnson & $\begin{array}{l}\text { Advanced Age Does Not Worsen Recovery or Long-Term Morbidity After } \\
\text { Postmastectomy Breast Reconstruction }\end{array}$ & Ann Plast Surg & NRCS not adjusted \\
\hline 439 & 23266307 & Johnson & $\begin{array}{l}\text { Cost minimisation analysis of using acellular dermal matrix (Strattice) for breast } \\
\text { reconstruction compared with standard techniques }\end{array}$ & Eur J Surg Oncol & NRCS $<30$ per arm \\
\hline 440 & 30795637 & Jonczyk & $\begin{array}{l}\text { Trending Towards Safer Breast Cancer Surgeries? Examining Acute Complication } \\
\text { Rates from A 13-Year NSQIP Analysis }\end{array}$ & Cancers (Basel) & $\begin{array}{l}\text { Copublication of } \\
\text { included study with no } \\
\text { new data }\end{array}$ \\
\hline 441 & 27018665 & Jordan & Seroma in Prosthetic Breast Reconstruction & $\begin{array}{l}\text { Plast Reconstr } \\
\text { Surg }\end{array}$ & $\begin{array}{l}\text { Does not address KQ1- } \\
\text { KQ6 }\end{array}$ \\
\hline 442 & $\begin{array}{l}\text { CN- } \\
01881181\end{array}$ & Jprn & $\begin{array}{l}\text { The study of assessing the safety of NEOVEIL sheet in immediate two-stage tissue } \\
\text { expander/implant breast reconstruction }\end{array}$ & $\begin{array}{l}\text { http://www.who.int/t } \\
\text { rialsearch/Trial2.as } \\
\text { px?TrialID=JPRN- } \\
\text { UMIN000018644 }\end{array}$ & $\begin{array}{l}\text { Protocol/methods with } \\
\text { no results }\end{array}$ \\
\hline 443 & 28543692 & Jubbal & $\begin{array}{l}\text { The impact of resident involvement in breast reconstruction surgery outcomes by } \\
\text { modality: An analysis of } 4,500 \text { cases }\end{array}$ & Microsurgery & $\begin{array}{l}\text { Does not address KQ1- } \\
\text { KQ6 }\end{array}$ \\
\hline
\end{tabular}




\begin{tabular}{|c|c|c|c|c|c|}
\hline No. & $\begin{array}{l}\text { PMID or } \\
\text { Other } \\
\text { Identifier }\end{array}$ & $\begin{array}{l}\text { First Author } \\
\text { Last Name }\end{array}$ & Title & Journal & Reason for Exclusion \\
\hline 444 & 28085525 & Juhl & $\begin{array}{l}\text { Unilateral breast reconstruction after mastectomy - patient satisfaction, } \\
\text { aesthetic outcome and quality of life }\end{array}$ & Acta Oncol & NRCS not adjusted \\
\hline 445 & 121307353 & Juhl & $\begin{array}{l}\text { Unilateral breast reconstruction after mastectomy - patient satisfaction, aesthetic } \\
\text { outcome and quality of life }\end{array}$ & Acta Oncologica & NRCS not adjusted \\
\hline 446 & 32007227 & Julien & $\begin{array}{l}\text { [Comparing outcomes of Immediate Breast reconstruction with and without use of } \\
\text { radiotherapy] }\end{array}$ & $\begin{array}{l}\text { Ann Chir Plast } \\
\text { Esthet }\end{array}$ & NRCS not adjusted \\
\hline 447 & 33482758 & Jung & $\begin{array}{l}\text { Does chemotherapy or radiotherapy affect the postoperative complication in breast } \\
\text { cancer patients who underwent immediate breast reconstruction with tissue } \\
\text { expander? }\end{array}$ & BMC Cancer & $\begin{array}{l}\text { Does not address KQ1- } \\
\text { KQ6 }\end{array}$ \\
\hline 448 & 26382872 & Kadle & $\begin{array}{l}\text { A 35-Year Evolution of Free Flap-Based Breast Reconstruction at a Large Urban } \\
\text { Academic Center }\end{array}$ & $\begin{array}{l}J \text { Reconstr } \\
\text { Microsurg }\end{array}$ & $\begin{array}{l}\text { Single group }>500 \text {, but } \\
\text { no complications data }\end{array}$ \\
\hline 449 & 20459373 & Kalaaji & Quality of life after breast reconstruction: comparison of three methods & $\begin{array}{l}\text { Scand J Plast } \\
\text { Reconstr Surg } \\
\text { Hand Surg }\end{array}$ & NRCS not adjusted \\
\hline 450 & 28409847 & Kamali & $\begin{array}{l}\text { Trends in immediate breast reconstruction and early complication rates among } \\
\text { older women: A big data analysis }\end{array}$ & J Surg Oncol & NRCS not adjusted \\
\hline 451 & 26986990 & Kamali & $\begin{array}{l}\text { Analyzing Regional Differences over a } 15-\text { Year Trend of One-Stage versus Two- } \\
\text { Stage Breast Reconstruction in 941,191 Postmastectomy Patients }\end{array}$ & $\begin{array}{l}\text { Plast Reconstr } \\
\text { Surg }\end{array}$ & $\begin{array}{l}\text { Does not address KQ1- } \\
\text { KQ6 }\end{array}$ \\
\hline 452 & 28338587 & Kamali & $\begin{array}{l}\text { Medial Row Perforators Are Associated with Higher Rates of Fat Necrosis in } \\
\text { Bilateral DIEP Flap Breast Reconstruction }\end{array}$ & $\begin{array}{l}\text { Plast Reconstr } \\
\text { Surg }\end{array}$ & $\begin{array}{l}\text { Single group N enrolled } \\
<500\end{array}$ \\
\hline 453 & 28079533 & Kamali & $\begin{array}{l}\text { National and Regional Differences in 32,248 Postmastectomy Autologous Breast } \\
\text { Reconstruction Using the Updated National Inpatient Survey }\end{array}$ & Ann Plast Surg & $\begin{array}{l}\text { Does not address KQ1- } \\
\text { KQ6 }\end{array}$ \\
\hline 454 & 29464148 & Kamali & $\begin{array}{l}\text { Immediate Breast Reconstruction among Patients with Medicare and Private } \\
\text { Insurance: A Matched Cohort Analysis }\end{array}$ & $\begin{array}{l}\text { Plast Reconstr } \\
\text { Surg Glob Open }\end{array}$ & $\begin{array}{l}\text { Does not address KQ1- } \\
\text { KQ6 }\end{array}$ \\
\hline 455 & 31764628 & Kamel & $\begin{array}{l}\text { Patient-Reported Satisfaction and Quality of Life in Obese Patients: A Comparison } \\
\text { between Microsurgical and Prosthetic Implant Recipients }\end{array}$ & $\begin{array}{l}\text { Plast Reconstr } \\
\text { Surg }\end{array}$ & $\begin{array}{l}\text { Single group } \mathrm{N} \text { enrolled } \\
<500\end{array}$ \\
\hline 456 & 30716775 & Kamel & $\begin{array}{l}\text { Patient-Reported Satisfaction and Quality of Life in Postmastectomy Radiated } \\
\text { Patients: A Comparison between Delayed and Delayed Immediate Autologous } \\
\text { Breast Reconstruction in a Predominantly Minority Patient Population }\end{array}$ & $\begin{array}{l}J \text { Reconstr } \\
\text { Microsurg }\end{array}$ & $\begin{array}{l}\text { Single group } \mathrm{N} \text { enrolled } \\
<500\end{array}$ \\
\hline 457 & 10724253 & Kaplan & $\begin{array}{l}\text { Cost-based comparison between perforator flaps and TRAM flaps for breast } \\
\text { reconstruction }\end{array}$ & $\begin{array}{l}\text { Plast Reconstr } \\
\text { Surg }\end{array}$ & NRCS $<30$ per arm \\
\hline 458 & 33462766 & Karadsheh & $\begin{array}{l}\text { Early postoperative outcomes in implant, pedicled, and free flap reconstruction for } \\
\text { breast cancer: an analysis of } 23,834 \text { patients from the ACS-NSQIP datasets }\end{array}$ & $\begin{array}{l}\text { Breast Cancer Res } \\
\text { Treat }\end{array}$ & $\begin{array}{l}\text { Does not address KQ1- } \\
\text { KQ6 }\end{array}$ \\
\hline 459 & 32892332 & Karamanos & $\begin{array}{l}\text { Impact of Blood Transfusion in Free Flap Breast Reconstruction Using Propensity } \\
\text { Score Matching }\end{array}$ & $\begin{array}{l}\text { J Reconstr } \\
\text { Microsurg }\end{array}$ & $\begin{array}{l}\text { Single group } \mathrm{N} \text { enrolled } \\
<500\end{array}$ \\
\hline 460 & 12601605 & Kassmann & $\begin{array}{l}\text { [Myosonographic evaluation of rectus abdominis muscle function after DIEP flap } \\
\text { breast reconstruction] }\end{array}$ & $\begin{array}{l}\text { Handchir Mikrochir } \\
\text { Plast Chir }\end{array}$ & $\begin{array}{l}\text { Single group N enrolled } \\
<500\end{array}$ \\
\hline 461 & 31800564 & Kaviani & $\begin{array}{l}\text { A Study on Breast Reconstruction in a Developing Country: A Comprehensive } \\
\text { Evaluation of the Techniques and Oncologic Outcomes }\end{array}$ & Ann Plast Surg & NRCS not adjusted \\
\hline 462 & 26526860 & Kearney & Timing of radiation and outcomes in implant-based breast reconstruction & $\begin{array}{l}\text { J Plast Reconstr } \\
\text { Aesthet Surg }\end{array}$ & NRCS not adjusted \\
\hline
\end{tabular}




\begin{tabular}{|c|c|c|c|c|c|}
\hline No. & $\begin{array}{l}\text { PMID or } \\
\text { Other } \\
\text { Identifier }\end{array}$ & $\begin{array}{l}\text { First Author } \\
\text { Last Name }\end{array}$ & Title & Journal & Reason for Exclusion \\
\hline 463 & 24473643 & Kelley & $\begin{array}{l}\text { A systematic review of morbidity associated with autologous breast reconstruction } \\
\text { before and after exposure to radiotherapy: are current practices ideal? }\end{array}$ & Ann Surg Oncol & Systematic review \\
\hline 464 & 21987043 & Kelley & $\begin{array}{l}\text { Tamoxifen increases the risk of microvascular flap complications in patients } \\
\text { undergoing microvascular breast reconstruction }\end{array}$ & $\begin{array}{l}\text { Plast Reconstr } \\
\text { Surg }\end{array}$ & $\begin{array}{l}\text { Does not address KQ1- } \\
\text { KQ6 }\end{array}$ \\
\hline 465 & 30794884 & Kellou & $\begin{array}{l}\text { [Limitations of breast reconstruction using exclusive lipofilling: A retrospective } \\
\text { study over } 10 \text { years] }\end{array}$ & $\begin{array}{l}\text { Gynecol Obstet } \\
\text { Fertil Senol }\end{array}$ & $\begin{array}{l}\text { Does not address KQ1- } \\
\text { KQ6 }\end{array}$ \\
\hline 466 & 24388599 & Kelly & $\begin{array}{l}\text { Lateralising paraumbilical medial row perforators: dangers and pitfalls in DIEP } \\
\text { FLAP planning: a systematic review of } 1116 \text { DIEP flaps }\end{array}$ & $\begin{array}{l}\text { J Plast Reconstr } \\
\text { Aesthet Surg }\end{array}$ & $\begin{array}{l}\text { Single group } \mathrm{N} \text { enrolled } \\
<500\end{array}$ \\
\hline 467 & 9283576 & Kern & Carcinogenic potential of silicone breast implants: a Connecticut statewide study & $\begin{array}{l}\text { Plast Reconstr } \\
\text { Surg }\end{array}$ & $\begin{array}{l}\text { Not mastectomy for } \\
\text { breast cancer }\end{array}$ \\
\hline 468 & 32207573 & Khajuria & $\begin{array}{l}\text { Immediate and delayed autologous abdominal microvascular flap breast } \\
\text { reconstruction in patients receiving adjuvant, neoadjuvant or no radiotherapy: a } \\
\text { meta-analysis of clinical and quality-of-life outcomes }\end{array}$ & BJS Open & $\begin{array}{l}\text { Narrative review/ } \\
\text { Commentary }\end{array}$ \\
\hline 469 & 31772906 & Khajuria & $\begin{array}{l}\text { A Meta-analysis of Clinical, Patient-Reported Outcomes and Cost of DIEP versus } \\
\text { Implant-based Breast Reconstruction }\end{array}$ & $\begin{array}{l}\text { Plast Reconstr } \\
\text { Surg Glob Open }\end{array}$ & Systematic review \\
\hline 470 & 29166926 & Khajuria & $\begin{array}{l}\text { Protocol for a systematic review and meta-analysis on the clinical outcomes and } \\
\text { cost of deep inferior epigastric perforator (DIEP) flap versus implants for breast } \\
\text { reconstruction }\end{array}$ & Syst Rev & $\begin{array}{l}\text { Narrative review/ } \\
\text { Commentary }\end{array}$ \\
\hline 471 & 21451371 & Khansa & $\begin{array}{l}\text { Postmastectomy breast reconstruction after previous lumpectomy and radiation } \\
\text { therapy: analysis of complications and satisfaction }\end{array}$ & Ann Plast Surg & $\begin{array}{l}\text { Does not address KQ1- } \\
\text { KQ6 }\end{array}$ \\
\hline 472 & 24123194 & Khansa & $\begin{array}{l}\text { Timing of prophylactic hysterectomy-oophorectomy, mastectomy, and } \\
\text { microsurgical breast reconstruction in BRCA1 and BRCA2 carriers }\end{array}$ & Microsurgery & $\begin{array}{l}\text { Does not address KQ1- } \\
\text { KQ6 }\end{array}$ \\
\hline 473 & 28445350 & Khavanin & $\begin{array}{l}\text { Shaped versus Round Implants in Breast Reconstruction: A Multi-Institutional } \\
\text { Comparison of Surgical and Patient-Reported Outcomes }\end{array}$ & $\begin{array}{l}\text { Plast Reconstr } \\
\text { Surg }\end{array}$ & $\begin{array}{l}\text { Copublication of } \\
\text { included study with no } \\
\text { new data }\end{array}$ \\
\hline 474 & 24121881 & Khavanin & $\begin{array}{l}\text { Tumescent technique does not increase the risk of complication following } \\
\text { mastectomy with immediate reconstruction }\end{array}$ & Ann Surg Oncol & $\begin{array}{l}\text { Single group } \mathrm{N} \text { enrolled } \\
<500\end{array}$ \\
\hline 475 & 24121881 & Khavanin & $\begin{array}{l}\text { Tumescent technique does not increase the risk of complication following } \\
\text { mastectomy with immediate reconstruction }\end{array}$ & $\begin{array}{l}\text { Annals of Surgical } \\
\text { Oncology }\end{array}$ & $\begin{array}{l}\text { Does not address KQ1- } \\
\text { KQ6 }\end{array}$ \\
\hline 476 & 24454465 & Khavanin & $\begin{array}{l}\text { Synergistic interactions with a high intraoperative expander fill volume increase the } \\
\text { risk for mastectomy flap necrosis }\end{array}$ & J Breast Cancer & $\begin{array}{l}\text { Does not address KQ1- } \\
\text { KQ6 }\end{array}$ \\
\hline 477 & 25311295 & Kilchenmann & $\begin{array}{l}\text { An evaluation of resource utilisation of single stage porcine acellular dermal matrix } \\
\text { assisted breast reconstruction: A comparative study }\end{array}$ & Breast & NRCS $<30$ per arm \\
\hline 478 & 23389902 & Kim & $\begin{array}{l}\text { Immediate transverse rectus abdominis musculocutaneous (TRAM) flap breast } \\
\text { reconstruction in underweight Asian patients }\end{array}$ & Breast Cancer & $\begin{array}{l}\text { Copublication of } \\
\text { included study with no } \\
\text { new data }\end{array}$ \\
\hline 479 & 120349978 & Kim & $\begin{array}{l}\text { Nipple areola skin-sparing mastectomy with TRAM flap reconstruction: Single- } \\
\text { center study }\end{array}$ & $\begin{array}{l}\text { Journal of Clinical } \\
\text { Oncology }\end{array}$ & $\begin{array}{l}\text { Duplicate of another } \\
\text { publication }\end{array}$ \\
\hline 480 & 23428931 & Kim & $\begin{array}{l}\text { Comparison of morbidity of donor site following pedicled muscle-sparing latissimus } \\
\text { dorsi flap versus extended latissimus dorsi flap breast reconstruction }\end{array}$ & $\begin{array}{l}\text { J Plast Reconstr } \\
\text { Aesthet Surg }\end{array}$ & NRCS not adjusted \\
\hline
\end{tabular}




\begin{tabular}{|c|c|c|c|c|c|}
\hline No. & $\begin{array}{l}\text { PMID or } \\
\text { Other } \\
\text { Identifier }\end{array}$ & $\begin{array}{l}\text { First Author } \\
\text { Last Name }\end{array}$ & Title & Journal & Reason for Exclusion \\
\hline 481 & 20134317 & Kim & $\begin{array}{l}\text { Nipple-areola skin-sparing mastectomy with immediate transverse rectus } \\
\text { abdominis musculocutaneous flap reconstruction is an oncologically safe } \\
\text { procedure }\end{array}$ & Breast Diseases & $\begin{array}{l}\text { Single group } N \text { enrolled } \\
<500\end{array}$ \\
\hline 482 & 30282415 & Kim & $\begin{array}{l}\text { Breast reconstruction statistics in Korea from the Big Data Hub of the Health } \\
\text { Insurance Review and Assessment Service }\end{array}$ & Arch Plast Surg & $\begin{array}{l}\text { Does not address KQ1- } \\
\text { KQ6 }\end{array}$ \\
\hline 483 & 22186498 & Kim & $\begin{array}{l}\text { A meta-analysis of human acellular dermis and submuscular tissue expander } \\
\text { breast reconstruction }\end{array}$ & $\begin{array}{l}\text { Plast Reconstr } \\
\text { Surg }\end{array}$ & $\begin{array}{l}\text { Does not address KQ1- } \\
\text { KQ6 }\end{array}$ \\
\hline 484 & 26090295 & Kim & $\begin{array}{l}\text { Individualized Risk of Surgical Complications: An Application of the Breast } \\
\text { Reconstruction Risk Assessment Score }\end{array}$ & $\begin{array}{l}\text { Plast Reconstr } \\
\text { Surg Glob Open }\end{array}$ & No outcome of interest \\
\hline 485 & 31775207 & Kim & $\begin{array}{l}\text { Inlay graft of acellular dermal matrix to prevent incisional dehiscence after } \\
\text { radiotherapy in prosthetic breast reconstruction }\end{array}$ & Arch Plast Surg & NRCS $<30$ per arm \\
\hline 486 & 28204936 & Kim & $\begin{array}{l}\text { Impact of Acellular Dermal Matrix (ADM) Use Under Mastectomy Flap Necrosis on } \\
\text { Perioperative Outcomes of Prosthetic Breast Reconstruction }\end{array}$ & $\begin{array}{l}\text { Aesthetic Plast } \\
\text { Surg }\end{array}$ & NRCS $<30$ per arm \\
\hline 487 & 32332387 & Kim & $\begin{array}{l}\text { Inframammary Fold Incision Can Reduce Skin Flap Necrosis in Immediate Breast } \\
\text { Reconstruction With Implant and Conjoined Fascial Flap }\end{array}$ & Ann Plast Surg & NRCS $<30$ per arm \\
\hline 488 & 32724804 & Kim & $\begin{array}{l}\text { Diametric Comparison between the Thoracodorsal Vessel and Deep Inferior } \\
\text { Epigastric Vessel in Breast Reconstruction }\end{array}$ & Biomed Res Int & $\begin{array}{l}\text { Single group } \mathrm{N} \text { enrolled } \\
<500\end{array}$ \\
\hline 489 & 22286439 & Kobraei & $\begin{array}{l}\text { Risk factors for adverse outcome following skin-sparing mastectomy and } \\
\text { immediate prosthetic reconstruction }\end{array}$ & $\begin{array}{l}\text { Plast Reconstr } \\
\text { Surg }\end{array}$ & $\begin{array}{l}\text { Does not address KQ1- } \\
\text { KQ6 }\end{array}$ \\
\hline 490 & 30173716 & Koh & Quality of life and shoulder function after latissimus dorsi breast reconstruction() & $\begin{array}{l}J \text { Plast Reconstr } \\
\text { Aesthet Surg }\end{array}$ & NRCS not adjusted \\
\hline 491 & 30859037 & $\begin{array}{l}\text { Komorowska- } \\
\text { Timek }\end{array}$ & $\begin{array}{l}\text { Subcutaneous Prosthetic Breast Reconstructions following Skin Reduction } \\
\text { Mastectomy }\end{array}$ & $\begin{array}{l}\text { Plast Reconstr } \\
\text { Surg Glob Open }\end{array}$ & NRCS $<30$ per arm \\
\hline 492 & 24636099 & Koolen & Effects of statins on ischemia-reperfusion complications in breast free flaps & J Surg Res & $\begin{array}{l}\text { Not breast } \\
\text { reconstruction }\end{array}$ \\
\hline 493 & 24636099 & Koolen & Effects of statins on ischemia-reperfusion complications in breast free flaps & $\begin{array}{l}\text { Journal of Surgical } \\
\text { Research }\end{array}$ & $\begin{array}{l}\text { Single group } \mathrm{N} \text { enrolled } \\
<500\end{array}$ \\
\hline 494 & 24512987 & Korwar & $\begin{array}{l}\text { Skin reducing mastectomy and immediate reconstruction: the effect of radiotherapy } \\
\text { on complications and patient reported outcomes }\end{array}$ & Eur J Surg Oncol & NRCS not adjusted \\
\hline 495 & 33002979 & Kotha & $\begin{array}{l}\text { A Critical Examination of Length of Stay in Autologous Breast Reconstruction: A } \\
\text { National Surgical Quality Improvement Program Analysis }\end{array}$ & $\begin{array}{l}\text { Plast Reconstr } \\
\text { Surg }\end{array}$ & $\begin{array}{l}\text { Does not address KQ1- } \\
\text { KQ6 }\end{array}$ \\
\hline 496 & 33349523 & Kouwenberg & Cost-utility analysis of four common surgical treatment pathways for breast cancer & $\begin{array}{l}\text { European Journal } \\
\text { of Surgical } \\
\text { Oncology }\end{array}$ & $\begin{array}{l}\text { Copublication of } \\
\text { included study with no } \\
\text { new data }\end{array}$ \\
\hline 497 & 30964213 & Kracoff & $\begin{array}{l}\text { Neo-adjuvant chemotherapy does not affect the immediate postoperative } \\
\text { complication rate after breast reconstruction }\end{array}$ & Breast J & $\begin{array}{l}\text { Does not address KQ1- } \\
\text { KQ6 }\end{array}$ \\
\hline 498 & 31066148 & Kracoff & $\begin{array}{l}\text { Does nipple sparing mastectomy affect the postoperative complication rate after } \\
\text { breast reconstruction? Comparison of postoperative complications after nipple } \\
\text { sparing mastectomy vs skin sparing mastectomy }\end{array}$ & Breast J & $\begin{array}{l}\text { Does not address KQ1- } \\
\text { KQ6 }\end{array}$ \\
\hline
\end{tabular}




\begin{tabular}{|c|c|c|c|c|c|}
\hline No. & $\begin{array}{l}\text { PMID or } \\
\text { Other } \\
\text { Identifier }\end{array}$ & $\begin{array}{l}\text { First Author } \\
\text { Last Name }\end{array}$ & Title & Journal & Reason for Exclusion \\
\hline 499 & 33614536 & Kraft & $\begin{array}{l}\text { Polypropylene Mesh Complications in the Sublay Position After Abdominally Based } \\
\text { Breast Reconstruction: Les complications des treillis de polypropylène en sous- } \\
\text { couche après une reconstruction mammaire par voie abdominale }\end{array}$ & Plast Surg (Oakv) & NRCS not adjusted \\
\hline 500 & 11050764 & Krause & $\begin{array}{l}\text { Skin sparing mastectomy and immediate autologous reconstruction: oncological } \\
\text { risks and aesthetic results }\end{array}$ & $\begin{array}{l}\text { Journal of cancer } \\
\text { research and } \\
\text { clinical oncology }\end{array}$ & $\begin{array}{l}\text { Single group N enrolled } \\
<500\end{array}$ \\
\hline 501 & 25811560 & Krishnan & $\begin{array}{l}\text { The cost effectiveness of the DIEP flap relative to the muscle-sparing TRAM flap in } \\
\text { postmastectomy breast reconstruction }\end{array}$ & $\begin{array}{l}\text { Plast Reconstr } \\
\text { Surg }\end{array}$ & $\begin{array}{l}\text { Does not address KQ1- } \\
\text { KQ6 }\end{array}$ \\
\hline 502 & 1387483 & Kroll & A comparison of outcomes using three different methods of breast reconstruction & $\begin{array}{l}\text { Plast Reconstr } \\
\text { Surg }\end{array}$ & NRCS not adjusted \\
\hline 503 & 8559819 & Kroll & $\begin{array}{l}\text { Comparison of resource costs between implant-based and TRAM flap breast } \\
\text { reconstruction }\end{array}$ & $\begin{array}{l}\text { Plast Reconstr } \\
\text { Surg }\end{array}$ & NRCS not adjusted \\
\hline 504 & 8559819 & Kroll & $\begin{array}{l}\text { Comparison of resource costs between implant-based and TRAM flap breast } \\
\text { reconstruction }\end{array}$ & $\begin{array}{l}\text { Plastic and } \\
\text { Reconstructive } \\
\text { Surgery } \\
\end{array}$ & $\begin{array}{l}\text { Duplicate of another } \\
\text { publication }\end{array}$ \\
\hline 505 & 7850550 & Kroll & $\begin{array}{l}\text { Rationale for elective contralateral mastectomy with immediate bilateral } \\
\text { reconstruction }\end{array}$ & Ann Surg Oncol & NRCS not adjusted \\
\hline 506 & 11335809 & Kroll & Comparison of cost for DIEP and free TRAM flap breast reconstructions & $\begin{array}{l}\text { Plast Reconstr } \\
\text { Surg }\end{array}$ & NRCS not adjusted \\
\hline 507 & 9618094 & Kroll & Does prior irradiation increase the risk of total or partial free-flap loss? & $\begin{array}{l}\text { J Reconstr } \\
\text { Microsurg }\end{array}$ & $\begin{array}{l}\text { Single group } \mathrm{N} \text { enrolled } \\
<500\end{array}$ \\
\hline 508 & 8700982 & Kroll & Choice of flap and incidence of free flap success & $\begin{array}{l}\text { Plast Reconstr } \\
\text { Surg }\end{array}$ & NRCS not adjusted \\
\hline 509 & 7638285 & Kroll & Abdominal wall strength, bulging, and hernia after TRAM flap breast reconstruction & $\begin{array}{l}\text { Plast Reconstr } \\
\text { Surg }\end{array}$ & NRCS not adjusted \\
\hline 510 & 22842406 & Kronowitz & $\begin{array}{l}\text { Current status of autologous tissue-based breast reconstruction in patients } \\
\text { receiving postmastectomy radiation therapy }\end{array}$ & $\begin{array}{l}\text { Plast Reconstr } \\
\text { Surg }\end{array}$ & $\begin{array}{l}\text { Narrative review/ } \\
\text { Commentary }\end{array}$ \\
\hline 511 & 16404237 & Kronowitz & Determining the optimal approach to breast reconstruction after partial mastectomy & $\begin{array}{l}\text { Plast Reconstr } \\
\text { Surg }\end{array}$ & NRCS $<30$ per arm \\
\hline 512 & 14663219 & Kronowitz & Optimizing autologous breast reconstruction in thin patients & $\begin{array}{l}\text { Plast Reconstr } \\
\text { Surg }\end{array}$ & NRCS not adjusted \\
\hline 513 & 20465509 & Kropf & $\begin{array}{l}\text { Influence of the recipient vessel on fat necrosis after breast reconstruction with a } \\
\text { free transverse rectus abdominis myocutaneous flap }\end{array}$ & $\begin{array}{l}\text { Scand J Plast } \\
\text { Reconstr Surg } \\
\text { Hand Surg }\end{array}$ & $\begin{array}{l}\text { Does not address KQ1- } \\
\text { KQ6 }\end{array}$ \\
\hline 514 & 29504033 & Kubo & $\begin{array}{l}\text { Complication analysis of complete versus partial coverage of tissue expanders } \\
\text { using serratus anterior musculofascial flaps in immediate breast reconstruction }\end{array}$ & Surg Today & NRCS not adjusted \\
\hline 515 & 26372685 & Kulkarni & $\begin{array}{l}\text { Venous Thrombosis in Handsewn versus Coupled Venous Anastomoses in } 857 \\
\text { Consecutive Breast Free Flaps }\end{array}$ & $\begin{array}{l}J \text { Reconstr } \\
\text { Microsurg }\end{array}$ & NRCS not adjusted \\
\hline 516 & 30231269 & Kung & $\begin{array}{l}\text { Radiation-Induced Skin Changes after Postmastectomy Radiation Therapy: A Pilot } \\
\text { Study on Indicators for Timing of Delayed Breast Reconstruction }\end{array}$ & $\begin{array}{l}\text { J Reconstr } \\
\text { Microsurg }\end{array}$ & NRCS $<30$ per arm \\
\hline
\end{tabular}




\begin{tabular}{|c|c|c|c|c|c|}
\hline No. & $\begin{array}{l}\text { PMID or } \\
\text { Other } \\
\text { Identifier }\end{array}$ & $\begin{array}{l}\text { First Author } \\
\text { Last Name }\end{array}$ & Title & Journal & Reason for Exclusion \\
\hline 517 & 31332635 & Kupstas & $\begin{array}{l}\text { Effect of Surgery Type on Time to Adjuvant Chemotherapy and Impact of Delay on } \\
\text { Breast Cancer Survival: A National Cancer Database Analysis }\end{array}$ & Ann Surg Oncol & $\begin{array}{l}\text { Single group }>500 \text {, but } \\
\text { no complications data }\end{array}$ \\
\hline 518 & 28328628 & Kuykendall & Unilateral Versus Bilateral Breast Reconstruction: Is Less Really More? & Ann Plast Surg & NRCS not adjusted \\
\hline 519 & 27714840 & Kwok & $\begin{array}{l}\text { An analysis of free flap failure using the ACS NSQIP database. Does flap site and } \\
\text { flap type matter? }\end{array}$ & Microsurgery & $\begin{array}{l}\text { Does not address KQ1- } \\
\text { KQ6 }\end{array}$ \\
\hline 520 & 29452440 & Kwok & $\begin{array}{l}\text { Operative Time and Flap Failure in Unilateral and Bilateral Free Flap Breast } \\
\text { Reconstruction }\end{array}$ & $\begin{array}{l}\text { Reconstr } \\
\text { Microsurg }\end{array}$ & $\begin{array}{l}\text { Does not address KQ1- } \\
\text { KQ6 }\end{array}$ \\
\hline 521 & 26054659 & Kwok & $\begin{array}{l}\text { National trends and complication rates after bilateral mastectomy and immediate } \\
\text { breast reconstruction from } 2005 \text { to } 2012\end{array}$ & $\begin{array}{l}\text { American Journal } \\
\text { of Surgery }\end{array}$ & $\begin{array}{l}\text { Duplicate of another } \\
\text { publication }\end{array}$ \\
\hline 522 & 26054659 & Kwok & $\begin{array}{l}\text { National trends and complication rates after bilateral mastectomy and immediate } \\
\text { breast reconstruction from } 2005 \text { to } 2012\end{array}$ & Am J Surg & $\begin{array}{l}\text { Does not address KQ1- } \\
\text { KQ6 }\end{array}$ \\
\hline 523 & 30085346 & Kwok & $\begin{array}{l}\text { Immediate Unilateral Breast Reconstruction using Abdominally Based Flaps: } \\
\text { Analysis of 3,310 Cases }\end{array}$ & $\begin{array}{l}\text { J Reconstr } \\
\text { Microsurg }\end{array}$ & NRCS not adjusted \\
\hline 524 & 109199616 & Kwok & $\begin{array}{l}\text { National trends and complication rates after bilateral mastectomy and immediate } \\
\text { breast reconstruction from } 2005 \text { to } 2012\end{array}$ & $\begin{array}{l}\text { American Journal } \\
\text { of Surgery }\end{array}$ & $\begin{array}{l}\text { Duplicate of another } \\
\text { publication }\end{array}$ \\
\hline 525 & 26794627 & $\begin{array}{l}\text { Lagares- } \\
\text { Borrego }\end{array}$ & $\begin{array}{l}\text { A comparison of long-term cost and clinical outcomes between the two-stage } \\
\text { sequence expander/prosthesis and autologous deep inferior epigastric flap } \\
\text { methods for breast reconstruction in a public hospital }\end{array}$ & $\begin{array}{l}J \text { Plast Reconstr } \\
\text { Aesthet Surg }\end{array}$ & NRCS not adjusted \\
\hline 526 & 30178391 & Lagendijk & Patient-Reported Outcome Measures May Add Value in Breast Cancer Surgery & Ann Surg Oncol & $\begin{array}{l}\text { Single group N enrolled } \\
<500\end{array}$ \\
\hline 527 & 29139613 & Lago & $\begin{array}{l}\text { Nipple-sparing mastectomy as treatment for patients with ductal carcinoma in } \\
\text { situ: A 10-year follow-up study }\end{array}$ & Breast Journal & $\begin{array}{l}\text { Single group } \mathrm{N} \text { enrolled } \\
<500\end{array}$ \\
\hline 528 & 30145648 & Lai & $\begin{array}{l}\text { Robotic Nipple-Sparing Mastectomy and Immediate Breast Reconstruction with } \\
\text { Gel Implant }\end{array}$ & Ann Surg Oncol & $\begin{array}{l}\text { Duplicate of another } \\
\text { publication }\end{array}$ \\
\hline 529 & 29750759 & Lam & $\begin{array}{l}\text { Immediate Two-Stage Prosthetic Breast Reconstruction Failure: Radiation Is Not } \\
\text { the Only Culprit }\end{array}$ & $\begin{array}{l}\text { Plast Reconstr } \\
\text { Surg }\end{array}$ & $\begin{array}{l}\text { Does not address KQ1- } \\
\text { KQ6 }\end{array}$ \\
\hline 530 & 23676964 & Lam & $\begin{array}{l}\text { The effects of postmastectomy adjuvant radiotherapy on immediate two-stage } \\
\text { prosthetic breast reconstruction: a systematic review }\end{array}$ & $\begin{array}{l}\text { Plast Reconstr } \\
\text { Surg }\end{array}$ & $\begin{array}{l}\text { Narrative review/ } \\
\text { Commentary }\end{array}$ \\
\hline 531 & 29062656 & Lam & $\begin{array}{l}\text { Two-Stage Prosthetic Breast Reconstruction after Mastectomy with or without Prior } \\
\text { Postmastectomy Radiotherapy }\end{array}$ & $\begin{array}{l}\text { Plast Reconstr } \\
\text { Surg Glob Open }\end{array}$ & $\begin{array}{l}\text { Single group N enrolled } \\
<500\end{array}$ \\
\hline 532 & 12560692 & Langstein & Breast cancer recurrence after immediate reconstruction: patterns and significance & $\begin{array}{l}\text { Plast Reconstr } \\
\text { Surg }\end{array}$ & $\begin{array}{l}\text { Does not address KQ1- } \\
\text { KQ6 }\end{array}$ \\
\hline 533 & 20395795 & Lanier & $\begin{array}{l}\text { The effect of acellular dermal matrix use on complication rates in tissue } \\
\text { expander/implant breast reconstruction }\end{array}$ & Ann Plast Surg & NRCS not adjusted \\
\hline 534 & $\begin{array}{l}\text { CN- } \\
01301217\end{array}$ & Laporta & $\begin{array}{l}\text { Breast Reconstruction in Elderly Patients: risk Factors, Clinical Outcomes, and } \\
\text { Aesthetic Results }\end{array}$ & $\begin{array}{l}\text { Journal of } \\
\text { reconstructive } \\
\text { microsurgery }\end{array}$ & $\begin{array}{l}\text { Duplicate of another } \\
\text { publication }\end{array}$ \\
\hline 535 & 28061518 & Laporta & $\begin{array}{l}\text { Breast Reconstruction in Elderly Patients: Risk Factors, Clinical Outcomes, and } \\
\text { Aesthetic Results }\end{array}$ & $\begin{array}{l}\text { Journal of } \\
\text { Reconstructive } \\
\text { Microsurgery } \\
\end{array}$ & $\begin{array}{l}\text { Duplicate of another } \\
\text { publication }\end{array}$ \\
\hline
\end{tabular}




\begin{tabular}{|c|c|c|c|c|c|}
\hline No. & $\begin{array}{l}\text { PMID or } \\
\text { Other } \\
\text { Identifier }\end{array}$ & $\begin{array}{l}\text { First Author } \\
\text { Last Name }\end{array}$ & Title & Journal & Reason for Exclusion \\
\hline 536 & 23559355 & Largent & Clinical trial outcomes of high- and extra high-profile breast implants & Aesthet Surg J & $\begin{array}{l}\text { Not breast } \\
\text { reconstruction }\end{array}$ \\
\hline 537 & 31316828 & Laura & $\begin{array}{l}\text { Postsurgical Ultrasound Evaluation of Patients with Prosthesis in Acellular Dermal } \\
\text { Matrix: Results from Monocentric Experience }\end{array}$ & Int J Surg Oncol & NRCS $<30$ per arm \\
\hline 538 & 33337991 & Lazzaroni & $\begin{array}{l}\text { Association of anti-RNA polymerase III antibody with silicone breast implants } \\
\text { rupture in a multicentre series of Italian patients with systemic sclerosis }\end{array}$ & $\begin{array}{l}\text { Clin Exp } \\
\text { Rheumatol }\end{array}$ & $\begin{array}{l}\text { Single group N enrolled } \\
<500\end{array}$ \\
\hline 539 & 32337778 & Le & $\begin{array}{l}\text { Impact of socioeconomic status on psychological functioning in survivorship } \\
\text { following breast cancer and reconstruction }\end{array}$ & Breast Journal & $\begin{array}{l}\text { Does not address KQ1- } \\
\text { KQ6 }\end{array}$ \\
\hline 540 & 26936318 & Leckenby & $\begin{array}{l}\text { Poly Implant Prothese (PIP) experience in the United Kingdom: A prospective } \\
\text { cohort study into the accuracy of diagnostic imaging findings in comparison to } \\
\text { operative findings of } 1029 \text { implants }\end{array}$ & $\begin{array}{l}J \text { Plast Reconstr } \\
\text { Aesthet Surg }\end{array}$ & $\begin{array}{l}\text { Not breast } \\
\text { reconstruction }\end{array}$ \\
\hline 541 & 26543648 & Leduey & $\begin{array}{l}\text { Comparison of the Explantation Rate of Poly Implant Prothese, Allergan, and } \\
\text { Perouse Silicone Breast Implants within the First Four Years after Reconstructive } \\
\text { Surgery before the Poly Implant Prothese Alert by the French Regulatory Authority }\end{array}$ & Int J Breast Cancer & $\begin{array}{l}>=10 \% \text { augmentation } \\
\text { reconstruction only }\end{array}$ \\
\hline 542 & 29068921 & Lee & $\begin{array}{l}\text { Evidence-Based Clinical Practice Guideline: Autologous Breast Reconstruction } \\
\text { with DIEP or Pedicled TRAM Abdominal Flaps }\end{array}$ & $\begin{array}{l}\text { Plast Reconstr } \\
\text { Surg }\end{array}$ & $\begin{array}{l}\text { Narrative review/ } \\
\text { Commentary }\end{array}$ \\
\hline 543 & 20395800 & Lee & $\begin{array}{l}\text { Postmastectomy radiation therapy and breast reconstruction: an analysis of } \\
\text { complications and patient satisfaction }\end{array}$ & Ann Plast Surg & NRCS not adjusted \\
\hline 544 & 20009825 & Lee & $\begin{array}{l}\text { Establishment of perforator flap programs for breast reconstruction: the New } \\
\text { England program experience }\end{array}$ & $\begin{array}{l}\text { Plast Reconstr } \\
\text { Surg }\end{array}$ & NRCS not adjusted \\
\hline 545 & 19651073 & Lee & $\begin{array}{l}\text { Patient-reported outcomes of breast reconstruction after mastectomy: a systematic } \\
\text { review }\end{array}$ & J Am Coll Surg & $\begin{array}{l}\text { Does not address KQ1- } \\
\text { KQ6 }\end{array}$ \\
\hline 546 & 30431446 & Lee & $\begin{array}{l}\text { Nipple-sparing Mastectomy and Immediate Breast Reconstruction After } \\
\text { Recurrence From Previous Breast Conservation Therapy }\end{array}$ & Ann Plast Surg & NRCS not adjusted \\
\hline 547 & 31964119 & Lee & $\begin{array}{l}\text { Ultrasonic dissection versus electrocautery for immediate prosthetic breast } \\
\text { reconstruction }\end{array}$ & Arch Plast Surg & $\begin{array}{l}\text { Single group } \mathrm{N} \text { enrolled } \\
<500\end{array}$ \\
\hline 548 & 26863006 & Lee & $\begin{array}{l}\text { Comparison of Long-Term Outcomes of Postmastectomy Radiotherapy between } \\
\text { Breast Cancer Patients with and without Immediate Flap Reconstruction }\end{array}$ & PLoS One & $\begin{array}{l}\text { Single group N enrolled } \\
<500\end{array}$ \\
\hline 549 & 26161312 & Lee & $\begin{array}{l}\text { Use of latissimus dorsi muscle onlay patch alternative to acellular dermal matrix in } \\
\text { implant-based breast reconstruction }\end{array}$ & Gland Surg & NRCS $<30$ per arm \\
\hline 550 & 28544393 & Lee & $\begin{array}{l}\text { Comparison of 5-year oncological outcomes of breast cancer based on surgery } \\
\text { type }\end{array}$ & ANZ J Surg & $\begin{array}{l}\text { Single group N enrolled } \\
<500\end{array}$ \\
\hline 551 & 22323920 & Lee & $\begin{array}{l}\text { Does Immediate Breast Reconstruction after Mastectomy affect the Initiation of } \\
\text { Adjuvant Chemotherapy? }\end{array}$ & J Breast Cancer & $\begin{array}{l}\text { Single group } \mathrm{N} \text { enrolled } \\
<500\end{array}$ \\
\hline 552 & 26791137 & Lee & $\begin{array}{l}\text { Technique and outcomes of laparoscopic bulge repair after abdominal free flap } \\
\text { reconstruction }\end{array}$ & Microsurgery & NRCS not adjusted \\
\hline 553 & 23094245 & Lee & $\begin{array}{l}\text { Reliability of reconstructed breast flap after chemotherapy and radiotherapy in } \\
\text { immediate breast reconstruction }\end{array}$ & Arch Plast Surg & $\begin{array}{l}\text { Single group N enrolled } \\
<500\end{array}$ \\
\hline 554 & 31246795 & Lee & $\begin{array}{l}\text { Predictors for Prolonged Drainage following Tissue Expander-Based Breast } \\
\text { Reconstruction }\end{array}$ & $\begin{array}{l}\text { Plast Reconstr } \\
\text { Surg }\end{array}$ & $\begin{array}{l}\text { Does not address KQ1- } \\
\text { KQ6 }\end{array}$ \\
\hline
\end{tabular}




\begin{tabular}{|c|c|c|c|c|c|}
\hline No. & $\begin{array}{l}\text { PMID or } \\
\text { Other } \\
\text { Identifier }\end{array}$ & $\begin{array}{l}\text { First Author } \\
\text { Last Name }\end{array}$ & Title & Journal & Reason for Exclusion \\
\hline 555 & 28255833 & Lee & $\begin{array}{l}\text { Optimal Sequencing of Postmastectomy Radiotherapy and Two Stages of } \\
\text { Prosthetic Reconstruction: A Meta-analysis }\end{array}$ & Ann Surg Oncol & $\begin{array}{l}\text { Does not address KQ1- } \\
\text { KQ6 }\end{array}$ \\
\hline 556 & 25536199 & Lee & $\begin{array}{l}\text { Effects of Obesity on Postoperative Complications After Breast Reconstruction } \\
\text { Using Free Muscle-Sparing Transverse Rectus Abdominis Myocutaneous, Deep } \\
\text { Inferior Epigastric Perforator, and Superficial Inferior Epigastric Artery Flap: A } \\
\text { Systematic Review and Meta-analysis }\end{array}$ & Ann Plast Surg & $\begin{array}{l}\text { Does not address KQ1- } \\
\text { KQ6 }\end{array}$ \\
\hline 557 & 26374273 & Lee & Prosthetic breast reconstruction in previously irradiated breasts: A meta-analysis & J Surg Oncol & $\begin{array}{l}\text { Does not address KQ1- } \\
\text { KQ6 }\end{array}$ \\
\hline 558 & 26499053 & Lee & $\begin{array}{l}\text { Comparison of one-stage vs two-stage prosthesis-based breast reconstruction: a } \\
\text { systematic review and meta-analysis }\end{array}$ & Am J Surg & Systematic review \\
\hline 559 & 26438439 & Lee & $\begin{array}{l}\text { Updated Evidence of Acellular Dermal Matrix Use for Implant-Based Breast } \\
\text { Reconstruction: A Meta-analysis }\end{array}$ & Ann Surg Oncol & $\begin{array}{l}\text { Narrative review/ } \\
\text { Commentary }\end{array}$ \\
\hline 560 & 28509698 & Lee & $\begin{array}{l}\text { A Meta-analysis of Studies Comparing Outcomes of Diverse Acellular Dermal } \\
\text { Matrices for Implant-Based Breast Reconstruction }\end{array}$ & Ann Plast Surg & $\begin{array}{l}\text { Does not address KQ1- } \\
\text { KQ6 }\end{array}$ \\
\hline 561 & 29718895 & Lee & $\begin{array}{l}\text { Long-term outcomes of patients with breast cancer after nipple-sparing } \\
\text { mastectomy/skin-sparing mastectomy followed by immediate transverse rectus } \\
\text { abdominis musculocutaneous flap reconstruction }\end{array}$ & $\begin{array}{l}\text { Medicine (United } \\
\text { States) }\end{array}$ & $\begin{array}{l}\text { Duplicate of another } \\
\text { publication }\end{array}$ \\
\hline 562 & 29718895 & Lee & $\begin{array}{l}\text { Long-term outcomes of patients with breast cancer after nipple-sparing } \\
\text { mastectomy/skin-sparing mastectomy followed by immediate transverse rectus } \\
\text { abdominis musculocutaneous flap reconstruction: Comparison with conventional } \\
\text { mastectomy in a single center study }\end{array}$ & $\begin{array}{l}\text { Medicine } \\
\text { (Baltimore) }\end{array}$ & $\begin{array}{l}\text { Single group }>500 \text {, but } \\
\text { no complications data }\end{array}$ \\
\hline 563 & 22872842 & Lee & $\begin{array}{l}\text { Outcome of management of local recurrence after immediate transverse rectus } \\
\text { abdominis myocutaneous flap breast reconstruction }\end{array}$ & Arch Plast Surg & NRCS $<30$ per arm \\
\hline 564 & 23283525 & Lee & $\begin{array}{l}\text { Adjuvant chemotherapy reduces the incidence of abdominal hypertrophic scarring } \\
\text { following immediate TRAM breast reconstruction }\end{array}$ & $\begin{array}{l}\text { Breast Cancer Res } \\
\text { Treat }\end{array}$ & $\begin{array}{l}\text { Copublication of } \\
\text { included study with no } \\
\text { new data }\end{array}$ \\
\hline 565 & 27121604 & Lee & $\begin{array}{l}\text { Risk factors of mastectomy skin flap necrosis in immediate breast reconstruction } \\
\text { using low abdominal flaps }\end{array}$ & $\begin{array}{l}J \text { Plast Surg Hand } \\
\text { Surg }\end{array}$ & No outcome of interest \\
\hline 566 & 33009147 & Lee & $\begin{array}{l}\text { The Hybrid Latissimus Dorsi Flap in Immediate Breast Reconstruction: A } \\
\text { Comparative Study With the Abdominal-Based Flap }\end{array}$ & Ann Plast Surg & $\begin{array}{l}\text { Single group } \mathrm{N} \text { enrolled } \\
<500\end{array}$ \\
\hline 567 & 32246465 & Lee & $\begin{array}{l}\text { Influence of complications following total mastectomy and immediate } \\
\text { reconstruction on breast cancer recurrence }\end{array}$ & Br J Surg & $\begin{array}{l}\text { Does not address KQ1- } \\
\text { KQ6 }\end{array}$ \\
\hline 568 & 23788143 & Lentz & $\begin{array}{l}\text { Radiation therapy and expander-implant breast reconstruction: an analysis of } \\
\text { timing and comparison of complications }\end{array}$ & Ann Plast Surg & NRCS $<30$ per arm \\
\hline 569 & 21136255 & Leone & $\begin{array}{l}\text { Factors affecting symmetrization of the contralateral breast: a 7-year unilateral } \\
\text { postmastectomy breast reconstruction experience }\end{array}$ & $\begin{array}{l}\text { Aesthetic Plast } \\
\text { Surg }\end{array}$ & No outcome of interest \\
\hline 570 & 32452097 & Leser & $\begin{array}{l}\text { Complication rates among women undergoing preventive mastectomy: An Austrian } \\
\text { registry }\end{array}$ & Breast J & $\begin{array}{l}\text { Single group } \mathrm{N} \text { enrolled } \\
<500\end{array}$ \\
\hline 571 & 21301299 & Levine & Perforator flap breast reconstruction after unsatisfactory implant reconstruction & Ann Plast Surg & $\begin{array}{l}\text { Single group } \mathrm{N} \text { enrolled } \\
<500\end{array}$ \\
\hline
\end{tabular}

B-32 


\begin{tabular}{|c|c|c|c|c|c|}
\hline No. & $\begin{array}{l}\text { PMID or } \\
\text { Other } \\
\text { Identifier }\end{array}$ & $\begin{array}{l}\text { First Author } \\
\text { Last Name }\end{array}$ & Title & Journal & Reason for Exclusion \\
\hline 572 & 22964681 & Levine & $\begin{array}{l}\text { Outcomes of delayed abdominal-based autologous reconstruction versus } \\
\text { latissimus dorsi flap plus implant reconstruction in previously irradiated patients }\end{array}$ & Ann Plast Surg & NRCS not adjusted \\
\hline 573 & 24076695 & Levine & $\begin{array}{l}\text { Buried flap reconstruction after nipple-sparing mastectomy: advancing toward } \\
\text { single-stage breast reconstruction }\end{array}$ & $\begin{array}{l}\text { Plastic and } \\
\text { reconstructive } \\
\text { surgery }\end{array}$ & $\begin{array}{l}\text { Single group } \mathrm{N} \text { enrolled } \\
<500\end{array}$ \\
\hline 574 & 32243319 & Levy & $\begin{array}{l}\text { Poly-4-Hydroxybutyric Acid Mesh Compares Favorably With Acellular Dermal } \\
\text { Matrix in Tissue Expander-Based Breast Reconstruction }\end{array}$ & Ann Plast Surg & NRCS not adjusted \\
\hline 575 & 31274754 & $\mathrm{Li}$ & Assessment of Factors for Complication in Autologous Breast Reconstruction.' & $\begin{array}{l}\text { Plast Reconstr } \\
\text { Surg }\end{array}$ & $\begin{array}{l}\text { Narrative review/ } \\
\text { Commentary }\end{array}$ \\
\hline 576 & 31256950 & $\mathrm{Li}$ & $\begin{array}{l}\text { Comparison of prepectoral and subpectoral breast reconstruction after } \\
\text { mastectomies: A systematic review and meta analysis }\end{array}$ & Eur J Surg Oncol & $\begin{array}{l}\text { Does not address KQ1- } \\
\text { KQ6 }\end{array}$ \\
\hline 577 & 31095530 & $\mathrm{Li}$ & $\begin{array}{l}\text { Pyoderma Gangrenosum After Abdominal Free Tissue Transfer for Breast } \\
\text { Reconstruction: Case Series and Management Guidelines }\end{array}$ & Ann Plast Surg & $\begin{array}{l}\text { Single group } \mathrm{N} \text { enrolled } \\
<500\end{array}$ \\
\hline 578 & 20578076 & Lim & $\begin{array}{l}\text { Oncological safety of skin sparing mastectomy followed by immediate } \\
\text { reconstruction for locally advanced breast cancer }\end{array}$ & J Surg Oncol & NRCS $<30$ per arm \\
\hline 579 & 22456353 & Lin & $\begin{array}{l}\text { Implant-based, two-stage breast reconstruction in the setting of radiation injury: an } \\
\text { outcome study }\end{array}$ & $\begin{array}{l}\text { Plast Reconstr } \\
\text { Surg }\end{array}$ & NRCS $<30$ per arm \\
\hline 580 & 22743867 & Lindegren & $\begin{array}{l}\text { Postmastectomy breast reconstruction in the irradiated breast: a comparative study } \\
\text { of DIEP and latissimus dorsi flap outcome }\end{array}$ & $\begin{array}{l}\text { Plast Reconstr } \\
\text { Surg }\end{array}$ & NRCS not adjusted \\
\hline 581 & 31538064 & Lindenblatt & $\begin{array}{l}\text { A systematic review of donor site aesthetic and complications after deep inferior } \\
\text { epigastric perforator flap breast reconstruction }\end{array}$ & Gland Surg & Systematic review \\
\hline 582 & 12621181 & Lipa & Breast reconstruction in older women: advantages of autogenous tissue & $\begin{array}{l}\text { Plast Reconstr } \\
\text { Surg }\end{array}$ & NRCS $<30$ per arm \\
\hline 583 & 26165574 & Lisa & $\begin{array}{l}\text { Comparison of Delayed and Immediate Tissue Expander Breast Reconstruction in } \\
\text { the Setting of Postmastectomy Radiation Therapy }\end{array}$ & Ann Plast Surg & $\begin{array}{l}\text { Narrative review/ } \\
\text { Commentary }\end{array}$ \\
\hline 584 & 24831775 & Liu & $\begin{array}{l}\text { Quality of life and patient satisfaction after microsurgical abdominal flap versus } \\
\text { staged expander/implant breast reconstruction: a critical study of unilateral } \\
\text { immediate breast reconstruction using patient-reported outcomes instrument } \\
\text { BREAST-Q }\end{array}$ & $\begin{array}{l}\text { Breast Cancer Res } \\
\text { Treat }\end{array}$ & NRCS not adjusted \\
\hline 585 & None & Liu & $\begin{array}{l}\text { Contraction of reconstructive effects following mastectomy between expandable } \\
\text { prosthesis and autologous tissue }\end{array}$ & $\begin{array}{l}\text { Chinese Journal of } \\
\text { Cancer Prevention } \\
\text { and Treatment }\end{array}$ & NRCS $<30$ per arm \\
\hline 586 & 25680100 & Liu & $\begin{array}{l}\text { Comparison of the postoperative incidence rate of capsular contracture among } \\
\text { different breast implants: a cumulative meta-analysis }\end{array}$ & PLoS One & $\begin{array}{l}\text { Not breast } \\
\text { reconstruction }\end{array}$ \\
\hline 587 & 31939242 & Liu & $\begin{array}{l}\text { [Multivariable analysis for flap-related complications in autologous breast } \\
\text { reconstruction and economic analysis of intraoperative indocyanine green } \\
\text { angiography] }\end{array}$ & $\begin{array}{l}\text { Zhongguo Xiu Fu } \\
\text { Chong Jian Wai Ke } \\
\text { Za Zhi }\end{array}$ & NRCS not adjusted \\
\hline 588 & 32095924 & $\begin{array}{l}\text { Livingston- } \\
\text { Rosanoff }\end{array}$ & $\begin{array}{l}\text { Evaluation of Long-Term Satisfaction with Breast Surgery in Patients Treated for } \\
\text { Ductal Carcinoma In Situ: A Population-Based Longitudinal Cohort Study }\end{array}$ & Ann Surg Oncol & No outcome of interest \\
\hline
\end{tabular}




\begin{tabular}{|c|c|c|c|c|c|}
\hline No. & $\begin{array}{l}\text { PMID or } \\
\text { Other } \\
\text { Identifier }\end{array}$ & $\begin{array}{l}\text { First Author } \\
\text { Last Name }\end{array}$ & Title & Journal & Reason for Exclusion \\
\hline 589 & 30308615 & Lohmander & $\begin{array}{l}\text { Implant Based Breast Reconstruction With Acellular Dermal Matrix: Safety Data } \\
\text { From an Open-label, Multicenter, Randomized, Controlled Trial in the Setting of } \\
\text { Breast Cancer Treatment }\end{array}$ & Ann Surg & $\begin{array}{l}\text { Does not address KQ1- } \\
\text { KQ6 }\end{array}$ \\
\hline 590 & 32762012 & Lohmander & $\begin{array}{l}\text { Quality of life and patient satisfaction after implant-based breast reconstruction with } \\
\text { or without acellular dermal matrix: randomized clinical trial }\end{array}$ & BJS Open & $\begin{array}{l}\text { Does not address KQ1- } \\
\text { KQ6 }\end{array}$ \\
\hline 591 & 29707460 & Loo & $\begin{array}{l}\text { Comparing the Outcome of Different Biologically Derived Acellular Dermal Matrices } \\
\text { in Implant-based Immediate Breast Reconstruction: A Meta-analysis of the } \\
\text { Literatures }\end{array}$ & $\begin{array}{l}\text { Plast Reconstr } \\
\text { Surg Glob Open }\end{array}$ & $\begin{array}{l}\text { Narrative review/ } \\
\text { Commentary }\end{array}$ \\
\hline 592 & 24867714 & Lopez & $\begin{array}{l}\text { The impact of conflicts of interest in plastic surgery: an analysis of acellular dermal } \\
\text { matrix, implant-based breast reconstruction }\end{array}$ & $\begin{array}{l}\text { Plastic and } \\
\text { reconstructive } \\
\text { surgery }\end{array}$ & $\begin{array}{l}\text { Narrative review/ } \\
\text { Commentary }\end{array}$ \\
\hline 593 & 24867714 & Lopez & $\begin{array}{l}\text { The impact of conflicts of interest in plastic surgery: an analysis of acellular dermal } \\
\text { matrix, implant-based breast reconstruction }\end{array}$ & $\begin{array}{l}\text { Plast Reconstr } \\
\text { Surg }\end{array}$ & $\begin{array}{l}\text { Does not address KQ1- } \\
\text { KQ6 }\end{array}$ \\
\hline 594 & 12087236 & Losken & $\begin{array}{l}\text { Trends in unilateral breast reconstruction and management of the contralateral } \\
\text { breast: the Emory experience }\end{array}$ & $\begin{array}{l}\text { Plast Reconstr } \\
\text { Surg }\end{array}$ & $\begin{array}{l}\text { Does not address KQ1- } \\
\text { KQ6 }\end{array}$ \\
\hline 595 & 15156978 & Losken & Factors that influence the completion of breast reconstruction & Ann Plast Surg & $\begin{array}{l}\text { Does not address KQ1- } \\
\text { KQ6 }\end{array}$ \\
\hline 596 & 23542858 & Losken & Time to completion of nipple reconstruction: what factors are involved? & Ann Plast Surg & NRCS $<30$ per arm \\
\hline 597 & 21451387 & Losken & Autologous fat grafting in secondary breast reconstruction & Ann Plast Surg & $\begin{array}{l}\text { Single group N enrolled } \\
<500\end{array}$ \\
\hline 598 & 25878934 & Luce & $\begin{array}{l}\text { Tissue Expander versus Tissue Expander and Latissimus Flap in Morbidly Obese } \\
\text { Breast Reconstruction Patients }\end{array}$ & $\begin{array}{l}\text { Plast Reconstr } \\
\text { Surg Glob Open }\end{array}$ & $\begin{array}{l}\text { Single group N enrolled } \\
<500\end{array}$ \\
\hline 599 & 25389715 & Lynch & $\begin{array}{l}\text { A Comparison of Dermal Autograft and Acellular Dermal Matrix in Tissue Expander } \\
\text { Breast Reconstruction: Long-term Aesthetic Outcomes and Capsular Contracture }\end{array}$ & Ann Plast Surg & NRCS $<30$ per arm \\
\hline 600 & 28743588 & Magill & $\begin{array}{l}\text { Determining the outcomes of post-mastectomy radiation therapy delivered to the } \\
\text { definitive implant in patients undergoing one- and two-stage implant-based breast } \\
\text { reconstruction: A systematic review and meta-analysis }\end{array}$ & $\begin{array}{l}J \text { Plast Reconstr } \\
\text { Aesthet Surg }\end{array}$ & $\begin{array}{l}\text { Does not address KQ1- } \\
\text { KQ6 }\end{array}$ \\
\hline 601 & 32172527 & Magno-Padron & $\begin{array}{l}\text { A Nationwide Analysis of Early and Late Readmissions following Free Tissue } \\
\text { Transfer for Breast Reconstruction }\end{array}$ & $\begin{array}{l}J \text { Reconstr } \\
\text { Microsurg }\end{array}$ & No outcome of interest \\
\hline 602 & 27826483 & Major & The Effect of Timing on Breast Reconstruction Outcomes in Diabetic Women & $\begin{array}{l}\text { Plast Reconstr } \\
\text { Surg Glob Open }\end{array}$ & $\begin{array}{l}\text { Does not address KQ1- } \\
\text { KQ6 }\end{array}$ \\
\hline 603 & 26579526 & Malata & $\begin{array}{l}\text { Decision Making in Double-Pedicled DIEP and SIEA Abdominal Free Flap Breast } \\
\text { Reconstructions: An Algorithmic Approach and Comprehensive Classification }\end{array}$ & Front Surg & $\begin{array}{l}\text { Single group N enrolled } \\
<500\end{array}$ \\
\hline 604 & 19342994 & Man & $\begin{array}{l}\text { Abdominal wall following free TRAM or DIEP flap reconstruction: a meta-analysis } \\
\text { and critical review }\end{array}$ & $\begin{array}{l}\text { Plast Reconstr } \\
\text { Surg }\end{array}$ & $\begin{array}{l}\text { Narrative review/ } \\
\text { Commentary }\end{array}$ \\
\hline 605 & 33627231 & Mandelbaum & National trends and predictors of mastectomy with immediate breast reconstruction & Am J Surg & $\begin{array}{l}\text { Does not address KQ1- } \\
\text { KQ6 }\end{array}$ \\
\hline 606 & 32712889 & Mandelbaum & $\begin{array}{l}\text { National Trends in Immediate Breast Reconstruction: An Analysis of Implant-Based } \\
\text { Versus Autologous Reconstruction After Mastectomy }\end{array}$ & Ann Surg Oncol & NRCS not adjusted \\
\hline 607 & 31044105 & Manrique & $\begin{array}{l}\text { Surgical Outcomes of Prepectoral Versus Subpectoral Implant-based Breast } \\
\text { Reconstruction in Young Women }\end{array}$ & $\begin{array}{l}\text { Plast Reconstr } \\
\text { Surg Glob Open }\end{array}$ & NRCS not adjusted \\
\hline
\end{tabular}




\begin{tabular}{|c|c|c|c|c|c|}
\hline No. & $\begin{array}{l}\text { PMID or } \\
\text { Other } \\
\text { Identifier }\end{array}$ & $\begin{array}{l}\text { First Author } \\
\text { Last Name }\end{array}$ & Title & Journal & Reason for Exclusion \\
\hline 608 & 31416221 & Manrique & $\begin{array}{l}\text { Two-Staged Implant-Based Breast Reconstruction: A Long-Term Outcome Study } \\
\text { in a Young Population }\end{array}$ & Medicina (Kaunas) & $\begin{array}{l}\text { Single group } \mathrm{N} \text { enrolled } \\
<500\end{array}$ \\
\hline 609 & 31985613 & Manrique & $\begin{array}{l}\text { Prepectoral Two-Stage Implant-Based Breast Reconstruction with and without } \\
\text { Acellular Dermal Matrix: Do We See a Difference? }\end{array}$ & $\begin{array}{l}\text { Plast Reconstr } \\
\text { Surg }\end{array}$ & NRCS $<30$ per arm \\
\hline 610 & 31633546 & Manrique & $\begin{array}{l}\text { Single-Stage Direct-to-Implant Breast Reconstruction: A Comparison Between } \\
\text { Subpectoral Versus Prepectoral Implant Placement }\end{array}$ & $\begin{array}{l}\text { Annals of plastic } \\
\text { surgery }\end{array}$ & NRCS not adjusted \\
\hline 611 & 31633546 & Manrique & $\begin{array}{l}\text { Single-Stage Direct-to-Implant Breast Reconstruction: A Comparison Between } \\
\text { Subpectoral Versus Prepectoral Implant Placement }\end{array}$ & Ann Plast Surg & NRCS not adjusted \\
\hline 612 & 31985613 & Manrique & $\begin{array}{l}\text { Prepectoral Two-Stage Implant-Based Breast Reconstruction with and without } \\
\text { Acellular Dermal Matrix: Do We See a Difference? }\end{array}$ & $\begin{array}{l}\text { Plastic and } \\
\text { reconstructive } \\
\text { surgery }\end{array}$ & NRCS $<30$ per arm \\
\hline 613 & 3768653 & Mansel & $\begin{array}{l}\text { Cosmetic results of immediate breast reconstruction post-mastectomy: a follow-up } \\
\text { study }\end{array}$ & Br J Surg & NRCS not adjusted \\
\hline 614 & 3768653 & Mansel & $\begin{array}{l}\text { Cosmetic results of immediate breast reconstruction post-mastectomy: A follow-up } \\
\text { study }\end{array}$ & $\begin{array}{l}\text { British Journal of } \\
\text { Surgery }\end{array}$ & NRCS not adjusted \\
\hline 615 & 31187585 & Manum & $\begin{array}{l}\text { Variables associated with length of stay in patients undergoing mastectomy and } \\
\text { delayed-immediate breast reconstruction with tissue expander }\end{array}$ & Breast J & $\begin{array}{l}\text { Single group } \mathrm{N} \text { enrolled } \\
<500\end{array}$ \\
\hline 616 & 31238166 & Manyam & $\begin{array}{l}\text { Long-Term Outcomes After Autologous or Tissue Expander/Implant-Based Breast } \\
\text { Reconstruction and Postmastectomy Radiation for Breast Cancer }\end{array}$ & Pract Radiat Oncol & $\begin{array}{l}\text { Duplicate of another } \\
\text { publication }\end{array}$ \\
\hline 617 & 31238166 & Manyam & $\begin{array}{l}\text { Long-Term Outcomes After Autologous or Tissue Expander/Implant-Based } \\
\text { Breast Reconstruction and Postmastectomy Radiation for Breast Cancer }\end{array}$ & $\begin{array}{l}\text { Practical Radiation } \\
\text { Oncology }\end{array}$ & NRCS not adjusted \\
\hline 618 & 31264293 & Manyam & $\begin{array}{l}\text { Long-term complications and reconstruction failures in previously radiated breast } \\
\text { cancer patients receiving salvage mastectomy with autologous reconstruction or } \\
\text { tissue expander/implant-based reconstruction }\end{array}$ & Breast J & NRCS not adjusted \\
\hline 619 & 139349741 & Manyam & $\begin{array}{l}\text { Long-term complications and reconstruction failures in previously radiated breast } \\
\text { cancer patients receiving salvage mastectomy with autologous reconstruction or } \\
\text { tissue expander/implant-based reconstruction }\end{array}$ & Breast Journal & $\begin{array}{l}\text { Duplicate of another } \\
\text { publication }\end{array}$ \\
\hline 620 & 21782310 & Marchac & [A cost analysis of DIEP flap in breast reconstruction] & $\begin{array}{l}\text { Ann Chir Plast } \\
\text { Esthet }\end{array}$ & No outcome of interest \\
\hline 621 & 29176408 & Marcusa & $\begin{array}{l}\text { Prescription Opioid Use among Opioid-Naive Women Undergoing Immediate } \\
\text { Breast Reconstruction }\end{array}$ & $\begin{array}{l}\text { Plast Reconstr } \\
\text { Surg }\end{array}$ & No outcome of interest \\
\hline 622 & None & Marongiu & $\begin{array}{l}\text { A human-derived acellular dermal matrix for breast reconstruction: The first } \\
\text { European experience }\end{array}$ & $\begin{array}{l}\text { European Journal } \\
\text { of Cancer }\end{array}$ & $\begin{array}{l}\text { Single group N enrolled } \\
<500\end{array}$ \\
\hline 623 & 27047779 & Marsh & $\begin{array}{l}\text { Three routine free flaps per day in a single operating theatre: principles of a } \\
\text { process mapping approach to improving surgical efficiency }\end{array}$ & Gland Surg & $\begin{array}{l}\text { Does not address KQ1- } \\
\text { KQ6 }\end{array}$ \\
\hline 624 & 25347626 & Martin & $\begin{array}{l}\text { Use of fenestrations in acellular dermal allograft in two-stage tissue } \\
\text { expander/implant breast reconstruction }\end{array}$ & $\begin{array}{l}\text { Plast Reconstr } \\
\text { Surg }\end{array}$ & $\begin{array}{l}\text { Single group N enrolled } \\
<500\end{array}$ \\
\hline 625 & 33133958 & Martinez & Outpatient Microsurgical Breast Reconstruction & $\begin{array}{l}\text { Plast Reconstr } \\
\text { Surg Glob Open }\end{array}$ & NRCS not adjusted \\
\hline 626 & 28051266 & Maruccia & $\begin{array}{l}\text { One-stage breast reconstruction techniques in elderly patients to preserve quality } \\
\text { of life }\end{array}$ & $\begin{array}{l}\text { Eur Rev Med } \\
\text { Pharmacol Sci }\end{array}$ & NRCS not adjusted \\
\hline
\end{tabular}




\begin{tabular}{|c|c|c|c|c|c|}
\hline No. & $\begin{array}{l}\text { PMID or } \\
\text { Other } \\
\text { Identifier }\end{array}$ & $\begin{array}{l}\text { First Author } \\
\text { Last Name }\end{array}$ & Title & Journal & Reason for Exclusion \\
\hline 627 & 24665051 & Masoomi & Predictive risk factors of free flap thrombosis in breast reconstruction surgery & Microsurgery & $\begin{array}{l}\text { Does not address KQ1- } \\
\text { KQ6 }\end{array}$ \\
\hline 628 & 31309057 & Masoomi & $\begin{array}{l}\text { Frequency and Predictors of 30-Day Surgical Site Complications in Autologous } \\
\text { Breast Reconstruction Surgery }\end{array}$ & World J Plast Surg & $\begin{array}{l}\text { Copublication of } \\
\text { included study with no } \\
\text { new data }\end{array}$ \\
\hline 629 & 31620328 & Masoomi & $\begin{array}{l}\text { Effect of Anemia in Postoperative Outcomes of Autologous Breast Reconstruction } \\
\text { Surgery }\end{array}$ & World J Plast Surg & $\begin{array}{l}\text { Does not address KQ1- } \\
\text { KQ6 }\end{array}$ \\
\hline 630 & 25642875 & Masoomi & $\begin{array}{l}\text { Does immediate tissue expander placement increase immediate postoperative } \\
\text { complications in patients with breast cancer? }\end{array}$ & Am Surg & $\begin{array}{l}\text { Does not address KQ1- } \\
\text { KQ6 }\end{array}$ \\
\hline 631 & 25357045 & Masoomi & $\begin{array}{l}\text { Perioperative outcomes of autologous breast reconstruction surgery in teaching } \\
\text { versus nonteaching hospitals }\end{array}$ & $\begin{array}{l}\text { Plast Reconstr } \\
\text { Surg }\end{array}$ & $\begin{array}{l}\text { Copublication of } \\
\text { included study with no } \\
\text { new data }\end{array}$ \\
\hline 632 & 26054302 & Masoomi & $\begin{array}{l}\text { Comparison of perioperative outcomes of autologous breast reconstruction } \\
\text { surgeries }\end{array}$ & $\begin{array}{l}\text { J Plast Reconstr } \\
\text { Aesthet Surg }\end{array}$ & NRCS not adjusted \\
\hline 633 & 104822047 & Massey & O-94 Patient reported outcomes following post mastectomy breast reconstruction & EJC Supplements & $\begin{array}{l}\text { Single group } \mathrm{N} \text { enrolled } \\
<500\end{array}$ \\
\hline 634 & 19730293 & Massey & $\begin{array}{l}\text { Perforator flaps: Recent experience, current trends, and future directions based on } \\
3974 \text { microsurgical breast reconstructions }\end{array}$ & $\begin{array}{l}\text { Plastic and } \\
\text { Reconstructive } \\
\text { Surgery }\end{array}$ & $\begin{array}{l}\text { Narrative review/ } \\
\text { Commentary }\end{array}$ \\
\hline 635 & 19730293 & Massey & $\begin{array}{l}\text { Perforator flaps: recent experience, current trends, and future directions based on } \\
3974 \text { microsurgical breast reconstructions }\end{array}$ & $\begin{array}{l}\text { Plast Reconstr } \\
\text { Surg }\end{array}$ & $\begin{array}{l}\text { Does not address KQ1- } \\
\text { KQ6 }\end{array}$ \\
\hline 636 & 23083621 & Matos & $\begin{array}{l}\text { Fat necrosis in the breast after reconstruction with transverse rectus abdominis } \\
\text { myocutaneous flap: MRI features }\end{array}$ & $\begin{array}{l}\text { European Journal } \\
\text { of Radiology }\end{array}$ & $\begin{array}{l}\text { Single group } \mathrm{N} \text { enrolled } \\
<500\end{array}$ \\
\hline 637 & 26193963 & Matsen & Skin Flap Necrosis After Mastectomy With Reconstruction: A Prospective Study & Ann Surg Oncol & $\begin{array}{l}\text { Single group N enrolled } \\
<500\end{array}$ \\
\hline 638 & 29617492 & Matsumoto & $\begin{array}{l}\text { Influence of advanced age on postoperative outcomes and total loss following } \\
\text { breast reconstruction: a critical assessment of } 560 \text { cases }\end{array}$ & Rev Col Bras Cir & $\begin{array}{l}\text { Does not address KQ1- } \\
\text { KQ6 }\end{array}$ \\
\hline 639 & 28195672 & Matthews & $\begin{array}{l}\text { Predictors of satisfaction and quality of life following post-mastectomy breast } \\
\text { reconstruction }\end{array}$ & Psychooncology & $\begin{array}{l}\text { Single group } \mathrm{N} \text { enrolled } \\
<500\end{array}$ \\
\hline 640 & 32332527 & Mauch & $\begin{array}{l}\text { Does Pregnancy Predict Incisional Hernia Repair after Abdominally Based } \\
\text { Autologous Breast Reconstruction? A Retrospective Review of } 890 \text { Free Flaps }\end{array}$ & $\begin{array}{l}\text { Plast Reconstr } \\
\text { Surg }\end{array}$ & $\begin{array}{l}\text { Copublication of } \\
\text { included study with no } \\
\text { new data }\end{array}$ \\
\hline 641 & 25717116 & Maxwell & $\begin{array}{l}\text { Ten-year results from the Natrelle } 410 \text { anatomical form-stable silicone breast } \\
\text { implant core study }\end{array}$ & Aesthet Surg J & $\begin{array}{l}\text { Single group } \mathrm{N} \text { enrolled } \\
<500\end{array}$ \\
\hline 642 & 32948492 & Mayer & $\begin{array}{l}\text { The value of preoperative computed tomography angiography (CT-A) in patients } \\
\text { undergoing delayed latissimus dorsi flap breast reconstruction after axillary lymph } \\
\text { node dissection or irradiation and suspicion of pedicle injury }\end{array}$ & $\begin{array}{l}\text { J Plast Reconstr } \\
\text { Aesthet Surg }\end{array}$ & $\begin{array}{l}\text { Single group } \mathrm{N} \text { enrolled } \\
<500\end{array}$ \\
\hline 643 & 109500657 & Mays & $\begin{array}{l}\text { Surgical Outcomes in Elderly Patients Undergoing Mastectomy With and Without } \\
\text { Reconstruction for Breast Cancer }\end{array}$ & $\begin{array}{l}\text { Journal of the } \\
\text { American College } \\
\text { of Surgeons }\end{array}$ & $\begin{array}{l}\text { Does not address KQ1- } \\
\text { KQ6 }\end{array}$ \\
\hline
\end{tabular}




\begin{tabular}{|c|c|c|c|c|c|}
\hline No. & $\begin{array}{l}\text { PMID or } \\
\text { Other } \\
\text { Identifier }\end{array}$ & $\begin{array}{l}\text { First Author } \\
\text { Last Name }\end{array}$ & Title & Journal & Reason for Exclusion \\
\hline 644 & 21136577 & McCarthy & $\begin{array}{l}\text { Patient satisfaction with postmastectomy breast reconstruction: a comparison of } \\
\text { saline and silicone implants }\end{array}$ & Cancer & $\begin{array}{l}\text { Duplicate of another } \\
\text { publication }\end{array}$ \\
\hline 645 & 3566108 & McCraw & $\begin{array}{l}\text { An early appraisal of the methods of tissue expansion and the transverse rectus } \\
\text { abdominis musculocutaneous flap in reconstruction of the breast following } \\
\text { mastectomy }\end{array}$ & Ann Plast Surg & $\begin{array}{l}\text { Single group } \mathrm{N} \text { enrolled } \\
<500\end{array}$ \\
\hline 646 & 3566108 & McCraw & $\begin{array}{l}\text { An early appraisal of the methods of tissue expansion and the transverse rectus } \\
\text { abdominis musculocutaneous flap in reconstruction of the breast following } \\
\text { mastectomy }\end{array}$ & $\begin{array}{l}\text { Annals of Plastic } \\
\text { Surgery }\end{array}$ & NRCS not adjusted \\
\hline 647 & 27627058 & McGuire & $\begin{array}{l}\text { Risk Factor Analysis for Capsular Contracture, Malposition, and Late Seroma in } \\
\text { Subjects Receiving Natrelle } 410 \text { Form-Stable Silicone Breast Implants }\end{array}$ & $\begin{array}{l}\text { Plast Reconstr } \\
\text { Surg }\end{array}$ & $\begin{array}{l}\text { Copublication of } \\
\text { included study with no } \\
\text { new data }\end{array}$ \\
\hline 648 & 12900602 & Mehrara & Alternative venous outflow vessels in microvascular breast reconstruction & $\begin{array}{l}\text { Plast Reconstr } \\
\text { Surg }\end{array}$ & NRCS $<30$ per arm \\
\hline 649 & 28847440 & Menez & $\begin{array}{l}\text { Multicenter evaluation of quality of life and patient satisfaction after breast } \\
\text { reconstruction, a long-term retrospective study }\end{array}$ & $\begin{array}{l}\text { Ann Chir Plast } \\
\text { Esthet }\end{array}$ & NRCS not adjusted \\
\hline 650 & 27776942 & Mennie & $\begin{array}{l}\text { National trends in immediate and delayed post-mastectomy reconstruction } \\
\text { procedures in England: A seven-year population-based cohort study }\end{array}$ & Eur J Surg Oncol & $\begin{array}{l}\text { Does not address KQ1- } \\
\text { KQ6 }\end{array}$ \\
\hline 651 & 30683449 & Mets & $\begin{array}{l}\text { Persistent disparities in breast cancer surgical outcomes among hispanic and } \\
\text { African American patients }\end{array}$ & Eur J Surg Oncol & $\begin{array}{l}\text { Does not address KQ1- } \\
\text { KQ6 }\end{array}$ \\
\hline 652 & 32823954 & Meyer & $\begin{array}{l}\text { The Value of Morphometric Measurements in Risk Assessment for Donor-Site } \\
\text { Complications after Microsurgical Breast Reconstruction }\end{array}$ & $\mathrm{J}$ Clin Med & NRCS $<30$ per arm \\
\hline 653 & 18235363 & Michy & $\begin{array}{l}\text { [What surgical procedure for immediate breast reconstruction after preoperative } \\
\text { radiotherapy and chemotherapy?] }\end{array}$ & J Chir (Paris) & NRCS not adjusted \\
\hline 654 & 22768687 & Mijatovic & [Quality of life after breast reconstruction] & Lijec Vjesn & Unable to retrieve article \\
\hline 655 & 29538000 & Mikhaylov & $\begin{array}{l}\text { Ketorolac and Hematoma Incidence in Postmastectomy Implant-Based Breast } \\
\text { Reconstruction }\end{array}$ & Ann Plast Surg & $\begin{array}{l}\text { Does not address KQ1- } \\
\text { KQ6 }\end{array}$ \\
\hline 656 & 9858162 & Miller & $\begin{array}{l}\text { Absence of longitudinal changes in rheumatologic parameters after silicone breast } \\
\text { implantation: a prospective 13-year study }\end{array}$ & $\begin{array}{l}\text { Plast Reconstr } \\
\text { Surg }\end{array}$ & NRCS not adjusted \\
\hline 657 & 25607768 & Miller & $\begin{array}{l}\text { Immediate Implant Reconstruction Is Associated With a Reduced Risk of } \\
\text { Lymphedema Compared to Mastectomy Alone: A Prospective Cohort Study }\end{array}$ & Ann Surg & $\begin{array}{l}\text { Does not address KQ1- } \\
\text { KQ6 }\end{array}$ \\
\hline 658 & 17255654 & Miller & Microvascular breast reconstruction in the diabetic patient & $\begin{array}{l}\text { Plast Reconstr } \\
\text { Surg }\end{array}$ & $\begin{array}{l}\text { Does not address KQ1- } \\
\text { KQ6 }\end{array}$ \\
\hline 659 & 31663934 & Mirhaidari & Prepectoral Versus Subpectoral Direct to Implant Immediate Breast Reconstruction & Ann Plast Surg & NRCS not adjusted \\
\hline 660 & 22094760 & Mirzabeigi & $\begin{array}{l}\text { Trials and tribulations with the inferior gluteal artery perforator flap in autologous } \\
\text { breast reconstruction }\end{array}$ & $\begin{array}{l}\text { Plast Reconstr } \\
\text { Surg }\end{array}$ & NRCS $<30$ per arm \\
\hline 661 & 30789475 & Mirzabeigi & $\begin{array}{l}\text { Locoregional Cancer Recurrence after Breast Reconstruction: Detection, } \\
\text { Management, and Secondary Reconstructive Strategies }\end{array}$ & $\begin{array}{l}\text { Plast Reconstr } \\
\text { Surg }\end{array}$ & NRCS $<30$ per arm \\
\hline 662 & 23542828 & Mirzabeigi & $\begin{array}{l}\text { An assessment of the risks and benefits of immediate autologous breast } \\
\text { reconstruction in patients undergoing postmastectomy radiation therapy }\end{array}$ & Ann Plast Surg & $\begin{array}{l}\text { Does not address KQ1- } \\
\text { KQ6 }\end{array}$ \\
\hline 663 & 25539292 & Mirzabeigi & $\begin{array}{l}\text { Predicting and managing donor-site wound complications in abdominally based } \\
\text { free flap breast reconstruction: improved outcomes with early reoperative closure }\end{array}$ & $\begin{array}{l}\text { Plast Reconstr } \\
\text { Surg }\end{array}$ & $\begin{array}{l}\text { Does not address KQ1- } \\
\text { KQ6 }\end{array}$ \\
\hline
\end{tabular}




\begin{tabular}{|c|c|c|c|c|c|}
\hline No. & $\begin{array}{l}\text { PMID or } \\
\text { Other } \\
\text { Identifier }\end{array}$ & $\begin{array}{l}\text { First Author } \\
\text { Last Name }\end{array}$ & Title & Journal & Reason for Exclusion \\
\hline 664 & 10863769 & Missana & $\begin{array}{l}\text { [Radiotherpay and immediate breast reconstruction with myocutaneous flap in } \\
\text { breast cancer of reserved prognosis] }\end{array}$ & $\begin{array}{l}\text { Ann Chir Plast } \\
\text { Esthet }\end{array}$ & Unable to retrieve article \\
\hline 665 & 10863769 & Missana & $\begin{array}{l}\text { Radiotherapy and immediate breast reconstruction with myocutaneous flap in } \\
\text { breast cancer of reserved prognosis }\end{array}$ & $\begin{array}{l}\text { Annales de } \\
\text { Chirurgie Plastique } \\
\text { Esthetique }\end{array}$ & Unable to retrieve article \\
\hline 666 & 24495186 & Mlodinow & $\begin{array}{l}\text { Risk factors for mastectomy flap necrosis following immediate tissue expander } \\
\text { breast reconstruction }\end{array}$ & $\begin{array}{l}J \text { Plast Surg Hand } \\
\text { Surg }\end{array}$ & $\begin{array}{l}\text { Does not address KQ1- } \\
\text { KQ6 }\end{array}$ \\
\hline 667 & 24025652 & Mlodinow & $\begin{array}{l}\text { Predictors of readmission after breast reconstruction: a multi-institutional analysis } \\
\text { of } 5012 \text { patients }\end{array}$ & Ann Plast Surg & $\begin{array}{l}\text { Duplicate of another } \\
\text { publication }\end{array}$ \\
\hline 668 & 7851550 & Modena & $\begin{array}{l}\text { Mastectomy and immediate breast reconstruction: Oncological considerations and } \\
\text { evaluation of two different methods relating to } 88 \text { cases }\end{array}$ & $\begin{array}{l}\text { European Journal } \\
\text { of Surgical } \\
\text { Oncology }\end{array}$ & NRCS not adjusted \\
\hline 669 & 22795362 & Mohan & $\begin{array}{l}\text { Trends in tertiary breast reconstruction: literature review and single centre } \\
\text { experience }\end{array}$ & Breast & $\begin{array}{l}\text { Narrative review/ } \\
\text { Commentary }\end{array}$ \\
\hline 670 & 32892804 & Mohan & $\begin{array}{l}\text { Autologous Breast Reconstruction in Low Body Mass Index Patients: Strategies for } \\
\text { Maximizing Skin Envelope and Breast Volume }\end{array}$ & Clin Plast Surg & $\begin{array}{l}\text { Single group } \mathrm{N} \text { enrolled } \\
<500\end{array}$ \\
\hline 671 & 31628083 & Moller & $\begin{array}{l}\text { The reconstructive journey: Description of the breast reconstruction pathway in a } \\
\text { high-volume UK-based microsurgical centre }\end{array}$ & $\begin{array}{l}\text { J Plast Reconstr } \\
\text { Aesthet Surg }\end{array}$ & NRCS not adjusted \\
\hline 672 & 31568276 & Momeni & $\begin{array}{l}\text { A Matched-Pair Analysis of Prepectoral with Subpectoral Breast Reconstruction: Is } \\
\text { There a Difference in Postoperative Complication Rate? }\end{array}$ & $\begin{array}{l}\text { Plast Reconstr } \\
\text { Surg }\end{array}$ & NRCS not adjusted \\
\hline 673 & 27355266 & Momoh & $\begin{array}{l}\text { Tradeoffs Associated With Contralateral Prophylactic Mastectomy in Women } \\
\text { Choosing Breast Reconstruction: Results of a Prospective Multicenter Cohort }\end{array}$ & Ann Surg & $\begin{array}{l}\text { Copublication of } \\
\text { included study with no } \\
\text { new data }\end{array}$ \\
\hline 674 & 21629047 & Momoh & $\begin{array}{l}\text { Delayed autologous breast reconstruction after postmastectomy radiation therapy: } \\
\text { is there an optimal time? }\end{array}$ & Ann Plast Surg & $\begin{array}{l}\text { Does not address KQ1- } \\
\text { KQ6 }\end{array}$ \\
\hline 675 & 21659842 & Momoh & $\begin{array}{l}\text { Analysis of complications and patient satisfaction in pedicled transverse rectus } \\
\text { abdominis myocutaneous and deep inferior epigastric perforator flap breast } \\
\text { reconstruction }\end{array}$ & Ann Plast Surg & NRCS not adjusted \\
\hline 676 & 110864088 & Momoh & $\begin{array}{l}\text { Breast reconstruction in patients with unilateral breast cancer who choose } \\
\text { contralateral prophylactic mastectomy: an assessment of postoperative morbidity }\end{array}$ & $\begin{array}{l}\text { Journal of the } \\
\text { American College } \\
\text { of Surgeons }\end{array}$ & $\begin{array}{l}\text { Does not address KQ1- } \\
\text { KQ6 }\end{array}$ \\
\hline 677 & 21843920 & Monrigal & $\begin{array}{l}\text { Mastectomy with immediate breast reconstruction after neoadjuvant chemotherapy } \\
\text { and radiation therapy. A new option for patients with operable invasive breast } \\
\text { cancer. Results of a } 20 \text { years single institution study }\end{array}$ & Eur J Surg Oncol & NRCS not adjusted \\
\hline 678 & 32205491 & Monroig & $\begin{array}{l}\text { Do Postoperative Prophylactic Antibiotics Reduce Highly Virulent Infections?: An } \\
\text { Analysis of } 660 \text { Tissue Expander Breast Reconstructions }\end{array}$ & Ann Plast Surg & $\begin{array}{l}\text { Does not address KQ1- } \\
\text { KQ6 }\end{array}$ \\
\hline 679 & 3432841 & Montoreano & $\begin{array}{l}\text { Latissimus dorsi and rectus abdominis breast reconstruction postmastectomy: } \\
\text { musculocutaneous breast reconstruction }\end{array}$ & Semin Surg Oncol & NRCS not adjusted \\
\hline 680 & 33220897 & Moon & $\begin{array}{l}\text { Adverse Events Associated with Breast Implants: The Role of Bacterial Infection } \\
\text { and Biofilm }\end{array}$ & Clin Plast Surg & $\begin{array}{l}\text { Narrative } \\
\text { review/Commentary }\end{array}$ \\
\hline
\end{tabular}




\begin{tabular}{|c|c|c|c|c|c|}
\hline No. & $\begin{array}{l}\text { PMID or } \\
\text { Other } \\
\text { Identifier }\end{array}$ & $\begin{array}{l}\text { First Author } \\
\text { Last Name }\end{array}$ & Title & Journal & Reason for Exclusion \\
\hline 681 & None & Moon & $\begin{array}{l}\text { Can pregnancy following muscle-sparing transverse abdominis myocutaneous } \\
\text { (MS-TRAM) flaps be safe on abdominal wall? }\end{array}$ & $\begin{array}{l}\text { Clinical and } \\
\text { Experimental } \\
\text { Obstetrics and } \\
\text { Gynecology }\end{array}$ & $\begin{array}{l}\text { Case report or series of } \\
\text { case reports }\end{array}$ \\
\hline 682 & 17312480 & Mosahebi & $\begin{array}{l}\text { Aesthetic outcome of different techniques of reconstruction following nipple-areola- } \\
\text { preserving envelope mastectomy with immediate reconstruction }\end{array}$ & $\begin{array}{l}\text { Plastic and } \\
\text { Reconstructive } \\
\text { Surgery }\end{array}$ & NRCS $<30$ per arm \\
\hline 683 & 32332524 & Mosharrafa & $\begin{array}{l}\text { Direct-to-Implant Breast Reconstruction with Simultaneous Nipple-Sparing } \\
\text { Mastopexy Utilizing an Inferiorly Based Adipodermal Flap: Our Experience with } \\
\text { Prepectoral and Subpectoral Techniques }\end{array}$ & $\begin{array}{l}\text { Plast Reconstr } \\
\text { Surg }\end{array}$ & $\begin{array}{l}\text { Narrative } \\
\text { review/Commentary }\end{array}$ \\
\hline 684 & 27798949 & Mull & $\begin{array}{l}\text { Impact of Time Interval between Radiation and Free Autologous Breast } \\
\text { Reconstruction }\end{array}$ & $\begin{array}{l}\text { J Reconstr } \\
\text { Microsurg }\end{array}$ & $\begin{array}{l}\text { Does not address KQ1- } \\
\text { KQ6 }\end{array}$ \\
\hline 685 & 28445351 & Mundy & Breast Cancer and Reconstruction: Normative Data for Interpreting the BREAST-Q & $\begin{array}{l}\text { Plast Reconstr } \\
\text { Surg }\end{array}$ & $\begin{array}{l}\text { Does not address KQ1- } \\
\text { KQ6 }\end{array}$ \\
\hline 686 & 32097289 & Mundy & $\begin{array}{l}\text { The Evolution of Breast Satisfaction and Well-Being after Breast Cancer: A } \\
\text { Propensity-Matched Comparison to the Norm }\end{array}$ & $\begin{array}{l}\text { Plast Reconstr } \\
\text { Surg }\end{array}$ & $\begin{array}{l}\text { Does not address KQ1- } \\
\text { KQ6 }\end{array}$ \\
\hline 687 & 32766077 & Mundy & Optimizing Intraoperative Evaluation of Mastectomy Skin Flap Viability & $\begin{array}{l}\text { Plast Reconstr } \\
\text { Surg Glob Open }\end{array}$ & $\begin{array}{l}\text { Case report or series of } \\
\text { case reports }\end{array}$ \\
\hline 688 & 18317121 & Munhoz & $\begin{array}{l}\text { Assessment of immediate conservative breast surgery reconstruction: a } \\
\text { classification system of defects revisited and an algorithm for selecting the } \\
\text { appropriate technique }\end{array}$ & $\begin{array}{l}\text { Plast Reconstr } \\
\text { Surg }\end{array}$ & $\begin{array}{l}\text { Single group } \mathrm{N} \text { enrolled } \\
<500\end{array}$ \\
\hline 689 & 30824169 & Murphy & Returns to the operating room after breast surgery at a tertiary care medical center & Am J Surg & $\begin{array}{l}\text { Not breast } \\
\text { reconstruction }\end{array}$ \\
\hline 690 & 31297826 & Murphy & Pain and opioid prescriptions vary by procedure after breast surgery & J Surg Oncol & $\begin{array}{l}\text { Does not address KQ1- } \\
\text { KQ6 }\end{array}$ \\
\hline 691 & 30745085 & Murphy & $\begin{array}{l}\text { A comparison of patient reported outcome measures in patients who received both } \\
\text { DIEP flap and PAP flap breast reconstructions }\end{array}$ & $\begin{array}{l}J \text { Plast Reconstr } \\
\text { Aesthet Surg }\end{array}$ & $\begin{array}{l}\text { Single group N enrolled } \\
<500\end{array}$ \\
\hline 692 & 29901587 & Myung & $\begin{array}{l}\text { Quantitative analysis of shoulder function and strength after breast reconstruction: } \\
\text { A retrospective cohort study }\end{array}$ & $\begin{array}{l}\text { Medicine } \\
\text { (Baltimore) }\end{array}$ & NRCS not adjusted \\
\hline 693 & 130212782 & Myung & $\begin{array}{l}\text { Quantitative analysis of shoulder function and strength after breast reconstruction: } \\
\text { A retrospective cohort study }\end{array}$ & Medicine & NRCS not adjusted \\
\hline 694 & 33692412 & Myung & $\begin{array}{l}\text { Validating machine learning approaches for prediction of donor related } \\
\text { complication in microsurgical breast reconstruction: a retrospective cohort study }\end{array}$ & Sci Rep & $\begin{array}{l}\text { Duplicate of another } \\
\text { publication }\end{array}$ \\
\hline 695 & 33692412 & Myung & $\begin{array}{l}\text { Validating machine learning approaches for prediction of donor related } \\
\text { complication in microsurgical breast reconstruction: a retrospective cohort study }\end{array}$ & Sci Rep & $\begin{array}{l}\text { Single group }>500 \text {, but } \\
\text { no harms data }\end{array}$ \\
\hline 696 & 19952629 & Nahabedian & $\begin{array}{l}\text { AlloDerm performance in the setting of prosthetic breast surgery, infection, and } \\
\text { irradiation }\end{array}$ & $\begin{array}{l}\text { Plast Reconstr } \\
\text { Surg }\end{array}$ & $\begin{array}{l}\text { Single group N enrolled } \\
<500\end{array}$ \\
\hline 697 & 29166344 & Nahabedian & $\begin{array}{l}\text { Two-Stage Prosthetic Breast Reconstruction: A Comparison Between Prepectoral } \\
\text { and Partial Subpectoral Techniques }\end{array}$ & $\begin{array}{l}\text { Plast Reconstr } \\
\text { Surg }\end{array}$ & NRCS not adjusted \\
\hline 698 & 11786798 & Nahabedian & $\begin{array}{l}\text { Contour abnormalities of the abdomen after breast reconstruction with abdominal } \\
\text { flaps: the role of muscle preservation }\end{array}$ & $\begin{array}{l}\text { Plast Reconstr } \\
\text { Surg }\end{array}$ & NRCS not adjusted \\
\hline
\end{tabular}




\begin{tabular}{|c|c|c|c|c|c|}
\hline No. & $\begin{array}{l}\text { PMID or } \\
\text { Other } \\
\text { Identifier }\end{array}$ & $\begin{array}{l}\text { First Author } \\
\text { Last Name }\end{array}$ & Title & Journal & Reason for Exclusion \\
\hline 699 & 21251120 & Nahabedian & Breast reconstruction in women under 30: a 10-year experience & Breast J & $\begin{array}{l}\text { Narrative review/ } \\
\text { Commentary }\end{array}$ \\
\hline 700 & 18443503 & Nahabedian & $\begin{array}{l}\text { The impact of breast reconstruction on the oncologic efficacy of radiation therapy: } \\
\text { a retrospective analysis }\end{array}$ & Ann Plast Surg & NRCS not adjusted \\
\hline 701 & 12142662 & Nahabedian & $\begin{array}{l}\text { Breast Reconstruction with the free TRAM or DIEP flap: patient selection, choice of } \\
\text { flap, and outcome }\end{array}$ & $\begin{array}{l}\text { Plast Reconstr } \\
\text { Surg }\end{array}$ & NRCS not adjusted \\
\hline 702 & 15220572 & Nahabedian & $\begin{array}{l}\text { Factors associated with anastomotic failure after microvascular reconstruction of } \\
\text { the breast }\end{array}$ & $\begin{array}{l}\text { Plast Reconstr } \\
\text { Surg }\end{array}$ & $\begin{array}{l}\text { Single group N enrolled } \\
<500\end{array}$ \\
\hline 703 & 15692347 & Nahabedian & $\begin{array}{l}\text { Breast reconstruction with the DIEP flap or the muscle-sparing (MS-2) free TRAM } \\
\text { flap: is there a difference? }\end{array}$ & $\begin{array}{l}\text { Plast Reconstr } \\
\text { Surg }\end{array}$ & NRCS not adjusted \\
\hline 704 & 33202009 & Nahabedian & What Are the Long-Term Aesthetic Issues in Prepectoral Breast Reconstruction? & Aesthet Surg J & NRCS $<30$ per arm \\
\hline 705 & 30847663 & Nakagomi & $\begin{array}{l}\text { Lateral thoracoaxillar dermal-fat flap for breast conserving surgery: the changes of } \\
\text { the indication and long-term results }\end{array}$ & Breast Cancer & $\begin{array}{l}\text { Not breast } \\
\text { reconstruction }\end{array}$ \\
\hline 706 & 15943735 & Nano & Qualitative assessment of breast reconstruction in a specialist breast unit & ANZ J Surg & NRCS not adjusted \\
\hline 707 & 31055108 & Naoum & $\begin{array}{l}\text { The Impact of Chest Wall Boost on Reconstruction Complications and Local } \\
\text { Control in Patients Treated for Breast Cancer }\end{array}$ & $\begin{array}{l}\text { Int J Radiat Oncol } \\
\text { Biol Phys }\end{array}$ & $\begin{array}{l}\text { Does not address KQ1- } \\
\text { KQ6 }\end{array}$ \\
\hline 708 & 32607638 & Naoum & $\begin{array}{l}\text { Optimal Reconstruction Type and PMRT Timing for Breast Cancer Patients treated } \\
\text { by Neoadjuvant Chemotherapy and Mastectomy }\end{array}$ & $\begin{array}{l}\text { International } \\
\text { Journal of } \\
\text { Radiation } \\
\text { Oncology, Biology, } \\
\text { Physics }\end{array}$ & $\begin{array}{l}\text { Does not address KQ1- } \\
\text { KQ6 }\end{array}$ \\
\hline 709 & $\begin{array}{l}\mathrm{CN}- \\
01580972\end{array}$ & Nct & Direct to Implant Breast Reconstruction Based Pre- or Retropectoral & $\begin{array}{l}\text { https://clinicaltrials. } \\
\text { gov/show/NCT0314 } \\
3335\end{array}$ & $\begin{array}{l}\text { Protocol/methods with } \\
\text { no results }\end{array}$ \\
\hline 710 & $\begin{array}{l}\mathrm{CN}- \\
01701127\end{array}$ & Nct & SEroma Reduction pOst MAstectomy 'SEROMA Study' & $\begin{array}{l}\text { https://clinicaltrials. } \\
\text { gov/show/NCT0373 } \\
8527\end{array}$ & $\begin{array}{l}\text { Not breast } \\
\text { reconstruction }\end{array}$ \\
\hline 711 & $\begin{array}{l}\text { CN- } \\
01517046\end{array}$ & Nct & The Use of an Acellular Dermal Matrix in a Two-Staged Breast Reconstruction & $\begin{array}{l}\text { https://clinicaltrials. } \\
\text { gov/show/NCT0061 } \\
6824\end{array}$ & $\begin{array}{l}\text { Protocol/methods with } \\
\text { no results }\end{array}$ \\
\hline 712 & $\begin{array}{l}\mathrm{CN}- \\
01581816\end{array}$ & Nct & $\begin{array}{l}\text { Prospective Trial of Subcutaneous Versus Subpectoral 2-Staged Implant-Based } \\
\text { Breast Reconstruction }\end{array}$ & $\begin{array}{l}\text { https://clinicaltrials. } \\
\text { gov/show/NCT0277 } \\
5409\end{array}$ & $\begin{array}{l}\text { Protocol/methods with } \\
\text { no results }\end{array}$ \\
\hline 713 & $\begin{array}{l}\text { CN- } \\
01794765\end{array}$ & Nct & $\begin{array}{l}\text { Delayed-immediate Versus Delayed Breast Reconstruction in Breast Cancer } \\
\text { Patients With Mastectomy and Radiation Therapy }\end{array}$ & $\begin{array}{l}\text { https://clinicaltrials. } \\
\text { gov/show/NCT0373 } \\
0922\end{array}$ & $\begin{array}{l}\text { Protocol/methods with } \\
\text { no results }\end{array}$ \\
\hline 714 & $\begin{array}{l}\mathrm{CN}- \\
01794855\end{array}$ & Nct & $\begin{array}{l}\text { Standard Silicone-based vs. B-Lite } ® \text { Light Weight Breast Implant After Total } \\
\text { Mastectomy and Radiotherapy for Breast Cancer }\end{array}$ & $\begin{array}{l}\text { https://clinicaltrials. } \\
\text { gov/show/NCT0373 } \\
7500\end{array}$ & $\begin{array}{l}\text { Protocol/methods with } \\
\text { no results }\end{array}$ \\
\hline
\end{tabular}




\begin{tabular}{|c|c|c|c|c|c|}
\hline No. & $\begin{array}{l}\text { PMID or } \\
\text { Other } \\
\text { Identifier }\end{array}$ & $\begin{array}{l}\text { First Author } \\
\text { Last Name }\end{array}$ & Title & Journal & Reason for Exclusion \\
\hline 715 & $\begin{array}{l}\text { CN- } \\
01543662\end{array}$ & Nct & $\begin{array}{l}\text { Breast Reconstruction With Acellular Dermal Matrix in the Setting of Breast Cancer } \\
\text { Treatment }\end{array}$ & $\begin{array}{l}\text { https://clinicaltrials. } \\
\text { gov/show/NCT0206 } \\
1527\end{array}$ & $\begin{array}{l}\text { Protocol/methods with } \\
\text { no results }\end{array}$ \\
\hline 716 & $\begin{array}{l}\text { CN- } \\
02082997\end{array}$ & Nct & $\begin{array}{l}\text { Pre- Versus Sub-pectoral Implant-based Breast Reconstruction After Skin-sparing } \\
\text { Mastectomy or Nipple-sparing Mastectomy }\end{array}$ & $\begin{array}{l}\text { https://clinicaltrials. } \\
\text { gov/show/NCT0429 } \\
3146\end{array}$ & $\begin{array}{l}\text { Protocol/methods with } \\
\text { no results }\end{array}$ \\
\hline 717 & $\begin{array}{l}\text { CN- } \\
02089009\end{array}$ & Nct & ERAS in Autologous Breast Reconstruction: a Pilot RCT & $\begin{array}{l}\text { https://clinicaltrials. } \\
\text { gov/show/NCT0430 } \\
6003\end{array}$ & $\begin{array}{l}\text { Protocol/methods with } \\
\text { no results }\end{array}$ \\
\hline 718 & $\begin{array}{l}\mathrm{CN}- \\
01522432\end{array}$ & Nct & $\begin{array}{l}\text { Hypofractionated Radiation Therapy After Mastectomy in Preventing Recurrence in } \\
\text { Patients With Stage Ila-Illa Breast Cancer }\end{array}$ & $\begin{array}{l}\text { https://clinicaltrials. } \\
\text { gov/show/NCT0341 } \\
4970\end{array}$ & $\begin{array}{l}\text { Protocol/methods with } \\
\text { no results }\end{array}$ \\
\hline 719 & $\begin{array}{l}\text { CN- } \\
01944992\end{array}$ & Nct & $\begin{array}{l}\text { Pre-pectoral Versus Sub-pectoral Implant Placement in Immediate Breast } \\
\text { Reconstruction }\end{array}$ & $\begin{array}{l}\text { https://clinicaltrials. } \\
\text { gov/show/NCT0395 } \\
9709\end{array}$ & $\begin{array}{l}\text { Protocol/methods with } \\
\text { no results }\end{array}$ \\
\hline 720 & 21601458 & Nedumpara & $\begin{array}{l}\text { Impact of immediate breast reconstruction on breast cancer recurrence and } \\
\text { survival }\end{array}$ & Breast & $\begin{array}{l}\text { Single group } \mathrm{N} \text { enrolled } \\
<500\end{array}$ \\
\hline 721 & $\begin{array}{l}\text { CN- } \\
01940884\end{array}$ & Negenborn & $\begin{array}{l}\text { Short-term cost-effectiveness of one-stage implant-based breast reconstruction } \\
\text { with an acellular dermal matrix versus two-stage expander-implant reconstruction } \\
\text { from a multicentre randomized clinical trial }\end{array}$ & $\begin{array}{l}\text { British journal of } \\
\text { surgery }\end{array}$ & $\begin{array}{l}\text { Duplicate of another } \\
\text { publication }\end{array}$ \\
\hline 722 & 30835827 & Negenborn & $\begin{array}{l}\text { Short-term cost-effectiveness of one-stage implant-based breast reconstruction } \\
\text { with an acellular dermal matrix versus two-stage expander-implant reconstruction } \\
\text { from a multicentre randomized clinical trial }\end{array}$ & Br J Surg & $\begin{array}{l}\text { Does not address KQ1- } \\
\text { KQ6 }\end{array}$ \\
\hline 723 & $\begin{array}{l}\text { CN- } \\
01930174\end{array}$ & Negenborn & $\begin{array}{l}\text { Quality of life and patient satisfaction after one-stage implant-based breast } \\
\text { reconstruction with an acellular dermal matrix versus two-stage breast } \\
\text { reconstruction (BRIOS): primary outcome of a randomised, controlled trial }\end{array}$ & $\begin{array}{l}\text { The lancet. } \\
\text { Oncology }\end{array}$ & $\begin{array}{l}\text { Duplicate of another } \\
\text { publication }\end{array}$ \\
\hline 724 & 30104147 & Negenborn & $\begin{array}{l}\text { Quality of life and patient satisfaction after one-stage implant-based breast } \\
\text { reconstruction with an acellular dermal matrix versus two-stage breast } \\
\text { reconstruction (BRIOS): primary outcome of a randomised, controlled trial }\end{array}$ & Lancet Oncol & $\begin{array}{l}\text { Does not address KQ1- } \\
\text { KQ6 }\end{array}$ \\
\hline 725 & 25557724 & Nelson & $\begin{array}{l}\text { Wound healing complications after autologous breast reconstruction: a model to } \\
\text { predict risk }\end{array}$ & $\begin{array}{l}J \text { Plast Reconstr } \\
\text { Aesthet Surg }\end{array}$ & $\begin{array}{l}\text { Copublication of } \\
\text { included study with no } \\
\text { new data }\end{array}$ \\
\hline 726 & 25456289 & Nelson & $\begin{array}{l}\text { Intraoperative perfusion management impacts postoperative outcomes: an analysis } \\
\text { of } 682 \text { autologous breast reconstruction patients }\end{array}$ & $\begin{array}{l}J \text { Plast Reconstr } \\
\text { Aesthet Surg }\end{array}$ & $\begin{array}{l}\text { Copublication of } \\
\text { included study with no } \\
\text { new data }\end{array}$ \\
\hline 727 & 28084138 & Nelson & $\begin{array}{l}\text { Intraoperative vasopressors and thrombotic complications in free flap breast } \\
\text { reconstruction }\end{array}$ & $\begin{array}{l}J \text { Plast Surg Hand } \\
\text { Surg }\end{array}$ & $\begin{array}{l}\text { Copublication of } \\
\text { included study with no } \\
\text { new data }\end{array}$ \\
\hline 728 & 23886556 & Nelson & $\begin{array}{l}\text { Delayed autologous breast reconstruction: factors which influence patient decision } \\
\text { making }\end{array}$ & $\begin{array}{l}J \text { Plast Reconstr } \\
\text { Aesthet Surg }\end{array}$ & $\begin{array}{l}\text { Does not address KQ1- } \\
\text { KQ6 }\end{array}$ \\
\hline
\end{tabular}




\begin{tabular}{|c|c|c|c|c|c|}
\hline No. & $\begin{array}{l}\text { PMID or } \\
\text { Other } \\
\text { Identifier }\end{array}$ & $\begin{array}{l}\text { First Author } \\
\text { Last Name }\end{array}$ & Title & Journal & Reason for Exclusion \\
\hline 729 & 21042098 & Nelson & $\begin{array}{l}\text { A Comparison between DIEP and muscle-sparing free TRAM flaps in breast } \\
\text { reconstruction: a single surgeon's recent experience }\end{array}$ & $\begin{array}{l}\text { Plast Reconstr } \\
\text { Surg }\end{array}$ & NRCS not adjusted \\
\hline 730 & 30431541 & Nelson & $\begin{array}{l}\text { Function and Strength after Free Abdominally Based Breast Reconstruction: A 10- } \\
\text { Year Follow-Up }\end{array}$ & $\begin{array}{l}\text { Plast Reconstr } \\
\text { Surg }\end{array}$ & NRCS $<30$ per arm \\
\hline 731 & 10560853 & Newman & Feasibility of immediate breast reconstruction for locally advanced breast cancer & Ann Surg Oncol & $\begin{array}{l}\text { Single group } \mathrm{N} \text { enrolled } \\
<500\end{array}$ \\
\hline 732 & 21184070 & Newman & $\begin{array}{l}\text { The true incidence of near-term postoperative complications in prosthetic breast } \\
\text { reconstruction utilizing human acellular dermal matrices: a meta-analysis }\end{array}$ & $\begin{array}{l}\text { Aesthetic Plast } \\
\text { Surg }\end{array}$ & $\begin{array}{l}\text { Narrative review/ } \\
\text { Commentary }\end{array}$ \\
\hline 733 & 27633549 & $\mathrm{Ng}$ & $\begin{array}{l}\text { Trends in Post-Mastectomy Reconstruction in an Asian Population: A 12-Year } \\
\text { Institutional Review }\end{array}$ & Breast J & $\begin{array}{l}\text { Does not address KQ1- } \\
\text { KQ6 }\end{array}$ \\
\hline 734 & 120533760 & $\mathrm{Ng}$ & $\begin{array}{l}\text { Trends in Post-Mastectomy Reconstruction in an Asian Population: A 12-Year } \\
\text { Institutional Review }\end{array}$ & Breast Journal & $\begin{array}{l}\text { Duplicate of another } \\
\text { publication }\end{array}$ \\
\hline 735 & 33634944 & $\mathrm{Ng}$ & $\begin{array}{l}\text { Immediate prepectoral implant reconstruction using TiLOOP Bra Pocket results in } \\
\text { improved patient satisfaction over dual plane reconstruction }\end{array}$ & $\begin{array}{l}\text { ANZ journal of } \\
\text { surgery }\end{array}$ & NRCS not adjusted \\
\hline 736 & 20628580 & Nguyen & $\begin{array}{l}\text { Infectious Complications Leading to Explantation in Implant-Based Breast } \\
\text { Reconstruction With AlloDerm }\end{array}$ & Eplasty & NRCS not adjusted \\
\hline 737 & 22791106 & Nguyen & Effect of immediate reconstruction on postmastectomy surgical site infection & Ann Surg & $\begin{array}{l}\text { Does not address KQ1- } \\
\text { KQ6 }\end{array}$ \\
\hline 738 & 27010582 & Nickel & $\begin{array}{l}\text { Effect of Noninfectious Wound Complications after Mastectomy on Subsequent } \\
\text { Surgical Procedures and Early Implant Loss }\end{array}$ & J Am Coll Surg & NRCS not adjusted \\
\hline 739 & 25455801 & Niddam & [Breast reconstruction by latissimus dorsi flap: Towards an evolution of ideas] & $\begin{array}{l}\text { Ann Chir Plast } \\
\text { Esthet }\end{array}$ & NRCS not adjusted \\
\hline 740 & 8004611 & Noda & Breast reconstruction & Cancer & NRCS not adjusted \\
\hline 741 & 108158587 & NR & Breast reconstruction improves well-being and quality of life & $\begin{array}{l}\text { Johns Hopkins } \\
\text { Medical Letter: } \\
\text { Health After } 50\end{array}$ & Unable to retrieve article \\
\hline 742 & 25840544 & Nwaogu & $\begin{array}{l}\text { Venous Thromboembolism after Breast Reconstruction in Patients Undergoing } \\
\text { Breast Surgery: An American College of Surgeons NSQIP Analysis }\end{array}$ & J Am Coll Surg & $\begin{array}{l}\text { Does not address KQ1- } \\
\text { KQ6 }\end{array}$ \\
\hline 743 & 9492663 & Nyren & $\begin{array}{l}\text { Risk of connective tissue disease and related disorders among women with breast } \\
\text { implants: a nation-wide retrospective cohort study in Sweden }\end{array}$ & $B m j$ & $\begin{array}{l}\text { Not breast } \\
\text { reconstruction }\end{array}$ \\
\hline 744 & 29948462 & O' Halloran & $\begin{array}{l}\text { Neoadjuvant chemoradiation and breast reconstruction: the potential for improved } \\
\text { outcomes in the treatment of breast cancer }\end{array}$ & Ir J Med Sci & $\begin{array}{l}\text { Single group } \mathrm{N} \text { enrolled } \\
<500\end{array}$ \\
\hline 745 & 29927832 & O'Connell & $\begin{array}{l}\text { Comparison of Immediate versus Delayed DIEP Flap Reconstruction in Women } \\
\text { Who Require Postmastectomy Radiotherapy }\end{array}$ & $\begin{array}{l}\text { Plast Reconstr } \\
\text { Surg }\end{array}$ & NRCS not adjusted \\
\hline 746 & 30923359 & O'Connell & $\begin{array}{l}\text { The impact of immediate breast reconstruction on the time to delivery of adjuvant } \\
\text { therapy: the iBRA- } 2 \text { study }\end{array}$ & Br J Cancer & $\begin{array}{l}\text { Does not address KQ1- } \\
\text { KQ6 }\end{array}$ \\
\hline 747 & 30923359 & O'Connell & $\begin{array}{l}\text { The impact of immediate breast reconstruction on the time to delivery of adjuvant } \\
\text { therapy: the iBRA-2 study }\end{array}$ & $\begin{array}{l}\text { British Journal of } \\
\text { Cancer }\end{array}$ & $\begin{array}{l}\text { Duplicate of another } \\
\text { publication }\end{array}$ \\
\hline 748 & 27013145 & O'Neill & $\begin{array}{l}\text { Usability of the internal mammary recipient vessels in microvascular breast } \\
\text { reconstruction }\end{array}$ & $\begin{array}{l}J \text { Plast Reconstr } \\
\text { Aesthet Surg }\end{array}$ & $\begin{array}{l}\text { Does not address KQ1- } \\
\text { KQ6 }\end{array}$ \\
\hline
\end{tabular}




\begin{tabular}{|c|c|c|c|c|c|}
\hline No. & $\begin{array}{l}\text { PMID or } \\
\text { Other } \\
\text { Identifier }\end{array}$ & $\begin{array}{l}\text { First Author } \\
\text { Last Name }\end{array}$ & Title & Journal & Reason for Exclusion \\
\hline 749 & 32152777 & O'Neill & $\begin{array}{l}\text { Development and Evaluation of a Machine Learning Prediction Model for Flap } \\
\text { Failure in Microvascular Breast Reconstruction }\end{array}$ & Ann Surg Oncol & $\begin{array}{l}\text { Does not address KQ1- } \\
\text { KQ6 }\end{array}$ \\
\hline 750 & 30489498 & Offodile & $\begin{array}{l}\text { Assessing the Quality of Microvascular Breast Reconstruction Performed in the } \\
\text { Urban Safety-Net Setting: A Doubly Robust Regression Analysis }\end{array}$ & $\begin{array}{l}\text { Plast Reconstr } \\
\text { Surg }\end{array}$ & $\begin{array}{l}\text { Does not address KQ1- } \\
\text { KQ6 }\end{array}$ \\
\hline 751 & 29044475 & Offodile & $\begin{array}{l}\text { The site of care matters: An examination of the relationship between high Medicaid } \\
\text { burden hospitals and the use, cost, and complications of immediate breast } \\
\text { reconstruction after mastectomy }\end{array}$ & Cancer & $\begin{array}{l}\text { Does not address KQ1- } \\
\text { KQ6 }\end{array}$ \\
\hline 752 & 25676466 & Offodile & Racial disparities in the type of postmastectomy reconstruction chosen & J Surg Res & $\begin{array}{l}\text { Does not address KQ1- } \\
\text { KQ6 }\end{array}$ \\
\hline 753 & 127216390 & Offodile & $\begin{array}{l}\text { The site of care matters: An examination of the relationship between high Medicaid } \\
\text { burden hospitals and the use, cost, and complications of immediate breast } \\
\text { reconstruction after mastectomy }\end{array}$ & Cancer (0008543X) & $\begin{array}{l}\text { Duplicate of another } \\
\text { publication }\end{array}$ \\
\hline 754 & 29052108 & Ogita & $\begin{array}{l}\text { Risk factors for complications among breast cancer patients treated with post- } \\
\text { mastectomy radiotherapy and immediate tissue-expander/permanent implant } \\
\text { reconstruction: a retrospective cohort study }\end{array}$ & Breast Cancer & $\begin{array}{l}\text { Does not address KQ1- } \\
\text { KQ6 }\end{array}$ \\
\hline 755 & 30225915 & Oh & $\begin{array}{l}\text { Patient-reported outcomes of breast reconstruction in older women: Audit of a } \\
\text { large metropolitan public/private practice in Sydney, Australia }\end{array}$ & Psychooncology & NRCS $<30$ per arm \\
\hline 756 & 26965305 & Oh & $\begin{array}{l}\text { Patterns and outcomes of breast reconstruction in older women - A systematic } \\
\text { review of the literature }\end{array}$ & Eur J Surg Oncol & $\begin{array}{l}\text { Does not address KQ1- } \\
\text { KQ6 }\end{array}$ \\
\hline 757 & 3308352 & Olbrisch & $\begin{array}{l}\text { [Tissue expander in breast reconstruction. Experiences and results with more than } \\
300 \text { expanders] }\end{array}$ & Chirurg & Unable to retrieve article \\
\hline 758 & 33214118 & Oleru & $\begin{array}{l}\text { The impact of hepatitis } \mathrm{B} \text { and } \mathrm{C} \text { diagnoses on surgical outcomes following } \\
\text { mastectomy and breast reconstruction }\end{array}$ & $\begin{array}{l}\text { J Plast Reconstr } \\
\text { Aesthet Surg }\end{array}$ & $\begin{array}{l}\text { Does not address KQ1- } \\
\text { KQ6 }\end{array}$ \\
\hline 759 & 32195865 & Olinger & $\begin{array}{l}\text { Outcomes of Immediate Implant-Based Mastectomy Reconstruction in Women with } \\
\text { Previous Breast Radiation }\end{array}$ & $\begin{array}{l}\text { Plast Reconstr } \\
\text { Surg }\end{array}$ & $\begin{array}{l}\text { Does not address KQ1- } \\
\text { KQ6 }\end{array}$ \\
\hline 760 & 32195865 & Olinger & $\begin{array}{l}\text { Outcomes of Immediate Implant-Based Mastectomy Reconstruction in Women with } \\
\text { Previous Breast Radiotherapy }\end{array}$ & $\begin{array}{l}\text { Plast Reconstr } \\
\text { Surg }\end{array}$ & $\begin{array}{l}\text { Duplicate of another } \\
\text { publication }\end{array}$ \\
\hline 761 & 31146506 & Oliver & $\begin{array}{l}\text { Postmastectomy Radiation Therapy (PMRT) before and after 2-Stage Expander- } \\
\text { Implant Breast Reconstruction: A Systematic Review }\end{array}$ & Medicina (Kaunas) & $\begin{array}{l}\text { Narrative review/ } \\
\text { Commentary }\end{array}$ \\
\hline 762 & 18209153 & Olsen & Hospital-associated costs due to surgical site infection after breast surgery & Arch Surg & $\begin{array}{l}\text { Does not address KQ1- } \\
\text { KQ6 }\end{array}$ \\
\hline 763 & 26036877 & Olsen & $\begin{array}{l}\text { Incidence of Surgical Site Infection Following Mastectomy With and Without } \\
\text { Immediate Reconstruction Using Private Insurer Claims Data }\end{array}$ & $\begin{array}{l}\text { Infect Control Hosp } \\
\text { Epidemiol }\end{array}$ & NRCS not adjusted \\
\hline 764 & 32221200 & Opsomer & Lumbar Flap versus the Gold Standard: Comparison to the DIEP Flap & $\begin{array}{l}\text { Plast Reconstr } \\
\text { Surg }\end{array}$ & NRCS not adjusted \\
\hline 765 & 30616243 & Orr & Bleeding After Free Flap-Based Breast Reconstruction: A NSQIP Analysis & $\begin{array}{l}J \text { Reconstr } \\
\text { Microsurg }\end{array}$ & $\begin{array}{l}\text { Does not address KQ1- } \\
\text { KQ6 }\end{array}$ \\
\hline 766 & 26612083 & Orzalesi & $\begin{array}{l}\text { Nipple sparing mastectomy: Surgical and oncological outcomes from a national } \\
\text { multicentric registry with } 913 \text { patients ( } 1006 \text { cases) over a six year period }\end{array}$ & Breast & $\begin{array}{l}\text { Does not address KQ1- } \\
\text { KQ6 }\end{array}$ \\
\hline 767 & 26287324 & Otte & $\begin{array}{l}\text { [The DIEP Flap as Method of Choice in Breast Reconstruction - Results and } \\
\text { Protocol for Succesful Reconstruction] }\end{array}$ & $\begin{array}{l}\text { Handchir Mikrochir } \\
\text { Plast Chir }\end{array}$ & NRCS not adjusted \\
\hline
\end{tabular}




\begin{tabular}{|c|c|c|c|c|c|}
\hline No. & $\begin{array}{l}\text { PMID or } \\
\text { Other } \\
\text { Identifier }\end{array}$ & $\begin{array}{l}\text { First Author } \\
\text { Last Name }\end{array}$ & Title & Journal & Reason for Exclusion \\
\hline 768 & 26855905 & Otte & $\begin{array}{l}\text { Conservative mastectomies and Immediate-DElayed AutoLogous (IDEAL) breast } \\
\text { reconstruction: the DIEP flap }\end{array}$ & Gland Surg & $\begin{array}{l}\text { Single group }>500 \text {, but } \\
\text { no complications data }\end{array}$ \\
\hline 769 & 25692294 & Ouyang & $\begin{array}{l}\text { Effect of implant vs. tissue reconstruction on cancer specific survival varies by } \\
\text { axillary lymph node status in breast cancer patients }\end{array}$ & PLoS One & NRCS not adjusted \\
\hline 770 & 32113960 & Oxley & $\begin{array}{l}\text { Successful same day discharge after immediate post-mastectomy alloplastic } \\
\text { breast reconstruction: A single tertiary centre retrospective audit }\end{array}$ & $\begin{array}{l}\text { J Plast Reconstr } \\
\text { Aesthet Surg }\end{array}$ & $\begin{array}{l}\text { Does not address KQ1- } \\
\text { KQ6 }\end{array}$ \\
\hline 771 & 23542834 & Ozturk & $\begin{array}{l}\text { Breast reconstruction with abdominal-based free flaps in high body mass index } \\
\text { population: postoperative complications and impact of weight loss }\end{array}$ & Ann Plast Surg & $\begin{array}{l}\text { Single group N enrolled } \\
<500\end{array}$ \\
\hline 772 & 22238939 & Pak & $\begin{array}{l}\text { [Results of single-stage reconstructive surgery in breast cancer patients (a report } \\
\text { of } 1143 \text { cases)] }\end{array}$ & Vopr Onkol & Unable to retrieve article \\
\hline 773 & 31438594 & Palubicka & $\begin{array}{l}\text { Surgical Site Infection after Breast Surgery: A Retrospective Analysis of 5-Year } \\
\text { Postoperative Data from a Single Center in Poland }\end{array}$ & Medicina (Kaunas) & $\begin{array}{l}\text { Single group N enrolled } \\
<500\end{array}$ \\
\hline 774 & 26817890 & Pan & $\begin{array}{l}\text { Predictors for Reconstruction and Mood Disorder Associated With Reconstruction } \\
\text { in Patients With Breast Cancer and Mastectomy: A Retrospective Cohort Study }\end{array}$ & $\begin{array}{l}\text { Medicine } \\
\text { (Baltimore) }\end{array}$ & $\begin{array}{l}\text { Does not address KQ1- } \\
\text { KQ6 }\end{array}$ \\
\hline 775 & 29510420 & Panayi & $\begin{array}{l}\text { Impact of Obesity on Outcomes in Breast Reconstruction: A Systematic Review } \\
\text { and Meta-Analysis }\end{array}$ & $\begin{array}{l}J \text { Reconstr } \\
\text { Microsurg }\end{array}$ & $\begin{array}{l}\text { Does not address KQ1- } \\
\text { KQ6 }\end{array}$ \\
\hline 776 & 26165884 & Pannucci & $\begin{array}{l}\text { Loupes-Only Microsurgery is a Safe Alternative to the Operating Microscope: An } \\
\text { Analysis of 1,649 Consecutive Free Flap Breast Reconstructions }\end{array}$ & $\begin{array}{l}J \text { Reconstr } \\
\text { Microsurg }\end{array}$ & $\begin{array}{l}\text { Does not address KQ1- } \\
\text { KQ6 }\end{array}$ \\
\hline 777 & 33567574 & Papa & $\begin{array}{l}\text { Protocol for Prevention and Monitoring of Surgical Site Infections in Implant-Based } \\
\text { Breast Reconstruction: Preliminary Results }\end{array}$ & Medicina (Kaunas) & $\begin{array}{l}\text { Single group N enrolled } \\
<500\end{array}$ \\
\hline 778 & 16437226 & Papadopulos & [Quality of life and patient satisfaction after breast reconstruction] & Chirurg & NRCS not adjusted \\
\hline 779 & 27187252 & Parabkaharan & $\begin{array}{l}\text { Comparison of Reconstructive Outcomes in Breast Cancer Patients With } \\
\text { Preexisting Subpectoral Implants: Implant-Sparing Mastectomy With Delayed } \\
\text { Implant Exchange Versus Immediate Tissue Expander Reconstruction }\end{array}$ & Ann Plast Surg & $\begin{array}{l}\text { Single group } \mathrm{N} \text { enrolled } \\
<500\end{array}$ \\
\hline 780 & 30881804 & Parikh & $\begin{array}{l}\text { Cortiva Versus AlloDerm Ready-to-use in Prepectoral and Submuscular Breast } \\
\text { Reconstruction: Prospective Randomized Clinical Trial Study Design and Early } \\
\text { Findings }\end{array}$ & $\begin{array}{l}\text { Plast Reconstr } \\
\text { Surg Glob Open }\end{array}$ & $\begin{array}{l}\text { Single group } \mathrm{N} \text { enrolled } \\
<500\end{array}$ \\
\hline 781 & 29511877 & Park & $\begin{array}{l}\text { The use of acellular dermal matrix in immediate two-stage prosthetic breast } \\
\text { reconstruction provides protection from postmastectomy radiation therapy: a } \\
\text { clinicopathologic perspective }\end{array}$ & $\begin{array}{l}\text { J Mater Sci Mater } \\
\text { Med }\end{array}$ & NRCS not adjusted \\
\hline 782 & 32629834 & Park & $\begin{array}{l}\text { Intraoperative Intercostal Nerve Block for Postoperative Pain Control in Pre- } \\
\text { Pectoral versus Subpectoral Direct-to-Implant Breast Reconstruction: A } \\
\text { Retrospective Study }\end{array}$ & Medicina (Kaunas) & NRCS not adjusted \\
\hline 783 & 33586091 & Park & $\begin{array}{l}\text { Is mastectomy with immediate reconstruction safe for patients undergoing } \\
\text { neoadjuvant chemotherapy? A nationwide study from Korean Breast Cancer } \\
\text { Society }\end{array}$ & Breast Cancer & $\begin{array}{l}\text { Does not address KQ1- } \\
\text { KQ6 }\end{array}$ \\
\hline 784 & 23018685 & Parks & $\begin{array}{l}\text { Human acellular dermis versus no acellular dermis in tissue expansion breast } \\
\text { reconstruction }\end{array}$ & $\begin{array}{l}\text { Plast Reconstr } \\
\text { Surg }\end{array}$ & NRCS not adjusted \\
\hline 785 & 18093828 & Patani & $\begin{array}{l}\text { Oncological safety and patient satisfaction with skin-sparing mastectomy and } \\
\text { immediate breast reconstruction }\end{array}$ & Surg Oncol & $\begin{array}{l}\text { Single group N enrolled } \\
<500\end{array}$ \\
\hline
\end{tabular}




\begin{tabular}{|c|c|c|c|c|c|}
\hline No. & $\begin{array}{l}\text { PMID or } \\
\text { Other } \\
\text { Identifier }\end{array}$ & $\begin{array}{l}\text { First Author } \\
\text { Last Name }\end{array}$ & Title & Journal & Reason for Exclusion \\
\hline 786 & 23897324 & Patel & $\begin{array}{l}\text { Microvascular autologous breast reconstruction in the context of radiation therapy: } \\
\text { comparing two reconstructive algorithms }\end{array}$ & $\begin{array}{l}\text { Plast Reconstr } \\
\text { Surg }\end{array}$ & $\begin{array}{l}\text { Single group } \mathrm{N} \text { enrolled } \\
<500\end{array}$ \\
\hline 787 & 21734543 & Patel & $\begin{array}{l}\text { Management of massive mastectomy skin flap necrosis following autologous } \\
\text { breast reconstruction }\end{array}$ & Ann Plast Surg & $\begin{array}{l}\text { Does not address KQ1- } \\
\text { KQ6 }\end{array}$ \\
\hline 788 & 24572859 & Patel & $\begin{array}{l}\text { Reinforcement of the abdominal wall following breast reconstruction with } \\
\text { abdominal flaps: a comparison of synthetic and biological mesh }\end{array}$ & $\begin{array}{l}\text { Plast Reconstr } \\
\text { Surg }\end{array}$ & $\begin{array}{l}\text { Does not address KQ1- } \\
\text { KQ6 }\end{array}$ \\
\hline 789 & 29761885 & Patel & $\begin{array}{l}\text { Immediate breast reconstruction for women having inflammatory breast cancer in } \\
\text { the United States }\end{array}$ & Cancer Med & $\begin{array}{l}\text { Single group } \mathrm{N} \text { enrolled } \\
<500\end{array}$ \\
\hline 790 & 32294076 & Patel & $\begin{array}{l}\text { Comparing Prepectoral Versus Subpectoral Tissue Expander Placement } \\
\text { Outcomes in Delayed-Immediate Autologous Breast Reconstruction }\end{array}$ & Ann Plast Surg & $\begin{array}{l}\text { Does not address KQ1- } \\
\text { KQ6 }\end{array}$ \\
\hline 791 & 31942389 & Patrinely & $\begin{array}{l}\text { Acellular Dermal Matrix Performance Compared with Latissimus Dorsi } \\
\text { Myocutaneous Flap in Expander-Based Breast Reconstruction }\end{array}$ & $\begin{array}{l}\text { Plast Reconstr } \\
\text { Surg Glob Open }\end{array}$ & NRCS not adjusted \\
\hline 792 & 28740767 & Peiris & $\begin{array}{l}\text { The Effect of the Timing of Radiotherapy on Clinical and Patient-Reported } \\
\text { Outcomes After Latissimus Dorsi Breast Reconstruction: A 10-Year Study }\end{array}$ & $\begin{array}{l}\text { Plast Reconstr } \\
\text { Surg Glob Open }\end{array}$ & NRCS not adjusted \\
\hline 793 & 29788682 & Pek & $\begin{array}{l}\text { Immediate breast reconstruction following nipple-sparing mastectomy in an asian } \\
\text { population: Aesthetic outcomes and mitigating nipple-areolar complex necrosis }\end{array}$ & $\begin{array}{l}\text { Archives of Plastic } \\
\text { Surgery }\end{array}$ & NRCS $<30$ per arm \\
\hline 794 & 29788682 & Pek & $\begin{array}{l}\text { Immediate breast reconstruction following nipple-sparing mastectomy in an Asian } \\
\text { population: Aesthetic outcomes and mitigating nipple-areolar complex necrosis }\end{array}$ & Arch Plast Surg & $\begin{array}{l}\text { Single group } \mathrm{N} \text { enrolled } \\
<500\end{array}$ \\
\hline 795 & 24732652 & Peled & $\begin{array}{l}\text { Impact of total skin-sparing mastectomy incision type on reconstructive } \\
\text { complications following radiation therapy }\end{array}$ & $\begin{array}{l}\text { Plast Reconstr } \\
\text { Surg }\end{array}$ & $\begin{array}{l}\text { Does not address KQ1- } \\
\text { KQ6 }\end{array}$ \\
\hline 796 & 22526909 & Peled & $\begin{array}{l}\text { Outcomes after total skin-sparing mastectomy and immediate reconstruction in } 657 \\
\text { breasts }\end{array}$ & $\begin{array}{l}\text { Annals of Surgical } \\
\text { Oncology }\end{array}$ & $\begin{array}{l}\text { Does not address KQ1- } \\
\text { KQ6 }\end{array}$ \\
\hline 797 & 20855759 & Peled & $\begin{array}{l}\text { Impact of chemotherapy on postoperative complications after mastectomy and } \\
\text { immediate breast reconstruction }\end{array}$ & Archives of Surgery & NRCS not adjusted \\
\hline 798 & 28671888 & Peled & $\begin{array}{l}\text { Complications After Total Skin-Sparing Mastectomy and Expander-Implant } \\
\text { Reconstruction: Effects of Radiation Therapy on the Stages of Reconstruction }\end{array}$ & Ann Plast Surg & NRCS not adjusted \\
\hline 799 & 26170194 & Peled & $\begin{array}{l}\text { Expanding the Indications for Total Skin-Sparing Mastectomy: Is It Safe for } \\
\text { Patients with Locally Advanced Disease? }\end{array}$ & Ann Surg Oncol & $\begin{array}{l}\text { Does not address KQ1- } \\
\text { KQ6 }\end{array}$ \\
\hline 800 & 19325334 & Persichetti & $\begin{array}{l}\text { Implant breast reconstruction after salvage mastectomy in previously irradiated } \\
\text { patients }\end{array}$ & Ann Plast Surg & NRCS not adjusted \\
\hline 801 & 23542851 & Pestana & Factors affecting complications in radiated breast reconstruction & Ann Plast Surg & NRCS not adjusted \\
\hline 802 & 8060065 & Peters & Factors affecting the rupture of silicone-gel breast implants & Ann Plast Surg & $\begin{array}{l}\text { Duplicate of another } \\
\text { publication }\end{array}$ \\
\hline 803 & 8060065 & Peters & Factors affecting the rupture of silicone-gel breast implants & Ann Plast Surg & $\begin{array}{l}\text { Single group } \mathrm{N} \text { enrolled } \\
<500\end{array}$ \\
\hline 804 & 7702307 & Peters & $\begin{array}{l}\text { Calcification of breast implant capsules: incidence, diagnosis, and contributing } \\
\text { factors }\end{array}$ & Ann Plast Surg & $\begin{array}{l}\text { Single group N enrolled } \\
<500\end{array}$ \\
\hline 805 & 1340174 & Petit & $\begin{array}{l}\text { [Immediate mammary reconstruction in the radical treatment of cancer of the } \\
\text { breast] }\end{array}$ & $\begin{array}{l}\text { Ann Chir Plast } \\
\text { Esthet }\end{array}$ & Unable to retrieve article \\
\hline 806 & 18210199 & Petit & $\begin{array}{l}\text { Oncological results of immediate breast reconstruction: long term follow-up of a } \\
\text { large series at a single institution }\end{array}$ & $\begin{array}{l}\text { Breast Cancer Res } \\
\text { Treat }\end{array}$ & $\begin{array}{l}\text { Single group N enrolled } \\
<500\end{array}$ \\
\hline
\end{tabular}




\begin{tabular}{|c|c|c|c|c|c|}
\hline No. & $\begin{array}{l}\text { PMID or } \\
\text { Other } \\
\text { Identifier }\end{array}$ & $\begin{array}{l}\text { First Author } \\
\text { Last Name }\end{array}$ & Title & Journal & Reason for Exclusion \\
\hline 807 & 31538071 & Phan & $\begin{array}{l}\text { The use of Patient Reported Outcome Measures in assessing patient outcomes } \\
\text { when comparing autologous to alloplastic breast reconstruction: a systematic } \\
\text { review }\end{array}$ & Gland Surg & $\begin{array}{l}\text { Does not address KQ1- } \\
\text { KQ6 }\end{array}$ \\
\hline 808 & 33299693 & Phillips & $\begin{array}{l}\text { Is Tissue Expansion Worth It? Comparative Outcomes of Skin-preserving versus } \\
\text { Delayed Autologous Breast Reconstruction }\end{array}$ & $\begin{array}{l}\text { Plast Reconstr } \\
\text { Surg Glob Open }\end{array}$ & $\begin{array}{l}\text { Single group } \mathrm{N} \text { enrolled } \\
<500\end{array}$ \\
\hline 809 & 26165569 & Pinell-White & $\begin{array}{l}\text { Patient-Reported Quality of Life After Breast Reconstruction: A One-Year } \\
\text { Longitudinal Study Using the WHO-QOL Survey }\end{array}$ & Ann Plast Surg & NRCS $<30$ per arm \\
\hline 810 & 16996422 & Pinsolle & Complications analysis of 266 immediate breast reconstructions & $\begin{array}{l}J \text { Plast Reconstr } \\
\text { Aesthet Surg }\end{array}$ & NRCS not adjusted \\
\hline 811 & 28619483 & Piper & $\begin{array}{l}\text { Characterizing infections in prosthetic breast reconstruction: A validity assessment } \\
\text { of national health databases }\end{array}$ & $\begin{array}{l}J \text { Plast Reconstr } \\
\text { Aesthet Surg }\end{array}$ & $\begin{array}{l}\text { Single group }>500 \text {, but } \\
\text { no complications data }\end{array}$ \\
\hline 812 & 23486127 & Piper & $\begin{array}{l}\text { Total skin-sparing mastectomy: a systematic review of oncologic outcomes and } \\
\text { postoperative complications }\end{array}$ & Ann Plast Surg & $\begin{array}{l}\text { Does not address KQ1- } \\
\text { KQ6 }\end{array}$ \\
\hline 813 & 19806332 & Piroth & $\begin{array}{l}\text { Immediate reconstruction with an expander/implant following ablatio mammae } \\
\text { because of breast cancer : side effects and cosmetic results after adjuvant chest } \\
\text { wall radiotherapy }\end{array}$ & Strahlenther Onkol & $\begin{array}{l}\text { Single group N enrolled } \\
<500\end{array}$ \\
\hline 814 & 10063590 & Plogmeier & [Breast reconstruction: autologous tissue versus implant] & Zentralb/ Chir & NRCS not adjusted \\
\hline 815 & 11711934 & Polednak & $\begin{array}{l}\text { Type of breast reconstructive surgery among breast cancer patients: a population- } \\
\text { based study }\end{array}$ & $\begin{array}{l}\text { Plast Reconstr } \\
\text { Surg }\end{array}$ & No outcome of interest \\
\hline 816 & 32420245 & Polotto & $\begin{array}{l}\text { One-step prepectoral breast reconstruction with porcine dermal matrix-covered } \\
\text { implant: a protective technique improving the outcome in post-mastectomy } \\
\text { radiation therapy setting }\end{array}$ & Gland Surg & $\begin{array}{l}\text { Does not address KQ1- } \\
\text { KQ6 }\end{array}$ \\
\hline 817 & 31868761 & Porter & Comparison of Saline Expanders and Air Expanders for Breast Reconstruction & Ann Plast Surg & $\begin{array}{l}\text { Does not address KQ1- } \\
\text { KQ6 }\end{array}$ \\
\hline 818 & 104983367 & Potter & Reporting clinical outcomes of breast reconstruction: a systematic review & $\begin{array}{l}\text { JNCl: Journal of } \\
\text { the National } \\
\text { Cancer Institute }\end{array}$ & $\begin{array}{l}\text { Does not address KQ1- } \\
\text { KQ6 }\end{array}$ \\
\hline 819 & 26109277 & Potter & $\begin{array}{l}\text { Systematic review and critical appraisal of the impact of acellular dermal matrix use } \\
\text { on the outcomes of implant-based breast reconstruction }\end{array}$ & Br J Surg & $\begin{array}{l}\text { Narrative review/ } \\
\text { Commentary }\end{array}$ \\
\hline 820 & 24011501 & Potter & $\begin{array}{l}\text { Early complications and implant loss in implant-based breast reconstruction with } \\
\text { and without acellular dermal matrix (Tecnoss Protexa(R)): a comparative study }\end{array}$ & Eur J Surg Oncol & NRCS not adjusted \\
\hline 821 & 30639093 & Potter & $\begin{array}{l}\text { Short-term safety outcomes of mastectomy and immediate implant-based breast } \\
\text { reconstruction with and without mesh (iBRA): a multicentre, prospective cohort } \\
\text { study }\end{array}$ & $\begin{array}{l}\text { The Lancet } \\
\text { Oncology }\end{array}$ & $\begin{array}{l}\text { Duplicate of another } \\
\text { publication }\end{array}$ \\
\hline 822 & 30507480 & Potter & Quality of life after breast reconstruction-the BRIOS study & Lancet Oncology & $\begin{array}{l}\text { Duplicate of another } \\
\text { publication }\end{array}$ \\
\hline 823 & 7761519 & Pouhaer & $\begin{array}{l}\text { Cosmetic results and complications in breast cancer patients after total } \\
\text { mastectomy with circular incision and immediate breast reconstruction }\end{array}$ & $\begin{array}{l}\text { Plast Reconstr } \\
\text { Surg }\end{array}$ & $\begin{array}{l}\text { Narrative review/ } \\
\text { Commentary }\end{array}$ \\
\hline 824 & 7761519 & Pouhaer & $\begin{array}{l}\text { Cosmetic results and complications in breast cancer patients after total } \\
\text { mastectomy with circular incision and immediate breast reconstruction }\end{array}$ & $\begin{array}{l}\text { Plastic and } \\
\text { Reconstructive } \\
\text { Surgery }\end{array}$ & $\begin{array}{l}\text { Single group N enrolled } \\
<500\end{array}$ \\
\hline
\end{tabular}




\begin{tabular}{|c|c|c|c|c|c|}
\hline No. & $\begin{array}{l}\text { PMID or } \\
\text { Other } \\
\text { Identifier }\end{array}$ & $\begin{array}{l}\text { First Author } \\
\text { Last Name }\end{array}$ & Title & Journal & Reason for Exclusion \\
\hline 825 & 32726819 & Prantl & $\begin{array}{l}\text { Impact of Smoking Status in Free Deep Inferior Epigastric Artery Perforator Flap } \\
\text { Breast Reconstruction: A Multicenter Study }\end{array}$ & $\begin{array}{l}\text { J Reconstr } \\
\text { Microsurg }\end{array}$ & $\begin{array}{l}\text { Copublication of } \\
\text { included study with no } \\
\text { new data }\end{array}$ \\
\hline 826 & 33346536 & Prantl & $\begin{array}{l}\text { Effect of Radiation Therapy on Microsurgical Deep Inferior Epigastric Perforator } \\
\text { Flap Breast Reconstructions: A Matched Cohort Analysis of } 4577 \text { Cases }\end{array}$ & Ann Plast Surg & $\begin{array}{l}\text { Copublication of } \\
\text { included study with no } \\
\text { new data }\end{array}$ \\
\hline 827 & 18434824 & Preminger & $\begin{array}{l}\text { The influence of AlloDerm on expander dynamics and complications in the setting } \\
\text { of immediate tissue expander/implant reconstruction: a matched-cohort study }\end{array}$ & Ann Plast Surg & NRCS not adjusted \\
\hline 828 & 29419662 & $\mathrm{Pu}$ & $\begin{array}{l}\text { The role of postmastectomy radiation therapy in patients with immediate prosthetic } \\
\text { breast reconstruction: A meta-analysis }\end{array}$ & $\begin{array}{l}\text { Medicine } \\
\text { (Baltimore) }\end{array}$ & $\begin{array}{l}\text { Does not address KQ1- } \\
\text { KQ6 }\end{array}$ \\
\hline 829 & 31495035 & Punglia & $\begin{array}{l}\text { Patient-preferred outcomes measurement after post-mastectomy radiation therapy } \\
\text { and immediate reconstruction }\end{array}$ & Breast J & $\begin{array}{l}\text { Single group } \mathrm{N} \text { enrolled } \\
<500\end{array}$ \\
\hline 830 & 124339287 & Pusic & $\begin{array}{l}\text { Patient-Reported Outcomes } 1 \text { Year After Immediate Breast Reconstruction: } \\
\text { Results of the Mastectomy Reconstruction Outcomes Consortium Study }\end{array}$ & $\begin{array}{l}\text { Journal of Clinical } \\
\text { Oncology }\end{array}$ & $\begin{array}{l}\text { Duplicate of another } \\
\text { publication }\end{array}$ \\
\hline 831 & 26219243 & Pyfer & $\begin{array}{l}\text { Early Postoperative Outcomes in Breast Conservation Surgery Versus Simple } \\
\text { Mastectomy with Implant Reconstruction: A NSQIP Analysis of 11,645 Patients }\end{array}$ & Ann Surg Oncol & $\begin{array}{l}\text { Does not address KQ1- } \\
\text { KQ6 }\end{array}$ \\
\hline 832 & 31668432 & Qi & $\begin{array}{l}\text { Does Choice of Reconstruction Type Affect Survival in Patients With Metastatic } \\
\text { Breast Cancer? }\end{array}$ & J Surg Res & $\begin{array}{l}\text { Does not address KQ1- } \\
\text { KQ6 }\end{array}$ \\
\hline 833 & 31467545 & Qian & $\begin{array}{l}\text { A Systematic Review and Meta-Analysis on Microsurgical Safety and Efficacy of } \\
\text { Profunda Artery Perforator Flap in Breast Reconstruction }\end{array}$ & J Oncol & $\begin{array}{l}\text { Does not address KQ1- } \\
\text { KQ6 }\end{array}$ \\
\hline 834 & 25652054 & Qin & $\begin{array}{l}\text { Assessing Outcomes and Safety of Inpatient Versus Outpatient Tissue Expander } \\
\text { Immediate Breast Reconstruction }\end{array}$ & Ann Surg Oncol & $\begin{array}{l}\text { Does not address KQ1- } \\
\text { KQ6 }\end{array}$ \\
\hline 835 & 25652054 & Qin & $\begin{array}{l}\text { Assessing Outcomes and Safety of Inpatient Versus Outpatient Tissue Expander } \\
\text { Immediate Breast Reconstruction }\end{array}$ & $\begin{array}{l}\text { Annals of Surgical } \\
\text { Oncology }\end{array}$ & $\begin{array}{l}\text { Duplicate of another } \\
\text { publication }\end{array}$ \\
\hline 836 & 24961932 & Qin & $\begin{array}{l}\text { Differential impact of non-insulin-dependent diabetes mellitus and insulin- } \\
\text { dependent diabetes mellitus on breast reconstruction outcomes }\end{array}$ & $\begin{array}{l}\text { Breast Cancer Res } \\
\text { Treat }\end{array}$ & NRCS not adjusted \\
\hline 837 & 29384865 & Qin & Postoperative outcomes of breast reconstruction after mastectomy & $\begin{array}{l}\text { Medicine (United } \\
\text { States) }\end{array}$ & $\begin{array}{l}\text { Duplicate of another } \\
\text { publication }\end{array}$ \\
\hline 838 & 28992647 & Qiu & $\begin{array}{l}\text { Surgical Duration Impacts Venous Thromboembolism Risk in Microsurgical Breast } \\
\text { Reconstruction }\end{array}$ & $\begin{array}{l}\text { J Reconstr } \\
\text { Microsurg }\end{array}$ & $\begin{array}{l}\text { Does not address KQ1- } \\
\text { KQ6 }\end{array}$ \\
\hline 839 & 33437474 & Quilichini & $\begin{array}{l}\text { Mastectomy with immediate breast reconstruction: Results of a mono-centric 4- } \\
\text { years cohort }\end{array}$ & $\begin{array}{l}\text { Ann Med Surg } \\
\text { (Lond) }\end{array}$ & No outcome of interest \\
\hline 840 & 27047785 & Quinn & Prosthetic breast reconstruction: indications and update & Gland Surg & $\begin{array}{l}\text { Narrative review/ } \\
\text { Commentary }\end{array}$ \\
\hline 841 & 27622099 & Qureshi & $\begin{array}{l}\text { Direct Hospital Cost of Outcome Pathways in Implant-Based Reconstruction with } \\
\text { Acellular Dermal Matrices }\end{array}$ & $\begin{array}{l}\text { Plast Reconstr } \\
\text { Surg Glob Open }\end{array}$ & $\begin{array}{l}\text { Does not address KQ1- } \\
\text { KQ6 }\end{array}$ \\
\hline 842 & 28235218 & Razdan & $\begin{array}{l}\text { National Breast Reconstruction Utilization in the Setting of Postmastectomy } \\
\text { Radiotherapy }\end{array}$ & $\begin{array}{l}J \text { Reconstr } \\
\text { Microsurg }\end{array}$ & $\begin{array}{l}\text { Does not address KQ1- } \\
\text { KQ6 }\end{array}$ \\
\hline 843 & 26910695 & Razdan & $\begin{array}{l}\text { Cost-Effectiveness Analysis of Breast Reconstruction Options in the Setting of } \\
\text { Postmastectomy Radiotherapy Using the BREAST-Q }\end{array}$ & $\begin{array}{l}\text { Plast Reconstr } \\
\text { Surg }\end{array}$ & No outcome of interest \\
\hline
\end{tabular}




\begin{tabular}{|c|c|c|c|c|c|}
\hline No. & $\begin{array}{l}\text { PMID or } \\
\text { Other } \\
\text { Identifier }\end{array}$ & $\begin{array}{l}\text { First Author } \\
\text { Last Name }\end{array}$ & Title & Journal & Reason for Exclusion \\
\hline 844 & 21617433 & Reddy & $\begin{array}{l}\text { Bilateral autologous reconstruction from different sites: indications and outcomes } \\
\text { after DIEP and SGAP flaps }\end{array}$ & $\begin{array}{l}\text { Plast Reconstr } \\
\text { Surg }\end{array}$ & $\begin{array}{l}\text { Case report or series of } \\
\text { case reports }\end{array}$ \\
\hline 845 & 20429922 & Reefy & $\begin{array}{l}\text { Oncological outcome and patient satisfaction with skin-sparing mastectomy and } \\
\text { immediate breast reconstruction: a prospective observational study }\end{array}$ & BMC Cancer & NRCS not adjusted \\
\hline 846 & 31858435 & Reinders & $\begin{array}{l}\text { Higher reconstruction failure and less patient-reported satisfaction after post } \\
\text { mastectomy radiotherapy with immediate implant-based breast reconstruction } \\
\text { compared to immediate autologous breast reconstruction }\end{array}$ & Breast Cancer & $\begin{array}{l}\text { Duplicate of another } \\
\text { publication }\end{array}$ \\
\hline 847 & 31858435 & Reinders & $\begin{array}{l}\text { Higher reconstruction failure and less patient-reported satisfaction after post } \\
\text { mastectomy radiotherapy with immediate implant-based breast reconstruction } \\
\text { compared to immediate autologous breast reconstruction }\end{array}$ & Breast Cancer & NRCS $<30$ per arm \\
\hline 848 & 23714788 & Reish & $\begin{array}{l}\text { Infection following implant-based reconstruction in } 1952 \text { consecutive breast } \\
\text { reconstructions: salvage rates and predictors of success }\end{array}$ & $\begin{array}{l}\text { Plastic and } \\
\text { reconstructive } \\
\text { surgery }\end{array}$ & $\begin{array}{l}\text { Duplicate of another } \\
\text { publication }\end{array}$ \\
\hline 849 & 23714788 & Reish & $\begin{array}{l}\text { Infection following implant-based reconstruction in } 1952 \text { consecutive breast } \\
\text { reconstructions: salvage rates and predictors of success }\end{array}$ & $\begin{array}{l}\text { Plast Reconstr } \\
\text { Surg }\end{array}$ & $\begin{array}{l}\text { Copublication of } \\
\text { included study with no } \\
\text { new data }\end{array}$ \\
\hline 850 & 25811561 & Reish & $\begin{array}{l}\text { Breast reconstruction outcomes after nipple-sparing mastectomy and radiation } \\
\text { therapy }\end{array}$ & $\begin{array}{l}\text { Plast Reconstr } \\
\text { Surg }\end{array}$ & $\begin{array}{l}\text { Does not address KQ1- } \\
\text { KQ6 }\end{array}$ \\
\hline 851 & 31620344 & Rezaei & Latissimus Dorsi Musculocutaneous Flap Inset Innovation in Breast Reconstruction & World J Plast Surg & NRCS $<30$ per arm \\
\hline 852 & 33470628 & Rhemtulla & $\begin{array}{l}\text { Incisional Hernia Incidence, Repair Techniques, and Outcomes Based on } 1600 \\
\text { Consecutive Patients Receiving Abdominally Based Autologous Breast } \\
\text { Reconstruction }\end{array}$ & Ann Plast Surg & $\begin{array}{l}\text { Copublication of } \\
\text { included study with no } \\
\text { new data }\end{array}$ \\
\hline 853 & 29383613 & Riba & $\begin{array}{l}\text { Surgical Risk Factors for the Delayed Initiation of Adjuvant Chemotherapy in } \\
\text { Breast Cancer }\end{array}$ & Ann Surg Oncol & $\begin{array}{l}\text { Does not address KQ1- } \\
\text { KQ6 }\end{array}$ \\
\hline 854 & 21780554 & Ribuffo & $\begin{array}{l}\text { Cagliari University Hospital (CUH) protocol for immediate alloplastic breast } \\
\text { reconstruction and unplanned radiotherapy. A preliminary report }\end{array}$ & $\begin{array}{l}\text { Eur Rev Med } \\
\text { Pharmacol Sci }\end{array}$ & $\begin{array}{l}\text { Single group N enrolled } \\
<500\end{array}$ \\
\hline 855 & 26166643 & Ribuffo & $\begin{array}{l}\text { Does postoperative radiation therapy represent a contraindication to expander- } \\
\text { implant based immediate breast reconstruction? An update 2012-2014 }\end{array}$ & $\begin{array}{l}\text { Eur Rev Med } \\
\text { Pharmacol Sci }\end{array}$ & $\begin{array}{l}\text { Narrative review/ } \\
\text { Commentary }\end{array}$ \\
\hline 856 & 32860077 & Ribuffo & $\begin{array}{l}\text { Dual-Plane Retro-pectoral Versus Pre-pectoral DTI Breast Reconstruction: An } \\
\text { Italian Multicenter Experience }\end{array}$ & $\begin{array}{l}\text { Aesthetic Plast } \\
\text { Surg }\end{array}$ & NRCS not adjusted \\
\hline 857 & 29341294 & Ricci & Topical nitroglycerin for the treatment of intraoperative microsurgical vasospasm & Microsurgery & $\begin{array}{l}\text { Does not address KQ1- } \\
\text { KQ6 }\end{array}$ \\
\hline 858 & 28296715 & Ricci & $\begin{array}{l}\text { Evaluating the Use of Tissue Oximetry to Decrease Intensive Unit Monitoring for } \\
\text { Free Flap Breast Reconstruction }\end{array}$ & Ann Plast Surg & $\begin{array}{l}\text { Does not address KQ1- } \\
\text { KQ6 }\end{array}$ \\
\hline 859 & 27135144 & Ricci & $\begin{array}{l}\text { A Novel Free Flap Monitoring System Using Tissue Oximetry with Text Message } \\
\text { Alerts }\end{array}$ & $\begin{array}{l}J \text { Reconstr } \\
\text { Microsurg }\end{array}$ & $\begin{array}{l}\text { Does not address KQ1- } \\
\text { KQ6 }\end{array}$ \\
\hline 860 & 23018695 & Richter & $\begin{array}{l}\text { A comparison of a new skin closure device and intradermal sutures in the closure } \\
\text { of full-thickness surgical incisions }\end{array}$ & $\begin{array}{l}\text { Plast Reconstr } \\
\text { Surg }\end{array}$ & $\begin{array}{l}\text { Single group N enrolled } \\
<500\end{array}$ \\
\hline 861 & 30691788 & Rifkin & $\begin{array}{l}\text { Impact of Diabetes on 30-Day Complications in Mastectomy and Implant-Based } \\
\text { Breast Reconstruction }\end{array}$ & J Surg Res & $\begin{array}{l}\text { Does not address KQ1- } \\
\text { KQ6 }\end{array}$ \\
\hline
\end{tabular}




\begin{tabular}{|c|c|c|c|c|c|}
\hline No. & $\begin{array}{l}\text { PMID or } \\
\text { Other } \\
\text { Identifier }\end{array}$ & $\begin{array}{l}\text { First Author } \\
\text { Last Name }\end{array}$ & Title & Journal & Reason for Exclusion \\
\hline 862 & 30691788 & Rifkin & $\begin{array}{l}\text { Impact of Diabetes on 30-D Complications in Mastectomy and Implant-Based } \\
\text { Breast Reconstruction }\end{array}$ & $\begin{array}{l}\text { Journal of Surgical } \\
\text { Research }\end{array}$ & $\begin{array}{l}\text { Duplicate of another } \\
\text { publication }\end{array}$ \\
\hline 863 & 26375459 & Rimler & $\begin{array}{l}\text { The effects of radiation therapy on perfusion of free versus pedicle transverse } \\
\text { rectus abdominis myocutaneous (TRAM) flaps in vivo }\end{array}$ & $\begin{array}{l}\text { J Plast Reconstr } \\
\text { Aesthet Surg }\end{array}$ & $\begin{array}{l}\text { Narrative review/ } \\
\text { Commentary }\end{array}$ \\
\hline 864 & 29724621 & Rindom & $\begin{array}{l}\text { Shoulder-related donor site morbidity and patient-reported satisfaction after } \\
\text { delayed breast reconstruction with pedicled flaps from the back: A comparative } \\
\text { analysis }\end{array}$ & $\begin{array}{l}\text { Journal of Plastic, } \\
\text { Reconstructive and } \\
\text { Aesthetic Surgery }\end{array}$ & NRCS not adjusted \\
\hline 865 & 26818271 & Rinker & $\begin{array}{l}\text { A Comparison of Methods to Assess Mastectomy Flap Viability in Skin-Sparing } \\
\text { Mastectomy and Immediate Reconstruction: A Prospective Cohort Study }\end{array}$ & $\begin{array}{l}\text { Plast Reconstr } \\
\text { Surg }\end{array}$ & NRCS $<30$ per arm \\
\hline 866 & 26202563 & Roberts & $\begin{array}{l}\text { Once is Rarely Enough: A Population-Based Study of Reoperations after } \\
\text { Postmastectomy Breast Reconstruction }\end{array}$ & Ann Surg Oncol & NRCS not adjusted \\
\hline 867 & 32964476 & Roberts & $\begin{array}{l}\text { Reoperation cascade in postmastectomy breast reconstruction and its associated } \\
\text { factors: Results from a long-term population-based study }\end{array}$ & J Surg Oncol & $\begin{array}{l}\text { Does not address KQ1- } \\
\text { KQ6 }\end{array}$ \\
\hline 868 & 32839117 & Robertson & $\begin{array}{l}\text { Reconstructive trends following mastectomies in Scotland: A comparison with } \\
\text { England }\end{array}$ & Surgeon & $\begin{array}{l}\text { Does not address KQ1- } \\
\text { KQ6 }\end{array}$ \\
\hline 869 & 27182693 & Rocco & Different types of implants for reconstructive breast surgery & $\begin{array}{l}\text { Cochrane } \\
\text { Database Syst Rev }\end{array}$ & Systematic review \\
\hline 870 & 25339608 & Rochlin & $\begin{array}{l}\text { Postmastectomy radiation therapy and immediate autologous breast } \\
\text { reconstruction: integrating perspectives from surgical oncology, radiation oncology, } \\
\text { and plastic and reconstructive surgery }\end{array}$ & J Surg Oncol & $\begin{array}{l}\text { Narrative review/ } \\
\text { Commentary }\end{array}$ \\
\hline 871 & 30973838 & Rochlin & $\begin{array}{l}\text { The Power of Patient Norms: Postoperative Pathway Associated With Shorter } \\
\text { Hospital Stay After Free Autologous Breast Reconstruction }\end{array}$ & Ann Plast Surg & $\begin{array}{l}\text { Does not address KQ1- } \\
\text { KQ6 }\end{array}$ \\
\hline 872 & 26275493 & $\begin{array}{l}\text { Rodriguez- } \\
\text { Unda }\end{array}$ & $\begin{array}{l}\text { Low incidence of complications using polyglactin } 910 \text { (Vicryl) mesh in breast } \\
\text { reconstruction: A systematic review }\end{array}$ & $\begin{array}{l}J \text { Plast Reconstr } \\
\text { Aesthet Surg }\end{array}$ & Systematic review \\
\hline 873 & 11994594 & Rogers & $\begin{array}{l}\text { Radiation effects on breast reconstruction with the deep inferior epigastric } \\
\text { perforator flap }\end{array}$ & $\begin{array}{l}\text { Plast Reconstr } \\
\text { Surg }\end{array}$ & $\begin{array}{l}\text { Single group } \mathrm{N} \text { enrolled } \\
<500\end{array}$ \\
\hline 874 & 29968023 & Romanoff & $\begin{array}{l}\text { A Comparison of Patient-Reported Outcomes After Nipple-Sparing Mastectomy } \\
\text { and Conventional Mastectomy with Reconstruction }\end{array}$ & Ann Surg Oncol & $\begin{array}{l}\text { Single group } \mathrm{N} \text { enrolled } \\
<500\end{array}$ \\
\hline 875 & 23395741 & Romics Jr & $\begin{array}{l}\text { Oncologic safety of skin-sparing mastectomy followed by immediate breast } \\
\text { reconstruction: Rate and localization of recurrences, and impact of reconstruction } \\
\text { techniques }\end{array}$ & Orvosi Hetilap & Unable to retrieve article \\
\hline 876 & 22634689 & Roostaeian & $\begin{array}{l}\text { Comparison of immediate implant placement versus the staged tissue expander } \\
\text { technique in breast reconstruction }\end{array}$ & $\begin{array}{l}\text { Plast Reconstr } \\
\text { Surg }\end{array}$ & NRCS not adjusted \\
\hline 877 & 27018662 & Roostaeian & $\begin{array}{l}\text { Impact of Prior Tissue Expander/Implant on Postmastectomy Free Flap Breast } \\
\text { Reconstruction }\end{array}$ & $\begin{array}{l}\text { Plast Reconstr } \\
\text { Surg }\end{array}$ & $\begin{array}{l}>=10 \% \text { revision } \\
\text { reconstruction only }\end{array}$ \\
\hline 878 & 24572867 & Roostaeian & $\begin{array}{l}\text { The effect of prior abdominal surgery on abdominally based free flaps in breast } \\
\text { reconstruction }\end{array}$ & $\begin{array}{l}\text { Plast Reconstr } \\
\text { Surg }\end{array}$ & $\begin{array}{l}\text { Copublication of } \\
\text { included study with no } \\
\text { new data }\end{array}$ \\
\hline 879 & 2147095 & Rosen & $\begin{array}{l}\text { Clinical experience with immediate breast reconstruction using tissue expansion or } \\
\text { transverse rectus abdominis musculocutaneous flaps }\end{array}$ & Ann Plast Surg & NRCS not adjusted \\
\hline
\end{tabular}




\begin{tabular}{|c|c|c|c|c|c|}
\hline No. & $\begin{array}{l}\text { PMID or } \\
\text { Other } \\
\text { Identifier }\end{array}$ & $\begin{array}{l}\text { First Author } \\
\text { Last Name }\end{array}$ & Title & Journal & Reason for Exclusion \\
\hline 880 & 11147117 & Rouzier & [Autologous breast reconstruction with latissimus dorsi flap] & $\begin{array}{l}\text { Ann Chir Plast } \\
\text { Esthet }\end{array}$ & Unable to retrieve article \\
\hline 881 & 10974078 & Rowland & $\begin{array}{l}\text { Role of breast reconstructive surgery in physical and emotional outcomes among } \\
\text { breast cancer survivors }\end{array}$ & $\begin{array}{l}\text { JNCl: Journal of } \\
\text { the National } \\
\text { Cancer Institute }\end{array}$ & $\begin{array}{l}\text { Single group } \mathrm{N} \text { enrolled } \\
<500\end{array}$ \\
\hline 882 & 31821317 & Rubilar & $\begin{array}{l}\text { Autologous versus prosthetic reconstruction for women with breast cancer who will } \\
\text { undergo post-reconstruction radiotherapy }\end{array}$ & Medwave & $\begin{array}{l}\text { Narrative review/ } \\
\text { Commentary }\end{array}$ \\
\hline 883 & 139349747 & Rudolph & $\begin{array}{l}\text { Operative risk stratification in the obese female undergoing implant-based breast } \\
\text { reconstruction }\end{array}$ & Breast Journal & $\begin{array}{l}>=10 \% \text { revision } \\
\text { reconstruction only }\end{array}$ \\
\hline 884 & 20223055 & Rusby & $\begin{array}{l}\text { Immediate breast reconstruction after mastectomy: what are the long-term } \\
\text { prospects? }\end{array}$ & $\begin{array}{l}\text { Ann R Coll Surg } \\
\text { Engl }\end{array}$ & NRCS $<30$ per arm \\
\hline 885 & 28382097 & Ryu & $\begin{array}{l}\text { Oncologic Outcomes after Immediate Breast Reconstruction Following Total } \\
\text { Mastectomy in Patients with Breast Cancer: A Matched Case-Control Study }\end{array}$ & $J$ Breast Cancer & $\begin{array}{l}\text { Does not address KQ1- } \\
\text { KQ6 }\end{array}$ \\
\hline 886 & 19407609 & Sacks & $\begin{array}{l}\text { Rib-sparing internal mammary vessel harvest for microvascular breast } \\
\text { reconstruction in } 100 \text { consecutive cases }\end{array}$ & $\begin{array}{l}\text { Plast Reconstr } \\
\text { Surg }\end{array}$ & NRCS not adjusted \\
\hline 887 & 28274406 & Sacotte & $\begin{array}{l}\text { Assessing long-term complications in patients undergoing immediate } \\
\text { postmastectomy breast reconstruction and adjuvant radiation }\end{array}$ & Pract Radiat Oncol & NRCS not adjusted \\
\hline 888 & 31395398 & Sada & $\begin{array}{l}\text { Mastectomy and immediate breast reconstruction in the elderly: Trends and } \\
\text { outcomes }\end{array}$ & Surgery & $\begin{array}{l}\text { Does not address KQ1- } \\
\text { KQ6 }\end{array}$ \\
\hline 889 & 29485605 & Sadideen & $\begin{array}{l}\text { The Safety of Early Adjuvant Internal Mammary Lymph Node Irradiation following } \\
\text { Mastectomy and Immediate Autologous Reconstruction }\end{array}$ & $\begin{array}{l}\text { Plast Reconstr } \\
\text { Surg }\end{array}$ & $\begin{array}{l}\text { Single group N enrolled } \\
<500\end{array}$ \\
\hline 890 & 129664178 & Sae Byul & $\begin{array}{l}\text { Long-term outcomes of patients with breast cancer after nipple-sparing } \\
\text { mastectomy/skin-sparing mastectomy followed by immediate transverse rectus } \\
\text { abdominis musculocutaneous flap reconstruction: Comparison with conventional } \\
\text { mastectomy in a single center study }\end{array}$ & Medicine & $\begin{array}{l}\text { Duplicate of another } \\
\text { publication }\end{array}$ \\
\hline 891 & 24354013 & Saha & Post-mastectomy reconstruction: a risk-stratified comparative analysis of outcomes & Breast & $\begin{array}{l}\text { Does not address KQ1- } \\
\text { KQ6 }\end{array}$ \\
\hline 892 & 24354013 & Saha & Post-mastectomy reconstruction: A risk-stratified comparative analysis of outcomes & Breast & $\begin{array}{l}\text { Does not address KQ1- } \\
\text { KQ6 }\end{array}$ \\
\hline 893 & 19387162 & Sailon & $\begin{array}{l}\text { Free transverse rectus abdominis myocutaneous and deep inferior epigastric } \\
\text { perforator flaps for breast reconstruction: a systematic review of flap complication } \\
\text { rates and donor-site morbidity }\end{array}$ & Ann Plast Surg & Systematic review \\
\hline 894 & 18090738 & Saint-Cyr & $\begin{array}{l}\text { Internal mammary perforator recipient vessels for breast reconstruction using free } \\
\text { TRAM, DIEP, and SIEA flaps }\end{array}$ & $\begin{array}{l}\text { Plast Reconstr } \\
\text { Surg }\end{array}$ & $\begin{array}{l}\text { Does not address KQ1- } \\
\text { KQ6 }\end{array}$ \\
\hline 895 & 17519690 & Saint-Cyr & $\begin{array}{l}\text { Changing trends in recipient vessel selection for microvascular autologous breast } \\
\text { reconstruction: an analysis of } 1483 \text { consecutive cases }\end{array}$ & $\begin{array}{l}\text { Plast Reconstr } \\
\text { Surg }\end{array}$ & $\begin{array}{l}\text { Does not address KQ1- } \\
\text { KQ6 }\end{array}$ \\
\hline 896 & 21705282 & Sajid & $\begin{array}{l}\text { Prevention of postoperative seroma-related morbidity by quilting of latissimus dorsi } \\
\text { flap donor site: a systematic review }\end{array}$ & Clin Breast Cancer & $\begin{array}{l}\text { Does not address KQ1- } \\
\text { KQ6 }\end{array}$ \\
\hline 897 & 21858596 & Salgarello & $\begin{array}{l}\text { DIEP flap donor site versus elective abdominoplasty short-term complication rates: } \\
\text { a meta-analysis }\end{array}$ & $\begin{array}{l}\text { Aesthetic Plast } \\
\text { Surg }\end{array}$ & $\begin{array}{l}\text { Does not address KQ1- } \\
\text { KQ6 }\end{array}$ \\
\hline
\end{tabular}




\begin{tabular}{|c|c|c|c|c|c|}
\hline No. & $\begin{array}{l}\text { PMID or } \\
\text { Other } \\
\text { Identifier }\end{array}$ & $\begin{array}{l}\text { First Author } \\
\text { Last Name }\end{array}$ & Title & Journal & Reason for Exclusion \\
\hline 898 & 21617451 & Salgarello & $\begin{array}{l}\text { Breast fat grafting with platelet-rich plasma: a comparative clinical study and } \\
\text { current state of the art }\end{array}$ & $\begin{array}{l}\text { Plast Reconstr } \\
\text { Surg }\end{array}$ & $\begin{array}{l}\text { Does not address KQ1- } \\
\text { KQ6 }\end{array}$ \\
\hline 899 & 16508729 & Salhab & $\begin{array}{l}\text { Skin-sparing mastectomy and immediate breast reconstruction: patient satisfaction } \\
\text { and clinical outcome }\end{array}$ & Int J Clin Oncol & NRCS $<30$ per arm \\
\hline 900 & 27975034 & Salibian & $\begin{array}{l}\text { Subcutaneous Implant-based Breast Reconstruction with Acellular Dermal } \\
\text { Matrix/Mesh: A Systematic Review }\end{array}$ & $\begin{array}{l}\text { Plast Reconstr } \\
\text { Surg Glob Open }\end{array}$ & $\begin{array}{l}\text { Does not address KQ1- } \\
\text { KQ6 }\end{array}$ \\
\hline 901 & 32997369 & Salibian & $\begin{array}{l}\text { Comparing outcomes between stacked/conjoined and non-stacked/conjoined } \\
\text { abdominal microvascular unilateral breast reconstruction }\end{array}$ & Microsurgery & $\begin{array}{l}\text { Does not address KQ1- } \\
\text { KQ6 }\end{array}$ \\
\hline 902 & 28949034 & Samargandi & $\begin{array}{l}\text { Comparing the thoracodorsal and internal mammary vessels as recipients for } \\
\text { microsurgical autologous breast reconstruction: A systematic review and meta- } \\
\text { analysis }\end{array}$ & Microsurgery & $\begin{array}{l}\text { Does not address KQ1- } \\
\text { KQ6 }\end{array}$ \\
\hline 903 & 26483861 & Sanati-Mehrizy & $\begin{array}{l}\text { A Comparison of Postoperative Outcomes in Immediate Versus Delayed } \\
\text { Reconstruction After Mastectomy }\end{array}$ & Eplasty & $\begin{array}{l}\text { Does not address KQ1- } \\
\text { KQ6 }\end{array}$ \\
\hline 904 & 28005734 & Sanati-Mehrizy & $\begin{array}{l}\text { Risk Factors Leading to Free Flap Failure: Analysis From the National Surgical } \\
\text { Quality Improvement Program Database }\end{array}$ & J Craniofac Surg & $\begin{array}{l}\text { Single group } \mathrm{N} \text { enrolled } \\
<500\end{array}$ \\
\hline 905 & 28234813 & Sandberg & Molecular Profiling Using Breast Cancer Subtype to Plan for Breast Reconstruction & $\begin{array}{l}\text { Plast Reconstr } \\
\text { Surg }\end{array}$ & $\begin{array}{l}\text { Does not address KQ1- } \\
\text { KQ6 }\end{array}$ \\
\hline 906 & 26872025 & Sando & $\begin{array}{l}\text { The Early Years of Practice: An Assessment of Operative Efficiency and Cost of } \\
\text { Free Flap and Implant Breast Reconstruction at an Academic Institution }\end{array}$ & $\begin{array}{l}\text { J Reconstr } \\
\text { Microsurg }\end{array}$ & NRCS not adjusted \\
\hline 907 & 27806906 & Santosa & $\begin{array}{l}\text { Effect of Patient Age on Outcomes in Breast Reconstruction: Results from a } \\
\text { Multicenter Prospective Study }\end{array}$ & J Am Coll Surg & $\begin{array}{l}\text { Copublication of } \\
\text { included study with no } \\
\text { new data }\end{array}$ \\
\hline 908 & 25289224 & Sarhane & $\begin{array}{l}\text { Preoperative Anemia and Postoperative Outcomes in Immediate Breast } \\
\text { Reconstructive Surgery: A Critical Analysis of 10,958 Patients from the ACS- } \\
\text { NSQIP Database }\end{array}$ & $\begin{array}{l}\text { Plast Reconstr } \\
\text { Surg Glob Open }\end{array}$ & $\begin{array}{l}\text { Does not address KQ1- } \\
\text { KQ6 }\end{array}$ \\
\hline 909 & 117554091 & Sari & $\begin{array}{l}\text { Radiation Therapy Outcomes After Skin-Sparing Mastectomy and Implant-Based } \\
\text { Breast Reconstruction }\end{array}$ & $\begin{array}{l}\text { International } \\
\text { Journal of } \\
\text { Radiation } \\
\text { Oncology, Biology, } \\
\text { Physics } \\
\end{array}$ & $\begin{array}{l}\text { Does not address KQ1- } \\
\text { KQ6 }\end{array}$ \\
\hline 910 & 17440339 & Saulis & $\begin{array}{l}\text { A retrospective analysis of patient satisfaction with immediate postmastectomy } \\
\text { breast reconstruction: comparison of three common procedures }\end{array}$ & $\begin{array}{l}\text { Plast Reconstr } \\
\text { Surg }\end{array}$ & NRCS not adjusted \\
\hline 911 & 30589770 & Sbitany & $\begin{array}{l}\text { Prepectoral Breast Reconstruction in the Setting of Postmastectomy Radiation } \\
\text { Therapy: An Assessment of Clinical Outcomes and Benefits }\end{array}$ & $\begin{array}{l}\text { Plast Reconstr } \\
\text { Surg }\end{array}$ & NRCS $<30$ per arm \\
\hline 912 & 22456352 & Sbitany & $\begin{array}{l}\text { Strategies for recognizing and managing intraoperative venous congestion in } \\
\text { abdominally based autologous breast reconstruction }\end{array}$ & $\begin{array}{l}\text { Plast Reconstr } \\
\text { Surg }\end{array}$ & No outcome of interest \\
\hline 913 & 28574950 & Sbitany & $\begin{array}{l}\text { Prepectoral Breast Reconstruction: A Safe Alternative to Submuscular Prosthetic } \\
\text { Reconstruction following Nipple-Sparing Mastectomy }\end{array}$ & $\begin{array}{l}\text { Plast Reconstr } \\
\text { Surg }\end{array}$ & NRCS not adjusted \\
\hline 914 & 19952627 & Sbitany & $\begin{array}{l}\text { Acellular dermis-assisted prosthetic breast reconstruction versus complete } \\
\text { submuscular coverage: a head-to-head comparison of outcomes }\end{array}$ & $\begin{array}{l}\text { Plast Reconstr } \\
\text { Surg }\end{array}$ & NRCS not adjusted \\
\hline
\end{tabular}




\begin{tabular}{|c|c|c|c|c|c|}
\hline No. & $\begin{array}{l}\text { PMID or } \\
\text { Other } \\
\text { Identifier }\end{array}$ & $\begin{array}{l}\text { First Author } \\
\text { Last Name }\end{array}$ & Title & Journal & Reason for Exclusion \\
\hline 915 & 22094735 & Sbitany & $\begin{array}{l}\text { Acellular dermis-assisted prosthetic breast reconstruction: a systematic and critical } \\
\text { review of efficacy and associated morbidity }\end{array}$ & $\begin{array}{l}\text { Plast Reconstr } \\
\text { Surg }\end{array}$ & $\begin{array}{l}\text { Does not address KQ1- } \\
\text { KQ6 }\end{array}$ \\
\hline 916 & 25057918 & Sbitany & $\begin{array}{l}\text { Tissue Expander Reconstruction After Total Skin-Sparing Mastectomy: Defining } \\
\text { the Effects of Coverage Technique on Nipple/Areola Preservation }\end{array}$ & Ann Plast Surg & NRCS not adjusted \\
\hline 917 & 25158699 & Sbitany & $\begin{array}{l}\text { Immediate implant-based breast reconstruction following total skin-sparing } \\
\text { mastectomy: defining the risk of preoperative and postoperative radiation therapy } \\
\text { for surgical outcomes }\end{array}$ & $\begin{array}{l}\text { Plast Reconstr } \\
\text { Surg }\end{array}$ & NRCS not adjusted \\
\hline 918 & 11981192 & Scevola & Drains and seromas in TRAM flap breast reconstruction & Ann Plast Surg & $\begin{array}{l}\text { Does not address KQ1- } \\
\text { KQ6 }\end{array}$ \\
\hline 919 & 30570559 & Schaeffer & $\begin{array}{l}\text { Early Functional Outcomes After Prepectoral Breast Reconstruction: A Case- } \\
\text { Matched Cohort Study }\end{array}$ & Ann Plast Surg & NRCS <30 per arm \\
\hline 920 & 28194591 & Schaverien & $\begin{array}{l}\text { Complications in DIEP Flap Breast Reconstruction After Mastectomy for Breast } \\
\text { Cancer: A Prospective Cohort Study Comparing Unilateral and Bilateral } \\
\text { Reconstructions }\end{array}$ & Ann Surg Oncol & $\begin{array}{l}\text { Narrative review/ } \\
\text { Commentary }\end{array}$ \\
\hline 921 & 23886555 & Schaverien & $\begin{array}{l}\text { Is immediate autologous breast reconstruction with postoperative radiotherapy } \\
\text { good practice?: a systematic review of the literature }\end{array}$ & $\begin{array}{l}\text { J Plast Reconstr } \\
\text { Aesthet Surg }\end{array}$ & $\begin{array}{l}\text { Does not address KQ1- } \\
\text { KQ6 }\end{array}$ \\
\hline 922 & 24652691 & Schaverien & $\begin{array}{l}\text { Effect of obesity on outcomes of free autologous breast reconstruction: a meta- } \\
\text { analysis }\end{array}$ & Microsurgery & $\begin{array}{l}\text { Does not address KQ1- } \\
\text { KQ6 }\end{array}$ \\
\hline 923 & 17720644 & Schaverien & $\begin{array}{l}\text { Comparison of outcomes and donor-site morbidity in unilateral free TRAM versus } \\
\text { DIEP flap breast reconstruction }\end{array}$ & $\begin{array}{l}\text { J Plast Reconstr } \\
\text { Aesthet Surg }\end{array}$ & NRCS not adjusted \\
\hline 924 & 19050512 & Scholz & $\begin{array}{l}\text { Long-term outcomes after primary breast reconstruction using a vertical skin } \\
\text { pattern for skin-sparing mastectomy }\end{array}$ & $\begin{array}{l}\text { Plast Reconstr } \\
\text { Surg }\end{array}$ & NRCS not adjusted \\
\hline 925 & 9531705 & Schondorf & $\begin{array}{l}\text { [Plastic reconstructive surgical methods in breast saving therapy of breast } \\
\text { carcinoma: our concept of modified quadrantectomy] }\end{array}$ & Zentralbl Gynakol & NRCS not adjusted \\
\hline 926 & 1325065 & Schuster & $\begin{array}{l}\text { Breast reconstruction in women treated with radiation therapy for breast cancer: } \\
\text { Cosmesis, complications, and tumor control }\end{array}$ & $\begin{array}{l}\text { Plastic and } \\
\text { Reconstructive } \\
\text { Surgery }\end{array}$ & NRCS $<30$ per arm \\
\hline 927 & 33425590 & Schwartz & $\begin{array}{l}\text { Early Expander-to-Implant Exchange after Postmastectomy Reconstruction } \\
\text { Reduces Rates of Subsequent Major Infectious Complications }\end{array}$ & $\begin{array}{l}\text { Plast Reconstr } \\
\text { Surg Glob Open }\end{array}$ & $\begin{array}{l}\text { Single group } \mathrm{N} \text { enrolled } \\
<500\end{array}$ \\
\hline 928 & 28538553 & Sebai & $\begin{array}{l}\text { The Effect of Resident Involvement on Postoperative Short-Term Surgical } \\
\text { Outcomes in Immediate Breast Reconstruction: A National Surgical Quality } \\
\text { Improvement Program Study of } 24,005 \text { Patients }\end{array}$ & $\begin{array}{l}\text { Plast Reconstr } \\
\text { Surg }\end{array}$ & $\begin{array}{l}\text { Does not address KQ1- } \\
\text { KQ6 }\end{array}$ \\
\hline 929 & None & Seddon & $\begin{array}{l}\text { Versatility, clinical outcomes and mammographic follow-up of Chest Wall } \\
\text { Perforator Flaps (CWPF): A single-centre experience }\end{array}$ & $\begin{array}{l}\text { European Journal } \\
\text { of Cancer }\end{array}$ & $\begin{array}{l}\text { Single group } \mathrm{N} \text { enrolled } \\
<500\end{array}$ \\
\hline 930 & 27049776 & $\begin{array}{l}\text { Seidenstuecke } \\
r\end{array}$ & $\begin{array}{l}\text { Myosonographic study of abdominal wall dynamics to assess donor site morbidity } \\
\text { after microsurgical breast reconstruction with a DIEP or an ms-2 TRAM flap }\end{array}$ & $\begin{array}{l}\text { J Plast Reconstr } \\
\text { Aesthet Surg }\end{array}$ & NRCS $<30$ per arm \\
\hline 931 & 21364411 & $\begin{array}{l}\text { Seidenstuecke } \\
r\end{array}$ & $\begin{array}{l}\text { Morbidity of microsurgical breast reconstruction in patients with comorbid } \\
\text { conditions }\end{array}$ & $\begin{array}{l}\text { Plast Reconstr } \\
\text { Surg }\end{array}$ & NRCS not adjusted \\
\hline 932 & 27894917 & Seigle-Murandi & $\begin{array}{l}\text { Incidence of breast implant rupture in a 12-year retrospective cohort: Evidence of } \\
\text { quality discrepancy depending on the range }\end{array}$ & $\begin{array}{l}\text { J Plast Reconstr } \\
\text { Aesthet Surg }\end{array}$ & $\begin{array}{l}>=10 \% \text { augmentation } \\
\text { reconstruction only }\end{array}$ \\
\hline
\end{tabular}




\begin{tabular}{|c|c|c|c|c|c|}
\hline No. & $\begin{array}{l}\text { PMID or } \\
\text { Other } \\
\text { Identifier }\end{array}$ & $\begin{array}{l}\text { First Author } \\
\text { Last Name }\end{array}$ & Title & Journal & Reason for Exclusion \\
\hline 933 & 21042100 & Selber & $\begin{array}{l}\text { A prospective study comparing the functional impact of SIEA, DIEP, and muscle- } \\
\text { sparing free TRAM flaps on the abdominal wall: Part II. Bilateral reconstruction }\end{array}$ & $\begin{array}{l}\text { Plast Reconstr } \\
\text { Surg }\end{array}$ & NRCS not adjusted \\
\hline 934 & 16641623 & Selber & Risk factors and complications in free TRAM flap breast reconstruction & Ann Plast Surg & $\begin{array}{l}\text { Single group }>500 \text {, but } \\
\text { no complications data }\end{array}$ \\
\hline 935 & 18626349 & Selber & $\begin{array}{l}\text { A head-to-head comparison between the muscle-sparing free TRAM and the SIEA } \\
\text { flaps: is the rate of flap loss worth the gain in abdominal wall function? }\end{array}$ & $\begin{array}{l}\text { Plast Reconstr } \\
\text { Surg }\end{array}$ & NRCS not adjusted \\
\hline 936 & 26111310 & Selber & $\begin{array}{l}\text { Critical Evaluation of Risk Factors and Early Complications in } 564 \text { Consecutive } \\
\text { Two-Stage Implant-Based Breast Reconstructions Using Acellular Dermal Matrix at } \\
\text { a Single Center }\end{array}$ & $\begin{array}{l}\text { Plast Reconstr } \\
\text { Surg }\end{array}$ & $\begin{array}{l}\text { Single group N enrolled } \\
<500\end{array}$ \\
\hline 937 & 28106627 & Seth & $\begin{array}{l}\text { Outcomes After Elevation of Serratus Anterior Fascia During Prosthetic Breast } \\
\text { Reconstruction }\end{array}$ & Ann Plast Surg & $\begin{array}{l}\text { Does not address KQ1- } \\
\text { KQ6 }\end{array}$ \\
\hline 938 & 32855081 & Sewart & $\begin{array}{l}\text { Does mesh improve patient satisfaction and health-related quality of life after } \\
\text { implant-based breast reconstruction? A multicentre prospective cohort study }\end{array}$ & $\begin{array}{l}\text { European Journal } \\
\text { of Cancer }\end{array}$ & $\begin{array}{l}\text { Does not address KQ1- } \\
\text { KQ6 }\end{array}$ \\
\hline 939 & 28954300 & Sewart & $\begin{array}{l}\text { The impact of radiotherapy on patient-reported outcomes of immediate implant- } \\
\text { based breast reconstruction: Results of a prospective multicentre cohort study }\end{array}$ & $\begin{array}{l}\text { European Journal } \\
\text { of Cancer }\end{array}$ & $\begin{array}{l}\text { Copublication of } \\
\text { included study with no } \\
\text { new data }\end{array}$ \\
\hline 940 & 33078212 & Sgarzani & $\begin{array}{l}\text { Sub-muscular Reconstruction after NAC Sparing Mastectomy: Direct to Implant } \\
\text { Breast Reconstruction with Human ADM Versus Tissue Expander }\end{array}$ & $\begin{array}{l}\text { Aesthetic Plast } \\
\text { Surg }\end{array}$ & NRCS not adjusted \\
\hline 941 & 22693373 & Shaikh & $\begin{array}{l}\text { Post mastectomy immediate breast reconstruction } 13 \text { years experience in a single } \\
\text { centre }\end{array}$ & Indian J Surg Oncol & $\begin{array}{l}\text { Duplicate of another } \\
\text { publication }\end{array}$ \\
\hline 942 & 15096928 & Shaikh-Naidu & $\begin{array}{l}\text { Determinants of aesthetic satisfaction following TRAM and implant breast } \\
\text { reconstruction }\end{array}$ & Ann Plast Surg & $\begin{array}{l}\text { Does not address KQ1- } \\
\text { KQ6 }\end{array}$ \\
\hline 943 & 32032723 & Shammas & Assessing the Utility of Post-Mastectomy Imaging after Breast Reconstruction & J Am Coll Surg & $\begin{array}{l}\text { Does not address KQ1- } \\
\text { KQ6 }\end{array}$ \\
\hline 944 & 32032723 & Shammas & Assessing the Utility of Post-Mastectomy Imaging after Breast Reconstruction & J Am Coll Surg & $\begin{array}{l}\text { Duplicate of another } \\
\text { publication }\end{array}$ \\
\hline 945 & 31460998 & Shammas & $\begin{array}{l}\text { Immediate Breast Reconstruction Allows for the Timely Initiation of Post- } \\
\text { Mastectomy Radiation Therapy }\end{array}$ & $\begin{array}{l}\text { Plast Reconstr } \\
\text { Surg }\end{array}$ & $\begin{array}{l}\text { Duplicate of another } \\
\text { publication }\end{array}$ \\
\hline 946 & 31460998 & Shammas & $\begin{array}{l}\text { Immediate Breast Reconstruction Allows for the Timely Initiation of } \\
\text { Postmastectomy Radiation Therapy }\end{array}$ & $\begin{array}{l}\text { Plast Reconstr } \\
\text { Surg }\end{array}$ & $\begin{array}{l}\text { Does not address KQ1- } \\
\text { KQ6 }\end{array}$ \\
\hline 947 & 142362112 & Shammas & Assessing the Utility of Post-Mastectomy Imaging after Breast Reconstruction & $\begin{array}{l}\text { Journal of the } \\
\text { American College } \\
\text { of Surgeons }\end{array}$ & $\begin{array}{l}\text { Duplicate of another } \\
\text { publication }\end{array}$ \\
\hline 948 & 29845051 & Shash & $\begin{array}{l}\text { Laparoscopic Harvesting of Omental Flaps for Breast Reconstruction-A Review of } \\
\text { the Literature and Outcome Analysis }\end{array}$ & Plast Surg (Oakv) & $\begin{array}{l}\text { Does not address KQ1- } \\
\text { KQ6 }\end{array}$ \\
\hline 949 & 31338643 & Sheckter & $\begin{array}{l}\text { The impact of hospital volume on patient safety indicators following post- } \\
\text { mastectomy breast reconstruction in the US }\end{array}$ & $\begin{array}{l}\text { Breast Cancer Res } \\
\text { Treat }\end{array}$ & No outcome of interest \\
\hline 950 & 32390251 & Shen & Prolonged Opioid Use After Surgery for Early-Stage Breast Cancer & Oncologist & $\begin{array}{l}\text { Does not address KQ1- } \\
\text { KQ6 }\end{array}$ \\
\hline 951 & 117523175 & Shoichiro & $\begin{array}{l}\text { The Impact of Chemotherapy on Complications Associated with Mastectomy and } \\
\text { Immediate Autologous Tissue Reconstruction }\end{array}$ & American Surgeon & $\begin{array}{l}\text { Does not address KQ1- } \\
\text { KQ6 }\end{array}$ \\
\hline
\end{tabular}




\begin{tabular}{|c|c|c|c|c|c|}
\hline No. & $\begin{array}{l}\text { PMID or } \\
\text { Other } \\
\text { Identifier }\end{array}$ & $\begin{array}{l}\text { First Author } \\
\text { Last Name }\end{array}$ & Title & Journal & Reason for Exclusion \\
\hline 952 & 20195965 & Shridharani & Breast sensation after breast reconstruction: a systematic review & $\begin{array}{l}J \text { Reconstr } \\
\text { Microsurg }\end{array}$ & $\begin{array}{l}\text { Does not address KQ1- } \\
\text { KQ6 }\end{array}$ \\
\hline 953 & 26910655 & Shubinets & $\begin{array}{l}\text { Surgically Treated Hernia following Abdominally Based Autologous Breast } \\
\text { Reconstruction: Prevalence, Outcomes, and Expenditures }\end{array}$ & $\begin{array}{l}\text { Plast Reconstr } \\
\text { Surg }\end{array}$ & NRCS not adjusted \\
\hline 954 & 25003427 & Shuck & $\begin{array}{l}\text { Impact of Connective Tissue Disease on Oncologic Breast Surgery and } \\
\text { Reconstruction }\end{array}$ & Ann Plast Surg & NRCS $<30$ per arm \\
\hline 955 & 32442071 & Shumway & Integration of Breast Reconstruction and Postmastectomy Radiotherapy & J Clin Oncol & Systematic review \\
\hline 956 & 26001862 & Silva & $\begin{array}{l}\text { The Effect of Contralateral Prophylactic Mastectomy on Perioperative } \\
\text { Complications in Women Undergoing Immediate Breast Reconstruction: A NSQIP } \\
\text { Analysis }\end{array}$ & Ann Surg Oncol & $\begin{array}{l}\text { Does not address KQ1- } \\
\text { KQ6 }\end{array}$ \\
\hline 957 & 31077489 & Simpson & $\begin{array}{l}\text { Incidence of complications following two-stage expander/implant breast } \\
\text { reconstruction: The impact of cancer diagnosis in prophylactic mastectomy }\end{array}$ & Breast $J$ & NRCS $<30$ per arm \\
\hline 958 & 28953716 & Singh & $\begin{array}{l}\text { Five-Year Safety Data for More than 55,000 Subjects following Breast } \\
\text { Implantation: Comparison of Rare Adverse Event Rates with Silicone Implants } \\
\text { versus National Norms and Saline Implants }\end{array}$ & $\begin{array}{l}\text { Plast Reconstr } \\
\text { Surg }\end{array}$ & $\begin{array}{l}>=10 \% \text { augmentation } \\
\text { reconstruction only }\end{array}$ \\
\hline 959 & 31342362 & Singh & $\begin{array}{l}\text { Neoadjuvant Radiotherapy to Facilitate Immediate Breast Reconstruction: A } \\
\text { Systematic Review and Current Clinical Trials }\end{array}$ & Ann Surg Oncol & $\begin{array}{l}\text { Does not address KQ1- } \\
\text { KQ6 }\end{array}$ \\
\hline 960 & 8790856 & Singletary & $\begin{array}{l}\text { Skin-sparing mastectomy with immediate breast reconstruction: the M. D. } \\
\text { Anderson Cancer Center experience }\end{array}$ & Ann Surg Oncol & $\begin{array}{l}\text { Does not address KQ1- } \\
\text { KQ6 }\end{array}$ \\
\hline 961 & 28027221 & Sinha & Late Surgical-Site Infection in Immediate Implant-Based Breast Reconstruction & $\begin{array}{l}\text { Plast Reconstr } \\
\text { Surg }\end{array}$ & $\begin{array}{l}\text { Copublication of } \\
\text { included study with no } \\
\text { new data }\end{array}$ \\
\hline 962 & 29978367 & Sinnott & $\begin{array}{l}\text { Impact of Postmastectomy Radiation Therapy in Prepectoral Versus Subpectoral } \\
\text { Implant-Based Breast Reconstruction }\end{array}$ & Ann Surg Oncol & $\begin{array}{l}\text { Does not address KQ1- } \\
\text { KQ6 }\end{array}$ \\
\hline 963 & 31348330 & Siotos & $\begin{array}{l}\text { Survival and Disease Recurrence Rates among Breast Cancer Patients following } \\
\text { Mastectomy with or without Breast Reconstruction }\end{array}$ & $\begin{array}{l}\text { Plast Reconstr } \\
\text { Surg }\end{array}$ & $\begin{array}{l}\text { Does not address KQ1- } \\
\text { KQ6 }\end{array}$ \\
\hline 964 & 30489499 & Siotos & $\begin{array}{l}\text { Cost-Effectiveness Analysis of Silicone versus Saline Implant-Based Breast } \\
\text { Reconstruction Using the BREAST-Q }\end{array}$ & $\begin{array}{l}\text { Plast Reconstr } \\
\text { Surg }\end{array}$ & NRCS not adjusted \\
\hline 965 & 29475791 & Siotos & Breast reconstruction and risk of arm lymphedema development: A meta-analysis & $\begin{array}{l}\text { J Plast Reconstr } \\
\text { Aesthet Surg }\end{array}$ & $\begin{array}{l}\text { Does not address KQ1- } \\
\text { KQ6 }\end{array}$ \\
\hline 966 & 26881927 & Skovsted Yde & Acellular dermal matrices in breast reconstructions - a literature review & $\begin{array}{l}\text { J Plast Surg Hand } \\
\text { Surg }\end{array}$ & $\begin{array}{l}\text { Narrative review/ } \\
\text { Commentary }\end{array}$ \\
\hline 967 & 27678203 & Smith & Cost and Complications of Local Therapies for Early-Stage Breast Cancer & J Natl Cancer Inst & $\begin{array}{l}\text { Does not address KQ1- } \\
\text { KQ6 }\end{array}$ \\
\hline 968 & 30093286 & Smith & $\begin{array}{l}\text { Human acellular dermis increases surgical site infection and overall complication } \\
\text { profile when compared with submuscular breast reconstruction: An updated meta- } \\
\text { analysis incorporating new products() }\end{array}$ & $\begin{array}{l}J \text { Plast Reconstr } \\
\text { Aesthet Surg }\end{array}$ & $\begin{array}{l}\text { Narrative review/ } \\
\text { Commentary }\end{array}$ \\
\hline 969 & 132785107 & Smith & $\begin{array}{l}\text { Early Toxicity and Patient Reported Outcomes of Post-Mastectomy Pencil-Beam } \\
\text { Scanning Proton Therapy in Women with Immediate Tissue Expander Breast } \\
\text { Reconstruction }\end{array}$ & $\begin{array}{l}\text { International } \\
\text { Journal of } \\
\text { Radiation }\end{array}$ & $\begin{array}{l}\text { Single group N enrolled } \\
<500\end{array}$ \\
\hline
\end{tabular}




\begin{tabular}{|c|c|c|c|c|c|}
\hline No. & $\begin{array}{l}\text { PMID or } \\
\text { Other } \\
\text { Identifier }\end{array}$ & $\begin{array}{l}\text { First Author } \\
\text { Last Name }\end{array}$ & Title & Journal & Reason for Exclusion \\
\hline & & & & $\begin{array}{l}\text { Oncology, Biology, } \\
\text { Physics }\end{array}$ & \\
\hline 970 & 25089217 & Smith & Functional morbidity following latissimus dorsi flap breast reconstruction & J Adv Pract Oncol & $\begin{array}{l}\text { Narrative review/ } \\
\text { Commentary }\end{array}$ \\
\hline 971 & 31980737 & Sobti & $\begin{array}{l}\text { Evaluation of capsular contracture following immediate prepectoral versus } \\
\text { subpectoral direct-to-implant breast reconstruction }\end{array}$ & Sci Rep & NRCS $<30$ per arm \\
\hline 972 & 7801137 & Solomon & $\begin{array}{l}\text { A clinical and laboratory profile of symptomatic women with silicone breast } \\
\text { implants }\end{array}$ & $\begin{array}{l}\text { Semin Arthritis } \\
\text { Rheum }\end{array}$ & $\begin{array}{l}\text { Single group } \mathrm{N} \text { enrolled } \\
<500\end{array}$ \\
\hline 973 & 24878776 & Song & $\begin{array}{l}\text { Impact of neoadjuvant chemotherapy on immediate breast reconstruction: a meta- } \\
\text { analysis }\end{array}$ & PLoS One & $\begin{array}{l}\text { Does not address KQ1- } \\
\text { KQ6 }\end{array}$ \\
\hline 974 & 29076316 & Song & Salvage of Infected Breast Implants & Arch Plast Surg & $\begin{array}{l}\text { Does not address KQ1- } \\
\text { KQ6 }\end{array}$ \\
\hline 975 & 32203988 & Song & Current status of and trends in post-mastectomy breast reconstruction in Korea & Arch Plast Surg & $\begin{array}{l}\text { Does not address KQ1- } \\
\text { KQ6 }\end{array}$ \\
\hline 976 & 29781241 & Sosin & $\begin{array}{l}\text { Timing of radiation therapy in nipple-sparing mastectomy influences outcomes and } \\
\text { patient-reported quality of life }\end{array}$ & Breast J & NRCS not adjusted \\
\hline 977 & 133048109 & Sosin & $\begin{array}{l}\text { Timing of radiation therapy in nipple-sparing mastectomy influences outcomes } \\
\text { and patient-reported quality of life }\end{array}$ & Breast Journal & NRCS $<30$ per arm \\
\hline 978 & 12420617 & Soubirac & $\begin{array}{l}\text { [Deflation of breast implants, pre-filled with saline or hydrogel. Results and analysis } \\
\text { of } 650 \text { treated patients] }\end{array}$ & $\begin{array}{l}\text { Ann Chir Plast } \\
\text { Esthet }\end{array}$ & NRCS not adjusted \\
\hline 979 & 127250790 & Soumian & $\begin{array}{l}\text { Early Outcomes Of Immediate Breast Reconstructions Using Acellular Dermal } \\
\text { Matrix After Mastectomy For Breast Cancer }\end{array}$ & $\begin{array}{l}\text { Journal of Cancer } \\
\text { Research \& } \\
\text { Therapeutics }\end{array}$ & NRCS not adjusted \\
\hline 980 & 18626353 & Spear & Options in reconstructing the irradiated breast & $\begin{array}{l}\text { Plast Reconstr } \\
\text { Surg }\end{array}$ & NRCS $<30$ per arm \\
\hline 981 & 19083539 & Spear & Considerations of previous augmentation in subsequent breast reconstruction & Aesthet Surg J & NRCS $<30$ per arm \\
\hline 982 & 15622237 & Spear & $\begin{array}{l}\text { The effect of radiation on pedicled TRAM flap breast reconstruction: outcomes and } \\
\text { implications }\end{array}$ & $\begin{array}{l}\text { Plast Reconstr } \\
\text { Surg }\end{array}$ & NRCS not adjusted \\
\hline 983 & 12832882 & Spear & $\begin{array}{l}\text { Resource cost comparison of implant-based breast reconstruction versus TRAM } \\
\text { flap breast reconstruction }\end{array}$ & $\begin{array}{l}\text { Plast Reconstr } \\
\text { Surg }\end{array}$ & NRCS not adjusted \\
\hline 984 & 23676517 & Spear & Long-term outcomes of failed prosthetic breast reconstruction & Ann Plast Surg & $\begin{array}{l}\text { Does not address KQ1- } \\
\text { KQ6 }\end{array}$ \\
\hline 985 & 24867717 & Spear & Natrelle round silicone breast implants: Core Study results at 10 years & $\begin{array}{l}\text { Plast Reconstr } \\
\text { Surg }\end{array}$ & $\begin{array}{l}\text { Single group N enrolled } \\
<500\end{array}$ \\
\hline 986 & 18626348 & Spear & $\begin{array}{l}\text { A retrospective analysis of outcomes using three common methods for immediate } \\
\text { breast reconstruction }\end{array}$ & $\begin{array}{l}\text { Plast Reconstr } \\
\text { Surg }\end{array}$ & NRCS $<30$ per arm \\
\hline 987 & 22743866 & Spear & $\begin{array}{l}\text { Two-stage prosthetic breast reconstruction using AlloDerm including outcomes of } \\
\text { different timings of radiotherapy }\end{array}$ & $\begin{array}{l}\text { Plast Reconstr } \\
\text { Surg }\end{array}$ & $\begin{array}{l}\text { Does not address KQ1- } \\
\text { KQ6 }\end{array}$ \\
\hline 988 & 12560691 & Spiegel & $\begin{array}{l}\text { Recurrence following treatment of ductal carcinoma in situ with skin-sparing } \\
\text { mastectomy and immediate breast reconstruction }\end{array}$ & $\begin{array}{l}\text { Plast Reconstr } \\
\text { Surg }\end{array}$ & NRCS not adjusted \\
\hline
\end{tabular}




\begin{tabular}{|c|c|c|c|c|c|}
\hline No. & $\begin{array}{l}\text { PMID or } \\
\text { Other } \\
\text { Identifier }\end{array}$ & $\begin{array}{l}\text { First Author } \\
\text { Last Name }\end{array}$ & Title & Journal & Reason for Exclusion \\
\hline 989 & 32097295 & Srinivasa & $\begin{array}{l}\text { Obesity and Breast Reconstruction: Complications and Patient-Reported } \\
\text { Outcomes in a Multicenter, Prospective Study }\end{array}$ & $\begin{array}{l}\text { Plast Reconstr } \\
\text { Surg }\end{array}$ & $\begin{array}{l}\text { Copublication of } \\
\text { included study with no } \\
\text { new data }\end{array}$ \\
\hline 990 & 29068918 & Srinivasa & $\begin{array}{l}\text { Direct-to-Implant versus Two-Stage Tissue Expander/Implant Reconstruction: 2- } \\
\text { Year Risks and Patient-Reported Outcomes from a Prospective, Multicenter Study }\end{array}$ & $\begin{array}{l}\text { Plast Reconstr } \\
\text { Surg }\end{array}$ & $\begin{array}{l}\text { Does not address KQ1- } \\
\text { KQ6 }\end{array}$ \\
\hline 991 & 30516558 & Steffenssen & $\begin{array}{l}\text { A Systematic Review and Meta-analysis of Functional Shoulder Impairment After } \\
\text { Latissimus Dorsi Breast Reconstruction }\end{array}$ & Ann Plast Surg & $\begin{array}{l}\text { Does not address KQ1- } \\
\text { KQ6 }\end{array}$ \\
\hline 992 & 30516558 & Steffenssen & $\begin{array}{l}\text { A Systematic Review and Meta-analysis of Functional Shoulder Impairment After } \\
\text { Latissimus Dorsi Breast Reconstruction }\end{array}$ & $\begin{array}{l}\text { Annals of plastic } \\
\text { surgery }\end{array}$ & $\begin{array}{l}\text { Does not address KQ1- } \\
\text { KQ6 }\end{array}$ \\
\hline 993 & 32195171 & Steiner & $\begin{array}{l}\text { Interdisciplinary Treatment of Breast Cancer After Mastectomy With Autologous } \\
\text { Breast Reconstruction Using Abdominal Free Flaps in a University Teaching } \\
\text { Hospital-A Standardized and Safe Procedure }\end{array}$ & Front Oncol & NRCS not adjusted \\
\hline 994 & 32195171 & Steiner & $\begin{array}{l}\text { Interdisciplinary Treatment of Breast Cancer After Mastectomy With Autologous } \\
\text { Breast Reconstruction Using Abdominal Free Flaps in a University Teaching } \\
\text { Hospital - A Standardized and Safe Procedure }\end{array}$ & $\begin{array}{l}\text { Frontiers in } \\
\text { Oncology }\end{array}$ & NRCS not adjusted \\
\hline 995 & 26961987 & Stevens & $\begin{array}{l}\text { Nine-Year Core Study Data for Sientra's FDA-Approved Round and Shaped } \\
\text { Implants with High-Strength Cohesive Silicone Gel }\end{array}$ & Aesthet Surg J & $\begin{array}{l}\text { Single group N enrolled } \\
<500\end{array}$ \\
\hline 996 & 25948657 & Stevens & $\begin{array}{l}\text { Eight-year follow-up data from the U.S. clinical trial for Sientra's FDA-approved } \\
\text { round and shaped implants with high-strength cohesive silicone gel }\end{array}$ & Aesthet Surg J & $\begin{array}{l}\text { Single group } \mathrm{N} \text { enrolled } \\
<500\end{array}$ \\
\hline 997 & 16772913 & Stevens & $\begin{array}{l}\text { A comparison of } 500 \text { prefilled textured saline breast implants versus } 500 \text { standard } \\
\text { textured saline breast implants: is there a difference in deflation rates? }\end{array}$ & $\begin{array}{l}\text { Plast Reconstr } \\
\text { Surg }\end{array}$ & NRCS not adjusted \\
\hline 998 & 8337271 & Stevenson & TRAM flap breast reconstruction and contralateral reduction or mastopexy & $\begin{array}{l}\text { Plast Reconstr } \\
\text { Surg }\end{array}$ & $\begin{array}{l}\text { Single group } \mathrm{N} \text { enrolled } \\
<500\end{array}$ \\
\hline 999 & 29489546 & Sue & $\begin{array}{l}\text { Mastectomy Skin Necrosis After Breast Reconstruction: A Comparative Analysis } \\
\text { Between Autologous Reconstruction and Implant-Based Reconstruction }\end{array}$ & Ann Plast Surg & NRCS not adjusted \\
\hline 1000 & 28301366 & Sue & $\begin{array}{l}\text { Management of Mastectomy Skin Necrosis in Implant Based Breast } \\
\text { Reconstruction }\end{array}$ & Ann Plast Surg & NRCS not adjusted \\
\hline 1001 & 33618944 & Suh & $\begin{array}{l}\text { A comparative study of pre- or subpectoral expander position with the fenestrated } \\
\text { Acellular dermal matrix anterior coverage, on drainage volume and Seroma } \\
\text { Formation after Non-Nipple-Sparing Mastectomy }\end{array}$ & $\begin{array}{l}\text { J Plast Reconstr } \\
\text { Aesthet Surg }\end{array}$ & NRCS $<30$ per arm \\
\hline 1002 & 18594356 & Sullivan & $\begin{array}{l}\text { True incidence of all complications following immediate and delayed breast } \\
\text { reconstruction }\end{array}$ & $\begin{array}{l}\text { Plast Reconstr } \\
\text { Surg }\end{array}$ & NRCS not adjusted \\
\hline 1003 & 18594356 & Sullivan & $\begin{array}{l}\text { True incidence of all complications following immediate and delayed breast } \\
\text { reconstruction }\end{array}$ & $\begin{array}{l}\text { Plastic and } \\
\text { Reconstructive } \\
\text { Surgery }\end{array}$ & NRCS not adjusted \\
\hline 1004 & 33482758 & Sung Mi & $\begin{array}{l}\text { Does chemotherapy or radiotherapy affect the postoperative complication in breast } \\
\text { cancer patients who underwent immediate breast reconstruction with tissue } \\
\text { expander? }\end{array}$ & BMC Cancer & $\begin{array}{l}\text { Duplicate of another } \\
\text { publication }\end{array}$ \\
\hline 1005 & 26098457 & Sutton & $\begin{array}{l}\text { Incidence of Internal Mammary Lymph Nodes with Silicone Breast Implants at MR } \\
\text { Imaging after Oncoplastic Surgery }\end{array}$ & Radiology & No outcome of interest \\
\hline
\end{tabular}




\begin{tabular}{|c|c|c|c|c|c|}
\hline No. & $\begin{array}{l}\text { PMID or } \\
\text { Other } \\
\text { Identifier }\end{array}$ & $\begin{array}{l}\text { First Author } \\
\text { Last Name }\end{array}$ & Title & Journal & Reason for Exclusion \\
\hline 1006 & NR & Syed & $\begin{array}{l}\text { Do modern methods of post-mastectomy immediate breast reconstruction for } \\
\text { breast cancer delay adjuvant therapy? }\end{array}$ & $\begin{array}{l}\text { European Journal } \\
\text { of Oncology }\end{array}$ & NRCS not adjusted \\
\hline 1007 & 21840780 & Tadiparthi & $\begin{array}{l}\text { Two-stage delayed breast reconstruction with an expander and free abdominal } \\
\text { tissue transfer: outcomes of } 65 \text { consecutive cases by a single surgeon }\end{array}$ & $\begin{array}{l}\text { J Plast Reconstr } \\
\text { Aesthet Surg }\end{array}$ & NRCS $<30$ per arm \\
\hline 1008 & 23806906 & Tadiparthi & $\begin{array}{l}\text { An analysis of the motivating and risk factors for conversion from implant-based to } \\
\text { total autologous breast reconstruction }\end{array}$ & $\begin{array}{l}\text { Plast Reconstr } \\
\text { Surg }\end{array}$ & $\begin{array}{l}\text { Single group N enrolled } \\
<500\end{array}$ \\
\hline 1009 & 26210234 & Taghizadeh & $\begin{array}{l}\text { Does post-mastectomy radiotherapy affect the outcome and prevalence of } \\
\text { complications in immediate DIEP breast reconstruction? A prospective cohort } \\
\text { study }\end{array}$ & $\begin{array}{l}\text { J Plast Reconstr } \\
\text { Aesthet Surg }\end{array}$ & $\begin{array}{l}\text { Single group } \mathrm{N} \text { enrolled } \\
<500\end{array}$ \\
\hline 1010 & 16529044 & Tamaki & [Immediate breast reconstruction following to skin-sparing mastectomy] & Nihon Rinsho & $\begin{array}{l}\text { Does not address KQ1- } \\
\text { KQ6 }\end{array}$ \\
\hline 1011 & 30562406 & Tan & $\begin{array}{l}\text { A cost-effectiveness analysis of DIEP vs free MS-TRAM flap for microsurgical } \\
\text { breast reconstruction }\end{array}$ & J Surg Oncol & NRCS not adjusted \\
\hline 1012 & 23730591 & Tan & $\begin{array}{l}\text { The deep inferior epigastric perforator and pedicled transverse rectus abdominis } \\
\text { myocutaneous flap in breast reconstruction: a comparative study }\end{array}$ & Arch Plast Surg & NRCS $<30$ per arm \\
\hline 1013 & 26202557 & Tang & $\begin{array}{l}\text { Nipple-Sparing Mastectomy in Irradiated Breasts: Selecting Patients to Minimize } \\
\text { Complications }\end{array}$ & Ann Surg Oncol & $\begin{array}{l}\text { Does not address KQ1- } \\
\text { KQ6 }\end{array}$ \\
\hline 1014 & 29697604 & Tang & $\begin{array}{l}\text { Facebook Facts: Breast Reconstruction Patient-Reported Outcomes Using Social } \\
\text { Media }\end{array}$ & $\begin{array}{l}\text { Plast Reconstr } \\
\text { Surg }\end{array}$ & NRCS not adjusted \\
\hline 1015 & 27014551 & Tanos & $\begin{array}{l}\text { Locally Advanced Breast Cancer: Autologous Versus Implant-based } \\
\text { Reconstruction }\end{array}$ & $\begin{array}{l}\text { Plast Reconstr } \\
\text { Surg Glob Open }\end{array}$ & NRCS not adjusted \\
\hline 1016 & 25940160 & Teisch & $\begin{array}{l}\text { Latissimus dorsi flap versus pedicled transverse rectus abdominis myocutaneous } \\
\text { breast reconstruction: outcomes }\end{array}$ & J Surg Res & No outcome of interest \\
\hline 1017 & 30708063 & $\begin{array}{l}\text { Tejera } \\
\text { Hernandez }\end{array}$ & Inverse radiotherapy planning in reconstructive surgery for breast cancer & Int J Surg & $\begin{array}{l}\text { Single group N enrolled } \\
<500\end{array}$ \\
\hline 1018 & 31140187 & Teoh & $\begin{array}{l}\text { Evaluation of the Role of Neoadjuvant Radiotherapy in the Management of } \\
\text { Patients Treated with Mastectomy and Immediate Autologous Breast } \\
\text { Reconstruction }\end{array}$ & $\begin{array}{l}J \text { Reconstr } \\
\text { Microsurg }\end{array}$ & $\begin{array}{l}\text { Narrative review/ } \\
\text { Commentary }\end{array}$ \\
\hline 1019 & 32892333 & Teotia & $\begin{array}{l}\text { Intraoperative Microvascular Complications in Autologous Breast Reconstruction: } \\
\text { The Effects of Resident Training on Microsurgical Outcomes }\end{array}$ & $\begin{array}{l}\text { J Reconstr } \\
\text { Microsurg }\end{array}$ & $\begin{array}{l}\text { Single group N enrolled } \\
<500\end{array}$ \\
\hline 1020 & 9739832 & Tepavicharova & $\begin{array}{l}\text { [The comparative characteristics of methods for breast reconstruction after a } \\
\text { mastectomy] }\end{array}$ & Khirurgiia (Sofiia) & Unable to retrieve article \\
\hline 1021 & 31280491 & Tevis & $\begin{array}{l}\text { Postoperative complications in combined gynecologic, plastic, and breast surgery: } \\
\text { An analysis from National Surgical Quality Improvement Program }\end{array}$ & Breast J & $\begin{array}{l}\text { Not breast } \\
\text { reconstruction }\end{array}$ \\
\hline 1022 & 31933584 & Thangarajah & $\begin{array}{l}\text { Comparison of Subpectoral versus Prepectoral Immediate Implant Reconstruction } \\
\text { after Skin- and Nipple-Sparing Mastectomy in Breast Cancer Patients: A } \\
\text { Retrospective Hospital-Based Cohort Study }\end{array}$ & Breast Care (Basel) & NRCS $<30$ per arm \\
\hline 1023 & 15114125 & Thoma & $\begin{array}{l}\text { Comparison of the deep inferior epigastric perforator flap and free transverse } \\
\text { rectus abdominis myocutaneous flap in postmastectomy reconstruction: a cost- } \\
\text { effectiveness analysis }\end{array}$ & $\begin{array}{l}\text { Plast Reconstr } \\
\text { Surg }\end{array}$ & NRCS not adjusted \\
\hline
\end{tabular}




\begin{tabular}{|c|c|c|c|c|c|}
\hline No. & $\begin{array}{l}\text { PMID or } \\
\text { Other } \\
\text { Identifier }\end{array}$ & $\begin{array}{l}\text { First Author } \\
\text { Last Name }\end{array}$ & Title & Journal & Reason for Exclusion \\
\hline 1024 & 31033841 & Thomas & $\begin{array}{l}\text { An Assessment of Bleeding Complications Necessitating Blood Transfusion across } \\
\text { Inpatient Plastic Surgery Procedures: A Nationwide Analysis Using the National } \\
\text { Surgical Quality Improvement Program Database }\end{array}$ & $\begin{array}{l}\text { Plast Reconstr } \\
\text { Surg }\end{array}$ & $\begin{array}{l}\text { Does not address KQ1- } \\
\text { KQ6 }\end{array}$ \\
\hline 1025 & 18224376 & Thomson & $\begin{array}{l}\text { A prospective longitudinal study of cosmetic outcome in immediate latissimus dorsi } \\
\text { breast reconstruction and the influence of radiotherapy }\end{array}$ & Ann Surg Oncol & NRCS $<30$ per arm \\
\hline 1026 & 26360138 & Thorarinsson & $\begin{array}{l}\text { A retrospective review of the incidence of various complications in different delayed } \\
\text { breast reconstruction methods }\end{array}$ & $\begin{array}{l}J \text { Plast Surg Hand } \\
\text { Surg }\end{array}$ & NRCS not adjusted \\
\hline 1027 & 28861376 & Thorarinsson & $\begin{array}{l}\text { Patient determinants as independent risk factors for postoperative complications of } \\
\text { breast reconstruction }\end{array}$ & Gland Surg & $\begin{array}{l}\text { Does not address KQ1- } \\
\text { KQ6 }\end{array}$ \\
\hline 1028 & 28740762 & Thorarinsson & $\begin{array}{l}\text { Long-Term Health-Related Quality of Life after Breast Reconstruction: Comparing } \\
4 \text { Different Methods of Reconstruction }\end{array}$ & $\begin{array}{l}\text { Plast Reconstr } \\
\text { Surg Glob Open }\end{array}$ & NRCS not adjusted \\
\hline 1029 & 28122466 & Thorarinsson & $\begin{array}{l}\text { Blood loss and duration of surgery are independent risk factors for complications } \\
\text { after breast reconstruction }\end{array}$ & $\begin{array}{l}\text { J Plast Surg Hand } \\
\text { Surg }\end{array}$ & $\begin{array}{l}\text { Does not address KQ1- } \\
\text { KQ6 }\end{array}$ \\
\hline 1030 & 32945960 & Ticha & $\begin{array}{l}\text { Patient-Reported Outcomes of Three Different Types of Breast Reconstruction with } \\
\text { Correlation to the Clinical Data } 5 \text { Years Postoperatively }\end{array}$ & $\begin{array}{l}\text { Aesthetic Plast } \\
\text { Surg }\end{array}$ & NRCS not adjusted \\
\hline 1031 & 28684286 & Tomouk & $\begin{array}{l}\text { Donor site morbidity in DIEP free flap breast reconstructions: A comparison of } \\
\text { unilateral, bilateral, and bipedicled surgical procedure types }\end{array}$ & $\begin{array}{l}J \text { Plast Reconstr } \\
\text { Aesthet Surg }\end{array}$ & $\begin{array}{l}\text { Single group } \mathrm{N} \text { enrolled } \\
<500\end{array}$ \\
\hline 1032 & 31987776 & Tondu & $\begin{array}{l}\text { Breast reconstruction after nipple-sparing mastectomy in the large and/or ptotic } \\
\text { breast: A systematic review of indications, techniques, and outcomes }\end{array}$ & $\begin{array}{l}\text { J Plast Reconstr } \\
\text { Aesthet Surg }\end{array}$ & $\begin{array}{l}\text { Does not address KQ1- } \\
\text { KQ6 }\end{array}$ \\
\hline 1033 & 23810309 & Tong & $\begin{array}{l}\text { Clinical outcomes of percutaneous drainage of breast fluid collections after } \\
\text { mastectomy with expander-based breast reconstruction }\end{array}$ & $\begin{array}{l}\text { Journal of Vascular } \\
\text { and Interventional } \\
\text { Radiology }\end{array}$ & $\begin{array}{l}\text { Does not address KQ1- } \\
\text { KQ6 }\end{array}$ \\
\hline 1034 & 104090246 & Tong & $\begin{array}{l}\text { Clinical outcomes of percutaneous drainage of breast fluid collections after } \\
\text { mastectomy with expander-based breast reconstruction }\end{array}$ & $\begin{array}{l}\text { Journal of Vascular } \\
\text { \& Interventional } \\
\text { Radiology }\end{array}$ & $\begin{array}{l}\text { Single group } \mathrm{N} \text { enrolled } \\
<500\end{array}$ \\
\hline 1035 & 22531404 & Tong & $\begin{array}{l}\text { The transition from pedicle transverse rectus abdominis myocutaneous to } \\
\text { perforator flap: what is the cost of opportunity? }\end{array}$ & Ann Plast Surg & NRCS not adjusted \\
\hline 1036 & 17701730 & Tonseth & $\begin{array}{l}\text { Patient-reported outcomes after breast reconstruction with deep inferior epigastric } \\
\text { perforator flaps }\end{array}$ & $\begin{array}{l}\text { Scand J Plast } \\
\text { Reconstr Surg } \\
\text { Hand Surg }\end{array}$ & NRCS not adjusted \\
\hline 1037 & 31711862 & Toyserkani & $\begin{array}{l}\text { Autologous versus implant-based breast reconstruction: A systematic review and } \\
\text { meta-analysis of Breast- } Q \text { patient-reported outcomes }\end{array}$ & $\begin{array}{l}\text { J Plast Reconstr } \\
\text { Aesthet Surg }\end{array}$ & Systematic review \\
\hline 1038 & 23692931 & Tran & $\begin{array}{l}\text { Risk factors associated with venous thromboembolism in } 49,028 \text { mastectomy } \\
\text { patients }\end{array}$ & Breast & $\begin{array}{l}\text { Does not address KQ1- } \\
\text { KQ6 }\end{array}$ \\
\hline 1039 & 23692931 & Tran & $\begin{array}{l}\text { Risk factors associated with venous thromboembolism in } 49,028 \text { mastectomy } \\
\text { patients }\end{array}$ & Breast & $\begin{array}{l}\text { Does not address KQ1- } \\
\text { KQ6 }\end{array}$ \\
\hline 1040 & 28591940 & Tran & $\begin{array}{l}\text { Cost analysis of postmastectomy reconstruction: A comparison of two staged } \\
\text { implant reconstruction using tissue expander and acellular dermal matrix with } \\
\text { abdominal-based perforator free flaps }\end{array}$ & J Surg Oncol & $\begin{array}{l}\text { Does not address KQ1- } \\
\text { KQ6 }\end{array}$ \\
\hline
\end{tabular}




\begin{tabular}{|c|c|c|c|c|c|}
\hline No. & $\begin{array}{l}\text { PMID or } \\
\text { Other } \\
\text { Identifier }\end{array}$ & $\begin{array}{l}\text { First Author } \\
\text { Last Name }\end{array}$ & Title & Journal & Reason for Exclusion \\
\hline 1041 & 28591935 & Tran & $\begin{array}{l}\text { Cost analysis of postmastectomy reconstruction: A comparison of two staged } \\
\text { implant reconstruction using tissue expander and acellular dermal matrix with } \\
\text { abdominal based perforator free flaps }\end{array}$ & J Surg Oncol & $\begin{array}{l}\text { Narrative review/ } \\
\text { Commentary }\end{array}$ \\
\hline 1042 & 10946929 & Tran & $\begin{array}{l}\text { Postoperative adjuvant irradiation: effects on tranverse rectus abdominis muscle } \\
\text { flap breast reconstruction }\end{array}$ & $\begin{array}{l}\text { Plast Reconstr } \\
\text { Surg }\end{array}$ & $\begin{array}{l}\text { Single group } \mathrm{N} \text { enrolled } \\
<500\end{array}$ \\
\hline 1043 & None & Tsai & Breast reconstruction modality and outcomes after mastectomy & $\begin{array}{l}\text { Formosan Journal } \\
\text { of Surgery }\end{array}$ & NRCS not adjusted \\
\hline 1044 & 28916881 & Tsay & $\begin{array}{l}\text { A 3D Mammometric Comparison of Implant-Based Breast Reconstruction With and } \\
\text { Without Acellular Dermal Matrix (ADM) }\end{array}$ & $\begin{array}{l}\text { Aesthetic Plast } \\
\text { Surg }\end{array}$ & NRCS $<30$ per arm \\
\hline 1045 & 24469159 & Tsoi & $\begin{array}{l}\text { Safety of tissue expander/implant versus autologous abdominal tissue breast } \\
\text { reconstruction in postmastectomy breast cancer patients: a systematic review and } \\
\text { meta-analysis }\end{array}$ & $\begin{array}{l}\text { Plast Reconstr } \\
\text { Surg }\end{array}$ & Systematic review \\
\hline 1046 & 24745568 & Tsoi & $\begin{array}{l}\text { Systematic review on the patient-reported outcomes of tissue-expander/implant vs } \\
\text { autologous abdominal tissue breast reconstruction in postmastectomy breast } \\
\text { cancer patients }\end{array}$ & J Am Coll Surg & $\begin{array}{l}\text { Narrative review/ } \\
\text { Commentary }\end{array}$ \\
\hline 1047 & 24679114 & Tuggle & $\begin{array}{l}\text { Increased hospital volume is associated with improved outcomes following } \\
\text { abdominal-based breast reconstruction }\end{array}$ & $\begin{array}{l}\text { J Plast Surg Hand } \\
\text { Surg }\end{array}$ & $\begin{array}{l}\text { Does not address KQ1- } \\
\text { KQ6 }\end{array}$ \\
\hline 1048 & 11391187 & Tzafetta & Evaluation of the factors related to postmastectomy breast reconstruction & $\begin{array}{l}\text { Plast Reconstr } \\
\text { Surg }\end{array}$ & NRCS $<30$ per arm \\
\hline 1049 & 28806290 & Uda & $\begin{array}{l}\text { Clinical and Quantitative Isokinetic Comparison of Abdominal Morbidity and } \\
\text { Dynamics following DIEP versus Muscle-Sparing Free TRAM Flap Breast } \\
\text { Reconstruction }\end{array}$ & $\begin{array}{l}\text { Plast Reconstr } \\
\text { Surg }\end{array}$ & NRCS not adjusted \\
\hline 1050 & 17051098 & Ulusal & $\begin{array}{l}\text { Simultaneous endoscope-assisted contralateral breast augmentation with implants } \\
\text { in patients undergoing postmastectomy breast reconstruction with abdominal flaps }\end{array}$ & $\begin{array}{l}\text { Plast Reconstr } \\
\text { Surg }\end{array}$ & $\begin{array}{l}\text { Single group } \mathrm{N} \text { enrolled } \\
<500\end{array}$ \\
\hline 1051 & 32381984 & Umezaki & [The Approach of Breast Reconstruction for Breast Cancer in Our Hospital] & $\begin{array}{l}\text { Gan To Kagaku } \\
\text { Ryoho }\end{array}$ & Unable to retrieve article \\
\hline 1052 & 29968032 & Upadhyaya & $\begin{array}{l}\text { Outcomes of Autologous Fat Grafting in Mastectomy Patients Following Breast } \\
\text { Reconstruction }\end{array}$ & Ann Surg Oncol & $\begin{array}{l}\text { Single group } \mathrm{N} \text { enrolled } \\
<500\end{array}$ \\
\hline 1053 & 24987526 & Valdatta & $\begin{array}{l}\text { Acellular dermal matrices and radiotherapy in breast reconstruction: a systematic } \\
\text { review and meta-analysis of the literature }\end{array}$ & Plast Surg Int & $\begin{array}{l}\text { Narrative review/ } \\
\text { Commentary }\end{array}$ \\
\hline 1054 & 26975786 & van Huizum & $\begin{array}{l}\text { Immediate breast reconstruction with a myocutaneous latissimus dorsi flap and } \\
\text { implant following skin-sparing salvage mastectomy after irradiation as part of } \\
\text { breast-conserving therapy }\end{array}$ & $\begin{array}{l}J \text { Plast Reconstr } \\
\text { Aesthet Surg }\end{array}$ & NRCS not adjusted \\
\hline 1055 & None & van Vuuren & $\begin{array}{l}\text { Patient satisfaction and complication rate after mastectomy with immediate two- } \\
\text { stage breast reconstruction as compared to mastectomy without immediate breast } \\
\text { reconstruction }\end{array}$ & Surgical Practice & $\begin{array}{l}\text { Single group N enrolled } \\
<500\end{array}$ \\
\hline 1056 & 31280700 & Vania & $\begin{array}{l}\text { Can pedicled TRAM flap be a satisfying alternative to free TRAM in developing } \\
\text { countries? - a systematic review and meta-analysis }\end{array}$ & Acta Chir Belg & $\begin{array}{l}\text { Does not address KQ1- } \\
\text { KQ6 }\end{array}$ \\
\hline 1057 & 27771262 & $\begin{array}{l}\text { Vanschoonbee } \\
\mathrm{k}\end{array}$ & $\begin{array}{l}\text { Outcome after urgent microvascular revision of free DIEP, SIEA and SGAP flaps } \\
\text { for autologous breast reconstruction }\end{array}$ & $\begin{array}{l}\text { J Plast Reconstr } \\
\text { Aesthet Surg }\end{array}$ & NRCS not adjusted \\
\hline
\end{tabular}




\begin{tabular}{|c|c|c|c|c|c|}
\hline No. & $\begin{array}{l}\text { PMID or } \\
\text { Other } \\
\text { Identifier }\end{array}$ & $\begin{array}{l}\text { First Author } \\
\text { Last Name }\end{array}$ & Title & Journal & Reason for Exclusion \\
\hline 1058 & 25913487 & Vargas & Mastectomy skin necrosis after microsurgical breast reconstruction & J Surg Res & $\begin{array}{l}\text { Single group N enrolled } \\
<500\end{array}$ \\
\hline 1059 & 25891675 & Vargas & Tumescent mastectomy technique in autologous breast reconstruction & J Surg Res & $\begin{array}{l}\text { Single group } \mathrm{N} \text { enrolled } \\
<500\end{array}$ \\
\hline 1060 & 25891675 & Vargas & Tumescent mastectomy technique in autologous breast reconstruction & $\begin{array}{l}\text { Journal of Surgical } \\
\text { Research }\end{array}$ & $\begin{array}{l}\text { Duplicate of another } \\
\text { publication }\end{array}$ \\
\hline 1061 & 31513715 & Vasconcelos & $\begin{array}{l}\text { Acellular dermal matrices safety in breast reconstruction-Is it truly associated with } \\
\text { higher rates of complications? A large single-surgeon cohort analysis }\end{array}$ & Breast J & NRCS not adjusted \\
\hline 1062 & 18626347 & Vega & $\begin{array}{l}500 \text { Consecutive patients with free TRAM flap breast reconstruction: A single } \\
\text { surgeon's experience }\end{array}$ & $\begin{array}{l}\text { Plastic and } \\
\text { Reconstructive } \\
\text { Surgery }\end{array}$ & $\begin{array}{l}\text { Duplicate of another } \\
\text { publication }\end{array}$ \\
\hline 1063 & 18626347 & Vega & $\begin{array}{l}500 \text { Consecutive patients with free TRAM flap breast reconstruction: a single } \\
\text { surgeon's experience }\end{array}$ & $\begin{array}{l}\text { Plast Reconstr } \\
\text { Surg }\end{array}$ & $\begin{array}{l}\text { Single group }>500 \text {, but } \\
\text { no complications data }\end{array}$ \\
\hline 1064 & 32705515 & Venkatesh & $\begin{array}{l}\text { Direct-to-Implant Breast Reconstruction in Patients Undergoing Post-Mastectomy } \\
\text { Radiotherapy }\end{array}$ & Ann Surg Oncol & $\begin{array}{l}\text { Narrative } \\
\text { review/Commentary }\end{array}$ \\
\hline 1065 & 25506538 & Vieira & $\begin{array}{l}\text { A Multi-institutional Analysis of Insurance Status as a Predictor of Morbidity } \\
\text { Following Breast Reconstruction }\end{array}$ & $\begin{array}{l}\text { Plast Reconstr } \\
\text { Surg Glob Open }\end{array}$ & $\begin{array}{l}\text { Does not address KQ1- } \\
\text { KQ6 }\end{array}$ \\
\hline 1066 & 31335468 & Viezel-Mathieu & $\begin{array}{l}\text { Acellular Dermal Matrix-sparing Direct-to-implant Prepectoral Breast } \\
\text { Reconstruction: A Comparative Study Including Cost Analysis }\end{array}$ & Ann Plast Surg & NRCS not adjusted \\
\hline 1067 & 21451370 & Vogel & $\begin{array}{l}\text { Breast cancer in women under age } 40 \text { years: treatment by total mastectomy and } \\
\text { reconstruction }\end{array}$ & Ann Plast Surg & $\begin{array}{l}\text { Does not address KQ1- } \\
\text { KQ6 }\end{array}$ \\
\hline 1068 & 31577663 & Voineskos & $\begin{array}{l}\text { Giving Meaning to Differences in BREAST-Q Scores: Minimal Important Difference } \\
\text { for Breast Reconstruction Patients }\end{array}$ & $\begin{array}{l}\text { Plast Reconstr } \\
\text { Surg }\end{array}$ & NRCS not adjusted \\
\hline 1069 & 28293504 & Vollbach & $\begin{array}{l}\text { An Appraisal of Internal Mammary Artery Perforators as Recipient Vessels in } \\
\text { Microvascular Breast Reconstruction-An Analysis of } 515 \text { Consecutive Cases }\end{array}$ & $\begin{array}{l}\text { Plast Reconstr } \\
\text { Surg Glob Open }\end{array}$ & $\begin{array}{l}\text { Single group N enrolled } \\
<500\end{array}$ \\
\hline 1070 & 27353390 & Wade & $\begin{array}{l}\text { The importance of the unit of analysis: Commentary on Beugels et al. (2016). } \\
\text { Complications in unilateral versus bilateral deep inferior epigastric artery perforator } \\
\text { flap breast reconstructions: A multicentre study }\end{array}$ & $\begin{array}{l}\text { J Plast Reconstr } \\
\text { Aesthet Surg }\end{array}$ & $\begin{array}{l}\text { Narrative review/ } \\
\text { Commentary }\end{array}$ \\
\hline 1071 & 23542852 & Wagner & A classification system for fat necrosis in autologous breast reconstruction & Ann Plast Surg & NRCS not adjusted \\
\hline 1072 & 31076195 & Wagner & A systematic review of complications in prepectoral breast reconstruction & $\begin{array}{l}\text { J Plast Reconstr } \\
\text { Aesthet Surg }\end{array}$ & $\begin{array}{l}\text { Does not address KQ1- } \\
\text { KQ6 }\end{array}$ \\
\hline 1073 & 29876176 & Walia & $\begin{array}{l}\text { Prepectoral Versus Subpectoral Tissue Expander Placement: A Clinical and } \\
\text { Quality of Life Outcomes Study }\end{array}$ & $\begin{array}{l}\text { Plast Reconstr } \\
\text { Surg Glob Open }\end{array}$ & NRCS $<30$ per arm \\
\hline 1074 & 20679822 & Wan & $\begin{array}{l}\text { Inclusion of mesh in donor-site repair of free TRAM and muscle-sparing free TRAM } \\
\text { flaps yields rates of abdominal complications comparable to those of DIEP flap } \\
\text { reconstruction }\end{array}$ & $\begin{array}{l}\text { Plast Reconstr } \\
\text { Surg }\end{array}$ & NRCS not adjusted \\
\hline 1075 & 25054245 & Wang & $\begin{array}{l}\text { Lessons learned from the American College of Surgeons National Surgical Quality } \\
\text { Improvement Program Database: has centralized data collection improved } \\
\text { immediate breast reconstruction outcomes and safety? }\end{array}$ & $\begin{array}{l}\text { Plast Reconstr } \\
\text { Surg }\end{array}$ & NRCS not adjusted \\
\hline 1076 & 25942235 & Wang & $\begin{array}{l}\text { Abstract 124: outcomes of total skin-sparing mastectomy and reconstruction in } 924 \\
\text { breasts over } 11 \text { years }\end{array}$ & $\begin{array}{l}\text { Plast Reconstr } \\
\text { Surg }\end{array}$ & $\begin{array}{l}\text { Does not address KQ1- } \\
\text { KQ6 }\end{array}$ \\
\hline
\end{tabular}




\begin{tabular}{|c|c|c|c|c|c|}
\hline No. & $\begin{array}{l}\text { PMID or } \\
\text { Other } \\
\text { Identifier }\end{array}$ & $\begin{array}{l}\text { First Author } \\
\text { Last Name }\end{array}$ & Title & Journal & Reason for Exclusion \\
\hline 1077 & 25052246 & Wang & $\begin{array}{l}\text { Total skin-sparing mastectomy and immediate breast reconstruction: an evolution } \\
\text { of technique and assessment of outcomes }\end{array}$ & Ann Surg Oncol & $\begin{array}{l}\text { Does not address KQ1- } \\
\text { KQ6 }\end{array}$ \\
\hline 1078 & 31020469 & Wang & $\begin{array}{l}\text { Post-mastectomy immediate breast reconstruction is oncologically safe in well- } \\
\text { selected T4 locally advanced breast cancer: a large population-based study and } \\
\text { matched case-control analysis }\end{array}$ & $\begin{array}{l}\text { Breast Cancer } \\
\text { Research and } \\
\text { Treatment }\end{array}$ & $\begin{array}{l}\text { Does not address KQ1- } \\
\text { KQ6 }\end{array}$ \\
\hline 1079 & 31020469 & Wang & $\begin{array}{l}\text { Post-mastectomy immediate breast reconstruction is oncologically safe in well- } \\
\text { selected T4 locally advanced breast cancer: a large population-based study and } \\
\text { matched case-control analysis }\end{array}$ & $\begin{array}{l}\text { Breast Cancer Res } \\
\text { Treat }\end{array}$ & $\begin{array}{l}\text { Does not address KQ1- } \\
\text { KQ6 }\end{array}$ \\
\hline 1080 & 24902911 & Wang & $\begin{array}{l}\text { Meta-analysis of the safety and factors contributing to complications of MS-TRAM, } \\
\text { DIEP, and SIEA flaps for breast reconstruction }\end{array}$ & $\begin{array}{l}\text { Aesthetic Plast } \\
\text { Surg }\end{array}$ & Systematic review \\
\hline 1081 & 32807619 & Wang & $\begin{array}{l}\text { Autologous tissue reconstruction after mastectomy - A cross-sectional survey of } \\
110 \text { hospitals in China }\end{array}$ & $\begin{array}{l}\text { European Journal } \\
\text { of Surgical } \\
\text { Oncology }\end{array}$ & $\begin{array}{l}\text { Does not address KQ1- } \\
\text { KQ6 }\end{array}$ \\
\hline 1082 & 20855759 & Warren Peled & $\begin{array}{l}\text { Impact of chemotherapy on postoperative complications after mastectomy and } \\
\text { immediate breast reconstruction }\end{array}$ & Arch Surg & NRCS not adjusted \\
\hline 1083 & 27187684 & Warschkow & $\begin{array}{l}\text { A population-based analysis of secondary malignancies in breast cancer patients } \\
\text { receiving breast reconstruction }\end{array}$ & Br J Cancer & No outcome of interest \\
\hline 1084 & 30329056 & Watad & $\begin{array}{l}\text { Silicone breast implants and the risk of autoimmune/rheumatic disorders: a real- } \\
\text { world analysis }\end{array}$ & Int J Epidemiol & $\begin{array}{l}\text { Not mastectomy for } \\
\text { breast cancer }\end{array}$ \\
\hline 1085 & 23783060 & Weichman & $\begin{array}{l}\text { Sterile 'ready-to-use' AlloDerm decreases postoperative infectious complications in } \\
\text { patients undergoing immediate implant-based breast reconstruction with acellular } \\
\text { dermal matrix }\end{array}$ & $\begin{array}{l}\text { Plast Reconstr } \\
\text { Surg }\end{array}$ & $\begin{array}{l}\text { Single group N enrolled } \\
<500\end{array}$ \\
\hline 1086 & 29369110 & Weinstein & $\begin{array}{l}\text { Moffitt Cancer Center Experience of Tissue Expander Breast Reconstruction: Does } \\
\text { Acellular Dermal Matrix Increase Return to the Operating Room? }\end{array}$ & Ann Plast Surg & NRCS not adjusted \\
\hline 1087 & 30109538 & Weiss & $\begin{array}{l}\text { Reconstruction in the Metastatic Breast Cancer Patient: Results from the National } \\
\text { Cancer Database }\end{array}$ & Ann Surg Oncol & $\begin{array}{l}\text { Does not address KQ1- } \\
\text { KQ6 }\end{array}$ \\
\hline 1088 & 32605294 & Weitgasser & $\begin{array}{l}\text { Bilateral Simultaneous Breast Reconstruction with DIEP- and TMG Flaps: Head to } \\
\text { Head Comparison, Risk and Complication Analysis }\end{array}$ & $\mathrm{J}$ Clin Med & NRCS not adjusted \\
\hline 1089 & 109550161 & Weller & $\begin{array}{l}\text { Effects of Radiation Therapy on Long-term Toxicity and Reconstruction Failure } \\
\text { Following Mastectomy and Autologous Reconstruction }\end{array}$ & $\begin{array}{l}\text { International } \\
\text { Journal of } \\
\text { Radiation } \\
\text { Oncology, Biology, } \\
\text { Physics }\end{array}$ & $\begin{array}{l}\text { Does not address KQ1- } \\
\text { KQ6 }\end{array}$ \\
\hline 1090 & 24691317 & Wes & $\begin{array}{l}\text { Do Prior Abdominal Surgeries Increase Complications in Abdominally Based } \\
\text { Breast Reconstructions? }\end{array}$ & Ann Plast Surg & $\begin{array}{l}\text { Copublication of } \\
\text { included study with no } \\
\text { new data }\end{array}$ \\
\hline 1091 & 11039373 & Wilkins & $\begin{array}{l}\text { Prospective analysis of psychosocial outcomes in breast reconstruction: one-year } \\
\text { postoperative results from the Michigan Breast Reconstruction Outcome Study }\end{array}$ & $\begin{array}{l}\text { Plast Reconstr } \\
\text { Surg }\end{array}$ & $\begin{array}{l}\text { Single group N enrolled } \\
<500\end{array}$ \\
\hline 1092 & 27906762 & Wilkins & $\begin{array}{l}\text { Complications in Postmastectomy Breast Reconstruction: One-year Outcomes of } \\
\text { the Mastectomy Reconstruction Outcomes Consortium (MROC) Study }\end{array}$ & Annals of Surgery & $\begin{array}{l}\text { Duplicate of another } \\
\text { publication }\end{array}$ \\
\hline
\end{tabular}




\begin{tabular}{|c|c|c|c|c|c|}
\hline No. & $\begin{array}{l}\text { PMID or } \\
\text { Other } \\
\text { Identifier }\end{array}$ & $\begin{array}{l}\text { First Author } \\
\text { Last Name }\end{array}$ & Title & Journal & Reason for Exclusion \\
\hline 1093 & 9326776 & Williams & The effects of radiation treatment after TRAM flap breast reconstruction & $\begin{array}{l}\text { Plastic and } \\
\text { Reconstructive } \\
\text { Surgery }\end{array}$ & $\begin{array}{l}\text { Copublication of } \\
\text { included study with no } \\
\text { new data }\end{array}$ \\
\hline 1094 & 9326776 & Williams & The effects of radiation treatment after TRAM flap breast reconstruction & $\begin{array}{l}\text { Plast Reconstr } \\
\text { Surg }\end{array}$ & NRCS not adjusted \\
\hline 1095 & 32113147 & Wilting & $\begin{array}{l}\text { Three-dimensional evaluation of breast volume changes following autologous free } \\
\text { flap breast reconstruction over six months' }\end{array}$ & Breast & $\begin{array}{l}\text { Single group } \mathrm{N} \text { enrolled } \\
<500\end{array}$ \\
\hline 1096 & 24673121 & Wink & $\begin{array}{l}\text { Direct-to-implant breast reconstruction: an analysis of } 1612 \text { cases from the ACS- } \\
\text { NSQIP surgical outcomes database }\end{array}$ & $\begin{array}{l}\text { J Plast Surg Hand } \\
\text { Surg }\end{array}$ & $\begin{array}{l}\text { Does not address KQ1- } \\
\text { KQ6 }\end{array}$ \\
\hline 1097 & 25798391 & Winocour & $\begin{array}{l}\text { Early Surgical Site Infection Following Tissue Expander Breast Reconstruction with } \\
\text { or without Acellular Dermal Matrix: National Benchmarking Using National Surgical } \\
\text { Quality Improvement Program }\end{array}$ & Arch Plast Surg & $\begin{array}{l}\text { Copublication of } \\
\text { included study with no } \\
\text { new data }\end{array}$ \\
\hline 1098 & 30507480 & Winters & Quality of life after breast reconstruction-the BRIOS study & Lancet Oncology & $\begin{array}{l}\text { Narrative review/ } \\
\text { Commentary }\end{array}$ \\
\hline 1099 & 31121016 & Wixtrom & $\begin{array}{l}\text { Device-Specific Findings of Imprinted-Texture Breast Implants: Characteristics, } \\
\text { Risks, and Benefits }\end{array}$ & Aesthet Surg J & $\begin{array}{l}\text { Single group N enrolled } \\
<500\end{array}$ \\
\hline 1100 & 31155830 & Wohlgemuth & $\begin{array}{l}\text { Risk of breast implant-associated anaplastic large cell lymphoma in patients } \\
\text { submitted to breast implantation: A systematic review }\end{array}$ & Breast $J$ & Systematic review \\
\hline 1101 & 10493686 & Wolfe & Silicone filled breast implants and the risk of fibromyalgia and rheumatoid arthritis & J Rheumatol & Unable to retrieve article \\
\hline 1102 & NR & Wolfswinkel & $\begin{array}{l}\text { Complications of abdominal-based free flaps for breast reconstruction in obese } \\
\text { patients: A meta-analysis and case series }\end{array}$ & $\begin{array}{l}\text { European Journal } \\
\text { of Plastic Surgery }\end{array}$ & $\begin{array}{l}\text { Does not address KQ1- } \\
\text { KQ6 }\end{array}$ \\
\hline 1103 & 138986907 & Wong & $\begin{array}{l}\text { IMRT is Associated with Lower Reconstruction Failure and Complication Rates } \\
\text { Following Post-Mastectomy Radiation to a Reconstructed Breast }\end{array}$ & $\begin{array}{l}\text { International } \\
\text { Journal of } \\
\text { Radiation } \\
\text { Oncology, Biology, } \\
\text { Physics }\end{array}$ & $\begin{array}{l}\text { Single group } \mathrm{N} \text { enrolled } \\
<500\end{array}$ \\
\hline 1104 & 31342383 & Wong & $\begin{array}{l}\text { National Patterns of Breast Reconstruction and Nipple-Sparing Mastectomy for } \\
\text { Breast Cancer, 2005-2015 }\end{array}$ & Ann Surg Oncol & $\begin{array}{l}\text { Does not address KQ1- } \\
\text { KQ6 }\end{array}$ \\
\hline 1105 & 32537347 & Wood & $\begin{array}{l}\text { Complications after Perforated versus Nonperforated Acellular Dermal Matrix Use } \\
\text { in Direct-to-Implant Breast Reconstruction: A Propensity Score Analysis }\end{array}$ & $\begin{array}{l}\text { Plast Reconstr } \\
\text { Surg Glob Open }\end{array}$ & $\begin{array}{l}\text { Single group N enrolled } \\
<500\end{array}$ \\
\hline 1106 & 33137841 & Woodward & Nipple-sparing mastectomy: A review of outcomes at a single institution & Breast J & $\begin{array}{l}\text { Single group N enrolled } \\
<500\end{array}$ \\
\hline 1107 & 24200701 & Wormald & $\begin{array}{l}\text { The increased risk of adverse outcomes in bilateral deep inferior epigastric artery } \\
\text { perforator flap breast reconstruction compared to unilateral reconstruction: a } \\
\text { systematic review and meta-analysis }\end{array}$ & $\begin{array}{l}\text { J Plast Reconstr } \\
\text { Aesthet Surg }\end{array}$ & $\begin{array}{l}\text { Does not address KQ1- } \\
\text { KQ6 }\end{array}$ \\
\hline 1108 & 31348326 & Wormer & $\begin{array}{l}\text { Reducing Expansion Visits in Immediate Implant-Based Breast Reconstruction: A } \\
\text { Comparative Study of Prepectoral and Subpectoral Expander Placement }\end{array}$ & $\begin{array}{l}\text { Plast Reconstr } \\
\text { Surg }\end{array}$ & NRCS not adjusted \\
\hline 1109 & 24691350 & Wu & $\begin{array}{l}\text { Racial differences in ischemic complications of pedicled versus free abdominal } \\
\text { flaps for breast reconstruction }\end{array}$ & Ann Plast Surg & $\begin{array}{l}\text { Case report or series of } \\
\text { case reports }\end{array}$ \\
\hline 1110 & 18953926 & $\mathrm{Wu}$ & $\begin{array}{l}\text { [A retrospective study of } 129 \text { cases with immediate breast reconstruction after skin- } \\
\text { sparing mastectomy for breast cancer] }\end{array}$ & $\begin{array}{l}\text { Zhonghua Wai Ke } \\
\text { Za Zhi }\end{array}$ & $\begin{array}{l}\text { Single group N enrolled } \\
<500\end{array}$ \\
\hline
\end{tabular}




\begin{tabular}{|c|c|c|c|c|c|}
\hline No. & $\begin{array}{l}\text { PMID or } \\
\text { Other } \\
\text { Identifier }\end{array}$ & $\begin{array}{l}\text { First Author } \\
\text { Last Name }\end{array}$ & Title & Journal & Reason for Exclusion \\
\hline 1111 & 18766032 & $\mathrm{Wu}$ & $\begin{array}{l}\text { Comparison of donor-site morbidity of SIEA, DIEP, and muscle-sparing TRAM } \\
\text { flaps for breast reconstruction }\end{array}$ & $\begin{array}{l}\text { Plast Reconstr } \\
\text { Surg }\end{array}$ & NRCS not adjusted \\
\hline 1112 & 29631098 & $\mathrm{Wu}$ & $\begin{array}{l}\text { Breast reconstruction with Alloderm Ready to Use: A meta-analysis of nine } \\
\text { observational cohorts }\end{array}$ & Breast & $\begin{array}{l}\text { Narrative review/ } \\
\text { Commentary }\end{array}$ \\
\hline 1113 & 30034254 & $\mathrm{Wu}$ & $\begin{array}{l}\text { Comparison of survival outcomes of locally advanced breast cancer patients } \\
\text { receiving postmastectomy radiotherapy with and without immediate breast } \\
\text { reconstruction: A population-based analysis }\end{array}$ & $\begin{array}{l}\text { Cancer } \\
\text { Management and } \\
\text { Research }\end{array}$ & $\begin{array}{l}\text { Does not address KQ1- } \\
\text { KQ6 }\end{array}$ \\
\hline 1114 & 30034254 & $\mathrm{Wu}$ & $\begin{array}{l}\text { Comparison of survival outcomes of locally advanced breast cancer patients } \\
\text { receiving post-mastectomy radiotherapy with and without immediate breast } \\
\text { reconstruction: a population-based analysis }\end{array}$ & Cancer Manag Res & $\begin{array}{l}\text { Does not address KQ1- } \\
\text { KQ6 }\end{array}$ \\
\hline 1115 & 29846217 & $\mathrm{Wu}$ & $\begin{array}{l}\text { Evaluating the Impact of Resident Participation and the July Effect on Outcomes in } \\
\text { Autologous Breast Reconstruction }\end{array}$ & Ann Plast Surg & $\begin{array}{l}\text { Does not address KQ1- } \\
\text { KQ6 }\end{array}$ \\
\hline 1116 & 31461141 & $\mathrm{Wu}$ & $\begin{array}{l}\text { Breast Cancer Recurrence in the Nipple-Areola Complex After Nipple-Sparing } \\
\text { Mastectomy With Immediate Breast Reconstruction for Invasive Breast Cancer }\end{array}$ & JAMA Surg & $\begin{array}{l}\text { Does not address KQ1- } \\
\text { KQ6 }\end{array}$ \\
\hline 1117 & 139809975 & $\mathrm{Wu}$ & $\begin{array}{l}\text { Breast Cancer Recurrence in the Nipple-Areola Complex After Nipple-Sparing } \\
\text { Mastectomy With Immediate Breast Reconstruction for Invasive Breast Cancer }\end{array}$ & JAMA Surgery & $\begin{array}{l}\text { Case report or series of } \\
\text { case reports }\end{array}$ \\
\hline 1118 & 33665246 & $\mathrm{Wu}$ & $\begin{array}{l}\text { Data on distant metastasis and survival after locoregional recurrence following } \\
\text { nipple-sparing mastectomy and immediate breast reconstruction }\end{array}$ & Data Brief & $\begin{array}{l}\text { Single group } \mathrm{N} \text { enrolled } \\
<500\end{array}$ \\
\hline 1119 & 33495030 & $\mathrm{Wu}$ & $\begin{array}{l}\text { Locoregional recurrence following nipple-sparing mastectomy with immediate } \\
\text { breast reconstruction: Patterns and prognostic significance }\end{array}$ & Eur J Surg Oncol & NRCS not adjusted \\
\hline 1120 & 25162244 & Wurzer & $\begin{array}{l}\text { [Is there a psychological and physiological difference between DIEP- and free } \\
\text { TRAM-flap? A retrospective patient survey] }\end{array}$ & $\begin{array}{l}\text { Handchir Mikrochir } \\
\text { Plast Chir }\end{array}$ & NRCS $<30$ per arm \\
\hline 1121 & 26285643 & $\begin{array}{l}\text { Xavier } \\
\text { Harmeling }\end{array}$ & $\begin{array}{l}\text { The effect of immediate breast reconstruction on the timing of adjuvant } \\
\text { chemotherapy: a systematic review }\end{array}$ & $\begin{array}{l}\text { Breast Cancer Res } \\
\text { Treat }\end{array}$ & $\begin{array}{l}\text { Narrative review/ } \\
\text { Commentary }\end{array}$ \\
\hline 1122 & 31985610 & Xue & $\begin{array}{l}\text { Follow-Up Study: One-Step Salvage of Infected Prosthetic Breast Reconstructions } \\
\text { Using Antibiotic-Impregnated Polymethylmethacrylate Plates and Concurrent } \\
\text { Tissue Expander Exchange }\end{array}$ & $\begin{array}{l}\text { Plast Reconstr } \\
\text { Surg }\end{array}$ & $\begin{array}{l}\text { Single group } \mathrm{N} \text { enrolled } \\
<500\end{array}$ \\
\hline 1123 & 33526379 & Yamashita & $\begin{array}{l}\text { Long-Term Oncologic Safety of Nipple-Sparing Mastectomy With Immediate } \\
\text { Reconstruction }\end{array}$ & Clin Breast Cancer & $\begin{array}{l}\text { Does not address KQ1- } \\
\text { KQ6 }\end{array}$ \\
\hline 1124 & 26562294 & Yang & $\begin{array}{l}\text { The Type of Breast Reconstruction May Not Influence Patient Satisfaction in the } \\
\text { Chinese Population: A Single Institutional Experience }\end{array}$ & PLoS One & NRCS not adjusted \\
\hline 1125 & 28833134 & Yang & $\begin{array}{l}\text { Changes in shoulder muscle activity pattern on surface electromyography after } \\
\text { breast cancer surgery }\end{array}$ & J Surg Oncol & NRCS $<30$ per arm \\
\hline 1126 & 22493623 & Yang & $\begin{array}{l}\text { Surgical techniques for personalized oncoplastic surgery in breast cancer patients } \\
\text { with small- to moderate-sized breasts (part 2): volume replacement }\end{array}$ & $J$ Breast Cancer & $\begin{array}{l}\text { Does not address KQ1- } \\
\text { KQ6 }\end{array}$ \\
\hline 1127 & 31775208 & Yang & $\begin{array}{l}\text { Considerations for patient selection: Prepectoral versus subpectoral implant-based } \\
\text { breast reconstruction }\end{array}$ & Arch Plast Surg & NRCS not adjusted \\
\hline 1128 & 33389980 & Yang & $\begin{array}{l}\text { Post-mastectomy radiation therapy in breast reconstruction: a patterns of care } \\
\text { study of the Korean Radiation Oncology Group }\end{array}$ & Radiat Oncol J & NRCS not adjusted \\
\hline
\end{tabular}




\begin{tabular}{|c|c|c|c|c|c|}
\hline No. & $\begin{array}{l}\text { PMID or } \\
\text { Other } \\
\text { Identifier }\end{array}$ & $\begin{array}{l}\text { First Author } \\
\text { Last Name }\end{array}$ & Title & Journal & Reason for Exclusion \\
\hline 1129 & 33483782 & Yazar & $\begin{array}{l}\text { Invited Response on: Nipple-Sparing Mastectomy and Immediate Implant-Based } \\
\text { Reconstruction with or Without Skin Reduction in Patients with Large Ptotic } \\
\text { Breasts: A Case-Matched Analysis }\end{array}$ & $\begin{array}{l}\text { Aesthetic Plast } \\
\text { Surg }\end{array}$ & $\begin{array}{l}\text { Does not address KQ1- } \\
\text { KQ6 }\end{array}$ \\
\hline 1130 & 9843345 & Yeh & Immediate breast reconstruction in breast cancer: morbidity and outcome & Am Surg & NRCS $<30$ per arm \\
\hline 1131 & $\begin{array}{l}\mathrm{CN}- \\
02175345\end{array}$ & Yehia & $\begin{array}{l}\text { Reconstruction Outcomes in a Multi-Institution Prospective Phase II } \\
\text { Hypofractionated Post-Mastectomy Radiation Therapy Trial }\end{array}$ & $\begin{array}{l}\text { International journal } \\
\text { of radiation } \\
\text { oncology biology } \\
\text { physics }\end{array}$ & NRCS not adjusted \\
\hline 1132 & 26618122 & Yim & $\begin{array}{l}\text { Outcomes of Take-Back Operations in Breast Reconstruction with Free Lower } \\
\text { Abdominal Flaps }\end{array}$ & Arch Plast Surg & $\begin{array}{l}\text { Does not address KQ1- } \\
\text { KQ6 }\end{array}$ \\
\hline 1133 & 25620484 & Ying & $\begin{array}{l}\text { Current trends of breast reconstruction after mastectomy for breast cancer patients } \\
\text { in China: A survey report }\end{array}$ & $\begin{array}{l}\text { Chinese Journal of } \\
\text { Oncology }\end{array}$ & $\begin{array}{l}\text { Duplicate of another } \\
\text { publication }\end{array}$ \\
\hline 1134 & 29102781 & Yoon & $\begin{array}{l}\text { Outcomes of immediate versus delayed breast reconstruction: Results of a } \\
\text { multicenter prospective study }\end{array}$ & Breast & $\begin{array}{l}\text { Does not address KQ1- } \\
\text { KQ6 }\end{array}$ \\
\hline 1135 & 31342370 & Young & $\begin{array}{l}\text { Outcomes of > } 1300 \text { Nipple-Sparing Mastectomies with Immediate Reconstruction: } \\
\text { The Impact of Expanding Indications on Complications }\end{array}$ & Ann Surg Oncol & $\begin{array}{l}\text { Does not address KQ1- } \\
\text { KQ6 }\end{array}$ \\
\hline 1136 & 27508508 & Youssef & $\begin{array}{l}\text { Use of Acellular Dermal Matrix versus Latissimus Dorsi Flap for Breast } \\
\text { Reconstruction: Clinical and Patient-Reported Outcomes }\end{array}$ & Breast J & NRCS $<30$ per arm \\
\hline 1137 & 27070347 & $\mathrm{Yu}$ & $\begin{array}{l}\text { Comparison of Histological Characteristics of Acellular Dermal Matrix Capsules to } \\
\text { Surrounding Breast Capsules in Acellular Dermal Matrix-Assisted Breast } \\
\text { Reconstruction }\end{array}$ & Ann Plast Surg & NRCS $<30$ per arm \\
\hline 1138 & 30132338 & Yun & Breast Reconstruction and Radiation Therapy & Cancer Control & $\begin{array}{l}\text { Narrative review/ } \\
\text { Commentary }\end{array}$ \\
\hline 1139 & 128167261 & Yun & $\begin{array}{l}\text { The role of postmastectomy radiation therapy in patients with immediate prosthetic } \\
\text { breast reconstruction: A meta-analysis }\end{array}$ & Medicine & $\begin{array}{l}\text { Does not address KQ1- } \\
\text { KQ6 }\end{array}$ \\
\hline 1140 & 138986910 & Zhang & $\begin{array}{l}\text { The Impact of Radiotherapy on Complications and Reconstruction Failures in } \\
\text { Patients Undergoing Mastectomy and Breast Reconstruction }\end{array}$ & $\begin{array}{l}\text { International } \\
\text { Journal of } \\
\text { Radiation } \\
\text { Oncology, Biology, } \\
\text { Physics }\end{array}$ & $\begin{array}{l}\text { Single group N enrolled } \\
<500\end{array}$ \\
\hline 1141 & 16883889 & Zhao & $\begin{array}{l}\text { [Clinic applications of primary breast reconstruction with a subpectoral silicone } \\
\text { tissue expander] }\end{array}$ & $\begin{array}{l}\text { Zhonghua Zheng } \\
\text { Xing Wai Ke Za Zhi }\end{array}$ & $\begin{array}{l}\text { Single group } \mathrm{N} \text { enrolled } \\
<500\end{array}$ \\
\hline 1142 & 26377821 & Zhao & $\begin{array}{l}\text { A Meta-analysis of Postoperative Complications of Tissue Expander/Implant Breast } \\
\text { Reconstruction Using Acellular Dermal Matrix }\end{array}$ & $\begin{array}{l}\text { Aesthetic Plast } \\
\text { Surg }\end{array}$ & Systematic review \\
\hline 1143 & 28000160 & Zheng & $\begin{array}{l}\text { Radiotherapy and nipple-areolar complex necrosis after nipple-sparing } \\
\text { mastectomy: a systematic review and meta-analysis }\end{array}$ & Radiol Med & $\begin{array}{l}\text { Does not address KQ1- } \\
\text { KQ6 }\end{array}$ \\
\hline 1144 & 24888814 & Zhong & $\begin{array}{l}\text { Barriers to immediate breast reconstruction in the Canadian Universal Health Care } \\
\text { System: Zhong T, Fernandes KA, Saskin R, et al (Univ Health Network, Toronto, } \\
\text { Ontario, Canada; Inst for Clinical Evaluative Sciences, Toronto, Ontario, Canada; } \\
\text { Et al) J Clin Oncol 32:2133-2141, } 2014\end{array}$ & Breast Diseases & $\begin{array}{l}\text { Does not address KQ1- } \\
\text { KQ6 }\end{array}$ \\
\hline
\end{tabular}




\begin{tabular}{|c|c|c|c|c|c|}
\hline No. & $\begin{array}{l}\text { PMID or } \\
\text { Other } \\
\text { Identifier }\end{array}$ & $\begin{array}{l}\text { First Author } \\
\text { Last Name }\end{array}$ & Title & Journal & Reason for Exclusion \\
\hline 1145 & $\begin{array}{l}\mathrm{CN}- \\
00915067\end{array}$ & Zhong & $\begin{array}{l}\text { The Multi Centre Canadian Acellular Dermal Matrix Trial (MCCAT): study protocol } \\
\text { for a randomized controlled trial in implant-based breast reconstruction }\end{array}$ & Trials & $\begin{array}{l}\text { Duplicate of another } \\
\text { publication }\end{array}$ \\
\hline 1146 & 24165392 & Zhong & $\begin{array}{l}\text { The Multi Centre Canadian Acellular Dermal Matrix Trial (MCCAT): study protocol } \\
\text { for a randomized controlled trial in implant-based breast reconstruction }\end{array}$ & Trials & $\begin{array}{l}\text { Protocol/methods with } \\
\text { no results }\end{array}$ \\
\hline 1147 & $\begin{array}{l}\mathrm{CN}- \\
01120691\end{array}$ & Zhong & $\begin{array}{l}\text { The Multi Centre Canadian Acellular Dermal Matrix Trial (MCCAT): study protocol } \\
\text { for a randomized controlled trial in implant-based breast reconstruction }\end{array}$ & Trials & $\begin{array}{l}\text { Duplicate of another } \\
\text { publication }\end{array}$ \\
\hline 1148 & 103996301 & Zhong & $\begin{array}{l}\text { The Multi Centre Canadian Acellular Dermal Matrix Trial (MCCAT): study protocol } \\
\text { for a randomized controlled trial in implant-based breast reconstruction }\end{array}$ & Trials & $\begin{array}{l}\text { Duplicate of another } \\
\text { publication }\end{array}$ \\
\hline 1149 & 26922050 & Zhu & $\begin{array}{l}\text { Comparison of subcutaneous versus submuscular expander placement in the first } \\
\text { stage of immediate breast reconstruction }\end{array}$ & $\begin{array}{l}\text { J Plast Reconstr } \\
\text { Aesthet Surg }\end{array}$ & NRCS $<30$ per arm \\
\hline 1150 & 22017572 & Zucatto & $\begin{array}{l}\text { Immediate breast reconstruction using free transverse rectus abdominis } \\
\text { myocutaneous flap: impact on breast cancer recurrence after mastectomy }\end{array}$ & Breast J & $\begin{array}{l}\text { Single group N enrolled } \\
<500\end{array}$ \\
\hline
\end{tabular}

Abbreviations: PMID = PubMed identifier, KQ = Key Question, $\mathrm{NR}=$ not reported, NRCS = nonrandomized comparative study.

Colors: Header rows are shaded orange. The color does not add unique information. 


\section{Appendix C. Results: Design, Arm, and Sample Details}

\section{Results of Literature Searches}

As illustrated by Figure C-1, our primary electronic search retrieved a combined 15,936 unique citations. Of these, 1,352 were deemed potentially relevant and retrieved in full text. After full-text screening, we identified 160 eligible studies that were reported in 202 articles.

Figure C-1. Flow diagram for studies

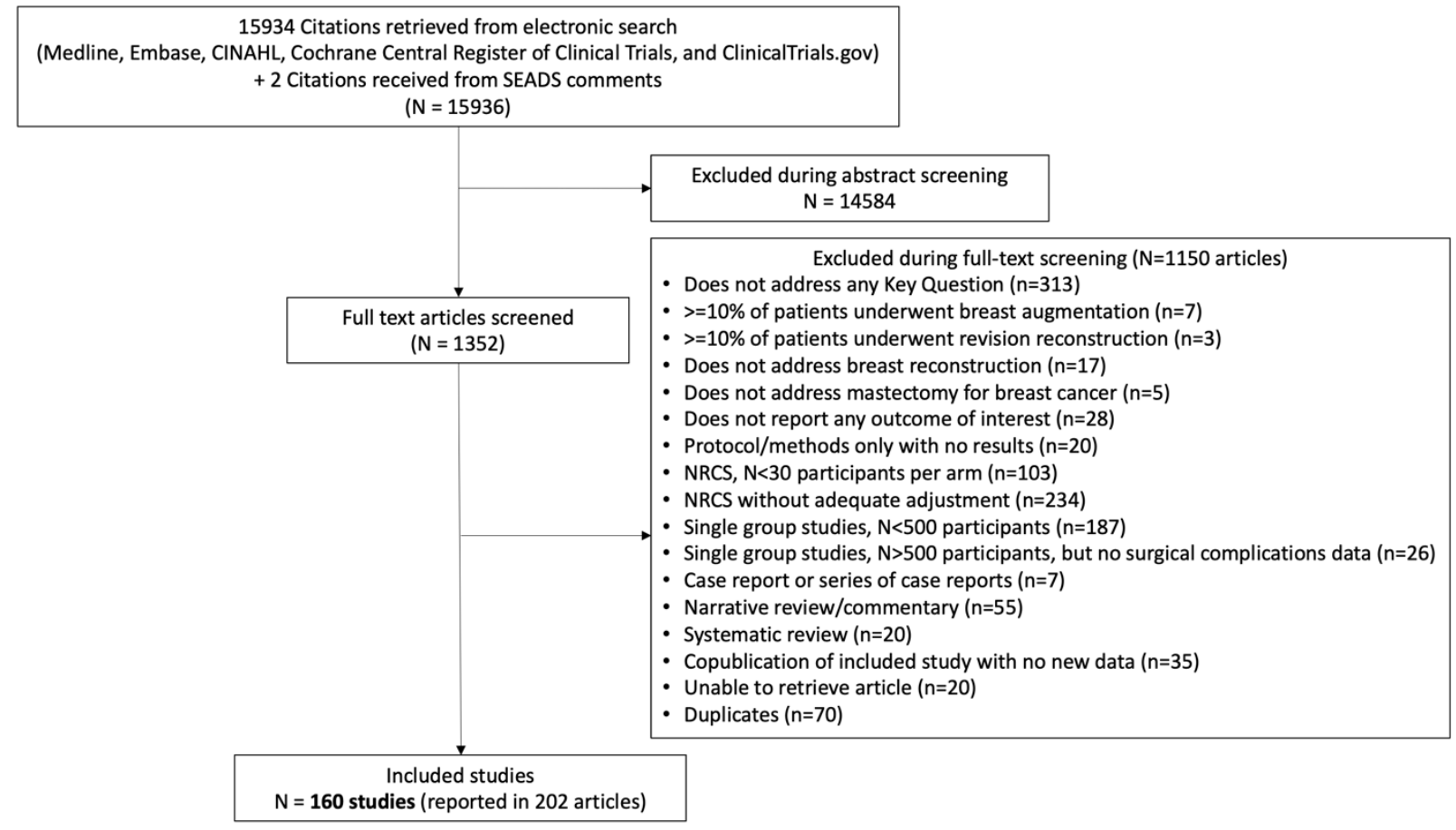

Abbreviations: NRCS = nonrandomized comparative study, SEADS = Submit Supplemental Evidence and Data for Systematic Reviews.

\section{Description of Included Studies}

\section{Overall Summary of Study Characteristics}

The 160 included studies (reported in 202 articles $^{1-202}$ ) were published between 1989 and 2021. All told, the studies enrolled and followed patients between 1977 and 2020. The 160 studies comprised eight RCTs, 83 NRCSs (observational cohort studies), and 69 single group studies.

The 160 included studies included a total of 459,228 patients. The 160 studies comprised eight RCTs with 570 patients (ranging from 34 to 150 patients each), 83 NRCSs with 202,862 patients (ranging from 70 to 32,897 patients each), and 69 single group studies with 275,218 patients (ranging from 501 to 56,522 patients each).

Appendix Tables C-1 to C-7 summarize the design and arm details of all 160 studies. Although the tables are organized by KQ, we describe all 160 studies here. Ninety studies (56\%) were conducted exclusively in the U.S., $12(8 \%)$ exclusively in South Korea, 7 (4\%) exclusively in Canada, 7 (4\%) exclusively in Sweden, $6(4 \%)$ exclusively in China, $5(3 \%)$ exclusively in the U.K., 4 (3\%) exclusively in France, 4 (3\%) exclusively in Germany, and 4 (3\%) exclusively in 
the Netherlands. Other common countries included Belgium and Italy (3 studies [2\%] each) and Japan (2 studies [1\%]). One study each (1\%) was conducted in Australia, Denmark, Finland, India, Portugal, Taiwan, and Turkey. Six studies (4\%) were conducted in multiple countries, each of which included the U.S. and Canada.

Among all 160 studies, 94 (59\%) were single-center studies, while 56 (35\%) involved multiple centers (10 studies [6\%] did not report number of centers). A large proportion of the studies either were not funded (48/160 studies; 30\%) or did report information about funding sources (72/160 studies; 45\%). Among the remaining 40 studies, 33 studies were funded by nonindustry sources, and 9 studies were funded by industry sources ( 2 studies were funded by both).

\section{Summary of Patient Characteristics}

Almost half of studies $(72 / 160 ; 45 \%)$ did not report any data about the ages for the entire study population. When reported, average patient ages ranged from 42.1 to 58.3 years. The youngest and oldest enrolled patients were 18 years and 83 years, respectively.

A large proportion of the studies $(96 / 160 ; 61 \%)$ did not report any data about body mass index (BMI) for the entire study population. When reported, average BMIs ranged from 21.9 to $34.5 \mathrm{~kg} / \mathrm{m}^{2}$. The lowest and highest BMIs among enrolled patients were $14.4 \mathrm{~kg} / \mathrm{m}^{2}$ and 60.3 $\mathrm{kg} / \mathrm{m}^{2}$, respectively.

Only 27 studies reported data about patient race. Most patients were white/Caucasian, with percentages ranging from $63.2 \%$ to $97.9 \%$. Black/African Americans were the next most common, ranging from $1.3 \%$ to $15.0 \%$.

Only 8 studies reported on whether the breast cancer being treated was the first or a repeat occurrence. All patients in all 8 studies were being treated for their first breast cancer.

Only 36 studies reported data about whether the mastectomy that patients (in the entire study population) received was for therapeutic or prophylactic purposes. Among most of these studies, the mastectomy was therapeutic for the majority of patients (ranging from $73.6 \%$ to $100 \%$ ). However, in 2 studies, the majority of patients received mastectomy for therapeutic purposes $(64.6 \%$ and $55.5 \%)$.

\section{Risk of Bias Assessments}

Appendix Tables D-1 to D-4 summarize the risk of bias assessment of all 160 studies. Among the 8 RCTs, we rated 4 at overall high risk of bias and 4 at overall moderate risk of bias. The main reasons for high risk of bias ratings were the lack of blinding of participants and care providers, incompleteness of outcome data, or evidence of selective outcome reporting. Among the 83 NRCSs, we rated 63 at overall high risk of bias, 19 at overall moderate risk of bias, and 1 at overall low risk of bias. The main reasons for high risk of bias ratings were the lack of blinding of participants, care providers, and outcomes assessors or evidence of serious risk of confounding. Among the 69 Single group studies, we rated 1 at overall high risk of bias, 14 at overall moderate risk of bias, and 54 at overall low risk of bias. The main reasons for moderate risk of bias ratings were because the interventions were not clearly described or consistently delivered or that the outcomes were not prespecified, clearly defined, and consistently assessed. 
Table C-1. Key Question 1: Implant-based versus autologous reconstruction - comparative studies, summary of design, arm, and sample details

\begin{tabular}{|c|c|c|c|c|c|c|c|c|c|c|c|}
\hline $\begin{array}{l}\text { Study, } \\
\text { Publication } \\
\text { Year, } \\
\text { PMID, } \\
\text { Country }\end{array}$ & $\begin{array}{l}\text { Design } \\
\text { (Funding) } \\
\text { (Study } \\
\text { Years) }\end{array}$ & $\begin{array}{l}\text { Risk } \\
\text { of } \\
\text { Bias }\end{array}$ & $\begin{array}{l}\text { Eligibility } \\
\text { Criteria }\end{array}$ & Arm & $\begin{array}{l}\text { Implant } \\
\text { Details (\%) } \\
\text { (Only } \\
\text { Reported } \\
\text { Details) } \\
\end{array}$ & $\begin{array}{l}\text { Reconstruction } \\
\text { Details (\%) } \\
\text { (Only Reported } \\
\text { Details) }\end{array}$ & $\mathbf{N}$ & $\begin{array}{l}\text { Age in } \\
\text { Years, } \\
\text { Mean (SD) } \\
\text { or as } \\
\text { Specified }\end{array}$ & $\begin{array}{l}\text { Race } \\
(\%)\end{array}$ & $\begin{array}{l}\text { Breast } \\
\text { Cancer } \\
\text { Occurrence } \\
\%\end{array}$ & $\begin{array}{l}\text { Cancer Stage } \\
\text { or } \\
\text { Mastectomy } \\
\text { Purpose (\%) }\end{array}$ \\
\hline \multirow[t]{5}{*}{$\begin{array}{l}\text { Abedi, 2016, } \\
25003437, \\
\text { Canada }\end{array}$} & $\begin{array}{l}\text { NRCS } \\
\text { (None) } \\
(2003- \\
2011)\end{array}$ & High & $\mathrm{I}: \mathrm{BR}$ & IBR & NR & $\begin{array}{l}\text { Timing: Imm (100) } \\
\text { Stages: >1 (87) } \\
\text { Chemo: Before } \\
\text { (28.7)/After (16.6) } \\
\text { Radio: Before } \\
(13.4) / \text { After (15.1) }\end{array}$ & 404 & $48.3(9.6)$ & NR & NR & NR \\
\hline & $\begin{array}{l}\text { NRCS } \\
\text { (None) } \\
(2003- \\
2011)\end{array}$ & High & I: BR & AR (all) & $\mathrm{N} / \mathrm{A}$ & $\begin{array}{l}\text { Timing: Imm (100) } \\
\text { Stages: NR } \\
\text { Chemo: Before } \\
\text { (47.5)/After (13.7) } \\
\text { Radio: Before } \\
\text { (38.9)/After (3.2) }\end{array}$ & 314 & $50(8.2)$ & NR & NR & NR \\
\hline & $\begin{array}{l}\text { NRCS } \\
\text { (None) } \\
(2003- \\
2011) \\
\end{array}$ & High & $\mathrm{I}: \mathrm{BR}$ & $\begin{array}{l}\text { AR with } \\
\text { DIEP }\end{array}$ & $\mathrm{N} / \mathrm{A}$ & NR & NR & NR & NR & NR & NR \\
\hline & $\begin{array}{l}\text { NRCS } \\
\text { (None) } \\
(2003- \\
2011) \\
\end{array}$ & High & $\mathrm{I}: \mathrm{BR}$ & $\begin{array}{l}\text { AR with } \\
\text { TRAM }\end{array}$ & $\mathrm{N} / \mathrm{A}$ & Timing: Imm (100) & NR & NR & NR & NR & NR \\
\hline & $\begin{array}{l}\text { NRCS } \\
\text { (None) } \\
(2003- \\
2011) \\
\end{array}$ & High & I: BR & Total & $\mathrm{N} / \mathrm{A}$ & . & 718 & NR & NR & NR & NR \\
\hline \multirow[t]{2}{*}{$\begin{array}{l}\text { Brito, 2020, } \\
\text { No PMID, } \\
\text { Portugal }\end{array}$} & $\begin{array}{l}\text { NRCS (NR) } \\
(2014- \\
2018)\end{array}$ & High & $\begin{array}{l}\text { I: IBR or AR with } \\
\text { pedicled TRAM or } \\
\text { LD-flap } \\
\text { E: Hybrid IBR and } \\
\text { AR (other than } \\
\text { LD) }\end{array}$ & IBR & NR & $\begin{array}{l}\text { TIMING: Imm } \\
\text { (95.6)/Del (4.4) } \\
\text { RADIO: Timing NR } \\
\text { (14.7) }\end{array}$ & 68 & $45.5(9.2)$ & NR & 1st: 100 & NR \\
\hline & $\begin{array}{l}\text { NRCS (NR) } \\
(2014- \\
2018)\end{array}$ & High & $\begin{array}{l}\text { I: IBR or AR with } \\
\text { pedicled TRAM or } \\
\text { LD-flap } \\
\text { E: Hybrid IBR and } \\
\text { AR (other than } \\
\text { LD) }\end{array}$ & AR & $\mathrm{N} / \mathrm{A}$ & $\begin{array}{l}\text { RADIO: Timing NR } \\
(61.3)\end{array}$ & 111 & $50.2(8.5)$ & NR & 1st: 100 & NR \\
\hline
\end{tabular}




\begin{tabular}{|c|c|c|c|c|c|c|c|c|c|c|c|}
\hline $\begin{array}{l}\text { Study, } \\
\text { Publication } \\
\text { Year, } \\
\text { PMID, } \\
\text { Country }\end{array}$ & $\begin{array}{l}\text { Design } \\
\text { (Funding) } \\
\text { (Study } \\
\text { Years) }\end{array}$ & $\begin{array}{l}\text { Risk } \\
\text { of } \\
\text { Bias }\end{array}$ & $\begin{array}{l}\text { Eligibility } \\
\text { Criteria }\end{array}$ & Arm & $\begin{array}{l}\text { Implant } \\
\text { Details (\%) } \\
\text { (Only } \\
\text { Reported } \\
\text { Details) } \\
\end{array}$ & $\begin{array}{l}\text { Reconstruction } \\
\text { Details (\%) } \\
\text { (Only Reported } \\
\text { Details) }\end{array}$ & $\mathbf{N}$ & $\begin{array}{l}\text { Age in } \\
\text { Years, } \\
\text { Mean (SD) } \\
\text { or as } \\
\text { Specified }\end{array}$ & $\begin{array}{l}\text { Race } \\
(\%)\end{array}$ & $\begin{array}{l}\text { Breast } \\
\text { Cancer } \\
\text { Occurrence } \\
\%\end{array}$ & $\begin{array}{l}\text { Cancer Stage } \\
\text { or } \\
\text { Mastectomy } \\
\text { Purpose (\%) }\end{array}$ \\
\hline & $\begin{array}{l}\text { NRCS (NR) } \\
(2014- \\
2018)\end{array}$ & High & $\begin{array}{l}\text { I: IBR or AR with } \\
\text { pedicled TRAM or } \\
\text { LD-flap } \\
\text { E: Hybrid IBR and } \\
\text { AR (other than } \\
\text { LD) }\end{array}$ & Total & $\mathrm{N} / \mathrm{A}$ & N/A & 179 & 49.1 & NR & 1st: 100 & NR \\
\hline \multirow[t]{3}{*}{$\begin{array}{l}\text { Brorson } \\
2020 a \\
32807615 \\
\text { Sweden }\end{array}$} & $\begin{array}{l}\text { RCT (Non- } \\
\text { industry) } \\
\text { (2008- } \\
2020)\end{array}$ & High & $\begin{array}{l}\text { I: Age } \geq 18 \text { years; } \\
\text { unilateral } \\
\text { mastectomy } \\
\text { E: Current } \\
\text { smoker; } \text { BMI >30 }\end{array}$ & IBR & NR & $\begin{array}{l}\text { Laterality: Uni (100) } \\
\text { Timing: Del (100) } \\
\text { Stages: } 1(100) \\
\text { Chemo: Before (18.6) }\end{array}$ & 80 & $55.7(9.0)$ & NR & NR & $\begin{array}{l}\text { Stage 0: } 19.1 \\
\text { Stage I: } 36.8 \\
\text { Stage II: } 42.6 \\
\text { Stage III: } 1.5\end{array}$ \\
\hline & $\begin{array}{l}\text { RCT (Non- } \\
\text { industry) } \\
\text { (2008- } \\
2020)\end{array}$ & High & $\begin{array}{l}\text { I: Age } \geq 18 \text { years; } \\
\text { unilateral } \\
\text { mastectomy } \\
\text { E: Current } \\
\text { smoker; BMI >30 }\end{array}$ & AR & $\mathrm{N} / \mathrm{A}$ & $\begin{array}{l}\text { Laterality: Uni (100) } \\
\text { Timing: Del }(100) \\
\text { Chemo: Before (23.6) }\end{array}$ & 70 & $55.1(7.0)$ & NR & NR & $\begin{array}{l}\text { Stage 0: } 14.5 \\
\text { Stage I: } 25.5 \\
\text { Stage II: } 60.0 \\
\text { Stage III: } 0\end{array}$ \\
\hline & $\begin{array}{l}\text { RCT (Non- } \\
\text { industry) } \\
\text { (2008- } \\
2020)\end{array}$ & High & $\begin{array}{l}\text { I: Age } \geq 18 \text { years; } \\
\text { unilateral } \\
\text { mastectomy } \\
\text { E: Current } \\
\text { smoker; BMI >30 }\end{array}$ & Total & $\mathrm{N} / \mathrm{A}$ & N/A & 150 & NR & NR & NR & NR \\
\hline \multirow[t]{4}{*}{$\begin{array}{l}\text { Carramaschi } \\
\text {, 1989, } \\
2602589, \\
\text { France }\end{array}$} & $\begin{array}{l}\text { NRCS (NR) } \\
(1982- \\
1986)\end{array}$ & High & $\begin{array}{l}\text { I: } \\
\text { Postmastectomy } \\
\text { BR }\end{array}$ & IBR & $\begin{array}{l}\text { MATERIAL } \\
\text { : Silicone } \\
(100)\end{array}$ & $\begin{array}{l}\text { Laterality: Uni } \\
\text { (97.8)/Bi }(2.2) \\
\text { Timing: Imm (11)/Del } \\
\text { (89) } \\
\text { Stages: } 1(92.2) />1 \\
\text { (7.8) } \\
\text { Chemo: NR } \\
\text { Radio: NR } \\
\end{array}$ & 166 & NR & NR & NR & NR \\
\hline & $\begin{array}{l}\text { NRCS (NR) } \\
(1982- \\
1986)\end{array}$ & High & $\begin{array}{l}\text { I: } \\
\text { Postmastectomy } \\
\text { BR }\end{array}$ & AR (all) & $\mathrm{N} / \mathrm{A}$ & NR & 74 & NR & NR & NR & NR \\
\hline & $\begin{array}{l}\text { NRCS (NR) } \\
(1982- \\
1986) \\
\end{array}$ & High & $\begin{array}{l}\text { I: } \\
\text { Postmastectomy } \\
\text { BR }\end{array}$ & $\begin{array}{l}\text { AR with } \\
\text { TRAM }\end{array}$ & $\mathrm{N} / \mathrm{A}$ & NR & 40 & NR & NR & NR & NR \\
\hline & $\begin{array}{l}\text { NRCS (NR) } \\
(1982- \\
1986)\end{array}$ & High & $\begin{array}{l}\text { I: } \\
\text { Postmastectomy } \\
\text { BR }\end{array}$ & AR with LD & $\mathrm{N} / \mathrm{A}$ & NR & 34 & NR & NR & NR & NR \\
\hline
\end{tabular}




\begin{tabular}{|c|c|c|c|c|c|c|c|c|c|c|c|}
\hline $\begin{array}{l}\text { Study, } \\
\text { Publication } \\
\text { Year, } \\
\text { PMID, } \\
\text { Country }\end{array}$ & $\begin{array}{l}\text { Design } \\
\text { (Funding) } \\
\text { (Study } \\
\text { Years) }\end{array}$ & $\begin{array}{l}\text { Risk } \\
\text { of } \\
\text { Bias }\end{array}$ & $\begin{array}{l}\text { Eligibility } \\
\text { Criteria }\end{array}$ & Arm & $\begin{array}{l}\text { Implant } \\
\text { Details (\%) } \\
\text { (Only } \\
\text { Reported } \\
\text { Details) }\end{array}$ & $\begin{array}{l}\text { Reconstruction } \\
\text { Details (\%) } \\
\text { (Only Reported } \\
\text { Details) }\end{array}$ & $\mathbf{N}$ & $\begin{array}{l}\text { Age in } \\
\text { Years, } \\
\text { Mean (SD) } \\
\text { or as } \\
\text { Specified }\end{array}$ & $\begin{array}{l}\text { Race } \\
(\%)\end{array}$ & $\begin{array}{l}\text { Breast } \\
\text { Cancer } \\
\text { Occurrence } \\
\%\end{array}$ & $\begin{array}{l}\text { Cancer Stage } \\
\text { or } \\
\text { Mastectomy } \\
\text { Purpose (\%) }\end{array}$ \\
\hline & $\begin{array}{l}\text { NRCS (NR) } \\
(1982- \\
1986)\end{array}$ & High & $\begin{array}{l}\text { I: } \\
\text { Postmastectomy } \\
\text { BR }\end{array}$ & Total & N/A & $\mathrm{N} / \mathrm{A}$ & 240 & $\begin{array}{l}46.6(\mathrm{NR}) \\
\text { Range: } 24, \\
70\end{array}$ & NR & NR & Stage III (18) \\
\hline \multirow[t]{3}{*}{$\begin{array}{l}\text { Chetta, } \\
2017 \\
28002254 \\
\text { US }\end{array}$} & $\begin{array}{l}\text { NRCS (NR) } \\
(2009- \\
2012)\end{array}$ & High & $\begin{array}{l}\text { I: Age >=18 years, } \\
\text { BR for breast } \\
\text { cancer/high } \\
\text { risk/family history } \\
\text { of breast cancer } \\
\text { E: Combination of } \\
\text { procedures (e.g., } \\
\text { IBR plus AR) }\end{array}$ & IBR & NR & $\begin{array}{l}\text { Timing: Imm (83)/Del } \\
\text { (17)/ } \\
\text { Radio: Before } \\
\text { (25)/After (75) }\end{array}$ & 3846 & $\begin{array}{l}18-34 \text { years } \\
(6 \%), 35-44 \\
\text { years }(26 \%) \text {, } \\
45-54 \text { years } \\
(40 \%), 55- \\
64 \text { years } \\
(22 \%), \geq 65 \\
\text { years }(21 \%)\end{array}$ & NR & NR & NR \\
\hline & $\begin{array}{l}\text { NRCS (NR) } \\
(2009- \\
2012)\end{array}$ & High & $\begin{array}{l}\text { I: Age >=18 years, } \\
\text { BR for breast } \\
\text { cancer/high } \\
\text { risk/family history } \\
\text { of breast cancer } \\
\text { E: Combination of } \\
\text { procedures (e.g., } \\
\text { IBR plus AR) }\end{array}$ & $\mathrm{AR}$ & $\mathrm{N} / \mathrm{A}$ & $\begin{array}{l}\text { Timing: Imm (52)/Del } \\
\text { (48) } \\
\text { Radio: Before } \\
\text { (64)/After (36) }\end{array}$ & 935 & $\begin{array}{l}18-34 \text { years } \\
(5 \%), 35-44 \\
\text { years }(23 \%), \\
45-54 \text { years } \\
(42 \%), 55- \\
64 \text { years } \\
(24 \%), \geq 65 \\
\text { years }(5 \%)\end{array}$ & NR & NR & NR \\
\hline & $\begin{array}{l}\text { NRCS (NR) } \\
(2009- \\
2012)\end{array}$ & High & $\begin{array}{l}\text { I: Age >=18 years, } \\
\text { BR for breast } \\
\text { cancer/high } \\
\text { risk/family history } \\
\text { of breast cancer } \\
\text { E: Combination of } \\
\text { procedures (e.g., } \\
\text { IBR plus AR) }\end{array}$ & Total & $\mathrm{N} / \mathrm{A}$ & $\mathrm{N} / \mathrm{A}$ & 4781 & NR & NR & NR & NR \\
\hline $\begin{array}{l}\text { Dauplat, } \\
2021, \\
33622886, \\
\text { France }\end{array}$ & $\begin{array}{l}\text { NRCS } \\
\text { (None) } \\
(2007- \\
2009)\end{array}$ & $\begin{array}{l}\text { Mode } \\
\text { rate }\end{array}$ & $\begin{array}{l}\text { I: Unilateral IBR or } \\
\text { AR after } \\
\text { therapeutic } \\
\text { mastectomy } \\
\text { E: Another } \\
\text { concurrent cancer }\end{array}$ & IBR & NR & $\begin{array}{l}\text { LATERALITY: Uni } \\
\text { (100) } \\
\text { TIMING: Imm (100) } \\
\text { CHEMO: Timing NR } \\
\text { (14) } \\
\text { RADIO: Timing NR } \\
\text { (10) }\end{array}$ & 205 & NR & NR & NR & Ther (100) \\
\hline
\end{tabular}




\begin{tabular}{|c|c|c|c|c|c|c|c|c|c|c|c|}
\hline $\begin{array}{l}\text { Study, } \\
\text { Publication } \\
\text { Year, } \\
\text { PMID, } \\
\text { Country }\end{array}$ & $\begin{array}{l}\text { Design } \\
\text { (Funding) } \\
\text { (Study } \\
\text { Years) }\end{array}$ & $\begin{array}{l}\text { Risk } \\
\text { of } \\
\text { Bias }\end{array}$ & $\begin{array}{l}\text { Eligibility } \\
\text { Criteria }\end{array}$ & Arm & $\begin{array}{l}\text { Implant } \\
\text { Details (\%) } \\
\text { (Only } \\
\text { Reported } \\
\text { Details) } \\
\end{array}$ & $\begin{array}{l}\text { Reconstruction } \\
\text { Details (\%) } \\
\text { (Only Reported } \\
\text { Details) }\end{array}$ & $\mathbf{N}$ & $\begin{array}{l}\text { Age in } \\
\text { Years, } \\
\text { Mean (SD) } \\
\text { or as } \\
\text { Specified } \\
\end{array}$ & $\begin{array}{l}\text { Race } \\
(\%)\end{array}$ & $\begin{array}{l}\text { Breast } \\
\text { Cancer } \\
\text { Occurrence } \\
\%\end{array}$ & $\begin{array}{l}\text { Cancer Stage } \\
\text { or } \\
\text { Mastectomy } \\
\text { Purpose (\%) }\end{array}$ \\
\hline & $\begin{array}{l}\text { NRCS } \\
\text { (None) } \\
(2007- \\
2009)\end{array}$ & $\begin{array}{l}\text { Mode } \\
\text { rate }\end{array}$ & $\begin{array}{l}\text { I: Unilateral IBR or } \\
\text { AR after } \\
\text { therapeutic } \\
\text { mastectomy } \\
\text { E: Another } \\
\text { concurrent cancer }\end{array}$ & $\begin{array}{l}\text { AR with } \\
\text { TRAM }\end{array}$ & N/A & $\begin{array}{l}\text { LATERALITY: Uni } \\
\text { (100) } \\
\text { TIMING: Imm (100) } \\
\text { CHEMO: Timing NR } \\
\text { (40) } \\
\text { RADIO: Timing NR } \\
(17)\end{array}$ & 30 & NR & NR & NR & Ther (100) \\
\hline & $\begin{array}{l}\text { NRCS } \\
\text { (None) } \\
(2007- \\
2009)\end{array}$ & $\begin{array}{l}\text { Mode } \\
\text { rate }\end{array}$ & $\begin{array}{l}\text { I: Unilateral IBR or } \\
\text { AR after } \\
\text { therapeutic } \\
\text { mastectomy } \\
\text { E: Another } \\
\text { concurrent cancer }\end{array}$ & $\begin{array}{l}\text { AR with LD } \\
\text { and implant }\end{array}$ & NR & $\begin{array}{l}\text { LATERALITY: Uni } \\
\text { (100) } \\
\text { TIMING: Imm (100) } \\
\text { CHEMO: Timing NR } \\
\text { (17) } \\
\text { RADIO: Timing NR } \\
\text { (14) }\end{array}$ & 91 & NR & NR & NR & Ther (100) \\
\hline & $\begin{array}{l}\text { NRCS } \\
\text { (None) } \\
(2007- \\
2009)\end{array}$ & $\begin{array}{l}\text { Mode } \\
\text { rate }\end{array}$ & $\begin{array}{l}\text { I: Unilateral IBR or } \\
\text { AR after } \\
\text { therapeutic } \\
\text { mastectomy } \\
\text { E: Another } \\
\text { concurrent cancer }\end{array}$ & $\begin{array}{l}\text { AR with LD } \\
\text { and no } \\
\text { implant }\end{array}$ & $\mathrm{N} / \mathrm{A}$ & $\begin{array}{l}\text { LATERALITY: Uni } \\
\text { (100) } \\
\text { TIMING: Imm (100) } \\
\text { CHEMO: Timing NR } \\
\text { (32) } \\
\text { RADIO: Timing NR } \\
\text { (7) }\end{array}$ & 78 & NR & NR & NR & Ther (100) \\
\hline & $\begin{array}{l}\text { NRCS } \\
\text { (None) } \\
(2007- \\
2009)\end{array}$ & $\begin{array}{l}\text { Mode } \\
\text { rate }\end{array}$ & $\begin{array}{l}\text { I: Unilateral IBR or } \\
\text { AR after } \\
\text { therapeutic } \\
\text { mastectomy } \\
\text { E: Another } \\
\text { concurrent cancer }\end{array}$ & Total & N/A & N/A & 404 & NR & NR & NR & Ther (100) \\
\hline $\begin{array}{l}\text { de Araujo, } \\
2016, \\
27673527, \\
\text { US }\end{array}$ & $\begin{array}{l}\text { NRCS (NR) } \\
(2002- \\
2012)\end{array}$ & High & $\begin{array}{l}\text { I: Age }>=18 \text { years } \\
\text { with unilateral } \\
\text { chest wall } \\
\text { radiotherapy } \\
\text { followed by } \\
\text { bilateral } \\
\text { mastectomy and } \\
\text { immediate } \\
\text { bilateral BR } \\
\text { E: Bilateral chest } \\
\text { wall radiotherapy }\end{array}$ & IBR & NR & $\begin{array}{l}\text { Laterality: Bi (100) } \\
\text { Radio: Before (100) }\end{array}$ & 38 & NR & NR & NR & $\begin{array}{l}\text { Stage NR } \\
(100)\end{array}$ \\
\hline
\end{tabular}




\begin{tabular}{|c|c|c|c|c|c|c|c|c|c|c|c|}
\hline $\begin{array}{l}\text { Study, } \\
\text { Publication } \\
\text { Year, } \\
\text { PMID, } \\
\text { Country }\end{array}$ & $\begin{array}{l}\text { Design } \\
\text { (Funding) } \\
\text { (Study } \\
\text { Years) }\end{array}$ & $\begin{array}{l}\text { Risk } \\
\text { of } \\
\text { Bias }\end{array}$ & $\begin{array}{l}\text { Eligibility } \\
\text { Criteria }\end{array}$ & Arm & $\begin{array}{l}\text { Implant } \\
\text { Details (\%) } \\
\text { (Only } \\
\text { Reported } \\
\text { Details) }\end{array}$ & $\begin{array}{l}\text { Reconstruction } \\
\text { Details (\%) } \\
\text { (Only Reported } \\
\text { Details) }\end{array}$ & $\mathbf{N}$ & $\begin{array}{l}\text { Age in } \\
\text { Years, } \\
\text { Mean (SD) } \\
\text { or as } \\
\text { Specified }\end{array}$ & $\begin{array}{l}\text { Race } \\
(\%)\end{array}$ & $\begin{array}{l}\text { Breast } \\
\text { Cancer } \\
\text { Occurrence } \\
\%\end{array}$ & $\begin{array}{l}\text { Cancer Stage } \\
\text { or } \\
\text { Mastectomy } \\
\text { Purpose (\%) }\end{array}$ \\
\hline & $\begin{array}{l}\text { NRCS (NR) } \\
(2002- \\
2012)\end{array}$ & High & $\begin{array}{l}\text { I: Age >=18 years } \\
\text { with unilateral } \\
\text { chest wall } \\
\text { radiotherapy } \\
\text { followed by } \\
\text { bilateral } \\
\text { mastectomy and } \\
\text { immediate } \\
\text { bilateral BR } \\
\text { E: Bilateral chest } \\
\text { wall radiotherapy }\end{array}$ & AR & $\mathrm{N} / \mathrm{A}$ & $\begin{array}{l}\text { Laterality: } \mathrm{Bi}(100) \\
\text { Stages: } 1(100) \\
\text { Radio: Before }(100)\end{array}$ & 32 & NR & NR & NR & $\begin{array}{l}\text { Stage NR } \\
(100)\end{array}$ \\
\hline & $\begin{array}{l}\text { NRCS (NR) } \\
(2002- \\
2012)\end{array}$ & High & $\begin{array}{l}\text { I: Age }>=18 \text { years } \\
\text { with unilateral } \\
\text { chest wall } \\
\text { radiotherapy } \\
\text { followed by } \\
\text { bilateral } \\
\text { mastectomy and } \\
\text { immediate } \\
\text { bilateral BR } \\
\text { E: Bilateral chest } \\
\text { wall radiotherapy }\end{array}$ & Total & $\mathrm{N} / \mathrm{A}$ & $\mathrm{N} / \mathrm{A}$ & 70 & $51.2(8.2)$ & NR & NR & $\begin{array}{l}\text { Stage NR } \\
(100)\end{array}$ \\
\hline \multirow[t]{2}{*}{$\begin{array}{l}\text { Eltahir, } \\
2015, \\
25539295, \\
\text { Netherlands }\end{array}$} & $\begin{array}{l}\text { NRCS (NR) } \\
(2006- \\
2010)\end{array}$ & $\begin{array}{l}\text { Mode } \\
\text { rate }\end{array}$ & $\begin{array}{l}\text { I: Age >=18 years } \\
\text { E: Presence of } \\
\text { metastasis or } \\
\text { severe illness, } \\
\text { reconstruction } \\
\text { failure }\end{array}$ & IBR & NR & Timing: Mixed (100) & 45 & $\begin{array}{l}\text { Median 42; } \\
\text { IQR 22, } 59\end{array}$ & NR & NR & $\begin{array}{l}\text { Stage II (95.3), } \\
\text { Stage III (4.7) }\end{array}$ \\
\hline & $\begin{array}{l}\text { NRCS (NR) } \\
(2006- \\
2010)\end{array}$ & $\begin{array}{l}\text { Mode } \\
\text { rate }\end{array}$ & $\begin{array}{l}\text { I: Age }>=18 \text { years } \\
\text { E: Presence of } \\
\text { metastasis or } \\
\text { severe illness, } \\
\text { reconstruction } \\
\text { failure }\end{array}$ & AR & N/A & Timing: Mixed (100) & 47 & $\begin{array}{l}\text { Median 49; } \\
\text { Range } 31 \\
74\end{array}$ & NR & NR & $\begin{array}{l}\text { Stage II (71.4), } \\
\text { Stage III (28.6) }\end{array}$ \\
\hline
\end{tabular}




\begin{tabular}{|c|c|c|c|c|c|c|c|c|c|c|c|}
\hline $\begin{array}{l}\text { Study, } \\
\text { Publication } \\
\text { Year, } \\
\text { PMID, } \\
\text { Country }\end{array}$ & $\begin{array}{l}\text { Design } \\
\text { (Funding) } \\
\text { (Study } \\
\text { Years) }\end{array}$ & $\begin{array}{l}\text { Risk } \\
\text { of } \\
\text { Bias }\end{array}$ & $\begin{array}{l}\text { Eligibility } \\
\text { Criteria }\end{array}$ & Arm & $\begin{array}{l}\text { Implant } \\
\text { Details (\%) } \\
\text { (Only } \\
\text { Reported } \\
\text { Details) } \\
\end{array}$ & $\begin{array}{l}\text { Reconstruction } \\
\text { Details (\%) } \\
\text { (Only Reported } \\
\text { Details) }\end{array}$ & $\mathbf{N}$ & $\begin{array}{l}\text { Age in } \\
\text { Years, } \\
\text { Mean (SD) } \\
\text { or as } \\
\text { Specified } \\
\end{array}$ & $\begin{array}{l}\text { Race } \\
(\%)\end{array}$ & $\begin{array}{l}\text { Breast } \\
\text { Cancer } \\
\text { Occurrence } \\
\%\end{array}$ & $\begin{array}{l}\text { Cancer Stage } \\
\text { or } \\
\text { Mastectomy } \\
\text { Purpose (\%) }\end{array}$ \\
\hline & $\begin{array}{l}\text { NRCS (NR) } \\
(2006- \\
2010)\end{array}$ & $\begin{array}{l}\text { Mode } \\
\text { rate }\end{array}$ & $\begin{array}{l}\text { l: Age }>=18 \text { years } \\
\text { E: Presence of } \\
\text { metastasis or } \\
\text { severe illness, } \\
\text { reconstruction } \\
\text { failure }\end{array}$ & Total & N/A & N/A & 92 & NR & NR & NR & NR \\
\hline \multirow[t]{3}{*}{$\begin{array}{l}\text { Fischer, } \\
2013, \\
23629074, \\
\text { US }\end{array}$} & $\begin{array}{l}\text { NRCS (NR) } \\
(2005- \\
2008)\end{array}$ & High & $\begin{array}{l}\text { I: Did not receive } \\
\text { postoperative } \\
\text { radiation therapy, } \\
\text { age<65 years, } \\
\text { BMl } 25-35 \mathrm{mg} / \mathrm{kg} 2\end{array}$ & IBR & $\begin{array}{l}\text { PLANE: } \\
\text { Total } \\
\text { submuscul } \\
\text { ar }(100)\end{array}$ & $\begin{array}{l}\text { Laterality: Uni (40)/Bi } \\
(60) \\
\text { Stages: } 1(100) \\
\text { Chemo: After (21.7) } \\
\text { Radio: Before (16.7) }\end{array}$ & 60 & $46.3(9.5)$ & NR & NR & NR \\
\hline & $\begin{array}{l}\text { NRCS (NR) } \\
(2005- \\
2008)\end{array}$ & High & $\begin{array}{l}\text { I: Did not receive } \\
\text { postoperative } \\
\text { radiation therapy, } \\
\text { age<65 years, } \\
\text { BMI } 25-35 \mathrm{mg} / \mathrm{kg} 2\end{array}$ & AR & $\mathrm{N} / \mathrm{A}$ & $\begin{array}{l}\text { Laterality: Uni } \\
(36.6) / \mathrm{Bi}(63.4) \\
\text { Stages: >1 (100) } \\
\text { Chemo: After (26.8) } \\
\text { Radio: Before (28.9) }\end{array}$ & 142 & $50(7.9)$ & NR & NR & NR \\
\hline & $\begin{array}{l}\text { NRCS (NR) } \\
(2005- \\
2008)\end{array}$ & High & $\begin{array}{l}\text { I: Did not receive } \\
\text { postoperative } \\
\text { radiation therapy, } \\
\text { age<65 years, } \\
\text { BMl } 25-35 \mathrm{mg} / \mathrm{kg} 2\end{array}$ & Total & $\mathrm{N} / \mathrm{A}$ & N/A & 202 & NR & NR & NR & NR \\
\hline $\begin{array}{l}\text { Fischer, } \\
2014, \\
24916480, \\
\text { US }\end{array}$ & $\begin{array}{l}\text { NRCS (NR) } \\
(2005- \\
2011)\end{array}$ & High & $\begin{array}{l}\text { I: AR with free flap } \\
\text { or TE/Implant BR }\end{array}$ & IBR & $\begin{array}{l}\text { PLANE: } \\
\text { Total } \\
\text { submuscul } \\
\text { ar }(100)\end{array}$ & $\begin{array}{l}\text { Laterality: Uni (40)/Bi } \\
\text { (60) } \\
\text { Timing: Imm } \\
\text { (90.3)/Del (7.1) } \\
\text { Stages: >1 (100) } \\
\text { Chemo: Before } \\
\text { (30.3)/After (47.7)/No } \\
\text { chemotherapy (22.0) } \\
\text { Radio: Before } \\
\text { (16.8)/After } \\
\text { (16.8)/None(66.4) }\end{array}$ & 155 & $47.9(11.6)$ & NR & NR & NR \\
\hline
\end{tabular}




\begin{tabular}{|c|c|c|c|c|c|c|c|c|c|c|c|}
\hline $\begin{array}{l}\text { Study, } \\
\text { Publication } \\
\text { Year, } \\
\text { PMID, } \\
\text { Country }\end{array}$ & $\begin{array}{l}\text { Design } \\
\text { (Funding) } \\
\text { (Study } \\
\text { Years) }\end{array}$ & $\begin{array}{l}\text { Risk } \\
\text { of } \\
\text { Bias }\end{array}$ & $\begin{array}{l}\text { Eligibility } \\
\text { Criteria }\end{array}$ & Arm & $\begin{array}{l}\text { Implant } \\
\text { Details (\%) } \\
\text { (Only } \\
\text { Reported } \\
\text { Details) }\end{array}$ & $\begin{array}{l}\text { Reconstruction } \\
\text { Details (\%) } \\
\text { (Only Reported } \\
\text { Details) }\end{array}$ & $\mathbf{N}$ & $\begin{array}{l}\text { Age in } \\
\text { Years, } \\
\text { Mean (SD) } \\
\text { or as } \\
\text { Specified }\end{array}$ & $\begin{array}{l}\text { Race } \\
(\%)\end{array}$ & $\begin{array}{l}\text { Breast } \\
\text { Cancer } \\
\text { Occurrence } \\
\%\end{array}$ & $\begin{array}{l}\text { Cancer Stage } \\
\text { or } \\
\text { Mastectomy } \\
\text { Purpose (\%) }\end{array}$ \\
\hline & $\begin{array}{l}\text { NRCS (NR) } \\
(2005- \\
2011)\end{array}$ & High & $\begin{array}{l}\text { I: AR with free flap } \\
\text { or TE/Implant BR }\end{array}$ & AR & $\mathrm{N} / \mathrm{A}$ & $\begin{array}{l}\text { Laterality: Uni } \\
\text { (38.1)/Bi }(61.9) \\
\text { Timing: Imm } \\
\text { (89.7)/Del (10.3) } \\
\text { Stages: } 1 \text { (100) } \\
\text { Chemo: Before } \\
\text { (25.2)/After (40.0)/No } \\
\text { chemotherapy (34.8) } \\
\text { Radio: Before } \\
\text { (16.8)/After } \\
\text { (28.4)/None(54.8) }\end{array}$ & 155 & $48.5(9.1)$ & NR & NR & NR \\
\hline & $\begin{array}{l}\text { NRCS (NR) } \\
(2005- \\
2011) \\
\end{array}$ & High & $\begin{array}{l}\text { I: AR with free flap } \\
\text { or TE/Implant BR }\end{array}$ & Total & $\mathrm{N} / \mathrm{A}$ & N/A & 310 & NR & NR & NR & NR \\
\hline \multirow[t]{2}{*}{$\begin{array}{l}\text { Fischer, } \\
2015, \\
26366550, \\
\text { US }\end{array}$} & $\begin{array}{l}\text { NRCS } \\
\text { (Non- } \\
\text { industry) } \\
\text { (2007- } \\
2012)\end{array}$ & High & $\begin{array}{l}\text { l: Age }>=18 \text { years } \\
\text { with } \\
\text { postmastectomy } \\
\text { BR } \\
\text { E: Known } \\
\text { metastatic } \\
\text { disease and } \\
\text { where the } \\
\text { discharge } \\
\text { disposition was } \\
\text { recorded as } \\
\text { unknown or death }\end{array}$ & $\begin{array}{l}\text { IBR (Direct } \\
\text { to Implant) }\end{array}$ & NR & $\begin{array}{l}\text { Laterality: Uni } \\
\text { (57.7)/Bi (42.3) } \\
\text { Timing: Imm (100) } \\
\text { Chemo: NR } \\
\text { Radio: NR }\end{array}$ & 1717 & $52.7(11.4)$ & NR & NR & $\begin{array}{l}\text { Stage } 0(23), \\
\text { Stage NR (77) }\end{array}$ \\
\hline & $\begin{array}{l}\text { NRCS } \\
\text { (Non- } \\
\text { industry) } \\
(2007- \\
2012)\end{array}$ & High & $\begin{array}{l}\text { l: Age >=18 years } \\
\text { with } \\
\text { postmastectomy } \\
\text { BR } \\
\text { E: Known } \\
\text { metastatic } \\
\text { disease and } \\
\text { where the } \\
\text { discharge } \\
\text { disposition was } \\
\text { recorded as } \\
\text { unknown or death }\end{array}$ & $\begin{array}{l}\text { IBR } \\
\text { (TE/IBR) }\end{array}$ & NR & $\begin{array}{l}\text { Laterality: Uni } \\
\text { (61.9)/Bi (38.1) } \\
\text { Timing: Imm (100) } \\
\text { Chemo: NR } \\
\text { Radio: NR }\end{array}$ & $\begin{array}{l}1069 \\
0\end{array}$ & $51.8(10.7)$ & NR & NR & $\begin{array}{l}\text { Stage } 0(20.2), \\
\text { Stage NR } \\
(79.8)\end{array}$ \\
\hline
\end{tabular}




\begin{tabular}{|c|c|c|c|c|c|c|c|c|c|c|c|}
\hline $\begin{array}{l}\text { Study, } \\
\text { Publication } \\
\text { Year, } \\
\text { PMID, } \\
\text { Country }\end{array}$ & $\begin{array}{l}\text { Design } \\
\text { (Funding) } \\
\text { (Study } \\
\text { Years) }\end{array}$ & $\begin{array}{l}\text { Risk } \\
\text { of } \\
\text { Bias }\end{array}$ & $\begin{array}{l}\text { Eligibility } \\
\text { Criteria }\end{array}$ & Arm & $\begin{array}{l}\text { Implant } \\
\text { Details (\%) } \\
\text { (Only } \\
\text { Reported } \\
\text { Details) }\end{array}$ & $\begin{array}{l}\text { Reconstruction } \\
\text { Details (\%) } \\
\text { (Only Reported } \\
\text { Details) }\end{array}$ & $\mathbf{N}$ & $\begin{array}{l}\text { Age in } \\
\text { Years, } \\
\text { Mean (SD) } \\
\text { or as } \\
\text { Specified }\end{array}$ & $\begin{array}{l}\text { Race } \\
(\%)\end{array}$ & $\begin{array}{l}\text { Breast } \\
\text { Cancer } \\
\text { Occurrence } \\
\%\end{array}$ & $\begin{array}{l}\text { Cancer Stage } \\
\text { or } \\
\text { Mastectomy } \\
\text { Purpose (\%) }\end{array}$ \\
\hline & $\begin{array}{l}\text { NRCS } \\
\text { (Non- } \\
\text { industry) } \\
\text { (2007- } \\
2012)\end{array}$ & High & $\begin{array}{l}\text { I: Age >=18 years } \\
\text { with } \\
\text { postmastectomy } \\
\text { BR } \\
\text { E: Known } \\
\text { metastatic } \\
\text { disease and } \\
\text { where the } \\
\text { discharge } \\
\text { disposition was } \\
\text { recorded as } \\
\text { unknown or death }\end{array}$ & AR & $\mathrm{N} / \mathrm{A}$ & $\begin{array}{l}\text { Laterality: Uni } \\
\text { (70.5)/Bi }(29.5) \\
\text { Timing: Imm (100) } \\
\text { Stages: } 1(100) \\
\text { Chemo: NR } \\
\text { Radio: NR }\end{array}$ & 2747 & $52.2(9.9)$ & NR & NR & $\begin{array}{l}\text { Stage } 0(20), \\
\text { Stage NR }(80)\end{array}$ \\
\hline & $\begin{array}{l}\text { NRCS } \\
\text { (Non- } \\
\text { industry) } \\
\text { (2007- } \\
2012)\end{array}$ & High & $\begin{array}{l}\text { I: Age >=18 years } \\
\text { with } \\
\text { postmastectomy } \\
\text { BR } \\
\text { E: Known } \\
\text { metastatic } \\
\text { disease and } \\
\text { where the } \\
\text { discharge } \\
\text { disposition was } \\
\text { recorded as } \\
\text { unknown or death }\end{array}$ & Total & $\mathrm{N} / \mathrm{A}$ & $\mathrm{N} / \mathrm{A}$ & $\begin{array}{l}1515 \\
4\end{array}$ & NR & NR & NR & NR \\
\hline \multirow[t]{4}{*}{$\begin{array}{l}\text { Garbay, } \\
1992, \\
1624727, \\
\text { France }\end{array}$} & $\begin{array}{l}\text { NRCS (NR) } \\
(1979- \\
1990)\end{array}$ & High & $\mathrm{I}: \mathrm{BR}$ & IBR & NR & $\begin{array}{l}\text { Timing: Imm (29)/Del } \\
\text { (71) } \\
\text { Stages: } 1(29) />1 \\
(71)\end{array}$ & 224 & NR & NR & NR & NR \\
\hline & $\begin{array}{l}\text { NRCS (NR) } \\
(1979- \\
1990) \\
\end{array}$ & High & I: BR & $\begin{array}{l}\text { AR with } \\
\text { TRAM }\end{array}$ & $\mathrm{N} / \mathrm{A}$ & NR & 63 & NR & NR & NR & NR \\
\hline & $\begin{array}{l}\text { NRCS (NR) } \\
(1979- \\
1990)\end{array}$ & High & $\mathrm{I}: \mathrm{BR}$ & AR with LD & $\mathrm{N} / \mathrm{A}$ & NR & 36 & NR & NR & NR & NR \\
\hline & $\begin{array}{l}\text { NRCS (NR) } \\
(1979- \\
1990)\end{array}$ & High & $\mathrm{I}: \mathrm{BR}$ & Total & $\mathrm{N} / \mathrm{A}$ & $\mathrm{N} / \mathrm{A}$ & 323 & NR & NR & NR & NR \\
\hline
\end{tabular}




\begin{tabular}{|c|c|c|c|c|c|c|c|c|c|c|c|}
\hline $\begin{array}{l}\text { Study, } \\
\text { Publication } \\
\text { Year, } \\
\text { PMID, } \\
\text { Country }\end{array}$ & $\begin{array}{l}\text { Design } \\
\text { (Funding) } \\
\text { (Study } \\
\text { Years) }\end{array}$ & $\begin{array}{l}\text { Risk } \\
\text { of } \\
\text { Bias }\end{array}$ & $\begin{array}{l}\text { Eligibility } \\
\text { Criteria }\end{array}$ & Arm & $\begin{array}{l}\text { Implant } \\
\text { Details (\%) } \\
\text { (Only } \\
\text { Reported } \\
\text { Details) }\end{array}$ & $\begin{array}{l}\text { Reconstruction } \\
\text { Details (\%) } \\
\text { (Only Reported } \\
\text { Details) }\end{array}$ & $\mathbf{N}$ & $\begin{array}{l}\text { Age in } \\
\text { Years, } \\
\text { Mean (SD) } \\
\text { or as } \\
\text { Specified }\end{array}$ & $\begin{array}{l}\text { Race } \\
(\%)\end{array}$ & $\begin{array}{l}\text { Breast } \\
\text { Cancer } \\
\text { Occurrence } \\
\%\end{array}$ & $\begin{array}{l}\text { Cancer Stage } \\
\text { or } \\
\text { Mastectomy } \\
\text { Purpose (\%) }\end{array}$ \\
\hline \multirow[t]{3}{*}{$\begin{array}{l}\text { Garvey, } \\
2012, \\
23096600, \\
\text { US }\end{array}$} & $\begin{array}{l}\text { NRCS (NR) } \\
(2005- \\
2013)\end{array}$ & $\begin{array}{l}\text { Mode } \\
\text { rate }\end{array}$ & $\begin{array}{l}\text { I: Obese patients } \\
\text { (BMl } \geq 30 \mathrm{~kg} / \mathrm{m} 2) \\
\text { E: "Delayed- } \\
\text { delayed" or } \\
\text { "delayed- } \\
\text { immediate" BR }\end{array}$ & IBR & $\begin{array}{l}\text { SIZE: } \\
\text { Mean } 702 \\
\text { cc, SD } 130\end{array}$ & $\begin{array}{l}\text { Timing: Imm } \\
\text { (91.4)/Del (8.6) } \\
\text { Stages: } 1 \text { (3.4)/>1 } \\
\text { (96.6) } \\
\text { Chemo: Before } \\
\text { (29.4)/After (13.1) } \\
\text { Radio: Before } \\
\text { (3.6)/After (11.1) }\end{array}$ & NR & $52.2(10.3)$ & NR & NR & NR \\
\hline & $\begin{array}{l}\text { NRCS (NR) } \\
(2005- \\
2013)\end{array}$ & $\begin{array}{l}\text { Mode } \\
\text { rate }\end{array}$ & $\begin{array}{l}\text { I: Obese patients } \\
\text { (BMI } \geq 30 \mathrm{~kg} / \mathrm{m} 2 \text { ) } \\
\text { E: "Delayed- } \\
\text { delayed" or } \\
\text { "delayed- } \\
\text { immediate" BR }\end{array}$ & AR & N/A & $\begin{array}{l}\text { Timing: Imm (71)/Del } \\
\text { (29) } \\
\text { Chemo: Before } \\
\text { (52.4)/After (7.1) } \\
\text { Radio: Before } \\
\text { (31)/Timing NR (6.2) }\end{array}$ & NR & $48.9(8.9)$ & NR & NR & NR \\
\hline & $\begin{array}{l}\text { NRCS (NR) } \\
(2005- \\
2013)\end{array}$ & $\begin{array}{l}\text { Mode } \\
\text { rate }\end{array}$ & $\begin{array}{l}\text { I: Obese patients } \\
\text { (BMI } \geq 30 \mathrm{~kg} / \mathrm{m} 2) \\
\text { E: "Delayed- } \\
\text { delayed" or } \\
\text { "delayed- } \\
\text { immediate" BR } \\
\end{array}$ & Total & $\mathrm{N} / \mathrm{A}$ & N/A & 700 & $\begin{array}{l}50 \text { (NR); } \\
\text { Range 26, } \\
78\end{array}$ & NR & NR & NR \\
\hline $\begin{array}{l}\text { Ha, 2020, } \\
32000718, \\
\text { South Korea }\end{array}$ & $\begin{array}{l}\text { NRCS (NR) } \\
(2010- \\
2014)\end{array}$ & High & $\begin{array}{l}\text { I: Immediate BR } \\
\text { E: Phyllodes } \\
\text { tumor, } \\
\text { angiosarcoma, or } \\
\text { metastatic cancer } \\
\text { at initial } \\
\text { presentation; } \\
\text { prophylactic } \\
\text { mastectomy; prior } \\
\text { history of breast } \\
\text { cancer; major } \\
\text { complications } \\
\text { such as flap loss } \\
\text { or implant loss } \\
\text { that may delay } \\
\text { adequate } \\
\text { postoperative } \\
\text { anti-cancer } \\
\text { treatment }\end{array}$ & IBR & NR & $\begin{array}{l}\text { Timing: Imm (100) } \\
\text { Chemo: Timing NR } \\
\text { (55)/No } \\
\text { chemotherapy (45) } \\
\text { Radio: Timing NR } \\
\text { (79)/None(21) }\end{array}$ & 247 & $41(8.73)$ & NR & NR & NR \\
\hline
\end{tabular}




\begin{tabular}{|c|c|c|c|c|c|c|c|c|c|c|c|}
\hline $\begin{array}{l}\text { Study, } \\
\text { Publication } \\
\text { Year, } \\
\text { PMID, } \\
\text { Country }\end{array}$ & $\begin{array}{l}\text { Design } \\
\text { (Funding) } \\
\text { (Study } \\
\text { Years) }\end{array}$ & $\begin{array}{l}\text { Risk } \\
\text { of } \\
\text { Bias }\end{array}$ & $\begin{array}{l}\text { Eligibility } \\
\text { Criteria }\end{array}$ & Arm & $\begin{array}{l}\text { Implant } \\
\text { Details (\%) } \\
\text { (Only } \\
\text { Reported } \\
\text { Details) } \\
\end{array}$ & $\begin{array}{l}\text { Reconstruction } \\
\text { Details (\%) } \\
\text { (Only Reported } \\
\text { Details) }\end{array}$ & $\mathbf{N}$ & $\begin{array}{l}\text { Age in } \\
\text { Years, } \\
\text { Mean (SD) } \\
\text { or as } \\
\text { Specified } \\
\end{array}$ & $\begin{array}{l}\text { Race } \\
(\%)\end{array}$ & $\begin{array}{l}\text { Breast } \\
\text { Cancer } \\
\text { Occurrence } \\
\%\end{array}$ & $\begin{array}{l}\text { Cancer Stage } \\
\text { or } \\
\text { Mastectomy } \\
\text { Purpose (\%) }\end{array}$ \\
\hline & $\begin{array}{l}\text { NRCS (NR) } \\
(2010- \\
2014)\end{array}$ & High & $\begin{array}{l}\text { I: Immediate BR } \\
\text { E: Phyllodes } \\
\text { tumor, } \\
\text { angiosarcoma, or } \\
\text { metastatic cancer } \\
\text { at initial } \\
\text { presentation; } \\
\text { prophylactic } \\
\text { mastectomy; prior } \\
\text { history of breast } \\
\text { cancer; major } \\
\text { complications } \\
\text { such as flap loss } \\
\text { or implant loss } \\
\text { that may delay } \\
\text { adequate } \\
\text { postoperative } \\
\text { anti-cancer } \\
\text { treatment }\end{array}$ & AR & $\mathrm{N} / \mathrm{A}$ & $\begin{array}{l}\text { Timing: Imm (100) } \\
\text { Chemo: Timing NR } \\
\text { (47)/No } \\
\text { chemotherapy (53) } \\
\text { Radio: Timing NR } \\
\text { (80)/None(20) }\end{array}$ & 249 & $43(6.99)$ & NR & NR & NR \\
\hline & $\begin{array}{l}\text { NRCS (NR) } \\
(2010- \\
2014)\end{array}$ & High & $\begin{array}{l}\text { I: Immediate BR } \\
\text { E: Phyllodes } \\
\text { tumor, } \\
\text { angiosarcoma, or } \\
\text { metastatic cancer } \\
\text { at initial } \\
\text { presentation; } \\
\text { prophylactic } \\
\text { mastectomy; prior } \\
\text { history of breast } \\
\text { cancer; major } \\
\text { complications } \\
\text { such as flap loss } \\
\text { or implant loss } \\
\text { that may delay } \\
\text { adequate } \\
\text { postoperative } \\
\text { anti-cancer } \\
\text { treatment }\end{array}$ & Total & N/A & $\mathrm{N} / \mathrm{A}$ & 496 & NR & NR & NR & NR \\
\hline
\end{tabular}




\begin{tabular}{|c|c|c|c|c|c|c|c|c|c|c|c|}
\hline $\begin{array}{l}\text { Study, } \\
\text { Publication } \\
\text { Year, } \\
\text { PMID, } \\
\text { Country }\end{array}$ & $\begin{array}{l}\text { Design } \\
\text { (Funding) } \\
\text { (Study } \\
\text { Years) }\end{array}$ & $\begin{array}{l}\text { Risk } \\
\text { of } \\
\text { Bias }\end{array}$ & $\begin{array}{l}\text { Eligibility } \\
\text { Criteria }\end{array}$ & Arm & $\begin{array}{l}\text { Implant } \\
\text { Details (\%) } \\
\text { (Only } \\
\text { Reported } \\
\text { Details) }\end{array}$ & $\begin{array}{l}\text { Reconstruction } \\
\text { Details (\%) } \\
\text { (Only Reported } \\
\text { Details) }\end{array}$ & $\mathbf{N}$ & $\begin{array}{l}\text { Age in } \\
\text { Years, } \\
\text { Mean (SD) } \\
\text { or as } \\
\text { Specified } \\
\end{array}$ & $\begin{array}{l}\text { Race } \\
(\%)\end{array}$ & $\begin{array}{l}\text { Breast } \\
\text { Cancer } \\
\text { Occurrence } \\
\%\end{array}$ & $\begin{array}{l}\text { Cancer Stage } \\
\text { or } \\
\text { Mastectomy } \\
\text { Purpose (\%) }\end{array}$ \\
\hline \multirow[t]{4}{*}{$\begin{array}{l}\text { Hangge, } \\
2019, \\
31606126, \\
\text { US }\end{array}$} & $\begin{array}{l}\text { NRCS } \\
\text { (None) } \\
\text { (2010- } \\
2017)\end{array}$ & High & $\begin{array}{l}\text { I: Mastectomy } \\
\text { with immediate 1- } \\
\text { stage IBR, 2- } \\
\text { stage IBR, or AR }\end{array}$ & $\begin{array}{l}\text { IBR (Direct } \\
\text { to Implant) }\end{array}$ & NR & $\begin{array}{l}\text { Laterality: Uni (31)/Bi } \\
\text { (69) } \\
\text { Timing: Imm (100) } \\
\text { Stages: } 1(100) \\
\text { Chemo: Timing NR } \\
\text { (24)/No } \\
\text { chemotherapy (76) } \\
\text { Radio: Timing NR } \\
\text { (17)/None(83) }\end{array}$ & 193 & $50.9(11)$ & $\begin{array}{l}\text { W (86), } \\
\text { B (3), A } \\
(6), H \\
(5), \\
\text { Others } \\
(1)\end{array}$ & NR & $\begin{array}{l}\text { Stage } 0(21), \\
\text { Stage I (50), } \\
\text { Stage II (34), } \\
\text { Stage III (13), } \\
\text { Stage IV (3) }\end{array}$ \\
\hline & $\begin{array}{l}\text { NRCS } \\
\text { (None) } \\
(2010- \\
2017)\end{array}$ & High & $\begin{array}{l}\text { I: Mastectomy } \\
\text { with immediate 1- } \\
\text { stage IBR, 2- } \\
\text { stage IBR, or AR }\end{array}$ & $\begin{array}{l}\text { IBR } \\
\text { (TE/IBR) }\end{array}$ & NR & $\begin{array}{l}\text { Laterality: Uni (29)/Bi } \\
\text { (71) } \\
\text { Timing: Imm (100) } \\
\text { Stages: } 1(100) \\
\text { Chemo: Timing NR } \\
\text { (23)/No } \\
\text { chemotherapy (77) } \\
\text { Radio: Timing NR } \\
\text { (21)/None(79) }\end{array}$ & 146 & $51.5(11.3)$ & $\begin{array}{l}W(87), \\
B(1), A \\
(3), H \\
(9)\end{array}$ & NR & $\begin{array}{l}\text { Stage } 0(20), \\
\text { Stage I (42), } \\
\text { Stage II (44), } \\
\text { Stage III (12), } \\
\text { Stage IV (2) }\end{array}$ \\
\hline & $\begin{array}{l}\text { NRCS } \\
\text { (None) } \\
\text { (2010- } \\
2017)\end{array}$ & High & $\begin{array}{l}\text { I: Mastectomy } \\
\text { with immediate 1- } \\
\text { stage IBR, 2- } \\
\text { stage IBR, or AR }\end{array}$ & AR & $\mathrm{N} / \mathrm{A}$ & $\begin{array}{l}\text { Laterality: Uni (47)/Bi } \\
\text { (53) } \\
\text { Timing: Imm (100) } \\
\text { Stages: >1 (100) } \\
\text { Chemo: Timing NR } \\
\text { (28)/No } \\
\text { chemotherapy (72) } \\
\text { Radio: Timing NR } \\
\text { (13)/None(87) }\end{array}$ & 60 & $55.9(7.8)$ & $\begin{array}{l}\text { W (77), } \\
\mathrm{B}(7), \mathrm{A} \\
(7), \mathrm{H} \\
(10)\end{array}$ & NR & $\begin{array}{l}\text { Stage } 0 \text { (25), } \\
\text { Stage I (49), } \\
\text { Stage II (33), } \\
\text { Stage III (13), } \\
\text { Stage IV (4) }\end{array}$ \\
\hline & $\begin{array}{l}\text { NRCS } \\
\text { (None) } \\
(2010- \\
2017) \\
\end{array}$ & High & $\begin{array}{l}\text { I: Mastectomy } \\
\text { with immediate 1- } \\
\text { stage IBR, 2- } \\
\text { stage IBR, or AR }\end{array}$ & Total & $\mathrm{N} / \mathrm{A}$ & $\mathrm{N} / \mathrm{A}$ & 399 & NR & NR & NR & NR \\
\hline
\end{tabular}




\begin{tabular}{|c|c|c|c|c|c|c|c|c|c|c|c|}
\hline $\begin{array}{l}\text { Study, } \\
\text { Publication } \\
\text { Year, } \\
\text { PMID, } \\
\text { Country }\end{array}$ & $\begin{array}{l}\text { Design } \\
\text { (Funding) } \\
\text { (Study } \\
\text { Years) }\end{array}$ & $\begin{array}{l}\text { Risk } \\
\text { of } \\
\text { Bias }\end{array}$ & $\begin{array}{l}\text { Eligibility } \\
\text { Criteria }\end{array}$ & Arm & $\begin{array}{l}\text { Implant } \\
\text { Details (\%) } \\
\text { (Only } \\
\text { Reported } \\
\text { Details) }\end{array}$ & $\begin{array}{l}\text { Reconstruction } \\
\text { Details (\%) } \\
\text { (Only Reported } \\
\text { Details) }\end{array}$ & $\mathbf{N}$ & $\begin{array}{l}\text { Age in } \\
\text { Years, } \\
\text { Mean (SD) } \\
\text { or as } \\
\text { Specified }\end{array}$ & $\begin{array}{l}\text { Race } \\
(\%)\end{array}$ & $\begin{array}{l}\text { Breast } \\
\text { Cancer } \\
\text { Occurrence } \\
\%\end{array}$ & $\begin{array}{l}\text { Cancer Stage } \\
\text { or } \\
\text { Mastectomy } \\
\text { Purpose (\%) }\end{array}$ \\
\hline \multirow[t]{3}{*}{$\begin{array}{l}\text { Jiang, } 2013, \\
24349366, \\
\text { US }\end{array}$} & $\begin{array}{l}\text { NRCS } \\
\text { (Non- } \\
\text { industry) } \\
(1998- \\
2002)\end{array}$ & High & $\begin{array}{l}\text { I: Unilateral breast } \\
\text { cancer } \\
\text { E: Partial or } \\
\text { subcutaneous } \\
\text { mastectomy; } \\
\text { Histological grade } \\
\text { IV (SEER } \\
\text { program code: } \\
\text { undifferentiated or } \\
\text { anaplastic) cancer }\end{array}$ & IBR & NR & $\begin{array}{l}\text { Laterality: Uni (100) } \\
\text { Timing: Imm (100) } \\
\text { Radio: Timing NR } \\
\text { (16.9)/None(79.7) }\end{array}$ & 1412 & $\begin{array}{l}<45 \text { years } \\
(34.9 \%), 45- \\
64 \text { years } \\
(55.9 \%), \\
>64 \text { years } \\
(9.1 \%)\end{array}$ & $\begin{array}{l}\text { W } \\
(88.6) \\
\text { B (5.8), } \\
\text { Others } \\
(5.5)\end{array}$ & NR & NR \\
\hline & $\begin{array}{l}\text { NRCS } \\
\text { (Non- } \\
\text { industry) } \\
\text { (1998- } \\
2002)\end{array}$ & High & $\begin{array}{l}\text { I: Unilateral breast } \\
\text { cancer } \\
\text { E: Partial or } \\
\text { subcutaneous } \\
\text { mastectomy; } \\
\text { Histological grade } \\
\text { IV (SEER } \\
\text { program code: } \\
\text { undifferentiated or } \\
\text { anaplastic) cancer }\end{array}$ & AR & $\mathrm{N} / \mathrm{A}$ & $\begin{array}{l}\text { Laterality: Uni (100) } \\
\text { Timing: Imm (100) } \\
\text { Stages: } 1(100) \\
\text { Radio: Timing NR } \\
(19.9) / \text { None(76.1) }\end{array}$ & 2649 & $\begin{array}{l}<45 \text { years } \\
(34.3 \%), 45- \\
64 \text { years } \\
(58.3 \%), \\
>64 \text { years } \\
(7.4 \%)\end{array}$ & $\begin{array}{l}\text { W } \\
(83.5), \\
\text { B } \\
(11.5), \\
\text { Others } \\
(5.1)\end{array}$ & NR & NR \\
\hline & $\begin{array}{l}\text { NRCS } \\
\text { (Non- } \\
\text { industry) } \\
\text { (1998- } \\
2002)\end{array}$ & High & $\begin{array}{l}\text { I: Unilateral breast } \\
\text { cancer } \\
\text { E: Partial or } \\
\text { subcutaneous } \\
\text { mastectomy; } \\
\text { Histological grade } \\
\text { IV (SEER } \\
\text { program code: } \\
\text { undifferentiated or } \\
\text { anaplastic) cancer }\end{array}$ & Total & $\mathrm{N} / \mathrm{A}$ & $\mathrm{N} / \mathrm{A}$ & 4061 & NR & NR & NR & NR \\
\hline $\begin{array}{l}\text { Kouwenberg } \\
\text {, 2019, } \\
30270015 \text {, } \\
\text { Netherlands }\end{array}$ & $\begin{array}{l}\text { NRCS } \\
\text { (None) } \\
\text { (2008- } \\
2017)\end{array}$ & $\begin{array}{l}\text { Mode } \\
\text { rate }\end{array}$ & $\begin{array}{l}\text { I: Mastectomy for } \\
\text { breast cancer } \\
\text { E: Distant } \\
\text { metastasis }\end{array}$ & IBR & NR & $\begin{array}{l}\text { Laterality: Uni } \\
\text { (67.2)/Bi (32.8) } \\
\text { Chemo: Before } \\
\text { (49.3)/After (50.7) } \\
\text { Radio: Timing NR } \\
\text { (28.4)/None(71.6) }\end{array}$ & 67 & 55 (11.63) & NR & NR & NR \\
\hline
\end{tabular}




\begin{tabular}{|c|c|c|c|c|c|c|c|c|c|c|c|}
\hline $\begin{array}{l}\text { Study, } \\
\text { Publication } \\
\text { Year, } \\
\text { PMID, } \\
\text { Country }\end{array}$ & $\begin{array}{l}\text { Design } \\
\text { (Funding) } \\
\text { (Study } \\
\text { Years) }\end{array}$ & $\begin{array}{l}\text { Risk } \\
\text { of } \\
\text { Bias }\end{array}$ & $\begin{array}{l}\text { Eligibility } \\
\text { Criteria }\end{array}$ & Arm & $\begin{array}{l}\text { Implant } \\
\text { Details (\%) } \\
\text { (Only } \\
\text { Reported } \\
\text { Details) }\end{array}$ & $\begin{array}{l}\text { Reconstruction } \\
\text { Details (\%) } \\
\text { (Only Reported } \\
\text { Details) }\end{array}$ & $\mathbf{N}$ & $\begin{array}{l}\text { Age in } \\
\text { Years, } \\
\text { Mean (SD) } \\
\text { or as } \\
\text { Specified }\end{array}$ & $\begin{array}{l}\text { Race } \\
(\%)\end{array}$ & $\begin{array}{l}\text { Breast } \\
\text { Cancer } \\
\text { Occurrence } \\
\%\end{array}$ & $\begin{array}{l}\text { Cancer Stage } \\
\text { or } \\
\text { Mastectomy } \\
\text { Purpose (\%) }\end{array}$ \\
\hline & $\begin{array}{l}\text { NRCS } \\
\text { (None) } \\
(2008- \\
2017)\end{array}$ & $\begin{array}{l}\text { Mode } \\
\text { rate }\end{array}$ & $\begin{array}{l}\text { I: Mastectomy for } \\
\text { breast cancer } \\
\text { E: Distant } \\
\text { metastasis }\end{array}$ & AR & $\mathrm{N} / \mathrm{A}$ & $\begin{array}{l}\text { Laterality: Uni } \\
\text { (65.7)/Bi (32.8) } \\
\text { Chemo: Timing NR } \\
\text { (59.7)/No } \\
\text { chemotherapy (40.3) } \\
\text { Radio: Timing NR } \\
\text { (23.9)/None(76.1) }\end{array}$ & 67 & $55(9.49)$ & NR & NR & NR \\
\hline & $\begin{array}{l}\text { NRCS } \\
\text { (None) } \\
(2008- \\
2017)\end{array}$ & $\begin{array}{l}\text { Mode } \\
\text { rate }\end{array}$ & $\begin{array}{l}\text { I: Mastectomy for } \\
\text { breast cancer } \\
\text { E: Distant } \\
\text { metastasis }\end{array}$ & Total & $\mathrm{N} / \mathrm{A}$ & $\mathrm{N} / \mathrm{A}$ & 134 & NR & NR & NR & NR \\
\hline \multirow[t]{3}{*}{$\begin{array}{l}\text { Kouwenberg } \\
\text {, 2020, } \\
32590633 \text {, } \\
\text { Netherlands }\end{array}$} & $\begin{array}{l}\text { NRCS } \\
\text { (None) } \\
(2008- \\
2018)\end{array}$ & $\begin{array}{l}\text { Mode } \\
\text { rate }\end{array}$ & $\begin{array}{l}\text { I: Mastectomy for } \\
\text { breast cancer }\end{array}$ & IBR & NR & $\begin{array}{l}\text { Laterality: Uni } \\
\text { (71.1)/Bi (28.9) } \\
\text { Timing: Imm } \\
\text { (47.6)/Del (52.0)/NR } \\
\text { (0.4) } \\
\text { Chemo: Timing NR } \\
\text { (43.9) } \\
\text { Radio: Timing NR } \\
\text { (22.6) }\end{array}$ & 296 & $60.1(10.0)$ & NR & NR & NR \\
\hline & $\begin{array}{l}\text { NRCS } \\
\text { (None) } \\
(2008- \\
2018)\end{array}$ & $\begin{array}{l}\text { Mode } \\
\text { rate }\end{array}$ & $\begin{array}{l}\text { I: Mastectomy for } \\
\text { breast cancer }\end{array}$ & AR & $\mathrm{N} / \mathrm{A}$ & $\begin{array}{l}\text { Laterality: Uni } \\
\text { (85.4)/Bi (14.6) } \\
\text { Timing: Imm } \\
\text { (15.6)/Del (82.6)/NR } \\
\text { (1.8) } \\
\text { Chemo: Timing NR } \\
\text { (47.6) } \\
\text { Radio: Timing NR } \\
\text { (30.6) }\end{array}$ & 179 & $59.6(9.7)$ & NR & NR & NR \\
\hline & $\begin{array}{l}\text { NRCS } \\
\text { (None) } \\
(2008- \\
2018)\end{array}$ & $\begin{array}{l}\text { Mode } \\
\text { rate }\end{array}$ & $\begin{array}{l}\text { I: Mastectomy for } \\
\text { breast cancer }\end{array}$ & Total & $\mathrm{N} / \mathrm{A}$ & N/A & 475 & NR & NR & NR & NR \\
\hline
\end{tabular}




\begin{tabular}{|c|c|c|c|c|c|c|c|c|c|c|c|}
\hline $\begin{array}{l}\text { Study, } \\
\text { Publication } \\
\text { Year, } \\
\text { PMID, } \\
\text { Country }\end{array}$ & $\begin{array}{l}\text { Design } \\
\text { (Funding) } \\
\text { (Study } \\
\text { Years) }\end{array}$ & $\begin{array}{l}\text { Risk } \\
\text { of } \\
\text { Bias }\end{array}$ & $\begin{array}{l}\text { Eligibility } \\
\text { Criteria }\end{array}$ & Arm & $\begin{array}{l}\text { Implant } \\
\text { Details (\%) } \\
\text { (Only } \\
\text { Reported } \\
\text { Details) }\end{array}$ & $\begin{array}{l}\text { Reconstruction } \\
\text { Details (\%) } \\
\text { (Only Reported } \\
\text { Details) }\end{array}$ & $\mathbf{N}$ & $\begin{array}{l}\text { Age in } \\
\text { Years, } \\
\text { Mean (SD) } \\
\text { or as } \\
\text { Specified } \\
\end{array}$ & $\begin{array}{l}\text { Race } \\
(\%)\end{array}$ & $\begin{array}{l}\text { Breast } \\
\text { Cancer } \\
\text { Occurrence } \\
\%\end{array}$ & $\begin{array}{l}\text { Cancer Stage } \\
\text { or } \\
\text { Mastectomy } \\
\text { Purpose (\%) }\end{array}$ \\
\hline \multirow[t]{5}{*}{$\begin{array}{l}\text { Kulkarni, } \\
2017, \\
28713853, \\
\text { US \& } \\
\text { Canada }\end{array}$} & $\begin{array}{l}\text { NRCS } \\
\text { (Non- } \\
\text { industry) } \\
(2011- \\
2016)\end{array}$ & $\begin{array}{l}\text { Mode } \\
\text { rate }\end{array}$ & $\begin{array}{l}\text { I: Age >=18 years; } \\
\text { first-time, } \\
\text { immediate or } \\
\text { delayed, bilateral } \\
\text { or unilateral } \\
\text { postmastectomy } \\
\text { BR }\end{array}$ & IBR (all) & NR & NR & 1846 & NR & NR & NR & NR \\
\hline & $\begin{array}{l}\text { NRCS } \\
\text { (Non- } \\
\text { industry) } \\
(2011- \\
2016)\end{array}$ & $\begin{array}{l}\text { Mode } \\
\text { rate }\end{array}$ & $\begin{array}{l}\text { I: Age >=18 years; } \\
\text { first-time, } \\
\text { immediate or } \\
\text { delayed, bilateral } \\
\text { or unilateral } \\
\text { postmastectomy } \\
\text { BR }\end{array}$ & $\begin{array}{l}\text { IBR (Direct } \\
\text { to Implant) }\end{array}$ & NR & NR & 79 & NR & NR & NR & NR \\
\hline & $\begin{array}{l}\text { NRCS } \\
\text { (Non- } \\
\text { industry) } \\
(2011- \\
2016)\end{array}$ & $\begin{array}{l}\text { Mode } \\
\text { rate }\end{array}$ & $\begin{array}{l}\text { I: Age >=18 years; } \\
\text { first-time, } \\
\text { immediate or } \\
\text { delayed, bilateral } \\
\text { or unilateral } \\
\text { postmastectomy } \\
\text { BR }\end{array}$ & $\begin{array}{l}\text { IBR } \\
\text { (TE/IBR) }\end{array}$ & NR & NR & 942 & NR & NR & NR & NR \\
\hline & $\begin{array}{l}\text { NRCS } \\
\text { (Non- } \\
\text { industry) } \\
(2011- \\
2016)\end{array}$ & $\begin{array}{l}\text { Mode } \\
\text { rate }\end{array}$ & $\begin{array}{l}\text { I: Age >=18 years; } \\
\text { first-time, } \\
\text { immediate or } \\
\text { delayed, bilateral } \\
\text { or unilateral } \\
\text { postmastectomy } \\
\text { BR }\end{array}$ & AR (all) & $\mathrm{N} / \mathrm{A}$ & NR & 821 & NR & NR & NR & NR \\
\hline & $\begin{array}{l}\text { NRCS } \\
\text { (Non- } \\
\text { industry) } \\
(2011- \\
2016)\end{array}$ & $\begin{array}{l}\text { Mode } \\
\text { rate }\end{array}$ & $\begin{array}{l}\text { I: Age >=18 years; } \\
\text { first-time, } \\
\text { immediate or } \\
\text { delayed, bilateral } \\
\text { or unilateral } \\
\text { postmastectomy } \\
\text { BR }\end{array}$ & $\begin{array}{l}\text { AR with } \\
\text { DIEP }\end{array}$ & $\mathrm{N} / \mathrm{A}$ & NR & 463 & NR & NR & NR & NR \\
\hline
\end{tabular}




\begin{tabular}{|c|c|c|c|c|c|c|c|c|c|c|c|}
\hline $\begin{array}{l}\text { Study, } \\
\text { Publication } \\
\text { Year, } \\
\text { PMID, } \\
\text { Country }\end{array}$ & $\begin{array}{l}\text { Design } \\
\text { (Funding) } \\
\text { (Study } \\
\text { Years) }\end{array}$ & $\begin{array}{l}\text { Risk } \\
\text { of } \\
\text { Bias }\end{array}$ & $\begin{array}{l}\text { Eligibility } \\
\text { Criteria }\end{array}$ & Arm & $\begin{array}{l}\text { Implant } \\
\text { Details (\%) } \\
\text { (Only } \\
\text { Reported } \\
\text { Details) } \\
\end{array}$ & $\begin{array}{l}\text { Reconstruction } \\
\text { Details (\%) } \\
\text { (Only Reported } \\
\text { Details) }\end{array}$ & $\mathbf{N}$ & $\begin{array}{l}\text { Age in } \\
\text { Years, } \\
\text { Mean (SD) } \\
\text { or as } \\
\text { Specified } \\
\end{array}$ & $\begin{array}{l}\text { Race } \\
(\%)\end{array}$ & $\begin{array}{l}\text { Breast } \\
\text { Cancer } \\
\text { Occurrence } \\
\%\end{array}$ & $\begin{array}{l}\text { Cancer Stage } \\
\text { or } \\
\text { Mastectomy } \\
\text { Purpose (\%) }\end{array}$ \\
\hline & $\begin{array}{l}\text { NRCS } \\
\text { (Non- } \\
\text { industry) } \\
\text { (2011- } \\
2016)\end{array}$ & $\begin{array}{l}\text { Mode } \\
\text { rate }\end{array}$ & $\begin{array}{l}\text { I: Age >=18 years; } \\
\text { first-time, } \\
\text { immediate or } \\
\text { delayed, bilateral } \\
\text { or unilateral } \\
\text { postmastectomy } \\
\text { BR }\end{array}$ & $\begin{array}{l}\text { AR with } \\
\text { TRAM }\end{array}$ & $\mathrm{N} / \mathrm{A}$ & NR & 94 & NR & NR & NR & NR \\
\hline & $\begin{array}{l}\text { NRCS } \\
\text { (Non- } \\
\text { industry) } \\
(2011- \\
2016)\end{array}$ & $\begin{array}{l}\text { Mode } \\
\text { rate }\end{array}$ & $\begin{array}{l}\text { I: Age >=18 years; } \\
\text { first-time, } \\
\text { immediate or } \\
\text { delayed, bilateral } \\
\text { or unilateral } \\
\text { postmastectomy } \\
\text { BR }\end{array}$ & $\begin{array}{l}\text { AR with } \\
\text { TRAM }\end{array}$ & $\mathrm{N} / \mathrm{A}$ & NR & 111 & NR & NR & NR & NR \\
\hline & $\begin{array}{l}\text { NRCS } \\
\text { (Non- } \\
\text { industry) } \\
\text { (2011- } \\
2016)\end{array}$ & $\begin{array}{l}\text { Mode } \\
\text { rate }\end{array}$ & $\begin{array}{l}\text { I: Age >=18 years; } \\
\text { first-time, } \\
\text { immediate or } \\
\text { delayed, bilateral } \\
\text { or unilateral } \\
\text { postmastectomy } \\
\text { BR }\end{array}$ & AR with LD & $\mathrm{N} / \mathrm{A}$ & NR & 80 & NR & NR & NR & NR \\
\hline & $\begin{array}{l}\text { NRCS } \\
\text { (Non- } \\
\text { industry) } \\
(2011- \\
2016)\end{array}$ & $\begin{array}{l}\text { Mode } \\
\text { rate }\end{array}$ & $\begin{array}{l}\text { I: Age >=18 years; } \\
\text { first-time, } \\
\text { immediate or } \\
\text { delayed, bilateral } \\
\text { or unilateral } \\
\text { postmastectomy } \\
\text { BR }\end{array}$ & $\begin{array}{l}\text { AR with } \\
\text { SIEA }\end{array}$ & $\mathrm{N} / \mathrm{A}$ & NR & 73 & NR & NR & NR & NR \\
\hline & $\begin{array}{l}\text { NRCS } \\
\text { (Non- } \\
\text { industry) } \\
\text { (2011- } \\
2016)\end{array}$ & $\begin{array}{l}\text { Mode } \\
\text { rate }\end{array}$ & $\begin{array}{l}\text { I: Age >=18 years; } \\
\text { first-time, } \\
\text { immediate or } \\
\text { delayed, bilateral } \\
\text { or unilateral } \\
\text { postmastectomy } \\
\text { BR }\end{array}$ & Total & $\mathrm{N} / \mathrm{A}$ & N/A & 2667 & $49.7(10.1)$ & $\begin{array}{l}\text { W } \\
(87.8), \\
\text { B (6.5), } \\
\text { A (4.7), } \\
\text { Others } \\
(1)\end{array}$ & NR & $\begin{array}{l}\text { Ther (89.7), } \\
\text { Proph (10.3) }\end{array}$ \\
\hline
\end{tabular}




\begin{tabular}{|c|c|c|c|c|c|c|c|c|c|c|c|}
\hline $\begin{array}{l}\text { Study, } \\
\text { Publication } \\
\text { Year, } \\
\text { PMID, } \\
\text { Country }\end{array}$ & $\begin{array}{l}\text { Design } \\
\text { (Funding) } \\
\text { (Study } \\
\text { Years) }\end{array}$ & $\begin{array}{l}\text { Risk } \\
\text { of } \\
\text { Bias }\end{array}$ & $\begin{array}{l}\text { Eligibility } \\
\text { Criteria }\end{array}$ & Arm & $\begin{array}{l}\text { Implant } \\
\text { Details (\%) } \\
\text { (Only } \\
\text { Reported } \\
\text { Details) } \\
\end{array}$ & $\begin{array}{l}\text { Reconstruction } \\
\text { Details (\%) } \\
\text { (Only Reported } \\
\text { Details) }\end{array}$ & $\mathbf{N}$ & $\begin{array}{l}\text { Age in } \\
\text { Years, } \\
\text { Mean (SD) } \\
\text { or as } \\
\text { Specified } \\
\end{array}$ & $\begin{array}{l}\text { Race } \\
(\%)\end{array}$ & $\begin{array}{l}\text { Breast } \\
\text { Cancer } \\
\text { Occurrence } \\
\%\end{array}$ & $\begin{array}{l}\text { Cancer Stage } \\
\text { or } \\
\text { Mastectomy } \\
\text { Purpose (\%) }\end{array}$ \\
\hline \multirow[t]{3}{*}{$\begin{array}{l}\text { Laporta, } \\
2017, \\
28061518, \\
\text { Italy }\end{array}$} & $\begin{array}{l}\text { NRCS } \\
\text { (None) } \\
\text { (2004- } \\
2014)\end{array}$ & High & $\begin{array}{l}\text { I: BR following } \\
\text { mastectomy } \\
\text { E: Local } \\
\text { recurrence } \\
\text { needing surgical } \\
\text { revision }\end{array}$ & IBR & NR & NR & NR & NR & NR & 1st: 100 & NR \\
\hline & $\begin{array}{l}\text { NRCS } \\
\text { (None) } \\
(2004- \\
2014)\end{array}$ & High & $\begin{array}{l}\text { I: BR following } \\
\text { mastectomy } \\
\text { E: Local } \\
\text { recurrence } \\
\text { needing surgical } \\
\text { revision }\end{array}$ & AR & N/A & Stages: 1 (100) & NR & NR & NR & 1st: 100 & NR \\
\hline & 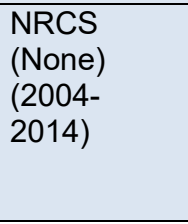 & High & $\begin{array}{l}\text { I: BR following } \\
\text { mastectomy } \\
\text { E: Local } \\
\text { recurrence } \\
\text { needing surgical } \\
\text { revision }\end{array}$ & Total & N/A & $\mathrm{N} / \mathrm{A}$ & 993 & 50.6 (NR) & NR & NR & NR \\
\hline \multirow[t]{3}{*}{$\begin{array}{l}\text { Lei, 2020, } \\
32481367, \\
\text { China }\end{array}$} & $\begin{array}{l}\text { NRCS (NR) } \\
(2012- \\
2016) \\
\end{array}$ & $\begin{array}{l}\text { Mode } \\
\text { rate }\end{array}$ & $\begin{array}{l}\text { I: Single-stage } \\
\text { IBR or AR after } \\
\text { mastectomy }\end{array}$ & IBR & NR & $\begin{array}{l}\text { Timing: Imm (100) } \\
\text { Chemo: Timing NR } \\
\text { (76.1) }\end{array}$ & 226 & NR & NR & NR & $\begin{array}{l}\text { Stage I: } 52.2 \\
\text { Stage II: } 47.8\end{array}$ \\
\hline & $\begin{array}{l}\text { NRCS (NR) } \\
(2012- \\
2016) \\
\end{array}$ & $\begin{array}{l}\text { Mode } \\
\text { rate }\end{array}$ & $\begin{array}{l}\text { I: Single-stage } \\
\text { IBR or AR after } \\
\text { mastectomy }\end{array}$ & AR & $\mathrm{N} / \mathrm{A}$ & $\begin{array}{l}\text { Timing: Imm (100) } \\
\text { Chemo: Timing NR } \\
\text { (72.9) }\end{array}$ & 83 & NR & NR & NR & $\begin{array}{l}\text { Stage I: } 45.9 \\
\text { Stage II: } 54.1\end{array}$ \\
\hline & $\begin{array}{l}\text { NRCS (NR) } \\
(2012- \\
2016) \\
\end{array}$ & $\begin{array}{l}\text { Mode } \\
\text { rate }\end{array}$ & $\begin{array}{l}\text { I: Single-stage } \\
\text { IBR or AR after } \\
\text { mastectomy }\end{array}$ & Total & N/A & N/A & 309 & NR & NR & NR & NR \\
\hline
\end{tabular}




\begin{tabular}{|c|c|c|c|c|c|c|c|c|c|c|c|}
\hline $\begin{array}{l}\text { Study, } \\
\text { Publication } \\
\text { Year, } \\
\text { PMID, } \\
\text { Country }\end{array}$ & $\begin{array}{l}\text { Design } \\
\text { (Funding) } \\
\text { (Study } \\
\text { Years) }\end{array}$ & $\begin{array}{l}\text { Risk } \\
\text { of } \\
\text { Bias }\end{array}$ & $\begin{array}{l}\text { Eligibility } \\
\text { Criteria }\end{array}$ & Arm & $\begin{array}{l}\text { Implant } \\
\text { Details (\%) } \\
\text { (Only } \\
\text { Reported } \\
\text { Details) }\end{array}$ & $\begin{array}{l}\text { Reconstruction } \\
\text { Details (\%) } \\
\text { (Only Reported } \\
\text { Details) }\end{array}$ & $\mathbf{N}$ & $\begin{array}{l}\text { Age in } \\
\text { Years, } \\
\text { Mean (SD) } \\
\text { or as } \\
\text { Specified } \\
\end{array}$ & $\begin{array}{l}\text { Race } \\
(\%)\end{array}$ & $\begin{array}{l}\text { Breast } \\
\text { Cancer } \\
\text { Occurrence } \\
\%\end{array}$ & $\begin{array}{l}\text { Cancer Stage } \\
\text { or } \\
\text { Mastectomy } \\
\text { Purpose (\%) }\end{array}$ \\
\hline \multirow[t]{2}{*}{$\begin{array}{l}\text { Liu, 2014, } \\
\text { 24558063, } \\
\text { US }\end{array}$} & $\begin{array}{l}\text { NRCS } \\
\text { (Non- } \\
\text { industry) } \\
\text { (2007- } \\
2011)\end{array}$ & High & $\begin{array}{l}\text { I: TE/IBR or AR; } \\
\text { without } \\
\text { pre/postoperative } \\
\text { radiotherapy } \\
\text { E: Patients with } \\
\text { previous aesthetic } \\
\text { or reconstructive } \\
\text { breast surgery, } \\
\text { patients who were } \\
\text { active smokers, } \\
\text { and patients with } \\
\text { a follow-up } \\
\text { duration of <6 } \\
\text { months after their } \\
\text { breast } \\
\text { reconstruction }\end{array}$ & IBR & NR & $\begin{array}{l}\text { Laterality: Uni } \\
\text { (45.8)/Bi (54.2) } \\
\text { Timing: Imm } \\
\text { (91.1)/Del (8.9) } \\
\text { Chemo: Timing NR } \\
\text { (34.1)/No } \\
\text { chemotherapy (65.9) } \\
\text { Radio: Timing NR } \\
\text { (100) }\end{array}$ & 179 & $46.9(10.1)$ & NR & NR & NR \\
\hline & $\begin{array}{l}\text { NRCS } \\
\text { (Non- } \\
\text { industry) } \\
\text { (2007- } \\
2011)\end{array}$ & High & $\begin{array}{l}\text { I: TE/IBR or AR; } \\
\text { without } \\
\text { pre/postoperative } \\
\text { radiotherapy } \\
\text { E: Patients with } \\
\text { previous aesthetic } \\
\text { or reconstructive } \\
\text { breast surgery, } \\
\text { patients who were } \\
\text { active smokers, } \\
\text { and patients with } \\
\text { a follow-up } \\
\text { duration of <6 } \\
\text { months after their } \\
\text { breast } \\
\text { reconstruction }\end{array}$ & AR & N/A & $\begin{array}{l}\text { Laterality: Uni (60)/Bi } \\
\text { (40) } \\
\text { Timing: Imm } \\
\text { (65.3)/Del (34.7) } \\
\text { Stages: } 1 \text { (100) } \\
\text { Chemo: Timing NR } \\
\text { (42.7)/No } \\
\text { chemotherapy (57.3) } \\
\text { Radio: Timing NR } \\
\text { (100) }\end{array}$ & 75 & $52.1(8)$ & NR & NR & NR \\
\hline
\end{tabular}




\begin{tabular}{|c|c|c|c|c|c|c|c|c|c|c|c|}
\hline $\begin{array}{l}\text { Study, } \\
\text { Publication } \\
\text { Year, } \\
\text { PMID, } \\
\text { Country }\end{array}$ & $\begin{array}{l}\text { Design } \\
\text { (Funding) } \\
\text { (Study } \\
\text { Years) }\end{array}$ & $\begin{array}{l}\text { Risk } \\
\text { of } \\
\text { Bias }\end{array}$ & $\begin{array}{l}\text { Eligibility } \\
\text { Criteria }\end{array}$ & Arm & $\begin{array}{l}\text { Implant } \\
\text { Details (\%) } \\
\text { (Only } \\
\text { Reported } \\
\text { Details) } \\
\end{array}$ & $\begin{array}{l}\text { Reconstruction } \\
\text { Details (\%) } \\
\text { (Only Reported } \\
\text { Details) }\end{array}$ & $\mathbf{N}$ & $\begin{array}{l}\text { Age in } \\
\text { Years, } \\
\text { Mean (SD) } \\
\text { or as } \\
\text { Specified }\end{array}$ & $\begin{array}{l}\text { Race } \\
(\%)\end{array}$ & $\begin{array}{l}\text { Breast } \\
\text { Cancer } \\
\text { Occurrence } \\
\%\end{array}$ & $\begin{array}{l}\text { Cancer Stage } \\
\text { or } \\
\text { Mastectomy } \\
\text { Purpose (\%) }\end{array}$ \\
\hline & $\begin{array}{l}\text { NRCS } \\
\text { (Non- } \\
\text { industry) } \\
(2007- \\
2011)\end{array}$ & High & $\begin{array}{l}\text { I: TE/IBR or AR; } \\
\text { without } \\
\text { pre/postoperative } \\
\text { radiotherapy } \\
\text { E: Patients with } \\
\text { previous aesthetic } \\
\text { or reconstructive } \\
\text { breast surgery, } \\
\text { patients who were } \\
\text { active smokers, } \\
\text { and patients with } \\
\text { a follow-up } \\
\text { duration of <6 } \\
\text { months after their } \\
\text { breast } \\
\text { reconstruction }\end{array}$ & Total & $\mathrm{N} / \mathrm{A}$ & $\mathrm{N} / \mathrm{A}$ & 254 & NR & NR & NR & NR \\
\hline \multirow[t]{3}{*}{$\begin{array}{l}\text { Mak, 2020, } \\
32665188, \\
\text { China }\end{array}$} & $\begin{array}{l}\text { NRCS (NR) } \\
(2008- \\
2013)\end{array}$ & $\begin{array}{l}\text { Mode } \\
\text { rate }\end{array}$ & $\begin{array}{l}\text { I: Immediate } \\
\text { reconstruction }\end{array}$ & IBR & NR & $\begin{array}{l}\text { Laterality: Uni (70)/Bi } \\
\text { (30) } \\
\text { Timing: Imm (100) } \\
\text { Chemo: Timing NR } \\
\text { (46.7) } \\
\text { Radio: Timing NR } \\
\text { (23.3) }\end{array}$ & 30 & $\begin{array}{l}\text { Median: } \\
39.2 \\
\text { SD: } 7.1\end{array}$ & NR & NR & NR \\
\hline & $\begin{array}{l}\text { NRCS (NR) } \\
(2008- \\
2013)\end{array}$ & $\begin{array}{l}\text { Mode } \\
\text { rate }\end{array}$ & $\begin{array}{l}\text { I: Immediate } \\
\text { reconstruction }\end{array}$ & AR & $\mathrm{N} / \mathrm{A}$ & $\begin{array}{l}\text { Timing: Uni (96.2)/Bi } \\
\text { (3.8) } \\
\text { Timing: Imm (100) } \\
\text { Chemo: Timing NR } \\
\text { (65.3) } \\
\text { Radio: Timing NR } \\
\text { (48.8) }\end{array}$ & 213 & $\begin{array}{l}\text { Median: } \\
46.3 \\
\text { SD: } 7.9\end{array}$ & NR & NR & NR \\
\hline & $\begin{array}{l}\text { NRCS (NR) } \\
(2008- \\
2013)\end{array}$ & $\begin{array}{l}\text { Mode } \\
\text { rate }\end{array}$ & $\begin{array}{l}\text { I: Immediate } \\
\text { reconstruction }\end{array}$ & Total & $\mathrm{N} / \mathrm{A}$ & $\mathrm{N} / \mathrm{A}$ & 243 & $\begin{array}{l}\text { Median: } \\
45.4 \\
\text { SD: } 8.1\end{array}$ & NR & NR & NR \\
\hline
\end{tabular}




\begin{tabular}{|c|c|c|c|c|c|c|c|c|c|c|c|}
\hline $\begin{array}{l}\text { Study, } \\
\text { Publication } \\
\text { Year, } \\
\text { PMID, } \\
\text { Country }\end{array}$ & $\begin{array}{l}\text { Design } \\
\text { (Funding) } \\
\text { (Study } \\
\text { Years) }\end{array}$ & $\begin{array}{l}\text { Risk } \\
\text { of } \\
\text { Bias }\end{array}$ & $\begin{array}{l}\text { Eligibility } \\
\text { Criteria }\end{array}$ & Arm & $\begin{array}{l}\text { Implant } \\
\text { Details (\%) } \\
\text { (Only } \\
\text { Reported } \\
\text { Details) }\end{array}$ & $\begin{array}{l}\text { Reconstruction } \\
\text { Details (\%) } \\
\text { (Only Reported } \\
\text { Details) }\end{array}$ & $\mathbf{N}$ & $\begin{array}{l}\text { Age in } \\
\text { Years, } \\
\text { Mean (SD) } \\
\text { or as } \\
\text { Specified }\end{array}$ & $\begin{array}{l}\text { Race } \\
(\%)\end{array}$ & $\begin{array}{l}\text { Breast } \\
\text { Cancer } \\
\text { Occurrence } \\
\%\end{array}$ & $\begin{array}{l}\text { Cancer Stage } \\
\text { or } \\
\text { Mastectomy } \\
\text { Purpose (\%) }\end{array}$ \\
\hline \multirow[t]{2}{*}{$\begin{array}{l}\text { McCarthy, } \\
2014 \\
24201740, \\
\text { US }\end{array}$} & $\begin{array}{l}\text { NRCS (NR) } \\
(2003- \\
2008)\end{array}$ & $\begin{array}{l}\text { Mode } \\
\text { rate }\end{array}$ & $\begin{array}{l}\text { I: Age >=21 years; } \\
\text { immediate } \\
\text { postmastectomy } \\
\text { two-stage TE/IBR, } \\
\text { or immediate } \\
\text { postmastectomy } \\
\text { AR } \\
\text { E: Prior } \\
\text { irradiation; } \\
\text { delayed } \\
\text { postmastectomy } \\
\text { BR; combined AR } \\
\text { and IBR; local } \\
\text { recurrence of } \\
\text { breast cancer; } \\
\text { and/or history of } \\
\text { complex regional } \\
\text { pain syndrome }\end{array}$ & IBR & NR & $\begin{array}{l}\text { Laterality: Uni } \\
\text { (51.8)/Bi (48.2) } \\
\text { Timing: Imm (100) } \\
\text { Stages: >1 (100) } \\
\text { Chemo: Timing NR } \\
\text { (62) } \\
\text { Radio: Timing NR } \\
\text { (100) }\end{array}$ & 141 & $\begin{array}{l}\text { Median 50; } \\
\text { Range 26, } \\
79\end{array}$ & NR & NR & NR \\
\hline & $\begin{array}{l}\text { NRCS (NR) } \\
(2003- \\
2008)\end{array}$ & $\begin{array}{l}\text { Mode } \\
\text { rate }\end{array}$ & $\begin{array}{l}\text { I: Age >=21 years; } \\
\text { immediate } \\
\text { postmastectomy } \\
\text { two-stage TE/IBR, } \\
\text { or immediate } \\
\text { postmastectomy } \\
\text { AR } \\
\text { E: Prior } \\
\text { irradiation; } \\
\text { delayed } \\
\text { postmastectomy } \\
\text { BR; combined AR } \\
\text { and IBR; local } \\
\text { recurrence of } \\
\text { breast cancer; } \\
\text { and/or history of } \\
\text { complex regional } \\
\text { pain syndrome }\end{array}$ & AR & $\mathrm{N} / \mathrm{A}$ & $\begin{array}{l}\text { Laterality: Uni } \\
\text { (78.9)/Bi }(21.1) \\
\text { Timing: Imm (100) } \\
\text { Stages: } 1(100) \\
\text { Chemo: Timing NR } \\
\text { (40.8) } \\
\text { Radio: Timing NR } \\
\text { (100) }\end{array}$ & 74 & $\begin{array}{l}\text { Median 52; } \\
\text { Range 25, } \\
69\end{array}$ & NR & NR & NR \\
\hline
\end{tabular}




\begin{tabular}{|c|c|c|c|c|c|c|c|c|c|c|c|}
\hline $\begin{array}{l}\text { Study, } \\
\text { Publication } \\
\text { Year, } \\
\text { PMID, } \\
\text { Country }\end{array}$ & $\begin{array}{l}\text { Design } \\
\text { (Funding) } \\
\text { (Study } \\
\text { Years) }\end{array}$ & $\begin{array}{l}\text { Risk } \\
\text { of } \\
\text { Bias }\end{array}$ & $\begin{array}{l}\text { Eligibility } \\
\text { Criteria }\end{array}$ & Arm & $\begin{array}{l}\text { Implant } \\
\text { Details (\%) } \\
\text { (Only } \\
\text { Reported } \\
\text { Details) } \\
\end{array}$ & $\begin{array}{l}\text { Reconstruction } \\
\text { Details (\%) } \\
\text { (Only Reported } \\
\text { Details) }\end{array}$ & $\mathbf{N}$ & $\begin{array}{l}\text { Age in } \\
\text { Years, } \\
\text { Mean (SD) } \\
\text { or as } \\
\text { Specified } \\
\end{array}$ & $\begin{array}{l}\text { Race } \\
(\%)\end{array}$ & $\begin{array}{l}\text { Breast } \\
\text { Cancer } \\
\text { Occurrence } \\
\%\end{array}$ & $\begin{array}{l}\text { Cancer Stage } \\
\text { or } \\
\text { Mastectomy } \\
\text { Purpose (\%) }\end{array}$ \\
\hline & $\begin{array}{l}\text { NRCS (NR) } \\
(2003- \\
2008)\end{array}$ & $\begin{array}{l}\text { Mode } \\
\text { rate }\end{array}$ & $\begin{array}{l}\text { I: Age >=21 years; } \\
\text { immediate } \\
\text { postmastectomy } \\
\text { two-stage TE/IBR, } \\
\text { or immediate } \\
\text { postmastectomy } \\
\text { AR } \\
\text { E: Prior } \\
\text { irradiation; } \\
\text { delayed } \\
\text { postmastectomy } \\
\text { BR; combined AR } \\
\text { and IBR; local } \\
\text { recurrence of } \\
\text { breast cancer; } \\
\text { and/or history of } \\
\text { complex regional } \\
\text { pain syndrome }\end{array}$ & Total & N/A & $\mathrm{N} / \mathrm{A}$ & 218 & NR & NR & NR & NR \\
\hline \multirow{3}{*}{$\begin{array}{l}\text { Merchant, } \\
2015, \\
26111325, \\
\text { US }\end{array}$} & $\begin{array}{l}\text { NRCS (NR) } \\
(2005- \\
2009) \\
\end{array}$ & High & $\begin{array}{l}\text { I: Immediate BR } \\
\text { E: Simultaneous } \\
\text { TE/IBR and AR }\end{array}$ & IBR & NR & Timing: Imm (100) & $\begin{array}{l}1043 \\
7\end{array}$ & NR & NR & NR & NR \\
\hline & $\begin{array}{l}\text { NRCS (NR) } \\
(2005- \\
2009) \\
\end{array}$ & High & $\begin{array}{l}\text { I: Immediate BR } \\
\text { E: Simultaneous } \\
\text { TE/IBR and AR }\end{array}$ & AR & $\mathrm{N} / \mathrm{A}$ & $\begin{array}{l}\text { Timing: Imm (100) } \\
\text { Stages: } 1(100)\end{array}$ & 2329 & NR & NR & NR & NR \\
\hline & $\begin{array}{l}\text { NRCS (NR) } \\
(2005- \\
2009)\end{array}$ & High & $\begin{array}{l}\text { I: Immediate BR } \\
\text { E: Simultaneous } \\
\text { TE/IBR and AR }\end{array}$ & Total & $\mathrm{N} / \mathrm{A}$ & $\mathrm{N} / \mathrm{A}$ & $\begin{array}{l}1276 \\
6\end{array}$ & $\begin{array}{l}18-39 \text { years } \\
(10.6 \%), 40- \\
59 \text { years } \\
(65.8 \%), \geq 60 \\
\text { years } \\
(23.5 \%)\end{array}$ & $\begin{array}{l}\text { W } \\
(70.9), \\
B(4.5), \\
\text { A (8.6), } \\
\text { H (9.9) }\end{array}$ & NR & NR \\
\hline $\begin{array}{l}\text { Mioton, } \\
2013, \\
23562485, \\
\text { US }\end{array}$ & $\begin{array}{l}\text { NRCS } \\
\text { (None) } \\
\text { (2006- } \\
2010)\end{array}$ & $\begin{array}{l}\text { Mode } \\
\text { rate }\end{array}$ & I: AR or TE/IBR & IBR & NR & $\begin{array}{l}\text { Timing: Imm } \\
\text { (92.1)/Del (7.9) }\end{array}$ & 9786 & $\begin{array}{l}51.02 \\
(10.56)\end{array}$ & $\begin{array}{l}\text { W } \\
(80.5), \\
B(6.3), \\
A(2.5), \\
\text { Others } \\
(10.8)\end{array}$ & NR & NR \\
\hline
\end{tabular}




\begin{tabular}{|c|c|c|c|c|c|c|c|c|c|c|c|}
\hline $\begin{array}{l}\text { Study, } \\
\text { Publication } \\
\text { Year, } \\
\text { PMID, } \\
\text { Country }\end{array}$ & $\begin{array}{l}\text { Design } \\
\text { (Funding) } \\
\text { (Study } \\
\text { Years) }\end{array}$ & $\begin{array}{l}\text { Risk } \\
\text { of } \\
\text { Bias }\end{array}$ & $\begin{array}{l}\text { Eligibility } \\
\text { Criteria }\end{array}$ & Arm & $\begin{array}{l}\text { Implant } \\
\text { Details (\%) } \\
\text { (Only } \\
\text { Reported } \\
\text { Details) } \\
\end{array}$ & $\begin{array}{l}\text { Reconstruction } \\
\text { Details (\%) } \\
\text { (Only Reported } \\
\text { Details) }\end{array}$ & $\mathbf{N}$ & $\begin{array}{l}\text { Age in } \\
\text { Years, } \\
\text { Mean (SD) } \\
\text { or as } \\
\text { Specified }\end{array}$ & $\begin{array}{l}\text { Race } \\
(\%)\end{array}$ & $\begin{array}{l}\text { Breast } \\
\text { Cancer } \\
\text { Occurrence } \\
\%\end{array}$ & $\begin{array}{l}\text { Cancer Stage } \\
\text { or } \\
\text { Mastectomy } \\
\text { Purpose (\%) }\end{array}$ \\
\hline & $\begin{array}{l}\text { NRCS } \\
\text { (None) } \\
(2006- \\
2010)\end{array}$ & $\begin{array}{l}\text { Mode } \\
\text { rate }\end{array}$ & I: AR or TE/IBR & AR & N/A & $\begin{array}{l}\text { Timing: Imm } \\
\text { (20.7)/Del (79.3) }\end{array}$ & 3296 & $51.8(9.702)$ & $\begin{array}{l}\text { W } \\
(76.8), \\
B(11), \\
A(2.9), \\
\text { Others } \\
(9.3)\end{array}$ & NR & NR \\
\hline & $\begin{array}{l}\text { NRCS } \\
\text { (None) } \\
\text { (2006- } \\
2010)\end{array}$ & $\begin{array}{l}\text { Mode } \\
\text { rate }\end{array}$ & I: AR or TE/IBR & Total & $\mathrm{N} / \mathrm{A}$ & $\mathrm{N} / \mathrm{A}$ & $\begin{array}{l}1308 \\
2\end{array}$ & NR & NR & NR & NR \\
\hline \multirow[t]{2}{*}{$\begin{array}{l}\text { Momeni, } \\
2018, \\
29095189, \\
\text { US }\end{array}$} & $\begin{array}{l}\text { NRCS } \\
\text { (None) } \\
(2008- \\
2013)\end{array}$ & High & $\begin{array}{l}\text { I: BR following } \\
\text { mastectomy for } \\
\text { breast cancer } \\
\text { E: Lumpectomy } \\
\text { and breast } \\
\text { reconstruction or } \\
\text { lumpectomy and } \\
\text { mastectomy; } \\
\text { multiple } \\
\text { reconstructive } \\
\text { procedures }\end{array}$ & IBR & NR & NR & $\begin{array}{l}1685 \\
1\end{array}$ & NR & NR & NR & NR \\
\hline & $\begin{array}{l}\text { NRCS } \\
\text { (None) } \\
\text { (2008- } \\
2013)\end{array}$ & High & $\begin{array}{l}\text { I: BR following } \\
\text { mastectomy for } \\
\text { breast cancer } \\
\text { E: Lumpectomy } \\
\text { and breast } \\
\text { reconstruction or } \\
\text { lumpectomy and } \\
\text { mastectomy; } \\
\text { multiple } \\
\text { reconstructive } \\
\text { procedures }\end{array}$ & AR & N/A & Stages: 1 (100) & 4622 & NR & NR & NR & NR \\
\hline
\end{tabular}




\begin{tabular}{|c|c|c|c|c|c|c|c|c|c|c|c|}
\hline $\begin{array}{l}\text { Study, } \\
\text { Publication } \\
\text { Year, } \\
\text { PMID, } \\
\text { Country }\end{array}$ & $\begin{array}{l}\text { Design } \\
\text { (Funding) } \\
\text { (Study } \\
\text { Years) }\end{array}$ & $\begin{array}{l}\text { Risk } \\
\text { of } \\
\text { Bias }\end{array}$ & $\begin{array}{l}\text { Eligibility } \\
\text { Criteria }\end{array}$ & Arm & $\begin{array}{l}\text { Implant } \\
\text { Details (\%) } \\
\text { (Only } \\
\text { Reported } \\
\text { Details) } \\
\end{array}$ & $\begin{array}{l}\text { Reconstruction } \\
\text { Details (\%) } \\
\text { (Only Reported } \\
\text { Details) }\end{array}$ & $\mathbf{N}$ & $\begin{array}{l}\text { Age in } \\
\text { Years, } \\
\text { Mean (SD) } \\
\text { or as } \\
\text { Specified }\end{array}$ & $\begin{array}{l}\text { Race } \\
(\%)\end{array}$ & $\begin{array}{l}\text { Breast } \\
\text { Cancer } \\
\text { Occurrence } \\
\%\end{array}$ & $\begin{array}{l}\text { Cancer Stage } \\
\text { or } \\
\text { Mastectomy } \\
\text { Purpose (\%) }\end{array}$ \\
\hline & $\begin{array}{l}\text { NRCS } \\
\text { (None) } \\
\text { (2008- } \\
2013)\end{array}$ & High & $\begin{array}{l}\text { I: BR following } \\
\text { mastectomy for } \\
\text { breast cancer } \\
\text { E: Lumpectomy } \\
\text { and breast } \\
\text { reconstruction or } \\
\text { lumpectomy and } \\
\text { mastectomy; } \\
\text { multiple } \\
\text { reconstructive } \\
\text { procedures }\end{array}$ & Total & $\mathrm{N} / \mathrm{A}$ & $\mathrm{N} / \mathrm{A}$ & $\begin{array}{l}2147 \\
3\end{array}$ & NR & NR & NR & NR \\
\hline \multirow[t]{3}{*}{$\begin{array}{l}\text { Naoum, } \\
2020 a, \\
31756414, \\
\text { US }\end{array}$} & $\begin{array}{l}\text { NRCS (NR) } \\
(1997- \\
2017)\end{array}$ & High & $\begin{array}{l}\text { I: BR } \\
\text { E: Neoadjuvant } \\
\text { chemotherapy; } \\
\text { local recurrence, } \\
\text { bilateral cancer; } \\
\text { different BR on } \\
\text { each breast }\end{array}$ & IBR & NR & $\begin{array}{l}\text { Timing: Imm } \\
\text { (99.8)/Del (0.2) } \\
\text { Stages: } 1(100) \\
\text { Chemo: After (38)/No } \\
\text { chemotherapy (62) } \\
\text { Radio: Timing NR } \\
\text { (41.1)/None(58.9) }\end{array}$ & 416 & $51.3(\mathrm{NR})$ & NR & NR & NR \\
\hline & $\begin{array}{l}\text { NRCS (NR) } \\
(1997- \\
2017)\end{array}$ & High & $\begin{array}{l}\text { I: BR } \\
\text { E: Neoadjuvant } \\
\text { chemotherapy; } \\
\text { local recurrence, } \\
\text { bilateral cancer; } \\
\text { different BR on } \\
\text { each breast }\end{array}$ & $A R$ & $\mathrm{~N} / \mathrm{A}$ & $\begin{array}{l}\text { Timing: Imm } \\
\text { (85.7)/Del (14.3) } \\
\text { Stages: } 1(100) \\
\text { Chemo: After } \\
\text { (62.7)/No } \\
\text { chemotherapy (37.3) } \\
\text { Radio: Timing NR } \\
\text { (39.2)/None(60.8) }\end{array}$ & 311 & 49.1 (NR) & NR & NR & NR \\
\hline & $\begin{array}{l}\text { NRCS (NR) } \\
(1997- \\
2017)\end{array}$ & High & $\begin{array}{l}\text { I: BR } \\
\text { E: Neoadjuvant } \\
\text { chemotherapy; } \\
\text { local recurrence, } \\
\text { bilateral cancer; } \\
\text { different BR on } \\
\text { each breast } \\
\end{array}$ & Total & $\mathrm{N} / \mathrm{A}$ & $\mathrm{N} / \mathrm{A}$ & 727 & NR & NR & NR & NR \\
\hline
\end{tabular}




\begin{tabular}{|c|c|c|c|c|c|c|c|c|c|c|c|}
\hline $\begin{array}{l}\text { Study, } \\
\text { Publication } \\
\text { Year, } \\
\text { PMID, } \\
\text { Country }\end{array}$ & $\begin{array}{l}\text { Design } \\
\text { (Funding) } \\
\text { (Study } \\
\text { Years) }\end{array}$ & $\begin{array}{l}\text { Risk } \\
\text { of } \\
\text { Bias }\end{array}$ & $\begin{array}{l}\text { Eligibility } \\
\text { Criteria }\end{array}$ & Arm & $\begin{array}{l}\text { Implant } \\
\text { Details (\%) } \\
\text { (Only } \\
\text { Reported } \\
\text { Details) } \\
\end{array}$ & $\begin{array}{l}\text { Reconstruction } \\
\text { Details (\%) } \\
\text { (Only Reported } \\
\text { Details) }\end{array}$ & $\mathbf{N}$ & $\begin{array}{l}\text { Age in } \\
\text { Years, } \\
\text { Mean (SD) } \\
\text { or as } \\
\text { Specified } \\
\end{array}$ & $\begin{array}{l}\text { Race } \\
(\%)\end{array}$ & $\begin{array}{l}\text { Breast } \\
\text { Cancer } \\
\text { Occurrence } \\
\%\end{array}$ & $\begin{array}{l}\text { Cancer Stage } \\
\text { or } \\
\text { Mastectomy } \\
\text { Purpose (\%) }\end{array}$ \\
\hline \multirow[t]{4}{*}{$\begin{array}{l}\text { Naoum, } \\
\text { 2020b, } \\
32607638, \\
\text { US }\end{array}$} & $\begin{array}{l}\text { NRCS } \\
\text { (None) } \\
(2000- \\
2017)\end{array}$ & High & $\begin{array}{l}\text { I: Reconstruction } \\
\text { with neoadjuvant } \\
\text { chemotherapy } \\
\text { and PMRT }\end{array}$ & IBR DTI & $\begin{array}{l}\text { ADM: Yes } \\
(98.4) / \text { No } \\
(1.6)\end{array}$ & $\begin{array}{l}\text { Laterality: Uni } \\
\text { (24.4)/Bi (75.6) } \\
\text { Timing: Imm } \\
\text { (99.2)/Del (0.8) } \\
\text { Stages: } 1(100) \\
\text { Chemo: After (100) } \\
\text { Radio: After (100) }\end{array}$ & 127 & $\begin{array}{l}\text { Median: } \\
42.9(\mathrm{IQR} \\
38.0,50.9)\end{array}$ & NR & NR & $\begin{array}{l}\text { Stage 0: } 23.6 \\
\text { Stage I: } 12.6 \\
\text { Stage II: } 38.6 \\
\text { Stage III: } 23.6 \\
\text { Stage IV: } 1.6\end{array}$ \\
\hline & $\begin{array}{l}\text { NRCS } \\
\text { (None) } \\
(2000- \\
2017)\end{array}$ & High & $\begin{array}{l}\text { I: Reconstruction } \\
\text { with neoadjuvant } \\
\text { chemotherapy } \\
\text { and PMRT }\end{array}$ & IBR TE/I & $\begin{array}{l}\text { ADM: Yes } \\
(60.0) / \mathrm{No} \\
(40.0)\end{array}$ & $\begin{array}{l}\text { Laterality: Uni } \\
\text { (34.1)/Bi (65.9) } \\
\text { Timing: Imm } \\
\text { (92.9)/Del (7.1) } \\
\text { Stages: >1 (100) } \\
\text { Chemo: After (100) } \\
\text { Radio: After (100) }\end{array}$ & 85 & $\begin{array}{l}\text { Median: } \\
44.5(\mathrm{IQR} \\
38.1,50.6)\end{array}$ & NR & NR & $\begin{array}{l}\text { Stage 0: } 12.9 \\
\text { Stage I: } 11.8 \\
\text { Stage II: } 50.6 \\
\text { Stage III: } 24.7 \\
\text { Stage IV: } 0\end{array}$ \\
\hline & $\begin{array}{l}\text { NRCS } \\
\text { (None) } \\
(2000- \\
2017)\end{array}$ & High & $\begin{array}{l}\text { I: Reconstruction } \\
\text { with neoadjuvant } \\
\text { chemotherapy } \\
\text { and PMRT }\end{array}$ & $\mathrm{AR}$ & $\mathrm{N} / \mathrm{A}$ & $\begin{array}{l}\text { Laterality: Uni } \\
\text { (67.0)/Bi (33.0) } \\
\text { Timing: Imm } \\
\text { (31.8)/Del (68.2) } \\
\text { Stages: } 1(100) \\
\text { Chemo: After (100) } \\
\text { Radio: After (100) }\end{array}$ & 88 & $\begin{array}{l}\text { Median: } \\
47.3(\mathrm{IQR} \\
40.8,52.0)\end{array}$ & NR & NR & $\begin{array}{l}\text { Stage 0: } 13.6 \\
\text { Stage I: } 17.0 \\
\text { Stage II: } 31.8 \\
\text { Stage III: } 36.4 \\
\text { Stage IV: } 1.1\end{array}$ \\
\hline & $\begin{array}{l}\text { NRCS } \\
\text { (None) } \\
(2000- \\
2017)\end{array}$ & High & $\begin{array}{l}\text { I: Reconstruction } \\
\text { with neoadjuvant } \\
\text { chemotherapy } \\
\text { and PMRT }\end{array}$ & Total & N/A & N/A & 300 & $\begin{array}{l}\text { Median: } \\
45.0(\mathrm{IQR} \\
38.6,51.2)\end{array}$ & NR & NR & $\begin{array}{l}\text { Stage 0: } 17.6 \\
\text { Stage I: } 13.6 \\
\text { Stage II: } 40.0 \\
\text { Stage III: } 27.6 \\
\text { Stage IV: } 1.0 \\
\end{array}$ \\
\hline \multirow[t]{2}{*}{$\begin{array}{l}\text { Nasser, } \\
2018 \\
30204678, \\
\text { US }\end{array}$} & $\begin{array}{l}\text { NRCS } \\
\text { (Non- } \\
\text { industry) } \\
\text { (2009- } \\
2012)\end{array}$ & High & $\begin{array}{l}\text { I: Adult women; } \\
\text { BR } \\
\text { E: Delayed BR; } \\
\text { LD flaps; } \\
\text { Simultaneous AR } \\
\text { and IBR }\end{array}$ & IBR & NR & Timing: Imm (100) & $\begin{array}{l}2812 \\
4\end{array}$ & NR & NR & NR & NR \\
\hline & $\begin{array}{l}\text { NRCS } \\
\text { (Non- } \\
\text { industry) } \\
\text { (2009- } \\
2012)\end{array}$ & High & $\begin{array}{l}\text { I: Adult women; } \\
\text { BR } \\
\text { E: Delayed BR; } \\
\text { LD flaps; } \\
\text { Simultaneous AR } \\
\text { and IBR }\end{array}$ & $\mathrm{AR}$ & N/A & $\begin{array}{l}\text { Timing: Imm (100) } \\
\text { Stages: } 1(100)\end{array}$ & 4773 & NR & NR & NR & NR \\
\hline
\end{tabular}




\begin{tabular}{|c|c|c|c|c|c|c|c|c|c|c|c|}
\hline \multirow[t]{2}{*}{$\begin{array}{l}\text { Study, } \\
\text { Publication } \\
\text { Year, } \\
\text { PMID, } \\
\text { Country } \\
\end{array}$} & $\begin{array}{l}\text { Design } \\
\text { (Funding) } \\
\text { (Study } \\
\text { Years) }\end{array}$ & $\begin{array}{l}\text { Risk } \\
\text { of } \\
\text { Bias }\end{array}$ & $\begin{array}{l}\text { Eligibility } \\
\text { Criteria }\end{array}$ & Arm & $\begin{array}{l}\text { Implant } \\
\text { Details (\%) } \\
\text { (Only } \\
\text { Reported } \\
\text { Details) } \\
\end{array}$ & $\begin{array}{l}\text { Reconstruction } \\
\text { Details (\%) } \\
\text { (Only Reported } \\
\text { Details) }\end{array}$ & $\mathbf{N}$ & $\begin{array}{l}\text { Age in } \\
\text { Years, } \\
\text { Mean (SD) } \\
\text { or as } \\
\text { Specified } \\
\end{array}$ & $\begin{array}{l}\text { Race } \\
\text { (\%) }\end{array}$ & $\begin{array}{l}\text { Breast } \\
\text { Cancer } \\
\text { Occurrence } \\
\%\end{array}$ & $\begin{array}{l}\text { Cancer Stage } \\
\text { or } \\
\text { Mastectomy } \\
\text { Purpose (\%) }\end{array}$ \\
\hline & $\begin{array}{l}\text { NRCS } \\
\text { (Non- } \\
\text { industry) } \\
\text { (2009- } \\
2012)\end{array}$ & High & $\begin{array}{l}\text { I: Adult women; } \\
\text { BR } \\
\text { E: Delayed BR; } \\
\text { LD flaps; } \\
\text { Simultaneous AR } \\
\text { and IBR }\end{array}$ & Total & N/A & N/A & $\begin{array}{l}3289 \\
7\end{array}$ & NR & NR & NR & NR \\
\hline \multirow[t]{3}{*}{$\begin{array}{l}\text { Nelson, } \\
2019 \\
31356276, \\
\text { US }\end{array}$} & $\begin{array}{l}\text { NRCS } \\
\text { (Non- } \\
\text { industry) } \\
\text { (2009- } \\
2017)\end{array}$ & High & $\begin{array}{l}\text { I: Age>=18 years; } \\
\text { BR after } \\
\text { therapeutic or } \\
\text { prophylactic } \\
\text { mastectomy }\end{array}$ & IBR & NR & $\begin{array}{l}\text { Laterality: Uni } \\
\text { (31.89)/Bi (68.11) } \\
\text { Timing: Imm } \\
\text { (99.93)/Del (0.07) } \\
\text { Chemo: Before } \\
\text { (41.41)/After } \\
\text { (0.27)/No } \\
\text { chemotherapy } \\
\text { (58.19) } \\
\text { Radio: Before } \\
\text { (8.15)/After } \\
\text { (17.67)/None(74.18) }\end{array}$ & 2932 & $\begin{array}{l}49.53 \\
(10.05)\end{array}$ & $\begin{array}{l}\text { W } \\
(85.54), \\
B \\
(6.55), \\
\text { A } \\
\text { (5.05), } \\
\text { Others } \\
(2.86)\end{array}$ & NR & NR \\
\hline & $\begin{array}{l}\text { NRCS } \\
\text { (Non- } \\
\text { industry) } \\
\text { (2009- } \\
2017)\end{array}$ & High & $\begin{array}{l}\text { I: Age>=18 years; } \\
\text { BR after } \\
\text { therapeutic or } \\
\text { prophylactic } \\
\text { mastectomy }\end{array}$ & AR & $\mathrm{N} / \mathrm{A}$ & $\begin{array}{l}\text { Laterality: Uni } \\
\text { (58.33)/Bi (41.67) } \\
\text { Timing: Imm } \\
\text { (58.93)/Del (39.29) } \\
\text { Chemo: Before } \\
\text { (30.95)/After } \\
\text { (3.27)/No } \\
\text { chemotherapy 65.48) } \\
\text { Radio: Before } \\
\text { (9.82)/After } \\
\text { (23.81)/Timing NR } \\
\text { (66.37)/None(66.37) }\end{array}$ & 336 & $29.92(8.11)$ & $\begin{array}{l}\text { W } \\
(74.11), \\
B \\
(13.39), \\
\text { A } \\
(4.46), \\
\text { Others } \\
\text { (8.04) }\end{array}$ & NR & NR \\
\hline & $\begin{array}{l}\text { NRCS } \\
\text { (Non- } \\
\text { industry) } \\
\text { (2009- } \\
2017)\end{array}$ & High & $\begin{array}{l}\text { I: Age>=18 years; } \\
\text { BR after } \\
\text { therapeutic or } \\
\text { prophylactic } \\
\text { mastectomy }\end{array}$ & Total & $\mathrm{N} / \mathrm{A}$ & N/A & 3268 & $49.57(9.87)$ & $\begin{array}{l}\text { W } \\
(63.19), \\
B \\
(7.25), \\
A \\
(4.99), \\
\text { Others } \\
(3.4)\end{array}$ & NR & NR \\
\hline
\end{tabular}




\begin{tabular}{|c|c|c|c|c|c|c|c|c|c|c|c|}
\hline $\begin{array}{l}\text { Study, } \\
\text { Publication } \\
\text { Year, } \\
\text { PMID, } \\
\text { Country }\end{array}$ & $\begin{array}{l}\text { Design } \\
\text { (Funding) } \\
\text { (Study } \\
\text { Years) }\end{array}$ & $\begin{array}{l}\text { Risk } \\
\text { of } \\
\text { Bias }\end{array}$ & $\begin{array}{l}\text { Eligibility } \\
\text { Criteria }\end{array}$ & Arm & $\begin{array}{l}\text { Implant } \\
\text { Details (\%) } \\
\text { (Only } \\
\text { Reported } \\
\text { Details) } \\
\end{array}$ & $\begin{array}{l}\text { Reconstruction } \\
\text { Details (\%) } \\
\text { (Only Reported } \\
\text { Details) }\end{array}$ & $\mathbf{N}$ & $\begin{array}{l}\text { Age in } \\
\text { Years, } \\
\text { Mean (SD) } \\
\text { or as } \\
\text { Specified }\end{array}$ & $\begin{array}{l}\text { Race } \\
(\%)\end{array}$ & $\begin{array}{l}\text { Breast } \\
\text { Cancer } \\
\text { Occurrence } \\
\%\end{array}$ & $\begin{array}{l}\text { Cancer Stage } \\
\text { or } \\
\text { Mastectomy } \\
\text { Purpose }(\%)\end{array}$ \\
\hline \multirow[t]{3}{*}{$\begin{array}{l}\text { Palve, 2020, } \\
32468337 \text {, } \\
\text { Finland }\end{array}$} & $\begin{array}{l}\text { NRCS } \\
\text { (Non- } \\
\text { industry) } \\
(2008- \\
2019) \\
\end{array}$ & $\begin{array}{l}\text { Mode } \\
\text { rate }\end{array}$ & $\begin{array}{l}\text { I: Breast } \\
\text { reconstruction }\end{array}$ & IBR & NR & $\begin{array}{l}\text { Timing: Imm (49)/Del } \\
\text { (51) }\end{array}$ & 51 & NR & NR & NR & NR \\
\hline & $\begin{array}{l}\text { NRCS } \\
\text { (Non- } \\
\text { industry) } \\
(2008- \\
2019) \\
\end{array}$ & $\begin{array}{l}\text { Mode } \\
\text { rate }\end{array}$ & $\begin{array}{l}\text { I: Breast } \\
\text { reconstruction }\end{array}$ & AR & $\mathrm{N} / \mathrm{A}$ & $\begin{array}{l}\text { Timing: Imm (15)/Del } \\
\text { (85) }\end{array}$ & 283 & $\mathrm{NR}$ & $\mathrm{NR}$ & $\mathrm{NR}$ & NR \\
\hline & $\begin{array}{l}\text { NRCS } \\
\text { (Non- } \\
\text { industry) } \\
(2008- \\
2019) \\
\end{array}$ & $\begin{array}{l}\text { Mode } \\
\text { rate }\end{array}$ & $\begin{array}{l}\text { I: Breast } \\
\text { reconstruction }\end{array}$ & Total & $\mathrm{N} / \mathrm{A}$ & $\mathrm{N} / \mathrm{A}$ & 334 & NR & NR & NR & NR \\
\hline $\begin{array}{l}\text { Qin, 2018, } \\
29384865 \text {, } \\
\text { China }\end{array}$ & $\begin{array}{l}\text { NRCS } \\
\text { (Non- } \\
\text { industry) } \\
(2009- \\
2015)\end{array}$ & High & $\begin{array}{l}\text { I: Immediate or } \\
\text { delayed unilateral } \\
\text { BR following } \\
\text { mastectomy } \\
\text { E: Not received } \\
\text { standard adjuvant } \\
\text { treatment; } \\
\text { bilateral BR; } \\
\text { synchronous } \\
\text { bilateral invasive } \\
\text { breast cancer or } \\
\text { metachronous } \\
\text { contralateral } \\
\text { breast cancer }\end{array}$ & IBR & NR & $\begin{array}{l}\text { Laterality: Uni (100) } \\
\text { Timing: Imm (100) } \\
\text { Stages: } 1(100) \\
\text { Chemo: Before } \\
\text { (16.7)/After (70.1) } \\
\text { Radio: Timing NR } \\
\text { (16.7) }\end{array}$ & 54 & $41.2(5.4)$ & NR & NR & NR \\
\hline
\end{tabular}




\begin{tabular}{|c|c|c|c|c|c|c|c|c|c|c|c|}
\hline $\begin{array}{l}\text { Study, } \\
\text { Publication } \\
\text { Year, } \\
\text { PMID, } \\
\text { Country }\end{array}$ & $\begin{array}{l}\text { Design } \\
\text { (Funding) } \\
\text { (Study } \\
\text { Years) }\end{array}$ & $\begin{array}{l}\text { Risk } \\
\text { of } \\
\text { Bias }\end{array}$ & $\begin{array}{l}\text { Eligibility } \\
\text { Criteria }\end{array}$ & Arm & $\begin{array}{l}\text { Implant } \\
\text { Details (\%) } \\
\text { (Only } \\
\text { Reported } \\
\text { Details) } \\
\end{array}$ & $\begin{array}{l}\text { Reconstruction } \\
\text { Details (\%) } \\
\text { (Only Reported } \\
\text { Details) }\end{array}$ & $\mathbf{N}$ & $\begin{array}{l}\text { Age in } \\
\text { Years, } \\
\text { Mean (SD) } \\
\text { or as } \\
\text { Specified } \\
\end{array}$ & $\begin{array}{l}\text { Race } \\
(\%)\end{array}$ & $\begin{array}{l}\text { Breast } \\
\text { Cancer } \\
\text { Occurrence } \\
\%\end{array}$ & $\begin{array}{l}\text { Cancer Stage } \\
\text { or } \\
\text { Mastectomy } \\
\text { Purpose (\%) }\end{array}$ \\
\hline & $\begin{array}{l}\text { NRCS } \\
\text { (Non- } \\
\text { industry) } \\
\text { (2009- } \\
2015)\end{array}$ & High & $\begin{array}{l}\text { I: Immediate or } \\
\text { delayed unilateral } \\
\text { BR following } \\
\text { mastectomy } \\
\text { E: Not received } \\
\text { standard adjuvant } \\
\text { treatment; } \\
\text { bilateral BR; } \\
\text { synchronous } \\
\text { bilateral invasive } \\
\text { breast cancer or } \\
\text { metachronous } \\
\text { contralateral } \\
\text { breast cancer }\end{array}$ & IBR & NR & $\begin{array}{l}\text { Laterality: Uni (100) } \\
\text { Timing: Del (100) } \\
\text { Stages: >1 (100) } \\
\text { Chemo: Before } \\
\text { (31.6)/After (73.7) } \\
\text { Radio: Timing NR } \\
\text { (73.7) }\end{array}$ & 38 & $38.7(4.8)$ & NR & NR & NR \\
\hline & $\begin{array}{l}\text { NRCS } \\
\text { (Non- } \\
\text { industry) } \\
\text { (2009- } \\
2015)\end{array}$ & High & $\begin{array}{l}\text { I: Immediate or } \\
\text { delayed unilateral } \\
\text { BR following } \\
\text { mastectomy } \\
\text { E: Not received } \\
\text { standard adjuvant } \\
\text { treatment; } \\
\text { bilateral BR; } \\
\text { synchronous } \\
\text { bilateral invasive } \\
\text { breast cancer or } \\
\text { metachronous } \\
\text { contralateral } \\
\text { breast cancer }\end{array}$ & AR & $\mathrm{N} / \mathrm{A}$ & $\begin{array}{l}\text { Laterality: Uni (100) } \\
\text { Chemo: Before } \\
\text { (18.6)/After (71.2) } \\
\text { Radio: Timing NR } \\
\text { (33.9) }\end{array}$ & 59 & $43.2(6.5)$ & NR & NR & NR \\
\hline
\end{tabular}




\begin{tabular}{|c|c|c|c|c|c|c|c|c|c|c|c|}
\hline $\begin{array}{l}\text { Study, } \\
\text { Publication } \\
\text { Year, } \\
\text { PMID, } \\
\text { Country }\end{array}$ & $\begin{array}{l}\text { Design } \\
\text { (Funding) } \\
\text { (Study } \\
\text { Years) }\end{array}$ & $\begin{array}{l}\text { Risk } \\
\text { of } \\
\text { Bias }\end{array}$ & $\begin{array}{l}\text { Eligibility } \\
\text { Criteria }\end{array}$ & Arm & $\begin{array}{l}\text { Implant } \\
\text { Details (\%) } \\
\text { (Only } \\
\text { Reported } \\
\text { Details) } \\
\end{array}$ & $\begin{array}{l}\text { Reconstruction } \\
\text { Details (\%) } \\
\text { (Only Reported } \\
\text { Details) }\end{array}$ & $\mathbf{N}$ & $\begin{array}{l}\text { Age in } \\
\text { Years, } \\
\text { Mean (SD) } \\
\text { or as } \\
\text { Specified } \\
\end{array}$ & $\begin{array}{l}\text { Race } \\
\text { (\%) }\end{array}$ & $\begin{array}{l}\text { Breast } \\
\text { Cancer } \\
\text { Occurrence } \\
\%\end{array}$ & $\begin{array}{l}\text { Cancer Stage } \\
\text { or } \\
\text { Mastectomy } \\
\text { Purpose (\%) }\end{array}$ \\
\hline & $\begin{array}{l}\text { NRCS } \\
\text { (Non- } \\
\text { industry) } \\
(2009- \\
2015)\end{array}$ & High & $\begin{array}{l}\text { I: Immediate or } \\
\text { delayed unilateral } \\
\text { BR following } \\
\text { mastectomy } \\
\text { E: Not received } \\
\text { standard adjuvant } \\
\text { treatment; } \\
\text { bilateral BR; } \\
\text { synchronous } \\
\text { bilateral invasive } \\
\text { breast cancer or } \\
\text { metachronous } \\
\text { contralateral } \\
\text { breast cancer }\end{array}$ & Total & N/A & $\mathrm{N} / \mathrm{A}$ & 151 & NR & NR & NR & NR \\
\hline \multirow[t]{3}{*}{$\begin{array}{l}\text { Roth, } 2007, \\
17413877, \\
\text { US }\end{array}$} & $\begin{array}{l}\text { NRCS (NR) } \\
(1994- \\
1998)\end{array}$ & High & $\begin{array}{l}\text { I: Unilateral or } \\
\text { bilateral TE/IBR or } \\
\text { AR with TRAM } \\
\text { flap }\end{array}$ & IBR & NR & NR & 69 & $48.5(9)$ & NR & NR & NR \\
\hline & $\begin{array}{l}\text { NRCS (NR) } \\
(1994- \\
1998)\end{array}$ & High & $\begin{array}{l}\text { I: Unilateral or } \\
\text { bilateral TE/IBR or } \\
\text { AR with TRAM } \\
\text { flap }\end{array}$ & AR & N/A & Stages: 1 (100) & 225 & $48.5(8.7)$ & NR & NR & NR \\
\hline & $\begin{array}{l}\text { NRCS (NR) } \\
(1994- \\
1998)\end{array}$ & High & $\begin{array}{l}\text { I: Unilateral or } \\
\text { bilateral TE/IBR or } \\
\text { AR with TRAM } \\
\text { flap }\end{array}$ & Total & & . & 294 & NR & $\begin{array}{l}\text { W (89), } \\
\text { B (4), A } \\
(1), H \\
(1), \\
\text { Others } \\
\text { (5) }\end{array}$ & NR & NR \\
\hline $\begin{array}{l}\text { Shiraishi, } \\
2020, \\
32589082, \\
\text { Japan }\end{array}$ & $\begin{array}{l}\text { NRCS } \\
\text { (None) } \\
\text { (NR-NR) }\end{array}$ & High & $\begin{array}{l}\text { I: First-time, } \\
\text { immediate IBR } \\
\text { with TE or AR } \\
\text { with DIEP } \\
\text { E: Implant or flap } \\
\text { loss }\end{array}$ & IBR & NR & $\begin{array}{l}\text { Timing: Imm (100) } \\
\text { Stages: >1 (100) }\end{array}$ & 56 & NR & NR & $1^{\text {st: }} 100$ & NR \\
\hline
\end{tabular}




\begin{tabular}{|c|c|c|c|c|c|c|c|c|c|c|c|}
\hline $\begin{array}{l}\text { Study, } \\
\text { Publication } \\
\text { Year, } \\
\text { PMID, } \\
\text { Country }\end{array}$ & $\begin{array}{l}\text { Design } \\
\text { (Funding) } \\
\text { (Study } \\
\text { Years) }\end{array}$ & $\begin{array}{l}\text { Risk } \\
\text { of } \\
\text { Bias }\end{array}$ & $\begin{array}{l}\text { Eligibility } \\
\text { Criteria }\end{array}$ & Arm & $\begin{array}{l}\text { Implant } \\
\text { Details (\%) } \\
\text { (Only } \\
\text { Reported } \\
\text { Details) } \\
\end{array}$ & $\begin{array}{l}\text { Reconstruction } \\
\text { Details (\%) } \\
\text { (Only Reported } \\
\text { Details) }\end{array}$ & $\mathbf{N}$ & $\begin{array}{l}\text { Age in } \\
\text { Years, } \\
\text { Mean (SD) } \\
\text { or as } \\
\text { Specified } \\
\end{array}$ & $\begin{array}{l}\text { Race } \\
(\%)\end{array}$ & $\begin{array}{l}\text { Breast } \\
\text { Cancer } \\
\text { Occurrence } \\
\%\end{array}$ & $\begin{array}{l}\text { Cancer Stage } \\
\text { or } \\
\text { Mastectomy } \\
\text { Purpose (\%) }\end{array}$ \\
\hline & $\begin{array}{l}\text { NRCS } \\
\text { (None) } \\
(20 X X- \\
20 X X)\end{array}$ & High & $\begin{array}{l}\text { I: First-time, } \\
\text { immediate IBR } \\
\text { with TE or AR } \\
\text { with DIEP } \\
\text { E: Implant or flap } \\
\text { loss }\end{array}$ & $\mathrm{AR}$ & N/A & Timing: Imm (100) & 34 & NR & NR & $1^{\text {st: }} 100$ & NR \\
\hline & $\begin{array}{l}\text { NRCS } \\
\text { (None) } \\
(20 X X- \\
20 X X)\end{array}$ & High & $\begin{array}{l}\text { I: First-time, } \\
\text { immediate IBR } \\
\text { with TE or AR } \\
\text { with DIEP } \\
\text { E: Implant or flap } \\
\text { loss }\end{array}$ & Total & $\mathrm{N} / \mathrm{A}$ & $\mathrm{N} / \mathrm{A}$ & 90 & $53.2(13.0)$ & NR & $1^{\text {st: }} 100$ & NR \\
\hline \multirow{3}{*}{$\begin{array}{l}\text { Simon, } \\
2020, \\
33363007, \\
\text { Italy }\end{array}$} & $\begin{array}{l}\text { NRCS (NR) } \\
(2016- \\
2019) \\
\end{array}$ & $\begin{array}{l}\text { Mode } \\
\text { rate }\end{array}$ & $\begin{array}{l}\text { I: IBR or AR with } \\
\text { LD flap }\end{array}$ & IBR & NR & $\begin{array}{l}\text { Laterality: Uni (100) } \\
\text { Timing: Imm (100) }\end{array}$ & 68 & NR & NR & NR & NR \\
\hline & $\begin{array}{l}\text { NRCS (NR) } \\
(2016- \\
2019) \\
\end{array}$ & $\begin{array}{l}\text { Mode } \\
\text { rate }\end{array}$ & $\begin{array}{l}\text { I: IBR or AR with } \\
\text { LD flap }\end{array}$ & AR & N/A & $\begin{array}{l}\text { Laterality: Uni (100) } \\
\text { Timing: Imm (100) }\end{array}$ & 139 & NR & NR & NR & NR \\
\hline & $\begin{array}{l}\text { NRCS (NR) } \\
(2016- \\
2019) \\
\end{array}$ & $\begin{array}{l}\text { Mode } \\
\text { rate }\end{array}$ & $\begin{array}{l}\text { I: IBR or AR with } \\
\text { LD flap }\end{array}$ & Total & $\mathrm{N} / \mathrm{A}$ & $\mathrm{N} / \mathrm{A}$ & 207 & NR & NR & NR & NR \\
\hline \multirow[t]{3}{*}{$\begin{array}{l}\text { Tallroth, } \\
2020, \\
33436336, \\
\text { Sweden }\end{array}$} & $\begin{array}{l}\text { RCT } \\
\text { (None) } \\
(2012- \\
2018) \\
\end{array}$ & $\begin{array}{l}\text { Mode } \\
\text { rate }\end{array}$ & $\begin{array}{l}\text { I: Delayed } \\
\text { reconstruction } \\
\text { without prior } \\
\text { radiation therapy }\end{array}$ & IBR & NR & $\begin{array}{l}\text { Laterality: Uni (100) } \\
\text { Timing: Del (100) } \\
\text { Chemo: Timing NR } \\
\text { (45)/No (55) }\end{array}$ & 29 & $55.8(8.9)$ & NR & NR & NR \\
\hline & $\begin{array}{l}\text { RCT } \\
\text { (None) } \\
(2012- \\
2018) \\
\end{array}$ & $\begin{array}{l}\text { Mode } \\
\text { rate }\end{array}$ & $\begin{array}{l}\text { I: Delayed } \\
\text { reconstruction } \\
\text { without prior } \\
\text { radiation therapy }\end{array}$ & AR & $\mathrm{N} / \mathrm{A}$ & $\begin{array}{l}\text { Laterality: Uni (100) } \\
\text { Timing: Del (100) } \\
\text { Chemo: Timing NR } \\
\text { (57)/No (43) }\end{array}$ & 29 & $52.3(10.0)$ & NR & NR & NR \\
\hline & $\begin{array}{l}\text { RCT } \\
\text { (None) } \\
\text { (2012- } \\
2018) \\
\end{array}$ & $\begin{array}{l}\text { Mode } \\
\text { rate }\end{array}$ & $\begin{array}{l}\text { I: Delayed } \\
\text { reconstruction } \\
\text { without prior } \\
\text { radiation therapy }\end{array}$ & Total & N/A & $\mathrm{N} / \mathrm{A}$ & 73 & $53.7(9.4)$ & NR & NR & NR \\
\hline
\end{tabular}




\begin{tabular}{|c|c|c|c|c|c|c|c|c|c|c|c|}
\hline $\begin{array}{l}\text { Study, } \\
\text { Publication } \\
\text { Year, } \\
\text { PMID, } \\
\text { Country }\end{array}$ & $\begin{array}{l}\text { Design } \\
\text { (Funding) } \\
\text { (Study } \\
\text { Years) }\end{array}$ & $\begin{array}{l}\text { Risk } \\
\text { of } \\
\text { Bias }\end{array}$ & $\begin{array}{l}\text { Eligibility } \\
\text { Criteria }\end{array}$ & Arm & $\begin{array}{l}\text { Implant } \\
\text { Details (\%) } \\
\text { (Only } \\
\text { Reported } \\
\text { Details) } \\
\end{array}$ & $\begin{array}{l}\text { Reconstruction } \\
\text { Details (\%) } \\
\text { (Only Reported } \\
\text { Details) }\end{array}$ & $\mathbf{N}$ & $\begin{array}{l}\text { Age in } \\
\text { Years, } \\
\text { Mean (SD) } \\
\text { or as } \\
\text { Specified }\end{array}$ & $\begin{array}{l}\text { Race } \\
(\%)\end{array}$ & $\begin{array}{l}\text { Breast } \\
\text { Cancer } \\
\text { Occurrence } \\
\%\end{array}$ & $\begin{array}{l}\text { Cancer Stage } \\
\text { or } \\
\text { Mastectomy } \\
\text { Purpose (\%) }\end{array}$ \\
\hline \multirow[t]{3}{*}{$\begin{array}{l}\text { Woo, 2018, } \\
30360958, \\
\text { South Korea }\end{array}$} & $\begin{array}{l}\text { NRCS (NR) } \\
(2008- \\
2013)\end{array}$ & High & $\begin{array}{l}\text { I: Immediate BR } \\
\text { E: Direct-to- } \\
\text { implant BR; } \\
\text { combination of AR } \\
\text { and TE/IBR (e.g., } \\
\text { LD flap); AR with } \\
\text { TRAM flap,; } \\
\text { history of shoulder } \\
\text { joint morbidity, } \\
\text { such as adhesive } \\
\text { capsulitis or } \\
\text { rotator cuff } \\
\text { disease }\end{array}$ & IBR & NR & $\begin{array}{l}\text { Timing: Imm (100) } \\
\text { Chemo: Before } \\
\text { (5.4)/After (48) } \\
\text { Radio: Before } \\
\text { (4)/After (13.5) }\end{array}$ & NR & NR & NR & NR & NR \\
\hline & $\begin{array}{l}\text { NRCS (NR) } \\
(2008- \\
2013)\end{array}$ & High & $\begin{array}{l}\text { I: Immediate BR } \\
\text { E: Direct-to- } \\
\text { implant BR; } \\
\text { combination of AR } \\
\text { and TE/IBR (e.g., } \\
\text { LD flap); AR with } \\
\text { TRAM flap; } \\
\text { history of shoulder } \\
\text { joint morbidity, } \\
\text { such as adhesive } \\
\text { capsulitis or } \\
\text { rotator cuff } \\
\text { disease }\end{array}$ & AR (all) & $\mathrm{N} / \mathrm{A}$ & Timing: Imm (100) & NR & NR & NR & NR & NR \\
\hline & $\begin{array}{l}\text { NRCS (NR) } \\
(2008- \\
2013)\end{array}$ & High & $\begin{array}{l}\text { I: Immediate BR } \\
\text { E: Direct-to- } \\
\text { implant BR; } \\
\text { combination of AR } \\
\text { and TE/IBR (e.g., } \\
\text { LD flap); AR with } \\
\text { TRAM flap,; } \\
\text { history of shoulder } \\
\text { joint morbidity, } \\
\text { such as adhesive } \\
\text { capsulitis or } \\
\text { rotator cuff } \\
\text { disease }\end{array}$ & $\begin{array}{l}\text { AR with } \\
\text { DIEP }\end{array}$ & $\mathrm{N} / \mathrm{A}$ & $\begin{array}{l}\text { Timing: Imm (100) } \\
\text { Chemo: Before } \\
\text { (2.3)/After (40.9) } \\
\text { Radio: Before } \\
\text { (2.2)/After (14.8) }\end{array}$ & NR & NR & NR & NR & NR \\
\hline
\end{tabular}




\begin{tabular}{|c|c|c|c|c|c|c|c|c|c|c|c|}
\hline $\begin{array}{l}\text { Study, } \\
\text { Publication } \\
\text { Year, } \\
\text { PMID, } \\
\text { Country }\end{array}$ & $\begin{array}{l}\text { Design } \\
\text { (Funding) } \\
\text { (Study } \\
\text { Years) }\end{array}$ & $\begin{array}{l}\text { Risk } \\
\text { of } \\
\text { Bias }\end{array}$ & $\begin{array}{l}\text { Eligibility } \\
\text { Criteria }\end{array}$ & Arm & $\begin{array}{l}\text { Implant } \\
\text { Details (\%) } \\
\text { (Only } \\
\text { Reported } \\
\text { Details) }\end{array}$ & $\begin{array}{l}\text { Reconstruction } \\
\text { Details (\%) } \\
\text { (Only Reported } \\
\text { Details) }\end{array}$ & $\mathbf{N}$ & $\begin{array}{l}\text { Age in } \\
\text { Years, } \\
\text { Mean (SD) } \\
\text { or as } \\
\text { Specified }\end{array}$ & $\begin{array}{l}\text { Race } \\
(\%)\end{array}$ & $\begin{array}{l}\text { Breast } \\
\text { Cancer } \\
\text { Occurrence } \\
\%\end{array}$ & $\begin{array}{l}\text { Cancer Stage } \\
\text { or } \\
\text { Mastectomy } \\
\text { Purpose (\%) }\end{array}$ \\
\hline & $\begin{array}{l}\text { NRCS (NR) } \\
(2008- \\
2013)\end{array}$ & High & $\begin{array}{l}\text { I: Immediate BR } \\
\text { E: Direct-to- } \\
\text { implant BR; } \\
\text { combination of AR } \\
\text { and TE/IBR (e.g., } \\
\text { LD flap); AR with } \\
\text { TRAM flap,; } \\
\text { history of shoulder } \\
\text { joint morbidity, } \\
\text { such as adhesive } \\
\text { capsulitis or } \\
\text { rotator cuff } \\
\text { disease }\end{array}$ & AR with LD & N/A & $\begin{array}{l}\text { Timing: Imm (100) } \\
\text { Chemo: Before } \\
\text { (8.6)/After (43.6) } \\
\text { Radio: After (9.4) }\end{array}$ & NR & NR & NR & NR & NR \\
\hline & $\begin{array}{l}\text { NRCS (NR) } \\
(2008- \\
2013)\end{array}$ & High & $\begin{array}{l}\text { I: Immediate BR } \\
\text { E: Direct-to- } \\
\text { implant BR; } \\
\text { combination of AR } \\
\text { and TE/IBR (e.g., } \\
\text { LD flap); AR with } \\
\text { TRAM flap,; } \\
\text { history of shoulder } \\
\text { joint morbidity, } \\
\text { such as adhesive } \\
\text { capsulitis or } \\
\text { rotator cuff } \\
\text { disease }\end{array}$ & Total & $\mathrm{N} / \mathrm{A}$ & N/A & 420 & $43(7.4)$ & NR & NR & NR \\
\hline \multirow[t]{2}{*}{$\begin{array}{l}\text { Wu, 2021, } \\
33740204, \\
\text { South Korea }\end{array}$} & $\begin{array}{l}\text { NRCS } \\
\text { (None) } \\
(2010- \\
2016)\end{array}$ & High & $\begin{array}{l}\text { I: Neoadjuvant } \\
\text { chemotherapy } \\
\text { followed by } \\
\text { mastectomy and } \\
\text { reconstruction }\end{array}$ & IBR & $\begin{array}{l}\text { Surface: } \\
\text { Smo(12.3)/ } \\
\text { Tex }(87.7)\end{array}$ & $\begin{array}{l}\text { Timing: Imm (100) } \\
\text { Stages: } 1(87) />1 \\
\text { (13) } \\
\text { Chemo: Before } \\
\text { (100)/After (13.8) } \\
\text { Chemo: After (45.7) }\end{array}$ & 138 & $\begin{array}{l}\text { Median 40 } \\
\text { (IQR 24, 60) }\end{array}$ & NR & $1^{\text {st: }} 100$ & NR \\
\hline & $\begin{array}{l}\text { NRCS } \\
\text { (None) } \\
(2010- \\
2016)\end{array}$ & High & $\begin{array}{l}\text { I: Neoadjuvant } \\
\text { chemotherapy } \\
\text { followed by } \\
\text { mastectomy and } \\
\text { reconstruction }\end{array}$ & AR & N/A & $\begin{array}{l}\text { Timing: Imm (100) } \\
\text { Chemo: Before } \\
\text { (100)/After (9.4) } \\
\text { Chemo: After (48.9) }\end{array}$ & 276 & $\begin{array}{l}\text { Median } 41 \\
(\text { IQR 23, 61) }\end{array}$ & NR & $1^{\text {st: }} 100$ & NR \\
\hline
\end{tabular}




\begin{tabular}{|c|c|c|c|c|c|c|c|c|c|c|c|}
\hline $\begin{array}{l}\text { Study, } \\
\text { Publication } \\
\text { Year, } \\
\text { PMID, } \\
\text { Country }\end{array}$ & $\begin{array}{l}\text { Design } \\
\text { (Funding) } \\
\text { (Study } \\
\text { Years) }\end{array}$ & $\begin{array}{l}\text { Risk } \\
\text { of } \\
\text { Bias }\end{array}$ & $\begin{array}{l}\text { Eligibility } \\
\text { Criteria }\end{array}$ & Arm & $\begin{array}{l}\text { Implant } \\
\text { Details (\%) } \\
\text { (Only } \\
\text { Reported } \\
\text { Details) }\end{array}$ & $\begin{array}{l}\text { Reconstruction } \\
\text { Details (\%) } \\
\text { (Only Reported } \\
\text { Details) }\end{array}$ & $\mathbf{N}$ & $\begin{array}{l}\text { Age in } \\
\text { Years, } \\
\text { Mean (SD) } \\
\text { or as } \\
\text { Specified }\end{array}$ & $\begin{array}{l}\text { Race } \\
(\%)\end{array}$ & $\begin{array}{l}\text { Breast } \\
\text { Cancer } \\
\text { Occurrence } \\
\%\end{array}$ & $\begin{array}{l}\text { Cancer Stage } \\
\text { or } \\
\text { Mastectomy } \\
\text { Purpose (\%) }\end{array}$ \\
\hline & 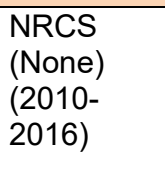 & High & $\begin{array}{l}\text { I: Neoadjuvant } \\
\text { chemotherapy } \\
\text { followed by } \\
\text { mastectomy and } \\
\text { reconstruction }\end{array}$ & Total & $\mathrm{N} / \mathrm{A}$ & $\mathrm{N} / \mathrm{A}$ & 414 & NR & NR & $1^{\text {st: }} 100$ & NR \\
\hline \multirow[t]{3}{*}{$\begin{array}{l}\text { Xu, 2018, } \\
30261115, \\
\text { China }\end{array}$} & $\begin{array}{l}\text { NRCS } \\
\text { (Non- } \\
\text { industry) } \\
(2012- \\
2016)\end{array}$ & $\begin{array}{l}\text { Mode } \\
\text { rate }\end{array}$ & $\begin{array}{l}\text { I: Immediate IBR } \\
\text { or AR } \\
\text { E: Delayed BR or } \\
\text { preoperative } \\
\text { neoadjuvant } \\
\text { chemotherapy; } \\
\text { more than one } \\
\text { type of BR }\end{array}$ & IBR & NR & $\begin{array}{l}\text { Timing: Imm (100) } \\
\text { Stages: } 1(100)\end{array}$ & 326 & $45.2(\mathrm{NR})$ & NR & NR & $\begin{array}{l}\text { Stage } 0(14.4), \\
\text { Stage NR (85) }\end{array}$ \\
\hline & $\begin{array}{l}\text { NRCS } \\
\text { (Non- } \\
\text { industry) } \\
(2012- \\
2016)\end{array}$ & $\begin{array}{l}\text { Mode } \\
\text { rate }\end{array}$ & $\begin{array}{l}\text { I: Immediate IBR } \\
\text { or AR } \\
\text { E: Delayed BR or } \\
\text { preoperative } \\
\text { neoadjuvant } \\
\text { chemotherapy; } \\
\text { more than one } \\
\text { type of BR }\end{array}$ & AR & $\mathrm{N} / \mathrm{A}$ & $\begin{array}{l}\text { Timing: Imm (100) } \\
\text { Stages: } 1(100)\end{array}$ & 100 & 50.8 (NR) & NR & NR & $\begin{array}{l}\text { Stage } 0(11), \\
\text { Stage NR (89) }\end{array}$ \\
\hline & $\begin{array}{l}\text { NRCS } \\
\text { (Non- } \\
\text { industry) } \\
(2012- \\
2016)\end{array}$ & $\begin{array}{l}\text { Mode } \\
\text { rate }\end{array}$ & $\begin{array}{l}\text { I: Immediate IBR } \\
\text { or AR } \\
\text { E: Delayed BR or } \\
\text { preoperative } \\
\text { neoadjuvant } \\
\text { chemotherapy; } \\
\text { more than one } \\
\text { type of BR }\end{array}$ & Total & $\mathrm{N} / \mathrm{A}$ & $\mathrm{N} / \mathrm{A}$ & 426 & NR & NR & NR & NR \\
\hline \multirow[t]{2}{*}{$\begin{array}{l}\text { Yueh, 2009, } \\
19228537, \\
\text { US }\end{array}$} & $\begin{array}{l}\text { NRCS } \\
\text { (Non- } \\
\text { industry) } \\
(1996- \\
2006) \\
\end{array}$ & High & $\begin{array}{l}\text { I: Therapeutic or } \\
\text { prophylactic } \\
\text { mastectomy } \\
\text { E: BR for breast } \\
\text { augmentation only }\end{array}$ & IBR & NR & NR & 87 & NR & NR & NR & NR \\
\hline & $\begin{array}{l}\text { NRCS } \\
\text { (Non- } \\
\text { industry) } \\
\text { (1996- } \\
2006) \\
\end{array}$ & High & $\begin{array}{l}\text { I: Therapeutic or } \\
\text { prophylactic } \\
\text { mastectomy } \\
\text { E: BR for breast } \\
\text { augmentation only }\end{array}$ & AR (all) & $\mathrm{N} / \mathrm{A}$ & Stages: 1 (100) & 675 & NR & NR & NR & NR \\
\hline
\end{tabular}




\begin{tabular}{|c|c|c|c|c|c|c|c|c|c|c|c|}
\hline $\begin{array}{l}\text { Study, } \\
\text { Publication } \\
\text { Year, } \\
\text { PMID, } \\
\text { Country }\end{array}$ & $\begin{array}{l}\text { Design } \\
\text { (Funding) } \\
\text { (Study } \\
\text { Years) }\end{array}$ & $\begin{array}{l}\text { Risk } \\
\text { of } \\
\text { Bias }\end{array}$ & $\begin{array}{l}\text { Eligibility } \\
\text { Criteria }\end{array}$ & Arm & $\begin{array}{l}\text { Implant } \\
\text { Details (\%) } \\
\text { (Only } \\
\text { Reported } \\
\text { Details) } \\
\end{array}$ & $\begin{array}{l}\text { Reconstruction } \\
\text { Details (\%) } \\
\text { (Only Reported } \\
\text { Details) }\end{array}$ & $\mathbf{N}$ & $\begin{array}{l}\text { Age in } \\
\text { Years, } \\
\text { Mean (SD) } \\
\text { or as } \\
\text { Specified } \\
\end{array}$ & $\begin{array}{l}\text { Race } \\
(\%)\end{array}$ & $\begin{array}{l}\text { Breast } \\
\text { Cancer } \\
\text { Occurrence } \\
\%\end{array}$ & $\begin{array}{l}\text { Cancer Stage } \\
\text { or } \\
\text { Mastectomy } \\
\text { Purpose (\%) }\end{array}$ \\
\hline & $\begin{array}{l}\text { NRCS } \\
\text { (Non- } \\
\text { industry) } \\
(1996- \\
2006)\end{array}$ & High & $\begin{array}{l}\text { I: Therapeutic or } \\
\text { prophylactic } \\
\text { mastectomy } \\
\text { E: BR for breast } \\
\text { augmentation only }\end{array}$ & $\begin{array}{l}\text { AR with } \\
\text { DIEP }\end{array}$ & N/A & Stages: 1 (100) & 420 & NR & NR & NR & NR \\
\hline & $\begin{array}{l}\text { NRCS } \\
\text { (Non- } \\
\text { industry) } \\
(1996- \\
2006) \\
\end{array}$ & High & $\begin{array}{l}\text { I: Therapeutic or } \\
\text { prophylactic } \\
\text { mastectomy } \\
\text { E: BR for breast } \\
\text { augmentation only }\end{array}$ & $\begin{array}{l}\text { AR with } \\
\text { TRAM }\end{array}$ & N/A & Stages: $1(100)$ & 143 & NR & $\mathrm{NR}$ & NR & NR \\
\hline & $\begin{array}{l}\text { NRCS } \\
\text { (Non- } \\
\text { industry) } \\
(1996- \\
2006)\end{array}$ & High & $\begin{array}{l}\text { I: Therapeutic or } \\
\text { prophylactic } \\
\text { mastectomy } \\
\text { E: BR for breast } \\
\text { augmentation only }\end{array}$ & AR with LD & N/A & Stages: 1 (100) & 112 & NR & NR & NR & NR \\
\hline & $\begin{array}{l}\text { NRCS } \\
\text { (Non- } \\
\text { industry) } \\
(1996- \\
2006) \\
\end{array}$ & High & $\begin{array}{l}\text { I: Therapeutic or } \\
\text { prophylactic } \\
\text { mastectomy } \\
\text { E: BR for breast } \\
\text { augmentation only }\end{array}$ & Total & N/A & N/A & 762 & NR & NR & NR & NR \\
\hline \multirow[t]{2}{*}{$\begin{array}{l}\text { Zhang, } \\
2019, \\
30675702, \\
\text { China }\end{array}$} & $\begin{array}{l}\text { NRCS (NR) } \\
(2001- \\
2015)\end{array}$ & High & $\begin{array}{l}\text { I: Age 18-65 } \\
\text { years; BR with or } \\
\text { without PMRT } \\
\text { E: Prophylactic } \\
\text { mastectomy and } \\
\text { BR; radiotherapy } \\
\text { for local-regional } \\
\text { recurrences; } \\
\text { bilateral BR }\end{array}$ & IBR & NR & Laterality: Uni (100) & 394 & NR & NR & NR & NR \\
\hline & $\begin{array}{l}\text { NRCS (NR) } \\
(2001- \\
2015)\end{array}$ & High & $\begin{array}{l}\text { I: Age 18-65 } \\
\text { years; BR with or } \\
\text { without PMRT } \\
\text { E: Prophylactic } \\
\text { mastectomy and } \\
\text { BR; radiotherapy } \\
\text { for local-regional } \\
\text { recurrences; } \\
\text { bilateral BR }\end{array}$ & $\mathrm{AR}$ & N/A & $\begin{array}{l}\text { Laterality: Uni (100) } \\
\text { Stages: } 1(100)\end{array}$ & 438 & NR & NR & NR & NR \\
\hline
\end{tabular}




\begin{tabular}{|c|c|c|c|c|c|c|c|c|c|c|c|}
\hline $\begin{array}{l}\text { Study, } \\
\text { Publication } \\
\text { Year, } \\
\text { PMID, } \\
\text { Country }\end{array}$ & $\begin{array}{l}\text { Design } \\
\text { (Funding) } \\
\text { (Study } \\
\text { Years) }\end{array}$ & $\begin{array}{l}\text { Risk } \\
\text { of } \\
\text { Bias }\end{array}$ & $\begin{array}{l}\text { Eligibility } \\
\text { Criteria }\end{array}$ & Arm & $\begin{array}{l}\text { Implant } \\
\text { Details (\%) } \\
\text { (Only } \\
\text { Reported } \\
\text { Details) } \\
\end{array}$ & $\begin{array}{l}\text { Reconstruction } \\
\text { Details (\%) } \\
\text { (Only Reported } \\
\text { Details) }\end{array}$ & $\mathbf{N}$ & $\begin{array}{l}\text { Age in } \\
\text { Years, } \\
\text { Mean (SD) } \\
\text { or as } \\
\text { Specified }\end{array}$ & $\begin{array}{l}\text { Race } \\
(\%)\end{array}$ & $\begin{array}{l}\text { Breast } \\
\text { Cancer } \\
\text { Occurrence } \\
\%\end{array}$ & $\begin{array}{l}\text { Cancer Stage } \\
\text { or } \\
\text { Mastectomy } \\
\text { Purpose (\%) }\end{array}$ \\
\hline & $\begin{array}{l}\text { NRCS (NR) } \\
(2001- \\
2015)\end{array}$ & High & $\begin{array}{l}\text { I: Age 18-65 } \\
\text { years; BR with or } \\
\text { without PMRT } \\
\text { E: Prophylactic } \\
\text { mastectomy and } \\
\text { BR; radiotherapy } \\
\text { for local-regional } \\
\text { recurrences; } \\
\text { bilateral BR }\end{array}$ & Total & N/A & $\mathrm{N} / \mathrm{A}$ & 832 & $\begin{array}{l}<40 \text { years } \\
(60 \%), \geq 40 \\
\text { years }(40 \%)\end{array}$ & NR & NR & NR \\
\hline
\end{tabular}

Blue coloring is only to visually separate different studies.

I: inclusion criteria, E: exclusion criteria

Laterality: whether the reconstruction was unilateral ("Uni”) or bilateral ("Bi"). Stages: Whether the reconstruction was completed in 1 stage or $>1$ stages. Timing: Timing of reconstruction relative to mastectomy, i.e., immediate ("Imm") or delayed ("Del"). Chemo: Timing of chemotherapy relative to reconstruction. Radio: Timing of radiation therapy relative to reconstruction.

$\mathrm{W}=$ White or Caucasian, $\mathrm{B}=$ Black or African American, $\mathrm{A}=$ Asian, $\mathrm{H}=$ Hispanic or Latino.

Proph $=$ prophylactic, Ther $=$ therapeutic.

Abbreviations: $\mathrm{AR}=$ autologous reconstruction, $\mathrm{BR}=$ breast reconstruction, $\mathrm{DIEP}=$ deep inferior epigastric perforator, $\mathrm{IBR}=\mathrm{implant}$-based reconstruction, $\mathrm{IQR}=$ interquartile range, $\mathrm{LD}=$ latissimus dorsi, $\mathrm{NR}=$ not reported, $\mathrm{NRCS}=$ nonrandomized comparative study, $\mathrm{PMID}=\mathrm{PubMed}$ identifier, $\mathrm{PMRT}=$ postmastectomy radiation therapy, $\mathrm{RCT}=$ randomized controlled trial, $\mathrm{SD}=$ standard deviation, $\mathrm{SIEA}=$ superficial inferior epigastric artery perforator, TE/I $=$ tissue expander/implant, Ther $=$ therapeutic, TRAM $=$ transverse rectus abdominis myocutaneous.

Colors: Header rows are shaded orange. Rows for every alternate study are shaded blue. The colors do not add unique information. 
Table C-2. Key Question 1: Implant-based versus autologous reconstruction - single group studies, summary of design, arm, and sample details

\begin{tabular}{|c|c|c|c|c|c|c|c|c|c|c|c|}
\hline $\begin{array}{l}\text { Study, } \\
\text { Publication } \\
\text { Year, } \\
\text { PMID, } \\
\text { Country }\end{array}$ & $\begin{array}{l}\text { Design } \\
\text { (Funding) } \\
\text { (Study } \\
\text { Years) }\end{array}$ & $\begin{array}{l}\text { Risk } \\
\text { of } \\
\text { Bias }\end{array}$ & Eligibility Criteria & Arm & $\begin{array}{l}\text { Implant } \\
\text { Details (\%) } \\
\text { (Only } \\
\text { Reported } \\
\text { Details) } \\
\end{array}$ & $\begin{array}{l}\text { Reconstruction } \\
\text { Details (\%) } \\
\text { (Only Reported } \\
\text { Details) }\end{array}$ & $\mathbf{N}$ & $\begin{array}{l}\text { Age in } \\
\text { Years, } \\
\text { Mean (SD) } \\
\text { or as } \\
\text { Specified }\end{array}$ & $\begin{array}{l}\text { Race } \\
(\%)\end{array}$ & $\begin{array}{l}\text { Breast } \\
\text { Cancer } \\
\text { Occurrence } \\
\%\end{array}$ & $\begin{array}{l}\text { Cancer } \\
\text { Stage or } \\
\text { Mastectomy } \\
\text { Purpose (\%) }\end{array}$ \\
\hline $\begin{array}{l}\text { Acosta, } \\
2011 \\
21046538 \\
\text { Sweden }\end{array}$ & $\begin{array}{l}\text { SGS (NR) } \\
(2000- \\
2009)\end{array}$ & High & $\begin{array}{l}\text { I: ASA classification } 1 \\
\text { or } 2\end{array}$ & AR & $\mathrm{N} / \mathrm{A}$ & $\begin{array}{l}\text { Laterality: Uni } \\
\text { (85.6)/Bi (14.4) } \\
\text { Timing: Imm (16)/Del } \\
\text { (84) } \\
\text { Radio: Before (54.7) }\end{array}$ & 543 & $51(8.7)$ & NR & NR & $\begin{array}{l}\text { Stage I (40), } \\
\text { Stage II (53), } \\
\text { Stage III (7) }\end{array}$ \\
\hline $\begin{array}{l}\text { Albornoz, } \\
2013 \\
23897346 \\
\text { US }\end{array}$ & $\begin{array}{l}\text { SGS } \\
\text { (None) } \\
(1998- \\
2010)\end{array}$ & Low & $\begin{array}{l}\text { I: Breast cancer or } \\
\text { increased risk of breast } \\
\text { cancer who underwent } \\
\text { mastectomy and } \\
\text { immediate AR } \\
\text { E: Delayed BR; LD } \\
\text { flaps; Simultaneous AR } \\
\text { and IBR }\end{array}$ & AR & $\mathrm{N} / \mathrm{A}$ & $\begin{array}{l}\text { Laterality: Uni } \\
\text { (85.4)/Bi (14.6) } \\
\text { Timing: Imm (100) } \\
\text { Stages: } 1(100)\end{array}$ & $\begin{array}{l}2101 \\
6\end{array}$ & $\begin{array}{l}\leq 39 \text { years } \\
(12 \%), 40- \\
49 \text { years } \\
(36.5 \%), \\
50-59 \text { years } \\
(35 \%), \geq 60 \\
\text { years } \\
(16.5 \%)\end{array}$ & $\begin{array}{l}\text { W (77.4), } \\
\text { B (10.5), } \\
\text { A }(2.7), \\
\text { H (6.4), } \\
\text { Others } \\
\text { (3) }\end{array}$ & NR & NR \\
\hline $\begin{array}{l}\text { Andree, } \\
2012, \\
23197233, \\
\text { Germany }\end{array}$ & $\begin{array}{l}\text { SGS (NR) } \\
(2004- \\
2011)\end{array}$ & Low & $\begin{array}{l}\text { I: Skin-sparing or } \\
\text { subcutaneous } \\
\text { mastectomy, with free } \\
\text { AR }\end{array}$ & AR & $\mathrm{N} / \mathrm{A}$ & $\begin{array}{l}\text { Timing: Imm (100) } \\
\text { Stages: } 1(100)\end{array}$ & 940 & NR & NR & NR & $\begin{array}{l}\text { Stage NR } \\
(100)\end{array}$ \\
\hline $\begin{array}{l}\text { Banuelos, } \\
2020, \\
31663932, \\
\text { US }\end{array}$ & $\begin{array}{l}\text { SGS } \\
\text { (None) } \\
(2015- \\
2017)\end{array}$ & Low & $\begin{array}{l}\text { I: Immediate tissue } \\
\text { expander/IBR }\end{array}$ & IBR & NR & Timing: Imm (100) & 768 & $50(10.9)$ & NR & NR & NR \\
\hline $\begin{array}{l}\text { Beugels, } \\
2018, \\
29399731, \\
\text { Netherlands }\end{array}$ & $\begin{array}{l}\text { SGS (NR) } \\
(2010- \\
2017)\end{array}$ & Low & $\begin{array}{l}\text { I: AR with DIEP flap } \\
\text { E: AR with stacked } \\
\text { unilateral or mixed } \\
\text { bilateral (immediate on } \\
\text { one side and delayed } \\
\text { on the other side) }\end{array}$ & AR & $\mathrm{N} / \mathrm{A}$ & $\begin{array}{l}\text { Laterality: Uni } \\
\text { (76.5)/Bi }(23.5) \\
\text { Timing: Imm } \\
\text { (39.5)/Del (60.5) } \\
\text { Stages: } 1 \text { (100) } \\
\text { Chemo: Timing NR } \\
\text { (56.3) } \\
\text { Radio: After } \\
\text { (3)/Timing NR (42.6) }\end{array}$ & 737 & $50.9(\mathrm{NR})$ & NR & NR & $\begin{array}{l}\text { Ther (76.5), } \\
\text { Proph (23.5) }\end{array}$ \\
\hline
\end{tabular}




\begin{tabular}{|c|c|c|c|c|c|c|c|c|c|c|c|}
\hline $\begin{array}{l}\text { Study, } \\
\text { Publication } \\
\text { Year, } \\
\text { PMID, } \\
\text { Country }\end{array}$ & $\begin{array}{l}\text { Design } \\
\text { (Funding) } \\
\text { (Study } \\
\text { Years) }\end{array}$ & $\begin{array}{l}\text { Risk } \\
\text { of } \\
\text { Bias }\end{array}$ & Eligibility Criteria & Arm & $\begin{array}{l}\text { Implant } \\
\text { Details (\%) } \\
\text { (Only } \\
\text { Reported } \\
\text { Details) } \\
\end{array}$ & $\begin{array}{l}\text { Reconstruction } \\
\text { Details (\%) } \\
\text { (Only Reported } \\
\text { Details) }\end{array}$ & $\mathbf{N}$ & $\begin{array}{l}\text { Age in } \\
\text { Years, } \\
\text { Mean (SD) } \\
\text { or as } \\
\text { Specified }\end{array}$ & $\begin{array}{l}\text { Race } \\
(\%)\end{array}$ & $\begin{array}{l}\text { Breast } \\
\text { Cancer } \\
\text { Occurrence } \\
\%\end{array}$ & $\begin{array}{l}\text { Cancer } \\
\text { Stage or } \\
\text { Mastectomy } \\
\text { Purpose (\%) }\end{array}$ \\
\hline $\begin{array}{l}\text { Brooks, } \\
2012, \\
22098451, \\
\text { US }\end{array}$ & $\begin{array}{l}\text { SGS (NR) } \\
(2000- \\
2006)\end{array}$ & Low & $\begin{array}{l}\text { I: Multi-stage IBR } \\
\text { E: Immediate IBR }\end{array}$ & IBR & $\begin{array}{l}\text { PLANE: Total } \\
\text { submuscular } \\
(100)\end{array}$ & $\begin{array}{l}\text { Laterality: Uni } \\
\text { (68.8)/Bi (31.2) } \\
\text { Timing: Del (100) } \\
\text { Stages: >1 (100) } \\
\text { Chemo: Timing NR } \\
\text { (49.4)/No } \\
\text { chemotherapy (50.6) } \\
\text { Radio: Before } \\
\text { (3.7)/After (9.5)/None } \\
(86.7)\end{array}$ & 560 & $\begin{array}{l}<50 \text { years } \\
(58.3 \%) \\
>50 \text { years } \\
(41.7 \%)\end{array}$ & NR & NR & $\begin{array}{l}\text { Ther (73.6), } \\
\text { Proph (22.4) }\end{array}$ \\
\hline $\begin{array}{l}\text { Chang, } \\
2000, \\
10809092, \\
\text { US }\end{array}$ & $\begin{array}{l}\text { SGS (NR) } \\
(1989- \\
1998)\end{array}$ & Low & I: AR with TRAM flap & AR & N/A & $\begin{array}{l}\text { Chemo: Before (78.8) } \\
\text { Radio: Before (13.3) }\end{array}$ & 718 & $\begin{array}{l}<40 \text { years } \\
(22.8 \%), 40- \\
49(44.8 \%) \\
\geq 50 \text { years } \\
(32.3 \%)\end{array}$ & & NR & NR \\
\hline $\begin{array}{l}\text { Chang, } \\
2011, \\
21407063, \\
\text { US }\end{array}$ & $\begin{array}{l}\text { SGS } \\
\text { (None) } \\
(2002- \\
2009)\end{array}$ & Low & I: AR with free flap & AR & $\mathrm{N} / \mathrm{A}$ & $\begin{array}{l}\text { Laterality: Uni } \\
(58.9) / \mathrm{Bi}(41.1)\end{array}$ & 650 & Mean 58.3 & NR & NR & NR \\
\hline $\begin{array}{l}\text { Chang, } \\
2016, \\
25003429, \\
\text { US }\end{array}$ & $\begin{array}{l}\text { SGS } \\
\text { (None) } \\
(2000- \\
2010)\end{array}$ & Low & I: AR with free flap & AR & $\mathrm{N} / \mathrm{A}$ & $\begin{array}{l}\text { Laterality: Uni } \\
\text { (54.3)/Bi (45.7) } \\
\text { Timing: Imm } \\
\text { (35.5)/Del (64.5) }\end{array}$ & 1608 & $49.04(9.1)$ & NR & NR & NR \\
\hline $\begin{array}{l}\text { Chen, 2014, } \\
25620484, \\
\text { China }\end{array}$ & $\begin{array}{l}\text { SGS (NR) } \\
(2012- \\
2012) \\
\end{array}$ & Low & $\begin{array}{l}\text { I: IBR with or without } \\
\text { ADM }\end{array}$ & IBR & NR & NR & 1860 & NR & NR & NR & NR \\
\hline $\begin{array}{l}\text { Chen, 2016, } \\
27930584, \\
\text { Taiwan }\end{array}$ & $\begin{array}{l}\text { SGS } \\
\text { (None) } \\
(1998- \\
2013)\end{array}$ & Low & $\begin{array}{l}\text { I: Immediate IBR } \\
\mathrm{E}: \text { AR or delayed BR }\end{array}$ & IBR & $\begin{array}{l}\text { PLANE: Total } \\
\text { submuscular } \\
(7.9)\end{array}$ & $\begin{array}{l}\text { Timing: Imm (100) } \\
\text { Stages: } 1(5) />1(95) \\
(52.2) / \text { No } \\
\text { chemotherapy (47.8) } \\
(7.4) / \text { None (92.6) }\end{array}$ & 569 & 42.1 (NR) & NR & NR & $\begin{array}{l}\text { Stage 0 } \\
(22.8), \text { Stage } \\
\text { I (32.7), } \\
\text { Stage II } \\
(32.2), \text { Stage } \\
\text { III (10.9), } \\
\text { Stage IV }(0.4)\end{array}$ \\
\hline
\end{tabular}




\begin{tabular}{|c|c|c|c|c|c|c|c|c|c|c|c|}
\hline $\begin{array}{l}\text { Study, } \\
\text { Publication } \\
\text { Year, } \\
\text { PMID, } \\
\text { Country }\end{array}$ & $\begin{array}{l}\text { Design } \\
\text { (Funding) } \\
\text { (Study } \\
\text { Years) }\end{array}$ & $\begin{array}{l}\text { Risk } \\
\text { of } \\
\text { Bias }\end{array}$ & Eligibility Criteria & Arm & $\begin{array}{l}\text { Implant } \\
\text { Details (\%) } \\
\text { (Only } \\
\text { Reported } \\
\text { Details) } \\
\end{array}$ & $\begin{array}{l}\text { Reconstruction } \\
\text { Details (\%) } \\
\text { (Only Reported } \\
\text { Details) }\end{array}$ & $\mathbf{N}$ & $\begin{array}{l}\text { Age in } \\
\text { Years, } \\
\text { Mean (SD) } \\
\text { or as } \\
\text { Specified } \\
\end{array}$ & $\begin{array}{l}\text { Race } \\
(\%)\end{array}$ & $\begin{array}{l}\text { Breast } \\
\text { Cancer } \\
\text { Occurrence } \\
\%\end{array}$ & $\begin{array}{l}\text { Cancer } \\
\text { Stage or } \\
\text { Mastectomy } \\
\text { Purpose (\%) }\end{array}$ \\
\hline $\begin{array}{l}\text { Chen, } \\
2018 a, \\
29596085, \\
\text { US }\end{array}$ & $\begin{array}{l}\text { SGS } \\
\text { (None) } \\
(2010- \\
2014)\end{array}$ & Low & $\begin{array}{l}\text { I: Age >=18 years, } \\
\text { immediate IBR }\end{array}$ & IBR & NR & $\begin{array}{l}\text { Laterality: Uni } \\
\text { (47.5)/Bi (52.5) } \\
\text { Timing: Imm (100) } \\
\text { Chemo: Before (5.2) } \\
\text { Radio: Before (5) }\end{array}$ & $\begin{array}{l}2304 \\
8\end{array}$ & $51.7(\mathrm{NR})$ & $\begin{array}{l}\text { W (77.4), } \\
\text { B (8.5), } \\
\text { A }(2.9), \\
\text { H (6.8), } \\
\text { Other } 1 \\
(0.3), \\
\text { Other } 2 \\
(4.2)\end{array}$ & NR & NR \\
\hline $\begin{array}{l}\text { Chen, } \\
2018 b, \\
29596085, \\
\text { US }\end{array}$ & $\begin{array}{l}\text { SGS } \\
\text { (None) } \\
(2010- \\
2014)\end{array}$ & Low & $\begin{array}{l}\text { I: Age >=18 years, } \\
\text { immediate AR }\end{array}$ & AR & N/A & $\begin{array}{l}\text { Laterality: Uni } \\
\text { (56.9)/Bi (43.1) } \\
\text { Timing: Imm (100) } \\
\text { Stages: } 1(100) \\
\text { Chemo: Before (5.9) } \\
\text { Radio: Before (75.2) }\end{array}$ & $\begin{array}{l}1949 \\
6\end{array}$ & 52.7 (NR) & $\begin{array}{l}\text { W (68.8), } \\
\text { B (14.3), } \\
\text { A (4.4), } \\
\text { H (8.4), } \\
\text { Other } 1 \\
(0.1), \\
\text { Other } 2 \\
(0.4)\end{array}$ & NR & NR \\
\hline $\begin{array}{l}\text { Cleveland, } \\
2013, \\
23945529, \\
\text { US }\end{array}$ & $\begin{array}{l}\text { SGS } \\
\text { (None) } \\
(2005- \\
2011)\end{array}$ & Low & $\mathrm{I}: \mathrm{AR}$ & AR & $\mathrm{N} / \mathrm{A}$ & $\begin{array}{l}\text { Laterality: Uni } \\
\text { (45.1)/Bi (54.9) } \\
\text { Timing: Imm } \\
\text { (75.5)/Del } \\
\text { (21.3)/Mixed (3.2) } \\
\text { Stages: } 1 \text { (100) }\end{array}$ & 812 & 50.2 (NR) & NR & NR & NR \\
\hline $\begin{array}{l}\text { Collier, } \\
2019, \\
31461001, \\
\text { US }\end{array}$ & $\begin{array}{l}\text { SGS (NR) } \\
(2013- \\
2014)\end{array}$ & $\begin{array}{l}\text { Mode } \\
\text { rate }\end{array}$ & I: Age >=18 years, IBR & IBR & NR & NR & $\begin{array}{l}1833 \\
8\end{array}$ & 51.68 (NR) & NR & NR & NR \\
\hline
\end{tabular}




\begin{tabular}{|c|c|c|c|c|c|c|c|c|c|c|c|}
\hline $\begin{array}{l}\text { Study, } \\
\text { Publication } \\
\text { Year, } \\
\text { PMID, } \\
\text { Country }\end{array}$ & $\begin{array}{l}\text { Design } \\
\text { (Funding) } \\
\text { (Study } \\
\text { Years) }\end{array}$ & $\begin{array}{l}\text { Risk } \\
\text { of } \\
\text { Bias }\end{array}$ & Eligibility Criteria & Arm & $\begin{array}{l}\text { Implant } \\
\text { Details (\%) } \\
\text { (Only } \\
\text { Reported } \\
\text { Details) } \\
\end{array}$ & $\begin{array}{l}\text { Reconstruction } \\
\text { Details (\%) } \\
\text { (Only Reported } \\
\text { Details) }\end{array}$ & $\mathbf{N}$ & $\begin{array}{l}\text { Age in } \\
\text { Years, } \\
\text { Mean (SD) } \\
\text { or as } \\
\text { Specified } \\
\end{array}$ & $\begin{array}{l}\text { Race } \\
\text { (\%) }\end{array}$ & $\begin{array}{l}\text { Breast } \\
\text { Cancer } \\
\text { Occurrence } \\
\%\end{array}$ & $\begin{array}{l}\text { Cancer } \\
\text { Stage or } \\
\text { Mastectomy } \\
\text { Purpose (\%) }\end{array}$ \\
\hline $\begin{array}{l}\text { Cordeiro, } \\
2006 \\
16980842 \\
\text { US }\end{array}$ & $\begin{array}{l}\text { SGS (NR) } \\
(1992- \\
2004)\end{array}$ & $\begin{array}{l}\text { Mode } \\
\text { rate }\end{array}$ & $\begin{array}{l}\text { I: Two-stage TE/implant } \\
\text { BR } \\
\text { E: Combined AR plus } \\
\text { TE/implant BR }\end{array}$ & IBR & $\begin{array}{l}\text { MATERIAL: } \\
\text { Silicone } \\
(12.2) / S a l i n e \\
87.8 \% \\
\text { SURFACE: } \\
\text { Textured } \\
\text { (100) } \\
\text { SHAPE: } \\
\text { Anatomic/Tea } \\
\text { rdrop (100) } \\
\text { SIZE: NR } \\
\text { PLANE: Total } \\
\text { submuscular } \\
\text { (100) }\end{array}$ & $\begin{array}{l}\text { Laterality: Uni } \\
\text { (75.3)/Bi (24.7) } \\
\text { Timing: Imm } \\
\text { (96.3)/Del (2.1)/Mixed } \\
\text { (1.6) } \\
\text { Stages: >1 (100) } \\
\text { Chemo: Before (38.2) } \\
\text { Radio: Before } \\
\text { (5.6)/After (15.3) }\end{array}$ & 1221 & NR & NR & NR & NR \\
\hline $\begin{array}{l}\text { Cordeiro, } \\
2012 \\
22286416 \\
\text { US }\end{array}$ & $\begin{array}{l}\text { SGS (NR) } \\
(1997- \\
2018)\end{array}$ & Low & $\begin{array}{l}\text { I: Two-stage TE/implant } \\
\text { BR } \\
\text { E: Combined AR plus } \\
\text { TE/implant BR, } \\
\text { postoperative/periopera } \\
\text { tive radiation therapy, } \\
\text { history of irradiation } \\
\text { because of Hodgkin } \\
\text { disease, delayed BR }\end{array}$ & IBR & $\begin{array}{l}\text { SURFACE: } \\
\text { Textured } \\
(100) \\
\text { PLANE: Total } \\
\text { submuscular } \\
(100)\end{array}$ & $\begin{array}{l}\text { Timing: Imm (100) } \\
\text { Stages: >1 (100) } \\
(7.1) / \text { None }(92.9)\end{array}$ & 1699 & NR & NR & NR & NR \\
\hline $\begin{array}{l}\text { Cordeiro, } \\
2015 b \\
26090764 \\
\text { US }\end{array}$ & $\begin{array}{l}\text { SGS } \\
\text { (Industry) } \\
(2005- \\
2012)\end{array}$ & Low & $\begin{array}{l}\text { I: Age }>=18 \text { years } \\
\text { E: Breast cancer } \\
\text { without mastectomy, } \\
\text { abscess/infection, any } \\
\text { disease known to } \\
\text { impact wound healing }\end{array}$ & IBR & $\begin{array}{l}\text { MATERIAL: } \\
\text { Silicone (100) } \\
\text { SURFACE: } \\
\text { Textured } \\
\text { (100) } \\
\text { SHAPE: } \\
\text { Anatomic/Tea } \\
\text { rdrop (100) } \\
\text { SIZE: NR } \\
\text { PLANE: } \\
\text { Partial } \\
\text { submuscular } \\
\text { (57.8)/Total } \\
\text { submuscular } \\
\text { (37.7) } \\
\end{array}$ & $\begin{array}{l}\text { Laterality: Uni } \\
\text { (24.2)/Bi (76) } \\
\text { Timing: Imm } \\
\text { (86.6)/Del }(12.8) \\
\text { Stages: } 1(8.2) />1 \\
\text { (91.8) }\end{array}$ & 2795 & $\begin{array}{l}\text { Median 50; } \\
\text { Range 18, } \\
82\end{array}$ & $\begin{array}{l}\text { W }(88.1), \\
\text { B }(3.7), \\
\text { A }(2.7), \\
\text { H }(2.3), \\
\text { Others } \\
(1)\end{array}$ & NR & Proph (40.9) \\
\hline
\end{tabular}




\begin{tabular}{|c|c|c|c|c|c|c|c|c|c|c|c|}
\hline $\begin{array}{l}\text { Study, } \\
\text { Publication } \\
\text { Year, } \\
\text { PMID, } \\
\text { Country }\end{array}$ & $\begin{array}{l}\text { Design } \\
\text { (Funding) } \\
\text { (Study } \\
\text { Years) }\end{array}$ & $\begin{array}{l}\text { Risk } \\
\text { of } \\
\text { Bias }\end{array}$ & Eligibility Criteria & Arm & $\begin{array}{l}\text { Implant } \\
\text { Details (\%) } \\
\text { (Only } \\
\text { Reported } \\
\text { Details) }\end{array}$ & $\begin{array}{l}\text { Reconstruction } \\
\text { Details (\%) } \\
\text { (Only Reported } \\
\text { Details) }\end{array}$ & $\mathbf{N}$ & $\begin{array}{l}\text { Age in } \\
\text { Years, } \\
\text { Mean (SD) } \\
\text { or as } \\
\text { Specified }\end{array}$ & $\begin{array}{l}\text { Race } \\
(\%)\end{array}$ & $\begin{array}{l}\text { Breast } \\
\text { Cancer } \\
\text { Occurrence } \\
\%\end{array}$ & $\begin{array}{l}\text { Cancer } \\
\text { Stage or } \\
\text { Mastectomy } \\
\text { Purpose (\%) }\end{array}$ \\
\hline $\begin{array}{l}\text { Coroneos, } \\
2019, \\
30222598, \\
\text { US }\end{array}$ & $\begin{array}{l}\text { SGS (NR) } \\
(2007- \\
2009)\end{array}$ & Low & I: Age >=18 years & IBR & $\begin{array}{l}\text { MATERIAL: } \\
\text { Silicone }(100)\end{array}$ & NR & 5031 & NR & NR & NR & NR \\
\hline $\begin{array}{l}\text { Daly, 2020, } \\
31994156, \\
\text { US }\end{array}$ & $\begin{array}{l}\text { SGS (NR) } \\
(2004- \\
2015)\end{array}$ & Low & I: AR with DIEP flap & AR & $\mathrm{N} / \mathrm{A}$ & $\begin{array}{l}\text { Laterality: Uni } \\
\text { (54.5)/Bi (45.5) } \\
\text { Timing: Imm (100) } \\
\text { Stages: } 1 \text { (100) } \\
\text { Radio: Before } \\
\text { (34.8)/After } \\
\text { (5.5)/None (59.7) }\end{array}$ & 818 & $50.1(\mathrm{NR})$ & NR & NR & NR \\
\hline $\begin{array}{l}\text { Enajat, } \\
2010, \\
19790180, \\
\text { Sweden }\end{array}$ & $\begin{array}{l}\text { SGS } \\
\text { (None) } \\
(2000- \\
2008)\end{array}$ & Low & $\begin{array}{l}\text { I: AR with DIEP flap } \\
\text { E: AR with flaps } \\
\text { supplied by more than } \\
\text { one artery (stacked or } \\
\text { bipedicled flaps) }\end{array}$ & AR & $\mathrm{N} / \mathrm{A}$ & $\begin{array}{l}\text { Laterality: Uni } \\
\text { (87.4)/Bi (12.6) } \\
\text { Timing: Imm } \\
\text { (13.8)/Del (86.2) }\end{array}$ & 501 & $50.6(8.7)$ & NR & NR & NR \\
\hline $\begin{array}{l}\text { Fitzgerald, } \\
2016, \\
27047776, \\
\text { UK }\end{array}$ & $\begin{array}{l}\text { SGS (NR) } \\
(2010- \\
2014)\end{array}$ & Low & I: Microsurgical BR & AR & $\mathrm{N} / \mathrm{A}$ & $\begin{array}{l}\text { Laterality: Uni } \\
\text { (89.2)/Bi (10.8) } \\
\text { Timing: Imm (70)/Del } \\
\text { (30) } \\
\text { Stages: } 1 \text { (100) }\end{array}$ & 1064 & $50(\mathrm{NR})$ & NR & NR & NR \\
\hline $\begin{array}{l}\text { Gfrerer, } \\
2015, \\
25626807, \\
\text { US }\end{array}$ & $\begin{array}{l}\text { SGS (NR) } \\
(2004- \\
2013)\end{array}$ & Low & $\begin{array}{l}\text { I: IBR } \\
\text { E: AR with or without } \\
\text { implants, bilateral } \\
\text { prophylactic } \\
\text { mastectomy for risk } \\
\text { reduction, h/o radiation } \\
\text { therapy to the chest } \\
\text { wall not associated with } \\
\text { breast cancer treatment }\end{array}$ & IBR & NR & $\begin{array}{l}\text { Laterality: Uni } \\
\text { (28.7)/Bi (72.2) } \\
\text { Timing: Imm } \\
\text { (59.8)/Del (40.2) } \\
\text { Chemo: Timing NR } \\
\text { (25) } \\
\text { Radio: Timing NR } \\
\text { (11.3) }\end{array}$ & 3142 & $47.6(9.6)$ & NR & NR & NR \\
\hline $\begin{array}{l}\text { Gill, 2004, } \\
\text { 15083015, } \\
\text { US }\end{array}$ & $\begin{array}{l}\text { SGS (NR) } \\
(1992- \\
2002)\end{array}$ & $\begin{array}{l}\text { Mode } \\
\text { rate }\end{array}$ & I: AR with DIEP flap & AR & $\mathrm{N} / \mathrm{A}$ & $\begin{array}{l}\text { Laterality: Uni } \\
\text { (60.7)/Bi (39.3) } \\
\text { Timing: Imm } \\
\text { (59.9)/Del (40.1) }\end{array}$ & 609 & $\begin{array}{l}48.9(\mathrm{NR}) \\
\text { Range } 16, \\
74\end{array}$ & NR & NR & $\begin{array}{l}\text { Ther (87.7), } \\
\text { Proph (12.3) }\end{array}$ \\
\hline $\begin{array}{l}\text { Haddock, } \\
2019 \\
31461004, \\
\text { US }\end{array}$ & $\begin{array}{l}\text { SGS (NR) } \\
(2010- \\
2018)\end{array}$ & $\begin{array}{l}\text { Mode } \\
\text { rate }\end{array}$ & I: AR with free flap & AR & $\mathrm{N} / \mathrm{A}$ & $\begin{array}{l}\text { Timing: Imm } \\
\text { (43.66)/Del (55.17) } \\
\text { Chemo: Before (12.4) } \\
\text { Radio: Timing NR } \\
\text { (19.8) }\end{array}$ & 509 & $54(\mathrm{NR})$ & NR & NR & NR \\
\hline
\end{tabular}




\begin{tabular}{|c|c|c|c|c|c|c|c|c|c|c|c|}
\hline $\begin{array}{l}\text { Study, } \\
\text { Publication } \\
\text { Year, } \\
\text { PMID, } \\
\text { Country }\end{array}$ & $\begin{array}{l}\text { Design } \\
\text { (Funding) } \\
\text { (Study } \\
\text { Years) }\end{array}$ & $\begin{array}{l}\text { Risk } \\
\text { of } \\
\text { Bias }\end{array}$ & Eligibility Criteria & Arm & $\begin{array}{l}\text { Implant } \\
\text { Details (\%) } \\
\text { (Only } \\
\text { Reported } \\
\text { Details) } \\
\end{array}$ & $\begin{array}{l}\text { Reconstruction } \\
\text { Details (\%) } \\
\text { (Only Reported } \\
\text { Details) }\end{array}$ & $\mathbf{N}$ & $\begin{array}{l}\text { Age in } \\
\text { Years, } \\
\text { Mean (SD) } \\
\text { or as } \\
\text { Specified } \\
\end{array}$ & $\begin{array}{l}\text { Race } \\
\text { (\%) }\end{array}$ & $\begin{array}{l}\text { Breast } \\
\text { Cancer } \\
\text { Occurrence } \\
\%\end{array}$ & $\begin{array}{l}\text { Cancer } \\
\text { Stage or } \\
\text { Mastectomy } \\
\text { Purpose (\%) }\end{array}$ \\
\hline $\begin{array}{l}\text { Haddock, } \\
2020 \\
33487570 \\
\text { US }\end{array}$ & $\begin{array}{l}\text { SGS } \\
\text { (None) } \\
(2009- \\
2018)\end{array}$ & Low & $\begin{array}{l}\text { I: IR with unilateral, } \\
\text { bilateral, or bipedicled } \\
\text { DIEP }\end{array}$ & AR & $\mathrm{N} / \mathrm{A}$ & Laterality: Uni (100) & 644 & NR & NR & NR & NR \\
\hline $\begin{array}{l}\text { Hamdi, } \\
2010, \\
20679823, \\
\text { US }\end{array}$ & $\begin{array}{l}\text { SGS (NR) } \\
(2002- \\
2009)\end{array}$ & Low & $\mathrm{I}: \mathrm{AR}$ & AR & $\mathrm{N} / \mathrm{A}$ & Stages: 1 (100) & 688 & NR & NR & NR & NR \\
\hline $\begin{array}{l}\text { Hamdi, } \\
2011, \\
20576480, \\
\text { Belgium } \\
\end{array}$ & $\begin{array}{l}\text { SGS (NR) } \\
(2002- \\
2009)\end{array}$ & Low & I: IBR & IBR & NR & NR & 688 & NR & NR & NR & NR \\
\hline $\begin{array}{l}\text { Hansen, } \\
2018, \\
29778821 \\
\text { US }\end{array}$ & $\begin{array}{l}\text { SGS (NR) } \\
(2004- \\
2015)\end{array}$ & Low & $\begin{array}{l}\text { I: Immediate 1-stage } \\
\text { BR }\end{array}$ & IBR & NR & $\begin{array}{l}\text { Laterality: Uni } \\
\text { (50.4)/Bi (49.6) } \\
\text { Timing: Imm (100) } \\
\text { Stages: } 1 \text { (100) } \\
\text { Radio: Before } \\
\text { (9.3)/After (31.7) }\end{array}$ & 903 & 49 (NR) & NR & NR & NR \\
\hline $\begin{array}{l}\text { Heo, 2018, } \\
30039735, \\
\text { South Korea }\end{array}$ & $\begin{array}{l}\text { SGS (NR) } \\
(2012- \\
2017)\end{array}$ & Low & $\begin{array}{l}\text { I: AR with a free TRAM } \\
\text { flap } \\
\text { E: AR with bi-pedicled } \\
\text { flap, bilateral } \\
\text { simultaneous } \\
\text { reconstruction, DIEP } \\
\text { flap, muscle-sparing } \\
\text { type } 0 \text { TRAM flap, and } \\
\text { use of foreign materials } \\
\text { such as ADM or mesh }\end{array}$ & AR & $\mathrm{N} / \mathrm{A}$ & $\begin{array}{l}\text { Laterality: Uni (100) } \\
\text { Timing: Imm (82)/Del } \\
\text { (18) } \\
\text { Stages: } 1 \text { (100) } \\
\text { Chemo: Before } \\
\text { (11.9)/After (76.4)/No } \\
\text { chemotherapy (11.7) }\end{array}$ & 615 & $48.4(7.8)$ & NR & NR & NR \\
\hline $\begin{array}{l}\text { Hunsicker, } \\
2017, \\
26849284 \\
\text { US }\end{array}$ & $\begin{array}{l}\text { SGS } \\
\text { (Industry) } \\
(2001- \\
2014)\end{array}$ & Low & $\begin{array}{l}\text { I: Immediate, direct-to- } \\
\text { implant, BR with ADM } \\
\text { E: Delayed, 2-stage } \\
\text { TE/IBR, expandable } \\
\text { implants }\end{array}$ & IBR & $\begin{array}{l}\text { SIZE: Mean } \\
\text { 484.8, SD } \\
\text { 123.8, Range } \\
\text { 100, 800 } \\
\text { PLANE: } \\
\text { Partial } \\
\text { submuscular } \\
(100)\end{array}$ & $\begin{array}{l}\text { Laterality: Uni (100) } \\
\text { Timing: Imm (100) } \\
\text { Stages: } 1(100) \\
\text { Chemo: Timing NR } \\
\text { (73.7) } \\
\text { Radio: Timing NR } \\
\text { (88.4) }\end{array}$ & 863 & $\begin{array}{l}47(10) ; \\
\text { Range 21, } \\
77\end{array}$ & NR & NR & $\begin{array}{l}\text { Ther (35.4), } \\
\text { Proph (64.6) }\end{array}$ \\
\hline
\end{tabular}




\begin{tabular}{|c|c|c|c|c|c|c|c|c|c|c|c|}
\hline $\begin{array}{l}\text { Study, } \\
\text { Publication } \\
\text { Year, } \\
\text { PMID, } \\
\text { Country }\end{array}$ & $\begin{array}{l}\text { Design } \\
\text { (Funding) } \\
\text { (Study } \\
\text { Years) }\end{array}$ & $\begin{array}{l}\text { Risk } \\
\text { of } \\
\text { Bias }\end{array}$ & Eligibility Criteria & Arm & $\begin{array}{l}\text { Implant } \\
\text { Details (\%) } \\
\text { (Only } \\
\text { Reported } \\
\text { Details) }\end{array}$ & $\begin{array}{l}\text { Reconstruction } \\
\text { Details (\%) } \\
\text { (Only Reported } \\
\text { Details) }\end{array}$ & $\mathbf{N}$ & $\begin{array}{l}\text { Age in } \\
\text { Years, } \\
\text { Mean (SD) } \\
\text { or as } \\
\text { Specified }\end{array}$ & $\begin{array}{l}\text { Race } \\
(\%)\end{array}$ & $\begin{array}{l}\text { Breast } \\
\text { Cancer } \\
\text { Occurrence } \\
\%\end{array}$ & $\begin{array}{l}\text { Cancer } \\
\text { Stage or } \\
\text { Mastectomy } \\
\text { Purpose (\%) }\end{array}$ \\
\hline $\begin{array}{l}\text { Jo, } 2020, \\
33386262, \\
\text { South Korea }\end{array}$ & $\begin{array}{l}\text { SGS } \\
\text { (None) } \\
(2010- \\
2019)\end{array}$ & Low & $\begin{array}{l}\text { I: AR with DIEP after } \\
\text { therapeutic mastectomy } \\
\text { without PMRT }\end{array}$ & AR & N/A & NR & 615 & $47.2(0.3)$ & NR & NR & NR \\
\hline $\begin{array}{l}\text { Huo, 2016, } \\
27697676, \\
\text { US }\end{array}$ & $\begin{array}{l}\text { SGS (Non- } \\
\text { industry) } \\
\text { (2009- } \\
2012)\end{array}$ & Low & $\begin{array}{l}\text { I: Age }<65 \text { years } \\
\text { E: Radiation therapy } \\
\text { within } 3 \text { months before } \\
\text { mastectomy; metastatic } \\
\text { disease }\end{array}$ & IBR & NR & NR & 1332 & NR & NR & NR & NR \\
\hline $\begin{array}{l}\text { Kanuri, } \\
2014, \\
24675199, \\
\text { US }\end{array}$ & $\begin{array}{l}\text { SGS (NR) } \\
(2004- \\
2011)\end{array}$ & Low & $\begin{array}{l}\text { I: Immediate IBR } \\
\text { E: Delayed IBR; AR }\end{array}$ & IBR & $\begin{array}{l}\text { ADM: Yes } \\
(66.3), \text { No } \\
(33.7)\end{array}$ & Timing: Imm (100) & 508 & NR & NR & NR & NR \\
\hline $\begin{array}{l}\text { Kato, 2013, } \\
24011080, \\
\text { Japan }\end{array}$ & $\begin{array}{l}\text { SGS (NR) } \\
(2005- \\
2011)\end{array}$ & Low & $\begin{array}{l}\text { I: TE/IBR } \\
\text { E: Bilateral breast } \\
\text { cancer; AR; cancer } \\
\text { recurrence; BR after } \\
\text { mastectomy for reasons } \\
\text { other than breast } \\
\text { cancer }\end{array}$ & IBR & $\begin{array}{l}\text { MATERIAL: } \\
\text { Silicone (100) }\end{array}$ & $\begin{array}{l}\text { Timing: Imm } \\
\text { (54.9)/Del (45.1) } \\
\text { Stages: >1 (100) } \\
\text { Chemo: Before } \\
\text { (9.8)/After (23.8)/No } \\
\text { chemotherapy (66.4) } \\
\text { Radio: Before } \\
\text { (0.1)/After } \\
\text { (5)/None(94.9) }\end{array}$ & 981 & $\begin{array}{l}<50 \text { years } \\
(64.7 \%), \geq 50 \\
\text { years } \\
(35.3 \%)\end{array}$ & NR & 1st: 100 & NR \\
\hline $\begin{array}{l}\text { Langer, } \\
2010, \\
20980954, \\
\text { Germany }\end{array}$ & $\begin{array}{l}\text { SGS (NR) } \\
(2004- \\
2009)\end{array}$ & Low & I: AR with free flaps & AR & $\mathrm{N} / \mathrm{A}$ & $\begin{array}{l}\text { Laterality: Uni } \\
\text { (88.7)/Bi (11.3) } \\
\text { Chemo: Timing NR } \\
\text { (53.9) } \\
\text { Radio: Timing NR } \\
\text { (43.7) }\end{array}$ & 635 & $\begin{array}{l}\text { Mean } 50.3 \\
\text { (NR); Range } \\
25,77\end{array}$ & NR & NR & NR \\
\hline $\begin{array}{l}\text { Lantieri, } \\
2015, \\
26238173, \\
\text { France }\end{array}$ & $\begin{array}{l}\text { SGS (NR) } \\
(1994- \\
2014)\end{array}$ & $\begin{array}{l}\text { Mode } \\
\text { rate }\end{array}$ & I: AR with DIEP flap & AR & $\mathrm{N} / \mathrm{A}$ & $\begin{array}{l}\text { Laterality: Uni } \\
(91.4) / \mathrm{Bi}(8.6)\end{array}$ & 1048 & NR & NR & NR & NR \\
\hline $\begin{array}{l}\text { Law, 2018, } \\
30463754 \\
\text { US }\end{array}$ & $\begin{array}{l}\text { SGS } \\
\text { (None) } \\
(2007- \\
2015)\end{array}$ & Low & $\begin{array}{l}\text { I: TE/IBR } \\
\text { E: AR; diabetes }\end{array}$ & IBR & NR & NR & $\begin{array}{l}1103 \\
9\end{array}$ & NR & NR & NR & NR \\
\hline
\end{tabular}




\begin{tabular}{|c|c|c|c|c|c|c|c|c|c|c|c|}
\hline $\begin{array}{l}\text { Study, } \\
\text { Publication } \\
\text { Year, } \\
\text { PMID, } \\
\text { Country }\end{array}$ & $\begin{array}{l}\text { Design } \\
\text { (Funding) } \\
\text { (Study } \\
\text { Years) }\end{array}$ & $\begin{array}{l}\text { Risk } \\
\text { of } \\
\text { Bias }\end{array}$ & Eligibility Criteria & Arm & $\begin{array}{l}\text { Implant } \\
\text { Details (\%) } \\
\text { (Only } \\
\text { Reported } \\
\text { Details) } \\
\end{array}$ & $\begin{array}{l}\text { Reconstruction } \\
\text { Details (\%) } \\
\text { (Only Reported } \\
\text { Details) }\end{array}$ & $\mathbf{N}$ & $\begin{array}{l}\text { Age in } \\
\text { Years, } \\
\text { Mean (SD) } \\
\text { or as } \\
\text { Specified } \\
\end{array}$ & $\begin{array}{l}\text { Race } \\
(\%)\end{array}$ & $\begin{array}{l}\text { Breast } \\
\text { Cancer } \\
\text { Occurrence } \\
\%\end{array}$ & $\begin{array}{l}\text { Cancer } \\
\text { Stage or } \\
\text { Mastectomy } \\
\text { Purpose (\%) }\end{array}$ \\
\hline $\begin{array}{l}\text { Lee, 2021a, } \\
32974692, \\
\text { South Korea }\end{array}$ & $\begin{array}{l}\text { SGS (NR) } \\
(2010- \\
2016)\end{array}$ & Low & $\begin{array}{l}\text { I: Immediate TE/IBR } \\
\text { E: Hybrid IBR and AR, } \\
\text { adjuvant chemotherapy }\end{array}$ & IBR & $\begin{array}{l}\text { Surface: } \\
\text { Smooth } \\
\text { (44.3)/Textur } \\
\text { ed (55.7) } \\
\text { Shape: } \\
\text { Round } \\
\text { (44.3)/Anato } \\
\text { mic (55.7) } \\
\text { Size: Mean } \\
\text { 331, SD } \\
\text { 102.1 } \\
\text { Plane: } \\
\text { Prepectoral } \\
\text { (100) } \\
\text { ADM: Yes } \\
\text { (60.0), No } \\
\text { (40.0) }\end{array}$ & $\begin{array}{l}\text { Laterality: Uni } \\
\text { (93.5)/Bi (6.5) } \\
\text { Timing: Imm (100) } \\
\text { Stages: } 2 \text { (100) } \\
\text { Chemo: Before } \\
\text { (2.3)/After (39.5) } \\
\text { Radio: After (10.9) }\end{array}$ & 568 & $43.7(7.3)$ & NR & NR & NR \\
\hline $\begin{array}{l}\text { Liao, 2008, } \\
18349626, \\
\text { US }\end{array}$ & $\begin{array}{l}\text { SGS (NR) } \\
(2000- \\
2006)\end{array}$ & Low & $\begin{array}{l}\text { I: AR with TRAM flap } \\
\text { E: AR with other flaps, } \\
\text { e.g., LD, perforator } \\
\text { flaps }\end{array}$ & AR & N/A & $\begin{array}{l}\text { Laterality: Uni } \\
\text { (84.5)/Bi (15.5) } \\
\text { Stages: } 1 \text { (100) }\end{array}$ & 679 & 49 (NR) & NR & NR & $\begin{array}{l}\text { Stage } 0 \\
(34.8), \text { Stage } \\
\text { I (28), Stage } \\
\text { II }(27.4), \\
\text { Stage III } \\
(8.8), \text { Stage } \\
\text { IV }(0.9)\end{array}$ \\
\hline $\begin{array}{l}\text { Lovecchio, } \\
2015, \\
24691330 \\
\text { US }\end{array}$ & $\begin{array}{l}\text { SGS } \\
\text { (None) } \\
(2004- \\
2013)\end{array}$ & Low & I: TE/IBR & IBR & NR & $\begin{array}{l}\text { Laterality: NR } \\
\text { Timing: NR } \\
\text { Stages: >1 (100) } \\
\text { Chemo: Timing NR } \\
\text { (51.8) } \\
\text { Radio: Before } \\
\text { (10)/After (20) }\end{array}$ & 1275 & $\begin{array}{l}47(\mathrm{NR}) \\
\text { Range 41, } \\
56\end{array}$ & NR & NR & NR \\
\hline $\begin{array}{l}\text { Masoomi, } \\
2019 \\
31331721 \\
\text { US }\end{array}$ & $\begin{array}{l}\text { SGS } \\
\text { (None) } \\
(2012- \\
2014)\end{array}$ & Low & $\mathrm{I}: \mathrm{AR}$ & AR & $\mathrm{N} / \mathrm{A}$ & $\begin{array}{l}\text { Stages: } 1(100) \\
\text { Chemo: Before }(11.7) \\
\text { Radio: Before }(16.7)\end{array}$ & $\begin{array}{l}5585 \\
0\end{array}$ & $\begin{array}{l}52(10) ;>65 \\
\text { years } \\
(11.3 \%)\end{array}$ & $\begin{array}{l}\text { W (71), } \\
\text { B (13.5), } \\
\text { A (3.1), } \\
\text { H (8.2), } \\
\text { Other } 1 \\
(0.3), \\
\text { Other } 2 \\
(3.9)\end{array}$ & NR & NR \\
\hline
\end{tabular}




\begin{tabular}{|c|c|c|c|c|c|c|c|c|c|c|c|}
\hline $\begin{array}{l}\text { Study, } \\
\text { Publication } \\
\text { Year, } \\
\text { PMID, } \\
\text { Country }\end{array}$ & $\begin{array}{l}\text { Design } \\
\text { (Funding) } \\
\text { (Study } \\
\text { Years) }\end{array}$ & $\begin{array}{l}\text { Risk } \\
\text { of } \\
\text { Bias }\end{array}$ & Eligibility Criteria & Arm & $\begin{array}{l}\text { Implant } \\
\text { Details (\%) } \\
\text { (Only } \\
\text { Reported } \\
\text { Details) } \\
\end{array}$ & $\begin{array}{l}\text { Reconstruction } \\
\text { Details (\%) } \\
\text { (Only Reported } \\
\text { Details) }\end{array}$ & $\mathbf{N}$ & $\begin{array}{l}\text { Age in } \\
\text { Years, } \\
\text { Mean (SD) } \\
\text { or as } \\
\text { Specified } \\
\end{array}$ & $\begin{array}{l}\text { Race } \\
(\%)\end{array}$ & $\begin{array}{l}\text { Breast } \\
\text { Cancer } \\
\text { Occurrence } \\
\%\end{array}$ & $\begin{array}{l}\text { Cancer } \\
\text { Stage or } \\
\text { Mastectomy } \\
\text { Purpose (\%) }\end{array}$ \\
\hline $\begin{array}{l}\text { Mehrara, } \\
2006, \\
17016173 \\
\text { US }\end{array}$ & $\begin{array}{l}\text { SGS (NR) } \\
(1991- \\
2002)\end{array}$ & $\begin{array}{l}\text { Mode } \\
\text { rate }\end{array}$ & I: Microvascular BR & AR & $\mathrm{N} / \mathrm{A}$ & $\begin{array}{l}\text { Laterality: Uni } \\
\text { (73.5)/Bi (26.5) } \\
\text { Timing: Imm (58)/Del } \\
\text { (42) } \\
\text { Stages: } 1(58) />1 \\
\text { (42) } \\
\text { Chemo: Before (7.7) }\end{array}$ & 952 & $\begin{array}{l}\text { Median } \\
48.9 ; \text { Range } \\
21,79\end{array}$ & NR & NR & NR \\
\hline $\begin{array}{l}\text { Mirzabeigi, } \\
2015 \\
25811579 \\
\text { US }\end{array}$ & $\begin{array}{l}\text { SGS (NR) } \\
(2008- \\
2012)\end{array}$ & Low & $\begin{array}{l}\text { I: AR with free flap } \\
\text { E: AR with other } \\
\text { commonly used free } \\
\text { flaps (e.g., gluteal or } \\
\text { transverse upper } \\
\text { gracilis) }\end{array}$ & AR & $\mathrm{N} / \mathrm{A}$ & $\begin{array}{l}\text { Laterality: Uni (43)/Bi } \\
\text { (57) } \\
\text { Stages: } 1(100) \\
\text { Chemo: Before (40.4) } \\
\text { Radio: Before (29.3) }\end{array}$ & 858 & $51.1(\mathrm{NR})$ & NR & NR & NR \\
\hline $\begin{array}{l}\text { Munder, } \\
2021 \\
32565553 \\
\text { Germany }\end{array}$ & $\begin{array}{l}\text { SGS (NR) } \\
(2004- \\
2014)\end{array}$ & Low & $\begin{array}{l}\text { I: AR with DIEP flap } \\
\text { E: Previous abdominal } \\
\text { or thoracic surgery and } \\
\text { abdominal scarring }\end{array}$ & AR & $\mathrm{N} / \mathrm{A}$ & $\begin{array}{l}\text { Laterality: Uni } \\
\text { (86.7)/Bi (13.3) } \\
\text { Timing: Imm (100) } \\
\text { Chemo: Timing NR } \\
\text { (67.6) } \\
\text { Radio: Timing NR } \\
\text { (58.7) }\end{array}$ & 1124 & $50.0(8.7)$ & NR & NR & NR \\
\hline $\begin{array}{l}\text { Nelson, } \\
2014 \\
25046665 \\
\text { US }\end{array}$ & $\begin{array}{l}\text { SGS } \\
\text { (None) } \\
(2005- \\
2011)\end{array}$ & $\begin{array}{l}\text { Mode } \\
\text { rate }\end{array}$ & I: AR with free flap & AR & $\mathrm{N} / \mathrm{A}$ & $\begin{array}{l}\text { Chemo: After (28.3) } \\
\text { Radio: After (19.1) }\end{array}$ & 848 & NR & $\begin{array}{l}\text { W (77.6), } \\
\text { B (15), A } \\
(2), H \\
(1.9), \\
\text { Others } \\
(3.5)\end{array}$ & NR & NR \\
\hline $\begin{array}{l}\text { O'Neill, } \\
2019 \\
31196805 \\
\text { Canada }\end{array}$ & $\begin{array}{l}\text { SGS } \\
\text { (None) } \\
(2009- \\
2018)\end{array}$ & Low & I: AR with free flap & AR & $\mathrm{N} / \mathrm{A}$ & $\begin{array}{l}\text { Laterality: Uni } \\
\text { (47.9)/Bi (52.1) } \\
\text { Timing: Imm (41)/Del } \\
\text { (59) } \\
\text { Chemo: Timing NR } \\
\text { (54.3) } \\
\text { Radio: Timing NR } \\
\text { (50.6) }\end{array}$ & 960 & $50.5(8.9)$ & NR & NR & NR \\
\hline
\end{tabular}




\begin{tabular}{|c|c|c|c|c|c|c|c|c|c|c|c|}
\hline $\begin{array}{l}\text { Study, } \\
\text { Publication } \\
\text { Year, } \\
\text { PMID, } \\
\text { Country }\end{array}$ & $\begin{array}{l}\text { Design } \\
\text { (Funding) } \\
\text { (Study } \\
\text { Years) }\end{array}$ & $\begin{array}{l}\text { Risk } \\
\text { of } \\
\text { Bias }\end{array}$ & Eligibility Criteria & Arm & $\begin{array}{l}\text { Implant } \\
\text { Details (\%) } \\
\text { (Only } \\
\text { Reported } \\
\text { Details) } \\
\end{array}$ & $\begin{array}{l}\text { Reconstruction } \\
\text { Details (\%) } \\
\text { (Only Reported } \\
\text { Details) }\end{array}$ & $\mathbf{N}$ & $\begin{array}{l}\text { Age in } \\
\text { Years, } \\
\text { Mean (SD) } \\
\text { or as } \\
\text { Specified }\end{array}$ & $\begin{array}{l}\text { Race } \\
(\%)\end{array}$ & $\begin{array}{l}\text { Breast } \\
\text { Cancer } \\
\text { Occurrence } \\
\%\end{array}$ & $\begin{array}{l}\text { Cancer } \\
\text { Stage or } \\
\text { Mastectomy } \\
\text { Purpose (\%) }\end{array}$ \\
\hline $\begin{array}{l}\text { Parikh, } \\
2018, \\
30204676, \\
\text { US }\end{array}$ & $\begin{array}{l}\text { SGS (NR) } \\
(2005- \\
2015)\end{array}$ & Low & $\begin{array}{l}\text { I: Immediate TE/IBR or } \\
\text { direct-to-implant BR, } \\
\text { with placement of } \\
\text { AlloDerm RTU or FD } \\
\text { ADM. } \\
\text { E: Delayed IBR without } \\
\text { use of ADM; ADM or } \\
\text { mesh product other } \\
\text { than AlloDerm RTU or } \\
\text { FD; Prepectoral BR; } \\
\text { Concomitant AR }\end{array}$ & IBR & NR & $\begin{array}{l}\text { Laterality: Uni } \\
\text { (41.3)/Bi (58.7) } \\
\text { Timing: Imm (100) } \\
\text { Stages: } 1(8.2) />1 \\
\text { (91.8) } \\
\text { Chemo: Before } \\
\text { (26.1)/After (35.6)/No } \\
\text { chemotherapy (38.3) } \\
\text { Radio: Before ()/After } \\
\text { (34.2)/None(65.8) }\end{array}$ & 1285 & $49.6(\mathrm{NR})$ & NR & NR & $\begin{array}{l}\text { Stage 0 } \\
(11.2), \text { Stage } \\
\text { I (32.4), } \\
\text { Stage II } \\
\text { (30.3), Stage } \\
\text { III (26.1) }\end{array}$ \\
\hline $\begin{array}{l}\text { Park, 2019, } \\
30863940, \\
\text { South Korea }\end{array}$ & $\begin{array}{l}\text { SGS (NR) } \\
(2009- \\
2017)\end{array}$ & $\begin{array}{l}\text { Mode } \\
\text { rate }\end{array}$ & $\begin{array}{l}\text { I: Immediate BR } \\
\text { E: Antihypertensive } \\
\text { medication only a } \\
\text { month before the } \\
\text { surgery or had } \\
\text { undergone a change in } \\
\text { their treatment regimen } \\
\text { within a month before } \\
\text { the surgery; patients } \\
\text { with hypertension not } \\
\text { on pertinent } \\
\text { medications }\end{array}$ & IBR & NR & $\begin{array}{l}\text { Laterality: Uni } \\
\text { (95.8)/Bi (4.2) } \\
\text { Timing: Imm (100) } \\
\text { Stages: >1 (100) } \\
\text { Chemo: Timing NR } \\
\text { (7.7)/No } \\
\text { chemotherapy (92.3) } \\
\text { Radio: Timing NR } \\
\text { (3.5)/None(96.5) }\end{array}$ & 999 & 43.6 (NR) & NR & NR & NR \\
\hline $\begin{array}{l}\text { Phan, 2020, } \\
31124177, \\
\text { UK }\end{array}$ & $\begin{array}{l}\text { SGS (NR) } \\
(2010- \\
2014)\end{array}$ & Low & I: AR with free flap & AR & $\mathrm{N} / \mathrm{A}$ & $\begin{array}{l}\text { Laterality: Uni (89)/Bi } \\
\text { (11) } \\
\text { Timing: Imm (66)/Del } \\
\text { (34) }\end{array}$ & 1070 & NR & NR & NR & NR \\
\hline $\begin{array}{l}\text { Polanco, } \\
2021 \\
33745850, \\
\text { US }\end{array}$ & $\begin{array}{l}\text { SGS (Non- } \\
\text { industry) } \\
\text { (2010- } \\
2017)\end{array}$ & Low & $\begin{array}{l}\text { I: Age } \geq 18 \text { years; AR } \\
\text { with free, muscle- } \\
\text { sparing, or pedicled } \\
\text { TRAM, DIEP, or SIEA } \\
\text { E: Hybrid IBR and AR }\end{array}$ & AR & $\mathrm{N} / \mathrm{A}$ & $\begin{array}{l}\text { Laterality: Uni } \\
\text { (57.5)/Bi (42.5) } \\
\text { Timing: Imm } \\
\text { (58.9)/Del (41.1) } \\
\text { Stages: } 1 \text { (100) }\end{array}$ & 777 & $\begin{array}{l}\text { Median: } 51 \\
(\text { IQR 44, 56) }\end{array}$ & NR & NR & NR \\
\hline
\end{tabular}




\begin{tabular}{|c|c|c|c|c|c|c|c|c|c|c|c|}
\hline $\begin{array}{l}\text { Study, } \\
\text { Publication } \\
\text { Year, } \\
\text { PMID, } \\
\text { Country }\end{array}$ & $\begin{array}{l}\text { Design } \\
\text { (Funding) } \\
\text { (Study } \\
\text { Years) }\end{array}$ & $\begin{array}{l}\text { Risk } \\
\text { of } \\
\text { Bias }\end{array}$ & Eligibility Criteria & Arm & $\begin{array}{l}\text { Implant } \\
\text { Details (\%) } \\
\text { (Only } \\
\text { Reported } \\
\text { Details) } \\
\end{array}$ & $\begin{array}{l}\text { Reconstruction } \\
\text { Details (\%) } \\
\text { (Only Reported } \\
\text { Details) }\end{array}$ & $\mathbf{N}$ & $\begin{array}{l}\text { Age in } \\
\text { Years, } \\
\text { Mean (SD) } \\
\text { or as } \\
\text { Specified }\end{array}$ & $\begin{array}{l}\text { Race } \\
(\%)\end{array}$ & $\begin{array}{l}\text { Breast } \\
\text { Cancer } \\
\text { Occurrence } \\
\%\end{array}$ & $\begin{array}{l}\text { Cancer } \\
\text { Stage or } \\
\text { Mastectomy } \\
\text { Purpose (\%) }\end{array}$ \\
\hline $\begin{array}{l}\text { Potter, } \\
2019, \\
30639093, \\
\text { UK }\end{array}$ & $\begin{array}{l}\text { SGS (Non- } \\
\text { industry) } \\
\text { (2014- } \\
2016)\end{array}$ & $\begin{array}{l}\text { Mode } \\
\text { rate }\end{array}$ & $\begin{array}{l}\text { I: Age >=16 years; } \\
\text { Immediate IBR } \\
\text { E: Combined IBR and } \\
\text { AR; delayed BR; } \\
\text { revisional surgery }\end{array}$ & IBR & $\begin{array}{l}\text { SIZE: Median } \\
390 \mathrm{~g}, \text { IQR } \\
260,583, \\
\text { Range 39, } \\
2300\end{array}$ & $\begin{array}{l}\text { Laterality: Uni (74)/Bi } \\
\text { (26) } \\
\text { Timing: Imm }(100) \\
\text { Stages: } 1(78) />1 \\
(21) \\
\text { Chemo: Before } \\
\text { (11)/No } \\
\text { chemotherapy (88) }\end{array}$ & 2108 & $\begin{array}{l}\text { Median 49; } \\
\text { IQR 43, 57; } \\
\text { Range 16; } \\
83\end{array}$ & NR & NR & $\begin{array}{l}\text { Stage I (59), } \\
\text { Stage II (37), } \\
\text { Stage III (3) }\end{array}$ \\
\hline $\begin{array}{l}\text { Prantl, 2020, } \\
32895743, \\
\text { Germany }\end{array}$ & $\begin{array}{l}\text { SGS (Non- } \\
\text { industry) } \\
\text { (2011- } \\
2019 \text { ) }\end{array}$ & Low & I: AR with DIEP flap & AR & $\mathrm{N} / \mathrm{A}$ & $\begin{array}{l}\text { Laterality: Uni } \\
\text { (58.6)/Bi (41.4) } \\
\text { Timing: Imm } \\
\text { (24.8)/Del (75.2) } \\
\text { Stages: } 1(100) \\
\text { Chemo: Before (58.9) } \\
\text { Radio: Before (18.5) }\end{array}$ & 3926 & 51.2 & NR & NR & NR \\
\hline $\begin{array}{l}\text { Rogoff, } \\
2020, \\
32243320, \\
\text { US }\end{array}$ & $\begin{array}{l}\text { SGS (NR) } \\
(2001- \\
2018)\end{array}$ & Low & I: TE/IBR & IBR & $\begin{array}{l}\text { ADM: Yes } \\
(64.9) / \text { No } \\
(35.1)\end{array}$ & $\begin{array}{l}\text { Laterality: Uni } \\
\text { (60.3)/Bi (39.7) } \\
\text { Timing: Imm (100) } \\
\text { Stages: } 2 \text { (100) } \\
\text { Chemo: Before } \\
\text { (16.9)/After (32.1) } \\
\text { Radio: Before (4.8) } \\
\text { /After (19.9) } \\
\end{array}$ & 627 & 53.1 & NR & NR & NR \\
\hline $\begin{array}{l}\text { Rubio, 2019, } \\
30665841, \\
\text { Belgium }\end{array}$ & $\begin{array}{l}\text { SGS } \\
\text { (None) } \\
(2008- \\
2011)\end{array}$ & Low & $\mathrm{I}: \mathrm{AR}$ & AR & $\mathrm{N} / \mathrm{A}$ & $\begin{array}{l}\text { Timing: Imm } \\
\text { (40.7)/Del (59.3) } \\
\text { Stages: } 1 \text { (100) }\end{array}$ & $\begin{array}{l}5652 \\
2\end{array}$ & $51.2(9.8)$ & $\begin{array}{l}\text { W (72.4), } \\
\text { B (11.7), } \\
\text { A (2.9), } \\
\text { H (9.8), } \\
\text { Other } 1 \\
(0.3), \\
\text { Other } 2 \\
(3)\end{array}$ & NR & NR \\
\hline $\begin{array}{l}\text { Salibian, } \\
2019, \\
31333984, \\
\text { US }\end{array}$ & $\begin{array}{l}\text { SGS (NR) } \\
(2006- \\
2018)\end{array}$ & Low & $\begin{array}{l}\text { I: Immediate alloplastic } \\
\text { BR }\end{array}$ & IBR & NR & $\begin{array}{l}\text { Laterality: NR } \\
\text { Timing: Imm (100) } \\
\text { Stages: NR } \\
\text { Chemo: NR } \\
\text { Radio: NR }\end{array}$ & 1045 & NR & NR & NR & NR \\
\hline
\end{tabular}




\begin{tabular}{|c|c|c|c|c|c|c|c|c|c|c|c|}
\hline $\begin{array}{l}\text { Study, } \\
\text { Publication } \\
\text { Year, } \\
\text { PMID, } \\
\text { Country }\end{array}$ & $\begin{array}{l}\text { Design } \\
\text { (Funding) } \\
\text { (Study } \\
\text { Years) }\end{array}$ & $\begin{array}{l}\text { Risk } \\
\text { of } \\
\text { Bias }\end{array}$ & Eligibility Criteria & Arm & $\begin{array}{l}\text { Implant } \\
\text { Details (\%) } \\
\text { (Only } \\
\text { Reported } \\
\text { Details) } \\
\end{array}$ & $\begin{array}{l}\text { Reconstruction } \\
\text { Details (\%) } \\
\text { (Only Reported } \\
\text { Details) }\end{array}$ & $\mathbf{N}$ & $\begin{array}{l}\text { Age in } \\
\text { Years, } \\
\text { Mean (SD) } \\
\text { or as } \\
\text { Specified } \\
\end{array}$ & $\begin{array}{l}\text { Race } \\
(\%)\end{array}$ & $\begin{array}{l}\text { Breast } \\
\text { Cancer } \\
\text { Occurrence } \\
\%\end{array}$ & $\begin{array}{l}\text { Cancer } \\
\text { Stage or } \\
\text { Mastectomy } \\
\text { Purpose (\%) }\end{array}$ \\
\hline $\begin{array}{l}\text { Seidenstuec } \\
\text { ker, 2016, } \\
27017243, \\
\text { Belgium } \\
\end{array}$ & $\begin{array}{l}\text { SGS } \\
\text { (None) } \\
(2004- \\
2010) \\
\end{array}$ & $\begin{array}{l}\text { Mode } \\
\text { rate }\end{array}$ & I: AR with DIEP flap & AR & N/A & $\begin{array}{l}\text { Timing: Imm (100) } \\
\text { Stages: } 1(100)\end{array}$ & 931 & NR & NR & NR & NR \\
\hline $\begin{array}{l}\text { Selber, } \\
2009 \\
19935283 \\
\text { US }\end{array}$ & $\begin{array}{l}\text { SGS } \\
\text { (None) } \\
(1992- \\
2007)\end{array}$ & Low & $\begin{array}{l}\text { I: AR with free TRAM, } \\
\text { DIEP, or SIEA flaps }\end{array}$ & AR & $\mathrm{N} / \mathrm{A}$ & $\begin{array}{l}\text { Laterality: Uni } \\
\text { (58.6)/Bi (41.3) } \\
\text { Timing: Imm } \\
\text { (77.7)/Del (19)/Mixed } \\
(2.3) \\
\text { Stages: } 1 \text { (100) } \\
\text { Chemo: After (16.2) } \\
\text { Radio: After (7.9) }\end{array}$ & 1031 & $\begin{array}{l}<65 \text { years } \\
(94.7 \%), \geq 65 \\
\text { years }(5.3 \%)\end{array}$ & NR & NR & NR \\
\hline $\begin{array}{l}\text { Seth, 2015, } \\
25180955, \\
\text { US }\end{array}$ & $\begin{array}{l}\text { SGS } \\
\text { (Industry; } \\
\text { Non- } \\
\text { industry) } \\
\text { (1999- } \\
2008) \\
\end{array}$ & Low & I: TE/IBR & IBR & NR & $\begin{array}{l}\text { Timing: Imm } \\
\text { (93.4)/Del (6.6) } \\
\text { Stages: >1 (100) } \\
\text { Radio: Timing NR } \\
\text { (25.1)/None(74.9) }\end{array}$ & 893 & 48.8 (NR) & NR & NR & NR \\
\hline $\begin{array}{l}\text { Sewart, } \\
2021, \\
33609398, \\
\text { UK }\end{array}$ & $\begin{array}{l}\text { SGS (Non- } \\
\text { industry) } \\
\text { (2014- } \\
2016)\end{array}$ & Low & $\begin{array}{l}\text { I: Age } \geq 18 \text { years; } \\
\text { Immediate IBR } \\
\text { E: Hybrid IBR and AR; } \\
\text { revision reconstruction }\end{array}$ & IBR & $\begin{array}{l}\text { Plane: } \\
\text { Prepectoral } \\
\text { (1.6)/Partial } \\
\text { submuscular } \\
(7.5)\end{array}$ & $\begin{array}{l}\text { Laterality: Uni } \\
\text { (75.1)/Bi }(24.9) \\
\text { Timing: Imm (100) } \\
\text { Stages: } 1(78.6) / 2 \\
\text { (21.4) } \\
\text { Chemo: Timing NR } \\
\text { (33.1) } \\
\text { Radio: Timing NR } \\
\text { (28.3) }\end{array}$ & 891 & $\begin{array}{l}\text { Median: } 50 \\
(\text { IQR 45, 58) }\end{array}$ & NR & NR & $\begin{array}{l}\text { Ther (82.2), } \\
\text { Proph (17.8) }\end{array}$ \\
\hline $\begin{array}{l}\text { Shaikh, } \\
2010, \\
22693373, \\
\text { India }\end{array}$ & $\begin{array}{l}\text { SGS (NR) } \\
(1996- \\
2008)\end{array}$ & Low & $\begin{array}{l}\text { I: AR with TRAM or LD } \\
\text { flaps }\end{array}$ & AR & $\mathrm{N} / \mathrm{A}$ & Timing: Imm (100) & 546 & NR & NR & NR & $\begin{array}{l}\text { Stage NR } \\
(100)\end{array}$ \\
\hline
\end{tabular}




\begin{tabular}{|c|c|c|c|c|c|c|c|c|c|c|c|}
\hline $\begin{array}{l}\text { Study, } \\
\text { Publication } \\
\text { Year, } \\
\text { PMID, } \\
\text { Country }\end{array}$ & $\begin{array}{l}\text { Design } \\
\text { (Funding) } \\
\text { (Study } \\
\text { Years) }\end{array}$ & $\begin{array}{l}\text { Risk } \\
\text { of } \\
\text { Bias }\end{array}$ & Eligibility Criteria & Arm & $\begin{array}{l}\text { Implant } \\
\text { Details (\%) } \\
\text { (Only } \\
\text { Reported } \\
\text { Details) } \\
\end{array}$ & $\begin{array}{l}\text { Reconstruction } \\
\text { Details (\%) } \\
\text { (Only Reported } \\
\text { Details) }\end{array}$ & $\mathbf{N}$ & $\begin{array}{l}\text { Age in } \\
\text { Years, } \\
\text { Mean (SD) } \\
\text { or as } \\
\text { Specified }\end{array}$ & $\begin{array}{l}\text { Race } \\
(\%)\end{array}$ & $\begin{array}{l}\text { Breast } \\
\text { Cancer } \\
\text { Occurrence } \\
\%\end{array}$ & $\begin{array}{l}\text { Cancer } \\
\text { Stage or } \\
\text { Mastectomy } \\
\text { Purpose (\%) }\end{array}$ \\
\hline $\begin{array}{l}\text { Singh, } 2012 \text {, } \\
22342636 \text {, } \\
\text { US }\end{array}$ & $\begin{array}{l}\text { SGS } \\
\text { (Industry) } \\
(2008- \\
2009)\end{array}$ & Low & $\begin{array}{l}\text { I: IBR } \\
\text { E: initial reconstruction } \\
\text { included a flap or other } \\
\text { AR procedure, } \\
\text { preexisting breast } \\
\text { implant complications, } \\
\text { unrelated surgery } \\
\text { concurrent with initial } \\
\text { reconstruction, death } \\
\text { during the } 18 \text { month } \\
\text { post-index period, TE } \\
\text { reconstruction in which } \\
\text { the tissue expander } \\
\text { exchange procedure } \\
\text { could not be identified } \\
\text { from available coded } \\
\text { claims. }\end{array}$ & IBR & NR & $\begin{array}{l}\text { Timing: Imm (100) } \\
\text { Stages: } 1(7.2) />1 \\
\text { (92.8) } \\
\text { Radio: Timing NR } \\
(17.8)\end{array}$ & 1316 & $49.1(\mathrm{NR})$ & NR & NR & NR \\
\hline $\begin{array}{l}\text { Singh, 2021, } \\
33564597, \\
\text { US }\end{array}$ & $\begin{array}{l}\text { SGS } \\
\text { (None) } \\
\text { (NR-2020) }\end{array}$ & Low & $\begin{array}{l}\text { I: Age } \geq 18 \text { years; } \\
\text { Immediate IBR } \\
\text { E: Pregnant }\end{array}$ & IBR & NR & $\begin{array}{l}\text { Timing: Imm (50)/Del } \\
(50) \\
\text { Stages: } 1(50) / 2(50)\end{array}$ & 1740 & 51.9 & $\mathrm{~W}(97.9)$ & NR & NR \\
\hline $\begin{array}{l}\text { Song, } 2016, \\
26637165, \\
\text { US }\end{array}$ & $\begin{array}{l}\text { SGS } \\
\text { (Industry; } \\
\text { Non- } \\
\text { industry) } \\
\text { (2002- } \\
2012)\end{array}$ & Low & I: AR & AR & $\mathrm{N} / \mathrm{A}$ & $\begin{array}{l}\text { Laterality: Uni } \\
\text { (68.5)/Bi (31.5) } \\
\text { Timing: Imm } \\
\text { (65.1)/Del } \\
\text { (32.2)/Mixed (2.5) } \\
\text { Stages: } 1 \text { (100) } \\
\text { Chemo: Before } \\
\text { (40.8)/After (14)/No } \\
\text { chemotherapy (46.4) } \\
\text { Radio: Before } \\
\text { (38.8)/After } \\
\text { (6.1)/None(54.5) }\end{array}$ & 1809 & $\begin{array}{l}\text { Range } 16, \\
78 ;<65 \\
\text { years } \\
(96.8 \%), \geq 65 \\
\text { years }(3.2 \%)\end{array}$ & $\begin{array}{l}\text { W (42.2), } \\
\text { B (1.9), } \\
\text { A }(4.5), \\
\text { H (0.4), } \\
\text { Other } 1 \\
(1.8), \\
\text { Other } 2 \\
(1.2)\end{array}$ & NR & $\begin{array}{l}\text { None (5.4), } \\
\text { Stage 0 } \\
\text { (26.6), Stage } \\
\text { NR (63.7) }\end{array}$ \\
\hline
\end{tabular}




\begin{tabular}{|c|c|c|c|c|c|c|c|c|c|c|c|}
\hline $\begin{array}{l}\text { Study, } \\
\text { Publication } \\
\text { Year, } \\
\text { PMID, } \\
\text { Country }\end{array}$ & $\begin{array}{l}\text { Design } \\
\text { (Funding) } \\
\text { (Study } \\
\text { Years) }\end{array}$ & $\begin{array}{l}\text { Risk } \\
\text { of } \\
\text { Bias }\end{array}$ & Eligibility Criteria & Arm & $\begin{array}{l}\text { Implant } \\
\text { Details (\%) } \\
\text { (Only } \\
\text { Reported } \\
\text { Details) } \\
\end{array}$ & $\begin{array}{l}\text { Reconstruction } \\
\text { Details (\%) } \\
\text { (Only Reported } \\
\text { Details) }\end{array}$ & $\mathbf{N}$ & $\begin{array}{l}\text { Age in } \\
\text { Years, } \\
\text { Mean (SD) } \\
\text { or as } \\
\text { Specified } \\
\end{array}$ & $\begin{array}{l}\text { Race } \\
(\%)\end{array}$ & $\begin{array}{l}\text { Breast } \\
\text { Cancer } \\
\text { Occurrence } \\
\%\end{array}$ & $\begin{array}{l}\text { Cancer } \\
\text { Stage or } \\
\text { Mastectomy } \\
\text { Purpose (\%) }\end{array}$ \\
\hline $\begin{array}{l}\text { Tran, 2018, } \\
29794694, \\
\text { US }\end{array}$ & $\begin{array}{l}\text { SGS (NR) } \\
(2004- \\
2015)\end{array}$ & Low & $\mathrm{I}: \mathrm{AR}$ & AR & $\mathrm{N} / \mathrm{A}$ & $\begin{array}{l}\text { Laterality: Uni } \\
\text { (52.3)/Bi (47.7) } \\
\text { Timing: Imm (NR)/Del } \\
\text { (34.8)/Mixed (NR) } \\
\text { Stages: } 1 \text { (100) } \\
\text { Chemo: Timing NR } \\
\text { (48.2)/No } \\
\text { chemotherapy (51.8) } \\
\text { Radio: Timing NR } \\
\text { (38.2)/None(61.8) }\end{array}$ & 853 & $50.1(8.6)$ & NR & NR & $\begin{array}{l}\text { Stage 0 } \\
(42.1), \text { Stage } \\
\text { I (23.7), } \\
\text { Stage II (21), } \\
\text { Stage III (10), } \\
\text { Stage IV (3.3) }\end{array}$ \\
\hline $\begin{array}{l}\text { Warren, } \\
2020, \\
33040748, \\
\text { US }\end{array}$ & $\begin{array}{l}\text { SGS (Non- } \\
\text { industry) } \\
(2011- \\
2015)\end{array}$ & $\begin{array}{l}\text { Mode } \\
\text { rate }\end{array}$ & $\begin{array}{l}\text { I: Age } \geq 18 \text { years } \\
\text { E: Likely to have } \\
\text { antibiotics at discharge; } \\
\text { length of stay } \geq 90 \text { days }\end{array}$ & IBR & NR & Timing: Imm (100) & 1924 & NR & NR & NR & NR \\
\hline $\begin{array}{l}\text { Watterson, } \\
1995, \\
7761505 \\
\text { Australia }\end{array}$ & $\begin{array}{l}\text { SGS (NR) } \\
(1981- \\
1991)\end{array}$ & $\begin{array}{l}\text { Mode } \\
\text { rate }\end{array}$ & NR & AR & $\mathrm{N} / \mathrm{A}$ & $\begin{array}{l}\text { Laterality: Uni } \\
\text { (68.9)/Bi (31.1) } \\
\text { Timing: Imm (27)/Del } \\
\text { (73) }\end{array}$ & 556 & $\begin{array}{l}46(N R) ; \\
\text { Range 24, } \\
69\end{array}$ & NR & NR & NR \\
\hline $\begin{array}{l}\text { Williams, } \\
1995, \\
7794079 \\
\text { US }\end{array}$ & $\begin{array}{l}\text { SGS (NR) } \\
(1981- \\
1993)\end{array}$ & $\begin{array}{l}\text { Mode } \\
\text { rate }\end{array}$ & I: AR with TRAM flap & AR & $\mathrm{N} / \mathrm{A}$ & NR & 680 & $\begin{array}{l}>60 \text { years } \\
(5.4 \%)\end{array}$ & NR & NR & NR \\
\hline $\begin{array}{l}\text { Yoo, 2014, } \\
24852813, \\
\text { South Korea }\end{array}$ & $\begin{array}{l}\text { SGS } \\
\text { (None) } \\
(2001- \\
2010)\end{array}$ & $\begin{array}{l}\text { Mode } \\
\text { rate }\end{array}$ & I: AR with TRAM flap & AR & $\mathrm{N} / \mathrm{A}$ & NR & 964 & NR & NR & NR & NR \\
\hline
\end{tabular}

Blue coloring is only to visually separate different studies.

I: inclusion criteria, E: exclusion criteria

Laterality: whether the reconstruction was unilateral ("Uni”) or bilateral ("Bi”). Stages: Whether the reconstruction was completed in 1 stage or $>1$ stages. Timing: Timing of reconstruction relative to mastectomy, i.e., immediate ("Imm") or delayed ("Del"). Chemo: Timing of chemotherapy relative to reconstruction. Radio: Timing of radiation therapy relative to reconstruction.

$\mathrm{W}=$ White or Caucasian, $\mathrm{B}=$ Black or African American, $\mathrm{A}=$ Asian, $\mathrm{H}=$ Hispanic or Latino.

Proph $=$ prophylactic, Ther $=$ therapeutic 
Abbreviations: $\mathrm{AR}=$ autologous reconstruction, $\mathrm{BR}=$ breast reconstruction, $\mathrm{DIEP}=$ Deep inferior epigastric perforator, $\mathrm{IBR}=\mathrm{implant}$-based reconstruction, $\mathrm{IQR}=$ interquartile range, $\mathrm{LD}=$ latissimus dorsi, $\mathrm{NR}=$ not reported, $\mathrm{NRCS}=$ nonrandomized comparative study, PMID $=$ PubMed identifier, $\mathrm{PMRT}=$ postmastectomy radiation therapy, $\mathrm{RCT}=$ randomized controlled trial, $\mathrm{SD}=$ standard deviation, $\mathrm{SIEA}=$ Superficial inferior epigastric artery perforator, $\mathrm{TE} / \mathrm{I}=$ tissue expander/implant, Ther $=$ therapeutic, TRAM $=$ Transverse rectus abdominis myocutaneous.

Colors: Header rows are shaded orange. Rows for every alternate study are shaded blue. The colors do not add unique information. 
Table C-3. Key Question 2: Timing of chemotherapy and radiation therapy relative to IBR or AR - summary of design, arm, and sample details

\begin{tabular}{|c|c|c|c|c|c|c|c|c|c|c|c|}
\hline $\begin{array}{l}\text { Study, } \\
\text { Publicati } \\
\text { on Year, } \\
\text { PMID, } \\
\text { Country }\end{array}$ & $\begin{array}{l}\text { Design } \\
\text { (Funding) } \\
\text { (Study } \\
\text { Years) }\end{array}$ & $\begin{array}{l}\text { Risk } \\
\text { of } \\
\text { Bias }\end{array}$ & Eligibility Criteria & Arm & $\begin{array}{l}\text { Implant } \\
\text { Details (\%) } \\
\text { (Only } \\
\text { Reported } \\
\text { Details) }\end{array}$ & $\begin{array}{l}\text { Reconstruction Details } \\
(\%) \\
\text { (Only Reported Details) }\end{array}$ & $\mathbf{N}$ & $\begin{array}{l}\text { Age in } \\
\text { Years, Mean } \\
\text { (SD) or as } \\
\text { Specified }\end{array}$ & $\begin{array}{l}\text { Race } \\
(\%)\end{array}$ & $\begin{array}{l}\text { Breast } \\
\text { Cance } \\
r \\
\text { Occur } \\
\text { rence } \\
\%\end{array}$ & $\begin{array}{l}\text { Cancer } \\
\text { Stage or } \\
\text { Mastectomy } \\
\text { Purpose (\%) }\end{array}$ \\
\hline \multirow[t]{3}{*}{$\begin{array}{l}\text { Cordeiro, } \\
2015 a \\
25742523 \\
\text {, US }\end{array}$} & $\begin{array}{l}\text { NRCS } \\
\text { (Industry) } \\
(2003- \\
2012)\end{array}$ & High & $\begin{array}{l}\text { I: IBR } \\
\text { E: Combined AR plus } \\
\text { TE/implant BR, } \\
\text { perioperative } \\
\text { radiation therapy, } \\
\text { delayed BR }\end{array}$ & $\begin{array}{l}\text { IBR } \\
\text { before } \\
\text { radiation }\end{array}$ & NR & $\begin{array}{l}\text { Laterality: Uni }(71.6) / \mathrm{Bi} \\
(28.4) \\
\text { Stages: }>1(100)\end{array}$ & NR & $46.3(8.9)$ & NR & NR & NR \\
\hline & $\begin{array}{l}\text { NRCS } \\
\text { (Industry) } \\
(2003- \\
2012)\end{array}$ & High & $\begin{array}{l}\text { I: IBR } \\
\text { E: Combined AR plus } \\
\text { TE/implant BR, } \\
\text { perioperative } \\
\text { radiation therapy, } \\
\text { delayed BR }\end{array}$ & $\begin{array}{l}\text { IBR after } \\
\text { radiation }\end{array}$ & NR & $\begin{array}{l}\text { Laterality: Uni (57)/Bi (43) } \\
\text { Stages: >1 (100) }\end{array}$ & NR & $46.1(10.6)$ & NR & NR & NR \\
\hline & $\begin{array}{l}\text { NRCS } \\
\text { (Industry) } \\
\text { (2003- } \\
2012)\end{array}$ & High & $\begin{array}{l}\text { I: IBR } \\
\text { E: Combined AR plus } \\
\text { TE/implant BR, } \\
\text { perioperative } \\
\text { radiation therapy, } \\
\text { delayed BR }\end{array}$ & Total & $\mathrm{N} / \mathrm{A}$ & $\mathrm{N} / \mathrm{A}$ & 1143 & NR & NR & NR & NR \\
\hline \multirow[t]{3}{*}{$\begin{array}{l}\text { Eriksson, } \\
2013, \\
24258257 \\
\text {, Sweden }\end{array}$} & $\begin{array}{l}\text { NRCS (NR) } \\
(2007- \\
2011)\end{array}$ & High & $\begin{array}{l}\text { I: Immediate IBR } \\
\text { E: Only risk-reducing } \\
\text { surgery }\end{array}$ & $\begin{array}{l}\text { IBR } \\
\text { before } \\
\text { radiation }\end{array}$ & $\begin{array}{l}\text { PLANE: } \\
\text { Partial } \\
\text { submuscular } \\
(100)\end{array}$ & $\begin{array}{l}\text { Timing: Imm (100) } \\
\text { Chemo: After (57.57)/No } \\
\text { chemotherapy }(42.11)\end{array}$ & 304 & $\begin{array}{l}\text { Median 46; } \\
\text { Range 21, } 74\end{array}$ & NR & NR & $\begin{array}{l}\text { Stage } 0 \\
(9.54), \text { Stage } \\
\text { NR }(90.46)\end{array}$ \\
\hline & $\begin{array}{l}\text { NRCS (NR) } \\
(2007- \\
2011)\end{array}$ & High & $\begin{array}{l}\text { I: Immediate IBR } \\
\text { E: Only risk-reducing } \\
\text { surgery }\end{array}$ & $\begin{array}{l}\text { IBR after } \\
\text { radiation }\end{array}$ & $\begin{array}{l}\text { PLANE: } \\
\text { Partial } \\
\text { submuscular } \\
(100)\end{array}$ & $\begin{array}{l}\text { Timing: Imm (100) } \\
\text { Chemo: After }(32.25) / \text { No } \\
\text { chemotherapy }(65.63)\end{array}$ & 64 & $\begin{array}{l}\text { Median 55; } \\
\text { Range 28, } 75\end{array}$ & NR & NR & $\begin{array}{l}\text { Stage } 0 \\
(28.13) \\
\text { Stage NR } \\
(70.31)\end{array}$ \\
\hline & $\begin{array}{l}\text { NRCS (NR) } \\
(2007- \\
2011) \\
\end{array}$ & High & $\begin{array}{l}\text { I: Immediate IBR } \\
\text { E: Only risk-reducing } \\
\text { surgery }\end{array}$ & Total & $\mathrm{N} / \mathrm{A}$ & $\mathrm{N} / \mathrm{A}$ & 368 & NR & NR & NR & NR \\
\hline \multirow{2}{*}{$\begin{array}{l}\text { Hirsch, } \\
2014, \\
25347643 \\
\text {, US }\end{array}$} & $\begin{array}{l}\text { NRCS (NR) } \\
(1998- \\
2008) \\
\end{array}$ & High & I: Immediate TE/IBR & $\begin{array}{l}\text { IBR } \\
\text { before } \\
\text { radiation }\end{array}$ & NR & $\begin{array}{l}\text { Timing: Imm (100) } \\
\text { Stages: }>1(100)\end{array}$ & NR & NR & NR & NR & NR \\
\hline & $\begin{array}{l}\text { NRCS (NR) } \\
(1998- \\
2008)\end{array}$ & High & I: Immediate TE/IBR & $\begin{array}{l}\text { IBR after } \\
\text { radiation }\end{array}$ & NR & $\begin{array}{l}\text { Timing: Imm (100) } \\
\text { Stages: >1 (100) }\end{array}$ & NR & NR & NR & NR & NR \\
\hline
\end{tabular}




\begin{tabular}{|c|c|c|c|c|c|c|c|c|c|c|c|}
\hline $\begin{array}{l}\text { Study, } \\
\text { Publicati } \\
\text { on Year, } \\
\text { PMID, } \\
\text { Country }\end{array}$ & $\begin{array}{l}\text { Design } \\
\text { (Funding) } \\
\text { (Study } \\
\text { Years) }\end{array}$ & $\begin{array}{l}\text { Risk } \\
\text { of } \\
\text { Bias }\end{array}$ & Eligibility Criteria & Arm & $\begin{array}{l}\text { Implant } \\
\text { Details (\%) } \\
\text { (Only } \\
\text { Reported } \\
\text { Details) }\end{array}$ & $\begin{array}{l}\text { Reconstruction Details } \\
\text { (\%) } \\
\text { (Only Reported Details) }\end{array}$ & $\mathbf{N}$ & $\begin{array}{l}\text { Age in } \\
\text { Years, Mean } \\
\text { (SD) or as } \\
\text { Specified }\end{array}$ & $\begin{array}{l}\text { Race } \\
(\%)\end{array}$ & $\begin{array}{l}\text { Breast } \\
\text { Cance } \\
\mathbf{r} \\
\text { Occur } \\
\text { rence } \\
\%\end{array}$ & $\begin{array}{l}\text { Cancer } \\
\text { Stage or } \\
\text { Mastectomy } \\
\text { Purpose (\%) }\end{array}$ \\
\hline & $\begin{array}{l}\text { NRCS (NR) } \\
(1998- \\
2008)\end{array}$ & High & I: Immediate TE/IBR & Total & $\mathrm{N} / \mathrm{A}$ & $\mathrm{N} / \mathrm{A}$ & 876 & $\begin{array}{l}\text { Mean } 48.1 ; \\
\text { Range 16.1, } \\
57.6\end{array}$ & NR & NR & NR \\
\hline \multirow[t]{3}{*}{$\begin{array}{l}\text { Stein, } \\
2020, \\
32561384 \\
\text {, Canada }\end{array}$} & $\begin{array}{l}\text { NRCS } \\
\text { (None) } \\
\text { (2010- } \\
2019)\end{array}$ & High & $\begin{array}{l}\mathrm{I}: \text { Immediate IBR with } \\
\text { radiation }\end{array}$ & $\begin{array}{l}\text { IBR } \\
\text { before } \\
\text { radiation }\end{array}$ & $\begin{array}{l}\text { SIZE: Mean } \\
406 \mathrm{cc}\end{array}$ & $\begin{array}{l}\text { Laterality: NR } \\
\text { Timing: Imm }(100) \\
\text { Stages: } 1(53.9) />1(46.1) \\
\text { Chemo: Before }(100) \\
\text { Radiation: Before }(100)\end{array}$ & 76 & 47.2 (NR) & NR & NR & NR \\
\hline & $\begin{array}{l}\text { NRCS } \\
\text { (None) } \\
\text { (2010- } \\
2019)\end{array}$ & High & $\begin{array}{l}\text { I: Immediate IBR with } \\
\text { radiation }\end{array}$ & $\begin{array}{l}\text { IBR after } \\
\text { radiation }\end{array}$ & $\begin{array}{l}\text { SIZE: Mean } \\
444 \mathrm{cc}\end{array}$ & $\begin{array}{l}\text { Laterality: NR } \\
\text { Timing: Imm (100) } \\
\text { Stages: } 1(50) />1(50) \\
\text { Chemo: After }(100) \\
\text { Radiation: After (100) }\end{array}$ & 54 & 54.7 (NR) & NR & NR & NR \\
\hline & $\begin{array}{l}\text { NRCS } \\
\text { (None) } \\
(2010- \\
2019)\end{array}$ & High & $\begin{array}{l}\text { I: Immediate IBR with } \\
\text { radiation }\end{array}$ & Total & N/A & N/A & 130 & $50.3(\mathrm{NR})$ & NR & NR & NR \\
\hline \multirow[t]{3}{*}{$\begin{array}{l}\text { Yoon, } \\
2020, \\
32332528 \\
\text {, US \& } \\
\text { Canada }\end{array}$} & $\begin{array}{l}\text { NRCS } \\
\text { (Non- } \\
\text { industry) } \\
\text { (2012- } \\
2015)\end{array}$ & $\begin{array}{l}\text { Mode } \\
\text { rate }\end{array}$ & $\begin{array}{l}\text { I: Immediate TE/ IBR } \\
\text { with PMRT }\end{array}$ & $\begin{array}{l}\text { IBR } \\
\text { before } \\
\text { radiation }\end{array}$ & NR & $\begin{array}{l}\text { Laterality: Uni (38.8)/Bi } \\
\text { (61.2) } \\
\text { Timing: Imm (100) } \\
\text { Stages: >1 (100) } \\
\text { Chemo: Before } \\
\text { (92.5)/After (7.5) } \\
\text { Radiation: After (100) }\end{array}$ & 80 & $45.3(10.1)$ & $\begin{array}{l}\text { W } \\
(93.6), \\
\text { Others } \\
(6.4)\end{array}$ & NR & NR \\
\hline & $\begin{array}{l}\text { NRCS } \\
\text { (Non- } \\
\text { industry) } \\
(2012- \\
2015)\end{array}$ & $\begin{array}{l}\text { Mode } \\
\text { rate }\end{array}$ & $\begin{array}{l}\text { I: Immediate TE/ IBR } \\
\text { with PMRT }\end{array}$ & $\begin{array}{l}\text { IBR after } \\
\text { radiation }\end{array}$ & NR & $\begin{array}{l}\text { Laterality: Uni (42.6)/Bi } \\
\text { (57.4) } \\
\text { Timing: Imm (100) } \\
\text { Stages: >1 (100) } \\
\text { Chemo: Before } \\
\text { (60.8)/After (39.2) } \\
\text { Radiation: Before (100) }\end{array}$ & 237 & $47.4(10.4)$ & $\begin{array}{l}\text { W } \\
(87.6), \\
\text { Others } \\
(12.4)\end{array}$ & NR & NR \\
\hline & $\begin{array}{l}\text { NRCS } \\
\text { (Non- } \\
\text { industry) } \\
\text { (2012- } \\
2015) \\
\end{array}$ & $\begin{array}{l}\text { Mode } \\
\text { rate }\end{array}$ & $\begin{array}{l}\text { I: Immediate TE/ IBR } \\
\text { with PMRT }\end{array}$ & Total & $\mathrm{N} / \mathrm{A}$ & $\mathrm{N} / \mathrm{A}$ & 317 & NR & NR & NR & NR \\
\hline
\end{tabular}

Note: Blue coloring is only to visually separate different studies. 
Laterality: whether the reconstruction was unilateral ("Uni") or bilateral ("Bi"). Stages: Whether the reconstruction was completed in 1 stage or $>1$ stages. Timing: Timing of reconstruction relative to mastectomy, i.e., immediate ("Imm") or delayed ("Del"). Chemo: Timing of chemotherapy relative to reconstruction.

Abbreviations: $\mathrm{BR}=$ breast reconstruction, $\mathrm{IBR}=$ implant-based reconstruction, $\mathrm{IQR}=$ interquartile range, $\mathrm{N} / \mathrm{A}=$ not applicable, $\mathrm{NR}=$ not reported, $\mathrm{NRCS}=$ nonrandomized comparative study, $\mathrm{PMID}=$ PubMed identifier, $\mathrm{SD}=$ standard deviation, $\mathrm{TE} / \mathrm{I}=$ tissue expander/implant, Ther $=$ therapeutic

Colors: Header rows are shaded orange. Rows for every alternate study are shaded blue. The colors do not add unique information. 
Table C-4. Key Question 3: Comparison of materials for IBR - summary of design, arm, and sample details

\begin{tabular}{|c|c|c|c|c|c|c|c|c|c|c|c|}
\hline $\begin{array}{l}\text { Study, } \\
\text { Publication } \\
\text { Year, } \\
\text { PMID, } \\
\text { Country }\end{array}$ & $\begin{array}{l}\text { Design } \\
\text { (Funding) } \\
\text { (Study } \\
\text { Years) }\end{array}$ & $\begin{array}{l}\text { Risk } \\
\text { of } \\
\text { Bias }\end{array}$ & $\begin{array}{l}\text { Eligibility } \\
\text { Criteria }\end{array}$ & Arm & $\begin{array}{l}\text { Implant } \\
\text { Details (\%) } \\
\text { (Only } \\
\text { Reported } \\
\text { Details) } \\
\end{array}$ & $\begin{array}{l}\text { Reconstruction Details (\%) } \\
\text { (Only Reported Details) }\end{array}$ & $\mathbf{N}$ & $\begin{array}{l}\text { Age in } \\
\text { Years, } \\
\text { Mean (SD) } \\
\text { or as } \\
\text { Specified }\end{array}$ & $\begin{array}{l}\text { Race } \\
(\%)\end{array}$ & $\begin{array}{l}\text { Breast } \\
\text { Cancer } \\
\text { Occurrence } \\
\%\end{array}$ & $\begin{array}{l}\text { Cancer } \\
\text { Stage or } \\
\text { Mastectom } \\
\text { y Purpose } \\
(\%)\end{array}$ \\
\hline \multirow[t]{3}{*}{$\begin{array}{l}\text { Antony, } \\
2014, \\
24135689, \\
\text { US }\end{array}$} & $\begin{array}{l}\text { NRCS } \\
\text { (None) } \\
(1997- \\
2007)\end{array}$ & High & $\begin{array}{l}\text { I: } 2 \text {-staged } \\
\text { bilateral IBR }\end{array}$ & $\begin{array}{l}\text { IBR with } \\
\text { silicone }\end{array}$ & NR & $\begin{array}{l}\text { Laterality: } \mathrm{Bi}(100) \\
\text { Stages: }>1(100)\end{array}$ & NR & NR & NR & NR & $\begin{array}{l}\text { Stage NR } \\
(100)\end{array}$ \\
\hline & $\begin{array}{l}\text { NRCS } \\
\text { (None) } \\
(1997- \\
2007) \\
\end{array}$ & High & $\begin{array}{l}\text { I: 2-staged } \\
\text { bilateral IBR }\end{array}$ & $\begin{array}{l}\text { IBR with } \\
\text { saline }\end{array}$ & NR & $\begin{array}{l}\text { Laterality: } \mathrm{Bi}(100) \\
\text { Stages: >1 (100) }\end{array}$ & NR & NR & NR & NR & $\begin{array}{l}\text { Stage NR } \\
(100)\end{array}$ \\
\hline & $\begin{array}{l}\text { NRCS } \\
\text { (None) } \\
(1997- \\
2007) \\
\end{array}$ & High & $\begin{array}{l}\text { I: 2-staged } \\
\text { bilateral IBR }\end{array}$ & Total & $\mathrm{N} / \mathrm{A}$ & $\mathrm{N} / \mathrm{A}$ & 365 & $47(9.4)$ & NR & NR & $\begin{array}{l}\text { Stage NR } \\
(100)\end{array}$ \\
\hline \multirow[t]{3}{*}{$\begin{array}{l}\text { Cordeiro, } \\
2015 a, \\
25742523, \\
\text { US }\end{array}$} & $\begin{array}{l}\text { NRCS } \\
\text { (Industry) } \\
\text { (2003- } \\
2012)\end{array}$ & High & $\begin{array}{l}\text { I: IBR } \\
\text { E: Combined } \\
\text { AR plus } \\
\text { TE/implant BR, } \\
\text { perioperative } \\
\text { radiation } \\
\text { therapy, } \\
\text { delayed BR }\end{array}$ & $\begin{array}{l}\text { IBR with } \\
\text { silicone }\end{array}$ & NR & Stages: >1 (100) & NR & NR & NR & NR & NR \\
\hline & $\begin{array}{l}\text { NRCS } \\
\text { (Industry) } \\
(2003- \\
2012)\end{array}$ & High & $\begin{array}{l}\text { I: IBR } \\
\text { E: Combined } \\
\text { AR plus } \\
\text { TE/implant BR, } \\
\text { perioperative } \\
\text { radiation } \\
\text { therapy, } \\
\text { delayed BR }\end{array}$ & $\begin{array}{l}\text { IBR with } \\
\text { saline }\end{array}$ & NR & Stages: >1 (100) & NR & NR & NR & NR & NR \\
\hline & $\begin{array}{l}\text { NRCS } \\
\text { (Industry) } \\
(2003- \\
2012)\end{array}$ & High & $\begin{array}{l}\text { I: IBR } \\
\text { E: Combined } \\
\text { AR plus } \\
\text { TE/implant BR, } \\
\text { perioperative } \\
\text { radiation } \\
\text { therapy, } \\
\text { delayed BR }\end{array}$ & Total & N/A & $\mathrm{N} / \mathrm{A}$ & $\begin{array}{l}114 \\
3\end{array}$ & NR & NR & NR & NR \\
\hline
\end{tabular}




\begin{tabular}{|c|c|c|c|c|c|c|c|c|c|c|c|}
\hline $\begin{array}{l}\text { Study, } \\
\text { Publication } \\
\text { Year, } \\
\text { PMID, } \\
\text { Country }\end{array}$ & $\begin{array}{l}\text { Design } \\
\text { (Funding) } \\
\text { (Study } \\
\text { Years) }\end{array}$ & $\begin{array}{l}\text { Risk } \\
\text { of } \\
\text { Bias }\end{array}$ & $\begin{array}{l}\text { Eligibility } \\
\text { Criteria }\end{array}$ & Arm & $\begin{array}{l}\text { Implant } \\
\text { Details (\%) } \\
\text { (Only } \\
\text { Reported } \\
\text { Details) }\end{array}$ & $\begin{array}{l}\text { Reconstruction Details (\%) } \\
\text { (Only Reported Details) }\end{array}$ & $\mathbf{N}$ & $\begin{array}{l}\text { Age in } \\
\text { Years, } \\
\text { Mean (SD) } \\
\text { or as } \\
\text { Specified }\end{array}$ & $\begin{array}{l}\text { Race } \\
(\%)\end{array}$ & $\begin{array}{l}\text { Breast } \\
\text { Cancer } \\
\text { Occurrence } \\
\%\end{array}$ & $\begin{array}{l}\text { Cancer } \\
\text { Stage or } \\
\text { Mastectom } \\
\text { y Purpose } \\
(\%)\end{array}$ \\
\hline \multirow[t]{2}{*}{$\begin{array}{l}\text { Le, } 2005 \\
15743498 \\
\text { US }\end{array}$} & $\begin{array}{l}\text { NRCS } \\
\text { (Non- } \\
\text { industry) } \\
(1993- \\
1994)\end{array}$ & High & $\begin{array}{l}\text { I: Age <65 } \\
\text { years; early- } \\
\text { stage/unstaged } \\
\text { first primary } \\
\text { breast cancer } \\
\text { treated with } \\
\text { mastectomy } \\
\text { E: Missing } \\
\text { implant } \\
\text { information; } \\
\text { bilateral } \\
\text { implants of } \\
\text { discordant } \\
\text { types }\end{array}$ & $\begin{array}{l}\text { IBR with } \\
\text { silicone }\end{array}$ & NR & NR & 333 & NR & NR & NR & NR \\
\hline & $\begin{array}{l}\text { NRCS } \\
\text { (Non- } \\
\text { industry) } \\
\text { (1993- } \\
1994)\end{array}$ & High & $\begin{array}{l}\text { I: Age <65 } \\
\text { years; early- } \\
\text { stage/unstaged } \\
\text { first primary } \\
\text { breast cancer } \\
\text { treated with } \\
\text { mastectomy } \\
\text { E: Missing } \\
\text { implant } \\
\text { information; } \\
\text { bilateral } \\
\text { implants of } \\
\text { discordant } \\
\text { types }\end{array}$ & $\begin{array}{l}\text { IBR with } \\
\text { saline }\end{array}$ & NR & NR & 149 & NR & NR & NR & NR \\
\hline
\end{tabular}




\begin{tabular}{|c|c|c|c|c|c|c|c|c|c|c|c|}
\hline $\begin{array}{l}\text { Study, } \\
\text { Publication } \\
\text { Year, } \\
\text { PMID, } \\
\text { Country }\end{array}$ & $\begin{array}{l}\text { Design } \\
\text { (Funding) } \\
\text { (Study } \\
\text { Years) }\end{array}$ & $\begin{array}{l}\text { Risk } \\
\text { of } \\
\text { Bias }\end{array}$ & $\begin{array}{l}\text { Eligibility } \\
\text { Criteria }\end{array}$ & Arm & $\begin{array}{l}\text { Implant } \\
\text { Details (\%) } \\
\text { (Only } \\
\text { Reported } \\
\text { Details) }\end{array}$ & $\begin{array}{l}\text { Reconstruction Details (\%) } \\
\text { (Only Reported Details) }\end{array}$ & $\mathbf{N}$ & $\begin{array}{l}\text { Age in } \\
\text { Years, } \\
\text { Mean (SD) } \\
\text { or as } \\
\text { Specified }\end{array}$ & $\begin{array}{l}\text { Race } \\
(\%)\end{array}$ & $\begin{array}{l}\text { Breast } \\
\text { Cancer } \\
\text { Occurrence } \\
\%\end{array}$ & $\begin{array}{l}\text { Cancer } \\
\text { Stage or } \\
\text { Mastectom } \\
\text { y Purpose } \\
(\%)\end{array}$ \\
\hline & $\begin{array}{l}\text { NRCS } \\
\text { (Non- } \\
\text { industry) } \\
(1993- \\
1994)\end{array}$ & High & $\begin{array}{l}\text { I: Age <65 } \\
\text { years; early- } \\
\text { stage/unstaged } \\
\text { first primary } \\
\text { breast cancer } \\
\text { treated with } \\
\text { mastectomy } \\
\text { E: Missing } \\
\text { implant } \\
\text { information; } \\
\text { bilateral } \\
\text { implants of } \\
\text { discordant } \\
\text { types }\end{array}$ & $\begin{array}{l}\text { IBR with } \\
\text { double } \\
\text { lumen } \\
\text { implants }\end{array}$ & NR & NR & 314 & NR & NR & NR & NR \\
\hline & $\begin{array}{l}\text { NRCS } \\
\text { (Non- } \\
\text { industry) } \\
(1993- \\
1994)\end{array}$ & High & $\begin{array}{l}\text { I: Age }<65 \\
\text { years; early- } \\
\text { stage/unstaged } \\
\text { first primary } \\
\text { breast cancer } \\
\text { treated with } \\
\text { mastectomy } \\
\text { E: Missing } \\
\text { implant } \\
\text { information; } \\
\text { bilateral } \\
\text { implants of } \\
\text { discordant } \\
\text { types }\end{array}$ & Total & N/A & $\mathrm{N} / \mathrm{A}$ & 796 & NR & $\begin{array}{l}\text { W (94.2), } \\
B(1.3), \\
A(1.6), \\
H(2)\end{array}$ & NR & NR \\
\hline $\begin{array}{l}\text { Macadam, } \\
2010, \\
20009795, \\
\text { Canada }\end{array}$ & $\begin{array}{l}\text { NRCS } \\
\text { (Non- } \\
\text { industry) } \\
\text { (NR) }\end{array}$ & High & I: IBR & $\begin{array}{l}\text { IBR with } \\
\text { silicone }\end{array}$ & NR & $\begin{array}{l}\text { Laterality: Uni (40)/Bi (60) } \\
\text { Timing: Imm (82.67)/Del } \\
\text { (17.33) } \\
\text { Chemo: Timing NR (60)/No } \\
\text { chemotherapy (40) } \\
\text { Radio: Timing NR } \\
\text { (37.33)/None(62.67) }\end{array}$ & 75 & $\begin{array}{l}52.27 \\
(9.54) ;<45 \\
\text { years } \\
(25.33 \%), \\
\geq 45 \text { years } \\
(74.67 \%)\end{array}$ & $\begin{array}{l}\text { W } \\
(34.78), \\
\text { A } \\
\text { (57.97), } \\
\text { Others } \\
(7.25)\end{array}$ & NR & $\begin{array}{l}\text { None (5.33), } \\
\text { Stage } 0 \\
(42.67), \\
\text { Stage NR } \\
(52)\end{array}$ \\
\hline
\end{tabular}




\begin{tabular}{|c|c|c|c|c|c|c|c|c|c|c|c|}
\hline $\begin{array}{l}\text { Study, } \\
\text { Publication } \\
\text { Year, } \\
\text { PMID, } \\
\text { Country }\end{array}$ & $\begin{array}{l}\text { Design } \\
\text { (Funding) } \\
\text { (Study } \\
\text { Years) }\end{array}$ & $\begin{array}{l}\text { Risk } \\
\text { of } \\
\text { Bias }\end{array}$ & $\begin{array}{l}\text { Eligibility } \\
\text { Criteria }\end{array}$ & Arm & $\begin{array}{l}\text { Implant } \\
\text { Details (\%) } \\
\text { (Only } \\
\text { Reported } \\
\text { Details) }\end{array}$ & $\begin{array}{l}\text { Reconstruction Details (\%) } \\
\text { (Only Reported Details) }\end{array}$ & $\mathbf{N}$ & $\begin{array}{l}\text { Age in } \\
\text { Years, } \\
\text { Mean (SD) } \\
\text { or as } \\
\text { Specified }\end{array}$ & $\begin{array}{l}\text { Race } \\
(\%)\end{array}$ & $\begin{array}{l}\text { Breast } \\
\text { Cancer } \\
\text { Occurrence } \\
\%\end{array}$ & $\begin{array}{l}\text { Cancer } \\
\text { Stage or } \\
\text { Mastectom } \\
\text { y Purpose } \\
(\%)\end{array}$ \\
\hline & $\begin{array}{l}\text { NRCS } \\
\text { (Non- } \\
\text { industry) } \\
\text { (NR) }\end{array}$ & High & I: IBR & $\begin{array}{l}\text { IBR with } \\
\text { saline }\end{array}$ & $\begin{array}{l}\text { SURFACE: } \\
\text { Smooth } \\
(100) \\
\text { SHAPE: } \\
\text { Round } \\
(100)\end{array}$ & $\begin{array}{l}\text { Laterality: Uni (44.12)/Bi } \\
\text { (55.88) } \\
\text { Timing: Imm (61.76)/Del } \\
\text { (35.29)/Mixed (2.94) } \\
\text { Chemo: Timing NR (47.06)/No } \\
\text { chemotherapy (52.94) } \\
\text { Radio: Timing NR } \\
\text { (38.24)/None(71.76) }\end{array}$ & 68 & $\begin{array}{l}55.62 \\
(9.14) ;<45 \\
\text { years } \\
(14.49 \%) \text {, } \\
\geq 45 \text { years } \\
(85.51 \%)\end{array}$ & $\begin{array}{l}\text { W } \\
(23.64), \\
\text { A (74.6), } \\
\text { Others } \\
(4.76)\end{array}$ & NR & $\begin{array}{l}\text { None (4.48), } \\
\text { Stage 0 } \\
(35.82), \\
\text { Stage NR } \\
(59.7)\end{array}$ \\
\hline & $\begin{array}{l}\text { NRCS } \\
\text { (Non- } \\
\text { industry) } \\
\text { (NR) }\end{array}$ & High & I: IBR & Total & $\mathrm{N} / \mathrm{A}$ & $\mathrm{N} / \mathrm{A}$ & 143 & NR & NR & NR & NR \\
\hline \multirow[t]{3}{*}{$\begin{array}{l}\text { McCarthy, } \\
2010, \\
21136577, \\
\text { US \& } \\
\text { Canada }\end{array}$} & $\begin{array}{l}\text { NRCS (NR) } \\
(2006- \\
2007)\end{array}$ & High & $\begin{array}{l}\mathrm{I}: \text { Age }>=21 \\
\text { years; IBR }\end{array}$ & $\begin{array}{l}\text { IBR with } \\
\text { silicone }\end{array}$ & NR & $\begin{array}{l}\text { Laterality: Uni (47.2)/Bi (52.8) } \\
\text { Timing: Imm (65.9)/Del (34.1) } \\
\text { Radio: Before (25)/After (23.3) }\end{array}$ & 176 & $53.7(11)$ & $\begin{array}{l}\text { W (92), } \\
B(1.1), \\
A(1.7), \\
\text { Others } \\
(5.2)\end{array}$ & NR & NR \\
\hline & $\begin{array}{l}\text { NRCS (NR) } \\
(2006- \\
2007)\end{array}$ & High & $\begin{array}{l}\mathrm{I}: \text { Age }>=21 \\
\text { years; IBR }\end{array}$ & $\begin{array}{l}\text { IBR with } \\
\text { saline }\end{array}$ & NR & $\begin{array}{l}\text { Laterality: Uni (60.1)/Bi (39.9) } \\
\text { Timing: Imm (74.2)/Del (25.8) } \\
\text { Radio: Before (20.3)/After } \\
\text { (19.3) }\end{array}$ & 306 & $51.3(10.4)$ & $\begin{array}{l}\text { W (87.8), } \\
\text { B (4), A } \\
(4), \\
\text { Others } \\
(4.2)\end{array}$ & NR & NR \\
\hline & $\begin{array}{l}\text { NRCS (NR) } \\
(2006- \\
2007)\end{array}$ & High & $\begin{array}{l}\text { I: Age }>=21 \\
\text { years; IBR }\end{array}$ & Total & $\mathrm{N} / \mathrm{A}$ & $\mathrm{N} / \mathrm{A}$ & 482 & NR & NR & NR & NR \\
\hline
\end{tabular}

Blue coloring is only to visually separate different studies.

I: inclusion criteria, E: exclusion criteria

Laterality: whether the reconstruction was unilateral ("Uni”) or bilateral ("Bi"). Stages: Whether the reconstruction was completed in 1 stage or $>1$ stages. Timing: Timing of reconstruction relative to mastectomy, i.e., immediate ("Imm") or delayed ("Del"). Chemo: Timing of chemotherapy relative to reconstruction. Radio: Timing of radiation therapy relative to reconstruction.

Abbreviations: $\mathrm{BR}=$ breast reconstruction, $\mathrm{IBR}=$ implant-based reconstruction, $\mathrm{IQR}=$ interquartile range, $\mathrm{N} / \mathrm{A}=$ not applicable, $\mathrm{NR}=$ not reported, $\mathrm{NRCS}=$ nonrandomized comparative study, $\mathrm{PMID}=$ PubMed identifier, $\mathrm{SD}=$ standard deviation, $\mathrm{TE} / \mathrm{I}=$ tissue expander/implant, $\mathrm{Ther}=$ therapeutic.

Colors: Header rows are shaded orange. Rows for every alternate study are shaded blue. The colors do not add unique information. 
Table C-5. Key Question 4: Comparison of anatomic planes for IBR - summary of design, arm, and sample details

\begin{tabular}{|c|c|c|c|c|c|c|c|c|c|c|c|}
\hline $\begin{array}{l}\text { Study, } \\
\text { Publication } \\
\text { Year, } \\
\text { PMID, } \\
\text { Country }\end{array}$ & $\begin{array}{l}\text { Design } \\
\text { (Funding) } \\
\text { (Study } \\
\text { Years) }\end{array}$ & $\begin{array}{l}\text { Risk } \\
\text { of } \\
\text { Bias }\end{array}$ & $\begin{array}{l}\text { Eligibility } \\
\text { Criteria }\end{array}$ & Arm & $\begin{array}{l}\text { Implant Details (\%) } \\
\text { (Only Reported } \\
\text { Details) }\end{array}$ & $\begin{array}{l}\text { Reconstruction Details } \\
(\%) \\
\text { (Only Reported Details) }\end{array}$ & $\mathbf{N}$ & $\begin{array}{l}\text { Age in } \\
\text { Years, } \\
\text { Mean } \\
\text { (SD) or } \\
\text { as } \\
\text { Specified }\end{array}$ & $\begin{array}{l}\text { Race } \\
(\%)\end{array}$ & $\begin{array}{l}\text { Breast } \\
\text { Cancer } \\
\text { Occurrence } \\
\%\end{array}$ & $\begin{array}{l}\text { Cancer } \\
\text { Stage or } \\
\text { Mastectomy } \\
\text { Purpose (\%) }\end{array}$ \\
\hline \multirow[t]{3}{*}{$\begin{array}{l}\text { Lee, } 2021 \mathrm{~b}, \\
33691448, \\
\text { South Korea }\end{array}$} & $\begin{array}{l}\text { RCT } \\
\text { (None) } \\
(2018- \\
2019)\end{array}$ & $\begin{array}{l}\text { Mode } \\
\text { rate }\end{array}$ & $\begin{array}{l}\text { I: Age } 30-60 ; \\
\text { Immediate IBR } \\
\text { E: Advanced- } \\
\text { stage III or IV } \\
\text { breast cancer }\end{array}$ & Prepectoral & $\begin{array}{l}\text { MATERIAL: Silicone } \\
(100) \\
\text { SURFACE: } \\
\text { Textured }(100) \\
\text { ADM: Yes }(100)\end{array}$ & $\begin{array}{l}\text { Laterality: Uni (100) } \\
\text { Timing: Imm (100) } \\
\text { Chemo: Timing NR (30) } \\
\text { Radio: Timing NR (25) }\end{array}$ & 20 & $46.2(7.1)$ & NR & NR & $\begin{array}{l}\text { Stage 0: } 30 \\
\text { Stage I: } 30 \\
\text { Stage II: } 40\end{array}$ \\
\hline & $\begin{array}{l}\text { RCT } \\
\text { (None) } \\
(2018- \\
2019)\end{array}$ & $\begin{array}{l}\text { Mode } \\
\text { rate }\end{array}$ & $\begin{array}{l}\text { I: Age } 30-60 ; \\
\text { Immediate IBR } \\
\text { E: Advanced- } \\
\text { stage III or IV } \\
\text { breast cancer }\end{array}$ & $\begin{array}{l}\text { Partial } \\
\text { submuscular }\end{array}$ & $\begin{array}{l}\text { MATERIAL: Silicone } \\
(100) \\
\text { SURFACE: } \\
\text { Textured }(100) \\
\text { ADM: Yes }(100)\end{array}$ & $\begin{array}{l}\text { Laterality: Uni (100) } \\
\text { Timing: Imm (100) } \\
\text { Chemo: Timing NR (42.9) } \\
\text { Radio: Timing NR (35.7) }\end{array}$ & 14 & $46.8(4.4)$ & NR & NR & $\begin{array}{l}\text { Stage 0: } 21.4 \\
\text { Stage I: } 42.9 \\
\text { Stage II: } 35.7\end{array}$ \\
\hline & $\begin{array}{l}\text { RCT } \\
\text { (None) } \\
(2018- \\
2019)\end{array}$ & $\begin{array}{l}\text { Mode } \\
\text { rate }\end{array}$ & $\begin{array}{l}\text { I: Age } 30-60 ; \\
\text { Immediate IBR } \\
\text { E: Advanced- } \\
\text { stage III or IV } \\
\text { breast cancer }\end{array}$ & Total & $\mathrm{N} / \mathrm{A}$ & $\mathrm{N} / \mathrm{A}$ & 34 & NR & NR & NR & NR \\
\hline \multirow[t]{3}{*}{$\begin{array}{l}\text { Avila, } 2020, \\
33234947 \\
\text { US }\end{array}$} & $\begin{array}{l}\text { NRCS } \\
\text { (None) } \\
\text { (2014- } \\
2018)\end{array}$ & High & I: IBR & Prepectoral & NR & $\begin{array}{l}\text { Laterality: Uni (14.8)/Bi } \\
\text { (85.2) } \\
\text { Timing: Imm (97)/Del (3) } \\
\text { Stages: } 1(73.9) / 2(26.1) \\
\text { Chemo: Before (15.3) } \\
\text { Radio: Timing NR (3.5) }\end{array}$ & 203 & $\begin{array}{l}46.5 \\
(10.0)\end{array}$ & NR & NR & NR \\
\hline & $\begin{array}{l}\text { NRCS } \\
\text { (None) } \\
\text { (2014- } \\
2018)\end{array}$ & High & I: IBR & $\begin{array}{l}\text { Total } \\
\text { submuscular }\end{array}$ & NR & $\begin{array}{l}\text { Laterality: Uni (12.9)/Bi } \\
\text { (87.1) } \\
\text { Timing: Imm (97)/Del (3) } \\
\text { Stages: } 1(33.2) / 2(66.8) \\
\text { Chemo: Before (14.4) } \\
\text { Radio: Timing NR (0.5) }\end{array}$ & 202 & $\begin{array}{l}45.9 \\
(10.4)\end{array}$ & NR & NR & NR \\
\hline & $\begin{array}{l}\text { NRCS } \\
\text { (None) } \\
\text { (2014- } \\
2018) \\
\end{array}$ & High & I: IBR & Total & N/A & $\mathrm{N} / \mathrm{A}$ & 405 & $\begin{array}{l}46.2 \\
(10.2)\end{array}$ & NR & NR & NR \\
\hline
\end{tabular}




\begin{tabular}{|c|c|c|c|c|c|c|c|c|c|c|c|}
\hline $\begin{array}{l}\text { Study, } \\
\text { Publication } \\
\text { Year, } \\
\text { PMID, } \\
\text { Country }\end{array}$ & $\begin{array}{l}\text { Design } \\
\text { (Funding) } \\
\text { (Study } \\
\text { Years) }\end{array}$ & $\begin{array}{l}\text { Risk } \\
\text { of } \\
\text { Bias }\end{array}$ & $\begin{array}{l}\text { Eligibility } \\
\text { Criteria }\end{array}$ & Arm & $\begin{array}{l}\text { Implant Details (\%) } \\
\text { (Only Reported } \\
\text { Details) }\end{array}$ & $\begin{array}{l}\text { Reconstruction Details } \\
\text { (\%) } \\
\text { (Only Reported Details) }\end{array}$ & $\mathbf{N}$ & $\begin{array}{l}\text { Age in } \\
\text { Years, } \\
\text { Mean } \\
\text { (SD) or } \\
\text { as } \\
\text { Specified }\end{array}$ & $\begin{array}{l}\text { Race } \\
(\%)\end{array}$ & $\begin{array}{l}\text { Breast } \\
\text { Cancer } \\
\text { Occurrence } \\
\%\end{array}$ & $\begin{array}{l}\text { Cancer } \\
\text { Stage or } \\
\text { Mastectomy } \\
\text { Purpose (\%) }\end{array}$ \\
\hline \multirow[t]{3}{*}{$\begin{array}{l}\text { Cattelani, } \\
2018, \\
29275104, \\
\text { Italy }\end{array}$} & $\begin{array}{l}\text { NRCS } \\
\text { (NR) } \\
(2015- \\
2016)\end{array}$ & High & $\begin{array}{l}\text { I: Age }<75 \\
\text { years, no } \\
\text { previous } \\
\text { radiation, BMI } \\
<30 \mathrm{~kg} / \mathrm{m} 2 \\
\mathrm{E}: \mathrm{T} 4 \text { or } \mathrm{Mp} \\
\text { breast tumors }\end{array}$ & Prepectoral & $\begin{array}{l}\text { MATERIAL: Silicone } \\
\text { (100) } \\
\text { SURFACE: } \\
\text { Textured (100) } \\
\text { SHAPE: } \\
\text { Anatomic/Teardrop } \\
\text { (100) } \\
\text { SIZE: Mean } 390.9 \\
\text { ml, Range } 180,570\end{array}$ & $\begin{array}{l}\text { Laterality: Uni (82.05)/Bi } \\
\text { (17.95) } \\
\text { Timing: Imm (100) } \\
\text { Stages: } 1(100) \\
\text { Chemo: Before } \\
\text { (10.26)/After }(28.21) / \text { No } \\
\text { chemotherapy (61.53) } \\
\text { Radio: Timing NR } \\
\text { (13.04)/None(86.96) }\end{array}$ & 39 & $\begin{array}{l}52.9(\mathrm{NR}) \\
\text { Range 36, } \\
71\end{array}$ & NR & NR & NR \\
\hline & $\begin{array}{l}\text { NRCS } \\
\text { (NR) } \\
(2015- \\
2016)\end{array}$ & High & $\begin{array}{l}\text { I: Age }<75 \\
\text { years, no } \\
\text { previous } \\
\text { radiation, BMI } \\
<30 \mathrm{~kg} / \mathrm{m} 2 \\
\mathrm{E}: \mathrm{T} 4 \text { or } \mathrm{Mp} \\
\text { breast tumors }\end{array}$ & $\begin{array}{l}\text { Total } \\
\text { submuscular }\end{array}$ & $\begin{array}{l}\text { SURFACE: } \\
\text { Textured } \\
(100) \\
\text { SIZE: Mean } 361.5 \\
\text { ml, Range } 190,650\end{array}$ & $\begin{array}{l}\text { Laterality: Uni }(82.22) / \mathrm{Bi} \\
(17.78) \\
\text { Timing: Imm }(100) \\
\text { Stages: } 1(73.3) />1(26.7) \\
\text { Chemo: Before } \\
\text { (8.89)/After (37.78)/No } \\
\text { chemotherapy (53.33) } \\
\text { Radio: Timing NR } \\
\text { (20.76)/None(79.24) }\end{array}$ & 45 & $\begin{array}{l}52.3 \text { (NR); } \\
\text { Range 26, } \\
75\end{array}$ & NR & NR & NR \\
\hline & $\begin{array}{l}\text { NRCS } \\
(\text { NR) } \\
(2015- \\
2016)\end{array}$ & High & $\begin{array}{l}\text { I: Age }<75 \\
\text { years, no } \\
\text { previous } \\
\text { radiation, BMI } \\
<30 \mathrm{~kg} / \mathrm{m} 2 \\
\mathrm{E}: \mathrm{T} 4 \text { or } \mathrm{Mp} \\
\text { breast tumors }\end{array}$ & Total & $\mathrm{N} / \mathrm{A}$ & $\mathrm{N} / \mathrm{A}$ & 84 & NR & NR & NR & NR \\
\hline \multirow[t]{2}{*}{$\begin{array}{l}\text { Gabriel, } \\
2020 \\
32195862, \\
\text { US }\end{array}$} & $\begin{array}{l}\text { NRCS } \\
\text { (None) } \\
2009- \\
2017 \text { ) }\end{array}$ & High & $\begin{array}{l}\text { I: Immediate } \\
\text { two-stage IBR; } \\
\text { BMI >=30 } \\
\text { E: Revision } \\
\text { reconstruction }\end{array}$ & Prepectoral & NR & $\begin{array}{l}\text { Laterality: Uni (52.7)/Bi } \\
\text { (47.3) } \\
\text { Timing: Imm (100) } \\
\text { Chemo: Before } \\
\text { (13.2)/After (5.9) } \\
\text { Radio: Before (3.9)/After } \\
\text { (1.6) }\end{array}$ & 68 & $\begin{array}{l}\text { Median: } \\
49(\text { IQR } \\
33,76)\end{array}$ & NR & NR & NR \\
\hline & $\begin{array}{l}\text { NRCS } \\
\text { (None) } \\
2009- \\
2017 \text { ) }\end{array}$ & High & $\begin{array}{l}\text { I: Immediate } \\
\text { two-stage IBR; } \\
\text { BMI >=30 } \\
\text { E: Revision } \\
\text { reconstruction }\end{array}$ & $\begin{array}{l}\text { Partial } \\
\text { submuscular }\end{array}$ & NR & $\begin{array}{l}\text { Laterality: Uni (50.8)/Bi } \\
\text { (49.2) } \\
\text { Chemo: Before } \\
\text { (12.3)/After (30.8) } \\
\text { Radio: Before (3.1)/After } \\
\text { (10.9) }\end{array}$ & 65 & $\begin{array}{l}\text { Median: } \\
53(\text { IQR } \\
28,73)\end{array}$ & NR & NR & NR \\
\hline
\end{tabular}




\begin{tabular}{|c|c|c|c|c|c|c|c|c|c|c|c|}
\hline $\begin{array}{l}\text { Study, } \\
\text { Publication } \\
\text { Year, } \\
\text { PMID, } \\
\text { Country }\end{array}$ & $\begin{array}{l}\text { Design } \\
\text { (Funding) } \\
\text { (Study } \\
\text { Years) }\end{array}$ & $\begin{array}{l}\text { Risk } \\
\text { of } \\
\text { Bias }\end{array}$ & $\begin{array}{l}\text { Eligibility } \\
\text { Criteria }\end{array}$ & Arm & $\begin{array}{l}\text { Implant Details (\%) } \\
\text { (Only Reported } \\
\text { Details) }\end{array}$ & $\begin{array}{l}\text { Reconstruction Details } \\
(\%) \\
\text { (Only Reported Details) }\end{array}$ & $\mathbf{N}$ & $\begin{array}{l}\text { Age in } \\
\text { Years, } \\
\text { Mean } \\
\text { (SD) or } \\
\text { as } \\
\text { Specified }\end{array}$ & $\begin{array}{l}\text { Race } \\
(\%)\end{array}$ & $\begin{array}{l}\text { Breast } \\
\text { Cancer } \\
\text { Occurrence } \\
\%\end{array}$ & $\begin{array}{l}\text { Cancer } \\
\text { Stage or } \\
\text { Mastectomy } \\
\text { Purpose (\%) }\end{array}$ \\
\hline & $\begin{array}{l}\text { NRCS } \\
\text { (None) } \\
2009- \\
2017 \text { ) }\end{array}$ & High & $\begin{array}{l}\text { I: Immediate } \\
\text { two-stage IBR; } \\
\text { BMI >=30 } \\
\text { E: Revision } \\
\text { reconstruction }\end{array}$ & Total & $\mathrm{N} / \mathrm{A}$ & $\mathrm{N} / \mathrm{A}$ & 133 & NR & NR & NR & NR \\
\hline \multirow[t]{3}{*}{$\begin{array}{l}\text { Kim, 2020, } \\
33066236, \\
\text { South Korea }\end{array}$} & $\begin{array}{l}\text { NRCS } \\
\text { (None) } \\
2015- \\
2020 \text { ) }\end{array}$ & $\begin{array}{l}\text { Mode } \\
\text { rate }\end{array}$ & $\begin{array}{l}\text { I: Immediate } \\
\text { unilateral } \\
\text { single-stage } \\
\text { IBR with ADM } \\
\text { E: Previous } \\
\text { breast surgery } \\
\text { or radiation } \\
\text { therapy }\end{array}$ & Prepectoral & $\begin{array}{l}\text { Size: Mean } 249.0 \text { cc } \\
\text { (SD 104.8) } \\
\text { ADM: Yes }(100)\end{array}$ & $\begin{array}{l}\text { Laterality: Uni }(100) \\
\text { Timing: Imm (100) } \\
\text { Stages: } 1(100) \\
\text { Chemo: Before (5.7)/After } \\
\text { (32.1) } \\
\text { Radio: Timing NR (11.3) }\end{array}$ & 53 & $47.7(7.5)$ & NR & $1^{\text {st: }} 100$ & $\begin{array}{l}\text { Stage I: } 73.6 \\
\text { Stage II: } 20.8 \\
\text { Stage III: } 5.7\end{array}$ \\
\hline & $\begin{array}{l}\text { NRCS } \\
\text { (None) } \\
2015- \\
2020 \text { ) }\end{array}$ & $\begin{array}{l}\text { Mode } \\
\text { rate }\end{array}$ & $\begin{array}{l}\text { I: Immediate } \\
\text { unilateral } \\
\text { single-stage } \\
\text { IBR with ADM } \\
\text { E: Previous } \\
\text { breast surgery } \\
\text { or radiation } \\
\text { therapy }\end{array}$ & $\begin{array}{l}\text { Partial } \\
\text { submuscular }\end{array}$ & $\begin{array}{l}\text { Size: Mean } 268.1 \mathrm{cc} \\
\text { (SD 103.0) } \\
\text { ADM: Yes }(100)\end{array}$ & $\begin{array}{l}\text { Laterality: Uni (100) } \\
\text { Timing: Imm (100) } \\
\text { Stages: } 1(100) \\
\text { Chemo: Before } \\
\text { (10.5)/After (43.0) } \\
\text { Radio: Timing NR (18.4) }\end{array}$ & 114 & $46.6(8.7)$ & NR & $1^{\text {st: }} 100$ & $\begin{array}{l}\text { Stage I: } 66.7 \\
\text { Stage II: } 21.0 \\
\text { Stage III: } \\
10.2\end{array}$ \\
\hline & $\begin{array}{l}\text { NRCS } \\
\text { (None) } \\
2015- \\
2020 \text { ) }\end{array}$ & $\begin{array}{l}\text { Mode } \\
\text { rate }\end{array}$ & $\begin{array}{l}\text { I: Immediate } \\
\text { unilateral } \\
\text { single-stage } \\
\text { IBR with ADM } \\
\text { E: Previous } \\
\text { breast surgery } \\
\text { or radiation } \\
\text { therapy }\end{array}$ & Total & $\mathrm{N} / \mathrm{A}$ & $\mathrm{N} / \mathrm{A}$ & 167 & NR & NR & $1^{\text {st: }} 100$ & NR \\
\hline $\begin{array}{l}\text { Kraenzlin, } \\
2021 \\
32568752 \\
\text { US }\end{array}$ & $\begin{array}{l}\text { NRCS } \\
\text { (None) } \\
2016- \\
2018 \text { ) }\end{array}$ & High & $\begin{array}{l}\text { I: Adults; IBR } \\
\text { with TE }\end{array}$ & Prepectoral & NR & $\begin{array}{l}\text { Laterality: Uni (40.8)/Bi } \\
(59.2) \\
\text { Timing: Del (100) } \\
\text { Stages: >1 (100) } \\
\text { Chemo: Before } \\
\text { (21.9)/After (13.6) } \\
\text { Radio: Before (11.8)/After } \\
(7.1)\end{array}$ & 169 & 48.8 & NR & NR & NR \\
\hline
\end{tabular}




\begin{tabular}{|c|c|c|c|c|c|c|c|c|c|c|c|}
\hline $\begin{array}{l}\text { Study, } \\
\text { Publication } \\
\text { Year, } \\
\text { PMID, } \\
\text { Country }\end{array}$ & $\begin{array}{l}\text { Design } \\
\text { (Funding) } \\
\text { (Study } \\
\text { Years) }\end{array}$ & $\begin{array}{l}\text { Risk } \\
\text { of } \\
\text { Bias }\end{array}$ & $\begin{array}{l}\text { Eligibility } \\
\text { Criteria }\end{array}$ & Arm & $\begin{array}{l}\text { Implant Details (\%) } \\
\text { (Only Reported } \\
\text { Details) }\end{array}$ & $\begin{array}{l}\text { Reconstruction Details } \\
\text { (\%) } \\
\text { (Only Reported Details) }\end{array}$ & $\mathbf{N}$ & $\begin{array}{l}\text { Age in } \\
\text { Years, } \\
\text { Mean } \\
\text { (SD) or } \\
\text { as } \\
\text { Specified }\end{array}$ & $\begin{array}{l}\text { Race } \\
(\%)\end{array}$ & $\begin{array}{l}\text { Breast } \\
\text { Cancer } \\
\text { Occurrence } \\
\%\end{array}$ & $\begin{array}{l}\text { Cancer } \\
\text { Stage or } \\
\text { Mastectomy } \\
\text { Purpose (\%) }\end{array}$ \\
\hline & $\begin{array}{l}\text { NRCS } \\
\text { (None) } \\
2016- \\
2018 \text { ) }\end{array}$ & High & $\begin{array}{l}\text { I: Adults; IBR } \\
\text { with TE }\end{array}$ & $\begin{array}{l}\text { Total } \\
\text { submuscular }\end{array}$ & NR & $\begin{array}{l}\text { Laterality: Uni (47.0)/Bi } \\
\text { (53.0) } \\
\text { Timing: Del (100) } \\
\text { Stages: >1 (100) } \\
\text { Chemo: Before } \\
\text { (26.5)/After (17.1) } \\
\text { Radio: Before (28.2)/After } \\
\text { (10.3) }\end{array}$ & 117 & 49.4 & NR & NR & NR \\
\hline & $\begin{array}{l}\text { NRCS } \\
\text { (None) } \\
2016- \\
2018 \text { ) } \\
\end{array}$ & High & $\begin{array}{l}\text { I: Adults; IBR } \\
\text { with TE }\end{array}$ & Total & $\mathrm{N} / \mathrm{A}$ & $\mathrm{N} / \mathrm{A}$ & 286 & NR & NR & NR & NR \\
\hline \multirow[t]{3}{*}{$\begin{array}{l}\text { Nealon, } \\
2020 a \\
32032345 \\
\text { US }\end{array}$} & $\begin{array}{l}\text { NRCS } \\
\text { (None) } \\
\text { (2014- } \\
2018)\end{array}$ & High & $\begin{array}{l}\text { I: Direct-to- } \\
\text { implant BR }\end{array}$ & Prepectoral & $\begin{array}{l}\text { SURFACE: Smooth } \\
\text { (100) } \\
\text { SHAPE: Round } \\
(100) \\
\text { SIZE: Mean } 468.2 \\
\text { ml, SD } 174.7\end{array}$ & $\begin{array}{l}\text { Laterality: Uni (39.5)/Bi } \\
\text { (60.5) } \\
\text { Timing: Imm (100) } \\
\text { Stages: } 1(100) \\
\text { Chemo: Timing NR } \\
\text { (38.6)/No chemotherapy } \\
\text { (61.4) } \\
\text { Radio: Timing NR } \\
\text { (36.8)/None(63.2) }\end{array}$ & 114 & $\begin{array}{l}52.7 \\
(12.4) ; \\
\text { Median } \\
51.5 ; \text { IQR } \\
47.8,62\end{array}$ & NR & NR & $\begin{array}{l}\text { Stage I } \\
(43.9), \text { Stage } \\
\text { II (17.5), } \\
\text { Stage III (6.1) }\end{array}$ \\
\hline & $\begin{array}{l}\text { NRCS } \\
\text { (None) } \\
\text { (2014- } \\
2018)\end{array}$ & High & $\begin{array}{l}\text { I: Direct-to- } \\
\text { implant BR }\end{array}$ & $\begin{array}{l}\text { Total } \\
\text { submuscular }\end{array}$ & $\begin{array}{l}\text { SURFACE: Smooth } \\
\text { (100) } \\
\text { SHAPE: Round } \\
\text { (100) } \\
\text { SIZE: Mean } 417.2 \\
\text { ml, SD } 141.8\end{array}$ & $\begin{array}{l}\text { Laterality: Uni (32.4)/Bi } \\
\text { (67.6) } \\
\text { Timing: Imm (100) } \\
\text { Stages: } 1(100) \\
\text { Chemo: Timing NR } \\
\text { (31.7)/No chemotherapy } \\
\text { (68.3) } \\
\text { Radio: Timing NR } \\
\text { (36.7)/None(63.3) }\end{array}$ & 142 & $\begin{array}{l}50.7 \\
(10.4) ; \\
\text { Median } \\
51 ; \text { IQR } \\
43.8,58\end{array}$ & NR & NR & $\begin{array}{l}\text { Stage I } \\
(44.4), \text { Stage } \\
\text { II (18.3), } \\
\text { Stage III (7), } \\
\text { Stage IV (1.4) }\end{array}$ \\
\hline & $\begin{array}{l}\text { NRCS } \\
\text { (None) } \\
(2014- \\
2018) \\
\end{array}$ & High & $\begin{array}{l}\text { I: Direct-to- } \\
\text { implant BR }\end{array}$ & Total & $\mathrm{N} / \mathrm{A}$ & $\mathrm{N} / \mathrm{A}$ & 256 & NR & NR & NR & NR \\
\hline
\end{tabular}




\begin{tabular}{|c|c|c|c|c|c|c|c|c|c|c|c|}
\hline $\begin{array}{l}\text { Study, } \\
\text { Publication } \\
\text { Year, } \\
\text { PMID, } \\
\text { Country }\end{array}$ & $\begin{array}{l}\text { Design } \\
\text { (Funding) } \\
\text { (Study } \\
\text { Years) }\end{array}$ & $\begin{array}{l}\text { Risk } \\
\text { of } \\
\text { Bias }\end{array}$ & $\begin{array}{l}\text { Eligibility } \\
\text { Criteria }\end{array}$ & Arm & $\begin{array}{l}\text { Implant Details (\%) } \\
\text { (Only Reported } \\
\text { Details) }\end{array}$ & $\begin{array}{l}\text { Reconstruction Details } \\
\text { (\%) } \\
\text { (Only Reported Details) }\end{array}$ & $\mathbf{N}$ & $\begin{array}{l}\text { Age in } \\
\text { Years, } \\
\text { Mean } \\
\text { (SD) or } \\
\text { as } \\
\text { Specified }\end{array}$ & $\begin{array}{l}\text { Race } \\
(\%)\end{array}$ & $\begin{array}{l}\text { Breast } \\
\text { Cancer } \\
\text { Occurrence } \\
\%\end{array}$ & $\begin{array}{l}\text { Cancer } \\
\text { Stage or } \\
\text { Mastectomy } \\
\text { Purpose (\%) }\end{array}$ \\
\hline \multirow[t]{3}{*}{$\begin{array}{l}\text { Ozgur, } \\
2020, \\
33223365 \\
\text { Turkey }\end{array}$} & $\begin{array}{l}\text { NRCS } \\
\text { (NR) } \\
(2012- \\
2015)\end{array}$ & High & $\begin{array}{l}\text { I: Immediate } \\
\text { single-staged } \\
\text { IBR after } \\
\text { therapeutic } \\
\text { mastectomy }\end{array}$ & $\begin{array}{l}\text { Partial } \\
\text { submuscular }\end{array}$ & $\begin{array}{l}\text { Size: Mean } 375.7 \\
\mathrm{~mm}^{3} \text { (SD 75.7) }\end{array}$ & $\begin{array}{l}\text { Laterality: Uni (82.4)/Bi } \\
\text { (17.6) } \\
\text { Timing: Imm (100) } \\
\text { Stages: } 1 \text { (100) } \\
\text { Chemo: Before (33.0) } \\
\text { Radio: Before (3.3)/After } \\
\text { (56.0) }\end{array}$ & 83 & $\begin{array}{l}43.7 \\
(10.2)\end{array}$ & NR & $\begin{array}{l}1^{\text {st: }} 96.7 \\
\text { Recurrent: } \\
3.3\end{array}$ & $\begin{array}{l}\text { Therapeutic: } \\
100\end{array}$ \\
\hline & $\begin{array}{l}\text { NRCS } \\
\text { (NR) } \\
(2012- \\
2015)\end{array}$ & High & $\begin{array}{l}\text { I: Immediate } \\
\text { single-staged } \\
\text { IBR after } \\
\text { therapeutic } \\
\text { mastectomy }\end{array}$ & $\begin{array}{l}\text { Total } \\
\text { submuscular }\end{array}$ & $\begin{array}{l}\text { Size: Mean } 373 \\
\mathrm{~mm}^{3} \text { (SD 80.9) }\end{array}$ & $\begin{array}{l}\text { Laterality: Uni (82.9)/Bi } \\
\text { (17.1) } \\
\text { Timing: Imm (100) } \\
\text { Stages: } 1(100) \\
\text { Chemo: Before (32.5) } \\
\text { Radio: Before (9.4)/After } \\
\text { (55.6) }\end{array}$ & 107 & $43.0(9.4)$ & NR & $\begin{array}{l}1^{\text {st: }} 90.6 \\
\text { Recurrent: } \\
9.4\end{array}$ & $\begin{array}{l}\text { Therapeutic: } \\
100\end{array}$ \\
\hline & $\begin{array}{l}\text { NRCS } \\
\text { (NR) } \\
(2012- \\
2015)\end{array}$ & High & $\begin{array}{l}\text { I: Immediate } \\
\text { single-staged } \\
\text { IBR after } \\
\text { therapeutic } \\
\text { mastectomy }\end{array}$ & Total & $\mathrm{N} / \mathrm{A}$ & $\mathrm{N} / \mathrm{A}$ & 190 & NR & NR & NR & $\begin{array}{l}\text { Therapeutic: } \\
100\end{array}$ \\
\hline
\end{tabular}

Blue coloring is only to visually separate different studies.

I: inclusion criteria, E: exclusion criteria

Laterality: whether the reconstruction was unilateral ("Uni”) or bilateral ("Bi"). Stages: Whether the reconstruction was completed in 1 stage or $>1$ stages. Timing: Timing of reconstruction relative to mastectomy, i.e., immediate ("Imm") or delayed ("Del”). Chemo: Timing of chemotherapy relative to reconstruction. Radio: Timing of radiation therapy relative to reconstruction.

Abbreviations: $\mathrm{BR}=$ breast reconstruction, $\mathrm{IBR}=$ implant-based reconstruction, $\mathrm{IQR}=$ interquartile range, $\mathrm{N} / \mathrm{A}=$ not applicable, $\mathrm{NR}=$ not reported, $\mathrm{NRCS}=$ nonrandomized comparative study, PMID = PubMed identifier, $\mathrm{SD}=$ standard deviation, SIEA $=$ Superficial inferior epigastric artery perforator, TE/I $=$ tissue expander/implant, Ther $=$ therapeutic, TRAM = Transverse rectus abdominis myocutaneous.

Colors: Header rows are shaded orange. Rows for every alternate study are shaded blue. The colors do not add unique information. 
Table C-6. Key Question 5: Use versus nonuse of human ADM for IBR - summary of design, arm, and sample details

\begin{tabular}{|c|c|c|c|c|c|c|c|c|c|c|c|}
\hline $\begin{array}{l}\text { Study, } \\
\text { Publication } \\
\text { Year, } \\
\text { PMID, } \\
\text { Country }\end{array}$ & $\begin{array}{l}\text { Design } \\
\text { (Funding) } \\
\text { (Study } \\
\text { Years) }\end{array}$ & $\begin{array}{l}\text { Risk } \\
\text { of } \\
\text { Bias }\end{array}$ & $\begin{array}{l}\text { Eligibility } \\
\text { Criteria }\end{array}$ & Arm & $\begin{array}{l}\text { Implant } \\
\text { Details (\%) } \\
\text { (Only } \\
\text { Reported } \\
\text { Details) }\end{array}$ & $\begin{array}{l}\text { Reconstruction } \\
\text { Details (\%) } \\
\text { (Only Reported } \\
\text { Details) }\end{array}$ & $\mathbf{N}$ & $\begin{array}{l}\text { Age in } \\
\text { Years, } \\
\text { Mean (SD) } \\
\text { or as } \\
\text { Specified }\end{array}$ & Race (\%) & $\begin{array}{l}\text { Breast } \\
\text { Cancer } \\
\text { Occurrence } \\
\%\end{array}$ & $\begin{array}{l}\text { Cancer } \\
\text { Stage or } \\
\text { Mastectomy } \\
\text { Purpose (\%) }\end{array}$ \\
\hline \multirow[t]{3}{*}{$\begin{array}{l}\text { McCarthy, } \\
2012, \\
23096987, \\
\text { NCT006391 } \\
\text { 06, US }\end{array}$} & $\begin{array}{l}\text { RCT (Non- } \\
\text { industry) } \\
\text { (NR) }\end{array}$ & $\begin{array}{l}\text { Mode } \\
\text { rate }\end{array}$ & $\begin{array}{l}\text { I: Age >=21 years; } \\
\text { immediate TE/IBR } \\
\text { E: Single-stage } \\
\text { IBR and/or } \\
\text { combined AR + } \\
\text { TE/IBR; prior } \\
\text { irradiation to the } \\
\text { ipsilateral } \\
\text { breast/chest; } \\
\text { history of prior } \\
\text { axillary lymph } \\
\text { node dissection }\end{array}$ & $\begin{array}{l}\text { IBR with } \\
\text { human } \\
\text { ADM }\end{array}$ & $\begin{array}{l}\text { PLANE: Total } \\
\text { submuscular } \\
(100)\end{array}$ & $\begin{array}{l}\text { Laterality: Uni (48)/Bi } \\
\text { (52) } \\
\text { Timing: Imm (100) } \\
\text { Stages: >1 (100) } \\
\text { Chemo: Before } \\
\text { (6)/After (30)/No } \\
\text { chemotherapy (64) }\end{array}$ & 36 & IQR 29, 69 & NR & NR & NR \\
\hline & $\begin{array}{l}\text { RCT (Non- } \\
\text { industry) } \\
\text { (NR) }\end{array}$ & $\begin{array}{l}\text { Mode } \\
\text { rate }\end{array}$ & $\begin{array}{l}\text { I: Age >=21 years; } \\
\text { immediate TE/IBR } \\
\text { E: Single-stage } \\
\text { IBR and/or } \\
\text { combined AR + } \\
\text { TE/IBR; prior } \\
\text { irradiation to the } \\
\text { ipsilateral } \\
\text { breast/chest; } \\
\text { history of prior } \\
\text { axillary lymph } \\
\text { node dissection }\end{array}$ & $\begin{array}{l}\text { IBR without } \\
\text { human } \\
\text { ADM }\end{array}$ & NR & $\begin{array}{l}\text { Laterality: Uni (44)/Bi } \\
\text { (56) } \\
\text { Timing: Imm (100) } \\
\text { Stages: >1 (100) } \\
\text { Chemo: Before } \\
\text { (6)/After (25)/No } \\
\text { chemotherapy (69) }\end{array}$ & 33 & IQR 32, 72 & NR & NR & NR \\
\hline & $\begin{array}{l}\text { RCT (Non- } \\
\text { industry) } \\
\text { (NR) }\end{array}$ & $\begin{array}{l}\text { Mode } \\
\text { rate }\end{array}$ & $\begin{array}{l}\text { I: Age >=21 years; } \\
\text { immediate TE/IBR } \\
\text { E: Single-stage } \\
\text { IBR and/or } \\
\text { combined AR + } \\
\text { TE/IBR; prior } \\
\text { irradiation to the } \\
\text { ipsilateral } \\
\text { breast/chest; } \\
\text { history of prior } \\
\text { axillary lymph } \\
\text { node dissection }\end{array}$ & Total & $\mathrm{N} / \mathrm{A}$ & $\mathrm{N} / \mathrm{A}$ & 69 & NR & NR & NR & NR \\
\hline
\end{tabular}




\begin{tabular}{|c|c|c|c|c|c|c|c|c|c|c|c|}
\hline $\begin{array}{l}\text { Study, } \\
\text { Publication } \\
\text { Year, } \\
\text { PMID, } \\
\text { Country } \\
\end{array}$ & $\begin{array}{l}\text { Design } \\
\text { (Funding) } \\
\text { (Study } \\
\text { Years) }\end{array}$ & $\begin{array}{l}\text { Risk } \\
\text { of } \\
\text { Bias }\end{array}$ & $\begin{array}{l}\text { Eligibility } \\
\text { Criteria }\end{array}$ & Arm & $\begin{array}{l}\text { Implant } \\
\text { Details (\%) } \\
\text { (Only } \\
\text { Reported } \\
\text { Details) } \\
\end{array}$ & $\begin{array}{l}\text { Reconstruction } \\
\text { Details (\%) } \\
\text { (Only Reported } \\
\text { Details) }\end{array}$ & $\mathbf{N}$ & $\begin{array}{l}\text { Age in } \\
\text { Years, } \\
\text { Mean (SD) } \\
\text { or as } \\
\text { Specified }\end{array}$ & Race (\%) & $\begin{array}{l}\text { Breast } \\
\text { Cancer } \\
\text { Occurrence } \\
\%\end{array}$ & $\begin{array}{l}\text { Cancer } \\
\text { Stage or } \\
\text { Mastectomy } \\
\text { Purpose (\%) }\end{array}$ \\
\hline \multirow[t]{3}{*}{$\begin{array}{l}\text { Wendel, } \\
\text { 2013, none, } \\
\text { US }\end{array}$} & $\begin{array}{l}\text { RCT (Non- } \\
\text { industry) } \\
\text { (2007- } \\
\text { 2011) } \\
\end{array}$ & High & I: TE/IBR & $\begin{array}{l}\text { IBR with } \\
\text { human } \\
\text { ADM }\end{array}$ & NR & NR & 20 & NR & NR & NR & NR \\
\hline & $\begin{array}{l}\text { RCT (Non- } \\
\text { industry) } \\
\text { (2007- } \\
\text { 2011) } \\
\end{array}$ & High & I: TE/IBR & $\begin{array}{l}\text { IBR without } \\
\text { human } \\
\text { ADM }\end{array}$ & NR & NR & 16 & NR & NR & NR & NR \\
\hline & $\begin{array}{l}\text { RCT (Non- } \\
\text { industry) } \\
\text { (2007- } \\
2011)\end{array}$ & High & I: TE/IBR & Total & $\mathrm{N} / \mathrm{A}$ & $\mathrm{N} / \mathrm{A}$ & 36 & $\begin{array}{l}18-65 \\
\text { years } \\
(83.3 \%) \\
\geq 65 \text { years } \\
(16.7 \%) \\
\end{array}$ & NR & NR & NR \\
\hline \multirow[t]{3}{*}{$\begin{array}{l}\text { Brooke, } \\
2012, \\
22868313, \\
\text { US }\end{array}$} & $\begin{array}{l}\text { NRCS } \\
\text { (None) } \\
(2000- \\
2010) \\
\end{array}$ & High & $\begin{array}{l}\text { I: TE/IBR } \\
\text { E: Prior major } \\
\text { breast surgery or } \\
\text { BR }\end{array}$ & $\begin{array}{l}\text { IBR with } \\
\text { use of } \\
\text { human } \\
\text { ADM }\end{array}$ & NR & $\begin{array}{l}\text { Laterality: Uni } \\
(31.3) / \mathrm{Bi}(68.7) \\
\text { Stages: >1 (100) }\end{array}$ & 131 & $50(12.1)$ & NR & NR & $\begin{array}{l}\text { Stage NR } \\
(100)\end{array}$ \\
\hline & $\begin{array}{l}\text { NRCS } \\
\text { (None) } \\
(2000- \\
2010)\end{array}$ & High & $\begin{array}{l}\mathrm{I}: \mathrm{TE} / \mathrm{IBR} \\
\mathrm{E}: \text { Prior major } \\
\text { breast surgery or } \\
\mathrm{BR}\end{array}$ & $\begin{array}{l}\text { IBR without } \\
\text { use of } \\
\text { human } \\
\text { ADM }\end{array}$ & NR & $\begin{array}{l}\text { Laterality: Uni } \\
(47.6) / \mathrm{Bi}(52.4) \\
\text { Stages: >1 (100) } \\
\text { Chemo: Timing NR } \\
(38)\end{array}$ & 42 & $46(10.7)$ & NR & NR & $\begin{array}{l}\text { Stage NR } \\
(100)\end{array}$ \\
\hline & $\begin{array}{l}\text { NRCS } \\
\text { (None) } \\
(2000- \\
2010) \\
\end{array}$ & High & $\begin{array}{l}\text { I: TE/IBR } \\
\text { E: Prior major } \\
\text { breast surgery or } \\
\text { BR }\end{array}$ & Total & $\mathrm{N} / \mathrm{A}$ & $\mathrm{N} / \mathrm{A}$ & 173 & $49.7(10.7)$ & NR & NR & $\begin{array}{l}\text { Stage NR } \\
(100)\end{array}$ \\
\hline
\end{tabular}




\begin{tabular}{|c|c|c|c|c|c|c|c|c|c|c|c|}
\hline $\begin{array}{l}\text { Study, } \\
\text { Publication } \\
\text { Year, } \\
\text { PMID, } \\
\text { Country }\end{array}$ & $\begin{array}{l}\text { Design } \\
\text { (Funding) } \\
\text { (Study } \\
\text { Years) }\end{array}$ & $\begin{array}{l}\text { Risk } \\
\text { of } \\
\text { Bias }\end{array}$ & $\begin{array}{l}\text { Eligibility } \\
\text { Criteria }\end{array}$ & Arm & $\begin{array}{l}\text { Implant } \\
\text { Details (\%) } \\
\text { (Only } \\
\text { Reported } \\
\text { Details) } \\
\end{array}$ & $\begin{array}{l}\text { Reconstruction } \\
\text { Details (\%) } \\
\text { (Only Reported } \\
\text { Details) }\end{array}$ & $\mathbf{N}$ & $\begin{array}{l}\text { Age in } \\
\text { Years, } \\
\text { Mean (SD) } \\
\text { or as } \\
\text { Specified }\end{array}$ & Race (\%) & $\begin{array}{l}\text { Breast } \\
\text { Cancer } \\
\text { Occurrence } \\
\%\end{array}$ & $\begin{array}{l}\text { Cancer } \\
\text { Stage or } \\
\text { Mastectomy } \\
\text { Purpose (\%) }\end{array}$ \\
\hline \multirow[t]{3}{*}{$\begin{array}{l}\text { Cattelani, } \\
2018, \\
29275104, \\
\text { Italy }\end{array}$} & $\begin{array}{l}\text { NRCS (NR) } \\
(2015- \\
2016)\end{array}$ & High & $\begin{array}{l}\text { I: Age }<75 \text { years, } \\
\text { no previous } \\
\text { radiotherapy, BMI } \\
<30 \mathrm{~kg} / \mathrm{m} 2 \\
\mathrm{E}: \mathrm{T} 4 \text { or } \mathrm{Mp} \\
\text { breast tumors, } \\
\text { previous surgery } \\
\text { of the same } \\
\text { breast }\end{array}$ & $\begin{array}{l}\text { IBR with } \\
\text { prepectoral } \\
\text { placement }\end{array}$ & $\begin{array}{l}\text { MATERIAL: } \\
\text { Silicone (100) } \\
\text { SURFACE: } \\
\text { Textured } \\
(100) \\
\text { SHAPE: } \\
\text { Anatomic/Tea } \\
\text { rdrop (100) } \\
\text { SIZE: Mean } \\
390.9 \text { ml, } \\
\text { Range 180, } \\
\text { 570 } \\
\text { PLANE: } \\
\text { Prepectoral } \\
\text { (100) }\end{array}$ & $\begin{array}{l}\text { Laterality: Uni } \\
\text { (82.05)/Bi (17.95) } \\
\text { Timing: Imm (100) } \\
\text { Stages: } 1(100) \\
\text { Chemo: Before } \\
\text { (10.26)/After } \\
\text { (28.21)/No } \\
\text { chemotherapy } \\
\text { (61.53) } \\
\text { Radio: Timing NR } \\
\text { (13.04)/None(86.96) }\end{array}$ & 39 & $\begin{array}{l}52.9 \text { (NR); } \\
\text { Range 36, } \\
71\end{array}$ & NR & NR & NR \\
\hline & $\begin{array}{l}\text { NRCS (NR) } \\
(2015- \\
2016)\end{array}$ & High & $\begin{array}{l}\text { I: Age }<75 \text { years, } \\
\text { no previous } \\
\text { radiotherapy, BMI } \\
<30 \mathrm{~kg} / \mathrm{m} 2 \\
\mathrm{E}: \mathrm{T} 4 \text { or } \mathrm{Mp} \\
\text { breast tumors, } \\
\text { previous surgery } \\
\text { of the same } \\
\text { breast }\end{array}$ & $\begin{array}{l}\text { IBR with } \\
\text { total } \\
\text { submuscul } \\
\text { ar } \\
\text { placement }\end{array}$ & $\begin{array}{l}\text { SURFACE: } \\
\text { Textured } \\
(100) \\
\text { SIZE: Mean } \\
361.5 \text { ml, } \\
\text { Range 190, } \\
650 \\
\text { PLANE: Total } \\
\text { submuscular } \\
(100)\end{array}$ & $\begin{array}{l}\text { Laterality: Uni } \\
\text { (82.22)/Bi }(17.78) \\
\text { Timing: Imm }(100) \\
\text { Stages: } 1(73.3) />1 \\
\text { (26.7) } \\
\text { Chemo: Before } \\
\text { (8.89)/After } \\
\text { (37.78)/No } \\
\text { chemotherapy } \\
\text { (53.33) } \\
\text { Radio: Timing NR } \\
\text { (20.76)/None(79.24) }\end{array}$ & 45 & $\begin{array}{l}52.3 \text { (NR); } \\
\text { Range 26, } \\
75\end{array}$ & NR & NR & NR \\
\hline & $\begin{array}{l}\text { NRCS (NR) } \\
(2015- \\
2016)\end{array}$ & High & $\begin{array}{l}\text { I: Age }<75 \text { years, } \\
\text { no previous } \\
\text { radiotherapy, BMI } \\
<30 \mathrm{~kg} / \mathrm{m} 2 \\
\mathrm{E}: \mathrm{T} 4 \text { or } \mathrm{Mp} \\
\text { breast tumors, } \\
\text { previous surgery } \\
\text { of the same } \\
\text { breast }\end{array}$ & Total & $\mathrm{N} / \mathrm{A}$ & $\mathrm{N} / \mathrm{A}$ & 84 & NR & NR & NR & NR \\
\hline
\end{tabular}




\begin{tabular}{|c|c|c|c|c|c|c|c|c|c|c|c|}
\hline $\begin{array}{l}\text { Study, } \\
\text { Publication } \\
\text { Year, } \\
\text { PMID, } \\
\text { Country }\end{array}$ & $\begin{array}{l}\text { Design } \\
\text { (Funding) } \\
\text { (Study } \\
\text { Years) }\end{array}$ & $\begin{array}{l}\text { Risk } \\
\text { of } \\
\text { Bias }\end{array}$ & $\begin{array}{l}\text { Eligibility } \\
\text { Criteria }\end{array}$ & Arm & $\begin{array}{l}\text { Implant } \\
\text { Details (\%) } \\
\text { (Only } \\
\text { Reported } \\
\text { Details) }\end{array}$ & $\begin{array}{l}\text { Reconstruction } \\
\text { Details (\%) } \\
\text { (Only Reported } \\
\text { Details) }\end{array}$ & $\mathbf{N}$ & $\begin{array}{l}\text { Age in } \\
\text { Years, } \\
\text { Mean (SD) } \\
\text { or as } \\
\text { Specified }\end{array}$ & Race (\%) & $\begin{array}{l}\text { Breast } \\
\text { Cancer } \\
\text { Occurrence } \\
\%\end{array}$ & $\begin{array}{l}\text { Cancer } \\
\text { Stage or } \\
\text { Mastectomy } \\
\text { Purpose (\%) }\end{array}$ \\
\hline \multirow[t]{3}{*}{$\begin{array}{l}\text { Chun, 2010, } \\
20124828, \\
\text { US }\end{array}$} & $\begin{array}{l}\text { NRCS (NR) } \\
(2002- \\
2008)\end{array}$ & High & $\begin{array}{l}\text { I: Immediate } \\
\text { TE/implant or AR } \\
+ \text { TE/implant } \\
\text { E: Delayed AR }\end{array}$ & $\begin{array}{l}\text { IBR with } \\
\text { use of } \\
\text { human } \\
\text { ADM }\end{array}$ & NR & $\begin{array}{l}\text { Timing: Imm (100) } \\
\text { Chemo: Before } \\
\text { (14.9)/After (19)/No } \\
\text { chemotherapy (66.1) } \\
\text { Radio: Before } \\
\text { (8.7)/After } \\
\text { (6.5)/None(85.9) }\end{array}$ & $\begin{array}{l}269 \\
\text { breasts }\end{array}$ & $47(10.5)$ & NR & NR & $\begin{array}{l}\text { Stage 0 } \\
(71.4), \text { Stage } \\
\text { I }(4.1), \text { Stage } \\
\text { II }(12.3), \\
\text { Stage III } \\
(12.3)\end{array}$ \\
\hline & $\begin{array}{l}\text { NRCS (NR) } \\
(2002- \\
2008)\end{array}$ & High & $\begin{array}{l}\text { I: Immediate } \\
\text { TE/implant or AR } \\
+ \text { TE/implant } \\
\text { E: Delayed AR }\end{array}$ & $\begin{array}{l}\text { IBR without } \\
\text { use of } \\
\text { human } \\
\text { ADM }\end{array}$ & NR & $\begin{array}{l}\text { Timing: Imm (100) } \\
\text { Chemo: Before } \\
\text { (8.2)/After }(30.8) / \text { No } \\
\text { chemotherapy (61) } \\
\text { Radio: Before } \\
\text { (5.2)/After } \\
\text { (8.6)/None(86.2) }\end{array}$ & $\begin{array}{l}146 \\
\text { breasts }\end{array}$ & $46.2(8.4)$ & NR & NR & $\begin{array}{l}\text { Stage 0 } \\
(66.4), \text { Stage } \\
\text { I (8.2), Stage } \\
\text { II (13.7), } \\
\text { Stage III } \\
(11.6)\end{array}$ \\
\hline & $\begin{array}{l}\text { NRCS (NR) } \\
(2002- \\
2008)\end{array}$ & High & $\begin{array}{l}\text { I: Immediate } \\
\text { TE/implant or AR } \\
+ \text { TE/implant } \\
\text { E: Delayed AR }\end{array}$ & Total & $\mathrm{N} / \mathrm{A}$ & $\mathrm{N} / \mathrm{A}$ & 283 & NR & NR & NR & NR \\
\hline \multirow[t]{3}{*}{$\begin{array}{l}\text { Craig, 2019, } \\
29800083, \\
\text { US }\end{array}$} & $\begin{array}{l}\text { NRCS } \\
\text { (None) } \\
(2004- \\
2014) \\
\end{array}$ & Low & $\begin{array}{l}\text { I: TE/implant BR } \\
\text { E: Pre- } \\
\text { mastectomy } \\
\text { radiation therapy }\end{array}$ & $\begin{array}{l}\text { IBR with } \\
\text { human } \\
\text { ADM }\end{array}$ & NR & $\begin{array}{l}\text { Timing: Imm (100) } \\
\text { Stages: >1 (100) } \\
\text { Radio: After (15.3) }\end{array}$ & NR & $\begin{array}{l}49(10.6) ; \\
\text { Range 29, } \\
68\end{array}$ & NR & NR & Ther (100) \\
\hline & $\begin{array}{l}\text { NRCS } \\
\text { (None) } \\
(2004- \\
2014)\end{array}$ & Low & $\begin{array}{l}\text { I: TE/implant BR } \\
\text { E: Pre- } \\
\text { mastectomy } \\
\text { radiation therapy }\end{array}$ & $\begin{array}{l}\text { IBR without } \\
\text { human } \\
\text { ADM }\end{array}$ & NR & $\begin{array}{l}\text { Timing: Imm (100) } \\
\text { Stages: >1 (100) } \\
\text { Radio: After (14.2) }\end{array}$ & NR & $\begin{array}{l}48.4(10.6) \\
\text { Range } 28 \\
72\end{array}$ & NR & NR & Ther (100) \\
\hline & $\begin{array}{l}\text { NRCS } \\
\text { (None) } \\
(2004- \\
2014)\end{array}$ & Low & $\begin{array}{l}\text { I: TE/implant BR } \\
\text { E: Pre- } \\
\text { mastectomy } \\
\text { radiation therapy }\end{array}$ & Total & $\mathrm{N} / \mathrm{A}$ & $\mathrm{N} / \mathrm{A}$ & 957 & NR & NR & NR & NR \\
\hline $\begin{array}{l}\text { Ganesh } \\
\text { Kumar, } \\
2021, \\
33172826, \\
\text { US \& } \\
\text { Canada }\end{array}$ & $\begin{array}{l}\text { NRCS } \\
\text { (Non- } \\
\text { industry) } \\
(2012- \\
2015)\end{array}$ & $\begin{array}{l}\text { Mode } \\
\text { rate }\end{array}$ & I: TE/IBR & $\begin{array}{l}\text { IBR with } \\
\text { human } \\
\text { ADM }\end{array}$ & NR & $\begin{array}{l}\text { Laterality: Uni } \\
\text { (38.9)/Bi }(61.1) \\
\text { Timing: Imm (100) } \\
\text { Stages: >1 (100) } \\
\text { Chemo: Before } \\
\text { (69.8)/After (30.2) } \\
\text { Radiation: Before } \\
\text { (3.7)/After (21.3) }\end{array}$ & 738 & $48.7(10.5)$ & NR & NR & $\begin{array}{l}\text { Ther (85.8), } \\
\text { Proph (14.2) }\end{array}$ \\
\hline
\end{tabular}




\begin{tabular}{|c|c|c|c|c|c|c|c|c|c|c|c|}
\hline $\begin{array}{l}\text { Study, } \\
\text { Publication } \\
\text { Year, } \\
\text { PMID, } \\
\text { Country }\end{array}$ & $\begin{array}{l}\text { Design } \\
\text { (Funding) } \\
\text { (Study } \\
\text { Years) }\end{array}$ & $\begin{array}{l}\text { Risk } \\
\text { of } \\
\text { Bias }\end{array}$ & $\begin{array}{l}\text { Eligibility } \\
\text { Criteria }\end{array}$ & Arm & $\begin{array}{l}\text { Implant } \\
\text { Details (\%) } \\
\text { (Only } \\
\text { Reported } \\
\text { Details) }\end{array}$ & $\begin{array}{l}\text { Reconstruction } \\
\text { Details (\%) } \\
\text { (Only Reported } \\
\text { Details) }\end{array}$ & $\mathbf{N}$ & $\begin{array}{l}\text { Age in } \\
\text { Years, } \\
\text { Mean (SD) } \\
\text { or as } \\
\text { Specified }\end{array}$ & Race (\%) & $\begin{array}{l}\text { Breast } \\
\text { Cancer } \\
\text { Occurrence } \\
\%\end{array}$ & $\begin{array}{l}\text { Cancer } \\
\text { Stage or } \\
\text { Mastectomy } \\
\text { Purpose (\%) }\end{array}$ \\
\hline & $\begin{array}{l}\text { NRCS } \\
\text { (Non- } \\
\text { industry) } \\
(2012- \\
2015)\end{array}$ & $\begin{array}{l}\text { Mode } \\
\text { rate }\end{array}$ & I: TE/IBR & $\begin{array}{l}\text { IBR without } \\
\text { human } \\
\text { ADM }\end{array}$ & NR & $\begin{array}{l}\text { Laterality: Uni } \\
\text { (38.8)/Bi (61.2) } \\
\text { Timing: Imm (100) } \\
\text { Stages: >1 (100) } \\
\text { Chemo: Before } \\
\text { (61.9)/After (38.1) } \\
\text { Radiation: Before } \\
\text { (5.9)/After (20.9) }\end{array}$ & 713 & $48.1(10.1)$ & NR & NR & $\begin{array}{l}\text { Ther (93.3), } \\
\text { Proph (6.7) }\end{array}$ \\
\hline & $\begin{array}{l}\text { NRCS } \\
\text { (Non- } \\
\text { industry) } \\
(2012- \\
2015)\end{array}$ & $\begin{array}{l}\text { Mode } \\
\text { rate }\end{array}$ & I: TE/IBR & Total & $\mathrm{N} / \mathrm{A}$ & N/A & 1451 & $48.4(10.3)$ & NR & NR & $\begin{array}{l}\text { Ther (89.5), } \\
\text { Proph (10.5) }\end{array}$ \\
\hline \multirow{3}{*}{$\begin{array}{l}\text { Hirsch, } \\
2014, \\
25347643, \\
\text { US }\end{array}$} & $\begin{array}{l}\text { NRCS (NR) } \\
(1998- \\
2008) \\
\end{array}$ & Low & $\begin{array}{l}\text { L: Immediate } \\
\text { TE/IBR }\end{array}$ & $\begin{array}{l}\text { IBR with } \\
\text { human } \\
\text { ADM }\end{array}$ & NR & $\begin{array}{l}\text { Timing: Imm (100) } \\
\text { Stages: >1 (100) }\end{array}$ & 201 & NR & NR & NR & NR \\
\hline & $\begin{array}{l}\text { NRCS (NR) } \\
(1998- \\
2008) \\
\end{array}$ & Low & $\begin{array}{l}\text { L: Immediate } \\
\text { TE/IBR }\end{array}$ & $\begin{array}{l}\text { IBR without } \\
\text { human } \\
\text { ADM }\end{array}$ & NR & $\begin{array}{l}\text { Timing: Imm (100) } \\
\text { Stages: >1 (100) }\end{array}$ & 675 & NR & NR & NR & NR \\
\hline & $\begin{array}{l}\text { NRCS (NR) } \\
(1998- \\
2008)\end{array}$ & Low & $\begin{array}{l}\text { L: Immediate } \\
\text { TE/IBR }\end{array}$ & Total & $\mathrm{N} / \mathrm{A}$ & $\mathrm{N} / \mathrm{A}$ & 876 & $\begin{array}{l}\text { Mean 48.1; } \\
\text { Range } \\
16.1,57.6\end{array}$ & NR & NR & NR \\
\hline $\begin{array}{l}\text { Ibrahim, } \\
2013, \\
24165587, \\
\text { US }\end{array}$ & $\begin{array}{l}\text { NRCS } \\
\text { (None) } \\
(2005- \\
2011)\end{array}$ & $\begin{array}{l}\text { Mode } \\
\text { rate }\end{array}$ & $\begin{array}{l}\text { I: Immediate or } \\
\text { delayed TE/IBR } \\
\text { E: AR with or } \\
\text { without ADM }\end{array}$ & $\begin{array}{l}\text { IBR with } \\
\text { human } \\
\text { ADM }\end{array}$ & NR & NR & 3283 & $50.7(10.6)$ & $\begin{array}{l}\text { W (83), B } \\
(5.7), A \\
(2.9), \mathrm{H} \\
(0.3), \\
\text { Other } 1 \\
(0.1), \\
\text { Other } 2 \\
(0.2), \\
\text { Other } 3 \\
(7.9)\end{array}$ & NR & NR \\
\hline
\end{tabular}




\begin{tabular}{|c|c|c|c|c|c|c|c|c|c|c|c|}
\hline $\begin{array}{l}\text { Study, } \\
\text { Publication } \\
\text { Year, } \\
\text { PMID, } \\
\text { Country }\end{array}$ & $\begin{array}{l}\text { Design } \\
\text { (Funding) } \\
\text { (Study } \\
\text { Years) }\end{array}$ & $\begin{array}{l}\text { Risk } \\
\text { of } \\
\text { Bias }\end{array}$ & $\begin{array}{l}\text { Eligibility } \\
\text { Criteria }\end{array}$ & Arm & $\begin{array}{l}\text { Implant } \\
\text { Details (\%) } \\
\text { (Only } \\
\text { Reported } \\
\text { Details) } \\
\end{array}$ & $\begin{array}{l}\text { Reconstruction } \\
\text { Details (\%) } \\
\text { (Only Reported } \\
\text { Details) }\end{array}$ & $\mathbf{N}$ & $\begin{array}{l}\text { Age in } \\
\text { Years, } \\
\text { Mean (SD) } \\
\text { or as } \\
\text { Specified }\end{array}$ & Race (\%) & $\begin{array}{l}\text { Breast } \\
\text { Cancer } \\
\text { Occurrence } \\
\%\end{array}$ & $\begin{array}{l}\text { Cancer } \\
\text { Stage or } \\
\text { Mastectomy } \\
\text { Purpose (\%) }\end{array}$ \\
\hline & $\begin{array}{l}\text { NRCS } \\
\text { (None) } \\
(2005- \\
2011)\end{array}$ & $\begin{array}{l}\text { Mode } \\
\text { rate }\end{array}$ & $\begin{array}{l}\text { I: Immediate or } \\
\text { delayed TE/IBR } \\
\text { E: AR with or } \\
\text { without ADM }\end{array}$ & $\begin{array}{l}\text { IBR without } \\
\text { human } \\
\text { ADM }\end{array}$ & NR & NR & 15714 & $51.3(10.8)$ & $\begin{array}{l}\text { W (78.7), } \\
\mathrm{B}(6.6), \mathrm{A} \\
(28), \mathrm{H} \\
(0.8), \\
\text { Other } 1 \\
(0.1), \\
\text { Other } 2 \\
(0.2), \\
\text { Other } 3 \\
(11)\end{array}$ & NR & NR \\
\hline & $\begin{array}{l}\text { NRCS } \\
\text { (None) } \\
(2005- \\
2011)\end{array}$ & $\begin{array}{l}\text { Mode } \\
\text { rate }\end{array}$ & $\begin{array}{l}\text { I: Immediate or } \\
\text { delayed TE/IBR } \\
\text { E: AR with or } \\
\text { without ADM }\end{array}$ & Total & $\mathrm{N} / \mathrm{A}$ & $\mathrm{N} / \mathrm{A}$ & 18977 & $51.2(10.7)$ & $\begin{array}{l}\text { W (79.4), } \\
\mathrm{B}(6.4), \mathrm{A} \\
(2.8), \mathrm{H} \\
(0.1), \\
\text { Other } 1 \\
(0.1), \\
\text { Other } 2 \\
(0.2), \\
\text { Other } 3 \\
(10.3)\end{array}$ & NR & NR \\
\hline \multirow[t]{3}{*}{$\begin{array}{l}\text { Lee, } 2020 \text {, } \\
\text { No PMID, } \\
\text { South Korea }\end{array}$} & $\begin{array}{l}\text { NRCS (NR) } \\
(27738380 \\
10- \\
2016938) \\
\end{array}$ & High & $\begin{array}{l}\text { I: Immediate } \\
\text { unilateral TE/IBR }\end{array}$ & $\begin{array}{l}\text { IBR with } \\
\text { human } \\
\text { ADM }\end{array}$ & NR & $\begin{array}{l}\text { Laterality: Uni (100) } \\
\text { Timing: Imm (100) } \\
\text { Stages: >1 (100) }\end{array}$ & 738 & NR & NR & NR & NR \\
\hline & $\begin{array}{l}\text { NRCS (NR) } \\
(2010- \\
2018)\end{array}$ & High & $\begin{array}{l}\text { I: Immediate } \\
\text { unilateral TE/IBR }\end{array}$ & $\begin{array}{l}\text { IBR without } \\
\text { human } \\
\text { ADM }\end{array}$ & NR & $\begin{array}{l}\text { Laterality: Uni (100) } \\
\text { Timing: Imm (100) } \\
\text { Stages: >1 (100) }\end{array}$ & 693 & NR & NR & NR & NR \\
\hline & $\begin{array}{l}\text { NRCS (NR) } \\
(2010- \\
2018) \\
\end{array}$ & High & $\begin{array}{l}\text { I: Immediate } \\
\text { unilateral TE/IBR }\end{array}$ & Total & $\mathrm{N} / \mathrm{A}$ & N/A & 1431 & $43.8(7.5)$ & NR & NR & NR \\
\hline \multirow[t]{2}{*}{$\begin{array}{l}\text { Liu, 2011, } \\
21228744, \\
\text { US }\end{array}$} & $\begin{array}{l}\text { NRCS (NR) } \\
(2004- \\
2011)\end{array}$ & High & $\begin{array}{l}\text { I: Immediate IBR } \\
\text { E: Delayed } \\
\text { reconstruction; } \\
\text { AR }\end{array}$ & $\begin{array}{l}\text { IBR with } \\
\text { human } \\
\text { ADM }\end{array}$ & NR & $\begin{array}{l}\text { Timing: Imm (100) } \\
\text { Radio: NR } \\
\text { (9.8)/None(90.2) }\end{array}$ & 266 & NR & $\mathrm{NR}$ & $\mathrm{NR}$ & NR \\
\hline & $\begin{array}{l}\text { NRCS (NR) } \\
(2004- \\
2011)\end{array}$ & High & $\begin{array}{l}\text { I: Immediate IBR } \\
\text { E: Delayed } \\
\text { reconstruction; } \\
\text { AR }\end{array}$ & $\begin{array}{l}\text { IBR without } \\
\text { human } \\
\text { ADM }\end{array}$ & NR & $\begin{array}{l}\text { Timing: Imm (100) } \\
\text { Radio: Timing NR } \\
(10.4) / \text { None(89.6) }\end{array}$ & 242 & NR & NR & NR & NR \\
\hline
\end{tabular}




\begin{tabular}{|c|c|c|c|c|c|c|c|c|c|c|c|}
\hline $\begin{array}{l}\text { Study, } \\
\text { Publication } \\
\text { Year, } \\
\text { PMID, } \\
\text { Country }\end{array}$ & $\begin{array}{l}\text { Design } \\
\text { (Funding) } \\
\text { (Study } \\
\text { Years) }\end{array}$ & $\begin{array}{l}\text { Risk } \\
\text { of } \\
\text { Bias }\end{array}$ & $\begin{array}{l}\text { Eligibility } \\
\text { Criteria }\end{array}$ & Arm & $\begin{array}{l}\text { Implant } \\
\text { Details (\%) } \\
\text { (Only } \\
\text { Reported } \\
\text { Details) }\end{array}$ & $\begin{array}{l}\text { Reconstruction } \\
\text { Details (\%) } \\
\text { (Only Reported } \\
\text { Details) }\end{array}$ & $\mathbf{N}$ & $\begin{array}{l}\text { Age in } \\
\text { Years, } \\
\text { Mean (SD) } \\
\text { or as } \\
\text { Specified }\end{array}$ & Race (\%) & $\begin{array}{l}\text { Breast } \\
\text { Cancer } \\
\text { Occurrence } \\
\%\end{array}$ & $\begin{array}{l}\text { Cancer } \\
\text { Stage or } \\
\text { Mastectomy } \\
\text { Purpose (\%) }\end{array}$ \\
\hline & $\begin{array}{l}\text { NRCS (NR) } \\
(2004- \\
2011)\end{array}$ & High & $\begin{array}{l}\text { I: Immediate IBR } \\
\text { E: Delayed } \\
\text { reconstruction; } \\
\text { AR }\end{array}$ & Total & N/A & $\mathrm{N} / \mathrm{A}$ & 508 & NR & NR & NR & NR \\
\hline \multirow[t]{3}{*}{$\begin{array}{l}\text { Nealon, } \\
2020 \mathrm{~b}, \\
31605310, \\
\text { US }\end{array}$} & $\begin{array}{l}\text { NRCS } \\
\text { (None) } \\
\text { (2008- } \\
2018)\end{array}$ & High & $\begin{array}{l}\text { I: Unilateral breast } \\
\text { cancer, bilateral } \\
\text { mastectomy, and } \\
\text { immediate IBR } \\
\text { E: Bilateral AR; } \\
\text { Delayed } \\
\text { contralateral } \\
\text { prophylactic } \\
\text { mastectomy and } \\
\text { BR }\end{array}$ & $\begin{array}{l}\text { IBR with } \\
\text { human } \\
\text { ADM }\end{array}$ & NR & Timing: Imm (100) & $\begin{array}{l}1488 \\
\text { breasts }\end{array}$ & NR & NR & NR & NR \\
\hline & $\begin{array}{l}\text { NRCS } \\
\text { (None) } \\
(2008- \\
2018)\end{array}$ & High & $\begin{array}{l}\text { I: Unilateral breast } \\
\text { cancer, bilateral } \\
\text { mastectomy, and } \\
\text { immediate IBR } \\
\text { E: Bilateral AR; } \\
\text { Delayed } \\
\text { contralateral } \\
\text { prophylactic } \\
\text { mastectomy and } \\
\text { BR }\end{array}$ & $\begin{array}{l}\text { IBR without } \\
\text { human } \\
\text { ADM }\end{array}$ & NR & Timing: Imm (100) & $\begin{array}{l}668 \\
\text { breasts }\end{array}$ & NR & NR & NR & NR \\
\hline & $\begin{array}{l}\text { NRCS } \\
\text { (None) } \\
(2008- \\
2018)\end{array}$ & High & $\begin{array}{l}\text { I: Unilateral breast } \\
\text { cancer, bilateral } \\
\text { mastectomy, and } \\
\text { immediate IBR } \\
\text { E: Bilateral AR; } \\
\text { Delayed } \\
\text { contralateral } \\
\text { prophylactic } \\
\text { mastectomy and } \\
\text { BR }\end{array}$ & Total & $\mathrm{N} / \mathrm{A}$ & $\mathrm{N} / \mathrm{A}$ & 1117 & NR & NR & NR & NR \\
\hline $\begin{array}{l}\text { Pannucci, } \\
2013, \\
23508050, \\
\text { US }\end{array}$ & $\begin{array}{l}\text { NRCS } \\
\text { (Non- } \\
\text { industry) } \\
(2008- \\
2011) \\
\end{array}$ & $\begin{array}{l}\text { Mode } \\
\text { rate }\end{array}$ & $\begin{array}{l}\text { I: TE/IBR } \\
\text { E: } \\
\text { Mastopexy/breast } \\
\text { augmentation }\end{array}$ & $\begin{array}{l}\text { IBR with } \\
\text { human } \\
\text { ADM }\end{array}$ & NR & NR & 3450 & NR & NR & NR & NR \\
\hline
\end{tabular}




\begin{tabular}{|c|c|c|c|c|c|c|c|c|c|c|c|}
\hline $\begin{array}{l}\text { Study, } \\
\text { Publication } \\
\text { Year, } \\
\text { PMID, } \\
\text { Country }\end{array}$ & $\begin{array}{l}\text { Design } \\
\text { (Funding) } \\
\text { (Study } \\
\text { Years) }\end{array}$ & $\begin{array}{l}\text { Risk } \\
\text { of } \\
\text { Bias }\end{array}$ & $\begin{array}{l}\text { Eligibility } \\
\text { Criteria }\end{array}$ & Arm & $\begin{array}{l}\text { Implant } \\
\text { Details (\%) } \\
\text { (Only } \\
\text { Reported } \\
\text { Details) } \\
\end{array}$ & $\begin{array}{l}\text { Reconstruction } \\
\text { Details (\%) } \\
\text { (Only Reported } \\
\text { Details) }\end{array}$ & $\mathbf{N}$ & $\begin{array}{l}\text { Age in } \\
\text { Years, } \\
\text { Mean (SD) } \\
\text { or as } \\
\text { Specified }\end{array}$ & Race (\%) & $\begin{array}{l}\text { Breast } \\
\text { Cancer } \\
\text { Occurrence } \\
\%\end{array}$ & $\begin{array}{l}\text { Cancer } \\
\text { Stage or } \\
\text { Mastectomy } \\
\text { Purpose (\%) }\end{array}$ \\
\hline & $\begin{array}{l}\text { NRCS } \\
\text { (Non- } \\
\text { industry) } \\
\text { (2008- } \\
2011)\end{array}$ & $\begin{array}{l}\text { Mode } \\
\text { rate }\end{array}$ & $\begin{array}{l}\text { I: TE/IBR } \\
\text { E: } \\
\text { Mastopexy/breast } \\
\text { augmentation }\end{array}$ & $\begin{array}{l}\text { IBR without } \\
\text { human } \\
\text { ADM }\end{array}$ & NR & NR & 10799 & NR & NR & NR & NR \\
\hline & $\begin{array}{l}\text { NRCS } \\
\text { (Non- } \\
\text { industry) } \\
(2008- \\
2011)\end{array}$ & $\begin{array}{l}\text { Mode } \\
\text { rate }\end{array}$ & $\begin{array}{l}\text { I: TE/IBR } \\
\text { E: } \\
\text { Mastopexy/breast } \\
\text { augmentation }\end{array}$ & Total & $\mathrm{N} / \mathrm{A}$ & $\mathrm{N} / \mathrm{A}$ & 14249 & $\begin{array}{l}<40 \text { years } \\
(15 \%), 40- \\
60 \text { years } \\
(57.5 \%), \\
\geq 60 \text { years } \\
(21.1 \%)\end{array}$ & NR & NR & NR \\
\hline \multirow[t]{3}{*}{$\begin{array}{l}\text { Peled, 2012, } \\
22634688, \\
\text { US }\end{array}$} & $\begin{array}{l}\text { NRCS (NR) } \\
(2006- \\
2010)\end{array}$ & High & $\begin{array}{l}\text { I: Immediate } \\
\text { TE/IBR }\end{array}$ & $\begin{array}{l}\text { IBR with } \\
\text { human } \\
\text { ADM }\end{array}$ & $\begin{array}{l}\text { SURFACE: } \\
\text { Textured } \\
\text { (100) } \\
\text { PLANE: Total } \\
\text { submuscular } \\
(100)\end{array}$ & $\begin{array}{l}\text { Timing: Imm (100) } \\
\text { Stages: >1 (100) } \\
\text { Chemo: Before } \\
\text { (36)/After (21)/No } \\
\text { chemotherapy (43) } \\
\text { Radio: Before } \\
\text { (9)/After } \\
\text { (14)/None(77) }\end{array}$ & 65 & 48.2 (NR) & NR & NR & $\begin{array}{l}\text { Ther (55), } \\
\text { Proph (45) }\end{array}$ \\
\hline & $\begin{array}{l}\text { NRCS (NR) } \\
(2006- \\
2010)\end{array}$ & High & $\begin{array}{l}\text { I: Immediate } \\
\text { TE/IBR }\end{array}$ & $\begin{array}{l}\text { IBR without } \\
\text { human } \\
\text { ADM }\end{array}$ & $\begin{array}{l}\text { PLANE: Total } \\
\text { submuscular } \\
(100)\end{array}$ & $\begin{array}{l}\text { Timing: Imm (100) } \\
\text { Stages: }>1(100) \\
\text { Chemo: Before } \\
\text { (44.4)/After (23.3)/No } \\
\text { chemotherapy (32.3) } \\
\text { Radio: Before } \\
\text { (4.4)/After } \\
\text { (23.3)/None(72.3) }\end{array}$ & 63 & 44.6 (NR) & NR & NR & $\begin{array}{l}\text { Ther (66.7), } \\
\text { Proph (33.3) }\end{array}$ \\
\hline & $\begin{array}{l}\text { NRCS (NR) } \\
(2006- \\
2010)\end{array}$ & High & $\begin{array}{l}\text { I: Immediate } \\
\text { TE/IBR }\end{array}$ & Total & $\mathrm{N} / \mathrm{A}$ & N/A & 128 & NR & NR & NR & NR \\
\hline
\end{tabular}




\begin{tabular}{|c|c|c|c|c|c|c|c|c|c|c|c|}
\hline $\begin{array}{l}\text { Study, } \\
\text { Publication } \\
\text { Year, } \\
\text { PMID, } \\
\text { Country }\end{array}$ & $\begin{array}{l}\text { Design } \\
\text { (Funding) } \\
\text { (Study } \\
\text { Years) }\end{array}$ & $\begin{array}{l}\text { Risk } \\
\text { of } \\
\text { Bias }\end{array}$ & $\begin{array}{l}\text { Eligibility } \\
\text { Criteria }\end{array}$ & Arm & $\begin{array}{l}\text { Implant } \\
\text { Details (\%) } \\
\text { (Only } \\
\text { Reported } \\
\text { Details) } \\
\end{array}$ & $\begin{array}{l}\text { Reconstruction } \\
\text { Details (\%) } \\
\text { (Only Reported } \\
\text { Details) }\end{array}$ & $\mathbf{N}$ & $\begin{array}{l}\text { Age in } \\
\text { Years, } \\
\text { Mean (SD) } \\
\text { or as } \\
\text { Specified }\end{array}$ & Race (\%) & $\begin{array}{l}\text { Breast } \\
\text { Cancer } \\
\text { Occurrence } \\
\%\end{array}$ & $\begin{array}{l}\text { Cancer } \\
\text { Stage or } \\
\text { Mastectomy } \\
\text { Purpose (\%) }\end{array}$ \\
\hline \multirow[t]{2}{*}{$\begin{array}{l}\text { Qureshi, } \\
2016, \\
27465177, \\
\text { US }\end{array}$} & $\begin{array}{l}\text { NRCS } \\
\text { (Industry) } \\
(2003- \\
2009)\end{array}$ & High & $\begin{array}{l}\text { I: TE/IBR } \\
\text { E: Concomitant or } \\
\text { prior ipsilateral } \\
\text { AR; immediate } \\
\text { implant; ADM } \\
\text { other than } \\
\text { AlloDerm } \\
\text { Regenerative } \\
\text { Tissue Matrix } \\
\text { (LifeCell Corp., } \\
\text { Branchburg, N.J.); } \\
\text { concurrent } \\
\text { congenital or } \\
\text { acquired } \\
\text { ipsilateral breast } \\
\text { deformity; patients } \\
\text { with plans for } \\
\text { future AR }\end{array}$ & $\begin{array}{l}\text { IBR with } \\
\text { human } \\
\text { ADM }\end{array}$ & $\begin{array}{l}\text { SURFACE: } \\
\text { Textured } \\
(100) \\
\text { SHAPE: } \\
\text { Round (100) }\end{array}$ & $\begin{array}{l}\text { Laterality: Uni } \\
\text { (53.6)/Bi (46.5) } \\
\text { Timing: Imm } \\
\text { (93.2)/Del (6.8) } \\
\text { Chemo: Timing NR } \\
\text { (47.5) } \\
\text { Radio: Timing NR } \\
\text { (24.4) }\end{array}$ & 295 & $49.6(10.3)$ & NR & NR & NR \\
\hline & $\begin{array}{l}\text { NRCS } \\
\text { (Industry) } \\
(2003- \\
2009)\end{array}$ & High & $\begin{array}{l}\text { I: TE/IBR } \\
\text { E: Concomitant or } \\
\text { prior ipsilateral } \\
\text { AR; immediate } \\
\text { implant; ADM } \\
\text { other than } \\
\text { AlloDerm } \\
\text { Regenerative } \\
\text { Tissue Matrix } \\
\text { (LifeCell Corp., } \\
\text { Branchburg, N.J.); } \\
\text { concurrent } \\
\text { congenital or } \\
\text { acquired } \\
\text { ipsilateral breast } \\
\text { deformity; patients } \\
\text { with plans for } \\
\text { future AR }\end{array}$ & $\begin{array}{l}\text { IBR without } \\
\text { human } \\
\text { ADM }\end{array}$ & $\begin{array}{l}\text { SURFACE: } \\
\text { Textured } \\
(100) \\
\text { SHAPE: } \\
\text { Round (100) }\end{array}$ & $\begin{array}{l}\text { Laterality: Uni } \\
\text { (55.9)/Bi (45) } \\
\text { Timing: Imm } \\
\text { (83.1)/Del (16.9) } \\
\text { Chemo: Timing NR } \\
\text { (51.7) } \\
\text { Radio: Timing NR } \\
\text { (35.6) }\end{array}$ & 118 & $50.8(9.7)$ & NR & NR & NR \\
\hline
\end{tabular}




\begin{tabular}{|c|c|c|c|c|c|c|c|c|c|c|c|}
\hline $\begin{array}{l}\text { Study, } \\
\text { Publication } \\
\text { Year, } \\
\text { PMID, } \\
\text { Country }\end{array}$ & $\begin{array}{l}\text { Design } \\
\text { (Funding) } \\
\text { (Study } \\
\text { Years) }\end{array}$ & $\begin{array}{l}\text { Risk } \\
\text { of } \\
\text { Bias }\end{array}$ & $\begin{array}{l}\text { Eligibility } \\
\text { Criteria }\end{array}$ & Arm & $\begin{array}{l}\text { Implant } \\
\text { Details (\%) } \\
\text { (Only } \\
\text { Reported } \\
\text { Details) } \\
\end{array}$ & $\begin{array}{l}\text { Reconstruction } \\
\text { Details (\%) } \\
\text { (Only Reported } \\
\text { Details) }\end{array}$ & $\mathbf{N}$ & $\begin{array}{l}\text { Age in } \\
\text { Years, } \\
\text { Mean (SD) } \\
\text { or as } \\
\text { Specified }\end{array}$ & Race (\%) & $\begin{array}{l}\text { Breast } \\
\text { Cancer } \\
\text { Occurrence } \\
\%\end{array}$ & $\begin{array}{l}\text { Cancer } \\
\text { Stage or } \\
\text { Mastectomy } \\
\text { Purpose (\%) }\end{array}$ \\
\hline & $\begin{array}{l}\text { NRCS } \\
\text { (Industry) } \\
\text { (2003- } \\
2009)\end{array}$ & High & $\begin{array}{l}\text { I: TE/IBR } \\
\text { E: Concomitant or } \\
\text { prior ipsilateral } \\
\text { AR; immediate } \\
\text { implant; ADM } \\
\text { other than } \\
\text { AlloDerm } \\
\text { Regenerative } \\
\text { Tissue Matrix } \\
\text { (LifeCell Corp., } \\
\text { Branchburg, N.J.); } \\
\text { concurrent } \\
\text { congenital or } \\
\text { acquired } \\
\text { ipsilateral breast } \\
\text { deformity; patients } \\
\text { with plans for } \\
\text { future AR }\end{array}$ & Total & $\mathrm{N} / \mathrm{A}$ & $\mathrm{N} / \mathrm{A}$ & 413 & NR & NR & NR & NR \\
\hline $\begin{array}{l}\text { Safran, } \\
2020, \\
32221195, \\
\text { Canada }\end{array}$ & $\begin{array}{l}\text { NRCS } \\
\text { (None) } \\
(2016- \\
2018)\end{array}$ & High & $\begin{array}{l}\text { I: Immediate, } \\
\text { direct-to-implant } \\
\text { prepectoral IBR } \\
\text { E: Previously } \\
\text { failed IBR; } \\
\text { extensive skin } \\
\text { envelope radiation } \\
\text { damage; locally } \\
\text { advanced breast } \\
\text { cancer; extensive } \\
\text { skin excision; } \\
\text { delayed IBR or } \\
\text { AR }\end{array}$ & $\begin{array}{l}\text { IBR with } \\
\text { human } \\
\text { ADM }\end{array}$ & $\begin{array}{l}\text { PLANE: } \\
\text { Prepectoral } \\
(100)\end{array}$ & $\begin{array}{l}\text { Timing: Imm (100) } \\
\text { Stages: } 1(100)\end{array}$ & 243 & NR & NR & NR & NR \\
\hline
\end{tabular}




\begin{tabular}{|c|c|c|c|c|c|c|c|c|c|c|c|}
\hline $\begin{array}{l}\text { Study, } \\
\text { Publication } \\
\text { Year, } \\
\text { PMID, } \\
\text { Country } \\
\end{array}$ & $\begin{array}{l}\text { Design } \\
\text { (Funding) } \\
\text { (Study } \\
\text { Years) }\end{array}$ & $\begin{array}{l}\text { Risk } \\
\text { of } \\
\text { Bias }\end{array}$ & $\begin{array}{l}\text { Eligibility } \\
\text { Criteria }\end{array}$ & Arm & $\begin{array}{l}\text { Implant } \\
\text { Details (\%) } \\
\text { (Only } \\
\text { Reported } \\
\text { Details) } \\
\end{array}$ & $\begin{array}{l}\text { Reconstruction } \\
\text { Details (\%) } \\
\text { (Only Reported } \\
\text { Details) }\end{array}$ & $\mathbf{N}$ & $\begin{array}{l}\text { Age in } \\
\text { Years, } \\
\text { Mean (SD) } \\
\text { or as } \\
\text { Specified }\end{array}$ & Race (\%) & $\begin{array}{l}\text { Breast } \\
\text { Cancer } \\
\text { Occurrence } \\
\%\end{array}$ & $\begin{array}{l}\text { Cancer } \\
\text { Stage or } \\
\text { Mastectomy } \\
\text { Purpose (\%) }\end{array}$ \\
\hline & $\begin{array}{l}\text { NRCS } \\
\text { (None) } \\
(2016- \\
2018)\end{array}$ & High & $\begin{array}{l}\text { I: Immediate, } \\
\text { direct-to-implant } \\
\text { prepectoral IBR } \\
\text { E: Previously } \\
\text { failed IBR; } \\
\text { extensive skin } \\
\text { envelope radiation } \\
\text { damage; locally } \\
\text { advanced breast } \\
\text { cancer; extensive } \\
\text { skin excision; } \\
\text { delayed IBR or } \\
\text { AR }\end{array}$ & $\begin{array}{l}\text { IBR without } \\
\text { human } \\
\text { ADM }\end{array}$ & $\begin{array}{l}\text { PLANE: } \\
\text { Prepectoral } \\
(100)\end{array}$ & $\begin{array}{l}\text { Timing: Imm (100) } \\
\text { Stages: } 1(100)\end{array}$ & 70 & NR & NR & NR & NR \\
\hline & $\begin{array}{l}\text { NRCS } \\
\text { (None) } \\
(2016- \\
2018)\end{array}$ & High & $\begin{array}{l}\text { I: Immediate, } \\
\text { direct-to-implant } \\
\text { prepectoral IBR } \\
\text { E: Previously } \\
\text { failed IBR; } \\
\text { extensive skin } \\
\text { envelope radiation } \\
\text { damage; locally } \\
\text { advanced breast } \\
\text { cancer; extensive } \\
\text { skin excision; } \\
\text { delayed IBR or } \\
\text { AR }\end{array}$ & Total & $\mathrm{N} / \mathrm{A}$ & $\mathrm{N} / \mathrm{A}$ & 313 & 48.6 (11.6) & NR & NR & $\begin{array}{l}\text { Ther (44.4), } \\
\text { Proph (55.6) }\end{array}$ \\
\hline \multirow[t]{2}{*}{$\begin{array}{l}\text { Seth, 2012, } \\
23018687, \\
\text { US }\end{array}$} & $\begin{array}{l}\text { NRCS } \\
\text { (Non- } \\
\text { industry) } \\
(2006- \\
2008)\end{array}$ & High & $\begin{array}{l}\text { I: Immediate } \\
\text { TE/IBR } \\
\text { E: Combination of } \\
\text { AR and TE/IBR } \\
\text { (e.g., LD flap) }\end{array}$ & $\begin{array}{l}\text { IBR with } \\
\text { human } \\
\text { ADM }\end{array}$ & $\begin{array}{l}\text { SIZE: Mean } \\
444.2 \text { ml, SD } \\
132.7 \\
\text { PLANE: Total } \\
\text { submuscular } \\
(100)\end{array}$ & $\begin{array}{l}\text { Laterality: Uni (55)/Bi } \\
\text { (45) } \\
\text { Timing: Imm (100) } \\
\text { Radio: Before } \\
\text { (4.5)/After (24.6) }\end{array}$ & $\begin{array}{l}199 \\
\text { Breasts }\end{array}$ & $49.5(11)$ & NR & NR & NR \\
\hline & $\begin{array}{l}\text { NRCS } \\
\text { (Non- } \\
\text { industry) } \\
(2006- \\
2008)\end{array}$ & High & $\begin{array}{l}\text { I: Immediate } \\
\text { TE/IBR } \\
\text { E: Combination of } \\
\text { AR and TE/IBR } \\
\text { (e.g., LD flap) }\end{array}$ & $\begin{array}{l}\text { IBR without } \\
\text { human } \\
\text { ADM }\end{array}$ & $\begin{array}{l}\text { SIZE: Mean } \\
437.3 \text { ml, SD } \\
132.2 \\
\text { PLANE: Total } \\
\text { submuscular } \\
(100)\end{array}$ & $\begin{array}{l}\text { Laterality: Uni (60)/Bi } \\
\text { (40) } \\
\text { Timing: Imm (100) } \\
\text { Radio: Before } \\
\text { (6.4)/After (18.8) }\end{array}$ & $\begin{array}{l}293 \\
\text { breasts }\end{array}$ & $47.4(10.1)$ & NR & NR & NR \\
\hline
\end{tabular}




\begin{tabular}{|c|c|c|c|c|c|c|c|c|c|c|c|}
\hline $\begin{array}{l}\text { Study, } \\
\text { Publication } \\
\text { Year, } \\
\text { PMID, } \\
\text { Country } \\
\end{array}$ & $\begin{array}{l}\text { Design } \\
\text { (Funding) } \\
\text { (Study } \\
\text { Years) }\end{array}$ & $\begin{array}{l}\text { Risk } \\
\text { of } \\
\text { Bias }\end{array}$ & $\begin{array}{l}\text { Eligibility } \\
\text { Criteria }\end{array}$ & Arm & $\begin{array}{l}\text { Implant } \\
\text { Details (\%) } \\
\text { (Only } \\
\text { Reported } \\
\text { Details) } \\
\end{array}$ & $\begin{array}{l}\text { Reconstruction } \\
\text { Details (\%) } \\
\text { (Only Reported } \\
\text { Details) }\end{array}$ & $\mathbf{N}$ & $\begin{array}{l}\text { Age in } \\
\text { Years, } \\
\text { Mean (SD) } \\
\text { or as } \\
\text { Specified } \\
\end{array}$ & Race (\%) & $\begin{array}{l}\text { Breast } \\
\text { Cancer } \\
\text { Occurrence } \\
\%\end{array}$ & $\begin{array}{l}\text { Cancer } \\
\text { Stage or } \\
\text { Mastectomy } \\
\text { Purpose (\%) }\end{array}$ \\
\hline & $\begin{array}{l}\text { NRCS } \\
\text { (Non- } \\
\text { industry) } \\
\text { (2006- } \\
2008)\end{array}$ & High & $\begin{array}{l}\text { I: Immediate } \\
\text { TE/IBR } \\
\text { E: Combination of } \\
\text { AR and TE/IBR } \\
\text { (e.g., LD flap) }\end{array}$ & Total & $\mathrm{N} / \mathrm{A}$ & $\mathrm{N} / \mathrm{A}$ & 417 & NR & NR & NR & NR \\
\hline \multirow[t]{3}{*}{$\begin{array}{l}\text { Sobti, } 2018, \\
29481386, \\
\text { US }\end{array}$} & $\begin{array}{l}\text { NRCS (NR) } \\
(2014- \\
2016)\end{array}$ & High & I: TE/IBR & $\begin{array}{l}\text { IBR with } \\
\text { human } \\
\text { ADM }\end{array}$ & NR & $\begin{array}{l}\text { Laterality: Uni } \\
\text { (25.1)/Bi (74.9) } \\
\text { Chemo: Timing NR } \\
\text { (13.9) } \\
\text { Radio: Timing NR } \\
\text { (9.5) }\end{array}$ & 338 & $46.4(9.8)$ & NR & NR & NR \\
\hline & $\begin{array}{l}\text { NRCS (NR) } \\
(2014- \\
2016)\end{array}$ & High & I: TE/IBR & $\begin{array}{l}\text { IBR without } \\
\text { human } \\
\text { ADM }\end{array}$ & NR & $\begin{array}{l}\text { Laterality: Uni } \\
\text { (43.4)/Bi (56.6) } \\
\text { Chemo: } \\
\text { Timing NR (17) } \\
\text { Radio: Timing NR } \\
\text { (3.7) }\end{array}$ & 376 & $46.7(9.4)$ & NR & NR & NR \\
\hline & $\begin{array}{l}\text { NRCS (NR) } \\
(2014- \\
2016) \\
\end{array}$ & High & I: TE/IBR & Total & $\mathrm{N} / \mathrm{A}$ & $\mathrm{N} / \mathrm{A}$ & 714 & $46.5(9.6)$ & NR & NR & NR \\
\hline \multirow[t]{3}{*}{$\begin{array}{l}\text { Stein, 2020, } \\
32561384, \\
\text { Canada }\end{array}$} & $\begin{array}{l}\text { NRCS } \\
\text { (None) } \\
(2010- \\
2019)\end{array}$ & High & $\begin{array}{l}\text { I: Immediate IBR } \\
\text { with radiation }\end{array}$ & $\begin{array}{l}\text { IBR with } \\
\text { human } \\
\text { ADM }\end{array}$ & $\begin{array}{l}\text { SIZE: Mean } \\
446 \text { cc }\end{array}$ & $\begin{array}{l}\text { Timing: Imm (100) } \\
\text { Stages: } 1(67.4) />1 \\
\text { (32.6) } \\
\text { Radiation: Before } \\
\text { (58.2)/After }(41.8)\end{array}$ & 89 & $51.1(\mathrm{NR})$ & NR & $\mathrm{NR}$ & NR \\
\hline & $\begin{array}{l}\text { NRCS } \\
\text { (None) } \\
(2010- \\
2019)\end{array}$ & High & $\begin{array}{l}\text { I: Immediate IBR } \\
\text { with radiation }\end{array}$ & $\begin{array}{l}\text { IBR without } \\
\text { human } \\
\text { ADM }\end{array}$ & $\begin{array}{l}\text { SIZE: Mean } \\
369 \text { cc }\end{array}$ & $\begin{array}{l}\text { Timing: Imm (100) } \\
\text { Stages: } 1(19.5) />1 \\
\text { (80.5) } \\
\text { Radiation: Before } \\
\text { (56.1)/After (43.9) }\end{array}$ & 41 & 48.6 (NR) & NR & NR & NR \\
\hline & $\begin{array}{l}\text { NRCS } \\
\text { (None) } \\
\text { (2010- } \\
2019) \\
\end{array}$ & High & $\begin{array}{l}\text { I: Immediate IBR } \\
\text { with radiation }\end{array}$ & Total & $\mathrm{N} / \mathrm{A}$ & $\mathrm{N} / \mathrm{A}$ & 130 & $50.3(\mathrm{NR})$ & NR & NR & NR \\
\hline $\begin{array}{l}\text { Vardanian, } \\
2011 \\
22030500, \\
\text { US }\end{array}$ & $\begin{array}{l}\text { NRCS } \\
\text { (None) } \\
(2000- \\
2008)\end{array}$ & High & $\begin{array}{l}\text { I: IBR } \\
\text { E: Delayed BR; } \\
\text { combination of AR } \\
\text { and TE/IBR }\end{array}$ & $\begin{array}{l}\text { IBR with } \\
\text { human } \\
\text { ADM }\end{array}$ & NR & $\begin{array}{l}\text { Laterality: Uni (31)/Bi } \\
\text { (69) } \\
\text { Timing: Imm (100) }\end{array}$ & 123 & $49(11)$ & NR & NR & $\begin{array}{l}\text { None (6.5), } \\
\text { Stage NR } \\
(93.5)\end{array}$ \\
\hline
\end{tabular}




\begin{tabular}{|c|c|c|c|c|c|c|c|c|c|c|c|}
\hline $\begin{array}{l}\text { Study, } \\
\text { Publication } \\
\text { Year, } \\
\text { PMID, } \\
\text { Country } \\
\end{array}$ & $\begin{array}{l}\text { Design } \\
\text { (Funding) } \\
\text { (Study } \\
\text { Years) }\end{array}$ & $\begin{array}{l}\text { Risk } \\
\text { of } \\
\text { Bias }\end{array}$ & $\begin{array}{l}\text { Eligibility } \\
\text { Criteria }\end{array}$ & Arm & $\begin{array}{l}\text { Implant } \\
\text { Details (\%) } \\
\text { (Only } \\
\text { Reported } \\
\text { Details) } \\
\end{array}$ & $\begin{array}{l}\text { Reconstruction } \\
\text { Details (\%) } \\
\text { (Only Reported } \\
\text { Details) }\end{array}$ & $\mathbf{N}$ & $\begin{array}{l}\text { Age in } \\
\text { Years, } \\
\text { Mean (SD) } \\
\text { or as } \\
\text { Specified }\end{array}$ & Race (\%) & $\begin{array}{l}\text { Breast } \\
\text { Cancer } \\
\text { Occurrence } \\
\%\end{array}$ & $\begin{array}{l}\text { Cancer } \\
\text { Stage or } \\
\text { Mastectomy } \\
\text { Purpose (\%) }\end{array}$ \\
\hline & $\begin{array}{l}\text { NRCS } \\
\text { (None) } \\
\text { (2000- } \\
2008) \\
\end{array}$ & High & $\begin{array}{l}\text { I: IBR } \\
\text { E: Delayed BR; } \\
\text { combination of AR } \\
\text { and TE/IBR } \\
\end{array}$ & $\begin{array}{l}\text { IBR without } \\
\text { human } \\
\text { ADM }\end{array}$ & NR & $\begin{array}{l}\text { Laterality: Uni (39)/Bi } \\
\text { (61) } \\
\text { Timing: Imm (100) }\end{array}$ & 80 & $47(10)$ & NR & NR & $\begin{array}{l}\text { None }(16.3), \\
\text { Stage } 0 \\
(83.7)\end{array}$ \\
\hline & $\begin{array}{l}\text { NRCS } \\
\text { (None) } \\
(2000- \\
2008) \\
\end{array}$ & High & $\begin{array}{l}\text { I: IBR } \\
\text { E: Delayed BR; } \\
\text { combination of AR } \\
\text { and TE/IBR }\end{array}$ & Total & $\mathrm{N} / \mathrm{A}$ & $\mathrm{N} / \mathrm{A}$ & 203 & NR & NR & NR & NR \\
\hline \multirow[t]{3}{*}{$\begin{array}{l}\text { Weichman, } \\
2012, \\
22544088, \\
\text { US }\end{array}$} & $\begin{array}{l}\text { NRCS (NR) } \\
(2007- \\
2010)\end{array}$ & $\begin{array}{l}\text { Mode } \\
\text { rate }\end{array}$ & $\begin{array}{l}\text { I: Immediate two- } \\
\text { stage, IBR } \\
\text { E: Immediate } \\
\text { permanent IBR, } \\
\text { AR, combination, } \\
\text { or delayed BR }\end{array}$ & $\begin{array}{l}\text { IBR with } \\
\text { human } \\
\text { ADM }\end{array}$ & NR & $\begin{array}{l}\text { Timing: Imm (100) } \\
\text { Stages: >1 (100) } \\
\text { Chemo: Before } \\
\text { (14.2)/After (31.3)/No } \\
\text { chemotherapy (54.5) } \\
\text { Radio: Before } \\
\text { (7.8)/After } \\
\text { (6.4)/None(85.8) }\end{array}$ & $\begin{array}{l}442 \\
\text { breasts }\end{array}$ & $\begin{array}{l}51.08 \\
(11.7)\end{array}$ & NR & NR & $\begin{array}{l}\text { Stage 0 } \\
(13.1), \text { Stage } \\
\text { I (19.2), } \\
\text { Stage II } \\
(17.4), \text { Stage } \\
\text { III (5.4), } \\
\text { Stage IV } \\
(0.045)\end{array}$ \\
\hline & $\begin{array}{l}\text { NRCS (NR) } \\
(2007- \\
2010)\end{array}$ & $\begin{array}{l}\text { Mode } \\
\text { rate }\end{array}$ & $\begin{array}{l}\text { I: Immediate two- } \\
\text { stage, IBR } \\
\text { E: Immediate } \\
\text { permanent IBR, } \\
\text { AR, combination, } \\
\text { or delayed BR }\end{array}$ & $\begin{array}{l}\text { IBR without } \\
\text { human } \\
\text { ADM }\end{array}$ & $\begin{array}{l}\text { PLANE: Total } \\
\text { submuscular } \\
(100)\end{array}$ & $\begin{array}{l}\text { Timing: Imm (100) } \\
\text { Stages: >1 (100) } \\
\text { Chemo: Before } \\
\text { (16.7)/After (28.6)/No } \\
\text { chemotherapy (54.7) } \\
\text { Radio: Before } \\
\text { (8.7)/After } \\
\text { (7.9)/None(83.4) }\end{array}$ & $\begin{array}{l}186 \\
\text { breasts }\end{array}$ & $\begin{array}{l}49.09 \\
(11.58)\end{array}$ & NR & NR & $\begin{array}{l}\text { Stage } 0 \\
(18.8), \text { Stage } \\
\text { I (18.8), } \\
\text { Stage II } \\
(17.7), \text { Stage } \\
\text { III }(8.6)\end{array}$ \\
\hline & $\begin{array}{l}\text { NRCS (NR) } \\
(2007- \\
2010)\end{array}$ & $\begin{array}{l}\text { Mode } \\
\text { rate }\end{array}$ & $\begin{array}{l}\text { I: Immediate two- } \\
\text { stage, IBR } \\
\text { E: Immediate } \\
\text { permanent IBR, } \\
\text { AR, combination, } \\
\text { or delayed BR }\end{array}$ & Total & $\mathrm{N} / \mathrm{A}$ & $\mathrm{N} / \mathrm{A}$ & 407 & NR & NR & NR & NR \\
\hline $\begin{array}{l}\text { Woo, 2017, } \\
28509694, \\
\text { South Korea }\end{array}$ & $\begin{array}{l}\text { NRCS } \\
\text { (None) } \\
\text { (2010- } \\
2016)\end{array}$ & High & $\begin{array}{l}\text { I: Immediate } \\
\text { TE/IBR } \\
\text { E: Direct-to- } \\
\text { implant BR; AR; } \\
\text { or delayed BR }\end{array}$ & $\begin{array}{l}\text { IBR with } \\
\text { human } \\
\text { ADM }\end{array}$ & NR & $\begin{array}{l}\text { Timing: Imm (100) } \\
\text { Stages: >1 (100) } \\
\text { Chemo: After (43.2) } \\
\text { Radio: Before (3)/After } \\
\text { (13.6)/None(83.4) }\end{array}$ & 199 & $42.9(6.9)$ & NR & NR & NR \\
\hline
\end{tabular}




\begin{tabular}{|c|c|c|c|c|c|c|c|c|c|c|c|}
\hline $\begin{array}{l}\text { Study, } \\
\text { Publication } \\
\text { Year, } \\
\text { PMID, } \\
\text { Country }\end{array}$ & $\begin{array}{l}\text { Design } \\
\text { (Funding) } \\
\text { (Study } \\
\text { Years) }\end{array}$ & $\begin{array}{l}\text { Risk } \\
\text { of } \\
\text { Bias }\end{array}$ & $\begin{array}{l}\text { Eligibility } \\
\text { Criteria }\end{array}$ & Arm & $\begin{array}{l}\text { Implant } \\
\text { Details (\%) } \\
\text { (Only } \\
\text { Reported } \\
\text { Details) }\end{array}$ & $\begin{array}{l}\text { Reconstruction } \\
\text { Details (\%) } \\
\text { (Only Reported } \\
\text { Details) }\end{array}$ & $\mathbf{N}$ & $\begin{array}{l}\text { Age in } \\
\text { Years, } \\
\text { Mean (SD) } \\
\text { or as } \\
\text { Specified }\end{array}$ & Race (\%) & $\begin{array}{l}\text { Breast } \\
\text { Cancer } \\
\text { Occurrence } \\
\%\end{array}$ & $\begin{array}{l}\text { Cancer } \\
\text { Stage or } \\
\text { Mastectomy } \\
\text { Purpose (\%) }\end{array}$ \\
\hline & $\begin{array}{l}\text { NRCS } \\
\text { (None) } \\
\text { (2010- } \\
2016)\end{array}$ & High & $\begin{array}{l}\text { I: Immediate } \\
\text { TE/IBR } \\
\text { E: Direct-to- } \\
\text { implant BR; AR; } \\
\text { or delayed BR }\end{array}$ & $\begin{array}{l}\text { IBR without } \\
\text { human } \\
\text { ADM }\end{array}$ & $\begin{array}{l}\text { PLANE: Total } \\
\text { submuscular } \\
(100)\end{array}$ & $\begin{array}{l}\text { Timing: Imm (100) } \\
\text { Stages: >1 (100) } \\
\text { Chemo: After (36.7) } \\
\text { Radio: Before } \\
\text { (3.0)/After } \\
\text { (16.6)/None(80.4) }\end{array}$ & 199 & $42.8(7.2)$ & NR & NR & NR \\
\hline & $\begin{array}{l}\text { NRCS } \\
\text { (None) } \\
\text { (2010- } \\
2016)\end{array}$ & High & $\begin{array}{l}\text { I: Immediate } \\
\text { TE/IBR } \\
\text { E: Direct-to- } \\
\text { implant BR; AR; } \\
\text { or delayed BR }\end{array}$ & Total & $\mathrm{N} / \mathrm{A}$ & N/A & 398 & NR & NR & NR & NR \\
\hline
\end{tabular}

Blue coloring is only to visually separate different studies.

I: inclusion criteria, E: exclusion criteria

Laterality: whether the reconstruction was unilateral ("Uni”) or bilateral ("Bi"). Stages: Whether the reconstruction was completed in 1 stage or $>1$ stages. Timing: Timing of reconstruction relative to mastectomy, i.e., immediate ("Imm") or delayed ("Del"). Chemo: Timing of chemotherapy relative to reconstruction. Radio: Timing of radiation therapy relative to reconstruction.

$\mathrm{W}=$ White or Caucasian, $\mathrm{B}=$ Black or African American, $\mathrm{A}=$ Asian, $\mathrm{H}=$ Hispanic or Latino.

Proph $=$ prophylactic, Ther $=$ therapeutic

Abbreviations: $\mathrm{AR}=$ autologous reconstruction, $\mathrm{BR}=$ breast reconstruction, $\mathrm{IBR}=$ implant-based reconstruction, $\mathrm{IQR}=$ interquartile range, $\mathrm{N} / \mathrm{A}=$ not applicable, $\mathrm{NR}=$ not reported, NRCS $=$ nonrandomized comparative study, PMID $=$ PubMed identifier, PMRT $=$ postmastectomy radiation therapy, $\mathrm{RCT}=$ randomized controlled trial, $\mathrm{SD}=$ standard deviation, $\mathrm{TE} / \mathrm{I}=$ tissue expander/implant, Ther $=$ therapeutic.

Colors: Header rows are shaded orange. Rows for every alternate study are shaded blue. The colors do not add unique information. 
Table C-7. Key Question 6: Comparison of flap types for autologous reconstruction - summary of design, arm, and sample details

\begin{tabular}{|c|c|c|c|c|c|c|c|c|c|c|}
\hline $\begin{array}{l}\text { Study, } \\
\text { Publication } \\
\text { Year, } \\
\text { PMID, } \\
\text { Country }\end{array}$ & $\begin{array}{l}\text { Design } \\
\text { (Funding) } \\
\text { (Study } \\
\text { Years) }\end{array}$ & $\begin{array}{l}\text { Risk } \\
\text { of } \\
\text { Bias }\end{array}$ & $\begin{array}{l}\text { Eligibility } \\
\text { Criteria }\end{array}$ & Arm & $\begin{array}{l}\text { Reconstruction } \\
\text { Details (\%) }\end{array}$ & $\mathbf{N}$ & $\begin{array}{l}\text { Age in Years, } \\
\text { Mean (SD) or } \\
\text { as Specified }\end{array}$ & Race (\%) & $\begin{array}{l}\text { Breast } \\
\text { Cancer } \\
\text { Occurrence } \\
\%\end{array}$ & $\begin{array}{l}\text { Cancer Stage } \\
\text { or Mastectomy } \\
\text { Purpose (\%) }\end{array}$ \\
\hline \multirow[t]{3}{*}{$\begin{array}{l}\text { Abedi, } 2016, \\
25003437, \\
\text { Canada }\end{array}$} & $\begin{array}{l}\text { NRCS } \\
\text { (None) } \\
(2003- \\
2011) \\
\end{array}$ & High & $\mathrm{I}: \mathrm{BR}$ & AR with DIEP & NR & NR & NR & NR & NR & NR \\
\hline & $\begin{array}{l}\text { NRCS } \\
\text { (None) } \\
(2003- \\
2011) \\
\end{array}$ & High & $\mathrm{I}: \mathrm{BR}$ & AR with TRAM & TIMING: Imm (100) & NR & NR & NR & NR & NR \\
\hline & $\begin{array}{l}\text { NRCS } \\
\text { (None) } \\
(2003- \\
2011)\end{array}$ & High & $\mathrm{I}: \mathrm{BR}$ & Total & $\begin{array}{l}\text { TIMING: Imm (100) } \\
\text { CHEMO: Before } \\
\text { (47.5)/After (13.7) } \\
\text { RADIO: Before } \\
\text { (38.9)/After (3.2) }\end{array}$ & 314 & $50(8.2)$ & NR & NR & NR \\
\hline \multirow{4}{*}{$\begin{array}{l}\text { Baumann, } \\
2010 \\
20440154 \\
\text { US }\end{array}$} & $\begin{array}{l}\text { NRCS (NR) } \\
(2001- \\
2006) \\
\end{array}$ & High & I: Free flap AR & AR with DIEP & STAGES: 1 (100) & 71 & NR & NR & NR & NR \\
\hline & $\begin{array}{l}\text { NRCS (NR) } \\
(2001- \\
2006) \\
\end{array}$ & High & I: Free flap AR & AR with TRAM & STAGES: 1 (100) & 120 & NR & NR & NR & NR \\
\hline & $\begin{array}{l}\text { NRCS (NR) } \\
(2001- \\
2006) \\
\end{array}$ & High & I: Free flap AR & AR with SIEA & STAGES: 1 (100) & 37 & NR & NR & NR & NR \\
\hline & $\begin{array}{l}\text { NRCS (NR) } \\
(2001- \\
2006) \\
\end{array}$ & High & I: Free flap AR & Total & $\mathrm{N} / \mathrm{A}$ & 228 & NR & NR & NR & NR \\
\hline $\begin{array}{l}\text { Brandberg, } \\
2000, \\
10626972, \\
\text { Sweden }\end{array}$ & $\begin{array}{l}\text { RCT (Non- } \\
\text { industry) } \\
\text { (1994-- } \\
1996)\end{array}$ & High & $\begin{array}{l}\text { I: Age <=79; free } \\
\text { of recurrence } \\
\text { E: Poorly } \\
\text { controlled } \\
\text { diabetes and } \\
\text { secondary } \\
\text { complications, } \\
\text { immunosuppressi } \\
\text { ve treatment, } \\
\text { family history or } \\
\text { previous } \\
\text { rheumatic disease }\end{array}$ & AR with TRAM & $\begin{array}{l}\text { LATERALITY: Uni (100) } \\
\text { TIMING: Del (100) } \\
\text { STAGES: >1 (100) } \\
\text { RADIO: Timing NR (48) }\end{array}$ & 29 & $52(9.2)$ & NR & 1st: 100 & Ther (100) \\
\hline
\end{tabular}




\begin{tabular}{|c|c|c|c|c|c|c|c|c|c|c|}
\hline $\begin{array}{l}\text { Study, } \\
\text { Publication } \\
\text { Year, } \\
\text { PMID, } \\
\text { Pountry }\end{array}$ & $\begin{array}{l}\text { Design } \\
\text { (Funding) } \\
\text { (Study } \\
\text { Years) }\end{array}$ & $\begin{array}{l}\text { Risk } \\
\text { of } \\
\text { Bias }\end{array}$ & $\begin{array}{l}\text { Eligibility } \\
\text { Criteria }\end{array}$ & Arm & $\begin{array}{l}\text { Reconstruction } \\
\text { Details (\%) }\end{array}$ & $\mathbf{N}$ & $\begin{array}{l}\text { Age in Years, } \\
\text { Mean (SD) or } \\
\text { as Specified }\end{array}$ & Race (\%) & $\begin{array}{l}\text { Breast } \\
\text { Cancer } \\
\text { Occurrence } \\
\%\end{array}$ & $\begin{array}{l}\text { Cancer Stage } \\
\text { or Mastectomy } \\
\text { Purpose }(\%)\end{array}$ \\
\hline & $\begin{array}{l}\text { RCT (Non- } \\
\text { industry) } \\
\text { (1994- } \\
1996)\end{array}$ & High & $\begin{array}{l}\text { I: Age <=79; free } \\
\text { of recurrence } \\
\text { E: Poorly } \\
\text { controlled } \\
\text { diabetes and } \\
\text { secondary } \\
\text { complications, } \\
\text { immunosuppressi } \\
\text { ve treatment, } \\
\text { family history or } \\
\text { previous } \\
\text { rheumatic disease }\end{array}$ & AR with LD & $\begin{array}{l}\text { LATERALITY: Uni (100) } \\
\text { TIMING: Del (100) } \\
\text { STAGES: >1 (100) } \\
\text { RADIO: Timing NR (47) }\end{array}$ & 30 & $54(8.9)$ & NR & 1st: 100 & Ther (100) \\
\hline & $\begin{array}{l}\text { RCT (Non- } \\
\text { industry) } \\
\text { (1994- } \\
1996)\end{array}$ & High & $\begin{array}{l}\text { I: Age <=79; free } \\
\text { of recurrence } \\
\text { E: Poorly } \\
\text { controlled } \\
\text { diabetes and } \\
\text { secondary } \\
\text { complications, } \\
\text { immunosuppressi } \\
\text { ve treatment, } \\
\text { family history or } \\
\text { previous } \\
\text { rheumatic disease }\end{array}$ & AR with LTD & $\begin{array}{l}\text { LATERALITY: Uni (100) } \\
\text { TIMING: Del (100) } \\
\text { STAGES: >1 (100) } \\
\text { RADIO: No }(100)\end{array}$ & 16 & $52(8.5)$ & NR & 1st: 100 & Ther (100) \\
\hline & $\begin{array}{l}\text { RCT (Non- } \\
\text { industry) } \\
\text { (1994- } \\
1996)\end{array}$ & High & $\begin{array}{l}\text { I: Age <=79; free } \\
\text { of recurrence } \\
\text { E: Poorly } \\
\text { controlled } \\
\text { diabetes and } \\
\text { secondary } \\
\text { complications, } \\
\text { immunosuppressi } \\
\text { ve treatment, } \\
\text { family history or } \\
\text { previous } \\
\text { rheumatic disease }\end{array}$ & Total & $\mathrm{N} / \mathrm{A}$ & 75 & NR & NR & 1st: 100 & Ther (100) \\
\hline
\end{tabular}




\begin{tabular}{|c|c|c|c|c|c|c|c|c|c|c|}
\hline $\begin{array}{l}\text { Study, } \\
\text { Publication } \\
\text { Year, } \\
\text { PMID, } \\
\text { Country }\end{array}$ & $\begin{array}{l}\text { Design } \\
\text { (Funding) } \\
\text { (Study } \\
\text { Years) }\end{array}$ & $\begin{array}{l}\text { Risk } \\
\text { of } \\
\text { Bias }\end{array}$ & $\begin{array}{l}\text { Eligibility } \\
\text { Criteria }\end{array}$ & Arm & $\begin{array}{l}\text { Reconstruction } \\
\text { Details (\%) }\end{array}$ & $\mathbf{N}$ & $\begin{array}{l}\text { Age in Years, } \\
\text { Mean (SD) or } \\
\text { as Specified }\end{array}$ & Race (\%) & $\begin{array}{l}\text { Breast } \\
\text { Cancer } \\
\text { Occurrence } \\
\%\end{array}$ & $\begin{array}{l}\text { Cancer Stage } \\
\text { or Mastectomy } \\
\text { Purpose (\%) }\end{array}$ \\
\hline \multirow[t]{3}{*}{$\begin{array}{l}\text { Brorson } \\
2020 b \\
32807615 \\
\text { Sweden }\end{array}$} & $\begin{array}{l}\text { RCT (Non- } \\
\text { industry) } \\
\text { (2008- } \\
2020)\end{array}$ & High & $\begin{array}{l}\text { I: Age 18-60; } \\
\text { Unilateral } \\
\text { mastectomy } \\
\text { E: Current } \\
\text { smoker; BMI >30 }\end{array}$ & AR with DIEP & $\begin{array}{l}\text { LATERALITY: Uni (100) } \\
\text { TIMING: Del (100) } \\
\text { STAGES: } 1(100) \\
\text { CHEMO: Before (91.2) }\end{array}$ & 44 & $49.3(6.4)$ & NR & NR & $\begin{array}{l}\text { Stage } 1(6.5) \\
\text { Stage } 2(45.2) \\
\text { Stage } 3(48.4)\end{array}$ \\
\hline & $\begin{array}{l}\text { RCT (Non- } \\
\text { industry) } \\
\text { (2008- } \\
2020)\end{array}$ & High & $\begin{array}{l}\text { I: Age 18-60; } \\
\text { Unilateral } \\
\text { mastectomy } \\
\text { E: Current } \\
\text { smoker; BMI >30 }\end{array}$ & AR with LD & $\begin{array}{l}\text { LATERALITY: Uni (100) } \\
\text { TIMING: Del (100) } \\
\text { STAGES: } 1(100) \\
\text { CHEMO: Before }(75.0)\end{array}$ & 39 & $51.9(8.3)$ & NR & NR & $\begin{array}{l}\text { Stage } 1(2.9) \\
\text { Stage } 2(32.4) \\
\text { Stage } 3(64.7)\end{array}$ \\
\hline & $\begin{array}{l}\text { RCT (Non- } \\
\text { industry) } \\
\text { (2008- } \\
2020)\end{array}$ & High & $\begin{array}{l}\text { I: Age 18-60; } \\
\text { Unilateral } \\
\text { mastectomy } \\
\text { E: Current } \\
\text { smoker; BMI >30 }\end{array}$ & Total & $\mathrm{N} / \mathrm{A}$ & 83 & NR & NR & NR & NR \\
\hline \multirow{3}{*}{$\begin{array}{l}\text { Carramaschi } \\
\text {, 1989, } \\
2602589, \\
\text { France }\end{array}$} & $\begin{array}{l}\text { NRCS (NR) } \\
(1982- \\
1986)\end{array}$ & High & $\begin{array}{l}\text { I: } \\
\text { Postmastectomy } \\
\text { BR }\end{array}$ & AR with TRAM & NR & 40 & NR & NR & NR & NR \\
\hline & $\begin{array}{l}\text { NRCS (NR) } \\
(1982- \\
1986)\end{array}$ & High & $\begin{array}{l}\text { I: } \\
\text { Postmastectomy } \\
\text { BR }\end{array}$ & AR with LD & NR & 34 & NR & NR & NR & NR \\
\hline & $\begin{array}{l}\text { NRCS (NR) } \\
(1982- \\
1986) \\
\end{array}$ & High & $\begin{array}{l}\text { I: } \\
\text { Postmastectomy } \\
\text { BR }\end{array}$ & Total & $\mathrm{N} / \mathrm{A}$ & 74 & NR & NR & NR & NR \\
\hline \multirow[t]{3}{*}{$\begin{array}{l}\text { Dauplat, } \\
2021, \\
33622886, \\
\text { France }\end{array}$} & $\begin{array}{l}\text { NRCS } \\
\text { (None) } \\
(2007- \\
2009)\end{array}$ & $\begin{array}{l}\text { Mode } \\
\text { rate }\end{array}$ & $\begin{array}{l}\text { I: Unilateral AR } \\
\text { after therapeutic } \\
\text { mastectomy } \\
\text { E: Another } \\
\text { concurrent cancer }\end{array}$ & AR with TRAM & $\begin{array}{l}\text { LATERALITY: Uni (100) } \\
\text { TIMING: Imm (100) } \\
\text { CHEMO: Timing NR } \\
\text { (40) } \\
\text { RADIO: Timing NR (17) }\end{array}$ & 30 & NR & NR & NR & Ther (100) \\
\hline & $\begin{array}{l}\text { NRCS } \\
\text { (None) } \\
(2007- \\
2009)\end{array}$ & $\begin{array}{l}\text { Mode } \\
\text { rate }\end{array}$ & $\begin{array}{l}\text { I: Unilateral AR } \\
\text { after therapeutic } \\
\text { mastectomy } \\
\text { E: Another } \\
\text { concurrent cancer }\end{array}$ & $\begin{array}{l}\text { AR with LD } \\
\text { and implant }\end{array}$ & $\begin{array}{l}\text { LATERALITY: Uni (100) } \\
\text { TIMING: Imm (100) } \\
\text { CHEMO: Timing NR } \\
\text { (17) } \\
\text { RADIO: Timing NR (14) }\end{array}$ & 91 & NR & NR & NR & Ther (100) \\
\hline & $\begin{array}{l}\text { NRCS } \\
\text { (None) } \\
\text { (2007- } \\
2009)\end{array}$ & $\begin{array}{l}\text { Mode } \\
\text { rate }\end{array}$ & $\begin{array}{l}\text { I: Unilateral AR } \\
\text { after therapeutic } \\
\text { mastectomy } \\
\text { E: Another } \\
\text { concurrent cancer }\end{array}$ & $\begin{array}{l}\text { AR with LD } \\
\text { and no implant }\end{array}$ & $\begin{array}{l}\text { LATERALITY: Uni (100) } \\
\text { TIMING: Imm (100) } \\
\text { CHEMO: Timing NR } \\
\text { (32) } \\
\text { RADIO: Timing NR (7) }\end{array}$ & 78 & NR & NR & NR & Ther (100) \\
\hline
\end{tabular}




\begin{tabular}{|c|c|c|c|c|c|c|c|c|c|c|}
\hline \multirow[t]{2}{*}{$\begin{array}{l}\text { Study, } \\
\text { Publication } \\
\text { Year, } \\
\text { PMID, } \\
\text { Country } \\
\end{array}$} & $\begin{array}{l}\text { Design } \\
\text { (Funding) } \\
\text { (Study } \\
\text { Years) }\end{array}$ & $\begin{array}{l}\text { Risk } \\
\text { of } \\
\text { Bias }\end{array}$ & $\begin{array}{l}\text { Eligibility } \\
\text { Criteria }\end{array}$ & Arm & $\begin{array}{l}\text { Reconstruction } \\
\text { Details (\%) }\end{array}$ & $\mathbf{N}$ & $\begin{array}{l}\text { Age in Years, } \\
\text { Mean (SD) or } \\
\text { as Specified }\end{array}$ & Race (\%) & $\begin{array}{l}\text { Breast } \\
\text { Cancer } \\
\text { Occurrence } \\
\%\end{array}$ & $\begin{array}{l}\text { Cancer Stage } \\
\text { or Mastectomy } \\
\text { Purpose (\%) }\end{array}$ \\
\hline & $\begin{array}{l}\text { NRCS } \\
\text { (None) } \\
(2007- \\
2009)\end{array}$ & $\begin{array}{l}\text { Mode } \\
\text { rate }\end{array}$ & $\begin{array}{l}\text { I: Unilateral IBR } \\
\text { after therapeutic } \\
\text { mastectomy } \\
\text { E: Another } \\
\text { concurrent cancer }\end{array}$ & Total & $\mathrm{N} / \mathrm{A}$ & 199 & NR & NR & NR & Ther (100) \\
\hline \multirow[t]{3}{*}{$\begin{array}{l}\text { Erdmann- } \\
\text { Sager, } \\
2018, \\
29019862, \\
\text { US, Canada }\end{array}$} & $\begin{array}{l}\text { NRCS } \\
\text { (Non- } \\
\text { industry) } \\
(2012- \\
2015)\end{array}$ & $\begin{array}{l}\text { Mode } \\
\text { rate }\end{array}$ & $\begin{array}{l}\text { I: First-time, } \\
\text { unilateral or } \\
\text { bilateral BR }\end{array}$ & AR with DIEP & $\begin{array}{l}\text { LATERALITY: Uni } \\
\text { (57.8)/Bi (42.2) } \\
\text { TIMING: Imm (84)/Del } \\
\text { (16) } \\
\text { CHEMO: After } \\
\text { (28.3)/No } \\
\text { chemotherapy (71.7) } \\
\text { RADIO: Before } \\
\text { (22.5)/After } \\
\text { (19.1)/None(58.4) }\end{array}$ & 445 & $51.1(8.8)$ & NR & NR & $\begin{array}{l}\text { Ther (88.5), } \\
\text { Proph (11.5) }\end{array}$ \\
\hline & $\begin{array}{l}\text { NRCS } \\
\text { (Non- } \\
\text { industry) } \\
(2012- \\
2015)\end{array}$ & $\begin{array}{l}\text { Mode } \\
\text { rate }\end{array}$ & $\begin{array}{l}\text { I: First-time, } \\
\text { unilateral or } \\
\text { bilateral BR }\end{array}$ & AR with TRAM & $\begin{array}{l}\text { LATERALITY: Uni } \\
\text { (63.5)/Bi (36.5) } \\
\text { TIMING: Imm (76.5)/Del } \\
\text { (23.5) } \\
\text { CHEMO: After } \\
\text { (46.5)/No } \\
\text { chemotherapy (53.5) } \\
\text { RADIO: Before } \\
\text { (15.5)/After } \\
\text { (36.6)/None(47.9) }\end{array}$ & 115 & $52.2(8.6)$ & NR & NR & $\begin{array}{l}\text { Ther (91.3), } \\
\text { Proph (8.7) }\end{array}$ \\
\hline & $\begin{array}{l}\text { NRCS } \\
\text { (Non- } \\
\text { industry) } \\
(2012- \\
2015)\end{array}$ & $\begin{array}{l}\text { Mode } \\
\text { rate }\end{array}$ & $\begin{array}{l}\text { I: First-time, } \\
\text { unilateral or } \\
\text { bilateral BR }\end{array}$ & AR with TRAM & $\begin{array}{l}\text { LATERALITY: Uni } \\
\text { (80.9)/Bi (19.1) } \\
\text { TIMING: Imm (88.8)/Del } \\
\text { (11.2) } \\
\text { CHEMO: After } \\
\text { (22.5)/No } \\
\text { chemotherapy (77.5) } \\
\text { RADIO: Before } \\
\text { (30.3)/After } \\
\text { (14.6)/None(55.1) }\end{array}$ & 89 & $53.6(8.5)$ & NR & NR & $\begin{array}{l}\text { Ther (95.5), } \\
\text { Proph (4.5) }\end{array}$ \\
\hline
\end{tabular}




\begin{tabular}{|c|c|c|c|c|c|c|c|c|c|c|}
\hline $\begin{array}{l}\text { Study, } \\
\text { Publication } \\
\text { Year, } \\
\text { PMID, } \\
\text { Country }\end{array}$ & $\begin{array}{l}\text { Design } \\
\text { (Funding) } \\
\text { (Study } \\
\text { Years) }\end{array}$ & $\begin{array}{l}\text { Risk } \\
\text { of } \\
\text { Bias }\end{array}$ & $\begin{array}{l}\text { Eligibility } \\
\text { Criteria }\end{array}$ & Arm & $\begin{array}{l}\text { Reconstruction } \\
\text { Details (\%) }\end{array}$ & $\mathbf{N}$ & $\begin{array}{l}\text { Age in Years, } \\
\text { Mean (SD) or } \\
\text { as Specified }\end{array}$ & Race (\%) & $\begin{array}{l}\text { Breast } \\
\text { Cancer } \\
\text { Occurrence } \\
\%\end{array}$ & $\begin{array}{l}\text { Cancer Stage } \\
\text { or Mastectomy } \\
\text { Purpose }(\%)\end{array}$ \\
\hline & $\begin{array}{l}\text { NRCS } \\
\text { (Non- } \\
\text { industry) } \\
(2012- \\
2015)\end{array}$ & $\begin{array}{l}\text { Mode } \\
\text { rate }\end{array}$ & $\begin{array}{l}\text { I: First-time, } \\
\text { unilateral or } \\
\text { bilateral BR }\end{array}$ & AR with SIEA & $\begin{array}{l}\text { LATERALITY: Uni } \\
\text { (66.2)/Bi (33.8) } \\
\text { TIMING: Imm (91.5)/Del } \\
\text { (8.5) } \\
\text { CHEMO: After } \\
\text { (11.3)/No } \\
\text { chemotherapy (88.7) } \\
\text { RADIO: Before } \\
\text { (40)/After } \\
\text { (1.7)/None(58.3) }\end{array}$ & 71 & $53.3(8.2)$ & NR & NR & $\begin{array}{l}\text { Ther (91.5), } \\
\text { Proph (8.5) }\end{array}$ \\
\hline & $\begin{array}{l}\text { NRCS } \\
\text { (Non- } \\
\text { industry) } \\
(2012- \\
2015)\end{array}$ & $\begin{array}{l}\text { Mode } \\
\text { rate }\end{array}$ & $\begin{array}{l}\text { I: First-time, } \\
\text { unilateral or } \\
\text { bilateral BR }\end{array}$ & Total & $\mathrm{N} / \mathrm{A}$ & 791 & NR & NR & NR & NR \\
\hline \multirow{3}{*}{$\begin{array}{l}\text { Garbay, } \\
1992, \\
1624727, \\
\text { France }\end{array}$} & $\begin{array}{l}\text { NRCS (NR) } \\
(1979- \\
1990)\end{array}$ & High & $\mathrm{I}: \mathrm{BR}$ & AR with TRAM & NR & 63 & NR & NR & NR & NR \\
\hline & $\begin{array}{l}\text { NRCS (NR) } \\
(1979- \\
1990) \\
\end{array}$ & High & I: BR & AR with LD & NR & 36 & NR & NR & NR & NR \\
\hline & $\begin{array}{l}\text { NRCS (NR) } \\
(1979- \\
1990)\end{array}$ & High & $\mathrm{I}: \mathrm{BR}$ & Total & $\mathrm{N} / \mathrm{A}$ & 99 & NR & NR & NR & NR \\
\hline \multirow[t]{3}{*}{$\begin{array}{l}\text { Israeli, } \\
2014, \\
24572840, \\
\text { US }\end{array}$} & $\begin{array}{l}\text { NRCS } \\
\text { (Industry) } \\
\text { (2008- } \\
2009)\end{array}$ & High & $\begin{array}{l}\text { I: AR } \\
\text { E: Revision BR; } \\
\text { AR with TRAM } \\
\text { flap involving an } \\
\text { TE/I }\end{array}$ & AR with TRAM & $\begin{array}{l}\text { RADIO: Before } \\
\text { (4.4)/After (13.5) }\end{array}$ & 252 & $50.7(7.65)$ & NR & NR & NR \\
\hline & $\begin{array}{l}\text { NRCS } \\
\text { (Industry) } \\
\text { (2008- } \\
2009)\end{array}$ & High & $\begin{array}{l}\text { I: AR } \\
\text { E: Revision BR; } \\
\text { AR with TRAM } \\
\text { flap involving an } \\
\text { TE/I }\end{array}$ & AR with LD & $\begin{array}{l}\text { RADIO: Before } \\
\text { (6.3)/After (11.6) }\end{array}$ & 302 & $50(8.94)$ & NR & NR & NR \\
\hline & $\begin{array}{l}\text { NRCS } \\
\text { (Industry) } \\
\text { (2008- } \\
2009)\end{array}$ & High & $\begin{array}{l}\text { I: AR } \\
\text { E: Revision BR; } \\
\text { AR with TRAM } \\
\text { flap involving an } \\
\text { TE/I }\end{array}$ & Total & $\mathrm{N} / \mathrm{A}$ & 554 & NR & NR & NR & NR \\
\hline
\end{tabular}




\begin{tabular}{|c|c|c|c|c|c|c|c|c|c|c|}
\hline $\begin{array}{l}\text { Study, } \\
\text { Publication } \\
\text { Year, } \\
\text { PMID, } \\
\text { Country }\end{array}$ & $\begin{array}{l}\text { Design } \\
\text { (Funding) } \\
\text { (Study } \\
\text { Years) }\end{array}$ & $\begin{array}{l}\text { Risk } \\
\text { of } \\
\text { Bias }\end{array}$ & $\begin{array}{l}\text { Eligibility } \\
\text { Criteria }\end{array}$ & Arm & $\begin{array}{l}\text { Reconstruction } \\
\text { Details (\%) }\end{array}$ & $\mathbf{N}$ & $\begin{array}{l}\text { Age in Years, } \\
\text { Mean (SD) or } \\
\text { as Specified }\end{array}$ & Race (\%) & $\begin{array}{l}\text { Breast } \\
\text { Cancer } \\
\text { Occurrence } \\
\%\end{array}$ & $\begin{array}{l}\text { Cancer Stage } \\
\text { or Mastectomy } \\
\text { Purpose (\%) }\end{array}$ \\
\hline \multirow[t]{3}{*}{$\begin{array}{l}\text { Knox, 2016, } \\
26267400, \\
\text { Canada }\end{array}$} & $\begin{array}{l}\text { NRCS (NR) } \\
(2002- \\
2013)\end{array}$ & High & $\begin{array}{l}\text { I: Unilateral or } \\
\text { bilateral AR with } \\
\text { DIEP or pedicled } \\
\text { TRAM flap } \\
\text { E: History of } \\
\text { abdominal hernia } \\
\text { or bulge }\end{array}$ & AR with DIEP & $\begin{array}{l}\text { LATERALITY: Uni } \\
\text { (59.3)/Bi (49.8) } \\
\text { TIMING: Imm (63.1)/Del } \\
\text { (33.9)/Mixed (3.1) } \\
\text { STAGES: } 1 \text { (100) } \\
\text { CHEMO: Before } \\
\text { (51.5)/After (9.2)/No } \\
\text { chemotherapy (39.3) } \\
\text { RADIO: Before } \\
\text { (47.7)/After } \\
\text { (7.7)/None(44.6) }\end{array}$ & 130 & $\begin{array}{l}49(8.4) ; \\
\text { Range 16, } 72\end{array}$ & NR & NR & $\begin{array}{l}\text { Stage } 0(16.2), \\
\text { Stage NR } \\
(77.7), \\
\text { Proph (5.4) }\end{array}$ \\
\hline & $\begin{array}{l}\text { NRCS (NR) } \\
(2002- \\
2013)\end{array}$ & High & $\begin{array}{l}\text { I: Unilateral or } \\
\text { bilateral AR with } \\
\text { DIEP or pedicled } \\
\text { TRAM flap } \\
\text { E: History of } \\
\text { abdominal hernia } \\
\text { or bulge }\end{array}$ & AR with TRAM & $\begin{array}{l}\text { LATERALITY: Uni } \\
\text { (82.2)/Bi (17.8) } \\
\text { TIMING: Imm (74.5)/Del } \\
\text { (24.9)/Mixed (0.5) } \\
\text { STAGES: } 1(100) \\
\text { CHEMO: Before } \\
\text { (48)/After (11.9)/No } \\
\text { chemotherapy (40.1) } \\
\text { RADIO: Before } \\
\text { (45.6)/After } \\
\text { (11.9)/None(42.5) }\end{array}$ & 377 & $\begin{array}{l}50.2(8) ; \\
\text { Range 29, } 71\end{array}$ & NR & NR & $\begin{array}{l}\text { Stage } 0(28.7) \text {, } \\
\text { Stage NR } \\
(58.1), \text { Proph } \\
(1.3)\end{array}$ \\
\hline & $\begin{array}{l}\text { NRCS (NR) } \\
(2002- \\
2013)\end{array}$ & High & $\begin{array}{l}\text { I: Unilateral or } \\
\text { bilateral AR with } \\
\text { DIEP or pedicled } \\
\text { TRAM flap } \\
\text { E: History of } \\
\text { abdominal hernia } \\
\text { or bulge }\end{array}$ & Total & $\mathrm{N} / \mathrm{A}$ & 507 & NR & NR & NR & NR \\
\hline \multirow[t]{3}{*}{$\begin{array}{l}\text { Kroll, } 2000 \text {, } \\
10987463, \\
\text { US }\end{array}$} & $\begin{array}{l}\text { NRCS (NR) } \\
(1989- \\
2000) \\
\end{array}$ & High & $\begin{array}{l}\text { I: AR with } \\
\text { DIEP/free TRAM } \\
\text { flap }\end{array}$ & AR with DIEP & NR & NR & NR & NR & NR & NR \\
\hline & $\begin{array}{l}\text { NRCS (NR) } \\
(1989- \\
2000)\end{array}$ & High & $\begin{array}{l}\text { I: AR with } \\
\text { DIEP/free TRAM } \\
\text { flap }\end{array}$ & AR with TRAM & NR & NR & NR & NR & NR & NR \\
\hline & $\begin{array}{l}\text { NRCS (NR) } \\
(1989- \\
2000)\end{array}$ & High & $\begin{array}{l}\text { I: AR with } \\
\text { DIEP/free TRAM } \\
\text { flap }\end{array}$ & Total & $\mathrm{N} / \mathrm{A}$ & 241 & NR & NR & NR & NR \\
\hline
\end{tabular}




\begin{tabular}{|c|c|c|c|c|c|c|c|c|c|c|}
\hline $\begin{array}{l}\text { Study, } \\
\text { Publication } \\
\text { Year, } \\
\text { PMID, } \\
\text { Country }\end{array}$ & $\begin{array}{l}\text { Design } \\
\text { (Funding) } \\
\text { (Study } \\
\text { Years) }\end{array}$ & $\begin{array}{l}\text { Risk } \\
\text { of } \\
\text { Bias }\end{array}$ & $\begin{array}{l}\text { Eligibility } \\
\text { Criteria }\end{array}$ & Arm & $\begin{array}{l}\text { Reconstruction } \\
\text { Details (\%) }\end{array}$ & $\mathbf{N}$ & $\begin{array}{l}\text { Age in Years, } \\
\text { Mean (SD) or } \\
\text { as Specified }\end{array}$ & Race (\%) & $\begin{array}{l}\text { Breast } \\
\text { Cancer } \\
\text { Occurrence } \\
\%\end{array}$ & $\begin{array}{l}\text { Cancer Stage } \\
\text { or Mastectomy } \\
\text { Purpose }(\%)\end{array}$ \\
\hline \multirow[t]{5}{*}{$\begin{array}{l}\text { Kulkarni, } \\
2017, \\
28713853, \\
\text { US, Canada }\end{array}$} & $\begin{array}{l}\text { NRCS } \\
\text { (Non- } \\
\text { industry) } \\
\text { (2011- } \\
2016)\end{array}$ & $\begin{array}{l}\text { Mode } \\
\text { rate }\end{array}$ & $\begin{array}{l}\text { I: Age >=18 years; } \\
\text { first-time, } \\
\text { immediate or } \\
\text { delayed, bilateral } \\
\text { or unilateral } \\
\text { postmastectomy } \\
\text { BR }\end{array}$ & AR with DIEP & NR & 463 & NR & NR & NR & NR \\
\hline & $\begin{array}{l}\text { NRCS } \\
\text { (Non- } \\
\text { industry) } \\
(2011- \\
2016)\end{array}$ & $\begin{array}{l}\text { Mode } \\
\text { rate }\end{array}$ & $\begin{array}{l}\text { I: Age >=18 years; } \\
\text { first-time, } \\
\text { immediate or } \\
\text { delayed, bilateral } \\
\text { or unilateral } \\
\text { postmastectomy } \\
\text { BR }\end{array}$ & AR with TRAM & NR & 94 & NR & NR & NR & NR \\
\hline & $\begin{array}{l}\text { NRCS } \\
\text { (Non- } \\
\text { industry) } \\
(2011- \\
2016)\end{array}$ & $\begin{array}{l}\text { Mode } \\
\text { rate }\end{array}$ & $\begin{array}{l}\text { I: Age >=18 years; } \\
\text { first-time, } \\
\text { immediate or } \\
\text { delayed, bilateral } \\
\text { or unilateral } \\
\text { postmastectomy } \\
\text { BR }\end{array}$ & AR with TRAM & NR & 111 & NR & NR & NR & NR \\
\hline & $\begin{array}{l}\text { NRCS } \\
\text { (Non- } \\
\text { industry) } \\
(2011- \\
2016)\end{array}$ & $\begin{array}{l}\text { Mode } \\
\text { rate }\end{array}$ & $\begin{array}{l}\text { I: Age >=18 years; } \\
\text { first-time, } \\
\text { immediate or } \\
\text { delayed, bilateral } \\
\text { or unilateral } \\
\text { postmastectomy } \\
\text { BR }\end{array}$ & AR with LD & NR & 80 & NR & NR & NR & NR \\
\hline & $\begin{array}{l}\text { NRCS } \\
\text { (Non- } \\
\text { industry) } \\
(2011- \\
2016)\end{array}$ & $\begin{array}{l}\text { Mode } \\
\text { rate }\end{array}$ & $\begin{array}{l}\text { I: Age >=18 years; } \\
\text { first-time, } \\
\text { immediate or } \\
\text { delayed, bilateral } \\
\text { or unilateral } \\
\text { postmastectomy } \\
\text { BR }\end{array}$ & AR with SIEA & NR & 73 & NR & NR & NR & NR \\
\hline
\end{tabular}




\begin{tabular}{|c|c|c|c|c|c|c|c|c|c|c|}
\hline \multirow[t]{2}{*}{$\begin{array}{l}\text { Study, } \\
\text { Publication } \\
\text { Year, } \\
\text { PMID, } \\
\text { Country } \\
\end{array}$} & $\begin{array}{l}\text { Design } \\
\text { (Funding) } \\
\text { (Study } \\
\text { Years) }\end{array}$ & $\begin{array}{l}\text { Risk } \\
\text { of } \\
\text { Bias }\end{array}$ & $\begin{array}{l}\text { Eligibility } \\
\text { Criteria }\end{array}$ & Arm & $\begin{array}{l}\text { Reconstruction } \\
\text { Details (\%) }\end{array}$ & $\mathbf{N}$ & $\begin{array}{l}\text { Age in Years, } \\
\text { Mean (SD) or } \\
\text { as Specified }\end{array}$ & Race (\%) & $\begin{array}{l}\text { Breast } \\
\text { Cancer } \\
\text { Occurrence } \\
\%\end{array}$ & $\begin{array}{l}\text { Cancer Stage } \\
\text { or Mastectomy } \\
\text { Purpose (\%) }\end{array}$ \\
\hline & $\begin{array}{l}\text { NRCS } \\
\text { (Non- } \\
\text { industry) } \\
(2011- \\
2016)\end{array}$ & $\begin{array}{l}\text { Mode } \\
\text { rate }\end{array}$ & $\begin{array}{l}\text { l: Age >=18 years; } \\
\text { first-time, } \\
\text { immediate or } \\
\text { delayed, bilateral } \\
\text { or unilateral } \\
\text { postmastectomy } \\
\text { BR }\end{array}$ & Total & $\mathrm{N} / \mathrm{A}$ & 821 & NR & NR & NR & NR \\
\hline \multirow[t]{2}{*}{$\begin{array}{l}\text { Macadam, } \\
2016, \\
26910656, \\
\text { US, } \\
\text { Canada, } \\
\text { Japan, } \\
\text { Lebanon }\end{array}$} & $\begin{array}{l}\text { NRCS } \\
\text { (Non- } \\
\text { industry) } \\
(2002- \\
2012)\end{array}$ & High & $\begin{array}{l}\text { I: AR } \\
\text { E: AR with a } \\
\text { combination of } \\
\text { flaps }\end{array}$ & AR with DIEP & $\begin{array}{l}\text { LATERALITY: Uni } \\
\text { (57.2)/Bi (42.8) } \\
\text { TIMING: Imm (58.2)/Del } \\
\text { (37.1)/Mixed (4.6) } \\
\text { STAGES: } 1(100) \\
\text { CHEMO: Before } \\
\text { (43.8)/After (8.8)/No } \\
\text { chemotherapy (48.6) } \\
\text { RADIO: Before } \\
\text { (39.2)/After } \\
\text { (3.1)/None(58) }\end{array}$ & 670 & $49(8.4)$ & $\begin{array}{l}\text { W (83.2), } \\
\text { B (3.5), A } \\
(8), \mathrm{H} \\
(1.3), \\
\text { Other } 1 \\
(2.4), \\
\text { Other } 2 \\
(1.6)\end{array}$ & NR & $\begin{array}{l}\text { None (8.8), } \\
\text { Stage } 0(23.4) \text {, } \\
\text { Stage NR } \\
(67.8) ; \text { Proph } \\
(8.5)\end{array}$ \\
\hline & $\begin{array}{l}\text { NRCS } \\
\text { (Non- } \\
\text { industry) } \\
(2002- \\
2012)\end{array}$ & High & $\begin{array}{l}\text { I: AR } \\
\text { E: AR with a } \\
\text { combination of } \\
\text { flaps }\end{array}$ & AR with TRAM & $\begin{array}{l}\text { LATERALITY: Uni } \\
\text { (79.2)/Bi (20.8) } \\
\text { TIMING: Imm (71.5)/Del } \\
\text { (27.8)/Mixed (0.7) } \\
\text { STAGES: } 1(100) \\
\text { CHEMO: Before } \\
\text { (31.7)/After (21.1)/No } \\
\text { chemotherapy (49.7) } \\
\text { RADIO: Before } \\
\text { (27.5)/After } \\
\text { (8.5)/None(64.1) }\end{array}$ & 144 & $47.8(7.7)$ & $\begin{array}{l}\text { W }(81.4), \\
\text { B (7.1), } \\
\text { Others } \\
(11.4)\end{array}$ & NR & $\begin{array}{l}\text { None (4.2), } \\
\text { Stage } 0(35.2) \text {, } \\
\text { Stage NR } \\
(60.6) ; \text { Proph } \\
(4.2)\end{array}$ \\
\hline
\end{tabular}




\begin{tabular}{|c|c|c|c|c|c|c|c|c|c|c|}
\hline $\begin{array}{l}\text { Study, } \\
\text { Publication } \\
\text { Year, } \\
\text { PMID, } \\
\text { Country }\end{array}$ & $\begin{array}{l}\text { Design } \\
\text { (Funding) } \\
\text { (Study } \\
\text { Years) }\end{array}$ & $\begin{array}{l}\text { Risk } \\
\text { of } \\
\text { Bias }\end{array}$ & $\begin{array}{l}\text { Eligibility } \\
\text { Criteria }\end{array}$ & Arm & $\begin{array}{l}\text { Reconstruction } \\
\text { Details (\%) }\end{array}$ & $\mathbf{N}$ & $\begin{array}{l}\text { Age in Years, } \\
\text { Mean (SD) or } \\
\text { as Specified }\end{array}$ & Race (\%) & $\begin{array}{l}\text { Breast } \\
\text { Cancer } \\
\text { Occurrence } \\
\%\end{array}$ & $\begin{array}{l}\text { Cancer Stage } \\
\text { or Mastectomy } \\
\text { Purpose (\%) }\end{array}$ \\
\hline & $\begin{array}{l}\text { NRCS } \\
\text { (Non- } \\
\text { industry) } \\
(2002- \\
2012)\end{array}$ & High & $\begin{array}{l}\text { I: AR } \\
\text { E: AR with a } \\
\text { combination of } \\
\text { flaps }\end{array}$ & AR with TRAM & $\begin{array}{l}\text { LATERALITY: Uni } \\
\text { (79.2)/Bi (20.8) } \\
\text { TIMING: Imm (70.7)/Del } \\
\text { (28)/Mixed (1.3) } \\
\text { STAGES: } 1(100) \\
\text { CHEMO: Before } \\
\text { (42.5)/After (16.7)/No } \\
\text { chemotherapy (44.4) } \\
\text { RADIO: Before } \\
\text { (40.2)/After } \\
\text { (9.8)/None(50.8) }\end{array}$ & 683 & $50.3(8.2)$ & $\begin{array}{l}\text { W (83.6), } \\
\text { B (2.5), A } \\
(7.9), H \\
(0.6), \\
\text { Other } 1 \\
(1.4), \\
\text { Other } 2 \\
\text { (4) }\end{array}$ & NR & $\begin{array}{l}\text { None (2.8), } \\
\text { Stage } 0(31.8) \text {, } \\
\text { Stage NR } \\
(65.5) ; \text { Proph } \\
(2.3)\end{array}$ \\
\hline & $\begin{array}{l}\text { NRCS } \\
\text { (Non- } \\
\text { industry) } \\
(2002- \\
2012)\end{array}$ & High & $\begin{array}{l}\text { I: AR } \\
\text { E: AR with a } \\
\text { combination of } \\
\text { flaps }\end{array}$ & AR with TRAM & $\begin{array}{l}\text { LATERALITY: Uni } \\
\text { (63.1)/Bi (36.9) } \\
\text { TIMING: Imm (65.1)/Del } \\
\text { (33.9)/Mixed (1) } \\
\text { STAGES: } 1 \text { (100) } \\
\text { CHEMO: Before } \\
\text { (36.3)/After (16.4)/No } \\
\text { chemotherapy (49) } \\
\text { RADIO: Before } \\
\text { (42.1)/After } \\
\text { (2.7)/None(55.5) }\end{array}$ & 293 & $49.6(7.9)$ & $\begin{array}{l}\text { W (80.2), } \\
B(6), A \\
(4.3), \\
\text { Other } 1 \\
(8.6), \\
\text { Other } 2 \\
(0.9)\end{array}$ & NR & $\begin{array}{l}\text { None (4.8), } \\
\text { Stage } 0(26.6) \text {, } \\
\text { Stage NR } \\
(68.5) ; \text { Proph } \\
(4.5)\end{array}$ \\
\hline & $\begin{array}{l}\text { NRCS } \\
\text { (Non- } \\
\text { industry) } \\
\text { (2002- } \\
2012) \\
\end{array}$ & High & $\begin{array}{l}\text { I: AR } \\
\text { E: AR with a } \\
\text { combination of } \\
\text { flaps }\end{array}$ & Total & $\mathrm{N} / \mathrm{A}$ & 1790 & NR & NR & NR & NR \\
\hline \multirow[t]{2}{*}{$\begin{array}{l}\text { Massenburg } \\
\text {, 2015, } \\
26487657 \text {, } \\
\text { US }\end{array}$} & $\begin{array}{l}\text { NRCS (NR) } \\
(2005- \\
2012)\end{array}$ & High & $\mathrm{I}: \mathrm{AR}$ & AR with TRAM & NR & 2464 & $52(9)$ & $\begin{array}{l}\text { W (82.3), } \\
\text { B (12.4), } \\
\text { A (3.3), H } \\
(1.4), \\
\text { Others } \\
(0.6)\end{array}$ & NR & NR \\
\hline & $\begin{array}{l}\text { NRCS (NR) } \\
(2005- \\
2012)\end{array}$ & High & I: AR & AR with LD & NR & 2085 & $52.8(10.9)$ & $\begin{array}{l}\text { W (83.8), } \\
\text { B (12.2), } \\
\text { A (2.6), H } \\
(0.4), \\
\text { Others } \\
(0.7)\end{array}$ & NR & NR \\
\hline
\end{tabular}




\begin{tabular}{|c|c|c|c|c|c|c|c|c|c|c|}
\hline $\begin{array}{l}\text { Study, } \\
\text { Publication } \\
\text { Year, } \\
\text { PMID, } \\
\text { Country }\end{array}$ & $\begin{array}{l}\text { Design } \\
\text { (Funding) } \\
\text { (Study } \\
\text { Years) }\end{array}$ & $\begin{array}{l}\text { Risk } \\
\text { of } \\
\text { Bias }\end{array}$ & $\begin{array}{l}\text { Eligibility } \\
\text { Criteria }\end{array}$ & Arm & $\begin{array}{l}\text { Reconstruction } \\
\text { Details (\%) }\end{array}$ & $\mathbf{N}$ & $\begin{array}{l}\text { Age in Years, } \\
\text { Mean (SD) or } \\
\text { as Specified }\end{array}$ & Race (\%) & $\begin{array}{l}\text { Breast } \\
\text { Cancer } \\
\text { Occurrence } \\
\%\end{array}$ & $\begin{array}{l}\text { Cancer Stage } \\
\text { or Mastectomy } \\
\text { Purpose (\%) }\end{array}$ \\
\hline & $\begin{array}{l}\text { NRCS (NR) } \\
(2005- \\
2012)\end{array}$ & High & I: AR & Total & $\mathrm{N} / \mathrm{A}$ & 4549 & $51.8(9.7)$ & $\begin{array}{l}\text { W (82.3), } \\
\text { B (13), A } \\
(3.4), \mathrm{H} \\
(0.7), \\
\text { Others } \\
(0.6)\end{array}$ & NR & NR \\
\hline \multirow[t]{4}{*}{$\begin{array}{l}\text { Mennie, } \\
2015, \\
25839173, \\
\text { UK }\end{array}$} & $\begin{array}{l}\text { NRCS } \\
\text { (Industry; } \\
\text { Non- } \\
\text { industry) } \\
\text { (2006- } \\
2012) \\
\end{array}$ & High & $\begin{array}{l}\text { I: AR } \\
\text { E: Other types of } \\
\text { immediate or } \\
\text { delayed BR }\end{array}$ & AR with DIEP & $\begin{array}{l}\text { LATERALITY: Uni } \\
\text { (87.6)/Bi }(12.4) \\
\text { STAGES: } 1(100)\end{array}$ & 5144 & NR & NR & NR & NR \\
\hline & $\begin{array}{l}\text { NRCS } \\
\text { (Industry; } \\
\text { Non- } \\
\text { industry) } \\
\text { (2006- } \\
2012) \\
\end{array}$ & High & $\begin{array}{l}\text { I: AR } \\
\text { E: Other types of } \\
\text { immediate or } \\
\text { delayed BR }\end{array}$ & AR with TRAM & $\begin{array}{l}\text { LATERALITY: Uni } \\
\text { (91.9)/Bi }(8.1) \\
\text { STAGES: } 1 \text { (100) }\end{array}$ & 1963 & NR & NR & NR & NR \\
\hline & $\begin{array}{l}\text { NRCS } \\
\text { (Industry; } \\
\text { Non- } \\
\text { industry) } \\
\text { (2006- } \\
2012) \\
\end{array}$ & High & $\begin{array}{l}\text { I: AR } \\
\text { E: Other types of } \\
\text { immediate or } \\
\text { delayed BR }\end{array}$ & AR with TRAM & $\begin{array}{l}\text { LATERALITY: Uni } \\
\text { (91.3)/Bi }(8.7) \\
\text { STAGES: } 1 \text { (100) }\end{array}$ & 922 & NR & NR & NR & NR \\
\hline & $\begin{array}{l}\text { NRCS } \\
\text { (Industry; } \\
\text { Non- } \\
\text { industry) } \\
\text { (2006- } \\
2012)\end{array}$ & High & $\begin{array}{l}\text { I: AR } \\
\text { E: Other types of } \\
\text { immediate or } \\
\text { delayed BR }\end{array}$ & Total & $\mathrm{N} / \mathrm{A}$ & 7929 & $\begin{array}{l}16-45 \text { years } \\
(29.2 \%), \\
46-50 \text { years } \\
(23.4 \%) \text {, } \\
51-55 \text { years } \\
(20.5 \%) \text {, } \\
56-60 \text { years } \\
(13.9 \%) \text {, } \\
\geq 60 \text { years } \\
(13 \%)\end{array}$ & NR & NR & NR \\
\hline
\end{tabular}




\begin{tabular}{|c|c|c|c|c|c|c|c|c|c|c|}
\hline $\begin{array}{l}\text { Study, } \\
\text { Publication } \\
\text { Year, } \\
\text { PMID, } \\
\text { Country }\end{array}$ & $\begin{array}{l}\text { Design } \\
\text { (Funding) } \\
\text { (Study } \\
\text { Years) }\end{array}$ & $\begin{array}{l}\text { Risk } \\
\text { of } \\
\text { Bias }\end{array}$ & $\begin{array}{l}\text { Eligibility } \\
\text { Criteria }\end{array}$ & Arm & $\begin{array}{l}\text { Reconstruction } \\
\text { Details (\%) }\end{array}$ & $\mathbf{N}$ & $\begin{array}{l}\text { Age in Years, } \\
\text { Mean (SD) or } \\
\text { as Specified }\end{array}$ & Race (\%) & $\begin{array}{l}\text { Breast } \\
\text { Cancer } \\
\text { Occurrence } \\
\%\end{array}$ & $\begin{array}{l}\text { Cancer Stage } \\
\text { or Mastectomy } \\
\text { Purpose }(\%)\end{array}$ \\
\hline \multirow[t]{3}{*}{$\begin{array}{l}\text { Rindom, } \\
\text { 2019, } \\
\text { 31515191, } \\
\text { Denmark }\end{array}$} & $\begin{array}{l}\text { RCT } \\
\text { (Industry) } \\
\text { (2013- } \\
2015)\end{array}$ & $\begin{array}{l}\text { Mode } \\
\text { rate }\end{array}$ & $\begin{array}{l}\text { I: Age }>=18, \\
\text { unilateral, delayed } \\
\text { BR }\end{array}$ & AR with LD & $\begin{array}{l}\text { LATERALITY: Uni (100) } \\
\text { TIMING: Del (100) } \\
\text { STAGES: } 1(100) \\
\text { CHEMO: Timing NR } \\
\text { (77.78) } \\
\text { RADIO: Timing NR (50) }\end{array}$ & 25 & $\begin{array}{l}\text { 54.2; Range } \\
41,71\end{array}$ & NR & NR & $\begin{array}{l}\text { Ther (94.45), } \\
\text { Proph (5.55) }\end{array}$ \\
\hline & $\begin{array}{l}\text { RCT } \\
\text { (Industry) } \\
\text { (2013- } \\
2015)\end{array}$ & $\begin{array}{l}\text { Mode } \\
\text { rate }\end{array}$ & $\begin{array}{l}\text { I: Age }>=18, \\
\text { unilateral, delayed } \\
\text { BR }\end{array}$ & AR with TAP & $\begin{array}{l}\text { LATERALITY: Uni (100) } \\
\text { TIMING: Del (100) } \\
\text { STAGES: >1 (13.64) } \\
\text { CHEMO: Timing NR } \\
\text { (68.18) } \\
\text { RADIO: Timing NR } \\
\text { (77.27) }\end{array}$ & 25 & $\begin{array}{l}\text { 55.8; Range } \\
35,70\end{array}$ & NR & NR & Ther (100) \\
\hline & $\begin{array}{l}\text { RCT } \\
\text { (Industry) } \\
\text { (2013- } \\
2015) \\
\end{array}$ & $\begin{array}{l}\text { Mode } \\
\text { rate }\end{array}$ & $\begin{array}{l}\text { I: Age }>=18, \\
\text { unilateral, delayed } \\
\text { BR }\end{array}$ & Total & $\mathrm{N} / \mathrm{A}$ & 50 & NR & NR & NR & \\
\hline $\begin{array}{l}\text { Woo, 2018, } \\
30360958, \\
\text { South Korea }\end{array}$ & $\begin{array}{l}\text { NRCS (NR) } \\
(2008- \\
2013)\end{array}$ & High & $\begin{array}{l}\text { I: Immediate BR } \\
\text { E: Direct-to- } \\
\text { implant BR; } \\
\text { combination of AR } \\
\text { and TE/IBR (e.g., } \\
\text { LD flap); AR with } \\
\text { TRAM flap,; } \\
\text { history of shoulder } \\
\text { joint morbidity, } \\
\text { such as adhesive } \\
\text { capsulitis or } \\
\text { rotator cuff } \\
\text { disease }\end{array}$ & AR with DIEP & $\begin{array}{l}\text { TIMING: Imm (100) } \\
\text { CHEMO: Before } \\
\text { (2.3)/After (40.9) } \\
\text { RADIO: Before } \\
\text { (2.2)/After (14.8) }\end{array}$ & NR & NR & NR & NR & NR \\
\hline
\end{tabular}




\begin{tabular}{|c|c|c|c|c|c|c|c|c|c|c|}
\hline $\begin{array}{l}\text { Study, } \\
\text { Publication } \\
\text { Year, } \\
\text { PMID, }\end{array}$ & $\begin{array}{l}\text { Design } \\
\text { (Funding) } \\
\text { (Study } \\
\text { Years) }\end{array}$ & $\begin{array}{l}\text { Risk } \\
\text { of } \\
\text { Bias }\end{array}$ & $\begin{array}{l}\text { Eligibility } \\
\text { Criteria }\end{array}$ & Arm & $\begin{array}{l}\text { Reconstruction } \\
\text { Details (\%) }\end{array}$ & $\mathbf{N}$ & $\begin{array}{l}\text { Age in Years, } \\
\text { Mean (SD) or } \\
\text { as Specified }\end{array}$ & Race (\%) & $\begin{array}{l}\text { Breast } \\
\text { Cancer } \\
\text { Occurrence } \\
\%\end{array}$ & $\begin{array}{l}\text { Cancer Stage } \\
\text { or Mastectomy } \\
\text { Purpose }(\%)\end{array}$ \\
\hline & $\begin{array}{l}\text { NRCS (NR) } \\
(2008- \\
2013)\end{array}$ & High & $\begin{array}{l}\text { I: Immediate BR } \\
\text { E: Direct-to- } \\
\text { implant BR; } \\
\text { combination of AR } \\
\text { and TE/IBR (e.g., } \\
\text { LD flap); AR with } \\
\text { TRAM flap,; } \\
\text { history of shoulder } \\
\text { joint morbidity, } \\
\text { such as adhesive } \\
\text { capsulitis or } \\
\text { rotator cuff } \\
\text { disease }\end{array}$ & AR with LD & $\begin{array}{l}\text { TIMING: Imm (100) } \\
\text { CHEMO: Before } \\
\text { (8.6)/After (43.6) } \\
\text { RADIO: After (9.4) }\end{array}$ & NR & NR & NR & NR & NR \\
\hline & $\begin{array}{l}\text { NRCS (NR) } \\
(2008- \\
2013)\end{array}$ & High & $\begin{array}{l}\text { I: Immediate BR } \\
\text { E: Direct-to- } \\
\text { implant BR; } \\
\text { combination of AR } \\
\text { and TE/IBR (e.g., } \\
\text { LD flap); AR with } \\
\text { TRAM flap,; } \\
\text { history of shoulder } \\
\text { joint morbidity, } \\
\text { such as adhesive } \\
\text { capsulitis or } \\
\text { rotator cuff } \\
\text { disease }\end{array}$ & Total & $\mathrm{N} / \mathrm{A}$ & NR & NR & NR & NR & NR \\
\hline \multirow[t]{2}{*}{$\begin{array}{l}\text { Yueh, 2009, } \\
\text { 19228537, } \\
\text { US }\end{array}$} & $\begin{array}{l}\text { NRCS } \\
\text { (Non- } \\
\text { industry) } \\
(1996- \\
2006)\end{array}$ & High & $\begin{array}{l}\text { I: Therapeutic or } \\
\text { prophylactic } \\
\text { mastectomy } \\
\text { E: BR for breast } \\
\text { augmentation only }\end{array}$ & AR with DIEP & STAGES: 1 (100) & 420 & NR & NR & NR & NR \\
\hline & $\begin{array}{l}\text { NRCS } \\
\text { (Non- } \\
\text { industry) } \\
(1996- \\
2006)\end{array}$ & High & $\begin{array}{l}\text { I: Therapeutic or } \\
\text { prophylactic } \\
\text { mastectomy } \\
\text { E: BR for breast } \\
\text { augmentation only }\end{array}$ & AR with TRAM & STAGES: 1 (100) & 143 & NR & NR & NR & NR \\
\hline
\end{tabular}




\begin{tabular}{|c|c|c|c|c|c|c|c|c|c|c|}
\hline $\begin{array}{l}\text { Study, } \\
\text { Publication } \\
\text { Year, } \\
\text { PMID, } \\
\text { Country }\end{array}$ & $\begin{array}{l}\text { Design } \\
\text { (Funding) } \\
\text { (Study } \\
\text { Years) }\end{array}$ & $\begin{array}{l}\text { Risk } \\
\text { of } \\
\text { Bias }\end{array}$ & $\begin{array}{l}\text { Eligibility } \\
\text { Criteria }\end{array}$ & Arm & $\begin{array}{l}\text { Reconstruction } \\
\text { Details (\%) }\end{array}$ & $\mathbf{N}$ & $\begin{array}{l}\text { Age in Years, } \\
\text { Mean (SD) or } \\
\text { as Specified }\end{array}$ & Race (\%) & $\begin{array}{l}\text { Breast } \\
\text { Cancer } \\
\text { Occurrence } \\
\%\end{array}$ & $\begin{array}{l}\text { Cancer Stage } \\
\text { or Mastectomy } \\
\text { Purpose (\%) }\end{array}$ \\
\hline & $\begin{array}{l}\text { NRCS } \\
\text { (Non- } \\
\text { industry) } \\
(1996- \\
2006) \\
\end{array}$ & High & $\begin{array}{l}\text { I: Therapeutic or } \\
\text { prophylactic } \\
\text { mastectomy } \\
\text { E: BR for breast } \\
\text { augmentation only }\end{array}$ & AR with LD & STAGES: 1 (100) & 112 & NR & NR & NR & NR \\
\hline & $\begin{array}{l}\text { NRCS } \\
\text { (Non- } \\
\text { industry) } \\
(1996- \\
2006) \\
\end{array}$ & High & $\begin{array}{l}\text { I: Therapeutic or } \\
\text { prophylactic } \\
\text { mastectomy } \\
\text { E: BR for breast } \\
\text { augmentation only }\end{array}$ & Total & $\mathrm{N} / \mathrm{A}$ & 675 & NR & NR & NR & NR \\
\hline \multirow[t]{3}{*}{$\begin{array}{l}\text { Zhong, } \\
2014, \\
24675183, \\
\text { Canada }\end{array}$} & $\begin{array}{l}\text { NRCS } \\
\text { (Non- } \\
\text { industry) } \\
(2009- \\
2012)\end{array}$ & High & $\begin{array}{l}\text { I: AR with DIEP or } \\
\text { free TRAM flaps }\end{array}$ & AR with DIEP & STAGES: 1 (100) & 244 & NR & NR & NR & NR \\
\hline & $\begin{array}{l}\text { NRCS } \\
\text { (Non- } \\
\text { industry) } \\
(2009- \\
2012) \\
\end{array}$ & High & $\begin{array}{l}\text { I: AR with DIEP or } \\
\text { free TRAM flaps }\end{array}$ & AR with TRAM & STAGES: 1 (100) & 48 & NR & NR & NR & NR \\
\hline & $\begin{array}{l}\text { NRCS } \\
\text { (Non- } \\
\text { industry) } \\
(2009- \\
2012) \\
\end{array}$ & High & $\begin{array}{l}\text { I: AR with DIEP or } \\
\text { free TRAM flaps }\end{array}$ & Total & $\mathrm{N} / \mathrm{A}$ & 292 & $50.1(8.6)$ & NR & NR & NR \\
\hline \multirow[t]{2}{*}{$\begin{array}{l}\text { Zoghbi Y, } \\
2017, \\
28052051, \\
\text { US }\end{array}$} & $\begin{array}{l}\text { NRCS } \\
\text { (None) } \\
(2010- \\
2011)\end{array}$ & High & $\begin{array}{l}\text { I: AR with DIEP or } \\
\text { free TRAM flaps }\end{array}$ & AR with DIEP & NR & 9699 & $50(13)$ & $\begin{array}{l}\text { W }(70.8), \\
\text { B (11.2), } \\
\text { A }(3.6), \mathrm{H} \\
(10.9), \\
\text { Others } \\
(3.5)\end{array}$ & NR & NR \\
\hline & $\begin{array}{l}\text { NRCS } \\
\text { (None) } \\
\text { (2010- } \\
2011)\end{array}$ & High & $\begin{array}{l}\text { I: AR with DIEP or } \\
\text { free TRAM flaps }\end{array}$ & AR with TRAM & NR & 6137 & $50(13)$ & $\begin{array}{l}\text { W (67.2), } \\
\text { B (13.8), } \\
\text { A (3.7), H } \\
(11.3), \\
\text { Others } \\
(3.6)\end{array}$ & NR & NR \\
\hline
\end{tabular}




\begin{tabular}{|c|c|c|c|c|c|c|c|c|c|c|}
\hline $\begin{array}{l}\text { Study, } \\
\text { Publication } \\
\text { Year, } \\
\text { PMID, } \\
\text { Country }\end{array}$ & $\begin{array}{l}\text { Design } \\
\text { (Funding) } \\
\text { (Study } \\
\text { Years) }\end{array}$ & $\begin{array}{l}\text { Risk } \\
\text { of } \\
\text { Bias }\end{array}$ & $\begin{array}{l}\text { Eligibility } \\
\text { Criteria }\end{array}$ & Arm & $\begin{array}{l}\text { Reconstruction } \\
\text { Details (\%) }\end{array}$ & $\mathbf{N}$ & $\begin{array}{l}\text { Age in Years, } \\
\text { Mean (SD) or } \\
\text { as Specified }\end{array}$ & Race (\%) & $\begin{array}{l}\text { Breast } \\
\text { Cancer } \\
\text { Occurrence } \\
\%\end{array}$ & $\begin{array}{l}\text { Cancer Stage } \\
\text { or Mastectomy } \\
\text { Purpose }(\%)\end{array}$ \\
\hline & $\begin{array}{l}\text { NRCS } \\
\text { (None) } \\
\text { (2010- } \\
2011)\end{array}$ & High & $\begin{array}{l}\text { I: AR with DIEP or } \\
\text { free TRAM flaps }\end{array}$ & Total & $\mathrm{N} / \mathrm{A}$ & 15836 & $\begin{array}{l}50(13) ; \\
\text { Median } 50\end{array}$ & $\begin{array}{l}\text { W (69.5), } \\
\text { B (12.2), } \\
\text { A (3.6), H } \\
(11.1), \\
\text { Others } \\
(3.6)\end{array}$ & NR & NR \\
\hline
\end{tabular}

Blue coloring is only to visually separate different studies.

I: inclusion criteria, E: exclusion criteria

Laterality: whether the reconstruction was unilateral ("Uni") or bilateral ("Bi"). Stages: Whether the reconstruction was completed in 1 stage or $>1$ stages. Timing: Timing of reconstruction relative to mastectomy, i.e., immediate ("Imm") or delayed ("Del"). Chemo: Timing of chemotherapy relative to reconstruction. Radio: Timing of radiation therapy relative to reconstruction.

$\mathrm{W}=$ White or Caucasian, $\mathrm{B}=$ Black or African American, $\mathrm{A}=$ Asian, $\mathrm{H}=$ Hispanic or Latino.

Proph $=$ prophylactic, Ther $=$ therapeutic.

Abbreviations: $\mathrm{AR}=$ autologous reconstruction, $\mathrm{BR}=$ breast reconstruction, $\mathrm{DIEP}=$ Deep inferior epigastric perforator, $\mathrm{IBR}=\mathrm{implant}-\mathrm{based}$ reconstruction, $\mathrm{IQR}=$ interquartile range, $\mathrm{LD}=$ latissimus dorsi, $\mathrm{NR}=$ not reported, $\mathrm{NRCS}=$ nonrandomized comparative study, $\mathrm{PMID}=$ PubMed identifier, $\mathrm{PMRT}=$ postmastectomy radiation therapy, $\mathrm{RCT}=$ randomized controlled trial, $\mathrm{SD}=$ standard deviation, $\mathrm{SIEA}=$ Superficial inferior epigastric artery perforator, TAP $=$ thoracodorsal artery perforator, TE/I $=$ tissue expander/implant, Ther $=$ therapeutic, TRAM $=$ Transverse rectus abdominis myocutaneous.

Colors: Header rows are shaded orange. Rows for every alternate study are shaded blue. The colors do not add unique information. 


\section{Appendix D. Results: Risk of Bias}

Table D-1. Risk of bias assessment for all Key Questions - randomized controlled trials

\begin{tabular}{|c|c|c|c|c|c|c|c|c|c|c|c|c|c|c|}
\hline KQ & $\begin{array}{l}\text { Study, } \\
\text { Year, PMID }\end{array}$ & $\begin{array}{l}\text { Random } \\
\text { Sequence } \\
\text { Generatio } \\
\text { n }\end{array}$ & $\begin{array}{l}\text { Allocation } \\
\text { Concealment }\end{array}$ & $\begin{array}{l}\text { Blinding of } \\
\text { Participants }\end{array}$ & $\begin{array}{l}\text { Blinding } \\
\text { of } \\
\text { Personnel/ } \\
\text { Care } \\
\text { Providers }\end{array}$ & $\begin{array}{l}\text { Blinding of } \\
\text { Outcome } \\
\text { Assessors } \\
\text { (Objective } \\
\text { Outcomes) }\end{array}$ & $\begin{array}{l}\text { Blinding of } \\
\text { Outcome } \\
\text { Assessors } \\
\text { (Subjective } \\
\text { Outcomes) }\end{array}$ & $\begin{array}{l}\text { Incomplete } \\
\text { Outcome } \\
\text { Data }\end{array}$ & $\begin{array}{l}\text { Selective } \\
\text { Outcome } \\
\text { Reporting }\end{array}$ & $\begin{array}{l}\text { Other } \\
\text { Bias }\end{array}$ & $\begin{array}{l}\text { Eligibility } \\
\text { Criteria } \\
\text { Prespecifi } \\
\text { ed and } \\
\text { Clearly } \\
\text { Described }\end{array}$ & $\begin{array}{l}\text { Intervention } \\
\text { Clearly } \\
\text { Described } \\
\text { and } \\
\text { Consistently } \\
\text { Delivered }\end{array}$ & $\begin{array}{l}\text { Outcomes } \\
\text { Prespecified, } \\
\text { Clearly } \\
\text { Defined, } \\
\text { Valid, } \\
\text { Reliable, and } \\
\text { Consistently } \\
\text { Assessed }\end{array}$ & $\begin{array}{l}\text { Overall } \\
\text { RoB }\end{array}$ \\
\hline 1 & $\begin{array}{l}\text { Brorson } \\
2020 a \text {, } \\
32807615\end{array}$ & Low & Low & High & High & High & $\mathrm{N} / \mathrm{A}$ & High & Low & Low & Yes & Yes & Yes & High \\
\hline 1 & $\begin{array}{l}\text { Tallroth, } \\
2020, \\
33436336\end{array}$ & Unclear & Unclear & High & High & Low & Unclear & Low & Low & Low & Yes & Yes & Yes & Moderate \\
\hline 4 & $\begin{array}{l}\text { Lee, } \\
2021 b \text {, } \\
33691448\end{array}$ & Unclear & Unclear & High & High & Unclear & N/A & Unclear & Unclear & Low & Yes & Yes & Yes & Moderate \\
\hline 5 & $\begin{array}{l}\text { McCarthy, } \\
2012 \text {, } \\
23096987\end{array}$ & Low & Low & High & High & Low & Low & Low & High & Low & Yes & Yes & Yes & Moderate \\
\hline 5 & $\begin{array}{l}\text { Wendel, } \\
2013, \\
\text { none }\end{array}$ & Unclear & Unclear & High & High & Low & $\mathrm{N} / \mathrm{A}$ & High & High & Low & No & No & No & High \\
\hline 6 & $\begin{array}{l}\text { Brandberg } \\
, 2000, \\
10626972\end{array}$ & Low & Low & High & High & High & High & High & High & Low & Yes & Yes & Yes & High \\
\hline 6 & $\begin{array}{l}\text { Brorson } \\
2020 b \text {, } \\
32807615\end{array}$ & Low & Low & High & High & High & $\mathrm{N} / \mathrm{A}$ & High & Low & Low & Yes & Yes & Yes & High \\
\hline 6 & $\begin{array}{l}\text { Rindom, } \\
2019, \\
31515191 \\
\end{array}$ & Low & Low & High & High & High & High & Low & Low & Low & Yes & Yes & Yes & Moderate \\
\hline
\end{tabular}

Abbreviations: KQ = Key Question, PMID = PubMed identifier. Ratings are color coded for emphasis only. The colors do not impart unique information.

From the Cochrane Risk of Bias Tool (each item rated as Low, High, Unclear, or N/A [not applicable])

- Random sequence generation (selection bias): Selection bias (biased allocation to interventions) due to inadequate generation of a randomized sequence.

- Allocation concealment (selection bias): Selection bias (biased allocation to interventions) due to inadequate concealment of allocations prior to assignment.

- Blinding of participants (performance bias): Performance bias due to knowledge of the allocated interventions by participants during the study.

- Blinding of personnel/care providers (performance bias): Performance bias due to knowledge of the allocated interventions by personnel/care providers during the study.

- Blinding of outcome assessor (detection bias): Detection bias due to knowledge of the allocated interventions by outcome assessors during the study.

- Incomplete outcome data (attrition bias): Attrition bias due to amount, nature, or handling of incomplete outcome data.

- Selective outcome reporting (outcome reporting bias): Bias arising from outcomes being selectively reported based on the direction and/or strength of the results. 
- Other Bias: Bias due to problems not covered elsewhere in the table.

From the National Heart, Lung, and Blood Institute (NHLBI) Quality Assessment Tool (each item rated as Yes, No, or Unclear)

- Eligibility criteria prespecified and clearly described: Potentially related to selection bias.

- Intervention clearly described and delivered consistently: Potentially related to performance bias.

- Outcomes prespecified, clearly defined, valid, reliable, and assessed consistently: Potentially related to detection bias.

Overall risk of bias assessed as HIGH, MODERATE, or LOW. 
Table D-2. Risk of bias assessment for all Key Questions - nonrandomized comparative studies, confounding and selection bias

\begin{tabular}{|c|c|c|c|c|c|c|c|c|c|c|c|c|c|c|}
\hline $\mathbf{K Q}$ & Study, Year, PMID & 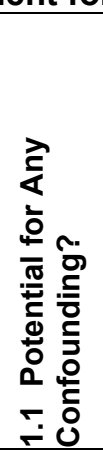 & 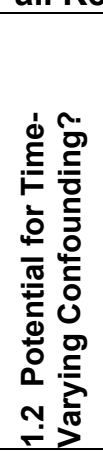 & 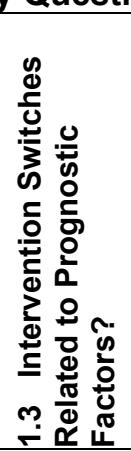 & 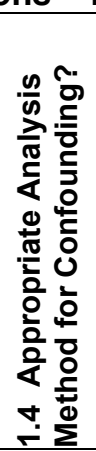 & 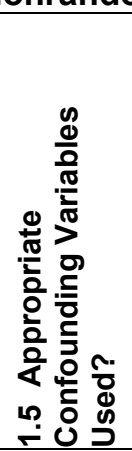 & 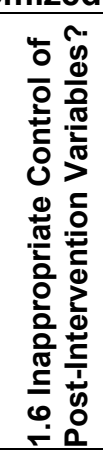 & 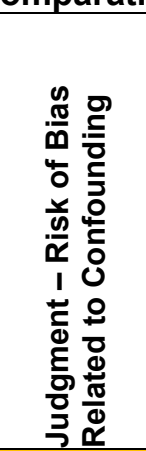 & 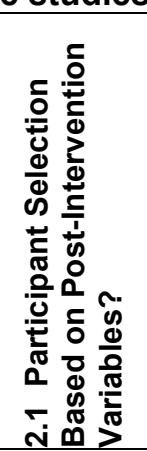 & 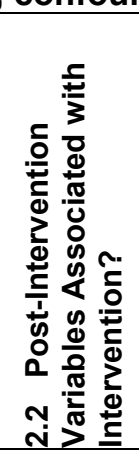 & 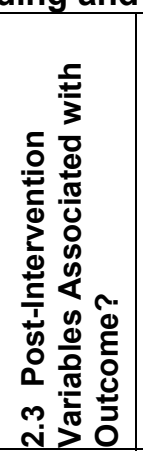 & 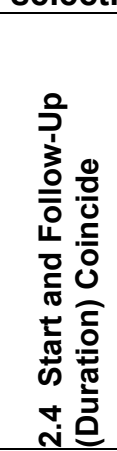 & 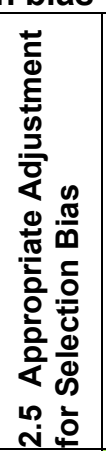 & 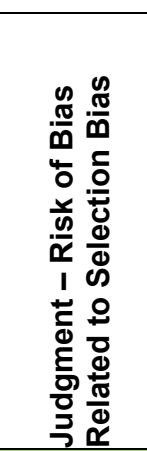 \\
\hline 1 & Brito, 2020, No PMID & Yes & No & $\mathrm{N} / \mathrm{A}$ & $\mathrm{N}$ & $\mathrm{N} / \mathrm{A}$ & $\mathrm{N}$ & Serious & $\mathrm{N}$ & $\mathrm{N} / \mathrm{A}$ & N/A & $\mathrm{Y}$ & N/A & Low \\
\hline 1 & Chetta, 2017, 28002254 & Yes & No & $\mathrm{N} / \mathrm{A}$ & PN & N/A & $\mathrm{N}$ & Serious & $\mathrm{N}$ & $\mathrm{N} / \mathrm{A}$ & $\mathrm{N} / \mathrm{A}$ & $\mathrm{Y}$ & N/A & Low \\
\hline 1 & de Araujo, 2016, 27673527 & Yes & No & $\mathrm{N} / \mathrm{A}$ & PN & $\mathrm{N} / \mathrm{A}$ & $\mathrm{N}$ & Serious & $\mathrm{N}$ & $\mathrm{N} / \mathrm{A}$ & $\mathrm{N} / \mathrm{A}$ & $\mathrm{Y}$ & $\mathrm{N} / \mathrm{A}$ & Low \\
\hline 1 & Eltahir, 2015,25539295 & Yes & No & $\mathrm{N} / \mathrm{A}$ & $\mathrm{Y}$ & $\mathrm{PY}$ & $\mathrm{N}$ & Low & $\mathrm{N}$ & $\mathrm{N} / \mathrm{A}$ & $\mathrm{N} / \mathrm{A}$ & PY & $\mathrm{N} / \mathrm{A}$ & Low \\
\hline 1 & Fischer, 2013, 23629074 & Yes & No & $\mathrm{N} / \mathrm{A}$ & $\mathrm{N}$ & $\mathrm{N} / \mathrm{A}$ & $\mathrm{N}$ & Serious & $\mathrm{N}$ & N/A & $\mathrm{N} / \mathrm{A}$ & $\mathrm{Y}$ & $\mathrm{N} / \mathrm{A}$ & Low \\
\hline 1 & Fischer, 2014, 24916480 & Yes & No & $\mathrm{N} / \mathrm{A}$ & $\mathrm{N}$ & $\mathrm{N} / \mathrm{A}$ & $\mathrm{N}$ & Serious & $\mathrm{N}$ & $\mathrm{N} / \mathrm{A}$ & $\mathrm{N} / \mathrm{A}$ & $\mathrm{Y}$ & $\mathrm{N} / \mathrm{A}$ & Low \\
\hline 1 & Fischer, 2015, 26366550 & Yes & No & N/A & $\mathrm{N}$ & N/A & $\mathrm{N}$ & Serious & $\mathrm{N}$ & $\mathrm{N} / \mathrm{A}$ & $\mathrm{N} / \mathrm{A}$ & $\mathrm{Y}$ & $\mathrm{N} / \mathrm{A}$ & Low \\
\hline 1 & Garvey, 2012, 23096600 & Yes & No & N/A & $\mathrm{Y}$ & $\mathrm{Y}$ & $\mathrm{N}$ & Low & $\mathrm{N}$ & $\mathrm{N} / \mathrm{A}$ & $\mathrm{N} / \mathrm{A}$ & $\mathrm{Y}$ & N/A & Low \\
\hline 1 & $\mathrm{Ha}, 2020,32000718$ & Yes & No & $\mathrm{N} / \mathrm{A}$ & $\mathrm{Y}$ & $\mathrm{Y}$ & $\mathrm{N}$ & Low & $\mathrm{N}$ & $\mathrm{N} / \mathrm{A}$ & $\mathrm{N} / \mathrm{A}$ & $\mathrm{N}$ & PN & Serious \\
\hline 1 & Hangge, 2019, 31606126 & Yes & No & $\mathrm{N} / \mathrm{A}$ & PN & $\mathrm{N} / \mathrm{A}$ & $\mathrm{N}$ & Serious & $\mathrm{N}$ & N/A & $\mathrm{N} / \mathrm{A}$ & PY & N/A & Low \\
\hline 1 & Jiang, 2013, 24349366 & Yes & No & $\mathrm{N} / \mathrm{A}$ & $\mathrm{N}$ & $\mathrm{N} / \mathrm{A}$ & $\mathrm{N}$ & Serious & $\mathrm{N}$ & $\mathrm{N} / \mathrm{A}$ & $\mathrm{N} / \mathrm{A}$ & $\mathrm{Y}$ & $\mathrm{N} / \mathrm{A}$ & Low \\
\hline 1 & Kouwenberg, 2019, 30270015 & Yes & No & $\mathrm{N} / \mathrm{A}$ & $\mathrm{Y}$ & PY & $\mathrm{N}$ & Low & $\mathrm{N}$ & $\mathrm{N} / \mathrm{A}$ & $\mathrm{N} / \mathrm{A}$ & $\mathrm{PN}$ & PY & Moderate \\
\hline 1 & Kouwenberg, 2020, 32590633 & Yes & No & $\mathrm{N} / \mathrm{A}$ & $\mathrm{Y}$ & $\mathrm{Y}$ & $\mathrm{N}$ & Low & $\mathrm{N}$ & $\mathrm{N} / \mathrm{A}$ & $\mathrm{N} / \mathrm{A}$ & PY & $\mathrm{N} / \mathrm{A}$ & Low \\
\hline 1 & Laporta, 2017, 28061518 & Yes & No & $\mathrm{N} / \mathrm{A}$ & PN & $\mathrm{N} / \mathrm{A}$ & $\mathrm{N}$ & Serious & $\mathrm{N}$ & N/A & N/A & $\mathrm{Y}$ & $\mathrm{N} / \mathrm{A}$ & Low \\
\hline 1 & Lei, 2020,32481367 & Yes & No & N/A & $\mathrm{Y}$ & $\mathrm{Y}$ & $\mathrm{Y}$ & Moderate & $\mathrm{N}$ & $\mathrm{N} / \mathrm{A}$ & $\mathrm{N} / \mathrm{A}$ & $\mathrm{Y}$ & $\mathrm{N} / \mathrm{A}$ & Low \\
\hline 1 & Liu, 2014, 24558063 & Yes & No & $\mathrm{N} / \mathrm{A}$ & PN & $\mathrm{N} / \mathrm{A}$ & $\mathrm{N}$ & Serious & $\mathrm{N}$ & $\mathrm{N} / \mathrm{A}$ & $\mathrm{N} / \mathrm{A}$ & $\mathrm{Y}$ & $\mathrm{N} / \mathrm{A}$ & Low \\
\hline 1 & Mak, 2020, 32665188 & Yes & No & $\mathrm{N} / \mathrm{A}$ & $\mathrm{N}$ & $\mathrm{N} / \mathrm{A}$ & $\mathrm{N}$ & Serious & $\mathrm{N}$ & N/A & N/A & $\mathrm{Y}$ & $\mathrm{N} / \mathrm{A}$ & Low \\
\hline 1 & McCarthy, 2014, 24201740 & Yes & No & $\mathrm{N} / \mathrm{A}$ & $\mathrm{Y}$ & $\mathrm{PY}$ & $\mathrm{Y}$ & Moderate & $\mathrm{PN}$ & $\mathrm{N} / \mathrm{A}$ & $\mathrm{N} / \mathrm{A}$ & $\mathrm{N}$ & $\mathrm{PN}$ & Moderate \\
\hline 1 & Merchant, 2015, 26111325 & Yes & No & $\mathrm{N} / \mathrm{A}$ & $\mathrm{N}$ & $\mathrm{N} / \mathrm{A}$ & $\mathrm{N}$ & Serious & $\mathrm{N}$ & $\mathrm{N} / \mathrm{A}$ & $\mathrm{N} / \mathrm{A}$ & $\mathrm{Y}$ & $\mathrm{N} / \mathrm{A}$ & Low \\
\hline 1 & Mioton, 2013,23562485 & Yes & No & N/A & $\mathrm{Y}$ & $\mathrm{Y}$ & $\mathrm{N}$ & Low & $\mathrm{PN}$ & N/A & N/A & PY & N/A & Low \\
\hline 1 & Momeni, 2018, 29095189 & Yes & No & N/A & $\mathrm{N}$ & $\mathrm{N} / \mathrm{A}$ & $\mathrm{N}$ & Serious & $\mathrm{N}$ & N/A & $\mathrm{N} / \mathrm{A}$ & $\mathrm{Y}$ & N/A & Low \\
\hline 1 & Naoum, 2020a, 31756414 & Yes & No & $\mathrm{N} / \mathrm{A}$ & PN & $\mathrm{N} / \mathrm{A}$ & $\mathrm{N}$ & Serious & $\mathrm{N}$ & $\mathrm{N} / \mathrm{A}$ & $\mathrm{N} / \mathrm{A}$ & $\mathrm{Y}$ & $\mathrm{N} / \mathrm{A}$ & Low \\
\hline 1 & Naoum, 2020b, 32607638 & Yes & No & $\mathrm{N} / \mathrm{A}$ & $\mathrm{N}$ & $\mathrm{N} / \mathrm{A}$ & $\mathrm{N}$ & Serious & $\mathrm{N}$ & N/A & N/A & PY & N/A & Moderate \\
\hline 1 & Nasser, 2018, 30204678 & Yes & No & $\mathrm{N} / \mathrm{A}$ & $\mathrm{N}$ & $\mathrm{N} / \mathrm{A}$ & $\mathrm{N}$ & Serious & $\mathrm{N}$ & $\mathrm{N} / \mathrm{A}$ & $\mathrm{N} / \mathrm{A}$ & $\mathrm{Y}$ & $\mathrm{N} / \mathrm{A}$ & Low \\
\hline 1 & Nelson, 2019, 31356276 & Yes & No & $\mathrm{N} / \mathrm{A}$ & $\mathrm{N}$ & $\mathrm{N} / \mathrm{A}$ & $\mathrm{N}$ & Serious & $\mathrm{N}$ & $\mathrm{N} / \mathrm{A}$ & $\mathrm{N} / \mathrm{A}$ & $\mathrm{Y}$ & $\mathrm{N} / \mathrm{A}$ & Low \\
\hline 1 & Palve, 2020, 32468337 & Yes & No & N/A & $\mathrm{Y}$ & $\mathrm{Y}$ & $\mathrm{Y}$ & Moderate & $\mathrm{N}$ & $\mathrm{N} / \mathrm{A}$ & $\mathrm{N} / \mathrm{A}$ & $\mathrm{Y}$ & $\mathrm{N} / \mathrm{A}$ & Low \\
\hline 1 & Qin, 2018, 29384865 & Yes & No & $\mathrm{N} / \mathrm{A}$ & $\mathrm{Y}$ & $\mathrm{PY}$ & $\mathrm{N}$ & Low & $\mathrm{N}$ & $\mathrm{N} / \mathrm{A}$ & $\mathrm{N} / \mathrm{A}$ & $\mathrm{N}$ & $\mathrm{PN}$ & Moderate \\
\hline 1 & Roth, 2007, 17413877 & Yes & No & $\mathrm{N} / \mathrm{A}$ & $\mathrm{N}$ & $\mathrm{N} / \mathrm{A}$ & $\mathrm{N}$ & Critical & $\mathrm{N}$ & $\mathrm{N} / \mathrm{A}$ & $\mathrm{N} / \mathrm{A}$ & $\mathrm{Y}$ & $\mathrm{N} / \mathrm{A}$ & Low \\
\hline 1 & Shiraishi, 2020, 32589082 & Yes & No & $\mathrm{N} / \mathrm{A}$ & $\mathrm{N}$ & $\mathrm{N} / \mathrm{A}$ & $\mathrm{N}$ & Serious & $\mathrm{N}$ & $\mathrm{N} / \mathrm{A}$ & $\mathrm{N} / \mathrm{A}$ & $\mathrm{Y}$ & $\mathrm{N} / \mathrm{A}$ & Low \\
\hline 1 & Simon, 2020, 33363007 & Yes & No & $\mathrm{N} / \mathrm{A}$ & $\mathrm{Y}$ & $\mathrm{PY}$ & $\mathrm{N}$ & Low & $\mathrm{N}$ & $\mathrm{N} / \mathrm{A}$ & $\mathrm{N} / \mathrm{A}$ & $\mathrm{Y}$ & $\mathrm{N} / \mathrm{A}$ & Low \\
\hline
\end{tabular}




\begin{tabular}{|c|c|c|c|c|c|c|c|c|c|c|c|c|c|c|}
\hline $\mathbf{K Q}$ & Study, Year, PMID & 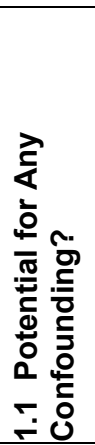 & 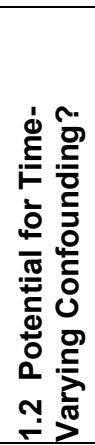 & 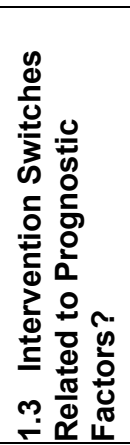 & 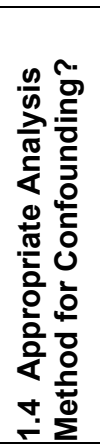 & 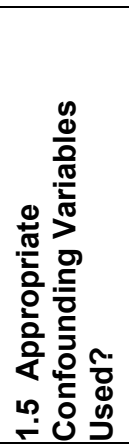 & 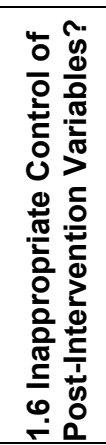 & 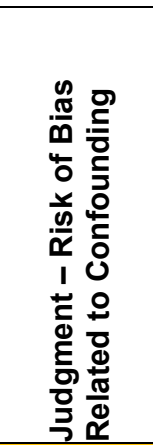 & 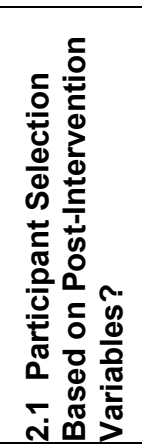 & 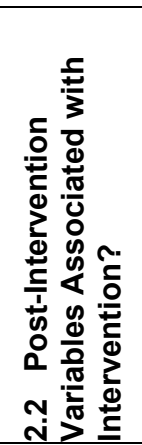 & 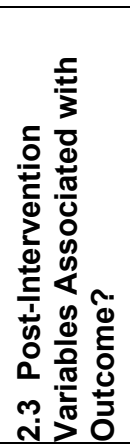 & 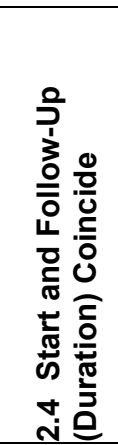 & 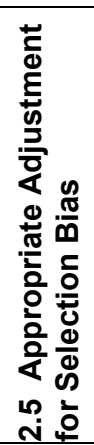 & 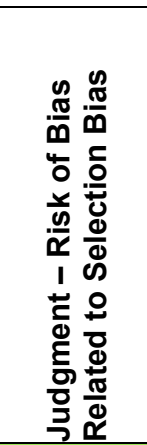 \\
\hline 1 & Wu, 2021, 33740204 & Yes & No & N/A & $\mathrm{N}$ & $\mathrm{N} / \mathrm{A}$ & $\mathrm{N}$ & Serious & $\mathrm{N}$ & $\mathrm{N} / \mathrm{A}$ & $\mathrm{N} / \mathrm{A}$ & $\mathrm{Y}$ & $\mathrm{N} / \mathrm{A}$ & Low \\
\hline 1 & $\mathrm{Xu}, 2018,30261115$ & Yes & No & $\mathrm{N} / \mathrm{A}$ & $\mathrm{Y}$ & PY & $\mathrm{N}$ & Low & $\mathrm{N}$ & $\mathrm{N} / \mathrm{A}$ & $\mathrm{N} / \mathrm{A}$ & $\mathrm{Y}$ & $\mathrm{N} / \mathrm{A}$ & Low \\
\hline 1 & Zhang, 2019, 30675702 & Yes & No & N/A & $\mathrm{N}$ & N/A & $\mathrm{N}$ & Serious & $\mathrm{N}$ & $\mathrm{N} / \mathrm{A}$ & N/A & $\mathrm{Y}$ & $\mathrm{N} / \mathrm{A}$ & Low \\
\hline 1,6 & Abedi, 2016,25003437 & Yes & No & $\mathrm{N} / \mathrm{A}$ & $\mathrm{N}$ & $\mathrm{N} / \mathrm{A}$ & $\mathrm{Y}$ & Serious & $\mathrm{N}$ & $\mathrm{N} / \mathrm{A}$ & $\mathrm{N} / \mathrm{A}$ & $\mathrm{Y}$ & $\mathrm{N} / \mathrm{A}$ & Low \\
\hline 1,6 & Carramaschi, 1989, 2602589 & Yes & No & N/A & $\mathrm{N}$ & N/A & $\mathrm{N}$ & Critical & $\mathrm{N}$ & $\mathrm{N} / \mathrm{A}$ & N/A & $\mathrm{N}$ & $\mathrm{N}$ & Low \\
\hline 1,6 & Dauplat, 2021, 33622886 & Yes & No & $\mathrm{N} / \mathrm{A}$ & $\mathrm{Y}$ & $\mathrm{Y}$ & $\mathrm{Y}$ & Moderate & $\mathrm{N}$ & $\mathrm{N} / \mathrm{A}$ & $\mathrm{N} / \mathrm{A}$ & $\mathrm{Y}$ & $\mathrm{N} / \mathrm{A}$ & Low \\
\hline 1,6 & Garbay, 1992, 1624727 & Yes & No & N/A & $\mathrm{N}$ & $\mathrm{N} / \mathrm{A}$ & $\mathrm{N}$ & Serious & $\mathrm{N}$ & N/A & N/A & $\mathrm{Y}$ & N/A & Low \\
\hline 1,6 & Kulkarni, 2017, 28713853 & Yes & No & $\mathrm{N} / \mathrm{A}$ & $\mathrm{Y}$ & $\mathrm{Y}$ & $\mathrm{PN}$ & Low & $\mathrm{PN}$ & $\mathrm{N} / \mathrm{A}$ & $\mathrm{N} / \mathrm{A}$ & $\mathrm{Y}$ & $\mathrm{N} / \mathrm{A}$ & Low \\
\hline 1,6 & Woo, 2018, 30360958 & Yes & No & $\mathrm{N} / \mathrm{A}$ & $\mathrm{N}$ & $\mathrm{N} / \mathrm{A}$ & $\mathrm{N}$ & Serious & $\mathrm{N}$ & $\mathrm{N} / \mathrm{A}$ & $\mathrm{N} / \mathrm{A}$ & PY & $\mathrm{N} / \mathrm{A}$ & Low \\
\hline 1,6 & Yueh, 2009, 19228537 & Yes & No & N/A & $\mathrm{N}$ & N/A & $\mathrm{N}$ & Critical & $\mathrm{N}$ & $\mathrm{N} / \mathrm{A}$ & $\mathrm{N} / \mathrm{A}$ & $\mathrm{Y}$ & $\mathrm{N} / \mathrm{A}$ & Low \\
\hline 2 & Eriksson, 2013,24258257 & Yes & No & $\mathrm{N} / \mathrm{A}$ & $\mathrm{Y}$ & $\mathrm{Y}$ & $\mathrm{N}$ & Low & $\mathrm{N}$ & $\mathrm{N} / \mathrm{A}$ & $\mathrm{N} / \mathrm{A}$ & $\mathrm{N}$ & PY & Moderate \\
\hline 2 & Yoon, 2020,32332528 & Yes & No & N/A & $\mathrm{Y}$ & PY & $\mathrm{N}$ & Low & $\mathrm{N}$ & $\mathrm{N} / \mathrm{A}$ & N/A & $\mathrm{N}$ & PY & Moderate \\
\hline 2,3 & Cordeiro, 2015a, 25742523 & Yes & No & $\mathrm{N} / \mathrm{A}$ & $\mathrm{N}$ & $\mathrm{N} / \mathrm{A}$ & $\mathrm{N}$ & Serious & $\mathrm{N}$ & $\mathrm{N} / \mathrm{A}$ & $\mathrm{N} / \mathrm{A}$ & $\mathrm{N}$ & PY & Moderate \\
\hline 2,5 & Hirsch, 2014, 25347643 & Yes & No & N/A & $\mathrm{N}$ & N/A & $\mathrm{N}$ & Serious & $\mathrm{N}$ & N/A & N/A & $\mathrm{Y}$ & N/A & Low \\
\hline 2,5 & Stein, 2020, 32561384 & Yes & No & $\mathrm{N} / \mathrm{A}$ & $\mathrm{N}$ & $\mathrm{N} / \mathrm{A}$ & $\mathrm{N}$ & Serious & $\mathrm{N}$ & $\mathrm{N} / \mathrm{A}$ & $\mathrm{N} / \mathrm{A}$ & $\mathrm{N}$ & PY & Moderate \\
\hline 3 & Antony, 2014, 24135689 & Yes & No & N/A & $\mathrm{PN}$ & N/A & $\mathrm{N}$ & Serious & $\mathrm{N}$ & N/A & N/A & $\mathrm{Y}$ & N/A & Low \\
\hline 3 & Le, 2005,15743498 & Yes & No & $\mathrm{N} / \mathrm{A}$ & $\mathrm{N}$ & $\mathrm{N} / \mathrm{A}$ & $\mathrm{N}$ & Serious & $\mathrm{N}$ & $\mathrm{N} / \mathrm{A}$ & $\mathrm{N} / \mathrm{A}$ & $\mathrm{Y}$ & $\mathrm{N} / \mathrm{A}$ & Low \\
\hline 3 & Macadam, 2010, 20009795 & Yes & No & $\mathrm{N} / \mathrm{A}$ & $\mathrm{N}$ & $\mathrm{N} / \mathrm{A}$ & $\mathrm{N}$ & Serious & $\mathrm{N}$ & $\mathrm{N} / \mathrm{A}$ & $\mathrm{N} / \mathrm{A}$ & $\mathrm{Y}$ & $\mathrm{N} / \mathrm{A}$ & Moderate \\
\hline 3 & McCarthy, 2010, 21136577 & Yes & No & N/A & $\mathrm{Y}$ & PY & $\mathrm{Y}$ & Moderate & $\mathrm{N}$ & N/A & $\mathrm{N} / \mathrm{A}$ & $\mathrm{N}$ & PY & Moderate \\
\hline 4 & Avila, 2020,33234947 & Yes & No & $\mathrm{N} / \mathrm{A}$ & $\mathrm{N}$ & $\mathrm{N} / \mathrm{A}$ & $\mathrm{N}$ & Serious & $\mathrm{N}$ & $\mathrm{N} / \mathrm{A}$ & $\mathrm{N} / \mathrm{A}$ & $\mathrm{Y}$ & $\mathrm{N} / \mathrm{A}$ & Low \\
\hline 4 & Gabriel, 2020, 32195862 & Yes & No & N/A & $\mathrm{N}$ & N/A & $\mathrm{N}$ & Serious & $\mathrm{N}$ & $\mathrm{N} / \mathrm{A}$ & N/A & $\mathrm{Y}$ & $\mathrm{N} / \mathrm{A}$ & Low \\
\hline 4 & Kim, 2020, 33066236 & Yes & No & $\mathrm{N} / \mathrm{A}$ & $\mathrm{Y}$ & $\mathrm{Y}$ & $\mathrm{Y}$ & Moderate & $\mathrm{N}$ & $\mathrm{N} / \mathrm{A}$ & $\mathrm{N} / \mathrm{A}$ & $\mathrm{Y}$ & $\mathrm{N} / \mathrm{A}$ & Low \\
\hline 4 & Kraenzlin, 2021, 32568752 & Yes & No & N/A & $\mathrm{N}$ & $\mathrm{N} / \mathrm{A}$ & $\mathrm{N}$ & Serious & $\mathrm{N}$ & N/A & N/A & $\mathrm{Y}$ & N/A & Low \\
\hline 4 & Nealon, 2020a, 32032345 & Yes & No & $\mathrm{N} / \mathrm{A}$ & $\mathrm{PN}$ & $\mathrm{N} / \mathrm{A}$ & $\mathrm{N}$ & Serious & $\mathrm{N}$ & $\mathrm{N} / \mathrm{A}$ & $\mathrm{N} / \mathrm{A}$ & $\mathrm{Y}$ & $\mathrm{N} / \mathrm{A}$ & Low \\
\hline 4 & Ozgur, 2020, 33223365 & Yes & No & N/A & $\mathrm{N}$ & N/A & $\mathrm{N}$ & Serious & $\mathrm{N}$ & N/A & $\mathrm{N} / \mathrm{A}$ & PY & N/A & Low \\
\hline 4,5 & Cattelani, 2018, 29275104 & Yes & No & $\mathrm{N} / \mathrm{A}$ & $\mathrm{PN}$ & $\mathrm{N} / \mathrm{A}$ & $\mathrm{N}$ & Critical & $\mathrm{N}$ & $\mathrm{N} / \mathrm{A}$ & $\mathrm{N} / \mathrm{A}$ & $\mathrm{Y}$ & $\mathrm{N} / \mathrm{A}$ & Low \\
\hline 5 & Brooke, 2012, 22868313 & Yes & No & $\mathrm{N} / \mathrm{A}$ & $\mathrm{N}$ & $\mathrm{Y}$ & $\mathrm{N}$ & Serious & $\mathrm{N}$ & $\mathrm{N} / \mathrm{A}$ & $\mathrm{N} / \mathrm{A}$ & PY & $\mathrm{N} / \mathrm{A}$ & Low \\
\hline 5 & Chun, 2010, 20124828 & Yes & No & N/A & $\mathrm{N}$ & $\mathrm{N} / \mathrm{A}$ & $\mathrm{N}$ & Serious & $\mathrm{N}$ & $\mathrm{N} / \mathrm{A}$ & N/A & $\mathrm{Y}$ & N/A & Low \\
\hline 5 & Craig, 2019,29800083 & Yes & No & $\mathrm{N} / \mathrm{A}$ & $\mathrm{Y}$ & $\mathrm{Y}$ & $\mathrm{N}$ & Low & PY & $\mathrm{Y}$ & $\mathrm{Y}$ & $\mathrm{N}$ & $\mathrm{N}$ & Moderate \\
\hline 5 & Ibrahim, 2013, 24165587 & Yes & No & N/A & $\mathrm{Y}$ & PY & PN & Low & PN & $\mathrm{N} / \mathrm{A}$ & $\mathrm{N} / \mathrm{A}$ & PY & $\mathrm{N} / \mathrm{A}$ & Low \\
\hline 5 & Ganesh Kumar, 2021, 33172826 & Yes & No & $\mathrm{N} / \mathrm{A}$ & $Y$ & $Y$ & PN & Low & $\mathrm{PN}$ & N/A & $\mathrm{N} / \mathrm{A}$ & PY & N/A & Low \\
\hline
\end{tabular}




\begin{tabular}{|c|c|c|c|c|c|c|c|c|c|c|c|c|c|c|}
\hline $\mathbf{K Q}$ & Study, Year, PMID & 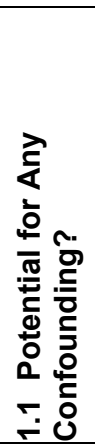 & 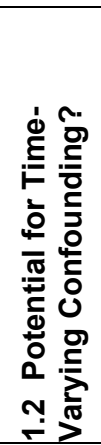 & 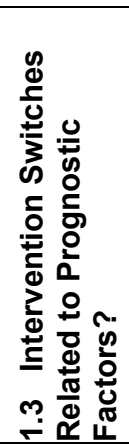 & 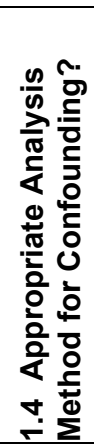 & 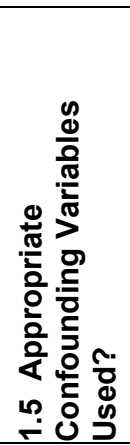 & 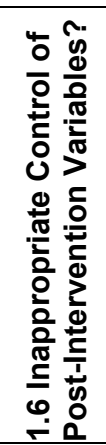 & 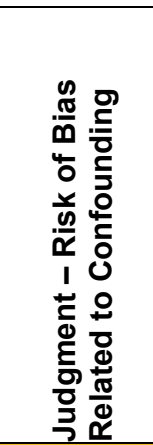 & 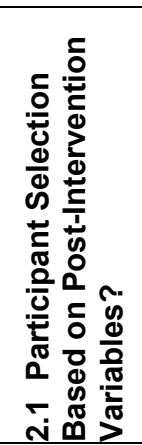 & 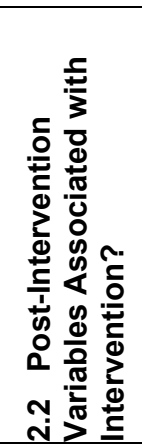 & 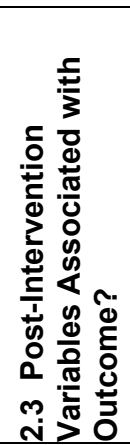 & 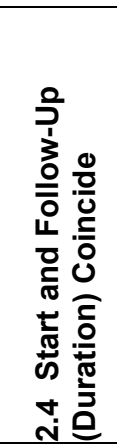 & 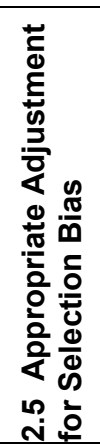 & 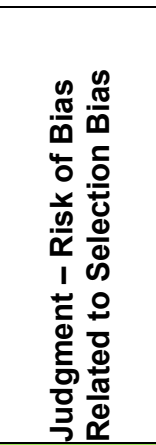 \\
\hline 5 & Lee, 2020, No PMID & Yes & No & $\mathrm{N} / \mathrm{A}$ & $\mathrm{N}$ & N/A & $\mathrm{N}$ & Serious & $\mathrm{N}$ & N/A & $\mathrm{N} / \mathrm{A}$ & $\mathrm{Y}$ & $\mathrm{N} / \mathrm{A}$ & Low \\
\hline 5 & Liu, 2011, 21228744 & Yes & No & $\mathrm{N} / \mathrm{A}$ & $\mathrm{N}$ & $\mathrm{N} / \mathrm{A}$ & $\mathrm{N}$ & Serious & $\mathrm{N}$ & $\mathrm{N} / \mathrm{A}$ & $\mathrm{N} / \mathrm{A}$ & PY & $\mathrm{N} / \mathrm{A}$ & Low \\
\hline 5 & Nealon, 2020b, 31605310 & Yes & No & $\mathrm{N} / \mathrm{A}$ & $\mathrm{N}$ & $\mathrm{N} / \mathrm{A}$ & $\mathrm{N}$ & Critical & $\mathrm{N}$ & $\mathrm{N} / \mathrm{A}$ & $\mathrm{N} / \mathrm{A}$ & $\mathrm{Y}$ & $\mathrm{N} / \mathrm{A}$ & Low \\
\hline 5 & Pannucci, 2013, 23508050 & Yes & No & $\mathrm{N} / \mathrm{A}$ & $\mathrm{Y}$ & $\mathrm{Y}$ & $\mathrm{N}$ & Low & $\mathrm{N}$ & $\mathrm{N} / \mathrm{A}$ & $\mathrm{N} / \mathrm{A}$ & $\mathrm{Y}$ & $\mathrm{N} / \mathrm{A}$ & Low \\
\hline 5 & Peled, 2012, 22634688 & Yes & No & $\mathrm{N} / \mathrm{A}$ & PN & $\mathrm{N} / \mathrm{A}$ & $\mathrm{N}$ & Serious & $\mathrm{N}$ & $\mathrm{N} / \mathrm{A}$ & $\mathrm{N} / \mathrm{A}$ & $\mathrm{Y}$ & $\mathrm{N} / \mathrm{A}$ & Low \\
\hline 5 & Qureshi, 2016, 27465177 & Yes & No & $\mathrm{N} / \mathrm{A}$ & PN & $\mathrm{N} / \mathrm{A}$ & $\mathrm{N}$ & Serious & $\mathrm{N}$ & $\mathrm{N} / \mathrm{A}$ & $\mathrm{N} / \mathrm{A}$ & $\mathrm{Y}$ & $\mathrm{N} / \mathrm{A}$ & Low \\
\hline 5 & Safran, 2020, 32221195 & Yes & No & $\mathrm{N} / \mathrm{A}$ & $\mathrm{PN}$ & $\mathrm{N} / \mathrm{A}$ & $\mathrm{N}$ & Serious & $\mathrm{N}$ & $\mathrm{N} / \mathrm{A}$ & $\mathrm{N} / \mathrm{A}$ & $\mathrm{Y}$ & $\mathrm{N} / \mathrm{A}$ & Moderate \\
\hline 5 & Seth, 2012,23018687 & Yes & No & $\mathrm{N} / \mathrm{A}$ & $\mathrm{N}$ & $\mathrm{N} / \mathrm{A}$ & $\mathrm{N}$ & Serious & $\mathrm{N}$ & $\mathrm{N} / \mathrm{A}$ & $\mathrm{N} / \mathrm{A}$ & $\mathrm{N}$ & $\mathrm{Y}$ & Low \\
\hline 5 & Sobti, 2018,29481386 & Yes & No & $\mathrm{N} / \mathrm{A}$ & $\mathrm{N}$ & $\mathrm{N} / \mathrm{A}$ & $\mathrm{Y}$ & Serious & $\mathrm{N}$ & $\mathrm{N} / \mathrm{A}$ & $\mathrm{N} / \mathrm{A}$ & $\mathrm{Y}$ & $\mathrm{N} / \mathrm{A}$ & Low \\
\hline 5 & Vardanian, 2011, 22030500 & Yes & No & $\mathrm{N} / \mathrm{A}$ & $\mathrm{PN}$ & $\mathrm{N} / \mathrm{A}$ & $\mathrm{N}$ & Serious & $\mathrm{Y}$ & $\mathrm{PN}$ & $\mathrm{N} / \mathrm{A}$ & $\mathrm{Y}$ & $\mathrm{N} / \mathrm{A}$ & Low \\
\hline 5 & Weichman, 2012, 22544088 & Yes & No & N/A & PY & PY & $\mathrm{N}$ & Low & $\mathrm{N}$ & $\mathrm{N} / \mathrm{A}$ & $\mathrm{N} / \mathrm{A}$ & $\mathrm{Y}$ & $\mathrm{N} / \mathrm{A}$ & Low \\
\hline 5 & Woo, 2017, 28509694 & Yes & No & $\mathrm{N} / \mathrm{A}$ & $\mathrm{PN}$ & $\mathrm{N} / \mathrm{A}$ & $\mathrm{N}$ & Serious & $\mathrm{N}$ & $\mathrm{N} / \mathrm{A}$ & $\mathrm{N} / \mathrm{A}$ & $\mathrm{Y}$ & $\mathrm{N} / \mathrm{A}$ & Low \\
\hline 6 & Baumann, 2010, 20440154 & Yes & No & $\mathrm{N} / \mathrm{A}$ & $\mathrm{PN}$ & $\mathrm{N} / \mathrm{A}$ & $\mathrm{N}$ & Serious & $\mathrm{N}$ & $\mathrm{N} / \mathrm{A}$ & $\mathrm{N} / \mathrm{A}$ & $\mathrm{Y}$ & $\mathrm{N} / \mathrm{A}$ & Low \\
\hline 6 & Erdmann-Sager, 2018, 29019862 & Yes & No & $\mathrm{N} / \mathrm{A}$ & $\mathrm{Y}$ & $\mathrm{Y}$ & $\mathrm{N}$ & Low & PN & $\mathrm{N} / \mathrm{A}$ & $\mathrm{N} / \mathrm{A}$ & PY & $\mathrm{N} / \mathrm{A}$ & Low \\
\hline 6 & Israeli, 2014, 24572840 & Yes & No & $\mathrm{N} / \mathrm{A}$ & $\mathrm{N}$ & $\mathrm{N} / \mathrm{A}$ & $\mathrm{N}$ & Serious & $\mathrm{Y}$ & $\mathrm{PN}$ & $\mathrm{N} / \mathrm{A}$ & $\mathrm{Y}$ & $\mathrm{N} / \mathrm{A}$ & Low \\
\hline 6 & Knox, 2016, 26267400 & Yes & No & $\mathrm{N} / \mathrm{A}$ & PN & $\mathrm{N} / \mathrm{A}$ & $\mathrm{N}$ & Serious & $\mathrm{N}$ & $\mathrm{N} / \mathrm{A}$ & $\mathrm{N} / \mathrm{A}$ & $\mathrm{Y}$ & $\mathrm{N} / \mathrm{A}$ & Low \\
\hline 6 & Kroll, 2000, 10987463 & Yes & No & $\mathrm{N} / \mathrm{A}$ & $\mathrm{N}$ & $\mathrm{N} / \mathrm{A}$ & $\mathrm{PN}$ & Serious & PY & $\mathrm{Y}$ & $\mathrm{PN}$ & PY & $\mathrm{N} / \mathrm{A}$ & Moderate \\
\hline 6 & Macadam, 2016, 26910656 & Yes & No & N/A & $\mathrm{PN}$ & $\mathrm{N} / \mathrm{A}$ & $\mathrm{N}$ & Serious & $\mathrm{N}$ & $\mathrm{N} / \mathrm{A}$ & $\mathrm{N} / \mathrm{A}$ & $\mathrm{Y}$ & $\mathrm{N} / \mathrm{A}$ & Low \\
\hline 6 & Massenburg, 2015, 26487657 & Yes & No & $\mathrm{N} / \mathrm{A}$ & $\mathrm{Y}$ & $\mathrm{Y}$ & $\mathrm{N}$ & Low & $\mathrm{N}$ & $\mathrm{N} / \mathrm{A}$ & $\mathrm{N} / \mathrm{A}$ & PY & $\mathrm{N} / \mathrm{A}$ & Low \\
\hline 6 & Mennie, 2015,25839173 & Yes & No & $\mathrm{N} / \mathrm{A}$ & $\mathrm{PN}$ & $\mathrm{N} / \mathrm{A}$ & $\mathrm{N}$ & Serious & $\mathrm{N}$ & $\mathrm{N} / \mathrm{A}$ & $\mathrm{N} / \mathrm{A}$ & $\mathrm{Y}$ & $\mathrm{N} / \mathrm{A}$ & Low \\
\hline 6 & Zhong, 2014, 24675183 & Yes & No & $\mathrm{N} / \mathrm{A}$ & $\mathrm{N}$ & $\mathrm{N} / \mathrm{A}$ & $\mathrm{N}$ & Serious & $\mathrm{N}$ & $\mathrm{N} / \mathrm{A}$ & $\mathrm{N} / \mathrm{A}$ & $\mathrm{Y}$ & $\mathrm{N} / \mathrm{A}$ & Low \\
\hline 6 & Zoghbi, 2017, 28052051 & Yes & No & $\mathrm{N} / \mathrm{A}$ & $\mathrm{Y}$ & $\mathrm{PN}$ & $\mathrm{N}$ & Moderate & $\mathrm{N}$ & $\mathrm{N} / \mathrm{A}$ & $\mathrm{N} / \mathrm{A}$ & PY & $\mathrm{N} / \mathrm{A}$ & Low \\
\hline
\end{tabular}


Table D-3. Risk of bias assessment for all Key Questions - nonrandomized comparative studies, assessment of remaining biases, quality, and overall risk of bias

\begin{tabular}{|c|c|c|c|c|c|c|c|c|c|c|c|c|}
\hline $\mathbf{K Q}$ & $\begin{array}{l}\text { Study, Year, } \\
\text { PMID }\end{array}$ & $\begin{array}{l}\text { Blinding of } \\
\text { Participants }\end{array}$ & $\begin{array}{l}\text { Blinding of } \\
\text { Personnel/ } \\
\text { Care } \\
\text { Providers }\end{array}$ & $\begin{array}{l}\text { Blinding of } \\
\text { Outcome } \\
\text { Assessors } \\
\text { (Objective } \\
\text { Outcomes) }\end{array}$ & $\begin{array}{l}\text { Blinding of } \\
\text { Outcome } \\
\text { Assessors } \\
\text { (Subjective } \\
\text { Outcomes) }\end{array}$ & $\begin{array}{l}\text { Incomplete } \\
\text { Outcome } \\
\text { Data }\end{array}$ & $\begin{array}{l}\text { Selective } \\
\text { Outcome } \\
\text { Reporting }\end{array}$ & $\begin{array}{l}\text { Other } \\
\text { Bias }\end{array}$ & $\begin{array}{l}\text { Eligibility } \\
\text { Criteria } \\
\text { Prespecified } \\
\text { and Clearly } \\
\text { Described }\end{array}$ & $\begin{array}{l}\text { Intervention } \\
\text { Clearly } \\
\text { Described } \\
\text { and } \\
\text { Consistently } \\
\text { Delivered }\end{array}$ & $\begin{array}{l}\text { Outcomes } \\
\text { Prespecified, } \\
\text { Clearly } \\
\text { Defined, } \\
\text { Valid, } \\
\text { Reliable, and } \\
\text { Consistently } \\
\text { Assessed }\end{array}$ & $\begin{array}{l}\text { Overall } \\
\text { RoB }\end{array}$ \\
\hline 1 & $\begin{array}{l}\text { Brito, 2020, No } \\
\text { PMID }\end{array}$ & High & High & High & Low & Low & Low & Low & Yes & Yes & Yes & High \\
\hline 1 & $\begin{array}{l}\text { Chetta, 2017, } \\
28002254\end{array}$ & High & High & Unclear & $\mathrm{N} / \mathrm{A}$ & Low & Low & Low & Yes & Yes & Yes & High \\
\hline 1 & $\begin{array}{l}\text { de Araujo, } \\
2016,27673527\end{array}$ & High & High & Unclear & $\mathrm{N} / \mathrm{A}$ & Low & Low & Low & Yes & Yes & Yes & High \\
\hline 1 & $\begin{array}{l}\text { Eltahir, 2015, } \\
25539295\end{array}$ & High & Unclear & N/A & Unclear & Low & Low & Low & Yes & Yes & Yes & $\begin{array}{l}\text { Modera } \\
\text { te }\end{array}$ \\
\hline 1 & $\begin{array}{l}\text { Fischer, 2013, } \\
23629074\end{array}$ & High & High & Low & $\mathrm{N} / \mathrm{A}$ & Low & Low & Low & Yes & Yes & Yes & High \\
\hline 1 & $\begin{array}{l}\text { Fischer, 2014, } \\
24916480\end{array}$ & High & High & Low & $\mathrm{N} / \mathrm{A}$ & Low & Low & Low & Yes & Yes & Yes & High \\
\hline 1 & $\begin{array}{l}\text { Fischer, 2015, } \\
26366550\end{array}$ & High & High & Low & N/A & Low & Low & Low & Yes & Yes & Yes & High \\
\hline 1 & $\begin{array}{l}\text { Garvey, 2012, } \\
23096600\end{array}$ & High & High & High & $\mathrm{N} / \mathrm{A}$ & Low & Low & Low & Yes & Unclear & Yes & $\begin{array}{l}\text { Modera } \\
\text { te }\end{array}$ \\
\hline 1 & $\begin{array}{l}\mathrm{Ha}, 2020 \\
32000718\end{array}$ & High & High & Unclear & $\mathrm{N} / \mathrm{A}$ & Low & Low & Low & Yes & Yes & Yes & High \\
\hline 1 & $\begin{array}{l}\text { Hangge, 2019, } \\
31606126\end{array}$ & High & High & Unclear & $\mathrm{N} / \mathrm{A}$ & Low & Low & Low & Yes & Yes & Yes & High \\
\hline 1 & $\begin{array}{l}\text { Jiang, 2013, } \\
24349366\end{array}$ & High & High & Low & $\mathrm{N} / \mathrm{A}$ & Low & Low & Low & Yes & Yes & Yes & High \\
\hline 1 & $\begin{array}{l}\text { Kouwenberg, } \\
2019,30270015\end{array}$ & High & High & $\mathrm{N} / \mathrm{A}$ & High & Low & Low & Low & Yes & Yes & Yes & $\begin{array}{l}\text { Modera } \\
\text { te }\end{array}$ \\
\hline 1 & $\begin{array}{l}\text { Kouwenberg, } \\
2020,32590633\end{array}$ & High & High & High & High & Unclear & Low & Low & Yes & Unclear & Yes & $\begin{array}{l}\text { Modera } \\
\text { te }\end{array}$ \\
\hline 1 & $\begin{array}{l}\text { Laporta, 2017, } \\
28061518\end{array}$ & High & High & Unclear & $\mathrm{N} / \mathrm{A}$ & Low & Low & Low & Yes & Yes & Yes & High \\
\hline 1 & $\begin{array}{l}\text { Lei, } 2020 \text {, } \\
32481367\end{array}$ & High & High & High & $\mathrm{N} / \mathrm{A}$ & Low & Low & Low & Yes & Yes & Yes & $\begin{array}{l}\text { Modera } \\
\text { te }\end{array}$ \\
\hline 1 & $\begin{array}{l}\text { Liu, 2014, } \\
24558063\end{array}$ & High & High & Unclear & $\mathrm{N} / \mathrm{A}$ & Low & Low & Low & Yes & Yes & Yes & High \\
\hline 1 & $\begin{array}{l}\text { Mak, 2020, } \\
32665188\end{array}$ & High & High & $\mathrm{N} / \mathrm{A}$ & Low & Low & Low & Low & No & Yes & Yes & $\begin{array}{l}\text { Modera } \\
\text { te }\end{array}$ \\
\hline
\end{tabular}




\begin{tabular}{|c|c|c|c|c|c|c|c|c|c|c|c|c|}
\hline $\mathbf{K Q}$ & $\begin{array}{l}\text { Study, Year, } \\
\text { PMID }\end{array}$ & $\begin{array}{l}\text { Blinding of } \\
\text { Participants }\end{array}$ & $\begin{array}{l}\text { Blinding of } \\
\text { Personnel/ } \\
\text { Care } \\
\text { Providers }\end{array}$ & $\begin{array}{l}\text { Blinding of } \\
\text { Outcome } \\
\text { Assessors } \\
\text { (Objective } \\
\text { Outcomes) }\end{array}$ & $\begin{array}{l}\text { Blinding of } \\
\text { Outcome } \\
\text { Assessors } \\
\text { (Subjective } \\
\text { Outcomes) }\end{array}$ & $\begin{array}{l}\text { Incomplete } \\
\text { Outcome } \\
\text { Data }\end{array}$ & $\begin{array}{l}\text { Selective } \\
\text { Outcome } \\
\text { Reporting }\end{array}$ & $\begin{array}{l}\text { Other } \\
\text { Bias }\end{array}$ & $\begin{array}{l}\text { Eligibility } \\
\text { Criteria } \\
\text { Prespecified } \\
\text { and Clearly } \\
\text { Described }\end{array}$ & $\begin{array}{l}\text { Intervention } \\
\text { Clearly } \\
\text { Described } \\
\text { and } \\
\text { Consistently } \\
\text { Delivered }\end{array}$ & $\begin{array}{l}\text { Outcomes } \\
\text { Prespecified, } \\
\text { Clearly } \\
\text { Defined, } \\
\text { Valid, } \\
\text { Reliable, and } \\
\text { Consistently } \\
\text { Assessed }\end{array}$ & $\begin{array}{l}\text { Overall } \\
\text { RoB }\end{array}$ \\
\hline 1 & $\begin{array}{l}\text { McCarthy, } \\
2014,24201740\end{array}$ & High & High & N/A & High & Low & Low & Low & Yes & Unclear & Yes & $\begin{array}{l}\text { Modera } \\
\text { te }\end{array}$ \\
\hline 1 & $\begin{array}{l}\text { Merchant, 2015, } \\
26111325\end{array}$ & High & High & Low & $\mathrm{N} / \mathrm{A}$ & Low & Low & $\begin{array}{l}\text { Uncle } \\
\text { ar }\end{array}$ & Yes & Yes & Yes & High \\
\hline 1 & $\begin{array}{l}\text { Mioton, 2013, } \\
23562485 \\
\end{array}$ & High & High & High & High & Low & Low & Low & Unclear & Unclear & Yes & $\begin{array}{l}\text { Modera } \\
\text { te }\end{array}$ \\
\hline 1 & $\begin{array}{l}\text { Momeni, 2018, } \\
29095189\end{array}$ & High & High & Low & $\mathrm{N} / \mathrm{A}$ & Low & Low & $\begin{array}{l}\text { Uncle } \\
\text { ar }\end{array}$ & Yes & Yes & Yes & High \\
\hline 1 & $\begin{array}{l}\text { Naoum, 2020a, } \\
31756414\end{array}$ & High & High & Low & N/A & Low & Low & Low & Yes & Yes & Yes & High \\
\hline 1 & $\begin{array}{l}\text { Naoum, 2020b, } \\
32607638\end{array}$ & High & High & $\mathrm{N} / \mathrm{A}$ & Low & Low & Low & Low & Yes & Yes & Yes & High \\
\hline 1 & $\begin{array}{l}\text { Nasser, 2018, } \\
30204678\end{array}$ & High & High & Low & $\mathrm{N} / \mathrm{A}$ & Low & Low & $\begin{array}{l}\text { Uncle } \\
\text { ar }\end{array}$ & Yes & Yes & Yes & High \\
\hline 1 & $\begin{array}{l}\text { Nelson, 2019, } \\
31356276\end{array}$ & High & High & $\mathrm{N} / \mathrm{A}$ & Unclear & Low & Low & High & Yes & Yes & Yes & High \\
\hline 1 & $\begin{array}{l}\text { Palve, 2020, } \\
32468337\end{array}$ & High & High & $\mathrm{N} / \mathrm{A}$ & High & Low & Low & Low & Yes & Yes & Yes & $\begin{array}{l}\text { Modera } \\
\text { te }\end{array}$ \\
\hline 1 & $\begin{array}{l}\text { Qin, 2018, } \\
29384865\end{array}$ & High & High & High & High & Low & High & Low & Yes & Unclear & No & High \\
\hline 1 & $\begin{array}{l}\text { Roth, 2007, } \\
17413877 \\
\end{array}$ & High & High & $\mathrm{N} / \mathrm{A}$ & Unclear & Low & Low & Low & Yes & Yes & Yes & High \\
\hline 1 & $\begin{array}{l}\text { Shiraishi, 2020, } \\
32589082\end{array}$ & High & High & Unclear & $\mathrm{N} / \mathrm{A}$ & Low & Low & Low & Yes & Yes & Yes & High \\
\hline 1 & $\begin{array}{l}\text { Simon, } 2020 \text {, } \\
33363007\end{array}$ & High & High & N/A & High & Low & Low & Low & Yes & Yes & Yes & $\begin{array}{l}\text { Modera } \\
\text { te }\end{array}$ \\
\hline 1 & $\begin{array}{l}\text { Wu, 2021, } \\
33740204\end{array}$ & High & High & N/A & Low & Unclear & Low & Low & Yes & Yes & Yes & High \\
\hline 1 & $\begin{array}{l}\text { Xu, 2018, } \\
30261115\end{array}$ & High & High & Unclear & N/A & Low & Low & Low & Yes & Yes & Yes & $\begin{array}{l}\text { Modera } \\
\text { te }\end{array}$ \\
\hline 1 & $\begin{array}{l}\text { Zhang, 2019, } \\
30675702\end{array}$ & High & High & Low & $\mathrm{N} / \mathrm{A}$ & Low & Low & Low & Yes & Yes & Yes & High \\
\hline 1,6 & $\begin{array}{l}\text { Abedi, 2016, } \\
25003437\end{array}$ & Unclear & Unclear & High & $\mathrm{N} / \mathrm{A}$ & Low & Low & Low & No & Yes & Yes & High \\
\hline 1,6 & $\begin{array}{l}\text { Carramaschi, } \\
1989,2602589\end{array}$ & Low & Low & Low & $\mathrm{N} / \mathrm{A}$ & Low & Low & Low & Yes & Yes & Unclear & High \\
\hline
\end{tabular}

D-7 


\begin{tabular}{|c|c|c|c|c|c|c|c|c|c|c|c|c|}
\hline $\mathbf{K Q}$ & $\begin{array}{l}\text { Study, Year, } \\
\text { PMID }\end{array}$ & $\begin{array}{l}\text { Blinding of } \\
\text { Participants }\end{array}$ & $\begin{array}{l}\text { Blinding of } \\
\text { Personnel/ } \\
\text { Care } \\
\text { Providers }\end{array}$ & $\begin{array}{l}\text { Blinding of } \\
\text { Outcome } \\
\text { Assessors } \\
\text { (Objective } \\
\text { Outcomes) }\end{array}$ & $\begin{array}{l}\text { Blinding of } \\
\text { Outcome } \\
\text { Assessors } \\
\text { (Subjective } \\
\text { Outcomes) }\end{array}$ & $\begin{array}{l}\text { Incomplete } \\
\text { Outcome } \\
\text { Data }\end{array}$ & $\begin{array}{l}\text { Selective } \\
\text { Outcome } \\
\text { Reporting }\end{array}$ & $\begin{array}{l}\text { Other } \\
\text { Bias }\end{array}$ & $\begin{array}{l}\text { Eligibility } \\
\text { Criteria } \\
\text { Prespecified } \\
\text { and Clearly } \\
\text { Described }\end{array}$ & $\begin{array}{l}\text { Intervention } \\
\text { Clearly } \\
\text { Described } \\
\text { and } \\
\text { Consistently } \\
\text { Delivered }\end{array}$ & $\begin{array}{l}\text { Outcomes } \\
\text { Prespecified, } \\
\text { Clearly } \\
\text { Defined, } \\
\text { Valid, } \\
\text { Reliable, and } \\
\text { Consistently } \\
\text { Assessed }\end{array}$ & $\begin{array}{l}\text { Overall } \\
\text { RoB }\end{array}$ \\
\hline 1,6 & $\begin{array}{l}\text { Dauplat, 2021, } \\
33622886\end{array}$ & High & High & N/A & High & Low & Low & Low & Yes & Yes & Yes & $\begin{array}{l}\text { Modera } \\
\text { te }\end{array}$ \\
\hline 1,6 & $\begin{array}{l}\text { Garbay, 1992, } \\
1624727\end{array}$ & High & High & $\mathrm{N} / \mathrm{A}$ & High & Low & Low & Low & No & No & Yes & High \\
\hline 1,6 & $\begin{array}{l}\text { Kulkarni, 2017, } \\
28713853\end{array}$ & High & High & High & High & Unclear & Low & Low & Yes & Yes & Yes & $\begin{array}{l}\text { Modera } \\
\text { te }\end{array}$ \\
\hline 1,6 & $\begin{array}{l}\text { Woo, 2018, } \\
30360958\end{array}$ & High & High & Unclear & $\mathrm{N} / \mathrm{A}$ & Low & Low & Low & Yes & Yes & Yes & High \\
\hline 1,6 & $\begin{array}{l}\text { Yueh, 2009, } \\
19228537\end{array}$ & High & High & N/A & Unclear & Low & Low & Low & Yes & Yes & Yes & High \\
\hline 2 & $\begin{array}{l}\text { Eriksson, 2013, } \\
24258257\end{array}$ & High & High & Low & Low & Low & Low & Low & Yes & No & Yes & High \\
\hline 2 & $\begin{array}{l}\text { Yoon, 2020, } \\
32332528\end{array}$ & High & High & High & High & Low & Low & Low & Yes & Yes & Unclear & $\begin{array}{l}\text { Modera } \\
\text { te }\end{array}$ \\
\hline 2,3 & $\begin{array}{l}\text { Cordeiro, } \\
2015 a, \\
25742523 \\
\end{array}$ & High & High & High & $\mathrm{N} / \mathrm{A}$ & Low & Low & Low & Yes & Yes & Yes & High \\
\hline 2,5 & $\begin{array}{l}\text { Hirsch, 2014, } \\
25347643\end{array}$ & High & High & Low & $\mathrm{N} / \mathrm{A}$ & Low & Low & Low & Yes & Yes & Yes & High \\
\hline 2,5 & $\begin{array}{l}\text { Stein, 2020, } \\
32561384\end{array}$ & High & High & N/A & Low & Low & Low & Low & Yes & Yes & Yes & High \\
\hline 3 & $\begin{array}{l}\text { Antony, } 2014, \\
24135689\end{array}$ & High & High & Low & $\mathrm{N} / \mathrm{A}$ & Low & Low & Low & Yes & Yes & Yes & High \\
\hline 3 & $\begin{array}{l}\text { Le, } 2005, \\
15743498\end{array}$ & High & High & Low & $\mathrm{N} / \mathrm{A}$ & Low & Low & $\begin{array}{l}\text { Uncle } \\
\text { ar }\end{array}$ & Yes & Yes & Yes & High \\
\hline 3 & $\begin{array}{l}\text { Macadam, } \\
2010,20009795\end{array}$ & High & High & $\mathrm{N} / \mathrm{A}$ & Unclear & Low & Low & Low & Yes & Yes & Yes & High \\
\hline 3 & $\begin{array}{l}\text { McCarthy, } \\
2010,21136577\end{array}$ & High & High & $\mathrm{N} / \mathrm{A}$ & High & Low & Low & Low & Unclear & Unclear & Yes & High \\
\hline 4 & $\begin{array}{l}\text { Avila, 2020, } \\
33234947\end{array}$ & High & High & Unclear & Low & Low & Low & Low & Yes & Yes & Yes & High \\
\hline 4 & $\begin{array}{l}\text { Gabriel, 2020, } \\
32195862\end{array}$ & High & High & $\mathrm{N} / \mathrm{A}$ & High & Low & Low & Low & Yes & Yes & Yes & High \\
\hline 4 & $\begin{array}{l}\text { Kim, 2020, } \\
33066236\end{array}$ & High & High & High & $\mathrm{N} / \mathrm{A}$ & Low & Low & Low & Yes & Yes & Yes & $\begin{array}{l}\text { Modera } \\
\text { te }\end{array}$ \\
\hline
\end{tabular}




\begin{tabular}{|c|c|c|c|c|c|c|c|c|c|c|c|c|}
\hline $\mathbf{K Q}$ & $\begin{array}{l}\text { Study, Year, } \\
\text { PMID }\end{array}$ & $\begin{array}{l}\text { Blinding of } \\
\text { Participants }\end{array}$ & $\begin{array}{l}\text { Blinding of } \\
\text { Personnel/ } \\
\text { Care } \\
\text { Providers }\end{array}$ & $\begin{array}{l}\text { Blinding of } \\
\text { Outcome } \\
\text { Assessors } \\
\text { (Objective } \\
\text { Outcomes) }\end{array}$ & $\begin{array}{l}\text { Blinding of } \\
\text { Outcome } \\
\text { Assessors } \\
\text { (Subjective } \\
\text { Outcomes) }\end{array}$ & $\begin{array}{l}\text { Incomplete } \\
\text { Outcome } \\
\text { Data }\end{array}$ & $\begin{array}{l}\text { Selective } \\
\text { Outcome } \\
\text { Reporting }\end{array}$ & $\begin{array}{l}\text { Other } \\
\text { Bias }\end{array}$ & $\begin{array}{l}\text { Eligibility } \\
\text { Criteria } \\
\text { Prespecified } \\
\text { and Clearly } \\
\text { Described }\end{array}$ & $\begin{array}{l}\text { Intervention } \\
\text { Clearly } \\
\text { Described } \\
\text { and } \\
\text { Consistently } \\
\text { Delivered }\end{array}$ & $\begin{array}{l}\text { Outcomes } \\
\text { Prespecified, } \\
\text { Clearly } \\
\text { Defined, } \\
\text { Valid, } \\
\text { Reliable, and } \\
\text { Consistently } \\
\text { Assessed }\end{array}$ & $\begin{array}{l}\text { Overall } \\
\text { RoB }\end{array}$ \\
\hline 4 & $\begin{array}{l}\text { Kraenzlin, 2021, } \\
32568752\end{array}$ & High & High & High & High & Low & High & Low & Yes & Yes & Yes & High \\
\hline 4 & $\begin{array}{l}\text { Nealon, 2020a, } \\
32032345\end{array}$ & High & High & Low & $\mathrm{N} / \mathrm{A}$ & Low & Low & Low & Yes & Yes & Yes & High \\
\hline 4 & $\begin{array}{l}\text { Ozgur, 2020, } \\
33223365\end{array}$ & High & High & $\mathrm{N} / \mathrm{A}$ & Low & Low & Low & Low & Yes & Yes & Yes & High \\
\hline 4,5 & $\begin{array}{l}\text { Cattelani, 2018, } \\
29275104\end{array}$ & High & High & Unclear & Unclear & Low & Low & Low & Yes & Yes & Yes & High \\
\hline 5 & $\begin{array}{l}\text { Brooke, 2012, } \\
22868313\end{array}$ & High & High & High & N/A & High & Low & Low & Yes & Yes & Yes & High \\
\hline 5 & $\begin{array}{l}\text { Chun, 2010, } \\
20124828\end{array}$ & High & High & Low & $\mathrm{N} / \mathrm{A}$ & Low & Low & Low & Yes & Yes & Yes & High \\
\hline 5 & $\begin{array}{l}\text { Craig, 2019, } \\
29800083\end{array}$ & Low & Low & Low & Low & Unclear & Low & Low & Yes & Unclear & Yes & Low \\
\hline 5 & $\begin{array}{l}\text { Ibrahim, 2013, } \\
24165587\end{array}$ & High & High & High & High & Low & Low & Low & Yes & Unclear & Yes & $\begin{array}{l}\text { Modera } \\
\text { te }\end{array}$ \\
\hline 5 & $\begin{array}{l}\text { Ganesh Kumar, } \\
2021,33172826\end{array}$ & High & High & High & High & Low & Low & Low & Yes & Unclear & Yes & $\begin{array}{l}\text { Modera } \\
\text { te }\end{array}$ \\
\hline 5 & $\begin{array}{l}\text { Lee, 2020, No } \\
\text { PMID }\end{array}$ & High & High & N/A & Low & Low & Low & Low & Yes & Yes & Yes & High \\
\hline 5 & $\begin{array}{l}\text { Liu, 2011, } \\
21228744 \\
\end{array}$ & High & High & Low & $\mathrm{N} / \mathrm{A}$ & Low & Low & Low & Yes & Yes & Yes & High \\
\hline 5 & $\begin{array}{l}\text { Nealon, 2020b, } \\
31605310\end{array}$ & High & High & Low & $\mathrm{N} / \mathrm{A}$ & Low & Low & Low & Yes & Yes & Yes & High \\
\hline 5 & $\begin{array}{l}\text { Pannucci, 2013, } \\
23508050\end{array}$ & High & High & Low & $\mathrm{N} / \mathrm{A}$ & Low & Low & $\begin{array}{l}\text { Uncle } \\
\text { ar }\end{array}$ & Yes & Yes & Yes & $\begin{array}{l}\text { Modera } \\
\text { te }\end{array}$ \\
\hline 5 & $\begin{array}{l}\text { Peled, 2012, } \\
22634688\end{array}$ & High & High & Low & $\mathrm{N} / \mathrm{A}$ & Low & Low & Low & Yes & Yes & Yes & High \\
\hline 5 & $\begin{array}{l}\text { Qureshi, 2016, } \\
27465177\end{array}$ & High & High & Unclear & N/A & Low & Low & Low & Yes & Yes & Yes & High \\
\hline 5 & $\begin{array}{l}\text { Safran, 2020, } \\
32221195\end{array}$ & High & High & Low & $\mathrm{N} / \mathrm{A}$ & Low & Low & Low & Yes & Yes & Yes & High \\
\hline 5 & $\begin{array}{l}\text { Seth, 2012, } \\
23018687\end{array}$ & High & High & Unclear & $\mathrm{N} / \mathrm{A}$ & Low & Low & Low & Yes & Yes & Yes & High \\
\hline 5 & $\begin{array}{l}\text { Sobti, 2018, } \\
29481386 \\
\end{array}$ & Low & High & Unclear & $\mathrm{N} / \mathrm{A}$ & Low & Low & Low & Yes & Yes & Yes & High \\
\hline
\end{tabular}




\begin{tabular}{|c|c|c|c|c|c|c|c|c|c|c|c|c|}
\hline KQ & $\begin{array}{l}\text { Study, Year, } \\
\text { PMID }\end{array}$ & $\begin{array}{l}\text { Blinding of } \\
\text { Participants }\end{array}$ & $\begin{array}{l}\text { Blinding of } \\
\text { Personnel/ } \\
\text { Care } \\
\text { Providers }\end{array}$ & $\begin{array}{l}\text { Blinding of } \\
\text { Outcome } \\
\text { Assessors } \\
\text { (Objective } \\
\text { Outcomes) }\end{array}$ & $\begin{array}{l}\text { Blinding of } \\
\text { Outcome } \\
\text { Assessors } \\
\text { (Subjective } \\
\text { Outcomes) }\end{array}$ & $\begin{array}{l}\text { Incomplete } \\
\text { Outcome } \\
\text { Data }\end{array}$ & $\begin{array}{l}\text { Selective } \\
\text { Outcome } \\
\text { Reporting }\end{array}$ & $\begin{array}{l}\text { Other } \\
\text { Bias }\end{array}$ & $\begin{array}{l}\text { Eligibility } \\
\text { Criteria } \\
\text { Prespecified } \\
\text { and Clearly } \\
\text { Described }\end{array}$ & $\begin{array}{l}\text { Intervention } \\
\text { Clearly } \\
\text { Described } \\
\text { and } \\
\text { Consistently } \\
\text { Delivered }\end{array}$ & $\begin{array}{l}\text { Outcomes } \\
\text { Prespecified, } \\
\text { Clearly } \\
\text { Defined, } \\
\text { Valid, } \\
\text { Reliable, and } \\
\text { Consistently } \\
\text { Assessed }\end{array}$ & $\begin{array}{l}\text { Overall } \\
\text { RoB }\end{array}$ \\
\hline 5 & $\begin{array}{l}\text { Vardanian, } \\
2011,22030500\end{array}$ & High & High & Low & $\mathrm{N} / \mathrm{A}$ & Low & Low & Low & Yes & Yes & Yes & High \\
\hline 5 & $\begin{array}{l}\text { Weichman, } \\
2012,22544088\end{array}$ & High & High & Unclear & $\mathrm{N} / \mathrm{A}$ & Low & Low & Low & Yes & Yes & Unclear & $\begin{array}{l}\text { Modera } \\
\text { te }\end{array}$ \\
\hline 5 & $\begin{array}{l}\text { Woo, 2017, } \\
28509694\end{array}$ & High & High & Low & $\mathrm{N} / \mathrm{A}$ & Low & Low & Low & Yes & Yes & Yes & High \\
\hline 6 & $\begin{array}{l}\text { Baumann, } \\
2010,20440154\end{array}$ & High & High & Unclear & $\mathrm{N} / \mathrm{A}$ & Low & Low & Low & Yes & Yes & Yes & High \\
\hline 6 & $\begin{array}{l}\text { Erdmann- } \\
\text { Sager, } 2018, \\
29019862\end{array}$ & High & High & High & High & Low & Low & Low & Yes & Unclear & Yes & $\begin{array}{l}\text { Modera } \\
\text { te }\end{array}$ \\
\hline 6 & $\begin{array}{l}\text { Israeli, 2014, } \\
24572840\end{array}$ & High & High & Unclear & $\mathrm{N} / \mathrm{A}$ & Low & Low & Low & Yes & Yes & Yes & High \\
\hline 6 & $\begin{array}{l}\text { Knox, 2016, } \\
26267400\end{array}$ & High & High & Unclear & $\mathrm{N} / \mathrm{A}$ & Low & Low & Low & Yes & Yes & Yes & High \\
\hline 6 & $\begin{array}{l}\text { Kroll, 2000, } \\
10987463\end{array}$ & High & High & $\mathrm{N} / \mathrm{A}$ & High & Low & Low & Low & Yes & Yes & Yes & High \\
\hline 6 & $\begin{array}{l}\text { Macadam, } \\
2016,26910656\end{array}$ & High & High & Low & Unclear & Low & Low & $\begin{array}{l}\text { Uncle } \\
\text { ar }\end{array}$ & Yes & Yes & Yes & High \\
\hline 6 & $\begin{array}{l}\text { Massenburg, } \\
2015,26487657\end{array}$ & High & High & High & High & Low & Unclear & Low & No & Unclear & Yes & High \\
\hline 6 & $\begin{array}{l}\text { Mennie, 2015, } \\
25839173\end{array}$ & High & High & Low & $\mathrm{N} / \mathrm{A}$ & Low & Low & Low & Yes & Yes & Yes & High \\
\hline 6 & $\begin{array}{l}\text { Zhong, 2014, } \\
24675183\end{array}$ & High & High & Low & N/A & Low & Low & $\begin{array}{l}\text { Uncle } \\
\text { ar }\end{array}$ & Yes & Yes & Yes & High \\
\hline 6 & $\begin{array}{l}\text { Zoghbi, 2017, } \\
28052051\end{array}$ & High & High & High & High & Unclear & Low & Low & Yes & Unclear & Yes & High \\
\hline
\end{tabular}

Abbreviations: $\mathrm{KQ}=$ Key Question, N/A = not applicable, $\mathrm{NRCS}=$ nonrandomized comparative study, PMID = PubMed identifier.

Ratings are color coded for emphasis only. The colors do not impart unique information.

From the Cochrane Risk of Bias Tool (each item rated as Low, High, Unclear, or N/A)

- Blinding of participants (performance bias): Performance bias due to knowledge of the allocated interventions by participants during the study.

- Blinding of personnel/care providers (performance bias): Performance bias due to knowledge of the allocated interventions by personnel/care providers during the study.

- Blinding of outcome assessor (detection bias): Detection bias due to knowledge of the allocated interventions by outcome assessors during the study.

- Incomplete outcome data (attrition bias): Attrition bias due to amount, nature or handling of incomplete outcome data.

- Selective outcome reporting (outcome reporting bias): Bias arising from outcomes being selectively reported based on the direction and/or strength of the results.

- Other BiaStages: Bias due to problems not covered elsewhere in the table. 
From the National Heart, Lung, and Blood Institute (NHLBI) Quality Assessment Tool (each item rated as Yes, No, Unclear, or No Data)

- Eligibility criteria prespecified and clearly described: potentially related to selection bias.

- Intervention clearly described and delivered consistently: potentially related to performance bias.

- Outcomes prespecified, clearly defined, valid, reliable, and assessed consistently: potentially related to detection bias.

Overall risk of bias assessed as HIGH, MODERATE, or LOW. 
Table D-4. Risk of bias and quality assessment for all Key Questions - single group studies

\begin{tabular}{|c|c|c|c|c|c|c|c|c|}
\hline $\mathbf{K Q}$ & Study, Year, PMID & $\begin{array}{l}\text { Incomplete } \\
\text { Outcome } \\
\text { Data }\end{array}$ & $\begin{array}{l}\text { Selective } \\
\text { Outcome } \\
\text { Reporting }\end{array}$ & $\begin{array}{l}\text { Other } \\
\text { Bias }\end{array}$ & $\begin{array}{l}\text { Eligibility } \\
\text { Criteria } \\
\text { Prespecified } \\
\text { and Clearly } \\
\text { Described }\end{array}$ & $\begin{array}{l}\text { Intervention } \\
\text { Clearly } \\
\text { Described and } \\
\text { Consistently } \\
\text { Delivered } \\
\end{array}$ & $\begin{array}{l}\text { Outcomes } \\
\text { Prespecified, Clearly } \\
\text { Defined, Valid, Reliable, } \\
\text { and Consistently } \\
\text { Assessed }\end{array}$ & $\begin{array}{l}\text { Overall } \\
\text { RoB }\end{array}$ \\
\hline 1 & Acosta, 2011, 21046538 & Low & High & Low & No & Yes & No & High \\
\hline 1 & Albornoz, 2013, 23897346 & Low & Low & Low & Yes & Yes & Yes & Low \\
\hline 1 & Andree, 2012,23197233 & Low & Low & Low & Yes & Yes & Yes & Low \\
\hline 1 & Banuelos, 2020, 31663932 & Low & Low & Low & Yes & Yes & Yes & Low \\
\hline 1 & Beugels, 2018, 29399731 & Low & Low & Low & Yes & Yes & Yes & Low \\
\hline 1 & Brooks, 2012, 22098451 & Low & Low & Low & Yes & Yes & Yes & Low \\
\hline 1 & Chang, 2000, 10809092 & Low & Low & Low & Yes & Yes & Yes & Low \\
\hline 1 & Chang, 2011, 21407063 & Low & Low & Low & Yes & Yes & Yes & Low \\
\hline 1 & Chang, 2016, 25003429 & Low & Low & Low & Yes & Yes & Yes & Low \\
\hline 1 & Chen, 2014,25620484 & Low & Low & Low & Yes & Yes & Yes & Low \\
\hline 1 & Chen, 2016, 27930584 & Low & Low & Low & Yes & Yes & Yes & Low \\
\hline 1 & Chen, 2018a, 29596085 & Low & Low & Low & Yes & Yes & Yes & Low \\
\hline 1 & Chen, 2018b, 29596085 & Low & Low & Low & Yes & Yes & Yes & Low \\
\hline 1 & Cleveland, 2013, 23945529 & Low & Low & Low & Yes & Yes & Yes & Low \\
\hline 1 & Collier, 2019, 31461001 & Low & Low & Unclear & Yes & Yes & Yes & Moderate \\
\hline 1 & Cordeiro, 2006, 16980842 & Unclear & Low & Low & Yes & Yes & Yes & Moderate \\
\hline 1 & Cordeiro, 2012, 22286416 & Low & Low & Low & Yes & Yes & Yes & Low \\
\hline 1 & Cordeiro, 2015b, 26090764 & Low & Low & Low & Yes & Yes & Yes & Low \\
\hline 1 & Coroneos, 2019, 30222598 & Low & Low & Low & Yes & Yes & Yes & Low \\
\hline 1 & Daly, 2020, 31994156 & Low & Low & Low & Yes & Yes & Yes & Low \\
\hline 1 & Enajat, 2010, 19790180 & Low & Low & Low & Yes & Yes & Yes & Low \\
\hline 1 & Fitzgerald, 2016, 27047776 & Low & Low & Low & Yes & Yes & Yes & Low \\
\hline 1 & Gfrerer, 2015, 25626807 & Low & Low & Low & Yes & Yes & Yes & Low \\
\hline 1 & Gill, 2004, 15083015 & Low & Low & Unclear & Yes & Unclear & Yes & Moderate \\
\hline 1 & Haddock, 2019, 31461004 & Low & Low & Low & No & Yes & Yes & Moderate \\
\hline 1 & Haddock, 2020, 33487570 & Low & Low & Low & Yes & Yes & Yes & Low \\
\hline 1 & Hamdi, 2010, 20679823 & Low & Low & Low & Yes & Yes & Yes & Low \\
\hline 1 & Hamdi, 2011, 20576480 & Low & Low & Low & Yes & Yes & Yes & Low \\
\hline 1 & Hansen, 2018, 29778821 & Low & Low & Low & Yes & Yes & Yes & Low \\
\hline 1 & Heo, 2018, 30039735 & Low & Low & Low & Yes & Yes & Yes & Low \\
\hline 1 & Hunsicker, 2017, 26849284 & Low & Low & Low & Yes & Yes & Yes & Low \\
\hline 1 & Huo, 2016, 27697676 & Low & Low & Low & Yes & Yes & Yes & Low \\
\hline 1 & Jo, 2020, 33386262 & Low & Low & Low & Yes & Yes & Yes & Low \\
\hline 1 & Kanuri, 2014, 24675199 & Low & Low & Low & Yes & Yes & Yes & Low \\
\hline 1 & Kato, 2013,24011080 & Low & Low & Low & Yes & Yes & Yes & Low \\
\hline 1 & Langer, 2010, 20980954 & Low & Low & Low & Yes & Yes & Yes & Low \\
\hline 1 & Lantieri, 2015,26238173 & Low & Low & Low & Unclear & Unclear & No & Moderate \\
\hline
\end{tabular}

D-12 


\begin{tabular}{|c|c|c|c|c|c|c|c|c|}
\hline KQ & Study, Year, PMID & $\begin{array}{l}\text { Incomplete } \\
\text { Outcome } \\
\text { Data }\end{array}$ & $\begin{array}{l}\text { Selective } \\
\text { Outcome } \\
\text { Reporting }\end{array}$ & $\begin{array}{l}\text { Other } \\
\text { Bias }\end{array}$ & \begin{tabular}{|l|} 
Eligibility \\
Criteria \\
Prespecified \\
and Clearly \\
Described \\
\end{tabular} & $\begin{array}{l}\text { Intervention } \\
\text { Clearly } \\
\text { Described and } \\
\text { Consistently } \\
\text { Delivered } \\
\end{array}$ & $\begin{array}{l}\text { Outcomes } \\
\text { Prespecified, Clearly } \\
\text { Defined, Valid, Reliable, } \\
\text { and Consistently } \\
\text { Assessed }\end{array}$ & $\begin{array}{l}\text { Overall } \\
\text { RoB }\end{array}$ \\
\hline 1 & Law, 2018, 30463754 & Low & Low & Low & Yes & Yes & Yes & Low \\
\hline 1 & Lee, 2021a, 32974692 & Low & Low & Low & Yes & Yes & Yes & Low \\
\hline 1 & Liao, 2008,18349626 & Low & Low & Low & Yes & Yes & Yes & Low \\
\hline 1 & Lovecchio, 2015, 24691330 & Low & Low & Low & Yes & Yes & Yes & Low \\
\hline 1 & Masoomi, 2019, 31331721 & Low & Low & Low & Yes & Yes & Yes & Low \\
\hline 1 & Mehrara, 2006, 17016173 & Low & Low & Low & Unclear & No & Yes & Moderate \\
\hline 1 & Mirzabeigi, 2015, 25811579 & Low & Low & Low & Yes & Yes & Yes & Low \\
\hline 1 & Munder, 2021, 32565553 & Low & Low & Low & Yes & Yes & Yes & Low \\
\hline 1 & Nelson, 2014, 25046665 & Low & Unclear & Low & Yes & Unclear & Yes & Moderate \\
\hline 1 & O'Neill, 2019, 31196805 & Low & Low & Low & Yes & Yes & Yes & Low \\
\hline 1 & Parikh, 2018, 30204676 & Low & Low & Low & Yes & Yes & Yes & Low \\
\hline 1 & Park, 2019, 30863940 & Low & Low & Low & Yes & Yes & Unclear & Moderate \\
\hline 1 & Phan, 2020, 31124177 & Low & Low & Low & Yes & Yes & Yes & Low \\
\hline 1 & Polanco, 2021, 33745850 & Low & Low & Low & Yes & Yes & Yes & Low \\
\hline 1 & Potter, 2019, 30639093 & Low & Low & Low & Yes & No & Yes & Moderate \\
\hline 1 & Prantl, 2020, 32895743 & Low & Low & Low & Yes & Yes & Yes & Low \\
\hline 1 & Rogoff, 2020, 32243320 & Low & Low & Low & Yes & Yes & Yes & Low \\
\hline 1 & Rubio, 2019, 30665841 & Low & Low & Low & Yes & Yes & Yes & Low \\
\hline 1 & Salibian, 2019, 31333984 & Low & Low & Low & Yes & Yes & Yes & Low \\
\hline 1 & Seidenstuecker, 2016, 27017243 & Unclear & Unclear & Low & Yes & Yes & Yes & Moderate \\
\hline 1 & Selber, 2009, 19935283 & Low & Low & Low & Yes & Yes & Yes & Low \\
\hline 1 & Seth, 2015,25180955 & Low & Low & Low & Yes & Yes & Yes & Low \\
\hline 1 & Sewart, 2021, 33609398 & Low & Low & Low & Yes & Yes & Yes & Low \\
\hline 1 & Shaikh, 2010, 22693373 & Low & Low & Low & Yes & Yes & Yes & Low \\
\hline 1 & Singh, 2012, 22342636 & Low & Low & Low & Yes & Yes & Yes & Low \\
\hline 1 & Singh, 2021, 33564597 & Low & Low & Low & Yes & Yes & Yes & Low \\
\hline 1 & Song, 2016,26637165 & Low & Low & Low & Yes & Yes & Yes & Low \\
\hline 1 & Tran, 2018, 29794694 & Low & Low & Low & Yes & Yes & Yes & Low \\
\hline 1 & Watterson, 1995, 7761505 & Low & Low & Low & No & Yes & Yes & Moderate \\
\hline 1 & Warren, 2020, 33040748 & Low & Low & Low & Yes & No & Yes & Moderate \\
\hline 1 & Williams, 1995, 7794079 & Low & Low & Low & No & Yes & Yes & Moderate \\
\hline 1 & Yoo, 2014, 24852813 & Low & Low & Low & Unclear & Unclear & Yes & Moderate \\
\hline
\end{tabular}

Abbreviations: KQ = Key Question, PMID = PubMed identifier, RoB = risk of bias.

Ratings are color coded for emphasis only.

From the Cochrane Risk of Bias Tool (each item rated as Low, High, Unclear, N/A)

- Incomplete outcome data (attrition bias): Attrition bias due to amount, nature or handling of incomplete outcome data.

- Selective outcome reporting (outcome reporting bias): Bias arising from outcomes being selectively reported based on the direction and/or strength of the results.

- Other BiaStages: Bias due to problems not covered elsewhere in the table. 
From the National Heart, Lung, and Blood Institute (NHLBI) Quality Assessment Tool (each item rated as Yes, No, Unclear, or No Data)

- Eligibility criteria prespecified and clearly described: potentially related to selection bias.

- Intervention clearly described and delivered consistently: potentially related to performance bias.

- Outcomes prespecified, clearly defined, valid, reliable, and assessed consistently: potentially related to detection bias.

Overall risk of bias assessed as HIGH, MODERATE, LOW. 


\section{Appendix E. Results: Summary Tables for Outcomes}

Table E-1.1. Summary Table - Key Question 1: IBR versus AR - continuous outcomes (general quality of life, psychosocial well-being, sexual well-being, patient satisfaction with breasts, and patient satisfaction with outcome)

\begin{tabular}{|c|c|c|c|c|c|c|c|c|c|c|c|c|}
\hline $\begin{array}{l}\text { Study, Year, } \\
\text { PMID, } \\
\text { Country }\end{array}$ & $\begin{array}{l}\text { Desig } \\
\mathrm{n}\end{array}$ & $\begin{array}{l}\text { Overall } \\
\text { RoB }\end{array}$ & Outcome & $\begin{array}{l}\text { Outcome } \\
\text { Measurement }\end{array}$ & \begin{tabular}{|l|} 
Time \\
Point
\end{tabular} & $\begin{array}{l}\text { Subgro } \\
\text { up }\end{array}$ & $\begin{array}{l}\text { IBR, } \\
\text { N }\end{array}$ & $\begin{array}{l}\text { IBR, Mean } \\
\text { (SD) }\end{array}$ & $\begin{array}{l}\text { AR } \\
, N\end{array}$ & $\begin{array}{l}\text { AR, Mean } \\
\text { (SD) }\end{array}$ & $\begin{array}{l}\text { AR Versus IBR, } \\
\text { Effect Size }(95 \% \mathrm{Cl})\end{array}$ & $\begin{array}{l}P \\
\text { Value }\end{array}$ \\
\hline $\begin{array}{l}\text { Kouwenberg, } \\
2019, \\
30270015, \\
\text { Netherlands }\end{array}$ & NRCS & Moderate & General QoL & $\begin{array}{l}\text { EQ-5D-5L utilities } \\
\text { score }(0-1)\end{array}$ & NR & All & 67 & $0.85(0.18)$ & 67 & $0.87(0.14)$ & NR & 0.7 \\
\hline \multirow{3}{*}{$\begin{array}{l}\text { Kouwenberg, } \\
2020, \\
32590633, \\
\text { Netherlands }\end{array}$} & NRCS & Moderate & General QoL & $\begin{array}{l}\text { EQ-5D-5L utilities } \\
\text { score }(0-1)\end{array}$ & $\begin{array}{l}>6 \\
\mathrm{mo}\end{array}$ & All & 296 & $0.85(0.30)$ & 179 & $0.85(0.20)$ & NR & NS \\
\hline & NRCS & Moderate & General QoL & $\begin{array}{l}\text { EQ-5D-5L VAS (0- } \\
100)\end{array}$ & $\begin{array}{l}>6 \\
\mathrm{mo}\end{array}$ & All & 296 & $77.6(18.4)$ & 179 & $79.2(16.7)$ & NR & NS \\
\hline & NRCS & Moderate & General QoL & $\begin{array}{l}\text { EORTC QLQC30: } \\
\text { Global health status } \\
(0-100)\end{array}$ & $\begin{array}{l}>6 \\
\text { mo }\end{array}$ & All & 296 & $80.2(18.4)$ & 179 & $81.4(14.7)$ & NR & NS \\
\hline \multirow{5}{*}{$\begin{array}{l}\text { Roth, 2007, } \\
17413877, \\
\text { US }\end{array}$} & NRCS & High & General QoL & $\begin{array}{l}\text { FACT-B: Functional } \\
\text { well-being }(0-28)\end{array}$ & $2 y$ & All & 35 & $23.3(\mathrm{NR})$ & 55 & $24.1(\mathrm{NR})$ & NR & NS \\
\hline & NRCS & High & General QoL & $\begin{array}{l}\text { SF-36: Role emotional } \\
(0-100)\end{array}$ & $2 y$ & All & 35 & 86.7 (NR) & 55 & 86.1 (NR) & NR & NS \\
\hline & NRCS & High & General QoL & SF-36: Vitality (0-100) & $2 y$ & All & 35 & 65.7 (NR) & 55 & $62.4(\mathrm{NR})$ & NR & NS \\
\hline & NRCS & High & General QoL & $\begin{array}{l}\text { SF-36: General mental } \\
\text { health }(0-100)\end{array}$ & $2 y$ & All & 35 & 77.6 (NR) & 55 & 77 (NR) & NR & NS \\
\hline & NRCS & High & General QoL & Body Image (9-45) & $2 y$ & All & 35 & $32.5(\mathrm{NR})$ & 55 & $35.3(\mathrm{NR})$ & NR & NS \\
\hline $\begin{array}{l}\text { Tallroth, } \\
2020, \\
33436336, \\
\text { Sweden }\end{array}$ & RCT & Moderate & $\begin{array}{l}\text { Psychosocial } \\
\text { WB }\end{array}$ & $\begin{array}{l}\text { BREAST-Q: } \\
\text { Psychosocial WB (0- } \\
100)\end{array}$ & $5.3 \mathrm{y}$ & All & 28 & $78.8(20.1)$ & 42 & $79.1(21.5)$ & MD $0.3(-6.7,7.3)$ & 0.93 \\
\hline \multirow[t]{2}{*}{$\begin{array}{l}\text { Eltahir, 2015, } \\
\text { 25539295, } \\
\text { Netherlands }\end{array}$} & NRCS & Moderate & $\begin{array}{l}\text { Psychosocial } \\
\text { WB }\end{array}$ & $\begin{array}{l}\text { BREAST-Q: } \\
\text { Psychosocial WB (0- } \\
\text { 100) }\end{array}$ & $2.2 \mathrm{y}$ & All & 45 & $77.2(18.1)$ & 47 & $74.0(17.8)$ & $\begin{array}{l}\text { adjMD } 4.6(-2.8, \\
12.0)\end{array}$ & 0.22 \\
\hline & NRCS & Moderate & $\begin{array}{l}\text { Psychosocial } \\
\text { WB }\end{array}$ & $\begin{array}{l}\text { SF-36: Social } \\
\text { functioning }(0-100)\end{array}$ & $2.2 \mathrm{y}$ & All & NR & NR & NR & NR & $\begin{array}{l}\text { adjMD -1.21 (-8.44, } \\
6.02)\end{array}$ & 0.74 \\
\hline
\end{tabular}




\begin{tabular}{|c|c|c|c|c|c|c|c|c|c|c|c|c|}
\hline $\begin{array}{l}\text { Study, Year, } \\
\text { PMID, } \\
\text { Country }\end{array}$ & $\begin{array}{l}\text { Desig } \\
\mathrm{n}\end{array}$ & $\begin{array}{l}\text { Overall } \\
\text { RoB }\end{array}$ & Outcome & $\begin{array}{l}\text { Outcome } \\
\text { Measurement }\end{array}$ & $\begin{array}{l}\text { Time } \\
\text { Point }\end{array}$ & $\begin{array}{l}\text { Subgro } \\
\text { up }\end{array}$ & $\begin{array}{l}\text { IBR, } \\
\text { N }\end{array}$ & $\begin{array}{l}\text { IBR, Mean } \\
\text { (SD) }\end{array}$ & $\begin{array}{l}\text { AR } \\
, N\end{array}$ & $\begin{array}{l}\text { AR, Mean } \\
\text { (SD) }\end{array}$ & $\begin{array}{l}\text { AR Versus IBR, } \\
\text { Effect Size }(95 \% \mathrm{CI})\end{array}$ & $\begin{array}{l}\mathrm{P} \\
\text { Value }\end{array}$ \\
\hline \multirow{5}{*}{$\begin{array}{l}\text { Kouwenberg, } \\
2020 \text {, } \\
32590633 \text {, } \\
\text { Netherlands }\end{array}$} & NRCS & Moderate & $\begin{array}{l}\text { Psychosocial } \\
\text { WB }\end{array}$ & $\begin{array}{l}\text { BREAST-Q: } \\
\text { Psychosocial WB (0- } \\
100)\end{array}$ & $\begin{array}{l}>6 \\
\mathrm{mo}\end{array}$ & All & 296 & $71.6(20.2)$ & 179 & $75.8(19.5)$ & NR & $<0.05$ \\
\hline & NRCS & Moderate & $\begin{array}{l}\text { Psychosocial } \\
\text { WB }\end{array}$ & $\begin{array}{l}\text { EORTC QLQC30: } \\
\text { Social function }(0-100)\end{array}$ & $\begin{array}{l}>6 \\
\mathrm{mo}\end{array}$ & All & 296 & $87.5(27.2)$ & 179 & $88.0(17.1)$ & NR & NS \\
\hline & NRCS & Moderate & $\begin{array}{l}\text { Psychosocial } \\
\text { WB }\end{array}$ & $\begin{array}{l}\text { EORTC QLQC30: } \\
\text { Emotional function (0- } \\
100 \text { ) }\end{array}$ & $\begin{array}{l}>6 \\
\mathrm{mo}\end{array}$ & All & 296 & $85.0(23.3)$ & 179 & $87.0(17.1)$ & NR & NS \\
\hline & NRCS & Moderate & $\begin{array}{l}\text { Psychosocial } \\
\text { WB }\end{array}$ & $\begin{array}{l}\text { EORTC QLQC30: } \\
\text { Cognitive function (0- } \\
100 \text { ) }\end{array}$ & $\begin{array}{l}>6 \\
\mathrm{mo}\end{array}$ & All & 296 & $85.0(23.7)$ & 179 & $83.7(21.5)$ & NR & NS \\
\hline & NRCS & Moderate & $\begin{array}{l}\text { Psychosocial } \\
\text { WB }\end{array}$ & $\begin{array}{l}\text { EORTC QLQC30: } \\
\text { Role function }(0-100)\end{array}$ & $\begin{array}{l}>6 \\
\mathrm{mo}\end{array}$ & All & 296 & $86.0(28.1)$ & 179 & $84.0(21.8)$ & NR & $<0.05$ \\
\hline \multirow{5}{*}{$\begin{array}{l}\text { Kulkarni, } \\
2017, \\
28713853, \\
\text { US \& Canada }\end{array}$} & NRCS & Moderate & $\begin{array}{l}\text { Psychosocial } \\
\text { WB }\end{array}$ & $\begin{array}{l}\text { BREAST-Q: } \\
\text { Psychosocial WB (0- } \\
100)\end{array}$ & $1 \mathrm{y}$ & All & 791 & $71.8(19)$ & 386 & $74.7(19.2)$ & $\begin{array}{l}\text { adjMD } 3.70(0.73, \\
6.76)\end{array}$ & 0.015 \\
\hline & NRCS & Moderate & $\begin{array}{l}\text { Psychosocial } \\
\text { WB }\end{array}$ & $\begin{array}{l}\text { BREAST-Q: } \\
\text { Psychosocial WB (0- } \\
100)\end{array}$ & $2 y$ & $\begin{array}{l}\text { Unilater } \\
\text { al }\end{array}$ & 600 & $74.6(18.7)$ & 317 & $76.8(18.9)$ & adjMD 3.84 (NR) & NR \\
\hline & NRCS & Moderate & $\begin{array}{l}\text { Psychosocial } \\
\text { WB }\end{array}$ & $\begin{array}{l}\text { BREAST-Q: } \\
\text { Psychosocial WB (0- } \\
100)\end{array}$ & $2 y$ & Bilateral & 994 & $74.5(19)$ & 224 & $73.4(19)$ & adjMD 0.91 (NR) & NR \\
\hline & NRCS & Moderate & $\begin{array}{l}\text { Psychosocial } \\
\text { WB }\end{array}$ & $\begin{array}{l}\text { PROMIS: Anxiety (0- } \\
100)\end{array}$ & $1 \mathrm{y}$ & All & 775 & $49.7(9.4)$ & 383 & $50.4(9.6)$ & $\begin{array}{l}\text { adjMD } 0.70(-0.75, \\
2.08)\end{array}$ & 0.36 \\
\hline & NRCS & Moderate & $\begin{array}{l}\text { Psychosocial } \\
\text { WB }\end{array}$ & $\begin{array}{l}\text { PROMIS: Depression } \\
(0-100)\end{array}$ & $1 \mathrm{y}$ & All & 776 & $47.3(8)$ & 385 & $47.9(8.2)$ & $\begin{array}{l}\text { adjMD } 0.40(-0.70, \\
1.45)\end{array}$ & 0.50 \\
\hline \multirow{2}{*}{$\begin{array}{l}\text { Roth, } 2007, \\
17413877, \\
\text { US }\end{array}$} & NRCS & High & $\begin{array}{l}\text { Psychosocial } \\
\text { WB }\end{array}$ & $\begin{array}{l}\text { FACT-B: Social/ family } \\
\text { WB }(0-28)\end{array}$ & $2 y$ & All & 35 & $19.3(\mathrm{NR})$ & 55 & $20.3(\mathrm{NR})$ & NR & 0.24 \\
\hline & NRCS & High & $\begin{array}{l}\text { Psychosocial } \\
\text { WB }\end{array}$ & $\begin{array}{l}\text { SF-36: Social } \\
\text { functioning }(0-100)\end{array}$ & $2 y$ & All & 35 & 87.9 (NR) & 55 & 87.7 (NR) & NR & $\geq 0.05$ \\
\hline
\end{tabular}




\begin{tabular}{|c|c|c|c|c|c|c|c|c|c|c|c|c|}
\hline $\begin{array}{l}\text { Study, Year, } \\
\text { PMID, } \\
\text { Country }\end{array}$ & $\begin{array}{l}\text { Desig } \\
n\end{array}$ & $\begin{array}{l}\text { Overall } \\
\text { RoB }\end{array}$ & Outcome & $\begin{array}{l}\text { Outcome } \\
\text { Measurement }\end{array}$ & $\begin{array}{l}\text { Time } \\
\text { Point }\end{array}$ & $\begin{array}{l}\text { Subgro } \\
\text { up }\end{array}$ & $\begin{array}{l}\text { IBR, } \\
\text { N }\end{array}$ & $\begin{array}{l}\text { IBR, Mean } \\
\text { (SD) }\end{array}$ & $\begin{array}{l}\text { AR } \\
, N\end{array}$ & $\begin{array}{l}\text { AR, Mean } \\
\text { (SD) }\end{array}$ & $\begin{array}{l}\text { AR Versus IBR, } \\
\text { Effect Size }(95 \% \mathrm{CI})\end{array}$ & $\begin{array}{l}P \\
\text { Value }\end{array}$ \\
\hline $\begin{array}{l}\text { Tallroth, } \\
2020, \\
33436336, \\
\text { Sweden } \\
\end{array}$ & RCT & Moderate & Sexual WB & $\begin{array}{l}\text { BREAST-Q: Sexual } \\
\text { well-being }(0-100)\end{array}$ & $5.3 \mathrm{y}$ & All & 28 & $58.4(23.1)$ & 42 & $67.1(28.1)$ & MD $8.7(0.2,17.2)$ & 0.046 \\
\hline $\begin{array}{l}\text { Eltahir, 2015, } \\
25539295, \\
\text { Netherlands }\end{array}$ & NRCS & Moderate & Sexual WB & $\begin{array}{l}\text { BREAST-Q: Sexual } \\
\text { well-being }(0-100)\end{array}$ & $2.2 \mathrm{y}$ & All & 45 & $\begin{array}{l}61.14 \\
(24.17)\end{array}$ & 47 & $\begin{array}{l}60.89 \\
(20.82)\end{array}$ & $\begin{array}{l}\text { adjMD } 6.44(-3.56 \\
16.5)\end{array}$ & 0.20 \\
\hline \multirow{3}{*}{$\begin{array}{l}\text { Kouwenberg, } \\
2020, \\
32590633, \\
\text { Netherlands }\end{array}$} & NRCS & Moderate & Sexual WB & $\begin{array}{l}\text { BREAST-Q: Sexual } \\
\text { well-being }(0-100)\end{array}$ & $\begin{array}{l}>6 \\
\mathrm{mo}\end{array}$ & All & 296 & $56.4(30.7)$ & 179 & $63.3(30.4)$ & NR & $<0.05$ \\
\hline & NRCS & Moderate & Sexual WB & $\begin{array}{l}\text { EORTC QLQBR23: } \\
\text { Sexual functioning (0- } \\
100)\end{array}$ & $\begin{array}{l}>6 \\
\mathrm{mo}\end{array}$ & All & 296 & $33.4(29.4)$ & 179 & $32.0(27.3)$ & NR & $<0.05$ \\
\hline & NRCS & Moderate & Sexual WB & $\begin{array}{l}\text { EORTC QLQBR23: } \\
\text { Sexual enjoyment (0- } \\
100)\end{array}$ & $\begin{array}{l}>6 \\
\mathrm{mo}\end{array}$ & All & 296 & $63.8(32.5)$ & 179 & $64.2(33.8)$ & NR & NS \\
\hline \multirow{4}{*}{$\begin{array}{l}\text { Kulkarni, } \\
2017, \\
28713853, \\
\text { US \& Canada }\end{array}$} & NRCS & Moderate & Sexual WB & $\begin{array}{l}\text { BREAST-Q: Sexual } \\
\text { WB }(0-100)\end{array}$ & $1 \mathrm{y}$ & All & 756 & $53(21.1)$ & 370 & $55.4(19.8)$ & $\begin{array}{l}\text { adjMD } 4.50(1.52, \\
7.48)\end{array}$ & 0.003 \\
\hline & NRCS & Moderate & Sexual WB & $\begin{array}{l}\text { BREAST-Q: Sexual } \\
\text { WB }(0-100)\end{array}$ & $2 y$ & All & $\begin{array}{l}149 \\
0\end{array}$ & $53.9(21.3)$ & 523 & $57.1(21.7)$ & $\begin{array}{l}\text { adjMD } 5.53(-2.95, \\
8.11)\end{array}$ & NR \\
\hline & NRCS & Moderate & Sexual WB & $\begin{array}{l}\text { BREAST-Q: Sexual } \\
\text { WB }(0-100)\end{array}$ & $2 y$ & $\begin{array}{l}\text { Unilater } \\
\text { al }\end{array}$ & 600 & $52.8(20.5)$ & 317 & $58.9(20.6)$ & adjMD $11.42(\mathrm{NR})$ & $<0.001$ \\
\hline & NRCS & Moderate & Sexual WB & $\begin{array}{l}\text { BREAST-Q: Sexual } \\
\text { WB }(0-100)\end{array}$ & $2 y$ & Bilateral & 994 & $54.7(21.5)$ & 214 & $54.4(23)$ & adjMD $4.2(\mathrm{NR})$ & $<0.001$ \\
\hline \multirow{2}{*}{$\begin{array}{l}\text { Tallroth, } \\
2020 \\
33436336 \\
\text { Sweden }\end{array}$} & RCT & Moderate & $\begin{array}{l}\text { Satisfaction } \\
\text { with breasts }\end{array}$ & $\begin{array}{l}\text { BREAST-Q: } \\
\text { Satisfaction with } \\
\text { breast }(0-100)\end{array}$ & $5.3 y$ & All & 28 & $63.4(11.8)$ & 42 & $72.1(17.7)$ & MD $8.7(3.8,13.6)$ & 0.001 \\
\hline & RCT & Moderate & $\begin{array}{l}\text { Satisfaction } \\
\text { with breasts }\end{array}$ & $\begin{array}{l}\text { BREAST-Q: } \\
\text { Satisfaction with } \\
\text { nipples }(0-100)\end{array}$ & $5.3 y$ & All & 28 & $65.4(21.8)$ & 42 & $67.7(24.9)$ & MD $2.3(-5.5,10.1)$ & 0.56 \\
\hline $\begin{array}{l}\text { Brito, } 2020, \\
\text { No PMID, } \\
\text { Portugal }\end{array}$ & NRCS & High & $\begin{array}{l}\text { Satisfaction } \\
\text { with breasts }\end{array}$ & $\begin{array}{l}\text { BREAST-Q: } \\
\text { Satisfaction with } \\
\text { breast }(0-100)\end{array}$ & NR & All & 68 & $56.3(17.1)$ & 111 & $64.1(17.1)$ & NR & 0.004 \\
\hline \multirow[t]{2}{*}{$\begin{array}{l}\text { Eltahir, 2015, } \\
25539295, \\
\text { Netherlands }\end{array}$} & NRCS & Moderate & $\begin{array}{l}\text { Satisfaction } \\
\text { with breasts }\end{array}$ & $\begin{array}{l}\text { BREAST-Q: } \\
\text { Satisfaction with } \\
\text { breast }(0-100)\end{array}$ & $2.2 \mathrm{y}$ & All & 45 & $\begin{array}{l}65.51 \\
(17.55)\end{array}$ & 47 & $\begin{array}{l}75.19 \\
(17.09)\end{array}$ & $\begin{array}{l}\text { adjMD } 8.16(1.18, \\
15.2)\end{array}$ & 0.023 \\
\hline & NRCS & Moderate & $\begin{array}{l}\text { Satisfaction } \\
\text { with breasts }\end{array}$ & $\begin{array}{l}\text { BREAST-Q: } \\
\text { Satisfaction with } \\
\text { nipples }(0-100)\end{array}$ & $2.2 \mathrm{y}$ & All & 45 & $\begin{array}{l}63.62 \\
(33.99)\end{array}$ & 47 & $\begin{array}{l}65.31 \\
(27.82)\end{array}$ & $\begin{array}{l}\operatorname{adjMD} 1.70(-14.2 \\
17.6)\end{array}$ & 0.83 \\
\hline $\begin{array}{l}\text { Kouwenberg, } \\
2020 \text {, }\end{array}$ & NRCS & Moderate & $\begin{array}{l}\text { Satisfaction } \\
\text { with breasts }\end{array}$ & $\begin{array}{l}\text { BREAST-Q: } \\
\text { Satisfaction with } \\
\text { breast }(0-100)\end{array}$ & $\begin{array}{l}>6 \\
\mathrm{mo}\end{array}$ & All & 296 & $59.4(19.3)$ & 179 & $71.3(17.7)$ & NR & $<0.05$ \\
\hline
\end{tabular}




\begin{tabular}{|c|c|c|c|c|c|c|c|c|c|c|c|c|}
\hline $\begin{array}{l}\text { Study, Year, } \\
\text { PMID, } \\
\text { Country }\end{array}$ & $\begin{array}{l}\text { Desig } \\
\mathrm{n}\end{array}$ & $\begin{array}{l}\text { Overall } \\
\text { RoB }\end{array}$ & Outcome & $\begin{array}{l}\text { Outcome } \\
\text { Measurement }\end{array}$ & $\begin{array}{l}\text { Time } \\
\text { Point }\end{array}$ & $\begin{array}{l}\text { Subgro } \\
\text { up }\end{array}$ & $\begin{array}{l}\text { IBR, } \\
\text { N }\end{array}$ & $\begin{array}{l}\text { IBR, Mean } \\
\text { (SD) }\end{array}$ & $\begin{array}{l}\text { AR } \\
, N\end{array}$ & $\begin{array}{l}\text { AR, Mean } \\
\text { (SD) }\end{array}$ & $\begin{array}{l}\text { AR Versus IBR, } \\
\text { Effect Size }(95 \% \mathrm{CI})\end{array}$ & $\begin{array}{l}\mathbf{P} \\
\text { Value }\end{array}$ \\
\hline $\begin{array}{l}32590633, \\
\text { Netherlands }\end{array}$ & NRCS & Moderate & $\begin{array}{l}\text { Satisfaction } \\
\text { with breasts }\end{array}$ & $\begin{array}{l}\text { BREAST-Q: } \\
\text { Satisfaction with } \\
\text { nipples }(0-100)\end{array}$ & $\begin{array}{l}>6 \\
\mathrm{mo}\end{array}$ & All & 296 & $55.0(48.7)$ & 179 & $63.0(29.0)$ & NR & $<0.05$ \\
\hline \multirow{4}{*}{$\begin{array}{l}\text { Kulkarni, } \\
2017, \\
28713853, \\
\text { US \& Canada }\end{array}$} & NRCS & Moderate & $\begin{array}{l}\text { Satisfaction } \\
\text { with breasts }\end{array}$ & $\begin{array}{l}\text { BREAST-Q: } \\
\text { Satisfaction with } \\
\text { breast }(0-100)\end{array}$ & $1 \mathrm{y}$ & All & 795 & $64.0(16.8)$ & 388 & $67.8(17.2)$ & $\begin{array}{l}\text { adjMD } 6.30 \text { (3.41, } \\
9.09)\end{array}$ & $<0.001$ \\
\hline & NRCS & Moderate & $\begin{array}{l}\text { Satisfaction } \\
\text { with breasts }\end{array}$ & $\begin{array}{l}\text { BREAST-Q: } \\
\text { Satisfaction with } \\
\text { breast }(0-100)\end{array}$ & $2 y$ & All & $\begin{array}{l}149 \\
0\end{array}$ & $64.2(18)$ & 523 & $68.5(18.3)$ & $\begin{array}{l}\text { adjMD } 7.94 \text { (5.68, } \\
10.2)\end{array}$ & NR \\
\hline & NRCS & Moderate & $\begin{array}{l}\text { Satisfaction } \\
\text { with breasts }\end{array}$ & $\begin{array}{l}\text { BREAST-Q: } \\
\text { Satisfaction with } \\
\text { breast }(0-100)\end{array}$ & $2 y$ & $\begin{array}{l}\text { Unilater } \\
\text { al }\end{array}$ & 600 & $61.2(18)$ & 317 & $68.3(18.1)$ & adjMD 9.85 (NR) & 0.001 \\
\hline & NRCS & Moderate & $\begin{array}{l}\text { Satisfaction } \\
\text { with breasts }\end{array}$ & $\begin{array}{l}\text { BREAST-Q: } \\
\text { Satisfaction with } \\
\text { breast }(0-100)\end{array}$ & $2 y$ & Bilateral & 994 & $66.1(17.7)$ & 214 & $68.9(18.6)$ & $\begin{array}{l}\text { adjMD } 5.13(2.07, \\
8.17)^{\star}\end{array}$ & 0.001 \\
\hline $\begin{array}{l}\text { Tallroth, } \\
2020, \\
33436336, \\
\text { Sweden }\end{array}$ & $\mathrm{RCT}$ & Moderate & $\begin{array}{l}\text { Satisfaction } \\
\text { with outcome }\end{array}$ & $\begin{array}{l}\text { BREAST-Q: } \\
\text { Satisfaction with } \\
\text { outcome }(0-100)\end{array}$ & $5.3 y$ & All & 28 & $79.4(14.2)$ & 42 & $82.3(21.4)$ & MD $2.9(-3.1,8.9)$ & 0.34 \\
\hline $\begin{array}{l}\text { Eltahir, 2015, } \\
25539295, \\
\text { Netherlands } \\
\end{array}$ & NRCS & Moderate & $\begin{array}{l}\text { Satisfaction } \\
\text { with outcome }\end{array}$ & $\begin{array}{l}\text { BREAST-Q: } \\
\text { Satisfaction with } \\
\text { outcome }(0-100)\end{array}$ & $2.2 \mathrm{y}$ & All & 45 & $74.5(19.0)$ & 47 & $81.8(18.7)$ & $\begin{array}{l}\operatorname{adjMD} 4.9(-3.1, \\
12.9)\end{array}$ & 0.23 \\
\hline $\begin{array}{l}\text { Kouwenberg, } \\
2020, \\
32590633, \\
\text { Netherlands }\end{array}$ & NRCS & Moderate & $\begin{array}{l}\text { Satisfaction } \\
\text { with outcome }\end{array}$ & $\begin{array}{l}\text { BREAST-Q: } \\
\text { Satisfaction with } \\
\text { outcome }(0-100)\end{array}$ & $\begin{array}{l}>6 \\
\mathrm{mo}\end{array}$ & All & 296 & $66.4(23.7)$ & 179 & $75.8(22.2)$ & NR & $<0.05$ \\
\hline
\end{tabular}

Abbreviations: adj $=$ adjusted, $\mathrm{CI}=$ confidence interval, $\mathrm{QoL}=$ quality of life, $\mathrm{MD}=$ mean difference, $\mathrm{mo}=$ months, $\mathrm{NR}=$ not reported, $\mathrm{NRCS}=$ nonrandomized comparative

study, $\mathrm{PMID}=$ PubMed identifier, $\mathrm{RCT}=$ randomized controlled trial, RoB $=$ risk of bias, $\mathrm{SD}=$ standard deviation, $\mathrm{VAS}=\mathrm{Visual}$ Analog Scale, $\mathrm{WB}=\mathrm{well}-\mathrm{being}, \mathrm{y}=\mathrm{years}$.

Colors: Header rows are shaded orange. Rows for every alternate outcome are shaded blue. The colors do not add unique information.

* Confidence interval calculated based on the reported $\mathrm{P}$ value. 
Table E-1.2. Summary Table - Key Question 1: IBR versus AR - continuous outcomes (physical well-being)

\begin{tabular}{|c|c|c|c|c|c|c|c|c|c|c|}
\hline $\begin{array}{l}\text { Study, Year, } \\
\text { PMID, } \\
\text { Country }\end{array}$ & Design & $\begin{array}{l}\text { Overall } \\
\text { RoB }\end{array}$ & $\begin{array}{l}\text { Outcome } \\
\text { Measurement }\end{array}$ & $\begin{array}{l}\text { Time } \\
\text { Point }\end{array}$ & Group & Subgroup & $\mathbf{N}$ & Mean (SD) & Effect Size (95\% Cl) & $\begin{array}{l}P \\
\text { Value }\end{array}$ \\
\hline \multirow{2}{*}{$\begin{array}{l}\text { Tallroth, } \\
2020, \\
33436336, \\
\text { Sweden }\end{array}$} & RCT & Moderate & $\begin{array}{l}\text { BREAST-Q: Chest } \\
(0-100)\end{array}$ & $5.3 y$ & IBR & All & 28 & $72.0(21.5)$ & Ref & Ref \\
\hline & RCT & Moderate & $\begin{array}{l}\text { BREAST-Q: Chest } \\
(0-100)\end{array}$ & $5.3 \mathrm{y}$ & AR & All & 42 & $79.6(21.1)$ & MD $7.6(0.3,14.9)$ & 0.041 \\
\hline \multirow{4}{*}{$\begin{array}{l}\text { Eltahir, 2015, } \\
\text { 25539295, } \\
\text { Netherlands }\end{array}$} & NRCS & Moderate & $\begin{array}{l}\text { BREAST-Q: } \\
\text { Physical WB }(0-100)\end{array}$ & $2.2 \mathrm{y}$ & IBR & All & 45 & $71.89(15.06)$ & Ref & Ref \\
\hline & NRCS & Moderate & $\begin{array}{l}\text { BREAST-Q: } \\
\text { Physical WB (0-100) }\end{array}$ & $2.2 \mathrm{y}$ & AR & All & 47 & $77.13(17.11)$ & adjMD -2.60 (-9.77, 4.57) & 0.47 \\
\hline & NRCS & Moderate & $\begin{array}{l}\text { SF-36: Physical } \\
\text { function }(0-100)\end{array}$ & $2.2 \mathrm{y}$ & IBR & All & 45 & NR & Ref & Ref \\
\hline & NRCS & Moderate & $\begin{array}{l}\text { SF-36: Physical } \\
\text { function }(0-100)\end{array}$ & $2.2 \mathrm{y}$ & AR & All & NR & NR & adjMD $2.13(-4.20,8.46)$ & 0.51 \\
\hline \multirow{4}{*}{$\begin{array}{l}\text { Kouwenberg, } \\
2020 \text {, } \\
32590633 \text {, } \\
\text { Netherlands }\end{array}$} & NRCS & Moderate & $\begin{array}{l}\text { BREAST-Q: Chest } \\
(0-100)\end{array}$ & $\begin{array}{l}>6 \\
\mathrm{mo}\end{array}$ & IBR & All & 296 & $72.6(17.8)$ & Ref & Ref \\
\hline & NRCS & Moderate & $\begin{array}{l}\text { BREAST-Q: Chest } \\
(0-100)\end{array}$ & $\begin{array}{l}>6 \\
\mathrm{mo}\end{array}$ & AR & All & 179 & $75.8(15.4)$ & NR & $<0.05$ \\
\hline & NRCS & Moderate & $\begin{array}{l}\text { EORTC QLQC30 } \\
(0-100)\end{array}$ & $\begin{array}{l}>6 \\
\mathrm{mo}\end{array}$ & IBR & All & 296 & $88.0(20.6)$ & Ref & Ref \\
\hline & NRCS & Moderate & $\begin{array}{l}\text { EORTC QLQC30 } \\
(0-100)\end{array}$ & $\begin{array}{l}>6 \\
\mathrm{mo}\end{array}$ & AR & All & 179 & $85.6(15.7)$ & NR & $<0.05$ \\
\hline \multirow{10}{*}{$\begin{array}{l}\text { Kulkarni, } \\
2017, \\
28713853, \\
\text { US \& } \\
\text { Canada }\end{array}$} & NRCS & Moderate & $\begin{array}{l}\text { BREAST-Q: Chest } \\
(0-100)\end{array}$ & $1 \mathrm{y}$ & IBR (all) & All & 791 & $76.7(14.5)$ & Ref & Ref \\
\hline & NRCS & Moderate & $\begin{array}{l}\text { BREAST-Q: Chest } \\
(0-100)\end{array}$ & $1 \mathrm{y}$ & AR (all) & All & 386 & $74.9(15.1)$ & adjMD $1.60(-0.57,3.68)$ & 0.003 \\
\hline & NRCS & Moderate & $\begin{array}{l}\text { BREAST-Q: Chest } \\
(0-100)\end{array}$ & $2 y$ & IBR (all) & All & NR & NR & NR & NR \\
\hline & NRCS & Moderate & $\begin{array}{l}\text { BREAST-Q: Chest } \\
(0-100)\end{array}$ & $2 y$ & IBR (all) & Unilateral & 600 & $77.2(13.8)$ & NR & NR \\
\hline & NRCS & Moderate & $\begin{array}{l}\text { BREAST-Q: Chest } \\
(0-100)\end{array}$ & $2 y$ & IBR (all) & Bilateral & 994 & $77.3(14.6)$ & NR & NR \\
\hline & NRCS & Moderate & $\begin{array}{l}\text { BREAST-Q: Chest } \\
(0-100)\end{array}$ & $2 y$ & AR (all) & All & NR & NR & NR & NR \\
\hline & NRCS & Moderate & $\begin{array}{l}\text { BREAST-Q: Chest } \\
(0-100)\end{array}$ & $2 y$ & AR (all) & Unilateral & 317 & $76.3(15.4)$ & $\begin{array}{l}\text { vs. IBR (Unilateral): adjMD } 1.77 \\
(-1.17,4.71)^{\star}\end{array}$ & 0.24 \\
\hline & NRCS & Moderate & $\begin{array}{l}\text { BREAST-Q: Chest } \\
(0-100)\end{array}$ & $2 y$ & AR (all) & Bilateral & 224 & $74.5(15.4)$ & $\begin{array}{l}\text { vs. IBR (Bilateral): adjMD } 0.57 \\
(-1.40,2.54)^{*}\end{array}$ & 0.57 \\
\hline & NRCS & Moderate & $\begin{array}{l}\text { BREAST-Q: Chest, } \\
\text { upper body }(0-100)\end{array}$ & $2 y$ & IBR (all) & All & 1490 & NR & NR & NR \\
\hline & NRCS & Moderate & $\begin{array}{l}\text { BREAST-Q: Chest, } \\
\text { upper body }(0-100)\end{array}$ & $2 y$ & IBR DTI & All & 93 & NR & Ref & Ref \\
\hline
\end{tabular}




\begin{tabular}{|c|c|c|c|c|c|c|c|c|c|c|}
\hline $\begin{array}{l}\text { Study, Year, } \\
\text { PMID, } \\
\text { Country }\end{array}$ & Design & $\begin{array}{l}\text { Overall } \\
\text { RoB }\end{array}$ & $\begin{array}{l}\text { Outcome } \\
\text { Measurement }\end{array}$ & $\begin{array}{l}\text { Time } \\
\text { Point }\end{array}$ & Group & Subgroup & $\mathbf{N}$ & Mean (SD) & Effect Size (95\% CI) & $\begin{array}{l}\mathbf{P} \\
\text { Value }\end{array}$ \\
\hline \multirow[t]{15}{*}{$\begin{array}{l}\text { Kulkarni, } \\
\text { continued }\end{array}$} & NRCS & Moderate & $\begin{array}{l}\text { BREAST-Q: Chest, } \\
\text { upper body }(0-100)\end{array}$ & $2 y$ & IBR TE & All & 1263 & NR & Ref & Ref \\
\hline & NRCS & Moderate & $\begin{array}{l}\text { BREAST-Q: Chest, } \\
\text { upper body }(0-100)\end{array}$ & $2 y$ & AR (all) & All & 1523 & $75.6(15.4)$ & $\begin{array}{l}\text { vs. IBR (all): adjMD } 1.69 \text { (0.13, } \\
3.24)\end{array}$ & NR \\
\hline & NRCS & Moderate & $\begin{array}{l}\text { BREAST-Q: Chest, } \\
\text { upper body }(0-100)\end{array}$ & $2 y$ & AR with DIEP & All & 350 & NR & $\begin{array}{l}\text { vs. IBR (TE): adjMD -1.44 } \\
(-4.11,1.23)^{*}\end{array}$ & 0.29 \\
\hline & NRCS & Moderate & $\begin{array}{l}\text { BREAST-Q: Chest, } \\
\text { upper body }(0-100)\end{array}$ & $2 y$ & $\begin{array}{l}\text { AR with free } \\
\text { TRAM }\end{array}$ & All & 87 & NR & $\begin{array}{l}\text { vs. IBR (TE): adjMD }-0.62 \\
(-4.78,3.54)^{\star}\end{array}$ & 0.77 \\
\hline & NRCS & Moderate & $\begin{array}{l}\text { BREAST-Q: Chest, } \\
\text { upper body }(0-100)\end{array}$ & $2 y$ & $\begin{array}{l}\text { AR with } \\
\text { pedicled } \\
\text { TRAM }\end{array}$ & All & 77 & NR & $\begin{array}{l}\text { vs. IBR (TE): adjMD -3.93 } \\
(-8.15,0.29)^{*}\end{array}$ & 0.068 \\
\hline & NRCS & Moderate & $\begin{array}{l}\text { PROMIS: Pain } \\
\text { interference }(0-100)\end{array}$ & $2 y$ & IBR (all) & All & 773 & $46(7.5)$ & Ref & Ref \\
\hline & NRCS & Moderate & $\begin{array}{l}\text { PROMIS: Pain } \\
\text { interference }(0-100)\end{array}$ & $2 y$ & AR (all) & All & 384 & $48.4(8.4)$ & adjMD $1.10(0.01,2.25)$ & 0.048 \\
\hline & NRCS & Moderate & $\begin{array}{l}\text { PROMIS: Physical } \\
\text { function }(0-100)\end{array}$ & $1 \mathrm{y}$ & IBR (all) & All & 777 & $52.2(6.8)$ & Ref & Ref \\
\hline & NRCS & Moderate & $\begin{array}{l}\text { PROMIS: Physical } \\
\text { function }(0-100)\end{array}$ & $1 \mathrm{y}$ & AR (all) & All & 385 & $50.1(7.2)$ & adjMD $-0.60(-1.51,0.39)$ & 0.25 \\
\hline & NRCS & Moderate & $\begin{array}{l}\text { PROMIS: Physical } \\
\text { function }(0-100)\end{array}$ & $2 y$ & IBR (all) & All & NR & NR & NR & NR \\
\hline & NRCS & Moderate & $\begin{array}{l}\text { PROMIS: Physical } \\
\text { function }(0-100)\end{array}$ & $2 y$ & IBR (all) & Unilateral & 600 & $52.6(6.5)$ & NR & NR \\
\hline & NRCS & Moderate & $\begin{array}{l}\text { PROMIS: Physical } \\
\text { function }(0-100)\end{array}$ & $2 y$ & IBR (all) & Bilateral & 994 & $52.8(6.3)$ & NR & NR \\
\hline & NRCS & Moderate & $\begin{array}{l}\text { PROMIS: Physical } \\
\text { function }(0-100)\end{array}$ & $2 y$ & AR (all) & All & NR & NR & NR & NR \\
\hline & NRCS & Moderate & $\begin{array}{l}\text { PROMIS: Physical } \\
\text { function }(0-100)\end{array}$ & $2 y$ & $\mathrm{AR}$ (all) & Unilateral & 317 & $51.3(7.3)$ & $\begin{array}{l}\text { vs. IBR (unilateral): adjMD }-0.14 \\
(-1.42,1.14)^{*}\end{array}$ & 0.83 \\
\hline & NRCS & Moderate & $\begin{array}{l}\text { PROMIS: Physical } \\
\text { function }(0-100)\end{array}$ & $2 y$ & AR (all) & Bilateral & 214 & $49.8(7.6)$ & $\begin{array}{l}\text { vs. IBR (bilateral): adjMD -1.21 } \\
(-2.47,0.05)^{*}\end{array}$ & 0.06 \\
\hline \multirow{2}{*}{$\begin{array}{l}\text { McCarthy, } \\
2014, \\
24201740, \\
\text { US }\end{array}$} & NRCS & Moderate & $\begin{array}{l}\text { BREAST-Q: } \\
\text { Physical WB (0-100) }\end{array}$ & $1-5 y$ & IBR & All & 141 & $76.5(16)$ & Ref & Ref \\
\hline & NRCS & Moderate & $\begin{array}{l}\text { BREAST-Q: } \\
\text { Physical WB (0-100) }\end{array}$ & $1-5 y$ & AR & All & 74 & $82.5(15.3)$ & NR & $<0.05$ \\
\hline
\end{tabular}

Abbreviations: adj $=$ adjusted, $\mathrm{CI}=$ confidence interval, $\mathrm{DIEP}=$ deep inferior epigastric perforator, $\mathrm{DTI}=$ direct to implant, $\mathrm{MD}=$ mean difference, $\mathrm{mo}=\mathrm{months}, \mathrm{NR}=$ not

reported, NRCS = nonrandomized comparative study, PMID = PubMed identifier, PROMIS: Patient Reported Outcomes Measurement Information System, RCT = randomized controlled trial, $\operatorname{Ref}=$ reference group, $\mathrm{RoB}=$ risk of bias, $\mathrm{SD}=$ standard deviation, $\mathrm{WB}=$ well-being, $\mathrm{y}=$ years.

Colors: Header rows are shaded orange. Rows for every alternate study are shaded blue. The colors do not add unique information.

* Confidence interval calculated based on the reported $\mathrm{P}$ value. 
Table E-1.3. Summary Table - Key Question 1: IBR versus AR - NRCSs, categorical outcomes (physical well-being and recurrence of breast cancer)

\begin{tabular}{|c|c|c|c|c|c|c|c|c|}
\hline $\begin{array}{l}\text { Study, Year, PMID, } \\
\text { Country }\end{array}$ & $\begin{array}{l}\text { Overall } \\
\text { RoB }\end{array}$ & Outcome & Outcome Measurement & $\begin{array}{l}\text { Time } \\
\text { Point }\end{array}$ & IBR, n/N (\%) & $A R, n / N(\%)$ & $\begin{array}{l}\text { Adjusted Odds } \\
\text { Ratio }(95 \% \mathrm{Cl})\end{array}$ & $\begin{array}{l}P \\
\text { Value }\end{array}$ \\
\hline \multirow{4}{*}{$\begin{array}{l}\text { Nelson, } 2019 \\
31356276, \text { US }\end{array}$} & High & Physical WB & Higher BREAST-Q: Chest & $1 \mathrm{y}$ & $\mathrm{NR} / 1342$ (NR) & NR/194 (NR) & $0.96(0.67,1.38)$ & NS \\
\hline & High & Physical WB & Higher BREAST-Q: Chest & $3 y$ & NR/1085 (NR) & NR/98 (NR) & $1.4(0.83,2.34)$ & NS \\
\hline & High & Physical WB & Higher BREAST-Q: Chest & $5 \mathrm{y}$ & $\mathrm{NR} / 743(\mathrm{NR})$ & $\mathrm{NR} / 41$ (NR) & $4.52(2.03,10.1)$ & $<0.001$ \\
\hline & High & Physical WB & Higher BREAST-Q: Chest & $7 \mathrm{y}$ & NR/377 (NR) & NR/19 (NR) & $3.08(1.03,9.15)$ & 0.043 \\
\hline \multirow{2}{*}{$\begin{array}{l}\text { Ha, 2020, } \\
32000718, \text { South } \\
\text { Korea }\end{array}$} & High & Breast cancer recurrence & $\begin{array}{l}\text { High histologic grade } \\
\text { (Grade III) breast cancer }\end{array}$ & $4.8 \mathrm{y}$ & $14 / 247(5.7)$ & $24 / 249(9.6)$ & $3.39(1.23,9.32)$ & 0.018 \\
\hline & High & Breast cancer recurrence & Locoregional breast cancer & $4.8 \mathrm{y}$ & $9 / 247(3.6)$ & $11 / 249(4.4)$ & NR & 0.70 \\
\hline \multirow{2}{*}{$\begin{array}{l}\text { Kouwenberg, 2020, } \\
32590633, \\
\text { Netherlands }\end{array}$} & Moderate & Breast cancer recurrence & Local recurrence & $>6 \mathrm{mo}$ & $13 / 296(4.5$ & $7 / 179(4.0)$ & $0.89(0.35,2.26)$ & 0.25 \\
\hline & Moderate & Breast cancer recurrence & Distant recurrence & $>6 \mathrm{mo}$ & $13 / 296(4.5)$ & $8 / 179(4.6)$ & $1.02(0.41,2.51)$ & 0.97 \\
\hline $\begin{array}{l}\text { Wu, 2021, } \\
33740204 \text {, South } \\
\text { Korea }\end{array}$ & High & Breast cancer recurrence & NR & $5.8 y$ & $29 / 138(21.0)$ & $64 / 276(23.2)$ & NR & 0.62 \\
\hline
\end{tabular}

Abbreviations: $\mathrm{AR}=$ autologous reconstruction, $\mathrm{CI}=$ confidence interval, $\mathrm{mo}=$ months, $\mathrm{NR}=$ not reported, $\mathrm{NRCS}=$ nonrandomized comparative study, $\mathrm{NS}=$ not significant, PMID $=$ PubMed identifier, RoB $=$ risk of bias, $\mathrm{WB}=$ well-being, $\mathrm{y}=$ years .

Colors: Header rows are shaded orange. Rows for every alternate outcome are shaded blue. The colors do not add unique information.

Table E-1.4. Summary Table - Key Question 1: IBR versus AR - NRCSs, categorical outcomes (patient satisfaction with breasts and with outcome)

\begin{tabular}{|c|c|c|c|c|c|c|c|c|}
\hline $\begin{array}{l}\text { Study, Year, } \\
\text { PMID, Country }\end{array}$ & $\begin{array}{l}\text { Overall } \\
\text { RoB }\end{array}$ & Outcome Measurement & $\begin{array}{l}\text { Time } \\
\text { Point }\end{array}$ & Group & $\mathrm{n} / \mathrm{N}(\%)$ & Comparison & $\begin{array}{l}\text { Adjusted Odds } \\
\text { Ratio }(95 \% \mathrm{Cl})\end{array}$ & $\begin{array}{l}\mathbf{P} \\
\text { Value }\end{array}$ \\
\hline \multirow{2}{*}{$\begin{array}{l}\text { Lei, 2020, } \\
32481367 \text {, China }\end{array}$} & High & Satisfied with breasts & $2 \mathrm{mo}$ & IBR & NR & Ref & Ref & Ref \\
\hline & High & Satisfied with breasts & $2 \mathrm{mo}$ & $\mathrm{AR}$ & NR & vS. IBR & $0.85(0.36,1.63)$ & 0.40 \\
\hline \multirow{4}{*}{$\begin{array}{l}\text { Yueh, 2009, } \\
\text { 19228537, Us }\end{array}$} & High & Satisfied with breasts & NR & IBR & $42 / 87(48.3)$ & Ref & Ref & Ref \\
\hline & High & Satisfied with breasts & NR & AR & NR/389 (NR) & vs. IBR & $1.43(1.18,1.73)$ & NR \\
\hline & High & Satisfied with breasts & NR & AR with TRAM & $102 / 143(71.3)$ & vs. IBR & $3.49(1.91,6.40)$ & NR \\
\hline & High & Satisfied with breasts & NR & AR with LD & $68 / 112(60.7)$ & vs. IBR & $1.99(1.09,3.65)$ & NR \\
\hline \multirow{2}{*}{$\begin{array}{l}\text { Lei, 2020, } \\
\text { 32481367, China }\end{array}$} & High & Satisfied with surgical outcome & $2 \mathrm{mo}$ & IBR & NR & Ref & Ref & Ref \\
\hline & High & Satisfied with surgical outcome & $2 \mathrm{mo}$ & $\mathrm{AR}$ & NR & vs. IBR & $0.69(0.45,1.67)$ & 0.33 \\
\hline \multirow{4}{*}{$\begin{array}{l}\text { Yueh, 2009, } \\
\text { 19228537, US }\end{array}$} & High & Satisfied with surgical outcome & NR & IBR & $49 / 87(56.3)$ & Ref & Ref & Ref \\
\hline & High & Satisfied with surgical outcome & NR & $\mathrm{AR}$ & NR/389 (NR) & vs. IBR & $1.83(1.11,3.03)$ & NR \\
\hline & High & Satisfied with surgical outcome & NR & AR with TRAM & $98 / 143(68.5)$ & VS. IBR & $2.05(1.13,3.72)$ & NR \\
\hline & High & Satisfied with surgical outcome & NR & AR with LD & $63 / 112(56.3)$ & vs. IBR & $1.12(0.64,2.12)$ & NR \\
\hline
\end{tabular}

Abbreviations: $\mathrm{AR}=$ autologous reconstruction, $\mathrm{CI}=$ confidence interval, $\mathrm{DIEP}=$ deep inferior epigastric perforator, $\mathrm{LD}=$ latissimus dorsi, $\mathrm{mo}=\mathrm{months}, \mathrm{RCT}=$ randomized controlled trial, PMID = PubMed identifier, Ref = reference group, RoB = risk of bias, TRAM = transverse rectus abdominis myocutaneous.

Colors: Header rows are shaded orange. Rows for every alternate outcome are shaded blue. The colors do not add unique information. 
Table E-1.5. Summary Table - Key Question 1: IBR versus AR - categorical outcomes, mortality, unplanned repeat hospitalizations, necrosis, thromboembolic events, wound dehiscence, delayed healing, seroma, and hematoma

\begin{tabular}{|c|c|c|c|c|c|c|c|c|c|}
\hline $\begin{array}{l}\text { Study, Year, } \\
\text { PMID, Country }\end{array}$ & Design & $\begin{array}{l}\text { Overall } \\
\text { RoB }\end{array}$ & Outcome & $\begin{array}{l}\text { Outcome } \\
\text { Description }\end{array}$ & $\begin{array}{l}\text { Time } \\
\text { Point }\end{array}$ & IBR n/N (\%) & AR n/N (\%) & $\begin{array}{l}\text { Adjusted Odds Ratio } \\
\text { (95\% CI) for AR versus } \\
\text { IBR }\end{array}$ & P Value \\
\hline \multirow[t]{2}{*}{$\begin{array}{l}\text { Jiang, 2013, } \\
24349366, \text { US }\end{array}$} & NRCS & High & Mortality & Overall mortality & $8.9 \mathrm{y}$ & $241 / 1412(17.1)$ & $\begin{array}{l}503 / 2649 \\
(19.0) \\
\end{array}$ & $0.96(0.89,1.04)$ & NR \\
\hline & NRCS & High & Mortality & $\begin{array}{l}\text { Breast cancer- } \\
\text { specific mortality }\end{array}$ & $8.9 \mathrm{y}$ & $209 / 1412(14.8)$ & $\begin{array}{l}432 / 2649 \\
(16.3)\end{array}$ & $0.95(0.87,1.04)$ & NR \\
\hline $\begin{array}{l}\text { Merchant, 2015, } \\
26111325, \text { US }\end{array}$ & NRCS & High & $\begin{array}{l}\text { Unplanned } \\
\text { repeat } \\
\text { hospitalizations }\end{array}$ & $\begin{array}{l}\text { Any unplanned } \\
\text { readmission }\end{array}$ & $1 \mathrm{mo}$ & $\begin{array}{l}338 / 10437 \\
(3.24)\end{array}$ & $95 / 2329(4.08)$ & $1.07(0.95,1.20)$ & NR \\
\hline $\begin{array}{l}\text { Mioton, 2013, } \\
\text { 23562485, US }\end{array}$ & NRCS & Moderate & $\begin{array}{l}\text { Unplanned } \\
\text { repeat } \\
\text { hospitalizations }\end{array}$ & $\begin{array}{l}\text { Any unplanned } \\
\text { readmission }\end{array}$ & $1 \mathrm{mo}$ & $172 / 3960(4.34)$ & $56 / 1052(5.32)$ & NR & NS \\
\hline \multirow[t]{2}{*}{$\begin{array}{l}\text { Nasser, 2018, } \\
30204678\end{array}$} & NRCS & High & $\begin{array}{l}\text { Unplanned } \\
\text { repeat } \\
\text { hospitalizations }\end{array}$ & $\begin{array}{l}\text { Unplanned ED } \\
\text { visits }\end{array}$ & $1 \mathrm{mo}$ & NR/28124 (NR) & NR/4773 (NR) & $1.11(0.91,1.25)$ & 0.18 \\
\hline & NRCS & High & $\begin{array}{l}\text { Unplanned } \\
\text { repeat } \\
\text { hospitalizations }\end{array}$ & $\begin{array}{l}\text { Unplanned ED } \\
\text { visits with pain- } \\
\text { related diagnosis }\end{array}$ & $1 \mathrm{mo}$ & NR/28124 (NR) & NR/4773 (NR) & $1.11(0.83,1.67)$ & 0.41 \\
\hline $\begin{array}{l}\text { Abedi, 2016, } \\
25003437, \\
\text { Canada }\end{array}$ & NRCS & High & Necrosis & $\begin{array}{l}\text { Mastectomy flap } \\
\text { necrosis }\end{array}$ & $1.6-1.9 y$ & 70/606 (11.6) & $60 / 395(15.2)$ & $0.66(0.38,1.16)$ & 0.15 \\
\hline $\begin{array}{l}\text { de Araujo, } \\
2016, \\
27673527, \text { US }\end{array}$ & NRCS & High & Necrosis & $\begin{array}{l}\text { Major } \\
\text { mastectomy flap } \\
\text { necrosis }\end{array}$ & $4.3 \mathrm{y}$ & NR/38 (NR) & NR/32 (NR) & $17.9(0.52,610.5)$ & 0.11 \\
\hline \multirow[t]{2}{*}{$\begin{array}{l}\text { Naoum, 2020a, } \\
\text { 31756414, US }\end{array}$} & NRCS & High & Necrosis & $\begin{array}{l}\text { Mastectomy flap } \\
\text { necrosis }\end{array}$ & $4-10 y$ & $\begin{array}{l}26 / 633 \text { breasts } \\
(4.1)\end{array}$ & $\begin{array}{l}16 / 342 \text { breasts } \\
(4.7)\end{array}$ & $0.83(0.19,3.50)$ & 0.8 \\
\hline & NRCS & High & Necrosis & Fat necrosis & $4-10 y$ & $\begin{array}{l}1 / 633 \text { breasts } \\
(0.2)\end{array}$ & $\begin{array}{l}24 / 342 \text { breasts } \\
(7.0)\end{array}$ & $21.2(2.5,174.5)$ & 0.004 \\
\hline $\begin{array}{l}\text { Woo, 2018, } \\
30360958, \\
\text { South Korea }\end{array}$ & NRCS & High & Necrosis & $\begin{array}{l}\text { Mastectomy flap } \\
\text { necrosis }\end{array}$ & NR & $14 / 60(23.3)$ & $7 / 70(10)$ & $0.31(0.11,0.86)$ & 0.02 \\
\hline \multirow{2}{*}{$\begin{array}{l}\text { Brorson 2020a, } \\
32807615, \\
\text { Sweden }\end{array}$} & RCT & High & $\begin{array}{l}\text { Thromboembolic } \\
\text { events }\end{array}$ & DVT & $1 \mathrm{mo}$ & $0 / 66(0)$ & $0 / 51(0)$ & No events & N/A \\
\hline & RCT & High & $\begin{array}{l}\text { Thromboembolic } \\
\text { events }\end{array}$ & $\mathrm{PE}$ & $1 \mathrm{mo}$ & $1 / 66(1.5)$ & $0 / 51(0)$ & $\begin{array}{l}\text { Not calculable (no } \\
\text { events in AR group) }\end{array}$ & $\begin{array}{l}\text { Not } \\
\text { calculable }\end{array}$ \\
\hline \multirow{3}{*}{$\begin{array}{l}\text { Tallroth, 2020, } \\
3346336, \\
\text { Sweden }\end{array}$} & RCT & Moderate & $\begin{array}{l}\text { Thromboembolic } \\
\text { events }\end{array}$ & DVT & $1 \mathrm{mo}$ & $1 / 28(3.6)$ & $0 / 42(0)$ & $\begin{array}{l}\text { Not calculable (no } \\
\text { events in AR group) }\end{array}$ & $\begin{array}{l}\text { Not } \\
\text { calculable }\end{array}$ \\
\hline & RCT & Moderate & $\begin{array}{l}\text { Thromboembolic } \\
\text { events }\end{array}$ & Arterial stop & $1 \mathrm{mo}$ & $0 / 28(0)$ & $2 / 42(4.8)$ & $\begin{array}{l}\text { Not calculable (no } \\
\text { events in IBR group) }\end{array}$ & $\begin{array}{l}\text { Not } \\
\text { calculable }\end{array}$ \\
\hline & RCT & Moderate & $\begin{array}{l}\text { Thromboembolic } \\
\text { events }\end{array}$ & Venous stasis & $1 \mathrm{mo}$ & $0 / 28(0)$ & $6 / 42(14.3)$ & $\begin{array}{l}\text { Not calculable (no } \\
\text { events in IBR group) }\end{array}$ & $\begin{array}{l}\text { Not } \\
\text { calculable }\end{array}$ \\
\hline
\end{tabular}




\begin{tabular}{|c|c|c|c|c|c|c|c|c|c|}
\hline $\begin{array}{l}\text { Study, Year, } \\
\text { PMID, Country }\end{array}$ & Design & $\begin{array}{l}\text { Overall } \\
\text { RoB }\end{array}$ & Outcome & $\begin{array}{l}\text { Outcome } \\
\text { Description }\end{array}$ & $\begin{array}{l}\text { Time } \\
\text { Point }\end{array}$ & IBR n/N (\%) & AR n/N (\%) & $\begin{array}{l}\text { Adjusted Odds Ratio } \\
\text { (95\% CI) for AR versus } \\
\text { IBR }\end{array}$ & P Value \\
\hline \multirow[t]{2}{*}{$\begin{array}{l}\text { Mioton, 2013, } \\
23562485, \text { US }\end{array}$} & NRCS & Moderate & $\begin{array}{l}\text { Thromboembolic } \\
\text { events }\end{array}$ & DVT & $1 \mathrm{mo}$ & $27 / 9786(0.28)$ & $20 / 3296(0.61)$ & $0.99(0.41,2.41)$ & NR \\
\hline & NRCS & Moderate & $\begin{array}{l}\text { Thromboembolic } \\
\text { events }\end{array}$ & $\mathrm{PE}$ & $1 \mathrm{mo}$ & $17 / 9786(0.17)$ & $17 / 3296(0.52)$ & $1.84(0.71,4.77)$ & NR \\
\hline $\begin{array}{l}\text { Momeni, 2018, } \\
\text { 29095189, US }\end{array}$ & NRCS & High & $\begin{array}{l}\text { Thromboembolic } \\
\text { events }\end{array}$ & DVT or PE & $3 \mathrm{mo}$ & $65 / 16851(3.85)$ & $\begin{array}{l}815 / 4622 \\
(17.63)\end{array}$ & $2.27(1.79,2.86)$ & NR \\
\hline $\begin{array}{l}\text { Garvey, 2012, } \\
23096600, \text { US }\end{array}$ & NRCS & Moderate & $\begin{array}{l}\text { Wound } \\
\text { dehiscence }\end{array}$ & NR & $1.5 \mathrm{y}$ & $\begin{array}{l}28 / 442 \text { breasts } \\
(6.3)\end{array}$ & $\begin{array}{l}25 / 548 \text { breasts } \\
(4.6)\end{array}$ & NR & 0.25 \\
\hline $\begin{array}{l}\text { Mioton, 2013, } \\
23562485, \text { US }\end{array}$ & NRCS & Moderate & $\begin{array}{l}\text { Wound } \\
\text { dehiscence }\end{array}$ & Wound disruption & $1 \mathrm{mo}$ & $44 * / 9786(0.44)$ & $41^{*} / 3296(1.24)$ & $1.79(0.83,3.84)$ & NR \\
\hline $\begin{array}{l}\text { Fischer, 2013, } \\
23629074, \text { US }\end{array}$ & NRCS & High & Delayed healing & $\begin{array}{l}\text { Delayed breast } \\
\text { wound healing }\end{array}$ & $4 y$ & $9 / 60(15)$ & $52 / 142(36.6)$ & $2.2(1.0,5.2)$ & 0.06 \\
\hline $\begin{array}{l}\text { Garvey, 2012, } \\
23096600, \text { US }\end{array}$ & NRCS & Moderate & Delayed healing & NR & $1.5 \mathrm{y}$ & $\begin{array}{l}19 / 442 \text { breasts } \\
(4.3)\end{array}$ & $\begin{array}{l}41 / 548 \text { breasts } \\
(7.5)\end{array}$ & NR & 0.01 \\
\hline $\begin{array}{l}\text { Fischer, 2014, } \\
24916480, \text { US }\end{array}$ & NRCS & High & Seroma & Breast seroma & $1.8-2.1 \mathrm{y}$ & $13 / 155(8.1)$ & $4 / 155(2.8)$ & NR & 0.009 \\
\hline $\begin{array}{l}\text { Garvey, 2012, } \\
23096600, \text { US }\end{array}$ & NRCS & Moderate & Seroma & $\begin{array}{l}\text { Breast seroma or } \\
\text { hematoma }\end{array}$ & $1.5 \mathrm{y}$ & $\begin{array}{l}\text { 61/442 breasts } \\
(13.8)\end{array}$ & $\begin{array}{l}27 / 548 \text { breasts } \\
(4.9)\end{array}$ & NR & $<0.001$ \\
\hline $\begin{array}{l}\text { Fischer, 2014, } \\
24916480, \text { US }\end{array}$ & NRCS & High & Hematoma & Breast hematoma & $\begin{array}{l}1.8-2.1 \\
\mathrm{mo}\end{array}$ & $4 / 155(2.4)$ & $4 / 155(2.8)$ & NR & 1.0 \\
\hline
\end{tabular}

Abbreviations: $\mathrm{AR}=$ autologous reconstruction, $\mathrm{CI}=$ confidence interval, $\mathrm{DVT}=$ deep vein thrombosis, $\mathrm{ED}=$ emergency department, $\mathrm{IBR}=$ implant-based reconstruction, $\mathrm{N} / \mathrm{A}=$ not applicable, $\mathrm{NR}=$ not reported, $\mathrm{NRCS}=$ nonrandomized comparative study, $\mathrm{PE}=$ pulmonary embolism, $\mathrm{PMID}=\mathrm{PubMed}$ identifier, RoB $=$ risk of bias, $\mathrm{y}=\mathrm{years}$. Colors: Header rows are shaded orange. Rows for every alternate outcome are shaded blue. The colors do not add unique information.

*calculated 
Table E-1.6. Summary Table - Key Question 1: IBR versus AR - NRCSs, categorical outcomes (unplanned repeat surgeries for revision, unplanned repeat surgeries for complications, pain, infections not explicitly implant related, and reconstructive failure)

\begin{tabular}{|c|c|c|c|c|c|c|c|c|c|}
\hline $\begin{array}{l}\text { Study, } \\
\text { Year, } \\
\text { PMID, } \\
\text { Country }\end{array}$ & $\begin{array}{l}\text { Overall } \\
\text { RoB }\end{array}$ & Outcome & Outcome Description & $\begin{array}{l}\text { Time } \\
\text { Point }\end{array}$ & Group & Subgroup & $n / N(\%)$ & $\begin{array}{l}\text { Adjusted Odds Ratio } \\
(95 \% \mathrm{Cl})\end{array}$ & $\begin{array}{l}P \\
\text { Value }\end{array}$ \\
\hline \multirow{6}{*}{$\begin{array}{l}\text { Fischer, } \\
2014, \\
24916480, \\
\text { US }\end{array}$} & High & $\begin{array}{l}\text { Unplanned repeat } \\
\text { surgery for revision }\end{array}$ & Unplanned revision & $\begin{array}{l}<6 \\
\mathrm{mo}\end{array}$ & IBR & All & $\begin{array}{l}8 / 155 \\
(5.2)\end{array}$ & Ref & Ref \\
\hline & High & $\begin{array}{l}\text { Unplanned repeat } \\
\text { surgery for revision }\end{array}$ & Unplanned revision & $\begin{array}{l}<6 \\
\mathrm{mo}\end{array}$ & AR & All & $\begin{array}{l}6 / 155 \\
(3.9)\end{array}$ & NR & 0.56 \\
\hline & High & $\begin{array}{l}\text { Unplanned repeat } \\
\text { surgery for revision }\end{array}$ & Unplanned revision & $<1 \mathrm{y}$ & IBR & All & $\begin{array}{l}17 / 155 \\
(11.0)\end{array}$ & Ref & Ref \\
\hline & High & $\begin{array}{l}\text { Unplanned repeat } \\
\text { surgery for revision }\end{array}$ & Unplanned revision & $<1 \mathrm{y}$ & $\mathrm{AR}$ & All & $\begin{array}{l}7 / 155 \\
(4.5)\end{array}$ & NR & 0.017 \\
\hline & High & $\begin{array}{l}\text { Unplanned repeat } \\
\text { surgery for revision }\end{array}$ & Unplanned revision & $<2 \mathrm{y}$ & IBR & All & $\begin{array}{l}21 / 155 \\
(13.5)\end{array}$ & Ref & Ref \\
\hline & High & $\begin{array}{l}\text { Unplanned repeat } \\
\text { surgery for revision }\end{array}$ & Unplanned revision & $<2 \mathrm{y}$ & AR & All & $\begin{array}{l}7 / 155 \\
(4.5)\end{array}$ & NR & 0.003 \\
\hline \multirow{7}{*}{$\begin{array}{l}\text { Kulkarni, } \\
2017, \\
28713853, \\
\text { US \& } \\
\text { Canada }\end{array}$} & Moderate & $\begin{array}{l}\text { Unplanned repeat } \\
\text { surgery for revision }\end{array}$ & Unplanned revision & $2 y$ & IBR DTI & All & $\begin{array}{l}31 / 93 \\
(33.3)\end{array}$ & - & - \\
\hline & Moderate & $\begin{array}{l}\text { Unplanned repeat } \\
\text { surgery for revision }\end{array}$ & Unplanned revision & $2 y$ & IBR TE & All & $\begin{array}{l}503 / 1263 \\
(39.8)\end{array}$ & Ref & Ref \\
\hline & Moderate & $\begin{array}{l}\text { Unplanned repeat } \\
\text { surgery for revision }\end{array}$ & Unplanned revision & $2 y$ & $\begin{array}{l}\text { AR with } \\
\text { DIEP }\end{array}$ & All & $\begin{array}{l}223 / 350 \\
(63.7)\end{array}$ & $\begin{array}{l}\text { vs. IBR with TE: } 2.66 \\
(1.83,3.86)\end{array}$ & $<0.001$ \\
\hline & Moderate & $\begin{array}{l}\text { Unplanned repeat } \\
\text { surgery for revision }\end{array}$ & Unplanned revision & $2 y$ & $\begin{array}{l}\text { AR with free } \\
\text { TRAM }\end{array}$ & All & $\begin{array}{l}56 / 87 \\
(64.4) \\
\end{array}$ & $\begin{array}{l}\text { vs. IBR with TE: } 2.26 \\
(1.35,3.78)\end{array}$ & 0.002 \\
\hline & Moderate & $\begin{array}{l}\text { Unplanned repeat } \\
\text { surgery for revision }\end{array}$ & Unplanned revision & $2 y$ & $\begin{array}{l}\text { AR with } \\
\text { pedicled } \\
\text { TRAM }\end{array}$ & All & $\begin{array}{l}40 / 77 \\
(57.1)\end{array}$ & $\begin{array}{l}\text { vs. IBR with TE: } 1.34 \\
(0.75,2.40)\end{array}$ & 0.33 \\
\hline & Moderate & $\begin{array}{l}\text { Unplanned repeat } \\
\text { surgery for revision }\end{array}$ & Unplanned revision & $2 y$ & AR with LD & All & $\begin{array}{l}41 / 64 \\
(64.1)\end{array}$ & $\begin{array}{l}\text { vs. IBR with TE: } 1.97 \\
(1.07,3.64)\end{array}$ & 0.031 \\
\hline & Moderate & $\begin{array}{l}\text { Unplanned repeat } \\
\text { surgery for revision }\end{array}$ & Unplanned revision & $2 y$ & $\begin{array}{l}\text { AR with } \\
\text { SIEA }\end{array}$ & All & $\begin{array}{l}33 / 62 \\
(53.2)\end{array}$ & $\begin{array}{l}\text { vs. IBR with TE: } 1.83 \\
(0.93,3.60)\end{array}$ & 0.079 \\
\hline \multirow{2}{*}{$\begin{array}{l}\text { Zhang, } \\
2019, \\
30675702, \\
\text { China }\end{array}$} & High & $\begin{array}{l}\text { Unplanned repeat } \\
\text { surgery for revision }\end{array}$ & Unplanned reoperation & $4.9 \mathrm{y}$ & IBR & All & $\begin{array}{l}230 / 394 \\
(58.4)\end{array}$ & Ref & Ref \\
\hline & High & $\begin{array}{l}\text { Unplanned repeat } \\
\text { surgery for revision }\end{array}$ & Unplanned reoperation & $4.9 \mathrm{y}$ & AR & All & $\begin{array}{l}154 / 438 \\
(35.2) \\
\end{array}$ & $\begin{array}{l}\text { vs. IBR: } 0.72(0.50, \\
1.06)\end{array}$ & 0.093 \\
\hline
\end{tabular}




\begin{tabular}{|c|c|c|c|c|c|c|c|c|c|}
\hline $\begin{array}{l}\text { Study, } \\
\text { Year, } \\
\text { PMID, } \\
\text { Country }\end{array}$ & $\begin{array}{l}\text { Overall } \\
\text { RoB }\end{array}$ & Outcome & Outcome Description & $\begin{array}{l}\text { Time } \\
\text { Point }\end{array}$ & Group & Subgroup & $\mathrm{n} / \mathrm{N}(\%)$ & $\begin{array}{l}\text { Adjusted Odds Ratio } \\
(95 \% \mathrm{Cl})\end{array}$ & $\begin{array}{l}\mathbf{P} \\
\text { Value }\end{array}$ \\
\hline \multirow{3}{*}{$\begin{array}{l}\text { Hangge, } \\
2013, \\
31606126, \\
\text { US }\end{array}$} & High & $\begin{array}{l}\text { Unplanned repeat } \\
\text { surgery for } \\
\text { complications }\end{array}$ & NR & NR & IBR DTI & All & $\begin{array}{l}81 / 193 \\
(42)\end{array}$ & $\begin{array}{l}\text { vs. AR: } 2.03 \text { (1.03, } \\
3.98)\end{array}$ & 0.042 \\
\hline & High & $\begin{array}{l}\text { Unplanned repeat } \\
\text { surgery for } \\
\text { complications }\end{array}$ & NR & NR & IBR TE & All & $\begin{array}{l}58 / 146 \\
(40)\end{array}$ & $\begin{array}{l}\text { vs. AR: } 1.81 \text { (0.90, } \\
3.64)\end{array}$ & 0.096 \\
\hline & High & $\begin{array}{l}\text { Unplanned repeat } \\
\text { surgery for } \\
\text { complications }\end{array}$ & NR & NR & AR & All & $17 / 60(28)$ & Ref & Ref \\
\hline \multirow{2}{*}{$\begin{array}{l}\text { Mioton, } \\
2013 \\
23562485 \\
\text { US }\end{array}$} & Moderate & $\begin{array}{l}\text { Unplanned repeat } \\
\text { surgery for } \\
\text { complications }\end{array}$ & NR & $1 \mathrm{mo}$ & IBR & All & $\begin{array}{l}662 / 9786 \\
(6.76)\end{array}$ & $1.08(0.88,1.32)$ & NR \\
\hline & Moderate & $\begin{array}{l}\text { Unplanned repeat } \\
\text { surgery for } \\
\text { complications }\end{array}$ & NR & $1 \mathrm{mo}$ & AR & All & $\begin{array}{l}316 / 3296 \\
(9.59)\end{array}$ & Ref & Ref \\
\hline \multirow{2}{*}{$\begin{array}{l}\text { Zhang, } \\
2019, \\
30675702, \\
\text { China }\end{array}$} & High & $\begin{array}{l}\text { Unplanned repeat } \\
\text { surgery for } \\
\text { complications }\end{array}$ & $\begin{array}{l}\text { Urgent surgery for a } \\
\text { compromised implant/flap }\end{array}$ & $4.9 \mathrm{y}$ & IBR & All & $\begin{array}{l}31 / 394 \\
(7.9)\end{array}$ & $\begin{array}{l}\text { vs. AR: } 0.63 \text { (0.29, } \\
1.37)\end{array}$ & NR \\
\hline & High & $\begin{array}{l}\text { Unplanned repeat } \\
\text { surgery for } \\
\text { complications }\end{array}$ & $\begin{array}{l}\text { Urgent surgery for a } \\
\text { compromised implant/flap }\end{array}$ & $4.9 \mathrm{y}$ & AR & All & $\begin{array}{l}33 / 438 \\
(7.5)\end{array}$ & Ref & Ref \\
\hline \multirow{7}{*}{$\begin{array}{l}\text { Kulkarni, } \\
2017, \\
28713853, \\
\text { US \& } \\
\text { Canada }\end{array}$} & Moderate & Pain & VAS: Moderate to severe & $2 y$ & IBR DTI & All & NR & - & - \\
\hline & Moderate & Pain & VAS: Moderate to severe & $2 y$ & IBR TE & All & NR & Ref & Ref \\
\hline & Moderate & Pain & VAS: Moderate to severe & $2 y$ & $\begin{array}{l}\text { AR with } \\
\text { DIEP }\end{array}$ & All & NR & $\begin{array}{l}\text { vs. IBR with TE: } 1.22 \\
(0.73,2.04)^{*}\end{array}$ & 0.45 \\
\hline & Moderate & Pain & VAS: Moderate to severe & $2 y$ & $\begin{array}{l}\text { AR with free } \\
\text { TRAM }\end{array}$ & All & NR & $\begin{array}{l}\text { vs. IBR with TE: } 1.73 \\
(0.73,4.08)^{*}\end{array}$ & 0.21 \\
\hline & Moderate & Pain & VAS: Moderate to severe & $2 y$ & $\begin{array}{l}\text { AR with } \\
\text { pedicled } \\
\text { TRAM } \\
\end{array}$ & All & NR & $\begin{array}{l}\text { vs. IBR with TE: } 1.64 \\
(0.68,3.95)^{\star}\end{array}$ & 0.27 \\
\hline & Moderate & Pain & VAS: Moderate to severe & $2 \mathrm{y}$ & AR with LD & All & NR & $\begin{array}{l}\text { vs. IBR with TE: } 0.94 \\
(0.28,3.14)^{*}\end{array}$ & 0.92 \\
\hline & Moderate & Pain & VAS: Moderate to severe & $2 y$ & $\begin{array}{l}\text { AR with } \\
\text { SIEA }\end{array}$ & All & NR & $\begin{array}{l}\text { vs. IBR with TE: } 1.43 \\
(0.57,3.62)^{*}\end{array}$ & 0.45 \\
\hline
\end{tabular}




\begin{tabular}{|c|c|c|c|c|c|c|c|c|c|}
\hline $\begin{array}{l}\text { Study, } \\
\text { Year, } \\
\text { PMID, } \\
\text { Country }\end{array}$ & $\begin{array}{l}\text { Overall } \\
\text { RoB }\end{array}$ & Outcome & Outcome Description & $\begin{array}{l}\text { Time } \\
\text { Point }\end{array}$ & Group & Subgroup & $n / N(\%)$ & $\begin{array}{l}\text { Adjusted Odds Ratio } \\
(95 \% \mathrm{Cl})\end{array}$ & \begin{tabular}{|l|}
$\mathbf{P}$ \\
Value
\end{tabular} \\
\hline \multirow{2}{*}{$\begin{array}{l}\text { de Araujo, } \\
2016, \\
27673527, \\
\text { US }\end{array}$} & High & $\begin{array}{l}\text { Infections not explicitly } \\
\text { implant-related }\end{array}$ & $\mathrm{SSI}$ & $4.3 \mathrm{y}$ & IBR & All & $\begin{array}{l}\text { NR/38 } \\
\text { (NR) }\end{array}$ & \begin{tabular}{|l|} 
vs. AR $0.86(0.18$, \\
$4.11)$ \\
\end{tabular} & 0.847 \\
\hline & High & $\begin{array}{l}\text { Infections not explicitly } \\
\text { implant-related }\end{array}$ & SSI & $4.3 \mathrm{y}$ & AR & All & $\begin{array}{l}\mathrm{NR} / 32 \\
(\mathrm{NR})\end{array}$ & Ref & Ref \\
\hline \multirow{2}{*}{$\begin{array}{l}\text { Garvey, } \\
2012, \\
23096600, \\
\text { US }\end{array}$} & Moderate & $\begin{array}{l}\text { Infections not explicitly } \\
\text { implant-related }\end{array}$ & Infections & $1.5 \mathrm{y}$ & IBR & All & $\begin{array}{l}50 / 442 \\
\text { breasts } \\
(11.3)\end{array}$ & Ref & Ref \\
\hline & Moderate & $\begin{array}{l}\text { Infections not explicitly } \\
\text { implant-related }\end{array}$ & Infections & $1.5 \mathrm{y}$ & AR & All & $\begin{array}{l}21 / 548 \\
\text { breasts } \\
(3.8)\end{array}$ & vs. IBR: NR & $<0.001$ \\
\hline \multirow{9}{*}{$\begin{array}{l}\text { Kulkarni, } \\
2017, \\
28713853, \\
\text { US \& } \\
\text { Canada }\end{array}$} & Moderate & $\begin{array}{l}\text { Infections not explicitly } \\
\text { implant-related }\end{array}$ & Breast WI & $2 y$ & IBR & All & NR & NR & NR \\
\hline & Moderate & $\begin{array}{l}\text { Infections not explicitly } \\
\text { implant-related }\end{array}$ & Breast WI & $2 y$ & IBR DTI & All & $\begin{array}{l}17 / 112 \\
(15.2) \\
\end{array}$ & - & - \\
\hline & Moderate & $\begin{array}{l}\text { Infections not explicitly } \\
\text { implant-related }\end{array}$ & Breast WI & $2 y$ & IBR TE & All & $\begin{array}{l}159 / 1525 \\
(10.4)\end{array}$ & Ref & Ref \\
\hline & Moderate & $\begin{array}{l}\text { Infections not explicitly } \\
\text { implant-related }\end{array}$ & Breast WI & $2 y$ & AR (all) & All & NR & NR & NR \\
\hline & Moderate & $\begin{array}{l}\text { Infections not explicitly } \\
\text { implant-related }\end{array}$ & Breast WI & $2 y$ & $\begin{array}{l}\text { AR with } \\
\text { DIEP }\end{array}$ & All & $\begin{array}{l}27 / 390 \\
(6.9)\end{array}$ & $\begin{array}{l}\text { vs IBR TE: } 0.44 \text { (0.25, } \\
0.78)\end{array}$ & 0.005 \\
\hline & Moderate & $\begin{array}{l}\text { Infections not explicitly } \\
\text { implant-related }\end{array}$ & Breast WI & $2 y$ & $\begin{array}{l}\text { AR with free } \\
\text { TRAM }\end{array}$ & All & $5 / 95(5.3)$ & $\begin{array}{l}\text { vs IBR TE: } 0.45 \text { (0.17, } \\
1.18)\end{array}$ & 0.10 \\
\hline & Moderate & $\begin{array}{l}\text { Infections not explicitly } \\
\text { implant-related }\end{array}$ & Breast WI & $2 y$ & $\begin{array}{l}\text { AR with } \\
\text { pedicled } \\
\text { TRAM }\end{array}$ & All & $8 / 85(9.4)$ & $\begin{array}{l}\text { vs IBR TE: } 0.73 \text { (0.31, } \\
1.70)\end{array}$ & 0.46 \\
\hline & Moderate & $\begin{array}{l}\text { Infections not explicitly } \\
\text { implant-related }\end{array}$ & Breast WI & $2 y$ & AR with LD & All & $6 / 71(8.5)$ & $\begin{array}{l}\text { vs IBR TE: } 0.50(0.15, \\
1.56)\end{array}$ & 0.23 \\
\hline & Moderate & $\begin{array}{l}\text { Infections not explicitly } \\
\text { implant-related }\end{array}$ & Breast WI & $2 y$ & $\begin{array}{l}\text { AR with } \\
\text { SIEA }\end{array}$ & All & $\begin{array}{l}8 / 65 \\
(12.3)\end{array}$ & $\begin{array}{l}\text { vs IBR TE: } 0.67 \text { (0.25, } \\
1.82)\end{array}$ & 0.43 \\
\hline \multirow{5}{*}{$\begin{array}{l}\text { Mioton, } \\
2013, \\
23562485, \\
\text { US }\end{array}$} & Moderate & $\begin{array}{l}\text { Infections not explicitly } \\
\text { implant-related }\end{array}$ & WI & $1 \mathrm{mo}$ & IBR & All & $\begin{array}{l}338 / 9786 \\
(3.45)\end{array}$ & Ref & Ref \\
\hline & Moderate & $\begin{array}{l}\text { Infections not explicitly } \\
\text { implant-related }\end{array}$ & WI & $1 \mathrm{mo}$ & AR & All & $\begin{array}{l}180 / 3296 \\
(5.46)\end{array}$ & $\begin{array}{l}\text { vs IBR: } 1.40 \text { (1.01, } \\
1.96)\end{array}$ & NR \\
\hline & Moderate & $\begin{array}{l}\text { Infections not explicitly } \\
\text { implant-related }\end{array}$ & Superficial SSI & $1 \mathrm{mo}$ & IBR & All & $\begin{array}{l}163 / 9786 \\
(1.67)\end{array}$ & Ref & Ref \\
\hline & Moderate & $\begin{array}{l}\text { Infections not explicitly } \\
\text { implant-related }\end{array}$ & Superficial SSI & $1 \mathrm{mo}$ & AR & All & $\begin{array}{l}97 / 3296 \\
(2.95) \\
\end{array}$ & $\begin{array}{l}\text { vs IBR: } 1.20 \text { (0.81, } \\
1.76)\end{array}$ & NR \\
\hline & Moderate & $\begin{array}{l}\text { Infections not explicitly } \\
\text { implant-related }\end{array}$ & Deep SSI & $1 \mathrm{mo}$ & IBR & All & $\begin{array}{l}195 / 9786 \\
(1.07)\end{array}$ & Ref & Ref \\
\hline
\end{tabular}

E-12 


\begin{tabular}{|c|c|c|c|c|c|c|c|c|c|}
\hline $\begin{array}{l}\text { Study, } \\
\text { Year, } \\
\text { PMID, } \\
\text { Country }\end{array}$ & $\begin{array}{l}\text { Overall } \\
\text { RoB }\end{array}$ & Outcome & Outcome Description & $\begin{array}{l}\text { Time } \\
\text { Point }\end{array}$ & Group & Subgroup & $n / N(\%)$ & $\begin{array}{l}\text { Adjusted Odds Ratio } \\
(95 \% \mathrm{Cl})\end{array}$ & $\begin{array}{l}\mathbf{P} \\
\text { Value }\end{array}$ \\
\hline & Moderate & $\begin{array}{l}\text { Infections not explicitly } \\
\text { implant-related }\end{array}$ & Deep SSI & $1 \mathrm{mo}$ & AR & All & $\begin{array}{l}65 / 3296 \\
(1.97) \\
\end{array}$ & $\begin{array}{l}\text { vs IBR: } 1.81 \text { (1.12, } \\
2.94)\end{array}$ & NR \\
\hline \multirow{2}{*}{$\begin{array}{l}\text { Naoum, } \\
2020 a, \\
31756414 \\
\text { US }\end{array}$} & High & $\begin{array}{l}\text { Infections not explicitly } \\
\text { implant-related }\end{array}$ & NR & $\begin{array}{l}4-10 \\
y\end{array}$ & IBR & All & $\begin{array}{l}23 / 633 \\
\text { breasts } \\
(3.6)\end{array}$ & Ref & Ref \\
\hline & High & $\begin{array}{l}\text { Infections not explicitly } \\
\text { implant-related }\end{array}$ & NR & $\begin{array}{l}4-10 \\
y\end{array}$ & AR & All & $\begin{array}{l}9 / 342 \\
\text { breasts } \\
(2.6)\end{array}$ & $\begin{array}{l}\text { vs IBR: } 0.77(0.20 \text {, } \\
2.50)\end{array}$ & 0.67 \\
\hline \multirow{3}{*}{$\begin{array}{l}\text { Naoum, } \\
2020 b \\
32607638 \\
\text { US }\end{array}$} & High & $\begin{array}{l}\text { Infections not explicitly } \\
\text { implant-related }\end{array}$ & NR & $\begin{array}{l}4.3- \\
6.3 y\end{array}$ & IBR DTI & All & $\begin{array}{l}7 / 127 \\
(5.5) \\
\end{array}$ & Ref & Ref \\
\hline & High & $\begin{array}{l}\text { Infections not explicitly } \\
\text { implant-related }\end{array}$ & NR & $\begin{array}{l}4.3- \\
6.3 y\end{array}$ & IBR with TE & All & $2 / 88(2.2)$ & Ref & Ref \\
\hline & High & $\begin{array}{l}\text { Infections not explicitly } \\
\text { implant-related }\end{array}$ & NR & $\begin{array}{l}4.3- \\
6.3 y\end{array}$ & AR & All & $\begin{array}{l}11 / 85 \\
(13.0)\end{array}$ & $\begin{array}{l}\text { vs. IBR DTI: } 3.2(0.6 \text {, } \\
16) \\
\text { vs. IBR with TE: } 8.1 \\
(1.7,39)\end{array}$ & $\begin{array}{l}0.20 \\
0.009\end{array}$ \\
\hline \multirow{2}{*}{$\begin{array}{l}\text { Chetta, } \\
2017 \\
28002254 \\
\text { US } \\
\end{array}$} & High & Reconstructive failure & NR & $\begin{array}{l}1.3 \\
\mathrm{mo}\end{array}$ & IBR & All & $\begin{array}{l}1101 / 3746 \\
(29.4) \\
\end{array}$ & Ref & Ref \\
\hline & High & Reconstructive failure & NR & $\begin{array}{l}1.3 \\
\mathrm{mo}\end{array}$ & AR & All & $\begin{array}{l}40 / 935 \\
(4.3) \\
\end{array}$ & $\begin{array}{l}\text { vs IBR: } 0.09(0.07, \\
0.13)\end{array}$ & $<0.001$ \\
\hline \multirow{2}{*}{$\begin{array}{l}\text { Fischer, } \\
2013, \\
23629074, \\
\text { US }\end{array}$} & High & Reconstructive failure & $\begin{array}{l}\text { Unplanned, nonaesthetic } \\
\text { TE/I removal related to a } \\
\text { complication }\end{array}$ & $4 y$ & IBR & All & $4 / 60(7.3)$ & Ref & Ref \\
\hline & High & Reconstructive failure & Flap loss & $4 y$ & AR & All & $\begin{array}{l}2 / 142 \\
(1.3) \\
\end{array}$ & $\begin{array}{l}\text { vs IBR: } 0.19(0.04, \\
0.80)\end{array}$ & 0.03 \\
\hline \multirow{2}{*}{$\begin{array}{l}\text { Garvey, } \\
2012 \\
23096600 \\
\text { US }\end{array}$} & Moderate & Reconstructive failure & NR & $1.5 \mathrm{y}$ & IBR & All & $\begin{array}{l}70 / 442 \\
\text { breasts } \\
(15.8) \\
\end{array}$ & Ref & Ref \\
\hline & Moderate & Reconstructive failure & NR & $1.5 \mathrm{y}$ & AR & All & $\begin{array}{l}8 / 548 \\
\text { breasts } \\
(1.5)\end{array}$ & vs IBR: NR & $<0.001$ \\
\hline
\end{tabular}




\begin{tabular}{|c|c|c|c|c|c|c|c|c|c|}
\hline $\begin{array}{l}\text { Study, } \\
\text { Year, } \\
\text { PMID, } \\
\text { Country }\end{array}$ & $\begin{array}{l}\text { Overall } \\
\text { RoB }\end{array}$ & Outcome & Outcome Description & $\begin{array}{l}\text { Time } \\
\text { Point }\end{array}$ & Group & Subgroup & $\mathrm{n} / \mathrm{N}(\%)$ & $\begin{array}{l}\text { Adjusted Odds Ratio } \\
(95 \% \mathrm{Cl})\end{array}$ & $\begin{array}{l}\mathbf{P} \\
\text { Value }\end{array}$ \\
\hline \multirow{6}{*}{$\begin{array}{l}\text { Kulkarni, } \\
2017, \\
28713853, \\
\text { US \& } \\
\text { Canada }\end{array}$} & Moderate & Reconstructive failure & NR & $2 y$ & IBR (all) & All & NR & Ref & Ref \\
\hline & Moderate & Reconstructive failure & NR & $2 y$ & IBR (all) & Unilateral & $\begin{array}{l}41 / 600 \\
(6.83)\end{array}$ & NR & NR \\
\hline & Moderate & Reconstructive failure & NR & $2 y$ & IBR (all) & Bilateral & $\begin{array}{l}74 / 994 \\
(7.44) \\
\end{array}$ & NR & NR \\
\hline & Moderate & Reconstructive failure & NR & $2 y$ & AR (all) & All & NR & NR & NR \\
\hline & Moderate & Reconstructive failure & NR & $2 y$ & AR (all) & Unilateral & $\begin{array}{l}4 / 317 \\
(1.26)\end{array}$ & $\begin{array}{l}\text { vs. IBR (all): } 0.12 \\
(0.04,0.36)\end{array}$ & $<0.001$ \\
\hline & Moderate & Reconstructive failure & NR & $2 y$ & AR (all) & Bilateral & $\begin{array}{l}4 / 224 \\
(1.87)\end{array}$ & $\begin{array}{l}\text { vs. IBR (all): } 0.14 \\
(0.05,0.45)\end{array}$ & 0.001 \\
\hline \multirow{2}{*}{$\begin{array}{l}\text { Mioton, } \\
2013, \\
23562485, \\
\text { US }\end{array}$} & Moderate & Reconstructive failure & Implant or flap failure & $1 \mathrm{mo}$ & IBR & All & $\begin{array}{l}83 / 9786 \\
(0.85)\end{array}$ & Ref & Ref \\
\hline & Moderate & Reconstructive failure & Implant or flap failure & $1 \mathrm{mo}$ & AR & All & $\begin{array}{l}103 / 3296 \\
(3.13)\end{array}$ & $\begin{array}{l}\text { vs. IBR: } 1.69 \text { (1.08, } \\
2.62)\end{array}$ & NR \\
\hline
\end{tabular}

Abbreviations: adj $=$ adjusted, $\mathrm{AR}=$ autologous reconstruction, $\mathrm{CI}=$ confidence interval, DIEP $=$ deep inferior epigastric perforator, $\mathrm{DTI}=$ direct to implant, $\mathrm{IBR}=$ implant-based reconstruction, $\mathrm{LD}=$ latissimus dorsi, $\mathrm{mo}=$ months, $\mathrm{N} / \mathrm{A}=$ not applicable, $\mathrm{NR}=$ not reported, $\mathrm{NRCS}=$ nonrandomized comparative study, $\mathrm{PMID}=\mathrm{PubMed}$ identifier, Ref $=$ reference group, $\mathrm{RoB}=$ risk of bias, $\mathrm{SIEA}=$ superficial inferior epigastric artery, $\mathrm{SSI}=$ surgical site infection, $\mathrm{TE}=$ tissue expander, TRAM $=$ transverse rectus abdominis myocutaneous, VAS = Visual Analog Scale, WI = wound infection, $\mathrm{y}=$ years.

Colors: Header rows are shaded orange. Rows for every alternate outcome are shaded blue. The colors do not add unique information.

* Confidence interval calculated based on the reported P value.

Table E-1.7. Summary Table - Key Question 1: IBR versus AR - NRCSs, continuous outcomes (pain and analgesic use)

\begin{tabular}{|c|c|c|c|c|c|c|c|c|c|}
\hline $\begin{array}{l}\text { Study, Year, } \\
\text { PMID, } \\
\text { Country }\end{array}$ & Outcome & Outcome Measurement & $\begin{array}{l}\text { Overall } \\
\text { RoB }\end{array}$ & $\begin{array}{l}\text { Time } \\
\text { Point }\end{array}$ & Group & $\mathbf{N}$ & Mean (SD) & $\begin{array}{l}\text { Adjusted Mean Difference } \\
(95 \% \mathrm{Cl})\end{array}$ & P Value \\
\hline \multirow{2}{*}{$\begin{array}{l}\text { Eltahir, 2015, } \\
25539295, \\
\text { Netherlands }\end{array}$} & Pain & SF-36: Pain (0-100) & Moderate & $2.2 \mathrm{y}$ & IBR & NR & NR & Ref & Ref \\
\hline & Pain & SF-36: Pain (0-100) & Moderate & $2.2 \mathrm{y}$ & AR & NR & NR & vs. IBR: $2.40(-5.37,10.2)$ & 0.54 \\
\hline \multirow{2}{*}{$\begin{array}{l}\text { Kouwenberg, } \\
2020 \text {, } \\
32590633 \text {, } \\
\text { Netherlands }\end{array}$} & Pain & EORTC QLQC30 Pain (0-100) & Moderate & $\begin{array}{l}>6 \\
\mathrm{mo}\end{array}$ & IBR & 296 & $15.9(26.3)$ & Ref & Ref \\
\hline & Pain & EORTC QLQC30 Pain (0-100) & Moderate & $\begin{array}{l}>6 \\
\mathrm{mo}\end{array}$ & AR & 179 & $17.2(27.2)$ & NR & NS \\
\hline \multirow{4}{*}{$\begin{array}{l}\text { Kulkarni, } \\
2017, \\
28713853, \\
\text { US \& Canada }\end{array}$} & Pain & MPQ-SF: Sensory (0-10) & Moderate & $1 w$ & IBR (all) & 1846 & NR & Ref & Ref \\
\hline & Pain & MPQ-SF: Sensory (0-10) & Moderate & $1 w$ & IBR Direct & NR & NR & NR & NR \\
\hline & Pain & MPQ-SF: Sensory (0-10) & Moderate & $1 \mathrm{w}$ & IBR TE & NR & NR & NR & NR \\
\hline & Pain & MPQ-SF: Sensory (0-10) & Moderate & $1 w$ & AR (all) & 463 & NR & NR & NR \\
\hline
\end{tabular}




\begin{tabular}{|c|c|c|c|c|c|c|c|c|c|}
\hline $\begin{array}{l}\text { Study, Year, } \\
\text { PMID, } \\
\text { Country }\end{array}$ & Outcome & Outcome Measurement & $\begin{array}{l}\text { Overall } \\
\text { RoB }\end{array}$ & $\begin{array}{l}\text { Time } \\
\text { Point }\end{array}$ & Group & $\mathbf{N}$ & Mean (SD) & $\begin{array}{l}\text { Adjusted Mean Difference } \\
(95 \% \mathrm{Cl})\end{array}$ & P Value \\
\hline & Pain & MPQ-SF: Sensory $(0-10)$ & Moderate & $1 \mathrm{w}$ & AR with DIEP & 111 & NR & $\begin{array}{l}\text { vs. IBR (all): }-1.20(-2.11 \\
-0.29)^{*}\end{array}$ & 0.01 \\
\hline & Pain & MPQ-SF: Sensory (0-10) & Moderate & $1 \mathrm{w}$ & AR with free TRAM & 94 & NR & vs. IBR (all): $0.26(-1.16,1.69)^{*}$ & 0.72 \\
\hline & Pain & MPQ-SF: Sensory (0-10) & Moderate & $1 \mathrm{w}$ & $\begin{array}{l}\text { AR with pedicled } \\
\text { TRAM }\end{array}$ & NR & NR & $\begin{array}{l}\text { vs. IBR (all): }-1.04(-2.53 \\
0.45)^{*}\end{array}$ & 0.17 \\
\hline & Pain & MPQ-SF: Sensory $(0-10)$ & Moderate & $1 \mathrm{w}$ & AR with LD & 80 & NR & vs. IBR (all): $0.35(-1.37,2.07)^{*}$ & 0.69 \\
\hline & Pain & MPQ-SF: Sensory $(0-10)$ & Moderate & $1 \mathrm{w}$ & AR with SIEA & 73 & NR & vs. IBR (all): $2.41(0.38,4.44)^{\star}$ & 0.02 \\
\hline & Pain & MPQ-SF: Sensory $(0-10)$ & Moderate & $3 \mathrm{mo}$ & IBR (all) & NR & NR & NR & NR \\
\hline & Pain & MPQ-SF: Sensory $(0-10)$ & Moderate & $3 \mathrm{mo}$ & IBR Direct & 96 & 4.2 (NR) & - & - \\
\hline & Pain & MPQ-SF: Sensory $(0-10)$ & Moderate & $3 \mathrm{mo}$ & IBR TE & 1329 & $5.7(\mathrm{NR})$ & Ref & Ref \\
\hline & Pain & MPQ-SF: Sensory $(0-10)$ & Moderate & $3 \mathrm{mo}$ & AR (all) & NR & NR & NR & NR \\
\hline & Pain & MPQ-SF: Sensory $(0-10)$ & Moderate & $3 \mathrm{mo}$ & AR with DIEP & 296 & $4.8(\mathrm{NR})$ & vs. IBR TE: $1.10(0.35,1.85)^{\star}$ & 0.004 \\
\hline & Pain & MPQ-SF: Sensory $(0-10)$ & Moderate & $3 \mathrm{mo}$ & AR with free TRAM & 83 & $6.7(\mathrm{NR})$ & vs. IBR TE: 2.48 (NR) & $<0.001$ \\
\hline & Pain & MPQ-SF: Sensory $(0-10)$ & Moderate & $3 \mathrm{mo}$ & $\begin{array}{l}\text { AR with pedicled } \\
\text { TRAM }\end{array}$ & 91 & $5(\mathrm{NR})$ & vs. IBR TE: $1.19(-0.14,2.52)^{\star}$ & 0.08 \\
\hline & Pain & MPQ-SF: Sensory $(0-10)$ & Moderate & $3 \mathrm{mo}$ & AR with LD & 62 & 5.4 (NR) & vs. IBR TE: $0.42(-1.19,2.03)^{*}$ & 0.61 \\
\hline & Pain & MPQ-SF: Sensory $(0-10)$ & Moderate & $3 \mathrm{mo}$ & AR with SIEA & 56 & NR & vs. IBR TE: $2.37(0.81,3.94)^{*}$ & 0.003 \\
\hline & Pain & MPQ-SF: Affective (0-10) & Moderate & $1 \mathrm{w}$ & IBR (all) & 1846 & NR & Ref & Ref \\
\hline & Pain & MPQ-SF: Affective (0-10) & Moderate & $1 \mathrm{w}$ & IBR Direct & NR & NR & NR & NR \\
\hline & Pain & MPQ-SF: Affective (0-10) & Moderate & $1 \mathrm{w}$ & IBR TE & NR & NR & NR & NR \\
\hline & Pain & MPQ-SF: Affective (0-10) & Moderate & $1 \mathrm{w}$ & AR (all) & NR & NR & NR & NR \\
\hline & Pain & MPQ-SF: Affective (0-10) & Moderate & $1 \mathrm{w}$ & AR with DIEP & 463 & NR & vs. IBR (all): $0.24(-0.10,0.58)^{*}$ & 0.16 \\
\hline & Pain & MPQ-SF: Affective (0-10) & Moderate & $1 \mathrm{w}$ & AR with free TRAM & 111 & NR & vs. IBR (all): $0.37(-0.15,0.89)^{*}$ & 0.16 \\
\hline & Pain & MPQ-SF: Affective (0-10) & Moderate & $1 \mathrm{w}$ & $\begin{array}{l}\text { AR with pedicled } \\
\text { TRAM }\end{array}$ & 94 & NR & $\begin{array}{l}\text { vs. IBR (all): }-0.01(-0.79 \\
0.77)^{*}\end{array}$ & 0.98 \\
\hline & Pain & MPQ-SF: Affective (0-10) & Moderate & $1 \mathrm{w}$ & AR with LD & 80 & NR & vs. IBR (all): $0.47(-0.17,1.11)^{*}$ & 0.15 \\
\hline & Pain & MPQ-SF: Affective (0-10) & Moderate & $1 \mathrm{w}$ & AR with SIEA & 73 & NR & $\begin{array}{l}\text { vs. IBR (all): }-0.03(-0.97 \\
0.91)\end{array}$ & 0.95 \\
\hline \multirow{10}{*}{$\begin{array}{l}\text { Kulkarni, } \\
\text { continued }\end{array}$} & Pain & MPQ-SF: Affective (0-10) & Moderate & $2 y$ & IBR (all) & 1263 & NR & NR & NR \\
\hline & Pain & MPQ-SF: Affective (0-10) & Moderate & $2 y$ & IBR Direct & 93 & NR & - & - \\
\hline & Pain & MPQ-SF: Affective (0-10) & Moderate & $2 y$ & IBR TE & 1263 & NR & Ref & Ref \\
\hline & Pain & MPQ-SF: Affective (0-10) & Moderate & $2 y$ & AR (all) & NR & NR & NR & NR \\
\hline & Pain & MPQ-SF: Affective (0-10) & Moderate & $2 y$ & AR with DIEP & 350 & NR & vs. IBR TE: $0.33(0.07,0.59)^{\star}$ & 0.013 \\
\hline & Pain & MPQ-SF: Affective (0-10) & Moderate & $2 y$ & AR with free TRAM & 87 & NR & vs. IBR TE: 0.84 (NR) & $<0.001$ \\
\hline & Pain & MPQ-SF: Affective (0-10) & Moderate & $2 y$ & $\begin{array}{l}\text { AR with pedicled } \\
\text { TRAM }\end{array}$ & 77 & NR & vs. IBR TE: $0.04(-0.47,0.55)^{\star}$ & 0.877 \\
\hline & Pain & MPQ-SF: Affective (0-10) & Moderate & $2 \mathrm{y}$ & AR with LD & 64 & NR & $\begin{array}{l}\text { vs. IBR TE: }-0.13(-0.66 \text {, } \\
0.40)^{*}\end{array}$ & 0.63 \\
\hline & Pain & MPQ-SF: Affective (0-10) & Moderate & $2 y$ & AR with SIEA & 62 & NR & vs. IBR TE: 1.24 (NR) & $<0.0001$ \\
\hline & Pain & VAS $(0-10)$ & Moderate & $1 \mathrm{w}$ & IBR (all) & 1846 & NR & Ref & Ref \\
\hline
\end{tabular}




\begin{tabular}{|c|c|c|c|c|c|c|c|c|c|}
\hline $\begin{array}{l}\text { Study, Year, } \\
\text { PMID, } \\
\text { Country }\end{array}$ & Outcome & Outcome Measurement & $\begin{array}{l}\text { Overall } \\
\text { RoB }\end{array}$ & $\begin{array}{l}\text { Time } \\
\text { Point }\end{array}$ & Group & $\mathbf{N}$ & Mean (SD) & $\begin{array}{l}\text { Adjusted Mean Difference } \\
(95 \% \mathrm{Cl})\end{array}$ & P Value \\
\hline & Pain & VAS $(0-10)$ & Moderate & $1 \mathrm{w}$ & IBR Direct & NR & NR & NR & NR \\
\hline & Pain & VAS (0-10) & Moderate & $1 \mathrm{w}$ & IBR TE & NR & NR & NR & NR \\
\hline & Pain & VAS (0-10) & Moderate & $1 \mathrm{w}$ & AR (all) & NR & NR & NR & NR \\
\hline & Pain & VAS (0-10) & Moderate & $1 \mathrm{w}$ & AR with DIEP & 463 & NR & $\begin{array}{l}\text { vs. IBR (all): }-0.18(-0.49 \\
0.13)^{*}\end{array}$ & 0.25 \\
\hline & Pain & VAS $(0-10)$ & Moderate & $1 \mathrm{w}$ & AR with free TRAM & 111 & NR & $\begin{array}{l}\text { vs. IBR (all): }-0.19(-0.68 \\
0.30)^{*}\end{array}$ & 0.45 \\
\hline & Pain & VAS (0-10) & Moderate & $1 \mathrm{w}$ & $\begin{array}{l}\text { AR with pedicled } \\
\text { TRAM }\end{array}$ & 94 & NR & $\begin{array}{l}\text { vs. IBR (all): }-0.72(-1.27 \\
-0.17)^{*}\end{array}$ & 0.01 \\
\hline & Pain & VAS $(0-10)$ & Moderate & $1 \mathrm{w}$ & AR with LD & 80 & NR & vs. IBR (all): $0.01(-0.51,0.53)^{*}$ & 0.97 \\
\hline & Pain & VAS $(0-10)$ & Moderate & $1 \mathrm{w}$ & AR with SIEA & 73 & NR & vs. IBR (all): $0.21(-0.42,0.84)^{*}$ & 0.51 \\
\hline \multirow{8}{*}{$\begin{array}{l}\text { Roth, 2007, } \\
17413877, \\
\text { US }\end{array}$} & Pain & VAS: Bodily pain (1-5) & High & $2 y$ & IBR & 48 & $2.2(1.2)$ & Ref & Ref \\
\hline & Pain & VAS: Bodily pain (1-5) & High & $2 y$ & $\mathrm{AR}$ & 159 & $2.2(1.2)$ & vs IBR: NR & NS \\
\hline & Pain & VAS: Breast pain (1-5) & High & $2 y$ & IBR & 48 & $2.1(1.3)$ & Ref & Ref \\
\hline & Pain & VAS: Breast pain (1-5) & High & $2 y$ & AR & 159 & $1.8(1.1)$ & vs IBR: NR & NS \\
\hline & Pain & VAS: Abdominal pain (1-5) & High & $2 y$ & IBR & 48 & $4.8(0.8)$ & Ref & Ref \\
\hline & Pain & VAS: Abdominal pain (1-5) & High & $2 y$ & AR & 159 & $4.0(1.2)$ & vs IBR: NR & $<0.0001$ \\
\hline & Pain & VAS: Back pain (1-5) & High & $2 y$ & IBR & 48 & $4.0(1.3)$ & Ref & Ref \\
\hline & Pain & VAS: Back pain (1-5) & High & $2 y$ & $\mathrm{AR}$ & 159 & $3.7(1.5)$ & vs IBR: NR & NS \\
\hline \multirow{6}{*}{$\begin{array}{l}\text { Shiraishi, } \\
2020, \\
32589082, \\
\text { Japan }\end{array}$} & Pain & MPQ-SF: Total $(0-10)$ & High & $1 \mathrm{y}$ & IBR & 56 & NR & Ref & Ref \\
\hline & Pain & MPQ-SF: Total (0-10) & High & $1 \mathrm{y}$ & AR & 34 & NR & $1.08(\mathrm{NR}, \mathrm{NR})$ & NR \\
\hline & Pain & MPQ-SF: Sensory $(0-10)$ & High & $1 \mathrm{y}$ & IBR & 56 & NR & Ref & Ref \\
\hline & Pain & MPQ-SF: Sensory (0-10) & High & $1 \mathrm{y}$ & AR & 34 & NR & 0.80 (NR, NR) & NR \\
\hline & Pain & MPQ-SF: Affective (0-10) & High & $1 \mathrm{y}$ & IBR & 56 & NR & Ref & Ref \\
\hline & Pain & MPQ-SF: Affective $(0-10)$ & High & $1 \mathrm{y}$ & $\mathrm{AR}$ & 34 & NR & 0.28 (NR, NR) & NR \\
\hline \multirow{2}{*}{$\begin{array}{l}\text { Shiraishi, } \\
2020, \\
32589082, \\
\text { Japan }\end{array}$} & $\begin{array}{l}\text { Analgesic } \\
\text { use }\end{array}$ & Analgesic use score $(0-5)$ & High & $1 \mathrm{y}$ & IBR & 56 & NR & Ref & Ref \\
\hline & $\begin{array}{l}\text { Analgesic } \\
\text { use }\end{array}$ & Analgesic use score (0-5) & High & $1 \mathrm{y}$ & AR & 34 & NR & 0.37 (NR, NR) & NR \\
\hline
\end{tabular}

Abbreviations: adj = adjusted, CI = confidence interval, DIEP = deep inferior epigastric perforator, LD = latissimus dorsi, MPQ-SF = McGill Pain Questionnaire-Short Form, $\mathrm{MD}=$ mean difference, MOS SF $=$ Medical Outcomes Study Short Form, MPQ-SF $=$ McGill Pain Questionnaire Short Form, NR $=$ not reported, NRCS $=$ nonrandomized comparative study, PMID = PubMed identifier, Ref = reference group, RoB = risk of bias, SD = standard deviation, SIEA = superficial inferior epigastric artery, TE = tissue expander, TRAM $=$ transverse rectus abdominis myocutaneous, VAS = Visual Analog Scale.

Colors: Header rows are shaded orange. Rows for every alternate outcome are shaded blue. The colors do not add unique information.

* Confidence interval calculated based on the reported $\mathrm{P}$ value. 
Table E-2.1. Summary Table - Key Question 2b: Timing of IBR in relation to radiation therapy - NRCSs, continuous outcomes (various)

\begin{tabular}{|c|c|c|c|c|c|c|c|c|c|c|}
\hline $\begin{array}{l}\text { Study, Year, } \\
\text { PMID, Country }\end{array}$ & $\begin{array}{l}\text { Overall } \\
\text { RoB }\end{array}$ & Outcome & Outcome Measurement & $\begin{array}{l}\text { Time } \\
\text { Point }\end{array}$ & $\begin{array}{l}\text { IBR } \\
\text { Before } \\
\text { Radiation, } \\
\text { N }\end{array}$ & $\begin{array}{l}\text { IBR } \\
\text { Before } \\
\text { Radiation, } \\
\text { Mean } \\
\text { (SD) }\end{array}$ & $\begin{array}{l}\text { IBR After } \\
\text { Radiation, } \\
\text { N }\end{array}$ & $\begin{array}{l}\text { IBR After } \\
\text { Radiation, } \\
\text { Mean } \\
\text { (SD) }\end{array}$ & $\begin{array}{l}\text { IBR Before } \\
\text { Versus After } \\
\text { Radiation, } \\
\text { Adjusted } \\
\text { MD ( } 95 \% \mathrm{Cl})\end{array}$ & $\begin{array}{l}P \\
\text { Value }\end{array}$ \\
\hline $\begin{array}{l}\text { Cordeiro, 2015, } \\
30270015, \text { US }\end{array}$ & High & Physical WB & $\begin{array}{l}\text { BREAST-Q: Physical WB } \\
(0-100)\end{array}$ & $3.3 \mathrm{y}$ & 84 & $72.5(2.6)$ & 22 & $73.4(1.9)$ & NR & NS \\
\hline \multirow{2}{*}{$\begin{array}{l}\text { Yoon, 2020, } \\
\text { 32332528, US \& } \\
\text { Canada }\end{array}$} & Moderate & Physical WB & $\begin{array}{l}\text { BREAST-Q: Physical WB } \\
(0-100)\end{array}$ & $2 y$ & 80 & NR & 237 & NR & $\begin{array}{l}-0.64(-7.19, \\
5.90)\end{array}$ & 0.84 \\
\hline & Moderate & Physical WB & $\begin{array}{l}\text { PROMIS: Physical } \\
\text { function }(0-100)\end{array}$ & $2 y$ & 80 & NR & 237 & NR & $\begin{array}{l}-0.04(-2.40, \\
2.32)\end{array}$ & 0.97 \\
\hline $\begin{array}{l}\text { Yoon, 2020, } \\
\text { 32332528, US \& } \\
\text { Canada }\end{array}$ & Moderate & $\begin{array}{l}\text { Psychosocial } \\
\text { WB }\end{array}$ & $\begin{array}{l}\text { BREAST-Q: Psychosocial } \\
\text { WB }(0-100)\end{array}$ & $2 y$ & 80 & NR & 237 & NR & $\begin{array}{l}0.48(-7.72, \\
8.68)\end{array}$ & 0.91 \\
\hline $\begin{array}{l}\text { Cordeiro, 2015, } \\
\text { 30270015, US }\end{array}$ & High & Sexual WB & $\begin{array}{l}\text { BREAST-Q: Sexual WB } \\
(0-100)\end{array}$ & $3.3 \mathrm{y}$ & 84 & $54.0(0.9)$ & 22 & $55.4(0.7)$ & NR & $<0.01$ \\
\hline \multirow{2}{*}{$\begin{array}{l}\text { Yoon, 2020, } \\
\text { 32332528, US \& } \\
\text { Canada }\end{array}$} & Moderate & Sexual WB & $\begin{array}{l}\text { BREAST-Q: Sexual WB } \\
(0-100)\end{array}$ & $2 y$ & 80 & NR & 237 & NR & $\begin{array}{l}-1.00(-8.41, \\
6.40)\end{array}$ & 0.78 \\
\hline & Moderate & Sexual WB & EORTC: Sexual function & $2 y$ & 80 & NR & 237 & NR & $\begin{array}{l}-1.40(-8.58 \\
5.77)\end{array}$ & 0.70 \\
\hline $\begin{array}{l}\text { Cordeiro, 2015, } \\
30270015, \text { US }\end{array}$ & High & $\begin{array}{l}\text { Satisfaction } \\
\text { with outcome }\end{array}$ & $\begin{array}{l}\text { BREAST-Q: Satisfaction } \\
\text { with outcome }(0-100)\end{array}$ & $3.3 \mathrm{y}$ & 84 & $68.4(3.8)$ & 22 & $70.2(3.0)$ & NR & 0.02 \\
\hline $\begin{array}{l}\text { Yoon, 2020, } \\
\text { 32332528, US \& } \\
\text { Canada }\end{array}$ & Moderate & Pain & $\begin{array}{l}\text { PROMIS: Pain } \\
\text { interference }(0-100)\end{array}$ & $2 y$ & 80 & NR & 237 & NR & $\begin{array}{l}2.86(-1.05, \\
6.77)\end{array}$ & 0.14 \\
\hline
\end{tabular}

Abbreviations: CI = confidence interval, EORTC $=$ European Organization for Research and Treatment of Cancer, IBR = implant-based reconstruction, NR $=$ not reported, $\mathrm{NRCS}=$ nonrandomized comparative study, $\mathrm{NS}=$ not significant, $\mathrm{PMID}=$ PubMed identifier, PROMIS $=$ Patient-Reported Outcomes Measurement Information System, RoB $=$ risk of bias, $\mathrm{SD}=$ standard deviation, $\mathrm{WB}=$ well-being, $\mathrm{y}=$ years.

Colors: Header rows are shaded orange. Rows for every alternate outcome are shaded blue. The colors do not add unique information. 
Table E-2.2. Summary Table - Key Question 2b: Timing of IBR in relation to radiation therapy - NRCSs, categorical outcomes (various)

\begin{tabular}{|c|c|c|c|c|c|c|c|c|}
\hline $\begin{array}{l}\text { Study, Year, PMID, } \\
\text { Country }\end{array}$ & $\begin{array}{l}\text { Overall } \\
\text { RoB }\end{array}$ & Outcome & $\begin{array}{l}\text { Outcome } \\
\text { Measurement }\end{array}$ & $\begin{array}{l}\text { Time } \\
\text { Point }\end{array}$ & $\begin{array}{l}\text { IBR Before } \\
\text { Radiation } \\
(\%)\end{array}$ & $\begin{array}{l}\text { IBR After } \\
\text { Radiation (\%) }\end{array}$ & $\begin{array}{l}\text { Effect Size }(95 \% \mathrm{Cl}) \\
\text { Before Versus After } \\
\text { Radiation }\end{array}$ & $\begin{array}{l}P \\
\text { Value }\end{array}$ \\
\hline $\begin{array}{l}\text { Eriksson, } 2013, \\
24258257, \text { Sweden }\end{array}$ & High & $\begin{array}{l}\text { Unplanned repeat surgeries } \\
\text { for revision }\end{array}$ & NR & $3.6 \mathrm{y}$ & NR & NR & adjHR $0.94(0.63,1.40)$ & NR \\
\hline $\begin{array}{l}\text { Hirsch, 2014, } \\
25347643, \text { US }\end{array}$ & High & Necrosis & NR & $3.1 \mathrm{y}$ & NR & NR & adjOR $0.96(0.68,1.35)$ & 0.94 \\
\hline \multirow{2}{*}{$\begin{array}{l}\text { Yoon, 2020, } \\
\text { 32332528, US \& } \\
\text { Canada }\end{array}$} & Moderate & $\begin{array}{l}\text { Infections (not explicitly } \\
\text { implant-related) }\end{array}$ & $\begin{array}{l}\text { Major (IV } \\
\text { antibiotics) }\end{array}$ & $2 y$ & $5 / 46(10.9 \%)$ & $7 / 104(6.7 \%)$ & NR & 0.40 \\
\hline & Moderate & $\begin{array}{l}\text { Infections (not explicitly } \\
\text { implant-related) }\end{array}$ & $\begin{array}{l}\text { Minor (oral } \\
\text { antibiotics) }\end{array}$ & $2 \mathrm{y}$ & $3 / 46(6.5 \%)$ & $7 / 104(6.7 \%)$ & NR & 0.96 \\
\hline $\begin{array}{l}\text { Yoon, 2020, } \\
\text { 32332528, US \& } \\
\text { Canada }\end{array}$ & Moderate & Wound dehiscence & NR & $2 y$ & $0 / 46(0 \%)$ & $5 / 104(4.8 \%)$ & NR & 0.32 \\
\hline $\begin{array}{l}\text { Yoon, 2020, } \\
\text { 32332528, US \& } \\
\text { Canada }\end{array}$ & Moderate & Seroma & NR & $2 y$ & $2 / 46(4.4 \%)$ & $8 / 104(7.7 \%)$ & NR & 0.46 \\
\hline $\begin{array}{l}\text { Yoon, 2020, } \\
\text { 32332528, US \& } \\
\text { Canada }\end{array}$ & Moderate & Capsular contracture & NR & $2 y$ & $1 / 46(2.2 \%)$ & $3 / 104(2.9 \%)$ & NR & 0.80 \\
\hline $\begin{array}{l}\text { Hirsch, 2014, } \\
\text { 25347643, US }\end{array}$ & High & Hematoma & NR & $3.1 \mathrm{y}$ & NR & NR & adjOR $0.56(0.22,1.45)$ & 0.39 \\
\hline $\begin{array}{l}\text { Yoon, 2020, } \\
\text { 32332528, US \& } \\
\text { Canada }\end{array}$ & Moderate & Hematoma & NR & $2 y$ & $1 / 46(2.2 \%)$ & $4 / 104(3.9 \%)$ & NR & 0.63 \\
\hline \multirow{3}{*}{$\begin{array}{l}\text { Hirsch, 2014, } \\
\text { 25347643, US }\end{array}$} & High & Composite/unspecified harms & Any complication & $3.5 \mathrm{y}$ & NR & NR & adjOR $0.81(0.56,1.17)$ & NR \\
\hline & High & Composite/unspecified harms & $\begin{array}{l}\text { Operative } \\
\text { complications }\end{array}$ & $3.5 y$ & NR & NR & adjOR $0.92(0.59,1.45)$ & NR \\
\hline & High & Composite/unspecified harms & $\begin{array}{l}\text { Nonoperative } \\
\text { complications }\end{array}$ & $3.5 \mathrm{y}$ & NR & NR & adjOR $0.90(0.60,1.34)$ & NR \\
\hline \multirow[t]{3}{*}{$\begin{array}{l}\text { Stein, 2020, } \\
\text { 32561384, Canada }\end{array}$} & High & Composite/unspecified harms & Any complication & $\begin{array}{l}10 \mathrm{mo}- \\
5 \mathrm{y}\end{array}$ & NR/76 (NR) & NR/54 (NR) & $\operatorname{adjOR~} 0.82(0.03,2.19)$ & 0.69 \\
\hline & High & Composite/unspecified harms & $\begin{array}{l}\text { Major } \\
\text { complications }\end{array}$ & $\begin{array}{l}10 \mathrm{mo}- \\
5 \mathrm{y}\end{array}$ & NR/76 (NR) & NR/54 (NR) & adjOR $0.62(0.21,1.86)$ & 0.40 \\
\hline & High & Composite/unspecified harms & $\begin{array}{l}\text { Minor } \\
\text { complications }\end{array}$ & $\begin{array}{l}10 \mathrm{mo}- \\
5 \mathrm{y}\end{array}$ & NR/76 (NR) & NR/54 (NR) & adjOR $1.29(0.41,4.03)$ & 0.65 \\
\hline \multirow{2}{*}{$\begin{array}{l}\text { Yoon, 2020, } \\
\text { 32332528, US \& } \\
\text { Canada }\end{array}$} & Moderate & Composite/unspecified harms & Any complication & $2 y$ & $33 / 80(41.3)$ & $95 / 237(40.1)$ & NR & 0.85 \\
\hline & Moderate & Composite/unspecified harms & $\begin{array}{l}\text { Major } \\
\text { complications }\end{array}$ & $2 y$ & $26 / 80(32.5)$ & $82 / 237(34.6)$ & NR & 0.73 \\
\hline
\end{tabular}

Abbreviations: adj = adjusted, $\mathrm{CI}=$ confidence interval, hosp. $=$ hospitalization, $\mathrm{HR}=$ hazard ratio, IBR $=$ implant-based reconstruction, $\mathrm{IV}=$ intravenous, $\mathrm{NR}=$ not reported, $\mathrm{NRCS}=$ nonrandomized comparative study, $\mathrm{NS}=$ not significant, $\mathrm{OR}=$ odds ratio, $\mathrm{PMID}=$ PubMed identifier, $\mathrm{RoB}=$ risk of bias, $\mathrm{y}=$ years

Colors: Header rows are shaded orange. Rows for every alternate outcome are shaded blue. The colors do not add unique information. 
Table E-3.1. Summary Table - Key Question 3: Comparisons of implant materials for IBR - NRCSs, continuous outcomes (general quality of life, physical well-being, psychosocial well-being, sexual well-being, satisfaction with outcome, and satisfaction with breasts)

\begin{tabular}{|c|c|c|c|c|c|c|c|c|c|c|}
\hline $\begin{array}{l}\text { Study, Year, } \\
\text { PMID }\end{array}$ & RoB & Outcome & Outcome Measurement & $\begin{array}{l}\text { Time } \\
\text { Point }(y)\end{array}$ & $\begin{array}{l}\text { Silicone, } \\
\mathbf{N}\end{array}$ & $\begin{array}{l}\text { Silicone, } \\
\text { Mean } \\
\text { (SD) }\end{array}$ & $\begin{array}{l}\text { Saline, } \\
\mathrm{N}\end{array}$ & $\begin{array}{l}\text { Saline, } \\
\text { Mean (SD) }\end{array}$ & $\begin{array}{l}\text { Effect Size } \\
(95 \% \mathrm{CI})\end{array}$ & Palue \\
\hline \multirow{6}{*}{$\begin{array}{l}\text { Macadam, 2010, } \\
\text { 20009795, } \\
\text { Canada }\end{array}$} & High & $\begin{array}{l}\text { General quality } \\
\text { of life }\end{array}$ & $\begin{array}{l}\text { EORTC QLQC30 (0- } \\
\text { 100): Global health status }\end{array}$ & $2.6-4.5$ & 72 & $79.9(18.1)$ & 67 & $74.9(20.9)$ & NR & 0.13 \\
\hline & High & $\begin{array}{l}\text { Physical } \\
\text { WB }\end{array}$ & $\begin{array}{l}\text { BREAST-Q (0-100): } \\
\text { Physical WB }\end{array}$ & $2.6-4.5$ & 74 & $76.2(14.9)$ & 68 & $73.4(16.3)$ & NR & 0.28 \\
\hline & High & $\begin{array}{l}\text { Psychosocial } \\
\text { WB }\end{array}$ & $\begin{array}{l}\text { BREAST-Q (0-100): } \\
\text { Psychosocial WB }\end{array}$ & $2.6-4.5$ & 75 & $77.6(18.6)$ & 67 & $70.8(18.8)$ & NR & 0.03 \\
\hline & High & $\begin{array}{l}\text { Sexual } \\
\text { WB }\end{array}$ & $\begin{array}{l}\text { BREAST-Q (0-100): } \\
\text { Sexual WB }\end{array}$ & $2.6-4.5$ & 71 & $54.4(19.8)$ & 65 & $47.6(20.9)$ & NR & 0.056 \\
\hline & High & $\begin{array}{l}\text { Satisfaction } \\
\text { with outcome }\end{array}$ & $\begin{array}{l}\text { BREAST-Q }(0-100) \text { : } \\
\text { Satisfaction with outcome }\end{array}$ & $2.6-4.5$ & 75 & $75.4(17.6)$ & 68 & $69.5(22.6)$ & NR & 0.082 \\
\hline & High & $\begin{array}{l}\text { Satisfaction } \\
\text { with breasts }\end{array}$ & $\begin{array}{l}\text { BREAST-Q }(0-100) \text { : } \\
\text { Satisfaction with breast }\end{array}$ & $2.6-4.5$ & 75 & $63.8(15.2)$ & 67 & $56.9(15.1)$ & NR & 0.008 \\
\hline $\begin{array}{l}\text { McCarthy, 2010, } \\
21136577, \text { US \& } \\
\text { Canada }\end{array}$ & High & $\begin{array}{l}\text { Satisfaction } \\
\text { with breasts }\end{array}$ & $\begin{array}{l}\text { BREAST-Q }(0-100) \text { : } \\
\text { Satisfaction with breast }\end{array}$ & $2.4-3.3$ & 176 & $58.0(20.3)$ & 306 & $52.5(20.4)$ & $\begin{array}{l}\text { adjMD } 4.1 \\
\left(1.31^{*}, 6.89^{*}\right)\end{array}$ & 0.004 \\
\hline
\end{tabular}

Abbreviations: adj = adjusted, CI = confidence interval, EORTC QLQC3 = European Organization for Research and Treatment of Cancer Quality of Life Questionnaire C30, $\mathrm{IBR}=$ implant-based reconstruction, $\mathrm{MD}=$ mean difference, $\mathrm{NR}=$ not reported, $\mathrm{NRCS}=$ nonrandomized comparative study, $\mathrm{PMID}=\mathrm{PubMed}$ identifier, $\mathrm{RoB}=$ risk of bias, $\mathrm{SD}=$ standard deviation, $\mathrm{WB}=$ well-being, $\mathrm{y}=$ years

Colors: Header rows are shaded orange. Rows for every alternate outcome are shaded blue. The colors do not add unique information.

* Calculated 
Table E-3.2. Summary Table - Key Question 3: Comparisons of implant materials for IBR - NRCSs, categorical outcomes (mortality, implant failure/loss, and capsular contracture)

\begin{tabular}{|c|c|c|c|c|c|c|c|c|}
\hline $\begin{array}{l}\text { Study, Year, } \\
\text { PMID, } \\
\text { Country }\end{array}$ & $\begin{array}{l}\text { Overall } \\
\text { RoB }\end{array}$ & Outcome & $\begin{array}{l}\text { Outcome } \\
\text { Measurement }\end{array}$ & $\begin{array}{l}\text { Time } \\
\text { Point } \\
\text { (y) }\end{array}$ & $\begin{array}{l}\text { Implant } \\
\text { Material }\end{array}$ & $\begin{array}{l}n / N \\
(\%)\end{array}$ & Effect Size (95\% Cl) & $\begin{array}{l}\mathbf{P} \\
\text { Value }\end{array}$ \\
\hline \multirow{6}{*}{$\begin{array}{l}\text { Le, 2005, } \\
15743498, \text { US }\end{array}$} & High & Mortality & Breast cancer mortality & 12.4 & Silicone & NR & Ref & Ref \\
\hline & High & Mortality & Breast cancer mortality & 12.4 & Saline & NR & vs. Silicone: adjHR $1.01(0.44,2.34)$ & NR \\
\hline & High & Mortality & Breast cancer mortality & 12.4 & $\begin{array}{l}\text { Double } \\
\text { lumen }\end{array}$ & NR & vs. Silicone: adjHR $1.49(0.83,2.70)$ & NR \\
\hline & High & Mortality & $\begin{array}{l}\text { Non-breast cancer } \\
\text { mortality }\end{array}$ & 12.4 & Silicone & NR & Ref & Ref \\
\hline & High & Mortality & $\begin{array}{l}\text { Non-breast cancer } \\
\text { mortality }\end{array}$ & 12.4 & Saline & NR & vs. Silicone: adjHR $1.75(0.29,10.39)$ & NR \\
\hline & High & Mortality & $\begin{array}{l}\text { Non-breast cancer } \\
\text { mortality }\end{array}$ & 12.4 & $\begin{array}{l}\text { Double } \\
\text { lumen }\end{array}$ & NR & vs. Silicone: adjHR $3.13(0.91,10.78)$ & NR \\
\hline \multirow{2}{*}{$\begin{array}{l}\text { Cordeiro, } \\
2015 a, \\
25742523 \text {, US }\end{array}$} & High & $\begin{array}{l}\text { Implant } \\
\text { failure/loss }\end{array}$ & TE and implant loss & 3.3 & Silicone & $\begin{array}{l}\text { NR/15 } \\
9\end{array}$ & adjOR $0.61(0.36,1.07)$ & NS \\
\hline & High & $\begin{array}{l}\text { Implant } \\
\text { failure/loss }\end{array}$ & TE and implant loss & 3.3 & Saline & $\begin{array}{l}\text { NR/12 } \\
9\end{array}$ & Ref & Ref \\
\hline \multirow[t]{2}{*}{$\begin{array}{l}\text { Antony, 2014, } \\
24135689, \text { US }\end{array}$} & High & $\begin{array}{l}\text { Capsular } \\
\text { contracture }\end{array}$ & Baker Grade III or IV & $3-5$ & Silicone & $\begin{array}{l}\text { NR/17 } \\
9\end{array}$ & Ref & Ref \\
\hline & High & $\begin{array}{l}\text { Capsular } \\
\text { contracture }\end{array}$ & Baker Grade III or IV & $3-5$ & Saline & $\begin{array}{l}\text { NR/16 } \\
6\end{array}$ & NR & NS \\
\hline
\end{tabular}

Abbreviations: adj = adjusted, CI = confidence interval, IBR = implant-based reconstruction, $\mathrm{HR}=$ hazard ratio, $\mathrm{NR}=$ not reported, NRCS $=$ nonrandomized comparative study, $\mathrm{NS}=$ not significant, $\mathrm{OR}=$ odds ratio, $\mathrm{PMID}=$ PubMed identifier, $\mathrm{Ref}=$ reference group, $\mathrm{RoB}=$ risk of bias, $\mathrm{y}=$ years

Colors: Header rows are shaded orange. Rows for every alternate outcome are shaded blue. The colors do not add unique information. 
Table E-4.1. Summary Table - Key Question 4: Comparisons of anatomic planes of implant placement for IBR - continuous outcomes (various)

\begin{tabular}{|c|c|c|c|c|c|c|c|c|c|c|}
\hline $\begin{array}{l}\text { Study, } \\
\text { Year, PMID, } \\
\text { Country }\end{array}$ & Design & RoB & Outcome & Outcome Measurement & $\begin{array}{l}\text { Time } \\
\text { Point }\end{array}$ & Arm & $\mathbf{N}$ & Mean (SD) & $\begin{array}{l}\text { Effect } \\
\text { Size } \\
(95 \% \mathrm{Cl}) \\
\end{array}$ & $\begin{array}{l}\text { P } \\
\text { Value }\end{array}$ \\
\hline \multirow{2}{*}{$\begin{array}{l}\text { Lee, 2021b, } \\
33691448, \\
\text { South } \\
\text { Korea }\end{array}$} & RCT & Moderate & Physical WB & SF-36 (0-100): PCS & $6 \mathrm{mo}$ & Prepectoral & 20 & $45.2(7.1)$ & Ref & Ref \\
\hline & RCT & Moderate & Physical WB & SF-36 (0-100): PCS & $6 \mathrm{mo}$ & $\begin{array}{l}\text { Partial } \\
\text { Submuscular }\end{array}$ & 14 & $45.2(7.1)$ & NR & 0.689 \\
\hline \multirow{6}{*}{$\begin{array}{l}\text { Cattelani, } \\
2018, \\
29275104, \\
\text { Italy }\end{array}$} & NRCS & High & Physical WB & $\begin{array}{l}\text { Constant Murley }(0-100) \text { : } \\
\text { Upper limb }\end{array}$ & $1 \mathrm{~d}$ & Prepectoral & 39 & $71.6(8.9)$ & Ref & Ref \\
\hline & NRCS & High & Physical WB & $\begin{array}{l}\text { Constant Murley }(0-100) \text { : } \\
\text { Upper limb }\end{array}$ & $1 \mathrm{~d}$ & Total Submuscular & 45 & $60.4(10.5)$ & NR & $<0.001$ \\
\hline & NRCS & High & Physical WB & $\begin{array}{l}\text { Constant Murley }(0-100) \text { : } \\
\text { Upper limb }\end{array}$ & $7 d$ & Prepectoral & 39 & $65.7(9.3)$ & Ref & Ref \\
\hline & NRCS & High & Physical WB & $\begin{array}{l}\text { Constant Murley }(0-100) \text { : } \\
\text { Upper limb }\end{array}$ & $7 d$ & Total Submuscular & 45 & $52.4(12.2)$ & NR & $<0.001$ \\
\hline & NRCS & High & Physical WB & DASH $(0-100)$ & $1 \mathrm{y}$ & Prepectoral & 39 & $9.9(17.9)$ & Ref & Ref \\
\hline & NRCS & High & Physical WB & DASH (0-100) & $1 \mathrm{y}$ & Total Submuscular & 45 & $29.2(16.9)$ & NR & $<0.001$ \\
\hline \multirow{6}{*}{$\begin{array}{l}\text { Lee, 2021b, } \\
33691448 \text {, } \\
\text { South } \\
\text { Korea }\end{array}$} & RCT & Moderate & Psychosocial WB & SF-36 (0-100): MCS & $6 \mathrm{mo}$ & Prepectoral & 20 & $40.5(10.5)$ & Ref & Ref \\
\hline & RCT & Moderate & Psychosocial WB & SF-36 (0-100): MCS & $6 \mathrm{mo}$ & $\begin{array}{l}\text { Partial } \\
\text { Submuscular }\end{array}$ & 14 & $40.5(10.5)$ & NR & 0.904 \\
\hline & RCT & Moderate & Psychosocial WB & HADS: Anxiety (0-21) & $6 \mathrm{mo}$ & Prepectoral & 20 & $6.3(3.3)$ & Ref & Ref \\
\hline & RCT & Moderate & Psychosocial WB & HADS: Anxiety (0-21) & $6 \mathrm{mo}$ & $\begin{array}{l}\text { Partial } \\
\text { Submuscular }\end{array}$ & 14 & $5.0(2.9)$ & NR & 0.959 \\
\hline & RCT & Moderate & Psychosocial WB & HADS: Depression (0-21) & $6 \mathrm{mo}$ & Prepectoral & 20 & $7.5(7.4)$ & Ref & Ref \\
\hline & RCT & Moderate & Psychosocial WB & HADS: Depression (0-21) & $6 \mathrm{mo}$ & $\begin{array}{l}\text { Partial } \\
\text { Submuscular }\end{array}$ & 14 & $6.3(3.8)$ & NR & 0.924 \\
\hline \multirow{2}{*}{$\begin{array}{l}\text { Cattelani, } \\
2018, \\
29275104, \\
\text { Italy }\end{array}$} & NRCS & High & Psychosocial WB & Return to usual work & NR & Prepectoral & 39 & $34.6 \mathrm{~d}(21)$ & Ref & Ref \\
\hline & NRCS & High & Psychosocial WB & Return to usual work & NR & Total Submuscular & 45 & $\begin{array}{l}57.3 \mathrm{~d} \\
(37.8)\end{array}$ & NR & $<0.001$ \\
\hline \multirow{2}{*}{$\begin{array}{l}\text { Cattelani, } \\
2018, \\
29275104, \\
\text { Italy }\end{array}$} & NRCS & High & $\begin{array}{l}\text { Satisfaction with } \\
\text { breasts }\end{array}$ & $\begin{array}{l}\text { BREAST-Q }(0-100): \\
\text { Satisfaction with breast }\end{array}$ & $1 \mathrm{y}$ & Prepectoral & 39 & $92.2(9.0)$ & Ref & Ref \\
\hline & NRCS & High & $\begin{array}{l}\text { Satisfaction with } \\
\text { breasts }\end{array}$ & $\begin{array}{l}\text { BREAST-Q (0-100): } \\
\text { Satisfaction with breast }\end{array}$ & $1 \mathrm{y}$ & Total Submuscular & 45 & $76.1(14.6)$ & NR & $<0.001$ \\
\hline \multirow{2}{*}{$\begin{array}{l}\text { Avila, 2020, } \\
33234947, \\
\text { US }\end{array}$} & NRCS & High & Pain & VAS $(0-10)$ & NR & Prepectoral & 73 & $3.94(0.83)$ & Ref & Ref \\
\hline & NRCS & High & Pain & VAS $(0-10)$ & NR & Total Submuscular & 73 & $5.25(0.81)$ & NR & $<0.001$ \\
\hline \multirow{4}{*}{$\begin{array}{l}\text { Cattelani, } \\
2018, \\
29275104, \\
\text { Italy }\end{array}$} & NRCS & High & Pain & $\mathrm{BPI}-\mathrm{SF}(0-100)$ & $1 \mathrm{~d}$ & Prepectoral & 39 & $17.6(15.5)$ & Ref & Ref \\
\hline & NRCS & High & Pain & BPI-SF $(0-100)$ & $1 \mathrm{~d}$ & Total Submuscular & 45 & $44.1(15.8)$ & NR & $<0.001$ \\
\hline & NRCS & High & Pain & BPI-SF (0-100) & $7 d$ & Prepectoral & 39 & $8.2(15.4)$ & Ref & Ref \\
\hline & NRCS & High & Pain & $\mathrm{BPI}-\mathrm{SF}(0-100)$ & $7 \mathrm{~d}$ & Total Submuscular & 45 & $22.0(18.6)$ & NR & $<0.001$ \\
\hline
\end{tabular}




\begin{tabular}{|c|c|c|c|c|c|c|c|c|c|c|}
\hline $\begin{array}{l}\text { Study, } \\
\text { Year, PMID, } \\
\text { Country }\end{array}$ & Design & RoB & Outcome & Outcome Measurement & $\begin{array}{l}\text { Time } \\
\text { Point }\end{array}$ & Arm & $\mathbf{N}$ & Mean (SD) & $\begin{array}{l}\text { Effect } \\
\text { Size } \\
(95 \% \mathrm{Cl})\end{array}$ & $\begin{array}{l}\mathbf{P} \\
\text { Value }\end{array}$ \\
\hline \multirow{4}{*}{$\begin{array}{l}\text { Kim, 2020, } \\
33066236 \text {, } \\
\text { South } \\
\text { Korea }\end{array}$} & NRCS & Moderate & Pain & VAS (0-10) & $1 \mathrm{~d}$ & Prepectoral & 53 & $2.66(1.82)$ & Ref & Ref \\
\hline & NRCS & Moderate & Pain & VAS $(0-10)$ & $1 \mathrm{~d}$ & $\begin{array}{l}\text { Partial } \\
\text { Submuscular }\end{array}$ & 114 & $2.26(1.38)$ & $\begin{array}{l}\text { adjMD } \\
-0.08\end{array}$ & 0.33 \\
\hline & NRCS & Moderate & Pain & VAS (0-10) & $7 d$ & Prepectoral & 53 & $1.08(1.19)$ & Ref & Ref \\
\hline & NRCS & Moderate & Pain & VAS (0-10) & $7 d$ & $\begin{array}{l}\text { Partial } \\
\text { Submuscular }\end{array}$ & 114 & $0.80(1.07)$ & -0.12 & 0.12 \\
\hline \multirow{2}{*}{$\begin{array}{l}\text { Avila, 2020, } \\
33234947, \\
\text { US }\end{array}$} & NRCS & High & Analgesic use & $\begin{array}{l}\text { Oral morphine } \\
\text { equivalents }\end{array}$ & NR & Prepectoral & 73 & $\begin{array}{l}17.4 \mathrm{mg} \\
(45.1)\end{array}$ & Ref & Ref \\
\hline & NRCS & High & Analgesic use & $\begin{array}{l}\text { Oral morphine } \\
\text { equivalents }\end{array}$ & NR & Total Submuscular & 73 & $\begin{array}{l}63.0 \mathrm{mg} \\
(44.9)\end{array}$ & NR & 0.03 \\
\hline
\end{tabular}

Abbreviations: adj = adjusted, BPI-SF = Brief Pain Inventory Short Form, CI = confidence interval, $d=$ days, DASH = Disabilities of the Arm, Shoulder, and Hand, HADS = Hospital Anxiety and Depression Scale, IBR $=$ implant-based reconstruction, MCS $=$ Mental Component Summary, NR = not reported, NRCS $=$ nonrandomized comparative study, PCS = Physical Component Summary, PMID $=$ PubMed identifier, Ref = reference, RoB $=$ risk of bias, $\mathrm{SD}=$ standard deviation, $\mathrm{SF}=\mathrm{Short} \mathrm{Form,} \mathrm{WB}=$ well-being, $\mathrm{y}=$ years.

Colors: Header rows are shaded orange. Rows for every alternate outcome are shaded blue. The colors do not add unique information.

Table E-4.2. Summary Table - Key Question 4: Comparisons of anatomic planes of implant placement for IBR - categorical outcomes (various)

\begin{tabular}{|c|c|c|c|c|c|c|c|c|c|}
\hline $\begin{array}{l}\text { Study, Year, } \\
\text { PMID, Country }\end{array}$ & $\begin{array}{l}\text { Desig } \\
\mathrm{n}\end{array}$ & $\begin{array}{l}\text { Overall } \\
\text { RoB }\end{array}$ & Outcome & $\begin{array}{l}\text { Outcome } \\
\text { Measurement }\end{array}$ & $\begin{array}{l}\text { Time } \\
\text { Point }\end{array}$ & Arm & $\mathrm{n} / \mathrm{N}(\%)$ & Effect Size $(95 \% \mathrm{CI})$ & $\begin{array}{l}\mathbf{P} \\
\text { Value }\end{array}$ \\
\hline \multirow[t]{2}{*}{$\begin{array}{l}\text { Avila, 2020, } \\
33234947 \text {, US }\end{array}$} & NRCS & High & $\begin{array}{l}\text { Unplanned repeat } \\
\text { surgeries for revision }\end{array}$ & NR & $1 \mathrm{mo}$ & Prepectoral & $8 / 203(3.94)$ & Ref & Ref \\
\hline & NRCS & High & $\begin{array}{l}\text { Unplanned repeat } \\
\text { surgeries for revision }\end{array}$ & NR & $1 \mathrm{mo}$ & Total submuscular & $17 / 202(8.42)$ & NR & NS \\
\hline \multirow{2}{*}{$\begin{array}{l}\text { Nealon, 2020a, } \\
\text { 32032345, US }\end{array}$} & NRCS & High & Necrosis & Skin necrosis & $1.7-2.4 \mathrm{y}$ & Prepectoral & $5 / 114(4.4)$ & Ref & Ref \\
\hline & NRCS & High & Necrosis & Skin necrosis & $1.7-2.4 \mathrm{y}$ & Total submuscular & $6 / 142(4.2)$ & adjOR $1.01(0.74,5.95)$ & 0.77 \\
\hline \multirow[t]{2}{*}{$\begin{array}{l}\text { Kraenzlin, 2021, } \\
32568752 \text {, US }\end{array}$} & NRCS & High & $\begin{array}{l}\text { Infections (not explicitly } \\
\text { implant-related) }\end{array}$ & NR & NR & Prepectoral & $34 / 169(11.0)$ & Ref & Ref \\
\hline & NRCS & High & $\begin{array}{l}\text { Infections (not explicitly } \\
\text { implant-related) }\end{array}$ & NR & NR & Total submuscular & $34 / 117(17.4)$ & NR & 0.21 \\
\hline \multirow[t]{2}{*}{$\begin{array}{l}\text { Nealon, 2020a, } \\
\text { 32032345, US }\end{array}$} & NRCS & High & $\begin{array}{l}\text { Infections (not explicitly } \\
\text { implant-related) }\end{array}$ & NR & $1.7-2.4 \mathrm{y}$ & Prepectoral & $2 / 114(1.8)$ & Ref & Ref \\
\hline & NRCS & High & $\begin{array}{l}\text { Infections (not explicitly } \\
\text { implant-related) }\end{array}$ & NR & $1.7-2.4 \mathrm{y}$ & Total submuscular & $6 / 142(4.2)$ & $\begin{array}{l}\text { adjOR } 0.31(<0.01, \\
8.65)\end{array}$ & 0.52 \\
\hline \multirow{3}{*}{$\begin{array}{l}\text { Nealon, } 2020 a \text {, } \\
32032345 \text {, US }\end{array}$} & NRCS & High & Need for explant surgery & NR & $1.7-2.4 \mathrm{y}$ & Prepectoral & $4 / 114(3.5)$ & Ref & Ref \\
\hline & NRCS & High & Need for explant surgery & NR & $1.7-2.4 \mathrm{y}$ & Total submuscular & $7 / 142(4.9)$ & adjOR $1.01(0.07,14.1)$ & 0.99 \\
\hline & $\mathrm{RCT}$ & $\begin{array}{l}\text { Moderat } \\
\mathrm{e}\end{array}$ & Capsular contracture & NR & $6 \mathrm{mo}$ & Prepectoral & $1 / 20(5.0)$ & Ref & Ref \\
\hline
\end{tabular}




\begin{tabular}{|c|c|c|c|c|c|c|c|c|c|}
\hline $\begin{array}{l}\text { Study, Year, } \\
\text { PMID, Country }\end{array}$ & $\begin{array}{l}\text { Desig } \\
\mathrm{n}\end{array}$ & $\begin{array}{l}\text { Overall } \\
\text { RoB }\end{array}$ & Outcome & $\begin{array}{l}\text { Outcome } \\
\text { Measurement }\end{array}$ & $\begin{array}{l}\text { Time } \\
\text { Point }\end{array}$ & Arm & $n / N(\%)$ & Effect Size $(95 \% \mathrm{CI})$ & $\begin{array}{l}\mathbf{P} \\
\text { Value }\end{array}$ \\
\hline $\begin{array}{l}\text { Lee, } 2021 \mathrm{~b}, \\
33691448, \\
\text { South Korea }\end{array}$ & RCT & $\begin{array}{l}\text { Moderat } \\
\mathrm{e}\end{array}$ & Capsular contracture & NR & $6 \mathrm{mo}$ & $\begin{array}{l}\text { Partial } \\
\text { submuscular }\end{array}$ & $0 / 14(0)$ & Not calculable & - \\
\hline \multirow{2}{*}{$\begin{array}{l}\text { Nealon, 2020a, } \\
32032345, \text { US }\end{array}$} & NRCS & High & Capsular contracture & NR & $1.7-2.4 \mathrm{y}$ & Prepectoral & $2 / 114(1.8)$ & Ref & Ref \\
\hline & NRCS & High & Capsular contracture & NR & $1.7-2.4 \mathrm{y}$ & Total submuscular & $12 / 142(8.5)$ & adjOR $0.30(0.03,1.55)$ & 0.16 \\
\hline \multirow{2}{*}{$\begin{array}{l}\text { Lee, 2021b, } \\
33691448, \\
\text { South Korea }\end{array}$} & $\mathrm{RCT}$ & $\begin{array}{l}\text { Moderat } \\
\mathrm{e}\end{array}$ & Seroma & NR & $6 \mathrm{mo}$ & Prepectoral & $3 / 20(15.0)$ & Ref & Ref \\
\hline & RCT & $\begin{array}{l}\text { Moderat } \\
\mathrm{e}\end{array}$ & Seroma & NR & $6 \mathrm{mo}$ & $\begin{array}{l}\text { Partial } \\
\text { submuscular }\end{array}$ & $2 / 14(14.3)$ & OR $1.06(0.15,7.34)$ & 0.95 \\
\hline \multirow{2}{*}{$\begin{array}{l}\text { Nealon, } 2020 a \text {, } \\
32032345, \text { US }\end{array}$} & NRCS & High & Seroma & NR & $1.7-2.4 \mathrm{y}$ & Prepectoral & $10 / 114(8.8)$ & Ref & Ref \\
\hline & NRCS & High & Seroma & NR & $1.7-2.4 \mathrm{y}$ & Total submuscular & $11 / 142(7.7)$ & $\operatorname{adjOR} 1.49(0.37,6.11)$ & 0.57 \\
\hline \multirow{2}{*}{$\begin{array}{l}\text { Nealon, 2020a, } \\
32032345 \text {, US }\end{array}$} & NRCS & High & Hematoma & NR & $1.7-2.4 \mathrm{y}$ & Prepectoral & $6 / 114(5.3)$ & Ref & Ref \\
\hline & NRCS & High & Hematoma & NR & $1.7-2.4 \mathrm{y}$ & Total submuscular & $7 / 142(4.9)$ & adjOR $5.18(0.39,7.05)$ & 0.23 \\
\hline \multirow[t]{2}{*}{$\begin{array}{l}\text { Avila, 2020, } \\
33234947, \text { US }\end{array}$} & NRCS & High & $\begin{array}{l}\text { Composite or unspecified } \\
\text { harms }\end{array}$ & $\begin{array}{l}\text { Necrosis/infecti } \\
\text { on, wound } \\
\text { dehiscence/ } \\
\text { hematoma/sero } \\
\text { ma }\end{array}$ & $1 \mathrm{mo}$ & Prepectoral & $12 / 203(5.91)$ & Ref & Ref \\
\hline & NRCS & High & $\begin{array}{l}\text { Composite or unspecified } \\
\text { harms }\end{array}$ & $\begin{array}{l}\text { Necrosis/infecti } \\
\text { on, wound } \\
\text { dehiscence/ } \\
\text { hematoma/sero } \\
\text { ma }\end{array}$ & $1 \mathrm{mo}$ & Total submuscular & 19/202 (9.41) & NR & NS \\
\hline \multirow[t]{2}{*}{$\begin{array}{l}\text { Gabriel, 2020, } \\
\text { 32195862, US }\end{array}$} & NRCS & High & $\begin{array}{l}\text { Composite or unspecified } \\
\text { harms }\end{array}$ & $\begin{array}{l}\text { Any } \\
\text { complication }\end{array}$ & $2 y$ & Prepectoral & $\begin{array}{l}19 / 129 \\
\text { breasts }(14.7)\end{array}$ & Ref & Ref \\
\hline & NRCS & High & $\begin{array}{l}\text { Composite or unspecified } \\
\text { harms }\end{array}$ & $\begin{array}{l}\text { Any } \\
\text { complication }\end{array}$ & $2 y$ & $\begin{array}{l}\text { Partial } \\
\text { submuscular }\end{array}$ & $\begin{array}{l}33 / 128 \\
\text { breasts (25.8) }\end{array}$ & adjOR $3.04(1.34,7.61)$ & 0.013 \\
\hline $\begin{array}{l}\text { Ozgur, 2020, } \\
33223365, \\
\text { Turkey }\end{array}$ & NRCS & High & $\begin{array}{l}\text { Composite or unspecified } \\
\text { harms }\end{array}$ & $\begin{array}{l}\text { Capsular } \\
\text { contracture, } \\
\text { inframammary } \\
\text { fold problems, } \\
\text { bottoming out, } \\
\text { rippling, } \\
\text { mechanical } \\
\text { shift, animation } \\
\text { deformity }\end{array}$ & $5.3-6.1 \mathrm{y}$ & $\begin{array}{l}\text { Partial } \\
\text { submuscular }\end{array}$ & $\begin{array}{l}8 / 91 \text { breasts } \\
(8.8)\end{array}$ & Ref & Ref \\
\hline
\end{tabular}




\begin{tabular}{|c|c|c|c|c|c|c|c|c|c|}
\hline $\begin{array}{l}\text { Study, Year, } \\
\text { PMID, Country }\end{array}$ & $\begin{array}{l}\text { Desig } \\
\mathrm{n}\end{array}$ & $\begin{array}{l}\text { Overall } \\
\text { RoB }\end{array}$ & Outcome & $\begin{array}{l}\text { Outcome } \\
\text { Measurement }\end{array}$ & $\begin{array}{l}\text { Time } \\
\text { Point }\end{array}$ & Arm & $n / N(\%)$ & Effect Size (95\% CI) & $\begin{array}{l}\mathbf{P} \\
\text { Value }\end{array}$ \\
\hline & NRCS & High & $\begin{array}{l}\text { Composite or unspecified } \\
\text { harms }\end{array}$ & $\begin{array}{l}\text { Capsular } \\
\text { contracture, } \\
\text { inframammary } \\
\text { fold problems, } \\
\text { bottoming out, } \\
\text { rippling, } \\
\text { mechanical } \\
\text { shift, animation } \\
\text { deformity }\end{array}$ & $5.3-6.1 \mathrm{y}$ & Total submuscular & $\begin{array}{l}29 / 117 \\
\text { breasts }(24.8)\end{array}$ & adjOR $3.28(1.39,7.76)$ & 0.007 \\
\hline
\end{tabular}

Abbreviations: adj $=$ adjusted, $\mathrm{CI}=$ confidence interval, $\mathrm{IBR}=$ implant-based reconstruction, $\mathrm{HR}=$ hazard ratio, $\mathrm{mo}=$ months, $\mathrm{NR}=$ not reported, $\mathrm{NRCS}=$ nonrandomized comparative study, $\mathrm{NS}=$ not significant, $\mathrm{OR}=$ odds ratio, $\mathrm{PMID}=$ PubMed identifier, $\mathrm{RoB}=$ risk of bias, $\mathrm{y}=$ years.

Colors: Header rows are shaded orange. Rows for every alternate outcome are shaded blue. The colors do not add unique information. 
Table E-5.1. Summary Table - Key Question 5: Use versus nonuse of human ADMs during IBR - continuous outcomes (physical wellbeing, psychosocial well-being, sexual well-being, and satisfaction with breasts)

\begin{tabular}{|c|c|c|c|c|c|c|c|c|c|c|c|}
\hline $\begin{array}{l}\text { Study, Year, } \\
\text { PMID, Country }\end{array}$ & Outcome & $\begin{array}{l}\text { Outcome } \\
\text { Measurement }\end{array}$ & Design & $\begin{array}{l}\text { Overall } \\
\text { RoB }\end{array}$ & $\begin{array}{l}\text { Time } \\
\text { Point }\end{array}$ & $\begin{array}{l}\text { Use of } \\
\text { ADM, } \\
\mathbf{N}\end{array}$ & $\begin{array}{l}\text { Use of } \\
\text { ADM, } \\
\text { Mean } \\
\text { (SD) }\end{array}$ & $\begin{array}{l}\text { Nonuse } \\
\text { of } \\
\text { ADM, N }\end{array}$ & $\begin{array}{l}\text { Nonuse of } \\
\text { ADM, Mean } \\
\text { (SD) }\end{array}$ & $\begin{array}{l}\text { Effect Size } \\
(95 \% \mathrm{Cl})\end{array}$ & $\begin{array}{l}P \\
\text { Value }\end{array}$ \\
\hline \multirow[t]{2}{*}{$\begin{array}{l}\text { McCarthy, 2012, } \\
23096987, \text { US }\end{array}$} & Physical WB & $\begin{array}{l}\text { BREAST-Q (0- } \\
\text { 100): Chest } \\
\text { and upper body }\end{array}$ & RCT & Moderate & Expansion & 36 & $\begin{array}{l}68.6 \\
(10.6)\end{array}$ & 33 & $69.3(7.9)$ & $\begin{array}{l}\text { NMD } 0.60(-4.87 \\
6.07)^{\star}\end{array}$ & 0.83 \\
\hline & Physical WB & $\begin{array}{l}\text { BREAST-Q (0- } \\
\text { 100): Chest } \\
\text { and upper body }\end{array}$ & RCT & Moderate & $\begin{array}{l}\text { After } \\
\text { expansion }\end{array}$ & 36 & $\begin{array}{l}79.7 \\
(15.1)\end{array}$ & 33 & $80.5(13.3)$ & $\begin{array}{l}\text { NMD } 0.50(-5.93 \\
6.93)^{*}\end{array}$ & 0.88 \\
\hline \multirow[t]{3}{*}{$\begin{array}{l}\text { Cattelani, } 2018, \\
29275104, \text { Italy }\end{array}$} & Physical WB & $\begin{array}{l}\text { Constant } \\
\text { Murley Score }\end{array}$ & NRCS & High & $1 \mathrm{~d}$ & 39 & $\begin{array}{l}71.62 \\
(8.87)\end{array}$ & 45 & $60.36(10.54)$ & NR & $<0.001$ \\
\hline & Physical WB & $\begin{array}{l}\text { Constant } \\
\text { Murley Score }\end{array}$ & NRCS & High & $7 \mathrm{~d}$ & 39 & $\begin{array}{l}65.67 \\
(9.31)\end{array}$ & 45 & $52.36(12.23)$ & NR & $<0.001$ \\
\hline & Physical WB & DASH score & NRCS & High & $1 \mathrm{y}$ & 39 & $\begin{array}{l}9.92 \\
(17.87)\end{array}$ & 45 & $29.18(16.91)$ & NR & $<0.001$ \\
\hline $\begin{array}{l}\text { Ganesh Kumar, } \\
\text { 2021, 33172826, } \\
\text { US \& Canada }\end{array}$ & Physical WB & $\begin{array}{l}\text { BREAST-Q (0- } \\
\text { 100): Physical } \\
\text { well-being }\end{array}$ & NRCS & Moderate & $2 y$ & 738 & NR & 713 & $\overline{N R}$ & $\begin{array}{l}\operatorname{adjMD}-0.82 \\
(-3.01,1.37)\end{array}$ & NR \\
\hline $\begin{array}{l}\text { Cattelani, 2018, } \\
29275104, \text { Italy }\end{array}$ & $\begin{array}{l}\text { Psychosocial } \\
\text { WB }\end{array}$ & $\begin{array}{l}\text { Return to usual } \\
\text { work }\end{array}$ & NRCS & High & NR & 39 & $\begin{array}{l}34.56 \mathrm{~d} \\
(21)\end{array}$ & 45 & $57.31 \mathrm{~d}(37.77)$ & NR & $<0.001$ \\
\hline $\begin{array}{l}\text { Ganesh Kumar, } \\
\text { 2021, 33172826, } \\
\text { US \& Canada }\end{array}$ & $\begin{array}{l}\text { Psychosocial } \\
\text { WB }\end{array}$ & $\begin{array}{l}\text { BREAST-Q (0- } \\
\text { 100): } \\
\text { Psychosocial } \\
\text { WB }\end{array}$ & NRCS & Moderate & $2 y$ & 738 & NR & 713 & NR & $\begin{array}{l}\text { adjMD }-0.26 \\
(-2.97,2.45)\end{array}$ & NR \\
\hline $\begin{array}{l}\text { Ganesh Kumar, } \\
\text { 2021, 33172826, } \\
\text { US \& Canada }\end{array}$ & Sexual WB & $\begin{array}{l}\text { BREAST-Q (0- } \\
\text { 100): Sexual } \\
\text { WB }\end{array}$ & NRCS & Moderate & $2 y$ & 738 & NR & 713 & NR & $\begin{array}{l}\text { adjMD }-2.28 \\
(-5.63,1.06)\end{array}$ & NR \\
\hline $\begin{array}{l}\text { Cattelani, 2018, } \\
29275104, \text { Italy }\end{array}$ & $\begin{array}{l}\text { Satisfaction } \\
\text { with breasts }\end{array}$ & $\begin{array}{l}\text { BREAST-Q: } \\
\text { Satisfaction } \\
\text { with breast }\end{array}$ & NRCS & High & $1 \mathrm{y}$ & 39 & $\begin{array}{l}92.2 \\
(9.03)\end{array}$ & 45 & $76.1(14.6)$ & NR & $<0.001$ \\
\hline $\begin{array}{l}\text { Ganesh Kumar, } \\
\text { 2021, 33172826, } \\
\text { US \& Canada }\end{array}$ & $\begin{array}{l}\text { Satisfaction } \\
\text { with breasts }\end{array}$ & $\begin{array}{l}\text { BREAST-Q: } \\
\text { Satisfaction } \\
\text { with breast }\end{array}$ & NRCS & Moderate & $2 y$ & 738 & NR & 713 & NR & $\begin{array}{l}\text { adjMD -1.95 } \\
(-4.96,1.06)\end{array}$ & $\overline{N R}$ \\
\hline
\end{tabular}

Abbreviations: adj = adjusted, ADM = acellular dermal matrix, CI = confidence interval, $\mathrm{d}=$ days, DASH = Disabilities of the Arm, Shoulder, and Hand, $\mathrm{h}=$ hours, IBR =

implant-based reconstruction, $\mathrm{MD}=$ mean difference, N/A = not applicable, NMD = net mean difference, $\mathrm{NR}=$ not reported, NRCS $=$ nonrandomized comparative study, $\mathrm{PMID}=$

PubMed identifier, $\mathrm{RCT}=$ randomized controlled trial, $\mathrm{RoB}=$ risk of bias, $\mathrm{SD}=$ standard deviation, $\mathrm{WB}=$ well-being, $\mathrm{y}=\mathrm{years}$.

Colors: Header rows are shaded orange. Rows for every alternate outcome are shaded blue. The colors do not add unique information.

*calculated 
Table E-5.2. Summary Table - Key Question 5: Use versus nonuse of human ADMs during IBR - categorical outcomes not metaanalyzed (various)

\begin{tabular}{|c|c|c|c|c|c|c|c|c|c|c|}
\hline $\begin{array}{l}\text { Study, Year, } \\
\text { PMID, Country }\end{array}$ & Design & $\begin{array}{l}\text { Overall } \\
\text { RoB }\end{array}$ & Outcome & $\begin{array}{l}\text { Outcome } \\
\text { Measurement }\end{array}$ & $\begin{array}{l}\text { Time } \\
\text { Point }\end{array}$ & Subgroup & $\begin{array}{l}\text { Use of } \\
\text { ADM, } \\
\text { n/N (\%) }\end{array}$ & $\begin{array}{l}\text { Nonuse of } \\
\text { ADM, n/N } \\
(\%)\end{array}$ & $\begin{array}{l}\text { Effect Size (95\% } \\
\mathrm{Cl})\end{array}$ & $\begin{array}{l}\mathbf{P} \\
\text { Value }\end{array}$ \\
\hline $\begin{array}{l}\text { Wendel, 2013, } \\
\text { None, US }\end{array}$ & RCT & High & Mortality & Death & $1 \mathrm{mo}$ & $\begin{array}{l}\text { All } \\
\text { patients }\end{array}$ & $0 / 20(0)$ & $0 / 16(0)$ & No events & $\mathrm{N} / \mathrm{A}$ \\
\hline $\begin{array}{l}\text { Ibrahim, 2013, } \\
24165587, \text { US }\end{array}$ & NRCS & Moderate & $\begin{array}{l}\text { Unplanned } \\
\text { repeat surgeries } \\
\text { for revision }\end{array}$ & NR & $6 \mathrm{mo}$ & $\begin{array}{l}\text { All } \\
\text { patients }\end{array}$ & $\begin{array}{l}237 / 3283 \\
(0.5)\end{array}$ & $\begin{array}{l}990 / 15714 \\
(0.6)\end{array}$ & NR & 0.14 \\
\hline $\begin{array}{l}\text { Nealon, 2020b, } \\
31605310, \text { US }\end{array}$ & NRCS & High & $\begin{array}{l}\text { Unplanned } \\
\text { repeat surgeries } \\
\text { for revision }\end{array}$ & NR & $5.3 y$ & $\begin{array}{l}\text { All } \\
\text { patients }\end{array}$ & NR & NR & $\begin{array}{l}\text { adjOR } 0.86 \text { (0.69, } \\
1.08)\end{array}$ & 0.19 \\
\hline $\begin{array}{l}\text { Sobti, 2018, } \\
\text { 29481386, US }\end{array}$ & NRCS & High & $\begin{array}{l}\text { Unplanned } \\
\text { repeat surgeries } \\
\text { for revision }\end{array}$ & $\begin{array}{l}\text { Revision for } \\
\text { malposition or } \\
\text { size }\end{array}$ & $5 \mathrm{y}$ & $\begin{array}{l}\text { All } \\
\text { patients }\end{array}$ & $\begin{array}{l}47 / 465 \\
\text { breasts } \\
(10.11)\end{array}$ & $\begin{array}{l}24 / 217 \\
\text { breasts } \\
(11.06)\end{array}$ & $\begin{array}{l}\text { adjOR } 1.10(0.63, \\
1.92)\end{array}$ & NR \\
\hline $\begin{array}{l}\text { Peled, 2012, } \\
\text { 22634688, US }\end{array}$ & NRCS & High & $\begin{array}{l}\text { Unplanned } \\
\text { repeat surgeries } \\
\text { for } \\
\text { complications }\end{array}$ & $\begin{array}{l}\text { For wound- } \\
\text { healing/infectious } \\
\text { complication }\end{array}$ & $\begin{array}{l}2.6- \\
3.3 \mathrm{y}\end{array}$ & $\begin{array}{l}\text { All } \\
\text { patients }\end{array}$ & $\begin{array}{l}11 / 100 \\
\text { breasts } \\
(11)\end{array}$ & $\begin{array}{l}21 / 90 \\
\text { breasts } \\
(23.3)\end{array}$ & NR & $<0.05$ \\
\hline \multirow[t]{2}{*}{$\begin{array}{l}\text { Ibrahim, 2013, } \\
24165587, \text { US }\end{array}$} & NRCS & Moderate & $\begin{array}{l}\text { Thromboembolic } \\
\text { events }\end{array}$ & $\begin{array}{l}\text { Deep vein } \\
\text { thrombosis }\end{array}$ & NR & $\begin{array}{l}\text { All } \\
\text { patients }\end{array}$ & $\begin{array}{l}9 / 3283 \\
(0.3)\end{array}$ & $\begin{array}{l}35 / 15714 \\
(0.2)\end{array}$ & NR & 0.47 \\
\hline & NRCS & Moderate & $\begin{array}{l}\text { Thromboembolic } \\
\text { events }\end{array}$ & $\begin{array}{l}\text { Pulmonary } \\
\text { embolism }\end{array}$ & NR & $\begin{array}{l}\text { All } \\
\text { patients }\end{array}$ & $\begin{array}{l}2 / 3283 \\
(0.06)\end{array}$ & $\begin{array}{l}29 / 15714 \\
(0.2)\end{array}$ & NR & 0.11 \\
\hline \multirow[t]{3}{*}{$\begin{array}{l}\text { Craig, 2019, } \\
29800083 \text {, US }\end{array}$} & NRCS & Low & $\begin{array}{l}\text { Wound } \\
\text { dehiscence }\end{array}$ & NR & $7 \mathrm{mo}$ & $\begin{array}{l}\text { All } \\
\text { patients }\end{array}$ & $\begin{array}{l}35 / 574 \\
\text { breasts } \\
(6.1)\end{array}$ & $\begin{array}{l}20 / 796 \\
\text { breasts } \\
(2.5)\end{array}$ & NR & NR \\
\hline & NRCS & Low & $\begin{array}{l}\text { Wound } \\
\text { dehiscence }\end{array}$ & NR & $7 \mathrm{mo}$ & $\begin{array}{l}\text { Postop } \\
\text { radiation }\end{array}$ & $\begin{array}{l}42 / 88 \\
\text { breasts } \\
(47.7)\end{array}$ & $\begin{array}{l}27 / 113 \\
\text { breasts } \\
(23.9)\end{array}$ & NR & NR \\
\hline & NRCS & Low & $\begin{array}{l}\text { Wound } \\
\text { dehiscence }\end{array}$ & NR & $7 \mathrm{mo}$ & $\begin{array}{l}\text { No postop } \\
\text { radiation }\end{array}$ & $\begin{array}{l}30 / 486 \\
\text { breasts } \\
(6.2)\end{array}$ & $\begin{array}{l}17 / 683 \\
\text { breasts } \\
(2.5)\end{array}$ & $\begin{array}{l}\text { adjOR } 2.46(1.23, \\
4.93)\end{array}$ & NR \\
\hline $\begin{array}{l}\text { Ganesh Kumar, } \\
\text { 2021, 33172826, } \\
\text { US \& Canada }\end{array}$ & NRCS & Moderate & $\begin{array}{l}\text { Wound } \\
\text { dehiscence }\end{array}$ & NR & $2 y$ & $\begin{array}{l}\text { All } \\
\text { patients }\end{array}$ & $\begin{array}{l}24 / 738 \\
(3.3)\end{array}$ & $5 / 713(0.7)$ & NR & 0.009 \\
\hline $\begin{array}{l}\text { Ibrahim, 2013, } \\
\text { 24165587, US }\end{array}$ & NRCS & Moderate & $\begin{array}{l}\text { Wound } \\
\text { dehiscence }\end{array}$ & NR & NR & $\begin{array}{l}\text { All } \\
\text { patients }\end{array}$ & $\begin{array}{l}15 / 3283 \\
(0.5)\end{array}$ & $\begin{array}{l}98 / 15714 \\
(0.6)\end{array}$ & NR & 0.26 \\
\hline $\begin{array}{l}\text { Qureshi, 2016, } \\
\text { 27465177, US }\end{array}$ & NRCS & High & $\begin{array}{l}\text { Wound } \\
\text { dehiscence }\end{array}$ & $\begin{array}{l}\text { Dehiscence } \\
\text { without necrosis }\end{array}$ & $2 y$ & $\begin{array}{l}\text { All } \\
\text { patients }\end{array}$ & $\begin{array}{l}\mathrm{NR} / 295 \\
(\mathrm{NR})\end{array}$ & $\begin{array}{l}\mathrm{NR} / 118 \\
(\mathrm{NR})\end{array}$ & adjOR 0.4 (NR, NR) & $<0.05$ \\
\hline $\begin{array}{l}\text { Woo, } 2017, \\
28509694, \text { South } \\
\text { Korea }\end{array}$ & NRCS & High & Delayed healing & $\begin{array}{l}\text { Delayed wound } \\
\text { healing or skin } \\
\text { flap necrosis }\end{array}$ & NR & $\begin{array}{l}\text { All } \\
\text { patients }\end{array}$ & $\begin{array}{l}32 / 199 \\
(16.1)\end{array}$ & $\begin{array}{l}32 / 199 \\
(16.1)\end{array}$ & $\begin{array}{l}\text { adjOR } 1.41 \text { (0.67, } \\
2.96)\end{array}$ & 0.37 \\
\hline
\end{tabular}




\begin{tabular}{|c|c|c|c|c|c|c|c|c|c|c|}
\hline $\begin{array}{l}\text { Study, Year, } \\
\text { PMID, Country }\end{array}$ & Design & $\begin{array}{l}\text { Overall } \\
\text { RoB }\end{array}$ & Outcome & $\begin{array}{l}\text { Outcome } \\
\text { Measurement }\end{array}$ & $\begin{array}{l}\text { Time } \\
\text { Point }\end{array}$ & Subgroup & $\begin{array}{l}\text { Use of } \\
\text { ADM, } \\
n / N(\%)\end{array}$ & $\begin{array}{l}\text { Nonuse of } \\
\text { ADM, n/N } \\
(\%)\end{array}$ & $\begin{array}{l}\text { Effect Size }(95 \% \\
\mathrm{Cl})\end{array}$ & $\begin{array}{l}P \\
\text { Value }\end{array}$ \\
\hline $\begin{array}{l}\text { Ganesh Kumar, } \\
\text { 2021, 33172826, } \\
\text { US \& Canada }\end{array}$ & NRCS & Moderate & Implant rupture & $\begin{array}{l}\text { Implant rupture, } \\
\text { leakage, or } \\
\text { deflation }\end{array}$ & $2 y$ & $\begin{array}{l}\text { All } \\
\text { patients }\end{array}$ & $\begin{array}{l}11 / 738 \\
(1.5)\end{array}$ & $7 / 713(1.0)$ & NR & 0.58 \\
\hline $\begin{array}{l}\text { Ganesh Kumar, } \\
\text { 2021, 33172826, } \\
\text { US \& Canada }\end{array}$ & NRCS & Moderate & $\begin{array}{l}\text { Implant } \\
\text { malposition }\end{array}$ & NR & $2 y$ & $\begin{array}{l}\text { All } \\
\text { patients }\end{array}$ & $\begin{array}{l}9 / 738 \\
(1.2)\end{array}$ & $4 / 713(0.6)$ & NR & 0.83 \\
\hline $\begin{array}{l}\text { Vardanian, } 2011 \text {, } \\
22030500, \text { US }\end{array}$ & NRCS & High & $\begin{array}{l}\text { Implant } \\
\text { malposition }\end{array}$ & NR & $2.4 \mathrm{y}$ & $\begin{array}{l}\text { All } \\
\text { patients }\end{array}$ & $\begin{array}{l}4 / 208 \\
\text { breasts } \\
(1.9)\end{array}$ & $\begin{array}{l}12 / 129 \\
\text { breasts } \\
(9.3)\end{array}$ & $\begin{array}{l}\text { adjOR } 0.23(0.06, \\
0.78)\end{array}$ & NR \\
\hline $\begin{array}{l}\text { Seth, 2012, } \\
23018687, \text { US }\end{array}$ & NRCS & High & $\begin{array}{l}\text { Implant } \\
\text { extrusion }\end{array}$ & NR & $2 y$ & $\begin{array}{l}\text { All } \\
\text { patients }\end{array}$ & $\begin{array}{l}2 / 199 \\
\text { breasts } \\
(1)\end{array}$ & $\begin{array}{l}9 / 293 \\
\text { breasts } \\
(2.3)\end{array}$ & $\begin{array}{l}\text { adjOR } 0.43 \text { (0.09, } \\
2.02)\end{array}$ & NR \\
\hline $\begin{array}{l}\text { Ganesh Kumar, } \\
\text { 2021, 33172826, } \\
\text { US \& Canada }\end{array}$ & NRCS & Moderate & $\begin{array}{l}\text { Capsular } \\
\text { contracture }\end{array}$ & NR & $2 y$ & $\begin{array}{l}\text { All } \\
\text { patients }\end{array}$ & $\begin{array}{l}14 / 738 \\
(1.9)\end{array}$ & $12 / 713(1.7)$ & NR & 0.24 \\
\hline $\begin{array}{l}\text { Nealon, 2020b, } \\
31605310 \text {, US }\end{array}$ & NRCS & High & $\begin{array}{l}\text { Capsular } \\
\text { contracture }\end{array}$ & NR & $5.3 y$ & $\begin{array}{l}\text { All } \\
\text { patients }\end{array}$ & NR & NR & $\begin{array}{l}\text { adjOR } 0.78(0.46, \\
1.36)\end{array}$ & 0.38 \\
\hline $\begin{array}{l}\text { Sobti, 2018, } \\
\text { 29481386, US }\end{array}$ & NRCS & High & $\begin{array}{l}\text { Capsular } \\
\text { contracture }\end{array}$ & NR & $5 y$ & $\begin{array}{l}\text { All } \\
\text { patients }\end{array}$ & $\begin{array}{l}21 / 465 \\
\text { breasts } \\
(4.52)\end{array}$ & $\begin{array}{l}7 / 217 \\
\text { breasts } \\
(3.23)\end{array}$ & $\begin{array}{l}\text { Nonuse vs use of } \\
\text { ADM: adjOR } 0.57 \\
(0.23,1.43)\end{array}$ & NR \\
\hline $\begin{array}{l}\text { Vardanian, 2011, } \\
22030500, \text { US }\end{array}$ & NRCS & High & $\begin{array}{l}\text { Capsular } \\
\text { contracture }\end{array}$ & NR & $2.4 \mathrm{y}$ & $\begin{array}{l}\text { All } \\
\text { patients }\end{array}$ & $\begin{array}{l}8 / 208 \\
\text { breasts } \\
(3.8)\end{array}$ & $\begin{array}{l}25 / 129 \\
\text { breasts } \\
(19.4)\end{array}$ & $\begin{array}{l}\text { adjOR } 0.18(0.08, \\
0.43)\end{array}$ & NR \\
\hline $\begin{array}{l}\text { Vardanian, 2011, } \\
22030500, \text { US }\end{array}$ & NRCS & High & $\begin{array}{l}\text { Harms to } \\
\text { inframammary } \\
\text { fold }\end{array}$ & $\begin{array}{l}\text { Inframammary } \\
\text { fold issues other } \\
\text { than bottoming- } \\
\text { out or shifting, } \\
\text { but related to the } \\
\text { integrity of the } \\
\text { fold }\end{array}$ & $2.4 \mathrm{y}$ & $\begin{array}{l}\text { All } \\
\text { patients }\end{array}$ & $\begin{array}{l}17 / 208 \\
\text { breasts } \\
(8.2)\end{array}$ & $\begin{array}{l}25 / 129 \\
\text { breasts } \\
(19.4)\end{array}$ & $\begin{array}{l}\text { adjOR } 0.49(0.23, \\
1.01)\end{array}$ & NR \\
\hline
\end{tabular}

Abbreviations: $\mathrm{ADM}=$ acellular dermal matrix, $\mathrm{CI}=$ confidence interval, $\mathrm{d}=$ days, $\mathrm{IBR}=$ implant-based reconstruction, $\mathrm{N} / \mathrm{A}=$ not applicable, $\mathrm{NR}=$ not reported, $\mathrm{NRCS}=$ nonrandomized comparative study, $\mathrm{PMID}=$ PubMed identifier, $\mathrm{RoB}=$ risk of bias.

Colors: Header rows are shaded orange. Rows for every alternate outcome are shaded blue. The colors do not add unique information. 
Table E-5.3. Summary Table - Key Question 5: Use versus nonuse of human ADMs during IBR - continuous outcomes (pain and analgesic use)

\begin{tabular}{|c|c|c|c|c|c|c|c|c|c|c|c|}
\hline $\begin{array}{l}\text { Study, Year, } \\
\text { PMID, } \\
\text { Country }\end{array}$ & Design & $\begin{array}{l}\text { Overall } \\
\text { RoB }\end{array}$ & Outcome & $\begin{array}{l}\text { Outcome } \\
\text { Measurement }\end{array}$ & $\begin{array}{l}\text { Time } \\
\text { Point }\end{array}$ & $\begin{array}{l}\text { Use } \\
\text { of } \\
\text { ADM, } \\
\mathbf{N}\end{array}$ & $\begin{array}{l}\text { Use of } \\
\text { ADM, } \\
\text { Mean (SD) }\end{array}$ & $\begin{array}{l}\text { Nonuse } \\
\text { of } \\
\text { ADM, N }\end{array}$ & $\begin{array}{l}\text { Nonuse of } \\
\text { ADM, Mean } \\
\text { (SD) }\end{array}$ & $\begin{array}{l}\text { Effect Size } \\
(95 \% \mathrm{Cl})\end{array}$ & $\begin{array}{l}P \\
\text { Value }\end{array}$ \\
\hline \multirow{3}{*}{$\begin{array}{l}\text { McCarthy, } \\
2012 \text {, } \\
23096987, \text { US }\end{array}$} & RCT & Moderate & Pain & $\begin{array}{l}\text { Visual analog } \\
\text { scale }(0-100)\end{array}$ & $24 \mathrm{~h}$ & 36 & $54.6(27.6)$ & 33 & $42.8(24.5)$ & $\begin{array}{l}\text { NMD } 6.2(-4.9 \\
17.3)^{*}\end{array}$ & 0.27 \\
\hline & RCT & Moderate & Pain & $\begin{array}{l}\text { Visual analog } \\
\text { scale }(0-100)\end{array}$ & $\begin{array}{l}\text { Expansion } \\
\text { phase }\end{array}$ & 36 & $17(15.9)$ & 33 & $4.6(8.9)$ & $\begin{array}{l}\text { NMD } 6.8(1.1, \\
12.5)^{\star}\end{array}$ & 0.019 \\
\hline & RCT & Moderate & Pain & $\begin{array}{l}\text { Visual analog } \\
\text { scale }(0-100) \\
\end{array}$ & $\begin{array}{l}\text { After } \\
\text { expansion }\end{array}$ & 36 & $5.6(11.6)$ & 33 & $4.6(8.9)$ & $\begin{array}{l}\text { NMD -4.6 (-9.8, } \\
0.6)^{*}\end{array}$ & 0.081 \\
\hline \multirow{2}{*}{$\begin{array}{l}\text { Cattelani, } \\
2018, \\
29275104, \text { Italy }\end{array}$} & NRCS & High & Pain & $\begin{array}{l}\text { BPI-SF (0- } \\
100)\end{array}$ & $1 \mathrm{~d}$ & 39 & $\begin{array}{l}17.56 \\
(15.52)\end{array}$ & 45 & $44.11(15.83)$ & NR & $<0.001$ \\
\hline & NRCS & High & Pain & $\begin{array}{l}\text { BPI-SF (0- } \\
100)\end{array}$ & $7 d$ & 39 & $\begin{array}{l}8.23 \\
(15.39) \\
\end{array}$ & 45 & $21.96(18.59)$ & NR & $<0.001$ \\
\hline \multirow{3}{*}{$\begin{array}{l}\text { McCarthy, } \\
2012 \text {, } \\
23096987, \text { US }\end{array}$} & RCT & Moderate & $\begin{array}{l}\text { Analgesic } \\
\text { use }\end{array}$ & $\begin{array}{l}\text { Oral codeine } \\
\text { equivalents }\end{array}$ & $0-6 \mathrm{~h}$ & 33 & $228(153)$ & 30 & 256 (197) & $\begin{array}{l}M D-28 \mathrm{mg} \mathrm{(-116,} \\
60)^{\star}\end{array}$ & 0.77 \\
\hline & RCT & Moderate & $\begin{array}{l}\text { Analgesic } \\
\text { use }\end{array}$ & $\begin{array}{l}\text { Oral codeine } \\
\text { equivalents }\end{array}$ & $6-24 \mathrm{~h}$ & 33 & 619 (519) & 30 & 715 (533) & $\begin{array}{l}\text { MD -96 mg (-356, } \\
164)^{*}\end{array}$ & 0.38 \\
\hline & RCT & Moderate & $\begin{array}{l}\text { Analgesic } \\
\text { use }\end{array}$ & $\begin{array}{l}\text { Oral codeine } \\
\text { equivalents }\end{array}$ & $0-24 \mathrm{~h}$ & 36 & 776 (602) & 32 & $910(634)$ & $\begin{array}{l}\text { MD -134 mg } \\
(-440,172)^{*}\end{array}$ & 0.38 \\
\hline
\end{tabular}

Abbreviations: ADM = acellular dermal matrix, BPI-SF = Brief Pain Inventory-Short Form, CI = confidence interval, $\mathrm{d}=$ days, $\mathrm{h}=$ hours, IBR $=$ implant-based reconstruction, $\mathrm{MD}=$ mean difference, $\mathrm{mg}=$ milligrams, $\mathrm{NR}=$ not reported, $\mathrm{NRCS}=$ nonrandomized comparative study, $\mathrm{PMID}=\mathrm{PubMed}$ identifier, $\mathrm{RCT}=$ randomized controlled trial, RoB $=$ risk of bias, $\mathrm{SD}=$ standard deviation.

Colors: Header rows are shaded orange. Rows for every alternate outcome are shaded blue. The colors do not add unique information.

* Calculated. 
Table E-5.4. Summary Table - Key Question 5: Use versus nonuse of human ADMs during IBR - categorical outcomes (composite or unspecified harms)

\begin{tabular}{|c|c|c|c|c|c|c|c|c|}
\hline $\begin{array}{l}\text { Study, Year, } \\
\text { PMID, Country }\end{array}$ & Design & $\begin{array}{l}\text { Overall } \\
\text { RoB }\end{array}$ & Outcome Measurement & $\begin{array}{l}\text { Time } \\
\text { Point }\end{array}$ & $\begin{array}{l}\text { Use of ADM, } \\
\mathrm{n} / \mathrm{N}(\%)\end{array}$ & $\begin{array}{l}\text { Nonuse of } \\
\text { ADM, n/N (\%) }\end{array}$ & Effect Size $(95 \% \mathrm{CI})$ & $\begin{array}{l}P \\
\text { Value }\end{array}$ \\
\hline $\begin{array}{l}\text { Wendel, 2013, } \\
\text { None, US }\end{array}$ & RCT & High & Serious adverse events & $1 \mathrm{mo}$ & $0 / 20(0)$ & $0 / 16(0)$ & $\mathrm{N} / \mathrm{A}$ & $\mathrm{N} / \mathrm{A}$ \\
\hline $\begin{array}{l}\text { Brooke, 2012, } \\
22868313, \text { US }\end{array}$ & NRCS & High & $\begin{array}{l}\text { Clinically significant complications, defined } \\
\text { as cellulitis, abscess, seroma, expander leak } \\
\text { or puncture, skin necrosis, wound } \\
\text { dehiscence, or hematoma requiring } \\
\text { readmission, reoperation, and/or expander } \\
\text { explantation }\end{array}$ & NR & $\begin{array}{l}37 / 221 \\
\text { breasts (17) }\end{array}$ & $\begin{array}{l}\text { 7/64 breasts } \\
\text { (11) }\end{array}$ & NR & 0.48 \\
\hline \multirow{2}{*}{$\begin{array}{l}\text { Ganesh } \\
\text { Kumar, 2021, } \\
33172826, \text { US } \\
\text { \& Canada }\end{array}$} & NRCS & Moderate & Any complication & $2 y$ & $\begin{array}{l}211 / 738 \\
(28.6) \\
\end{array}$ & $\begin{array}{l}178 / 713 \\
(25.0) \\
\end{array}$ & adjOR $1.21(0.86,1.70)$ & 0.26 \\
\hline & NRCS & Moderate & Major complications & $2 y$ & $\begin{array}{l}169 / 738 \\
(22.9)\end{array}$ & $\begin{array}{l}117 / 713 \\
(16.4)\end{array}$ & adjOR $1.43(1.00,2.05)$ & 0.052 \\
\hline \multirow{3}{*}{$\begin{array}{l}\text { Hirsch, 2014, } \\
25347643, \text { US }\end{array}$} & NRCS & Low & Any complication & $3.5 \mathrm{y}$ & NR & NR & adjOR $0.90(0.60,1.34)$ & 0.6 \\
\hline & NRCS & Low & Operative complication except explantation & $3.5 \mathrm{y}$ & NR & NR & adjOR $0.68(0.46,1.02)$ & 0.46 \\
\hline & NRCS & Low & Nonoperative complication & $3.5 y$ & NR & NR & adjOR $0.67(0.26,1.74)$ & 0.43 \\
\hline $\begin{array}{l}\text { Liu, 2011, } \\
21228744 \text {, US }\end{array}$ & NRCS & High & Surgical complications & NR & $52 / 266(19.5)$ & $25 / 204(12.3)$ & adjOR $1.76(1.03,3.01)$ & 0.036 \\
\hline $\begin{array}{l}\text { Safran, } 2020, \\
32221195 \\
\text { Canada }\end{array}$ & NRCS & High & $\begin{array}{l}\text { Any complication, including hematoma, } \\
\text { infection, seroma, implant displacement, } \\
\text { NAC full-thickness necrosis, superficial } \\
\text { cellulitis, red breast syndrome, incision } \\
\text { necrosis, delayed healing, hypergranulation, } \\
\text { and NAC superficial necrosis. }\end{array}$ & NR & $\begin{array}{l}\text { NR/243 } \\
\text { breasts (NR) }\end{array}$ & $\begin{array}{l}\text { NR/70 } \\
\text { breasts (NR) }\end{array}$ & adjOR $1.59(0.56,4.50)$ & NR \\
\hline \multirow{3}{*}{$\begin{array}{l}\text { Stein, 2020, } \\
32561384 \\
\text { Canada }\end{array}$} & NRCS & High & Any complication & $\begin{array}{l}10 \mathrm{mo} \\
-5 \mathrm{y} \\
\end{array}$ & 16/41 (39.0) & $37 / 89(42.1)$ & adjOR $0.86(0.26,2.78)$ & NR \\
\hline & NRCS & High & Major complications & $\begin{array}{l}10 \mathrm{mo} \\
-5 \mathrm{y}\end{array}$ & $10 / 41(24.4)$ & $22 / 89(24.7)$ & adjOR $0.83(0.22,3.08)$ & NR \\
\hline & NRCS & High & Minor complications & $\begin{array}{l}10 \mathrm{mo} \\
-5 \mathrm{y}\end{array}$ & $10 / 41(24.4)$ & $20 / 89(22.5)$ & adjOR $0.83(0.21,3.29)$ & NR \\
\hline $\begin{array}{l}\text { Weichman, } \\
2012 \text {, } \\
22544088, \text { US }\end{array}$ & NRCS & Moderate & $\begin{array}{l}\text { Complications including mastectomy skin flap } \\
\text { necrosis, mastectomy skin flap necrosis and } \\
\text { associated infection, infection alone, seroma, } \\
\text { and hematoma }\end{array}$ & $3 y$ & $\begin{array}{l}\text { NR/442 } \\
\text { breasts (NR) }\end{array}$ & $\begin{array}{l}\text { NR/186 } \\
\text { breasts (NR) }\end{array}$ & NR & $<0.05$ \\
\hline $\begin{array}{l}\text { Woo, } 2017, \\
28509694, \\
\text { South Korea }\end{array}$ & NRCS & High & $\begin{array}{l}\text { Major complication: Complications } \\
\text { necessitating additional surgery or } \\
\text { intervention }\end{array}$ & NR & $26 / 199(13.1)$ & $38 / 199(19.1)$ & adjOR $1.1(0.5,2.4)$ & 0.81 \\
\hline
\end{tabular}

Abbreviations: adj = adjusted, $\mathrm{ADM}=$ acellular dermal matrix, $\mathrm{CI}=$ confidence interval, $\mathrm{IBR}=$ implant-based reconstruction, $\mathrm{mo}=\mathrm{months}, \mathrm{N} / \mathrm{A}=\mathrm{not}$ applicable, $\mathrm{NR}=$ not reported, NRCS $=$ nonrandomized comparative study, $\mathrm{OR}=$ odds ratio, $\mathrm{PMID}=$ PubMed identifier, $\mathrm{RCT}=$ randomized controlled trial, $\mathrm{RoB}=$ risk of bias, $\mathrm{y}=\mathrm{years}$. 
Colors: Header rows are shaded orange. Rows for every alternate study are shaded blue. The colors do not add unique information. 
Table E-6.1. Summary Table - Key Question 6: Comparisons of flap types for AR - continuous outcomes (physical well-being)

\begin{tabular}{|c|c|c|c|c|c|c|c|c|c|}
\hline $\begin{array}{l}\text { Study, Year, } \\
\text { PMID, } \\
\text { Country }\end{array}$ & Design & $\begin{array}{l}\text { Overall } \\
\text { RoB }\end{array}$ & Outcome Measurement & $\begin{array}{l}\text { Time } \\
\text { Point }\end{array}$ & Flap Type & $\mathbf{N}$ & $\begin{array}{l}\text { Mean (SD } \\
\text { or } 95 \% \\
\mathrm{Cl})\end{array}$ & Effect Size $(95 \%$ Cl) & P Value \\
\hline \multirow{10}{*}{$\begin{array}{l}\text { Rindom, } \\
2019, \\
31515191, \\
\text { Denmark }\end{array}$} & RCT & Moderate & CMSS: Total score $(0-100)$ & $1 \mathrm{y}$ & LD & 18 & $\begin{array}{l}68.1(58.2 \\
79.9)\end{array}$ & Ref & Ref \\
\hline & RCT & Moderate & CMSS: Total score $(0-100)$ & $1 \mathrm{y}$ & TAP & 22 & $\begin{array}{l}78.7(70.9, \\
86.4)\end{array}$ & adjNMD $6.2(0.5,12.0)$ & 0.033 \\
\hline & RCT & Moderate & CMSS: Pain (0-15) & $1 \mathrm{y}$ & LD & 18 & $\begin{array}{l}11.6(9.8 \\
13.4)\end{array}$ & Ref & Ref \\
\hline & RCT & Moderate & CMSS: Pain (0-15) & $1 \mathrm{y}$ & TAP & 22 & $\begin{array}{l}14.0(12.8, \\
15.2)\end{array}$ & adjNMD $1.8(0.2,3.4)$ & 0.023 \\
\hline & RCT & Moderate & CMSS: Activity in daily life (0-20) & $1 \mathrm{y}$ & LD & 18 & $\begin{array}{l}\text { 17.1 (14.9, } \\
19.2)\end{array}$ & Ref & Ref \\
\hline & RCT & Moderate & CMSS: Activity in daily life $(0-20)$ & $1 \mathrm{y}$ & TAP & 22 & $\begin{array}{l}18.7(17.3 \\
20.0)\end{array}$ & adjNMD $2.6(1.1,4.2)$ & $<0.0001$ \\
\hline & RCT & Moderate & CMSS: Range of motion (0-40) & $1 \mathrm{y}$ & LD & 18 & $\begin{array}{l}29.6(24.6, \\
34.5)\end{array}$ & Ref & Ref \\
\hline & RCT & Moderate & CMSS: Range of motion (0-40) & $1 \mathrm{y}$ & TAP & 22 & $\begin{array}{l}34.8(31.0 \\
38.6)\end{array}$ & adjNMD $0.9(-1.4,3.2)$ & 0.45 \\
\hline & RCT & Moderate & CMSS: Strength (0-25) & $1 \mathrm{y}$ & LD & 18 & $\begin{array}{l}9.9(7.8 \\
12.0)\end{array}$ & Ref & Ref \\
\hline & RCT & Moderate & CMSS: Strength (0-25) & $1 \mathrm{y}$ & TAP & 22 & $\begin{array}{l}11.2(9.3 \\
13.1)\end{array}$ & adjNMD $1.2(-1.0,3.3)$ & 0.29 \\
\hline \multirow{10}{*}{$\begin{array}{l}\text { Erdmann- } \\
\text { Sager, 2018, } \\
29019862, \\
\text { US \& } \\
\text { Canada }\end{array}$} & NRCS & Moderate & BREAST-Q: Abdomen (0-100) & $1 \mathrm{y}$ & DIEP & NR & NR & Ref & Ref \\
\hline & NRCS & Moderate & BREAST-Q: Abdomen (0-100) & $1 \mathrm{y}$ & Free TRAM & NR & NR & $\begin{array}{l}\text { vs. DIEP: adjMD }-4.16 \\
(-8.33,0.02)\end{array}$ & NR \\
\hline & NRCS & Moderate & BREAST-Q: Abdomen (0-100) & $1 \mathrm{y}$ & Pedicled TRAM & NR & NR & $\begin{array}{l}\text { vs. DIEP: adjMD -4.01 } \\
(-8.48,0.45)\end{array}$ & NR \\
\hline & NRCS & Moderate & BREAST-Q: Abdomen (0-100) & $1 \mathrm{y}$ & SIEA & NR & NR & $\frac{\text { vs. DIEP: adjMD } 4.72(-0.07,}{9.52)}$ & NR \\
\hline & NRCS & Moderate & BREAST-Q: Abdomen (0-100) & $2 y$ & DIEP & NR & NR & Ref & Ref \\
\hline & NRCS & Moderate & BREAST-Q: Abdomen (0-100) & $2 y$ & Free TRAM & NR & NR & $\begin{array}{l}\frac{\text { vs. DIEP: }}{-0.31)} \text { adjMD }-4.9(-9.50 \text {, } \\
\text { - }\end{array}$ & NR \\
\hline & NRCS & Moderate & BREAST-Q: Abdomen (0-100) & $2 y$ & Pedicled TRAM & NR & NR & $\begin{array}{l}\text { vs. DIEP: adjMD }-7.22 \\
(-12.30,-2.14)\end{array}$ & NR \\
\hline & NRCS & Moderate & BREAST-Q: Abdomen (0-100) & $2 y$ & SIEA & NR & NR & $\frac{\text { vs. DIEP: }}{5.95)}$ adjMD $0.58(-4.79$, & NR \\
\hline & NRCS & Moderate & $\begin{array}{l}\text { BREAST-Q: Chest, upper body (0- } \\
\text { 100) }\end{array}$ & $1 \mathrm{y}$ & DIEP & NR & NR & Ref & Ref \\
\hline & NRCS & Moderate & $\begin{array}{l}\text { BREAST -Q: Chest, upper body (0- } \\
100)\end{array}$ & $1 \mathrm{y}$ & Free TRAM & NR & NR & $\begin{array}{l}\text { vs. DIEP: adjMD }-1.55 \\
(-5.35,2.24)\end{array}$ & NR \\
\hline
\end{tabular}




\begin{tabular}{|c|c|c|c|c|c|c|c|c|c|}
\hline $\begin{array}{l}\text { Study, Year, } \\
\text { PMID, } \\
\text { Country }\end{array}$ & Design & $\begin{array}{l}\text { Overall } \\
\text { RoB }\end{array}$ & Outcome Measurement & $\begin{array}{l}\text { Time } \\
\text { Point }\end{array}$ & Flap Type & $\mathbf{N}$ & $\begin{array}{l}\text { Mean (SD } \\
\text { or } 95 \% \\
\mathrm{Cl}) \\
\end{array}$ & Effect Size (95\% Cl) & P Value \\
\hline \multirow{22}{*}{$\begin{array}{l}\text { Erdmann- } \\
\text { Sager, } \\
\text { continued }\end{array}$} & NRCS & Moderate & $\begin{array}{l}\text { BREAST -Q: Chest, upper body (0- } \\
100)\end{array}$ & $1 \mathrm{y}$ & Pedicled TRAM & NR & NR & $\frac{\text { vs. DIEP: }}{4.99)}$ adjMD 1.52 (-1.94, & NR \\
\hline & NRCS & Moderate & $\begin{array}{l}\text { BREAST -Q: Chest, upper body (0- } \\
\text { 100) }\end{array}$ & $1 \mathrm{y}$ & SIEA & NR & NR & $\frac{\text { vs. DIEP: }}{7.05)}$ adjMD $3.42(-0.22$, & NR \\
\hline & NRCS & Moderate & $\begin{array}{l}\text { BREAST -Q: Chest, upper body (0- } \\
\text { 100) }\end{array}$ & $2 y$ & DIEP & NR & NR & Ref & Ref \\
\hline & NRCS & Moderate & $\begin{array}{l}\text { BREAST -Q: Chest, upper body (0- } \\
100)\end{array}$ & $2 y$ & Free TRAM & NR & NR & $\begin{array}{l}\text { vs. DIEP: adjMD }-2.22 \\
(-5.89,1.45)\end{array}$ & NR \\
\hline & NRCS & Moderate & $\begin{array}{l}\text { BREAST -Q: Chest, upper body (0- } \\
100)\end{array}$ & $2 y$ & Pedicled TRAM & NR & NR & $\begin{array}{l}\text { vs. DIEP: adjMD }-3.92 \\
(-7.66,-0.18)\end{array}$ & NR \\
\hline & NRCS & Moderate & $\begin{array}{l}\text { BREAST -Q: Chest, upper body (0- } \\
100)\end{array}$ & $2 y$ & SIEA & NR & NR & $\frac{\text { vs. DIEP: }}{4.95)}$ adjMD $0.76(-3.44$, & NR \\
\hline & NRCS & Moderate & PROMIS: Physical functioning (0-100) & $1 \mathrm{y}$ & DIEP & NR & NR & Ref & Ref \\
\hline & NRCS & Moderate & PROMIS: Physical functioning (0-100) & $1 \mathrm{y}$ & Free TRAM & NR & NR & $\begin{array}{l}\text { vs. DIEP: adjMD }-0.03 \\
(-2.36,2.30)\end{array}$ & NR \\
\hline & NRCS & Moderate & PROMIS: Physical functioning (0-100) & $1 \mathrm{y}$ & Pedicled TRAM & NR & NR & $\frac{\text { vs. DIEP: }}{2.10)}$ adjMD $0.00(-2.09$, & NR \\
\hline & NRCS & Moderate & PROMIS: Physical functioning (0-100) & $1 \mathrm{y}$ & SIEA & NR & NR & $\frac{\text { vs. DIEP: }}{3.26)}$ adjMD $1.26(-0.74$, & NR \\
\hline & NRCS & Moderate & PROMIS: Physical functioning (0-100) & $2 y$ & DIEP & NR & NR & Ref & Ref \\
\hline & NRCS & Moderate & PROMIS: Physical functioning (0-100) & $2 y$ & Free TRAM & NR & NR & $\frac{\text { vs. DIEP: }}{2.36)}$ adjMD $0.42(-1.52$, & NR \\
\hline & NRCS & Moderate & PROMIS: Physical functioning (0-100) & $2 y$ & Pedicled TRAM & NR & NR & $\frac{\text { vs. DIEP: }}{3.55)}$ adjMD $1.48(-0.59$, & NR \\
\hline & NRCS & Moderate & PROMIS: Physical functioning (0-100) & $2 y$ & SIEA & NR & NR & $\frac{\text { vs. DIEP: }}{3.49)}$ adjMD $1.20(-1.09$ & NR \\
\hline & NRCS & Moderate & PROMIS: Pain interference $(0-100)$ & $1 \mathrm{y}$ & DIEP & NR & NR & Ref & Ref \\
\hline & NRCS & Moderate & PROMIS: Pain interference (0-100) & $1 \mathrm{y}$ & Free TRAM & NR & NR & $\frac{\text { vs. DIEP: }}{2.60)}$ adjMD $0.34(-1.91$, & NR \\
\hline & NRCS & Moderate & PROMIS: Pain interference (0-100) & $1 \mathrm{y}$ & Pedicled TRAM & NR & NR & $\begin{array}{l}\text { vs. DIEP: adjMD }-0.07 \\
(-2.10,1.97)\end{array}$ & NR \\
\hline & NRCS & Moderate & PROMIS: Pain interference $(0-100)$ & $1 \mathrm{y}$ & SIEA & NR & NR & $\begin{array}{l}\text { vs. DIEP: adjMD }-0.60 \\
(-2.54,1.34)\end{array}$ & NR \\
\hline & NRCS & Moderate & PROMIS: Pain interference $(0-100)$ & $2 y$ & DIEP & NR & NR & Ref & Ref \\
\hline & NRCS & Moderate & PROMIS: Pain interference $(0-100)$ & $2 y$ & Free TRAM & NR & NR & $\begin{array}{l}\text { vs. DIEP: adjMD }-0.50 \\
(-2.57,1.57)\end{array}$ & NR \\
\hline & NRCS & Moderate & PROMIS: Pain interference $(0-100)$ & $2 y$ & Pedicled TRAM & NR & NR & $\frac{\text { vs. DIEP: }}{3.13)}$ adjMD $0.44(-2.25$, & NR \\
\hline & NRCS & Moderate & PROMIS: Pain interference $(0-100)$ & $2 y$ & SIEA & NR & NR & $\begin{array}{l}\text { vs. DIEP: } \\
2.57)\end{array}$ & NR \\
\hline
\end{tabular}


Abbreviations: adj $=$ adjusted, $\mathrm{CI}=$ confidence interval, $\mathrm{CMSS}=$ Constant Murley Shoulder Score, $\mathrm{DIEP}=$ deep inferior epigastric perforator, $\mathrm{LD}=$ latissimus dorsi, $\mathrm{MD}=$ mean difference, NMD = net mean difference, NR = not reported, NRCS = nonrandomized comparative study, PMID = PubMed identifier, PROMIS = Patient-Reported Outcomes Measurement Information System, RCT = randomized controlled trial, Ref $=$ reference group, $\mathrm{RoB}=$ risk of bias, $\mathrm{SD}=$ standard deviation, $\mathrm{SIEA}=$ superficial inferior epigastric artery perforator, $\mathrm{TRAM}=$ transverse rectus abdominis myocutaneous, $\mathrm{TAP}=$ thoracodorsal artery perforator, $\mathrm{y}=$ years.

Colors: Header rows are shaded orange. Rows for every alternate study are shaded blue. The colors do not add unique information

Table E-6.2. Summary Table - Key Question 6: Comparisons of flap types for AR - NRCS, continuous outcomes (psychosocial and sexual well-being)

\begin{tabular}{|c|c|c|c|c|c|c|c|c|}
\hline $\begin{array}{l}\text { Study, Year, } \\
\text { PMID, } \\
\text { Country }\end{array}$ & $\begin{array}{l}\text { Overall } \\
\text { RoB }\end{array}$ & Outcome Measurement & $\begin{array}{l}\text { Time } \\
\text { Point }\end{array}$ & Flap Type & $\mathbf{N}$ & $\begin{array}{l}\text { Mean } \\
\text { (SD) }\end{array}$ & Effect Size $(95 \% \mathrm{Cl})$ & $\begin{array}{l}P \\
\text { Value }\end{array}$ \\
\hline \multirow{16}{*}{$\begin{array}{l}\text { Erdmann- } \\
\text { Sager, 2018, } \\
29019862 \text {, } \\
\text { US \& Canada }\end{array}$} & Moderate & BREAST-Q: Psychosocial WB (0-100) & $1 \mathrm{y}$ & DIEP & NR & NR & Ref & Ref \\
\hline & Moderate & BREAST-Q: Psychosocial WB (0-100) & $1 \mathrm{y}$ & Free TRAM & NR & NR & vs. DIEP: adjMD -1.14 (-5.33, 3.05) & NR \\
\hline & Moderate & BREAST-Q: Psychosocial WB (0-100) & $1 \mathrm{y}$ & $\begin{array}{l}\text { Pedicled } \\
\text { TRAM }\end{array}$ & NR & NR & vs. DIEP: adjMD $1.27(-3.43,5.97)$ & NR \\
\hline & Moderate & BREAST-Q: Psychosocial WB (0-100) & $1 \mathrm{y}$ & SIEA & NR & NR & vs. DIEP: adjMD $-0.67(-5.66,4.32)$ & NR \\
\hline & Moderate & BREAST-Q: Psychosocial WB (0-100) & $2 y$ & DIEP & NR & NR & Ref & Ref \\
\hline & Moderate & BREAST-Q: Psychosocial WB (0-100) & $2 y$ & Free TRAM & NR & NR & vs. DIEP: adjMD $-0.08(-5.33,5.18)$ & NR \\
\hline & Moderate & BREAST-Q: Psychosocial WB (0-100) & $2 y$ & $\begin{array}{l}\text { Pedicled } \\
\text { TRAM }\end{array}$ & NR & NR & vs. DIEP: adjMD $0.62(-3.95,5.20)$ & NR \\
\hline & Moderate & BREAST-Q: Psychosocial WB (0-100) & $2 y$ & SIEA & NR & NR & vs. DIEP: adjMD $1.64(-3.64,6.91)$ & NR \\
\hline & Moderate & BREAST-Q: Sexual WB (0-100) & $1 \mathrm{y}$ & DIEP & NR & NR & Ref & Ref \\
\hline & Moderate & BREAST-Q: Sexual WB $(0-100)$ & $1 \mathrm{y}$ & Free TRAM & NR & NR & vs. DIEP: adjMD $-2.33(-7.10,2.44)$ & NR \\
\hline & Moderate & BREAST-Q: Sexual WB (0-100) & $1 \mathrm{y}$ & $\begin{array}{l}\text { Pedicled } \\
\text { TRAM }\end{array}$ & NR & NR & vs. DIEP: adjMD $0.81(-4.31,5.93)$ & NR \\
\hline & Moderate & BREAST-Q: Sexual WB $(0-100)$ & $1 \mathrm{y}$ & SIEA & NR & NR & vs. DIEP: adjMD -2.66 $(-8.63,3.31)$ & NR \\
\hline & Moderate & BREAST-Q: Sexual WB $(0-100)$ & $2 y$ & DIEP & NR & NR & Ref & Ref \\
\hline & Moderate & BREAST-Q: Sexual WB $(0-100)$ & $2 y$ & Free TRAM & NR & NR & vs. DIEP: adjMD $2.35(-3.40,8.10)$ & NR \\
\hline & Moderate & BREAST-Q: Sexual WB (0-100) & $2 y$ & $\begin{array}{l}\text { Pedicled } \\
\text { TRAM }\end{array}$ & NR & NR & vs. DIEP: adjMD -2.61 $(-8.97,3.75)$ & NR \\
\hline & Moderate & BREAST-Q: Sexual WB (0-100) & $2 y$ & SIEA & NR & NR & vs. DIEP: adjMD $1.97(-4.15,8.09)$ & NR \\
\hline
\end{tabular}

Abbreviations: adj = adjusted, $\mathrm{CI}=$ confidence interval, DIEP $=$ deep inferior epigastric perforator, $\mathrm{LD}=$ latissimus dorsi, $\mathrm{MD}=$ mean difference, $\mathrm{NR}=$ not reported, $\mathrm{NRCS}=$ nonrandomized comparative study, $\mathrm{PMID}=\mathrm{PubMed}$ identifier, $\mathrm{RCT}=$ randomized controlled trial, $\mathrm{Ref}=$ reference group, $\mathrm{RoB}=$ risk of bias, $\mathrm{SD}=$ standard deviation, $\mathrm{SIEA}=$ superficial inferior epigastric artery perforator, TRAM $=$ transverse rectus abdominis myocutaneous, $\mathrm{WB}=$ well-being, $\mathrm{y}=\mathrm{years}$.

Colors: Header rows are shaded orange. Rows for every alternate outcome are shaded blue. The colors do not add unique information. 
Table E-6.3. Summary Table - Key Question 6: Comparisons of flap types for AR - continuous outcomes (patient satisfaction with breasts and duration of initial hospitalization)

\begin{tabular}{|c|c|c|c|c|c|c|c|c|c|c|}
\hline $\begin{array}{l}\text { Study, } \\
\text { Year, } \\
\text { PMID, } \\
\text { Country }\end{array}$ & Design & $\begin{array}{l}\text { Overall } \\
\text { RoB }\end{array}$ & Outcome & $\begin{array}{l}\text { Outcome } \\
\text { Measurement }\end{array}$ & $\begin{array}{l}\text { Time } \\
\text { Point }\end{array}$ & Flap Type & $\mathbf{N}$ & $\begin{array}{l}\text { Mean } \\
\text { (SD or } \\
\text { Range) }\end{array}$ & Effect Size $(95 \% \mathrm{Cl})$ & $\begin{array}{l}\text { P } \\
\text { Value }\end{array}$ \\
\hline \multirow{18}{*}{$\begin{array}{l}\text { Brandberg, } \\
2000, \\
10626972, \\
\text { Sweden }\end{array}$} & RCT & High & $\begin{array}{l}\text { Patient satisfaction } \\
\text { with breasts }\end{array}$ & Cosmetic (1-6) & $1 \mathrm{y}$ & TRAM & 26 & $5.6(\mathrm{NR})$ & vs. LD: MD $0.36^{*}$ & NR \\
\hline & RCT & High & $\begin{array}{l}\text { Patient satisfaction } \\
\text { with breasts }\end{array}$ & Cosmetic (1-6) & $1 \mathrm{y}$ & LD & 23 & $\begin{array}{l}5.14 \\
(\mathrm{NR}) \\
\end{array}$ & vs. LTD: MD -0.05* & NR \\
\hline & RCT & High & $\begin{array}{l}\text { Patient satisfaction } \\
\text { with breasts }\end{array}$ & Cosmetic (1-6) & $1 \mathrm{y}$ & LTD & 12 & $\begin{array}{l}5.19 \\
\text { (NR) }\end{array}$ & vs. TRAM: $-0.46^{*}$ & NR \\
\hline & RCT & High & $\begin{array}{l}\text { Patient satisfaction } \\
\text { with breasts }\end{array}$ & Shape (1-6) & $1 \mathrm{y}$ & TRAM & 26 & $5.3(\mathrm{NR})$ & vs. LD: MD 0.36* & NR \\
\hline & RCT & High & $\begin{array}{l}\text { Patient satisfaction } \\
\text { with breasts }\end{array}$ & Shape (1-6) & $1 \mathrm{y}$ & LD & 23 & $\begin{array}{l}4.94 \\
(\mathrm{NR})\end{array}$ & vs. LTD: MD $0.35^{*}$ & NR \\
\hline & RCT & High & $\begin{array}{l}\text { Patient satisfaction } \\
\text { with breasts }\end{array}$ & Shape (1-6) & $1 \mathrm{y}$ & LTD & 12 & $\begin{array}{l}4.59 \\
(\mathrm{NR})\end{array}$ & vs. TRAM: MD $-0.71^{*}$ & NR \\
\hline & RCT & High & $\begin{array}{l}\text { Patient satisfaction } \\
\text { with breasts }\end{array}$ & Size (1-6) & $1 \mathrm{y}$ & TRAM & 26 & $\begin{array}{l}5.43 \\
(\mathrm{NR})\end{array}$ & vs. LD: MD 0.50* & NR \\
\hline & RCT & High & $\begin{array}{l}\text { Patient satisfaction } \\
\text { with breasts }\end{array}$ & Size (1-6) & $1 \mathrm{y}$ & LD & 23 & $\begin{array}{l}4.93 \\
(\mathrm{NR})\end{array}$ & vs. LTD: MD -0.18* & NR \\
\hline & RCT & High & $\begin{array}{l}\text { Patient satisfaction } \\
\text { with breasts }\end{array}$ & Size (1-6) & $1 \mathrm{y}$ & LTD & 12 & $\begin{array}{l}5.11 \\
(\mathrm{NR})\end{array}$ & vs. TRAM: MD $-0.32^{*}$ & NR \\
\hline & RCT & High & $\begin{array}{l}\text { Patient satisfaction } \\
\text { with breasts }\end{array}$ & $\begin{array}{l}\text { Scars on the breast (1- } \\
6)\end{array}$ & $1 \mathrm{y}$ & TRAM & 26 & $\begin{array}{l}4.83 \\
(\mathrm{NR}) \\
\end{array}$ & vs. LD: MD $0.36^{*}$ & NR \\
\hline & RCT & High & $\begin{array}{l}\text { Patient satisfaction } \\
\text { with breasts }\end{array}$ & $\begin{array}{l}\text { Scars on the breast (1- } \\
6)\end{array}$ & $1 \mathrm{y}$ & LD & 23 & $\begin{array}{l}4.47 \\
(\mathrm{NR})\end{array}$ & vs. LTD: MD $-0.65^{*}$ & NR \\
\hline & RCT & High & $\begin{array}{l}\text { Patient satisfaction } \\
\text { with breasts }\end{array}$ & $\begin{array}{l}\text { Scars on the breast (1- } \\
6 \text { ) }\end{array}$ & $1 \mathrm{y}$ & LTD & 12 & $\begin{array}{l}5.12 \\
(\mathrm{NR})\end{array}$ & vs. TRAM: MD 0.29* & NR \\
\hline & RCT & High & $\begin{array}{l}\text { Patient satisfaction } \\
\text { with breasts }\end{array}$ & Donor site scars (1-6) & $1 \mathrm{y}$ & TRAM & 26 & $\begin{array}{l}4.76 \\
\text { (NR) }\end{array}$ & vs. LD: MD $0.07^{*}$ & NR \\
\hline & RCT & High & $\begin{array}{l}\text { Patient satisfaction } \\
\text { with breasts }\end{array}$ & Donor site scars (1-6) & $1 \mathrm{y}$ & LD & 23 & $\begin{array}{l}4.69 \\
(\mathrm{NR})\end{array}$ & vs. LTD: MD $-0.26^{*}$ & NR \\
\hline & RCT & High & $\begin{array}{l}\text { Patient satisfaction } \\
\text { with breasts }\end{array}$ & Donor site scars (1-6) & $1 \mathrm{y}$ & LTD & 12 & $\begin{array}{l}4.95 \\
(\mathrm{NR})\end{array}$ & vs. TRAM: MD 0.19* & NR \\
\hline & RCT & High & $\begin{array}{l}\text { Patient satisfaction } \\
\text { with breasts }\end{array}$ & $\begin{array}{l}\text { Similarity with } \\
\text { contralateral breast (1-6) }\end{array}$ & $1 \mathrm{y}$ & TRAM & 26 & $\begin{array}{l}4.76 \\
\text { (NR) }\end{array}$ & vs. LD: MD 0.10* & NR \\
\hline & RCT & High & $\begin{array}{l}\text { Patient satisfaction } \\
\text { with breasts }\end{array}$ & $\begin{array}{l}\text { Similarity with } \\
\text { contralateral breast (1-6) }\end{array}$ & $1 \mathrm{y}$ & LD & 23 & $\begin{array}{l}4.66 \\
(\mathrm{NR})\end{array}$ & vs. LTD: MD $0.81^{*}$ & NR \\
\hline & RCT & High & $\begin{array}{l}\text { Patient satisfaction } \\
\text { with breasts }\end{array}$ & $\begin{array}{l}\text { Similarity with } \\
\text { contralateral breast (1-6) }\end{array}$ & $1 \mathrm{y}$ & LTD & 12 & $\begin{array}{l}3.85 \\
(\mathrm{NR})\end{array}$ & vs. TRAM: MD -0.91* & NR \\
\hline
\end{tabular}




\begin{tabular}{|c|c|c|c|c|c|c|c|c|c|c|}
\hline $\begin{array}{l}\text { Study, } \\
\text { Year, } \\
\text { PMID, } \\
\text { Country }\end{array}$ & Design & $\begin{array}{l}\text { Overall } \\
\text { RoB }\end{array}$ & Outcome & $\begin{array}{l}\text { Outcome } \\
\text { Measurement }\end{array}$ & $\begin{array}{l}\text { Time } \\
\text { Point }\end{array}$ & Flap Type & $\mathbf{N}$ & $\begin{array}{l}\text { Mean } \\
\text { (SD or } \\
\text { Range) }\end{array}$ & Effect Size $(95 \% \mathrm{CI})$ & $\begin{array}{l}\mathbf{P} \\
\text { Value }\end{array}$ \\
\hline \multirow{8}{*}{$\begin{array}{l}\text { Erdmann- } \\
\text { Sager, } \\
2018, \\
29019862, \\
\text { US \& } \\
\text { Canada }\end{array}$} & NRCS & Moderate & $\begin{array}{l}\text { Patient satisfaction } \\
\text { with breasts }\end{array}$ & $\begin{array}{l}\text { BREAST-Q: Satisfaction } \\
\text { with breast }(0-100)\end{array}$ & $1 \mathrm{y}$ & DIEP & NR & NR & Ref & Ref \\
\hline & NRCS & Moderate & $\begin{array}{l}\text { Patient satisfaction } \\
\text { with breasts }\end{array}$ & $\begin{array}{l}\text { BREAST-Q: Satisfaction } \\
\text { with breast }(0-100)\end{array}$ & $1 \mathrm{y}$ & \begin{tabular}{|l|} 
Free \\
TRAM \\
\end{tabular} & NR & NR & $\begin{array}{l}\text { vs. DIEP: adjMD } 0.04 \\
(-4.56,4.63)\end{array}$ & NR \\
\hline & NRCS & Moderate & $\begin{array}{l}\text { Patient satisfaction } \\
\text { with breasts }\end{array}$ & $\begin{array}{l}\text { BREAST-Q: Satisfaction } \\
\text { with breast }(0-100)\end{array}$ & $1 \mathrm{y}$ & $\begin{array}{l}\text { Pedicled } \\
\text { TRAM } \\
\end{array}$ & NR & NR & $\begin{array}{l}\text { vs. DIEP: adjMD } 1.36 \\
(-3.45,6.17)\end{array}$ & NR \\
\hline & NRCS & Moderate & $\begin{array}{l}\text { Patient satisfaction } \\
\text { with breasts }\end{array}$ & $\begin{array}{l}\text { BREAST-Q: Satisfaction } \\
\text { with breast }(0-100)\end{array}$ & $1 \mathrm{y}$ & SIEA & NR & NR & $\begin{array}{l}\text { vs. DIEP: adjMD -1.82 } \\
(-6.37,2.72)\end{array}$ & NR \\
\hline & NRCS & Moderate & $\begin{array}{l}\text { Patient satisfaction } \\
\text { with breasts }\end{array}$ & $\begin{array}{l}\text { BREAST-Q: Satisfaction } \\
\text { with breast }(0-100)\end{array}$ & $2 y$ & DIEP & NR & NR & Ref & Ref \\
\hline & NRCS & Moderate & $\begin{array}{l}\text { Patient satisfaction } \\
\text { with breasts }\end{array}$ & $\begin{array}{l}\text { BREAST-Q: Satisfaction } \\
\text { with breast }(0-100)\end{array}$ & $2 y$ & \begin{tabular}{|l|} 
Free \\
TRAM \\
\end{tabular} & NR & NR & $\begin{array}{l}\text { vs. DIEP: adjMD }-2.61 \\
(-8.97,3.75)\end{array}$ & NR \\
\hline & NRCS & Moderate & $\begin{array}{l}\text { Patient satisfaction } \\
\text { with breasts }\end{array}$ & $\begin{array}{l}\text { BREAST-Q: Satisfaction } \\
\text { with breast }(0-100)\end{array}$ & $2 y$ & $\begin{array}{l}\text { Pedicled } \\
\text { TRAM } \\
\end{array}$ & NR & NR & $\begin{array}{l}\text { vs. DIEP: adjMD } 1.36 \\
(-3.45,6.17)\end{array}$ & NR \\
\hline & NRCS & Moderate & $\begin{array}{l}\text { Patient satisfaction } \\
\text { with breasts }\end{array}$ & $\begin{array}{l}\text { BREAST-Q: Satisfaction } \\
\text { with breast }(0-100)\end{array}$ & $2 y$ & SIEA & NR & NR & $\begin{array}{l}\text { vs. DIEP: adjMD } 0.42 \\
(-5.56,6.4)\end{array}$ & NR \\
\hline \multirow{2}{*}{$\begin{array}{l}\text { Rindom, } \\
2019, \\
31515191, \\
\text { Denmark }\end{array}$} & $\mathrm{RCT}$ & Moderate & $\begin{array}{l}\text { Duration of initial } \\
\text { hospitalization }\end{array}$ & Number of days & \begin{tabular}{|l|} 
Post- \\
op
\end{tabular} & LD & 18 & $\begin{array}{l}6.4 \mathrm{~d}(3- \\
12)\end{array}$ & Ref & Ref \\
\hline & $\mathrm{RCT}$ & Moderate & $\begin{array}{l}\text { Duration of initial } \\
\text { hospitalization }\end{array}$ & Number of days & $\begin{array}{l}\text { Post- } \\
\text { op }\end{array}$ & TAP & 22 & $\begin{array}{l}6.5 \mathrm{~d}(4- \\
14)\end{array}$ & $\begin{array}{l}\text { vs. TAP: } 0.9 \mathrm{~d}(-1.4 \text {, } \\
3.2)\end{array}$ & 0.45 \\
\hline \multirow{2}{*}{$\begin{array}{l}\text { Zoghbi, } \\
2017, \\
28052051, \\
\text { US }\end{array}$} & NRCS & High & $\begin{array}{l}\text { Duration of initial } \\
\text { hospitalization }\end{array}$ & Number of days & \begin{tabular}{|l|} 
Post- \\
op \\
\end{tabular} & DIEP & 9699 & $\begin{array}{l}4.68 \mathrm{~d} \\
(2.80) \\
\end{array}$ & Ref & Ref \\
\hline & NRCS & High & $\begin{array}{l}\text { Duration of initial } \\
\text { hospitalization }\end{array}$ & Number of days & $\begin{array}{l}\text { Post- } \\
\text { op }\end{array}$ & TRAM & 6137 & $\begin{array}{l}4.79 \mathrm{~d} \\
(2.69)\end{array}$ & vs. DIEP: NR & $<0.001$ \\
\hline
\end{tabular}

Abbreviations: adj $=$ adjusted, $\mathrm{CI}=$ confidence interval, $\mathrm{DIEP}=$ deep inferior epigastric perforator, $\mathrm{LD}=$ latissimus dorsi, $\mathrm{LTD}=$ lateral thoracodorsal flap, $\mathrm{MD}=$ mean difference, $\mathrm{NR}=$ not reported, NRCS $=$ nonrandomized comparative study, PMID $=$ PubMed identifier, $\mathrm{RCT}=$ randomized controlled trial, $\mathrm{Ref}=$ reference group, RoB $=$ risk of bias, $\mathrm{SD}=$ standard deviation, SIEA = superficial inferior epigastric artery perforator, TRAM $=$ transverse rectus abdominis myocutaneous, $\mathrm{y}=$ years.

Colors: Header rows are shaded orange. Rows for every alternate outcome are shaded blue. The colors do not add unique information.

* calculated. 
Table E-6.4. Summary Table - Key Question 6: Comparisons of flap types for AR - categorical outcomes (patient satisfaction with breasts, patient satisfaction with outcome, recurrence of breast cancer, and duration of initial hospitalization)

\begin{tabular}{|c|c|c|c|c|c|c|c|c|c|}
\hline $\begin{array}{l}\text { Study, } \\
\text { Year, } \\
\text { PMID, } \\
\text { Country }\end{array}$ & Design & $\begin{array}{l}\text { Overall } \\
\text { RoB }\end{array}$ & Outcome & $\begin{array}{l}\text { Outcome } \\
\text { Measurement }\end{array}$ & $\begin{array}{l}\text { Time } \\
\text { Point }\end{array}$ & $\begin{array}{l}\text { Flap } \\
\text { Type }\end{array}$ & $n / N(\%)$ & $\begin{array}{l}\text { Adjusted Odds Ratio (95\% } \\
\mathrm{CI})\end{array}$ & $\begin{array}{l}P \\
\text { Value }\end{array}$ \\
\hline \multirow{6}{*}{$\begin{array}{l}\text { Yueh, } \\
2009, \\
19228537, \\
\text { US }\end{array}$} & NRCS & High & $\begin{array}{l}\text { Patient satisfaction } \\
\text { with breasts }\end{array}$ & Satisfied with breasts & NR & DIEP & $\begin{array}{l}\text { NR/117 } \\
\text { (NR) }\end{array}$ & $\begin{array}{l}\text { vs. TRAM: } 0.67(0.37,1.23) \\
\text { vs. LD: } 0.90(0.60,1.34)^{*}\end{array}$ & NR \\
\hline & NRCS & High & $\begin{array}{l}\text { Patient satisfaction } \\
\text { with breasts }\end{array}$ & Satisfied with breasts & NR & TRAM & $\begin{array}{l}102 / 143 \\
(71.3)\end{array}$ & Ref & Ref \\
\hline & NRCS & High & $\begin{array}{l}\text { Patient satisfaction } \\
\text { with breasts }\end{array}$ & Satisfied with breasts & NR & LD & $\begin{array}{l}68 / 112 \\
(60.7) \\
\end{array}$ & vs. TRAM: $0.78(0.54,1.14)$ & NR \\
\hline & NRCS & High & $\begin{array}{l}\text { Patient satisfaction } \\
\text { with outcome }\end{array}$ & $\begin{array}{l}\text { Generally satisfied with } \\
\text { outcome }\end{array}$ & NR & DIEP & $\begin{array}{l}\mathrm{NR} / 117 \\
(\mathrm{NR})\end{array}$ & $\begin{array}{l}\text { vs. TRAM: } 0.82(0.33,2.01) \\
\text { vs. LD: } 1.05(0.70,1.57)^{*}\end{array}$ & NS \\
\hline & NRCS & High & $\begin{array}{l}\text { Patient satisfaction } \\
\text { with outcome }\end{array}$ & $\begin{array}{l}\text { Generally satisfied with } \\
\text { outcome }\end{array}$ & NR & TRAM & $\begin{array}{l}98 / 143 \\
(68.5)\end{array}$ & Ref & Ref \\
\hline & NRCS & High & $\begin{array}{l}\text { Patient satisfaction } \\
\text { with outcome }\end{array}$ & $\begin{array}{l}\text { Generally satisfied with } \\
\text { outcome }\end{array}$ & NR & LD & $\begin{array}{l}63 / 112 \\
(56.3)\end{array}$ & vs. TRAM: $0.77(0.53,1.11)$ & NS \\
\hline \multirow{3}{*}{$\begin{array}{l}\text { Brandberg, } \\
2000, \\
10626972, \\
\text { Sweden }\end{array}$} & RCT & High & $\begin{array}{l}\text { Recurrence of breast } \\
\text { cancer }\end{array}$ & NR & $1 \mathrm{y}$ & TRAM & $\begin{array}{l}2 / 29 \\
(6.9)\end{array}$ & vs. LD: $2.15(0.18,25.07)^{\star}$ & 0.54 \\
\hline & RCT & High & $\begin{array}{l}\text { Recurrence of breast } \\
\text { cancer }\end{array}$ & NR & $1 \mathrm{y}$ & LD & $\begin{array}{l}1 / 30 \\
(3.33)\end{array}$ & vs. LTD: $1.07(0.03,33.69)^{\star}$ & 0.97 \\
\hline & RCT & High & $\begin{array}{l}\text { Recurrence of breast } \\
\text { cancer }\end{array}$ & NR & $1 \mathrm{y}$ & LTD & $0 / 16(0)$ & $\begin{array}{l}\text { vs. TRAM: } 0.44(0.02, \\
10.28)^{*}\end{array}$ & 0.61 \\
\hline \multirow{2}{*}{$\begin{array}{l}\text { Zoghbi, } \\
2017, \\
28052051, \\
\text { US }\end{array}$} & NRCS & High & $\begin{array}{l}\text { Duration of initial } \\
\text { hospitalization }\end{array}$ & $\begin{array}{l}\text { Increased length of } \\
\text { stay }\end{array}$ & Postop & DIEP & $\begin{array}{l}\text { NR/9699 } \\
\text { (NR) }\end{array}$ & Ref & Ref \\
\hline & NRCS & High & $\begin{array}{l}\text { Duration of initial } \\
\text { hospitalization }\end{array}$ & $\begin{array}{l}\text { Increased length of } \\
\text { stay }\end{array}$ & Postop & TRAM & $\begin{array}{l}\text { NR/6137 } \\
\text { (NR) }\end{array}$ & vs. DIEP: $1.59(1.45,1.72)$ & $<0.001$ \\
\hline
\end{tabular}

Abbreviations: $\mathrm{AR}=$ autologous reconstruction, $\mathrm{CI}=$ confidence interval, $\mathrm{DIEP}=$ deep inferior epigastric perforator, $\mathrm{LD}=$ latissimus dorsi, $\mathrm{NRCS}=$ nonrandomized comparative study, PMID = PubMed identifier, Ref = reference group, $\mathrm{RoB}=$ risk of bias, TRAM = transverse rectus abdominis myocutaneous.

Colors: Header rows are shaded orange. Rows for every alternate outcome are shaded blue. The colors do not add unique information.

* Calculated. 
Table E-6.5. Summary Table - Key Question 6: Comparisons of flap types for AR - categorical outcomes (various)

\begin{tabular}{|c|c|c|c|c|c|c|c|c|c|}
\hline $\begin{array}{l}\text { Study, Year, } \\
\text { PMID, Country }\end{array}$ & Design & $\begin{array}{l}\text { Overall } \\
\text { RoB }\end{array}$ & Outcome & $\begin{array}{l}\text { Outcome } \\
\text { Measurement }\end{array}$ & $\begin{array}{l}\text { Time } \\
\text { Point }\end{array}$ & $\begin{array}{l}\text { Flap } \\
\text { Type }\end{array}$ & $\mathrm{n} / \mathrm{N}(\%)$ & Effect Size $(95 \% \mathrm{Cl})$ & P Value \\
\hline \multirow{3}{*}{$\begin{array}{l}\text { Brandberg, } \\
\text { 2000, 10626972, } \\
\text { Sweden }\end{array}$} & RCT & High & Mortality & NR & $1 \mathrm{y}$ & TRAM & $1 / 29(3.5)$ & $\begin{array}{l}\text { vs. LD: OR } 0.50(0.040 \\
5.83)^{\star}\end{array}$ & 0.58 \\
\hline & $\mathrm{RCT}$ & High & Mortality & NR & $1 \mathrm{y}$ & LD & $2 / 30(6.7)$ & $\begin{array}{l}\text { vs. LTD: OR } 2.21(0.09 \\
52.22)^{*}\end{array}$ & 0.62 \\
\hline & $\mathrm{RCT}$ & High & Mortality & NR & $1 \mathrm{y}$ & LTD & $0 / 16(0)$ & $\begin{array}{l}\text { vs. TRAM: OR } 0.9 \\
(0.03,28.48)^{\star}\end{array}$ & 0.95 \\
\hline \multirow{2}{*}{$\begin{array}{l}\text { Massenburg, } \\
2015,26487657 \\
\text { US }\end{array}$} & NRCS & High & $\begin{array}{l}\text { Unpanned repeat } \\
\text { surgeries for revision }\end{array}$ & NR & $1 \mathrm{mo}$ & $\begin{array}{l}\text { Pedicled } \\
\text { TRAM }\end{array}$ & $\begin{array}{l}159 / 1608 \\
(9.9)\end{array}$ & $\begin{array}{l}\text { vs. LD: adjOR } 1.71 \\
(1.25,2.33)\end{array}$ & NR \\
\hline & NRCS & High & $\begin{array}{l}\text { Unpanned repeat } \\
\text { surgeries for revision }\end{array}$ & NR & $1 \mathrm{mo}$ & LD & $62 / 1079(5.7)$ & Ref & Ref \\
\hline \multirow{2}{*}{$\begin{array}{l}\text { Rindom, 2019, } \\
\text { 31515191, } \\
\text { Denmark }\end{array}$} & RCT & Moderate & Pain & $\begin{array}{l}\text { Shoulder related pain } \\
\text { (Yes/No) }\end{array}$ & $1 \mathrm{y}$ & LD & $13 / 18(72)$ & Ref & Ref \\
\hline & $\mathrm{RCT}$ & Moderate & Pain & $\begin{array}{l}\text { Shoulder related pain } \\
\text { (Yes/No) }\end{array}$ & $1 \mathrm{y}$ & TAP & $7 / 22(32)$ & $\begin{array}{l}\text { vs. LD: adjOR } 0.05 \\
(0.005,0.51)\end{array}$ & 0.011 \\
\hline \multirow[t]{4}{*}{$\begin{array}{l}\text { Rindom, 2019, } \\
\text { 31515191, } \\
\text { Denmark }\end{array}$} & RCT & Moderate & Necrosis & $\begin{array}{l}\text { Major necrosis } \\
\text { requiring removal of } \\
\text { the implant }\end{array}$ & $1 \mathrm{y}$ & LD & $0 / 18(0)$ & Ref & Ref \\
\hline & RCT & Moderate & Necrosis & $\begin{array}{l}\text { Major necrosis } \\
\text { requiring removal of } \\
\text { the implant }\end{array}$ & $1 \mathrm{y}$ & TAP & $1 / 22(4.54)$ & $\begin{array}{l}\text { vs. LD: adjOR } 1.67 \\
(0.05,52.7)^{*}\end{array}$ & $0.77^{*}$ \\
\hline & RCT & Moderate & Necrosis & $\begin{array}{l}\text { Minor necrosis: } \\
\text { epidermolysis or } \\
\text { small necrosis of } \\
\text { most distal part of } \\
\text { flap }\end{array}$ & $1 \mathrm{y}$ & LD & $0 / 18(0)$ & Ref & Ref \\
\hline & RCT & Moderate & Necrosis & $\begin{array}{l}\text { Minor necrosis: } \\
\text { epidermolysis or } \\
\text { small necrosis of } \\
\text { most distal part of } \\
\text { flap }\end{array}$ & $1 \mathrm{y}$ & TAP & $2 / 22(13.6)$ & $\begin{array}{l}\text { vs. LD: adjOR } 5.53 \\
(0.26,118.3)^{*}\end{array}$ & $0.27^{*}$ \\
\hline \multirow{2}{*}{$\begin{array}{l}\text { Abedi, 2016, } \\
25003437, \\
\text { Canada }\end{array}$} & NRCS & High & Necrosis & $\begin{array}{l}\text { Mastectomy flap } \\
\text { necrosis }\end{array}$ & $\begin{array}{l}1.6-1.9 \\
y\end{array}$ & DIEP & $20 / 83(24.1)$ & vs. TRAM: NR & 0.61 \\
\hline & NRCS & High & Necrosis & $\begin{array}{l}\text { Mastectomy flap } \\
\text { necrosis }\end{array}$ & $\begin{array}{l}1.6-1.9 \\
y\end{array}$ & TRAM & $40 / 312(12.8)$ & Ref & Ref \\
\hline \multirow[t]{2}{*}{$\begin{array}{l}\text { Kroll, 2000, } \\
\text { 10987463, US }\end{array}$} & NRCS & High & Necrosis & Fat necrosis & $3 \mathrm{mo}$ & DIEP & $\begin{array}{l}36 / 279 \\
\text { breasts (12.9) }\end{array}$ & $\begin{array}{l}\text { Vs. TRAM: adjOR } 2.10 \\
(0.87,5.10)\end{array}$ & 0.10 \\
\hline & NRCS & High & Necrosis & Fat necrosis & $3 \mathrm{mo}$ & TRAM & $\begin{array}{l}9 / 31 \text { breasts } \\
(29)\end{array}$ & Ref & Ref \\
\hline
\end{tabular}




\begin{tabular}{|c|c|c|c|c|c|c|c|c|c|}
\hline $\begin{array}{l}\text { Study, Year, } \\
\text { PMID, Country }\end{array}$ & Design & $\begin{array}{l}\text { Overall } \\
\text { RoB }\end{array}$ & Outcome & \begin{tabular}{|l} 
Outcome \\
Measurement
\end{tabular} & $\begin{array}{l}\text { Time } \\
\text { Point }\end{array}$ & $\begin{array}{l}\text { Flap } \\
\text { Type }\end{array}$ & $n / N(\%)$ & Effect Size $(95 \% \mathrm{Cl})$ & P Value \\
\hline \multirow{4}{*}{$\begin{array}{l}\text { Erdmann-Sager, } \\
\text { 2018, 29019862, } \\
\text { US \& Canada }\end{array}$} & NRCS & Moderate & $\begin{array}{l}\text { Harms to area of flap } \\
\text { harvest }\end{array}$ & $\begin{array}{l}\text { Any donor site } \\
\text { complication }\end{array}$ & $2 \mathrm{y}$ & DIEP & 99/355 (27.9) & Ref & Ref \\
\hline & NRCS & Moderate & $\begin{array}{l}\text { Harms to area of flap } \\
\text { harvest }\end{array}$ & $\begin{array}{l}\text { Any donor site } \\
\text { complication }\end{array}$ & $2 y$ & \begin{tabular}{|l|} 
Free \\
TRAM \\
\end{tabular} & $14 / 92(15.2)$ & $\begin{array}{l}\text { vs. DIEP: adjOR } 0.52 \\
(0.27,1.02)\end{array}$ & 0.057 \\
\hline & NRCS & Moderate & $\begin{array}{l}\text { Harms to area of flap } \\
\text { harvest }\end{array}$ & $\begin{array}{l}\text { Any donor site } \\
\text { complication }\end{array}$ & $2 y$ & \begin{tabular}{|l|} 
Pedicled \\
TRAM \\
\end{tabular} & $14 / 78(18.0)$ & $\begin{array}{l}\text { vs. DIEP: adjOR } 0.63 \\
(0.32,1.24)\end{array}$ & 0.18 \\
\hline & NRCS & Moderate & $\begin{array}{l}\text { Harms to area of flap } \\
\text { harvest }\end{array}$ & $\begin{array}{l}\text { Any donor site } \\
\text { complication }\end{array}$ & $2 y$ & SIEA & $33 / 62(53.2)$ & $\begin{array}{l}\text { vs. DIEP: adjOR } 2.73 \\
(1.51,4.96)\end{array}$ & 0.001 \\
\hline \multirow{2}{*}{$\begin{array}{l}\text { Knox, 2016, } \\
26267400, \\
\text { Canada }\end{array}$} & NRCS & High & $\begin{array}{l}\text { Harms to area of flap } \\
\text { harvest }\end{array}$ & $\begin{array}{l}\text { Abdominal bulge or } \\
\text { hernia }\end{array}$ & \begin{tabular}{|l|}
$1.7-2.3$ \\
$y$
\end{tabular} & DIEP & $4 / 130(3.10)$ & Ref & Ref \\
\hline & NRCS & High & $\begin{array}{l}\text { Harms to area of flap } \\
\text { harvest }\end{array}$ & $\begin{array}{l}\text { Abdominal bulge or } \\
\text { hernia }\end{array}$ & \begin{tabular}{|l|}
$1.7-2.3$ \\
$y$
\end{tabular} & TRAM & $80 / 377(21.2)$ & $\begin{array}{l}\text { vs. DIEP: adjOR } 5.2 \\
(1.3,20.9)\end{array}$ & 0.002 \\
\hline \multirow[t]{3}{*}{$\begin{array}{l}\text { Mennie, 2015, } \\
\text { 25839173, UK }\end{array}$} & NRCS & High & $\begin{array}{l}\text { Harms to area of flap } \\
\text { harvest }\end{array}$ & Hernia repair & $3 y$ & DIEP & $\begin{array}{l}63 / 5144 \\
(1.22) \\
\end{array}$ & Ref & Ref \\
\hline & NRCS & High & $\begin{array}{l}\text { Harms to area of flap } \\
\text { harvest }\end{array}$ & Hernia repair & $3 y$ & \begin{tabular}{|l|} 
Free \\
TRAM \\
\end{tabular} & $\begin{array}{l}50 / 1963 \\
(2.55) \\
\end{array}$ & $\begin{array}{l}\text { vs. DIEP: adjOR } 1.81 \\
(1.24,2.64)\end{array}$ & NR \\
\hline & NRCS & High & $\begin{array}{l}\text { Harms to area of flap } \\
\text { harvest }\end{array}$ & Hernia repair & $3 y$ & \begin{tabular}{|l|} 
Pedicled \\
TRAM \\
\end{tabular} & $36 / 822(4.38)$ & $\begin{array}{l}\text { vs. DIEP: adjOR } 2.89 \\
(1.91,4.37)\end{array}$ & NR \\
\hline \multirow{2}{*}{$\begin{array}{l}\text { Zhong, 2014, } \\
\text { 24675183, } \\
\text { Canada }\end{array}$} & NRCS & High & $\begin{array}{l}\text { Harms to area of flap } \\
\text { harvest }\end{array}$ & $\begin{array}{l}\text { Abdominal bulge or } \\
\text { hernia }\end{array}$ & NR & DIEP & $15 / 244(6)$ & Ref & Ref \\
\hline & NRCS & High & $\begin{array}{l}\text { Harms to area of flap } \\
\text { harvest }\end{array}$ & $\begin{array}{l}\text { Abdominal bulge or } \\
\text { hernia }\end{array}$ & NR & TRAM & $8 / 48(17)$ & $\begin{array}{l}\text { vs. DIEP: adjOR } 2.73 \\
(1.01,7.07)\end{array}$ & 0.04 \\
\hline \multirow{4}{*}{$\begin{array}{l}\text { Brorson 2020b, } \\
32807615, \\
\text { Sweden }\end{array}$} & $\mathrm{RCT}$ & High & $\begin{array}{l}\text { Thromboembolic } \\
\text { events }\end{array}$ & DVT & $1 \mathrm{mo}$ & DIEP & $0 / 24(0)$ & No events & $\mathrm{N} / \mathrm{A}$ \\
\hline & RCT & High & $\begin{array}{l}\text { Thromboembolic } \\
\text { events }\end{array}$ & DVT & $1 \mathrm{mo}$ & LD & $0 / 32(0)$ & No events & $\mathrm{N} / \mathrm{A}$ \\
\hline & $\mathrm{RCT}$ & High & $\begin{array}{l}\text { Thromboembolic } \\
\text { events }\end{array}$ & $\mathrm{PE}$ & $1 \mathrm{mo}$ & DIEP & $0 / 24(0)$ & No events & $\mathrm{N} / \mathrm{A}$ \\
\hline & RCT & High & $\begin{array}{l}\text { Thromboembolic } \\
\text { events }\end{array}$ & $\mathrm{PE}$ & $1 \mathrm{mo}$ & LD & $0 / 32(0)$ & No events & $\mathrm{N} / \mathrm{A}$ \\
\hline \multirow{2}{*}{$\begin{array}{l}\text { Rindom, 2019, } \\
\text { 31515191, } \\
\text { Denmark }\end{array}$} & RCT & Moderate & Infections & Any infection & $1 \mathrm{y}$ & LD & $1 / 18(5.6)$ & Ref & Ref \\
\hline & RCT & Moderate & Infections & Any infection & $1 \mathrm{y}$ & TAP & $1 / 22(4.5)$ & $\begin{array}{l}\text { vs. LD: OR } 1.24 \text { (0.07, } \\
21.2)^{*}\end{array}$ & $0.88^{*}$ \\
\hline \multirow[t]{2}{*}{$\begin{array}{l}\text { Zoghbi, 2017, } \\
28052051, \text { US }\end{array}$} & NRCS & High & Infections & Wound infections & Postop & TRAM & $\begin{array}{l}\text { NR/6137 } \\
\text { (NR) }\end{array}$ & $\begin{array}{l}\text { vs. DIEP: adjOR } 1.67 \\
(1.23,2.27)\end{array}$ & 0.001 \\
\hline & NRCS & High & Infections & Wound infections & Postop & DIEP & $\begin{array}{l}\text { NR/9699 } \\
\text { (NR) }\end{array}$ & Ref & Ref \\
\hline \multirow[t]{2}{*}{$\begin{array}{l}\text { Zoghbi, 2017, } \\
\text { 28052051, US }\end{array}$} & NRCS & High & Wound dehiscence & NR & NR & DIEP & $\begin{array}{l}\text { NR/9699 } \\
\text { (NR) }\end{array}$ & Ref & Ref \\
\hline & NRCS & High & Wound dehiscence & NR & NR & TRAM & $\begin{array}{l}\text { NR/6137 } \\
\text { (NR) }\end{array}$ & $\begin{array}{l}\text { vs. DIEP: adjOR } 4.3 \\
\text { (NR) }\end{array}$ & $<0.00001$ \\
\hline
\end{tabular}




\begin{tabular}{|c|c|c|c|c|c|c|c|c|c|}
\hline $\begin{array}{l}\text { Study, Year, } \\
\text { PMID, Country }\end{array}$ & Design & $\begin{array}{l}\text { Overall } \\
\text { RoB }\end{array}$ & Outcome & $\begin{array}{l}\text { Outcome } \\
\text { Measurement }\end{array}$ & $\begin{array}{l}\text { Time } \\
\text { Point }\end{array}$ & $\begin{array}{l}\text { Flap } \\
\text { Type }\end{array}$ & $\mathrm{n} / \mathrm{N}(\%)$ & Effect Size (95\% CI) & P Value \\
\hline \multirow{2}{*}{$\begin{array}{l}\text { Rindom, 2019, } \\
\text { 31515191, } \\
\text { Denmark }\end{array}$} & RCT & Moderate & Seroma & NR & $1 \mathrm{y}$ & LD & $1 / 18(5.6)$ & Ref & Ref \\
\hline & $\mathrm{RCT}$ & Moderate & Seroma & NR & $1 \mathrm{y}$ & TAP & $0 / 22(0)$ & $\begin{array}{l}\text { vs. LD: OR } 2.53(0.08 \\
80.0)^{*}\end{array}$ & $0.59^{*}$ \\
\hline \multirow{2}{*}{$\begin{array}{l}\text { Rindom, 2019, } \\
31515191, \\
\text { Denmark }\end{array}$} & RCT & Moderate & Hematoma & NR & $1 \mathrm{y}$ & LD & $0 / 18(0)$ & Ref & Ref \\
\hline & RCT & Moderate & Hematoma & NR & $1 \mathrm{y}$ & TAP & $1 / 22(4.5)$ & $\begin{array}{l}\text { Not calculable (no } \\
\text { events in Ref group) }\end{array}$ & $\begin{array}{l}\text { Not } \\
\text { calculable }\end{array}$ \\
\hline \multirow[t]{2}{*}{$\begin{array}{l}\text { Kroll, 2000, } \\
\text { 10987463, us }\end{array}$} & NRCS & High & Flap failure/loss & Partial flap loss & $3 \mathrm{mo}$ & DIEP & $\begin{array}{l}5 / 31 \text { breasts } \\
(16.1)\end{array}$ & $\begin{array}{l}\text { vs. TRAM: adjOR } 6.74 \\
(1.83,24.7)\end{array}$ & 0.004 \\
\hline & NRCS & High & Flap failure/loss & Partial flap loss & $3 \mathrm{mo}$ & TRAM & $\begin{array}{l}6 / 279 \text { breasts } \\
(2.20)\end{array}$ & Ref & Ref \\
\hline \multirow{2}{*}{$\begin{array}{l}\text { Massenburg, } \\
2015,26487657, \\
\text { US }\end{array}$} & NRCS & High & Flap failure/loss & NR & $1 \mathrm{mo}$ & $\begin{array}{l}\text { Pedicled } \\
\text { TRAM }\end{array}$ & $67 / 2464(2.7)$ & $\begin{array}{l}\text { vs. LD: adjOR } 2.28 \\
(1.38,3.77)\end{array}$ & 0.001 \\
\hline & NRCS & High & Flap failure/loss & NR & $1 \mathrm{mo}$ & LD & $22 / 2085(1.1)$ & Ref & Ref \\
\hline
\end{tabular}

Abbreviations: adj $=$ adjusted $\mathrm{AR}=$ autologous reconstruction, $\mathrm{CI}=$ confidence interval, $\mathrm{DIEP}=$ deep inferior epigastric perforator, $\mathrm{DVT}=$ deep vein thrombosis, $\mathrm{LD}=$ latissimus dorsi, $\mathrm{LTD}=$ lateral thoracodorsal flap, $\mathrm{OR}=$ odds ratio, $\mathrm{PE}=$ pulmonary embolism, $\mathrm{RCT}=$ randomized controlled trial, $\mathrm{PMID}=\mathrm{PubMed}$ identifier, Ref $=$ reference group, RoB $=$ risk of bias, TRAM $=$ transverse rectus abdominis myocutaneous, $\mathrm{y}=$ years.

Colors: Header rows are shaded orange. Rows for every alternate outcome are shaded blue. The colors do not add unique information.

* Calculated. 
Table E-6.6. Summary Table - Key Question 6: Comparisons of flap types for AR - categorical outcomes (composite or unspecified harms)

\begin{tabular}{|c|c|c|c|c|c|c|c|c|c|}
\hline $\begin{array}{l}\text { Study, Year, } \\
\text { PMID, } \\
\text { Country }\end{array}$ & $\begin{array}{l}\text { Desig } \\
n\end{array}$ & $\begin{array}{l}\text { Overall } \\
\text { RoB }\end{array}$ & Outcome Description & $\begin{array}{l}\text { Time } \\
\text { Point }\end{array}$ & $\begin{array}{l}\text { Flap } \\
\text { Type }\end{array}$ & $\mathrm{n} / \mathrm{N}(\%)$ & $\begin{array}{l}\text { Compariso } \\
\mathrm{n}\end{array}$ & $\begin{array}{l}\text { Adjusted Odds } \\
\text { Ratio }(95 \% \mathrm{Cl})\end{array}$ & P Value \\
\hline \multirow{12}{*}{$\begin{array}{l}\text { Brorson } \\
2020 b \\
32807615 \\
\text { Sweden }\end{array}$} & RCT & High & Clavien-Dindo Grade I complications & $1 \mathrm{mo}$ & DIEP & $16 / 34(47)$ & Ref & Ref & Ref \\
\hline & RCT & High & Clavien-Dindo Grade I complications & $1 \mathrm{mo}$ & LD & $20 / 33(63)$ & vs. DIEP & $1.73(0.66,4.57)$ & 0.27 \\
\hline & RCT & High & Clavien-Dindo Grade II complications & $1 \mathrm{mo}$ & DIEP & $14 / 34(41)$ & Ref & Ref & Ref \\
\hline & RCT & High & Clavien-Dindo Grade II complications & $1 \mathrm{mo}$ & LD & $16 / 32(50)$ & vs. DIEP & $1.29(0.48,3.44)$ & 0.76 \\
\hline & RCT & High & Clavien-Dindo Grade IIIa complications & $1 \mathrm{mo}$ & DIEP & $9 / 34(26)$ & Ref & Ref & Ref \\
\hline & RCT & High & Clavien-Dindo Grade IIla complications & $1 \mathrm{mo}$ & LD & $7 / 32(22)$ & vs. DIEP & $0.85(0.27,2.64)$ & 0.77 \\
\hline & RCT & High & Clavien-Dindo Grade IIIb complications & $1 \mathrm{mo}$ & DIEP & $11 / 34(32)$ & Ref & Ref & Ref \\
\hline & RCT & High & Clavien-Dindo Grade IIIb complications & $1 \mathrm{mo}$ & LD & $3 / 32(9)$ & vs. DIEP & $0.22(0.05,0.87)$ & 0.031 \\
\hline & RCT & High & Clavien-Dindo Grade IV complications & $1 \mathrm{mo}$ & DIEP & $0 / 34(0)$ & Ref & No events & $\mathrm{N} / \mathrm{A}$ \\
\hline & RCT & High & Clavien-Dindo Grade IV complications & $1 \mathrm{mo}$ & LD & $0 / 32(0)$ & Vs. DIEP & No events & $\mathrm{N} / \mathrm{A}$ \\
\hline & RCT & High & Clavien-Dindo Grade V complications & $1 \mathrm{mo}$ & DIEP & $0 / 34(0)$ & Ref & No events & $\mathrm{N} / \mathrm{A}$ \\
\hline & RCT & High & Clavien-Dindo Grade V complications & $1 \mathrm{mo}$ & LD & $0 / 32(0)$ & vs. DIEP & No events & $\mathrm{N} / \mathrm{A}$ \\
\hline \multirow{4}{*}{$\begin{array}{l}\text { Rindom, 2019, } \\
\text { 31515191, } \\
\text { Denmark }\end{array}$} & RCT & Moderate & $\begin{array}{l}\text { Minor complications treated } \\
\text { conservatively }\end{array}$ & $1 \mathrm{y}$ & LD & $2 / 18(11.1)$ & Ref & Ref & Ref \\
\hline & RCT & Moderate & $\begin{array}{l}\text { Minor complications treated } \\
\text { conservatively }\end{array}$ & $1 \mathrm{y}$ & TAP & $2 / 22(9.09)$ & vs. LD & $0.8(0.1,6.32)^{*}$ & $0.83^{*}$ \\
\hline & RCT & Moderate & $\begin{array}{l}\text { Major complications requiring surgical } \\
\text { intervention }\end{array}$ & $1 \mathrm{y}$ & LD & $0 / 18(0)$ & Ref & Ref & Ref \\
\hline & RCT & Moderate & $\begin{array}{l}\text { Major complications requiring surgical } \\
\text { intervention }\end{array}$ & $1 \mathrm{y}$ & TAP & $4 / 22(18)$ & vs. LD & $0.13(0.01,2.62)^{*}$ & $0.18^{*}$ \\
\hline \multirow{3}{*}{$\begin{array}{l}\text { Dauplat, 2021, } \\
33622886, \\
\text { France }\end{array}$} & NRCS & Moderate & $\begin{array}{l}\text { Major complications requiring surgical } \\
\text { intervention or readmission }\end{array}$ & $1 \mathrm{y}$ & TRAM & $10 / 30(30)$ & Ref & Ref & Ref \\
\hline & NRCS & Moderate & $\begin{array}{l}\text { Major complications requiring surgical } \\
\text { intervention or readmission }\end{array}$ & $1 \mathrm{y}$ & $\begin{array}{l}\text { LD } \\
\text { with } \\
\text { implant }\end{array}$ & 9/91 (9) & Ref & Ref & Ref \\
\hline & NRCS & Moderate & $\begin{array}{l}\text { Major complications requiring surgical } \\
\text { intervention or readmission }\end{array}$ & $1 \mathrm{y}$ & $\begin{array}{l}\text { LD } \\
\text { without } \\
\text { implant }\end{array}$ & $7 / 78(9)$ & $\begin{array}{l}\text { vs. TRAM } \\
\text { vs. LD with } \\
\text { implant }\end{array}$ & $\begin{array}{l}1.69(1.19,2.41) \\
4.85(1.67,14.1)\end{array}$ & $\begin{array}{l}\text { NR } \\
\text { NR }\end{array}$ \\
\hline \multirow{4}{*}{$\begin{array}{l}\text { Erdmann- } \\
\text { Sager, 2018, } \\
\text { 29019862, US } \\
\text { \& Canada }\end{array}$} & NRCS & Moderate & Any breast complication & $2 y$ & DIEP & NR & Ref & Ref & Ref \\
\hline & NRCS & Moderate & Any breast complication & $2 y$ & $\begin{array}{l}\text { Free } \\
\text { TRAM }\end{array}$ & NR & vs. DIEP & $0.51(0.25,1.02)$ & 0.58 \\
\hline & NRCS & Moderate & Any breast complication & $2 y$ & $\begin{array}{l}\text { Pedicl } \\
\text { ed } \\
\text { TRAM }\end{array}$ & NR & vs. DIEP & $0.94(0.46,1.94)$ & 0.87 \\
\hline & NRCS & Moderate & Any breast complication & $2 y$ & SIEA & NR & vs. DIEP & $1.15(0.61,2.17)$ & 0.67 \\
\hline
\end{tabular}




\begin{tabular}{|c|c|c|c|c|c|c|c|c|c|}
\hline $\begin{array}{l}\text { Study, Year, } \\
\text { PMID, } \\
\text { Country }\end{array}$ & $\begin{array}{l}\text { Desig } \\
n\end{array}$ & $\begin{array}{l}\text { Overall } \\
\text { RoB }\end{array}$ & Outcome Description & $\begin{array}{l}\text { Time } \\
\text { Point }\end{array}$ & $\begin{array}{l}\text { Flap } \\
\text { Type }\end{array}$ & $n / N(\%)$ & $\begin{array}{l}\text { Compariso } \\
\mathbf{n}\end{array}$ & $\begin{array}{l}\text { Adjusted Odds } \\
\text { Ratio (95\% Cl) }\end{array}$ & P Value \\
\hline \multirow[t]{4}{*}{$\begin{array}{l}\text { Massenburg, } \\
2015, \\
26487657, \text { US }\end{array}$} & NRCS & High & Any complication & $1 \mathrm{mo}$ & $\begin{array}{l}\text { Pedicl } \\
\text { ed } \\
\text { TRAM }\end{array}$ & $\begin{array}{l}216 / 1608 \\
(13.4)\end{array}$ & vs. LD & $1.92(1.45,2.55)$ & NR \\
\hline & NRCS & High & Any complication & $1 \mathrm{mo}$ & LD & $77 / 1079(7.1)$ & Ref & Ref & Ref \\
\hline & NRCS & High & $\begin{array}{l}\text { Superficial SSI, deep SSI, organ space } \\
\text { infection, or wound disruption/ } \\
\text { dehiscence }\end{array}$ & $1 \mathrm{mo}$ & $\begin{array}{l}\text { Pedicl } \\
\text { ed } \\
\text { TRAM }\end{array}$ & $\begin{array}{l}199 / 2464 \\
(8.1)\end{array}$ & vs. LD & $1.80(1.29,2.51)$ & 0.001 \\
\hline & NRCS & High & $\begin{array}{l}\text { Superficial SSI, deep SSI, organ space } \\
\text { infection, or wound disruption/ } \\
\text { dehiscence }\end{array}$ & $1 \mathrm{mo}$ & LD & $90 / 2085(4.3)$ & Ref & Ref & Ref \\
\hline \multirow[t]{2}{*}{$\begin{array}{l}\text { Zhong, 2014, } \\
24675183, \\
\text { Canada }\end{array}$} & NRCS & High & $\begin{array}{l}\text { Major breast complications (total/partial } \\
\text { flap loss, fat necrosis, or breast } \\
\text { hematoma) }\end{array}$ & NR & DIEP & $50 / 244(20.5)$ & Ref & Ref & Ref \\
\hline & NRCS & High & $\begin{array}{l}\text { Major breast complications (total/partial } \\
\text { flap loss, fat necrosis, or breast } \\
\text { hematoma) }\end{array}$ & NR & TRAM & $10 / 48(20.8)$ & vs. DIEP & $0.98(0.4,2.14)$ & 0.95 \\
\hline
\end{tabular}

Abbreviations: $\mathrm{AR}=$ autologous reconstruction, $\mathrm{CI}=$ confidence interval, $\mathrm{DIEP}=$ deep inferior epigastric perforator, $\mathrm{LD}=$ latissimus dorsi, $\mathrm{NR}=$ not reported, $\mathrm{NRCS}=$ nonrandomized comparative study, PMID $=$ PubMed identifier, RoB $=$ risk of bias, TRAM $=$ transverse rectus abdominis myocutaneous, SIEA $=$ superficial inferior epigastric artery, $\mathrm{SSI}=$ surgical site infection, $\mathrm{TAP}=$ thoracodorsal artery perforator, $\mathrm{y}=$ years.

Colors: Header rows are shaded orange. Rows for every alternate study are shaded blue. The colors do not add unique information.

* Calculated. 


\section{Appendix F. Results: Full Evidence Tables for Outcomes}

Table F-1.1. Full Evidence Table - Key Question 1: IBR versus AR - continuous outcomes (general quality of life)

\begin{tabular}{|c|c|c|c|c|c|c|c|c|c|}
\hline Study, Year, PMID, Country & Outcome Measurement & Design & Overall RoB & Time Point & Arm & $\mathrm{N}$ & Mean (SD) & Effect Size $(95 \% \mathrm{Cl})$ & P Value \\
\hline \multirow{2}{*}{$\begin{array}{l}\text { Kouwenberg, 2019, } \\
30270015, \text { Netherlands }\end{array}$} & EQ-5D-5L utilities score $(0-1)$ & NRCS & Moderate & NR & IBR & 67 & $0.853(0.18)$ & Ref & Ref \\
\hline & EQ-5D-5L utilities score (0-1) & NRCS & Moderate & NR & AR & 67 & $0.872(0.14)$ & NR & 0.7 \\
\hline \multirow{6}{*}{$\begin{array}{l}\text { Kouwenberg, 2020, } \\
\text { 32590633, Netherlands }\end{array}$} & EQ-5D-5L utilities score $(0-1)$ & NRCS & Moderate & $>6 \mathrm{mo}$ & IBR & 296 & $0.85(0.30)$ & Ref & Ref \\
\hline & EQ-5D-5L utilities score (0-1) & NRCS & Moderate & $>6 \mathrm{mo}$ & AR & 179 & $0.85(0.20)$ & NR & NS \\
\hline & EQ-5D-5L VAS $(0-100)$ & NRCS & Moderate & $>6 \mathrm{mo}$ & IBR & 296 & $77.6(18.4)$ & Ref & Ref \\
\hline & EQ-5D-5L VAS $(0-100)$ & NRCS & Moderate & $>6 \mathrm{mo}$ & AR & 179 & $79.2(16.7)$ & NR & NS \\
\hline & $\begin{array}{l}\text { EORTC QLQC30: Global health } \\
\text { status }(0-100)\end{array}$ & NRCS & Moderate & $>6 \mathrm{mo}$ & IBR & 296 & $80.2(18.4)$ & Ref & Ref \\
\hline & $\begin{array}{l}\text { EORTC QLQC30: Global health } \\
\text { status }(0-100)\end{array}$ & NRCS & Moderate & $>6 \mathrm{mo}$ & AR & 179 & $81.4(14.7)$ & NR & NS \\
\hline \multirow[t]{20}{*}{ Roth, 2007, 17413877, US } & SF-36: Role emotional (0-100) & NRCS & High & Pre-op & IBR & 35 & $69.5(\mathrm{NR})$ & Ref & Ref \\
\hline & SF-36: Role emotional (0-100) & NRCS & High & Pre-op & AR & 55 & $60.6(\mathrm{NR})$ & NR & NR \\
\hline & SF-36: Role emotional $(0-100)$ & NRCS & High & $2 y$ & IBR & 35 & $86.7(\mathrm{NR})$ & Ref & Ref \\
\hline & SF-36: Role emotional $(0-100)$ & NRCS & High & $2 y$ & AR & 55 & $86.1(\mathrm{NR})$ & NR & $\geq 0.05$ \\
\hline & SF-36: Vitality $(0-100)$ & NRCS & High & Pre-op & IBR & 35 & $57.5(\mathrm{NR})$ & Ref & Ref \\
\hline & SF-36: Vitality $(0-100)$ & NRCS & High & Pre-op & AR & 55 & $55.8(\mathrm{NR})$ & NR & NR \\
\hline & SF-36: Vitality $(0-100)$ & NRCS & High & $2 y$ & IBR & 35 & $65.7(\mathrm{NR})$ & Ref & Ref \\
\hline & SF-36: Vitality $(0-100)$ & NRCS & High & $2 y$ & $\mathrm{AR}$ & 55 & $62.4(\mathrm{NR})$ & NR & NR \\
\hline & SF-36: General mental health $(0-100)$ & NRCS & High & Pre-op & IBR & 35 & $67.5(\mathrm{NR})$ & Ref & Ref \\
\hline & SF-36: General mental health $(0-100)$ & NRCS & High & Pre-op & AR & 55 & $65.9(\mathrm{NR})$ & NR & NR \\
\hline & SF-36: General mental health (0-100) & NRCS & High & $2 y$ & IBR & 35 & $77.6(\mathrm{NR})$ & Ref & Ref \\
\hline & SF-36: General mental health $(0-100)$ & NRCS & High & $2 y$ & AR & 55 & $77(\mathrm{NR})$ & NR & $\geq 0.05$ \\
\hline & FACT-B: Functional well-being $(0-28)$ & NRCS & High & Pre-op & IBR & 35 & $20.9(\mathrm{NR})$ & Ref & Ref \\
\hline & FACT-B: Functional well-being $(0-28)$ & NRCS & High & Pre-op & AR & 55 & $20.7(\mathrm{NR})$ & NR & NR \\
\hline & FACT-B: Functional well-being $(0-28)$ & NRCS & High & $2 \mathrm{y}$ & IBR & 35 & $23.3(\mathrm{NR})$ & Ref & Ref \\
\hline & FACT-B: Functional well-being $(0-28)$ & NRCS & High & $2 y$ & AR & 55 & $24.1(\mathrm{NR})$ & NR & $\geq 0.05$ \\
\hline & Body Image (9-45) & NRCS & High & Pre-op & IBR & 35 & $34.2(\mathrm{NR})$ & Ref & Ref \\
\hline & Body Image (9-45) & NRCS & High & Pre-op & AR & 55 & 34.7 (NR) & NR & NR \\
\hline & Body Image (9-45) & NRCS & High & $2 y$ & IBR & 35 & $32.5(\mathrm{NR})$ & Ref & Ref \\
\hline & Body Image (9-45) & NRCS & High & $2 y$ & AR & 55 & $35.3(\mathrm{NR})$ & NR & $\geq 0.05$ \\
\hline
\end{tabular}

Abbreviations: adj $=$ adjusted, $\mathrm{CI}=$ confidence interval, EQ-5D-5L $=5$-level European Quality of Life-5D version, $\mathrm{MD}=$ mean difference, $\mathrm{NR}=$ not reported, $\mathrm{NMD}=$ net mean difference, NRCS = nonrandomized comparative study, PMID = Pubmed identifier, Ref = reference group, RoB = risk of bias, $\mathrm{SD}=\mathrm{standard}$ deviation, $\mathrm{SF}=\mathrm{Short}$ Form.

Colors: Header rows are shaded orange. Rows for every alternate study are shaded blue. The colors do not add unique information. 
Table F-1.2. Full Evidence Table - Key Question 1: IBR versus AR - continuous outcomes (physical well-being)

\begin{tabular}{|c|c|c|c|c|c|c|c|c|c|c|}
\hline $\begin{array}{l}\text { Study, Year, } \\
\text { PMID, Country }\end{array}$ & $\begin{array}{l}\text { Outcome } \\
\text { Measurement }\end{array}$ & Design & $\begin{array}{l}\text { Overall } \\
\text { RoB }\end{array}$ & $\begin{array}{l}\text { Time } \\
\text { Point }\end{array}$ & Arm & Subgroup & $\mathbf{N}$ & Mean (SD) & Effect Size $(95 \% \mathrm{Cl})$ & $\begin{array}{l}\mathbf{P} \\
\text { Value }\end{array}$ \\
\hline \multirow{2}{*}{$\begin{array}{l}\text { Tallroth, 2020, } \\
33436336, \\
\text { Sweden }\end{array}$} & $\begin{array}{l}\text { BREAST-Q: Chest } \\
(0-100)\end{array}$ & RCT & Moderate & $5.3 y$ & IBR & All & 28 & $72.0(21.5)$ & Ref & Ref \\
\hline & $\begin{array}{l}\text { BREAST-Q: Chest } \\
(0-100)\end{array}$ & RCT & Moderate & $5.3 \mathrm{y}$ & AR & All & 42 & $79.6(21.1)$ & MD $7.6(0.3,14.9)$ & 0.041 \\
\hline \multirow[t]{4}{*}{$\begin{array}{l}\text { Eltahir, 2015, } \\
25539295, \\
\text { Netherlands }\end{array}$} & $\begin{array}{l}\text { BREAST-Q: } \\
\text { Physical well-being } \\
(0-100)\end{array}$ & NRCS & Moderate & $2.2 \mathrm{y}$ & IBR & All & 45 & $71.89(15.06)$ & Ref & Ref \\
\hline & $\begin{array}{l}\text { BREAST-Q: } \\
\text { Physical well-being } \\
(0-100)\end{array}$ & NRCS & Moderate & $2.2 \mathrm{y}$ & AR & All & 47 & $77.13(17.11)$ & $\begin{array}{l}\text { adjMD -2.6 (-9.77, } \\
4.57)\end{array}$ & 0.473 \\
\hline & $\begin{array}{l}\text { RAND R1: physical } \\
\text { functioning }(0-100)\end{array}$ & NRCS & Moderate & $2.2 \mathrm{y}$ & IBR & All & 45 & NR & Ref & Ref \\
\hline & $\begin{array}{l}\text { RAND R1: physical } \\
\text { functioning }(0-100)\end{array}$ & NRCS & Moderate & $2.2 \mathrm{y}$ & AR & All & NR & NR & $\operatorname{adjMD} 2.13(-4.2,8.46)$ & 0.506 \\
\hline \multirow{4}{*}{$\begin{array}{l}\text { Kouwenberg, } \\
2020, \\
32590633, \\
\text { Netherlands }\end{array}$} & $\begin{array}{l}\text { BREAST-Q: Chest } \\
(0-100)\end{array}$ & NRCS & Moderate & $>6 \mathrm{mo}$ & IBR & All & 296 & $72.6(17.8)$ & Ref & Ref \\
\hline & $\begin{array}{l}\text { BREAST-Q: Chest } \\
(0-100)\end{array}$ & NRCS & Moderate & $>6 \mathrm{mo}$ & AR & All & 179 & $75.8(15.4)$ & NR & $<0.05$ \\
\hline & $\begin{array}{l}\text { EORTC QLQC30 } \\
(0-100)\end{array}$ & NRCS & Moderate & $>6 \mathrm{mo}$ & IBR & All & 296 & $88.0(20.6)$ & Ref & Ref \\
\hline & $\begin{array}{l}\text { EORTC QLQC30 } \\
(0-100)\end{array}$ & NRCS & Moderate & $>6 \mathrm{mo}$ & AR & All & 179 & 85.6 (15.7) & NR & $<0.05$ \\
\hline \multirow{9}{*}{$\begin{array}{l}\text { Kulkarni, 2017, } \\
\text { 28713853, US } \\
\text { \& Canada }\end{array}$} & $\begin{array}{l}\text { BREAST-Q: Chest } \\
(0-100)\end{array}$ & NRCS & Moderate & Baseline & IBR (all) & All & 1132 & $80(14)$ & NR & NR \\
\hline & $\begin{array}{l}\text { BREAST-Q: Chest } \\
(0-100)\end{array}$ & NRCS & Moderate & Baseline & IBR (all) & Unilateral & 600 & $80.3(13.9)$ & NR & NR \\
\hline & $\begin{array}{l}\text { BREAST-Q: Chest } \\
(0-100)\end{array}$ & NRCS & Moderate & Baseline & IBR (all) & Bilateral & 994 & $80.3(14.6)$ & NR & NR \\
\hline & $\begin{array}{l}\text { BREAST-Q: Chest } \\
(0-100)\end{array}$ & NRCS & Moderate & Baseline & IBR Direct & All & NR & NR & NR & NR \\
\hline & $\begin{array}{l}\text { BREAST-Q: Chest } \\
(0-100)\end{array}$ & NRCS & Moderate & Baseline & IBR TE & All & NR & NR & NR & NR \\
\hline & $\begin{array}{l}\text { BREAST-Q: Chest } \\
(0-100)\end{array}$ & NRCS & Moderate & Baseline & AR (all) & All & 493 & $76.5(15.5)$ & NR & NR \\
\hline & $\begin{array}{l}\text { BREAST-Q: Chest } \\
(0-100)\end{array}$ & NRCS & Moderate & Baseline & AR (all) & Unilateral & 317 & $77.4(15.8)$ & NR & NR \\
\hline & $\begin{array}{l}\text { BREAST-Q: Chest } \\
(0-100)\end{array}$ & NRCS & Moderate & Baseline & AR (all) & Bilateral & 224 & $77.1(14.7)$ & NR & NR \\
\hline & $\begin{array}{l}\text { BREAST-Q: Chest } \\
(0-100)\end{array}$ & NRCS & Moderate & Baseline & AR with DIEP & All & NR & NR & NR & NR \\
\hline
\end{tabular}




\begin{tabular}{|c|c|c|c|c|c|c|c|c|c|c|}
\hline $\begin{array}{l}\text { Study, Year, } \\
\text { PMID, Country }\end{array}$ & $\begin{array}{l}\text { Outcome } \\
\text { Measurement }\end{array}$ & Design & $\begin{array}{l}\text { Overall } \\
\text { RoB }\end{array}$ & $\begin{array}{l}\text { Time } \\
\text { Point }\end{array}$ & Arm & Subgroup & $\mathbf{N}$ & Mean (SD) & Effect Size (95\% CI) & $\begin{array}{l}\mathbf{P} \\
\text { Value }\end{array}$ \\
\hline & $\begin{array}{l}\text { BREAST-Q: Chest } \\
(0-100)\end{array}$ & NRCS & Moderate & Baseline & AR with free TRAM & All & NR & NR & NR & NR \\
\hline & $\begin{array}{l}\text { BREAST-Q: Chest } \\
(0-100)\end{array}$ & NRCS & Moderate & Baseline & AR with pedicled TRAM & All & NR & NR & NR & NR \\
\hline & $\begin{array}{l}\text { BREAST-Q: Chest } \\
(0-100)\end{array}$ & NRCS & Moderate & Baseline & AR with LD & All & NR & NR & NR & NR \\
\hline & $\begin{array}{l}\text { BREAST-Q: Chest } \\
(0-100)\end{array}$ & NRCS & Moderate & Baseline & AR with SIEA & All & NR & NR & NR & NR \\
\hline & $\begin{array}{l}\text { BREAST-Q: Chest } \\
(0-100)\end{array}$ & NRCS & Moderate & $1 \mathrm{y}$ & IBR (all) & All & 791 & $76.7(14.5)$ & Ref & Ref \\
\hline & $\begin{array}{l}\text { BREAST-Q: Chest } \\
(0-100)\end{array}$ & NRCS & Moderate & $1 \mathrm{y}$ & IBR Direct & All & NR & NR & NR & NR \\
\hline & $\begin{array}{l}\text { BREAST-Q: Chest } \\
(0-100)\end{array}$ & NRCS & Moderate & $1 \mathrm{y}$ & IBR TE & All & NR & NR & NR & NR \\
\hline & $\begin{array}{l}\text { BREAST-Q: Chest } \\
(0-100)\end{array}$ & NRCS & Moderate & $1 \mathrm{y}$ & AR (all) & All & 386 & $74.9(15.1)$ & $\begin{array}{l}\text { vs IBR (all): adjMD } 1.6 \\
(-0.57,3.68)\end{array}$ & 0.003 \\
\hline & $\begin{array}{l}\text { BREAST-Q: Chest } \\
(0-100)\end{array}$ & NRCS & Moderate & $1 \mathrm{y}$ & AR with DIEP & All & NR & NR & NR & NR \\
\hline & $\begin{array}{l}\text { BREAST-Q: Chest } \\
(0-100)\end{array}$ & NRCS & Moderate & $1 \mathrm{y}$ & AR with free TRAM & All & NR & NR & NR & NR \\
\hline & $\begin{array}{l}\text { BREAST-Q: Chest } \\
(0-100)\end{array}$ & NRCS & Moderate & $1 \mathrm{y}$ & AR with pedicled TRAM & All & NR & NR & NR & NR \\
\hline & $\begin{array}{l}\text { BREAST-Q: Chest } \\
(0-100)\end{array}$ & NRCS & Moderate & $1 \mathrm{y}$ & AR with LD & All & NR & NR & NR & NR \\
\hline & $\begin{array}{l}\text { BREAST-Q: Chest } \\
(0-100)\end{array}$ & NRCS & Moderate & $1 \mathrm{y}$ & AR with SIEA & All & NR & NR & NR & NR \\
\hline & $\begin{array}{l}\text { BREAST-Q: Chest } \\
(0-100)\end{array}$ & NRCS & Moderate & $2 y$ & IBR (all) & All & NR & NR & NR & NR \\
\hline & $\begin{array}{l}\text { BREAST-Q: Chest } \\
(0-100)\end{array}$ & NRCS & Moderate & $2 y$ & IBR (all) & Unilateral & 600 & $77.2(13.8)$ & Ref & Ref \\
\hline & $\begin{array}{l}\text { BREAST-Q: Chest } \\
(0-100)\end{array}$ & NRCS & Moderate & $2 y$ & IBR (all) & Bilateral & 994 & $77.3(14.6)$ & Ref & Ref \\
\hline & $\begin{array}{l}\text { BREAST-Q: Chest } \\
(0-100)\end{array}$ & NRCS & Moderate & $2 y$ & IBR Direct & All & NR & NR & NR & NR \\
\hline & $\begin{array}{l}\text { BREAST-Q: Chest } \\
(0-100)\end{array}$ & NRCS & Moderate & $2 y$ & IBR TE & All & NR & NR & NR & NR \\
\hline & $\begin{array}{l}\text { BREAST-Q: Chest } \\
(0-100)\end{array}$ & NRCS & Moderate & $2 y$ & AR (all) & All & NR & NR & NR & NR \\
\hline & $\begin{array}{l}\text { BREAST-Q: Chest } \\
(0-100)\end{array}$ & NRCS & Moderate & $2 y$ & AR (all) & Unilateral & 317 & $76.3(15.4)$ & $\begin{array}{l}\text { Vs IBR (Unilateral): } \\
\operatorname{adjMD~} 1.77(\mathrm{NR}, \mathrm{NR})\end{array}$ & 0.238 \\
\hline & $\begin{array}{l}\text { BREAST-Q: Chest } \\
(0-100)\end{array}$ & NRCS & Moderate & $2 y$ & AR (all) & Bilateral & 224 & $74.5(15.4)$ & $\begin{array}{l}\text { Vs IBR (Bilateral): } \\
\text { adjMD } 0.57(\mathrm{NR}, \mathrm{NR})\end{array}$ & 0.57 \\
\hline
\end{tabular}




\begin{tabular}{|c|c|c|c|c|c|c|c|c|c|c|}
\hline $\begin{array}{l}\text { Study, Year, } \\
\text { PMID, Country }\end{array}$ & $\begin{array}{l}\text { Outcome } \\
\text { Measurement }\end{array}$ & Design & $\begin{array}{l}\text { Overall } \\
\text { RoB }\end{array}$ & $\begin{array}{l}\text { Time } \\
\text { Point }\end{array}$ & Arm & Subgroup & $\mathbf{N}$ & Mean (SD) & Effect Size $(95 \% \mathrm{Cl})$ & $\begin{array}{l}\mathbf{P} \\
\text { Value }\end{array}$ \\
\hline & $\begin{array}{l}\text { BREAST-Q: Chest } \\
(0-100)\end{array}$ & NRCS & Moderate & $2 y$ & AR with DIEP & All & NR & NR & NR & NR \\
\hline & $\begin{array}{l}\text { BREAST-Q: Chest } \\
(0-100)\end{array}$ & NRCS & Moderate & $2 y$ & AR with free TRAM & All & NR & NR & NR & NR \\
\hline & $\begin{array}{l}\text { BREAST-Q: Chest } \\
(0-100)\end{array}$ & NRCS & Moderate & $2 y$ & AR with pedicled TRAM & All & NR & NR & NR & NR \\
\hline & $\begin{array}{l}\text { BREAST-Q: Chest } \\
(0-100)\end{array}$ & NRCS & Moderate & $2 y$ & AR with LD & All & NR & NR & NR & NR \\
\hline & $\begin{array}{l}\text { BREAST-Q: Chest } \\
(0-100)\end{array}$ & NRCS & Moderate & $2 y$ & AR with SIEA & All & NR & NR & NR & NR \\
\hline & $\begin{array}{l}\text { BREAST-Q: Chest } \\
\text { and upper body (0- } \\
100)\end{array}$ & NRCS & Moderate & $1 \mathrm{w}$ & IBR (all) & All & 1846 & NR & Ref & Ref \\
\hline & $\begin{array}{l}\text { BREAST-Q: Chest } \\
\text { and upper body (0- } \\
100)\end{array}$ & NRCS & Moderate & $1 w$ & IBR Direct & All & NR & NR & NR & NR \\
\hline & $\begin{array}{l}\text { BREAST-Q: Chest } \\
\text { and upper body (0- } \\
100)\end{array}$ & NRCS & Moderate & $1 \mathrm{w}$ & IBR TE & All & NR & NR & NR & NR \\
\hline & $\begin{array}{l}\text { BREAST-Q: Chest } \\
\text { and upper body (0- } \\
100)\end{array}$ & NRCS & Moderate & $1 \mathrm{w}$ & AR (all) & All & NR & NR & NR & NR \\
\hline & $\begin{array}{l}\text { BREAST-Q: Chest } \\
\text { and upper body (0- } \\
100)\end{array}$ & NRCS & Moderate & $1 \mathrm{w}$ & AR with DIEP & All & 463 & NR & $\frac{\text { vs IBR (all): }}{\text { (NR, NR) }}$ adjMD 4.89 & 0.57 \\
\hline & $\begin{array}{l}\text { BREAST-Q: Chest } \\
\text { and upper body (0- } \\
100)\end{array}$ & NRCS & Moderate & $1 \mathrm{w}$ & AR with free TRAM & All & 111 & NR & $\frac{\text { vs IBR (all): }}{\text { (NR, NR) }}$ & 0.06 \\
\hline & $\begin{array}{l}\text { BREAST-Q: Chest } \\
\text { and upper body (0- } \\
100)\end{array}$ & NRCS & Moderate & $1 w$ & AR with pedicled TRAM & All & 94 & NR & $\frac{\text { vs IBR (all): }}{(\mathrm{NR}, \mathrm{NR})}$ adjMD 6.71 & NR \\
\hline & $\begin{array}{l}\text { BREAST-Q: Chest } \\
\text { and upper body (0- } \\
100)\end{array}$ & NRCS & Moderate & $1 w$ & AR with LD & All & 80 & NR & $\begin{array}{l}\text { vs IBR (all): adjMD } \\
-0.79 \text { (NR, NR) }\end{array}$ & 0.56 \\
\hline & $\begin{array}{l}\text { BREAST-Q: Chest } \\
\text { and upper body (0- } \\
100)\end{array}$ & NRCS & Moderate & $1 w$ & AR with SIEA & All & 73 & NR & $\frac{\text { vs IBR (all): }}{\text { (NR, NR) }}$ & 0.01 \\
\hline & $\begin{array}{l}\text { BREAST-Q: Chest } \\
\text { and upper body (0- } \\
100)\end{array}$ & NRCS & Moderate & $3 \mathrm{mo}$ & IBR (all) & All & NR & NR & NR & NR \\
\hline
\end{tabular}




\begin{tabular}{|c|c|c|c|c|c|c|c|c|c|c|}
\hline $\begin{array}{l}\text { Study, Year, } \\
\text { PMID, Country }\end{array}$ & $\begin{array}{l}\text { Outcome } \\
\text { Measurement }\end{array}$ & Design & $\begin{array}{l}\text { Overall } \\
\text { RoB }\end{array}$ & $\begin{array}{l}\text { Time } \\
\text { Point }\end{array}$ & Arm & Subgroup & $\mathbf{N}$ & Mean (SD) & Effect Size (95\% CI) & $\begin{array}{l}P \\
\text { Value }\end{array}$ \\
\hline & $\begin{array}{l}\text { BREAST-Q: Chest } \\
\text { and upper body (0- } \\
100)\end{array}$ & NRCS & Moderate & $3 \mathrm{mo}$ & IBR Direct & All & 96 & 70.6 (NR) & NR & NR \\
\hline & $\begin{array}{l}\text { BREAST-Q: Chest } \\
\text { and upper body (0- } \\
100)\end{array}$ & NRCS & Moderate & $3 \mathrm{mo}$ & IBR TE & All & 1329 & $67.5(\mathrm{NR})$ & NR & NR \\
\hline & $\begin{array}{l}\text { BREAST-Q: Chest } \\
\text { and upper body (0- } \\
100)\end{array}$ & NRCS & Moderate & $3 \mathrm{mo}$ & AR (all) & All & NR & NR & NR & NR \\
\hline & $\begin{array}{l}\text { BREAST-Q: Chest } \\
\text { and upper body (0- } \\
100)\end{array}$ & NRCS & Moderate & $3 \mathrm{mo}$ & AR with DIEP & All & 296 & 72.9 (NR) & NR & NR \\
\hline & $\begin{array}{l}\text { BREAST-Q: Chest } \\
\text { and upper body (0- } \\
100)\end{array}$ & NRCS & Moderate & $3 \mathrm{mo}$ & AR with free TRAM & All & 83 & $68.3(\mathrm{NR})$ & NR & NR \\
\hline & $\begin{array}{l}\text { BREAST-Q: Chest } \\
\text { and upper body (0- } \\
100)\end{array}$ & NRCS & Moderate & $3 \mathrm{mo}$ & AR with pedicled TRAM & All & 91 & $70.9(\mathrm{NR})$ & NR & NR \\
\hline & $\begin{array}{l}\text { BREAST-Q: Chest } \\
\text { and upper body (0- } \\
100)\end{array}$ & NRCS & Moderate & $3 \mathrm{mo}$ & AR with LD & All & 62 & $69.3(\mathrm{NR})$ & NR & NR \\
\hline & $\begin{array}{l}\text { BREAST-Q: Chest } \\
\text { and upper body (0- } \\
100)\end{array}$ & NRCS & Moderate & $3 \mathrm{mo}$ & AR with SIEA & All & 56 & 73 (NR) & NR & NR \\
\hline & $\begin{array}{l}\text { BREAST-Q: Chest } \\
\text { and upper body (0- } \\
100)\end{array}$ & NRCS & Moderate & $2 y$ & IBR (all) & All & 1490 & NR & Ref & Ref \\
\hline & $\begin{array}{l}\text { BREAST-Q: Chest } \\
\text { and upper body (0- } \\
100)\end{array}$ & NRCS & Moderate & $2 y$ & IBR Direct & All & 93 & NR & Ref & Ref \\
\hline & $\begin{array}{l}\text { BREAST-Q: Chest } \\
\text { and upper body (0- } \\
100)\end{array}$ & NRCS & Moderate & $2 y$ & IBR TE & All & 1263 & NR & $\frac{\text { vs IBR (direct): adjMD }}{0.92 \text { (NR, NR) }}$ & 0.629 \\
\hline & $\begin{array}{l}\text { BREAST-Q: Chest } \\
\text { and upper body (0- } \\
100)\end{array}$ & NRCS & Moderate & $2 y$ & AR (all) & All & 1523 & $75.6(15.4)$ & $\frac{\text { vs IBR (all): }}{(0.13,3.24)}$ & NR \\
\hline & $\begin{array}{l}\text { BREAST-Q: Chest } \\
\text { and upper body (0- } \\
100)\end{array}$ & NRCS & Moderate & $2 y$ & AR with DIEP & All & 350 & NR & $\begin{array}{l}\frac{\text { vs IBR (TE): adjMD }}{-1.44(\mathrm{NR}, \mathrm{NR})} \\
\end{array}$ & 0.289 \\
\hline & $\begin{array}{l}\text { BREAST-Q: Chest } \\
\text { and upper body (0- } \\
100)\end{array}$ & NRCS & Moderate & $2 y$ & AR with free TRAM & All & 87 & NR & $\begin{array}{l}\frac{\text { vs IBR (TE): adjMD }}{-0.62(N R, N R)} \\
\text { - NR }\end{array}$ & 0.774 \\
\hline
\end{tabular}




\begin{tabular}{|c|c|c|c|c|c|c|c|c|c|c|}
\hline $\begin{array}{l}\text { Study, Year, } \\
\text { PMID, Country }\end{array}$ & $\begin{array}{l}\text { Outcome } \\
\text { Measurement }\end{array}$ & Design & $\begin{array}{l}\text { Overall } \\
\text { RoB }\end{array}$ & $\begin{array}{l}\text { Time } \\
\text { Point }\end{array}$ & Arm & Subgroup & $\mathbf{N}$ & Mean (SD) & Effect Size (95\% Cl) & $\begin{array}{l}P \\
\text { Value }\end{array}$ \\
\hline & $\begin{array}{l}\text { BREAST-Q: Chest } \\
\text { and upper body (0- } \\
100)\end{array}$ & NRCS & Moderate & $2 y$ & AR with pedicled TRAM & All & 77 & NR & $\begin{array}{l}\text { vs IBR (TE): adjMD } \\
-3.93(\mathrm{NR}, \mathrm{NR})\end{array}$ & 0.068 \\
\hline & $\begin{array}{l}\text { BREAST-Q: Chest } \\
\text { and upper body (0- } \\
100)\end{array}$ & NRCS & Moderate & $2 y$ & AR with LD & All & 64 & NR & $\begin{array}{l}\text { vs IBR (TE): adjMD } \\
-0.22 \text { (NR, NR) }\end{array}$ & 0.93 \\
\hline & $\begin{array}{l}\text { BREAST-Q: Chest } \\
\text { and upper body (0- } \\
100)\end{array}$ & NRCS & Moderate & $2 y$ & AR with SIEA & All & 62 & NR & $\frac{\text { vs IBR (TE): adjMD }}{2.83 \text { (NR, NR) }}$ & 0.273 \\
\hline & $\begin{array}{l}\text { BREAST-Q: } \\
\text { Abdomen }\end{array}$ & NRCS & Moderate & Baseline & IBR (all) & All & 1129 & $91.3(12.4)$ & & \\
\hline & $\begin{array}{l}\text { BREAST-Q: } \\
\text { Abdomen }\end{array}$ & NRCS & Moderate & Baseline & IBR Direct & All & NR & NR & NR & NR \\
\hline & $\begin{array}{l}\text { BREAST-Q: } \\
\text { Abdomen }\end{array}$ & NRCS & Moderate & Baseline & IBR TE & All & NR & NR & NR & NR \\
\hline & $\begin{array}{l}\text { BREAST-Q: } \\
\text { Abdomen }\end{array}$ & NRCS & Moderate & Baseline & AR (all) & All & 491 & $87.6(15)$ & NR & NR \\
\hline & $\begin{array}{l}\text { BREAST-Q: } \\
\text { Abdomen }\end{array}$ & NRCS & Moderate & Baseline & AR with DIEP & All & NR & NR & NR & NR \\
\hline & $\begin{array}{l}\text { BREAST-Q: } \\
\text { Abdomen }\end{array}$ & NRCS & Moderate & Baseline & AR with free TRAM & All & NR & NR & NR & NR \\
\hline & $\begin{array}{l}\text { BREAST-Q: } \\
\text { Abdomen }\end{array}$ & NRCS & Moderate & Baseline & AR with pedicled TRAM & All & NR & NR & NR & NR \\
\hline & $\begin{array}{l}\text { BREAST-Q: } \\
\text { Abdomen }\end{array}$ & NRCS & Moderate & Baseline & AR with LD & All & NR & NR & NR & NR \\
\hline & $\begin{array}{l}\text { BREAST-Q: } \\
\text { Abdomen }\end{array}$ & NRCS & Moderate & Baseline & AR with SIEA & All & NR & NR & NR & NR \\
\hline & $\begin{array}{l}\text { BREAST-Q: } \\
\text { Abdomen }\end{array}$ & NRCS & Moderate & $1 \mathrm{y}$ & IBR (all) & All & NR & NR & NR & NR \\
\hline & $\begin{array}{l}\text { BREAST-Q: } \\
\text { Abdomen }\end{array}$ & NRCS & Moderate & $1 \mathrm{y}$ & IBR Direct & All & NR & NR & NR & NR \\
\hline & $\begin{array}{l}\text { BREAST-Q: } \\
\text { Abdomen }\end{array}$ & NRCS & Moderate & $1 \mathrm{y}$ & IBR TE & All & NR & NR & NR & NR \\
\hline & $\begin{array}{l}\text { BREAST-Q: } \\
\text { Abdomen }\end{array}$ & NRCS & Moderate & $1 \mathrm{y}$ & AR (all) & All & 378 & $74.5(19.1)$ & NR & NR \\
\hline & $\begin{array}{l}\text { BREAST-Q: } \\
\text { Abdomen }\end{array}$ & NRCS & Moderate & $1 \mathrm{y}$ & AR with DIEP & All & NR & NR & NR & NR \\
\hline & $\begin{array}{l}\text { BREAST-Q: } \\
\text { Abdomen }\end{array}$ & NRCS & Moderate & $1 \mathrm{y}$ & AR with free TRAM & All & NR & NR & NR & NR \\
\hline & $\begin{array}{l}\text { BREAST-Q: } \\
\text { Abdomen }\end{array}$ & NRCS & Moderate & $1 \mathrm{y}$ & AR with pedicled TRAM & All & NR & NR & NR & NR \\
\hline
\end{tabular}




\begin{tabular}{|c|c|c|c|c|c|c|c|c|c|c|}
\hline $\begin{array}{l}\text { Study, Year, } \\
\text { PMID, Country }\end{array}$ & $\begin{array}{l}\text { Outcome } \\
\text { Measurement }\end{array}$ & Design & $\begin{array}{l}\text { Overall } \\
\text { RoB }\end{array}$ & $\begin{array}{l}\text { Time } \\
\text { Point }\end{array}$ & Arm & Subgroup & $\mathbf{N}$ & Mean (SD) & Effect Size (95\% CI) & $\begin{array}{l}\mathbf{P} \\
\text { Value }\end{array}$ \\
\hline & $\begin{array}{l}\text { BREAST-Q: } \\
\text { Abdomen }\end{array}$ & NRCS & Moderate & $1 \mathrm{y}$ & AR with LD & All & NR & NR & NR & NR \\
\hline & $\begin{array}{l}\text { BREAST-Q: } \\
\text { Abdomen }\end{array}$ & NRCS & Moderate & $1 \mathrm{y}$ & AR with SIEA & All & NR & NR & NR & NR \\
\hline & $\begin{array}{l}\text { BREAST-Q: } \\
\text { Abdomen }\end{array}$ & NRCS & Moderate & $2 y$ & IBR (all) & All & NR & NR & NR & NR \\
\hline & $\begin{array}{l}\text { BREAST-Q: } \\
\text { Abdomen }\end{array}$ & NRCS & Moderate & $2 y$ & IBR Direct & All & NR & NR & NR & NR \\
\hline & $\begin{array}{l}\text { BREAST-Q: } \\
\text { Abdomen }\end{array}$ & NRCS & Moderate & $2 y$ & IBR TE & All & NR & NR & NR & NR \\
\hline & $\begin{array}{l}\text { BREAST-Q: } \\
\text { Abdomen }\end{array}$ & NRCS & Moderate & $2 y$ & AR (all) & All & 763 & $76.3(19.8)$ & NR & NR \\
\hline & $\begin{array}{l}\text { BREAST-Q: } \\
\text { Abdomen }\end{array}$ & NRCS & Moderate & $2 y$ & AR with DIEP & All & NR & NR & NR & NR \\
\hline & $\begin{array}{l}\text { BREAST-Q: } \\
\text { Abdomen }\end{array}$ & NRCS & Moderate & $2 y$ & AR with free TRAM & All & NR & NR & NR & NR \\
\hline & $\begin{array}{l}\text { BREAST-Q: } \\
\text { Abdomen }\end{array}$ & NRCS & Moderate & $2 y$ & AR with pedicled TRAM & All & NR & NR & NR & NR \\
\hline & $\begin{array}{l}\text { BREAST-Q: } \\
\text { Abdomen }\end{array}$ & NRCS & Moderate & $2 y$ & AR with LD & All & NR & NR & NR & NR \\
\hline & $\begin{array}{l}\text { BREAST-Q: } \\
\text { Abdomen }\end{array}$ & NRCS & Moderate & $2 y$ & AR with SIEA & All & NR & NR & NR & NR \\
\hline & $\begin{array}{l}\text { PROMIS: Pain } \\
\text { interference }(0-100)\end{array}$ & NRCS & Moderate & $1 \mathrm{y}$ & IBR (all) & All & 1133 & $45.5(7.1)$ & NR & NR \\
\hline & $\begin{array}{l}\text { PROMIS: Pain } \\
\text { interference }(0-100)\end{array}$ & NRCS & Moderate & $1 \mathrm{y}$ & IBR Direct & All & NR & NR & NR & NR \\
\hline & $\begin{array}{l}\text { PROMIS: Pain } \\
\text { interference }(0-100)\end{array}$ & NRCS & Moderate & $1 \mathrm{y}$ & IBR TE & All & NR & NR & NR & NR \\
\hline & $\begin{array}{l}\text { PROMIS: Pain } \\
\text { interference }(0-100)\end{array}$ & NRCS & Moderate & $1 \mathrm{y}$ & AR (all) & All & 493 & $46.4(7.7)$ & NR & NR \\
\hline & $\begin{array}{l}\text { PROMIS: Pain } \\
\text { interference }(0-100)\end{array}$ & NRCS & Moderate & $1 \mathrm{y}$ & AR with DIEP & All & NR & NR & NR & NR \\
\hline & $\begin{array}{l}\text { PROMIS: Pain } \\
\text { interference }(0-100)\end{array}$ & NRCS & Moderate & $1 \mathrm{y}$ & AR with free TRAM & All & NR & NR & NR & NR \\
\hline & $\begin{array}{l}\text { PROMIS: Pain } \\
\text { interference }(0-100)\end{array}$ & NRCS & Moderate & $1 \mathrm{y}$ & AR with pedicled TRAM & All & NR & NR & NR & NR \\
\hline & $\begin{array}{l}\text { PROMIS: Pain } \\
\text { interference }(0-100)\end{array}$ & NRCS & Moderate & $1 \mathrm{y}$ & AR with LD & All & NR & NR & NR & NR \\
\hline & $\begin{array}{l}\text { PROMIS: Pain } \\
\text { interference }(0-100)\end{array}$ & NRCS & Moderate & $1 \mathrm{y}$ & AR with SIEA & All & NR & NR & NR & NR \\
\hline & $\begin{array}{l}\text { PROMIS: Pain } \\
\text { interference (0-100) }\end{array}$ & NRCS & Moderate & $2 y$ & IBR (all) & All & 773 & $46(7.5)$ & Ref & Ref \\
\hline
\end{tabular}




\begin{tabular}{|c|c|c|c|c|c|c|c|c|c|c|}
\hline $\begin{array}{l}\text { Study, Year, } \\
\text { PMID, Country }\end{array}$ & $\begin{array}{l}\text { Outcome } \\
\text { Measurement }\end{array}$ & Design & $\begin{array}{l}\text { Overall } \\
\text { RoB }\end{array}$ & $\begin{array}{l}\text { Time } \\
\text { Point }\end{array}$ & Arm & Subgroup & $\mathbf{N}$ & Mean (SD) & Effect Size (95\% CI) & $\begin{array}{l}P \\
\text { Value }\end{array}$ \\
\hline & $\begin{array}{l}\text { PROMIS: Pain } \\
\text { interference }(0-100)\end{array}$ & NRCS & Moderate & $2 y$ & IBR Direct & All & NR & NR & NR & NR \\
\hline & $\begin{array}{l}\text { PROMIS: Pain } \\
\text { interference }(0-100)\end{array}$ & NRCS & Moderate & $2 y$ & IBR TE & All & NR & NR & NR & NR \\
\hline & $\begin{array}{l}\text { PROMIS: Pain } \\
\text { interference }(0-100)\end{array}$ & NRCS & Moderate & $2 y$ & AR (all) & All & 384 & $48.4(8.4)$ & $\frac{\text { vs IBR (all): adjMD } 1.1}{(0.01,2.25)}$ & 0.048 \\
\hline & $\begin{array}{l}\text { PROMIS: Pain } \\
\text { interference }(0-100)\end{array}$ & NRCS & Moderate & $2 y$ & AR with DIEP & All & NR & NR & . & . \\
\hline & $\begin{array}{l}\text { PROMIS: Pain } \\
\text { interference (0-100) }\end{array}$ & NRCS & Moderate & $2 y$ & AR with free TRAM & All & NR & NR & . & . \\
\hline & $\begin{array}{l}\text { PROMIS: Pain } \\
\text { interference }(0-100)\end{array}$ & NRCS & Moderate & $2 y$ & AR with pedicled TRAM & All & NR & NR & . & . \\
\hline & $\begin{array}{l}\text { PROMIS: Pain } \\
\text { interference }(0-100)\end{array}$ & NRCS & Moderate & $2 y$ & AR with LD & All & NR & NR & . & . \\
\hline & $\begin{array}{l}\text { PROMIS: Pain } \\
\text { interference }(0-100)\end{array}$ & NRCS & Moderate & $2 y$ & AR with SIEA & All & NR & NR & . & . \\
\hline & $\begin{array}{l}\text { PROMIS: Physical } \\
\text { function }(0-100)\end{array}$ & NRCS & Moderate & Baseline & IBR (all) & All & 1135 & $53.3(6.6)$ & NR & NR \\
\hline & $\begin{array}{l}\text { PROMIS: Physical } \\
\text { function }(0-100)\end{array}$ & NRCS & Moderate & Baseline & IBR (all) & Unilateral & 600 & $53.1(6.6)$ & NR & NR \\
\hline & $\begin{array}{l}\text { PROMIS: Physical } \\
\text { function }(0-100)\end{array}$ & NRCS & Moderate & Baseline & IBR (all) & Bilateral & 994 & $53.5(6.6)$ & NR & NR \\
\hline & $\begin{array}{l}\text { PROMIS: Physical } \\
\text { function }(0-100)\end{array}$ & NRCS & Moderate & Baseline & IBR Direct & All & NR & NR & NR & NR \\
\hline & $\begin{array}{l}\text { PROMIS: Physical } \\
\text { function }(0-100)\end{array}$ & NRCS & Moderate & Baseline & IBR TE & All & NR & NR & NR & NR \\
\hline & $\begin{array}{l}\text { PROMIS: Physical } \\
\text { function }(0-100)\end{array}$ & NRCS & Moderate & Baseline & AR (all) & All & 492 & $52.4(7.1)$ & NR & NR \\
\hline & $\begin{array}{l}\text { PROMIS: Physical } \\
\text { function }(0-100)\end{array}$ & NRCS & Moderate & Baseline & AR (all) & Unilateral & 317 & $52.5(6.9)$ & NR & NR \\
\hline & $\begin{array}{l}\text { PROMIS: Physical } \\
\text { function }(0-100)\end{array}$ & NRCS & Moderate & Baseline & AR (all) & Bilateral & 214 & $52.4(7.1)$ & NR & NR \\
\hline & $\begin{array}{l}\text { PROMIS: Physical } \\
\text { function }(0-100)\end{array}$ & NRCS & Moderate & Baseline & AR with DIEP & All & NR & NR & NR & NR \\
\hline & $\begin{array}{l}\text { PROMIS: Physical } \\
\text { function }(0-100)\end{array}$ & NRCS & Moderate & Baseline & AR with free TRAM & All & NR & NR & NR & NR \\
\hline & $\begin{array}{l}\text { PROMIS: Physical } \\
\text { function }(0-100)\end{array}$ & NRCS & Moderate & Baseline & AR with pedicled TRAM & All & NR & NR & NR & NR \\
\hline & $\begin{array}{l}\text { PROMIS: Physical } \\
\text { function }(0-100)\end{array}$ & NRCS & Moderate & Baseline & AR with LD & All & NR & NR & NR & NR \\
\hline & $\begin{array}{l}\text { PROMIS: Physical } \\
\text { function }(0-100)\end{array}$ & NRCS & Moderate & Baseline & AR with SIEA & All & NR & NR & NR & NR \\
\hline
\end{tabular}




\begin{tabular}{|c|c|c|c|c|c|c|c|c|c|c|}
\hline $\begin{array}{l}\text { Study, Year, } \\
\text { PMID, Country }\end{array}$ & $\begin{array}{l}\text { Outcome } \\
\text { Measurement }\end{array}$ & Design & $\begin{array}{l}\text { Overall } \\
\text { RoB }\end{array}$ & $\begin{array}{l}\text { Time } \\
\text { Point }\end{array}$ & Arm & Subgroup & $\mathbf{N}$ & Mean (SD) & Effect Size $(95 \% \mathrm{CI})$ & $\begin{array}{l}\mathbf{P} \\
\text { Value }\end{array}$ \\
\hline & $\begin{array}{l}\text { PROMIS: Physical } \\
\text { function }(0-100)\end{array}$ & NRCS & Moderate & $1 \mathrm{y}$ & IBR (all) & All & 777 & $52.2(6.8)$ & Ref & Ref \\
\hline & $\begin{array}{l}\text { PROMIS: Physical } \\
\text { function }(0-100)\end{array}$ & NRCS & Moderate & $1 \mathrm{y}$ & IBR (all) & Unilateral & NR & NR & NR & NR \\
\hline & $\begin{array}{l}\text { PROMIS: Physical } \\
\text { function }(0-100)\end{array}$ & NRCS & Moderate & $1 \mathrm{y}$ & IBR (all) & Bilateral & NR & NR & NR & NR \\
\hline & $\begin{array}{l}\text { PROMIS: Physical } \\
\text { function }(0-100)\end{array}$ & NRCS & Moderate & $1 \mathrm{y}$ & IBR Direct & All & NR & NR & NR & NR \\
\hline & $\begin{array}{l}\text { PROMIS: Physical } \\
\text { function }(0-100)\end{array}$ & NRCS & Moderate & $1 \mathrm{y}$ & IBR TE & All & NR & NR & NR & NR \\
\hline & $\begin{array}{l}\text { PROMIS: Physical } \\
\text { function }(0-100)\end{array}$ & NRCS & Moderate & $1 \mathrm{y}$ & AR (all) & All & 385 & $50.1(7.2)$ & $\begin{array}{l}\text { vs IBR (all): adjMD }-0.6 \\
(-1.51,0.39)\end{array}$ & 0.249 \\
\hline & $\begin{array}{l}\text { PROMIS: Physical } \\
\text { function }(0-100)\end{array}$ & NRCS & Moderate & $1 \mathrm{y}$ & AR (all) & Unilateral & NR & NR & NR & NR \\
\hline & $\begin{array}{l}\text { PROMIS: Physical } \\
\text { function }(0-100)\end{array}$ & NRCS & Moderate & $1 \mathrm{y}$ & AR (all) & Bilateral & NR & NR & NR & NR \\
\hline & $\begin{array}{l}\text { PROMIS: Physical } \\
\text { function }(0-100)\end{array}$ & NRCS & Moderate & $1 \mathrm{y}$ & AR with DIEP & All & NR & NR & NR & NR \\
\hline & $\begin{array}{l}\text { PROMIS: Physical } \\
\text { function }(0-100)\end{array}$ & NRCS & Moderate & $1 \mathrm{y}$ & AR with free TRAM & All & NR & NR & NR & NR \\
\hline & $\begin{array}{l}\text { PROMIS: Physical } \\
\text { function }(0-100)\end{array}$ & NRCS & Moderate & $1 \mathrm{y}$ & AR with pedicled TRAM & All & NR & NR & NR & NR \\
\hline & $\begin{array}{l}\text { PROMIS: Physical } \\
\text { function }(0-100)\end{array}$ & NRCS & Moderate & $1 \mathrm{y}$ & AR with LD & All & NR & NR & NR & NR \\
\hline & $\begin{array}{l}\text { PROMIS: Physical } \\
\text { function }(0-100)\end{array}$ & NRCS & Moderate & $1 \mathrm{y}$ & AR with SIEA & All & NR & NR & NR & NR \\
\hline & $\begin{array}{l}\text { PROMIS: Physical } \\
\text { function }(0-100)\end{array}$ & NRCS & Moderate & $2 y$ & IBR (all) & All & NR & NR & NR & NR \\
\hline & $\begin{array}{l}\text { PROMIS: Physical } \\
\text { function }(0-100)\end{array}$ & NRCS & Moderate & $2 y$ & IBR (all) & Unilateral & 600 & $52.6(6.5)$ & Ref & Ref \\
\hline & $\begin{array}{l}\text { PROMIS: Physical } \\
\text { function }(0-100)\end{array}$ & NRCS & Moderate & $2 y$ & IBR (all) & Bilateral & 994 & $52.8(6.3)$ & Ref & Ref \\
\hline & $\begin{array}{l}\text { PROMIS: Physical } \\
\text { function }(0-100)\end{array}$ & NRCS & Moderate & $2 y$ & IBR Direct & All & NR & NR & NR & NR \\
\hline & $\begin{array}{l}\text { PROMIS: Physical } \\
\text { function }(0-100)\end{array}$ & NRCS & Moderate & $2 y$ & IBR TE & All & NR & NR & NR & NR \\
\hline & $\begin{array}{l}\text { PROMIS: Physical } \\
\text { function }(0-100)\end{array}$ & NRCS & Moderate & $2 y$ & AR (all) & All & NR & NR & NR & NR \\
\hline & $\begin{array}{l}\text { PROMIS: Physical } \\
\text { function }(0-100)\end{array}$ & NRCS & Moderate & $2 y$ & AR (all) & Unilateral & 317 & $51.3(7.3)$ & $\begin{array}{l}\text { vs IBR (unilateral): } \\
\text { adjMD -0.14 (NR, NR) }\end{array}$ & 0.830 \\
\hline & $\begin{array}{l}\text { PROMIS: Physical } \\
\text { function }(0-100)\end{array}$ & NRCS & Moderate & $2 y$ & AR (all) & Bilateral & 214 & $49.8(7.6)$ & $\begin{array}{l}\text { vs IBR (bilateral): } \\
\text { adjMD }-1.21(\mathrm{NR}, \mathrm{NR})\end{array}$ & 0.06 \\
\hline
\end{tabular}




\begin{tabular}{|c|c|c|c|c|c|c|c|c|c|c|}
\hline $\begin{array}{l}\text { Study, Year, } \\
\text { PMID, Country }\end{array}$ & $\begin{array}{l}\text { Outcome } \\
\text { Measurement }\end{array}$ & Design & $\begin{array}{l}\text { Overall } \\
\text { RoB }\end{array}$ & $\begin{array}{l}\text { Time } \\
\text { Point }\end{array}$ & Arm & Subgroup & $\mathbf{N}$ & Mean (SD) & Effect Size $(95 \% \mathrm{Cl})$ & $\begin{array}{l}\mathbf{P} \\
\text { Value }\end{array}$ \\
\hline & $\begin{array}{l}\text { PROMIS: Physical } \\
\text { function }(0-100)\end{array}$ & NRCS & Moderate & $2 y$ & AR with DIEP & All & NR & NR & NR & NR \\
\hline & $\begin{array}{l}\text { PROMIS: Physical } \\
\text { function }(0-100)\end{array}$ & NRCS & Moderate & $2 y$ & AR with free TRAM & All & NR & NR & NR & NR \\
\hline & $\begin{array}{l}\text { PROMIS: Physical } \\
\text { function }(0-100)\end{array}$ & NRCS & Moderate & $2 y$ & AR with pedicled TRAM & All & NR & NR & NR & NR \\
\hline & $\begin{array}{l}\text { PROMIS: Physical } \\
\text { function }(0-100)\end{array}$ & NRCS & Moderate & $2 y$ & AR with LD & All & NR & NR & NR & NR \\
\hline & $\begin{array}{l}\text { PROMIS: Physical } \\
\text { function }(0-100)\end{array}$ & NRCS & Moderate & $2 y$ & AR with SIEA & All & NR & NR & NR & NR \\
\hline \multirow[t]{2}{*}{$\begin{array}{l}\text { McCarthy, } \\
2014, \\
24201740, \text { US }\end{array}$} & $\begin{array}{l}\text { BREAST-Q: } \\
\text { Physical well-being } \\
(0-100)\end{array}$ & NRCS & Moderate & $1-5 y$ & IBR & All & 141 & $76.5(16)$ & Ref & Ref \\
\hline & $\begin{array}{l}\text { BREAST-Q: } \\
\text { Physical well-being } \\
(0-100)\end{array}$ & NRCS & Moderate & $1-5 y$ & AR & . & 74 & $82.5(15.3)$ & NR & $<0.05$ \\
\hline \multirow[t]{8}{*}{$\begin{array}{l}\text { Nelson, 2019, } \\
31356276, \text { US }\end{array}$} & $\begin{array}{l}\text { BREAST-Q: Chest } \\
(0-100)\end{array}$ & NRCS & High & $1 \mathrm{y}$ & IBR & . & 1342 & $73.62(15.95)$ & NR & NR \\
\hline & $\begin{array}{l}\text { BREAST-Q: Chest } \\
(0-100)\end{array}$ & NRCS & High & $1 \mathrm{y}$ & AR & . & 194 & $74.52(17.28)$ & NR & NR \\
\hline & $\begin{array}{l}\text { BREAST-Q: Chest } \\
(0-100)\end{array}$ & NRCS & High & $3 y$ & IBR & . & 1085 & $75.62(16.09)$ & NR & NR \\
\hline & $\begin{array}{l}\text { BREAST-Q: Chest } \\
(0-100)\end{array}$ & NRCS & High & $3 y$ & AR & . & 98 & $77.71(14.81)$ & NR & NR \\
\hline & $\begin{array}{l}\text { BREAST-Q: Chest } \\
(0-100)\end{array}$ & NRCS & High & $5 y$ & IBR & . & 743 & $76.98(16.46)$ & NR & NR \\
\hline & $\begin{array}{l}\text { BREAST-Q: Chest } \\
(0-100)\end{array}$ & NRCS & High y & $5 y$ & AR & . & 41 & $84.12(15.86)$ & NR & NR \\
\hline & $\begin{array}{l}\text { BREAST-Q: Chest } \\
(0-100)\end{array}$ & NRCS & High & $7 y$ & IBR & . & 377 & $76.44(17.23)$ & NR & NR \\
\hline & $\begin{array}{l}\text { BREAST-Q: Chest } \\
(0-100)\end{array}$ & NRCS & High & $7 y$ & AR & . & 19 & $78.37(14.47)$ & NR & NR \\
\hline
\end{tabular}

Abbreviations: adj = adjusted, $\mathrm{CI}=$ confidence interval, $\mathrm{DIEP}=$ deep inferior epigastric perforator, $\mathrm{MD}=$ mean difference, $\mathrm{mo}=\mathrm{months}, \mathrm{NR}=$ not reported, $\mathrm{NRCS}=$

nonrandomized comparative study, OR = odds ratio (reported as odds of scoring higher), PMID = Pubmed identifier, PROMIS: Patient Reported Outcomes Measurement

Information System, RCT = randomized controlled trial, $\mathrm{Ref}=$ reference group, $\mathrm{RoB}=$ risk of bias, $\mathrm{SD}=$ standard deviation, $\mathrm{y}=\mathrm{years}$.

Colors: Header rows are shaded orange. Rows for every alternate study are shaded blue. The colors do not add unique information. 
Table F-1.3. Full Evidence Table - Key Question 1: IBR versus AR - continuous outcomes (psychosocial well-being)

\begin{tabular}{|c|c|c|c|c|c|c|c|c|c|c|}
\hline $\begin{array}{l}\text { Study, Year, } \\
\text { PMID, } \\
\text { Country }\end{array}$ & Outcome Measurement & Design & $\begin{array}{l}\text { Overall } \\
\text { RoB }\end{array}$ & $\begin{array}{l}\text { Time } \\
\text { Point }\end{array}$ & Arm & Subgroup & $\mathbf{N}$ & Mean (SD) & Effect Size $(95 \% \mathrm{Cl})$ & $\begin{array}{l}\text { Palue } \\
\text { Valu }\end{array}$ \\
\hline \multirow{2}{*}{$\begin{array}{l}\text { Tallroth, } \\
2020, \\
33436336, \\
\text { Sweden }\end{array}$} & $\begin{array}{l}\text { BREAST-Q: Psychosocial } \\
\text { WB (0-100) }\end{array}$ & RCT & Moderate & $5.3 y$ & IBR & All & 28 & $78.8(20.1)$ & Ref & Ref \\
\hline & $\begin{array}{l}\text { BREAST-Q: Psychosocial } \\
\text { WB }(0-100)\end{array}$ & RCT & Moderate & $5.3 \mathrm{y}$ & AR & All & 42 & $79.1(21.5)$ & MD $0.3(-6.7,7.3)$ & 0.93 \\
\hline \multirow{4}{*}{$\begin{array}{l}\text { Eltahir, } \\
2015, \\
25539295, \\
\text { Netherlands }\end{array}$} & $\begin{array}{l}\text { BREAST-Q: Psychosocial } \\
\text { well-being }(0-100)\end{array}$ & NRCS & Moderate & $2.2 \mathrm{y}$ & IBR & All & 45 & $77.18(18.1)$ & Ref & Ref \\
\hline & $\begin{array}{l}\text { BREAST-Q: Psychosocial } \\
\text { well-being }(0-100)\end{array}$ & NRCS & Moderate & $2.2 \mathrm{y}$ & AR & All & 47 & $73.96(17.8)$ & adjMD $4.61(-2.8,12.01)$ & 0.22 \\
\hline & $\begin{array}{l}\text { RAND R1: Social } \\
\text { functioning }(0-100)\end{array}$ & NRCS & Moderate & $2.2 \mathrm{y}$ & IBR & All & NR & NR & Ref & Ref \\
\hline & $\begin{array}{l}\text { RAND R1: Social } \\
\text { functioning }(0-100)\end{array}$ & NRCS & Moderate & $2.2 \mathrm{y}$ & AR & All & NR & NR & adjMD -1.21 $(-8.44,6.02)$ & 0.741 \\
\hline \multirow{10}{*}{$\begin{array}{l}\text { Kouwenberg } \\
\text {, 2020, } \\
32590633 \text {, } \\
\text { Netherlands }\end{array}$} & $\begin{array}{l}\text { BREAST-Q: Psychosocial } \\
\text { WB }(0-100)\end{array}$ & NRCS & Moderate & $>6 \mathrm{mo}$ & IBR & All & 296 & $71.6(20.2)$ & Ref & Ref \\
\hline & $\begin{array}{l}\text { BREAST-Q: Psychosocial } \\
\text { WB }(0-100)\end{array}$ & NRCS & Moderate & $>6 \mathrm{mo}$ & AR & All & 179 & 75.8 (19.5) & NR & $<0.05$ \\
\hline & $\begin{array}{l}\text { EORTC QLQC30: Social } \\
\text { function }(0-100)\end{array}$ & NRCS & Moderate & $>6 \mathrm{mo}$ & IBR & All & 296 & $87.5(27.2)$ & Ref & Ref \\
\hline & $\begin{array}{l}\text { EORTC QLQC30: Social } \\
\text { function }(0-100)\end{array}$ & NRCS & Moderate & $>6 \mathrm{mo}$ & AR & All & 179 & $88.0(17.1)$ & NR & NS \\
\hline & $\begin{array}{l}\text { EORTC QLQC30: } \\
\text { Emotional function }(0-100)\end{array}$ & NRCS & Moderate & $>6 \mathrm{mo}$ & IBR & All & 296 & $85.0(23.3)$ & Ref & Ref \\
\hline & $\begin{array}{l}\text { EORTC QLQC30: } \\
\text { Emotional function (0-100) }\end{array}$ & NRCS & Moderate & $>6 \mathrm{mo}$ & AR & All & 179 & $87.0(17.1)$ & NR & NS \\
\hline & $\begin{array}{l}\text { EORTC QLQC30: } \\
\text { Cognitive function }(0-100)\end{array}$ & NRCS & Moderate & $>6 \mathrm{mo}$ & IBR & All & 296 & $85.0(23.7)$ & Ref & Ref \\
\hline & $\begin{array}{l}\text { EORTC QLQC30: } \\
\text { Cognitive function }(0-100)\end{array}$ & NRCS & Moderate & $>6 \mathrm{mo}$ & AR & All & 179 & $83.7(21.5)$ & NR & NS \\
\hline & $\begin{array}{l}\text { EORTC QLQC30: Role } \\
\text { function }(0-100)\end{array}$ & NRCS & Moderate & $>6 \mathrm{mo}$ & IBR & All & 296 & $86.0(28.1)$ & Ref & Ref \\
\hline & $\begin{array}{l}\text { EORTC QLQC30: Role } \\
\text { function }(0-100)\end{array}$ & NRCS & Moderate & $>6 \mathrm{mo}$ & AR & All & 179 & $84.0(21.8)$ & NR & $<0.05$ \\
\hline \multirow{4}{*}{$\begin{array}{l}\text { Kulkarni, } \\
2017, \\
28713853, \\
\text { US \& } \\
\text { Canada }\end{array}$} & $\begin{array}{l}\text { BREAST-Q: Psychosocial } \\
\text { well-being }(0-100)\end{array}$ & NRCS & Moderate & Baseline & IBR (all) & All & 1131 & $72.4(17.5)$ & NR & NR \\
\hline & $\begin{array}{l}\text { BREAST-Q: Psychosocial } \\
\text { well-being }(0-100)\end{array}$ & NRCS & Moderate & Baseline & IBR (all) & Unilateral & 600 & $73(17.5)$ & NR & NR \\
\hline & $\begin{array}{l}\text { BREAST-Q: Psychosocial } \\
\text { well-being }(0-100)\end{array}$ & NRCS & Moderate & Baseline & IBR (all) & Bilateral & 994 & $71.2(17.5)$ & NR & NR \\
\hline & $\begin{array}{l}\text { BREAST-Q: Psychosocial } \\
\text { well-being }(0-100)\end{array}$ & NRCS & Moderate & Baseline & IBR Direct & All & NR & NR & NR & NR \\
\hline
\end{tabular}




\begin{tabular}{|c|c|c|c|c|c|c|c|c|c|c|}
\hline $\begin{array}{l}\text { Study, Year, } \\
\text { PMID, } \\
\text { Country }\end{array}$ & Outcome Measurement & Design & $\begin{array}{l}\text { Overall } \\
\text { RoB }\end{array}$ & $\begin{array}{l}\text { Time } \\
\text { Point }\end{array}$ & Arm & Subgroup & $\mathbf{N}$ & Mean (SD) & Effect Size (95\% CI) & $\begin{array}{l}P \\
\text { Value }\end{array}$ \\
\hline & $\begin{array}{l}\text { BREAST-Q: Psychosocial } \\
\text { well-being }(0-100)\end{array}$ & NRCS & Moderate & Baseline & IBR TE & All & NR & NR & NR & NR \\
\hline & $\begin{array}{l}\text { BREAST-Q: Psychosocial } \\
\text { well-being }(0-100)\end{array}$ & NRCS & Moderate & Baseline & AR (all) & All & 492 & $68.4(18.3)$ & NR & NR \\
\hline & $\begin{array}{l}\text { BREAST-Q: Psychosocial } \\
\text { well-being }(0-100)\end{array}$ & NRCS & Moderate & Baseline & AR (all) & Unilateral & 317 & $69(18.3)$ & NR & NR \\
\hline & $\begin{array}{l}\text { BREAST-Q: Psychosocial } \\
\text { well-being }(0-100)\end{array}$ & NRCS & Moderate & Baseline & AR (all) & Bilateral & 224 & $66.5(18.1)$ & NR & NR \\
\hline & $\begin{array}{l}\text { BREAST-Q: Psychosocial } \\
\text { well-being }(0-100)\end{array}$ & NRCS & Moderate & Baseline & AR with DIEP & All & NR & NR & NR & NR \\
\hline & $\begin{array}{l}\text { BREAST-Q: Psychosocial } \\
\text { well-being }(0-100)\end{array}$ & NRCS & Moderate & Baseline & AR with free TRAM & All & NR & NR & NR & NR \\
\hline & $\begin{array}{l}\text { BREAST-Q: Psychosocial } \\
\text { well-being }(0-100)\end{array}$ & NRCS & Moderate & Baseline & $\begin{array}{l}\text { AR with pedicled } \\
\text { TRAM }\end{array}$ & All & NR & NR & NR & NR \\
\hline & $\begin{array}{l}\text { BREAST-Q: Psychosocial } \\
\text { well-being }(0-100)\end{array}$ & NRCS & Moderate & Baseline & AR with LD & All & NR & NR & NR & NR \\
\hline & $\begin{array}{l}\text { BREAST-Q: Psychosocial } \\
\text { well-being }(0-100)\end{array}$ & NRCS & Moderate & Baseline & AR with SIEA & All & NR & NR & NR & NR \\
\hline & $\begin{array}{l}\text { BREAST-Q: Psychosocial } \\
\text { well-being }(0-100)\end{array}$ & NRCS & Moderate & $1 \mathrm{y}$ & IBR (all) & All & 791 & $71.8(19)$ & Ref & Ref \\
\hline & $\begin{array}{l}\text { BREAST-Q: Psychosocial } \\
\text { well-being }(0-100)\end{array}$ & NRCS & Moderate & $1 \mathrm{y}$ & IBR Direct & All & NR & NR & NR & NR \\
\hline & $\begin{array}{l}\text { BREAST-Q: Psychosocial } \\
\text { well-being }(0-100)\end{array}$ & NRCS & Moderate & $1 \mathrm{y}$ & IBR TE & All & NR & NR & NR & NR \\
\hline & $\begin{array}{l}\text { BREAST-Q: Psychosocial } \\
\text { well-being }(0-100)\end{array}$ & NRCS & Moderate & $1 \mathrm{y}$ & AR (all) & All & 386 & $74.7(19.2)$ & $\frac{\text { vs IBR (all): }}{6.76)}$ adjMD 3.7 (0.73, & 0.015 \\
\hline & $\begin{array}{l}\text { BREAST-Q: Psychosocial } \\
\text { well-being }(0-100)\end{array}$ & NRCS & Moderate & $1 \mathrm{y}$ & AR with DIEP & All & NR & NR & NR & NR \\
\hline & $\begin{array}{l}\text { BREAST-Q: Psychosocial } \\
\text { well-being }(0-100)\end{array}$ & NRCS & Moderate & $1 \mathrm{y}$ & AR with free TRAM & All & NR & NR & NR & NR \\
\hline & $\begin{array}{l}\text { BREAST-Q: Psychosocial } \\
\text { well-being }(0-100)\end{array}$ & NRCS & Moderate & $1 \mathrm{y}$ & $\begin{array}{l}\text { AR with pedicled } \\
\text { TRAM }\end{array}$ & All & NR & NR & NR & NR \\
\hline & $\begin{array}{l}\text { BREAST-Q: Psychosocial } \\
\text { well-being }(0-100)\end{array}$ & NRCS & Moderate & $1 \mathrm{y}$ & AR with LD & All & NR & NR & NR & NR \\
\hline & $\begin{array}{l}\text { BREAST-Q: Psychosocial } \\
\text { well-being }(0-100)\end{array}$ & NRCS & Moderate & $1 \mathrm{y}$ & AR with SIEA & All & NR & NR & NR & NR \\
\hline & $\begin{array}{l}\text { BREAST-Q: Psychosocial } \\
\text { well-being }(0-100)\end{array}$ & NRCS & Moderate & $2 y$ & IBR (all) & All & 1490 & 74.5 (18.9) & Ref & Ref \\
\hline & $\begin{array}{l}\text { BREAST-Q: Psychosocial } \\
\text { well-being }(0-100)\end{array}$ & NRCS & Moderate & $2 y$ & IBR (all) & Unilateral & 600 & $74.6(18.7)$ & Ref & Ref \\
\hline
\end{tabular}

F-12 


\begin{tabular}{|c|c|c|c|c|c|c|c|c|c|c|}
\hline $\begin{array}{l}\text { Study, Year, } \\
\text { PMID, } \\
\text { Country }\end{array}$ & Outcome Measurement & Design & $\begin{array}{l}\text { Overall } \\
\text { RoB }\end{array}$ & \begin{tabular}{|l|} 
Time \\
Point
\end{tabular} & Arm & Subgroup & $\mathbf{N}$ & Mean (SD) & Effect Size (95\% CI) & $\begin{array}{l}P \\
\text { Value }\end{array}$ \\
\hline & $\begin{array}{l}\text { BREAST-Q: Psychosocial } \\
\text { well-being }(0-100)\end{array}$ & NRCS & Moderate & $2 y$ & IBR (all) & Bilateral & 994 & $74.5(19)$ & Ref & Ref \\
\hline & $\begin{array}{l}\text { BREAST-Q: Psychosocial } \\
\text { well-being }(0-100)\end{array}$ & NRCS & Moderate & $2 y$ & IBR Direct & All & NR & NR & NR & NR \\
\hline & $\begin{array}{l}\text { BREAST-Q: Psychosocial } \\
\text { well-being }(0-100)\end{array}$ & NRCS & Moderate & $2 y$ & IBR TE & All & NR & NR & NR & NR \\
\hline & $\begin{array}{l}\text { BREAST-Q: Psychosocial } \\
\text { well-being }(0-100)\end{array}$ & NRCS & Moderate & $2 y$ & AR (all) & All & 523 & $75.8(19)$ & $\frac{\text { vs IBR (all): }}{5.29)}$ adjMD 3.27 (1.25, & NR \\
\hline & $\begin{array}{l}\text { BREAST-Q: Psychosocial } \\
\text { well-being }(0-100)\end{array}$ & NRCS & Moderate & $2 y$ & AR (all) & Unilateral & 317 & $76.8(18.9)$ & $\begin{array}{l}\text { vs IBR (unilateral): adjMD } 4.84 \\
\text { (NR, NR) }\end{array}$ & NR \\
\hline & $\begin{array}{l}\text { BREAST-Q: Psychosocial } \\
\text { well-being }(0-100)\end{array}$ & NRCS & Moderate & $2 y$ & AR (all) & Bilateral & 224 & $73.4(19)$ & $\begin{array}{l}\text { vs IBR (bilateral): adjMD } 0.91 \\
\text { (NR, NR) }\end{array}$ & NR \\
\hline & $\begin{array}{l}\text { BREAST-Q: Psychosocial } \\
\text { well-being }(0-100)\end{array}$ & NRCS & Moderate & $2 y$ & AR with DIEP & All & NR & NR & NR & NR \\
\hline & $\begin{array}{l}\text { BREAST-Q: Psychosocial } \\
\text { well-being }(0-100)\end{array}$ & NRCS & Moderate & $2 y$ & AR with free TRAM & All & NR & NR & NR & NR \\
\hline & $\begin{array}{l}\text { BREAST-Q: Psychosocial } \\
\text { well-being }(0-100)\end{array}$ & NRCS & Moderate & $2 y$ & $\begin{array}{l}\text { AR with pedicled } \\
\text { TRAM }\end{array}$ & All & NR & NR & NR & NR \\
\hline & $\begin{array}{l}\text { BREAST-Q: Psychosocial } \\
\text { well-being }(0-100)\end{array}$ & NRCS & Moderate & $2 y$ & AR with LD & All & NR & NR & NR & NR \\
\hline & $\begin{array}{l}\text { BREAST-Q: Psychosocial } \\
\text { well-being }(0-100)\end{array}$ & NRCS & Moderate & $2 y$ & AR with SIEA & All & NR & NR & NR & NR \\
\hline & PROMIS: Anxiety $(0-100)$ & NRCS & Moderate & Baseline & IBR (all) & All & 1136 & $59.1(8.8)$ & NR & NR \\
\hline & PROMIS: Anxiety (0-100) & NRCS & Moderate & Baseline & IBR Direct & All & NR & NR & NR & NR \\
\hline & PROMIS: Anxiety (0-100) & NRCS & Moderate & Baseline & IBR TE & All & NR & NR & NR & NR \\
\hline & PROMIS: Anxiety (0-100) & NRCS & Moderate & Baseline & AR (all) & All & 493 & $58.3(8.8)$ & NR & NR \\
\hline & PROMIS: Anxiety (0-100) & NRCS & Moderate & Baseline & AR with DIEP & All & NR & NR & NR & NR \\
\hline & PROMIS: Anxiety (0-100) & NRCS & Moderate & Baseline & AR with free TRAM & All & NR & NR & NR & NR \\
\hline & PROMIS: Anxiety (0-100) & NRCS & Moderate & Baseline & $\begin{array}{l}\text { AR with pedicled } \\
\text { TRAM }\end{array}$ & All & NR & NR & NR & NR \\
\hline & PROMIS: Anxiety (0-100) & NRCS & Moderate & Baseline & AR with LD & All & NR & NR & NR & NR \\
\hline & PROMIS: Anxiety (0-100) & NRCS & Moderate & Baseline & AR with SIEA & All & NR & NR & NR & NR \\
\hline & PROMIS: Anxiety (0-100) & NRCS & Moderate & $1 \mathrm{y}$ & IBR (all) & All & 775 & $49.7(9.4)$ & Ref & Ref \\
\hline & PROMIS: Anxiety (0-100) & NRCS & Moderate & $1 \mathrm{y}$ & IBR Direct & All & NR & NR & NR & NR \\
\hline & PROMIS: Anxiety (0-100) & NRCS & Moderate & $1 \mathrm{y}$ & IBR TE & All & NR & NR & NR & NR \\
\hline & PROMIS: Anxiety (0-100) & NRCS & Moderate & $1 \mathrm{y}$ & AR (all) & All & 383 & $50.4(9.6)$ & 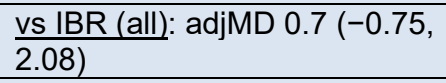 & 0.356 \\
\hline & PROMIS: Anxiety (0-100) & NRCS & Moderate & $1 \mathrm{y}$ & AR with DIEP & All & NR & NR & NR & NR \\
\hline & PROMIS: Anxiety (0-100) & NRCS & Moderate & $1 \mathrm{y}$ & AR with free TRAM & All & NR & NR & NR & NR \\
\hline
\end{tabular}




\begin{tabular}{|c|c|c|c|c|c|c|c|c|c|c|}
\hline $\begin{array}{l}\text { Study, Year, } \\
\text { PMID, } \\
\text { Country }\end{array}$ & Outcome Measurement & Design & $\begin{array}{l}\text { Overall } \\
\text { RoB }\end{array}$ & $\begin{array}{l}\text { Time } \\
\text { Point }\end{array}$ & Arm & Subgroup & $\mathbf{N}$ & Mean (SD) & Effect Size $(95 \% \mathrm{Cl})$ & $\begin{array}{l}\mathbf{P} \\
\text { Value }\end{array}$ \\
\hline & PROMIS: Anxiety (0-100) & NRCS & Moderate & $1 \mathrm{y}$ & $\begin{array}{l}\text { AR with pedicled } \\
\text { TRAM }\end{array}$ & All & NR & NR & NR & NR \\
\hline & PROMIS: Anxiety (0-100) & NRCS & Moderate & $1 \mathrm{y}$ & AR with LD & All & NR & NR & NR & NR \\
\hline & PROMIS: Anxiety (0-100) & NRCS & Moderate & $1 \mathrm{y}$ & AR with SIEA & All & NR & NR & NR & NR \\
\hline & $\begin{array}{l}\text { PROMIS: Depression (0- } \\
\text { 100) }\end{array}$ & NRCS & Moderate & Baseline & IBR (all) & All & 1135 & $49.8(8.3)$ & NR & NR \\
\hline & $\begin{array}{l}\text { PROMIS: Depression (0- } \\
\text { 100) }\end{array}$ & NRCS & Moderate & Baseline & IBR Direct & All & NR & NR & NR & NR \\
\hline & $\begin{array}{l}\text { PROMIS: Depression (0- } \\
\text { 100) }\end{array}$ & NRCS & Moderate & Baseline & IBR TE & All & NR & NR & NR & NR \\
\hline & $\begin{array}{l}\text { PROMIS: Depression (0- } \\
\text { 100) }\end{array}$ & NRCS & Moderate & Baseline & AR (all) & All & 493 & $49.7(8.5)$ & NR & NR \\
\hline & $\begin{array}{l}\text { PROMIS: Depression (0- } \\
\text { 100) }\end{array}$ & NRCS & Moderate & Baseline & AR with DIEP & All & NR & NR & NR & NR \\
\hline & $\begin{array}{l}\text { PROMIS: Depression (0- } \\
\text { 100) }\end{array}$ & NRCS & Moderate & Baseline & AR with free TRAM & All & NR & NR & NR & NR \\
\hline & $\begin{array}{l}\text { PROMIS: Depression (0- } \\
\text { 100) }\end{array}$ & NRCS & Moderate & Baseline & $\begin{array}{l}\text { AR with pedicled } \\
\text { TRAM }\end{array}$ & All & NR & NR & NR & NR \\
\hline & $\begin{array}{l}\text { PROMIS: Depression (0- } \\
\text { 100) }\end{array}$ & NRCS & Moderate & Baseline & AR with LD & All & NR & NR & NR & NR \\
\hline & $\begin{array}{l}\text { PROMIS: Depression (0- } \\
\text { 100) }\end{array}$ & NRCS & Moderate & Baseline & AR with SIEA & All & NR & NR & NR & NR \\
\hline & $\begin{array}{l}\text { PROMIS: Depression (0- } \\
\text { 100) }\end{array}$ & NRCS & Moderate & $1 \mathrm{y}$ & IBR (all) & All & 776 & $47.3(8)$ & Ref & Ref \\
\hline & $\begin{array}{l}\text { PROMIS: Depression (0- } \\
\text { 100) }\end{array}$ & NRCS & Moderate & $1 \mathrm{y}$ & IBR Direct & All & NR & NR & NR & NR \\
\hline & $\begin{array}{l}\text { PROMIS: Depression (0- } \\
\text { 100) }\end{array}$ & NRCS & Moderate & $1 \mathrm{y}$ & IBR TE & All & NR & NR & NR & NR \\
\hline & $\begin{array}{l}\text { PROMIS: Depression (0- } \\
\text { 100) }\end{array}$ & NRCS & Moderate & $1 \mathrm{y}$ & AR (all) & All & 385 & $47.9(8.2)$ & $\frac{\text { vs IBR (all): }}{1.45)}$ adjMD $0.4(-0.7$ & 0.497 \\
\hline & $\begin{array}{l}\text { PROMIS: Depression (0- } \\
\text { 100) }\end{array}$ & NRCS & Moderate & $1 \mathrm{y}$ & AR with DIEP & All & NR & NR & NR & NR \\
\hline & $\begin{array}{l}\text { PROMIS: Depression (0- } \\
\text { 100) }\end{array}$ & NRCS & Moderate & $1 \mathrm{y}$ & AR with free TRAM & All & NR & NR & NR & NR \\
\hline & $\begin{array}{l}\text { PROMIS: Depression (0- } \\
\text { 100) }\end{array}$ & NRCS & Moderate & $1 \mathrm{y}$ & $\begin{array}{l}\text { AR with pedicled } \\
\text { TRAM }\end{array}$ & All & NR & NR & NR & NR \\
\hline & $\begin{array}{l}\text { PROMIS: Depression (0- } \\
\text { 100) }\end{array}$ & NRCS & Moderate & $1 \mathrm{y}$ & AR with LD & All & NR & NR & NR & NR \\
\hline & $\begin{array}{l}\text { PROMIS: Depression (0- } \\
\text { 100) }\end{array}$ & NRCS & Moderate & $1 \mathrm{y}$ & AR with SIEA & All & NR & NR & NR & NR \\
\hline
\end{tabular}




\begin{tabular}{|c|c|c|c|c|c|c|c|c|c|c|}
\hline $\begin{array}{l}\text { Study, Year, } \\
\text { PMID, } \\
\text { Country }\end{array}$ & Outcome Measurement & Design & $\begin{array}{l}\text { Overall } \\
\text { RoB }\end{array}$ & $\begin{array}{l}\text { Time } \\
\text { Point }\end{array}$ & Arm & Subgroup & $\mathbf{N}$ & Mean (SD) & Effect Size $(95 \% \mathrm{CI})$ & $\begin{array}{l}P \\
\text { Value }\end{array}$ \\
\hline \multirow{8}{*}{$\begin{array}{l}\text { Roth, 2007, } \\
17413877, \\
\text { US }\end{array}$} & $\begin{array}{l}\text { SF-36: Social functioning } \\
(0-100)\end{array}$ & NRCS & High & Pre-op & IBR & All & 35 & $74.3(\mathrm{NR})$ & Ref & Ref \\
\hline & $\begin{array}{l}\text { SF-36: Social functioning } \\
(0-100)\end{array}$ & NRCS & High & Pre-op & AR & $\overline{A l l}$ & 55 & $78.2(\mathrm{NR})$ & $\overline{N R}$ & NR \\
\hline & $\begin{array}{l}\text { SF-36: Social functioning } \\
(0-100)\end{array}$ & NRCS & High & $2 y$ & IBR & All & 35 & 87.9 (NR) & Ref & Ref \\
\hline & $\begin{array}{l}\text { SF-36: Social functioning } \\
(0-100)\end{array}$ & NRCS & High & $2 y$ & AR & All & 55 & 87.7 (NR) & NR & $\geq 0.05$ \\
\hline & $\begin{array}{l}\text { FACT-B: Social/family } \\
\text { well-being }(0-28)\end{array}$ & NRCS & High & Pre-op & IBR & All & 35 & 20.8 (NR) & Ref & Ref \\
\hline & $\begin{array}{l}\text { FACT-B: Social/family } \\
\text { well-being }(0-28)\end{array}$ & NRCS & High & Pre-op & AR & All & 55 & $21.6(\mathrm{NR})$ & NR & NR \\
\hline & $\begin{array}{l}\text { FACT-B: Social/family } \\
\text { well-being }(0-28)\end{array}$ & NRCS & High & $2 y$ & IBR & All & 35 & $19.3(\mathrm{NR})$ & Ref & Ref \\
\hline & $\begin{array}{l}\text { FACT-B: Social/family } \\
\text { well-being }(0-28)\end{array}$ & NRCS & High & $2 y$ & AR & All & 55 & 20.3 (NR) & NR & 0.24 \\
\hline
\end{tabular}

Abbreviations: adj $=$ adjusted, $\mathrm{CI}=$ confidence interval, DIEP $=$ deep inferior epigastric perforator, FACT-B $=$ Functional Assessment of Cancer Therapy $-\mathrm{Breast}, \mathrm{LD}=$ latissimus dorsi, $\mathrm{MD}=$ mean difference, $\mathrm{mo}=$ months, $\mathrm{NMD}=$ net mean difference, $\mathrm{NR}=$ not reported, NRCS $=$ nonrandomized comparative study, $\mathrm{PMID}=\mathrm{Pubmed}$ identifier, $\mathrm{RCT}=$ randomized controlled trial, $\mathrm{Ref}=$ reference group, $\mathrm{RoB}=$ risk of bias, $\mathrm{SD}=$ standard deviation, $\mathrm{SIEA}=$ superficial inferior epigastric artery perforator, $\mathrm{TRAM}=$ transverse rectus abdominis myocutaneous, $\mathrm{y}=$ years.

Colors: Header rows are shaded orange. Rows for every alternate study are shaded blue. The colors do not add unique information.

Table F-1.4. Full Evidence Table - Key Question 1: IBR versus AR - continuous outcomes (sexual well-being)

\begin{tabular}{|c|c|c|c|c|c|c|c|c|c|c|}
\hline $\begin{array}{l}\text { Study, Year, } \\
\text { PMID, } \\
\text { Country }\end{array}$ & $\begin{array}{l}\text { Outcome } \\
\text { Measurement }\end{array}$ & Design & $\begin{array}{l}\text { Overall } \\
\text { RoB }\end{array}$ & $\begin{array}{l}\text { Time } \\
\text { Point }\end{array}$ & Arm & Subgroup & $\mathbf{N}$ & Mean (SD) & Effect Size $(95 \% \mathrm{CI})$ & $\begin{array}{l}P \\
\text { Value }\end{array}$ \\
\hline \multirow{2}{*}{$\begin{array}{l}\text { Tallroth, } \\
2020, \\
33436336, \\
\text { Sweden }\end{array}$} & $\begin{array}{l}\text { BREAST-Q: Sexual } \\
\text { well-being }(0-100)\end{array}$ & RCT & Moderate & $5.3 \mathrm{y}$ & IBR & All & 28 & $58.4(23.1)$ & Ref & Ref \\
\hline & $\begin{array}{l}\text { BREAST-Q: Sexual } \\
\text { well-being }(0-100)\end{array}$ & RCT & Moderate & $5.3 \mathrm{y}$ & AR & All & 42 & $67.1(28.1)$ & MD $8.7(0.2,17.2)$ & 0.046 \\
\hline \multirow{2}{*}{$\begin{array}{l}\text { Eltahir, } \\
2015, \\
25539295, \\
\text { Netherlands }\end{array}$} & $\begin{array}{l}\text { BREAST-Q: Sexual } \\
\text { well-being }(0-100)\end{array}$ & NRCS & Moderate & $2.2 \mathrm{y}$ & IBR & All & 45 & $61.14(24.17)$ & Ref & Ref \\
\hline & $\begin{array}{l}\text { BREAST-Q: Sexual } \\
\text { well-being }(0-100)\end{array}$ & NRCS & Moderate & $2.2 \mathrm{y}$ & AR & All & 47 & $60.89(20.82)$ & adjMD $6.44(-3.56,16.45)$ & 0.204 \\
\hline \multirow{3}{*}{$\begin{array}{l}\text { Kouwenberg } \\
\text {, 2020, } \\
32590633 \text {, } \\
\text { Netherlands }\end{array}$} & $\begin{array}{l}\text { BREAST-Q: Sexual } \\
\text { well-being }(0-100)\end{array}$ & NRCS & Moderate & $>6 \mathrm{mo}$ & IBR & All & 296 & $56.4(30.7)$ & Ref & Ref \\
\hline & $\begin{array}{l}\text { BREAST-Q: Sexual } \\
\text { well-being }(0-100)\end{array}$ & NRCS & Moderate & $>6 \mathrm{mo}$ & AR & All & 179 & $63.3(30.4)$ & NR & $<0.05$ \\
\hline & $\begin{array}{l}\text { EORTC QLQBR23: } \\
\text { Sexual functioning (0- } \\
100)\end{array}$ & NRCS & Moderate & $>6 \mathrm{mo}$ & IBR & All & 296 & $33.4(29.4)$ & Ref & Ref \\
\hline
\end{tabular}




\begin{tabular}{|c|c|c|c|c|c|c|c|c|c|c|}
\hline $\begin{array}{l}\text { Study, Year, } \\
\text { PMID, }\end{array}$ & $\begin{array}{l}\text { Outcome } \\
\text { Measurement }\end{array}$ & Design & $\begin{array}{l}\text { Overall } \\
\text { RoB }\end{array}$ & $\begin{array}{l}\text { Time } \\
\text { Point }\end{array}$ & Arm & Subgroup & $\mathbf{N}$ & Mean (SD) & Effect Size $(95 \% \mathrm{CI})$ & $\begin{array}{l}\mathbf{P} \\
\text { Value }\end{array}$ \\
\hline & $\begin{array}{l}\text { EORTC QLQBR23: } \\
\text { Sexual functioning (0- } \\
100)\end{array}$ & NRCS & Moderate & $>6 \mathrm{mo}$ & AR & All & 179 & $32.0(27.3)$ & NR & $<0.05$ \\
\hline & $\begin{array}{l}\text { EORTC QLQBR23: } \\
\text { Sexual enjoyment (0- } \\
100)\end{array}$ & NRCS & Moderate & $>6 \mathrm{mo}$ & IBR & All & 296 & $63.8(32.5)$ & Ref & Ref \\
\hline & $\begin{array}{l}\text { EORTC QLQBR23: } \\
\text { Sexual enjoyment (0- } \\
100)\end{array}$ & NRCS & Moderate & $>6 \mathrm{mo}$ & AR & All & 179 & $64.2(33.8)$ & NR & NS \\
\hline \multirow{16}{*}{$\begin{array}{l}\text { Kulkarni, } \\
2017, \\
28713853, \\
\text { US \& } \\
\text { Canada }\end{array}$} & $\begin{array}{l}\text { BREAST-Q: Sexual } \\
\text { well-being }(0-100)\end{array}$ & NRCS & Moderate & Baseline & IBR (all) & All & 1104 & $59.1(18.3)$ & NR & NR \\
\hline & $\begin{array}{l}\text { BREAST-Q: Sexual } \\
\text { well-being }(0-100)\end{array}$ & NRCS & Moderate & Baseline & . & Unilateral & 600 & $58.4(19.7)$ & NR & NR \\
\hline & $\begin{array}{l}\text { BREAST-Q: Sexual } \\
\text { well-being }(0-100)\end{array}$ & NRCS & Moderate & Baseline & & Bilateral & 994 & $59(18.5)$ & NR & NR \\
\hline & $\begin{array}{l}\text { BREAST-Q: Sexual } \\
\text { well-being }(0-100)\end{array}$ & NRCS & Moderate & Baseline & IBR Direct & All & NR & NR & NR & NR \\
\hline & $\begin{array}{l}\text { BREAST-Q: Sexual } \\
\text { well-being }(0-100)\end{array}$ & NRCS & Moderate & Baseline & IBR TE & All & NR & NR & NR & NR \\
\hline & $\begin{array}{l}\text { BREAST-Q: Sexual } \\
\text { well-being }(0-100)\end{array}$ & NRCS & Moderate & Baseline & AR (all) & All & 477 & $54(20.9)$ & NR & NR \\
\hline & $\begin{array}{l}\text { BREAST-Q: Sexual } \\
\text { well-being }(0-100)\end{array}$ & NRCS & Moderate & Baseline & . & Unilateral & 317 & $54(20.4)$ & NR & NR \\
\hline & $\begin{array}{l}\text { BREAST-Q: Sexual } \\
\text { well-being }(0-100)\end{array}$ & NRCS & Moderate & Baseline & . & Bilateral & 214 & $52.9(21.5)$ & NR & NR \\
\hline & $\begin{array}{l}\text { BREAST-Q: Sexual } \\
\text { well-being }(0-100)\end{array}$ & NRCS & Moderate & Baseline & AR with DIEP & All & NR & NR & NR & NR \\
\hline & $\begin{array}{l}\text { BREAST-Q: Sexual } \\
\text { well-being }(0-100)\end{array}$ & NRCS & Moderate & Baseline & AR with free TRAM & All & NR & NR & NR & NR \\
\hline & $\begin{array}{l}\text { BREAST-Q: Sexual } \\
\text { well-being }(0-100)\end{array}$ & NRCS & Moderate & Baseline & $\begin{array}{l}\text { AR with pedicled } \\
\text { TRAM }\end{array}$ & All & NR & NR & NR & NR \\
\hline & $\begin{array}{l}\text { BREAST-Q: Sexual } \\
\text { well-being }(0-100)\end{array}$ & NRCS & Moderate & Baseline & AR with LD & All & NR & NR & NR & NR \\
\hline & $\begin{array}{l}\text { BREAST-Q: Sexual } \\
\text { well-being }(0-100)\end{array}$ & NRCS & Moderate & Baseline & AR with SIEA & All & NR & NR & NR & NR \\
\hline & $\begin{array}{l}\text { BREAST-Q: Sexual } \\
\text { well-being }(0-100)\end{array}$ & NRCS & Moderate & $1 \mathrm{y}$ & IBR (all) & All & 756 & $53(21.1)$ & Ref & Ref \\
\hline & $\begin{array}{l}\text { BREAST-Q: Sexual } \\
\text { well-being }(0-100)\end{array}$ & NRCS & Moderate & $1 \mathrm{y}$ & IBR Direct & All & NR & NR & NR & NR \\
\hline & $\begin{array}{l}\text { BREAST-Q: Sexual } \\
\text { well-being }(0-100)\end{array}$ & NRCS & Moderate & $1 \mathrm{y}$ & IBR TE & All & NR & NR & NR & NR \\
\hline
\end{tabular}




\begin{tabular}{|c|c|c|c|c|c|c|c|c|c|c|}
\hline $\begin{array}{l}\text { Study, Year, } \\
\text { PMID, }\end{array}$ & $\begin{array}{l}\text { Outcome } \\
\text { Measurement }\end{array}$ & Design & $\begin{array}{l}\text { Overall } \\
\text { RoB }\end{array}$ & $\begin{array}{l}\text { Time } \\
\text { Point }\end{array}$ & Arm & Subgroup & $\mathbf{N}$ & Mean (SD) & Effect Size (95\% Cl) & $\begin{array}{l}\mathbf{P} \\
\text { Value }\end{array}$ \\
\hline & $\begin{array}{l}\text { BREAST-Q: Sexual } \\
\text { well-being }(0-100)\end{array}$ & NRCS & Moderate & $1 \mathrm{y}$ & AR (all) & All & 370 & $55.4(19.8)$ & $\frac{\text { vs IBR (all): }}{(1.52,7.48)}$ adjMD 4.50 & 0.003 \\
\hline & $\begin{array}{l}\text { BREAST-Q: Sexual } \\
\text { well-being }(0-100)\end{array}$ & NRCS & Moderate & $1 \mathrm{y}$ & AR with DIEP & All & NR & NR & NR & NR \\
\hline & $\begin{array}{l}\text { BREAST-Q: Sexual } \\
\text { well-being }(0-100)\end{array}$ & NRCS & Moderate & $1 \mathrm{y}$ & AR with free TRAM & All & NR & NR & NR & NR \\
\hline & $\begin{array}{l}\text { BREAST-Q: Sexual } \\
\text { well-being }(0-100)\end{array}$ & NRCS & Moderate & $1 \mathrm{y}$ & $\begin{array}{l}\text { AR with pedicled } \\
\text { TRAM }\end{array}$ & All & NR & NR & NR & NR \\
\hline & $\begin{array}{l}\text { BREAST-Q: Sexual } \\
\text { well-being }(0-100)\end{array}$ & NRCS & Moderate & $1 \mathrm{y}$ & AR with LD & All & NR & NR & NR & NR \\
\hline & $\begin{array}{l}\text { BREAST-Q: Sexual } \\
\text { well-being }(0-100)\end{array}$ & NRCS & Moderate & $1 \mathrm{y}$ & AR with SIEA & All & NR & NR & NR & NR \\
\hline & $\begin{array}{l}\text { BREAST-Q: Sexual } \\
\text { well-being }(0-100)\end{array}$ & NRCS & Moderate & $2 y$ & IBR (all) & All & 1490 & $53.9(21.3)$ & Ref & Ref \\
\hline & $\begin{array}{l}\text { BREAST-Q: Sexual } \\
\text { well-being }(0-100)\end{array}$ & NRCS & Moderate & $2 y$ & . & Unilateral & 600 & $52.8(20.5)$ & Ref & Ref \\
\hline & $\begin{array}{l}\text { BREAST-Q: Sexual } \\
\text { well-being (0-100) }\end{array}$ & NRCS & Moderate & $2 y$ & & Bilateral & 994 & $54.7(21.5)$ & Ref & Ref \\
\hline & $\begin{array}{l}\text { BREAST-Q: Sexual } \\
\text { well-being }(0-100)\end{array}$ & NRCS & Moderate & $2 y$ & IBR Direct & All & NR & NR & NR & NR \\
\hline & $\begin{array}{l}\text { BREAST-Q: Sexual } \\
\text { well-being }(0-100)\end{array}$ & NRCS & Moderate & $2 y$ & IBR TE & All & NR & NR & NR & NR \\
\hline & $\begin{array}{l}\text { BREAST-Q: Sexual } \\
\text { well-being }(0-100)\end{array}$ & NRCS & Moderate & $2 y$ & AR (all) & All & 523 & $57.1(21.7)$ & $\frac{\text { vs IBR (all): }}{(2.95,8.11)}$ adjMD 5.53 & NR \\
\hline & $\begin{array}{l}\text { BREAST-Q: Sexual } \\
\text { well-being }(0-100)\end{array}$ & NRCS & Moderate & $2 y$ & . & Unilateral & 317 & $58.9(20.6)$ & $\frac{\text { vs IBR (unilateral): }}{11.42(\mathrm{NR}, \mathrm{NR})}$ adjMD & $<0.001$ \\
\hline & $\begin{array}{l}\text { BREAST-Q: Sexual } \\
\text { well-being }(0-100)\end{array}$ & NRCS & Moderate & $2 y$ & . & Bilateral & 214 & $54.4(23)$ & $\frac{\text { vs IBR (bilateral): }}{\text { (NR, NR) }}$ & $<0.001$ \\
\hline & $\begin{array}{l}\text { BREAST-Q: Sexual } \\
\text { well-being }(0-100)\end{array}$ & NRCS & Moderate & $2 y$ & AR with DIEP & All & NR & NR & NR & NR \\
\hline & $\begin{array}{l}\text { BREAST-Q: Sexual } \\
\text { well-being }(0-100)\end{array}$ & NRCS & Moderate & $2 y$ & AR with free TRAM & All & NR & NR & NR & NR \\
\hline & $\begin{array}{l}\text { BREAST-Q: Sexual } \\
\text { well-being }(0-100)\end{array}$ & NRCS & Moderate & $2 y$ & $\begin{array}{l}\text { AR with pedicled } \\
\text { TRAM }\end{array}$ & All & NR & NR & NR & NR \\
\hline & $\begin{array}{l}\text { BREAST-Q: Sexual } \\
\text { well-being }(0-100)\end{array}$ & NRCS & Moderate & $2 y$ & AR with LD & All & NR & NR & NR & NR \\
\hline & $\begin{array}{l}\text { BREAST-Q: Sexual } \\
\text { well-being }(0-100)\end{array}$ & NRCS & Moderate & $2 y$ & AR with SIEA & All & NR & NR & NR & NR \\
\hline
\end{tabular}

Abbreviations: adj $=$ adjusted, $\mathrm{CI}=$ confidence interval, $\mathrm{DIEP}=$ deep inferior epigastric perforator, $\mathrm{LD}=$ latissimus dorsi, $\mathrm{MD}=$ mean difference, $\mathrm{mo}=$ months, $\mathrm{NR}=$ not

reported, $\mathrm{NRCS}=$ nonrandomized comparative study, $\mathrm{PMID}=$ Pubmed identifier, $\mathrm{RCT}=$ randomized controlled trial, $\mathrm{Ref}=$ reference group, $\mathrm{RoB}=$ risk of bias, $\mathrm{SD}=\mathrm{standard}$

deviation, SIEA = superficial inferior epigastric artery perforator, TRAM $=$ transverse rectus abdominis myocutaneous, $y=$ years. 
Colors: Header rows are shaded orange. Rows for every alternate study are shaded blue. The colors do not add unique information.

Table F-1.5. Full Evidence Table - Key Question 1: IBR versus AR - continuous outcomes (patient satisfaction with breast)

\begin{tabular}{|c|c|c|c|c|c|c|c|c|c|c|}
\hline $\begin{array}{l}\text { Study, Year, } \\
\text { PMID, } \\
\text { Country }\end{array}$ & $\begin{array}{l}\text { Outcome } \\
\text { Measurement }\end{array}$ & Design & $\begin{array}{l}\text { Overall } \\
\text { RoB }\end{array}$ & $\begin{array}{l}\text { Time } \\
\text { Point }\end{array}$ & Arm & Subgroup & $\mathbf{N}$ & Mean (SD) & Effect Size $(95 \% \mathrm{CI})$ & $\begin{array}{l}P \\
\text { Value }\end{array}$ \\
\hline \multirow{4}{*}{$\begin{array}{l}\text { Tallroth, } \\
2020 \\
33436336 \\
\text { Sweden }\end{array}$} & $\begin{array}{l}\text { BREAST-Q: } \\
\text { Satisfaction with } \\
\text { breast }(0-100)\end{array}$ & RCT & Moderate & $5.3 \mathrm{y}$ & IBR & All & 28 & $63.4(11.8)$ & Ref & Ref \\
\hline & $\begin{array}{l}\text { BREAST-Q: } \\
\text { Satisfaction with } \\
\text { breast }(0-100)\end{array}$ & RCT & Moderate & $5.3 \mathrm{y}$ & AR & All & 42 & $72.1(17.7)$ & MD $8.7(3.8,13.6)$ & 0.001 \\
\hline & $\begin{array}{l}\text { BREAST-Q: } \\
\text { Satisfaction with } \\
\text { nipples }(0-100)\end{array}$ & RCT & Moderate & $5.3 y$ & IBR & All & 28 & $65.4(21.8)$ & Ref & Ref \\
\hline & $\begin{array}{l}\text { BREAST-Q: } \\
\text { Satisfaction with } \\
\text { nipples }(0-100)\end{array}$ & RCT & Moderate & $5.3 \mathrm{y}$ & AR & All & 42 & $67.7(24.9)$ & MD $2.3(-5.5,10.1)$ & 0.56 \\
\hline \multirow[t]{2}{*}{$\begin{array}{l}\text { Brito, 2020, } \\
\text { No PMID, } \\
\text { Portugal }\end{array}$} & $\begin{array}{l}\text { BREAST-Q: } \\
\text { Satisfaction with } \\
\text { breast }(0-100)\end{array}$ & NRCS & High & NR & IBR & All & 68 & $56.3(17.1)$ & Ref & Ref \\
\hline & $\begin{array}{l}\text { BREAST-Q: } \\
\text { Satisfaction with } \\
\text { breast }(0-100)\end{array}$ & NRCS & High & NR & AR & All & 111 & $64.1(17.1)$ & NR & 0.004 \\
\hline \multirow[t]{4}{*}{$\begin{array}{l}\text { Eltahir, 2015, } \\
25539295, \\
\text { Netherlands }\end{array}$} & $\begin{array}{l}\text { BREAST-Q: } \\
\text { Satisfaction with } \\
\text { breast }(0-100)\end{array}$ & NRCS & Moderate & $2.2 \mathrm{y}$ & IBR & All & 45 & $65.51(17.55)$ & Ref & Ref \\
\hline & $\begin{array}{l}\text { BREAST-Q: } \\
\text { Satisfaction with } \\
\text { breast }(0-100)\end{array}$ & NRCS & Moderate & $2.2 \mathrm{y}$ & AR & All & 47 & $75.19(17.09)$ & adjMD $-8.16(-15.15,-1.18)$ & 0.023 \\
\hline & $\begin{array}{l}\text { BREAST-Q: } \\
\text { Satisfaction with } \\
\text { nipples }(0-100)\end{array}$ & NRCS & Moderate & $2.2 \mathrm{y}$ & IBR & All & 45 & 63.62 (33.99) & Ref & Ref \\
\hline & $\begin{array}{l}\text { BREAST-Q: } \\
\text { Satisfaction with } \\
\text { nipples }(0-100)\end{array}$ & NRCS & Moderate & $2.2 \mathrm{y}$ & AR & All & 47 & $65.31(27.82)$ & adjMD -1.7 (-17.55, 14.15) & 0.831 \\
\hline \multirow{2}{*}{$\begin{array}{l}\text { Kouwenberg, } \\
2020 \text {, } \\
32590633 \text {, } \\
\text { Netherlands }\end{array}$} & $\begin{array}{l}\text { BREAST-Q: } \\
\text { Satisfaction with } \\
\text { breast }(0-100)\end{array}$ & NRCS & Moderate & $>6 \mathrm{mo}$ & IBR & All & 296 & $59.4(19.3)$ & Ref & Ref \\
\hline & $\begin{array}{l}\text { BREAST-Q: } \\
\text { Satisfaction with } \\
\text { breast }(0-100)\end{array}$ & NRCS & Moderate & $>6 \mathrm{mo}$ & AR & All & 179 & $71.3(17.7)$ & NR & $<0.05$ \\
\hline
\end{tabular}




\begin{tabular}{|c|c|c|c|c|c|c|c|c|c|c|}
\hline $\begin{array}{l}\text { Study, Year, } \\
\text { PMID, }\end{array}$ & $\begin{array}{l}\text { Outcome } \\
\text { Measurement }\end{array}$ & Design & $\begin{array}{l}\text { Overall } \\
\text { RoB }\end{array}$ & $\begin{array}{l}\text { Time } \\
\text { Point }\end{array}$ & Arm & Subgroup & $\mathbf{N}$ & Mean (SD) & Effect Size (95\% CI) & $\begin{array}{l}\mathbf{P} \\
\text { Value }\end{array}$ \\
\hline & $\begin{array}{l}\text { BREAST-Q: } \\
\text { Satisfaction with } \\
\text { nipples }(0-100)\end{array}$ & NRCS & Moderate & $>6 \mathrm{mo}$ & IBR & All & 296 & $55.0(48.7)$ & Ref & Ref \\
\hline & $\begin{array}{l}\text { BREAST-Q: } \\
\text { Satisfaction with } \\
\text { nipples }(0-100)\end{array}$ & NRCS & Moderate & $>6 \mathrm{mo}$ & AR & All & 179 & $63.0(29.0)$ & NR & $<0.05$ \\
\hline \multirow{11}{*}{$\begin{array}{l}\text { Kulkarni, } \\
2017, \\
28713853, \\
\text { US \& Canada }\end{array}$} & $\begin{array}{l}\text { BREAST-Q: } \\
\text { Satisfaction with } \\
\text { breast }(0-100)\end{array}$ & NRCS & Moderate & Baseline & IBR (all) & All & 1132 & $64.9(21.2)$ & Ref & Ref \\
\hline & $\begin{array}{l}\text { BREAST-Q: } \\
\text { Satisfaction with } \\
\text { breast }(0-100)\end{array}$ & NRCS & Moderate & Baseline & IBR (all) & Unilateral & 600 & $65.8(21.7)$ & NR & NR \\
\hline & $\begin{array}{l}\text { BREAST-Q: } \\
\text { Satisfaction with } \\
\text { breast }(0-100)\end{array}$ & NRCS & Moderate & Baseline & IBR (all) & Bilateral & 994 & $62.8(21)$ & NR & NR \\
\hline & $\begin{array}{l}\text { BREAST-Q: } \\
\text { Satisfaction with } \\
\text { breast }(0-100)\end{array}$ & NRCS & Moderate & Baseline & IBR Direct & All & NR & NR & NR & NR \\
\hline & $\begin{array}{l}\text { BREAST-Q: } \\
\text { Satisfaction with } \\
\text { breast }(0-100)\end{array}$ & NRCS & Moderate & Baseline & IBR TE & All & NR & NR & NR & NR \\
\hline & $\begin{array}{l}\text { BREAST-Q: } \\
\text { Satisfaction with } \\
\text { breast }(0-100)\end{array}$ & NRCS & Moderate & Baseline & AR (all) & All & 491 & $59(20.7)$ & NR & NR \\
\hline & $\begin{array}{l}\text { BREAST-Q: } \\
\text { Satisfaction with } \\
\text { breast }(0-100)\end{array}$ & NRCS & Moderate & Baseline & AR (all) & Unilateral & 317 & $59.6(21.1)$ & NR & NR \\
\hline & $\begin{array}{l}\text { BREAST-Q: } \\
\text { Satisfaction with } \\
\text { breast }(0-100)\end{array}$ & NRCS & Moderate & Baseline & AR (all) & Bilateral & 214 & $56.1(18.5)$ & NR & NR \\
\hline & $\begin{array}{l}\text { BREAST-Q: } \\
\text { Satisfaction with } \\
\text { breast }(0-100)\end{array}$ & NRCS & Moderate & Baseline & AR with DIEP & All & NR & NR & NR & NR \\
\hline & $\begin{array}{l}\text { BREAST-Q: } \\
\text { Satisfaction with } \\
\text { breast }(0-100)\end{array}$ & NRCS & Moderate & Baseline & AR with free TRAM & All & NR & NR & NR & NR \\
\hline & $\begin{array}{l}\text { BREAST-Q: } \\
\text { Satisfaction with } \\
\text { breast }(0-100)\end{array}$ & NRCS & Moderate & Baseline & $\begin{array}{l}\text { AR with pedicled } \\
\text { TRAM }\end{array}$ & All & NR & NR & NR & NR \\
\hline
\end{tabular}




\begin{tabular}{|c|c|c|c|c|c|c|c|c|c|c|}
\hline $\begin{array}{l}\text { Study, Year, } \\
\text { PMID, } \\
\text { Country }\end{array}$ & $\begin{array}{l}\text { Outcome } \\
\text { Measurement }\end{array}$ & Design & $\begin{array}{l}\text { Overall } \\
\text { RoB }\end{array}$ & $\begin{array}{l}\text { Time } \\
\text { Point }\end{array}$ & Arm & Subgroup & $\mathbf{N}$ & Mean (SD) & Effect Size $(95 \% \mathrm{Cl})$ & $\begin{array}{l}\mathbf{P} \\
\text { Value }\end{array}$ \\
\hline & $\begin{array}{l}\text { BREAST-Q: } \\
\text { Satisfaction with } \\
\text { breast }(0-100)\end{array}$ & NRCS & Moderate & Baseline & AR with LD & All & NR & NR & NR & NR \\
\hline & $\begin{array}{l}\text { BREAST-Q: } \\
\text { Satisfaction with } \\
\text { breast }(0-100)\end{array}$ & NRCS & Moderate & Baseline & AR with SIEA & All & NR & NR & NR & NR \\
\hline & $\begin{array}{l}\text { BREAST-Q: } \\
\text { Satisfaction with } \\
\text { breast }(0-100)\end{array}$ & NRCS & Moderate & $1 \mathrm{y}$ & IBR (all) & All & 795 & $64(16.8)$ & Ref & Ref \\
\hline & $\begin{array}{l}\text { BREAST-Q: } \\
\text { Satisfaction with } \\
\text { breast }(0-100)\end{array}$ & NRCS & Moderate & $1 \mathrm{y}$ & IBR Direct & All & NR & NR & NR & NR \\
\hline & $\begin{array}{l}\text { BREAST-Q: } \\
\text { Satisfaction with } \\
\text { breast }(0-100)\end{array}$ & NRCS & Moderate & $1 \mathrm{y}$ & IBR TE & All & NR & NR & NR & NR \\
\hline & $\begin{array}{l}\text { BREAST-Q: } \\
\text { Satisfaction with } \\
\text { breast }(0-100)\end{array}$ & NRCS & Moderate & $1 \mathrm{y}$ & AR (all) & All & 388 & $67.8(17.2)$ & $\frac{\text { vs IBR (all): }}{9.09)}$ adjMD 6.3 (3.41, & $<0.001$ \\
\hline & $\begin{array}{l}\text { BREAST-Q: } \\
\text { Satisfaction with } \\
\text { breast }(0-100)\end{array}$ & NRCS & Moderate & $1 \mathrm{y}$ & AR with DIEP & All & NR & NR & NR & NR \\
\hline & $\begin{array}{l}\text { BREAST-Q: } \\
\text { Satisfaction with } \\
\text { breast }(0-100)\end{array}$ & NRCS & Moderate & $1 \mathrm{y}$ & AR with free TRAM & All & NR & NR & NR & NR \\
\hline & $\begin{array}{l}\text { BREAST-Q: } \\
\text { Satisfaction with } \\
\text { breast }(0-100)\end{array}$ & NRCS & Moderate & $1 \mathrm{y}$ & $\begin{array}{l}\text { AR with pedicled } \\
\text { TRAM }\end{array}$ & All & NR & NR & NR & NR \\
\hline & $\begin{array}{l}\text { BREAST-Q: } \\
\text { Satisfaction with } \\
\text { breast }(0-100)\end{array}$ & NRCS & Moderate & $1 \mathrm{y}$ & AR with LD & All & NR & NR & NR & NR \\
\hline & $\begin{array}{l}\text { BREAST-Q: } \\
\text { Satisfaction with } \\
\text { breast }(0-100)\end{array}$ & NRCS & Moderate & $1 \mathrm{y}$ & AR with SIEA & All & NR & NR & NR & NR \\
\hline & $\begin{array}{l}\text { BREAST-Q: } \\
\text { Satisfaction with } \\
\text { breast }(0-100)\end{array}$ & NRCS & Moderate & $2 y$ & IBR (all) & All & 1490 & $64.2(18)$ & Ref & Ref \\
\hline & $\begin{array}{l}\text { BREAST-Q: } \\
\text { Satisfaction with } \\
\text { breast }(0-100)\end{array}$ & NRCS & Moderate & $2 y$ & IBR (all) & Unilateral & 600 & $61.2(18)$ & Ref & Ref \\
\hline
\end{tabular}




\begin{tabular}{|c|c|c|c|c|c|c|c|c|c|c|}
\hline $\begin{array}{l}\text { Study, Year, } \\
\text { PMID, } \\
\text { Country }\end{array}$ & $\begin{array}{l}\text { Outcome } \\
\text { Measurement }\end{array}$ & Design & $\begin{array}{l}\text { Overall } \\
\text { RoB }\end{array}$ & $\begin{array}{l}\text { Time } \\
\text { Point }\end{array}$ & Arm & Subgroup & $\mathbf{N}$ & Mean (SD) & Effect Size (95\% CI) & $\begin{array}{l}\mathbf{P} \\
\text { Value }\end{array}$ \\
\hline & $\begin{array}{l}\text { BREAST-Q: } \\
\text { Satisfaction with } \\
\text { breast }(0-100)\end{array}$ & NRCS & Moderate & $2 y$ & IBR (all) & Bilateral & 994 & $66.1(17.7)$ & Ref & Ref \\
\hline & $\begin{array}{l}\text { BREAST-Q: } \\
\text { Satisfaction with } \\
\text { breast }(0-100)\end{array}$ & NRCS & Moderate & $2 \mathrm{y}$ & IBR Direct & All & NR & NR & NR & NR \\
\hline & $\begin{array}{l}\text { BREAST-Q: } \\
\text { Satisfaction with } \\
\text { breast }(0-100)\end{array}$ & NRCS & Moderate & $2 y$ & IBR TE & All & NR & NR & NR & NR \\
\hline & $\begin{array}{l}\text { BREAST-Q: } \\
\text { Satisfaction with } \\
\text { breast }(0-100)\end{array}$ & NRCS & Moderate & $2 y$ & AR (all) & All & 523 & $68.5(18.3)$ & 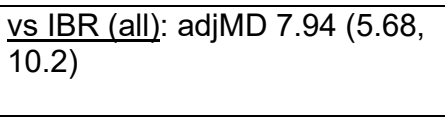 & NR \\
\hline & $\begin{array}{l}\text { BREAST-Q: } \\
\text { Satisfaction with } \\
\text { breast }(0-100)\end{array}$ & NRCS & Moderate & $2 y$ & AR (all) & Unilateral & 317 & $68.3(18.1)$ & 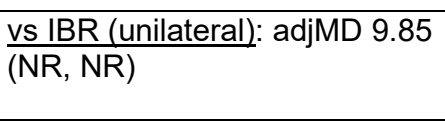 & NR \\
\hline & $\begin{array}{l}\text { BREAST-Q: } \\
\text { Satisfaction with } \\
\text { breast }(0-100)\end{array}$ & NRCS & Moderate & $2 y$ & AR (all) & Bilateral & 214 & $68.9(18.6)$ & 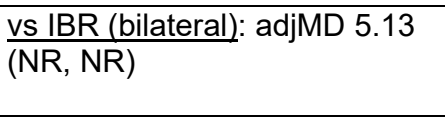 & 0.001 \\
\hline & $\begin{array}{l}\text { BREAST-Q: } \\
\text { Satisfaction with } \\
\text { breast }(0-100)\end{array}$ & NRCS & Moderate & $2 y$ & AR with DIEP & All & NR & NR & NR & NR \\
\hline & $\begin{array}{l}\text { BREAST-Q: } \\
\text { Satisfaction with } \\
\text { breast }(0-100)\end{array}$ & NRCS & Moderate & $2 y$ & AR with free TRAM & All & NR & NR & NR & NR \\
\hline & $\begin{array}{l}\text { BREAST-Q: } \\
\text { Satisfaction with } \\
\text { breast }(0-100)\end{array}$ & NRCS & Moderate & $2 y$ & $\begin{array}{l}\text { AR with pedicled } \\
\text { TRAM }\end{array}$ & All & NR & NR & NR & NR \\
\hline & $\begin{array}{l}\text { BREAST-Q: } \\
\text { Satisfaction with } \\
\text { breast }(0-100)\end{array}$ & NRCS & Moderate & $2 y$ & AR with LD & All & NR & NR & NR & NR \\
\hline & $\begin{array}{l}\text { BREAST-Q: } \\
\text { Satisfaction with } \\
\text { breast }(0-100)\end{array}$ & NRCS & Moderate & $2 y$ & AR with SIEA & All & NR & NR & NR & NR \\
\hline \multirow[t]{2}{*}{$\begin{array}{l}\text { Nelson, 2019, } \\
31356276, \\
\text { US }\end{array}$} & $\begin{array}{l}\text { BREAST-Q: } \\
\text { Satisfaction with } \\
\text { breast }(0-100)\end{array}$ & NRCS & High & $1 \mathrm{y}$ & IBR & & 1342 & $64.17(18.68)$ & Ref & Ref \\
\hline & $\begin{array}{l}\text { BREAST-Q: } \\
\text { Satisfaction with } \\
\text { breast }(0-100)\end{array}$ & NRCS & High & $1 \mathrm{y}$ & AR & & 194 & $68.25(20.24)$ & NR & NR \\
\hline
\end{tabular}




\begin{tabular}{|c|c|c|c|c|c|c|c|c|c|c|}
\hline $\begin{array}{l}\text { Study, Year, } \\
\text { PMID, } \\
\text { Country }\end{array}$ & $\begin{array}{l}\text { Outcome } \\
\text { Measurement }\end{array}$ & Design & $\begin{array}{l}\text { Overall } \\
\text { RoB }\end{array}$ & $\begin{array}{l}\text { Time } \\
\text { Point }\end{array}$ & Arm & Subgroup & $\mathbf{N}$ & Mean (SD) & Effect Size (95\% CI) & $\begin{array}{l}\mathbf{P} \\
\text { Value }\end{array}$ \\
\hline & $\begin{array}{l}\text { BREAST-Q: } \\
\text { Satisfaction with } \\
\text { breast }(0-100)\end{array}$ & NRCS & High & $3 y$ & IBR & & 1085 & $63.7(17.66)$ & Ref & Ref \\
\hline & $\begin{array}{l}\text { BREAST-Q: } \\
\text { Satisfaction with } \\
\text { breast }(0-100)\end{array}$ & NRCS & High & $3 y$ & AR & & 98 & $73.48(17.07)$ & NR & NR \\
\hline & $\begin{array}{l}\text { BREAST-Q: } \\
\text { Satisfaction with } \\
\text { breast }(0-100)\end{array}$ & NRCS & High & $5 y$ & IBR & & 743 & $63.06(17.25)$ & Ref & Ref \\
\hline & $\begin{array}{l}\text { BREAST-Q: } \\
\text { Satisfaction with } \\
\text { breast }(0-100)\end{array}$ & NRCS & High & $5 y$ & AR & & 41 & $79.65(19.49)$ & NR & NR \\
\hline & $\begin{array}{l}\text { BREAST-Q: } \\
\text { Satisfaction with } \\
\text { breast }(0-100)\end{array}$ & NRCS & High & $7 y$ & IBR & & 377 & $64.67(19.19)$ & Ref & Ref \\
\hline & $\begin{array}{l}\text { BREAST-Q: } \\
\text { Satisfaction with } \\
\text { breast }(0-100)\end{array}$ & NRCS & High & $7 y$ & AR & & 19 & $76(18.24)$ & NR & NR \\
\hline
\end{tabular}

Abbreviations: adj $=$ adjusted, $\mathrm{CI}=$ confidence interval, $\mathrm{DIEP}=$ deep inferior epigastric perforator, $\mathrm{LD}=$ latissimus dorsi, $\mathrm{LTD}=$ lateral thoracodorsal flap, $\mathrm{MD}=$ mean difference, mo $=$ months, $\mathrm{NR}=$ not reported, $\mathrm{NRCS}=$ nonrandomized comparative study, PMID $=$ Pubmed identifier, $\mathrm{RCT}=$ randomized controlled trial, $\mathrm{Ref}=$ reference group, RoB $=$ risk of bias, $\mathrm{SD}=$ standard deviation, $\mathrm{SIEA}=$ superficial inferior epigastric artery perforator, TRAM $=$ transverse rectus abdominis myocutaneous, $\mathrm{y}=$ years

Colors: Header rows are shaded orange. Rows for every alternate study are shaded blue. The colors do not add unique information.

Table F-1.6. Full Evidence Table - Key Question 1: IBR versus AR - categorical outcomes (physical well-being)

\begin{tabular}{|l|l|l|l|l|l|l|l|l|}
$\begin{array}{l}\text { Study, Year, } \\
\text { PMID, Country }\end{array}$ & Outcome Measurement & Design & Overall RoB & Time Point & IBR, n/N (\%) & AR, n/N (\%) & Adjusted Odds Ratio (95\% Cl) & P Value \\
$\begin{array}{l}\text { Nelson, 2019, } \\
31356276, \text { US }\end{array}$ & $\begin{array}{l}\text { Likely to have higher BREAST-Q: Chest } \\
\text { scores }\end{array}$ & NRCS & High & 1 y & NR/1342 (NR) & NR/194 (NR) & $0.96(0.67,1.38)$ \\
\cline { 2 - 8 } & $\begin{array}{l}\text { Likely to have higher BREAST-Q: Chest } \\
\text { scores }\end{array}$ & NRCS & High & $3 \mathrm{y}$ & NR/1085 (NR) & NR/98 (NR) & $1.4(0.83,2.34)$ \\
\cline { 2 - 8 } & $\begin{array}{l}\text { Likely to have higher BREAST-Q: Chest } \\
\text { scores }\end{array}$ & NRCS & High & $5 \mathrm{y}$ & NR/743 (NR) & NR/41 (NR) & $4.52(2.03,10.1)$ \\
\cline { 2 - 8 } \\
$\begin{array}{l}\text { Likely to have higher BREAST-Q: Chest } \\
\text { scores }\end{array}$ & NRCS & High & $7 \mathrm{y}$ & NR/377 (NR) & NR/19 (NR) & $3.08(1.03,9.15)$ \\
\hline
\end{tabular}

Abbreviations: AR = autologous reconstruction, $\mathrm{CI}=$ confidence interval, $\mathrm{NR}=$ not reported, NRCS $=$ nonrandomized comparative study, $\mathrm{NS}=$ not significant, $\mathrm{PMID}=$ PubMed identifier, $\mathrm{RoB}=$ risk of bias.

Colors: Header rows are shaded orange. The color does not add unique information. 
Table F-1.7. Full Evidence Table - Key Question 1: IBR versus AR - categorical outcomes (patient satisfaction with breast)

\begin{tabular}{|c|c|c|c|c|c|c|c|c|c|}
\hline $\begin{array}{l}\text { Study, Year, } \\
\text { PMID, Country }\end{array}$ & Outcome Measurement & Design & $\begin{array}{l}\text { Overall } \\
\text { RoB }\end{array}$ & $\begin{array}{l}\text { Time } \\
\text { Point }\end{array}$ & Arm & $\mathrm{n} / \mathrm{N}(\%)$ & Comparison & $\begin{array}{l}\text { Odds Ratio } \\
(95 \% \mathrm{Cl})\end{array}$ & P Value \\
\hline \multirow{2}{*}{$\begin{array}{l}\text { Lei, } 2020, \\
32481367, \\
\text { China }\end{array}$} & Satisfied with breasts & NRCS & High & $2 \mathrm{mo}$ & IBR & NR & Ref & Ref & Ref \\
\hline & Satisfied with breasts & NRCS & High & $2 \mathrm{mo}$ & AR & NR & vs. IBR & $0.85(0.36,1.63)$ & 0.40 \\
\hline \multirow{5}{*}{$\begin{array}{l}\text { Yueh, 2009, } \\
\text { 19228537, US }\end{array}$} & Satisfied with breasts & NRCS & High & NR & IBR & $42 / 87(48.3)$ & Ref & Ref & Ref \\
\hline & Satisfied with breasts & NRCS & High & NR & AR & NR/389 (NR) & vs. IBR & $1.43(1.18,1.73)$ & NR \\
\hline & Satisfied with breasts & NRCS & High & NR & AR with DIEP & NR/117 (NR) & vs. AR with TRAM & $0.67(0.37,1.23)$ & NR \\
\hline & Satisfied with breasts & NRCS & High & NR & AR with TRAM & $102 / 143(71.3)$ & vs. IBR & $3.49(1.91,6.40)$ & NR \\
\hline & Satisfied with breasts & NRCS & High & NR & AR with LD & $68 / 112(60.7)$ & $\begin{array}{l}\text { vs. IBR } \\
\text { vs. AR with TRAM }\end{array}$ & $\begin{array}{l}1.99(1.09,3.65) \\
0.78(0.54,1.14)\end{array}$ & $\begin{array}{l}\text { NR } \\
\text { NR }\end{array}$ \\
\hline
\end{tabular}

Abbreviations: $\mathrm{AR}=$ autologous reconstruction, $\mathrm{CI}=$ confidence interval, $\mathrm{LD}=$ latissimus dorsi, $\mathrm{LTD}=$ lateral thoracodorsal flap, $\mathrm{PMID}=\mathrm{PubMed}$ identifier, $\mathrm{RCT}=$ randomized

controlled trial, $\mathrm{Ref}=$ reference group, $\mathrm{RoB}=$ risk of bias, TRAM $=$ transverse rectus abdominis myocutaneous.

Colors: Header rows are shaded orange. Rows for every alternate study are shaded blue. The colors do not add unique information.

Table F-1.8. Full Evidence Table - Key Question 1: IBR versus AR - continuous outcomes (patient satisfaction with outcome)

\begin{tabular}{|c|c|c|c|c|c|c|c|c|c|}
\hline $\begin{array}{l}\text { Study, Year, } \\
\text { PMID, } \\
\text { Country }\end{array}$ & Outcome Measurement & $\begin{array}{l}\text { Desig } \\
\mathrm{n}\end{array}$ & $\begin{array}{l}\text { Overall } \\
\text { RoB }\end{array}$ & $\begin{array}{l}\text { Time } \\
\text { Point }\end{array}$ & Arm & $\mathbf{N}$ & Mean (SD) & Effect Size $(95 \% \mathrm{Cl})$ & P Value \\
\hline \multirow{2}{*}{$\begin{array}{l}\text { Tallroth, 2020, } \\
\text { 33436336, } \\
\text { Sweden }\end{array}$} & $\begin{array}{l}\text { BREAST-Q: Satisfaction } \\
\text { with outcome }(0-100)\end{array}$ & RCT & Moderate & $5.3 y$ & IBR & 28 & $79.4(14.2)$ & Ref & Ref \\
\hline & $\begin{array}{l}\text { BREAST-Q: Satisfaction } \\
\text { with outcome }(0-100)\end{array}$ & RCT & Moderate & $5.3 y$ & AR & 42 & $82.3(21.4)$ & MD $2.9(-3.1,8.9)$ & 0.34 \\
\hline \multirow{2}{*}{$\begin{array}{l}\text { Eltahir, 2015, } \\
\text { 25539295, } \\
\text { Netherlands }\end{array}$} & $\begin{array}{l}\text { BREAST-Q: Satisfaction } \\
\text { with outcome }(0-100)\end{array}$ & NRCS & Moderate & $2.2 \mathrm{y}$ & IBR & 45 & $74.53(18.98)$ & Ref & Ref \\
\hline & $\begin{array}{l}\text { BREAST-Q: Satisfaction } \\
\text { with outcome }(0-100)\end{array}$ & NRCS & Moderate & $2.2 \mathrm{y}$ & AR & 47 & $81.82(18.69)$ & adjMD $4.9(-3.09,12.89)$ & 0.226 \\
\hline \multirow{2}{*}{$\begin{array}{l}\text { Kouwenberg, } \\
2020 \text {, } \\
32590633 \text {, } \\
\text { Netherlands }\end{array}$} & $\begin{array}{l}\text { BREAST-Q: Satisfaction } \\
\text { with outcome }(0-100)\end{array}$ & NRCS & Moderate & $>6 \mathrm{mo}$ & IBR & 296 & $66.4(23.7)$ & Ref & Ref \\
\hline & $\begin{array}{l}\text { BREAST-Q: Satisfaction } \\
\text { with outcome }(0-100)\end{array}$ & NRCS & Moderate & $>6 \mathrm{mo}$ & AR & 179 & $75.8(22.2)$ & NR & $<0.05$ \\
\hline \multirow{5}{*}{$\begin{array}{l}\text { Kulkarni, 2017, } \\
\text { 28713853, US } \\
\text { \& Canada }\end{array}$} & $\begin{array}{l}\text { BREAST-Q: Satisfaction } \\
\text { with information }(0-100)\end{array}$ & NRCS & Moderate & $3 \mathrm{mo}$ & IBR (all) & NR & NR & NR & NR \\
\hline & $\begin{array}{l}\text { BREAST-Q: Satisfaction } \\
\text { with information }(0-100)\end{array}$ & NRCS & Moderate & $3 \mathrm{mo}$ & IBR Direct & 79 & $73.4(16.8)$ & NR & NR \\
\hline & $\begin{array}{l}\text { BREAST-Q: Satisfaction } \\
\text { with information }(0-100)\end{array}$ & NRCS & Moderate & $3 \mathrm{mo}$ & IBR TE & 942 & $72.6(18.1)$ & NR & NR \\
\hline & $\begin{array}{l}\text { BREAST-Q: Satisfaction } \\
\text { with information }(0-100)\end{array}$ & NRCS & Moderate & $3 \mathrm{mo}$ & AR (all) & NR & NR & NR & NR \\
\hline & $\begin{array}{l}\text { BREAST-Q: Satisfaction } \\
\text { with information }(0-100)\end{array}$ & NRCS & Moderate & $3 \mathrm{mo}$ & AR with DIEP & 395 & $72.8(13)$ & NR & NR \\
\hline
\end{tabular}




\begin{tabular}{|c|c|c|c|c|c|c|c|c|c|}
\hline $\begin{array}{l}\text { Study, Year, } \\
\text { PMID, } \\
\text { Country }\end{array}$ & Outcome Measurement & $\begin{array}{l}\text { Desig } \\
\mathrm{n}\end{array}$ & $\begin{array}{l}\text { Overall } \\
\text { RoB }\end{array}$ & $\begin{array}{l}\text { Time } \\
\text { Point }\end{array}$ & Arm & $\mathbf{N}$ & Mean (SD) & Effect Size (95\% CI) & P Value \\
\hline & $\begin{array}{l}\text { BREAST-Q: Satisfaction } \\
\text { with information }(0-100)\end{array}$ & NRCS & Moderate & $3 \mathrm{mo}$ & AR with free TRAM & NR & $72.8(16.5)$ & NR & NR \\
\hline & $\begin{array}{l}\text { BREAST-Q: Satisfaction } \\
\text { with information }(0-100)\end{array}$ & NRCS & Moderate & $3 \mathrm{mo}$ & $\begin{array}{l}\text { AR with pedicled } \\
\text { TRAM }\end{array}$ & 65 & $76.2(19.9)$ & NR & NR \\
\hline & $\begin{array}{l}\text { BREAST-Q: Satisfaction } \\
\text { with information }(0-100)\end{array}$ & NRCS & Moderate & $3 \mathrm{mo}$ & AR with LD & 53 & $69.3(19.1)$ & NR & NR \\
\hline & $\begin{array}{l}\text { BREAST-Q: Satisfaction } \\
\text { with information }(0-100)\end{array}$ & NRCS & Moderate & $3 \mathrm{mo}$ & AR with SIEA & NR & NR & NR & NR \\
\hline & $\begin{array}{l}\text { BREAST-Q: Satisfaction } \\
\text { with surgeon }(0-100)\end{array}$ & NRCS & Moderate & $3 \mathrm{mo}$ & IBR (all) & NR & NR & NR & NR \\
\hline & $\begin{array}{l}\text { BREAST-Q: Satisfaction } \\
\text { with surgeon }(0-100)\end{array}$ & NRCS & Moderate & $3 \mathrm{mo}$ & IBR Direct & 79 & $87.5(17.1)$ & NR & NR \\
\hline & $\begin{array}{l}\text { BREAST-Q: Satisfaction } \\
\text { with surgeon }(0-100)\end{array}$ & NRCS & Moderate & $3 \mathrm{mo}$ & IBR TE & 942 & $88.5(17)$ & NR & NR \\
\hline & $\begin{array}{l}\text { BREAST-Q: Satisfaction } \\
\text { with surgeon }(0-100)\end{array}$ & NRCS & Moderate & $3 \mathrm{mo}$ & AR (all) & NR & NR & NR & NR \\
\hline & $\begin{array}{l}\text { BREAST-Q: Satisfaction } \\
\text { with surgeon }(0-100)\end{array}$ & NRCS & Moderate & $3 \mathrm{mo}$ & AR with DIEP & 395 & $91.6(14)$ & NR & NR \\
\hline & $\begin{array}{l}\text { BREAST-Q: Satisfaction } \\
\text { with surgeon }(0-100)\end{array}$ & NRCS & Moderate & $3 \mathrm{mo}$ & AR with free TRAM & NR & NR & NR & NR \\
\hline & $\begin{array}{l}\text { BREAST-Q: Satisfaction } \\
\text { with surgeon }(0-100)\end{array}$ & NRCS & Moderate & $3 \mathrm{mo}$ & $\begin{array}{l}\text { AR with pedicled } \\
\text { TRAM }\end{array}$ & 65 & $91(15.9)$ & NR & NR \\
\hline & $\begin{array}{l}\text { BREAST-Q: Satisfaction } \\
\text { with surgeon }(0-100)\end{array}$ & NRCS & Moderate & $3 \mathrm{mo}$ & AR with LD & 53 & $92.5(14.4)$ & NR & NR \\
\hline & $\begin{array}{l}\text { BREAST-Q: Satisfaction } \\
\text { with surgeon }(0-100)\end{array}$ & NRCS & Moderate & $3 \mathrm{mo}$ & AR with SIEA & NR & NR & NR & NR \\
\hline & $\begin{array}{l}\text { BREAST-Q: Satisfaction } \\
\text { with medical team (0- } \\
100)\end{array}$ & NRCS & Moderate & $3 \mathrm{mo}$ & IBR (all) & NR & NR & NR & NR \\
\hline & $\begin{array}{l}\text { BREAST-Q: Satisfaction } \\
\text { with medical team (0- } \\
100)\end{array}$ & NRCS & Moderate & $3 \mathrm{mo}$ & IBR Direct & 79 & $90.1(18.4)$ & NR & NR \\
\hline & $\begin{array}{l}\text { BREAST-Q: Satisfaction } \\
\text { with medical team (0- } \\
100)\end{array}$ & NRCS & Moderate & $3 \mathrm{mo}$ & IBR TE & 942 & $93.2(15.2)$ & NR & NR \\
\hline & $\begin{array}{l}\text { BREAST-Q: Satisfaction } \\
\text { with medical team (0- } \\
100)\end{array}$ & NRCS & Moderate & $3 \mathrm{mo}$ & AR (all) & NR & NR & NR & NR \\
\hline & $\begin{array}{l}\text { BREAST-Q: Satisfaction } \\
\text { with medical team (0- } \\
100)\end{array}$ & NRCS & Moderate & $3 \mathrm{mo}$ & AR with DIEP & 395 & 90 (19) & NR & NR \\
\hline
\end{tabular}




\begin{tabular}{|c|c|c|c|c|c|c|c|c|c|}
\hline $\begin{array}{l}\text { Study, Year, } \\
\text { PMID, } \\
\text { Country }\end{array}$ & Outcome Measurement & $\begin{array}{l}\text { Desig } \\
\mathrm{n}\end{array}$ & $\begin{array}{l}\text { Overall } \\
\text { RoB }\end{array}$ & $\begin{array}{l}\text { Time } \\
\text { Point }\end{array}$ & Arm & $\mathbf{N}$ & Mean (SD) & Effect Size (95\% CI) & P Value \\
\hline & $\begin{array}{l}\text { BREAST-Q: Satisfaction } \\
\text { with medical team (0- } \\
100)\end{array}$ & NRCS & Moderate & $3 \mathrm{mo}$ & AR with free TRAM & NR & NR & NR & NR \\
\hline & $\begin{array}{l}\text { BREAST-Q: Satisfaction } \\
\text { with medical team (0- } \\
100)\end{array}$ & NRCS & Moderate & $3 \mathrm{mo}$ & $\begin{array}{l}\text { AR with pedicled } \\
\text { TRAM }\end{array}$ & 65 & $91(13.8)$ & NR & NR \\
\hline & $\begin{array}{l}\text { BREAST-Q: Satisfaction } \\
\text { with medical team (0- } \\
100)\end{array}$ & NRCS & Moderate & $3 \mathrm{mo}$ & AR with LD & 53 & $94.5(12.7)$ & NR & NR \\
\hline & $\begin{array}{l}\text { BREAST-Q: Satisfaction } \\
\text { with medical team (0- } \\
100)\end{array}$ & NRCS & Moderate & $3 \mathrm{mo}$ & AR with SIEA & NR & NR & NR & NR \\
\hline & $\begin{array}{l}\text { BREAST-Q: Satisfaction } \\
\text { with office staff }(0-100)\end{array}$ & NRCS & Moderate & $3 \mathrm{mo}$ & IBR (all) & NR & NR & NR & NR \\
\hline & $\begin{array}{l}\text { BREAST-Q: Satisfaction } \\
\text { with office staff }(0-100)\end{array}$ & NRCS & Moderate & $3 \mathrm{mo}$ & IBR Direct & 79 & $92.6(15.8)$ & NR & NR \\
\hline & $\begin{array}{l}\text { BREAST-Q: Satisfaction } \\
\text { with office staff }(0-100)\end{array}$ & NRCS & Moderate & $3 \mathrm{mo}$ & IBR TE & 942 & $96.6(10.4)$ & NR & NR \\
\hline & $\begin{array}{l}\text { BREAST-Q: Satisfaction } \\
\text { with office staff }(0-100)\end{array}$ & NRCS & Moderate & $3 \mathrm{mo}$ & AR (all) & NR & NR & NR & NR \\
\hline & $\begin{array}{l}\text { BREAST-Q: Satisfaction } \\
\text { with office staff }(0-100)\end{array}$ & NRCS & Moderate & $3 \mathrm{mo}$ & AR with DIEP & 395 & $93.8(13.4)$ & NR & NR \\
\hline & $\begin{array}{l}\text { BREAST-Q: Satisfaction } \\
\text { with office staff }(0-100)\end{array}$ & NRCS & Moderate & $3 \mathrm{mo}$ & AR with free TRAM & NR & NR & NR & NR \\
\hline & $\begin{array}{l}\text { BREAST-Q: Satisfaction } \\
\text { with office staff }(0-100)\end{array}$ & NRCS & Moderate & $3 \mathrm{mo}$ & $\begin{array}{l}\text { AR with pedicled } \\
\text { TRAM }\end{array}$ & 65 & $93.5(14.6)$ & NR & NR \\
\hline & $\begin{array}{l}\text { BREAST-Q: Satisfaction } \\
\text { with office staff }(0-100)\end{array}$ & NRCS & Moderate & $3 \mathrm{mo}$ & AR with LD & 53 & $94.7(14.6)$ & NR & NR \\
\hline & $\begin{array}{l}\text { BREAST-Q: Satisfaction } \\
\text { with office staff }(0-100)\end{array}$ & NRCS & Moderate & $3 \mathrm{mo}$ & AR with SIEA & NR & NR & NR & NR \\
\hline
\end{tabular}

Abbreviations: adj = adjusted, $\mathrm{CI}=$ confidence interval, $\mathrm{MD}=$ mean difference, mo $=$ months, $\mathrm{NR}=$ not reported, NRCS $=$ nonrandomized comparative study, $\mathrm{PMID}=\mathrm{Pubmed}$ identifier, $\mathrm{RCT}=$ randomized controlled trial, $\mathrm{Ref}=$ reference group, $\mathrm{RoB}=$ risk of bias, $\mathrm{SD}=$ standard deviation, $\mathrm{y}=\mathrm{years}$.

Colors: Header rows are shaded orange. Rows for every alternate study are shaded blue. The colors do not add unique information.

Table F-1.9. Full Evidence Table - Key Question 1: IBR versus AR - categorical outcomes (patient satisfaction with outcome)

\begin{tabular}{|l|l|l|l|l|l|l|l|l|l|}
\hline $\begin{array}{l}\text { Study, Year, } \\
\text { PMID, } \\
\text { Country }\end{array}$ & Outcome Measurement & Design & $\begin{array}{l}\text { Overall } \\
\text { RoB }\end{array}$ & $\begin{array}{l}\text { Time } \\
\text { Point }\end{array}$ & Arm & n/N (\%) & Comparison & $\begin{array}{l}\text { Adjusted Odds } \\
\text { Ratio (95\% Cl) }\end{array}$ & $\begin{array}{l}\text { P } \\
\text { Value }\end{array}$ \\
\hline & $\begin{array}{l}\text { Satisfied with surgical } \\
\text { outcome }\end{array}$ & NRCS & High & $2 \mathrm{mo}$ & IBR & NR & Ref & Ref \\
\hline
\end{tabular}




\begin{tabular}{|c|c|c|c|c|c|c|c|c|c|}
\hline $\begin{array}{l}\text { Study, Year, } \\
\text { PMID, } \\
\text { Country }\end{array}$ & Outcome Measurement & Design & $\begin{array}{l}\text { Overall } \\
\text { RoB }\end{array}$ & $\begin{array}{l}\text { Time } \\
\text { Point }\end{array}$ & Arm & $\mathrm{n} / \mathrm{N}(\%)$ & Comparison & $\begin{array}{l}\text { Adjusted Odds } \\
\text { Ratio }(95 \% \mathrm{CI})\end{array}$ & $\begin{array}{l}\mathbf{P} \\
\text { Value }\end{array}$ \\
\hline $\begin{array}{l}\text { Lei, 2020, } \\
32481367, \\
\text { China }\end{array}$ & $\begin{array}{l}\text { Satisfied with surgical } \\
\text { outcome }\end{array}$ & NRCS & High & $2 \mathrm{mo}$ & AR & NR & vs. IBR & $0.69(0.45,1.67)$ & 0.33 \\
\hline \multirow[t]{5}{*}{$\begin{array}{l}\text { Yueh, 2009, } \\
\text { 19228537, US }\end{array}$} & $\begin{array}{l}\text { Satisfied with surgical } \\
\text { outcome }\end{array}$ & NRCS & High & NR & IBR & $49 / 87(56.3)$ & Ref & Ref & Ref \\
\hline & $\begin{array}{l}\text { Satisfied with surgical } \\
\text { outcome }\end{array}$ & NRCS & High & NR & AR & NR/389 (NR) & vs. IBR & $1.83(1.11,3.03)$ & NR \\
\hline & $\begin{array}{l}\text { Satisfied with surgical } \\
\text { outcome }\end{array}$ & NRCS & High & NR & AR with DIEP & $\mathrm{NR} / 117$ (NR) & vs. AR with TRAM & $0.82(0.33,2.01)$ & NR \\
\hline & $\begin{array}{l}\text { Satisfied with surgical } \\
\text { outcome }\end{array}$ & NRCS & High & NR & AR with TRAM & $98 / 143(68.5)$ & vs. IBR & $2.05(1.13,3.72)$ & NR \\
\hline & $\begin{array}{l}\text { Satisfied with surgical } \\
\text { outcome }\end{array}$ & NRCS & High & NR & AR with LD & $63 / 112(56.3)$ & $\begin{array}{l}\text { vs. IBR } \\
\text { vs. AR with TRAM }\end{array}$ & $\begin{array}{l}1.12(0.64,2.12) \\
0.77(0.53,1.11)\end{array}$ & $\begin{array}{l}\text { NR } \\
\text { NR }\end{array}$ \\
\hline
\end{tabular}

Abbreviations: $\mathrm{AR}=$ autologous reconstruction, $\mathrm{CI}=$ confidence interval, $\mathrm{IBR}=$ implant-based reconstruction, $\mathrm{LD}=$ latissimus dorsi, $\mathrm{LTD}=$ lateral thoracodorsal flap, $\mathrm{PMID}=$

PubMed identifier, RCT $=$ randomized controlled trial, $\mathrm{Ref}=$ reference group, $\mathrm{RoB}=$ risk of bias, $\mathrm{TRAM}=$ transverse rectus abdominis myocutaneous.

Colors: Header rows are shaded orange. Rows for every alternate study are shaded blue. The colors do not add unique information.

Table F-1.10. Full Evidence Table - Key Question 1: IBR versus AR - categorical outcomes (recurrence of breast cancer)

\begin{tabular}{|c|c|c|c|c|c|c|c|c|}
\hline $\begin{array}{l}\text { Study, Year, } \\
\text { PMID, } \\
\text { Country }\end{array}$ & Outcome Measurement & Design & $\begin{array}{l}\text { Overall } \\
\text { RoB }\end{array}$ & $\begin{array}{l}\text { Time } \\
\text { Point }\end{array}$ & Arm & $\mathrm{n} / \mathrm{N}(\%)$ & Effect Size (95\% Cl) & $\begin{array}{l}\mathbf{P} \\
\text { Value }\end{array}$ \\
\hline \multirow{4}{*}{$\begin{array}{l}\mathrm{Ha}, 2020 \text {, } \\
32000718, \\
\text { South Korea }\end{array}$} & $\begin{array}{l}\text { Locoregional recurrence detected by biopsy or } \\
\text { imaging }\end{array}$ & NRCS & High & $4.8 \mathrm{y}$ & IBR & 9/247 (3.6) & Ref & Ref \\
\hline & $\begin{array}{l}\text { Locoregional recurrence detected by biopsy or } \\
\text { imaging }\end{array}$ & NRCS & High & $4.8 \mathrm{y}$ & $\mathrm{AR}$ & $11 / 249(4.4)$ & NR & 0.704 \\
\hline & Any relapse & NRCS & High & $4.8 \mathrm{y}$ & IBR & $14 / 247(5.7)$ & Ref & Ref \\
\hline & Any relapse & NRCS & High & $4.8 \mathrm{y}$ & AR & $24 / 249(9.6)$ & $\operatorname{adjHR} 3.39(1.23,9.32)$ & 0.018 \\
\hline \multirow{4}{*}{$\begin{array}{l}\text { Kouwenberg, } \\
2020 \text {, } \\
32590633 \text {, } \\
\text { Netherlands }\end{array}$} & Local recurrence & NRCS & Moderate & $>6 \mathrm{mo}$ & IBR & $13 / 296(4.5$ & Ref & Ref \\
\hline & Local recurrence & NRCS & Moderate & $>6 \mathrm{mo}$ & AR & $7 / 179(4.0)$ & $\operatorname{adjOR} 0.89(0.35,2.26)$ & 0.25 \\
\hline & Distant recurrence & NRCS & Moderate & $>6 \mathrm{mo}$ & IBR & $13 / 296(4.5)$ & Ref & Ref \\
\hline & Distant recurrence & NRCS & Moderate & $>6 \mathrm{mo}$ & AR & $8 / 179(4.6)$ & adjOR $1.02(0.41,2.51)$ & 0.97 \\
\hline \multirow{2}{*}{$\begin{array}{l}\text { Wu, 2021, } \\
33740204 \text {, } \\
\text { South Korea }\end{array}$} & NR & NRCS & High & $5.8 \mathrm{y}$ & IBR & $29 / 138(21.0)$ & Ref & Ref \\
\hline & NR & NRCS & High & $5.8 \mathrm{y}$ & AR & $64 / 276(23.2)$ & NR & 0.62 \\
\hline
\end{tabular}

Abbreviations: adj $=$ adjusted, $\mathrm{AR}=$ autologous reconstruction, $\mathrm{CI}=$ confidence interval, $\mathrm{HR}=$ hazard ratio, $\mathrm{IBR}=$ implant-based reconstruction, $\mathrm{N} / \mathrm{A}=$ not applicable, $\mathrm{OR}=$ odds ratio, PMID $=$ PubMed identifier, $\mathrm{RCT}=$ randomized controlled trial, Ref $=$ reference group, $\mathrm{RoB}=$ risk of bias, $\mathrm{PMID}=\mathrm{PubMed}$ identifier.

Colors: Header rows are shaded orange. Rows for every alternate study are shaded blue. The colors do not add unique information. 
Table F-1.11. Full Evidence Table - Key Question 1: IBR versus AR - continuous outcomes (harms, pain, and analgesic use)

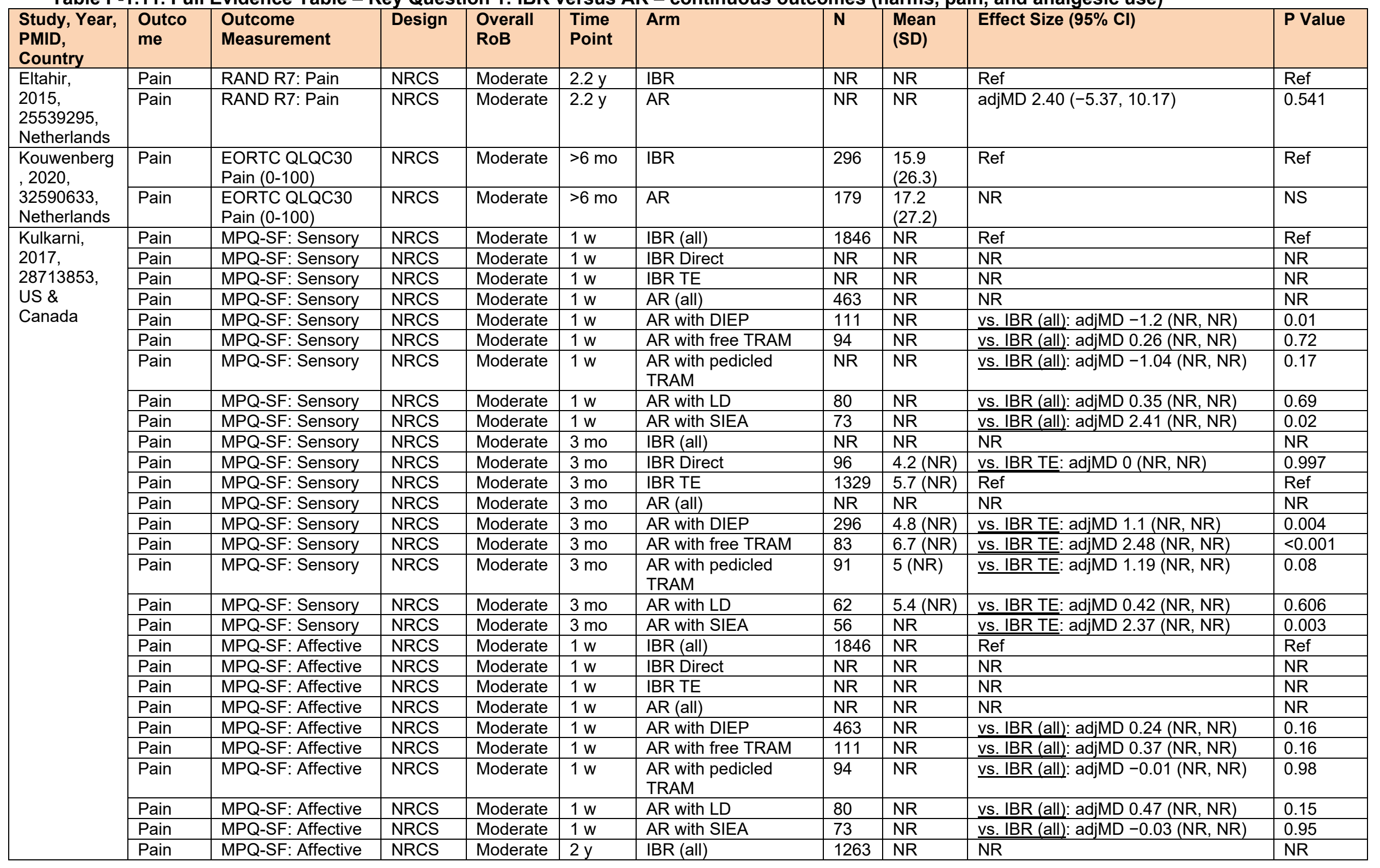




\begin{tabular}{|c|c|c|c|c|c|c|c|c|c|c|}
\hline $\begin{array}{l}\text { Study, Year, } \\
\text { PMID, } \\
\text { Country }\end{array}$ & $\begin{array}{l}\text { Outco } \\
\text { me }\end{array}$ & $\begin{array}{l}\text { Outcome } \\
\text { Measurement }\end{array}$ & Design & $\begin{array}{l}\text { Overall } \\
\text { RoB }\end{array}$ & $\begin{array}{l}\text { Time } \\
\text { Point }\end{array}$ & Arm & $\mathbf{N}$ & $\begin{array}{l}\text { Mean } \\
\text { (SD) }\end{array}$ & Effect Size (95\% CI) & P Value \\
\hline & Pain & MPQ-SF: Affective & NRCS & Moderate & $2 y$ & IBR Direct & 93 & NR & vs. IBR TE: adjMD 0.02 (NR, NR) & 0.919 \\
\hline & Pain & MPQ-SF: Affective & NRCS & Moderate & $2 y$ & IBR TE & 1263 & NR & Ref & Ref \\
\hline & Pain & MPQ-SF: Affective & NRCS & Moderate & $2 y$ & AR (all) & NR & NR & NR & NR \\
\hline & Pain & MPQ-SF: Affective & NRCS & Moderate & $2 y$ & AR with DIEP & 350 & NR & vs. IBR TE: adjMD 0.33 (NR, NR) & 0.013 \\
\hline & Pain & MPQ-SF: Affective & NRCS & Moderate & $2 y$ & AR with free TRAM & 87 & NR & vs. IBR TE: adjMD 0.84 (NR, NR) & $<0.001$ \\
\hline & Pain & MPQ-SF: Affective & NRCS & Moderate & $2 y$ & $\begin{array}{l}\text { AR with pedicled } \\
\text { TRAM }\end{array}$ & 77 & NR & vs. IBR TE: adjMD 0.04 (NR, NR) & 0.877 \\
\hline & Pain & MPQ-SF: Affective & NRCS & Moderate & $2 \mathrm{y}$ & AR with LD & 64 & NR & vs. IBR TE: adjMD -0.13 (NR, NR) & 0.63 \\
\hline & Pain & MPQ-SF: Affective & NRCS & Moderate & $2 y$ & AR with SIEA & 62 & NR & vs. IBR TE: adjMD 1.24 (NR, NR) & $<0.0001$ \\
\hline & Pain & NPRS & NRCS & Moderate & $1 \mathrm{w}$ & IBR (all) & 1846 & NR & Ref & Ref \\
\hline & Pain & NPRS & NRCS & Moderate & $1 \mathrm{w}$ & IBR Direct & NR & NR & NR & NR \\
\hline & Pain & NPRS & NRCS & Moderate & $1 \mathrm{w}$ & IBR TE & NR & NR & NR & NR \\
\hline & Pain & NPRS & NRCS & Moderate & $1 \mathrm{w}$ & AR (all) & NR & NR & NR & NR \\
\hline & Pain & NPRS & NRCS & Moderate & $1 \mathrm{w}$ & AR with DIEP & 463 & NR & vs. IBR (all): adjMD -0.18 (NR, NR) & 0.25 \\
\hline & Pain & NPRS & NRCS & Moderate & $1 \mathrm{w}$ & AR with free TRAM & 111 & NR & vs. IBR (all): adjMD -0.19 (NR, NR) & 0.45 \\
\hline & Pain & NPRS & NRCS & Moderate & $1 \mathrm{w}$ & $\begin{array}{l}\text { AR with pedicled } \\
\text { TRAM }\end{array}$ & 94 & NR & vs. IBR (all): adjMD -0.72 (NR, NR) & 0.01 \\
\hline & Pain & NPRS & NRCS & Moderate & $1 \mathrm{w}$ & AR with LD & 80 & NR & vs. IBR (all): adjMD 0.01 (NR, NR) & 0.97 \\
\hline & Pain & NPRS & NRCS & Moderate & $1 \mathrm{w}$ & AR with SIEA & 73 & NR & vs. IBR (all): adjMD 0.21 (NR, NR) & 0.51 \\
\hline & Pain & NPRS & NRCS & Moderate & $3 \mathrm{mo}$ & IBR (all) & NR & NR & NR & NR \\
\hline & Pain & NPRS & NRCS & Moderate & $3 \mathrm{mo}$ & IBR Direct & 96 & $1.3(\mathrm{NR})$ & NR & NR \\
\hline & Pain & NPRS & NRCS & Moderate & $3 \mathrm{mo}$ & IBR TE & 1329 & $2(\mathrm{NR})$ & NR & NR \\
\hline & Pain & NPRS & NRCS & Moderate & $3 \mathrm{mo}$ & AR (all) & NR & NR & NR & NR \\
\hline & Pain & NPRS & NRCS & Moderate & $3 \mathrm{mo}$ & AR with DIEP & 296 & $1.5(\mathrm{NR})$ & NR & NR \\
\hline & Pain & NPRS & NRCS & Moderate & $3 \mathrm{mo}$ & AR with free TRAM & 83 & $1.5(\mathrm{NR})$ & NR & NR \\
\hline & Pain & NPRS & NRCS & Moderate & $3 \mathrm{mo}$ & $\begin{array}{l}\text { AR with pedicled } \\
\text { TRAM }\end{array}$ & 91 & $1.8(\mathrm{NR})$ & NR & NR \\
\hline & Pain & NPRS & NRCS & Moderate & $3 \mathrm{mo}$ & AR with LD & 62 & $2(\mathrm{NR})$ & NR & NR \\
\hline & Pain & NPRS & NRCS & Moderate & $3 \mathrm{mo}$ & AR with SIEA & 56 & $1.4(\mathrm{NR})$ & NR & NR \\
\hline \multirow{8}{*}{$\begin{array}{l}\text { Roth, 2007, } \\
17413877, \\
\text { US }\end{array}$} & Pain & Bodily pain & NRCS & High & $2 y$ & IBR & 48 & $2.2(1.2)$ & Ref & Ref \\
\hline & Pain & Bodily pain & NRCS & High & $2 y$ & AR & 159 & $2.2(1.2)$ & NR & NS \\
\hline & Pain & Breast pain & NRCS & High & $2 y$ & IBR & 48 & $2.1(1.3)$ & Ref & Ref \\
\hline & Pain & Breast pain & NRCS & High & $2 y$ & AR & 159 & $1.8(1.1)$ & NR & NS \\
\hline & Pain & Abdominal pain & NRCS & High & $2 y$ & IBR & 48 & $4.8(0.8)$ & Ref & Ref \\
\hline & Pain & Abdominal pain & NRCS & High & $2 y$ & AR & 159 & $4(1.2)$ & NR & $<0.0001$ \\
\hline & Pain & Back pain & NRCS & High & $2 y$ & IBR & 48 & $4(1.3)$ & Ref & Ref \\
\hline & Pain & Back pain & NRCS & High & $2 y$ & $\mathrm{AR}$ & 159 & $3.7(1.5)$ & NR & NS \\
\hline $\begin{array}{l}\text { Shiraishi, } \\
2020 \text {, }\end{array}$ & Pain & $\begin{array}{l}\text { MPQ-SF: Total (0- } \\
\text { 10) }\end{array}$ & NRCS & High & $1 \mathrm{y}$ & IBR & 56 & NR & Ref & Ref \\
\hline
\end{tabular}




\begin{tabular}{|c|c|c|c|c|c|c|c|c|c|c|}
\hline $\begin{array}{l}\text { Study, Year, } \\
\text { PMID, } \\
\text { Country }\end{array}$ & $\begin{array}{l}\text { Outco } \\
\text { me }\end{array}$ & $\begin{array}{l}\text { Outcome } \\
\text { Measurement }\end{array}$ & Design & $\begin{array}{l}\text { Overall } \\
\text { RoB }\end{array}$ & $\begin{array}{l}\text { Time } \\
\text { Point }\end{array}$ & Arm & $\mathbf{N}$ & $\begin{array}{l}\text { Mean } \\
\text { (SD) }\end{array}$ & Effect Size $(95 \% \mathrm{Cl})$ & P Value \\
\hline \multirow[t]{5}{*}{$\begin{array}{l}32589082, \\
\text { Japan }\end{array}$} & Pain & $\begin{array}{l}\text { MPQ-SF: Total (0- } \\
\text { 10) }\end{array}$ & NRCS & High & $1 \mathrm{y}$ & AR & 34 & NR & $1.08(\mathrm{NR}, \mathrm{NR})$ & NR \\
\hline & Pain & $\begin{array}{l}\text { MPQ-SF: Sensory } \\
(0-10)\end{array}$ & NRCS & High & $1 \mathrm{y}$ & IBR & 56 & NR & Ref & Ref \\
\hline & Pain & $\begin{array}{l}\text { MPQ-SF: Sensory } \\
(0-10)\end{array}$ & NRCS & High & $1 \mathrm{y}$ & AR & 34 & NR & 0.80 (NR, NR) & NR \\
\hline & Pain & $\begin{array}{l}\text { MPQ-SF: Affective } \\
(0-10)\end{array}$ & NRCS & High & $1 \mathrm{y}$ & IBR & 56 & NR & Ref & Ref \\
\hline & Pain & $\begin{array}{l}\text { MPQ-SF: Affective } \\
(0-10)\end{array}$ & NRCS & High & $1 \mathrm{y}$ & AR & 34 & NR & $0.28(\mathrm{NR}, \mathrm{NR})$ & NR \\
\hline \multirow{2}{*}{$\begin{array}{l}\text { Shiraishi, } \\
2020, \\
32589082, \\
\text { Japan }\end{array}$} & $\begin{array}{l}\text { Analges } \\
\text { ic use }\end{array}$ & $\begin{array}{l}\text { Analgesic use } \\
\text { score }(0-5)\end{array}$ & NRCS & High & $1 \mathrm{y}$ & IBR & 56 & NR & Ref & Ref \\
\hline & $\begin{array}{l}\text { Analges } \\
\text { ic use }\end{array}$ & $\begin{array}{l}\text { Analgesic use } \\
\text { score }(0-5)\end{array}$ & NRCS & High & $1 \mathrm{y}$ & AR & 34 & NR & 0.37 (NR, NR) & NR \\
\hline
\end{tabular}

Abbreviations: adj = adjusted, CI = confidence interval, DIEP = deep inferior epigastric perforator, LD = latissimus dorsi, MPQ-SF = McGill Pain Questionnaire-Short Form, MD

$=$ mean difference, NPRS $=$ Numerical Pain Rating Scale, NR = not reported, NRCS $=$ nonrandomized comparative study, PMID $=$ Pubmed identifier, Ref $=$ reference group, RoB

$=$ risk of bias, $\mathrm{SD}=$ standard deviation, $\mathrm{SIEA}=$ superficial inferior epigastric artery, $\mathrm{TE}=$ tissue expander, TRAM $=$ transverse rectus abdominis myocutaneous.

Colors: Header rows are shaded orange. Rows for every alternate outcome are shaded blue. The colors do not add unique information.

Table F-1.12. Full Evidence Table - Key Question 1: IBR versus AR - categorical outcomes, mortality

\begin{tabular}{|c|c|c|c|c|c|c|c|c|}
\hline $\begin{array}{l}\text { Study, Year, PMID, } \\
\text { Country }\end{array}$ & $\begin{array}{l}\text { Outcome } \\
\text { Description }\end{array}$ & Design & $\begin{array}{l}\text { Overall } \\
\text { RoB }\end{array}$ & $\begin{array}{l}\text { Time } \\
\text { Point }\end{array}$ & IBR n/N (\%) & AR n/N (\%) & $\begin{array}{l}\text { Effect Size }(95 \% \mathrm{Cl}) \text { for } \\
\text { AR Versus IBR }\end{array}$ & P Value \\
\hline \multirow{2}{*}{$\begin{array}{l}\text { Jiang, 2013, 24349366, } \\
\text { US }\end{array}$} & Overall mortality & NRCS & High & $8.9 \mathrm{y}$ & $241 / 1412(17.1)$ & $503 / 2649(19.0)$ & adjOR $0.96(0.89,1.04)$ & NR \\
\hline & $\begin{array}{l}\text { Breast cancer- } \\
\text { specific mortality }\end{array}$ & NRCS & High & $8.9 \mathrm{y}$ & $209 / 1412(14.8)$ & $432 / 2649(16.3)$ & adjOR $0.95(0.87,1.04)$ & NR \\
\hline
\end{tabular}

Abbreviations: adj $=$ adjusted, $\mathrm{AR}=$ autologous reconstruction, $\mathrm{CI}=$ confidence interval, $\mathrm{IBR}=$ implant-based reconstruction, $\mathrm{N} / \mathrm{A}=$ not applicable, $\mathrm{NR}=$ not reported, $\mathrm{NRCS}=$ nonrandomized comparative study, $\mathrm{OR}=$ odds ratio, $\mathrm{PMID}=$ PubMed identifier, $\mathrm{RoB}=$ risk of bias, $\mathrm{y}=$ years

Colors: Header rows are shaded orange. The color does not add unique information.

Table F-1.13. Full Evidence Table - Key Question 1: IBR versus AR - categorical outcomes (harms - unplanned repeat hospitalizations)

\begin{tabular}{|c|c|c|c|c|c|c|c|c|}
\hline $\begin{array}{l}\text { Study, Year, } \\
\text { PMID, Country }\end{array}$ & Outcome Description & Design & Overall RoB & $\begin{array}{l}\text { Time } \\
\text { Point }\end{array}$ & IBR $\mathrm{n} / \mathrm{N}(\%)$ & AR n/N (\%) & $\begin{array}{l}\text { Effect Size }(95 \% \mathrm{CI}) \\
\text { for AR Versus IBR }\end{array}$ & $\begin{array}{l}\mathrm{P} \\
\text { Value } \\
\end{array}$ \\
\hline $\begin{array}{l}\text { Fischer, 2014, } \\
\text { 24916480, US }\end{array}$ & Any unplanned readmission & NRCS & High & $1 \mathrm{mo}$ & $7 / 155(4.5)$ & $10 / 155(6.5)$ & NR & NR \\
\hline $\begin{array}{l}\text { Merchant, 2015, } \\
26111325 \text {, US }\end{array}$ & Any unplanned readmission & NRCS & High & $1 \mathrm{mo}$ & $338 / 10437(3.24)$ & $95 / 2329(4.08)$ & adjOR $1.07(0.95,1.20)$ & NR \\
\hline
\end{tabular}




\begin{tabular}{|c|c|c|c|c|c|c|c|c|}
\hline $\begin{array}{l}\text { Study, Year, } \\
\text { PMID, Country }\end{array}$ & Outcome Description & Design & Overall RoB & $\begin{array}{l}\text { Time } \\
\text { Point }\end{array}$ & IBR n/N (\%) & AR n/N (\%) & $\begin{array}{l}\text { Effect Size }(95 \% \mathrm{CI}) \\
\text { for AR Versus IBR }\end{array}$ & $\begin{array}{l}\mathbf{P} \\
\text { Value }\end{array}$ \\
\hline $\begin{array}{l}\text { Mioton, 2013, } \\
23562485, \text { US }\end{array}$ & Any unplanned readmission & NRCS & Moderate & $1 \mathrm{mo}$ & $172 / 3960(4.34)$ & $56 / 1052(5.32)$ & NR & NS \\
\hline \multirow{2}{*}{$\begin{array}{l}\text { Nasser, } 2018 \text {, } \\
30204678\end{array}$} & Unplanned ED visits & NRCS & High & $1 \mathrm{mo}$ & NR/28124 (NR) & NR/4773 (NR) & adjOR $1.11(0.91,1.25)$ & 0.18 \\
\hline & $\begin{array}{l}\text { Unplanned ED visits with } \\
\text { pain-related diagnosis }\end{array}$ & NRCS & High & $1 \mathrm{mo}$ & NR/28124 (NR) & NR/4773 (NR) & adjOR $1.11(0.83,1.67)$ & 0.41 \\
\hline $\begin{array}{l}\text { Potter, 2019, } \\
\text { 30639093, UK }\end{array}$ & Any unplanned readmission & Single group & Moderate & $3 \mathrm{mo}$ & $372 / 2081(18)$ & $\mathrm{N} / \mathrm{A}$ & $\mathrm{N} / \mathrm{A}$ & $\mathrm{N} / \mathrm{A}$ \\
\hline $\begin{array}{l}\text { Sewart, 2021, } \\
\text { 33609398, UK }\end{array}$ & Any unplanned readmission & Single group & Low & $3 \mathrm{mo}$ & $147 / 891(16.5)$ & $\mathrm{N} / \mathrm{A}$ & $\mathrm{N} / \mathrm{A}$ & $\mathrm{N} / \mathrm{A}$ \\
\hline $\begin{array}{l}\text { Tran, 2018, } \\
30665841\end{array}$ & Any unplanned readmission & Single group & Low & NR & $35 / 879(2.8)$ & $\mathrm{N} / \mathrm{A}$ & $\mathrm{N} / \mathrm{A}$ & $\mathrm{N} / \mathrm{A}$ \\
\hline $\begin{array}{l}\text { Polanco, 2021, } \\
33745850, \text { US }\end{array}$ & Any unplanned readmission & Single group & Low & $1 \mathrm{mo}$ & $\mathrm{N} / \mathrm{A}$ & $52 / 777(6.7)$ & $\mathrm{N} / \mathrm{A}$ & $\mathrm{N} / \mathrm{A}$ \\
\hline
\end{tabular}

Abbreviations: adj $=$ adjusted, $\mathrm{AR}=$ autologous reconstruction, $\mathrm{CI}=$ confidence interval, $\mathrm{IBR}=$ implant-based reconstruction, $\mathrm{N} / \mathrm{A}=$ not applicable, $\mathrm{NR}=\mathrm{not}$ reported, $\mathrm{NRCS}=$

nonrandomized comparative study, $\mathrm{OR}=$ odds ratio, $\mathrm{PMID}=$ PubMed identifier, $\mathrm{RoB}=$ risk of bias

Colors: Header rows are shaded orange. Rows for every alternate study are shaded blue. The colors do not add unique information.

Table F-1.14. Full Evidence Table - Key Question 1: IBR versus AR - categorical outcomes (harms - unplanned repeat surgery for

revision of reconstruction)

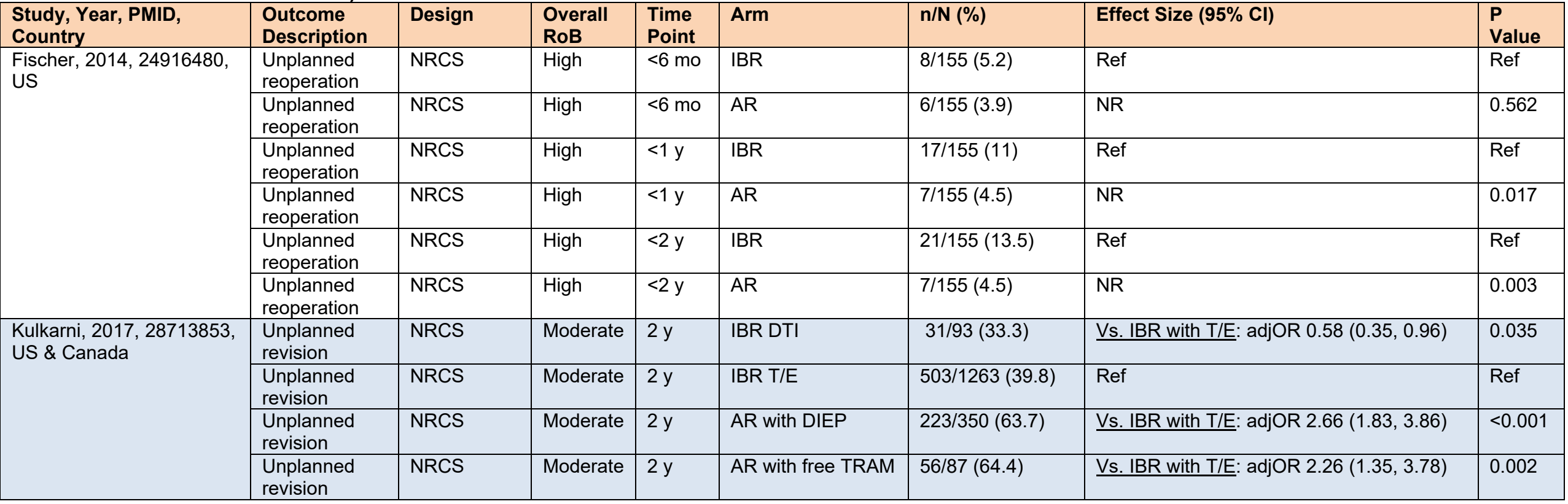




\begin{tabular}{|c|c|c|c|c|c|c|c|c|}
\hline $\begin{array}{l}\text { Study, Year, PMID, } \\
\text { Country }\end{array}$ & $\begin{array}{l}\text { Outcome } \\
\text { Description }\end{array}$ & Design & $\begin{array}{l}\text { Overall } \\
\text { RoB }\end{array}$ & $\begin{array}{l}\text { Time } \\
\text { Point }\end{array}$ & Arm & $n / N(\%)$ & Effect Size $(95 \% \mathrm{Cl})$ & $\begin{array}{l}\mathbf{P} \\
\text { Value }\end{array}$ \\
\hline & $\begin{array}{l}\text { Unplanned } \\
\text { revision }\end{array}$ & NRCS & Moderate & $2 y$ & $\begin{array}{l}\text { AR with pedicled } \\
\text { TRAM }\end{array}$ & $40 / 77(57.1)$ & Vs. IBR with T/E: adjOR $1.34(0.75,2.4)$ & 0.33 \\
\hline & $\begin{array}{l}\text { Unplanned } \\
\text { revision }\end{array}$ & NRCS & Moderate & $2 y$ & AR with LD & $41 / 64(64.1)$ & Vs. IBR with T/E: adjOR $1.97(1.07,3.64)$ & 0.031 \\
\hline & $\begin{array}{l}\text { Unplanned } \\
\text { revision }\end{array}$ & NRCS & Moderate & $2 y$ & AR with SIEA & $33 / 62(53.2)$ & Vs. IBR with T/E: adjOR $1.83(0.93,3.6)$ & 0.079 \\
\hline \multirow{2}{*}{$\begin{array}{l}\text { Zhang, 2019, 30675702, } \\
\text { China }\end{array}$} & Reoperation & NRCS & High & $4.9 \mathrm{y}$ & IBR & $230 / 394(58.4)$ & adjOR $0.72(0.5,1.06)$ & 0.093 \\
\hline & Reoperation & NRCS & High & $4.9 \mathrm{y}$ & AR & $154 / 438(35.2)$ & Ref & Ref \\
\hline $\begin{array}{l}\text { Coroneos, } 2019 \text {, } \\
\text { 30222598, US }\end{array}$ & Reoperation & Single group & Low & $3 y$ & IBR & $1026 / 5031(20.4)$ & $\mathrm{N} / \mathrm{A}$ & $\mathrm{N} / \mathrm{A}$ \\
\hline $\begin{array}{l}\text { Park, 2019, } 30863940, \\
\text { South Korea }\end{array}$ & Reoperation & Single group & Moderate & $6 \mathrm{mo}$ & IBR & $87 / 999(8.7)$ & $\mathrm{N} / \mathrm{A}$ & $\mathrm{N} / \mathrm{A}$ \\
\hline $\begin{array}{l}\text { Rogoff, 2020, 32243320, } \\
\text { US }\end{array}$ & Reoperation & Single group & Low & NR & IBR & $4 / 627(0.6)$ & $\mathrm{N} / \mathrm{A}$ & \\
\hline $\begin{array}{l}\text { Albornoz, 2013, } \\
23897346, \text { US }\end{array}$ & Flap revision & Single group & Low & NR & AR & $210 / 21016(1.0)$ & $\mathrm{N} / \mathrm{A}$ & $\mathrm{N} / \mathrm{A}$ \\
\hline $\begin{array}{l}\text { Beugels, 2018, 29399731, } \\
\text { Netherlands }\end{array}$ & $\begin{array}{l}\text { Flap re- } \\
\text { exploration }\end{array}$ & Single group & Low & $\begin{array}{l}9-10 \\
\text { mo }\end{array}$ & AR & 67/910 flaps (7.4) & $\mathrm{N} / \mathrm{A}$ & $\mathrm{N} / \mathrm{A}$ \\
\hline $\begin{array}{l}\text { Fitzgerald, 2016, } \\
27047776, \text { UK }\end{array}$ & Reoperation & Single group & Low & NR & AR & $135 / 1064(12.7)$ & N/A & N/A \\
\hline Gill, 2004, 15083015, US & Reoperation & Single group & Moderate & $10 y$ & AR & $45 / 758(5.9)$ & N/A & N/A \\
\hline $\begin{array}{l}\text { Selber, 2009, 19935283, } \\
\text { US }\end{array}$ & Revision & Single group & Low & $5.6 \mathrm{mo}$ & $\mathrm{AR}$ & $197 / 1031(19.1)$ & $\mathrm{N} / \mathrm{A}$ & $\mathrm{N} / \mathrm{A}$ \\
\hline Tran, 2018, 29794694, US & $\begin{array}{l}\text { Unplanned } \\
\text { reoperation }\end{array}$ & Single group & Low & NR & AR & $44 / 879(5.0)$ & $\mathrm{N} / \mathrm{A}$ & $\mathrm{N} / \mathrm{A}$ \\
\hline
\end{tabular}

Abbreviations: adj $=$ adjusted, $\mathrm{AR}=$ autologous reconstruction, $\mathrm{CI}=$ confidence interval, $\mathrm{IBR}=$ implant-based reconstruction, $\mathrm{mo}=\mathrm{months}, \mathrm{N} / \mathrm{A}=\mathrm{not}$ applicable, $\mathrm{NR}=$ not reported, $\mathrm{NRCS}=$ nonrandomized comparative study, $\mathrm{OR}=$ odds ratio, $\mathrm{PMID}=$ PubMed identifier, $\mathrm{Ref}=$ reference group, $\mathrm{RoB}=$ risk of bias, $\mathrm{y}=\mathrm{years}$.

Colors: Header rows are shaded orange. Rows for every alternate study are shaded blue. The colors do not add unique information.

* Secondary breast procedures: grouped into 4 broad categories: procedures for new or ongoing disease (mastectomy, lumpectomy, or biopsy); balancing procedures (mastopexy and reduction mammoplasty); planned secondary procedures (nipple-areolar reconstruction and exchange of tissue expander (TE) for permanent implant); and unplanned revisions (breast implant removal, revision, or exchange; TE removal without replacement; reconstruction with different modality; and revision of the reconstruction breast without further specification).

Table F-1.15. Full Evidence Table - Key Question 1: IBR versus AR - categorical outcomes (harms - unplanned repeat surgery for complications)

\begin{tabular}{|c|c|c|c|c|c|c|c|c|}
\hline $\begin{array}{l}\text { Study, Year, PMID, } \\
\text { Country }\end{array}$ & Outcome Description & Design & $\begin{array}{l}\text { Overall } \\
\text { RoB }\end{array}$ & $\begin{array}{l}\text { Time } \\
\text { Point }\end{array}$ & Arm & $\mathrm{n} / \mathrm{N}(\%)$ & Effect Size $(95 \% \mathrm{Cl})$ & $\begin{array}{l}\mathbf{P} \\
\text { Value }\end{array}$ \\
\hline \multirow{2}{*}{$\begin{array}{l}\text { Hangge, 2013, } \\
31606126, \text { US }\end{array}$} & NR & NRCS & High & NR & IBR DTI & $81 / 193(42)$ & vs. AR: adjOR $2.03(1.03,3.98)$ & 0.042 \\
\hline & NR & NRCS & High & NR & IBR T/E & $58 / 146(40)$ & vs. AR: adjOR $1.81(0.90,3.64)$ & 0.096 \\
\hline
\end{tabular}




\begin{tabular}{|c|c|c|c|c|c|c|c|c|}
\hline $\begin{array}{l}\text { Study, Year, PMID, } \\
\text { Country }\end{array}$ & Outcome Description & Design & $\begin{array}{l}\text { Overall } \\
\text { RoB }\end{array}$ & $\begin{array}{l}\text { Time } \\
\text { Point }\end{array}$ & Arm & $n / N(\%)$ & Effect Size $(95 \% \mathrm{CI})$ & $\begin{array}{l}\mathbf{P} \\
\text { Value }\end{array}$ \\
\hline & NR & NRCS & High & NR & AR & $17 / 60(28)$ & Ref & Ref \\
\hline \multirow{2}{*}{$\begin{array}{l}\text { Mioton, 2013, 23562485, } \\
\text { US }\end{array}$} & NR & NRCS & Moderate & $1 \mathrm{mo}$ & IBR & $662 / 9786(6.76)$ & $1.08(0.88,1.32)$ & NR \\
\hline & NR & NRCS & Moderate & $1 \mathrm{mo}$ & AR & $316 / 3296(9.59)$ & Ref & Ref \\
\hline \multirow[t]{2}{*}{$\begin{array}{l}\text { Zhang, 2011, 30675702, } \\
\text { China }\end{array}$} & $\begin{array}{l}\text { Urgent surgery for a } \\
\text { compromised implant/flap }\end{array}$ & NRCS & High & $4.9 \mathrm{y}$ & IBR & $31 / 394(7.9)$ & $0.63(0.29,1.37)$ & NR \\
\hline & $\begin{array}{l}\text { Urgent surgery for a } \\
\text { compromised implant/flap }\end{array}$ & NRCS & High & $4.9 \mathrm{y}$ & AR & $33 / 438(7.5)$ & Ref & Ref \\
\hline $\begin{array}{l}\text { Acosta, 2019, } \\
21046538, \text { Sweden }\end{array}$ & Wound revision & Single group & High & $9 y$ & IBR & $62 / 543(11.4)$ & $\mathrm{N} / \mathrm{A}$ & $\mathrm{N} / \mathrm{A}$ \\
\hline $\begin{array}{l}\text { Hamdi, 2011, 20576480, } \\
\text { Belgium }\end{array}$ & $\begin{array}{l}\text { Reoperation with autologous } \\
\text { tissue after failure of IBR }\end{array}$ & Single group & Low & $2.6 \mathrm{y}$ & IBR & $54 / 688(7.8)$ & $\mathrm{N} / \mathrm{A}$ & $\mathrm{N} / \mathrm{A}$ \\
\hline $\begin{array}{l}\text { Sewart, 2021, } \\
\text { 33609398, UK }\end{array}$ & Any & Single group & Low & $3 \mathrm{mo}$ & IBR & $150 / 891(16.8)$ & $\mathrm{N} / \mathrm{A}$ & $\mathrm{N} / \mathrm{A}$ \\
\hline $\begin{array}{l}\text { Hamdi, 2010, 20679823, } \\
\text { US }\end{array}$ & $\begin{array}{l}\text { Reoperation after failure of free } \\
\text { flap breast reconstruction }\end{array}$ & Single group & Low & $3.1 \mathrm{y}$ & AR & $14 / 688(2.0)$ & N/A & $\mathrm{N} / \mathrm{A}$ \\
\hline O'Neill, 2006, 31196805 & $\begin{array}{l}\text { Intraoperative complications } \\
\text { requiring revision of/additional } \\
\text { anastomoses }\end{array}$ & Single group & Low & $3 \mathrm{mo}$ & AR & $56 / 912(6.1)$ & $\mathrm{N} / \mathrm{A}$ & $\mathrm{N} / \mathrm{A}$ \\
\hline $\begin{array}{l}\text { Park, 2019, 30863940, } \\
\text { South Korea }\end{array}$ & Any & Single group & Moderate & $>6 \mathrm{mo}$ & AR & $62 / 999(6.2)$ & $\mathrm{N} / \mathrm{A}$ & $\mathrm{N} / \mathrm{A}$ \\
\hline $\begin{array}{l}\text { Polanco, 2021, } \\
\text { 33745850, US }\end{array}$ & Any & Single group & Low & $1 \mathrm{mo}$ & AR & $79 / 777(10.2)$ & $\mathrm{N} / \mathrm{A}$ & $\mathrm{N} / \mathrm{A}$ \\
\hline $\begin{array}{l}\text { Potter, 2019, 30639093, } \\
\text { UK }\end{array}$ & NR & Single group & Moderate & $3 \mathrm{mo}$ & AR & $370 / 2081(18.0)$ & $\mathrm{N} / \mathrm{A}$ & $\mathrm{N} / \mathrm{A}$ \\
\hline
\end{tabular}

Abbreviations: adj $=$ adjusted, $\mathrm{AR}=$ autologous reconstruction, $\mathrm{CI}=$ confidence interval, $\mathrm{IBR}=$ implant-based reconstruction, $\mathrm{mo}=$ months, $\mathrm{N} / \mathrm{A}=$ not applicable, $\mathrm{NR}=$ not reported, NRCS = nonrandomized comparative study, $\mathrm{OR}=$ odds ratio, $\mathrm{PMID}=$ PubMed identifier, $\mathrm{Ref}=$ reference group, $\mathrm{RoB}=$ risk of bias, $\mathrm{y}=$ years.

Colors: Header rows are shaded orange. Rows for every alternate study are shaded blue. The colors do not add unique information.

Table F-1.16. Full Evidence Table - Key Question 1: IBR versus AR - categorical outcomes (harms - pain, including chronic pain)

\begin{tabular}{|c|c|c|c|c|c|c|c|c|c|}
\hline $\begin{array}{l}\text { Study, Year, } \\
\text { PMID, } \\
\text { Country }\end{array}$ & $\begin{array}{l}\text { Outcome } \\
\text { Description }\end{array}$ & Design & $\begin{array}{l}\text { Overall } \\
\text { RoB }\end{array}$ & $\begin{array}{l}\text { Time } \\
\text { Point }\end{array}$ & Arm & Subgroup & $\mathrm{n} / \mathrm{N}(\%)$ & Effect Size $(95 \% \mathrm{Cl})$ & $\begin{array}{l}\mathrm{P} \\
\text { Value }\end{array}$ \\
\hline \multirow[t]{3}{*}{$\begin{array}{l}\text { Kulkarni, 2017, } \\
\text { 28713853, US } \\
\text { \& Canada }\end{array}$} & $\begin{array}{l}\text { NPRS: } \\
\text { Moderate to } \\
\text { severe }\end{array}$ & NRCS & Moderate & $2 y$ & IBR DTI & All & NR & vs. IBR with T/E: adjOR 0.54 (NR, NR) & 0.292 \\
\hline & $\begin{array}{l}\text { NPRS: } \\
\text { Moderate to } \\
\text { severe }\end{array}$ & NRCS & Moderate & $2 y$ & IBR T/E & All & NR & Ref & Ref \\
\hline & $\begin{array}{l}\text { NPRS: } \\
\text { Moderate to } \\
\text { severe }\end{array}$ & NRCS & Moderate & $2 y$ & AR with DIEP & All & NR & vs. IBR with T/E: adjOR 1.22 (NR, NR) & 0.454 \\
\hline
\end{tabular}




\begin{tabular}{|c|c|c|c|c|c|c|c|c|c|}
\hline $\begin{array}{l}\text { Study, Year, } \\
\text { PMID, } \\
\text { Country }\end{array}$ & $\begin{array}{l}\text { Outcome } \\
\text { Description }\end{array}$ & Design & $\begin{array}{l}\text { Overall } \\
\text { RoB }\end{array}$ & $\begin{array}{l}\text { Time } \\
\text { Point }\end{array}$ & Arm & Subgroup & $\mathrm{n} / \mathrm{N}(\%)$ & Effect Size (95\% Cl) & $\begin{array}{l}\mathbf{P} \\
\text { Value }\end{array}$ \\
\hline & $\begin{array}{l}\text { NPRS: } \\
\text { Moderate to } \\
\text { severe }\end{array}$ & NRCS & Moderate & $2 y$ & AR with free TRAM & All & NR & vs. IBR with T/E: adjOR 1.73 (NR, NR) & 0.205 \\
\hline & $\begin{array}{l}\text { NPRS: } \\
\text { Moderate to } \\
\text { severe }\end{array}$ & NRCS & Moderate & $2 \mathrm{y}$ & $\begin{array}{l}\text { AR with pedicled } \\
\text { TRAM }\end{array}$ & All & NR & vs. IBR with T/E: adjOR 1.64 (NR, NR) & 0.266 \\
\hline & $\begin{array}{l}\text { NPRS: } \\
\text { Moderate to } \\
\text { severe }\end{array}$ & NRCS & Moderate & $2 \mathrm{y}$ & AR with LD & All & NR & vs. IBR with T/E: adjOR 0.94 (NR, NR) & 0.915 \\
\hline & $\begin{array}{l}\text { NPRS: } \\
\text { Moderate to } \\
\text { severe }\end{array}$ & NRCS & Moderate & $2 y$ & AR with SIEA & All & NR & vs. IBR with T/E: adjOR 1.43 (NR, NR) & 0.445 \\
\hline $\begin{array}{l}\text { Cordeiro, } \\
\text { 2015b, } \\
26090764 \text {, US }\end{array}$ & Breast pain & $\begin{array}{l}\text { Single } \\
\text { group }\end{array}$ & Low & $2 y$ & IBR & All & $\begin{array}{l}\text { 88/4912 implants } \\
(1.79)\end{array}$ & N/A & N/A \\
\hline \multirow[t]{3}{*}{$\begin{array}{l}\text { Huo, 2016, } \\
27697676, \text { US }\end{array}$} & Breast pain & $\begin{array}{l}\text { Single } \\
\text { group }\end{array}$ & Low & $1 \mathrm{y}$ & IBR & All & $68 / 1332(5.1)$ & $\mathrm{N} / \mathrm{A}$ & $\mathrm{N} / \mathrm{A}$ \\
\hline & Breast pain & $\begin{array}{l}\text { Single } \\
\text { group }\end{array}$ & Low & $1 \mathrm{y}$ & IBR & Obese & $22 / 383(5.8)$ & $\mathrm{N} / \mathrm{A}$ & $\mathrm{N} / \mathrm{A}$ \\
\hline & Breast pain & $\begin{array}{l}\text { Single } \\
\text { group }\end{array}$ & Low & $1 \mathrm{y}$ & IBR & Nonobese & $46 / 949(4.8)$ & $\mathrm{N} / \mathrm{A}$ & $\mathrm{N} / \mathrm{A}$ \\
\hline $\begin{array}{l}\text { Seth, 2015, } \\
25180955, \text { US }\end{array}$ & $\begin{array}{l}\text { Breast pain or } \\
\text { tightness }\end{array}$ & $\begin{array}{l}\text { Single } \\
\text { group }\end{array}$ & Low & NR & IBR & All & $42 / 893(4.7)$ & $\mathrm{N} / \mathrm{A}$ & $\mathrm{N} / \mathrm{A}$ \\
\hline
\end{tabular}

Abbreviations: adj = adjusted, $\mathrm{AR}=$ autologous reconstruction, $\mathrm{CI}=$ confidence interval, DIEP $=$ deep inferior epigastric perforator, DTI $=$ direct to implant, IBR $=$ implant-based reconstruction, $\mathrm{LD}=$ latissimus dorsi, N/A = not applicable, NPRS $=$ Numerical Pain Rating Scale, NR $=$ not reported, NRCS $=$ nonrandomized comparative study, OR $=$ odds ratio, PMID $=$ PubMed identifier, Ref $=$ reference group, $\mathrm{RoB}=$ risk of bias, $\mathrm{SIEA}=$ superficial inferior epigastric artery perforator, $\mathrm{T} / \mathrm{E}=$ tissue expander, $\mathrm{TRAM}=$ transverse rectus abdominis myocutaneous.

Colors: Header rows are shaded orange. Rows for every alternate study are shaded blue. The colors do not add unique information.

Table F-1.17. Full Evidence Table - Key Question 1: IBR versus AR - categorical outcomes (harms - necrosis)

\begin{tabular}{|c|c|c|c|c|c|c|c|c|c|}
\hline $\begin{array}{l}\text { Study, Year, } \\
\text { PMID, Country }\end{array}$ & $\begin{array}{l}\text { Outcome } \\
\text { Description }\end{array}$ & Design & $\begin{array}{l}\text { Overall } \\
\text { RoB }\end{array}$ & $\begin{array}{l}\text { Time } \\
\text { Point }\end{array}$ & Arm & Subgroup & $\mathrm{n} / \mathrm{N}(\%)$ & Effect Size (95\% CI) & $\begin{array}{l}P \\
\text { Value }\end{array}$ \\
\hline \multirow{2}{*}{$\begin{array}{l}\text { Abedi, } 2016, \\
25003437, \\
\text { Canada }\end{array}$} & $\begin{array}{l}\text { Mastectomy flap } \\
\text { necrosis }\end{array}$ & NRCS & High & $\begin{array}{l}1.6-1.9 \\
y\end{array}$ & IBR & All & $70 / 606(11.6)$ & adjOR $0.66(0.38,1.16)$ & 0.15 \\
\hline & $\begin{array}{l}\text { Mastectomy flap } \\
\text { necrosis }\end{array}$ & NRCS & High & $\begin{array}{l}1.6-1.9 \\
y\end{array}$ & AR & All & $60 / 395$ (15.2) & Ref & Ref \\
\hline \multirow{2}{*}{$\begin{array}{l}\text { Carramaschi, } \\
\text { 1989, 2602589, } \\
\text { France }\end{array}$} & $\begin{array}{l}\text { More than local } \\
\text { necrosis }\end{array}$ & NRCS & High & NR & IBR & All & $5 / 166(3)$ & NR & NR \\
\hline & $\begin{array}{l}\text { More than local } \\
\text { necrosis }\end{array}$ & NRCS & High & NR & AR (all) & All & $1 / 74(1.4)$ & NR & NR \\
\hline
\end{tabular}




\begin{tabular}{|c|c|c|c|c|c|c|c|c|c|}
\hline $\begin{array}{l}\text { Study, Year, } \\
\text { PMID, Country }\end{array}$ & $\begin{array}{l}\text { Outcome } \\
\text { Description }\end{array}$ & Design & $\begin{array}{l}\text { Overall } \\
\text { RoB }\end{array}$ & $\begin{array}{l}\text { Time } \\
\text { Point }\end{array}$ & Arm & Subgroup & $\mathrm{n} / \mathrm{N}(\%)$ & Effect Size $(95 \% \mathrm{Cl})$ & $\begin{array}{l}\text { P } \\
\text { Value }\end{array}$ \\
\hline & $\begin{array}{l}\text { More than local } \\
\text { necrosis }\end{array}$ & NRCS & High & NR & AR with TRAM & All & $0 / 40(0)$ & NR & NR \\
\hline & $\begin{array}{l}\text { More than local } \\
\text { necrosis }\end{array}$ & NRCS & High & NR & AR with LD & All & $1 / 34(2.9)$ & NR & NR \\
\hline & Local necrosis & NRCS & High & NR & IBR & All & NR & NR & NR \\
\hline & Local necrosis & NRCS & High & NR & AR (all) & All & $7 / 74(9.5)$ & NR & NR \\
\hline & Local necrosis & NRCS & High & NR & AR with TRAM & All & $5 / 40(12.5)$ & NR & NR \\
\hline & Local necrosis & NRCS & High & NR & AR with LD & All & $2 / 34(5.9)$ & NR & NR \\
\hline \multirow[t]{2}{*}{$\begin{array}{l}\text { de Araujo, 2016, } \\
27673527, \text { US }\end{array}$} & Major skin necrosis & NRCS & High & $4.3 \mathrm{y}$ & IBR & All & $\mathrm{NR} / 38(\mathrm{NR})$ & $\begin{array}{l}\text { adjOR 17.894 (0.524, } \\
610.484)\end{array}$ & 0.1092 \\
\hline & Major skin necrosis & NRCS & High & $4.3 \mathrm{y}$ & AR & All & NR/32 (NR) & Ref & Ref \\
\hline \multirow{4}{*}{$\begin{array}{l}\text { Garvey, 2012, } \\
23096600, \text { US }\end{array}$} & Fat necrosis & NRCS & Moderate & $1.5 \mathrm{y}$ & IBR & All & NR & NR & NR \\
\hline & Fat necrosis & NRCS & Moderate & $1.5 \mathrm{y}$ & AR & All & $38 / 548$ breasts $(6.9)$ & NR & NR \\
\hline & Partial flap necrosis & NRCS & Moderate & $1.5 \mathrm{y}$ & IBR & All & NR & NR & NR \\
\hline & Partial flap necrosis & NRCS & Moderate & $1.5 \mathrm{y}$ & AR & All & $21 / 548$ breasts (3.8) & NR & NR \\
\hline \multirow{4}{*}{$\begin{array}{l}\text { Naoum, 2020a, } \\
31756414 \text {, US }\end{array}$} & Skin necrosis & NRCS & High & $4-10 y$ & IBR & All & 26/633 breasts (4.1) & Ref & Ref \\
\hline & Skin necrosis & NRCS & High & $4-10 y$ & AR & All & $16 / 342$ breasts (4.7) & adjOR $0.83(0.19,3.5)$ & 0.8 \\
\hline & Fat necrosis & NRCS & High & $4-10 y$ & IBR & All & $\begin{array}{l}1 \text { breast/633 breasts } \\
(0.2)\end{array}$ & Ref & Ref \\
\hline & Fat necrosis & NRCS & High & $4-10 y$ & AR & All & $24 / 342$ breasts $(7)$ & adjOR $21.2(2.5,174.46)$ & 0.004 \\
\hline \multirow[t]{2}{*}{$\begin{array}{l}\text { Nelson, 2019, } \\
31356276, \text { US }\end{array}$} & $\begin{array}{l}\text { Mastectomy flap } \\
\text { necrosis }\end{array}$ & NRCS & High & $<3 \mathrm{mo}$ & IBR & All & $\begin{array}{l}\text { 65/1211 breasts } \\
(13.6)\end{array}$ & NR & NR \\
\hline & $\begin{array}{l}\text { Mastectomy flap } \\
\text { necrosis }\end{array}$ & NRCS & High & $<3 \mathrm{mo}$ & AR & All & NR & NR & NR \\
\hline \multirow{2}{*}{$\begin{array}{l}\text { Woo, 2018, } \\
30360958, \\
\text { South Korea }\end{array}$} & Skin flap necrosis & NRCS & High & NR & IBR & All & $14 / 60(23.3)$ & Ref & Ref \\
\hline & Skin flap necrosis & NRCS & High & NR & AR & All & $7 / 70(10)$ & adjOR $0.66(0.38,1.16)$ & 0.38 \\
\hline \multirow[t]{2}{*}{$\begin{array}{l}\text { Banuelos, 2020, } \\
\text { 31663932, US }\end{array}$} & $\begin{array}{l}\text { Mastectomy flap } \\
\text { necrosis }\end{array}$ & $\begin{array}{l}\text { Single } \\
\text { group }\end{array}$ & Low & $\leq 1 \mathrm{mo}$ & IBR & All & $15 / 768(1.8)$ & $\mathrm{N} / \mathrm{A}$ & $\mathrm{N} / \mathrm{A}$ \\
\hline & $\begin{array}{l}\text { Mastectomy flap } \\
\text { necrosis }\end{array}$ & $\begin{array}{l}\text { Single } \\
\text { group }\end{array}$ & Low & $>1 \mathrm{mo}$ & IBR & All & $4 / 768(0.5)$ & $\mathrm{N} / \mathrm{A}$ & $\mathrm{N} / \mathrm{A}$ \\
\hline \multirow[t]{3}{*}{$\begin{array}{l}\text { Brooks, 2012, } \\
22098451, \text { US }\end{array}$} & NR & $\begin{array}{l}\text { Single } \\
\text { group }\end{array}$ & Low & $3.4 \mathrm{mo}$ & IBR & All & $15 / 733(2.0)$ & $\mathrm{N} / \mathrm{A}$ & $\mathrm{N} / \mathrm{A}$ \\
\hline & NR & $\begin{array}{l}\text { Single } \\
\text { group }\end{array}$ & Low & $3.4 \mathrm{mo}$ & IBR & No radiation & $14 / 636(2.2)$ & $\mathrm{N} / \mathrm{A}$ & N/A \\
\hline & NR & $\begin{array}{l}\text { Single } \\
\text { group }\end{array}$ & Low & $3.4 \mathrm{mo}$ & IBR & Radiation & $1 / 97(1.0)$ & $\mathrm{N} / \mathrm{A}$ & $\mathrm{N} / \mathrm{A}$ \\
\hline \multirow[t]{2}{*}{$\begin{array}{l}\text { Cordeiro, 2006, } \\
\text { 16980842, US }\end{array}$} & $\begin{array}{l}\text { Native skin flap } \\
\text { necrosis }\end{array}$ & $\begin{array}{l}\text { Single } \\
\text { group }\end{array}$ & Moderate & NR & IBR & All & $\begin{array}{l}\text { 45/2276 procedures } \\
(2.0)\end{array}$ & $\mathrm{N} / \mathrm{A}$ & N/A \\
\hline & $\begin{array}{l}\text { Native skin flap } \\
\text { necrosis }\end{array}$ & $\begin{array}{l}\text { Single } \\
\text { group }\end{array}$ & Moderate & NR & IBR & $\begin{array}{l}\text { T/E placement } \\
\text { procedure }\end{array}$ & $\begin{array}{l}\text { 44/1221 procedures } \\
(3.6)\end{array}$ & $\mathrm{N} / \mathrm{A}$ & N/A \\
\hline
\end{tabular}




\begin{tabular}{|c|c|c|c|c|c|c|c|c|c|}
\hline $\begin{array}{l}\text { Study, Year, } \\
\text { PMID, Country }\end{array}$ & $\begin{array}{l}\text { Outcome } \\
\text { Description }\end{array}$ & Design & $\begin{array}{l}\text { Overall } \\
\text { RoB }\end{array}$ & $\begin{array}{l}\text { Time } \\
\text { Point }\end{array}$ & Arm & Subgroup & n/N (\%) & Effect Size $(95 \% \mathrm{Cl})$ & $\begin{array}{l}\mathbf{P} \\
\text { Value }\end{array}$ \\
\hline & $\begin{array}{l}\text { Native skin flap } \\
\text { necrosis }\end{array}$ & $\begin{array}{l}\text { Single } \\
\text { group }\end{array}$ & Moderate & NR & IBR & $\begin{array}{l}\text { Exchange } \\
\text { procedure }\end{array}$ & $\begin{array}{l}\text { 1/1055 procedures } \\
(0.1)\end{array}$ & $\mathrm{N} / \mathrm{A}$ & N/A \\
\hline & $\begin{array}{l}\text { Native skin flap } \\
\text { necrosis }\end{array}$ & $\begin{array}{l}\text { Single } \\
\text { group }\end{array}$ & Moderate & NR & IBR & Prior radiation & $\begin{array}{l}5 / 136 \text { procedures } \\
(3.7)\end{array}$ & $\mathrm{N} / \mathrm{A}$ & N/A \\
\hline & $\begin{array}{l}\text { Native skin flap } \\
\text { necrosis }\end{array}$ & $\begin{array}{l}\text { Single } \\
\text { group }\end{array}$ & Moderate & NR & IBR & $\begin{array}{l}\text { No prior } \\
\text { radiation }\end{array}$ & $\begin{array}{l}40 / 2140 \text { procedures } \\
(1.9)\end{array}$ & $\mathrm{N} / \mathrm{A}$ & N/A \\
\hline & $\begin{array}{l}\text { Native skin flap } \\
\text { necrosis }\end{array}$ & $\begin{array}{l}\text { Single } \\
\text { group }\end{array}$ & Moderate & NR & IBR & Immediate & $\begin{array}{l}\text { 44/1176 procedures } \\
(3.7)\end{array}$ & $\mathrm{N} / \mathrm{A}$ & $\mathrm{N} / \mathrm{A}$ \\
\hline & $\begin{array}{l}\text { Native skin flap } \\
\text { necrosis }\end{array}$ & $\begin{array}{l}\text { Single } \\
\text { group }\end{array}$ & Moderate & NR & IBR & Delayed & $0 / 26$ procedures $(0)$ & $\mathrm{N} / \mathrm{A}$ & N/A \\
\hline \multirow[t]{3}{*}{$\begin{array}{l}\text { Cordeiro, 2012, } \\
22286416, \text { US }\end{array}$} & Skin flap necrosis & $\begin{array}{l}\text { Single } \\
\text { group }\end{array}$ & Low & $<1 \mathrm{y}$ & IBR & All & $144 / 1699(8.5)$ & $\mathrm{N} / \mathrm{A}$ & $\mathrm{N} / \mathrm{A}$ \\
\hline & Skin flap necrosis & $\begin{array}{l}\text { Single } \\
\text { group }\end{array}$ & Low & $<1 \mathrm{y}$ & IBR & Prior radiation & $22 / 121(18.2)$ & $\mathrm{N} / \mathrm{A}$ & $\mathrm{N} / \mathrm{A}$ \\
\hline & Skin flap necrosis & $\begin{array}{l}\text { Single } \\
\text { group }\end{array}$ & Low & $<1 \mathrm{y}$ & IBR & $\begin{array}{l}\text { No prior } \\
\text { radiation }\end{array}$ & $122 / 1578(7.7)$ & $\mathrm{N} / \mathrm{A}$ & N/A \\
\hline $\begin{array}{l}\text { Gfrerer, 2015, } \\
25626807, \text { US }\end{array}$ & Skin necrosis & $\begin{array}{l}\text { Single } \\
\text { group }\end{array}$ & Low & $7 y$ & IBR & All & $97 / 3142(3.1)$ & $\mathrm{N} / \mathrm{A}$ & $\mathrm{N} / \mathrm{A}$ \\
\hline \multirow[t]{2}{*}{$\begin{array}{l}\text { Hansen, 2018, } \\
\text { 29778821, US }\end{array}$} & NR & $\begin{array}{l}\text { Single } \\
\text { group }\end{array}$ & Low & $1 \mathrm{mo}$ & IBR & All & $14 / 930(1.5)$ & $\mathrm{N} / \mathrm{A}$ & $\mathrm{N} / \mathrm{A}$ \\
\hline & NR & $\begin{array}{l}\text { Single } \\
\text { group }\end{array}$ & Low & $1 \mathrm{y}$ & IBR & All & $115 / 930(12.4)$ & $\mathrm{N} / \mathrm{A}$ & $\mathrm{N} / \mathrm{A}$ \\
\hline $\begin{array}{l}\text { Hunsicker, } \\
2017, \\
26849284 \text {, US }\end{array}$ & $\begin{array}{l}\text { Skin } \\
\text { necrosis/breakdown }\end{array}$ & $\begin{array}{l}\text { Single } \\
\text { group }\end{array}$ & Low & $<1 \mathrm{y}$ & IBR & All & 94/1584 breasts (5.9) & $\mathrm{N} / \mathrm{A}$ & $\mathrm{N} / \mathrm{A}$ \\
\hline \multirow[t]{6}{*}{$\begin{array}{l}\text { Huo, 2016, } \\
27697676, \text { US }\end{array}$} & Fat necrosis & $\begin{array}{l}\text { Single } \\
\text { group }\end{array}$ & Low & $1 \mathrm{y}$ & IBR & All & $66 / 1332(5.0)$ & $\mathrm{N} / \mathrm{A}$ & $\mathrm{N} / \mathrm{A}$ \\
\hline & Fat necrosis & $\begin{array}{l}\text { Single } \\
\text { group }\end{array}$ & Low & $1 \mathrm{y}$ & IBR & Non-obese & $39 / 949(4.1)$ & $\mathrm{N} / \mathrm{A}$ & $\mathrm{N} / \mathrm{A}$ \\
\hline & Fat necrosis & $\begin{array}{l}\text { Single } \\
\text { group }\end{array}$ & Low & $1 \mathrm{y}$ & IBR & Obese & $27 / 383(7.1)$ & $\mathrm{N} / \mathrm{A}$ & $\mathrm{N} / \mathrm{A}$ \\
\hline & Skin flap necrosis & $\begin{array}{l}\text { Single } \\
\text { group }\end{array}$ & Low & $1 \mathrm{y}$ & IBR & All & $13 / 1332(0.1)$ & $\mathrm{N} / \mathrm{A}$ & N/A \\
\hline & Skin flap necrosis & $\begin{array}{l}\text { Single } \\
\text { group }\end{array}$ & Low & $1 \mathrm{y}$ & IBR & Non-obese & $9 / 949(1.0)$ & $\mathrm{N} / \mathrm{A}$ & $\mathrm{N} / \mathrm{A}$ \\
\hline & Skin flap necrosis & $\begin{array}{l}\text { Single } \\
\text { group }\end{array}$ & Low & $1 \mathrm{y}$ & IBR & Obese & $4 / 383(1.0)$ & $\mathrm{N} / \mathrm{A}$ & $\mathrm{N} / \mathrm{A}$ \\
\hline $\begin{array}{l}\text { Kanuri, 2014, } \\
24675199, \text { US }\end{array}$ & $\begin{array}{l}\text { Mastectomy skin } \\
\text { flap necrosis } \\
\text { requiring excision } \\
\text { and reclosure }\end{array}$ & $\begin{array}{l}\text { Single } \\
\text { group }\end{array}$ & Low & NR & IBR & All & $79 / 710$ breasts (11.1) & $\mathrm{N} / \mathrm{A}$ & N/A \\
\hline
\end{tabular}




\begin{tabular}{|c|c|c|c|c|c|c|c|c|c|}
\hline $\begin{array}{l}\text { Study, Year, } \\
\text { PMID, Country }\end{array}$ & $\begin{array}{l}\text { Outcome } \\
\text { Description }\end{array}$ & Design & $\begin{array}{l}\text { Overall } \\
\text { RoB }\end{array}$ & $\begin{array}{l}\text { Time } \\
\text { Point }\end{array}$ & Arm & Subgroup & $\mathrm{n} / \mathrm{N}(\%)$ & Effect Size (95\% Cl) & $\begin{array}{l}P \\
\text { Value }\end{array}$ \\
\hline $\begin{array}{l}\text { Lovecchio, } \\
2015, \\
24691330, \text { US }\end{array}$ & $\begin{array}{l}\text { Necrosis or } \\
\text { dehiscence }\end{array}$ & $\begin{array}{l}\text { Single } \\
\text { group }\end{array}$ & Low & $2.3 \mathrm{y}$ & IBR & All & $159 / 1639(9.7)$ & $\mathrm{N} / \mathrm{A}$ & $\mathrm{N} / \mathrm{A}$ \\
\hline $\begin{array}{l}\text { Parikh, 2018, } \\
30204676, \text { US }\end{array}$ & $\begin{array}{l}\text { Mastectomy flap } \\
\text { necrosis }\end{array}$ & $\begin{array}{l}\text { Single } \\
\text { group }\end{array}$ & Low & $>2 y$ & IBR & All & $20 / 1285(1.6)$ & $\mathrm{N} / \mathrm{A}$ & $\mathrm{N} / \mathrm{A}$ \\
\hline $\begin{array}{l}\text { Park, 2019, } \\
30863940, \\
\text { South Korea }\end{array}$ & $\begin{array}{l}\text { Mastectomy flap } \\
\text { necrosis }\end{array}$ & $\begin{array}{l}\text { Single } \\
\text { group }\end{array}$ & Moderate & $>6 \mathrm{mo}$ & IBR & All & $94 / 999(9.4)$ & $\mathrm{N} / \mathrm{A}$ & $\mathrm{N} / \mathrm{A}$ \\
\hline $\begin{array}{l}\text { Rogoff, 2020, } \\
32243320, \text { US }\end{array}$ & $\begin{array}{l}\text { Mastectomy flap } \\
\text { necrosis }\end{array}$ & $\begin{array}{l}\text { Single } \\
\text { group }\end{array}$ & Low & NR & IBR & All & $77 / 627(12.3)$ & $\mathrm{N} / \mathrm{A}$ & \\
\hline $\begin{array}{l}\text { Seth, 2015, } \\
\text { 25180955, US }\end{array}$ & $\begin{array}{l}\text { Mastectomy flap } \\
\text { necrosis: requiring } \\
\text { surgical excision } \\
\text { with or without } \\
\text { closure in the office } \\
\text { or in the operating } \\
\text { room }\end{array}$ & $\begin{array}{l}\text { Single } \\
\text { group }\end{array}$ & Low & NR & IBR & All & $96 / 893(10.8)$ & $\mathrm{N} / \mathrm{A}$ & $\mathrm{N} / \mathrm{A}$ \\
\hline \multirow[t]{3}{*}{$\begin{array}{l}\text { Singh, 2012, } \\
22342636, \text { US }\end{array}$} & Breast necrosis & $\begin{array}{l}\text { Single } \\
\text { group }\end{array}$ & Low & $6 \mathrm{mo}$ & IBR & All & $41 / 1316(3.1)$ & $\mathrm{N} / \mathrm{A}$ & $\mathrm{N} / \mathrm{A}$ \\
\hline & Breast necrosis & $\begin{array}{l}\text { Single } \\
\text { group }\end{array}$ & Low & $6 \mathrm{mo}$ & IBR & DTI & $1 / 95(1.1)$ & $\mathrm{N} / \mathrm{A}$ & $\mathrm{N} / \mathrm{A}$ \\
\hline & Breast necrosis & $\begin{array}{l}\text { Single } \\
\text { group }\end{array}$ & Low & $6 \mathrm{mo}$ & IBR & $\mathrm{T} / \mathrm{E}$ & $40 / 1221(3.3)$ & $\mathrm{N} / \mathrm{A}$ & $\mathrm{N} / \mathrm{A}$ \\
\hline \multirow[t]{3}{*}{$\begin{array}{l}\text { Singh, 2021, } \\
\text { 33564597, US }\end{array}$} & NR & $\begin{array}{l}\text { Single } \\
\text { group }\end{array}$ & Low & $3 \mathrm{mo}$ & IBR & All & $56 / 1740(3.23)$ & $\mathrm{N} / \mathrm{A}$ & $\mathrm{N} / \mathrm{A}$ \\
\hline & NR & $\begin{array}{l}\text { Single } \\
\text { group }\end{array}$ & Low & $3 \mathrm{mo}$ & IBR & DTI & $23 / 870(2.67)$ & $\mathrm{N} / \mathrm{A}$ & $\mathrm{N} / \mathrm{A}$ \\
\hline & NR & $\begin{array}{l}\text { Single } \\
\text { group }\end{array}$ & Low & $3 \mathrm{mo}$ & IBR & $\mathrm{T} / \mathrm{E}$ & $33 / 870(3.8)$ & $\mathrm{N} / \mathrm{A}$ & $\mathrm{N} / \mathrm{A}$ \\
\hline \multirow{2}{*}{$\begin{array}{l}\text { Acosta, 2011, } \\
\text { 21046538, } \\
\text { Sweden }\end{array}$} & Partial necrosis & $\begin{array}{l}\text { Single } \\
\text { group }\end{array}$ & High & $9 y$ & AR & All & $11 / 543(1.9)$ & $\mathrm{N} / \mathrm{A}$ & $\mathrm{N} / \mathrm{A}$ \\
\hline & Complete necrosis & $\begin{array}{l}\text { Single } \\
\text { group }\end{array}$ & High & $9 y$ & AR & All & $14 / 543(2.6)$ & $\mathrm{N} / \mathrm{A}$ & $\mathrm{N} / \mathrm{A}$ \\
\hline $\begin{array}{l}\text { Andree, 2012, } \\
\text { 23197233, } \\
\text { Germany }\end{array}$ & Margin necrosis & $\begin{array}{l}\text { Single } \\
\text { group }\end{array}$ & Low & $1 \mathrm{y}$ & $\mathrm{AR}$ & All & $27 / 1068$ flaps (2.5) & $\mathrm{N} / \mathrm{A}$ & $\mathrm{N} / \mathrm{A}$ \\
\hline $\begin{array}{l}\text { Beugels, 2018, } \\
29399731, \\
\text { Netherlands }\end{array}$ & Fat necrosis & $\begin{array}{l}\text { Single } \\
\text { group }\end{array}$ & Low & $\begin{array}{l}9-10 \\
\text { mo }\end{array}$ & AR & All & 101/910 flaps (11.1) & $\mathrm{N} / \mathrm{A}$ & $\mathrm{N} / \mathrm{A}$ \\
\hline $\begin{array}{l}\text { Chang, 2000, } \\
\text { 10809092, US }\end{array}$ & Flap fat necrosis & $\begin{array}{l}\text { Single } \\
\text { group }\end{array}$ & Low & NR & AR & All & 55/936 flaps (5.9) & $\mathrm{N} / \mathrm{A}$ & $\mathrm{N} / \mathrm{A}$ \\
\hline
\end{tabular}




\begin{tabular}{|c|c|c|c|c|c|c|c|c|c|}
\hline $\begin{array}{l}\text { Study, Year, } \\
\text { PMID, Country }\end{array}$ & $\begin{array}{l}\text { Outcome } \\
\text { Description }\end{array}$ & Design & $\begin{array}{l}\text { Overall } \\
\text { RoB }\end{array}$ & $\begin{array}{l}\text { Time } \\
\text { Point }\end{array}$ & Arm & Subgroup & $n / N(\%)$ & Effect Size (95\% CI) & $\begin{array}{l}P \\
\text { Value }\end{array}$ \\
\hline & $\begin{array}{l}\text { Mastectomy flap } \\
\text { necrosis }\end{array}$ & $\begin{array}{l}\text { Single } \\
\text { group }\end{array}$ & Low & NR & AR & All & 99/936 flaps (10.3) & $\mathrm{N} / \mathrm{A}$ & N/A \\
\hline \multirow[t]{5}{*}{$\begin{array}{l}\text { Chang, 2011, } \\
21407063, \text { US }\end{array}$} & Fat necrosis & $\begin{array}{l}\text { Single } \\
\text { group }\end{array}$ & Low & NR & AR & All & $135 / 818(16.5)$ & $\mathrm{N} / \mathrm{A}$ & N/A \\
\hline & Fat necrosis & $\begin{array}{l}\text { Single } \\
\text { group }\end{array}$ & Low & NR & AR & Age $<50$ & $71 / 411(17.3)$ & $\mathrm{N} / \mathrm{A}$ & N/A \\
\hline & Fat necrosis & $\begin{array}{l}\text { Single } \\
\text { group }\end{array}$ & Low & NR & AR & Age 50-59 & $43 / 285(15.1)$ & $\mathrm{N} / \mathrm{A}$ & $\mathrm{N} / \mathrm{A}$ \\
\hline & Fat necrosis & $\begin{array}{l}\text { Single } \\
\text { group }\end{array}$ & Low & NR & AR & Age 60-69 & $16 / 103(15.5)$ & $\mathrm{N} / \mathrm{A}$ & N/A \\
\hline & Fat necrosis & $\begin{array}{l}\text { Single } \\
\text { group }\end{array}$ & Low & NR & AR & Age $>70$ & $5 / 19(26.3)$ & $\mathrm{N} / \mathrm{A}$ & $\mathrm{N} / \mathrm{A}$ \\
\hline \multirow[t]{2}{*}{$\begin{array}{l}\text { Daly, 2020, } \\
\text { 31994156, US }\end{array}$} & Fat necrosis $\geq 2 \mathrm{~cm}$ & $\begin{array}{l}\text { Single } \\
\text { group }\end{array}$ & Low & $12 \mathrm{y}$ & AR & All & 172/1187 flaps (14.5) & $\mathrm{N} / \mathrm{A}$ & $\mathrm{N} / \mathrm{A}$ \\
\hline & Umbilical necrosis & $\begin{array}{l}\text { Single } \\
\text { group }\end{array}$ & Low & $12 \mathrm{y}$ & AR & All & $29 / 818(3.5)$ & $\mathrm{N} / \mathrm{A}$ & $\mathrm{N} / \mathrm{A}$ \\
\hline $\begin{array}{l}\text { Enajat, 2010, } \\
\text { 19790180, } \\
\text { Sweden }\end{array}$ & Fat necrosis & $\begin{array}{l}\text { Single } \\
\text { group }\end{array}$ & Low & $1-7 d$ & AR & All & 56/564 flaps (10.0) & $\mathrm{N} / \mathrm{A}$ & N/A \\
\hline $\begin{array}{l}\text { Gill, 2004, } \\
\text { 15083015, US }\end{array}$ & Fat necrosis & $\begin{array}{l}\text { Single } \\
\text { group }\end{array}$ & Moderate & $10 y$ & AR & All & $98 / 758(12.9)$ & $\mathrm{N} / \mathrm{A}$ & $\mathrm{N} / \mathrm{A}$ \\
\hline $\begin{array}{l}\text { Haddock, } 2020 \text {, } \\
33487570, \text { US }\end{array}$ & Fat necrosis & $\begin{array}{l}\text { Single } \\
\text { group }\end{array}$ & Low & $1.6 \mathrm{y}$ & AR & All & $66 / 506(13)$ & $\mathrm{N} / \mathrm{A}$ & $\mathrm{N} / \mathrm{A}$ \\
\hline $\begin{array}{l}\text { Jo, 2020, } \\
33386262, \\
\text { South Korea }\end{array}$ & $\begin{array}{l}\text { Mastectomy flap } \\
\text { necrosis }\end{array}$ & $\begin{array}{l}\text { Single } \\
\text { group }\end{array}$ & Low & $\begin{array}{l}11 \mathrm{mo} \\
-1.5 \mathrm{y}\end{array}$ & AR & All & $89 / 823(14.3)$ & $\mathrm{N} / \mathrm{A}$ & N/A \\
\hline $\begin{array}{l}\text { Langer, 2010, } \\
\text { 20980954, } \\
\text { Germany }\end{array}$ & $\begin{array}{l}\text { Fat necrosis but } \\
\text { intact skin island }\end{array}$ & $\begin{array}{l}\text { Single } \\
\text { group }\end{array}$ & Low & $5 y$ & AR & All & 2/670 flaps (0.3) & $\mathrm{N} / \mathrm{A}$ & N/A \\
\hline $\begin{array}{l}\text { Lantieri, 2015, } \\
26238173, \\
\text { France }\end{array}$ & $\begin{array}{l}\text { Partial necrosis of } \\
\text { flap with delayed } \\
\text { healing }\end{array}$ & $\begin{array}{l}\text { Single } \\
\text { group }\end{array}$ & Moderate & NR & AR & All & $52 / 952(5.5)$ & $\mathrm{N} / \mathrm{A}$ & $\mathrm{N} / \mathrm{A}$ \\
\hline $\begin{array}{l}\text { Masoomi, 2019, } \\
31331721 \text {, US }\end{array}$ & Fat necrosis & $\begin{array}{l}\text { Single } \\
\text { group }\end{array}$ & Low & NR & AR & All & $490 / 55840(0.9)$ & $\mathrm{N} / \mathrm{A}$ & $\mathrm{N} / \mathrm{A}$ \\
\hline $\begin{array}{l}\text { Mehrara, 2006, } \\
17016173, \text { US }\end{array}$ & Fat necrosis & $\begin{array}{l}\text { Single } \\
\text { group }\end{array}$ & Moderate & $1 \mathrm{mo}$ & AR & All & $134 / 1195$ (11.2) & $\mathrm{N} / \mathrm{A}$ & $\mathrm{N} / \mathrm{A}$ \\
\hline \multirow{2}{*}{$\begin{array}{l}\text { Munder, 2020, } \\
32565553 \text {, } \\
\text { Germany }\end{array}$} & AR flap necrosis & $\begin{array}{l}\text { Single } \\
\text { group }\end{array}$ & Low & $2 y$ & AR & All & 32/1274 flaps (2.5) & $\mathrm{N} / \mathrm{A}$ & $\mathrm{N} / \mathrm{A}$ \\
\hline & Fat necrosis & $\begin{array}{l}\text { Single } \\
\text { group }\end{array}$ & Low & $2 y$ & AR & All & 47/1274 flaps (3.6) & $\mathrm{N} / \mathrm{A}$ & $\mathrm{N} / \mathrm{A}$ \\
\hline $\begin{array}{l}\text { Nelson, 2014, } \\
25046665, \text { US }\end{array}$ & Breast fat necrosis & $\begin{array}{l}\text { Single } \\
\text { group }\end{array}$ & Moderate & NR & AR & All & 71/1293 flaps (5.5) & $\mathrm{N} / \mathrm{A}$ & $\mathrm{N} / \mathrm{A}$ \\
\hline
\end{tabular}




\begin{tabular}{|c|c|c|c|c|c|c|c|c|c|}
\hline $\begin{array}{l}\text { Study, Year, } \\
\text { PMID, Country }\end{array}$ & $\begin{array}{l}\text { Outcome } \\
\text { Description }\end{array}$ & Design & $\begin{array}{l}\text { Overall } \\
\text { RoB }\end{array}$ & $\begin{array}{l}\text { Time } \\
\text { Point }\end{array}$ & Arm & Subgroup & $n / N(\%)$ & Effect Size $(95 \% \mathrm{Cl})$ & $\begin{array}{l}\mathbf{P} \\
\text { Value }\end{array}$ \\
\hline & $\begin{array}{l}\text { Donor site fat } \\
\text { necrosis }\end{array}$ & $\begin{array}{l}\text { Single } \\
\text { group }\end{array}$ & Moderate & NR & AR & All & 3/1293 flaps (0.2) & $\mathrm{N} / \mathrm{A}$ & N/A \\
\hline & $\begin{array}{l}\text { Breast skin flap } \\
\text { necrosis }\end{array}$ & $\begin{array}{l}\text { Single } \\
\text { group }\end{array}$ & Moderate & NR & AR & All & 194/1293 flaps (15.0) & $\mathrm{N} / \mathrm{A}$ & N/A \\
\hline & $\begin{array}{l}\text { Donor site skin flap } \\
\text { necrosis }\end{array}$ & $\begin{array}{l}\text { Single } \\
\text { group }\end{array}$ & Moderate & NR & AR & All & $6 / 1293$ flaps $(0.5)$ & $\mathrm{N} / \mathrm{A}$ & N/A \\
\hline \multirow{2}{*}{$\begin{array}{l}\text { 'Neill, 2019, } \\
31196805, \\
\text { Canada }\end{array}$} & Flap necrosis & $\begin{array}{l}\text { Single } \\
\text { group }\end{array}$ & Low & $<3 \mathrm{mo}$ & AR & All & $50 / 912(5.5)$ & N/A & N/A \\
\hline & Fat necrosis & $\begin{array}{l}\text { Single } \\
\text { group }\end{array}$ & Low & $<3 \mathrm{mo}$ & AR & All & $17 / 912(1.3)$ & N/A & N/A \\
\hline $\begin{array}{l}\text { Rubio, 2019, } \\
30665841, \\
\text { Belgium }\end{array}$ & Fat necrosis & $\begin{array}{l}\text { Single } \\
\text { group }\end{array}$ & Low & NR & AR & All & $509 / 56522(0.9)$ & N/A & N/A \\
\hline \multirow[t]{2}{*}{$\begin{array}{l}\text { Selber, 2009, } \\
\text { 19935283, US }\end{array}$} & Fat necrosis & $\begin{array}{l}\text { Single } \\
\text { group }\end{array}$ & Low & $5.6 \mathrm{mo}$ & AR & All & $35 / 1031(3.4)$ & $\mathrm{N} / \mathrm{A}$ & N/A \\
\hline & $\begin{array}{l}\text { Mastectomy flap } \\
\text { necrosis }\end{array}$ & $\begin{array}{l}\text { Single } \\
\text { group }\end{array}$ & Low & $5.6 \mathrm{mo}$ & AR & All & 4/1031 (0.4) & N/A & N/A \\
\hline $\begin{array}{l}\text { Shaikh, 2010, } \\
\text { 22693373, India }\end{array}$ & $\begin{array}{l}\text { Superficial skin } \\
\text { necrosis }\end{array}$ & $\begin{array}{l}\text { Single } \\
\text { group }\end{array}$ & Low & NR & AR & All & $20 / 546(3.7)$ & $\mathrm{N} / \mathrm{A}$ & $\mathrm{N} / \mathrm{A}$ \\
\hline \multirow[t]{2}{*}{$\begin{array}{l}\text { Song, 2016, } \\
26637165 \text {, US }\end{array}$} & Fat necrosis & $\begin{array}{l}\text { Single } \\
\text { group }\end{array}$ & Low & $\begin{array}{l}4.3-5.4 \\
y\end{array}$ & AR & All & $353 / 1809$ (19.5) & N/A & N/A \\
\hline & $\begin{array}{l}\text { Mastectomy flap } \\
\text { necrosis requiring } \\
\text { surgery }\end{array}$ & $\begin{array}{l}\text { Single } \\
\text { group }\end{array}$ & Low & $\begin{array}{l}4.3-5.4 \\
y\end{array}$ & AR & All & $165 / 1809(9.1)$ & $\mathrm{N} / \mathrm{A}$ & N/A \\
\hline \multirow[t]{2}{*}{$\begin{array}{l}\text { Tran, 2018, } \\
\text { 29794694, US }\end{array}$} & Fat necrosis & $\begin{array}{l}\text { Single } \\
\text { group }\end{array}$ & Low & NR & AR & All & 174/1253 flaps (13.9) & $\mathrm{N} / \mathrm{A}$ & N/A \\
\hline & Umbilical necrosis & $\begin{array}{l}\text { Single } \\
\text { group }\end{array}$ & Low & NR & AR & All & $29 / 879(3.3)$ & N/A & N/A \\
\hline $\begin{array}{l}\text { Watterson, } \\
\text { 1995, } 7761505, \\
\text { Australia }\end{array}$ & Fat necrosis & $\begin{array}{l}\text { Single } \\
\text { group }\end{array}$ & Moderate & $2.7 y$ & AR & All & $59 / 556(10.6)$ & $\mathrm{N} / \mathrm{A}$ & N/A \\
\hline \multirow[t]{2}{*}{$\begin{array}{l}\text { Williams, } 1995, \\
7794079, \text { US }\end{array}$} & Fat necrosis & $\begin{array}{l}\text { Single } \\
\text { group }\end{array}$ & Moderate & $2.4 y$ & AR & All & $76 / 607$ (12.5) & $\mathrm{N} / \mathrm{A}$ & N/A \\
\hline & $\begin{array}{l}\text { Full thickness skin } \\
\text { loss }\end{array}$ & $\begin{array}{l}\text { Single } \\
\text { group }\end{array}$ & Moderate & $2.4 \mathrm{y}$ & $\mathrm{AR}$ & All & $8 / 607(6.3)$ & $\mathrm{N} / \mathrm{A}$ & N/A \\
\hline \multirow{3}{*}{$\begin{array}{l}\text { Yoo, 2014, } \\
\text { 24852813, } \\
\text { South Korea }\end{array}$} & Breast skin necrosis & $\begin{array}{l}\text { Single } \\
\text { group }\end{array}$ & Moderate & NR & AR & All & $75 / 500(15)$ & N/A & N/A \\
\hline & $\begin{array}{l}\text { Donor site skin } \\
\text { necrosis }\end{array}$ & $\begin{array}{l}\text { Single } \\
\text { group }\end{array}$ & Moderate & NR & AR & All & $16 / 500(3.2)$ & N/A & N/A \\
\hline & Breast fat necrosis & $\begin{array}{l}\text { Single } \\
\text { group }\end{array}$ & Moderate & NR & AR & All & $71 / 500(14.2)$ & $\mathrm{N} / \mathrm{A}$ & N/A \\
\hline
\end{tabular}




\begin{tabular}{|c|c|c|c|c|c|c|c|c|c|}
\hline $\begin{array}{l}\text { Study, Year, } \\
\text { PMID, Country }\end{array}$ & $\begin{array}{l}\text { Outcome } \\
\text { Description }\end{array}$ & Design & $\begin{array}{l}\text { Overall } \\
\text { RoB }\end{array}$ & $\begin{array}{l}\text { Time } \\
\text { Point }\end{array}$ & Arm & Subgroup & $n / N(\%)$ & Effect Size (95\% Cl) & $\begin{array}{l}\mathbf{P} \\
\text { Value }\end{array}$ \\
\hline & $\begin{array}{l}\text { Donor site fat } \\
\text { necrosis }\end{array}$ & $\begin{array}{l}\text { Single } \\
\text { group }\end{array}$ & Moderate & NR & AR & All & $4 / 500(0.8)$ & $\mathrm{N} / \mathrm{A}$ & $\mathrm{N} / \mathrm{A}$ \\
\hline
\end{tabular}

Abbreviations: adj = adjusted, $\mathrm{AR}=$ autologous reconstruction, $\mathrm{CI}=$ confidence interval, $\mathrm{DIEP}=$ deep inferior epigastric perforator, IBR $=$ implant-based reconstruction, $\mathrm{LD}=$

latissimus dorsi, $\mathrm{NR}=$ not reported, $\mathrm{NRCS}=$ nonrandomized comparative study, $\mathrm{OR}=$ odds ratio, $\mathrm{PMID}=\mathrm{PubMed}$ identifier, $\mathrm{Ref}=$ reference group, RoB $=$ risk of bias, $\mathrm{y}=\mathrm{years}$.

Colors: Header rows are shaded orange. Rows for every alternate study are shaded blue. The colors do not add unique information.

Table F-1.18. Full Evidence Table - Key Question 1: IBR versus AR - categorical outcomes (harms - thromboembolic events)

\begin{tabular}{|c|c|c|c|c|c|c|c|c|c|}
\hline $\begin{array}{l}\text { Study, Year, PMID, } \\
\text { Country }\end{array}$ & $\begin{array}{l}\text { Outcome } \\
\text { Description }\end{array}$ & Design & $\begin{array}{l}\text { Overall } \\
\text { RoB }\end{array}$ & $\begin{array}{l}\text { Time } \\
\text { Point }\end{array}$ & Arm & Subgroup & $\mathrm{n} / \mathrm{N}(\%)$ & Effect Size $(95 \% \mathrm{Cl})$ & P Value \\
\hline \multirow{4}{*}{$\begin{array}{l}\text { Brorson 2020a, } \\
32807615, \text { Sweden }\end{array}$} & DVT & RCT & High & $1 \mathrm{mo}$ & IBR & All & $0 / 66(0)$ & Ref & Ref \\
\hline & DVT & RCT & High & $1 \mathrm{mo}$ & $\mathrm{AR}$ & All & $0 / 51(0)$ & No events & $\mathrm{N} / \mathrm{A}$ \\
\hline & $\mathrm{PE}$ & RCT & High & $1 \mathrm{mo}$ & IBR & All & $1 / 66(1.5)$ & Ref & Ref \\
\hline & PE & RCT & High & $1 \mathrm{mo}$ & AR & All & $0 / 51(0)$ & $\begin{array}{l}\text { Not calculable (no events } \\
\text { in AR group) }\end{array}$ & $\begin{array}{l}\text { Not } \\
\text { calculable }\end{array}$ \\
\hline \multirow{6}{*}{$\begin{array}{l}\text { Tallroth, 2020, 3346336, } \\
\text { Sweden }\end{array}$} & DVT & RCT & Moderate & $1 \mathrm{mo}$ & IBR & All & $1 / 28(3.6)$ & Ref & Ref \\
\hline & DVT & RCT & Moderate & $1 \mathrm{mo}$ & AR & All & $0 / 42(0)$ & $\begin{array}{l}\text { Not calculable (no events } \\
\text { in AR group) }\end{array}$ & $\begin{array}{l}\text { Not } \\
\text { calculable }\end{array}$ \\
\hline & Arterial stop & RCT & Moderate & $1 \mathrm{mo}$ & IBR & All & $0 / 28(0)$ & Ref & Ref \\
\hline & Arterial stop & RCT & Moderate & $1 \mathrm{mo}$ & AR & All & $2 / 42(4.8)$ & $\begin{array}{l}\text { Not calculable (no events } \\
\text { in IBR group) }\end{array}$ & $\begin{array}{l}\text { Not } \\
\text { calculable }\end{array}$ \\
\hline & Venous stasis & RCT & Moderate & $1 \mathrm{mo}$ & IBR & All & $0 / 28(0)$ & Ref & Ref \\
\hline & Venous stasis & RCT & Moderate & $1 \mathrm{mo}$ & AR & All & $6 / 42(14.3)$ & $\begin{array}{l}\text { Not calculable (no events } \\
\text { in IBR group) }\end{array}$ & $\begin{array}{l}\text { Not } \\
\text { calculable }\end{array}$ \\
\hline \multirow{4}{*}{$\begin{array}{l}\text { Carramaschi, 1989, } \\
\text { 2602589, France }\end{array}$} & $\mathrm{PE}$ & NRCS & High & $\mathrm{NR}$ & IBR & All & $0 / 166(0)$ & NR & NR \\
\hline & $\mathrm{PE}$ & NRCS & High & NR & AR (all) & All & $2 / 74(2.7)$ & NR & NR \\
\hline & $\mathrm{PE}$ & NRCS & High & NR & AR with TRAM & All & $2 / 40(5)$ & NR & NR \\
\hline & $\mathrm{PE}$ & NRCS & High & NR & AR with LD & All & $0 / 34(0)$ & NR & NR \\
\hline \multirow{10}{*}{$\begin{array}{l}\text { Kulkarni, 2017, } \\
\text { 28713853, US \& Canada }\end{array}$} & DVT & NRCS & Moderate & $1 \mathrm{y}$ & IBR & All & $5 / 1615(0.3)$ & NR & NR \\
\hline & DVT & NRCS & Moderate & $1 \mathrm{y}$ & AR (all) & All & NR & NR & NR \\
\hline & DVT & NRCS & Moderate & $1 \mathrm{y}$ & AR with DIEP & All & $1 / 365(0.3)$ & NR & NR \\
\hline & DVT & NRCS & Moderate & $1 \mathrm{y}$ & $\begin{array}{l}\text { AR with free } \\
\text { TRAM }\end{array}$ & All & $1 / 97(1)$ & NR & NR \\
\hline & DVT & NRCS & Moderate & $1 \mathrm{y}$ & $\begin{array}{l}\text { AR with } \\
\text { pedicled TRAM }\end{array}$ & All & $0 / 84(0)$ & NR & NR \\
\hline & DVT & NRCS & Moderate & $1 \mathrm{y}$ & AR with LD & All & $1 / 73(1.4)$ & NR & NR \\
\hline & $\mathrm{PE}$ & NRCS & Moderate & $1 \mathrm{y}$ & IBR & All & $4 / 1615(0.3)$ & NR & NR \\
\hline & $\mathrm{PE}$ & NRCS & Moderate & $1 \mathrm{y}$ & AR (all) & All & NR & NR & NR \\
\hline & $\mathrm{PE}$ & NRCS & Moderate & $1 \mathrm{y}$ & AR with DIEP & All & $4 / 365(1.1)$ & NR & NR \\
\hline & PE & NRCS & Moderate & $1 \mathrm{y}$ & $\begin{array}{l}\text { AR with free } \\
\text { TRAM }\end{array}$ & All & $2 / 97(2.1)$ & NR & NR \\
\hline
\end{tabular}




\begin{tabular}{|c|c|c|c|c|c|c|c|c|c|}
\hline $\begin{array}{l}\text { Study, Year, PMID, } \\
\text { Country }\end{array}$ & $\begin{array}{l}\text { Outcome } \\
\text { Description }\end{array}$ & Design & $\begin{array}{l}\text { Overall } \\
\text { RoB }\end{array}$ & $\begin{array}{l}\text { Time } \\
\text { Point }\end{array}$ & Arm & Subgroup & $\mathrm{n} / \mathrm{N}(\%)$ & Effect Size $(95 \% \mathrm{CI})$ & P Value \\
\hline & $\mathrm{PE}$ & NRCS & Moderate & $1 \mathrm{y}$ & $\begin{array}{l}\text { AR with } \\
\text { pedicled TRAM }\end{array}$ & All & $0 / 84(0)$ & NR & NR \\
\hline & $\mathrm{PE}$ & NRCS & Moderate & $1 \mathrm{y}$ & AR with LD & All & $0 / 73(0)$ & NR & NR \\
\hline \multirow{4}{*}{$\begin{array}{l}\text { Mioton, 2013, 23562485, } \\
\text { US }\end{array}$} & DVT & NRCS & Moderate & $1 \mathrm{mo}$ & IBR & All & $27 / 9786(0.28)$ & Ref & Ref \\
\hline & DVT & NRCS & Moderate & $1 \mathrm{mo}$ & AR & All & $20 / 3296(0.61)$ & adjOR $0.992(0.41,2.41)$ & NR \\
\hline & $\mathrm{PE}$ & NRCS & Moderate & $1 \mathrm{mo}$ & IBR & All & $17 / 9786(0.17)$ & Ref & Ref \\
\hline & $\mathrm{PE}$ & NRCS & Moderate & $1 \mathrm{mo}$ & AR & All & $17 / 3296(0.52)$ & adjOR $1.84(0.71,4.77)$ & NR \\
\hline \multirow{2}{*}{$\begin{array}{l}\text { Momeni, 2018, } \\
\text { 29095189, US }\end{array}$} & DVT/PE & NRCS & High & $3 \mathrm{mo}$ & IBR & All & $65 / 16851(3.85)$ & adjOR $0.44(0.35,0.56)$ & \\
\hline & DVT/PE & NRCS & High & $3 \mathrm{mo}$ & $\mathrm{AR}$ & All & $815 / 4622(17.63)$ & Ref & Ref \\
\hline \multirow{2}{*}{$\begin{array}{l}\text { Chen, 2018a, 29596085, } \\
\text { US }\end{array}$} & DVT & Single group & Low & NR & IBR & All & $9 / 23048(0.04)$ & $\mathrm{N} / \mathrm{A}$ & $\mathrm{N} / \mathrm{A}$ \\
\hline & DVT & Single group & Low & NR & IBR & All & $20 / 23048(0.09)$ & N/A & $\mathrm{N} / \mathrm{A}$ \\
\hline $\begin{array}{l}\text { Albornoz, 2013, } \\
\text { 23897346, US }\end{array}$ & DVT or PE & Single group & Low & NR & AR & All & $231 / 21016(1.1)$ & $\mathrm{N} / \mathrm{A}$ & $\mathrm{N} / \mathrm{A}$ \\
\hline $\begin{array}{l}\text { Beugels, 2018, } \\
\text { 29399731, Netherlands }\end{array}$ & $\begin{array}{l}\text { Venous } \\
\text { congestion }\end{array}$ & Single group & Low & $9-10 \mathrm{mo}$ & AR & All & 40/910 flaps (4.4) & $\mathrm{N} / \mathrm{A}$ & $\mathrm{N} / \mathrm{A}$ \\
\hline $\begin{array}{l}\text { Chang, 2000, 10809092, } \\
\text { US }\end{array}$ & $\begin{array}{l}\text { Vessel } \\
\text { thrombosis }\end{array}$ & Single group & Low & NR & AR & All & 34/936 flaps (3.6) & $\mathrm{N} / \mathrm{A}$ & $\mathrm{N} / \mathrm{A}$ \\
\hline \multirow{5}{*}{$\begin{array}{l}\text { Chang, 2011, 21407063, } \\
\text { US }\end{array}$} & Thrombosis & Single group & Low & NR & AR & All & $29 / 818(3.5)$ & $\mathrm{N} / \mathrm{A}$ & $\mathrm{N} / \mathrm{A}$ \\
\hline & Thrombosis & Single group & Low & NR & AR & Age $<50$ & $14 / 411(3.4)$ & $\mathrm{N} / \mathrm{A}$ & $\mathrm{N} / \mathrm{A}$ \\
\hline & Thrombosis & Single group & Low & NR & AR & Age 50-59 & $11 / 285(3.9)$ & N/A & N/A \\
\hline & Thrombosis & Single group & Low & NR & AR & Age 60-69 & $3 / 103(2.9)$ & $\mathrm{N} / \mathrm{A}$ & $\mathrm{N} / \mathrm{A}$ \\
\hline & Thrombosis & Single group & Low & NR & AR & Age $>70$ & $1 / 19(5.3)$ & $\mathrm{N} / \mathrm{A}$ & $\mathrm{N} / \mathrm{A}$ \\
\hline \multirow[t]{3}{*}{$\begin{array}{l}\text { Chang, 2016, 25003429, } \\
\text { US }\end{array}$} & $\begin{array}{l}\text { Venous } \\
\text { thrombosis }\end{array}$ & Single group & Low & NR & AR & All & 69/1773 flaps (3.9) & $\mathrm{N} / \mathrm{A}$ & $\mathrm{N} / \mathrm{A}$ \\
\hline & $\begin{array}{l}\text { Arterial } \\
\text { thrombosis }\end{array}$ & Single group & Low & NR & $\mathrm{AR}$ & All & $68 / 1773$ flaps (3.8) & $\mathrm{N} / \mathrm{A}$ & $\mathrm{N} / \mathrm{A}$ \\
\hline & $\begin{array}{l}\text { Venous or } \\
\text { arterial } \\
\text { thrombosis }\end{array}$ & Single group & Low & NR & AR & All & 14/1773 flaps $(0.8)$ & $\mathrm{N} / \mathrm{A}$ & $\mathrm{N} / \mathrm{A}$ \\
\hline \multirow{2}{*}{$\begin{array}{l}\text { Chen, 2018b, 29596085, } \\
\text { US }\end{array}$} & DVT & Single group & Low & NR & $\mathrm{AR}$ & All & $24 / 19496(0.1)$ & $\mathrm{N} / \mathrm{A}$ & $\mathrm{N} / \mathrm{A}$ \\
\hline & PE & Single group & Low & NR & AR & All & $44 / 19496(0.2)$ & $\mathrm{N} / \mathrm{A}$ & $\mathrm{N} / \mathrm{A}$ \\
\hline $\begin{array}{l}\text { Daly, 2020, 31994156, } \\
\text { US }\end{array}$ & $\mathrm{PE}$ & Single group & Low & $12 y$ & AR & All & $1 / 818(0.1)$ & $\mathrm{N} / \mathrm{A}$ & $\mathrm{N} / \mathrm{A}$ \\
\hline \multirow[t]{3}{*}{$\begin{array}{l}\text { Enajat, 2010, 19790180, } \\
\text { Sweden }\end{array}$} & $\begin{array}{l}\text { Venous } \\
\text { congestion }\end{array}$ & Single group & Low & $1-7 d$ & $\mathrm{AR}$ & All & 7/564 flaps (1.2) & $\mathrm{N} / \mathrm{A}$ & $\mathrm{N} / \mathrm{A}$ \\
\hline & $\begin{array}{l}\text { Arterial } \\
\text { thrombosis }\end{array}$ & Single group & Low & $1-7 d$ & AR & All & 18/564 (implants) (3.2) & $\mathrm{N} / \mathrm{A}$ & $\mathrm{N} / \mathrm{A}$ \\
\hline & $\begin{array}{l}\text { Venous } \\
\text { thrombosis }\end{array}$ & Single group & Low & $1-7 d$ & AR & All & 10/564 flaps (5.6) & $\mathrm{N} / \mathrm{A}$ & $\mathrm{N} / \mathrm{A}$ \\
\hline
\end{tabular}




\begin{tabular}{|c|c|c|c|c|c|c|c|c|c|}
\hline $\begin{array}{l}\text { Study, Year, PMID, } \\
\text { Country }\end{array}$ & $\begin{array}{l}\text { Outcome } \\
\text { Description }\end{array}$ & Design & $\begin{array}{l}\text { Overall } \\
\text { RoB }\end{array}$ & $\begin{array}{l}\text { Time } \\
\text { Point }\end{array}$ & Arm & Subgroup & $n / N(\%)$ & Effect Size $(95 \% \mathrm{Cl})$ & P Value \\
\hline \multirow[t]{2}{*}{ Gill, 2004, 15083015, US } & $\begin{array}{l}\text { Venous } \\
\text { occlusion }\end{array}$ & Single group & Moderate & $10 y$ & AR & All & $29 / 758(3.8)$ & N/A & N/A \\
\hline & $\begin{array}{l}\text { Arterial } \\
\text { occlusion }\end{array}$ & Single group & Moderate & $10 y$ & AR & All & $4 / 758(0.5)$ & $\mathrm{N} / \mathrm{A}$ & $\mathrm{N} / \mathrm{A}$ \\
\hline $\begin{array}{l}\text { Haddock, 2019, } \\
31461004, \text { US }\end{array}$ & DVT & Single group & Moderate & NR & AR & All & $5 / 509(1.0)$ & $\mathrm{N} / \mathrm{A}$ & $\mathrm{N} / \mathrm{A}$ \\
\hline $\begin{array}{l}\text { Langer, 2010, 20980954, } \\
\text { Germany }\end{array}$ & $\begin{array}{l}\text { Venous } \\
\text { congestion }\end{array}$ & Single group & Low & $5 y$ & AR & All & 4/670 flaps (0.6) & N/A & $\mathrm{N} / \mathrm{A}$ \\
\hline $\begin{array}{l}\text { Liao, 2008, 18349626, } \\
\text { US }\end{array}$ & DVT or PE & Single group & Low & NR & AR & All & $7 / 679(1.0)$ & $\mathrm{N} / \mathrm{A}$ & $\mathrm{N} / \mathrm{A}$ \\
\hline \multirow{2}{*}{$\begin{array}{l}\text { Masoomi, 2019, } \\
31331721, \text { US }\end{array}$} & DVT & Single group & Low & NR & AR & All & $203 / 7991(2.5)$ & $\mathrm{N} / \mathrm{A}$ & $\mathrm{N} / \mathrm{A}$ \\
\hline & VTE & Single group & Low & NR & AR & All & $60 / 55840(0.1)$ & N/A & N/A \\
\hline \multirow{5}{*}{$\begin{array}{l}\text { Mehrara, 2006, } \\
17016173, \text { US }\end{array}$} & DVT & Single group & Moderate & $1 \mathrm{mo}$ & AR & All & 9/1195 (0.1) & $\mathrm{N} / \mathrm{A}$ & $\mathrm{N} / \mathrm{A}$ \\
\hline & $\begin{array}{l}\text { Arterial } \\
\text { thrombosis }\end{array}$ & Single group & Moderate & $1 \mathrm{mo}$ & AR & All & 9/1195 (0.8) & N/A & N/A \\
\hline & $\begin{array}{l}\text { Arterial } \\
\text { thrombosis }\end{array}$ & Single group & Moderate & $1 \mathrm{mo}$ & AR & TRAM & $7 / 978(0.7)$ & N/A & N/A \\
\hline & $\begin{array}{l}\text { Venous } \\
\text { thrombosis }\end{array}$ & Single group & Moderate & $1 \mathrm{mo}$ & AR & All & $15 / 1195(1.3)$ & N/A & $\mathrm{N} / \mathrm{A}$ \\
\hline & $\begin{array}{l}\text { Venous } \\
\text { thrombosis }\end{array}$ & Single group & Moderate & $1 \mathrm{mo}$ & AR & TRAM & $8 / 978(0.8)$ & $\mathrm{N} / \mathrm{A}$ & $\mathrm{N} / \mathrm{A}$ \\
\hline \multirow[t]{4}{*}{$\begin{array}{l}\text { Mirzabeigi, } 2015, \\
25811579, \text { US }\end{array}$} & $\begin{array}{l}\text { Intraoperative } \\
\text { arterial } \\
\text { thrombosis }\end{array}$ & Single group & Low & NR & AR & All & $36 / 1347$ flaps (2.7) & N/A & $\mathrm{N} / \mathrm{A}$ \\
\hline & $\begin{array}{l}\text { Intraoperative } \\
\text { venous } \\
\text { thrombosis }\end{array}$ & Single group & Low & NR & AR & All & 7/1347 flaps (0.5) & N/A & N/A \\
\hline & $\begin{array}{l}\text { Postoperative } \\
\text { arterial } \\
\text { thrombosis }\end{array}$ & Single group & Low & NR & AR & All & 18/1347 flaps (1.3) & N/A & N/A \\
\hline & $\begin{array}{l}\text { Postoperative } \\
\text { venous } \\
\text { thrombosis }\end{array}$ & Single group & Low & NR & AR & All & 16/1347 flaps (1.2) & N/A & N/A \\
\hline \multirow[t]{2}{*}{$\begin{array}{l}\text { Munder, 2020, } \\
\text { 32565553, Germany }\end{array}$} & $\begin{array}{l}\text { Insufficient } \\
\text { arterial supply }\end{array}$ & Single group & Low & $2 y$ & AR & All & 1/1274 flaps $(0.1)$ & $\mathrm{N} / \mathrm{A}$ & $\mathrm{N} / \mathrm{A}$ \\
\hline & $\begin{array}{l}\text { Insufficient } \\
\text { venous } \\
\text { supply } \\
\end{array}$ & Single group & Low & $2 y$ & AR & All & 10/1274 flaps (0.8) & $\mathrm{N} / \mathrm{A}$ & $\mathrm{N} / \mathrm{A}$ \\
\hline \multirow[t]{2}{*}{$\begin{array}{l}\text { Nelson, 2014, 25046665, } \\
\text { US }\end{array}$} & $\begin{array}{l}\text { Arterial } \\
\text { thrombosis }\end{array}$ & Single group & Moderate & Intra-op & AR & All & 42/1293 flaps (3.3) & $\mathrm{N} / \mathrm{A}$ & $\mathrm{N} / \mathrm{A}$ \\
\hline & $\begin{array}{l}\text { Arterial } \\
\text { thrombosis }\end{array}$ & Single group & Moderate & Delayed & AR & All & 18/1293 flaps (1.4) & $\mathrm{N} / \mathrm{A}$ & $\mathrm{N} / \mathrm{A}$ \\
\hline
\end{tabular}




\begin{tabular}{|c|c|c|c|c|c|c|c|c|c|c|}
\hline $\begin{array}{l}\text { Study, Year, PMID, } \\
\text { Country }\end{array}$ & $\begin{array}{l}\text { Outcome } \\
\text { Description }\end{array}$ & Design & $\begin{array}{l}\text { Overall } \\
\text { RoB }\end{array}$ & $\begin{array}{l}\text { Time } \\
\text { Point }\end{array}$ & Arm & Subgroup & $n / N(\%)$ & Eff & ect Size $(95 \% \mathrm{Cl})$ & P Value \\
\hline & $\begin{array}{l}\text { Venous } \\
\text { thrombosis }\end{array}$ & Single group & Moderate & Intra-op & AR & All & 7/1293 flaps (0.5) & $\mathrm{N} / \mathrm{A}$ & & N/A \\
\hline & $\begin{array}{l}\text { Venous } \\
\text { thrombosis }\end{array}$ & Single group & Moderate & Delayed & AR & All & 16/1293 flaps (1.2) & $\mathrm{N} / \mathrm{A}$ & & N/A \\
\hline \multirow{2}{*}{$\begin{array}{l}\text { O'Neill, 2019, 31196805, } \\
\text { Canada }\end{array}$} & DVT or PE & Single group & Low & 1 day & AR & All & $2 / 512(0.4)$ & $\mathrm{N} / \mathrm{A}$ & & $\mathrm{N} / \mathrm{A}$ \\
\hline & DVT or PE & Single group & Low & $<2 \mathrm{mo}$ & AR & All & $7 / 960(0.5)$ & $\mathrm{N} / \mathrm{A}$ & & $\mathrm{N} / \mathrm{A}$ \\
\hline \multirow[t]{2}{*}{$\begin{array}{l}\text { Prantl, 2020, 32895743, } \\
\text { Germany }\end{array}$} & $\begin{array}{l}\text { Arterial } \\
\text { thrombosis }\end{array}$ & Single group & Low & $3 \mathrm{mo}$ & AR & All & 74/4577 flaps (1.6) & $\mathrm{N} / \mathrm{A}$ & & N/A \\
\hline & $\begin{array}{l}\text { Venous } \\
\text { thrombosis }\end{array}$ & Single group & Low & $3 \mathrm{mo}$ & AR & All & 123/4577 flaps (2.7) & $\mathrm{N} / \mathrm{A}$ & & N/A \\
\hline \multirow{3}{*}{$\begin{array}{l}\text { Rubio, 2019, 30665841, } \\
\text { Belgium }\end{array}$} & PE & Single group & Low & NR & AR & All & $113 / 56522(0.2)$ & $\mathrm{N} / \mathrm{A}$ & & $\mathrm{N} / \mathrm{A}$ \\
\hline & DVT & Single group & Low & NR & AR & All & $226 / 56522(0.4)$ & $\mathrm{N} / \mathrm{A}$ & & N/A \\
\hline & DVT or PE & Single group & Low & NR & AR & All & $48 / 35883(0.1)$ & $\mathrm{N} / \mathrm{A}$ & & N/A \\
\hline $\begin{array}{l}\text { Seidenstuecker, 2016, } \\
\text { 27017243, Belgium }\end{array}$ & $\begin{array}{l}\text { DVT, PE, or } \\
\text { cardiac or } \\
\text { pulmonary } \\
\text { failure }\end{array}$ & Single group & Moderate & NR & AR & All & 0/931 flaps (0) & $\mathrm{N} / \mathrm{A}$ & & N/A \\
\hline \multirow{2}{*}{$\begin{array}{l}\text { Selber, 2009, 19935283, } \\
\text { US }\end{array}$} & $\mathrm{PE}$ & Single group & Low & $5.6 \mathrm{mo}$ & AR & All & $2 / 1031(0.2)$ & $\mathrm{N} / \mathrm{A}$ & & $\mathrm{N} / \mathrm{A}$ \\
\hline & $\begin{array}{l}\text { Vessel } \\
\text { thrombosis }\end{array}$ & Single group & Low & $5.6 \mathrm{mo}$ & AR & All & $1 / 1031(0.1)$ & $\mathrm{N} / \mathrm{A}$ & & N/A \\
\hline $\begin{array}{l}\text { Song, 2016, 26637165, } \\
\text { US }\end{array}$ & VTE & Single group & Low & $\begin{array}{l}4.3-5.4 \\
y\end{array}$ & AR & All & $25 / 1809(1.4)$ & $\mathrm{N} / /$ & & N/A \\
\hline \multirow{2}{*}{$\begin{array}{l}\text { Tran, 2018, 29794694, } \\
\text { US }\end{array}$} & $\mathrm{PE}$ & Single group & Low & NR & AR & All & $1 / 879(0.08)$ & $\mathrm{N} / \mathrm{A}$ & & $\mathrm{N} / \mathrm{A}$ \\
\hline & DVT & Single group & Low & NR & AR & All & $1 / 879(0.08)$ & $\mathrm{N} / \mathrm{A}$ & & $\mathrm{N} / \mathrm{A}$ \\
\hline $\begin{array}{l}\text { Yoo, 2014, 24852813, } \\
\text { South Korea }\end{array}$ & $\mathrm{PE}$ & Single group & Moderate & NR & AR & All & $17 / 504(3.4)$ & $\mathrm{N} / \mathrm{h}$ & & N/A \\
\hline \multicolumn{11}{|c|}{$\begin{array}{l}\text { Abbreviations: adj }=\text { adjusted, } \mathrm{AR}=\text { autologous reconstruction, } \mathrm{CI}=\text { confidence interval, } \mathrm{DIEP}=\text { deep inferior epigastric perforator, } \mathrm{IBR}=\mathrm{implant-based} \text { reconstruction, } \mathrm{LD}= \\
\text { latissimus dorsi, } \mathrm{NR}=\text { not reported, } \mathrm{NRCS}=\text { nonrandomized comparative study, } \mathrm{OR}=\text { odds ratio, } \mathrm{PMID}=\mathrm{PubMed} \text { identifier, Ref }=\text { reference group, RoB }=\text { risk of bias, } \mathrm{y}=\mathrm{years.} \\
\text { Colors: Header rows are shaded orange. Rows for every alternate study are shaded blue. The colors do not add unique information. }\end{array}$} \\
\hline $\begin{array}{l}\text { Study, Year, PMID, } \\
\text { Country }\end{array}$ & $\begin{array}{l}\text { Outcome } \\
\text { Description }\end{array}$ & Design & $\begin{array}{l}\text { Overall } \\
\text { RoB }\end{array}$ & $\begin{array}{l}\text { Time } \\
\text { Point }\end{array}$ & Arm & Subgroup & $\mathrm{n} / \mathrm{N}(\%)$ & & Effect Size $(95 \% \mathrm{Cl})$ & $\begin{array}{l}\mathbf{P} \\
\text { Value }\end{array}$ \\
\hline \multirow{2}{*}{$\begin{array}{l}\text { de Araujo, 2016, } \\
27673527, \text { US }\end{array}$} & SSI & NRCS & High & $4.3 \mathrm{y}$ & IBR & All & NR/38 (NR) & & adjOR $0.86(0.18,4.11)$ & 0.847 \\
\hline & SSI & NRCS & High & $4.3 \mathrm{y}$ & AR & All & NR/32 (NR) & & Ref & Ref \\
\hline \multirow{2}{*}{$\begin{array}{l}\text { Garvey, 2012, 23096600, } \\
\text { US }\end{array}$} & Infections & NRCS & Moderate & $1.5 \mathrm{y}$ & IBR & All & $50 / 442$ breasts $(11.3)$ & & Ref & Ref \\
\hline & Infections & NRCS & Moderate & $1.5 \mathrm{y}$ & AR & All & $21 / 548$ breasts (3.8) & & NR & $<0.001$ \\
\hline \multirow{3}{*}{$\begin{array}{l}\text { Kulkarni, 2017, 28713853, } \\
\text { US \& Canada }\end{array}$} & Breast WI & NRCS & Moderate & $1 \mathrm{y}$ & IBR (all) & All & $162 / 1615(10)$ & & NR & NR \\
\hline & Breast WI & NRCS & Moderate & $1 \mathrm{y}$ & IBR DTI & All & NR & & NR & NR \\
\hline & Breast WI & NRCS & Moderate & $1 \mathrm{y}$ & IBR T/E & All & NR & & NR & NR \\
\hline
\end{tabular}




\begin{tabular}{|c|c|c|c|c|c|c|c|c|c|}
\hline $\begin{array}{l}\text { Study, Year, PMID, } \\
\text { Country }\end{array}$ & $\begin{array}{l}\text { Outcome } \\
\text { Description }\end{array}$ & Design & $\begin{array}{l}\text { Overall } \\
\text { RoB }\end{array}$ & $\begin{array}{l}\text { Time } \\
\text { Point }\end{array}$ & Arm & Subgroup & $\mathrm{n} / \mathrm{N}(\%)$ & Effect Size $(95 \% \mathrm{CI})$ & $\begin{array}{l}\mathbf{P} \\
\text { Value }\end{array}$ \\
\hline & Breast WI & NRCS & Moderate & $1 \mathrm{y}$ & AR (all) & All & NR & NR & NR \\
\hline & Breast WI & NRCS & Moderate & $1 \mathrm{y}$ & AR with DIEP & All & $14 / 365(3.8)$ & NR & NR \\
\hline & Breast WI & NRCS & Moderate & $1 \mathrm{y}$ & $\begin{array}{l}\text { AR with free } \\
\text { TRAM }\end{array}$ & All & $4 / 97(4.1)$ & NR & NR \\
\hline & Breast WI & NRCS & Moderate & $1 \mathrm{y}$ & $\begin{array}{l}\text { AR with } \\
\text { pedicled TRAM }\end{array}$ & All & $5 / 84(6)$ & NR & NR \\
\hline & Breast WI & NRCS & Moderate & $1 \mathrm{y}$ & AR with LD & All & $6 / 73(8.2)$ & NR & NR \\
\hline & Breast WI & NRCS & Moderate & $1 \mathrm{y}$ & AR with SIEA & All & NR & NR & NR \\
\hline & Breast WI & NRCS & Moderate & $2 y$ & IBR & All & NR & NR & NR \\
\hline & Breast WI & NRCS & Moderate & $2 y$ & IBR DTI & All & $17 / 112(15.2)$ & $\begin{array}{l}\text { vs IBR T/E: adjOR } 1.70 \\
(0.91,3.18)\end{array}$ & 0.1 \\
\hline & Breast WI & NRCS & Moderate & $2 \mathrm{y}$ & IBR T/E & All & $159 / 1525(10.4)$ & Ref & Ref \\
\hline & Breast WI & NRCS & Moderate & $2 y$ & AR (all) & All & NR & NR & NR \\
\hline & Breast WI & NRCS & Moderate & $2 y$ & AR with DIEP & All & $27 / 390(6.9)$ & $\begin{array}{l}\text { vs IBR T/E: adjOR } 0.44 \\
(0.25,0.78)\end{array}$ & 0.005 \\
\hline & Breast WI & NRCS & Moderate & $2 y$ & $\begin{array}{l}\text { AR with free } \\
\text { TRAM }\end{array}$ & All & $5 / 95(5.3)$ & $\begin{array}{l}\text { vs IBR T/E: adjOR } 0.45 \\
(0.17,1.18)\end{array}$ & 0.10 \\
\hline & Breast WI & NRCS & Moderate & $2 y$ & $\begin{array}{l}\text { AR with } \\
\text { pedicled TRAM }\end{array}$ & All & $8 / 85(9.4)$ & $\begin{array}{l}\text { vs IBR T/E: adjOR } 0.73 \\
(0.31,1.70)\end{array}$ & 0.46 \\
\hline & Breast WI & NRCS & Moderate & $2 y$ & AR with LD & All & $6 / 71(8.5)$ & $\begin{array}{l}\text { vs IBR T/E: adjOR } 0.50 \\
(0.15,1.56)\end{array}$ & 0.23 \\
\hline & Breast WI & NRCS & Moderate & $2 y$ & AR with SIEA & All & $8 / 65(12.3)$ & $\begin{array}{l}\text { vs IBR T/E: adjOR } 0.67 \\
(0.25,1.82)\end{array}$ & 0.43 \\
\hline \multirow{6}{*}{$\begin{array}{l}\text { Mioton, 2013, 23562485, } \\
\text { US }\end{array}$} & WI & NRCS & Moderate & $1 \mathrm{mo}$ & IBR & All & $338 / 9786(3.45)$ & Ref & Ref \\
\hline & WI & NRCS & Moderate & $1 \mathrm{mo}$ & AR & All & $180 / 3296(5.46)$ & adjOR $1.40(1.01,1.96)$ & NR \\
\hline & Superficial SSI & NRCS & Moderate & $1 \mathrm{mo}$ & IBR & All & $163 / 9786(1.67)$ & Ref & Ref \\
\hline & Superficial SSI & NRCS & Moderate & $1 \mathrm{mo}$ & $\mathrm{AR}$ & All & $97 / 3296(2.95)$ & adjOR $1.20(0.81,1.76)$ & NR \\
\hline & Deep SSI & NRCS & Moderate & $1 \mathrm{mo}$ & IBR & All & $195 / 9786(1.07)$ & Ref & Ref \\
\hline & Deep SSI & NRCS & Moderate & $1 \mathrm{mo}$ & $\mathrm{AR}$ & All & $65 / 3296(1.97)$ & adjOR $1.81(1.12,2.94)$ & NR \\
\hline \multirow{2}{*}{$\begin{array}{l}\text { Naoum, 2020a, 31756414, } \\
\text { US }\end{array}$} & NR & NRCS & High & $4-10 y$ & IBR & All & 23/633 breasts (3.6) & Ref & Ref \\
\hline & NR & NRCS & High & $4-10 y$ & AR & All & 9/342 breasts $(2.6)$ & adjOR $0.77(0.20,2.50)$ & 0.67 \\
\hline \multirow[t]{3}{*}{$\begin{array}{l}\text { Naoum, 2020b, 32607638, } \\
\text { US }\end{array}$} & NR & NRCS & High & $\begin{array}{l}4.3-6.3 \\
y\end{array}$ & IBR DTI & All & $7 / 127(5.5)$ & Ref & Ref \\
\hline & NR & NRCS & High & $\begin{array}{l}4.3-6.3 \\
y\end{array}$ & IBR with TE & All & $2 / 88(2.2)$ & Ref & Ref \\
\hline & NR & NRCS & High & $\begin{array}{l}4.3-6.3 \\
y\end{array}$ & AR & All & $11 / 85(13.0)$ & $\begin{array}{l}\text { vs. IBR DTI: } 3.2(0.6 \text {, } \\
16) \\
\text { vs. IBR with TE: } 8.1 \\
(1.7,39)\end{array}$ & $\begin{array}{l}0.20 \\
0.009\end{array}$ \\
\hline \multirow{2}{*}{$\begin{array}{l}\text { Nelson, 2019, 31356276, } \\
\text { US }\end{array}$} & NR & NRCS & High & $<3 \mathrm{mo}$ & IBR & All & $82 / 1211$ breasts (6.7) & NR & NR \\
\hline & NR & NRCS & High & $<3 \mathrm{mo}$ & AR & All & NR & NR & NR \\
\hline
\end{tabular}




\begin{tabular}{|c|c|c|c|c|c|c|c|c|c|}
\hline $\begin{array}{l}\text { Study, Year, PMID, } \\
\text { Country }\end{array}$ & $\begin{array}{l}\text { Outcome } \\
\text { Description }\end{array}$ & Design & $\begin{array}{l}\text { Overall } \\
\text { RoB }\end{array}$ & $\begin{array}{l}\text { Time } \\
\text { Point }\end{array}$ & Arm & Subgroup & $n / N(\%)$ & Effect Size (95\% Cl) & $\begin{array}{l}P \\
\text { Value }\end{array}$ \\
\hline \multirow[t]{2}{*}{$\begin{array}{l}\text { Banuelos, 2020, 31663932, } \\
\text { US }\end{array}$} & SSI & $\begin{array}{l}\text { Single } \\
\text { group }\end{array}$ & Low & $\leq 1 \mathrm{mo}$ & IBR & All & $13 / 768(1.7)$ & $\mathrm{N} / \mathrm{A}$ & N/A \\
\hline & SSI & $\begin{array}{l}\text { Single } \\
\text { group }\end{array}$ & Low & $>1 \mathrm{mo}$ & IBR & All & $32 / 768(4.2)$ & $\mathrm{N} / \mathrm{A}$ & $\mathrm{N} / \mathrm{A}$ \\
\hline \multirow[t]{3}{*}{$\begin{array}{l}\text { Brooks, 2012, 22098451, } \\
\text { US }\end{array}$} & NR & $\begin{array}{l}\text { Single } \\
\text { group }\end{array}$ & Low & $3.4 \mathrm{y}$ & IBR & All & $74 / 733(10.1)$ & $\mathrm{N} / \mathrm{A}$ & $\mathrm{N} / \mathrm{A}$ \\
\hline & NR & $\begin{array}{l}\text { Single } \\
\text { group }\end{array}$ & Low & $3.4 \mathrm{y}$ & IBR & Radiation & 10/97 (9.7) & $\mathrm{N} / \mathrm{A}$ & $\mathrm{N} / \mathrm{A}$ \\
\hline & NR & $\begin{array}{l}\text { Single } \\
\text { group }\end{array}$ & Low & $3.4 \mathrm{y}$ & IBR & $\begin{array}{l}\text { No } \\
\text { radiation }\end{array}$ & $64 / 636(10.1)$ & $\mathrm{N} / \mathrm{A}$ & N/A \\
\hline $\begin{array}{l}\text { Chen, 2014, 25620484, } \\
\text { China }\end{array}$ & NR & $\begin{array}{l}\text { Single } \\
\text { group }\end{array}$ & Low & NR & IBR & All & $27 / 1860(1.5)$ & $\mathrm{N} / \mathrm{A}$ & $\mathrm{N} / \mathrm{A}$ \\
\hline $\begin{array}{l}\text { Chen, 2016, 27930584, } \\
\text { Taiwan }\end{array}$ & NR & $\begin{array}{l}\text { Single } \\
\text { group }\end{array}$ & Low & $\geq 2 \mathrm{y}$ & IBR & All & $815 / 569$ breasts $(5.1)$ & $\mathrm{N} / \mathrm{A}$ & N/A \\
\hline $\begin{array}{l}\text { Chen, 2018a, 29596085, } \\
\text { US }\end{array}$ & NR & $\begin{array}{l}\text { Single } \\
\text { group }\end{array}$ & Low & NR & IBR & All & $67 / 23048(0.3)$ & $\mathrm{N} / \mathrm{A}$ & N/A \\
\hline \multirow[t]{2}{*}{$\begin{array}{l}\text { Collier, 2019, 31461001, } \\
\text { US }\end{array}$} & $\begin{array}{l}\text { Readmitted for } \\
\text { infection }\end{array}$ & $\begin{array}{l}\text { Single } \\
\text { group }\end{array}$ & Moderate & $1 \mathrm{mo}$ & IBR & All & $374 / 18338(2.0)$ & $\mathrm{N} / \mathrm{A}$ & $\mathrm{N} / \mathrm{A}$ \\
\hline & $\begin{array}{l}\text { Readmitted for } \\
\text { infection }\end{array}$ & $\begin{array}{l}\text { Single } \\
\text { group }\end{array}$ & Moderate & $3 \mathrm{mo}$ & IBR & All & $385 / 18338(2.1)$ & $\mathrm{N} / \mathrm{A}$ & $\mathrm{N} / \mathrm{A}$ \\
\hline \multirow[t]{7}{*}{$\begin{array}{l}\text { Cordeiro, 2006, 16980842, } \\
\text { US }\end{array}$} & NR & $\begin{array}{l}\text { Single } \\
\text { group }\end{array}$ & Moderate & NR & IBR & All & $58 / 2276$ procedures (2.5) & $\mathrm{N} / \mathrm{A}$ & $\mathrm{N} / \mathrm{A}$ \\
\hline & NR & $\begin{array}{l}\text { Single } \\
\text { group }\end{array}$ & Moderate & NR & IBR & $\begin{array}{l}\text { Expander } \\
\text { placement } \\
\text { procedure }\end{array}$ & $38 / 1221$ procedures (3.1) & $\mathrm{N} / \mathrm{A}$ & $\mathrm{N} / \mathrm{A}$ \\
\hline & NR & $\begin{array}{l}\text { Single } \\
\text { group }\end{array}$ & Moderate & NR & IBR & $\begin{array}{l}\text { Exchange } \\
\text { procedure }\end{array}$ & 20/1055 procedures (1.9) & $\mathrm{N} / \mathrm{A}$ & $\mathrm{N} / \mathrm{A}$ \\
\hline & NR & $\begin{array}{l}\text { Single } \\
\text { group }\end{array}$ & Moderate & NR & IBR & $\begin{array}{l}\text { Prior } \\
\text { radiation }\end{array}$ & $7 / 136$ procedures $(5.1)$ & $\mathrm{N} / \mathrm{A}$ & $\mathrm{N} / \mathrm{A}$ \\
\hline & NR & $\begin{array}{l}\text { Single } \\
\text { group }\end{array}$ & Moderate & NR & IBR & $\begin{array}{l}\text { No prior } \\
\text { radiation }\end{array}$ & $51 / 2140$ procedures $(2.4)$ & $\mathrm{N} / \mathrm{A}$ & N/A \\
\hline & NR & $\begin{array}{l}\text { Single } \\
\text { group }\end{array}$ & Moderate & NR & IBR & Immediate & $37 / 1176$ procedures $(3.1)$ & $\mathrm{N} / \mathrm{A}$ & N/A \\
\hline & NR & $\begin{array}{l}\text { Single } \\
\text { group }\end{array}$ & Moderate & NR & IBR & Delayed & $1 / 26$ procedures (3.8) & $\mathrm{N} / \mathrm{A}$ & $\mathrm{N} / \mathrm{A}$ \\
\hline \multirow[t]{3}{*}{$\begin{array}{l}\text { Cordeiro, 2012, 22286416, } \\
\text { US }\end{array}$} & NR & $\begin{array}{l}\text { Single } \\
\text { group }\end{array}$ & Low & $<1 \mathrm{y}$ & IBR & All & $85 / 1699(5.0)$ & $\mathrm{N} / \mathrm{A}$ & N/A \\
\hline & NR & $\begin{array}{l}\text { Single } \\
\text { group }\end{array}$ & Low & $<1 \mathrm{y}$ & IBR & $\begin{array}{l}\text { Prior } \\
\text { radiation }\end{array}$ & $10 / 121(8.3)$ & $\mathrm{N} / \mathrm{A}$ & $\mathrm{N} / \mathrm{A}$ \\
\hline & NR & $\begin{array}{l}\text { Single } \\
\text { group }\end{array}$ & Low & $<1 \mathrm{y}$ & IBR & $\begin{array}{l}\text { No prior } \\
\text { radiation }\end{array}$ & $75 / 1578(4.8)$ & $\mathrm{N} / \mathrm{A}$ & N/A \\
\hline
\end{tabular}




\begin{tabular}{|c|c|c|c|c|c|c|c|c|c|}
\hline $\begin{array}{l}\text { Study, Year, PMID, } \\
\text { Country }\end{array}$ & $\begin{array}{l}\text { Outcome } \\
\text { Description }\end{array}$ & Design & $\begin{array}{l}\text { Overall } \\
\text { RoB }\end{array}$ & $\begin{array}{l}\text { Time } \\
\text { Point }\end{array}$ & Arm & Subgroup & $\mathrm{n} / \mathrm{N}(\%)$ & Effect Size $(95 \% \mathrm{Cl})$ & $\begin{array}{l}\mathbf{P} \\
\text { Value }\end{array}$ \\
\hline $\begin{array}{l}\text { Cordeiro, 2015b, } \\
26090764, \text { US }\end{array}$ & NR & $\begin{array}{l}\text { Single } \\
\text { group }\end{array}$ & Low & $2 y$ & IBR & All & 152/4912 implants (3.1) & $\mathrm{N} / \mathrm{A}$ & N/A \\
\hline $\begin{array}{l}\text { Gfrerer, 2015, 25626807, } \\
\text { US }\end{array}$ & NR & $\begin{array}{l}\text { Single } \\
\text { group }\end{array}$ & Low & $7 y$ & IBR & All & $92 / 3142(2.9)$ & $\mathrm{N} / \mathrm{A}$ & $\mathrm{N} / \mathrm{A}$ \\
\hline \multirow[t]{2}{*}{$\begin{array}{l}\text { Hansen, 2018, 29778821, } \\
\text { US }\end{array}$} & NR & $\begin{array}{l}\text { Single } \\
\text { group }\end{array}$ & Low & $1 \mathrm{mo}$ & IBR & All & $28 / 930(3.0)$ & $\mathrm{N} / \mathrm{A}$ & $\mathrm{N} / \mathrm{A}$ \\
\hline & NR & $\begin{array}{l}\text { Single } \\
\text { group }\end{array}$ & Low & $1 \mathrm{mo}$ & IBR & All & $64 / 930(6.9)$ & $\mathrm{N} / \mathrm{A}$ & $\mathrm{N} / \mathrm{A}$ \\
\hline $\begin{array}{l}\text { Hunsicker, 2017, } \\
26849284, \text { US }\end{array}$ & NR & $\begin{array}{l}\text { Single } \\
\text { group }\end{array}$ & Low & $<1 \mathrm{y}$ & IBR & All & $48 / 1584$ breasts $(3.0)$ & $\mathrm{N} / \mathrm{A}$ & N/A \\
\hline Huo, 2016, 27697676, US & NR & $\begin{array}{l}\text { Single } \\
\text { group }\end{array}$ & Low & $1 \mathrm{y}$ & IBR & All & $257 / 1332(19.3)$ & $\mathrm{N} / \mathrm{A}$ & $\mathrm{N} / \mathrm{A}$ \\
\hline $\begin{array}{l}\text { Kato, 2013, 24011080, } \\
\text { Japan }\end{array}$ & $\begin{array}{l}\text { Redness, pain, } \\
\text { or fever, or } \\
\text { positive } \\
\text { bacterial culture }\end{array}$ & $\begin{array}{l}\text { Single } \\
\text { group }\end{array}$ & Low & $<1 \mathrm{y}$ & IBR & All & $47 / 981(4.8)$ & $\mathrm{N} / \mathrm{A}$ & $\mathrm{N} / \mathrm{A}$ \\
\hline \multirow[t]{2}{*}{ Law, 2018, 30463754, US } & NR & $\begin{array}{l}\text { Single } \\
\text { group }\end{array}$ & Low & $3 \mathrm{mo}$ & IBR & All & 2086/11039 (18.9) & $\mathrm{N} / \mathrm{A}$ & N/A \\
\hline & NR & $\begin{array}{l}\text { Single } \\
\text { group }\end{array}$ & Low & $1 \mathrm{y}$ & IBR & All & $2661 / 11039(24.1)$ & $\mathrm{N} / \mathrm{A}$ & $\mathrm{N} / \mathrm{A}$ \\
\hline $\begin{array}{l}\text { Lee, 2021a, 32974692, } \\
\text { South Korea }\end{array}$ & NR & $\begin{array}{l}\text { Single } \\
\text { group }\end{array}$ & Low & $5 y$ & IBR & All & $10 / 605$ breasts $(1.7)$ & $\mathrm{N} / \mathrm{A}$ & $\mathrm{N} / \mathrm{A}$ \\
\hline $\begin{array}{l}\text { Lovecchio, 2015, } \\
\text { 24691330, US }\end{array}$ & $\begin{array}{l}\text { Infections } \\
\text { requiring IV } \\
\text { antibiotics }\end{array}$ & $\begin{array}{l}\text { Single } \\
\text { group }\end{array}$ & Low & $2.3 \mathrm{y}$ & IBR & All & $67 / 1639(4.1)$ & $\mathrm{N} / \mathrm{A}$ & $\mathrm{N} / \mathrm{A}$ \\
\hline $\begin{array}{l}\text { Parikh, 2018, 30204676, } \\
\text { US }\end{array}$ & SSI & $\begin{array}{l}\text { Single } \\
\text { group }\end{array}$ & Low & $>2 y$ & IBR & All & $139 / 1285(10.8)$ & $\mathrm{N} / \mathrm{A}$ & $\mathrm{N} / \mathrm{A}$ \\
\hline $\begin{array}{l}\text { Park, 2019, 30863940, } \\
\text { South Korea }\end{array}$ & NR & $\begin{array}{l}\text { Single } \\
\text { group }\end{array}$ & Moderate & $>6 \mathrm{mo}$ & IBR & All & 29/999 (2.9) & $\mathrm{N} / \mathrm{A}$ & $\mathrm{N} / \mathrm{A}$ \\
\hline $\begin{array}{l}\text { Rogoff, 2020, 32243320, } \\
\text { US }\end{array}$ & NR & $\begin{array}{l}\text { Single } \\
\text { group }\end{array}$ & Low & NR & IBR & All & $155 / 627(24.7)$ & $\mathrm{N} / \mathrm{A}$ & $\mathrm{N} / \mathrm{A}$ \\
\hline Seth, 2015,25180955, US & $\begin{array}{l}\text { Infections } \\
\text { requiring IV } \\
\text { antibiotics } \\
\text { and/or } \\
\text { readmission }\end{array}$ & $\begin{array}{l}\text { Single } \\
\text { group }\end{array}$ & Low & NR & IBR & All & $59 / 893(6.6)$ & $\mathrm{N} / \mathrm{A}$ & $\mathrm{N} / \mathrm{A}$ \\
\hline $\begin{array}{l}\text { Sewart, 2021, 33609398, } \\
\text { UK }\end{array}$ & NR & $\begin{array}{l}\text { Single } \\
\text { group }\end{array}$ & Low & $3 \mathrm{mo}$ & IBR & All & $229 / 691(25.7)$ & $\mathrm{N} / \mathrm{A}$ & $\mathrm{N} / \mathrm{A}$ \\
\hline \multirow[t]{2}{*}{ Singh, 2012, 22342636, US } & $\mathrm{NR}$ & $\begin{array}{l}\text { Single } \\
\text { group }\end{array}$ & Low & $6 \mathrm{mo}$ & IBR & All & $161 / 1316(12.2)$ & $\mathrm{N} / \mathrm{A}$ & $\mathrm{N} / \mathrm{A}$ \\
\hline & NR & $\begin{array}{l}\text { Single } \\
\text { group }\end{array}$ & Low & $6 \mathrm{mo}$ & IBR & DTI & $9 / 95(9.5)$ & $\mathrm{N} / \mathrm{A}$ & $\mathrm{N} / \mathrm{A}$ \\
\hline
\end{tabular}




\begin{tabular}{|c|c|c|c|c|c|c|c|c|c|}
\hline $\begin{array}{l}\text { Study, Year, PMID, } \\
\text { Country }\end{array}$ & $\begin{array}{l}\text { Outcome } \\
\text { Description }\end{array}$ & Design & $\begin{array}{l}\text { Overall } \\
\text { RoB }\end{array}$ & $\begin{array}{l}\text { Time } \\
\text { Point }\end{array}$ & Arm & Subgroup & $\mathrm{n} / \mathrm{N}(\%)$ & Effect Size $(95 \% \mathrm{CI})$ & $\begin{array}{l}P \\
\text { Value }\end{array}$ \\
\hline & NR & $\begin{array}{l}\text { Single } \\
\text { group }\end{array}$ & Low & $6 \mathrm{mo}$ & IBR & T/E & $152 / 1221(12.4)$ & $\mathrm{N} / \mathrm{A}$ & $\mathrm{N} / \mathrm{A}$ \\
\hline \multirow[t]{3}{*}{ Singh, 2021, 33564597, US } & NR & $\begin{array}{l}\text { Single } \\
\text { group }\end{array}$ & Low & $3 \mathrm{mo}$ & IBR & All & $95 / 1740(5.47)$ & $\mathrm{N} / \mathrm{A}$ & $\mathrm{N} / \mathrm{A}$ \\
\hline & NR & $\begin{array}{l}\text { Single } \\
\text { group }\end{array}$ & Low & $3 \mathrm{mo}$ & IBR & $\mathrm{DTI}$ & $41 / 870(4.66)$ & $\mathrm{N} / \mathrm{A}$ & $\mathrm{N} / \mathrm{A}$ \\
\hline & NR & $\begin{array}{l}\text { Single } \\
\text { group }\end{array}$ & Low & $3 \mathrm{mo}$ & IBR & $\mathrm{T} / \mathrm{E}$ & $55 / 870(6.27)$ & $\mathrm{N} / \mathrm{A}$ & $\mathrm{N} / \mathrm{A}$ \\
\hline $\begin{array}{l}\text { Warren, 2020, 33040748, } \\
\text { US }\end{array}$ & $\begin{array}{l}\text { Surgical site } \\
\text { infection }\end{array}$ & $\begin{array}{l}\text { Single } \\
\text { group }\end{array}$ & Moderate & NR & IBR & All & $82 / 1924(4.3)$ & $\mathrm{N} / \mathrm{A}$ & $\mathrm{N} / \mathrm{A}$ \\
\hline $\begin{array}{l}\text { Beugels, 2018, 29399731, } \\
\text { Netherlands }\end{array}$ & NR & $\begin{array}{l}\text { Single } \\
\text { group }\end{array}$ & Low & $\begin{array}{l}9-10 \\
\text { mo }\end{array}$ & AR & All & $63 / 910$ flaps $(6.9)$ & $\mathrm{N} / \mathrm{A}$ & $\mathrm{N} / \mathrm{A}$ \\
\hline $\begin{array}{l}\text { Chang, 2000, 10809092, } \\
\text { US }\end{array}$ & Flap infection & $\begin{array}{l}\text { Single } \\
\text { group }\end{array}$ & Low & NR & AR & All & 17/936 flaps (1.8) & $\mathrm{N} / \mathrm{A}$ & $\mathrm{N} / \mathrm{A}$ \\
\hline \multirow[t]{5}{*}{$\begin{array}{l}\text { Chang, 2011, 21407063, } \\
\text { US }\end{array}$} & NR & $\begin{array}{l}\text { Single } \\
\text { group }\end{array}$ & Low & NR & AR & All & $3 / 818(0.4)$ & $\mathrm{N} / \mathrm{A}$ & $\mathrm{N} / \mathrm{A}$ \\
\hline & NR & $\begin{array}{l}\text { Single } \\
\text { group }\end{array}$ & Low & NR & AR & Age $<50$ & $2 / 411(0.5)$ & $\mathrm{N} / \mathrm{A}$ & $\mathrm{N} / \mathrm{A}$ \\
\hline & NR & $\begin{array}{l}\text { Single } \\
\text { group }\end{array}$ & Low & NR & AR & Age 50-59 & $0 / 285(0)$ & $\mathrm{N} / \mathrm{A}$ & $\mathrm{N} / \mathrm{A}$ \\
\hline & NR & $\begin{array}{l}\text { Single } \\
\text { group }\end{array}$ & Low & NR & AR & Age 60-69 & $1 / 103(1)$ & $\mathrm{N} / \mathrm{A}$ & $\mathrm{N} / \mathrm{A}$ \\
\hline & NR & $\begin{array}{l}\text { Single } \\
\text { group }\end{array}$ & Low & NR & AR & Age $>70$ & $0 / 19(0)$ & $\mathrm{N} / \mathrm{A}$ & $\mathrm{N} / \mathrm{A}$ \\
\hline $\begin{array}{l}\text { Chen, 2018b, 29596085, } \\
\text { US }\end{array}$ & NR & $\begin{array}{l}\text { Single } \\
\text { group }\end{array}$ & Low & NR & AR & All & $158 / 19496(0.8)$ & $\mathrm{N} / \mathrm{A}$ & $\mathrm{N} / \mathrm{A}$ \\
\hline $\begin{array}{l}\text { Cleveland, 2013, } \\
\text { 23945529, US }\end{array}$ & $\begin{array}{l}\text { Cellulitis at } \\
\text { donor site } \\
\text { treated with } \\
\text { antibiotics }\end{array}$ & $\begin{array}{l}\text { Single } \\
\text { group }\end{array}$ & Low & $1 \mathrm{mo}$ & AR & All & $60 / 812(7.4)$ & $\mathrm{N} / \mathrm{A}$ & $\mathrm{N} / \mathrm{A}$ \\
\hline Daly, 2020, 31994156, US & NR & $\begin{array}{l}\text { Single } \\
\text { group }\end{array}$ & Low & $12 \mathrm{y}$ & AR & All & $66 / 818(8.1)$ & $\mathrm{N} / \mathrm{A}$ & $\mathrm{N} / \mathrm{A}$ \\
\hline $\begin{array}{l}\text { Enajat, 2010, 19790180, } \\
\text { Sweden }\end{array}$ & NR & $\begin{array}{l}\text { Single } \\
\text { group }\end{array}$ & Low & $1-7 d$ & AR & All & 60/564 flaps (10.6) & $\mathrm{N} / \mathrm{A}$ & $\mathrm{N} / \mathrm{A}$ \\
\hline Gill, 2004, 15083015, US & Any infection & $\begin{array}{l}\text { Single } \\
\text { group }\end{array}$ & Moderate & $10 y$ & AR & All & $21 / 758(2.8)$ & $\mathrm{N} / \mathrm{A}$ & $\mathrm{N} / \mathrm{A}$ \\
\hline $\begin{array}{l}\text { Haddock, 2019, 31461004, } \\
\text { US }\end{array}$ & NR & $\begin{array}{l}\text { Single } \\
\text { group }\end{array}$ & Moderate & NR & AR & All & $40 / 509(7.8)$ & $\mathrm{N} / \mathrm{A}$ & $\mathrm{N} / \mathrm{A}$ \\
\hline $\begin{array}{l}\text { Jo, 2020, 33386262, South } \\
\text { Korea }\end{array}$ & NR & $\begin{array}{l}\text { Single } \\
\text { group }\end{array}$ & Low & $\begin{array}{l}11 \mathrm{mo} \\
-1.5 \mathrm{y}\end{array}$ & AR & All & $7 / 623(1.12)$ & $\mathrm{N} / \mathrm{A}$ & $\mathrm{N} / \mathrm{A}$ \\
\hline $\begin{array}{l}\text { Masoomi, 2019, 31331721, } \\
\text { US }\end{array}$ & $\mathrm{WI}$ & $\begin{array}{l}\text { Single } \\
\text { group }\end{array}$ & Low & NR & $\mathrm{AR}$ & All & $750 / 55840(1.3)$ & $\mathrm{N} / \mathrm{A}$ & $\mathrm{N} / \mathrm{A}$ \\
\hline
\end{tabular}




\begin{tabular}{|c|c|c|c|c|c|c|c|c|c|}
\hline $\begin{array}{l}\text { Study, Year, PMID, } \\
\text { Country }\end{array}$ & $\begin{array}{l}\text { Outcome } \\
\text { Description }\end{array}$ & Design & $\begin{array}{l}\text { Overall } \\
\text { RoB }\end{array}$ & $\begin{array}{l}\text { Time } \\
\text { Point }\end{array}$ & Arm & Subgroup & $\mathrm{n} / \mathrm{N}(\%)$ & Effect Size $(95 \% \mathrm{Cl})$ & $\begin{array}{l}\mathbf{P} \\
\text { Value }\end{array}$ \\
\hline \multirow[t]{2}{*}{$\begin{array}{l}\text { Nelson, 2014, 25046665, } \\
\text { US }\end{array}$} & Breast WI & $\begin{array}{l}\text { Single } \\
\text { group }\end{array}$ & Moderate & NR & AR & All & $60 / 1293$ flaps (4.6) & $\mathrm{N} / \mathrm{A}$ & $\mathrm{N} / \mathrm{A}$ \\
\hline & Donor site WI & $\begin{array}{l}\text { Single } \\
\text { group }\end{array}$ & Moderate & NR & AR & All & 25/1293 flaps (1.9) & $\mathrm{N} / \mathrm{A}$ & $\mathrm{N} / \mathrm{A}$ \\
\hline \multirow[t]{2}{*}{$\begin{array}{l}\text { O'Neill, 2019, 31196805, } \\
\text { Canada }\end{array}$} & NR & $\begin{array}{l}\text { Single } \\
\text { group }\end{array}$ & Low & $1 \mathrm{~d}$ & AR & All & $12 / 512(2.3)$ & $\mathrm{N} / \mathrm{A}$ & $\mathrm{N} / \mathrm{A}$ \\
\hline & NR & $\begin{array}{l}\text { Single } \\
\text { group }\end{array}$ & Low & $<2 \mathrm{mo}$ & AR & All & $39 / 960(4.0)$ & $\mathrm{N} / \mathrm{A}$ & $\mathrm{N} / \mathrm{A}$ \\
\hline \multirow[t]{2}{*}{$\begin{array}{l}\text { Prantl, 2020, 32895743, } \\
\text { Germany }\end{array}$} & Breast WI & $\begin{array}{l}\text { Single } \\
\text { group }\end{array}$ & Low & $3 \mathrm{mo}$ & AR & All & $20 / 4577$ flaps $(0.4)$ & $\mathrm{N} / \mathrm{A}$ & $\mathrm{N} / \mathrm{A}$ \\
\hline & Donor site WI & $\begin{array}{l}\text { Single } \\
\text { group }\end{array}$ & Low & $3 \mathrm{mo}$ & AR & All & $23 / 4577$ flaps $(0.5)$ & $\mathrm{N} / \mathrm{A}$ & $\mathrm{N} / \mathrm{A}$ \\
\hline $\begin{array}{l}\text { Rubio, 2019, 30665841, } \\
\text { Belgium }\end{array}$ & WI & $\begin{array}{l}\text { Single } \\
\text { group }\end{array}$ & Low & NR & AR & All & $735 / 56522(1.3)$ & $\mathrm{N} / \mathrm{A}$ & $\mathrm{N} / \mathrm{A}$ \\
\hline $\begin{array}{l}\text { Seidenstuecker, } 2016 \text {, } \\
27017243 \text {, Belgium }\end{array}$ & $\begin{array}{l}\text { Systemic } \\
\text { infection }\end{array}$ & $\begin{array}{l}\text { Single } \\
\text { group }\end{array}$ & Moderate & NR & AR & All & 0/931 flaps $(0)$ & $\mathrm{N} / \mathrm{A}$ & $\mathrm{N} / \mathrm{A}$ \\
\hline $\begin{array}{l}\text { Selber, 2009, 19935283, } \\
\text { US }\end{array}$ & WI & $\begin{array}{l}\text { Single } \\
\text { group }\end{array}$ & Low & $5.6 \mathrm{mo}$ & AR & All & $37 / 1031(3.6)$ & $\mathrm{N} / \mathrm{A}$ & $\mathrm{N} / \mathrm{A}$ \\
\hline Song, 2016, 26637165, US & NR & $\begin{array}{l}\text { Single } \\
\text { group }\end{array}$ & Low & $\begin{array}{l}4.3-5.4 \\
y\end{array}$ & AR & All & $186 / 1809(10.3)$ & $\mathrm{N} / \mathrm{A}$ & $\mathrm{N} / \mathrm{A}$ \\
\hline Tran, 2018, 29794694, US & NR & $\begin{array}{l}\text { Single } \\
\text { group }\end{array}$ & Low & NR & AR & All & $67 / 879(5.3)$ & $\mathrm{N} / \mathrm{A}$ & $\mathrm{N} / \mathrm{A}$ \\
\hline $\begin{array}{l}\text { Watterson, 1995, 7761505, } \\
\text { Australia }\end{array}$ & WI & $\begin{array}{l}\text { Single } \\
\text { group }\end{array}$ & Moderate & $2.7 \mathrm{y}$ & AR & All & $28 / 556(5.0)$ & $\mathrm{N} / \mathrm{A}$ & $\mathrm{N} / \mathrm{A}$ \\
\hline $\begin{array}{l}\text { Williams, 1995, 7794079, } \\
\text { US }\end{array}$ & Major infection & $\begin{array}{l}\text { Single } \\
\text { group }\end{array}$ & Moderate & $2.4 \mathrm{y}$ & AR & All & $23 / 607$ (3.8) & $\mathrm{N} / \mathrm{A}$ & $\mathrm{N} / \mathrm{A}$ \\
\hline \multirow[t]{2}{*}{$\begin{array}{l}\text { Yoo, 2014, 24852813, } \\
\text { South Korea }\end{array}$} & Breast WI & $\begin{array}{l}\text { Single } \\
\text { group }\end{array}$ & Moderate & NR & AR & All & $4 / 500(0.8)$ & $\mathrm{N} / \mathrm{A}$ & $\mathrm{N} / \mathrm{A}$ \\
\hline & Donor WI & $\begin{array}{l}\text { Single } \\
\text { group }\end{array}$ & Moderate & NR & AR & All & $5 / 500(1.0)$ & $\mathrm{N} / \mathrm{A}$ & $\mathrm{N} / \mathrm{A}$ \\
\hline
\end{tabular}

Abbreviations: adj $=$ adjusted, $\mathrm{AR}=$ autologous reconstruction, $\mathrm{CI}=$ confidence interval, $\mathrm{d}=$ days, $\mathrm{DIEP}=$ deep inferior epigastric perforator, $\mathrm{DTI}=$ direct to implant, $\mathrm{IBR}=$

implant-based reconstruction, $\mathrm{LD}=$ latissimus dorsi, $\mathrm{mo}=$ months, $\mathrm{NR}=$ not reported, $\mathrm{NRCS}=$ nonrandomized comparative study, $\mathrm{OR}=$ odds ratio, $\mathrm{PMID}=\mathrm{PubMed}$ identifier,

Ref $=$ reference group, $\mathrm{RoB}=$ risk of bias, $\mathrm{SSI}=$ surgical site infection, $\mathrm{T} / \mathrm{E}=$ tissue expander, $\mathrm{WI}=$ wound infection, $\mathrm{y}=\mathrm{years}$.

Colors: Header rows are shaded orange. Rows for every alternate study are shaded blue. The colors do not add unique information.

Table F-1.20. Full Evidence Table - Key Question 1: IBR versus AR - categorical outcomes (harms - wound dehiscence)

\begin{tabular}{|c|c|c|c|c|c|c|c|c|c|}
\hline $\begin{array}{l}\text { Study, Year, PMID, } \\
\text { Country }\end{array}$ & $\begin{array}{l}\text { Outcome } \\
\text { Description }\end{array}$ & Design & $\begin{array}{l}\text { Overall } \\
\text { RoB }\end{array}$ & $\begin{array}{l}\text { Time } \\
\text { Point }\end{array}$ & Arm & Subgroup & $n / N(\%)$ & Effect Size $(95 \% \mathrm{Cl})$ & $\begin{array}{l}\mathbf{P} \\
\text { Value }\end{array}$ \\
\hline \multirow{3}{*}{$\begin{array}{l}\text { Garvey, 2012, } \\
23096600, \text { US }\end{array}$} & NR & NRCS & Moderate & $1.5 \mathrm{y}$ & IBR & All & $28 / / 442$ breasts $(6.3)$ & Ref & Ref \\
\hline & NR & NRCS & Moderate & $1.5 \mathrm{y}$ & AR & All & $25 / 548$ breasts (4.6) & NR & 0.25 \\
\hline & Breast WD & NRCS & Moderate & $1 \mathrm{y}$ & IBR & All & $26 / 1615(1.6)$ & NR & NR \\
\hline
\end{tabular}




\begin{tabular}{|c|c|c|c|c|c|c|c|c|c|}
\hline $\begin{array}{l}\text { Study, Year, PMID, } \\
\text { Country }\end{array}$ & $\begin{array}{l}\text { Outcome } \\
\text { Description }\end{array}$ & Design & $\begin{array}{l}\text { Overall } \\
\text { RoB }\end{array}$ & $\begin{array}{l}\text { Time } \\
\text { Point }\end{array}$ & Arm & Subgroup & $\mathrm{n} / \mathrm{N}(\%)$ & Effect Size $(95 \% \mathrm{Cl})$ & $\begin{array}{l}\mathbf{P} \\
\text { Value }\end{array}$ \\
\hline \multirow{6}{*}{$\begin{array}{l}\text { Kulkarni, 2017, } \\
\text { 28713853, US \& } \\
\text { Canada }\end{array}$} & Breast WD & NRCS & Moderate & $1 \mathrm{y}$ & AR (all) & All & NR & NR & NR \\
\hline & Breast WD & NRCS & Moderate & $1 \mathrm{y}$ & AR with DIEP & All & $13 / 365(3.6)$ & NR & NR \\
\hline & Breast WD & NRCS & Moderate & $1 \mathrm{y}$ & $\begin{array}{l}\text { AR with free } \\
\text { TRAM }\end{array}$ & All & $1 / 97(1)$ & NR & NR \\
\hline & Breast WD & NRCS & Moderate & $1 \mathrm{y}$ & $\begin{array}{l}\text { AR with } \\
\text { pedicled } \\
\text { TRAM } \\
\end{array}$ & All & $1 / 84(1.2)$ & NR & NR \\
\hline & Breast WD & NRCS & Moderate & $1 \mathrm{y}$ & AR with LD & All & $1 / 73(1.4)$ & NR & NR \\
\hline & Breast WD & NRCS & Moderate & $1 \mathrm{y}$ & AR with SIEA & All & NR & NR & NR \\
\hline \multirow[t]{2}{*}{$\begin{array}{l}\text { Mioton, 2013, } \\
\text { 23562485, US }\end{array}$} & $\begin{array}{l}\text { Wound } \\
\text { disruption }\end{array}$ & NRCS & Moderate & $1 \mathrm{mo}$ & IBR & All & $44 * / 9786(0.44)$ & Ref & Ref \\
\hline & $\begin{array}{l}\text { Wound } \\
\text { disruption }\end{array}$ & NRCS & Moderate & $1 \mathrm{mo}$ & AR & All & $41 * / 3296(1.24)$ & adjOR $1.79(0.83,3.84)$ & NR \\
\hline \multirow{2}{*}{$\begin{array}{l}\text { Banuelos, 2020, } \\
\text { 31663932, US }\end{array}$} & NR & Single group & Low & $\leq 1 \mathrm{mo}$ & IBR & All & $15 / 768(1.9)$ & $\mathrm{N} / \mathrm{A}$ & $\mathrm{N} / \mathrm{A}$ \\
\hline & NR & Single group & Low & $>1 \mathrm{mo}$ & IBR & All & $12 / 768(1.6)$ & $\mathrm{N} / \mathrm{A}$ & $\mathrm{N} / \mathrm{A}$ \\
\hline \multirow{3}{*}{$\begin{array}{l}\text { Brooks, 2012, } \\
22098451, \text { US }\end{array}$} & NR & Single group & Low & $3.4 \mathrm{y}$ & IBR & All & $15 / 733(2.1)$ & $\mathrm{N} / \mathrm{A}$ & $\mathrm{N} / \mathrm{A}$ \\
\hline & NR & Single group & Low & $3.4 \mathrm{y}$ & IBR & $\begin{array}{l}\text { Prior } \\
\text { radiation }\end{array}$ & $8 / 97(11.9)$ & $\mathrm{N} / \mathrm{A}$ & $\mathrm{N} / \mathrm{A}$ \\
\hline & NR & Single group & Low & $3.4 \mathrm{y}$ & IBR & $\begin{array}{l}\text { No prior } \\
\text { radiation }\end{array}$ & $7 / 636(1.1)$ & $\mathrm{N} / \mathrm{A}$ & $\mathrm{N} / \mathrm{A}$ \\
\hline $\begin{array}{l}\text { Chen, 2018a, } \\
\text { 29596085, US }\end{array}$ & $\begin{array}{l}\text { Wound } \\
\text { disruption/compl } \\
\text { ication }\end{array}$ & Single group & Low & NR & IBR & All & $154 / 23048(0.7)$ & $\mathrm{N} / \mathrm{A}$ & $\mathrm{N} / \mathrm{A}$ \\
\hline \multirow[t]{3}{*}{$\begin{array}{l}\text { Cordeiro, 2012, } \\
22286416, \text { US }\end{array}$} & $\begin{array}{l}\text { WD or delayed } \\
\text { healing }\end{array}$ & Single group & Low & $<1 \mathrm{y}$ & IBR & All & $5 / 1699(0.3)$ & $\mathrm{N} / \mathrm{A}$ & $\mathrm{N} / \mathrm{A}$ \\
\hline & $\begin{array}{l}\text { WD or delayed } \\
\text { healing }\end{array}$ & Single group & Low & $<1 \mathrm{y}$ & IBR & $\begin{array}{l}\text { Prior } \\
\text { radiation }\end{array}$ & $1 / 121(0.8)$ & $\mathrm{N} / \mathrm{A}$ & $\mathrm{N} / \mathrm{A}$ \\
\hline & $\begin{array}{l}\text { WD or delayed } \\
\text { healing }\end{array}$ & Single group & Low & $<1 \mathrm{y}$ & IBR & $\begin{array}{l}\text { No prior } \\
\text { radiation }\end{array}$ & $4 / 1578(0.3)$ & $\mathrm{N} / \mathrm{A}$ & $\mathrm{N} / \mathrm{A}$ \\
\hline \multirow{3}{*}{$\begin{array}{l}\text { Huo, 2016, 27697676, } \\
\text { US }\end{array}$} & NR & Single group & Low & $1 \mathrm{y}$ & IBR & All & $41 / 1332(3.1)$ & $\mathrm{N} / \mathrm{A}$ & $\mathrm{N} / \mathrm{A}$ \\
\hline & NR & Single group & Low & $1 \mathrm{y}$ & IBR & Non-obese & $27 / 949(2.8)$ & $\mathrm{N} / \mathrm{A}$ & N/A \\
\hline & NR & Single group & Low & $1 \mathrm{y}$ & IBR & Obese & $14 / 383(3.6)$ & $\mathrm{N} / \mathrm{A}$ & $\mathrm{N} / \mathrm{A}$ \\
\hline $\begin{array}{l}\text { Lee, 2021a, 32974692, } \\
\text { South Korea }\end{array}$ & NR & & Low & $5 y$ & IBR & All & $13 / 605$ breasts $(2.1)$ & $\mathrm{N} / \mathrm{A}$ & $\mathrm{N} / \mathrm{A}$ \\
\hline $\begin{array}{l}\text { Parikh, 2018, } \\
30204676, \text { US }\end{array}$ & NR & Single group & Low & $>2 y$ & IBR & All & $21 / 1285(1.6)$ & $\mathrm{N} / \mathrm{A}$ & N/A \\
\hline $\begin{array}{l}\text { Park, 2019, 30863940, } \\
\text { South Korea }\end{array}$ & NR & Single group & Moderate & $>6 \mathrm{mo}$ & IBR & All & $49 / 999(4.9)$ & $\mathrm{N} / \mathrm{A}$ & $\mathrm{N} / \mathrm{A}$ \\
\hline \multirow[t]{2}{*}{$\begin{array}{l}\text { Rogoff, } 2020 \\
32243320, \text { US }\end{array}$} & NR & Single group & Low & NR & IBR & All & $69 / 627(11)$ & $\mathrm{N} / \mathrm{A}$ & $\mathrm{N} / \mathrm{A}$ \\
\hline & NR & Single group & Low & $3 \mathrm{mo}$ & IBR & All & $58 / 1740(3.35)$ & $\mathrm{N} / \mathrm{A}$ & $\mathrm{N} / \mathrm{A}$ \\
\hline
\end{tabular}




\begin{tabular}{|c|c|c|c|c|c|c|c|c|c|}
\hline $\begin{array}{l}\text { Study, Year, PMID, } \\
\text { Country }\end{array}$ & $\begin{array}{l}\text { Outcome } \\
\text { Description }\end{array}$ & Design & $\begin{array}{l}\text { Overall } \\
\text { RoB }\end{array}$ & $\begin{array}{l}\text { Time } \\
\text { Point }\end{array}$ & Arm & Subgroup & $n / N(\%)$ & Effect Size (95\% Cl) & $\begin{array}{l}\mathbf{P} \\
\text { Value }\end{array}$ \\
\hline \multirow{2}{*}{$\begin{array}{l}\text { Singh, 2021, } \\
33564597, \text { US }\end{array}$} & NR & Single group & Low & $3 \mathrm{mo}$ & IBR & DTI & $22 / 870(2.54)$ & N/A & N/A \\
\hline & NR & Single group & Low & $3 \mathrm{mo}$ & & $\mathrm{T} / \mathrm{E}$ & $36 / 870(4.15)$ & $\mathrm{N} / \mathrm{A}$ & $\mathrm{N} / \mathrm{A}$ \\
\hline $\begin{array}{l}\text { Albornoz, 2013, } \\
23897346, \text { US }\end{array}$ & NR & Single group & Low & NR & AR & All & $84 / 21016(0.4)$ & N/A & N/A \\
\hline \multirow{5}{*}{$\begin{array}{l}\text { Chang, 2011, } \\
21407063, \text { us }\end{array}$} & NR & Single group & Low & NR & AR & All & $1 / 818(0.1)$ & $\mathrm{N} / \mathrm{A}$ & $\mathrm{N} / \mathrm{A}$ \\
\hline & NR & Single group & Low & NR & AR & Age $<50$ & $0 / 411(0)$ & N/A & N/A \\
\hline & NR & Single group & Low & NR & AR & Age 50-59 & $1 / 285(0.4)$ & N/A & $\mathrm{N} / \mathrm{A}$ \\
\hline & NR & Single group & Low & NR & $\mathrm{AR}$ & Age $60-69$ & $0 / 103(0)$ & N/A & $\mathrm{N} / \mathrm{A}$ \\
\hline & NR & Single group & Low & NR & $\mathrm{AR}$ & Age $>70$ & $0 / 19(0)$ & N/A & $\mathrm{N} / \mathrm{A}$ \\
\hline $\begin{array}{l}\text { Chen, 2018b, } \\
\text { 29596085, US }\end{array}$ & NR & Single group & Low & NR & AR & All & $84 / 19496(0.4)$ & N/A & N/A \\
\hline $\begin{array}{l}\text { Daly, 2020, 31994156, } \\
\text { US }\end{array}$ & NR & Single group & Low & $12 \mathrm{y}$ & AR & All & 93/818 (11.4) & N/A & N/A \\
\hline $\begin{array}{l}\text { Heo, 2018, 30039735, } \\
\text { South Korea }\end{array}$ & $\begin{array}{l}\text { Wound } \\
\text { problems (i.e. } \\
\text { wound } \\
\text { dehiscence, } \\
\text { wound infection, } \\
\text { wound necrosis, } \\
\text { delayed wound } \\
\text { healing) }\end{array}$ & Single group & Low & $1 \mathrm{y}$ & AR & All & $23 / 615(3.7)$ & $\mathrm{N} / \mathrm{A}$ & $\mathrm{N} / \mathrm{A}$ \\
\hline $\begin{array}{l}\text { Masoomi, 2019, } \\
\text { 31331721, US }\end{array}$ & NR & Single group & Low & NR & AR & All & $436 / 55840(0.8)$ & $\mathrm{N} / \mathrm{A}$ & N/A \\
\hline \multirow{3}{*}{$\begin{array}{l}\text { O'Neill, 2019, } \\
\text { 31196805, Canada }\end{array}$} & NR & Single group & Low & $1 \mathrm{~d}$ & $\mathrm{AR}$ & All & $6 / 512(1.2)$ & N/A & N/A \\
\hline & NR & Single group & Low & $<2 \mathrm{mo}$ & AR & All & $31 / 912(3.4)$ & N/A & $\mathrm{N} / \mathrm{A}$ \\
\hline & NR & Single group & Low & $<3 \mathrm{mo}$ & $\mathrm{AR}$ & All & $38 / 960(3.9)$ & N/A & N/A \\
\hline \multirow{2}{*}{$\begin{array}{l}\text { Prantl, } 2020 \text {, } \\
\text { 32895743, Germany }\end{array}$} & NR & Single group & Low & $3 \mathrm{mo}$ & $\mathrm{AR}$ & All & 80/4577 flaps (1.7) & $\mathrm{N} / \mathrm{A}$ & $\mathrm{N} / \mathrm{A}$ \\
\hline & NR & Single group & Low & $3 \mathrm{mo}$ & AR & All & 70/4577 flaps (1.5) & $\mathrm{N} / \mathrm{A}$ & N/A \\
\hline $\begin{array}{l}\text { Rubio, 2019, } \\
\text { 30665841, Belgium }\end{array}$ & NR & Single group & Low & NR & AR & All & $452 / 56522(0.8)$ & N/A & N/A \\
\hline $\begin{array}{l}\text { Selber, 2009, } \\
\text { 19935283, US }\end{array}$ & NR & Single group & Low & $5.6 \mathrm{mo}$ & AR & All & $3 / 1031(0.3)$ & $\mathrm{N} / \mathrm{A}$ & $\mathrm{N} / \mathrm{A}$ \\
\hline \multirow{2}{*}{$\begin{array}{l}\text { Yoo, 2014, 24852813, } \\
\text { South Korea }\end{array}$} & Breast WD & Single group & Moderate & NR & AR & All & $5 / 500(1.0)$ & $\mathrm{N} / \mathrm{A}$ & $\mathrm{N} / \mathrm{A}$ \\
\hline & Donor site WD & Single group & Moderate & NR & AR & All & $7 / 500(1.4)$ & N/A & N/A \\
\hline
\end{tabular}

Abbreviations: adj = adjusted, $\mathrm{AR}=$ autologous reconstruction, $\mathrm{CI}=$ confidence interval, $\mathrm{d}=$ days, $\mathrm{DIEP}=$ deep inferior epigastric perforator, $\mathrm{DTI}=$ direct to implant, IBR $=$ implant-based reconstruction, $\mathrm{LD}=$ latissimus dorsi, $\mathrm{mo}=$ months, $\mathrm{NR}=$ not reported, NRCS $=$ nonrandomized comparative study, OR $=$ odds ratio, $\mathrm{PMID}=\mathrm{PubMed}$ identifier, Ref $=$ reference group, $\mathrm{RoB}=$ risk of bias, $\mathrm{SSI}=$ surgical site infection, $\mathrm{T} / \mathrm{E}=$ tissue expander, $\mathrm{WD}=$ wound dehiscence, $\mathrm{y}=$ years.

Colors: Header rows are shaded orange. Rows for every alternate study are shaded blue. The colors do not add unique information. 
Table F-1.21. Full Evidence Table - Key Question 1: IBR versus AR - categorical outcomes (harms - delayed healing)

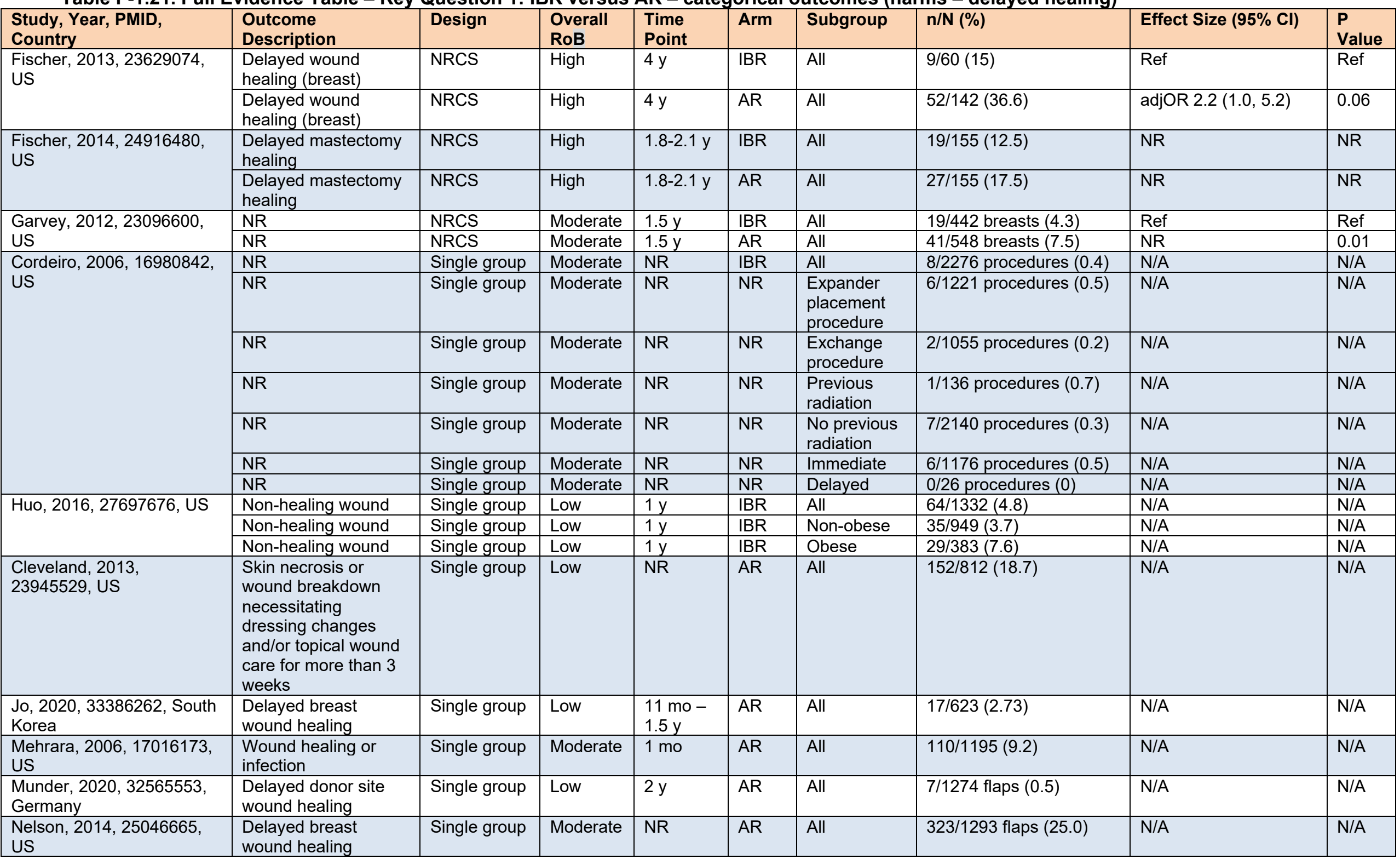




\begin{tabular}{|c|c|c|c|c|c|c|c|c|c|}
\hline $\begin{array}{l}\text { Study, Year, PMID, } \\
\text { Country }\end{array}$ & $\begin{array}{l}\text { Outcome } \\
\text { Description }\end{array}$ & Design & $\begin{array}{l}\text { Overall } \\
\text { RoB }\end{array}$ & $\begin{array}{l}\text { Time } \\
\text { Point }\end{array}$ & Arm & Subgroup & $\mathrm{n} / \mathrm{N}(\%)$ & Effect Size (95\% CI) & $\begin{array}{l}\mathbf{P} \\
\text { Value }\end{array}$ \\
\hline & $\begin{array}{l}\text { Delayed donor site } \\
\text { wound healing }\end{array}$ & Single group & Moderate & NR & AR & All & 149/1293 flaps (11.5) & $\mathrm{N} / \mathrm{A}$ & $\mathrm{N} / \mathrm{A}$ \\
\hline \multirow[t]{2}{*}{$\begin{array}{l}\text { Prantl, 2020, 32895743, } \\
\text { Germany }\end{array}$} & $\begin{array}{l}\text { Delayed breast } \\
\text { wound healing }\end{array}$ & Single group & Low & $3 \mathrm{mo}$ & AR & All & $70 / 4577(1.5)$ & $\mathrm{N} / \mathrm{A}$ & $\mathrm{N} / \mathrm{A}$ \\
\hline & $\begin{array}{l}\text { Delayed donor site } \\
\text { wound healing }\end{array}$ & Single group & Low & $3 \mathrm{mo}$ & AR & All & $80 / 4577(1.7)$ & $\mathrm{N} / \mathrm{A}$ & $\mathrm{N} / \mathrm{A}$ \\
\hline $\begin{array}{l}\text { Rubio, 2019, 30665841, } \\
\text { Belgium }\end{array}$ & NR & Single group & Low & NR & AR & All & $678 / 56522(1.2)$ & $\mathrm{N} / \mathrm{A}$ & $\mathrm{N} / \mathrm{A}$ \\
\hline \multirow[t]{2}{*}{$\begin{array}{l}\text { Selber, 2009, 19935283, } \\
\text { US }\end{array}$} & $\begin{array}{l}\text { Delayed breast } \\
\text { wound healing }\end{array}$ & Single group & Low & $5.6 \mathrm{mo}$ & AR & All & $78 / 1031(7.6)$ & $\mathrm{N} / \mathrm{A}$ & $\mathrm{N} / \mathrm{A}$ \\
\hline & $\begin{array}{l}\text { Delayed donor site } \\
\text { wound healing }\end{array}$ & Single group & Low & $5.6 \mathrm{mo}$ & AR & All & $35 / 1031(3.4)$ & N/A & $\mathrm{N} / \mathrm{A}$ \\
\hline Song, 2016, 26637165, US & NR & Single group & Low & $4.3-5.4 y$ & $\mathrm{AR}$ & All & $357 / 1809(19.7)$ & $\mathrm{N} / \mathrm{A}$ & $\mathrm{N} / \mathrm{A}$ \\
\hline
\end{tabular}

Abbreviations: adj $=$ adjusted, $\mathrm{AR}=$ autologous reconstruction, $\mathrm{CI}=$ confidence interval, $\mathrm{d}=$ days, $\mathrm{DIEP}=$ deep inferior epigastric perforator, $\mathrm{DTI}=$ direct to implant, $\mathrm{IBR}=$

implant-based reconstruction, $\mathrm{LD}=$ latissimus dorsi, $\mathrm{mo}=$ months, $\mathrm{NR}=$ not reported, $\mathrm{NRCS}=$ nonrandomized comparative study, $\mathrm{OR}=$ odds ratio, $\mathrm{PMID}=\mathrm{PubMed}$ identifier,

$\mathrm{Ref}=$ reference group, $\mathrm{RoB}=$ risk of bias, $\mathrm{SSI}=$ surgical site infection, $\mathrm{T} / \mathrm{E}=$ tissue expander, $\mathrm{WD}=$ wound dehiscence, $\mathrm{y}=\mathrm{years}$.

Colors: Header rows are shaded orange. Rows for every alternate study are shaded blue. The colors do not add unique information.

Table F-1.22. Full Evidence Table - Key Question 1: IBR versus AR - categorical outcomes (harms - seroma)

\begin{tabular}{|c|c|c|c|c|c|c|c|c|c|}
\hline $\begin{array}{l}\text { Study, Year, PMID, } \\
\text { Country }\end{array}$ & $\begin{array}{l}\text { Outcome } \\
\text { Description }\end{array}$ & Design & $\begin{array}{l}\text { Overall } \\
\text { RoB }\end{array}$ & $\begin{array}{l}\text { Time } \\
\text { Point }\end{array}$ & Arm & Subgroup & $\mathrm{n} / \mathrm{N}(\%)$ & Effect Size $(95 \% \mathrm{Cl})$ & $\begin{array}{l}\mathbf{P} \\
\text { Value }\end{array}$ \\
\hline \multirow[t]{2}{*}{$\begin{array}{l}\text { Fischer, 2014, } \\
\text { 24916480, US }\end{array}$} & Breast seroma & NRCS & High & $\begin{array}{l}1.8-2.1 \\
y\end{array}$ & IBR & All & $13 / 155(8.1)$ & Ref & Ref \\
\hline & Breast seroma & NRCS & High & $\begin{array}{l}1.8-2.1 \\
y\end{array}$ & $\mathrm{AR}$ & All & $4 / 155(2.8)$ & NR & 0.009 \\
\hline \multirow[t]{2}{*}{$\begin{array}{l}\text { Garvey, 2012, 23096600, } \\
\text { US }\end{array}$} & $\begin{array}{l}\text { Seroma or } \\
\text { hematoma }\end{array}$ & NRCS & Moderate & $1.5 \mathrm{y}$ & IBR & All & $61 / 442$ breasts $(13.8)$ & Ref & Ref \\
\hline & $\begin{array}{l}\text { Seroma or } \\
\text { hematoma }\end{array}$ & NRCS & Moderate & $1.5 y$ & AR & All & $27 / 548$ breasts $(4.9)$ & NR & $<0.001$ \\
\hline \multirow{9}{*}{$\begin{array}{l}\text { Kulkarni, 2017, } \\
\text { 28713853, US \& Canada }\end{array}$} & Breast seroma & NRCS & Moderate & $1 \mathrm{y}$ & IBR & All & $47 / 1615(2.9)$ & NR & NR \\
\hline & Breast seroma & NRCS & Moderate & $1 \mathrm{y}$ & AR (all) & All & NR & NR & NR \\
\hline & Breast seroma & NRCS & Moderate & $1 \mathrm{y}$ & AR with DIEP & All & $3 / 365(0.8)$ & NR & NR \\
\hline & Breast seroma & NRCS & Moderate & $1 \mathrm{y}$ & AR with free TRAM & All & $0 / 97(0)$ & NR & NR \\
\hline & Breast seroma & NRCS & Moderate & $1 \mathrm{y}$ & $\begin{array}{l}\text { AR with pedicled } \\
\text { TRAM }\end{array}$ & All & $2 / 84(2.4)$ & NR & NR \\
\hline & Breast seroma & NRCS & Moderate & $1 \mathrm{y}$ & AR with LD & All & $2 / 73(2.7)$ & NR & NR \\
\hline & Breast seroma & NRCS & Moderate & $1 \mathrm{y}$ & AR with SIEA & All & NR & NR & NR \\
\hline & $\begin{array}{l}\text { Donor site } \\
\text { seroma }\end{array}$ & NRCS & Moderate & $1 \mathrm{y}$ & IBR & All & NR & NR & NR \\
\hline & $\begin{array}{l}\text { Donor site } \\
\text { seroma }\end{array}$ & NRCS & Moderate & $1 \mathrm{y}$ & AR (all) & All & NR & NR & NR \\
\hline
\end{tabular}




\begin{tabular}{|c|c|c|c|c|c|c|c|c|c|}
\hline $\begin{array}{l}\text { Study, Year, PMID, } \\
\text { Country }\end{array}$ & $\begin{array}{l}\text { Outcome } \\
\text { Description }\end{array}$ & Design & $\begin{array}{l}\text { Overall } \\
\text { RoB }\end{array}$ & $\begin{array}{l}\text { Time } \\
\text { Point }\end{array}$ & Arm & Subgroup & $n / N(\%)$ & Effect Size $(95 \% \mathrm{CI})$ & $\begin{array}{l}\mathbf{P} \\
\text { Value }\end{array}$ \\
\hline & $\begin{array}{l}\text { Donor site } \\
\text { seroma }\end{array}$ & NRCS & Moderate & $1 \mathrm{y}$ & AR with DIEP & All & 19/365 (5.2) & NR & NR \\
\hline & $\begin{array}{l}\text { Donor site } \\
\text { seroma }\end{array}$ & NRCS & Moderate & $1 \mathrm{y}$ & AR with free TRAM & All & $2 / 97(2.1)$ & NR & NR \\
\hline & $\begin{array}{l}\text { Donor site } \\
\text { seroma }\end{array}$ & NRCS & Moderate & $1 \mathrm{y}$ & $\begin{array}{l}\text { AR with pedicled } \\
\text { TRAM }\end{array}$ & All & $0 / 84(0)$ & NR & NR \\
\hline & $\begin{array}{l}\text { Donor site } \\
\text { seroma }\end{array}$ & NRCS & Moderate & $1 \mathrm{y}$ & AR with LD & All & $14 / 73(19.2)$ & NR & NR \\
\hline & $\begin{array}{l}\text { Donor site } \\
\text { seroma }\end{array}$ & NRCS & Moderate & $1 \mathrm{y}$ & AR with SIEA & All & NR & NR & NR \\
\hline \multirow{2}{*}{$\begin{array}{l}\text { Nelson, 2019, 31356276, } \\
\text { US }\end{array}$} & NR & NRCS & High & $<3 \mathrm{mo}$ & IBR & All & 49/1211 breasts (4.04) & NR & NR \\
\hline & NR & NRCS & High & $<3 \mathrm{mo}$ & AR & All & NR & NR & NR \\
\hline $\begin{array}{l}\text { Chen, 2018a, 29596085, } \\
\text { US }\end{array}$ & NR & $\begin{array}{l}\text { Single } \\
\text { group }\end{array}$ & Low & NR & IBR & All & $139 / 23048(0.6)$ & $\mathrm{N} / \mathrm{A}$ & $\mathrm{N} / \mathrm{A}$ \\
\hline \multirow[t]{7}{*}{$\begin{array}{l}\text { Cordeiro, } 2006 \\
16980842, \text { US }\end{array}$} & NR & $\begin{array}{l}\text { Single } \\
\text { group }\end{array}$ & Moderate & NR & IBR & All & $5 / 2276$ procedures $(0.2)$ & N/A & N/A \\
\hline & NR & $\begin{array}{l}\text { Single } \\
\text { group }\end{array}$ & Moderate & NR & IBR & $\begin{array}{l}\text { Expander } \\
\text { placement } \\
\text { procedure }\end{array}$ & $3 / 1221$ procedures $(0.2)$ & $\mathrm{N} / \mathrm{A}$ & $\mathrm{N} / \mathrm{A}$ \\
\hline & NR & $\begin{array}{l}\text { Single } \\
\text { group }\end{array}$ & Moderate & NR & IBR & $\begin{array}{l}\text { Exchange } \\
\text { procedure }\end{array}$ & 2/1055 procedures $(0.2)$ & N/A & N/A \\
\hline & NR & $\begin{array}{l}\text { Single } \\
\text { group }\end{array}$ & Moderate & NR & IBR & $\begin{array}{l}\text { Previous } \\
\text { radiation }\end{array}$ & 1/136 procedures $(0.7)$ & $\mathrm{N} / \mathrm{A}$ & N/A \\
\hline & NR & $\begin{array}{l}\text { Single } \\
\text { group }\end{array}$ & Moderate & NR & IBR & $\begin{array}{l}\text { No } \\
\text { previous } \\
\text { radiation }\end{array}$ & $6 / 2140$ procedures $(0.3)$ & $\mathrm{N} / \mathrm{A}$ & $\mathrm{N} / \mathrm{A}$ \\
\hline & NR & $\begin{array}{l}\text { Single } \\
\text { group }\end{array}$ & Moderate & NR & IBR & Immediate & $3 / 1176$ procedures $(0.3)$ & $\mathrm{N} / \mathrm{A}$ & $\mathrm{N} / \mathrm{A}$ \\
\hline & NR & $\begin{array}{l}\text { Single } \\
\text { group }\end{array}$ & Moderate & NR & IBR & Delayed & $0 / 26$ procedures $(0)$ & N/A & N/A \\
\hline \multirow[t]{3}{*}{$\begin{array}{l}\text { Cordeiro, 2012, } \\
\text { 22286416, US }\end{array}$} & NR & $\begin{array}{l}\text { Single } \\
\text { group }\end{array}$ & Low & $<1 \mathrm{y}$ & IBR & All & $24 / 1699(1.4)$ & $\mathrm{N} / \mathrm{A}$ & N/A \\
\hline & NR & $\begin{array}{l}\text { Single } \\
\text { group }\end{array}$ & Low & $<1 \mathrm{y}$ & IBR & $\begin{array}{l}\text { Prior } \\
\text { radiation }\end{array}$ & $3 / 121(2.5)$ & $\mathrm{N} / \mathrm{A}$ & $\mathrm{N} / \mathrm{A}$ \\
\hline & NR & $\begin{array}{l}\text { Single } \\
\text { group }\end{array}$ & Low & $<1 \mathrm{y}$ & IBR & $\begin{array}{l}\text { No Prior } \\
\text { radiation }\end{array}$ & $21 / 1578(1.3)$ & $\mathrm{N} / \mathrm{A}$ & N/A \\
\hline $\begin{array}{l}\text { Cordeiro, } 2015 \mathrm{~b} \\
26090764, \text { US }\end{array}$ & NR & $\begin{array}{l}\text { Single } \\
\text { group }\end{array}$ & Low & $2 y$ & IBR & All & $54 / 4912$ implants (1.1) & N/A & N/A \\
\hline \multirow[t]{2}{*}{$\begin{array}{l}\text { Hansen, 2018, } \\
\text { 29778821, US }\end{array}$} & NR & $\begin{array}{l}\text { Single } \\
\text { group }\end{array}$ & Low & $1 \mathrm{mo}$ & IBR & All & $15 / 930(1.6)$ & $\mathrm{N} / \mathrm{A}$ & N/A \\
\hline & NR & $\begin{array}{l}\text { Single } \\
\text { group }\end{array}$ & Low & $1 \mathrm{y}$ & IBR & All & $28 / 930(3.0)$ & N/A & N/A \\
\hline
\end{tabular}




\begin{tabular}{|c|c|c|c|c|c|c|c|c|c|}
\hline $\begin{array}{l}\text { Study, Year, PMID, } \\
\text { Country }\end{array}$ & $\begin{array}{l}\text { Outcome } \\
\text { Description }\end{array}$ & Design & $\begin{array}{l}\text { Overall } \\
\text { RoB }\end{array}$ & $\begin{array}{l}\text { Time } \\
\text { Point }\end{array}$ & Arm & Subgroup & $\mathrm{n} / \mathrm{N}(\%)$ & Effect Size $(95 \% \mathrm{CI})$ & $\begin{array}{l}P \\
\text { Value }\end{array}$ \\
\hline $\begin{array}{l}\text { Hunsicker, 2017, } \\
\text { 26849284, US }\end{array}$ & NR & $\begin{array}{l}\text { Single } \\
\text { group }\end{array}$ & Low & $<1 \mathrm{y}$ & IBR & All & $17 / 1584$ breasts (1.1) & $\mathrm{N} / \mathrm{A}$ & $\mathrm{N} / \mathrm{A}$ \\
\hline \multirow[t]{3}{*}{$\begin{array}{l}\text { Huo, 2016, 27697676, } \\
\text { US }\end{array}$} & NR & $\begin{array}{l}\text { Single } \\
\text { group }\end{array}$ & Low & $1 \mathrm{y}$ & IBR & All & $89 / 1332(6.7)$ & $\mathrm{N} / \mathrm{A}$ & $\mathrm{N} / \mathrm{A}$ \\
\hline & NR & $\begin{array}{l}\text { Single } \\
\text { group }\end{array}$ & Low & $1 \mathrm{y}$ & IBR & $\begin{array}{l}\text { Non-obese } \\
\text { patients }\end{array}$ & $45 / 949(4.7)$ & $\mathrm{N} / \mathrm{A}$ & $\mathrm{N} / \mathrm{A}$ \\
\hline & NR & $\begin{array}{l}\text { Single } \\
\text { group }\end{array}$ & Low & $1 \mathrm{y}$ & IBR & $\begin{array}{l}\text { Obese } \\
\text { patients }\end{array}$ & $44 / 383(11.4)$ & $\mathrm{N} / \mathrm{A}$ & $\mathrm{N} / \mathrm{A}$ \\
\hline $\begin{array}{l}\text { Lee, 2021a, 32974692, } \\
\text { South Korea }\end{array}$ & $\begin{array}{l}\text { Seroma or } \\
\text { hematoma }\end{array}$ & $\begin{array}{l}\text { Single } \\
\text { group }\end{array}$ & Low & $5 y$ & IBR & All & $2 / 605$ breasts $(0.3)$ & $\mathrm{N} / \mathrm{A}$ & N/A \\
\hline $\begin{array}{l}\text { Lovecchio, 2015, } \\
24691330, \text { US }\end{array}$ & NR & $\begin{array}{l}\text { Single } \\
\text { group }\end{array}$ & Low & $6.4 \mathrm{y}$ & IBR & All & $46 / 1639(2.8)$ & $\mathrm{N} / \mathrm{A}$ & $\mathrm{N} / \mathrm{A}$ \\
\hline $\begin{array}{l}\text { Parikh, 2018, 30204676, } \\
\text { US }\end{array}$ & NR & $\begin{array}{l}\text { Single } \\
\text { group }\end{array}$ & Low & $>2 y$ & IBR & All & $72 / 1285(5.6)$ & $\mathrm{N} / \mathrm{A}$ & $\mathrm{N} / \mathrm{A}$ \\
\hline $\begin{array}{l}\text { Park, 2019, } 30863940, \\
\text { South Korea }\end{array}$ & NR & $\begin{array}{l}\text { Single } \\
\text { group }\end{array}$ & Moderate & $>6 \mathrm{mo}$ & IBR & All & $38 / 999(3.8)$ & $\mathrm{N} / \mathrm{A}$ & $\mathrm{N} / \mathrm{A}$ \\
\hline $\begin{array}{l}\text { Seth, 2015, 25180955, } \\
\text { US }\end{array}$ & $\begin{array}{l}\text { Seroma requiring } \\
\text { drainage }\end{array}$ & $\begin{array}{l}\text { Single } \\
\text { group }\end{array}$ & Low & NR & IBR & All & $40 / 893(4.5)$ & N/A & $\mathrm{N} / \mathrm{A}$ \\
\hline \multirow[t]{3}{*}{$\begin{array}{l}\text { Singh, 2012, 22342636, } \\
\text { US }\end{array}$} & NR & $\begin{array}{l}\text { Single } \\
\text { group }\end{array}$ & Low & $1 \mathrm{mo}$ & IBR & All & $61 / 1316(4.6)$ & & \\
\hline & NR & $\begin{array}{l}\text { Single } \\
\text { group }\end{array}$ & Low & $1 \mathrm{mo}$ & IBR & DTI & $6 / 95(6.3)$ & $\mathrm{N} / \mathrm{A}$ & $\mathrm{N} / \mathrm{A}$ \\
\hline & NR & $\begin{array}{l}\text { Single } \\
\text { group }\end{array}$ & Low & $1 \mathrm{mo}$ & IBR & $\mathrm{T} / \mathrm{E}$ & $55 / 1221(4.5)$ & $\mathrm{N} / \mathrm{A}$ & $\mathrm{N} / \mathrm{A}$ \\
\hline $\begin{array}{l}\text { Albornoz, 2013, } \\
23897346, \text { US }\end{array}$ & $\begin{array}{l}\text { Seroma or } \\
\text { wound infection }\end{array}$ & $\begin{array}{l}\text { Single } \\
\text { group }\end{array}$ & Low & NR & AR & All & $189 / 21016(0.9)$ & $\mathrm{N} / \mathrm{A}$ & $\mathrm{N} / \mathrm{A}$ \\
\hline \multirow[t]{3}{*}{$\begin{array}{l}\text { Singh, } 2021,33564597 \\
\text { US }\end{array}$} & NR & $\begin{array}{l}\text { Single } \\
\text { group }\end{array}$ & Low & $3 \mathrm{mo}$ & IBR & All & $219 / 1740(12.59)$ & N/A & $\mathrm{N} / \mathrm{A}$ \\
\hline & NR & $\begin{array}{l}\text { Single } \\
\text { group }\end{array}$ & Low & $3 \mathrm{mo}$ & IBR & DTI & $98 / 870(11.22)$ & $\mathrm{N} / \mathrm{A}$ & $\mathrm{N} / \mathrm{A}$ \\
\hline & NR & $\begin{array}{l}\text { Single } \\
\text { group }\end{array}$ & Low & $3 \mathrm{mo}$ & IBR & $\mathrm{T} / \mathrm{E}$ & $122 / 870(13.97)$ & $\mathrm{N} / \mathrm{A}$ & $\mathrm{N} / \mathrm{A}$ \\
\hline $\begin{array}{l}\text { Beugels, 2018, } \\
\text { 29399731, Netherlands }\end{array}$ & NR & $\begin{array}{l}\text { Single } \\
\text { group }\end{array}$ & Low & $9-10 \mathrm{mo}$ & AR & All & 15/910 flaps (1.6) & $\mathrm{N} / \mathrm{A}$ & $\mathrm{N} / \mathrm{A}$ \\
\hline $\begin{array}{l}\text { Chang, 2000, 10809092, } \\
\text { US }\end{array}$ & Flap seroma & $\begin{array}{l}\text { Single } \\
\text { group }\end{array}$ & Low & NR & AR & All & 38/936 flaps (4.1) & $\mathrm{N} / \mathrm{A}$ & $\mathrm{N} / \mathrm{A}$ \\
\hline \multirow[t]{2}{*}{$\begin{array}{l}\text { Chang, 2011, 21407063, } \\
\text { US }\end{array}$} & $\begin{array}{l}\text { Donor site } \\
\text { seroma }\end{array}$ & $\begin{array}{l}\text { Single } \\
\text { group }\end{array}$ & Low & NR & AR & All & $4 / 818(0.4)$ & N/A & $\mathrm{N} / \mathrm{A}$ \\
\hline & $\begin{array}{l}\text { Donor site } \\
\text { seroma }\end{array}$ & $\begin{array}{l}\text { Single } \\
\text { group }\end{array}$ & Low & NR & AR & Age $<50$ & $3 / 411(0.7)$ & $\mathrm{N} / \mathrm{A}$ & $\mathrm{N} / \mathrm{A}$ \\
\hline
\end{tabular}




\begin{tabular}{|c|c|c|c|c|c|c|c|c|c|}
\hline $\begin{array}{l}\text { Study, Year, PMID, } \\
\text { Country }\end{array}$ & $\begin{array}{l}\text { Outcome } \\
\text { Description }\end{array}$ & Design & $\begin{array}{l}\text { Overall } \\
\text { RoB }\end{array}$ & $\begin{array}{l}\text { Time } \\
\text { Point }\end{array}$ & Arm & Subgroup & $\mathrm{n} / \mathrm{N}(\%)$ & Effect Size (95\% CI) & $\begin{array}{l}\mathbf{P} \\
\text { Value }\end{array}$ \\
\hline & $\begin{array}{l}\text { Donor site } \\
\text { seroma }\end{array}$ & $\begin{array}{l}\text { Single } \\
\text { group }\end{array}$ & Low & NR & AR & Age $50-59$ & $1 / 285(0.4)$ & $\mathrm{N} / \mathrm{A}$ & $\mathrm{N} / \mathrm{A}$ \\
\hline & $\begin{array}{l}\text { Donor site } \\
\text { seroma }\end{array}$ & $\begin{array}{l}\text { Single } \\
\text { group }\end{array}$ & Low & NR & AR & Age 60-69 & $0 / 103(0)$ & $\mathrm{N} / \mathrm{A}$ & $\mathrm{N} / \mathrm{A}$ \\
\hline & $\begin{array}{l}\text { Donor site } \\
\text { seroma }\end{array}$ & $\begin{array}{l}\text { Single } \\
\text { group }\end{array}$ & Low & NR & AR & Age $>70$ & $0 / 19(0)$ & $\mathrm{N} / \mathrm{A}$ & $\mathrm{N} / \mathrm{A}$ \\
\hline $\begin{array}{l}\text { Chen, 2018b, 29596085, } \\
\text { US }\end{array}$ & NR & $\begin{array}{l}\text { Single } \\
\text { group }\end{array}$ & Low & NR & AR & All & $118 / 19496(0.6)$ & $\mathrm{N} / \mathrm{A}$ & $\mathrm{N} / \mathrm{A}$ \\
\hline \multirow[t]{2}{*}{$\begin{array}{l}\text { Daly, 2020, 31994156, } \\
\text { US }\end{array}$} & Breast seroma & $\begin{array}{l}\text { Single } \\
\text { group }\end{array}$ & Low & $12 \mathrm{y}$ & AR & All & $25 / 818(3.1)$ & $\mathrm{N} / \mathrm{A}$ & N/A \\
\hline & $\begin{array}{l}\text { Donor site } \\
\text { seroma }\end{array}$ & $\begin{array}{l}\text { Single } \\
\text { group }\end{array}$ & Low & $12 y$ & AR & All & $45 / 818(5.5)$ & $\mathrm{N} / \mathrm{A}$ & $\mathrm{N} / \mathrm{A}$ \\
\hline $\begin{array}{l}\text { Enajat, 2010, 19790180, } \\
\text { Sweden }\end{array}$ & NR & $\begin{array}{l}\text { Single } \\
\text { group }\end{array}$ & Low & $1-7 d$ & AR & All & 11/564 implants (1.9) & N/A & $\mathrm{N} / \mathrm{A}$ \\
\hline Gill, 2004, 15083015, US & NR & $\begin{array}{l}\text { Single } \\
\text { group }\end{array}$ & Moderate & $10 y$ & AR & All & $35 / 758(4.6)$ & N/A & $\mathrm{N} / \mathrm{A}$ \\
\hline $\begin{array}{l}\text { Heo, 2018, 30039735, } \\
\text { South Korea }\end{array}$ & $\begin{array}{l}\text { Seroma or } \\
\text { hematoma }\end{array}$ & $\begin{array}{l}\text { Single } \\
\text { group }\end{array}$ & Low & $1 \mathrm{y}$ & AR & All & $2 / 615(0.3)$ & N/A & $\mathrm{N} / \mathrm{A}$ \\
\hline $\begin{array}{l}\text { Jo, } 2020,33386262 \text {, } \\
\text { South Korea }\end{array}$ & NR & $\begin{array}{l}\text { Single } \\
\text { group }\end{array}$ & Low & $\begin{array}{l}11 \mathrm{mo}- \\
1.5 \mathrm{y}\end{array}$ & AR & All & $24 / 623(3.85)$ & $\mathrm{N} / \mathrm{A}$ & $\mathrm{N} / \mathrm{A}$ \\
\hline $\begin{array}{l}\text { Masoomi, 2019, } \\
\text { 31331721, US }\end{array}$ & NR & $\begin{array}{l}\text { Single } \\
\text { group }\end{array}$ & Low & NR & AR & All & $660 / 55840(1.2)$ & N/A & $\mathrm{N} / \mathrm{A}$ \\
\hline $\begin{array}{l}\text { Munder, 32565553, } \\
\text { 2020, Germany }\end{array}$ & $\begin{array}{l}\text { Donor site } \\
\text { seroma }\end{array}$ & $\begin{array}{l}\text { Single } \\
\text { group }\end{array}$ & Low & $2 y$ & AR & All & 6/1274 flaps $(0.5)$ & N/A & $\mathrm{N} / \mathrm{A}$ \\
\hline \multirow[t]{2}{*}{$\begin{array}{l}\text { Nelson, } 2014,25046665 \\
\text { US }\end{array}$} & Breast seroma & $\begin{array}{l}\text { Single } \\
\text { group }\end{array}$ & Moderate & NR & AR & All & 35/1293 flaps (2.7) & N/A & $\mathrm{N} / \mathrm{A}$ \\
\hline & $\begin{array}{l}\text { Donor site } \\
\text { seroma }\end{array}$ & $\begin{array}{l}\text { Single } \\
\text { group }\end{array}$ & Moderate & NR & AR & All & 11/1293 flaps $(0.9)$ & $\mathrm{N} / \mathrm{A}$ & $\mathrm{N} / \mathrm{A}$ \\
\hline $\begin{array}{l}\text { O'Neill, 2019, 31196805, } \\
\text { Canada }\end{array}$ & NR & $\begin{array}{l}\text { Single } \\
\text { group }\end{array}$ & Low & $<3 \mathrm{mo}$ & AR & All & $17 / 912(1.9)$ & N/A & $\mathrm{N} / \mathrm{A}$ \\
\hline $\begin{array}{l}\text { Rubio, 2019, 30665841, } \\
\text { Belgium }\end{array}$ & NR & $\begin{array}{l}\text { Single } \\
\text { group }\end{array}$ & Low & NR & AR & All & $622 / 56522(1.1)$ & $\mathrm{N} / \mathrm{A}$ & $\mathrm{N} / \mathrm{A}$ \\
\hline $\begin{array}{l}\text { Selber, 2009, 19935283, } \\
\text { US }\end{array}$ & NR & $\begin{array}{l}\text { Single } \\
\text { group }\end{array}$ & Low & $5.6 \mathrm{mo}$ & AR & All & $30 / 1031(2.9)$ & $\mathrm{N} / \mathrm{A}$ & $\mathrm{N} / \mathrm{A}$ \\
\hline $\begin{array}{l}\text { Song, 2016, 26637165, } \\
\text { US }\end{array}$ & NR & $\begin{array}{l}\text { Single } \\
\text { group }\end{array}$ & Low & $\begin{array}{l}4.3-5.4 \\
y\end{array}$ & AR & All & $151 / 1809(8.3)$ & $\mathrm{N} / \mathrm{A}$ & $\mathrm{N} / \mathrm{A}$ \\
\hline $\begin{array}{l}\text { Tran, 2018, 29794694, } \\
\text { US }\end{array}$ & NR & $\begin{array}{l}\text { Single } \\
\text { group }\end{array}$ & Low & NR & AR & All & $26 / 1253$ flaps (2.1) & N/A & $\mathrm{N} / \mathrm{A}$ \\
\hline $\begin{array}{l}\text { Williams, 1995, 7794079, } \\
\text { US }\end{array}$ & NR & $\begin{array}{l}\text { Single } \\
\text { group }\end{array}$ & Moderate & $2.4 \mathrm{y}$ & $\mathrm{AR}$ & All & $8 / 607$ (1.3) & $\mathrm{N} / \mathrm{A}$ & $\mathrm{N} / \mathrm{A}$ \\
\hline
\end{tabular}


Abbreviations: adj $=$ adjusted, $\mathrm{AR}=$ autologous reconstruction, $\mathrm{CI}=$ confidence interval, $\mathrm{d}=$ days, $\mathrm{DIEP}=$ deep inferior epigastric perforator, $\mathrm{DTI}=$ direct to implant, $\mathrm{IBR}=$

implant-based reconstruction, $\mathrm{LD}=$ latissimus dorsi, $\mathrm{mo}=$ months, $\mathrm{N} / \mathrm{A}=$ not applicable, $\mathrm{NR}=$ not reported, NRCS $=$ nonrandomized comparative study, $\mathrm{OR}=$ odds ratio, $\mathrm{PMID}$

$=$ PubMed identifier, $\operatorname{Ref}=$ reference group, $\mathrm{RoB}=$ risk of bias, $\mathrm{SSI}=$ surgical site infection, $\mathrm{T} / \mathrm{E}=$ tissue expander, $\mathrm{y}=\mathrm{years}$.

Colors: Header rows are shaded orange. Rows for every alternate study are shaded blue. The colors do not add unique information.

Table F-1.23. Full Evidence Table - Key Question 1: IBR versus AR - categorical outcomes (harms - scarring)

\begin{tabular}{|l|l|l|l|l|l|l|l|}
\hline $\begin{array}{l}\text { Study, Year, PMID, } \\
\text { Country }\end{array}$ & Outcome Description & Design & $\begin{array}{l}\text { Overall } \\
\text { RoB }\end{array}$ & $\begin{array}{l}\text { Time } \\
\text { Point }\end{array}$ & Arm & n/N (\%) & $\begin{array}{l}\text { Effect Size (95\% } \\
\text { Cl) }\end{array}$ \\
\hline $\begin{array}{l}\text { Cordeiro, 2015b, } \\
26090764, \text { US }\end{array}$ & $\begin{array}{l}\text { Hypertrophic or other } \\
\text { scarring }\end{array}$ & Single group & Low & 2 y & IBR & $69 / 4912$ implants (1.4) \\
\hline $\begin{array}{l}\text { Yoo, 2014, 24852813, } \\
\text { South Korea }\end{array}$ & $\begin{array}{l}\text { Breast hypertrophic } \\
\text { scarring }\end{array}$ & Single group & Moderate & NR & AR & $13 / 500(2.6)$ \\
\cline { 2 - 7 } & $\begin{array}{l}\text { Donor site } \\
\text { hypertrophic scarring }\end{array}$ & Single group & Moderate & NR & AR & 26/500 (5.2) \\
\hline
\end{tabular}

Abbreviations: adj $=$ adjusted, $\mathrm{AR}=$ autologous reconstruction, $\mathrm{CI}=$ confidence interval, $\mathrm{IBR}=$ implant-based reconstruction, $\mathrm{N} / \mathrm{A}=$ not applicable, $\mathrm{NR}=$ not reported, $\mathrm{NRCS}=$ nonrandomized comparative study, $\mathrm{OR}=$ odds ratio, $\mathrm{PMID}=$ PubMed identifier, $\mathrm{RoB}=$ risk of bias, $\mathrm{y}=$ years

Colors: Header rows are shaded orange. Rows for every alternate study are shaded blue. The colors do not add unique information.

Table F-1.24. Full Evidence Table - Key Question 1: IBR versus AR - categorical outcomes (harms - reconstructive failure)

\begin{tabular}{|c|c|c|c|c|c|c|c|c|c|}
\hline $\begin{array}{l}\text { Study, Year, } \\
\text { PMID, Country }\end{array}$ & Outcome Description & Design & $\begin{array}{l}\text { Overall } \\
\text { RoB }\end{array}$ & $\begin{array}{l}\text { Time } \\
\text { Point }\end{array}$ & Arm & Subgroup & $n / N(\%)$ & $\begin{array}{l}\text { Adjusted Odds Ratio (95\% } \\
\text { Cl) }\end{array}$ & $\begin{array}{l}\mathbf{P} \\
\text { Value }\end{array}$ \\
\hline \multirow[t]{2}{*}{$\begin{array}{l}\text { Chetta, 2017, } \\
\text { 28002254, US }\end{array}$} & NR & NRCS & High & $1.3 \mathrm{mo}$ & IBR & All & $\begin{array}{l}1101 / 3746 \\
(29.4)\end{array}$ & $0.09(0.07,0.13)$ & $<0.001$ \\
\hline & NR & NRCS & High & $1.3 \mathrm{mo}$ & AR & All & $40 / 935(4.3)$ & Ref & Ref \\
\hline \multirow[t]{2}{*}{$\begin{array}{l}\text { Fischer, 2013, } \\
\text { 23629074, US }\end{array}$} & $\begin{array}{l}\text { Flap loss in AR cohort or an } \\
\text { unplanned, nonaesthetic TE/I } \\
\text { removal related to a complication } \\
\text { in TE/I cohort }\end{array}$ & NRCS & High & $4 y$ & IBR & All & $4 / 60(7.3)$ & Ref & Ref \\
\hline & $\begin{array}{l}\text { Flap loss in AR cohort or an } \\
\text { unplanned, nonaesthetic TE/I } \\
\text { removal related to a complication } \\
\text { in TE/I cohort }\end{array}$ & NRCS & High & $4 y$ & AR & All & $2 / 142(1.3)$ & $0.19(0.04,0.8)$ & 0.03 \\
\hline \multirow[t]{4}{*}{$\begin{array}{l}\text { Fischer, 2014, } \\
\text { 24916480, US }\end{array}$} & $\begin{array}{l}\text { Total flap loss or TE/I removal } \\
\text { secondary to infection or } \\
\text { exposure }\end{array}$ & NRCS & High & $\begin{array}{l}1.8-2.1 \\
y\end{array}$ & IBR & All & $9 / 155(5.6)$ & NR & NR \\
\hline & $\begin{array}{l}\text { Total flap loss or TE/I removal } \\
\text { secondary to infection or } \\
\text { exposure }\end{array}$ & NRCS & High & $\begin{array}{l}1.8-2.1 \\
y\end{array}$ & AR & All & 2/155 (1.2) & NR & NR \\
\hline & Implant/flap failure & NRCS & High & $11 \mathrm{mo}$ & IBR & All & $14 / 155(5.6)$ & NR & NR \\
\hline & Implant/flap failure & NRCS & High & $11 \mathrm{mo}$ & AR & All & $3 / 155(1.2)$ & NR & NR \\
\hline
\end{tabular}




\begin{tabular}{|c|c|c|c|c|c|c|c|c|c|}
\hline $\begin{array}{l}\text { Study, Year, } \\
\text { PMID, Country }\end{array}$ & Outcome Description & Design & $\begin{array}{l}\text { Overall } \\
\text { RoB }\end{array}$ & $\begin{array}{l}\text { Time } \\
\text { Point }\end{array}$ & Arm & Subgroup & $\mathrm{n} / \mathrm{N}(\%)$ & $\begin{array}{l}\text { Adjusted Odds Ratio (95\% } \\
\text { Cl) }\end{array}$ & $\begin{array}{l}\mathbf{P} \\
\text { Value }\end{array}$ \\
\hline \multirow[t]{2}{*}{$\begin{array}{l}\text { Garvey, 2012, } \\
23096600, \text { US }\end{array}$} & NR & NRCS & Moderate & $1.5 \mathrm{y}$ & IBR & All & $\begin{array}{l}70 / 442 \text { breasts } \\
(15.8)\end{array}$ & Ref & Ref \\
\hline & NR & NRCS & Moderate & $1.5 \mathrm{y}$ & AR & All & $\begin{array}{l}8 / 548 \text { breasts } \\
(1.5)\end{array}$ & NR & $<0.001$ \\
\hline \multirow{13}{*}{$\begin{array}{l}\text { Kulkarni, 2017, } \\
\text { 28713853, US } \\
\text { \& Canada }\end{array}$} & NR & NRCS & Moderate & $2 y$ & IBR (all) & All & NR & NR & NR \\
\hline & NR & NRCS & Moderate & $2 y$ & IBR (all) & Unilateral & $41 / 600(6.83)$ & Ref & Ref \\
\hline & NR & NRCS & Moderate & $2 y$ & IBR (all) & Bilateral & $74 / 994(7.44)$ & Ref & Ref \\
\hline & NR & NRCS & Moderate & $2 y$ & IBR DTI & All & $8 / 112(7.1)$ & NR & NR \\
\hline & NR & NRCS & Moderate & $2 y$ & IBR T/E & All & $108 / 1525(7.1)$ & NR & NR \\
\hline & NR & NRCS & Moderate & $2 y$ & AR (all) & All & NR & NR & NR \\
\hline & NR & NRCS & Moderate & $2 y$ & AR (all) & Unilateral & $4 / 317(1.26)$ & $\frac{\text { vs. IBR (all): }}{0.36)} 0.12(0.04$ & $<0.001$ \\
\hline & NR & NRCS & Moderate & $2 y$ & AR (all) & Bilateral & $4 / 224(1.87)$ & $\begin{array}{l}\text { vs. IBR (all): } 0.14(0.05, \\
0.45)\end{array}$ & 0.001 \\
\hline & NR & NRCS & Moderate & $2 y$ & $\begin{array}{l}\text { AR with } \\
\text { DIEP }\end{array}$ & All & $5 / 390(1.3)$ & NR & NR \\
\hline & NR & NRCS & Moderate & $2 y$ & $\begin{array}{l}\text { AR with } \\
\text { free } \\
\text { TRAM }\end{array}$ & All & $2 / 95(2.1)$ & NR & NR \\
\hline & NR & NRCS & Moderate & $2 y$ & $\begin{array}{l}\text { AR with } \\
\text { pedicled } \\
\text { TRAM } \\
\end{array}$ & All & $1 / 85(1.2)$ & NR & NR \\
\hline & NR & NRCS & Moderate & $2 y$ & $\begin{array}{l}\text { AR with } \\
\text { LD }\end{array}$ & All & $2 / 71(2.8)$ & NR & NR \\
\hline & NR & NRCS & Moderate & $2 y$ & $\begin{array}{l}\text { AR with } \\
\text { SIEA }\end{array}$ & All & $0 / 65(0)$ & NR & NR \\
\hline \multirow{2}{*}{$\begin{array}{l}\text { Mioton, 2013, } \\
23562485, \text { US }\end{array}$} & Implant/flap failure & NRCS & Moderate & $1 \mathrm{mo}$ & IBR & All & $83 / 9786(0.85)$ & Ref & Ref \\
\hline & Implant/flap failure & NRCS & Moderate & $1 \mathrm{mo}$ & AR & All & $\begin{array}{l}103 / 3296 \\
(3.13)\end{array}$ & $1.69(1.08,2.62)$ & NR \\
\hline
\end{tabular}

Abbreviations: adj $=$ adjusted, $\mathrm{AR}=$ autologous reconstruction, $\mathrm{CI}=$ confidence interval, $\mathrm{IBR}=$ implant-based reconstruction, $\mathrm{N} / \mathrm{A}=$ not applicable, $\mathrm{NR}=$ not reported, $\mathrm{NRCS}=$

nonrandomized comparative study, $\mathrm{OR}=$ odds ratio, $\mathrm{PMID}=$ PubMed identifier, $\mathrm{Ref}=$ reference group, $\mathrm{RoB}=$ risk of bias, $\mathrm{y}=\mathrm{years}$.

Colors: Header rows are shaded orange. Rows for every alternate study are shaded blue. The colors do not add unique information.

Table F-1.25. Full Evidence Table - Key Question 1: IBR versus AR - categorical outcomes (harms - hematoma or hemorrhage)

\begin{tabular}{|c|c|c|c|c|c|c|c|c|c|}
\hline $\begin{array}{l}\text { Study, Year, } \\
\text { PMID, Country }\end{array}$ & $\begin{array}{l}\text { Outcome } \\
\text { Description }\end{array}$ & Design & $\begin{array}{l}\text { Overall } \\
\text { RoB }\end{array}$ & $\begin{array}{l}\text { Time } \\
\text { Point }\end{array}$ & Arm & Subgroup & $\mathrm{n} / \mathrm{N}(\%)$ & $\begin{array}{l}\text { Effect Size }(95 \% \\
\mathrm{Cl})\end{array}$ & P Value \\
\hline \multirow[t]{2}{*}{$\begin{array}{l}\text { Fischer, 2014, } \\
24916480, \text { US }\end{array}$} & $\begin{array}{l}\text { Breast } \\
\text { hematoma }\end{array}$ & NRCS & High & $1.8-2.1 \mathrm{mo}$ & IBR & All & $4 / 155(2.4)$ & Ref & Ref \\
\hline & $\begin{array}{l}\text { Breast } \\
\text { hematoma }\end{array}$ & NRCS & High & $1.8-2.1 \mathrm{mo}$ & AR & All & $4 / 155(2.8)$ & NR & 1.0 \\
\hline
\end{tabular}




\begin{tabular}{|c|c|c|c|c|c|c|c|c|c|}
\hline $\begin{array}{l}\text { Study, Year, } \\
\text { PMID, Country }\end{array}$ & $\begin{array}{l}\text { Outcome } \\
\text { Description }\end{array}$ & Design & \begin{tabular}{|l|} 
Overall \\
RoB \\
\end{tabular} & $\begin{array}{l}\text { Time } \\
\text { Point }\end{array}$ & Arm & Subgroup & $\mathrm{n} / \mathrm{N}(\%)$ & $\begin{array}{l}\text { Effect Size }(95 \% \\
\mathrm{Cl})\end{array}$ & P Value \\
\hline \multirow{12}{*}{$\begin{array}{l}\text { Kulkarni, 2017, } \\
28713853, \text { US \& } \\
\text { Canada }\end{array}$} & $\begin{array}{l}\text { Breast } \\
\text { hematoma }\end{array}$ & NRCS & Moderate & $1 \mathrm{y}$ & IBR & All & $56 / 1615(3.5)$ & NR & NR \\
\hline & $\begin{array}{l}\text { Breast } \\
\text { hematoma }\end{array}$ & NRCS & Moderate & $1 \mathrm{y}$ & AR (all) & All & NR & NR & NR \\
\hline & $\begin{array}{l}\text { Breast } \\
\text { hematoma }\end{array}$ & NRCS & Moderate & $1 \mathrm{y}$ & AR with DIEP & All & $22 / 365(6)$ & NR & NR \\
\hline & $\begin{array}{l}\text { Breast } \\
\text { hematoma }\end{array}$ & NRCS & Moderate & $1 \mathrm{y}$ & AR with free TRAM & All & $4 / 97(4.1)$ & NR & NR \\
\hline & $\begin{array}{l}\text { Breast } \\
\text { hematoma }\end{array}$ & NRCS & Moderate & $1 \mathrm{y}$ & $\begin{array}{l}\text { AR with pedicled } \\
\text { TRAM }\end{array}$ & All & $3 / 84(3.6)$ & NR & NR \\
\hline & $\begin{array}{l}\text { Breast } \\
\text { hematoma }\end{array}$ & NRCS & Moderate & $1 \mathrm{y}$ & AR with LD & All & $3 / 73(4.1)$ & NR & NR \\
\hline & $\begin{array}{l}\text { Donor site } \\
\text { hematoma }\end{array}$ & NRCS & Moderate & $1 \mathrm{y}$ & IBR & All & $\mathrm{N} / \mathrm{A}$ & $\mathrm{N} / \mathrm{A}$ & $\mathrm{N} / \mathrm{A}$ \\
\hline & $\begin{array}{l}\text { Donor site } \\
\text { hematoma }\end{array}$ & NRCS & Moderate & $1 \mathrm{y}$ & AR (all) & All & NR & NR & NR \\
\hline & $\begin{array}{l}\text { Donor site } \\
\text { hematoma }\end{array}$ & NRCS & Moderate & $1 \mathrm{y}$ & AR with DIEP & All & $10 / 365(2.7)$ & NR & NR \\
\hline & $\begin{array}{l}\text { Donor site } \\
\text { hematoma }\end{array}$ & NRCS & Moderate & $1 \mathrm{y}$ & AR with free TRAM & All & $0 / 97(0)$ & NR & NR \\
\hline & $\begin{array}{l}\text { Donor site } \\
\text { hematoma }\end{array}$ & NRCS & Moderate & $1 \mathrm{y}$ & $\begin{array}{l}\text { AR with pedicled } \\
\text { TRAM }\end{array}$ & All & $0 / 84(0)$ & NR & NR \\
\hline & $\begin{array}{l}\text { Donor site } \\
\text { hematoma }\end{array}$ & NRCS & Moderate & $1 \mathrm{y}$ & AR with LD & All & $0 / 73(0)$ & NR & NR \\
\hline \multirow{2}{*}{$\begin{array}{l}\text { Nelson, 2019, } \\
31356276, \text { US } \\
\end{array}$} & Hematoma & NRCS & High & $<3 \mathrm{mo}$ & IBR & All & $15 / 1211$ breasts $(1.2)$ & NR & NR \\
\hline & Hematoma & NRCS & High & $<3 \mathrm{mo}$ & $\mathrm{AR}$ & All & NR & NR & NR \\
\hline \multirow{2}{*}{$\begin{array}{l}\text { Banuelos, 2020, } \\
\text { 31663932, US }\end{array}$} & Hematoma & Single group & Low & $\leq 1 \mathrm{mo}$ & IBR & All & $21 / 768(2.7)$ & $\mathrm{N} / \mathrm{A}$ & $\mathrm{N} / \mathrm{A}$ \\
\hline & Hematoma & Single group & Low & $>1 \mathrm{mo}$ & IBR & All & $0 / 768(0)$ & $\mathrm{N} / \mathrm{A}$ & $\mathrm{N} / \mathrm{A}$ \\
\hline \multirow{3}{*}{$\begin{array}{l}\text { Brooks, 2012, } \\
22098451 \text {, US }\end{array}$} & Hematoma & Single group & Low & $3.4 \mathrm{y}$ & IBR & All & $18 / 733(2.5)$ & & \\
\hline & Hematoma & Single group & Low & $3.4 \mathrm{y}$ & IBR & Radiation & $0 / 97(0)$ & $\mathrm{N} / \mathrm{A}$ & $\mathrm{N} / \mathrm{A}$ \\
\hline & Hematoma & Single group & Low & $3.4 \mathrm{y}$ & IBR & No radiation & $18 / 636(2.8)$ & $\mathrm{N} / \mathrm{A}$ & $\mathrm{N} / \mathrm{A}$ \\
\hline $\begin{array}{l}\text { Chen, 2018a, } \\
\text { 29596085, US }\end{array}$ & $\begin{array}{l}\text { Hematoma or } \\
\text { hemorrhage }\end{array}$ & Single group & Low & NR & IBR & All & $495 / 23048(2.1)$ & $\mathrm{N} / \mathrm{A}$ & N/A \\
\hline \multirow[t]{3}{*}{$\begin{array}{l}\text { Cordeiro, 2006, } \\
16980842, \text { US }\end{array}$} & Hematoma & Single group & Moderate & NR & IBR & All & $\begin{array}{l}\text { 10/2276 procedures } \\
(0.4)\end{array}$ & $\mathrm{N} / \mathrm{A}$ & $\mathrm{N} / \mathrm{A}$ \\
\hline & Hematoma & Single group & Moderate & NR & IBR & $\begin{array}{l}\text { Expander } \\
\text { placement } \\
\text { procedure }\end{array}$ & $\begin{array}{l}7 / 1221 \text { procedures } \\
(0.6)\end{array}$ & $\mathrm{N} / \mathrm{A}$ & $\mathrm{N} / \mathrm{A}$ \\
\hline & Hematoma & Single group & Moderate & NR & IBR & $\begin{array}{l}\text { Exchange } \\
\text { procedure }\end{array}$ & $\begin{array}{l}\text { 3/1055 procedures } \\
(0.3)\end{array}$ & $\mathrm{N} / \mathrm{A}$ & $\mathrm{N} / \mathrm{A}$ \\
\hline
\end{tabular}




\begin{tabular}{|c|c|c|c|c|c|c|c|c|c|}
\hline $\begin{array}{l}\text { Study, Year, } \\
\text { PMID, Country }\end{array}$ & $\begin{array}{l}\text { Outcome } \\
\text { Description }\end{array}$ & Design & $\begin{array}{l}\text { Overall } \\
\text { RoB }\end{array}$ & $\begin{array}{l}\text { Time } \\
\text { Point }\end{array}$ & Arm & Subgroup & $\mathrm{n} / \mathrm{N}(\%)$ & $\begin{array}{l}\text { Effect Size }(95 \% \\
\mathrm{Cl})\end{array}$ & P Value \\
\hline & Hematoma & Single group & Moderate & NR & IBR & $\begin{array}{l}\text { Previous } \\
\text { radiation }\end{array}$ & 0/136 procedures $(0)$ & N/A & $\mathrm{N} / \mathrm{A}$ \\
\hline & Hematoma & Single group & Moderate & NR & IBR & $\begin{array}{l}\text { No previous } \\
\text { radiation }\end{array}$ & $\begin{array}{l}\text { 10/2140 procedures } \\
(0.5)\end{array}$ & $\mathrm{N} / \mathrm{A}$ & $\mathrm{N} / \mathrm{A}$ \\
\hline & Hematoma & Single group & Moderate & NR & IBR & Immediate & $\begin{array}{l}7 / 1176 \text { procedures } \\
(0.6)\end{array}$ & $\mathrm{N} / \mathrm{A}$ & $\mathrm{N} / \mathrm{A}$ \\
\hline & Hematoma & Single group & Moderate & NR & IBR & Delayed & $0 / 26$ procedures $(0)$ & $\mathrm{N} / \mathrm{A}$ & $\mathrm{N} / \mathrm{A}$ \\
\hline \multirow{3}{*}{$\begin{array}{l}\text { Cordeiro, 2012, } \\
22286416, \text { US }\end{array}$} & Hematoma & Single group & Low & $<1 \mathrm{y}$ & IBR & All & $29 / 1699(1.7)$ & $\mathrm{N} / \mathrm{A}$ & $\mathrm{N} / \mathrm{A}$ \\
\hline & Hematoma & Single group & Low & $<1 \mathrm{y}$ & IBR & $\begin{array}{l}\text { Prior } \\
\text { radiation }\end{array}$ & $1 / 121(0.8)$ & N/A & $\mathrm{N} / \mathrm{A}$ \\
\hline & Hematoma & Single group & Low & $<1 \mathrm{y}$ & IBR & $\begin{array}{l}\text { No prior } \\
\text { radiation }\end{array}$ & $28 / 1578(1.8)$ & $\mathrm{N} / \mathrm{A}$ & $\mathrm{N} / \mathrm{A}$ \\
\hline $\begin{array}{l}\text { Hunsicker, 2017, } \\
\text { 26849284, US }\end{array}$ & Hematoma & Single group & Low & $<1 \mathrm{y}$ & IBR & All & $15 / 1584$ breasts $(0.9)$ & $\mathrm{N} / \mathrm{A}$ & $\mathrm{N} / \mathrm{A}$ \\
\hline \multirow{3}{*}{$\begin{array}{l}\text { Huo, 2016, } \\
\text { 27697676, US }\end{array}$} & Hematoma & Single group & Low & $1 \mathrm{y}$ & IBR & All & $35 / 1332(2.6)$ & $\mathrm{N} / \mathrm{A}$ & $\mathrm{N} / \mathrm{A}$ \\
\hline & Hematoma & Single group & Low & $1 \mathrm{y}$ & IBR & Non-obese & $26 / 949(2.7)$ & N/A & $\mathrm{N} / \mathrm{A}$ \\
\hline & Hematoma & Single group & Low & $1 \mathrm{y}$ & IBR & Obese & $9 / 383(2.3)$ & $\mathrm{N} / \mathrm{A}$ & $\mathrm{N} / \mathrm{A}$ \\
\hline $\begin{array}{l}\text { Lovecchio, 2015, } \\
\text { 24691330, US }\end{array}$ & Hematoma & Single group & Low & $2.3 y$ & IBR & All & $33 / 1639(2.0)$ & $\mathrm{N} / \mathrm{A}$ & $\mathrm{N} / \mathrm{A}$ \\
\hline $\begin{array}{l}\text { Park, 2019, } \\
\text { 30863940, South } \\
\text { Korea }\end{array}$ & Hematoma & Single group & Moderate & $>6 \mathrm{mo}$ & IBR & All & $11 / 999(1.1)$ & N/A & $\mathrm{N} / \mathrm{A}$ \\
\hline $\begin{array}{l}\text { Rogoff, } 2020, \\
32243320, \text { US }\end{array}$ & Hematoma & Single group & Low & NR & IBR & All & $26 / 627(4.1)$ & $\mathrm{N} / \mathrm{A}$ & $\mathrm{N} / \mathrm{A}$ \\
\hline $\begin{array}{l}\text { Seth, 2015, } \\
\text { 25180955, US }\end{array}$ & $\begin{array}{l}\text { Hematoma } \\
\text { requiring } \\
\text { reoperation }\end{array}$ & Single group & Low & NR & IBR & All & $29 / 893$ (3.2) & $\mathrm{N} / \mathrm{A}$ & $\mathrm{N} / \mathrm{A}$ \\
\hline \multirow{2}{*}{$\begin{array}{l}\text { Singh, 2012, } \\
\text { 22342636, US }\end{array}$} & Hematoma & Single group & Low & $1 \mathrm{mo}$ & IBR & DTI & $6 / 95(6.3)$ & $\mathrm{N} / \mathrm{A}$ & $\mathrm{N} / \mathrm{A}$ \\
\hline & Hematoma & Single group & Low & $1 \mathrm{mo}$ & IBR & $\mathrm{T} / \mathrm{E}$ & $36 / 1221(2.9)$ & $\mathrm{N} / \mathrm{A}$ & $\mathrm{N} / \mathrm{A}$ \\
\hline \multirow{3}{*}{$\begin{array}{l}\text { Singh, 2021, } \\
\text { 33564597, US }\end{array}$} & Hematoma & Single group & Low & $3 \mathrm{mo}$ & IBR & All & $219 / 1740(12.6)$ & N/A & $\mathrm{N} / \mathrm{A}$ \\
\hline & Hematoma & Single group & Low & $3 \mathrm{mo}$ & IBR & DTI & $97 / 870(11.2)$ & $\mathrm{N} / \mathrm{A}$ & $\mathrm{N} / \mathrm{A}$ \\
\hline & Hematoma & Single group & Low & $3 \mathrm{mo}$ & IBR & $\mathrm{T} / \mathrm{E}$ & $122 / 870(14.0)$ & $\mathrm{N} / \mathrm{A}$ & $\mathrm{N} / \mathrm{A}$ \\
\hline \multirow[t]{2}{*}{$\begin{array}{l}\text { Albornoz, 2013, } \\
23897346, \text { US }\end{array}$} & $\begin{array}{l}\text { Hematoma or } \\
\text { hemorrhage }\end{array}$ & Single group & Low & NR & $\mathrm{AR}$ & All & $651 / 21016(3.1)$ & $\mathrm{N} / \mathrm{A}$ & $\mathrm{N} / \mathrm{A}$ \\
\hline & Transfusion & Single group & Low & NR & & All & $1681 / 21016(8.0)$ & $\mathrm{N} / \mathrm{A}$ & $\mathrm{N} / \mathrm{A}$ \\
\hline \multirow{2}{*}{$\begin{array}{l}\text { Andree, 2012, } \\
\text { 23197233, } \\
\text { Germany }\end{array}$} & $\begin{array}{l}\text { Breast } \\
\text { hematoma }\end{array}$ & Single group & Low & $1 \mathrm{mo}$ & AR & All & $26 / 1068$ flaps (2.4) & N/A & $\mathrm{N} / \mathrm{A}$ \\
\hline & $\begin{array}{l}\text { Donor site } \\
\text { hematoma }\end{array}$ & Single group & Low & $1 \mathrm{y}$ & AR & All & 9/1068 flaps $(0.8)$ & $\mathrm{N} / \mathrm{A}$ & $\mathrm{N} / \mathrm{A}$ \\
\hline
\end{tabular}




\begin{tabular}{|c|c|c|c|c|c|c|c|c|c|}
\hline $\begin{array}{l}\text { Study, Year, } \\
\text { PMID, Country }\end{array}$ & $\begin{array}{l}\text { Outcome } \\
\text { Description }\end{array}$ & Design & $\begin{array}{l}\text { Overall } \\
\text { RoB }\end{array}$ & $\begin{array}{l}\text { Time } \\
\text { Point }\end{array}$ & Arm & Subgroup & $\mathrm{n} / \mathrm{N}(\%)$ & $\begin{array}{l}\text { Effect Size }(95 \% \\
\mathrm{Cl})\end{array}$ & P Value \\
\hline $\begin{array}{l}\text { Beugels, 2018, } \\
29399731, \\
\text { Netherlands }\end{array}$ & $\begin{array}{l}\text { Breast } \\
\text { hematoma }\end{array}$ & Single group & Low & $9-10 \mathrm{mo}$ & AR & All & 53/910 flaps (5.8) & $\mathrm{N} / \mathrm{A}$ & $\mathrm{N} / \mathrm{A}$ \\
\hline $\begin{array}{l}\text { Chang, 2000, } \\
\text { 10809092, US }\end{array}$ & $\begin{array}{l}\text { Flap } \\
\text { hematoma }\end{array}$ & Single group & Low & NR & AR & All & 16/936 flaps (1.7) & $\mathrm{N} / \mathrm{A}$ & $\mathrm{N} / \mathrm{A}$ \\
\hline \multirow{5}{*}{$\begin{array}{l}\text { Chang, 2011, } \\
21407063, \text { US }\end{array}$} & Hematoma & Single group & Low & NR & $\mathrm{AR}$ & All & $7 / 818(0.9)$ & $\mathrm{N} / \mathrm{A}$ & $\mathrm{N} / \mathrm{A}$ \\
\hline & Hematoma & Single group & Low & NR & $\mathrm{AR}$ & Age $<50$ & $2 / 411(0.5)$ & $\mathrm{N} / \mathrm{A}$ & $\mathrm{N} / \mathrm{A}$ \\
\hline & Hematoma & Single group & Low & NR & $\mathrm{AR}$ & Age 50-59 & $4 / 285(1.4)$ & $\mathrm{N} / \mathrm{A}$ & $\mathrm{N} / \mathrm{A}$ \\
\hline & Hematoma & Single group & Low & NR & $\mathrm{AR}$ & Age 60-69 & $0 / 103(0)$ & $\mathrm{N} / \mathrm{A}$ & $\mathrm{N} / \mathrm{A}$ \\
\hline & Hematoma & Single group & Low & NR & $\mathrm{AR}$ & Age $>70$ & $1 / 19(5.3)$ & N/A & N/A \\
\hline $\begin{array}{l}\text { Chen, 2018b, } \\
\text { 29596085, US }\end{array}$ & $\begin{array}{l}\text { Bleeding } \\
\text { (hematoma/ } \\
\text { hemorrhage) }\end{array}$ & Single group & Low & NR & AR & All & $688 / 19496(3.5)$ & N/A & $\mathrm{N} / \mathrm{A}$ \\
\hline $\begin{array}{l}\text { Cleveland, 2013, } \\
\text { 23945529, US }\end{array}$ & $\begin{array}{l}\text { Postop } \\
\text { transfusion }\end{array}$ & Single group & Low & NR & AR & All & $65 / 812(8.0)$ & $\mathrm{N} / \mathrm{A}$ & $\mathrm{N} / \mathrm{A}$ \\
\hline $\begin{array}{l}\text { Enajat, 2010, } \\
19790180, \text { Sweden }\end{array}$ & Hematoma & Single group & Low & $1-7 d$ & $\mathrm{AR}$ & All & 44/564 flaps (7.8) & $\mathrm{N} / \mathrm{A}$ & $\mathrm{N} / \mathrm{A}$ \\
\hline $\begin{array}{l}\text { Gill, 2004, } \\
\text { 15083015, US }\end{array}$ & Hematoma & Single group & Moderate & $10 \mathrm{y}$ & $\mathrm{AR}$ & All & $14 / 758(1.8)$ & $\mathrm{N} / \mathrm{A}$ & $\mathrm{N} / \mathrm{A}$ \\
\hline $\begin{array}{l}\text { Haddock, 2019, } \\
31461004, \text { US }\end{array}$ & Hematoma & Single group & Moderate & NR & $\mathrm{AR}$ & All & $15 / 509(2.9)$ & $\mathrm{N} / \mathrm{A}$ & $\mathrm{N} / \mathrm{A}$ \\
\hline $\begin{array}{l}\text { Jo, } 2020 \text {, } \\
33386262 \text {, South } \\
\text { Korea }\end{array}$ & $\begin{array}{l}\text { Breast } \\
\text { hematoma }\end{array}$ & Single group & Low & $\begin{array}{l}11 \mathrm{mo}- \\
1.5 \mathrm{y}\end{array}$ & AR & All & $36 / 623(5.78)$ & $\mathrm{N} / \mathrm{A}$ & $\mathrm{N} / \mathrm{A}$ \\
\hline $\begin{array}{l}\text { Liao, 2008, } \\
\text { 18349626, US }\end{array}$ & Hematoma & Single group & Low & NR & AR & All & $5 / 679(0.7)$ & $\mathrm{N} / \mathrm{A}$ & $\mathrm{N} / \mathrm{A}$ \\
\hline \multirow{2}{*}{$\begin{array}{l}\text { Masoomi, 2019, } \\
\text { 31331721, US }\end{array}$} & Hematoma & Single group & Low & NR & AR & All & $1465 / 55840(2.6)$ & $\mathrm{N} / \mathrm{A}$ & $\mathrm{N} / \mathrm{A}$ \\
\hline & $\begin{array}{l}\text { Blood } \\
\text { transfusion }\end{array}$ & Single group & Low & NR & AR & All & $687 / 7991$ (8.6) & $\mathrm{N} / \mathrm{A}$ & $\mathrm{N} / \mathrm{A}$ \\
\hline \multirow{2}{*}{$\begin{array}{l}\text { Munder, 2020, } \\
32565553, \\
\text { Germany }\end{array}$} & $\begin{array}{l}\text { Breast } \\
\text { hematoma }\end{array}$ & Single group & Low & $2 y$ & AR & All & 17/1274 flaps (1.3) & $\mathrm{N} / \mathrm{A}$ & $\mathrm{N} / \mathrm{A}$ \\
\hline & $\begin{array}{l}\text { Donor site } \\
\text { hematoma }\end{array}$ & Single group & Low & $2 y$ & $\mathrm{AR}$ & All & 5/1274 flaps $(0.4)$ & $\mathrm{N} / \mathrm{A}$ & $\mathrm{N} / \mathrm{A}$ \\
\hline $\begin{array}{l}\text { Mehrara, 2006, } \\
17016173, \text { US }\end{array}$ & Hematoma & Single group & Moderate & $1 \mathrm{mo}$ & $\mathrm{AR}$ & All & 19/1195 (1.6) & $\mathrm{N} / \mathrm{A}$ & $\mathrm{N} / \mathrm{A}$ \\
\hline \multirow[t]{2}{*}{$\begin{array}{l}\text { Nelson, 2014, } \\
25046665, \text { US }\end{array}$} & $\begin{array}{l}\text { Breast } \\
\text { hematoma }\end{array}$ & Single group & Moderate & NR & AR & All & 29/1293 flaps (2.2) & $\mathrm{N} / \mathrm{A}$ & $\mathrm{N} / \mathrm{A}$ \\
\hline & $\begin{array}{l}\text { Donor site } \\
\text { hematoma }\end{array}$ & Single group & Moderate & NR & $\mathrm{AR}$ & All & $\begin{array}{l}1 \text { flap/1293 flaps } \\
(0.1)\end{array}$ & $\mathrm{N} / \mathrm{A}$ & $\mathrm{N} / \mathrm{A}$ \\
\hline \multirow{2}{*}{$\begin{array}{l}\text { O'Neill, 2019, } \\
\text { 31196805, Canada }\end{array}$} & Hematoma & Single group & Low & $1 \mathrm{~d}$ & $\mathrm{AR}$ & All & $24 / 512(4.7)$ & $\mathrm{N} / \mathrm{A}$ & $\mathrm{N} / \mathrm{A}$ \\
\hline & Hematoma & Single group & Low & $<2 \mathrm{mo}$ & $\mathrm{AR}$ & All & $42 / 960(4.4)$ & $\mathrm{N} / \mathrm{A}$ & $\mathrm{N} / \mathrm{A}$ \\
\hline
\end{tabular}




\begin{tabular}{|c|c|c|c|c|c|c|c|c|c|}
\hline $\begin{array}{l}\text { Study, Year, } \\
\text { PMID, Country }\end{array}$ & $\begin{array}{l}\text { Outcome } \\
\text { Description }\end{array}$ & Design & $\begin{array}{l}\text { Overall } \\
\text { RoB }\end{array}$ & $\begin{array}{l}\text { Time } \\
\text { Point }\end{array}$ & Arm & Subgroup & $\mathrm{n} / \mathrm{N}(\%)$ & $\begin{array}{l}\text { Effect Size }(95 \% \\
\mathrm{Cl})\end{array}$ & P Value \\
\hline & Hematoma & Single group & Low & $3 \mathrm{mo}$ & AR & All & $62 / 912(6.8)$ & $\mathrm{N} / \mathrm{A}$ & $\mathrm{N} / \mathrm{A}$ \\
\hline \multirow{2}{*}{$\begin{array}{l}\text { Phan, 2020, } \\
\text { 31124177, UK }\end{array}$} & Hematoma & Single group & Low & NR & $\mathrm{AR}$ & All & $69 / 1070(6.4)$ & $\mathrm{N} / \mathrm{A}$ & $\mathrm{N} / \mathrm{A}$ \\
\hline & $\begin{array}{l}\text { Hematoma } \\
\text { requiring } \\
\text { postop blood } \\
\text { transfusion }\end{array}$ & Single group & Low & NR & $\mathrm{AR}$ & All & $10 / 1070(0.9)$ & $\mathrm{N} / \mathrm{A}$ & $\mathrm{N} / \mathrm{A}$ \\
\hline \multirow{2}{*}{$\begin{array}{l}\text { Prantl, 2020, } \\
32895743, \\
\text { Germany }\end{array}$} & $\begin{array}{l}\text { Breast } \\
\text { hematoma }\end{array}$ & Single group & Low & $3 \mathrm{mo}$ & AR & All & $\begin{array}{l}148 \text { flaps/4577 flaps } \\
\text { (3.2) }\end{array}$ & $\mathrm{N} / \mathrm{A}$ & $\mathrm{N} / \mathrm{A}$ \\
\hline & $\begin{array}{l}\text { Donor site } \\
\text { hematoma }\end{array}$ & Single group & Low & $3 \mathrm{mo}$ & AR & All & $\begin{array}{l}37 \text { flaps/4577 flaps } \\
(0.8)\end{array}$ & $\mathrm{N} / \mathrm{A}$ & $\mathrm{N} / \mathrm{A}$ \\
\hline $\begin{array}{l}\text { Rubio, 2019, } \\
\text { 30665841, Belgium }\end{array}$ & Hematoma & Single group & Low & NR & AR & All & $1357 / 56522(2.4)$ & $\mathrm{N} / \mathrm{A}$ & $\mathrm{N} / \mathrm{A}$ \\
\hline $\begin{array}{l}\text { Selber, 2009, } \\
\text { 19935283, US }\end{array}$ & Hematoma & Single group & Low & $5.6 \mathrm{mo}$ & AR & All & $13 / 1031(1.3)$ & $\mathrm{N} / \mathrm{A}$ & $\mathrm{N} / \mathrm{A}$ \\
\hline $\begin{array}{l}\text { Song, 2016, } \\
26637165 \text {, US }\end{array}$ & $\begin{array}{l}\text { Hematoma } \\
\text { requiring } \\
\text { reoperation }\end{array}$ & Single group & Low & $4.3-5.4 y$ & AR & All & $103 / 1809(5.7)$ & $\mathrm{N} / \mathrm{A}$ & $\mathrm{N} / \mathrm{A}$ \\
\hline $\begin{array}{l}\text { Tran, 2018, } \\
\text { 29794694, US }\end{array}$ & Hematoma & Single group & Low & NR & AR & All & $50 / 1253$ flaps (4.0) & $\mathrm{N} / \mathrm{A}$ & $\mathrm{N} / \mathrm{A}$ \\
\hline $\begin{array}{l}\text { Watterson, 1995, } \\
7761505, \text { Australia }\end{array}$ & Hematoma & Single group & Moderate & $2.7 \mathrm{mo}$ & AR & All & $7 / 556(1.3)$ & $\mathrm{N} / \mathrm{A}$ & $\mathrm{N} / \mathrm{A}$ \\
\hline
\end{tabular}

7761505, Australia

Abbreviations: adj $=$ adjusted, $\mathrm{AR}=$ autologous reconstruction, $\mathrm{CI}=$ confidence interval, $\mathrm{IBR}=$ implant-based reconstruction, $\mathrm{N} / \mathrm{A}=$ not applicable, $\mathrm{NR}=$ not reported, $\mathrm{NRCS}=$ nonrandomized comparative study, $\mathrm{OR}=$ odds ratio, $\mathrm{PMID}=$ PubMed identifier, $\mathrm{Ref}=$ reference group, $\mathrm{RoB}=$ risk of bias, $\mathrm{y}=\mathrm{years}$.

Colors: Header rows are shaded orange. Rows for every alternate study are shaded blue. The colors do not add unique information.

Table F-1.26. Full Evidence Table - Key Question 1: IBR versus AR - categorical outcomes (composite/unspecified harms)

\begin{tabular}{|c|c|c|c|c|c|c|c|c|c|}
\hline $\begin{array}{l}\text { Study, Year, } \\
\text { PMID, Country }\end{array}$ & Outcome & Design & $\begin{array}{l}\text { Overall } \\
\text { RoB }\end{array}$ & $\begin{array}{l}\text { Time } \\
\text { Point }\end{array}$ & Arm & Subgroup & $n / N(\%)$ & Effect Size $(95 \% \mathrm{Cl})$ & $\begin{array}{l}P \\
\text { Value }\end{array}$ \\
\hline \multirow{12}{*}{$\begin{array}{l}\text { Brorson 2020a, } \\
32807615, \\
\text { Sweden }\end{array}$} & Clavien-Dindo Grade I complications & RCT & High & $1 \mathrm{mo}$ & IBR & All & $29 / 70(41)$ & Ref & Ref \\
\hline & Clavien-Dindo Grade I complications & RCT & High & $1 \mathrm{mo}$ & AR & All & $23 / 55(42)$ & OR $1.26(0.63,2.55)$ & 0.65 \\
\hline & Clavien-Dindo Grade II complications & RCT & High & $1 \mathrm{mo}$ & IBR & All & $18 / 70(26)$ & Ref & Ref \\
\hline & Clavien-Dindo Grade II complications & RCT & High & $1 \mathrm{mo}$ & AR & All & $14 / 55(25)$ & OR $0.99(0.44,2.22)$ & 0.53 \\
\hline & Clavien-Dindo Grade IIla complications & RCT & High & $1 \mathrm{mo}$ & IBR & All & $1 / 70(1.4)$ & Ref & Ref \\
\hline & Clavien-Dindo Grade IIla complications & RCT & High & $1 \mathrm{mo}$ & AR & All & $1 / 55(1.8)$ & OR $1.28(0.08,20.9)$ & 0.86 \\
\hline & Clavien-Dindo Grade IIlb complications & RCT & High & $1 \mathrm{mo}$ & IBR & All & $4 / 70(5.7)$ & Ref & Ref \\
\hline & Clavien-Dindo Grade IIIb complications & RCT & High & $1 \mathrm{mo}$ & AR & All & $1 / 55(1.8)$ & OR $0.31(0.03,2.82)$ & 0.30 \\
\hline & Clavien-Dindo Grade IV complications & RCT & High & $1 \mathrm{mo}$ & IBR & All & $0 / 70(0)$ & Ref & Ref \\
\hline & Clavien-Dindo Grade IV complications & RCT & High & $1 \mathrm{mo}$ & AR & All & $0 / 54(0)$ & No events & $\mathrm{N} / \mathrm{A}$ \\
\hline & Clavien-Dindo Grade V complications & RCT & High & $1 \mathrm{mo}$ & IBR & All & $0 / 70(0)$ & Ref & Ref \\
\hline & Clavien-Dindo Grade V complications & RCT & High & $1 \mathrm{mo}$ & AR & All & $0 / 54(0)$ & No events & $\mathrm{N} / \mathrm{A}$ \\
\hline
\end{tabular}




\begin{tabular}{|c|c|c|c|c|c|c|c|c|c|}
\hline $\begin{array}{l}\text { Study, Year, } \\
\text { PMID, Country }\end{array}$ & Outcome & Design & $\begin{array}{l}\text { Overall } \\
\text { RoB }\end{array}$ & $\begin{array}{l}\text { Time } \\
\text { Point }\end{array}$ & Arm & Subgroup & $\mathrm{n} / \mathrm{N}(\%)$ & Effect Size $(95 \% \mathrm{Cl})$ & $\begin{array}{l}P \\
\text { Value }\end{array}$ \\
\hline \multirow[t]{2}{*}{$\begin{array}{l}\text { Chetta, 2017, } \\
28002254, \text { US }\end{array}$} & $\begin{array}{l}\text { Any complication: infection, wound } \\
\text { complications, hematoma, mechanical } \\
\text { implant complications (rupture), } \\
\text { capsular contractures of implant, fat } \\
\text { necrosis and flap venous congestion }\end{array}$ & NRCS & High & $1.3 y$ & IBR & All & $\begin{array}{l}1742 / 3846 \\
(45.3)\end{array}$ & adjOR $0.48(0.4,0.57)$ & NR \\
\hline & $\begin{array}{l}\text { Any complication: infection, wound } \\
\text { complications, hematoma, mechanical } \\
\text { implant complications (rupture), } \\
\text { capsular contractures of implant, fat } \\
\text { necrosis and flap venous congestion }\end{array}$ & NRCS & High & $1.3 \mathrm{y}$ & AR & All & $189 / 935(30.8)$ & Ref & Ref \\
\hline \multirow{2}{*}{$\begin{array}{l}\text { Dauplat, 2021, } \\
33622886, \\
\text { France }\end{array}$} & $\begin{array}{l}\text { Major complications (rehospitalization } \\
\text { or reoperation) }\end{array}$ & NRCS & Moderate & $1 \mathrm{y}$ & IBR & All & $37 / 205(18)$ & Ref & Ref \\
\hline & $\begin{array}{l}\text { Major complications (rehospitalization } \\
\text { or reoperation) }\end{array}$ & NRCS & Moderate & $1 \mathrm{y}$ & $\begin{array}{l}\text { AR with } \\
\text { LD } \\
\text { without } \\
\text { implant }\end{array}$ & All & $7 / 78(9)$ & adjOR $2.86(1.41,5.83)$ & NR \\
\hline \multirow[t]{2}{*}{$\begin{array}{l}\text { Fischer, 2014, } \\
24916480, \text { US }\end{array}$} & Major surgical complication & NRCS & High & $\begin{array}{l}1.8-2.1 \\
y\end{array}$ & IBR & All & $31 / 155(20)$ & Ref & Ref \\
\hline & Major surgical complication & NRCS & High & $\begin{array}{l}1.8-2.1 \\
y\end{array}$ & $\mathrm{AR}$ & All & $17 / 155(11)$ & NR & 0.08 \\
\hline \multirow{3}{*}{$\begin{array}{l}\text { Fischer, 2015, } \\
\text { 26366550, US }\end{array}$} & Complications requiring hospitalization & NRCS & High & $3 \mathrm{mo}$ & IBR DTI & All & $113 / 1717(6.6)$ & Ref & Ref \\
\hline & Complications requiring hospitalization & NRCS & High & $3 \mathrm{mo}$ & $\begin{array}{l}\text { IBR with } \\
\text { T/E }\end{array}$ & All & $695 / 10690(6.5)$ & Ref & Ref \\
\hline & Complications requiring hospitalization & NRCS & High & $3 \mathrm{mo}$ & AR & All & $360 / 2747(13.1)$ & $\begin{array}{l}\text { vs. IBR DTI: adjOR } \\
1.36(1.22,1.52) \\
\text { vs. IBR with T/E: adjOR } \\
2.09(1.82,2.41)\end{array}$ & NR \\
\hline \multirow{2}{*}{$\begin{array}{l}\text { Kouwenberg, } \\
2020, \\
32590633, \\
\text { Netherlands }\end{array}$} & Any complication & NRCS & NRCS & $>6 \mathrm{mo}$ & IBR & All & $94 / 296(31.6)$ & Ref & Ref \\
\hline & Any complication & NRCS & NRCS & $>6 \mathrm{mo}$ & AR & All & $83 / 179(46.6)$ & adjOR $1.86(1.27,2.72)$ & 0.002 \\
\hline \multirow{8}{*}{$\begin{array}{l}\text { Kulkarni, 2017, } \\
\text { 28713853, US } \\
\text { \& Canada }\end{array}$} & Any complication & NRCS & Moderate & $1 \mathrm{y}$ & IBR (all) & All & NR & Ref & Ref \\
\hline & Any complication & NRCS & Moderate & $1 \mathrm{y}$ & IBR (all) & Unilateral & NR & NR & NR \\
\hline & Any complication & NRCS & Moderate & $1 \mathrm{y}$ & IBR (all) & Bilateral & NR & NR & NR \\
\hline & Any complication & NRCS & Moderate & $1 \mathrm{y}$ & IBR DTI & All & NR & NR & NR \\
\hline & Any complication & NRCS & Moderate & $1 \mathrm{y}$ & IBR DTI & Unilateral & NR & NR & NR \\
\hline & Any complication & NRCS & Moderate & $1 \mathrm{y}$ & IBR DTI & Bilateral & NR & NR & NR \\
\hline & Any complication & NRCS & Moderate & $1 \mathrm{y}$ & $\begin{array}{l}\text { IBR with } \\
\text { T/E }\end{array}$ & All & NR & NR & NR \\
\hline & Any complication & NRCS & Moderate & $1 \mathrm{y}$ & $\begin{array}{l}\text { IBR with } \\
\text { T/E }\end{array}$ & Unilateral & NR & NR & NR \\
\hline
\end{tabular}




\begin{tabular}{|c|c|c|c|c|c|c|c|c|c|}
\hline $\begin{array}{l}\text { Study, Year, } \\
\text { PMID, Country }\end{array}$ & Outcome & Design & $\begin{array}{l}\text { Overall } \\
\text { RoB }\end{array}$ & $\begin{array}{l}\text { Time } \\
\text { Point }\end{array}$ & Arm & Subgroup & $n / N(\%)$ & Effect Size $(95 \% \mathrm{CI})$ & $\begin{array}{l}\mathbf{P} \\
\text { Value }\end{array}$ \\
\hline & Any complication & NRCS & Moderate & $1 \mathrm{y}$ & $\begin{array}{l}\text { IBR with } \\
\text { T/E }\end{array}$ & Bilateral & NR & NR & NR \\
\hline & Any complication & NRCS & Moderate & $1 \mathrm{y}$ & AR (all) & All & NR & NR & NR \\
\hline & Any complication & NRCS & Moderate & $1 \mathrm{y}$ & AR (all) & Unilateral & NR & NR & NR \\
\hline & Any complication & NRCS & Moderate & $1 \mathrm{y}$ & AR (all) & Bilateral & NR & NR & NR \\
\hline & Any complication & NRCS & Moderate & $1 \mathrm{y}$ & $\begin{array}{l}\text { AR with } \\
\text { DIEP }\end{array}$ & All & NR/365 (NR) & $\frac{\text { vs. IBR (all): adjOR }}{2.22(1.57,3.13)}$ & $<0.001$ \\
\hline & Any complication & NRCS & Moderate & $1 \mathrm{y}$ & $\begin{array}{l}\text { AR with } \\
\text { DIEP }\end{array}$ & Unilateral & NR & NR & NR \\
\hline & Any complication & NRCS & Moderate & $1 \mathrm{y}$ & $\begin{array}{l}\text { AR with } \\
\text { DIEP }\end{array}$ & Bilateral & NR & NR & NR \\
\hline & Any complication & NRCS & Moderate & $1 \mathrm{y}$ & $\begin{array}{l}\text { AR with } \\
\text { free } \\
\text { TRAM }\end{array}$ & All & NR & $\begin{array}{l}\text { vs. IBR (all): adjOR } \\
1.94(1.17,3.23)\end{array}$ & 0.011 \\
\hline & Any complication & NRCS & Moderate & $1 \mathrm{y}$ & $\begin{array}{l}\text { AR with } \\
\text { free } \\
\text { TRAM }\end{array}$ & Unilateral & NR & NR & NR \\
\hline & Any complication & NRCS & Moderate & $1 \mathrm{y}$ & $\begin{array}{l}\text { AR with } \\
\text { free } \\
\text { TRAM }\end{array}$ & Bilateral & NR & NR & NR \\
\hline & Any complication & NRCS & Moderate & $1 \mathrm{y}$ & $\begin{array}{l}\text { AR with } \\
\text { pedicled } \\
\text { TRAM }\end{array}$ & All & NR & $\frac{\text { vs. IBR (all): adjOR }}{1.89(1.08,3.30)}$ & 0.025 \\
\hline & Any complication & NRCS & Moderate & $1 \mathrm{y}$ & $\begin{array}{l}\text { AR with } \\
\text { pedicled } \\
\text { TRAM }\end{array}$ & Unilateral & NR & NR & NR \\
\hline & Any complication & NRCS & Moderate & $1 \mathrm{y}$ & $\begin{array}{l}\text { AR with } \\
\text { pedicled } \\
\text { TRAM }\end{array}$ & Bilateral & NR & NR & NR \\
\hline & Any complication & NRCS & Moderate & $1 \mathrm{y}$ & $\begin{array}{l}\text { AR with } \\
\text { LD }\end{array}$ & All & NR & $\begin{array}{l}\text { vs. IBR (all): adjOR } \\
1.95(1.08,3.51)\end{array}$ & 0.026 \\
\hline & Any complication & NRCS & Moderate & $1 \mathrm{y}$ & $\begin{array}{l}\text { AR with } \\
\text { LD }\end{array}$ & Unilateral & NR & NR & NR \\
\hline & Any complication & NRCS & Moderate & $1 \mathrm{y}$ & $\begin{array}{l}\text { AR with } \\
\text { LD }\end{array}$ & Bilateral & NR & NR & NR \\
\hline & Any complication & NRCS & Moderate & $1 \mathrm{y}$ & $\begin{array}{l}\text { AR with } \\
\text { SIEA }\end{array}$ & All & NR & NR & NR \\
\hline & Any complication & NRCS & Moderate & $1 \mathrm{y}$ & $\begin{array}{l}\text { AR with } \\
\text { SIEA }\end{array}$ & Unilateral & NR & NR & NR \\
\hline & Any complication & NRCS & Moderate & $1 \mathrm{y}$ & $\begin{array}{l}\text { AR with } \\
\text { SIEA }\end{array}$ & All & NR & NR & NR \\
\hline & Any complication & NRCS & Moderate & $2 y$ & IBR & All & NR & NR & NR \\
\hline
\end{tabular}




\begin{tabular}{|c|c|c|c|c|c|c|c|c|c|}
\hline $\begin{array}{l}\text { Study, Year, } \\
\text { PMID, Country }\end{array}$ & Outcome & Design & $\begin{array}{l}\text { Overall } \\
\text { RoB }\end{array}$ & $\begin{array}{l}\text { Time } \\
\text { Point }\end{array}$ & Arm & Subgroup & $n / N(\%)$ & Effect Size (95\% Cl) & $\begin{array}{l}\mathbf{P} \\
\text { Value }\end{array}$ \\
\hline & Any complication & NRCS & Moderate & $2 y$ & IBR & Unilateral & $145 / 600(24.2)$ & NR & NR \\
\hline & Any complication & NRCS & Moderate & $2 y$ & IBR & Bilateral & $287 / 994(28.9)$ & NR & NR \\
\hline & Any complication & NRCS & Moderate & $2 y$ & IBR DTI & All & $35 / 112(31.3)$ & $\frac{\text { vs. IBR with T/E: adjOR }}{1.08(0.65,1.77)}$ & 0.78 \\
\hline & Any complication & NRCS & Moderate & $2 y$ & IBR DTI & Unilateral & NR & NR & NR \\
\hline & Any complication & NRCS & Moderate & $2 y$ & IBR DTI & Bilateral & NR & NR & NR \\
\hline & Any complication & NRCS & Moderate & $2 y$ & $\begin{array}{l}\text { IBR with } \\
\text { T/E }\end{array}$ & All & $406 / 1525(26.6)$ & Ref & Ref \\
\hline & Any complication & NRCS & Moderate & $2 \mathrm{y}$ & $\begin{array}{l}\text { IBR with } \\
\text { T/E }\end{array}$ & Unilateral & NR & NR & NR \\
\hline & Any complication & NRCS & Moderate & $2 y$ & $\begin{array}{l}\text { IBR with } \\
\text { T/E }\end{array}$ & Bilateral & NR & NR & NR \\
\hline & Any complication & NRCS & Moderate & $2 y$ & AR (all) & All & NR & NR & NR \\
\hline & Any complication & NRCS & Moderate & $2 y$ & AR (all) & Unilateral & $147 / 317(46.4)$ & NR & NR \\
\hline & Any complication & NRCS & Moderate & $2 y$ & AR (all) & Bilateral & $123 / 214(57.5)$ & NR & NR \\
\hline & Any complication & NRCS & Moderate & $2 y$ & $\begin{array}{l}\text { AR with } \\
\text { DIEP }\end{array}$ & All & $185 / 390(47.4)$ & $\begin{array}{l}\text { vs. IBR with T/E: adjOR } \\
1.97(1.41,2.76)\end{array}$ & $<0.001$ \\
\hline & Any complication & NRCS & Moderate & $2 y$ & $\begin{array}{l}\text { AR with } \\
\text { DIEP }\end{array}$ & Unilateral & NR & NR & NR \\
\hline & Any complication & NRCS & Moderate & $2 y$ & $\begin{array}{l}\text { AR with } \\
\text { DIEP }\end{array}$ & Bilateral & NR & NR & NR \\
\hline & Any complication & NRCS & Moderate & $2 y$ & $\begin{array}{l}\text { AR with } \\
\text { free } \\
\text { TRAM }\end{array}$ & All & $34 / 95$ (35.8) & $\frac{\text { vs. IBR with T/E: adjOR }}{2.48(1.33,4.64)}$ & 0.005 \\
\hline & Any complication & NRCS & Moderate & $2 y$ & $\begin{array}{l}\text { AR with } \\
\text { free } \\
\text { TRAM }\end{array}$ & Unilateral & NR & NR & NR \\
\hline & Any complication & NRCS & Moderate & $2 y$ & $\begin{array}{l}\text { AR with } \\
\text { free } \\
\text { TRAM }\end{array}$ & Bilateral & NR & NR & NR \\
\hline & Any complication & NRCS & Moderate & $2 y$ & $\begin{array}{l}\text { AR with } \\
\text { pedicled } \\
\text { TRAM }\end{array}$ & All & $35 / 85$ (41.2) & $\frac{\text { vs. IBR with T/E: }}{1.91(1.01,1.31)}$ & 0.005 \\
\hline & Any complication & NRCS & Moderate & $2 y$ & $\begin{array}{l}\text { AR with } \\
\text { pedicled } \\
\text { TRAM }\end{array}$ & Unilateral & NR & NR & NR \\
\hline & Any complication & NRCS & Moderate & $2 y$ & $\begin{array}{l}\text { AR with } \\
\text { pedicled } \\
\text { TRAM }\end{array}$ & Bilateral & NR & NR & NR \\
\hline & Any complication & NRCS & Moderate & $2 y$ & $\begin{array}{l}\text { AR with } \\
\text { LD }\end{array}$ & All & 28/71 (39.4) & $\frac{\text { vs. IBR with T/E: adjOR }}{1.87(1.03,3.4)}$ & 0.04 \\
\hline
\end{tabular}




\begin{tabular}{|c|c|c|c|c|c|c|c|c|c|}
\hline $\begin{array}{l}\text { Study, Year, } \\
\text { PMID, Country }\end{array}$ & Outcome & Design & $\begin{array}{l}\text { Overall } \\
\text { RoB }\end{array}$ & $\begin{array}{l}\text { Time } \\
\text { Point }\end{array}$ & Arm & Subgroup & $n / N(\%)$ & Effect Size $(95 \% \mathrm{CI})$ & $\begin{array}{l}\mathbf{P} \\
\text { Value }\end{array}$ \\
\hline & Any complication & NRCS & Moderate & $2 \mathrm{y}$ & $\begin{array}{l}\text { AR with } \\
\text { LD }\end{array}$ & Unilateral & NR & NR & NR \\
\hline & Any complication & NRCS & Moderate & $2 y$ & $\begin{array}{l}\text { AR with } \\
\text { LD }\end{array}$ & Bilateral & NR & NR & NR \\
\hline & Any complication & NRCS & Moderate & $2 y$ & $\begin{array}{l}\text { AR with } \\
\text { SIEA }\end{array}$ & All & 48/65 (73.9) & $\begin{array}{l}\text { vs. IBR with T/E: adjOR } \\
4.71(2.32,9.54)\end{array}$ & $<0.001$ \\
\hline & Any complication & NRCS & Moderate & $2 y$ & $\begin{array}{l}\text { AR with } \\
\text { SIEA }\end{array}$ & Unilateral & NR & NR & NR \\
\hline & Any complication & NRCS & Moderate & $2 y$ & $\begin{array}{l}\text { AR with } \\
\text { SIEA }\end{array}$ & All & NR & NR & NR \\
\hline & Major complications & NRCS & Moderate & $1 \mathrm{y}$ & IBR (all) & All & NR & NR & NR \\
\hline & Major complications & NRCS & Moderate & $1 \mathrm{y}$ & IBR (all) & Unilateral & $105 / 600(17.5)$ & Ref & Ref \\
\hline & Major complications & NRCS & Moderate & $1 \mathrm{y}$ & IBR (all) & Bilateral & $215 / 994(21.6)$ & Ref & Ref \\
\hline & Major complications & NRCS & Moderate & $1 \mathrm{y}$ & IBR DTI & All & NR & NR & NR \\
\hline & Major complications & NRCS & Moderate & $1 \mathrm{y}$ & IBR DTI & Unilateral & NR & NR & NR \\
\hline & Major complications & NRCS & Moderate & $1 \mathrm{y}$ & IBR DTI & Bilateral & NR & NR & NR \\
\hline & Major complications & NRCS & Moderate & $1 \mathrm{y}$ & $\begin{array}{l}\text { IBR with } \\
\text { T/E }\end{array}$ & All & NR & NR & NR \\
\hline & Major complications & NRCS & Moderate & $1 \mathrm{y}$ & $\begin{array}{l}\text { IBR with } \\
\text { T/E }\end{array}$ & Unilateral & NR & NR & NR \\
\hline & Major complications & NRCS & Moderate & $1 \mathrm{y}$ & $\begin{array}{l}\text { IBR with } \\
\text { T/E }\end{array}$ & Bilateral & NR & NR & NR \\
\hline & Major complications & NRCS & Moderate & $1 \mathrm{y}$ & AR (all) & All & NR & NR & NR \\
\hline & Major complications & NRCS & Moderate & $1 \mathrm{y}$ & AR (all) & Unilateral & $87 / 317(27.4)$ & $\frac{\text { vs. IBR (all): adjOR }}{2.19(1.39,3.47)}$ & 0.001 \\
\hline & Major complications & NRCS & Moderate & $1 \mathrm{y}$ & AR (all) & Bilateral & $81 / 224(37.9)$ & $\begin{array}{l}\text { vs. IBR (all): adjOR } \\
1.69(1.11,2.56)\end{array}$ & 0.014 \\
\hline & Major complications & NRCS & Moderate & $1 \mathrm{y}$ & $\begin{array}{l}\text { AR with } \\
\text { DIEP }\end{array}$ & All & NR & $\begin{array}{l}\text { vs. IBR (all): adjOR } \\
1.75(1.19,2.58)\end{array}$ & 0.004 \\
\hline & Major complications & NRCS & Moderate & $1 \mathrm{y}$ & $\begin{array}{l}\text { AR with } \\
\text { DIEP }\end{array}$ & Unilateral & NR & NR & NR \\
\hline & Major complications & NRCS & Moderate & $1 \mathrm{y}$ & $\begin{array}{l}\text { AR with } \\
\text { DIEP }\end{array}$ & Bilateral & NR & NR & NR \\
\hline & Major complications & NRCS & Moderate & $1 \mathrm{y}$ & $\begin{array}{l}\text { AR with } \\
\text { free } \\
\text { TRAM }\end{array}$ & All & NR & $\begin{array}{l}\text { vs. IBR (all): adjOR } \\
1.75(1.19,2.58)\end{array}$ & 0.12 \\
\hline & Major complications & NRCS & Moderate & $1 \mathrm{y}$ & $\begin{array}{l}\text { AR with } \\
\text { free } \\
\text { TRAM }\end{array}$ & Unilateral & NR & NR & NR \\
\hline & Major complications & NRCS & Moderate & $1 \mathrm{y}$ & $\begin{array}{l}\text { AR with } \\
\text { free } \\
\text { TRAM }\end{array}$ & Bilateral & NR & NR & NR \\
\hline
\end{tabular}




\begin{tabular}{|c|c|c|c|c|c|c|c|c|c|}
\hline $\begin{array}{l}\text { Study, Year, } \\
\text { PMID, Country }\end{array}$ & Outcome & Design & $\begin{array}{l}\text { Overall } \\
\text { RoB }\end{array}$ & $\begin{array}{l}\text { Time } \\
\text { Point }\end{array}$ & Arm & Subgroup & $n / N(\%)$ & Effect Size $(95 \% \mathrm{CI})$ & $\begin{array}{l}\mathbf{P} \\
\text { Value }\end{array}$ \\
\hline & Major complications & NRCS & Moderate & $1 \mathrm{y}$ & $\begin{array}{l}\text { AR with } \\
\text { pedicled } \\
\text { TRAM }\end{array}$ & All & NR & $\frac{\text { vs. IBR (all): adjOR }}{1.86(1.02,3.4)}$ & 0.044 \\
\hline & Major complications & NRCS & Moderate & $1 \mathrm{y}$ & $\begin{array}{l}\text { AR with } \\
\text { pedicled } \\
\text { TRAM }\end{array}$ & Unilateral & NR & NR & NR \\
\hline & Major complications & NRCS & Moderate & $1 \mathrm{y}$ & $\begin{array}{l}\text { AR with } \\
\text { pedicled } \\
\text { TRAM }\end{array}$ & Bilateral & NR & NR & NR \\
\hline & Major complications & NRCS & Moderate & $1 \mathrm{y}$ & $\begin{array}{l}\text { AR with } \\
\text { LD }\end{array}$ & All & NR & $\begin{array}{l}\text { vs. IBR (all): adjOR } \\
0.98(0.47,2)\end{array}$ & 0.953 \\
\hline & Major complications & NRCS & Moderate & $1 \mathrm{y}$ & $\begin{array}{l}\text { AR with } \\
\text { LD }\end{array}$ & Unilateral & NR & NR & NR \\
\hline & Major complications & NRCS & Moderate & $1 \mathrm{y}$ & $\begin{array}{l}\text { AR with } \\
\text { LD }\end{array}$ & Bilateral & NR & NR & NR \\
\hline & Major complications & NRCS & Moderate & $1 \mathrm{y}$ & $\begin{array}{l}\text { AR with } \\
\text { SIEA }\end{array}$ & All & NR & NR & NR \\
\hline & Major complications & NRCS & Moderate & $1 \mathrm{y}$ & $\begin{array}{l}\text { AR with } \\
\text { SIEA }\end{array}$ & Unilateral & NR & NR & NR \\
\hline & Major complications & NRCS & Moderate & $1 \mathrm{y}$ & $\begin{array}{l}\text { AR with } \\
\text { SIEA }\end{array}$ & Bilateral & NR & NR & NR \\
\hline & Reoperative complications & NRCS & Moderate & $2 y$ & IBR & All & NR & NR & NR \\
\hline & Reoperative complications & NRCS & Moderate & $2 y$ & IBR DTI & All & $21 / 112(18 . .8)$ & $\begin{array}{l}\text { vs. IBR with T/E: adjOR } \\
1.06(0.56,1.99)\end{array}$ & 0.87 \\
\hline & Reoperative complications & NRCS & Moderate & $2 \mathrm{y}$ & $\begin{array}{l}\text { IBR with } \\
\text { T/E }\end{array}$ & All & $237 / 1525(15.5)$ & Ref & Ref \\
\hline & Reoperative complications & NRCS & Moderate & $2 y$ & AR (all) & All & NR & NR & NR \\
\hline & Reoperative complications & NRCS & Moderate & $2 y$ & $\begin{array}{l}\text { AR with } \\
\text { DIEP }\end{array}$ & All & $114 / 390(29.2)$ & $\frac{\text { vs. IBR with T/E: }}{2.76(1.87,4.07)}$ & $<0.001$ \\
\hline & Reoperative complications & NRCS & Moderate & $2 \mathrm{y}$ & $\begin{array}{l}\text { AR with } \\
\text { free } \\
\text { TRAM }\end{array}$ & All & $26 / 95(27.4)$ & $\frac{\text { vs. IBR with T/E: }}{3.02(1.73,5.29)}$ & $<0.01$ \\
\hline & Reoperative complications & NRCS & Moderate & $2 y$ & $\begin{array}{l}\text { AR with } \\
\text { pedicled } \\
\text { TRAM }\end{array}$ & All & 25/85 (19.4) & $\frac{\text { vs. IBR with T/E: adjOR }}{2.48(1.33,4.64)}$ & 0.005 \\
\hline & Reoperative complications & NRCS & Moderate & $2 \mathrm{y}$ & $\begin{array}{l}\text { AR with } \\
\text { LD }\end{array}$ & All & $10 / 71(14.1)$ & $\frac{\text { vs. IBR with T/E: }}{1.03(0.46,2.29)}$ & 0.94 \\
\hline & Reoperative complications & NRCS & Moderate & $2 y$ & $\begin{array}{l}\text { AR with } \\
\text { SIEA }\end{array}$ & All & $20 / 65(30.8)$ & $\begin{array}{l}\text { vs. IBR with T/E: adjOR } \\
2.62(1.24,5.52)\end{array}$ & 0.01 \\
\hline
\end{tabular}




\begin{tabular}{|c|c|c|c|c|c|c|c|c|c|}
\hline $\begin{array}{l}\text { Study, Year, } \\
\text { PMID, Country }\end{array}$ & Outcome & Design & $\begin{array}{l}\text { Overall } \\
\text { RoB }\end{array}$ & $\begin{array}{l}\text { Time } \\
\text { Point }\end{array}$ & Arm & Subgroup & $\mathrm{n} / \mathrm{N}(\%)$ & Effect Size (95\% Cl) & $\begin{array}{l}\mathbf{P} \\
\text { Value }\end{array}$ \\
\hline \multirow[t]{2}{*}{$\begin{array}{l}\text { Laporta, } 2017, \\
28061518, \text { Italy }\end{array}$} & $\begin{array}{l}\text { Overall surgical complication: including } \\
\text { mastectomy skin flap and NAC } \\
\text { necrosis, vascular thrombosis, flap } \\
\text { loss, partial flap loss, infection, } \\
\text { hematoma, donor site seroma, and } \\
\text { wound dehiscence, whereas late } \\
\text { surgical complications consisted of fat } \\
\text { necrosis, an abdominal bulge or hernia, } \\
\text { capsular contracture or implant leak, } \\
\text { implant loss; number of take backs to } \\
\text { the theater for secondary surgery. }\end{array}$ & NRCS & High & $>6 \mathrm{mo}$ & IBR & All & $\begin{array}{l}\text { NR/356 breasts } \\
\text { (NR) }\end{array}$ & adjOR $1.6(0.6,4.1)$ & 0.034 \\
\hline & $\begin{array}{l}\text { Overall surgical complication: including } \\
\text { mastectomy skin flap and NAC } \\
\text { necrosis, vascular thrombosis, flap } \\
\text { loss, partial flap loss, infection, } \\
\text { hematoma, donor site seroma, and } \\
\text { wound dehiscence, whereas late } \\
\text { surgical complications consisted of fat } \\
\text { necrosis, an abdominal bulge or hernia, } \\
\text { capsular contracture or implant leak, } \\
\text { implant loss; number of take backs to } \\
\text { the theater for secondary surgery. }\end{array}$ & NRCS & High & $>6 \mathrm{mo}$ & AR & All & $\begin{array}{l}\text { NR/895 breasts } \\
\text { (NR) }\end{array}$ & Ref & Ref \\
\hline \multirow[t]{2}{*}{$\begin{array}{l}\text { Liu, 2014, } \\
\text { 24558063, US }\end{array}$} & $\begin{array}{l}\text { Major complications: defined as } \\
\text { requiring subsequent unanticipated } \\
\text { surgical interventions }\end{array}$ & NRCS & High & $>6 \mathrm{mo}$ & IBR & All & $67 / 179(37.4)$ & Ref & Ref \\
\hline & $\begin{array}{l}\text { Major complications: defined as } \\
\text { requiring subsequent unanticipated } \\
\text { surgical interventions }\end{array}$ & NRCS & High & $>6 \mathrm{mo}$ & AR & All & $16 / 75(21.3)$ & $\begin{array}{l}\text { adjOR } 5.363 \text { (1.128, } \\
25.507)\end{array}$ & 0.035 \\
\hline \multirow{4}{*}{$\begin{array}{l}\text { Mak, 2020, } \\
32665188, \\
\text { China }\end{array}$} & Any complication & NRCS & Moderate & $1 \mathrm{mo}$ & IBR & All & $15 / 30(50.0)$ & Ref & Ref \\
\hline & Any complication & NRCS & Moderate & $1 \mathrm{mo}$ & $\mathrm{AR}$ & All & $52 / 213(24.4)$ & $2.24(1.09,5.39)$ & 0.030 \\
\hline & Any complication requiring reoperation & NRCS & Moderate & $1 \mathrm{mo}$ & IBR & All & $2 / 30(6.7)$ & $\overline{\text { Ref }}$ & Ref \\
\hline & Any complication requiring reoperation & NRCS & Moderate & $1 \mathrm{mo}$ & AR & All & $14 / 213(6.6)$ & $\underline{\mathrm{NR}}$ & 0.99 \\
\hline \multirow{2}{*}{$\begin{array}{l}\text { Palve, } 2020, \\
32468337 \\
\text { Finland }\end{array}$} & Any complication & NRCS & Moderate & $3 \mathrm{mo}$ & IBR & All & $\overline{N R / 51(N R)}$ & $\overline{\text { Ref }}$ & Ref \\
\hline & Any complication & NRCS & Moderate & $3 \mathrm{mo}$ & AR & All & $\mathrm{NR} / 283$ (NR) & adjOR $4.05(2.10,7.81)$ & $<0.001$ \\
\hline $\begin{array}{l}\text { Qin, 2018, } \\
\text { 29384865, } \\
\text { China }\end{array}$ & $\begin{array}{l}\text { postoperative complications(infection, } \\
\text { any of these: marginal necrosis of } \\
\text { incision, dehiscence of incisions, upper } \\
\text { limb lymphedema, bleeding, nipple } \\
\text { necrosis, seroma, capsular contracture }\end{array}$ & NRCS & High & $3.7 \mathrm{y}$ & IBR DTI & All & $15 / 54(27.8)$ & $\begin{array}{l}\text { vs. IBR with T/E and } \\
\frac{\text { AR: adjOR } 1.13(0.84,}{1.36)}\end{array}$ & NR \\
\hline
\end{tabular}




\begin{tabular}{|c|c|c|c|c|c|c|c|c|c|}
\hline $\begin{array}{l}\text { Study, Year, } \\
\text { PMID, Country }\end{array}$ & Outcome & Design & $\begin{array}{l}\text { Overall } \\
\text { RoB }\end{array}$ & $\begin{array}{l}\text { Time } \\
\text { Point }\end{array}$ & Arm & Subgroup & $\mathrm{n} / \mathrm{N}(\%)$ & Effect Size (95\% Cl) & $\begin{array}{l}P \\
\text { Value }\end{array}$ \\
\hline & $\begin{array}{l}\text { postoperative complications(infection, } \\
\text { any of these: marginal necrosis of } \\
\text { incision, dehiscence of incisions, upper } \\
\text { limb lymphedema, bleeding, nipple } \\
\text { necrosis, seroma, capsular contracture }\end{array}$ & NRCS & High & $3.7 \mathrm{y}$ & $\begin{array}{l}\text { IBR with } \\
\text { T/E }\end{array}$ & All & $5 / 38(13.2)$ & $\begin{array}{l}\text { vs. IBR DTI and AR: } \\
\text { adjOR } 1.07(0.92,1.16)\end{array}$ & NR \\
\hline & $\begin{array}{l}\text { postoperative complications(infection, } \\
\text { any of these: marginal necrosis of } \\
\text { incision, dehiscence of incisions, upper } \\
\text { limb lymphedema, bleeding, nipple } \\
\text { necrosis, seroma, capsular contracture }\end{array}$ & NRCS & High & $3.7 \mathrm{y}$ & AR & All & $20 / 59(33.9)$ & $\begin{array}{l}\frac{\text { vs. IBR DTI and IBR }}{\text { with T/E: adjOR } 1.58} \\
(1.32,2.75)\end{array}$ & NR \\
\hline \multirow{2}{*}{$\begin{array}{l}\text { Simon, 2020, } \\
\text { 33363007, Italy }\end{array}$} & Any complication & Ref & Moderate & $3.3 \mathrm{y}$ & IBR & All & NR/68 (NR) & Ref & Ref \\
\hline & All complication & Ref & Moderate & $3.3 \mathrm{y}$ & AR & All & $\mathrm{NR} / 139$ (NR) & $\operatorname{adjOR} 8.28(1.71,4.01)$ & 0.009 \\
\hline \multirow[t]{2}{*}{$\begin{array}{l}\text { Xu, 2018, } \\
30261115, \\
\text { China }\end{array}$} & $\begin{array}{l}\text { Overall complications, including } \\
\text { capsular contracture, hematoma, } \\
\text { wound infection, wound dehiscence, } \\
\text { seroma, fat liquefaction/necrosis, } \\
\text { implant rupture, flap/nipple areola } \\
\text { necrosis, abdominal bulge/hernia, and } \\
\text { implant/flap failure }\end{array}$ & NRCS & Moderate & $1.2 \mathrm{y}$ & IBR & All & $57 / 326(17.5)$ & $\begin{array}{l}\text { adjOR } 0.747 \text { (0.366, } \\
1.525)\end{array}$ & 0.424 \\
\hline & $\begin{array}{l}\text { Overall complications, including } \\
\text { capsular contracture, hematoma, } \\
\text { wound infection, wound dehiscence, } \\
\text { seroma, fat liquefaction/necrosis, } \\
\text { implant rupture, flap/nipple areola } \\
\text { necrosis, abdominal bulge/hernia, and } \\
\text { implant/flap failure }\end{array}$ & NRCS & Moderate & $1.2 \mathrm{y}$ & AR & All & $27 / 100(27.0)$ & Ref & Ref \\
\hline $\begin{array}{l}\text { Salibian, 2019, } \\
\text { 31333984, US }\end{array}$ & Major ischemic complications & $\begin{array}{l}\text { Single } \\
\text { group }\end{array}$ & Low & $3.3 \mathrm{y}$ & IBR & All & $70 / 1045(6.7)$ & $\mathrm{N} / \mathrm{A}$ & $\mathrm{N} / \mathrm{A}$ \\
\hline $\begin{array}{l}\text { Acosta, 2011, } \\
21046538, \\
\text { Sweden }\end{array}$ & Overall complications & $\begin{array}{l}\text { Single } \\
\text { group }\end{array}$ & High & $9 y$ & AR & All & $105 / 543(19.3)$ & $\mathrm{N} / \mathrm{A}$ & $\mathrm{N} / \mathrm{A}$ \\
\hline $\begin{array}{l}\text { Beugels, 2018, } \\
29399731, \\
\text { Netherlands }\end{array}$ & $\begin{array}{l}\text { Wound problems: included wound } \\
\text { dehiscence and superficial skin } \\
\text { necrosis related to the breast } \\
\text { reconstruction, but not necrosis of } \\
\text { mastectomy skin }\end{array}$ & $\begin{array}{l}\text { Single } \\
\text { group }\end{array}$ & Low & $\begin{array}{l}9-10 \\
\text { mo }\end{array}$ & AR & All & $\begin{array}{l}\text { 115/910 flaps } \\
(12.6)\end{array}$ & $\mathrm{N} / \mathrm{A}$ & $\mathrm{N} / \mathrm{A}$ \\
\hline
\end{tabular}




\begin{tabular}{|c|c|c|c|c|c|c|c|c|c|}
\hline $\begin{array}{l}\text { Study, Year, } \\
\text { PMID, Country }\end{array}$ & Outcome & Design & $\begin{array}{l}\text { Overall } \\
\text { RoB }\end{array}$ & $\begin{array}{l}\text { Time } \\
\text { Point }\end{array}$ & Arm & Subgroup & $\mathrm{n} / \mathrm{N}(\%)$ & Effect Size (95\% Cl) & $\begin{array}{l}\mathbf{P} \\
\text { Value }\end{array}$ \\
\hline $\begin{array}{l}\text { Langer, } 2010 \text {, } \\
20980954, \\
\text { Germany }\end{array}$ & $\begin{array}{l}\text { Breast seroma, hematoma, or wound } \\
\text { infection }\end{array}$ & $\begin{array}{l}\text { Single } \\
\text { group }\end{array}$ & Low & $5 y$ & AR & All & $\begin{array}{l}23 / 670 \text { flaps } \\
(3.4)\end{array}$ & $\mathrm{N} / \mathrm{A}$ & $\mathrm{N} / \mathrm{A}$ \\
\hline $\begin{array}{l}\text { Prantl, 2020, } \\
32895743, \\
\text { Germany }\end{array}$ & $\begin{array}{l}\text { Deep vein thrombosis, pulmonary } \\
\text { embolism, myocardial infarct and } \\
\text { others }\end{array}$ & $\begin{array}{l}\text { Single } \\
\text { group }\end{array}$ & Low & $3 \mathrm{mo}$ & AR & All & $\begin{array}{l}\text { 294/4577 flaps } \\
(6.4)\end{array}$ & $\mathrm{N} / \mathrm{A}$ & $\mathrm{N} / \mathrm{A}$ \\
\hline
\end{tabular}

Abbreviations: adj = adjusted, $\mathrm{AR}=$ autologous reconstruction, $\mathrm{CI}=$ confidence interval, $\mathrm{DIEP}=$ deep inferior epigastric perforator, $\mathrm{IBR}=$ implant-based reconstruction, $\mathrm{LD}=$

latissimus dorsi, $\mathrm{NR}=$ not reported, $\mathrm{NRCS}=$ nonrandomized comparative study, $\mathrm{OR}=$ odds ratio, $\mathrm{PMID}=\mathrm{PubMed}$ identifier, $\mathrm{Ref}=$ reference group, $\mathrm{RoB}=$ risk of bias, $\mathrm{y}=\mathrm{years}$.

Colors: Header rows are shaded orange. Rows for every alternate study are shaded blue. The colors do not add unique information. 
Table F-2.1. Full Evidence Table - Key Question 2: Timing of radiation therapy relative to IBR - continuous outcomes (physical wellbeing)

\begin{tabular}{|c|c|c|c|c|c|c|c|c|c|c|}
\hline $\begin{array}{l}\text { Study, Year, } \\
\text { PMID }\end{array}$ & $\begin{array}{l}\text { Outcome } \\
\text { Measurement }\end{array}$ & $\begin{array}{l}\text { Desig } \\
n\end{array}$ & $\begin{array}{l}\text { Overall } \\
\text { RoB }\end{array}$ & $\begin{array}{l}\text { Time } \\
\text { Point }\end{array}$ & $\begin{array}{l}\text { IBR Before } \\
\text { Radiation, } \\
\text { N }\end{array}$ & $\begin{array}{l}\text { IBR Before } \\
\text { Radiation, } \\
\text { Mean (SD) }\end{array}$ & $\begin{array}{l}\text { IBR After } \\
\text { Radiation, } \\
\text { N }\end{array}$ & $\begin{array}{l}\text { IBR After } \\
\text { Radiation, } \\
\text { Mean (SD) }\end{array}$ & $\begin{array}{l}\text { IBR Before Versus } \\
\text { After Radiation, } \\
\text { Effect Size } \\
(95 \% \mathrm{Cl})\end{array}$ & $\begin{array}{l}P \\
\text { Value }\end{array}$ \\
\hline $\begin{array}{l}\text { Cordeiro, 2015, } \\
30270015, \text { US }\end{array}$ & $\begin{array}{l}\text { EORTC QLQC30: } \\
\text { Global health status }\end{array}$ & NRCS & High & $3.3 \mathrm{y}$ & 84 & $72.5(2.6)$ & 22 & $73.4(1.9)$ & NR & NS \\
\hline \multirow{2}{*}{$\begin{array}{l}\text { Yoon, 2020, } \\
\text { 32332528, US } \\
\text { \& Canada }\end{array}$} & $\begin{array}{l}\text { BREAST-Q: Physical } \\
\text { WB }(0-100)\end{array}$ & NRCS & Moderate & $2 y$ & 80 & NR & 237 & NR & $-0.64(-7.19,5.90)$ & 0.84 \\
\hline & $\begin{array}{l}\text { PROMIS: Physical } \\
\text { function }(0-100)\end{array}$ & NRCS & Moderate & $2 y$ & 80 & NR & 237 & NR & $-0.04(-2.40,2.32)$ & 0.97 \\
\hline
\end{tabular}

Abbreviations: CI = confidence interval, EORTC $=$ European Organization for Research and Treatment of Cancer, IBR $=$ implant-based reconstruction, NR $=$ not reported,

$\mathrm{NRCS}=$ nonrandomized comparative study, NS = not significant, PMID = PubMed identifier, PROMIS = Patient-Reported Outcomes Measurement Information System, RoB = risk of bias, $\mathrm{SD}=$ standard deviation, $\mathrm{WB}=$ well-being, $\mathrm{y}=$ years.

Colors: Header rows are shaded orange. Rows for every alternate study are shaded blue. The colors do not add unique information.

Table F-2.2. Full Evidence Table - Key Question 2: Timing of radiation therapy relative to IBR - continuous outcomes (psychosocial well-being)

\begin{tabular}{|c|c|c|c|c|c|c|c|c|c|c|}
\hline $\begin{array}{l}\text { Study, Year, } \\
\text { PMID }\end{array}$ & Outcome Measurement & $\begin{array}{l}\text { Desig } \\
n\end{array}$ & $\begin{array}{l}\text { Overall } \\
\text { RoB }\end{array}$ & $\begin{array}{l}\text { Time } \\
\text { Point }\end{array}$ & $\begin{array}{l}\text { IBR Before } \\
\text { Radiation, } \\
\text { N }\end{array}$ & $\begin{array}{l}\text { IBR Before } \\
\text { Radiation, } \\
\text { Mean (SD) }\end{array}$ & $\begin{array}{l}\text { IBR After } \\
\text { Radiation, } \\
\text { N }\end{array}$ & $\begin{array}{l}\text { IBR After } \\
\text { Radiation, } \\
\text { Mean (SD) }\end{array}$ & $\begin{array}{l}\text { IBR Before Versus } \\
\text { After Radiation, } \\
\text { Effect Size } \\
(95 \% \mathrm{CI})\end{array}$ & $\begin{array}{l}\mathbf{P} \\
\text { Value }\end{array}$ \\
\hline $\begin{array}{l}\text { Cordeiro, } \\
2015, \\
30270015, \text { US } \\
\end{array}$ & $\begin{array}{l}\text { BREAST-Q: } \\
\text { Psychosocial WB (0-100) }\end{array}$ & NRCS & High & $3.3 \mathrm{y}$ & 84 & $71.1(1.4)$ & 22 & $72.3(1.2)$ & NR & $<0.01$ \\
\hline $\begin{array}{l}\text { Yoon, 2020, } \\
32332528, \text { US } \\
\text { \& Canada }\end{array}$ & $\begin{array}{l}\text { BREAST-Q: } \\
\text { Psychosocial WB (0-100) }\end{array}$ & NRCS & Moderate & $2 y$ & 80 & NR & 237 & NR & $0.48(-7.72,8.68)$ & 0.91 \\
\hline
\end{tabular}

Abbreviations: $\mathrm{CI}=$ confidence interval, IBR $=$ implant-based reconstruction, $\mathrm{NR}=$ not reported, $\mathrm{NRCS}=$ nonrandomized comparative study, $\mathrm{NS}=$ not significant, $\mathrm{PMID}=$

PubMed identifier, $\mathrm{RoB}=$ risk of bias, $\mathrm{SD}=$ standard deviation, $\mathrm{WB}=$ well-being, $\mathrm{y}=$ years.

Colors: Header rows are shaded orange. Rows for every alternate study are shaded blue. The colors do not add unique information. 
Table F-2.3. Full Evidence Table - Key Question 2: Timing of radiation therapy relative to IBR - continuous outcomes (sexual well-being)

\begin{tabular}{|c|c|c|c|c|c|c|c|c|c|c|}
\hline $\begin{array}{l}\text { Study, Year, } \\
\text { PMID }\end{array}$ & $\begin{array}{l}\text { Outcome } \\
\text { Measurement }\end{array}$ & $\begin{array}{l}\text { Desig } \\
n\end{array}$ & $\begin{array}{l}\text { Overall } \\
\text { RoB }\end{array}$ & $\begin{array}{l}\text { Time } \\
\text { Point }\end{array}$ & $\begin{array}{l}\text { IBR Before } \\
\text { Radiation, } \\
\text { N }\end{array}$ & $\begin{array}{l}\text { IBR Before } \\
\text { Radiation, } \\
\text { Mean (SD) }\end{array}$ & $\begin{array}{l}\text { IBR After } \\
\text { Radiation, } \\
\text { N }\end{array}$ & $\begin{array}{l}\text { IBR After } \\
\text { Radiation, } \\
\text { Mean (SD) }\end{array}$ & $\begin{array}{l}\text { IBR Before Versus } \\
\text { After Radiation, } \\
\text { Effect Size } \\
(95 \% \mathrm{Cl})\end{array}$ & $\begin{array}{l}P \\
\text { Value }\end{array}$ \\
\hline $\begin{array}{l}\text { Cordeiro, 2015, } \\
30270015 \text {, US }\end{array}$ & $\begin{array}{l}\text { BREAST-Q: Sexual WB } \\
(0-100)\end{array}$ & NRCS & High & $3.3 \mathrm{y}$ & 84 & $54.0(0.9)$ & 22 & $55.4(0.7)$ & NR & $<0.01$ \\
\hline \multirow{2}{*}{$\begin{array}{l}\text { Yoon, 2020, } \\
\text { 32332528, US } \\
\text { \& Canada }\end{array}$} & $\begin{array}{l}\text { BREAST-Q: Sexual WB } \\
(0-100)\end{array}$ & NRCS & Moderate & $2 y$ & 80 & NR & 237 & NR & $-1.00(-8.41,6.40)$ & 0.78 \\
\hline & $\begin{array}{l}\text { EORTC: Sexual } \\
\text { function }\end{array}$ & NRCS & Moderate & $2 y$ & 80 & NR & 237 & NR & $-1.40(-8.58,5.77)$ & 0.70 \\
\hline
\end{tabular}

Abbreviations: CI = confidence interval, EORTC $=$ European Organization for Research and Treatment of Cancer, IBR $=$ implant-based reconstruction, NR $=$ not reported,

NRCS = nonrandomized comparative study, NS = not significant, $\mathrm{PMID}=$ PubMed identifier, $\mathrm{RoB}=$ risk of bias, $\mathrm{SD}=\mathrm{standard}$ deviation, $\mathrm{WB}=\mathrm{well}-\mathrm{being}, \mathrm{y}=\mathrm{years}$.

Colors: Header rows are shaded orange. Rows for every alternate study are shaded blue. The colors do not add unique information.

Table F-2.4. Full Evidence Table - Key Question 2: Timing of radiation therapy relative to IBR - continuous outcomes (patient satisfaction with breast)

\begin{tabular}{|c|c|c|c|c|c|c|c|c|c|c|}
\hline $\begin{array}{l}\text { Study, Year, } \\
\text { PMID }\end{array}$ & Outcome Measurement & $\begin{array}{l}\text { Desig } \\
\mathrm{n}\end{array}$ & $\begin{array}{l}\text { Overall } \\
\text { RoB }\end{array}$ & $\begin{array}{l}\text { Time } \\
\text { Point }\end{array}$ & $\begin{array}{l}\text { IBR Before } \\
\text { Radiation, } \\
\text { N }\end{array}$ & $\begin{array}{l}\text { IBR Before } \\
\text { Radiation, } \\
\text { Mean (SD) }\end{array}$ & $\begin{array}{l}\text { IBR After } \\
\text { Radiation, } \\
\text { N }\end{array}$ & $\begin{array}{l}\text { IBR After } \\
\text { Radiation, } \\
\text { Mean (SD) }\end{array}$ & $\begin{array}{l}\text { IBR Before Versus } \\
\text { After Radiation, } \\
\text { Effect Size } \\
(95 \% \mathrm{Cl})\end{array}$ & $\begin{array}{l}P \\
\text { Value }\end{array}$ \\
\hline $\begin{array}{l}\text { Cordeiro, } \\
2015, \\
30270015, \text { US } \\
\end{array}$ & $\begin{array}{l}\text { BREAST-Q: Satisfaction } \\
\text { with breast }(0-100)\end{array}$ & NRCS & High & $3.3 \mathrm{y}$ & 84 & $56.2(3.3)$ & 22 & $57.2(3.1)$ & NR & NS \\
\hline $\begin{array}{l}\text { Yoon, 2020, } \\
\text { 32332528, US } \\
\text { \& Canada }\end{array}$ & $\begin{array}{l}\text { BREAST-Q: Satisfaction } \\
\text { with breast }(0-100)\end{array}$ & NRCS & Moderate & $2 y$ & 80 & NR & 237 & NR & $-3.89(-11.0,3.23)$ & 0.28 \\
\hline
\end{tabular}

Abbreviations: $\mathrm{CI}=$ confidence interval, $\mathrm{IBR}=$ implant-based reconstruction, $\mathrm{NR}=$ not reported, $\mathrm{NRCS}=$ nonrandomized comparative study, $\mathrm{NS}=$ not significant, $\mathrm{PMID}=$

PubMed identifier, $\mathrm{RoB}=$ risk of bias, $\mathrm{SD}=$ standard deviation, $\mathrm{y}=$ years

Colors: Header rows are shaded orange. The color does not add unique information.

Table F-2.5. Full Evidence Table - Key Question 2: Timing of radiation therapy relative to IBR - continuous outcomes (patient satisfaction with outcome)

\begin{tabular}{|c|c|c|c|c|c|c|c|c|c|c|}
\hline $\begin{array}{l}\text { Study, Year, } \\
\text { PMID }\end{array}$ & Outcome Measurement & $\begin{array}{l}\text { Desig } \\
\mathrm{n}\end{array}$ & $\begin{array}{l}\text { Overall } \\
\text { RoB }\end{array}$ & $\begin{array}{l}\text { Time } \\
\text { Point }\end{array}$ & $\begin{array}{l}\text { IBR Before } \\
\text { Radiation, } \\
\text { N }\end{array}$ & $\begin{array}{l}\text { IBR Before } \\
\text { Radiation, } \\
\text { Mean (SD) }\end{array}$ & $\begin{array}{l}\text { IBR After } \\
\text { Radiation, } \\
\text { N }\end{array}$ & $\begin{array}{l}\text { IBR After } \\
\text { Radiation, } \\
\text { Mean (SD) }\end{array}$ & $\begin{array}{l}\text { IBR Before Versus } \\
\text { After Radiation, } \\
\text { Effect Size } \\
(95 \% \mathrm{Cl})\end{array}$ & $\begin{array}{l}\mathbf{P} \\
\text { Value }\end{array}$ \\
\hline $\begin{array}{l}\text { Cordeiro, } \\
2015, \\
30270015 \text {, US }\end{array}$ & $\begin{array}{l}\text { BREAST-Q: Satisfaction } \\
\text { with outcome }(0-100)\end{array}$ & NRCS & High & $3.3 \mathrm{y}$ & 84 & $68.4(3.8)$ & 22 & $70.2(3.0)$ & NR & 0.02 \\
\hline
\end{tabular}

30270015, US

Abbreviations: $\mathrm{CI}=$ confidence interval, $\mathrm{IBR}=$ implant-based reconstruction, $\mathrm{NR}=$ not reported, NRCS $=$ nonrandomized comparative study, NS $=$ not significant, $\mathrm{PMID}=$

PubMed identifier, $\mathrm{RoB}=$ risk of bias, $\mathrm{SD}=$ standard deviation, $\mathrm{y}=$ years.

Colors: Header rows are shaded orange. The color does not add unique information. 
Table F-2.6. Full Evidence Table - Key Question 2: Timing of radiation therapy relative to IBR - continuous outcomes (pain)

\begin{tabular}{|c|c|c|c|c|c|c|c|c|c|c|}
\hline $\begin{array}{l}\text { Study, Year, } \\
\text { PMID }\end{array}$ & $\begin{array}{l}\text { Outcome } \\
\text { Measurement }\end{array}$ & Design & $\begin{array}{l}\text { Overall } \\
\text { RoB }\end{array}$ & $\begin{array}{l}\text { Time } \\
\text { Point }\end{array}$ & $\begin{array}{l}\text { IBR Before } \\
\text { Radiation, } \\
\text { N }\end{array}$ & $\begin{array}{l}\text { IBR Before } \\
\text { Radiation, } \\
\text { Mean (SD) }\end{array}$ & $\begin{array}{l}\text { IBR After } \\
\text { Radiation, } \\
\mathbf{N}\end{array}$ & $\begin{array}{l}\text { IBR After } \\
\text { Radiation, } \\
\text { Mean (SD) }\end{array}$ & $\begin{array}{l}\text { IBR Before Versus } \\
\text { After Radiation, } \\
\text { Effect Size } \\
(95 \% \mathrm{CI})\end{array}$ & $\begin{array}{l}P \\
\text { Value }\end{array}$ \\
\hline $\begin{array}{l}\text { Yoon, 2020, } \\
\text { 32332528, US } \\
\text { \& Canada }\end{array}$ & $\begin{array}{l}\text { PROMIS: Pain } \\
\text { interference }(0-100)\end{array}$ & NRCS & Moderate & $3.3 y$ & 80 & NR & 237 & NR & $2.86(-1.05,6.77)$ & 0.14 \\
\hline
\end{tabular}

\& Canada

Abbreviations: $\mathrm{CI}=$ confidence interval, IBR $=$ implant-based reconstruction, NR $=$ not reported, NRCS $=$ nonrandomized comparative study, NS $=$ not significant, $\mathrm{PMID}=$

PubMed identifier, $\mathrm{RoB}=$ risk of bias, $\mathrm{SD}=$ standard deviation, $\mathrm{y}=$ years.

Colors: Header rows are shaded orange. The color does not add unique information.

Table F-2.7. Full Evidence Table - Key Question 2: Timing of radiation therapy relative to IBR - categorical outcomes (unplanned repeat surgeries for revision of reconstruction)

\begin{tabular}{|c|c|c|c|c|c|c|c|}
\hline Study, Year, PMID & Design & $\begin{array}{l}\text { Overall } \\
\text { RoB }\end{array}$ & $\begin{array}{l}\text { Time } \\
\text { Point }\end{array}$ & $\begin{array}{l}\text { IBR Before Radiation, } \\
\mathrm{n} / \mathrm{N}(\%)\end{array}$ & $\begin{array}{l}\text { IBR After Radiation, } \\
\mathrm{n} / \mathrm{N}(\%)\end{array}$ & $\begin{array}{l}\text { IBR Before Versus After Radiation, } \\
\text { Effect Size }(95 \% \mathrm{CI})\end{array}$ & $\begin{array}{l}\mathbf{P} \\
\text { Value }\end{array}$ \\
\hline Eriksson, 2013, 24258257 & NRCS & High & $3.6 \mathrm{y}$ & NR & NR & adjHR $0.94(0.63,1.40)^{*}$ & NR \\
\hline
\end{tabular}

Abbreviations: adj $=$ adjusted, $\mathrm{CI}=$ confidence interval, $\mathrm{HR}=$ hazard ratio, IBR $=$ implant-based reconstruction, $\mathrm{NR}=$ not reported, $\mathrm{NRCS}=$ nonrandomized comparative study,

PMID $=$ Pubmed identifier, $\mathrm{RoB}=$ risk of bias, $\mathrm{y}=$ years.

Colors: Header rows are shaded orange. The color does not add unique information.

* Calculated

Table F-2.8. Full Evidence Table - Key Question 2: Timing of radiation therapy relative to IBR - categorical outcomes (necrosis)

\begin{tabular}{|l|l|l|l|l|l|l|l|}
\hline Study, Year, PMID & Design & $\begin{array}{l}\text { Overall } \\
\text { RoB }\end{array}$ & $\begin{array}{l}\text { Time } \\
\text { Point }\end{array}$ & $\begin{array}{l}\text { IBR Before } \\
\text { Radiation, n/N (\%) }\end{array}$ & $\begin{array}{l}\text { IBR After Radiation, n/N } \\
\text { (\%) }\end{array}$ & $\begin{array}{l}\text { IBR After Versus Before Radiation, } \\
\text { Effect Size (95\% Cl) }\end{array}$ & $\begin{array}{l}\text { P } \\
\text { Value }\end{array}$ \\
\hline Hirsch, 2014, 25347643 & NRCS & High & $3.1 \mathrm{y}$ & NR & NR & adjOR 0.96 (0.68, 1.35) \\
\hline
\end{tabular}

Abbreviations: adj $=$ adjusted, $\mathrm{CI}=$ confidence interval, $\mathrm{IBR}=$ implant-based reconstruction, $\mathrm{NR}=$ not reported, $\mathrm{NRCS}=$ nonrandomized comparative study, $\mathrm{OR}=$ odds ratio,

PMID $=$ Pubmed identifier, $\mathrm{RoB}=$ risk of bias, $\mathrm{y}=$ years.

Colors: Header rows are shaded orange. The color does not add unique information.

Table F-2.9. Full Evidence Table - Key Question 2: Timing of radiation therapy relative to IBR - categorical outcomes (infections not explicitly implant related)

\begin{tabular}{|c|c|c|c|c|c|c|c|c|}
\hline $\begin{array}{l}\text { Study, Year, } \\
\text { PMID }\end{array}$ & Outcome Description & Design & $\begin{array}{l}\text { Overall } \\
\text { RoB }\end{array}$ & $\begin{array}{l}\text { Time } \\
\text { Point }\end{array}$ & $\begin{array}{l}\text { IBR Before } \\
\text { Radiation, n/N (\%) }\end{array}$ & $\begin{array}{l}\text { IBR After } \\
\text { Radiation, n/N (\%) }\end{array}$ & $\begin{array}{l}\text { IBR After Versus Before } \\
\text { Radiation, Effect Size }(95 \% \mathrm{Cl})\end{array}$ & $\begin{array}{l}\mathbf{P} \\
\text { Value }\end{array}$ \\
\hline \multirow[t]{2}{*}{$\begin{array}{l}\text { Yoon, 2020, } \\
\text { 32332528, US } \\
\text { \& Canada }\end{array}$} & $\begin{array}{l}\text { Major (IV antibiotics } \\
\text { with or without return to } \\
\text { surgery) }\end{array}$ & NRCS & Moderate & $2 y$ & $5 / 46(10.9)$ & $7 / 104(6.7)$ & NR & 0.40 \\
\hline & Minor (oral antibiotics) & NRCS & Moderate & $2 y$ & $3 / 46(6.5)$ & $7 / 104(6.7)$ & NR & 0.96 \\
\hline
\end{tabular}

Abbreviations: $\mathrm{CI}=$ confidence interval, $\mathrm{IBR}=$ implant-based reconstruction, $\mathrm{NR}=$ not reported, $\mathrm{NRCS}=$ nonrandomized comparative study, $\mathrm{PMID}=\mathrm{Pubmed}$ identifier, $\mathrm{RoB}=$

risk of bias, IV = intravenous, $\mathrm{y}=$ years.

Colors: Header rows are shaded orange. The color does not add unique information. 
Table F-2.10. Full Evidence Table - Key Question 2: Timing of radiation therapy relative to IBR - categorical outcomes (wound dehiscence)

\begin{tabular}{|c|c|c|c|c|c|c|c|c|}
\hline Study, Year, PMID & Outcome & Design & $\begin{array}{l}\text { Overall } \\
\text { RoB }\end{array}$ & $\begin{array}{l}\text { Time } \\
\text { Point }\end{array}$ & $\begin{array}{l}\text { IBR Before Radiation, } \\
\text { n/N (\%) }\end{array}$ & $\begin{array}{l}\text { IBR After Radiation, } \\
\text { n/N (\%) }\end{array}$ & $\begin{array}{l}\text { IBR After Versus } \\
\text { Before Radiation, } \\
\text { Effect Size }(95 \% \mathrm{Cl})\end{array}$ & $\begin{array}{l}\mathbf{P} \\
\text { Value }\end{array}$ \\
\hline $\begin{array}{l}\text { Yoon, 2020, 32332528, } \\
\text { US \& Canada }\end{array}$ & Wound dehiscence & NRCS & Moderate & $2 \mathrm{y}$ & $0 / 46(0)$ & $5 / 104(4.8)$ & NR & 0.32 \\
\hline
\end{tabular}

Abbreviations: $\mathrm{CI}=$ confidence interval, $\mathrm{IBR}=$ implant-based reconstruction, $\mathrm{NR}=$ not reported, $\mathrm{NRCS}=$ nonrandomized comparative study, $\mathrm{PMID}=\mathrm{Pubmed}$ identifier, RoB $=$ risk of bias, $\mathrm{y}=$ years.

Colors: Header rows are shaded orange. The color does not add unique information.

Table F-2.11. Full Evidence Table - Key Question 2: Timing of radiation therapy relative to IBR - categorical outcomes (seroma)

\begin{tabular}{|c|c|c|c|c|c|c|c|c|}
\hline Study, Year, PMID & Outcome & Design & $\begin{array}{l}\text { Overall } \\
\text { RoB }\end{array}$ & $\begin{array}{l}\text { Time } \\
\text { Point }\end{array}$ & $\begin{array}{l}\text { IBR Before } \\
\text { Radiation, n/N (\%) }\end{array}$ & $\begin{array}{l}\text { IBR After Radiation, } \\
\mathrm{n} / \mathrm{N}(\%)\end{array}$ & $\begin{array}{l}\text { IBR After Versus Before } \\
\text { Radiation, Effect Size (95\% Cl) }\end{array}$ & $\begin{array}{l}\mathbf{P} \\
\text { Value }\end{array}$ \\
\hline $\begin{array}{l}\text { Yoon, 2020, 32332528, } \\
\text { US \& Canada }\end{array}$ & Seroma & NRCS & Moderate & $2 y$ & $2 / 46(4.4)$ & $8 / 104(7.7)$ & NR & 0.47 \\
\hline
\end{tabular}

Abbreviations: $\mathrm{CI}=$ confidence interval, IBR $=$ implant-based reconstruction, $\mathrm{NR}=$ not reported, $\mathrm{NRCS}=$ nonrandomized comparative study, $\mathrm{PMID}=\mathrm{Pubmed}$ identifier, $\mathrm{RoB}=$

risk of bias, $\mathrm{y}=$ years.

Colors: Header rows are shaded orange. The color does not add unique information.

Table F-2.12. Full Evidence Table - Key Question 2: Timing of radiation therapy relative to IBR - categorical outcomes (implant

failure/loss)

\begin{tabular}{|c|c|c|c|c|c|c|c|c|}
\hline Study, Year, PMID & $\begin{array}{l}\text { Outcome } \\
\text { Description }\end{array}$ & Design & $\begin{array}{l}\text { Overall } \\
\text { RoB }\end{array}$ & $\begin{array}{l}\text { Time } \\
\text { Point }\end{array}$ & $\begin{array}{l}\text { IBR Before } \\
\text { Radiation, n/N (\%) }\end{array}$ & $\begin{array}{l}\text { IBR After } \\
\text { Radiation, n/N (\%) }\end{array}$ & $\begin{array}{l}\text { IBR Before Versus After } \\
\text { Radiation, Effect Size }(95 \% \mathrm{Cl})\end{array}$ & $\begin{array}{l}\text { P } \\
\text { Value }\end{array}$ \\
\hline $\begin{array}{l}\text { Cordeiro, 2015a, } \\
25742523\end{array}$ & Implant loss & NRCS & High & $3.3 \mathrm{y}$ & $26 / 210(12.4)$ & $17 / 94(18.1)$ & adjOR $0.96(0.64,1.43)^{*}$ & NR \\
\hline Eriksson, 2013, 24258257 & IBR failure & NRCS & High & $3.6 \mathrm{y}$ & NR & NR & $\operatorname{adjHR} 0.62(0.41,0.93)^{*}$ & NR \\
\hline Hirsch, 2014, 25347643 & Explantation & NRCS & High & $3.5 \mathrm{y}$ & NR & NR & adjOR $1.12(0.75,1.68)^{\star}$ & 0.09 \\
\hline
\end{tabular}

Abbreviations: adj $=$ adjusted, $\mathrm{CI}=$ confidence interval, $\mathrm{HR}=$ Hazard ratio, $\mathrm{IBR}=$ implant-based reconstruction, $\mathrm{NR}=$ not reported, $\mathrm{NRCS}=$ nonrandomized comparative study,

$\mathrm{OR}=$ odds ratio, $\mathrm{PMID}=$ Pubmed identifier, $\mathrm{RoB}=$ risk of bias, $\mathrm{y}=$ year.

Colors: Header rows are shaded orange. Rows for every alternate study are shaded blue. The colors do not add unique information.

* Calculated.

Table F-2.13. Full Evidence Table - Key Question 2: Timing of radiation therapy relative to IBR - categorical outcomes (capsular contracture)

\begin{tabular}{|c|c|c|c|c|c|c|c|c|}
\hline Study, Year, PMID & Outcome & Design & $\begin{array}{l}\text { Overall } \\
\text { RoB }\end{array}$ & $\begin{array}{l}\text { Time } \\
\text { Point }\end{array}$ & $\begin{array}{l}\text { IBR Before } \\
\text { Radiation, n/N (\%) }\end{array}$ & $\begin{array}{l}\text { IBR After } \\
\text { Radiation, n/N (\%) }\end{array}$ & $\begin{array}{l}\text { IBR Before Versus After } \\
\text { Radiation, Effect Size }(95 \% \mathrm{CI})\end{array}$ & $\begin{array}{l}\mathbf{P} \\
\text { Value }\end{array}$ \\
\hline $\begin{array}{l}\text { Yoon, 2020, 32332528, } \\
\text { US \& Canada }\end{array}$ & Capsular contracture & NRCS & Moderate & $2 y$ & $1 / 46(2.2)$ & $3 / 104(2.9)$ & NR & 0.80 \\
\hline
\end{tabular}

Abbreviations: $\mathrm{CI}=$ confidence interval, $\mathrm{IBR}=$ implant-based reconstruction, $\mathrm{NR}=$ not reported, $\mathrm{NRCS}=$ nonrandomized comparative study, $\mathrm{PMID}=\mathrm{Pubmed}$ identifier, RoB $=$

risk of bias, $\mathrm{y}=$ years.

Colors: Header rows are shaded orange. The color does not add unique information. 
Table F-2.14. Full Evidence Table - Key Question 2: Timing of radiation therapy relative to IBR - categorical outcomes (hematoma)

\begin{tabular}{|c|c|c|c|c|c|c|c|}
\hline Study, Year, PMID & Design & $\begin{array}{l}\text { Overall } \\
\text { RoB }\end{array}$ & $\begin{array}{l}\text { Time } \\
\text { Point }\end{array}$ & $\begin{array}{l}\text { IBR Before } \\
\text { Radiation, n/N (\%) }\end{array}$ & $\begin{array}{l}\text { IBR After } \\
\text { Radiation, n/N (\%) }\end{array}$ & $\begin{array}{l}\text { IBR After Versus Before Radiation, } \\
\text { Effect Size }(95 \% \mathrm{Cl})\end{array}$ & $\begin{array}{l}P \\
\text { Value }\end{array}$ \\
\hline Hirsch, 2014, 25347643 & NRCS & High & $3.1 \mathrm{y}$ & NR & NR & adjOR $0.56(0.22,1.45)$ & 0.39 \\
\hline $\begin{array}{l}\text { Yoon, 2020, 32332528, } \\
\text { US \& Canada }\end{array}$ & NRCS & Moderate & $2 y$ & $1 / 46(2.2)$ & $4 / 104(3.9)$ & NR & 0.63 \\
\hline
\end{tabular}

Abbreviations: adj $=$ adjusted, $\mathrm{CI}=$ confidence interval, $\mathrm{IBR}=$ implant-based reconstruction, $\mathrm{NR}=$ not reported, $\mathrm{NRCS}=$ nonrandomized comparative study, $\mathrm{OR}=$ odds ratio,

PMID = Pubmed identifier, RoB $=$ risk of bias, $\mathrm{y}=$ years.

Colors: Header rows are shaded orange. Rows for every alternate study are shaded blue. The colors do not add unique information.

Table F-2.15. Full Evidence Table - Key Question 2: Timing of radiation therapy relative to IBR - categorical outcomes (combined/unspecified harms)

\begin{tabular}{|c|c|c|c|c|c|c|c|c|}
\hline Study, Year, PMID & $\begin{array}{l}\text { Outcome } \\
\text { Description }\end{array}$ & Design & $\begin{array}{l}\text { Overall } \\
\text { RoB }\end{array}$ & Time Point & $\begin{array}{l}\text { IBR Before } \\
\text { Radiation, n/N } \\
(\%)\end{array}$ & $\begin{array}{l}\text { IBR After } \\
\text { Radiation, n/N (\%) }\end{array}$ & $\begin{array}{l}\text { IBR Before Versus After } \\
\text { Radiation, Effect Size } \\
(95 \% \mathrm{Cl})\end{array}$ & P Value \\
\hline \multirow[t]{3}{*}{ Hirsch, 2014, 25347643} & Any complication & NRCS & High & $3.5 \mathrm{y}$ & NR & NR & adjOR $0.81(0.56,1.17)$ & NR \\
\hline & $\begin{array}{l}\text { Operative } \\
\text { complications }\end{array}$ & NRCS & High & $3.5 \mathrm{y}$ & NR & NR & adjOR $0.92(0.59,1.45)$ & NR \\
\hline & $\begin{array}{l}\text { Nonoperative } \\
\text { complications }\end{array}$ & NRCS & High & $3.5 \mathrm{y}$ & NR & NR & adjOR $0.90(0.60,1.34)$ & NR \\
\hline \multirow{3}{*}{$\begin{array}{l}\text { Stein, 2020, 32561384, } \\
\text { Canada }\end{array}$} & Any complication & NRCS & High & $10 \mathrm{mo}-5 \mathrm{y}$ & NR/76 (NR) & NR/54 (NR) & adjOR $0.82(0.03,2.19)$ & 0.69 \\
\hline & Major complications & NRCS & High & $10 \mathrm{mo}-5 \mathrm{y}$ & NR/76 (NR) & NR/54 (NR) & adjOR $0.62(0.21,1.86)$ & 0.40 \\
\hline & Minor complications & NRCS & High & $10 \mathrm{mo}-5 \mathrm{y}$ & NR/76 (NR) & NR/54 (NR) & adjOR $1.29(0.41,4.03)$ & 0.65 \\
\hline \multirow{2}{*}{$\begin{array}{l}\text { Yoon, 2020, 32332528, } \\
\text { US \& Canada }\end{array}$} & Any complication & NRCS & Moderate & $2 y$ & $33 / 80(41.3)$ & $95 / 237(40.1)$ & NR & 0.85 \\
\hline & Major complications & NRCS & Moderate & $2 y$ & $26 / 80(32.5)$ & $82 / 237(34.6)$ & NR & 0.73 \\
\hline
\end{tabular}

Abbreviations: adj $=$ adjusted, $\mathrm{CI}=$ confidence interval, $\mathrm{IBR}=$ implant-based reconstruction, $\mathrm{NR}=$ not reported, $\mathrm{NRCS}=$ nonrandomized comparative study, $\mathrm{OR}=$ odds ratio, PMID $=$ Pubmed identifier, $\mathrm{RoB}=$ risk of bias, $\mathrm{y}=$ years.

Colors: Header rows are shaded orange. Rows for every alternate study are shaded blue. The colors do not add unique information. 
Table F-3.1. Full Evidence Table - Key Question 3: Comparison of materials for IBR - continuous outcomes (general quality of life)

\begin{tabular}{|c|c|c|c|c|c|c|c|c|c|c|}
\hline $\begin{array}{l}\text { Study, Year, } \\
\text { PMID }\end{array}$ & $\begin{array}{l}\text { Outcome } \\
\text { Measurement }\end{array}$ & Design & $\begin{array}{l}\text { Overall } \\
\text { RoB }\end{array}$ & $\begin{array}{l}\text { Time } \\
\text { Point }\end{array}$ & $\begin{array}{l}\text { IBR With } \\
\text { Silicone, N }\end{array}$ & $\begin{array}{l}\text { IBR With Silicone, } \\
\text { Mean (SD) }\end{array}$ & $\begin{array}{l}\text { IBR With } \\
\text { Saline, N }\end{array}$ & $\begin{array}{l}\text { IBR With Saline, } \\
\text { Mean (SD) }\end{array}$ & $\begin{array}{l}\text { Effect Size } \\
(95 \% \mathrm{Cl})\end{array}$ & $\begin{array}{l}\mathbf{P} \\
\text { Value }\end{array}$ \\
\hline $\begin{array}{l}\text { Macadam, } \\
2010,20009795\end{array}$ & $\begin{array}{l}\text { EORTC QLQC30: } \\
\text { Global health status }\end{array}$ & NRCS & High & $2.6-4.5 y$ & 72 & $79.9(18.1)$ & 67 & $74.9(20.9)$ & NR & 0.13 \\
\hline
\end{tabular}

Abbreviations: CI = confidence interval, EORTC QLQC3 = European Organization for Research and Treatment of Cancer Quality of Life Questionnaire C30, IBR = implant-

based reconstruction, $\mathrm{NR}=$ not reported, $\mathrm{NRCS}=$ nonrandomized comparative study, $\mathrm{PMID}=$ Pubmed identifier, $\mathrm{RoB}=$ risk of bias, $\mathrm{SD}=$ standard deviation, $\mathrm{y}=\mathrm{years}$.

Colors: Header rows are shaded orange. The color does not add unique information.

Table F-3.2. Full Evidence Table - Key Question 3: Comparison of materials for IBR - continuous outcomes (physical well-being)

\begin{tabular}{|l|l|l|l|l|l|l|l|l|l|l|}
\hline $\begin{array}{l}\text { Study, Year, } \\
\text { PMID }\end{array}$ & $\begin{array}{l}\text { Outcome } \\
\text { Measurement }\end{array}$ & Design & $\begin{array}{l}\text { Overall } \\
\text { RoB }\end{array}$ & $\begin{array}{l}\text { Time } \\
\text { Point }\end{array}$ & $\begin{array}{l}\text { IBR With } \\
\text { Silicone, N }\end{array}$ & $\begin{array}{l}\text { IBR With Silicone, } \\
\text { Mean (SD) }\end{array}$ & $\begin{array}{l}\text { IBR With } \\
\text { Saline, N }\end{array}$ & $\begin{array}{l}\text { IBR With Saline, } \\
\text { Mean (SD) }\end{array}$ & $\begin{array}{l}\text { Silicone Versus } \\
\text { Saline, Effect Size } \\
\text { (95\% Cl) }\end{array}$ & $\begin{array}{l}\text { P } \\
\text { Value }\end{array}$ \\
\hline $\begin{array}{l}\text { Macadam, } \\
\text { 2010, 20009795 }\end{array}$ & $\begin{array}{l}\text { BREAST-Q: } \\
\text { Physical well-being }\end{array}$ & NRCS & High & $\begin{array}{l}2.6-4.5 \\
\mathrm{y}\end{array}$ & 74 & $76.2(14.9)$ & 68 & $73.4(16.3)$ & NR \\
\hline
\end{tabular}

Abbreviations: $\mathrm{CI}=$ confidence interval, IBR $=$ implant-based reconstruction, $\mathrm{NR}=$ not reported, NRCS $=$ nonrandomized comparative study, PMID $=$ Pubmed identifier, RoB $=$

risk of bias, $\mathrm{SD}=$ standard deviation, $\mathrm{y}=$ years.

Colors: Header rows are shaded orange. The color does not add unique information.

Table F-3.3. Full Evidence Table - Key Question 3: Comparison of materials for IBR - continuous outcomes (psychosocial well-being)

\begin{tabular}{|c|c|c|c|c|c|c|c|c|c|c|}
\hline $\begin{array}{l}\text { Study, Year, } \\
\text { PMID }\end{array}$ & $\begin{array}{l}\text { Outcome } \\
\text { Measurement }\end{array}$ & Design & $\begin{array}{l}\text { Overall } \\
\text { RoB }\end{array}$ & $\begin{array}{l}\text { Time } \\
\text { Point }\end{array}$ & $\begin{array}{l}\text { IBR With } \\
\text { Silicone, N }\end{array}$ & $\begin{array}{l}\text { IBR With Silicone, } \\
\text { Mean (SD) }\end{array}$ & $\begin{array}{l}\text { IBR With } \\
\text { Saline, } \mathbf{N}\end{array}$ & $\begin{array}{l}\text { IBR With Saline, } \\
\text { Mean (SD) }\end{array}$ & $\begin{array}{l}\text { Effect Size } \\
(95 \% \mathrm{Cl})\end{array}$ & $\begin{array}{l}\text { P } \\
\text { Value }\end{array}$ \\
\hline $\begin{array}{l}\text { Macadam, } \\
2010,20009795\end{array}$ & $\begin{array}{l}\text { BREAST-Q: } \\
\text { Psychosocial well-being }\end{array}$ & NRCS & High & $\begin{array}{l}2.6- \\
4.5 y\end{array}$ & 75 & $77.6(18.6)$ & 67 & $70.8(18.8)$ & NR & 0.004 \\
\hline
\end{tabular}

2010, 20009795 Psychosocial well-being

risk of bias, $\mathrm{SD}=$ standard deviation, $\mathrm{y}=$ years

Colors: Header rows are shaded orange. The color does not add unique information.

Table F-3.4. Full Evidence Table - Key Question 3: Comparison of materials for IBR - continuous outcomes (sexual well-being)

\begin{tabular}{|c|c|c|c|c|c|c|c|c|c|c|}
\hline $\begin{array}{l}\text { Study, Year, } \\
\text { PMID }\end{array}$ & $\begin{array}{l}\text { Outcome } \\
\text { Measurement }\end{array}$ & Design & $\begin{array}{l}\text { Overall } \\
\text { RoB }\end{array}$ & $\begin{array}{l}\text { Time } \\
\text { Point }\end{array}$ & $\begin{array}{l}\text { IBR With } \\
\text { Silicone, N }\end{array}$ & $\begin{array}{l}\text { IBR With Silicone, } \\
\text { Mean (SD) }\end{array}$ & $\begin{array}{l}\text { IBR With } \\
\text { Saline, N }\end{array}$ & $\begin{array}{l}\text { IBR With Saline, } \\
\text { Mean (SD) }\end{array}$ & $\begin{array}{l}\text { Effect Size } \\
(95 \% \mathrm{Cl})\end{array}$ & $\begin{array}{l}\mathbf{P} \\
\text { Value }\end{array}$ \\
\hline $\begin{array}{l}\text { Macadam, 2010, } \\
20009795\end{array}$ & $\begin{array}{l}\text { BREAST-Q: Sexual } \\
\text { well-being }\end{array}$ & NRCS & High & $\begin{array}{l}2.6-4.5 \\
y\end{array}$ & 71 & $54.4(19.8)$ & 65 & $47.6(20.9)$ & NR & 0.0562 \\
\hline
\end{tabular}

Abbreviations: $\mathrm{CI}=$ confidence interval, IBR $=$ implant-based reconstruction, $\mathrm{NR}=$ not reported, $\mathrm{NRCS}=$ nonrandomized comparative study, $\mathrm{PMID}=\mathrm{Pubmed}$ identifier, RoB $=$

risk of bias, $\mathrm{SD}=$ standard deviation, $\mathrm{y}=$ years

Colors: Header rows are shaded orange. The color does not add unique information. 
Table F-3.5. Full Evidence Table - Key Question 3: Comparison of materials for IBR - continuous outcomes (patient satisfaction with breast)

\begin{tabular}{|c|c|c|c|c|c|c|c|c|c|c|}
\hline $\begin{array}{l}\text { Study, Year, } \\
\text { PMID }\end{array}$ & $\begin{array}{l}\text { Outcome Measure } \\
\text { ment }\end{array}$ & Design & $\begin{array}{l}\text { Overall } \\
\text { RoB }\end{array}$ & $\begin{array}{l}\text { Time } \\
\text { Point }\end{array}$ & $\begin{array}{l}\text { IBR With } \\
\text { Silicone, N }\end{array}$ & $\begin{array}{l}\text { IBR With Silicone, } \\
\text { Mean (SD) }\end{array}$ & $\begin{array}{l}\text { IBR With } \\
\text { Saline, N }\end{array}$ & $\begin{array}{l}\text { IBR With Saline, } \\
\text { Mean (SD) }\end{array}$ & $\begin{array}{l}\text { Silicone Versus } \\
\text { Saline, Effect Size } \\
(95 \% \mathrm{Cl})\end{array}$ & $\begin{array}{l}\mathbf{P} \\
\text { Value }\end{array}$ \\
\hline $\begin{array}{l}\text { Macadam, 2010, } \\
20009795\end{array}$ & $\begin{array}{l}\text { BREAST-Q: } \\
\text { Satisfaction with breast }\end{array}$ & NRCS & High & $\begin{array}{l}2.6- \\
4.5 \mathrm{y}\end{array}$ & 75 & $63.8(15.2)$ & 67 & $56.9(15.1)$ & NR & 0.0083 \\
\hline $\begin{array}{l}\text { McCarthy, 2010, } \\
21136577\end{array}$ & $\begin{array}{l}\text { BREAST-Q: } \\
\text { Satisfaction with breast }\end{array}$ & NRCS & High & $\begin{array}{l}2.4- \\
3.3 y\end{array}$ & 176 & $58(20.3)$ & 306 & $52.5(20.4)$ & $\begin{array}{l}\operatorname{adjMD} 4.1\left(1.31^{*},\right. \\
\left.6.89^{*}\right)\end{array}$ & 0.032 \\
\hline
\end{tabular}

21136577

Satisfaction with breast

$3.3 \mathrm{y}$

Abbreviations: adj = adjusted, $\mathrm{CI}=$ confidence interval, $\mathrm{IBR}=$ implant-based reconstruction, $\mathrm{MD}=$ mean difference, $\mathrm{NR}=$ not reported, $\mathrm{NRCS}=$ nonrandomized comparative

study, $\mathrm{PMID}=$ Pubmed identifier, $\mathrm{RoB}=$ risk of bias, $\mathrm{SD}=$ standard deviation, $\mathrm{y}=$ years.

Colors: Header rows are shaded orange. Rows for every alternate study are shaded blue. The colors do not add unique information.

* Calculated.

Table F-3.6. Full Evidence Table - Key Question 3: Comparison of materials for IBR - continuous outcomes (patient satisfaction with outcome)

\begin{tabular}{|c|c|c|c|c|c|c|c|c|c|c|}
\hline $\begin{array}{l}\text { Study, Year, } \\
\text { PMID }\end{array}$ & Outcome Measurement & Design & $\begin{array}{l}\text { Overall } \\
\text { RoB }\end{array}$ & $\begin{array}{l}\text { Time } \\
\text { Point }\end{array}$ & $\begin{array}{l}\text { IBR With } \\
\text { Silicone, N }\end{array}$ & $\begin{array}{l}\text { IBR With } \\
\text { Silicone, } \\
\text { Mean (SD) }\end{array}$ & $\begin{array}{l}\text { IBR With } \\
\text { Saline, N }\end{array}$ & $\begin{array}{l}\text { IBR With } \\
\text { Saline, } \\
\text { Mean (SD) }\end{array}$ & $\begin{array}{l}\text { Effect Size } \\
(95 \% \mathrm{Cl})\end{array}$ & $\begin{array}{l}\text { P } \\
\text { Value }\end{array}$ \\
\hline $\begin{array}{l}\text { Macadam, 2010, } \\
20009795\end{array}$ & $\begin{array}{l}\text { BREAST-Q: Satisfaction with } \\
\text { outcome }\end{array}$ & NRCS & High & $\begin{array}{l}2.6-4.5 \\
y\end{array}$ & 75 & $75.4(17.6)$ & 68 & $69.5(22.6)$ & NR & 0.0815 \\
\hline
\end{tabular}

Abbreviations: $\mathrm{CI}=$ confidence interval, $\mathrm{IBR}=$ implant-based reconstruction, $\mathrm{NR}=$ not reported, $\mathrm{NRCS}=$ nonrandomized comparative study, PMID $=$ Pubmed identifier, RoB $=$

risk of bias, $\mathrm{SD}=$ standard deviation, $\mathrm{y}=$ years.

Colors: Header rows are shaded orange. The color does not add unique information.

Table F-3.7. Full Evidence Table - Key Question 3: Comparison of materials for IBR - categorical outcomes (mortality)

\begin{tabular}{|c|c|c|c|c|c|c|c|c|}
\hline Study, Year, PMID & Outcome Measurement & Design & Overall RoB & Time Point & Arm & $\mathrm{n} / \mathrm{N}(\%)$ & Effect Size $(95 \% \mathrm{Cl})$ & P Value \\
\hline \multirow{6}{*}{$\begin{array}{l}\text { Le, } 2005, \\
15743498\end{array}$} & Breast cancer mortality & NRCS & High & $12.4 \mathrm{y}$ & IBR with silicone & NR & Ref & Ref \\
\hline & Breast cancer mortality & NRCS & High & $12.4 \mathrm{y}$ & IBR with saline & NR & vs. Silicone: adjHR $1.01(0.44,2.34)$ & NR \\
\hline & Breast cancer mortality & NRCS & High & $12.4 \mathrm{y}$ & IBR with double lumen & NR & vs. Silicone: adjHR $1.49(0.83,2.7)$ & NR \\
\hline & $\begin{array}{l}\text { Non-breast cancer } \\
\text { mortality }\end{array}$ & NRCS & High & $12.4 \mathrm{y}$ & IBR with silicone & NR & Ref & Ref \\
\hline & $\begin{array}{l}\text { Non-breast cancer } \\
\text { mortality }\end{array}$ & NRCS & High & $12.4 \mathrm{y}$ & IBR with saline & NR & vs. Silicone: adjHR $1.75(0.29,10.39)$ & NR \\
\hline & $\begin{array}{l}\text { Non-breast cancer } \\
\text { mortality }\end{array}$ & NRCS & High & $12.4 \mathrm{y}$ & IBR with double lumen & NR & vs. Silicone: adjHR $3.13(0.91,10.78)$ & NR \\
\hline
\end{tabular}

Abbreviations: adj = adjusted, $\mathrm{CI}=$ confidence interval, $\mathrm{IBR}=$ implant-based reconstruction, $\mathrm{HR}=$ hazard ratio, $\mathrm{NR}=$ not reported, NRCS $=$ nonrandomized comparative study,

PMID $=$ Pubmed identifier, Ref $=$ reference group, $\mathrm{RoB}=$ risk of bias, $\mathrm{y}=$ years.

Colors: Header rows are shaded orange. The color does not add unique information. 
Table F-3.8. Full Evidence Table - Key Question 3: Comparison of materials for IBR - categorical outcomes (capsular contracture)

\begin{tabular}{|c|c|c|c|c|c|c|c|c|}
\hline Study, Year, PMID & Outcome Measurement & Design & $\begin{array}{l}\text { Overall } \\
\text { RoB }\end{array}$ & $\begin{array}{l}\text { Time } \\
\text { Point }\end{array}$ & $\begin{array}{l}\text { IBR With Silicone, } \\
\text { n/N (\%) }\end{array}$ & $\begin{array}{l}\text { IBR With } \\
\text { Saline, n/N (\%) }\end{array}$ & $\begin{array}{l}\text { Effect Size } \\
(95 \% \mathrm{Cl})\end{array}$ & P Value \\
\hline Antony, 2014, 24135689 & Baker Grade III or IV & NRCS & High & $3-5 y$ & $\mathrm{NR} / 179$ (NR) & $\mathrm{NR} / 166$ (NR) & NR & NS \\
\hline
\end{tabular}

Abbreviations: $\mathrm{CI}=$ confidence interval, IBR $=$ implant-based reconstruction, $\mathrm{NR}=$ not reported, $\mathrm{NRCS}=$ nonrandomized comparative study, $\mathrm{NS}=$ not significant, $\mathrm{PMID}=$

Pubmed identifier, $\mathrm{RoB}=$ risk of bias, $\mathrm{y}=$ years.

Colors: Header rows are shaded orange. The color does not add unique information.

Table F-3.9. Full Evidence Table - Key Question 3: Comparison of materials for IBR - categorical outcomes (implant failure/loss)

\begin{tabular}{|c|c|c|c|c|c|c|c|c|}
\hline Study, Year, PMID & $\begin{array}{l}\text { Outcome } \\
\text { Measurement }\end{array}$ & Design & $\begin{array}{l}\text { Overall } \\
\text { RoB }\end{array}$ & $\begin{array}{l}\text { Time } \\
\text { Point }\end{array}$ & $\begin{array}{l}\text { IBR With Silicone, } \\
\mathrm{n} / \mathrm{N}(\%)\end{array}$ & $\begin{array}{l}\text { IBR With Saline, } \\
\text { n/N (\%) }\end{array}$ & $\begin{array}{l}\text { Silicone Versus } \\
\text { Saline, Effect Size } \\
(95 \% \mathrm{Cl})\end{array}$ & P Value \\
\hline $\begin{array}{l}\text { Cordeiro, 2015a, } \\
25742523, \text { US }\end{array}$ & TE and implant loss & NRCS & High & $3.3 \mathrm{y}$ & NR/159 (NR) & $\mathrm{NR} / 129(\mathrm{NR})$ & adjOR $0.61(0.36,1.07)$ & NS \\
\hline
\end{tabular}

Abbreviations: adj $=$ adjusted, $\mathrm{CI}=$ confidence interval, $\mathrm{IBR}=$ implant-based reconstruction, $\mathrm{NR}=$ not reported, $\mathrm{NRCS}=$ nonrandomized comparative study, $\mathrm{NS}=$ not significant,

$\mathrm{OR}=$ odds ratio, $\mathrm{PMID}=$ Pubmed identifier, $\mathrm{RoB}=$ risk of bias, $\mathrm{TE}=$ tissue expander, $\mathrm{y}=$ years

Colors: Header rows are shaded orange. The color does not add unique information.

Table F-4.1. Full Evidence Table - Key Question 4: Comparison of anatomic planes for IBR - continuous outcomes (physical well-being)

\begin{tabular}{|c|c|c|c|c|c|c|c|c|c|}
\hline $\begin{array}{l}\text { Study, Year, } \\
\text { PMID, Country }\end{array}$ & Outcome Measurement & Design & $\begin{array}{l}\text { Overall } \\
\text { RoB }\end{array}$ & $\begin{array}{l}\text { Time } \\
\text { Point }\end{array}$ & Arm & $\mathbf{N}$ & Mean (SD) & $\begin{array}{l}\text { Effect Size } \\
(95 \% \mathrm{Cl})\end{array}$ & $\begin{array}{l}\mathbf{P} \\
\text { Value } \\
\end{array}$ \\
\hline \multirow{2}{*}{$\begin{array}{l}\text { Lee, 2021b, 33691448, } \\
\text { South Korea }\end{array}$} & SF-36 (0-100): PCS & RCT & Moderate & $6 \mathrm{mo}$ & Prepectoral & 20 & $45.2(7.1)$ & Ref & Ref \\
\hline & SF-36 (0-100): PCS & RCT & Moderate & $6 \mathrm{mo}$ & Partial Submuscular & 14 & $45.2(7.1)$ & NR & 0.689 \\
\hline \multirow{6}{*}{$\begin{array}{l}\text { Cattelani, 2018, } \\
\text { 29275104, Italy }\end{array}$} & Constant Murley $(0-100)$ : Upper limb & NRCS & High & $1 \mathrm{~d}$ & Prepectoral & 39 & $71.6(8.9)$ & Ref & Ref \\
\hline & Constant Murley $(0-100)$ : Upper limb & NRCS & High & $1 \mathrm{~d}$ & Total submuscular & 45 & $60.4(10.5)$ & NR & $<0.001$ \\
\hline & Constant Murley $(0-100)$ : Upper limb & NRCS & High & $7 \mathrm{~d}$ & Prepectoral & 39 & $65.7(9.3)$ & Ref & Ref \\
\hline & Constant Murley $(0-100)$ : Upper limb & NRCS & High & $7 \mathrm{~d}$ & Total submuscular & 45 & $52.4(12.2)$ & NR & $<0.001$ \\
\hline & DASH score & NRCS & High & $1 \mathrm{y}$ & Prepectoral & 39 & $9.9(17.9)$ & Ref & Ref \\
\hline & DASH score & NRCS & High & $1 \mathrm{y}$ & Total submuscular & 45 & $29.2(16.9)$ & NR & $<0.001$ \\
\hline
\end{tabular}

Abbreviations: $\mathrm{CI}=$ confidence interval, $\mathrm{d}=$ days, DASH $=$ Disabilities of the Arm, Shoulder, and Hand, IBR = implant-based reconstruction, NR $=$ not reported, NRCS $=$

nonrandomized comparative study, $\mathrm{PCS}=$ Physical Component Summary, PMID $=$ Pubmed identifier, $\mathrm{RoB}=$ risk of bias, $\mathrm{SD}=$ standard deviation, $\mathrm{y}=$ years.

Colors: Header rows are shaded orange. Rows for every alternate study are shaded blue. The colors do not add unique information.

Table F-4.2. Full Evidence Table - Key Question 4: Comparison of anatomic planes for IBR - continuous outcomes (psychosocial wellbeing)

\begin{tabular}{|c|c|c|c|c|c|c|c|c|c|}
\hline $\begin{array}{l}\text { Study, Year, } \\
\text { PMID, Country }\end{array}$ & Outcome Measurement & Design & $\begin{array}{l}\text { Overall } \\
\text { RoB }\end{array}$ & $\begin{array}{l}\text { Time } \\
\text { Point }\end{array}$ & Arm & $\mathbf{N}$ & Mean (SD) & $\begin{array}{l}\text { Effect Size } \\
(95 \% \mathrm{Cl})\end{array}$ & P Value \\
\hline \multirow{4}{*}{$\begin{array}{l}\text { Lee, } 2021 \mathrm{~b}, \\
33691448 \text {, South } \\
\text { Korea }\end{array}$} & SF-36 (0-100): MCS & RCT & Moderate & $6 \mathrm{mo}$ & Prepectoral & 20 & $40.5(10.5)$ & Ref & Ref \\
\hline & SF-36 (0-100): MCS & RCT & Moderate & $6 \mathrm{mo}$ & Partial Submuscular & 14 & $40.5(10.5)$ & NR & 0.904 \\
\hline & HADS: Anxiety (0-21) & RCT & Moderate & $6 \mathrm{mo}$ & Prepectoral & 20 & $6.3(3.3)$ & Ref & Ref \\
\hline & HADS: Anxiety (0-21) & RCT & Moderate & $6 \mathrm{mo}$ & Partial Submuscular & 14 & $5.0(2.9)$ & NR & 0.959 \\
\hline
\end{tabular}




\begin{tabular}{|c|c|c|c|c|c|c|c|c|c|}
\hline $\begin{array}{l}\text { Study, Year, } \\
\text { PMID, Country }\end{array}$ & Outcome Measurement & Design & $\begin{array}{l}\text { Overall } \\
\text { RoB }\end{array}$ & $\begin{array}{l}\text { Time } \\
\text { Point }\end{array}$ & Arm & $\mathbf{N}$ & Mean (SD) & $\begin{array}{l}\text { Effect Size } \\
(95 \% \mathrm{Cl})\end{array}$ & P Value \\
\hline & HADS: Depression (0-21) & RCT & Moderate & $6 \mathrm{mo}$ & Prepectoral & 20 & $7.5(7.4)$ & Ref & Ref \\
\hline & HADS: Depression (0-21) & RCT & Moderate & $6 \mathrm{mo}$ & Partial Submuscular & 14 & $6.3(3.8)$ & NR & 0.924 \\
\hline \multirow{2}{*}{$\begin{array}{l}\text { Cattelani, 2018, } \\
29275104, \text { Italy }\end{array}$} & Return to usual work & NRCS & High & NR & Prepectoral & 39 & $34.6(21)$ & Ref & Ref \\
\hline & Return to usual work & NRCS & High & NR & Total submuscular & 45 & $57.3(37.8)$ & NR & $<0.001$ \\
\hline
\end{tabular}

Abbreviations: $\mathrm{CI}=$ confidence interval, $\mathrm{d}=$ days, $\mathrm{IBR}=$ implant-based reconstruction, $\mathrm{NR}=$ not reported, NRCS $=$ nonrandomized comparative study, $\mathrm{PMID}=\mathrm{Pubmed}$

identifier, RoB = risk of bias, $\mathrm{SD}=$ standard deviation.

Colors: Header rows are shaded orange. Rows for every alternate study are shaded blue. The colors do not add unique information.

Table F-4.3. Full Evidence Table - Key Question 4: Comparison of anatomic planes for IBR - continuous outcomes (patient satisfaction with breast)

\begin{tabular}{|c|c|c|c|c|c|c|c|c|c|}
\hline $\begin{array}{l}\text { Study, Year, PMID, } \\
\text { Country }\end{array}$ & $\begin{array}{l}\text { Outcome } \\
\text { Measurement }\end{array}$ & Design & $\begin{array}{l}\text { Overall } \\
\text { RoB }\end{array}$ & $\begin{array}{l}\text { Time } \\
\text { Point }\end{array}$ & Arm & $\mathbf{N}$ & Mean (SD) & $\begin{array}{l}\text { Effect Size } \\
(95 \% \mathrm{Cl})\end{array}$ & P Value \\
\hline \multirow{2}{*}{$\begin{array}{l}\text { Cattelani, 2018, } \\
29275104, \text { Italy }\end{array}$} & BREAST-Q & NRCS & High & $1 \mathrm{y}$ & Prepectoral & 39 & $92.2(9.0)$ & Ref & Ref \\
\hline & BREAST-Q & NRCS & High & $1 \mathrm{y}$ & Total submuscular & 45 & $76.1(14.6)$ & NR & $<0.001$ \\
\hline
\end{tabular}

Abbreviations: $\mathrm{CI}=$ confidence interval, $\mathrm{IBR}=$ implant-based reconstruction, $\mathrm{NR}=$ not reported, $\mathrm{NRCS}=$ nonrandomized comparative study, $\mathrm{PMID}=\mathrm{Pubmed}$ identifier, $\mathrm{RoB}=$

risk of bias, $\mathrm{SD}=$ standard deviation, $\mathrm{y}=$ years

Colors: Header rows are shaded orange. The color does not add unique information.

Table F-4.4. Full Evidence Table - Key Question 4: Comparison of anatomic planes for IBR - continuous outcomes (pain, including chronic pain and analgesic use)

\begin{tabular}{|c|c|c|c|c|c|c|c|c|c|c|}
\hline $\begin{array}{l}\text { Study, Year, } \\
\text { PMID, Country }\end{array}$ & Outcome & Outcome Measurement & Design & $\begin{array}{l}\text { Overall } \\
\text { RoB }\end{array}$ & $\begin{array}{l}\text { Time } \\
\text { Point }\end{array}$ & Arm & $\mathbf{N}$ & Mean (SD) & $\begin{array}{l}\text { Effect Size } \\
(95 \% \mathrm{Cl})\end{array}$ & $\begin{array}{l}\mathbf{P} \\
\text { Value }\end{array}$ \\
\hline \multirow{2}{*}{$\begin{array}{l}\text { Avila, 2020, } \\
33234947 \text {, Us }\end{array}$} & Pain & VAS (0-10) & NRCS & High & NR & Prepectoral & 73 & $3.94(0.83)$ & Ref & Ref \\
\hline & Pain & VAS $(0-10)$ & NRCS & High & NR & Total Submuscular & 73 & $5.25(0.81)$ & NR & $<0.001$ \\
\hline \multirow{4}{*}{$\begin{array}{l}\text { Cattelani, } 2018, \\
29275104, \text { Italy }\end{array}$} & Pain & BPI-SF & NRCS & High & $1 \mathrm{~d}$ & Prepectoral & 39 & $17.6(15.5)$ & Ref & Ref \\
\hline & Pain & BPI-SF & NRCS & High & $1 \mathrm{~d}$ & Total submuscular & 45 & $44.1(15.8)$ & NR & $<0.001$ \\
\hline & Pain & BPI-SF & NRCS & High & $7 d$ & Prepectoral & 39 & $8.2(15.4)$ & Ref & Ref \\
\hline & Pain & $\mathrm{BPI}-\mathrm{SF}$ & NRCS & High & $7 d$ & Total submuscular & 45 & $22(18.6)$ & NR & $<0.001$ \\
\hline \multirow{4}{*}{$\begin{array}{l}\text { Kim, 2020, } \\
33066236, \text { South } \\
\text { Korea }\end{array}$} & Pain & VAS (0-10) & NRCS & Moderate & $1 \mathrm{~d}$ & Prepectoral & 53 & $2.66(1.82)$ & Ref & Ref \\
\hline & Pain & VAS $(0-10)$ & NRCS & Moderate & $1 \mathrm{~d}$ & Partial Submuscular & 114 & $2.26(1.38)$ & adjMD -0.08 & 0.33 \\
\hline & Pain & VAS (0-10) & NRCS & Moderate & $7 d$ & Prepectoral & 53 & $1.08(1.19)$ & Ref & Ref \\
\hline & Pain & VAS $(0-10)$ & NRCS & Moderate & $7 d$ & Partial Submuscular & 114 & $0.80(1.07)$ & -0.12 & 0.12 \\
\hline \multirow{2}{*}{$\begin{array}{l}\text { Avila, 2020, } \\
33234947 \text {, US }\end{array}$} & Analgesic use & Oral morphine equivalents & NRCS & High & NR & Prepectoral & 73 & $17.4 \mathrm{mg}(45.1)$ & Ref & Ref \\
\hline & Analgesic use & Oral morphine equivalents & NRCS & High & NR & Total Submuscular & 73 & $63.0 \mathrm{mg} \mathrm{(44.9)}$ & NR & 0.03 \\
\hline
\end{tabular}

Abbreviations: BPI-SF = Brief Pain Inventory-Short Form, CI = confidence interval, $\mathrm{d}=$ days, IBR $=$ implant-based reconstruction, NR $=$ not reported, NRCS $=$ nonrandomized comparative study, $\mathrm{PMID}=$ Pubmed identifier, $\mathrm{RoB}=$ risk of bias, $\mathrm{SD}=$ standard deviation, $\mathrm{VAS}=$ visual analog scale.

Colors: Header rows are shaded orange. Rows for every alternate outcome are shaded blue. The colors do not add unique information. 
Table F-4.5. Full Evidence Table - Key Question 4: Comparison of anatomic planes for IBR - categorical outcomes (analgesic use)

\begin{tabular}{|c|c|c|c|c|c|c|c|c|}
\hline $\begin{array}{l}\text { Study, Year, } \\
\text { PMID, Country }\end{array}$ & Outcome Measurement & Design & $\begin{array}{l}\text { Overall } \\
\text { RoB }\end{array}$ & $\begin{array}{l}\text { Time } \\
\text { Point }\end{array}$ & Arm & $\mathrm{n} / \mathrm{N}(\%)$ & $\begin{array}{l}\text { Effect Size } \\
(95 \% \mathrm{Cl})\end{array}$ & P Value \\
\hline \multirow{8}{*}{$\begin{array}{l}\text { Cattelani, 2018, } \\
\text { 29275104, Italy }\end{array}$} & Paracetamol, ketoprofen, or opioid use & NRCS & High & $1 \mathrm{~d}$ & Prepectoral & $39 / 39(100)$ & Ref & Ref \\
\hline & Paracetamol, ketoprofen, or opioid use & NRCS & High & $1 \mathrm{~d}$ & Total Submuscular & $45 / 45(100)$ & NR & NR \\
\hline & Paracetamol, ketoprofen, or opioid use & NRCS & High & $7 d$ & Prepectoral & $9 / 39(23.1)$ & Ref & Ref \\
\hline & Paracetamol, ketoprofen, or opioid use & NRCS & High & $7 \mathrm{~d}$ & Total Submuscular & $33 / 45(73.3)$ & NR & NR \\
\hline & Opioid use & NRCS & High & $1 \mathrm{~d}$ & Prepectoral & $3 / 39(7.7)$ & Ref & Ref \\
\hline & Opioid use & NRCS & High & $1 \mathrm{~d}$ & Total Submuscular & $11 / 45(24.4)$ & NR & NR \\
\hline & Opioid use & NRCS & High & $7 d$ & Prepectoral & $0 / 39(0)$ & Ref & Ref \\
\hline & Opioid use & NRCS & High & $7 d$ & Total Submuscular & $0 / 45(0)$ & NR & NR \\
\hline
\end{tabular}

Abbreviations: $\mathrm{d}=$ days, $\mathrm{CI}=$ confidence interval, $\mathrm{d}=$ days, IBR $=$ implant-based reconstruction, $\mathrm{NR}=$ not reported, $\mathrm{NRCS}=$ nonrandomized comparative study, $\mathrm{PMID}=$

Pubmed identifier, RoB = risk of bias

Colors: Header rows are shaded orange. The color does not add unique information.

Table F-4.6. Key Question 4: Comparison of anatomic planes for IBR - categorical outcomes (unplanned repeat surgeries for revision)

\begin{tabular}{|c|c|c|c|c|c|c|c|c|}
\hline Study, Year, PMID, Country & $\begin{array}{l}\text { Outcome } \\
\text { Measurement }\end{array}$ & Design & $\begin{array}{l}\text { Overall } \\
\text { RoB }\end{array}$ & $\begin{array}{l}\text { Time } \\
\text { Point }\end{array}$ & Arm & $n / N(\%)$ & $\begin{array}{l}\text { Effect Size }(95 \% \\
\mathrm{Cl})\end{array}$ & P Value \\
\hline \multirow[t]{2}{*}{ Avila, 2020, 33234947, US } & NR & NRCS & High & $1 \mathrm{mo}$ & Prepectoral & $8 / 203(3.94)$ & Ref & Ref \\
\hline & NR & NRCS & High & $1 \mathrm{mo}$ & Total submuscular & $17 / 202(8.42)$ & NR & NS \\
\hline
\end{tabular}

Abbreviations: adj = adjusted, $\mathrm{CI}=$ confidence interval, $\mathrm{IBR}=$ implant-based reconstruction, $\mathrm{NR}=$ not reported, $\mathrm{NRCS}=$ nonrandomized comparative study, $\mathrm{OR}=$ odds ratio, PMID $=$ Pubmed identifier, $\mathrm{RoB}=$ risk of bias, $\mathrm{y}=$ years.

Colors: Header rows are shaded orange. The color does not add unique information.

Table F-4.7. Full Evidence Table - Key Question 4: Comparison of anatomic planes for IBR - categorical outcomes (necrosis)

\begin{tabular}{|c|c|c|c|c|c|c|c|c|}
\hline Study, Year, PMID, Country & $\begin{array}{l}\text { Outcome } \\
\text { Measurement }\end{array}$ & Design & $\begin{array}{l}\text { Overall } \\
\text { RoB }\end{array}$ & $\begin{array}{l}\text { Time } \\
\text { Point }\end{array}$ & Arm & $\mathrm{n} / \mathrm{N}(\%)$ & Effect Size (95\% CI) & P Value \\
\hline \multirow[t]{2}{*}{ Nealon, 2020, 32032345, US } & Skin necrosis & NRCS & High & $1.7-2.4 \mathrm{y}$ & Prepectoral & $5 / 114(4.4)$ & Ref & Ref \\
\hline & Skin necrosis & NRCS & High & $1.7-2.4 \mathrm{y}$ & Total Submuscular & $6 / 142(4.2)$ & adjOR $1.01(0.74,5.95)$ & 0.77 \\
\hline
\end{tabular}

Abbreviations: adj $=$ adjusted, $\mathrm{CI}=$ confidence interval, $\mathrm{IBR}=$ implant-based reconstruction, $\mathrm{NR}=$ not reported, $\mathrm{NRCS}=$ nonrandomized comparative study, $\mathrm{OR}=$ odds ratio, PMID $=$ Pubmed identifier, $\mathrm{RoB}=$ risk of bias, $\mathrm{y}=$ years.

Colors: Header rows are shaded orange. The color does not add unique information. 
Table F-4.8. Full Evidence Table - Key Question 4: Comparison of anatomic planes for IBR - categorical outcomes (infections)

\begin{tabular}{|c|c|c|c|c|c|c|c|c|c|}
\hline $\begin{array}{l}\text { Study, Year, } \\
\text { PMID, Country }\end{array}$ & Outcome & $\begin{array}{l}\text { Outcome } \\
\text { Measurement }\end{array}$ & Design & $\begin{array}{l}\text { Overall } \\
\text { RoB }\end{array}$ & $\begin{array}{l}\text { Time } \\
\text { Point }\end{array}$ & Arm & $n / N(\%)$ & Effect Size $(95 \% \mathrm{CI})$ & $\begin{array}{l}\mathbf{P} \\
\text { Value }\end{array}$ \\
\hline \multirow{2}{*}{$\begin{array}{l}\text { Kraenzlin, } \\
2021, \\
32568752, \text { US }\end{array}$} & $\begin{array}{l}\text { Infections (not explicitly } \\
\text { implant-related) }\end{array}$ & NR & NRCS & High & NR & Prepectoral & $\begin{array}{l}34 / 169 \\
(11.0) \\
\end{array}$ & Ref & Ref \\
\hline & $\begin{array}{l}\text { Infections (not explicitly } \\
\text { implant-related) }\end{array}$ & NR & NRCS & High & NR & Total submuscular & $\begin{array}{l}34 / 117 \\
(17.4) \\
\end{array}$ & NR & 0.21 \\
\hline \multirow[t]{2}{*}{$\begin{array}{l}\text { Nealon, 2020, } \\
32032345 \text {, US }\end{array}$} & $\begin{array}{l}\text { Infections (not explicitly } \\
\text { implant-related) }\end{array}$ & NR & NRCS & High & $1.7-2.4 \mathrm{y}$ & Prepectoral & $2 / 114(1.8)$ & Ref & Ref \\
\hline & $\begin{array}{l}\text { Infections (not explicitly } \\
\text { implant-related) }\end{array}$ & NR & NRCS & High & $1.7-2.4 \mathrm{y}$ & $\begin{array}{l}\text { Total } \\
\text { Submuscular }\end{array}$ & $6 / 142(4.2)$ & adjOR $0.31(0,8.65)$ & 0.52 \\
\hline
\end{tabular}

Abbreviations: adj = adjusted, $\mathrm{CI}=$ confidence interval, $\mathrm{IBR}=$ implant-based reconstruction, $\mathrm{NR}=$ not reported, NRCS $=$ nonrandomized comparative study, $\mathrm{OR}=$ odds ratio, PMID $=$ Pubmed identifier, RoB $=$ risk of bias, $\mathrm{y}=$ years.

Colors: Header rows are shaded orange. Rows for every alternate study are shaded blue. The colors do not add unique information.

Table F-4.9. Full Evidence Table - Key Question 4: Comparison of anatomic planes for IBR - categorical outcomes (seroma)

\begin{tabular}{|c|c|c|c|c|c|c|c|c|}
\hline Study, Year, PMID, Country & $\begin{array}{l}\text { Outcome } \\
\text { Measurement }\end{array}$ & Design & $\begin{array}{l}\text { Overall } \\
\text { RoB }\end{array}$ & $\begin{array}{l}\text { Time } \\
\text { Point }\end{array}$ & Arm & $\mathrm{n} / \mathrm{N}(\%)$ & Effect Size $(95 \% \mathrm{Cl})$ & P Value \\
\hline \multirow{2}{*}{$\begin{array}{l}\text { Lee, 2021b, 33691448, South } \\
\text { Korea }\end{array}$} & NR & RCT & Moderate & $6 \mathrm{mo}$ & Prepectoral & $3 / 20(15.0)$ & Ref & Ref \\
\hline & NR & RCT & Moderate & $6 \mathrm{mo}$ & Partial submuscular & $2 / 14(14.3)$ & OR $1.06(0.15,7.34)$ & 0.95 \\
\hline \multirow[t]{2}{*}{ Nealon, 2020, 32032345, US } & NR & NRCS & High & $1.7-2.4 \mathrm{y}$ & Prepectoral & $10 / 114(8.8)$ & Ref & Ref \\
\hline & NR & NRCS & High & $1.7-2.4 \mathrm{y}$ & Total Submuscular & $11 / 142(7.7)$ & adjOR $1.49(0.37,6.11)$ & 0.57 \\
\hline
\end{tabular}

Abbreviations: adj = adjusted, $\mathrm{CI}=$ confidence interval, $\mathrm{IBR}=$ implant-based reconstruction, $\mathrm{NR}=$ not reported, $\mathrm{NRCS}=$ nonrandomized comparative study, $\mathrm{OR}=$ odds ratio, PMID $=$ Pubmed identifier, $\mathrm{RoB}=$ risk of bias, $\mathrm{y}=$ years.

Colors: Header rows are shaded orange. Rows for every alternate study are shaded blue. The colors do not add unique information.

Table F-4.10. Full Evidence Table - Key Question 4: Comparison of anatomic planes for IBR - categorical outcomes (need for explant surgery)

\begin{tabular}{|c|c|c|c|c|c|c|c|c|}
\hline Study, Year, PMID, Country & $\begin{array}{l}\text { Outcome } \\
\text { Measurement }\end{array}$ & Design & $\begin{array}{l}\text { Overall } \\
\text { RoB }\end{array}$ & $\begin{array}{l}\text { Time } \\
\text { Point }\end{array}$ & Arm & $\mathrm{n} / \mathrm{N}(\%)$ & Effect Size (95\% CI) & P Value \\
\hline \multirow[t]{2}{*}{ Nealon, 2020, 32032345, US } & NR & NRCS & High & $1.7-2.4 \mathrm{y}$ & Prepectoral & $4 / 114(3.5)$ & Ref & Ref \\
\hline & NR & NRCS & High & $1.7-2.4 \mathrm{y}$ & Total Submuscular & $7 / 142(4.9)$ & adjOR $1.01(0.07,14.11)$ & 0.99 \\
\hline
\end{tabular}

Abbreviations: adj $=$ adjusted, $\mathrm{CI}=$ confidence interval, $\mathrm{IBR}=$ implant-based reconstruction, $\mathrm{NR}=$ not reported, NRCS $=$ nonrandomized comparative study, $\mathrm{OR}=$ odds ratio, PMID $=$ Pubmed identifier, $\mathrm{RoB}=$ risk of bias, $\mathrm{y}=$ years.

Colors: Header rows are shaded orange. The color does not add unique information. 
Table F-4.11. Full Evidence Table - Key Question 4: Comparison of anatomic planes for IBR - categorical outcomes (capsular contracture)

\begin{tabular}{|c|c|c|c|c|c|c|c|c|}
\hline Study, Year, PMID, Country & $\begin{array}{l}\text { Outcome } \\
\text { Measurement }\end{array}$ & Design & $\begin{array}{l}\text { Overall } \\
\text { RoB }\end{array}$ & $\begin{array}{l}\text { Time } \\
\text { Point }\end{array}$ & Arm & $\mathrm{n} / \mathrm{N}(\%)$ & Effect Size $(95 \% \mathrm{Cl})$ & $\begin{array}{l}P \\
\text { Value }\end{array}$ \\
\hline \multirow{2}{*}{$\begin{array}{l}\text { Lee, 2021b, 33691448, South } \\
\text { Korea }\end{array}$} & NR & RCT & Moderate & $6 \mathrm{mo}$ & Prepectoral & $1 / 20(5.0)$ & Ref & Ref \\
\hline & NR & RCT & Moderate & $6 \mathrm{mo}$ & Partial submuscular & $0 / 14(0)$ & Not calculable & - \\
\hline \multirow[t]{2}{*}{ Nealon, 2020, 32032345, US } & NR & NRCS & High & $1.7-2.4 y$ & Prepectoral & $2 / 114(1.8)$ & Ref & Ref \\
\hline & NR & NRCS & High & $1.7-2.4 y$ & Total Submuscular & $12 / 142(8.5)$ & adjOR $0.30(0.03,1.55)$ & 0.16 \\
\hline
\end{tabular}

Abbreviations: adj $=$ adjusted, $\mathrm{CI}=$ confidence interval, $\mathrm{IBR}=$ implant-based reconstruction, $\mathrm{NR}=$ not reported, $\mathrm{NRCS}=$ nonrandomized comparative study, $\mathrm{OR}=$ odds ratio, PMID $=$ Pubmed identifier, $\mathrm{RoB}=$ risk of bias, $\mathrm{y}=$ years.

Colors: Header rows are shaded orange. Rows for every alternate study are shaded blue. The colors do not add unique information.

Table F-4.12. Full Evidence Table - Key Question 4: Comparison of anatomic planes for IBR - categorical outcomes (hematoma)

\begin{tabular}{|c|c|c|c|c|c|c|c|c|}
\hline Study, Year, PMID, Country & \begin{tabular}{|l|} 
Outcome \\
Measurement
\end{tabular} & Design & $\begin{array}{l}\text { Overall } \\
\text { RoB }\end{array}$ & Time Point & Arm & $\mathrm{n} / \mathrm{N}(\%)$ & Effect Size $(95 \% \mathrm{Cl})$ & \begin{tabular}{|l}
$\mathbf{P}$ \\
Value \\
\end{tabular} \\
\hline \multirow[t]{2}{*}{ Nealon, 2020, 32032345, US } & NR & & High & & Prepec & & Ref & Ref \\
\hline & \begin{tabular}{|l|}
$N R$ \\
\end{tabular} & NRCS & High & $1.7-2.4 \mathrm{y}$ & Total Submuscular & $7 / 142(4.9)$ & adjOR $5.18(0.39,7.05$ & 0.23 \\
\hline
\end{tabular}

Abbreviations: adj $=$ adjusted, $\mathrm{CI}=$ confidence interval, $\mathrm{IBR}=$ implant-based reconstruction, $\mathrm{NR}=$ not reported, $\mathrm{NRCS}=$ nonrandomized comparative study, $\mathrm{OR}=$ odds ratio,

PMID $=$ Pubmed identifier, $\mathrm{RoB}=$ risk of bias, $\mathrm{y}=$ years.

Colors: Header rows are shaded orange. The color does not add unique information.

Table F-4.13. Full Evidence Table - Key Question 4: Comparison of anatomic planes for IBR - categorical outcomes (combined/unspecified harms)

\begin{tabular}{|c|c|c|c|c|c|c|c|c|}
\hline $\begin{array}{l}\text { Study, Year, } \\
\text { PMID, Country }\end{array}$ & Outcome Measurement & Design & $\begin{array}{l}\text { Overall } \\
\text { RoB }\end{array}$ & $\begin{array}{l}\text { Time } \\
\text { Point }\end{array}$ & Arm & $\mathrm{n} / \mathrm{N}(\%)$ & $\begin{array}{l}\text { Effect Size } \\
(95 \% \mathrm{Cl})\end{array}$ & $\begin{array}{l}\mathbf{P} \\
\text { Value }\end{array}$ \\
\hline \multirow[t]{2}{*}{$\begin{array}{l}\text { Avila, 2020, } \\
33234947 \text {, US }\end{array}$} & $\begin{array}{l}\text { Necrosis/infection, wound dehiscence/ } \\
\text { hematoma/seroma }\end{array}$ & NRCS & High & $1 \mathrm{mo}$ & Prepectoral & $12 / 203(5.91)$ & Ref & Ref \\
\hline & $\begin{array}{l}\text { Necrosis/infection, wound dehiscence/ } \\
\text { hematoma/seroma }\end{array}$ & NRCS & High & $1 \mathrm{mo}$ & $\begin{array}{l}\text { Total } \\
\text { submuscular }\end{array}$ & $19 / 202(9.41)$ & NR & NS \\
\hline \multirow{2}{*}{$\begin{array}{l}\text { Gabriel, 2020, } \\
\text { 32195862, US }\end{array}$} & Any complication & NRCS & High & $2 y$ & Prepectoral & $19 / 129$ breasts (14.7) & Ref & Ref \\
\hline & Any complication & NRCS & High & $2 y$ & $\begin{array}{l}\text { Partial } \\
\text { submuscular }\end{array}$ & $33 / 128$ breasts $(25.8)$ & $\begin{array}{l}\text { adjOR } 3.04 \\
(1.34,7.61)\end{array}$ & 0.013 \\
\hline \multirow[t]{2}{*}{$\begin{array}{l}\text { Ozgur, 2020, } \\
33223365 \text {, Turkey }\end{array}$} & $\begin{array}{l}\text { Capsular contracture, inframammary fold problems, } \\
\text { bottoming out, rippling, mechanical shift, animation } \\
\text { deformity }\end{array}$ & NRCS & High & $\begin{array}{l}5.3- \\
6.1 \mathrm{y}\end{array}$ & $\begin{array}{l}\text { Partial } \\
\text { submuscular }\end{array}$ & $8 / 91$ breasts $(8.8)$ & Ref & Ref \\
\hline & $\begin{array}{l}\text { Capsular contracture, inframammary fold problems, } \\
\text { bottoming out, rippling, mechanical shift, animation } \\
\text { deformity }\end{array}$ & NRCS & High & $\begin{array}{l}5.3- \\
6.1 \mathrm{y}\end{array}$ & $\begin{array}{l}\text { Total } \\
\text { submuscular }\end{array}$ & $29 / 117$ breasts $(24.8)$ & $\begin{array}{l}\text { adjOR } 3.28 \\
(1.39,7.76)\end{array}$ & 0.007 \\
\hline
\end{tabular}

Abbreviations: BPI-SF $=$ Brief Pain Inventory-Short Form, $\mathrm{CI}=$ confidence interval, $\mathrm{d}=$ days, IBR $=$ implant-based reconstruction, $\mathrm{NR}=$ not reported, NRCS $=$ nonrandomized

comparative study, PMID = Pubmed identifier, $\mathrm{RoB}=$ risk of bias, $\mathrm{SD}=$ standard deviation, $\mathrm{VAS}=$ visual analog scale

Colors: Header rows are shaded orange. Rows for every alternate study are shaded blue. The colors do not add unique information. 
Table F-5.1. Full Evidence Table - Key Question 5: Use versus nonuse of human ADM during IBR - continuous outcomes (physical wellbeing)

\begin{tabular}{|c|c|c|c|c|c|c|c|c|c|c|}
\hline $\begin{array}{l}\text { Study, Year, } \\
\text { PMID, Country }\end{array}$ & $\begin{array}{l}\text { Outcome } \\
\text { Measurement }\end{array}$ & $\begin{array}{l}\text { Desig } \\
\mathrm{n}\end{array}$ & $\begin{array}{l}\text { Overall } \\
\text { RoB }\end{array}$ & Time Point & $\begin{array}{l}\text { Use of } \\
\text { ADM, } \\
\mathrm{N}\end{array}$ & $\begin{array}{l}\text { Use of } \\
\text { ADM, Mean } \\
\text { (SD) }\end{array}$ & $\begin{array}{l}\text { Nonuse } \\
\text { of ADM, } \\
\mathrm{N}\end{array}$ & $\begin{array}{l}\text { Nonuse of } \\
\text { ADM, Mean } \\
\text { (SD) }\end{array}$ & $\begin{array}{l}\text { Effect Size } \\
(95 \% \mathrm{Cl})\end{array}$ & $\begin{array}{l}\mathbf{P} \\
\text { Value }\end{array}$ \\
\hline \multirow[t]{4}{*}{$\begin{array}{l}\text { McCarthy, 2012, } \\
\text { 23096987, US }\end{array}$} & $\begin{array}{l}\text { BREAST-Q: Chest } \\
\text { and Upper Body }\end{array}$ & RCT & Moderate & Baseline & 36 & $85.6(13.5)$ & 33 & $86.9(12.4)$ & $\mathrm{N} / \mathrm{A}$ & $\mathrm{N} / \mathrm{A}$ \\
\hline & $\begin{array}{l}\text { BREAST-Q: Chest } \\
\text { and Upper Body }\end{array}$ & RCT & Moderate & $24 \mathrm{~h}$ & 36 & $65.8(12.7)$ & 33 & $68.2(13.7)$ & NMD -1.1 $(-7.3,5.1)^{\star}$ & $0.73^{*}$ \\
\hline & $\begin{array}{l}\text { BREAST-Q: Chest } \\
\text { and Upper Body }\end{array}$ & RCT & Moderate & Expansion phase & 36 & $68.6(10.6)$ & 33 & $69.3(7.9)$ & NMD $0.6(-4.9,6.1)^{*}$ & $0.83^{*}$ \\
\hline & $\begin{array}{l}\text { BREAST-Q: Chest } \\
\text { and Upper Body }\end{array}$ & RCT & Moderate & After expansion & 36 & $79.7(15.1)$ & 33 & $80.5(13.3)$ & NMD $0.5(-5.9,6.9)^{*}$ & $0.88^{*}$ \\
\hline \multirow[t]{4}{*}{$\begin{array}{l}\text { Cattelani, 2018, } \\
29275104, \text { Italy }\end{array}$} & $\begin{array}{l}\text { Constant Murley } \\
\text { Score }\end{array}$ & NRCS & High & $1 \mathrm{~d}$ & 39 & $71.62(8.87)$ & 45 & $60.36(10.54)$ & NR & $<0.001$ \\
\hline & $\begin{array}{l}\text { Constant Murley } \\
\text { Score }\end{array}$ & NRCS & High & $7 d$ & 39 & $65.67(9.31)$ & 45 & $52.36(12.23)$ & NR & $<0.001$ \\
\hline & DASH score & NRCS & High & $1 \mathrm{y}$ & 39 & $9.92(17.87)$ & 45 & $29.18(16.91)$ & NR & $<0.001$ \\
\hline & Return to usual work & NRCS & High & $N R(d)$ & 39 & $34.56(21)$ & 45 & $57.31(37.77)$ & NR & $<0.001$ \\
\hline $\begin{array}{l}\text { Ganesh Kumar, } \\
\text { 2021, 33172826, } \\
\text { US \& Canada }\end{array}$ & $\begin{array}{l}\text { BREAST-Q (0-100): } \\
\text { Physical well-being }\end{array}$ & NRCS & Moderate & $2 y$ & 738 & NR & 713 & NR & $\begin{array}{l}\operatorname{adjMD}-0.82(-3.01 \\
1.37)\end{array}$ & NR \\
\hline
\end{tabular}

US \& Canada

Abbreviations: $\mathrm{ADM}=$ acellular dermal matrix, $\mathrm{CI}=$ confidence interval, $\mathrm{d}=$ days, DASH $=$ Disabilities of the Arm, Shoulder, and Hand, $\mathrm{h}=$ hours, IBR $=$ implant-based

reconstruction, $\mathrm{N} / \mathrm{A}=$ not applicable, $\mathrm{NMD}=$ net mean difference, $\mathrm{NR}=$ not reported, NRCS $=$ nonrandomized comparative study, $\mathrm{PMID}=\mathrm{Pubmed}$ identifier, $\mathrm{RCT}=$ randomized controlled trial, $\mathrm{RoB}=$ risk of bias, $\mathrm{SD}=$ standard deviation, $\mathrm{y}=$ years.

Colors: Header rows are shaded orange. Rows for every alternate study are shaded blue. The colors do not add unique information.

* Calculated.

Table F-5.2. Full Evidence Table - Key Question 5: Use versus nonuse of human ADM during IBR - continuous outcomes (psychosocial well-being)

\begin{tabular}{|c|c|c|c|c|c|c|c|c|c|c|}
\hline $\begin{array}{l}\text { Study, Year, PMID, } \\
\text { Country }\end{array}$ & Outcome Measurement & Design & $\begin{array}{l}\text { Overall } \\
\text { RoB }\end{array}$ & $\begin{array}{l}\text { Time } \\
\text { Point }\end{array}$ & $\begin{array}{l}\text { Use of } \\
\text { ADM, } N\end{array}$ & $\begin{array}{l}\text { Use of ADM, } \\
\text { Mean (SD) }\end{array}$ & $\begin{array}{l}\text { Nonuse } \\
\text { of ADM, N }\end{array}$ & $\begin{array}{l}\text { Nonuse of ADM, } \\
\text { Mean (SD) }\end{array}$ & $\begin{array}{l}\text { Effect Size } \\
(95 \% \mathrm{Cl})\end{array}$ & $\begin{array}{l}\mathbf{P} \\
\text { Value }\end{array}$ \\
\hline $\begin{array}{l}\text { Cattelani, 2018, } \\
29275104, \text { Italy }\end{array}$ & Return to usual work & NRCS & High & NR & 39 & $34.56 \mathrm{~d}(21)$ & 45 & $57.31 \mathrm{~d}(37.77)$ & NR & $\begin{array}{l}<0.00 \\
1\end{array}$ \\
\hline $\begin{array}{l}\text { Ganesh Kumar, 2021, } \\
33172826, \text { US \& Canada }\end{array}$ & $\begin{array}{l}\text { BREAST-Q (0-100): } \\
\text { Psychosocial WB }\end{array}$ & NRCS & Moderate & $2 y$ & 738 & NR & 713 & NR & $\begin{array}{l}\text { adjMD }-0.26 \\
(-2.97,2.45)\end{array}$ & NR \\
\hline
\end{tabular}

33172826, US \& Canada Psychosocial WB

Abbreviations: adj $=$ adjusted, $\mathrm{ADM}=$ acellular dermal matrix, $\mathrm{CI}=$ confidence interval, $\mathrm{IBR}=$ implant-based reconstruction, $\mathrm{N} / \mathrm{A}=$ not applicable, $\mathrm{NRCS}=$ nonrandomized

comparative study, $\mathrm{PMID}=$ Pubmed identifier, $\mathrm{RoB}=$ risk of bias, $\mathrm{SD}=$ standard deviation, $\mathrm{MD}=$ mean difference, $\mathrm{y}=\mathrm{years}$.

Colors: Header rows are shaded orange. Rows for every alternate study are shaded blue. The colors do not add unique information. 
Table F-5.3. Full Evidence Table - Key Question 5: Use versus nonuse of human ADM during IBR - continuous outcomes (sexual wellbeing)

\begin{tabular}{|c|c|c|c|c|c|c|c|c|c|c|}
\hline $\begin{array}{l}\text { Study, Year, PMID, } \\
\text { Country }\end{array}$ & $\begin{array}{l}\text { Outcome } \\
\text { Measurement }\end{array}$ & Design & $\begin{array}{l}\text { Overall } \\
\text { RoB }\end{array}$ & $\begin{array}{l}\text { Time } \\
\text { Point }\end{array}$ & $\begin{array}{l}\text { Use of } \\
\text { ADM, } N\end{array}$ & $\begin{array}{l}\text { Use of ADM, } \\
\text { Mean (SD) }\end{array}$ & $\begin{array}{l}\text { Nonuse } \\
\text { of ADM, N }\end{array}$ & $\begin{array}{l}\text { Nonuse of ADM, } \\
\text { Mean (SD) }\end{array}$ & $\begin{array}{l}\text { Effect Size } \\
(95 \% \mathrm{CI})\end{array}$ & $\begin{array}{l}\text { P } \\
\text { Value }\end{array}$ \\
\hline $\begin{array}{l}\text { Ganesh Kumar, 2021, } \\
33172826, \text { US \& Canada }\end{array}$ & $\begin{array}{l}\text { BREAST-Q: Sexual } \\
\text { well-being }\end{array}$ & NRCS & Moderate & $2 y$ & 738 & NR & 713 & NR & $\begin{array}{l}\text { adjMD }-2.28 \\
(-5.63,1.06)\end{array}$ & NR \\
\hline
\end{tabular}

Abbreviations: adj = adjusted, $\mathrm{ADM}=$ acellular dermal matrix, $\mathrm{CI}=$ confidence interval, $\mathrm{IBR}=$ implant-based reconstruction, $\mathrm{N} / \mathrm{A}=$ not applicable, $\mathrm{NRCS}=$ nonrandomized comparative study, $\mathrm{PMID}=$ Pubmed identifier, $\mathrm{RoB}=$ risk of bias, $\mathrm{SD}=$ standard deviation, $\mathrm{MD}=$ mean difference, $\mathrm{y}=\mathrm{years}$.

Colors: Header rows are shaded orange. The color does not add unique information.

Table F-5.4. Full Evidence Table - Key Question 5: Use versus nonuse of human ADM during IBR - continuous outcomes (patient satisfaction with breast)

\begin{tabular}{|c|c|c|c|c|c|c|c|c|c|c|}
\hline $\begin{array}{l}\text { Study, Year, PMID, } \\
\text { Country }\end{array}$ & $\begin{array}{l}\text { Outcome } \\
\text { Measurement }\end{array}$ & Design & $\begin{array}{l}\text { Overall } \\
\text { RoB }\end{array}$ & $\begin{array}{l}\text { Time } \\
\text { Point }\end{array}$ & $\begin{array}{l}\text { Use of } \\
\text { ADM, N }\end{array}$ & $\begin{array}{l}\text { Use of ADM, } \\
\text { Mean (SD) }\end{array}$ & $\begin{array}{l}\text { Nonuse } \\
\text { of ADM, N }\end{array}$ & $\begin{array}{l}\text { Nonuse of ADM, } \\
\text { Mean (SD) }\end{array}$ & $\begin{array}{l}\text { Effect Size } \\
(95 \% \mathrm{Cl})\end{array}$ & $\begin{array}{l}\text { P } \\
\text { Value }\end{array}$ \\
\hline $\begin{array}{l}\text { Cattelani, 2018, } \\
\text { 29275104, Italy }\end{array}$ & $\begin{array}{l}\text { BREAST-Q: } \\
\text { Satisfaction with breast }\end{array}$ & NRCS & High & $1 \mathrm{y}$ & 39 & $92.2(9.03)$ & 45 & $76.1(14.6)$ & NR & $<0.001$ \\
\hline $\begin{array}{l}\text { Ganesh Kumar, 2021, } \\
\text { 33172826, US \& Canada }\end{array}$ & $\begin{array}{l}\text { BREAST-Q: } \\
\text { Satisfaction with breast }\end{array}$ & NRCS & Moderate & $2 y$ & 738 & NR & 713 & NR & $\begin{array}{l}\text { adjMD }-1.95 \\
(-4.96,1.06)\end{array}$ & NR \\
\hline
\end{tabular}

33172826, US \& Canada Satisfaction with breast

Abbreviations: adj $=$ adjusted, $\mathrm{ADM}=$ acellular dermal matrix, $\mathrm{CI}=$ confidence interval, $\mathrm{IBR}=$ implant-based reconstruction, $\mathrm{MD}=$ mean difference, $\mathrm{N} / \mathrm{A}=\mathrm{not}$ applicable, $\mathrm{NR}=$

not reported, NRCS = nonrandomized comparative study, PMID = Pubmed identifier, RoB $=$ risk of bias, $\mathrm{SD}=$ standard deviation, $\mathrm{y}=\mathrm{years}$.

Colors: Header rows are shaded orange. Rows for every alternate study are shaded blue. The colors do not add unique information.

Table F-5.5. Full Evidence Table - Key Question 5: Use versus nonuse of human ADM during IBR - continuous outcomes (analgesic use)

\begin{tabular}{|c|c|c|c|c|c|c|c|c|c|c|}
\hline $\begin{array}{l}\text { Study, Year, } \\
\text { PMID, Country }\end{array}$ & $\begin{array}{l}\text { Outcome } \\
\text { Measurement }\end{array}$ & Design & $\begin{array}{l}\text { Overall } \\
\text { RoB }\end{array}$ & $\begin{array}{l}\text { Time } \\
\text { Point }\end{array}$ & $\begin{array}{l}\text { Use of } \\
\text { ADM, N }\end{array}$ & $\begin{array}{l}\text { Use of ADM, } \\
\text { Mean (SD) }\end{array}$ & $\begin{array}{l}\text { Nonuse } \\
\text { of ADM, N }\end{array}$ & $\begin{array}{l}\text { Nonuse of ADM, } \\
\text { Mean (SD) }\end{array}$ & Effect Size (95\% CI) & $\begin{array}{l}\mathbf{P} \\
\text { Value } \\
\end{array}$ \\
\hline \multirow[t]{3}{*}{$\begin{array}{l}\text { McCarthy, 2012, } \\
23096987, \text { US }\end{array}$} & $\begin{array}{l}\text { Oral codeine } \\
\text { equivalents }\end{array}$ & RCT & Moderate & $0-6 \mathrm{~h}$ & 33 & $228(153)$ & 30 & $256(197)$ & $\mathrm{MD}-28 \mathrm{mg}(-116,60)^{*}$ & 0.77 \\
\hline & $\begin{array}{l}\text { Oral codeine } \\
\text { equivalents }\end{array}$ & RCT & Moderate & $6-24 \mathrm{~h}$ & 33 & $619(519)$ & 30 & $715(533)$ & MD -96 mg $(-356,164)^{*}$ & 0.38 \\
\hline & $\begin{array}{l}\text { Oral codeine } \\
\text { equivalents }\end{array}$ & RCT & Moderate & $0-24 \mathrm{~h}$ & 36 & $776(602)$ & 32 & $910(634)$ & $\mathrm{MD}-134 \mathrm{mg}(-440,172)^{*}$ & 0.38 \\
\hline
\end{tabular}

Abbreviations: $\mathrm{ADM}=$ acellular dermal matrix, $\mathrm{CI}=$ confidence interval, $\mathrm{h}=$ hours, $\mathrm{IBR}=$ implant-based reconstruction, $\mathrm{MD}=\mathrm{mean}$ difference, $\mathrm{mg}=\mathrm{milligrams}, \mathrm{MD}=\mathrm{mean}$

difference, PMID = Pubmed identifier, RCT $=$ randomized controlled trial, RoB $=$ risk of bias, $\mathrm{SD}=$ standard deviation.

Colors: Header rows are shaded orange. The color does not add unique information.

* Calculated. 
Table F-5.6. Full Evidence Table - Key Question 5: Use versus nonuse of human ADM during IBR - categorical outcomes (analgesic use)

\begin{tabular}{|c|c|c|c|c|c|c|c|c|}
\hline Study, Year, PMID, Country & Outcome Measurement & Design & $\begin{array}{l}\text { Overall } \\
\text { RoB }\end{array}$ & $\begin{array}{l}\text { Time } \\
\text { Point }\end{array}$ & $\begin{array}{l}\text { Use of ADM, } \\
n / N(\%)\end{array}$ & $\begin{array}{l}\text { Nonuse of } \\
\text { ADM, } n / N(\%)\end{array}$ & $\begin{array}{l}\text { Effect Size } \\
(95 \% \mathrm{Cl})\end{array}$ & P Value \\
\hline \multirow[t]{4}{*}{ Cattelani, 2018, 29275104, Italy } & Use of paracetamol, ketoprofen, or opioids & NRCS & High & $1 \mathrm{~d}$ & $39 / 39(100)$ & $45 / 45(100)$ & NR & NR \\
\hline & Use of paracetamol, ketoprofen, or opioids & NRCS & High & $7 \mathrm{~d}$ & $9 / 39(23.1)$ & $33 / 45(73.3)$ & NR & NR \\
\hline & Opioid use & NRCS & High & $1 \mathrm{~d}$ & $3 / 39(7.7)$ & $11 / 45(24.4)$ & NR & NR \\
\hline & Opioid use & NRCS & High & $7 \mathrm{~d}$ & $0 / 39(0)$ & $0 / 39(0)$ & NR & NR \\
\hline
\end{tabular}

Abbreviations: $\mathrm{ADM}=$ acellular dermal matrix, $\mathrm{CI}=$ confidence interval, $\mathrm{d}=$ days, $\mathrm{IBR}=$ implant-based reconstruction, $\mathrm{NR}=$ not reported, $\mathrm{NRCS}=$ nonrandomized comparative study, PMID $=$ Pubmed identifier, RoB $=$ risk of bias.

Colors: Header rows are shaded orange. The color does not add unique information.

Table F-5.7. Full Evidence Table - Key Question 5: Use versus nonuse of human ADM during IBR - categorical outcomes (mortality)

\begin{tabular}{|l|l|l|l|l|l|l}
\hline Study, Year, PMID, Country & Design & Overall RoB & Time Point & Use of ADM, n/N (\%) & Nonuse of ADM, n/N (\%) & Effect Size (95\% CI) \\
\hline Wendel, 2013, None, US & RCT & High & $1 \mathrm{mo}$ & $0 / 20(0)$ & $0 / 16(0)$
\end{tabular}

Abbreviations: $\mathrm{ADM}=$ acellular dermal matrix, $\mathrm{CI}=$ confidence interval, $\mathrm{IBR}=$ implant-based reconstruction, $\mathrm{mo}=$ months, $\mathrm{N} / \mathrm{A}=$ not applicable, $\mathrm{PMID}=\mathrm{Pubmed}$ identifier,

$\mathrm{RCT}=$ randomized controlled trial, $\mathrm{RoB}=$ risk of bias.

Colors: Header rows are shaded orange. The color does not add unique information.

Table F-5.8. Full Evidence Table - Key Question 5: Use versus nonuse of human ADM during IBR - categorical outcomes (unplanned repeat surgeries)

\begin{tabular}{|c|c|c|c|c|c|c|c|c|}
\hline $\begin{array}{l}\text { Study, Year, } \\
\text { PMID, Country }\end{array}$ & Outcome Measurement & Design & $\begin{array}{l}\text { Overall } \\
\text { RoB }\end{array}$ & $\begin{array}{l}\text { Time } \\
\text { Point }\end{array}$ & $\begin{array}{l}\text { Use of ADM, } \\
\mathrm{n} / \mathrm{N}(\%)\end{array}$ & $\begin{array}{l}\text { Nonuse of } \\
\text { ADM, } n / N(\%)\end{array}$ & $\begin{array}{l}\text { Effect Size } \\
(95 \% \mathrm{Cl})\end{array}$ & $\begin{array}{l}\mathbf{P} \\
\text { Value }\end{array}$ \\
\hline $\begin{array}{l}\text { Ibrahim, 2013, } \\
24165587 \text {, US }\end{array}$ & For revision of reconstruction (e.g., for asymmetry) & NRCS & Moderate & $6 \mathrm{mo}$ & $237 / 3283(0.5)$ & $\begin{array}{l}990 / 15714 \\
(0.6)\end{array}$ & NR & 0.14 \\
\hline $\begin{array}{l}\text { Nealon, 2020b, } \\
31605310 \text {, US }\end{array}$ & Revision of reconstruction & NRCS & High & $5.3 \mathrm{y}$ & NR & NR & $\begin{array}{l}\text { adjOR } 0.86 \\
(0.69,1.08)\end{array}$ & 0.19 \\
\hline $\begin{array}{l}\text { Sobti, 2018, } \\
29481386, \text { US }\end{array}$ & Revision of reconstruction for malposition or size & NRCS & High & $5 \mathrm{y}$ & $\begin{array}{l}\text { 47/465 breasts } \\
(10.11)\end{array}$ & $\begin{array}{l}24 / 217 \text { breasts } \\
(11.06)\end{array}$ & $\begin{array}{l}\text { adjOR } 1.10 \\
(0.63,1.92)\end{array}$ & NR \\
\hline $\begin{array}{l}\text { Peled, 2012, } \\
\text { 22634688, US }\end{array}$ & $\begin{array}{l}\text { For wound-healing or infectious complication, but not other } \\
\text { indications, such as hematoma, oncologic indications, or revision } \\
\text { procedures }\end{array}$ & NRCS & High & $\begin{array}{l}2.6- \\
3.3 \mathrm{y}\end{array}$ & $\begin{array}{l}11 / 100 \text { breasts } \\
\text { (11) }\end{array}$ & $\begin{array}{l}21 / 90 \text { breasts } \\
(23.3)\end{array}$ & NR & $<0.05$ \\
\hline
\end{tabular}

Abbreviations: adj $=$ adjusted, $\mathrm{ADM}=$ acellular dermal matrix, $\mathrm{CI}=$ confidence interval, $\mathrm{IBR}=$ implant - based reconstruction, $\mathrm{mo}=$ months, $\mathrm{NR}=$ not reported, $\mathrm{NRCS}=$ nonrandomized comparative study, $\mathrm{OR}=$ odds ratio, $\mathrm{PMID}=$ Pubmed identifier, $\mathrm{RoB}=$ risk of bias, $\mathrm{y}=$ years

Colors: Header rows are shaded orange. Rows for every alternate study are shaded blue. The colors do not add unique information. 
Table F-5.9. Full Evidence Table - Key Question 5: Use versus nonuse of human ADM during IBR - continuous outcomes (pain, including chronic pain)

\begin{tabular}{|c|c|c|c|c|c|c|c|c|c|c|}
\hline $\begin{array}{l}\text { Study, Year, } \\
\text { PMID, Country }\end{array}$ & $\begin{array}{l}\text { Outcome } \\
\text { Measurement }\end{array}$ & Design & $\begin{array}{l}\text { Overall } \\
\text { RoB }\end{array}$ & Time Point & $\begin{array}{l}\text { Use of } \\
\text { ADM, N }\end{array}$ & $\begin{array}{l}\text { Use of ADM, } \\
\text { Mean (SD) }\end{array}$ & $\begin{array}{l}\text { Nonuse } \\
\text { of ADM, } \mathbf{N}\end{array}$ & $\begin{array}{l}\text { Nonuse of ADM, } \\
\text { Mean (SD) }\end{array}$ & $\begin{array}{l}\text { Effect Size } \\
(95 \% \mathrm{Cl})\end{array}$ & $\begin{array}{l}P \\
\text { Value }\end{array}$ \\
\hline \multirow{4}{*}{$\begin{array}{l}\text { McCarthy, 2012, } \\
23096987, \text { US }\end{array}$} & VAS & $\mathrm{RCT}$ & Moderate & Baseline & 36 & $7(14.9)$ & 33 & $1.4(3.2)$ & NR & NR \\
\hline & VAS & RCT & Moderate & $24 \mathrm{~h}$ & 36 & $54.6(27.6)$ & 33 & $42.8(24.5)$ & NMD $6.2(-4.9,17.3)^{*}$ & $0.27^{*}$ \\
\hline & VAS & RCT & Moderate & Expansion phase & 36 & $17(15.9)$ & 33 & $4.6(8.9)$ & NMD $6.8(1.1,12.5)^{\star}$ & 0.019 \\
\hline & VAS & RCT & Moderate & After expansion & 36 & $5.6(11.6)$ & 33 & $4.6(8.9)$ & NMD -4.6 $(-9.8,0.6)^{*}$ & 0.081 \\
\hline \multirow{2}{*}{$\begin{array}{l}\text { Cattelani, 2018, } \\
29275104, \text { Italy }\end{array}$} & BPI-SF & NRCS & High & $1 \mathrm{~d}$ & 39 & $17.56(15.52)$ & 45 & $44.11(15.83)$ & NR & $<0.001$ \\
\hline & BPI-SF & NRCS & High & $7 d$ & 39 & $8.23(15.39)$ & 45 & $21.96(18.59)$ & NR & $<0.001$ \\
\hline
\end{tabular}

Abbreviations: $\mathrm{ADM}=$ acellular dermal matrix, BPI-SF $=$ Brief Pain Inventory-Short Form, $\mathrm{CI}=$ confidence interval, $\mathrm{d}=\mathrm{days}, \mathrm{h}=\mathrm{hours}, \mathrm{IBR}=$ implant-based reconstruction,

$\mathrm{NMD}=$ net mean difference, $\mathrm{NR}=$ not reported, $\mathrm{NRCS}=$ nonrandomized comparative study, $\mathrm{PMID}=$ Pubmed identifier, $\mathrm{RCT}=$ randomized controlled trial, RoB $=$ risk of bias,

$\mathrm{SD}=$ standard deviation, VAS $=$ Visual Analog Scale.

Colors: Header rows are shaded orange. Rows for every alternate study are shaded blue. The colors do not add unique information.

* Calculated.

Table F-5.10. Full Evidence Table - Key Question 5: Use versus nonuse of human ADM during IBR - categorical outcomes (necrosis)

\begin{tabular}{|c|c|c|c|c|c|c|c|c|c|}
\hline $\begin{array}{l}\text { Study, Year, PMID, } \\
\text { Country }\end{array}$ & $\begin{array}{l}\text { Outcome } \\
\text { Measurement }\end{array}$ & Design & $\begin{array}{l}\text { Overall } \\
\text { RoB }\end{array}$ & $\begin{array}{l}\text { Time } \\
\text { Point }\end{array}$ & Subgroup & $\begin{array}{l}\text { Use of ADM, n/N } \\
(\%)\end{array}$ & $\begin{array}{l}\text { Nonuse of ADM, n/N } \\
(\%)\end{array}$ & Effect Size $(95 \% \mathrm{Cl})$ & $\begin{array}{l}\mathbf{P} \\
\text { Value }\end{array}$ \\
\hline \multirow[t]{3}{*}{$\begin{array}{l}\text { Craig, 2019, 29800083, } \\
\text { US }\end{array}$} & NR & NRCS & Low & $7 \mathrm{mo}$ & All patients & $\begin{array}{l}145 / 574 \text { breasts } \\
(25.3)\end{array}$ & $60 / 796$ breasts $(7.5)$ & NR & NR \\
\hline & NR & NRCS & Low & $7 \mathrm{mo}$ & $\begin{array}{l}\text { Post-op } \\
\text { radiation }\end{array}$ & $\begin{array}{l}21 / 88 \text { breasts } \\
(23.9)\end{array}$ & $15 / 113$ breasts $(13.3)$ & NR & 0.37 \\
\hline & NR & NRCS & Low & $7 \mathrm{mo}$ & $\begin{array}{l}\text { No post-op } \\
\text { radiation }\end{array}$ & $\begin{array}{l}\text { 124/486 breasts } \\
(25.5)\end{array}$ & $45 / 683$ breasts $(6.6)$ & adjOR $4.99(3.28,8.03)$ & NR \\
\hline $\begin{array}{l}\text { Hirsch, 2014, 25347643, } \\
\text { US }\end{array}$ & $\begin{array}{l}\text { Major flap } \\
\text { necrosis }\end{array}$ & NRCS & Low & $3.1 \mathrm{y}$ & All patients & NR & NR & adjOR $0.98(0.58,1.66)$ & 0.94 \\
\hline $\begin{array}{l}\text { Nealon, 2020b, } \\
31605310, \text { US }\end{array}$ & Skin necrosis & NRCS & High & $5.3 y$ & All patients & NR & NR & adjOR $0.87(0.51,1.52)$ & 0.62 \\
\hline $\begin{array}{l}\text { Qureshi, 2016, } \\
\text { 27465177, US }\end{array}$ & $\begin{array}{l}\text { Mastectomy flap } \\
\text { necrosis }\end{array}$ & NRCS & High & $2 y$ & All patients & NR/295 (NR) & NR/118 (NR) & adjOR $3.1(1.00,9.61)^{*}$ & $<0.05$ \\
\hline $\begin{array}{l}\text { Seth, 2012, 23018687, } \\
\text { US }\end{array}$ & $\begin{array}{l}\text { Major flap } \\
\text { necrosis }\end{array}$ & NRCS & High & $2 y$ & All patients & $\begin{array}{l}17 / 199 \text { breasts } \\
(8.5)\end{array}$ & $26 / 393$ breasts $(6.6)$ & adjOR $1.32(0.70,2.49)$ & NR \\
\hline $\begin{array}{l}\text { Sobti, 2018, 29481386, } \\
\text { US }\end{array}$ & Tissue necrosis & NRCS & High & $5 y$ & All patients & $14 / 338(2.4)$ & $30 / 376(5.1)$ & adjOR $0.53(0.27,1.02)$ & NR \\
\hline $\begin{array}{l}\text { Sorkin, 2017, 28806288, } \\
\text { US \& Canada }\end{array}$ & $\begin{array}{l}\text { Mastectomy skin } \\
\text { flap necrosis }\end{array}$ & NRCS & Moderate & $2 y$ & All patients & $44 / 655(6.7)$ & $34 / 642(5.3)$ & NR & 0.23 \\
\hline
\end{tabular}

Abbreviations: adj = adjusted, $\mathrm{ADM}=$ acellular dermal matrix, $\mathrm{CI}=$ confidence interval, $\mathrm{IBR}=$ implant-based reconstruction, $\mathrm{mo}=$ months, $\mathrm{NR}=$ not reported, $\mathrm{NRCS}=$

nonrandomized comparative study, $\mathrm{OR}=$ odds ratio, $\mathrm{PMID}=$ Pubmed identifier, $\mathrm{RoB}=$ risk of bias, $\mathrm{y}=$ years 
Colors: Header rows are shaded orange. Rows for every alternate study are shaded blue. The colors do not add unique information.

* Calculated.

Table F-5.11. Full Evidence Table - Key Question 5: Use versus nonuse of human ADM during IBR - categorical outcomes

(thromboembolic events)

\begin{tabular}{|c|c|c|c|c|c|c|c|c|}
\hline Study, Year, PMID, Country & Outcome Measurement & Design & Overall RoB & Time Point & Use of ADM, n/N (\%) & $\begin{array}{l}\text { Nonuse of ADM, } \\
\mathrm{n} / \mathrm{N}(\%)\end{array}$ & $\begin{array}{l}\text { Effect Size } \\
(95 \% \mathrm{Cl})\end{array}$ & P Value \\
\hline \multirow[t]{2}{*}{ Ibrahim, 2013, 24165587, US } & Deep vein thrombosis & NRCS & Moderate & NR & $9 / 3283(0.3)$ & $35 / 15714(0.2)$ & NR & 0.47 \\
\hline & Pulmonary embolism & NRCS & Moderate & NR & $2 / 3283(0.06)$ & $29 / 15714(0.2)$ & NR & 0.11 \\
\hline
\end{tabular}

Abbreviations: $\mathrm{ADM}=$ acellular dermal matrix, $\mathrm{CI}=$ confidence interval, $\mathrm{IBR}=$ implant-based reconstruction, $\mathrm{NR}=$ not reported, $\mathrm{NRCS}=$ nonrandomized comparative study, $\mathrm{OR}$

$=$ odds ratio, $\mathrm{PMID}=$ Pubmed identifier, $\mathrm{RoB}=$ risk of bias.

Colors: Header rows are shaded orange. The color does not add unique information.

Table F-5.12. Full Evidence Table - Key Question 5: Use versus nonuse of human ADM during IBR - categorical outcomes (wound dehiscence)

\begin{tabular}{|c|c|c|c|c|c|c|c|c|c|}
\hline Study, Year, PMID, Country & $\begin{array}{l}\text { Outcome } \\
\text { Measurement }\end{array}$ & Subgroup & Design & $\begin{array}{l}\text { Overall } \\
\text { RoB }\end{array}$ & $\begin{array}{l}\text { Time } \\
\text { Point }\end{array}$ & Use of ADM, n/N (\%) & $\begin{array}{l}\text { Nonuse of ADM, n/N } \\
(\%)\end{array}$ & $\begin{array}{l}\text { Effect Size } \\
(95 \% \mathrm{Cl})\end{array}$ & $\begin{array}{l}\text { P } \\
\text { Value }\end{array}$ \\
\hline \multirow[t]{3}{*}{ Craig, 2019, 29800083, US } & NR & All patients & NRCS & Low & $7 \mathrm{mo}$ & $35 / 574$ breasts $(6.1)$ & 20/796 breasts (2.5) & NR & NR \\
\hline & NR & $\begin{array}{l}\text { Post-op } \\
\text { radiation }\end{array}$ & NRCS & Low & $7 \mathrm{mo}$ & $42 / 88$ breasts (47.7) & $27 / 113$ breasts $(23.9)$ & NR & NR \\
\hline & NR & $\begin{array}{l}\text { No post-op } \\
\text { radiation }\end{array}$ & NRCS & Low & $7 \mathrm{mo}$ & $30 / 486$ breasts $(6.2)$ & $17 / 683$ breasts $(2.5)$ & $\begin{array}{l}\text { adjOR } 2.46 \\
(1.23,4.93)\end{array}$ & NR \\
\hline $\begin{array}{l}\text { Ganesh Kumar, 2021, } \\
33172826, \text { US \& Canada }\end{array}$ & NR & All patients & NRCS & Moderate & $2 y$ & $24 / 738(3.3)$ & $5 / 713(0.7)$ & NR & 0.009 \\
\hline Ibrahim, 2013, 24165587, US & NR & All patients & NRCS & Moderate & NR & $15 / 3283(0.5)$ & $98 / 15714(0.6)$ & NR & 0.26 \\
\hline Qureshi, 2016, 27465177, US & $\begin{array}{l}\text { Dehiscence without } \\
\text { necrosis }\end{array}$ & All patients & NRCS & High & $2 y$ & NR/295 (NR) & NR/118 (NR) & $\begin{array}{l}\text { adjOR } 0.4 \\
\text { (NR. NR) }\end{array}$ & $<0.05$ \\
\hline
\end{tabular}

Abbreviations: adj $=$ adjusted, $\mathrm{ADM}=$ acellular dermal matrix, $\mathrm{CI}=$ confidence interval, $\mathrm{IBR}=$ implant-based reconstruction, $\mathrm{mo}=$ months, $\mathrm{NR}=$ not reported, $\mathrm{NRCS}=$ nonrandomized comparative study, $\mathrm{OR}=$ odds ratio, $\mathrm{PMID}=$ Pubmed identifier, $\mathrm{RoB}=$ risk of bias, $\mathrm{y}=$ years.

Colors: Header rows are shaded orange. Rows for every alternate study are shaded blue. The colors do not add unique information.

Table F-5.13. Full Evidence Table - Key Question 5: Use versus nonuse of human ADM during IBR - categorical outcomes (delayed healing)

\begin{tabular}{|c|c|c|c|c|c|c|c|c|}
\hline Study, Year, PMID, Country & Outcome Measurement & Design & $\begin{array}{l}\text { Overall } \\
\text { RoB }\end{array}$ & $\begin{array}{l}\text { Time } \\
\text { Point }\end{array}$ & $\begin{array}{l}\text { Use of ADM, } \\
n / N(\%)\end{array}$ & $\begin{array}{l}\text { Nonuse of } \\
\text { ADM, } n / N(\%)\end{array}$ & Effect Size (95\% CI) & $\begin{array}{l}\mathbf{P} \\
\text { Value }\end{array}$ \\
\hline Woo, 2017, 28509694, South Korea & $\begin{array}{l}\text { Delayed wound healing or skin flap } \\
\text { necrosis }\end{array}$ & NRCS & High & NR & $32 / 199(16.1)$ & $32 / 199(16.1)$ & $\operatorname{adjOR} 1.41(0.67,2.96)$ & 0.37 \\
\hline
\end{tabular}

Abbreviations: adj = adjusted, ADM = acellular dermal matrix, $\mathrm{CI}=$ confidence interval, $\mathrm{IBR}=$ implant-based reconstruction, $\mathrm{NRCS}=$ nonrandomized comparative study, OR = odds ratio, PMID = Pubmed identifier, RoB = risk of bias. 
Colors: Header rows are shaded orange. The color does not add unique information.

Table F-5.14. Full Evidence Table - Key Question 5: Use versus nonuse of human ADM during IBR - categorical outcomes (seroma)

\begin{tabular}{|c|c|c|c|c|c|c|c|c|}
\hline Study, Year, PMID, Country & Subgroup & Design & $\begin{array}{l}\text { Overall } \\
\text { RoB }\end{array}$ & $\begin{array}{l}\text { Time } \\
\text { Point }\end{array}$ & Use of ADM, $n / N(\%)$ & $\begin{array}{l}\text { Nonuse of ADM, } \\
\mathrm{n} / \mathrm{N}(\%)\end{array}$ & Effect Size $(95 \% \mathrm{CI})$ & $\begin{array}{l}\mathbf{P} \\
\text { Value }\end{array}$ \\
\hline McCarthy, 2012, 23096987, US & All patients & RCT & Moderate & NR & $1 / 36(2.78)$ & $3 / 33(9.09)$ & OR $0.29(0.03,2.89)^{*}$ & $0.29^{*}$ \\
\hline Chun, 2010, 20124828, US & All patients & NRCS & High & NR & $38 / 269$ breasts $(14.1)$ & $4 / 146$ breasts $(2.7)$ & adjOR $4.24(1.28,14)$ & 0.018 \\
\hline \multirow[t]{3}{*}{ Craig, 2019, 29800083, US } & All patients & NRCS & Low & $7 \mathrm{mo}$ & $65 / 574$ breasts (11.3) & $32 / 796$ breasts $(4)$ & NR & NR \\
\hline & Post-op radiation & NRCS & Low & $7 \mathrm{mo}$ & 12/88 breasts (13.6) & $6 / 113$ breasts $(5.3)$ & NR & NR \\
\hline & $\begin{array}{l}\text { No post-op } \\
\text { radiation }\end{array}$ & NRCS & Low & $7 \mathrm{mo}$ & $53 / 486$ breasts $(10.9)$ & $\begin{array}{l}26 / 683 \text { breasts } \\
(3.8)\end{array}$ & $\operatorname{adjOR} 3.19(1.85,5.52)$ & NR \\
\hline Seth, 2012, 23018687, US & All patients & NRCS & High & $2 y$ & $8 / 199$ breasts (4) & $8 / 393$ breasts $(2)$ & adjOR $2.02(0.75,5.45)$ & NR \\
\hline Sorkin, 2017, 28806288, US \& Canada & All patients & NRCS & Moderate & $2 y$ & $21 / 655(3.2)$ & $20 / 642(3.1)$ & NR & 0.97 \\
\hline Woo, 2017, 28509694, South Korea & All patients & NRCS & High & NR & $8 / 199(4)$ & $17 / 199(8.5)$ & adjOR $0.89(0.33,2.39)$ & 0.81 \\
\hline
\end{tabular}

Woo, 2017, 28509694, South Korea

Abbreviations: adj $=$ adjusted, $\mathrm{ADM}=$ acellular dermal matrix, $\mathrm{CI}=$ confidence interval, $\mathrm{IBR}=$ implant-based reconstruction, $\mathrm{mo}=$ months, $\mathrm{NR}=$ not reported, $\mathrm{NRCS}=$

nonrandomized comparative study, $\mathrm{OR}=$ odds ratio, $\mathrm{PMID}=$ Pubmed identifier, $\mathrm{RCT}=$ randomized controlled trial, $\mathrm{RoB}=$ risk of bias, $\mathrm{y}=\mathrm{years}$.

Colors: Header rows are shaded orange. Rows for every alternate study are shaded blue. The colors do not add unique information.

* Calculated.

Table F-5.15. Full Evidence Table - Key Question 5: Use versus nonuse of human ADM during IBR - categorical outcomes (infections not explicitly implant related)

\begin{tabular}{|c|c|c|c|c|c|c|c|c|c|}
\hline $\begin{array}{l}\text { Study, Year, } \\
\text { PMID, Country }\end{array}$ & Outcome Measurement & Design & $\begin{array}{l}\text { Overall } \\
\text { RoB }\end{array}$ & $\begin{array}{l}\text { Time } \\
\text { Point }\end{array}$ & Subgroup & $\begin{array}{l}\text { Use of ADM, } \\
n / N(\%)\end{array}$ & $\begin{array}{l}\text { Nonuse of } \\
\text { ADM, } n / N(\%)\end{array}$ & $\begin{array}{l}\text { Effect Size } \\
(95 \% \mathrm{Cl})\end{array}$ & $\begin{array}{l}\mathbf{P} \\
\text { Value }\end{array}$ \\
\hline $\begin{array}{l}\text { McCarthy, 2012, } \\
23096987, \text { US }\end{array}$ & NR & RCT & Moderate & NR & All patients & $3 / 36(8.3)$ & $1 / 33(3.0)$ & $\begin{array}{l}\text { OR 2.91 } \\
(0.29,29.45)^{*}\end{array}$ & $0.37^{*}$ \\
\hline $\begin{array}{l}\text { Wendel, 2013, } \\
\text { None, US }\end{array}$ & NR & RCT & High & $1 \mathrm{mo}$ & All patients & $6 / 20(30)$ & $2 / 16(12.5)$ & $\begin{array}{l}\text { OR } 3.00 \\
(0.51,17.50)^{*}\end{array}$ & $0.22^{*}$ \\
\hline $\begin{array}{l}\text { Brooke, } 2012, \\
22868313, \text { US }\end{array}$ & NR & NRCS & High & NR & All patients & $\begin{array}{l}22 / 221 \\
\text { breasts }(10)\end{array}$ & $\begin{array}{l}1 \text { breast/64 } \\
\text { breasts }(2)\end{array}$ & NR & 0.09 \\
\hline \multirow[t]{2}{*}{$\begin{array}{l}\text { Chun, 2010, } \\
20124828 \text {, US }\end{array}$} & Overall infection & NRCS & High & NR & All patients & $\begin{array}{l}24 / 269 \\
\text { breasts }(8.9)\end{array}$ & $\begin{array}{l}3 / 146 \text { breasts } \\
(2.1)\end{array}$ & $\begin{array}{l}\text { adjOR } 5.37 \\
(1.63,17.6)\end{array}$ & 0.006 \\
\hline & $\begin{array}{l}\text { Major infection requiring admission } \\
\text { for IV antibiotics and/or surgery }\end{array}$ & NRCS & High & NR & All patients & $\begin{array}{l}22 / 269 \\
\text { breasts }(8.2)\end{array}$ & $\begin{array}{l}1 / 146 \text { breasts } \\
(0.68)\end{array}$ & NR & 0.0016 \\
\hline \multirow[t]{3}{*}{$\begin{array}{l}\text { Craig, 2019, } \\
\text { 29800083, US }\end{array}$} & NR & NRCS & Low & $7 \mathrm{mo}$ & All patients & $\begin{array}{l}70 / 574 \\
\text { breasts (12.2) }\end{array}$ & $\begin{array}{l}38 / 796 \text { breasts } \\
(4.8)\end{array}$ & NR & NR \\
\hline & NR & NRCS & Low & $7 \mathrm{mo}$ & Post-op radiation & $\begin{array}{l}14 / 88 \text { breasts } \\
(15.9)\end{array}$ & $\begin{array}{l}13 / 113 \text { breasts } \\
(11.5)\end{array}$ & NR & 0.77 \\
\hline & NR & NRCS & Low & $7 \mathrm{mo}$ & No post-op radiation & $\begin{array}{l}56 / 486 \\
\text { breasts }(11.5)\end{array}$ & $\begin{array}{l}25 / 683 \text { breasts } \\
(3.7)\end{array}$ & $\begin{array}{l}\text { adjOR } 2.68 \\
(1.54,5.06)\end{array}$ & NR \\
\hline
\end{tabular}




\begin{tabular}{|c|c|c|c|c|c|c|c|c|c|}
\hline $\begin{array}{l}\text { Study, Year, } \\
\text { PMID, Country }\end{array}$ & Outcome Measurement & Design & $\begin{array}{l}\text { Overall } \\
\text { RoB }\end{array}$ & $\begin{array}{l}\text { Time } \\
\text { Point }\end{array}$ & Subgroup & $\begin{array}{l}\text { Use of ADM, } \\
\mathrm{n} / \mathrm{N}(\%)\end{array}$ & $\begin{array}{l}\text { Nonuse of } \\
\text { ADM, } n / N(\%)\end{array}$ & $\begin{array}{l}\text { Effect Size } \\
(95 \% \mathrm{Cl})\end{array}$ & $\begin{array}{l}\mathbf{P} \\
\text { Value }\end{array}$ \\
\hline \multirow[t]{4}{*}{$\begin{array}{l}\text { Ibrahim, 2013, } \\
24165587, \text { US }\end{array}$} & Superficial surgical-site infection & NRCS & Moderate & NR & All patients & $70 / 3283(2.1)$ & $\begin{array}{l}246 / 15714 \\
(1.6)\end{array}$ & NR & 0.021 \\
\hline & $\begin{array}{l}\text { Deep incisional surgical-site } \\
\text { infection }\end{array}$ & NRCS & Moderate & NR & All patients & $39 / 3283(1.2)$ & $159 / 15714(1)$ & NR & 0.37 \\
\hline & Organ space infection & NRCS & Moderate & NR & All patients & $17 / 1717(1)$ & $55 / 7442(0.7)$ & NR & 0.29 \\
\hline & Sepsis & NRCS & Moderate & NR & All patients & $17 / 3283(0.5)$ & $61 / 15714(0.4)$ & NR & 0.52 \\
\hline $\begin{array}{l}\text { Liu, 2011, } \\
21228744, \text { US }\end{array}$ & $\begin{array}{l}\text { Wound infection: including major } \\
\text { infection and minor infection }\end{array}$ & NRCS & High & NR & All patients & $18 / 266(6.8)$ & $5 / 204(2.5)$ & $\begin{array}{l}\text { adjOR } 3.25 \\
(0.8,13.12)\end{array}$ & 0.097 \\
\hline $\begin{array}{l}\text { Nealon, 2020b, } \\
31605310, \text { US }\end{array}$ & NR & NRCS & High & $5.3 y$ & All patients & NR & NR & $\begin{array}{l}\text { adjOR } 0.96 \\
(0.57,1.65)\end{array}$ & 0.57 \\
\hline $\begin{array}{l}\text { Peled, 2012, } \\
\text { 22634688, US }\end{array}$ & $\begin{array}{l}\text { Localized or systemic infection } \\
\text { treated with oral antibiotics or } \\
\text { admission for IV antibiotics. }\end{array}$ & NRCS & High & $\begin{array}{l}2.6- \\
3.3 y\end{array}$ & All patients & $\begin{array}{l}20 / 100 \\
\text { breasts }(20)\end{array}$ & $\begin{array}{l}25 / 90 \text { breasts } \\
(27.8)\end{array}$ & NR & $<0.05$ \\
\hline $\begin{array}{l}\text { Seth, 2012, } \\
23018687, \text { US }\end{array}$ & NR & NRCS & High & $2 y$ & All patients & $\begin{array}{l}14 / 199 \\
\text { breasts }(7)\end{array}$ & $\begin{array}{l}17 / 393 \text { breasts } \\
(4.3)\end{array}$ & $\begin{array}{l}\text { adjOR } 1.67 \\
(0.81,3.47)\end{array}$ & 0.81 \\
\hline $\begin{array}{l}\text { Sobti, 2018, } \\
29481386, \text { US }\end{array}$ & NR & NRCS & High & $5 y$ & All patients & $56 / 338(4.6)$ & $29 / 376(4.9)$ & $\begin{array}{l}\text { adjOR } 0.88 \\
(0.51,1.53)\end{array}$ & 0.51 \\
\hline \multirow{3}{*}{$\begin{array}{l}\text { Sorkin, } 2017, \\
28806288, \text { US \& } \\
\text { Canada }\end{array}$} & Wound infection - Any & NRCS & Moderate & $2 y$ & All patients & $74 / 655$ (11.3) & $61 / 642(9.5)$ & NR & 0.11 \\
\hline & $\begin{array}{l}\text { Wound infection requiring IV } \\
\text { antibiotics or reoperation }\end{array}$ & NRCS & Moderate & $2 y$ & All patients & $46 / 655(7)$ & $29 / 642(4.5)$ & NR & 0.045 \\
\hline & $\begin{array}{l}\text { Wound infection requiring oral } \\
\text { antibiotics }\end{array}$ & NRCS & Moderate & $2 y$ & All patients & $32 / 655(4.9)$ & $34 / 642(5.3)$ & $\begin{array}{l}\text { adjOR } 1.49 \\
(0.9,2.44)\end{array}$ & 0.12 \\
\hline $\begin{array}{l}\text { Woo, 2017, } \\
28509694, \text { South } \\
\text { Korea }\end{array}$ & NR & NRCS & High & NR & All patients & 6/199 (3) & 7/199 (3.5) & $\begin{array}{l}\text { adjOR } 2.33 \\
(0.61,8.91)\end{array}$ & 0.22 \\
\hline
\end{tabular}

Abbreviations: adj = adjusted, $\mathrm{ADM}=$ acellular dermal matrix, $\mathrm{CI}=$ confidence interval, $\mathrm{IBR}=$ implant-based reconstruction, $\mathrm{IV}=$ intravenous, $\mathrm{mo}=$ months, $\mathrm{NR}=$ not reported,

$\mathrm{NRCS}=$ nonrandomized comparative study, $\mathrm{OR}=$ odds ratio, $\mathrm{PMID}=$ Pubmed identifier, $\mathrm{RCT}=$ randomized controlled trial, $\mathrm{RoB}=$ risk of bias, $\mathrm{y}=\mathrm{years}$.

Colors: Header rows are shaded orange. Rows for every alternate study are shaded blue. The colors do not add unique information.

* Calculated. 
Table F-5.16. Full Evidence Table - Key Question 5: Use versus nonuse of human ADM during IBR - categorical outcomes (implant failure/loss or need for explant surgery)

\begin{tabular}{|c|c|c|c|c|c|c|c|c|c|}
\hline $\begin{array}{l}\text { Study, Year, PMID, } \\
\text { Country }\end{array}$ & Outcome Measurement & Subgroup & Design & Overall RoB & $\begin{array}{l}\text { Time } \\
\text { Point }\end{array}$ & $\begin{array}{l}\text { Use of ADM, n/N } \\
(\%)\end{array}$ & $\begin{array}{l}\text { Nonuse of } \\
\text { ADM, } n / N(\%)\end{array}$ & Effect Size (95\% Cl) & $\begin{array}{l}\mathbf{P} \\
\text { Value }\end{array}$ \\
\hline \multirow[t]{3}{*}{$\begin{array}{l}\text { Craig, 2019, 29800083, } \\
\text { US }\end{array}$} & Need for explant surgery & All patients & NRCS & Low & $7 \mathrm{mo}$ & $\begin{array}{l}56 / 574 \text { breasts } \\
(9.8)\end{array}$ & $\begin{array}{l}63 / 796 \text { breasts } \\
(7.9)\end{array}$ & NR & NR \\
\hline & Need for explant surgery & $\begin{array}{l}\text { Post-op } \\
\text { radiation }\end{array}$ & NRCS & Low & $7 \mathrm{mo}$ & $\begin{array}{l}10 / 88 \text { breasts } \\
(11.4)\end{array}$ & $\begin{array}{l}23 / 113 \text { breasts } \\
(20.4)\end{array}$ & adjOR $0.38(0.11,0.96)$ & 0.04 \\
\hline & Need for explant surgery & $\begin{array}{l}\text { No post-op } \\
\text { radiation }\end{array}$ & NRCS & Low & $7 \mathrm{mo}$ & $\begin{array}{l}46 / 486 \text { breasts } \\
(9.5)\end{array}$ & $\begin{array}{l}40 / 683 \text { breasts } \\
(5.9)\end{array}$ & adjOR $1.90(1.03,3.51)$ & NR \\
\hline $\begin{array}{l}\text { Hirsch, 2014, 25347643, } \\
\text { US }\end{array}$ & $\begin{array}{l}\text { Explantation with/without } \\
\text { conversion to AR }\end{array}$ & All patients & NRCS & Low & $3.5 \mathrm{y}$ & NR & NR & adjOR $0.41(0.15,1.17)$ & 0.09 \\
\hline $\begin{array}{l}\text { Ibrahim, 2013, } \\
\text { 24165587, US }\end{array}$ & Graft/prosthesis failure & All patients & NRCS & Moderate & NR & $25 / 3283(0.8)$ & $121 / 15714(0.8)$ & NR & 0.9 \\
\hline $\begin{array}{l}\text { Nealon, 2020b, } \\
31605310, \text { US }\end{array}$ & Need for explant surgery & All patients & NRCS & High & $5.3 y$ & NR & NR & adjOR $1.92(0.41,8.33)$ & 0.39 \\
\hline $\begin{array}{l}\text { Pannucci, 2013, } \\
\text { 23508050, US }\end{array}$ & Expander/Implant loss & All patients & NRCS & Moderate & NR & $89 / 3450(2.6)$ & NR/10799 (NR) & adjOR $1.42(1.04,1.94)$ & 0.026 \\
\hline $\begin{array}{l}\text { Peled, 2012, 22634688, } \\
\text { US }\end{array}$ & Expander-implant loss & All patients & NRCS & High & $\begin{array}{l}2.6- \\
3.3 y\end{array}$ & $7 / 100$ breasts $(7)$ & $\begin{array}{l}\text { 16/90 breasts } \\
(17.8)\end{array}$ & NR & $<0.05$ \\
\hline $\begin{array}{l}\text { Qureshi, 2016, } \\
27465177, \text { US }\end{array}$ & Explantation & All patients & NRCS & High & $2 y$ & NR/296 (NR) & $\mathrm{NR} / 118(\mathrm{NR})$ & adjOR 1.2 (NR, NR) & NR \\
\hline $\begin{array}{l}\text { Seth, 2012, 23018687, } \\
\text { US }\end{array}$ & $\begin{array}{l}\text { ECF, explantation or } \\
\text { conversion to flap }\end{array}$ & All patients & NRCS & High & $2 y$ & $\begin{array}{l}17 / 199 \text { breasts } \\
(8.5)\end{array}$ & $\begin{array}{l}29 / 293 \text { breasts } \\
(7.4)\end{array}$ & adjOR $1.17(0.63,2.19)$ & NR \\
\hline $\begin{array}{l}\text { Sorkin, 2017, 28806288, } \\
\text { US \& Canada }\end{array}$ & $\begin{array}{l}\text { Reconstructive/implant } \\
\text { failure }\end{array}$ & All patients & NRCS & Moderate & $2 y$ & $60 / 655(9.2)$ & $37 / 642(5.8)$ & adjOR $1.55(0.93,2.58)$ & 0.089 \\
\hline $\begin{array}{l}\text { Woo, 2017, 28509694, } \\
\text { South Korea }\end{array}$ & Implant loss & All patients & NRCS & High & NR & $4 / 199(2)$ & $4 / 199(2)$ & $\operatorname{adjOR} 1(0.3,3.35)$ & 1.0 \\
\hline
\end{tabular}

South Korea

Abbreviations: adj $=$ adjusted, $\mathrm{ADM}=$ acellular dermal matrix, $\mathrm{CI}=$ confidence interval, $\mathrm{IBR}=$ implant-based reconstruction, $\mathrm{mo}=$ months, $\mathrm{NR}=$ not reported, $\mathrm{NRCS}=$

nonrandomized comparative study, $\mathrm{OR}=$ odds ratio, $\mathrm{PMID}=$ Pubmed identifier, $\mathrm{RoB}=$ risk of bias, $\mathrm{y}=$ years.

Colors: Header rows are shaded orange. Rows for every alternate study are shaded blue. The colors do not add unique information.

Table F-5.17. Full Evidence Table - Key Question 5: Use versus nonuse of human ADM during IBR - categorical outcomes (implant complications except for implant failure)

\begin{tabular}{|c|c|c|c|c|c|c|c|c|c|}
\hline $\begin{array}{l}\text { Study, Year, PMID, } \\
\text { Country }\end{array}$ & Outcome & Outcome Measurement & Design & $\begin{array}{l}\text { Overall } \\
\text { RoB }\end{array}$ & $\begin{array}{l}\text { Time } \\
\text { Point }\end{array}$ & $\begin{array}{l}\text { Use of ADM, } \\
\mathrm{n} / \mathrm{N}(\%)\end{array}$ & $\begin{array}{l}\text { Nonuse of } \\
\text { ADM, n/N (\%) }\end{array}$ & $\begin{array}{l}\text { Effect Size } \\
(95 \% \mathrm{Cl})\end{array}$ & $\begin{array}{l}\mathbf{P} \\
\text { Value }\end{array}$ \\
\hline Seth, 2012, 23018687, US & $\begin{array}{l}\text { Implant } \\
\text { extrusion/exposure }\end{array}$ & Extrusion & NRCS & High & $2 y$ & $\begin{array}{l}2 / 199 \text { breasts } \\
\text { (1) }\end{array}$ & $\begin{array}{l}\text { 9/293 breasts } \\
(2.3)\end{array}$ & $\begin{array}{l}\text { adjOR } 0.43 \\
(0.09,2.02)\end{array}$ & NR \\
\hline $\begin{array}{l}\text { Ganesh Kumar, 2021, } \\
\text { 33172826, US \& Canada }\end{array}$ & Implant rupture & $\begin{array}{l}\text { Implant rupture, leakage, or } \\
\text { deflation }\end{array}$ & NRCS & Moderate & $2 y$ & $11 / 738(1.5)$ & $7 / 713(1.0)$ & NR & 0.58 \\
\hline
\end{tabular}




\begin{tabular}{|c|c|c|c|c|c|c|c|c|c|}
\hline $\begin{array}{l}\text { Study, Year, PMID, } \\
\text { Country }\end{array}$ & Outcome & Outcome Measurement & Design & $\begin{array}{l}\text { Overall } \\
\text { RoB }\end{array}$ & $\begin{array}{l}\text { Time } \\
\text { Point }\end{array}$ & $\begin{array}{l}\text { Use of ADM, } \\
\mathrm{n} / \mathrm{N}(\%)\end{array}$ & $\begin{array}{l}\text { Nonuse of } \\
\text { ADM, } n / N(\%)\end{array}$ & $\begin{array}{l}\text { Effect Size } \\
(95 \% \mathrm{Cl})\end{array}$ & $\begin{array}{l}\mathbf{P} \\
\text { Value }\end{array}$ \\
\hline $\begin{array}{l}\text { Ganesh Kumar, 2021, } \\
33172826, \text { US \& Canada }\end{array}$ & Implant malposition & NR & NRCS & Moderate & $2 y$ & $9 / 738(1.2)$ & $4 / 713(0.6)$ & NR & 0.83 \\
\hline $\begin{array}{l}\text { Vardanian, 2011, } \\
22030500, \text { US }\end{array}$ & Implant malposition & NR & NRCS & High & $2.4 \mathrm{y}$ & $\begin{array}{l}4 / 208 \text { breasts } \\
(1.9)\end{array}$ & $\begin{array}{l}12 / 129 \text { breasts } \\
(9.3)\end{array}$ & $\begin{array}{l}\text { adjOR } 0.23 \\
(0.06,0.78)\end{array}$ & NR \\
\hline
\end{tabular}

22030500, US

Abbreviations: adj $=$ adjusted, $\mathrm{ADM}=$ acellular dermal matrix, $\mathrm{CI}=$ confidence interval, $\mathrm{IBR}=$ implant-based reconstruction, $\mathrm{mo}=\mathrm{months}, \mathrm{NR}=$ not reported, $\mathrm{NRCS}=$

nonrandomized comparative study, $\mathrm{OR}=$ odds ratio, $\mathrm{PMID}=$ Pubmed identifier, $\mathrm{RoB}=$ risk of bias, $\mathrm{y}=$ years.

Colors: Header rows are shaded orange. Rows for every alternate outcome are shaded blue. The colors do not add unique information.

Table F-5.18. Full Evidence Table - Key Question 5: Use versus nonuse of human ADM during IBR - categorical outcomes (capsular contracture)

\begin{tabular}{|c|c|c|c|c|c|c|c|}
\hline Study, Year, PMID, Country & Design & $\begin{array}{l}\text { Overall } \\
\text { RoB }\end{array}$ & $\begin{array}{l}\text { Time } \\
\text { Point }\end{array}$ & Use of ADM, n/N (\%) & $\begin{array}{l}\text { Nonuse of ADM, } n / N \\
(\%)\end{array}$ & Effect Size $(95 \% \mathrm{CI})$ & $\begin{array}{l}P \\
\text { Value }\end{array}$ \\
\hline Nealon, 2020b, 31605310, US & NRCS & High & $5.3 y$ & NR & NR & adjOR $0.78(0.46,1.36)$ & 0.38 \\
\hline $\begin{array}{l}\text { Ganesh Kumar, 2021, 33172826, US } \\
\text { \& Canada }\end{array}$ & NRCS & Moderate & $2 y$ & $14 / 738(1.9)$ & $12 / 713(1.7)$ & NR & 0.24 \\
\hline Sobti, 2018, 29481386, US & NRCS & High & $5 y$ & $21 / 465$ breasts $(4.52)$ & $7 / 217$ breasts $(3.23)$ & Nonuse vs use of ADM: adjOR $0.57(0.23,1.43)$ & NR \\
\hline Vardanian, 2011,22030500, US & NRCS & High & $2.4 \mathrm{y}$ & $8 / 208$ breasts $(3.8)$ & $25 / 129$ breasts (19.4) & adjOR $0.18(0.08,0.43)$ & NR \\
\hline
\end{tabular}

Abbreviations: adj = adjusted, ADM = acellular dermal matrix, $\mathrm{CI}=$ confidence interval, $\mathrm{IBR}=$ implant-based reconstruction, $\mathrm{NR}=$ not reported, $\mathrm{NRCS}=$ nonrandomized comparative study, $\mathrm{OR}=$ odds ratio, $\mathrm{PMID}=$ Pubmed identifier, $\mathrm{RoB}=$ risk of bias, $\mathrm{y}=$ years.

Colors: Header rows are shaded orange. Rows for every alternate study are shaded blue. The colors do not add unique information.

Table F-5.19. Full Evidence Table - Key Question 5: Use versus nonuse of human ADM during IBR - categorical outcomes (hematoma)

\begin{tabular}{|c|c|c|c|c|c|c|c|}
\hline Study, Year, PMID, Country & Design & $\begin{array}{l}\text { Overall } \\
\text { RoB }\end{array}$ & $\begin{array}{l}\text { Time } \\
\text { Point }\end{array}$ & Use of ADM, $n / N(\%)$ & $\begin{array}{l}\text { Nonuse of ADM, n/N } \\
(\%)\end{array}$ & Effect Size $(95 \% \mathrm{Cl})$ & P Value \\
\hline Hirsch, 2014, 25347643, US & NRCS & Low & $3.1 \mathrm{y}$ & NR & NR & adjOR $1.47(0.62,3.49)$ & 0.39 \\
\hline Nealon, 2020b, 31605310, US & NRCS & High & $5.3 y$ & NR & NR & adjOR $0.84(0.40,1.84)$ & 0.64 \\
\hline Seth, 2012, 23018687, US & NRCS & High & $2 \mathrm{y}$ & $6 / 199$ breasts $(3)$ & $6 / 393$ breasts $(1.5)$ & adjOR $0.78(0.12,7.09)$ & NR \\
\hline Sobti, 2018,29481386, US & NRCS & High & $5 y$ & $2 / 338(0.3)$ & $3 / 376(0.8)$ & adjOR $0.50(0.0,1.28)$ & NR \\
\hline Sorkin, 2017, 28806288, US \& Canada & NRCS & Moderate & $2 y$ & $17 / 655(2.6)$ & $26 / 642(4)$ & NR & 0.15 \\
\hline
\end{tabular}

\begin{tabular}{|l|l|l|l|l}
\hline Sorkin, 2017, 28806288, US \& Canada & NRCS & Moderate & 2 y & $17 / 655(2.6)$ \\
\hline
\end{tabular}

Abbreviations: adj = adjusted, $\mathrm{ADM}=$ acellular dermal matrix, $\mathrm{CI}=$ confidence interval, $\mathrm{IBR}=$ implant-based reconstruction, NR $=$ not reported, NRCS $=$ nonrandomized

comparative study, $\mathrm{OR}=$ odds ratio, $\mathrm{PMID}=$ Pubmed identifier, $\mathrm{RoB}=$ risk of bias, $\mathrm{y}=$ years.

Colors: Header rows are shaded orange. Rows for every alternate study are shaded blue. The colors do not add unique information. 
Table F-5.20. Full Evidence Table - Key Question 5: Use versus nonuse of human ADM during IBR - categorical outcomes

\section{(combined/unspecified harms)}

\begin{tabular}{|c|c|c|c|c|c|c|c|c|}
\hline $\begin{array}{l}\text { Study, Year, PMID, } \\
\text { Country }\end{array}$ & Outcome Measurement & Design & $\begin{array}{l}\text { Overall } \\
\text { RoB }\end{array}$ & $\begin{array}{l}\text { Time } \\
\text { Point }\end{array}$ & $\begin{array}{l}\text { Use of ADM, } \\
\mathrm{n} / \mathrm{N}(\%)\end{array}$ & $\begin{array}{l}\text { Nonuse of } \\
\text { ADM, } n / N(\%)\end{array}$ & Effect Size $(95 \% \mathrm{CI})$ & $\begin{array}{l}P \\
\text { Value }\end{array}$ \\
\hline Wendel, 2013, None, US & Serious adverse events & $\mathrm{RCT}$ & High & $1 \mathrm{mo}$ & $0 / 20(0)$ & $0 / 16(0)$ & N/A (No events) & $\mathrm{N} / \mathrm{A}$ \\
\hline $\begin{array}{l}\text { Brooke, } 2012, \\
22868313, \text { US }\end{array}$ & $\begin{array}{l}\text { Clinically significant complications, defined as } \\
\text { cellulitis, abscess, seroma, expander leak or } \\
\text { puncture, skin necrosis, wound dehiscence, or } \\
\text { hematoma requiring readmission, reoperation, } \\
\text { and/or expander explantation }\end{array}$ & NRCS & High & NR & $\begin{array}{l}37 / 221 \\
\text { breasts (17) }\end{array}$ & $\begin{array}{l}7 / 64 \text { breasts } \\
(11)\end{array}$ & $\mathrm{NR}$ & 0.48 \\
\hline \multirow[t]{2}{*}{$\begin{array}{l}\text { Ganesh Kumar, 2021, } \\
\text { 33172826, US \& Canada }\end{array}$} & Any complication & NRCS & Moderate & $2 y$ & $\begin{array}{l}211 / 738 \\
(28.6)\end{array}$ & $\begin{array}{l}178 / 713 \\
(25.0) \\
\end{array}$ & adjOR $1.21(0.86,1.70)$ & 0.26 \\
\hline & Major complications & NRCS & Moderate & $2 y$ & $\begin{array}{l}169 / 738 \\
(22.9)\end{array}$ & $\begin{array}{l}117 / 713 \\
(16.4)\end{array}$ & adjOR $1.43(1.00,2.05)$ & 0.052 \\
\hline \multirow{3}{*}{$\begin{array}{l}\text { Hirsch, 2014, 25347643, } \\
\text { US }\end{array}$} & Any complication & NRCS & Low & $3.5 \mathrm{y}$ & NR & NR & adjOR $0.9(0.6,1.34)$ & 0.60 \\
\hline & Operative complication except explantation & NRCS & Low & $3.5 \mathrm{y}$ & NR & NR & adjOR $0.68(0.46,1.02)$ & 0.46 \\
\hline & Nonoperative complication & NRCS & Low & $3.5 \mathrm{y}$ & NR & NR & adjOR $0.67(0.26,1.74)$ & 0.43 \\
\hline Liu, 2011, 21228744, US & Surgical complications & NRCS & High & NR & $52 / 266(19.5)$ & $25 / 204(12.3)$ & adjOR $1.76(1.03,3.01)$ & 0.036 \\
\hline $\begin{array}{l}\text { Safran, 2020, 32221195, } \\
\text { Canada }\end{array}$ & $\begin{array}{l}\text { Any complication, including hematoma, infection, } \\
\text { seroma, implant displacement, NAC full-thickness } \\
\text { necrosis, superficial cellulitis, red breast } \\
\text { syndrome, incision necrosis, delayed healing, } \\
\text { hypergranulation, and NAC superficial necrosis. }\end{array}$ & NRCS & High & NR & $\begin{array}{l}\text { NR/243 } \\
\text { breasts (NR) }\end{array}$ & $\begin{array}{l}\text { NR/70 } \\
\text { breasts (NR) }\end{array}$ & adjOR $1.59(0.56,4.50)$ & NR \\
\hline \multirow[t]{3}{*}{$\begin{array}{l}\text { Stein, 2020, 32561384, } \\
\text { Canada }\end{array}$} & Any complication & NRCS & High & $\begin{array}{l}10 \\
\mathrm{mo}- \\
5 \mathrm{y}\end{array}$ & $16 / 41(39.0)$ & $37 / 89(42.1)$ & adjOR $0.86(0.26,2.78)$ & NR \\
\hline & Major complications & NRCS & High & $\begin{array}{l}10 \\
\mathrm{mo}- \\
5 \mathrm{y}\end{array}$ & $10 / 41(24.4)$ & $22 / 89(24.7)$ & adjOR $0.83(0.22,3.08)$ & NR \\
\hline & Minor complications & NRCS & High & $\begin{array}{l}10 \\
\mathrm{mo}- \\
5 \mathrm{y}\end{array}$ & $10 / 41(24.4)$ & $20 / 89(22.5)$ & adjOR $0.83(0.21,3.29)$ & NR \\
\hline $\begin{array}{l}\text { Weichman, 2012, } \\
\text { 22544088, US }\end{array}$ & $\begin{array}{l}\text { Complications including mastectomy skin flap } \\
\text { necrosis, mastectomy skin flap necrosis and } \\
\text { associated infection, infection alone, seroma, and } \\
\text { hematoma }\end{array}$ & NRCS & Moderate & $3 y$ & $\begin{array}{l}\text { NR/442 } \\
\text { breasts (NR) }\end{array}$ & $\begin{array}{l}\text { NR/186 } \\
\text { breasts (NR) }\end{array}$ & NR & $<0.05$ \\
\hline $\begin{array}{l}\text { Woo, 2017, } 28509694, \\
\text { South Korea }\end{array}$ & $\begin{array}{l}\text { Major complication: defined as complications that } \\
\text { necessitated additional surgery or intervention }\end{array}$ & NRCS & High & NR & $26 / 199(13.1)$ & $38 / 199(19.1)$ & adjOR $1.1(0.5,2.4)$ & 0.81 \\
\hline
\end{tabular}

South Korea necessitated additional surgery or intervention

Abbreviations: adj $=$ adjusted, $\mathrm{ADM}=$ acellular dermal matrix, $\mathrm{CI}=$ confidence interval, $\mathrm{IBR}=$ implant-based reconstruction, $\mathrm{mo}=$ months, $\mathrm{N} / \mathrm{A}=$ not applicable, $\mathrm{NR}=$ not

reported, $\mathrm{NRCS}=$ nonrandomized comparative study, $\mathrm{OR}=$ odds ratio, $\mathrm{PMID}=$ Pubmed identifier, $\mathrm{RCT}=$ randomized controlled trial, RoB $=$ risk of bias, $\mathrm{y}=\mathrm{years}$.

Colors: Header rows are shaded orange. Rows for every alternate study are shaded blue. The colors do not add unique information. 
Table F-5.21: Full Evidence Table - Key Question 5: Use versus nonuse of human ADM during IBR - categorical outcomes (harms to the inframammary fold)

\begin{tabular}{|c|c|c|c|c|c|c|c|c|}
\hline $\begin{array}{l}\text { Study, Year, PMID, } \\
\text { Country }\end{array}$ & Outcome Measurement & Design & $\begin{array}{l}\text { Overall } \\
\text { RoB }\end{array}$ & $\begin{array}{l}\text { Time } \\
\text { Point }\end{array}$ & $\begin{array}{l}\text { Use of ADM, } \\
\mathrm{n} / \mathrm{N}(\%)\end{array}$ & $\begin{array}{l}\text { Nonuse of } \\
\text { ADM, } n / N(\%)\end{array}$ & $\begin{array}{l}\text { Effect Size } \\
(95 \% \mathrm{Cl})\end{array}$ & $\begin{array}{l}\mathbf{P} \\
\text { Value }\end{array}$ \\
\hline $\begin{array}{l}\text { Vardanian, 2011, } \\
22030500, \text { US }\end{array}$ & $\begin{array}{l}\text { Problems of the inframammary fold: defined as inframammary fold } \\
\text { issues other than bottoming-out or shifting, but related to the } \\
\text { integrity of the fold }\end{array}$ & NRCS & High & $2.4 \mathrm{y}$ & $\begin{array}{l}17 / 208 \\
\text { breasts (8.2) }\end{array}$ & $\begin{array}{l}25 / 129 \\
\text { breasts (19.4) }\end{array}$ & $\begin{array}{l}\text { adjOR } 0.49 \\
(0.23,1.01)\end{array}$ & NR \\
\hline
\end{tabular}

Abbreviations: adj = adjusted, ADM = acellular dermal matrix, $\mathrm{CI}=$ confidence interval, $\mathrm{IBR}=$ implant-based reconstruction, $\mathrm{NR}=$ not reported, NRCS $=$ nonrandomized comparative study, $\mathrm{OR}=$ odds ratio, $\mathrm{PMID}=$ Pubmed identifier, $\mathrm{RoB}=$ risk of bias, $\mathrm{y}=$ years

Colors: Header rows are shaded orange. The color does not add unique information. 
Table F-6.1. Full Evidence Table - Key Question 6: Comparison of flap types for AR - continuous outcomes (physical well-being)

\begin{tabular}{|c|c|c|c|c|c|c|c|c|c|}
\hline $\begin{array}{l}\text { Study, Year, PMID, } \\
\text { Country }\end{array}$ & Outcome Measurement & Design & $\begin{array}{l}\text { Overall } \\
\text { RoB }\end{array}$ & $\begin{array}{l}\text { Time } \\
\text { Point }\end{array}$ & Flap & $\mathbf{N}$ & $\begin{array}{l}\text { Mean (SD or } \\
95 \% \mathrm{Cl})\end{array}$ & Effect Size $(95 \% \mathrm{CI})$ & P Value \\
\hline \multirow[t]{10}{*}{$\begin{array}{l}\text { Rindom, 2019, 31515191, } \\
\text { Denmark }\end{array}$} & $\begin{array}{l}\text { Constant Murley Shoulder Score: } \\
\text { Total }(0-100)\end{array}$ & RCT & Moderate & $1 \mathrm{y}$ & LD & 18 & $68.1(58.2,79.9)$ & Ref & Ref \\
\hline & $\begin{array}{l}\text { Constant Murley Shoulder Score: } \\
\text { Total }(0-100)\end{array}$ & $\mathrm{RCT}$ & Moderate & $1 \mathrm{y}$ & TAP & 22 & $78.7(70.9,86.4)$ & adjNMD $6.2(0.5,12.0)$ & 0.033 \\
\hline & $\begin{array}{l}\text { Constant Murley Shoulder Score: } \\
\text { Pain }(0-15)\end{array}$ & RCT & Moderate & $1 \mathrm{y}$ & LD & 18 & $11.6(9.8,13.4)$ & Ref & Ref \\
\hline & $\begin{array}{l}\text { Constant Murley Shoulder Score: } \\
\text { Pain }(0-15)\end{array}$ & RCT & Moderate & $1 \mathrm{y}$ & TAP & 22 & $14.0(12.8,15.2)$ & $\operatorname{adjNMD} 1.8(0.2,3.4)$ & 0.023 \\
\hline & $\begin{array}{l}\text { Constant Murley Shoulder Score: } \\
\text { Activity in daily life }(0-20)\end{array}$ & RCT & Moderate & $1 \mathrm{y}$ & LD & 18 & $17.1(14.9,19.2)$ & Ref & Ref \\
\hline & $\begin{array}{l}\text { Constant Murley Shoulder Score: } \\
\text { Activity in daily life }(0-20)\end{array}$ & RCT & Moderate & $1 \mathrm{y}$ & TAP & 22 & $18.7(17.3,20.0)$ & adjNMD $2.6(1.1,4.2)$ & $<0.0001$ \\
\hline & $\begin{array}{l}\text { Constant Murley Shoulder Score: } \\
\text { Range of motion }(0-40)\end{array}$ & $\mathrm{RCT}$ & Moderate & $1 \mathrm{y}$ & LD & 18 & $29.6(24.6,34.5)$ & Ref & Ref \\
\hline & $\begin{array}{l}\text { Constant Murley Shoulder Score: } \\
\text { Range of motion }(0-40)\end{array}$ & $\mathrm{RCT}$ & Moderate & $1 \mathrm{y}$ & TAP & 22 & $34.8(31.0,38.6)$ & adjNMD $0.9(-1.4,3.2)$ & 0.45 \\
\hline & $\begin{array}{l}\text { Constant Murley Score: Strength } \\
(0-25)\end{array}$ & $\mathrm{RCT}$ & Moderate & $1 \mathrm{y}$ & LD & 18 & $9.9(7.8,12.0)$ & Ref & Ref \\
\hline & $\begin{array}{l}\text { Constant Murley Score: Strength } \\
(0-25)\end{array}$ & RCT & Moderate & $1 \mathrm{y}$ & TAP & 22 & $11.2(9.3,13.1)$ & $\operatorname{adjNMD~} 1.2(-1.0,3.3)$ & 0.29 \\
\hline \multirow{11}{*}{$\begin{array}{l}\text { Erdmann-Sager, 2018, } \\
\text { 29019862, US \& Canada }\end{array}$} & BREAST-Q: Abdomen (0-100) & NRCS & Moderate & $1 \mathrm{y}$ & DIEP & NR & NR & Ref & Ref \\
\hline & BREAST-Q: Abdomen (0-100) & NRCS & Moderate & $1 \mathrm{y}$ & Free TRAM & NR & NR & $\begin{array}{l}\text { vs. DIEP: adjMD }-4.16 \\
(-8.33,0.02)\end{array}$ & NR \\
\hline & BREAST-Q: Abdomen (0-100) & NRCS & Moderate & $1 \mathrm{y}$ & Pedicled TRAM & NR & NR & $\begin{array}{l}\text { vs. DIEP: adjMD }-4.01 \\
(-8.48,0.45)\end{array}$ & NR \\
\hline & BREAST-Q: Abdomen (0-100) & NRCS & Moderate & $1 \mathrm{y}$ & AR with SIEA & NR & NR & $\begin{array}{l}\text { vs. DIEP: adjMD } 4.72 \\
(-0.07,9.52)\end{array}$ & NR \\
\hline & BREAST-Q: Abdomen (0-100) & NRCS & Moderate & $2 y$ & DIEP & NR & NR & Ref & Ref \\
\hline & BREAST-Q: Abdomen (0-100) & NRCS & Moderate & $2 y$ & Free TRAM & NR & NR & $\begin{array}{l}\text { vs. DIEP: adjMD }-4.9 \\
(-9.50,-0.31)\end{array}$ & NR \\
\hline & BREAST-Q: Abdomen (0-100) & NRCS & Moderate & $2 y$ & Pedicled TRAM & NR & NR & $\begin{array}{l}\text { vs. DIEP: adjMD }-7.22 \\
(-12.30,-2.14)\end{array}$ & NR \\
\hline & BREAST-Q: Abdomen (0-100) & NRCS & Moderate & $2 y$ & AR with SIEA & NR & NR & $\begin{array}{l}\text { vs. DIEP: adjMD } 0.58 \\
(-4.79,5.95)\end{array}$ & NR \\
\hline & $\begin{array}{l}\text { BREAST-Q: Chest and upper } \\
\text { body }(0-100)\end{array}$ & NRCS & Moderate & $1 \mathrm{y}$ & DIEP & NR & NR & Ref & Ref \\
\hline & $\begin{array}{l}\text { BREAST-Q: Chest and upper } \\
\text { body }(0-100)\end{array}$ & NRCS & Moderate & $1 \mathrm{y}$ & Free TRAM & NR & NR & $\begin{array}{l}\text { vs. DIEP: adjMD }-1.55 \\
(-5.35,2.24)\end{array}$ & NR \\
\hline & $\begin{array}{l}\text { BREAST-Q: Chest and upper } \\
\text { body }(0-100)\end{array}$ & NRCS & Moderate & $1 \mathrm{y}$ & Pedicled TRAM & NR & NR & $\begin{array}{l}\text { vs. DIEP: adjMD } 1.52 \\
(-1.94,4.99)\end{array}$ & NR \\
\hline
\end{tabular}




\begin{tabular}{|c|c|c|c|c|c|c|c|c|c|}
\hline $\begin{array}{l}\text { Study, Year, PMID, } \\
\text { Country }\end{array}$ & Outcome Measurement & Design & $\begin{array}{l}\text { Overall } \\
\text { RoB }\end{array}$ & $\begin{array}{l}\text { Time } \\
\text { Point }\end{array}$ & Flap & $\mathbf{N}$ & $\begin{array}{l}\text { Mean (SD or } \\
95 \% \mathrm{Cl} \text { ) }\end{array}$ & Effect Size $(95 \% \mathrm{CI})$ & P Value \\
\hline & $\begin{array}{l}\text { BREAST-Q: Chest and upper } \\
\text { body }(0-100)\end{array}$ & NRCS & Moderate & $1 \mathrm{y}$ & AR with SIEA & NR & NR & $\begin{array}{l}\text { vs. DIEP: adjMD } 3.42 \\
(-0.22,7.05)\end{array}$ & NR \\
\hline & $\begin{array}{l}\text { BREAST-Q: Chest and upper } \\
\text { body }(0-100)\end{array}$ & NRCS & Moderate & $2 y$ & DIEP & NR & NR & Ref & Ref \\
\hline & $\begin{array}{l}\text { BREAST-Q: Chest and upper } \\
\text { body }(0-100)\end{array}$ & NRCS & Moderate & $2 y$ & Free TRAM & NR & NR & $\begin{array}{l}\text { vs. DIEP: adjMD }-2.22 \\
(-5.89,1.45)\end{array}$ & NR \\
\hline & $\begin{array}{l}\text { BREAST-Q: Chest and upper } \\
\text { body }(0-100)\end{array}$ & NRCS & Moderate & $2 y$ & Pedicled TRAM & NR & NR & $\begin{array}{l}\text { vs. DIEP: adjMD }-3.92 \\
(-7.66,-0.18)\end{array}$ & NR \\
\hline & $\begin{array}{l}\text { BREAST-Q: Chest and upper } \\
\text { body }(0-100)\end{array}$ & NRCS & Moderate & $2 y$ & AR with SIEA & NR & NR & $\begin{array}{l}\text { vs. DIEP: adjMD } 0.76 \\
(-3.44,4.95)\end{array}$ & NR \\
\hline & $\begin{array}{l}\text { PROMIS: Physical functioning } \\
(0-100)\end{array}$ & NRCS & Moderate & $1 \mathrm{y}$ & DIEP & NR & NR & Ref & Ref \\
\hline & $\begin{array}{l}\text { PROMIS: Physical functioning } \\
(0-100)\end{array}$ & NRCS & Moderate & $1 \mathrm{y}$ & Free TRAM & NR & NR & $\begin{array}{l}\text { vs. DIEP: adjMD }-0.03 \\
(-2.36,2.30)\end{array}$ & NR \\
\hline & $\begin{array}{l}\text { PROMIS: Physical functioning } \\
(0-100)\end{array}$ & NRCS & Moderate & $1 \mathrm{y}$ & Pedicled TRAM & NR & NR & $\begin{array}{l}\text { vs. DIEP: adjMD } 0.00 \\
(-2.09,2.10)\end{array}$ & NR \\
\hline & $\begin{array}{l}\text { PROMIS: Physical functioning } \\
(0-100)\end{array}$ & NRCS & Moderate & $1 \mathrm{y}$ & AR with SIEA & NR & NR & $\begin{array}{l}\text { vs. DIEP: adjMD } 1.26 \\
(-0.74,3.26)\end{array}$ & NR \\
\hline & $\begin{array}{l}\text { PROMIS: Physical functioning } \\
(0-100)\end{array}$ & NRCS & Moderate & $2 y$ & DIEP & NR & NR & Ref & Ref \\
\hline & $\begin{array}{l}\text { PROMIS: Physical functioning } \\
(0-100)\end{array}$ & NRCS & Moderate & $2 y$ & Free TRAM & NR & NR & $\begin{array}{l}\text { vs. DIEP: adjMD } 0.42 \\
(-1.52,2.36)\end{array}$ & NR \\
\hline & $\begin{array}{l}\text { PROMIS: Physical functioning } \\
(0-100)\end{array}$ & NRCS & Moderate & $2 y$ & Pedicled TRAM & NR & NR & $\begin{array}{l}\text { vs. DIEP: adjMD } 1.48 \\
(-0.59,3.55)\end{array}$ & NR \\
\hline & $\begin{array}{l}\text { PROMIS: Physical functioning } \\
(0-100)\end{array}$ & NRCS & Moderate & $2 y$ & AR with SIEA & NR & NR & $\begin{array}{l}\text { VS. DIEP: adjMD } 1.20 \\
(-1.09,3.49)\end{array}$ & NR \\
\hline & $\begin{array}{l}\text { PROMIS: Pain interference (0- } \\
100)\end{array}$ & NRCS & Moderate & $1 \mathrm{y}$ & DIEP & NR & NR & Ref & Ref \\
\hline & $\begin{array}{l}\text { PROMIS: Pain interference (0- } \\
100)\end{array}$ & NRCS & Moderate & $1 \mathrm{y}$ & Free TRAM & NR & NR & $\begin{array}{l}\text { vs. DIEP: adjMD } 0.34 \\
(-1.91,2.60)\end{array}$ & NR \\
\hline & $\begin{array}{l}\text { PROMIS: Pain interference (0- } \\
100)\end{array}$ & NRCS & Moderate & $1 \mathrm{y}$ & Pedicled TRAM & NR & NR & $\begin{array}{l}\text { vs. DIEP: adjMD }-0.07 \\
(-2.10,1.97)\end{array}$ & NR \\
\hline & $\begin{array}{l}\text { PROMIS: Pain interference (0- } \\
100)\end{array}$ & NRCS & Moderate & $1 \mathrm{y}$ & AR with SIEA & NR & NR & $\begin{array}{l}\text { vs. DIEP: adjMD }-0.6 \\
(-2.54,1.34)\end{array}$ & NR \\
\hline & $\begin{array}{l}\text { PROMIS: Pain interference (0- } \\
\text { 100) }\end{array}$ & NRCS & Moderate & $2 y$ & DIEP & NR & NR & Ref & Ref \\
\hline & $\begin{array}{l}\text { PROMIS: Pain interference (0- } \\
100)\end{array}$ & NRCS & Moderate & $2 y$ & Free TRAM & NR & NR & $\begin{array}{l}\text { vs. DIEP: adjMD }-0.5 \\
(-2.57,1.57)\end{array}$ & NR \\
\hline & $\begin{array}{l}\text { PROMIS: Pain interference (0- } \\
100)\end{array}$ & NRCS & Moderate & $2 y$ & Pedicled TRAM & NR & NR & $\begin{array}{l}\text { vs. DIEP: adjMD } 0.44 \\
(-2.25,3.13)\end{array}$ & NR \\
\hline & $\begin{array}{l}\text { PROMIS: Pain interference (0- } \\
100)\end{array}$ & NRCS & Moderate & $2 y$ & AR with SIEA & NR & NR & $\begin{array}{l}\text { vs. DIEP: adjMD } 0.11 \\
(-2.34,2.57)\end{array}$ & NR \\
\hline
\end{tabular}




\begin{tabular}{|c|c|c|c|c|c|c|c|c|c|}
\hline $\begin{array}{l}\text { Study, Year, PMID, } \\
\text { Country }\end{array}$ & Outcome Measurement & Design & $\begin{array}{l}\text { Overall } \\
\text { RoB }\end{array}$ & $\begin{array}{l}\text { Time } \\
\text { Point }\end{array}$ & Flap & $\mathbf{N}$ & $\begin{array}{l}\text { Mean (SD or } \\
95 \% \mathrm{Cl})\end{array}$ & Effect Size (95\% CI) & P Value \\
\hline \multirow[t]{5}{*}{$\begin{array}{l}\text { Kulkarni, 2017, 28713853, } \\
\text { US \& Canada }\end{array}$} & $\begin{array}{l}\text { BREAST-Q: Chest and upper } \\
\text { body }(0-100)\end{array}$ & NRCS & Moderate & $3 \mathrm{mo}$ & DIEP & 296 & 72.9 (NR) & NR & NR \\
\hline & $\begin{array}{l}\text { BREAST-Q: Chest and upper } \\
\text { body }(0-100)\end{array}$ & NRCS & Moderate & $3 \mathrm{mo}$ & Free TRAM & 83 & $68.3(\mathrm{NR})$ & NR & NR \\
\hline & $\begin{array}{l}\text { BREAST-Q: Chest and upper } \\
\text { body }(0-100)\end{array}$ & NRCS & Moderate & $3 \mathrm{mo}$ & Pedicled TRAM & 91 & 70.9 (NR) & NR & NR \\
\hline & $\begin{array}{l}\text { BREAST-Q: Chest and upper } \\
\text { body }(0-100)\end{array}$ & NRCS & Moderate & $3 \mathrm{mo}$ & LD & 62 & $69.3(\mathrm{NR})$ & NR & NR \\
\hline & $\begin{array}{l}\text { BREAST-Q: Chest and upper } \\
\text { body }(0-100)\end{array}$ & NRCS & Moderate & $3 \mathrm{mo}$ & AR with SIEA & 56 & $73(\mathrm{NR})$ & NR & NR \\
\hline \multirow{4}{*}{$\begin{array}{l}\text { Macadam, 2016, } \\
26910656, \text { US, Canada, \& } \\
\text { Japan }\end{array}$} & BREAST-Q: Abdomen (0-100) & NRCS & High & $\begin{array}{l}4.5- \\
7.3 y\end{array}$ & DIEP & 387 & $83.5(17.4)$ & NR & NR \\
\hline & BREAST-Q: Abdomen (0-100) & NRCS & High & $\begin{array}{l}4.5- \\
7.3 y\end{array}$ & Free TRAM & 74 & $78.6(23.4)$ & NR & NR \\
\hline & BREAST-Q: Abdomen (0-100) & NRCS & High & $\begin{array}{l}4.5- \\
7.3 y\end{array}$ & Pedicled TRAM & 359 & $76.2(21.8)$ & NR & NR \\
\hline & BREAST-Q: Abdomen (0-100) & NRCS & High & $\begin{array}{l}4.5- \\
7.3 y\end{array}$ & $\begin{array}{l}\text { Muscle-sparing } \\
\text { TRAM }\end{array}$ & 123 & $78.1(22.8)$ & NR & NR \\
\hline
\end{tabular}

Abbreviations: adj = adjusted, $\mathrm{AR}=$ autologous reconstruction, $\mathrm{CI}=$ confidence interval, $\mathrm{DIEP}=$ deep inferior epigastric perforator, $\mathrm{LD}=$ latissimus dorsi, $\mathrm{MD}=$ mean difference, mo $=$ months, NMD = net mean difference, NR = not reported, NRCS = nonrandomized comparative study, PMID = Pubmed identifier, PROMIS = Patient-Reported Outcomes Measurement Information System, RCT = randomized controlled trial, Ref $=$ reference group, RoB $=$ risk of bias, $\mathrm{SD}=$ standard deviation, $\mathrm{SIEA}=$ superficial inferior epigastric artery perforator, $\mathrm{TAP}=$ thoracodorsal artery perforator, TRAM $=$ transverse rectus abdominis myocutaneous, $\mathrm{y}=$ years.

Colors: Header rows are shaded orange. Rows for every alternate study are shaded blue. The colors do not add unique information.

Table F-6.2. Full Evidence Table - Key Question 6: Comparison of flap types for AR - continuous outcomes (psychosocial well-being)

\begin{tabular}{|c|c|c|c|c|c|c|c|c|c|}
\hline $\begin{array}{l}\text { Study, Year, PMID, } \\
\text { Country }\end{array}$ & $\begin{array}{l}\text { Outcome } \\
\text { Measurement }\end{array}$ & Design & $\begin{array}{l}\text { Overall } \\
\text { RoB }\end{array}$ & $\begin{array}{l}\text { Time } \\
\text { Point }\end{array}$ & Arm & $\mathbf{N}$ & Mean (SD) & Effect Size $(95 \% \mathrm{CI})$ & $\begin{array}{l}\mathbf{P} \\
\text { Value }\end{array}$ \\
\hline \multirow[t]{4}{*}{$\begin{array}{l}\text { Erdmann-Sager, 2018, } \\
\text { 29019862, US \& } \\
\text { Canada }\end{array}$} & $\begin{array}{l}\text { BREAST-Q: } \\
\text { Psychosocial well-being } \\
(0-100)\end{array}$ & NRCS & Moderate & $1 \mathrm{y}$ & DIEP & NR & NR & Ref & Ref \\
\hline & $\begin{array}{l}\text { BREAST-Q: } \\
\text { Psychosocial well-being } \\
(0-100)\end{array}$ & NRCS & Moderate & $1 \mathrm{y}$ & Free TRAM & NR & NR & vs. DIEP: adjMD -1.14 $(-5.33,3.05)$ & NR \\
\hline & $\begin{array}{l}\text { BREAST-Q: } \\
\text { Psychosocial well-being } \\
(0-100)\end{array}$ & NRCS & Moderate & $1 \mathrm{y}$ & Pedicled TRAM & NR & NR & vs. DIEP: adjMD $1.27(-3.43,5.97)$ & NR \\
\hline & $\begin{array}{l}\text { BREAST-Q: } \\
\text { Psychosocial well-being } \\
(0-100)\end{array}$ & NRCS & Moderate & $1 \mathrm{y}$ & AR with SIEA & NR & NR & vs. DIEP: adjMD -0.67 $(-5.66,4.32)$ & NR \\
\hline
\end{tabular}




\begin{tabular}{|c|c|c|c|c|c|c|c|c|c|}
\hline $\begin{array}{l}\text { Study, Year, PMID, } \\
\text { Country }\end{array}$ & $\begin{array}{l}\text { Outcome } \\
\text { Measurement }\end{array}$ & Design & $\begin{array}{l}\text { Overall } \\
\text { RoB }\end{array}$ & $\begin{array}{l}\text { Time } \\
\text { Point }\end{array}$ & Arm & $\mathbf{N}$ & Mean (SD) & Effect Size (95\% Cl) & $\begin{array}{l}\mathbf{P} \\
\text { Value }\end{array}$ \\
\hline & $\begin{array}{l}\text { BREAST-Q: } \\
\text { Psychosocial well-being } \\
(0-100)\end{array}$ & NRCS & Moderate & $2 \mathrm{y}$ & DIEP & NR & NR & Ref & Ref \\
\hline & $\begin{array}{l}\text { BREAST-Q: } \\
\text { Psychosocial well-being } \\
(0-100)\end{array}$ & NRCS & Moderate & $2 y$ & Free TRAM & NR & NR & vs. DIEP: adjMD $-0.08(-5.33,5.18)$ & NR \\
\hline & $\begin{array}{l}\text { BREAST-Q: } \\
\text { Psychosocial well-being } \\
(0-100)\end{array}$ & NRCS & Moderate & $2 y$ & Pedicled TRAM & NR & NR & vs. DIEP: adjMD $0.62(-3.95,5.20)$ & NR \\
\hline & $\begin{array}{l}\text { BREAST-Q: } \\
\text { Psychosocial well-being } \\
(0-100)\end{array}$ & NRCS & Moderate & $2 \mathrm{y}$ & AR with SIEA & NR & NR & vs. DIEP: adjMD $1.64(-3.64,6.91)$ & NR \\
\hline \multirow[t]{3}{*}{$\begin{array}{l}\text { Macadam, 2016, } \\
\text { 26910656, US, } \\
\text { Canada, \& Japan }\end{array}$} & $\begin{array}{l}\text { BREAST-Q: } \\
\text { Psychosocial well-being } \\
(0-100)\end{array}$ & NRCS & High & $\begin{array}{l}4.5-7.3 \\
y\end{array}$ & DIEP & 387 & $79.9(18.4)$ & NR & NR \\
\hline & $\begin{array}{l}\text { BREAST-Q: } \\
\text { Psychosocial well-being } \\
(0-100)\end{array}$ & NRCS & High & $\begin{array}{l}4.5-7.3 \\
y\end{array}$ & Free TRAM & 74 & $79.1(21.7)$ & NR & NR \\
\hline & $\begin{array}{l}\text { BREAST-Q: } \\
\text { Psychosocial well-being } \\
(0-100)\end{array}$ & NRCS & High & $\begin{array}{l}4.5-7.3 \\
y\end{array}$ & Muscle-sparing TRAM & 123 & $75.9(22.7)$ & NR & NR \\
\hline
\end{tabular}

Abbreviations: adj = adjusted, $\mathrm{AR}=$ autologous reconstruction, $\mathrm{CI}=$ confidence interval, $\mathrm{DIEP}=$ deep inferior epigastric perforator, $\mathrm{LD}=$ latissimus dorsi, $\mathrm{MD}=$ mean difference,

$\mathrm{NR}=$ not reported, NRCS $=$ nonrandomized comparative study, PMID $=$ Pubmed identifier, $\mathrm{RCT}=$ randomized controlled trial, $\mathrm{Ref}=$ reference group, $\mathrm{RoB}=$ risk of bias, $\mathrm{SD}=$

standard deviation, SIEA = superficial inferior epigastric artery perforator, TRAM $=$ transverse rectus abdominis myocutaneous, $\mathrm{y}=$ years.

Colors: Header rows are shaded orange. Rows for every alternate study are shaded blue. The colors do not add unique information.

Table F-6.3. Full Evidence Table - Key Question 6: Comparison of flap types for AR - continuous outcomes (sexual well-being)

\begin{tabular}{|c|c|c|c|c|c|c|c|c|c|}
\hline $\begin{array}{l}\text { Study, Year, PMID, } \\
\text { Country }\end{array}$ & $\begin{array}{l}\text { Outcome } \\
\text { Measurement }\end{array}$ & Design & $\begin{array}{l}\text { Overall } \\
\text { RoB }\end{array}$ & $\begin{array}{l}\text { Time } \\
\text { Point }\end{array}$ & Arm & $\mathbf{N}$ & Mean (SD) & Effect Size $(95 \% \mathrm{CI})$ & $\begin{array}{l}\mathbf{P} \\
\text { Value }\end{array}$ \\
\hline \multirow[t]{3}{*}{$\begin{array}{l}\text { Erdmann-Sager, 2018, } \\
\text { 29019862, US \& Canada }\end{array}$} & $\begin{array}{l}\text { BREAST-Q: } \\
\text { Sexual well-being } \\
(0-100)\end{array}$ & NRCS & Moderate & $1 \mathrm{y}$ & DIEP & NR & NR & Ref & Ref \\
\hline & $\begin{array}{l}\text { BREAST-Q: } \\
\text { Sexual well-being } \\
(0-100)\end{array}$ & NRCS & Moderate & $1 y$ & Free TRAM & NR & NR & vs. DIEP: adjMD -2.33 $(-7.10,2.44)$ & NR \\
\hline & $\begin{array}{l}\text { BREAST-Q: } \\
\text { Sexual well-being } \\
(0-100)\end{array}$ & NRCS & Moderate & $1 \mathrm{y}$ & Pedicled TRAM & NR & NR & vs. DIEP: adjMD $0.81(-4.31,5.93)$ & NR \\
\hline
\end{tabular}




\begin{tabular}{|c|c|c|c|c|c|c|c|c|c|}
\hline $\begin{array}{l}\text { Study, Year, PMID, } \\
\text { Country }\end{array}$ & $\begin{array}{l}\text { Outcome } \\
\text { Measurement }\end{array}$ & Design & $\begin{array}{l}\text { Overall } \\
\text { RoB }\end{array}$ & $\begin{array}{l}\text { Time } \\
\text { Point }\end{array}$ & Arm & $\mathbf{N}$ & Mean (SD) & Effect Size $(95 \% \mathrm{Cl})$ & $\begin{array}{l}\mathbf{P} \\
\text { Value }\end{array}$ \\
\hline & $\begin{array}{l}\text { BREAST-Q: } \\
\text { Sexual well-being } \\
(0-100)\end{array}$ & NRCS & Moderate & $1 \mathrm{y}$ & AR with SIEA & NR & NR & vs. DIEP: adjMD -2.66 (-8.63, 3.31) & NR \\
\hline & $\begin{array}{l}\text { BREAST-Q: } \\
\text { Sexual well-being } \\
(0-100)\end{array}$ & NRCS & Moderate & $2 \mathrm{y}$ & DIEP & NR & NR & Ref & Ref \\
\hline & $\begin{array}{l}\text { BREAST-Q: } \\
\text { Sexual well-being } \\
(0-100)\end{array}$ & NRCS & Moderate & $2 y$ & Free TRAM & NR & NR & vs. DIEP: adjMD $2.35(-3.40,8.10)$ & NR \\
\hline & $\begin{array}{l}\text { BREAST-Q: } \\
\text { Sexual well-being } \\
(0-100)\end{array}$ & NRCS & Moderate & $2 y$ & Pedicled TRAM & NR & NR & vs. DIEP: adjMD -2.61 (-8.97, 3.75) & NR \\
\hline & $\begin{array}{l}\text { BREAST-Q: } \\
\text { Sexual well-being } \\
(0-100)\end{array}$ & NRCS & Moderate & $2 y$ & AR with SIEA & NR & NR & vs. DIEP: adjMD $1.97(-4.15,8.09)$ & NR \\
\hline \multirow[t]{3}{*}{$\begin{array}{l}\text { Macadam, 2016, } \\
26910656, \text { US, Canada, } \\
\text { \& Japan }\end{array}$} & $\begin{array}{l}\text { BREAST-Q: } \\
\text { Sexual well-being } \\
(0-100)\end{array}$ & NRCS & High & $\begin{array}{l}4.5- \\
7.3 \mathrm{y}\end{array}$ & DIEP & 387 & $59.0(21.5)$ & NR & NR \\
\hline & $\begin{array}{l}\text { BREAST-Q: } \\
\text { Sexual well-being } \\
(0-100)\end{array}$ & NRCS & High & $\begin{array}{l}4.5- \\
7.3 \mathrm{y}\end{array}$ & Pedicled TRAM & 359 & $57.3(24)$ & NR & NR \\
\hline & $\begin{array}{l}\text { BREAST-Q: } \\
\text { Sexual well-being } \\
(0-100)\end{array}$ & NRCS & High & $\begin{array}{l}4.5- \\
7.3 \mathrm{y}\end{array}$ & Muscle-sparing TRAM & 123 & $56.0(23.8)$ & NR & NR \\
\hline
\end{tabular}

Abbreviations: adj $=$ adjusted, $\mathrm{AR}=$ autologous reconstruction, $\mathrm{CI}=$ confidence interval, $\mathrm{DIEP}=$ deep inferior epigastric perforator, $\mathrm{LD}=$ latissimus dorsi, $\mathrm{MD}=$ mean difference,

$\mathrm{NR}=$ not reported, $\mathrm{NRCS}=$ nonrandomized comparative study, $\mathrm{PMID}=$ PubMed identifier, $\mathrm{RCT}=$ randomized controlled trial, $\mathrm{Ref}=$ reference group, RoB $=$ risk of bias, $\mathrm{SD}=$

standard deviation, SIEA = superficial inferior epigastric artery perforator, TRAM $=$ transverse rectus abdominis myocutaneous, $\mathrm{y}=$ years.

Colors: Header rows are shaded orange. Rows for every alternate study are shaded blue. The colors do not add unique information.

Table F-6.4. Full Evidence Table - Key Question 6: Comparison of flap types for AR - continuous outcomes (patient satisfaction with breast)

\begin{tabular}{|c|c|c|c|c|c|c|c|c|c|}
\hline $\begin{array}{l}\text { Study, Year, PMID, } \\
\text { Country }\end{array}$ & Outcome Measurement & Design & $\begin{array}{l}\text { Overall } \\
\text { RoB }\end{array}$ & $\begin{array}{l}\text { Time } \\
\text { Point }\end{array}$ & Arm & $\mathbf{N}$ & $\begin{array}{l}\text { Mean } \\
\text { (SD) }\end{array}$ & Effect Size $(95 \% \mathrm{CI})$ & $\begin{array}{l}P \\
\text { Value }\end{array}$ \\
\hline \multirow{3}{*}{$\begin{array}{l}\text { Brandberg, 2000, } \\
\text { 10626972, Sweden }\end{array}$} & Cosmetic (1-6) & RCT & High & $1 \mathrm{y}$ & AR with TRAM & 26 & $5.6(\mathrm{NR})$ & vs. LD: MD $0.36^{*}$ & NR \\
\hline & Cosmetic (1-6) & RCT & High & $1 \mathrm{y}$ & LD & 23 & $5.14(\mathrm{NR})$ & vs. LTD: MD -0.05* & NR \\
\hline & Cosmetic (1-6) & RCT & High & $1 \mathrm{y}$ & AR with LTD & 12 & $5.19(\mathrm{NR})$ & vs. TRAM: $-0.46^{*}$ & NR \\
\hline
\end{tabular}




\begin{tabular}{|c|c|c|c|c|c|c|c|c|c|}
\hline $\begin{array}{l}\text { Study, Year, PMID, } \\
\text { Country }\end{array}$ & Outcome Measurement & Design & $\begin{array}{l}\text { Overall } \\
\text { RoB }\end{array}$ & $\begin{array}{l}\text { Time } \\
\text { Point }\end{array}$ & Arm & $\mathbf{N}$ & $\begin{array}{l}\text { Mean } \\
\text { (SD) }\end{array}$ & Effect Size $(95 \% \mathrm{CI})$ & $\begin{array}{l}\mathbf{P} \\
\text { Value }\end{array}$ \\
\hline & Shape (1-6) & $\mathrm{RCT}$ & High & $1 \mathrm{y}$ & AR with TRAM & 26 & 5.3 (NR) & vs. LD: MD $0.36^{*}$ & NR \\
\hline & Shape (1-6) & RCT & High & $1 \mathrm{y}$ & LD & 23 & $4.94(\mathrm{NR})$ & vs. LTD: MD $0.35^{*}$ & NR \\
\hline & Shape (1-6) & RCT & High & $1 \mathrm{y}$ & AR with LTD & 12 & $4.59(\mathrm{NR})$ & vs. TRAM: MD $-0.71^{*}$ & NR \\
\hline & Size (1-6) & RCT & High & $1 \mathrm{y}$ & AR with TRAM & 26 & $5.43(\mathrm{NR})$ & vs. LD: MD $0.50^{*}$ & NR \\
\hline & Size $(1-6)$ & RCT & High & $1 \mathrm{y}$ & LD & 23 & 4.93 (NR) & vs. LTD: MD $-0.18^{*}$ & NR \\
\hline & Size (1-6) & RCT & High & $1 \mathrm{y}$ & AR with LTD & 12 & $5.11(\mathrm{NR})$ & vs. TRAM: MD $-0.32^{*}$ & NR \\
\hline & Scars on the breast (1-6) & RCT & High & $1 \mathrm{y}$ & AR with TRAM & 26 & 4.83 (NR) & vs. LD: MD $0.36^{*}$ & NR \\
\hline & Scars on the breast (1-6) & RCT & High & $1 \mathrm{y}$ & LD & 23 & $4.47(\mathrm{NR})$ & vs. LTD: MD $-0.65^{*}$ & NR \\
\hline & Scars on the breast (1-6) & RCT & High & $1 \mathrm{y}$ & AR with LTD & 12 & $5.12(\mathrm{NR})$ & vs. TRAM: MD 0.29* & NR \\
\hline & Donor site scars (1-6) & RCT & High & $1 \mathrm{y}$ & AR with TRAM & 26 & $4.76(\mathrm{NR})$ & vs. LD: MD $0.07^{*}$ & NR \\
\hline & Donor site scars (1-6) & RCT & High & $1 \mathrm{y}$ & LD & 23 & 4.69 (NR) & vs. LTD: MD $-0.26^{*}$ & NR \\
\hline & Donor site scars (1-6) & RCT & High & $1 \mathrm{y}$ & AR with LTD & 12 & $4.95(\mathrm{NR})$ & vs. TRAM: MD $0.19^{*}$ & NR \\
\hline & $\begin{array}{l}\text { Similarity with contralateral } \\
\text { breast }(1-6)\end{array}$ & $\mathrm{RCT}$ & High & $1 \mathrm{y}$ & AR with TRAM & 26 & 4.76 (NR) & vs. LD: MD $0.10^{*}$ & NR \\
\hline & $\begin{array}{l}\text { Similarity with contralateral } \\
\text { breast }(1-6)\end{array}$ & $\mathrm{RCT}$ & High & $1 \mathrm{y}$ & LD & 23 & 4.66 (NR) & vs. LTD: MD $0.81^{*}$ & NR \\
\hline & $\begin{array}{l}\text { Similarity with contralateral } \\
\text { breast }(1-6)\end{array}$ & RCT & High & $1 \mathrm{y}$ & AR with LTD & 12 & $3.85(\mathrm{NR})$ & vs. TRAM: MD -0.91* & NR \\
\hline \multirow[t]{8}{*}{$\begin{array}{l}\text { Erdmann-Sager, 2018, } \\
\text { 29019862, US \& Canada }\end{array}$} & $\begin{array}{l}\text { BREAST-Q: Satisfaction } \\
\text { with breast }(0-100)\end{array}$ & NRCS & Moderate & $1 \mathrm{y}$ & DIEP & NR & NR & Ref & Ref \\
\hline & $\begin{array}{l}\text { BREAST-Q: Satisfaction } \\
\text { with breast }(0-100)\end{array}$ & NRCS & Moderate & $1 \mathrm{y}$ & Free TRAM & NR & NR & $\begin{array}{l}\text { vs. DIEP: adjMD } 0.04 \\
(-4.56,4.63)\end{array}$ & NR \\
\hline & $\begin{array}{l}\text { BREAST-Q: Satisfaction } \\
\text { with breast }(0-100)\end{array}$ & NRCS & Moderate & $1 \mathrm{y}$ & Pedicled TRAM & NR & NR & $\begin{array}{l}\text { vs. DIEP: adjMD } 1.36 \\
(-3.45,6.17)\end{array}$ & NR \\
\hline & $\begin{array}{l}\text { BREAST-Q: Satisfaction } \\
\text { with breast }(0-100)\end{array}$ & NRCS & Moderate & $1 \mathrm{y}$ & AR with SIEA & NR & NR & $\begin{array}{l}\text { vs. DIEP: adjMD }-1.82 \\
(-6.37,2.72)\end{array}$ & NR \\
\hline & $\begin{array}{l}\text { BREAST-Q: Satisfaction } \\
\text { with breast }(0-100)\end{array}$ & NRCS & Moderate & $2 y$ & DIEP & NR & NR & Ref & Ref \\
\hline & $\begin{array}{l}\text { BREAST-Q: Satisfaction } \\
\text { with breast }(0-100)\end{array}$ & NRCS & Moderate & $2 y$ & Free TRAM & NR & NR & $\begin{array}{l}\text { vs. DIEP: adjMD -2.61 } \\
(-8.97,3.75)\end{array}$ & NR \\
\hline & $\begin{array}{l}\text { BREAST-Q: Satisfaction } \\
\text { with breast }(0-100)\end{array}$ & NRCS & Moderate & $2 y$ & Pedicled TRAM & NR & NR & $\begin{array}{l}\text { vs. DIEP: adjMD } 1.36 \\
(-3.45,6.17)\end{array}$ & NR \\
\hline & $\begin{array}{l}\text { BREAST-Q: Satisfaction } \\
\text { with breast }(0-100)\end{array}$ & NRCS & Moderate & $2 y$ & AR with SIEA & NR & NR & $\begin{array}{l}\text { vs. DIEP: adjMD } 0.42 \\
(-5.56,6.4)\end{array}$ & NR \\
\hline \multirow{3}{*}{$\begin{array}{l}\text { Macadam, 2016, } \\
\text { 26910656, US, Canada, \& } \\
\text { Japan }\end{array}$} & $\begin{array}{l}\text { BREAST-Q: Satisfaction } \\
\text { with breast }(0-100)\end{array}$ & NRCS & High & $\begin{array}{l}4.5- \\
7.3 \mathrm{y}\end{array}$ & DIEP & 387 & $\begin{array}{l}71.9 \\
(17.3) \\
\end{array}$ & NR & NR \\
\hline & $\begin{array}{l}\text { BREAST-Q: Satisfaction } \\
\text { with breast }(0-100)\end{array}$ & NRCS & High & $\begin{array}{l}4.5- \\
7.3 y\end{array}$ & Free TRAM & 74 & $71.7(21)$ & NR & NR \\
\hline & $\begin{array}{l}\text { BREAST-Q: Satisfaction } \\
\text { with breast }(0-100)\end{array}$ & NRCS & High & $\begin{array}{l}4.5- \\
7.3 y\end{array}$ & Pedicled TRAM & 359 & $\begin{array}{l}69.8 \\
(20.7) \\
\end{array}$ & NR & NR \\
\hline
\end{tabular}




\begin{tabular}{|c|c|c|c|c|c|c|c|c|c|}
\hline $\begin{array}{l}\text { Study, Year, PMID, } \\
\text { Country }\end{array}$ & Outcome Measurement & Design & $\begin{array}{l}\text { Overall } \\
\text { RoB }\end{array}$ & $\begin{array}{l}\text { Time } \\
\text { Point }\end{array}$ & Arm & $\mathbf{N}$ & $\begin{array}{l}\text { Mean } \\
\text { (SD) }\end{array}$ & Effect Size (95\% CI) & $\begin{array}{l}\mathbf{P} \\
\text { Value }\end{array}$ \\
\hline & $\begin{array}{l}\text { BREAST-Q: Satisfaction } \\
\text { with breast }(0-100)\end{array}$ & NRCS & High & $\begin{array}{l}4.5- \\
7.3 y\end{array}$ & Muscle-sparing TRAM & 123 & $\begin{array}{l}68.7 \\
(18.7)\end{array}$ & NR & NR \\
\hline
\end{tabular}

Abbreviations: adj = adjusted, $\mathrm{AR}=$ autologous reconstruction, $\mathrm{CI}=$ confidence interval, $\mathrm{DIEP}=$ deep inferior epigastric perforator, $\mathrm{LD}=$ latissimus dorsi, $\mathrm{LTD}=$ lateral

thoracodorsal flap, $\mathrm{MD}=$ mean difference, $\mathrm{NR}=$ not reported, NRCS $=$ nonrandomized comparative study, PMID $=\mathrm{PubMed}$ identifier, RCT $=$ randomized controlled trial, Ref $=$

reference group, $\mathrm{RoB}=$ risk of bias, $\mathrm{SD}=$ standard deviation, $\mathrm{SIEA}=$ superficial inferior epigastric artery perforator, $\mathrm{TRAM}=$ transverse rectus abdominis $\mathrm{myocutaneous,} \mathrm{y}=$

years.

Colors: Header rows are shaded orange. Rows for every alternate study are shaded blue. The colors do not add unique information.

* calculated

Table F-6.5. Full Evidence Table - Key Question 6: Comparison of flap types for AR - categorical outcomes (patient satisfaction with breast)

\begin{tabular}{|c|c|c|c|c|c|c|c|c|c|}
\hline Study, Year, PMID, Country & Outcome Measurement & Design & $\begin{array}{l}\text { Overall } \\
\text { RoB }\end{array}$ & $\begin{array}{l}\text { Time } \\
\text { Point }\end{array}$ & Arm & $\mathrm{n} / \mathrm{N}(\%)$ & Comparison & $\begin{array}{l}\text { Adjusted Odds } \\
\text { Ratio }(95 \% \mathrm{Cl})\end{array}$ & $\begin{array}{l}\mathbf{P} \\
\text { Value }\end{array}$ \\
\hline \multirow[t]{3}{*}{ Yueh, 2009, 19228537, US } & $\begin{array}{l}\text { Satisfied with breasts } \\
\text { (Yes/No) }\end{array}$ & NRCS & High & NR & DIEP & NR/117 (NR) & vs. TRAM & $0.67(0.37,1.23)$ & NR \\
\hline & $\begin{array}{l}\text { Satisfied with breasts } \\
\text { (Yes/No) }\end{array}$ & NRCS & High & NR & AR with TRAM & $102 / 143(71.3)$ & Ref & Ref & Ref \\
\hline & $\begin{array}{l}\text { Satisfied with breasts } \\
\text { (Yes/No) }\end{array}$ & NRCS & High & NR & LD & $68 / 112(60.7)$ & vs. TRAM & $0.78(0.54,1.14)$ & NR \\
\hline
\end{tabular}

Abbreviations: $\mathrm{AR}=$ autologous reconstruction, $\mathrm{CI}=$ confidence interval, $\mathrm{DIEP}=$ deep inferior epigastric perforator, $\mathrm{LD}=$ latissimus dorsi, $\mathrm{NRCS}=$ nonrandomized comparative

study, PMID = PubMed identifier, Ref = reference group, RoB = risk of bias, TRAM = transverse rectus abdominis myocutaneous.

Colors: Header rows are shaded orange. The color does not add unique information.

Table F-6.6. Full Evidence Table - Key Question 6: Comparison of flap types for AR - continuous outcomes (patient satisfaction with outcome)

\begin{tabular}{|c|c|c|c|c|c|c|c|c|c|}
\hline $\begin{array}{l}\text { Study, Year, PMID, } \\
\text { Country }\end{array}$ & Outcome Measurement & Design & $\begin{array}{l}\text { Overall } \\
\text { RoB }\end{array}$ & $\begin{array}{l}\text { Time } \\
\text { Point }\end{array}$ & Arm & $\mathbf{N}$ & Mean (SD) & $\begin{array}{l}\text { Effect Size } \\
(95 \% \mathrm{Cl})\end{array}$ & $\begin{array}{l}\mathbf{P} \\
\text { Value } \\
\end{array}$ \\
\hline \multirow[t]{7}{*}{$\begin{array}{l}\text { Kulkarni, 2017, 28713853, } \\
\text { US \& Canada }\end{array}$} & $\begin{array}{l}\text { BREAST-Q: Satisfaction } \\
\text { with information }(0-100)\end{array}$ & NRCS & Moderate & $3 \mathrm{mo}$ & DIEP & 395 & $72.8(13)$ & NR & NR \\
\hline & $\begin{array}{l}\text { BREAST-Q: Satisfaction } \\
\text { with information }(0-100)\end{array}$ & NRCS & Moderate & $3 \mathrm{mo}$ & Free TRAM & NR & $72.8(16.5)$ & NR & NR \\
\hline & $\begin{array}{l}\text { BREAST-Q: Satisfaction } \\
\text { with information }(0-100)\end{array}$ & NRCS & Moderate & $3 \mathrm{mo}$ & Pedicled TRAM & 65 & $76.2(19.9)$ & NR & NR \\
\hline & $\begin{array}{l}\text { BREAST-Q: Satisfaction } \\
\text { with information }(0-100)\end{array}$ & NRCS & Moderate & $3 \mathrm{mo}$ & LD & 53 & $69.3(19.1)$ & NR & NR \\
\hline & $\begin{array}{l}\text { BREAST-Q: Satisfaction } \\
\text { with information }(0-100)\end{array}$ & NRCS & Moderate & $3 \mathrm{mo}$ & AR with SIEA & NR & NR & NR & NR \\
\hline & $\begin{array}{l}\text { BREAST-Q: Satisfaction } \\
\text { with surgeon }(0-100)\end{array}$ & NRCS & Moderate & $3 \mathrm{mo}$ & DIEP & 395 & $91.6(14)$ & NR & NR \\
\hline & $\begin{array}{l}\text { BREAST-Q: Satisfaction } \\
\text { with surgeon }(0-100)\end{array}$ & NRCS & Moderate & $3 \mathrm{mo}$ & Free TRAM & NR & NR & NR & NR \\
\hline
\end{tabular}




\begin{tabular}{|c|c|c|c|c|c|c|c|c|c|}
\hline $\begin{array}{l}\text { Study, Year, PMID, } \\
\text { Country }\end{array}$ & Outcome Measurement & Design & $\begin{array}{l}\text { Overall } \\
\text { RoB }\end{array}$ & $\begin{array}{l}\text { Time } \\
\text { Point }\end{array}$ & Arm & $\mathbf{N}$ & Mean (SD) & $\begin{array}{l}\text { Effect Size } \\
(95 \% \mathrm{Cl})\end{array}$ & $\begin{array}{l}\mathbf{P} \\
\text { Value }\end{array}$ \\
\hline & $\begin{array}{l}\text { BREAST-Q: Satisfaction } \\
\text { with surgeon }(0-100)\end{array}$ & NRCS & Moderate & $3 \mathrm{mo}$ & Pedicled TRAM & 65 & $91(15.9)$ & NR & NR \\
\hline & $\begin{array}{l}\text { BREAST-Q: Satisfaction } \\
\text { with surgeon }(0-100)\end{array}$ & NRCS & Moderate & $3 \mathrm{mo}$ & LD & 53 & $92.5(14.4)$ & NR & NR \\
\hline & $\begin{array}{l}\text { BREAST-Q: Satisfaction } \\
\text { with surgeon }(0-100)\end{array}$ & NRCS & Moderate & $3 \mathrm{mo}$ & AR with SIEA & NR & NR & NR & NR \\
\hline & $\begin{array}{l}\text { BREAST-Q: Satisfaction } \\
\text { with medical team (0- } \\
100)\end{array}$ & NRCS & Moderate & $3 \mathrm{mo}$ & DIEP & 395 & $90(19)$ & NR & NR \\
\hline & $\begin{array}{l}\text { BREAST-Q: Satisfaction } \\
\text { with medical team (0- } \\
100)\end{array}$ & NRCS & Moderate & $3 \mathrm{mo}$ & Free TRAM & NR & NR & NR & NR \\
\hline & $\begin{array}{l}\text { BREAST-Q: Satisfaction } \\
\text { with medical team (0- } \\
100)\end{array}$ & NRCS & Moderate & $3 \mathrm{mo}$ & Pedicled TRAM & 65 & $91(13.8)$ & NR & NR \\
\hline & $\begin{array}{l}\text { BREAST-Q: Satisfaction } \\
\text { with medical team (0- } \\
100)\end{array}$ & NRCS & Moderate & $3 \mathrm{mo}$ & LD & 53 & $94.5(12.7)$ & NR & NR \\
\hline & $\begin{array}{l}\text { BREAST-Q: Satisfaction } \\
\text { with medical team (0- } \\
100)\end{array}$ & NRCS & Moderate & $3 \mathrm{mo}$ & AR with SIEA & NR & NR & NR & NR \\
\hline & $\begin{array}{l}\text { BREAST-Q: Satisfaction } \\
\text { with office staff }(0-100)\end{array}$ & NRCS & Moderate & $3 \mathrm{mo}$ & DIEP & 395 & $93.8(13.4)$ & NR & NR \\
\hline & $\begin{array}{l}\text { BREAST-Q: Satisfaction } \\
\text { with office staff }(0-100)\end{array}$ & NRCS & Moderate & $3 \mathrm{mo}$ & Free TRAM & NR & NR & NR & NR \\
\hline & $\begin{array}{l}\text { BREAST-Q: Satisfaction } \\
\text { with office staff }(0-100)\end{array}$ & NRCS & Moderate & $3 \mathrm{mo}$ & Pedicled TRAM & 65 & $93.5(14.6)$ & NR & NR \\
\hline & $\begin{array}{l}\text { BREAST-Q: Satisfaction } \\
\text { with office staff }(0-100)\end{array}$ & NRCS & Moderate & $3 \mathrm{mo}$ & LD & 53 & $94.7(14.6)$ & NR & NR \\
\hline & $\begin{array}{l}\text { BREAST-Q: Satisfaction } \\
\text { with office staff }(0-100)\end{array}$ & NRCS & Moderate & $3 \mathrm{mo}$ & AR with SIEA & NR & NR & NR & NR \\
\hline \multirow[t]{4}{*}{$\begin{array}{l}\text { Macadam, 2016, 26910656, } \\
\text { US, Canada, \& Japan }\end{array}$} & $\begin{array}{l}\text { BREAST-Q: Satisfaction } \\
\text { with outcome }(0-100)\end{array}$ & NRCS & High & $\begin{array}{l}4.5- \\
7.3 y\end{array}$ & DIEP & 387 & $78.6(19.3)$ & NR & NR \\
\hline & $\begin{array}{l}\text { BREAST-Q: Satisfaction } \\
\text { with outcome }(0-100)\end{array}$ & NRCS & High & $\begin{array}{l}4.5- \\
7.3 y\end{array}$ & Free TRAM & 74 & $76.4(22.2)$ & NR & NR \\
\hline & $\begin{array}{l}\text { BREAST-Q: Satisfaction } \\
\text { with outcome }(0-100)\end{array}$ & NRCS & High & $\begin{array}{l}4.5- \\
7.3 y\end{array}$ & Pedicled TRAM & 359 & $73.9(24.2)$ & NR & NR \\
\hline & $\begin{array}{l}\text { BREAST-Q: Satisfaction } \\
\text { with outcome }(0-100)\end{array}$ & NRCS & High & $\begin{array}{l}4.5- \\
7.3 y\end{array}$ & Muscle-sparing TRAM & 123 & $72.9(23.3)$ & NR & NR \\
\hline
\end{tabular}

Abbreviations: $\mathrm{Adj}=$ adjusted, $\mathrm{AR}=$ autologous reconstruction, $\mathrm{CI}=$ confidence interval, DIEP $=$ deep inferior epigastric perforator, $\mathrm{LD}=$ latissimus dorsi, $\mathrm{LTD}=$ lateral thoracodorsal flap, $\mathrm{MD}=$ mean difference, $\mathrm{mo}=$ months, $\mathrm{NR}=$ not reported, $\mathrm{NRCS}=$ nonrandomized comparative study, $\mathrm{PMID}=\mathrm{Pubmed}$ identifier, $\mathrm{RCT}=$ randomized 
controlled trial, $\mathrm{RoB}=$ risk of bias, $\mathrm{SD}=$ standard deviation, $\mathrm{SIEA}=$ superficial inferior epigastric artery perforator, $\mathrm{TRAM}=$ transverse rectus abdominis myocutaneous, $\mathrm{y}=$ years.

Colors: Header rows are shaded orange. Rows for every alternate study are shaded blue. The colors do not add unique information.

Table F-6.7. Full Evidence Table - Key Question 1: IBR versus AR - categorical outcomes (patient satisfaction with outcome)

\begin{tabular}{|c|c|c|c|c|c|c|c|c|c|}
\hline Study, Year, PMID, Country & Outcome Measurement & Design & $\begin{array}{l}\text { Overall } \\
\text { RoB }\end{array}$ & $\begin{array}{l}\text { Time } \\
\text { Point }\end{array}$ & Arm & $\mathrm{n} / \mathrm{N}(\%)$ & Comparison & $\begin{array}{l}\text { Adjusted Odds } \\
\text { Ratio }(95 \% \mathrm{Cl}) \\
\end{array}$ & $\begin{array}{l}\mathbf{P} \\
\text { Value }\end{array}$ \\
\hline \multirow[t]{3}{*}{ Yueh, 2009, 19228537, US } & $\begin{array}{l}\text { Generally satisfied with } \\
\text { outcome (Yes/No) }\end{array}$ & NRCS & High & NR & DIEP & NR/117 (NR) & vs. TRAM & $0.82(0.33,2.01)$ & NS \\
\hline & $\begin{array}{l}\text { Generally satisfied with } \\
\text { outcome (Yes/No) }\end{array}$ & NRCS & High & NR & TRAM & $98 / 143(68.5)$ & Ref & Ref & Ref \\
\hline & $\begin{array}{l}\text { Generally satisfied with } \\
\text { outcome (Yes/No) }\end{array}$ & NRCS & High & NR & LD & $63 / 112(56.3)$ & vs. TRAM & $0.77(0.53,1.11)$ & NS \\
\hline
\end{tabular}

Abbreviations: $\mathrm{AR}=$ autologous reconstruction, $\mathrm{CI}=$ confidence interval, $\mathrm{DIEP}=$ deep inferior epigastric perforator, $\mathrm{LD}=$ latissimus dorsi, $\mathrm{NRCS}=$ nonrandomized comparative

study, NR = not reported, NS = not significant, PMID $=$ PubMed identifier, Ref $=$ reference group, RoB $=$ risk of bias, TRAM $=$ transverse rectus abdominis myocutaneous.

Colors: Header rows are shaded orange. The color does not add unique information.

Table F-6.8. Full Evidence Table - Key Question 6: Comparison of flap types for AR - categorical outcomes (recurrence of breast cancer)

\begin{tabular}{|c|c|c|c|c|c|c|c|c|}
\hline Study, Year, PMID, Country & Design & Overall RoB & $\begin{array}{l}\text { Time } \\
\text { Point }\end{array}$ & Arm & $\mathrm{n} / \mathrm{N}(\%)$ & Comparison & Odds Ratio $(95 \% \mathrm{Cl})$ & P Value \\
\hline \multirow[t]{3}{*}{ Brandberg, 2000, 10626972, Sweden } & RCT & High & $1 \mathrm{y}$ & AR with TRAM & $2 / 29(6.9 \%)$ & vs. LD & $2.15(0.18,25.07)^{*}$ & 0.54 \\
\hline & $\mathrm{RCT}$ & High & $1 \mathrm{y}$ & LD & $1 / 30(3.33 \%)$ & vs. LTD & $1.07(0.03,33.69)^{*}$ & 0.97 \\
\hline & RCT & High & $1 \mathrm{y}$ & AR with LTD & $0 / 16(0 \%)$ & vs. TRAM & $0.44(0.02,10.28)^{*}$ & 0.61 \\
\hline
\end{tabular}

Abbreviations: $\mathrm{AR}=$ autologous reconstruction, $\mathrm{CI}=$ confidence interval, $\mathrm{LD}=$ latissimus dorsi, $\mathrm{LTD}=$ lateral thoracodorsal flap, $\mathrm{RCT}=$ randomized controlled trial, $\mathrm{PMID}=$

PubMed identifier, RoB $=$ risk of bias, TRAM $=$ transverse rectus abdominis myocutaneous.

* calculated

Colors: Header rows are shaded orange. The color does not add unique information.

Table F-6.9. Full Evidence Table - Key Question 6: Comparison of flap types for AR - continuous outcomes (duration of initial hospitalization)

\begin{tabular}{|c|c|c|c|c|c|c|c|c|}
\hline Study, Year, PMID, Country & Design & $\begin{array}{l}\text { Overall } \\
\text { RoB }\end{array}$ & $\begin{array}{l}\text { Time } \\
\text { Point }\end{array}$ & Arm & $\mathbf{N}$ & Mean (SD or Range) & $\begin{array}{l}\text { Adjusted Mean } \\
\text { Difference }(95 \% \mathrm{CI})\end{array}$ & $\begin{array}{l}P \\
\text { Value }\end{array}$ \\
\hline \multirow[t]{2}{*}{ Rindom, 2019, 31515191, Denmark } & RCT & Moderate & Post-op & LD & 18 & $6.4 \mathrm{~d}$ (range 3-12 d) & Ref & Ref \\
\hline & RCT & Moderate & Post-op & TAP & 22 & $6.5 \mathrm{~d}$ (range 4-14 d) & $0.9 \mathrm{~d}(-1.4,3.2)$ & 0.45 \\
\hline \multirow[t]{2}{*}{ Zoghbi, 2017, 28052051, US } & NRCS & High & Post-op & DIEP & 9699 & $4.68 \mathrm{~d}(2.80 \mathrm{~d})$ & Ref & Ref \\
\hline & NRCS & High & Post-op & AR with TRAM & 6137 & $4.79 \mathrm{~d}(2.69 \mathrm{~d})$ & NR & $<0.001$ \\
\hline
\end{tabular}

Abbreviations: $\mathrm{CI}=$ confidence interval, $\mathrm{d}=$ days, DIEP $=$ deep inferior epigastric perforator, $\mathrm{LD}=$ latissimus dorsi, $\mathrm{NR}=$ not reported, $\mathrm{NRCS}=$ nonrandomized comparative study, $\mathrm{PMID}=$ Pubmed identifier, $\mathrm{RCT}=$ randomized controlled trial, $\mathrm{Ref}=$ reference group, $\mathrm{RoB}=$ risk of bias, $\mathrm{SD}=$ standard deviation, $\mathrm{TAP}=$ thoracodorsal artery perforator, TRAM $=$ transverse rectus abdominis myocutaneous.

Colors: Header rows are shaded orange. Rows for every alternate study are shaded blue. The colors do not add unique information. 
Table F-6.10. Full Evidence Table - Key Question 6: Comparison of flap types for AR - categorical outcomes (duration of initial hospitalization)

\begin{tabular}{|c|c|c|c|c|c|c|c|c|}
\hline Study, Year, PMID, Country & Outcome Measurement & Design & $\begin{array}{l}\text { Overall } \\
\text { RoB }\end{array}$ & Time Point & Arm & $\mathrm{n} / \mathrm{N}(\%)$ & $\begin{array}{l}\text { Adjusted Odds } \\
\text { Ratio }(95 \% \mathrm{Cl})\end{array}$ & $\begin{array}{l}P \\
\text { Value }\end{array}$ \\
\hline \multirow[t]{2}{*}{ Zoghbi, 2017, 28052051, US } & Increased length of stay & NRCS & High & Post-surgery & DIEP & NR/9699 (NR) & Ref & Ref \\
\hline & Increased length of stay & NRCS & High & Post-surgery & AR with TRAM & NR/6137 (NR) & $1.59(1.45,1.72)$ & $<0.001$ \\
\hline
\end{tabular}

Abbreviations: $\mathrm{AR}=$ autologous reconstruction, $\mathrm{CI}=$ confidence interval, $\mathrm{DIEP}=$ deep inferior epigastric perforator, $\mathrm{NR}=$ not reported, $\mathrm{NRCS}=$ nonrandomized comparative study, PMID = PubMed identifier, Ref = reference group, RoB = risk of bias, TRAM = transverse rectus abdominis myocutaneous.

Colors: Header rows are shaded orange. The color does not add unique information.

Table F-6.11. Full Evidence Table - Question 6: Comparison of flap types for AR - categorical outcomes (mortality)

\begin{tabular}{|c|c|c|c|c|c|c|c|c|}
\hline Study, Year, PMID, Country & Design & Overall RoB & $\begin{array}{l}\text { Time } \\
\text { Point }\end{array}$ & Arm & $\mathrm{n} / \mathrm{N}(\%)$ & Comparison & Odds Ratio (95\% CI) & P Value \\
\hline \multirow[t]{3}{*}{ Brandberg, 2000, 10626972, Sweden } & RCT & High & $1 \mathrm{y}$ & AR with TRAM & $1 / 29(3.5)$ & vs. LD & $0.50(0.04,5.83)^{\star}$ & 0.58 \\
\hline & RCT & High & $1 \mathrm{y}$ & LD & $2 / 30(6.7)$ & vs. LTD & $2.21(0.09,52.2)^{\star}$ & 0.62 \\
\hline & RCT & High & $1 \mathrm{y}$ & AR with LTD & $0 / 16(0)$ & vs. TRAM & $0.9(0.03,28.5)^{*}$ & 0.95 \\
\hline
\end{tabular}

Abbreviations: $\mathrm{AR}=$ autologous reconstruction, $\mathrm{CI}=$ confidence interval, $\mathrm{LD}=$ latissimus dorsi, $\mathrm{LTD}=$ lateral thoracodorsal flap, $\mathrm{RCT}=$ randomized controlled trial, $\mathrm{PMID}=$

PubMed identifier, $\mathrm{RoB}=$ risk of bias, TRAM = transverse rectus abdominis myocutaneous, $\mathrm{y}=$ years

Colors: Header rows are shaded orange. The color does not add unique information.

* calculated

Table F-6.12. Full Evidence Table - Question 6: Comparison of flap types for AR - categorical outcomes (unplanned repeat surgeries for revision of reconstruction)

\begin{tabular}{|c|c|c|c|c|c|c|c|c|}
\hline Study, Year, PMID, Country & Design & $\begin{array}{l}\text { Overall } \\
\text { RoB }\end{array}$ & $\begin{array}{l}\text { Time } \\
\text { Point }\end{array}$ & Arm & $\mathrm{n} / \mathrm{N}(\%)$ & Comparison & $\begin{array}{l}\text { Adjusted Odds } \\
\text { Ratio }(95 \% \mathrm{Cl})\end{array}$ & $\begin{array}{l}P \\
\text { Value }\end{array}$ \\
\hline \multirow{5}{*}{ Kulkarni, 2017, 28713853, US \& Canada } & NRCS & Moderate & $2 \mathrm{y}$ & DIEP & $223 / 350(63.7)$ & NR & NR & NR \\
\hline & NRCS & Moderate & $2 y$ & Free TRAM & $56 / 87(64.4)$ & NR & NR & NR \\
\hline & NRCS & Moderate & $2 y$ & Pedicled TRAM & $40 / 77(57.1)$ & NR & NR & NR \\
\hline & NRCS & Moderate & $2 y$ & LD & $41 / 64(64.1)$ & NR & NR & NR \\
\hline & NRCS & Moderate & $2 y$ & AR with SIEA & $33 / 62(53.2)$ & NR & NR & NR \\
\hline \multirow[t]{3}{*}{ Massenburg, 2015, 26487657, US } & NRCS & High & $2 y$ & Free TRAM & $95 / 609(15.6)$ & vs. LD & $2.03(1.39,2.96)$ & NR \\
\hline & NRCS & High & $2 y$ & Pedicled TRAM & $159 / 1608(9.9)$ & vs. LD & $1.71(1.25,2.33)$ & NR \\
\hline & NRCS & High & $2 y$ & LD & $62 / 1079(5.7)$ & Ref & Ref & Ref \\
\hline
\end{tabular}

Abbreviations: $\mathrm{AR}=$ autologous reconstruction, $\mathrm{CI}=$ confidence interval, $\mathrm{DIEP}=$ deep inferior epigastric perforator, $\mathrm{LD}=$ latissimus dorsi, $\mathrm{NR}=$ not reported, $\mathrm{NRCS}=$

nonrandomized comparative study, PMID = PubMed identifier, Ref = reference group, RoB $=$ risk of bias, SIEA = superficial inferior epigastric artery, TRAM $=$ transverse rectus abdominis myocutaneous, $\mathrm{y}=$ years.

Colors: Header rows are shaded orange. Rows for every alternate study are shaded blue. The colors do not add unique information. 
Table F-6.13. Full Evidence Table - Key Question 6: IBR versus AR - comparison of flap types for AR - continuous outcomes (pain, including chronic pain)

\begin{tabular}{|c|c|c|c|c|c|c|c|c|c|}
\hline Study, Year, PMID, Country & Outcome Measurement & Design & $\begin{array}{l}\text { Overall } \\
\text { RoB }\end{array}$ & $\begin{array}{l}\text { Time } \\
\text { Point }\end{array}$ & Arm & $\mathbf{N}$ & $\begin{array}{l}\text { Mean } \\
\text { (SD) }\end{array}$ & $\begin{array}{l}\text { Effect Size } \\
(95 \% \mathrm{Cl})\end{array}$ & $\begin{array}{l}P \\
\text { Value }\end{array}$ \\
\hline \multirow{10}{*}{$\begin{array}{l}\text { Kulkarni, 2017, 28713853, US \& } \\
\text { Canada }\end{array}$} & MPQ-SF: Sensory (0-10) & NRCS & Moderate & $3 \mathrm{mo}$ & DIEP & 296 & 4.8 (NR) & NR & NR \\
\hline & MPQ-SF: Sensory $(0-10)$ & NRCS & Moderate & $3 \mathrm{mo}$ & Free TRAM & 83 & $6.7(\mathrm{NR})$ & NR & NR \\
\hline & MPQ-SF: Sensory (0-10) & NRCS & Moderate & $3 \mathrm{mo}$ & Pedicled TRAM & 91 & $5(\mathrm{NR})$ & NR & NR \\
\hline & MPQ-SF: Sensory $(0-10)$ & NRCS & Moderate & $3 \mathrm{mo}$ & LD & 62 & $5.4(\mathrm{NR})$ & NR & NR \\
\hline & MPQ-SF: Sensory (0-10) & NRCS & Moderate & $3 \mathrm{mo}$ & AR with SIEA & 56 & NR & NR & NR \\
\hline & NPRS (0-10) & NRCS & Moderate & $3 \mathrm{mo}$ & DIEP & 296 & $1.5(\mathrm{NR})$ & NR & NR \\
\hline & NPRS (0-10) & NRCS & Moderate & $3 \mathrm{mo}$ & Free TRAM & 83 & $1.5(\mathrm{NR})$ & NR & NR \\
\hline & NPRS (0-10) & NRCS & Moderate & $3 \mathrm{mo}$ & Pedicled TRAM & 91 & $1.8(\mathrm{NR})$ & NR & NR \\
\hline & NPRS (0-10) & NRCS & Moderate & $3 \mathrm{mo}$ & LD & 62 & 2 (NR) & NR & NR \\
\hline & NPRS (0-10) & NRCS & Moderate & $3 \mathrm{mo}$ & AR with SIEA & 56 & $1.4(\mathrm{NR})$ & NR & NR \\
\hline
\end{tabular}

Abbreviations: $\mathrm{AR}=$ autologous reconstruction, $\mathrm{CI}=$ confidence interval, $\mathrm{DIEP}=$ deep inferior epigastric perforator, $\mathrm{LD}=$ latissimus dorsi, $\mathrm{mo}=\mathrm{months}, \mathrm{MPQ}-\mathrm{SF}=\mathrm{McGill}$ Pain Questionnaire-Short Form, NPRS $=$ Numerical Pain Rating Scale, NR = not reported, NRCS $=$ nonrandomized comparative study, PMID $=$ Pubmed identifier, RoB $=$ risk of bias, $\mathrm{SD}=$ standard deviation, SIEA = superficial inferior epigastric artery, TRAM = transverse rectus abdominis myocutaneous.

Colors: Header rows are shaded orange. The color does not add unique information.

Table F-6.14. Full Evidence Table - Key Question 6: IBR versus AR - comparison of flap types for AR - categorical outcomes (pain, including chronic pain)

\begin{tabular}{|c|c|c|c|c|c|c|c|c|c|}
\hline Study, Year, PMID, Country & Outcome Measurement & Design & $\begin{array}{l}\text { Overall } \\
\text { RoB }\end{array}$ & $\begin{array}{l}\text { Time } \\
\text { Point }\end{array}$ & Arm & $\mathrm{n} / \mathrm{N}(\%)$ & Comparison & $\begin{array}{l}\text { Odds Ratio (95\% } \\
\mathrm{Cl})\end{array}$ & P Value \\
\hline \multirow[t]{2}{*}{$\begin{array}{l}\text { Rindom, 2019, 31515191, } \\
\text { Denmark }\end{array}$} & $\begin{array}{l}\text { Shoulder related pain } \\
\text { (Yes/No) }\end{array}$ & $\mathrm{RCT}$ & Moderate & $1 \mathrm{y}$ & LD & $13 / 18(72 \%)$ & Ref & Ref & Ref \\
\hline & $\begin{array}{l}\text { Shoulder related pain } \\
\text { (Yes/No) }\end{array}$ & $\mathrm{RCT}$ & Moderate & $1 \mathrm{y}$ & TAP & $7 / 22(32 \%)$ & vs. LD & $0.05(0.005,0.51)$ & 0.011 \\
\hline
\end{tabular}

Abbreviations: $\mathrm{AR}=$ autologous reconstruction, $\mathrm{CI}=$ confidence interval, $\mathrm{LD}=$ latissimus dorsi, $\mathrm{PMID}=$ Pubmed identifier, $\mathrm{RCT}=$ randomized controlled trial, Ref $=$ reference group, $\mathrm{RoB}=$ risk of bias, TAP $=$ thoracodorsal artery perforator, $\mathrm{y}=$ years.

Colors: Header rows are shaded orange. The color does not add unique information.

Table F-6.15. Full Evidence Table - Key Question 6: Comparison of flap types for AR - categorical outcomes (necrosis)

\begin{tabular}{|c|c|c|c|c|c|c|c|c|c|}
\hline $\begin{array}{l}\text { Study, Year, PMID, } \\
\text { Country }\end{array}$ & Outcome Description & Design & $\begin{array}{l}\text { Overall } \\
\text { RoB }\end{array}$ & $\begin{array}{l}\text { Time } \\
\text { Point }\end{array}$ & Arm & $\mathrm{n} / \mathrm{N}(\%)$ & Comparison & $\begin{array}{l}\text { Adjusted Odds } \\
\text { Ratio }(95 \% \mathrm{CI})\end{array}$ & $\begin{array}{l}\mathbf{P} \\
\text { Value }\end{array}$ \\
\hline \multirow[t]{3}{*}{$\begin{array}{l}\text { Rindom, 2019, 31515191, } \\
\text { Denmark }\end{array}$} & $\begin{array}{l}\text { Major necrosis requiring } \\
\text { removal of the implant }\end{array}$ & RCT & Moderate & $1 \mathrm{y}$ & LD & $0 / 18(0)$ & Ref & Ref & Ref \\
\hline & $\begin{array}{l}\text { Major necrosis requiring } \\
\text { removal of the implant }\end{array}$ & RCT & Moderate & $1 \mathrm{y}$ & TAP & $1 / 22(4.54)$ & vs. LD & $1.67(0.05,52.7)^{\star}$ & $0.77^{*}$ \\
\hline & $\begin{array}{l}\text { Minor necrosis: } \\
\text { epidermolysis or small } \\
\text { necrosis of most distal } \\
\text { part of flap }\end{array}$ & RCT & Moderate & $1 \mathrm{y}$ & LD & $0 / 18(0)$ & Ref & Ref & Ref \\
\hline
\end{tabular}




\begin{tabular}{|c|c|c|c|c|c|c|c|c|c|}
\hline $\begin{array}{l}\text { Study, Year, PMID, } \\
\text { Country }\end{array}$ & Outcome Description & Design & \begin{tabular}{|l|} 
Overall \\
RoB
\end{tabular} & $\begin{array}{l}\text { Time } \\
\text { Point }\end{array}$ & Arm & $\mathrm{n} / \mathrm{N}(\%)$ & Comparison & $\begin{array}{l}\text { Adjusted Odds } \\
\text { Ratio (95\% CI) }\end{array}$ & $\begin{array}{l}\mathbf{P} \\
\text { Value }\end{array}$ \\
\hline & $\begin{array}{l}\text { Minor necrosis: } \\
\text { epidermolysis or small } \\
\text { necrosis of most distal } \\
\text { part of flap }\end{array}$ & RCT & Moderate & $1 \mathrm{y}$ & TAP & $2 / 22(13.6)$ & vs. LD & $5.53(0.26,118.3)^{\star}$ & $0.27^{*}$ \\
\hline \multirow{2}{*}{$\begin{array}{l}\text { Abedi, 2016, 25003437, } \\
\text { Canada }\end{array}$} & Mastectomy flap necrosis & NRCS & High & $1.6-1.9 y$ & DIEP & $20 / 83(24.1)$ & vs. TRAM & NR & 0.61 \\
\hline & Mastectomy flap necrosis & NRCS & High & $1.6-1.9 \mathrm{y}$ & AR with TRAM & $40 / 312(12.8)$ & Ref & Ref & Ref \\
\hline \multirow[t]{3}{*}{$\begin{array}{l}\text { Baumann, 2010, } \\
\text { 20440154, US }\end{array}$} & Fat necrosis & NRCS & High & $\begin{array}{l}\text { Mean } 1.2 \\
\mathrm{y}\end{array}$ & DIEP & $14 / 71(20)$ & NR & NR & NR \\
\hline & Fat necrosis & NRCS & High & $\begin{array}{l}\text { Mean } 1.2 \\
\mathrm{y}\end{array}$ & AR with TRAM & $13 / 120(11)$ & NR & NR & NR \\
\hline & Fat necrosis & NRCS & High & $\begin{array}{l}\text { Mean } 1.2 \\
\mathrm{y}\end{array}$ & AR with SIEA & $5 / 37(14)$ & NR & NR & NR \\
\hline \multirow{4}{*}{$\begin{array}{l}\text { Carramaschi, 1989, } \\
\text { 2602589, France }\end{array}$} & Local necrosis & NRCS & High & NR & AR with TRAM & $0 / 40(0)$ & NR & NR & NR \\
\hline & Local necrosis & NRCS & High & NR & LD & $1 / 34(2.9)$ & NR & NR & NR \\
\hline & More than local necrosis & NRCS & High & NR & AR with TRAM & $5 / 40(12.5)$ & NR & NR & NR \\
\hline & More than local necrosis & NRCS & High & NR & LD & $2 / 34(5.9)$ & NR & NR & NR \\
\hline \multirow{14}{*}{$\begin{array}{l}\text { Erdmann-Sager, 2018, } \\
\text { 29019862, US \& Canada }\end{array}$} & Donor site necrosis & NRCS & Moderate & $2 y$ & DIEP & $25 / 355(7)$ & NR & NR & NR \\
\hline & Donor site necrosis & NRCS & Moderate & $2 y$ & Free TRAM & $2 / 92(2.2)$ & NR & NR & NR \\
\hline & Donor site necrosis & NRCS & Moderate & $2 y$ & Pedicled TRAM & $2 / 78(2.6)$ & NR & NR & NR \\
\hline & Donor site necrosis & NRCS & Moderate & $2 y$ & AR with SIEA & $5 / 62(8.1)$ & NR & NR & NR \\
\hline & $\begin{array}{l}\text { Donor site chronic fat } \\
\text { necrosis }\end{array}$ & NRCS & Moderate & $2 y$ & DIEP & $12 / 355(3.4)$ & NR & NR & NR \\
\hline & $\begin{array}{l}\text { Donor site chronic fat } \\
\text { necrosis }\end{array}$ & NRCS & Moderate & $2 y$ & Free TRAM & $1 / 92(1.1)$ & NR & NR & NR \\
\hline & $\begin{array}{l}\text { Donor site chronic fat } \\
\text { necrosis }\end{array}$ & NRCS & Moderate & $2 y$ & Pedicled TRAM & $1 / 78(1.3)$ & NR & NR & NR \\
\hline & $\begin{array}{l}\text { Donor site chronic fat } \\
\text { necrosis }\end{array}$ & NRCS & Moderate & $2 y$ & AR with SIEA & $2 / 62(3.2)$ & NR & NR & NR \\
\hline & $\begin{array}{l}\text { Breast chronic fat } \\
\text { necrosis }\end{array}$ & NRCS & Moderate & $2 y$ & DIEP & $34 / 355(9.6)$ & NR & NR & NR \\
\hline & $\begin{array}{l}\text { Breast chronic fat } \\
\text { necrosis }\end{array}$ & NRCS & Moderate & $2 y$ & Free TRAM & $6 / 92(6.5)$ & NR & NR & NR \\
\hline & $\begin{array}{l}\text { Breast chronic fat } \\
\text { necrosis }\end{array}$ & NRCS & Moderate & $2 y$ & Pedicled TRAM & $8 / 78(10.3)$ & NR & NR & NR \\
\hline & $\begin{array}{l}\text { Breast chronic fat } \\
\text { necrosis }\end{array}$ & NRCS & Moderate & $2 y$ & AR with SIEA & $7 / 62(11.3)$ & NR & NR & NR \\
\hline & $\begin{array}{l}\text { Mastectomy skin flap } \\
\text { necrosis }\end{array}$ & NRCS & Moderate & $2 y$ & DIEP & $31 / 355(8.7)$ & NR & NR & NR \\
\hline & $\begin{array}{l}\text { Mastectomy skin flap } \\
\text { necrosis }\end{array}$ & NRCS & Moderate & $2 y$ & Free TRAM & $6 / 92(6.5)$ & NR & NR & NR \\
\hline
\end{tabular}




\begin{tabular}{|c|c|c|c|c|c|c|c|c|c|}
\hline $\begin{array}{l}\text { Study, Year, PMID, } \\
\text { Country }\end{array}$ & Outcome Description & Design & $\begin{array}{l}\text { Overall } \\
\text { RoB }\end{array}$ & $\begin{array}{l}\text { Time } \\
\text { Point }\end{array}$ & Arm & $\mathrm{n} / \mathrm{N}(\%)$ & Comparison & $\begin{array}{l}\text { Adjusted Odds } \\
\text { Ratio }(95 \% \mathrm{Cl})\end{array}$ & $\begin{array}{l}\mathbf{P} \\
\text { Value }\end{array}$ \\
\hline & $\begin{array}{l}\text { Mastectomy skin flap } \\
\text { necrosis }\end{array}$ & NRCS & Moderate & $2 y$ & Pedicled TRAM & $6 / 78(7.7)$ & NR & NR & NR \\
\hline & $\begin{array}{l}\text { Mastectomy skin flap } \\
\text { necrosis }\end{array}$ & NRCS & Moderate & $2 y$ & AR with SIEA & $6 / 62(9.7)$ & NR & NR & NR \\
\hline \multirow{2}{*}{$\begin{array}{l}\text { Israeli, 2014, 24572840, } \\
\text { US }\end{array}$} & Skin or fat necrosis & NRCS & High & $1.5 y$ & AR with TRAM & $16 / 252(6.4)$ & NR & NR & NR \\
\hline & & & & & LD & $5 / 302(1.7)$ & NR & NR & NR \\
\hline \multirow[t]{16}{*}{$\begin{array}{l}\text { Kulkarni, 2017, 28713853, } \\
\text { US \& Canada }\end{array}$} & $\begin{array}{l}\text { Chronic fat necrosis: } \\
\text { Breast }\end{array}$ & NRCS & Moderate & $1 \mathrm{y}$ & DIEP & $33 / 365(9)$ & NR & NR & NR \\
\hline & $\begin{array}{l}\text { Chronic fat necrosis: } \\
\text { Breast }\end{array}$ & NRCS & Moderate & $1 \mathrm{y}$ & Free TRAM & $5 / 97(5.2)$ & NR & NR & NR \\
\hline & $\begin{array}{l}\text { Chronic fat necrosis: } \\
\text { Breast }\end{array}$ & NRCS & Moderate & $1 \mathrm{y}$ & Pedicled TRAM & $6 / 84(7.1)$ & $\mathrm{NR}$ & NR & NR \\
\hline & $\begin{array}{l}\text { Chronic fat necrosis: } \\
\text { Breast }\end{array}$ & NRCS & Moderate & $1 \mathrm{y}$ & LD & $0 / 73(0)$ & NR & NR & NR \\
\hline & $\begin{array}{l}\text { Chronic fat necrosis: } \\
\text { Donor site }\end{array}$ & NRCS & Moderate & $1 \mathrm{y}$ & DIEP & $7 / 365$ (1.9) & NR & NR & NR \\
\hline & $\begin{array}{l}\text { Chronic fat necrosis: } \\
\text { Donor site }\end{array}$ & NRCS & Moderate & $1 \mathrm{y}$ & Free TRAM & $0 / 97(0)$ & NR & NR & NR \\
\hline & $\begin{array}{l}\text { Chronic fat necrosis: } \\
\text { Donor site }\end{array}$ & NRCS & Moderate & $1 \mathrm{y}$ & Pedicled TRAM & $2 / 84(2.4)$ & NR & NR & NR \\
\hline & $\begin{array}{l}\text { Chronic fat necrosis: } \\
\text { Donor site }\end{array}$ & NRCS & Moderate & $1 \mathrm{y}$ & LD & $0 / 73(0)$ & $\mathrm{NR}$ & NR & NR \\
\hline & Donor site necrosis & NRCS & Moderate & $1 \mathrm{y}$ & DIEP & $19 / 365(5.2)$ & NR & NR & NR \\
\hline & Donor site necrosis & NRCS & Moderate & $1 \mathrm{y}$ & Free TRAM & $2 / 97(2.1)$ & NR & NR & NR \\
\hline & Donor site necrosis & NRCS & Moderate & $1 y$ & Pedicled TRAM & $1 / 84(1.2)$ & NR & NR & NR \\
\hline & Donor site necrosis & NRCS & Moderate & $1 \mathrm{y}$ & LD & $0 / 73(0)$ & NR & NR & NR \\
\hline & $\begin{array}{l}\text { Acute partial flap } \\
\text { necrosis }\end{array}$ & NRCS & Moderate & $1 \mathrm{y}$ & DIEP & $9 / 365(2.5)$ & NR & NR & NR \\
\hline & $\begin{array}{l}\text { Acute partial flap } \\
\text { necrosis }\end{array}$ & NRCS & Moderate & $1 \mathrm{y}$ & Free TRAM & $5 / 97(5.2)$ & NR & NR & NR \\
\hline & $\begin{array}{l}\text { Acute partial flap } \\
\text { necrosis }\end{array}$ & NRCS & Moderate & $1 \mathrm{y}$ & Pedicled TRAM & $10 / 84(11.9)$ & NR & NR & NR \\
\hline & $\begin{array}{l}\text { Acute partial flap } \\
\text { necrosis }\end{array}$ & NRCS & Moderate & $1 \mathrm{y}$ & LD & $1 / 73(1.4)$ & NR & NR & NR \\
\hline \multirow[t]{2}{*}{ Kroll, 2000, 10987463, US } & Fat necrosis & NRCS & High & $3 \mathrm{mo}$ & DIEP & $\begin{array}{l}36 / 279 \\
\text { breasts (12.9) }\end{array}$ & vs. TRAM & $2.10(0.87,5.10)$ & 0.101 \\
\hline & Fat necrosis & NRCS & High & $3 \mathrm{mo}$ & AR with TRAM & $\begin{array}{l}9 / 31 \text { breasts } \\
(29)\end{array}$ & Ref & Ref & Ref \\
\hline \multirow{2}{*}{$\begin{array}{l}\text { Macadam, 2016, } \\
\text { 26910656, US, Canada, \& } \\
\text { Japan }\end{array}$} & Fat necrosis & NRCS & High & $4.5-7.3 y$ & DIEP & $\begin{array}{l}109 / 670 \\
(16.3)\end{array}$ & NR & NR & NR \\
\hline & Fat necrosis & NRCS & High & $4.5-7.3 \mathrm{y}$ & Free TRAM & $24 / 144(16.7)$ & NR & NR & NR \\
\hline
\end{tabular}




\begin{tabular}{|c|c|c|c|c|c|c|c|c|c|}
\hline $\begin{array}{l}\text { Study, Year, PMID, } \\
\text { Country }\end{array}$ & Outcome Description & Design & $\begin{array}{l}\text { Overall } \\
\text { RoB }\end{array}$ & $\begin{array}{l}\text { Time } \\
\text { Point }\end{array}$ & Arm & $\mathrm{n} / \mathrm{N}(\%)$ & Comparison & $\begin{array}{l}\text { Adjusted Odds } \\
\text { Ratio }(95 \% \mathrm{Cl})\end{array}$ & $\begin{array}{l}\mathbf{P} \\
\text { Value }\end{array}$ \\
\hline & Fat necrosis & NRCS & High & $4.5-7.3$ y & Pedicled TRAM & $\begin{array}{l}171 / 683 \\
(25.3)\end{array}$ & NR & NR & NR \\
\hline & Fat necrosis & NRCS & High & $4.5-7.3 y$ & $\begin{array}{l}\text { Muscle-sparing } \\
\text { TRAM }\end{array}$ & $44 / 293(15)$ & NR & NR & NR \\
\hline & $\begin{array}{l}\text { Membranous fat necrosis } \\
\text { of breast requiring } \\
\text { dressing }\end{array}$ & NRCS & High & $4.5-7.3$ y & DIEP & $79 / 670(11.8)$ & NR & NR & NR \\
\hline & $\begin{array}{l}\text { Membranous fat necrosis } \\
\text { of breast requiring } \\
\text { dressing }\end{array}$ & NRCS & High & $4.5-7.3 y$ & Free TRAM & $12 / 144(8.3)$ & NR & NR & NR \\
\hline & $\begin{array}{l}\text { Membranous fat necrosis } \\
\text { of breast requiring } \\
\text { dressing }\end{array}$ & NRCS & High & $4.5-7.3 y$ & Pedicled TRAM & $72 / 683(10.7)$ & NR & NR & NR \\
\hline & $\begin{array}{l}\text { Membranous fat necrosis } \\
\text { of breast requiring } \\
\text { dressing }\end{array}$ & NRCS & High & $4.5-7.3 \mathrm{y}$ & $\begin{array}{l}\text { Muscle-sparing } \\
\text { TRAM }\end{array}$ & $35 / 293(11.9)$ & NR & NR & NR \\
\hline & $\begin{array}{l}\text { Membranous fat necrosis } \\
\text { of breast requiring } \\
\text { surgery }\end{array}$ & NRCS & High & $4.5-7.3 \mathrm{y}$ & DIEP & $55 / 670(8.2)$ & NR & NR & NR \\
\hline & $\begin{array}{l}\text { Membranous fat necrosis } \\
\text { of breast requiring } \\
\text { surgery }\end{array}$ & NRCS & High & $4.5-7.3 y$ & Free TRAM & $6 / 144(4.2)$ & NR & NR & NR \\
\hline & $\begin{array}{l}\text { Membranous fat necrosis } \\
\text { of breast requiring } \\
\text { surgery }\end{array}$ & NRCS & High & $4.5-7.3 y$ & Pedicled TRAM & $82 / 683(12.1)$ & NR & NR & NR \\
\hline & $\begin{array}{l}\text { Membranous fat necrosis } \\
\text { of breast requiring } \\
\text { surgery }\end{array}$ & NRCS & High & $4.5-7.3 \mathrm{y}$ & $\begin{array}{l}\text { Muscle-sparing } \\
\text { TRAM }\end{array}$ & $21 / 293(7.2)$ & NR & NR & NR \\
\hline
\end{tabular}

Abbreviations: $\mathrm{AR}=$ autologous reconstruction, $\mathrm{CI}=$ confidence interval, $\mathrm{DIEP}=$ deep inferior epigastric perforator, $\mathrm{LD}=$ latissimus dorsi, $\mathrm{NR}=$ not reported, $\mathrm{NRCS}=$ nonrandomized comparative study, PMID $=$ PubMed identifier, RCT $=$ randomized controlled trial, Ref $=$ reference group, RoB $=$ risk of bias, SIEA $=$ superficial inferior epigastric artery, TAP $=$ thoracodorsal artery perforator, TRAM $=$ transverse rectus abdominis myocutaneous, $\mathrm{y}=$ years.

Colors: Header rows are shaded orange. Rows for every alternate study are shaded blue. The colors do not add unique information.

* calculated

Table F-6.16. Full Evidence Table - Key Question 6: Comparison of flap types for AR - categorical outcomes (thromboembolic events)

\begin{tabular}{|c|c|c|c|c|c|c|c|c|}
\hline $\begin{array}{l}\text { Study, Year, PMID, } \\
\text { Country }\end{array}$ & Outcome Description & Design & Overall RoB & Time Point & Arm & $\mathrm{n} / \mathrm{N}(\%)$ & $\begin{array}{l}\text { Effect Size } \\
(95 \% \mathrm{Cl})\end{array}$ & $\begin{array}{l}P \\
\text { Value }\end{array}$ \\
\hline \multirow{2}{*}{$\begin{array}{l}\text { Brorson 2020b, 32807615, } \\
\text { Sweden }\end{array}$} & Thromboembolic events & RCT & High & $1 \mathrm{mo}$ & DIEP & $0 / 24(0)$ & No events & $\mathrm{N} / \mathrm{A}$ \\
\hline & Thromboembolic events & RCT & High & $1 \mathrm{mo}$ & LD & $0 / 32(0)$ & No events & N/A \\
\hline
\end{tabular}




\begin{tabular}{|c|c|c|c|c|c|c|c|c|}
\hline $\begin{array}{l}\text { Study, Year, PMID, } \\
\text { Country }\end{array}$ & Outcome Description & Design & Overall RoB & Time Point & Arm & $\mathrm{n} / \mathrm{N}(\%)$ & $\begin{array}{l}\text { Effect Size } \\
(95 \% \mathrm{Cl})\end{array}$ & $\begin{array}{l}\mathbf{P} \\
\text { Value }\end{array}$ \\
\hline & Thromboembolic events & RCT & High & $1 \mathrm{mo}$ & DIEP & $0 / 24(0)$ & No events & $\mathrm{N} / \mathrm{A}$ \\
\hline & Thromboembolic events & RCT & High & $1 \mathrm{mo}$ & LD & $0 / 32(0)$ & No events & $\mathrm{N} / \mathrm{A}$ \\
\hline \multirow[t]{2}{*}{$\begin{array}{l}\text { Carramaschi, 1989, } \\
\text { 2602589, France }\end{array}$} & $\begin{array}{l}\text { Deep vein thrombosis or } \\
\text { pulmonary embolism }\end{array}$ & NRCS & High & NR & AR with TRAM & $2 / 40(5)$ & NR & NR \\
\hline & $\begin{array}{l}\text { Deep vein thrombosis or } \\
\text { pulmonary embolism }\end{array}$ & NRCS & High & NR & LD & $0 / 34(0)$ & NR & NR \\
\hline \multirow{8}{*}{$\begin{array}{l}\text { Kulkarni, 2017, 28713853, } \\
\text { US \& Canada }\end{array}$} & Deep venous thrombosis & NRCS & Moderate & $1 \mathrm{y}$ & DIEP & $1 / 365(0.3)$ & NR & NR \\
\hline & Deep venous thrombosis & NRCS & Moderate & $1 \mathrm{y}$ & Free TRAM & $0 / 97(0)$ & NR & NR \\
\hline & Deep venous thrombosis & NRCS & Moderate & $1 \mathrm{y}$ & Pedicled TRAM & $0 / 84(0)$ & NR & NR \\
\hline & Deep venous thrombosis & NRCS & Moderate & $1 \mathrm{y}$ & LD & $1 / 73(1.4)$ & NR & NR \\
\hline & Pulmonary embolism & NRCS & Moderate & $1 \mathrm{y}$ & DIEP & $4 / 365(1.1)$ & NR & NR \\
\hline & Pulmonary embolism & NRCS & Moderate & $1 \mathrm{y}$ & Free TRAM & $1 / 97(1)$ & NR & NR \\
\hline & Pulmonary embolism & NRCS & Moderate & $1 \mathrm{y}$ & Pedicled TRAM & $0 / 84(0)$ & NR & NR \\
\hline & Pulmonary embolism & NRCS & Moderate & $1 \mathrm{y}$ & LD & $0 / 73(0)$ & NR & NR \\
\hline \multirow[t]{4}{*}{$\begin{array}{l}\text { Macadam, 2016, 26910656, } \\
\text { US, Canada, \& Japan }\end{array}$} & $\begin{array}{l}\text { Deep vein thrombosis or } \\
\text { pulmonary embolism }\end{array}$ & NRCS & High & $4.5-7.3 y$ & DIEP & $8 / 670(1.2)$ & NR & NR \\
\hline & $\begin{array}{l}\text { Deep vein thrombosis or } \\
\text { pulmonary embolism }\end{array}$ & NRCS & High & $4.5-7.3 y$ & Free TRAM & $2 / 97(2.1)$ & NR & NR \\
\hline & $\begin{array}{l}\text { Deep vein thrombosis or } \\
\text { pulmonary embolism }\end{array}$ & NRCS & High & $4.5-7.3 y$ & Pedicled TRAM & $11 / 683(1.6)$ & NR & NR \\
\hline & $\begin{array}{l}\text { Deep vein thrombosis or } \\
\text { pulmonary embolism }\end{array}$ & NRCS & High & $4.5-7.3 y$ & Muscle-sparing TRAM & $4 / 293(1.4)$ & NR & NR \\
\hline
\end{tabular}

Abbreviations: $\mathrm{AR}=$ autologous reconstruction, $\mathrm{CI}=$ confidence interval, $\mathrm{DIEP}=$ deep inferior epigastric perforator, $\mathrm{LD}=$ latissimus dorsi $\mathrm{NR}=$ not reported, $\mathrm{NRCS}=$

nonrandomized comparative study, PMID $=$ PubMed identifier, RoB $=$ risk of bias, TRAM $=$ transverse rectus abdominis myocutaneous, $\mathrm{y}=$ years.

Colors: Header rows are shaded orange. Rows for every alternate study are shaded blue. The colors do not add unique information.

Table F-6.17. Full Evidence Table - Key Question 6: Comparison of flap types for AR - categorical outcomes (infections not explicitly implant related)

\begin{tabular}{|c|c|c|c|c|c|c|c|c|}
\hline Study, Year, PMID, Country & $\begin{array}{l}\text { Outcome } \\
\text { Description }\end{array}$ & Design & $\begin{array}{l}\text { Overall } \\
\text { RoB }\end{array}$ & $\begin{array}{l}\text { Time } \\
\text { Point }\end{array}$ & Arm & $\mathrm{n} / \mathrm{N}(\%)$ & $\begin{array}{l}\text { Adjusted Odds } \\
\text { Ratio }(95 \% \mathrm{Cl})\end{array}$ & $\begin{array}{l}\mathbf{P} \\
\text { Value }\end{array}$ \\
\hline \multirow{2}{*}{$\begin{array}{l}\text { Rindom, 2019, 31515191, } \\
\text { Denmark }\end{array}$} & Any infection & RCT & Moderate & $1 \mathrm{y}$ & LD & $1 / 18(5.6)$ & Ref & Ref \\
\hline & Any infection & RCT & Moderate & $1 \mathrm{y}$ & TAP & $1 / 22(4.5)$ & $1.24(0.07,21.2)^{*}$ & $0.88^{*}$ \\
\hline \multirow[t]{4}{*}{$\begin{array}{l}\text { Erdmann-Sager, 2018, } \\
\text { 29019862, US \& Canada }\end{array}$} & $\begin{array}{l}\text { Donor site wound } \\
\text { infection }\end{array}$ & NRCS & Moderate & $2 y$ & DIEP & $10 / 355(2.8)$ & NR & NR \\
\hline & $\begin{array}{l}\text { Donor site wound } \\
\text { infection }\end{array}$ & NRCS & Moderate & $2 y$ & Free TRAM & $3 / 92(3.3)$ & NR & NR \\
\hline & $\begin{array}{l}\text { Donor site wound } \\
\text { infection }\end{array}$ & NRCS & Moderate & $2 y$ & Pedicled TRAM & $6 / 78(7.7)$ & NR & NR \\
\hline & $\begin{array}{l}\text { Donor site wound } \\
\text { infection }\end{array}$ & NRCS & Moderate & $2 y$ & AR with SIEA & $9 / 62(14.5)$ & NR & NR \\
\hline
\end{tabular}




\begin{tabular}{|c|c|c|c|c|c|c|c|c|}
\hline Study, Year, PMID, Country & $\begin{array}{l}\text { Outcome } \\
\text { Description }\end{array}$ & Design & $\begin{array}{l}\text { Overall } \\
\text { RoB }\end{array}$ & $\begin{array}{l}\text { Time } \\
\text { Point }\end{array}$ & Arm & $\mathrm{n} / \mathrm{N}(\%)$ & $\begin{array}{l}\text { Adjusted Odds } \\
\text { Ratio }(95 \% \mathrm{Cl})\end{array}$ & \begin{tabular}{|l|}
$P$ \\
Value
\end{tabular} \\
\hline & $\begin{array}{l}\text { Breast wound } \\
\text { infection }\end{array}$ & NRCS & Moderate & $2 y$ & DIEP & $22 / 355(6.2)$ & NR & NR \\
\hline & $\begin{array}{l}\text { Breast wound } \\
\text { infection }\end{array}$ & NRCS & Moderate & $2 y$ & Free TRAM & $4 / 92(4.4)$ & NR & NR \\
\hline & $\begin{array}{l}\text { Breast wound } \\
\text { infection }\end{array}$ & NRCS & Moderate & $2 y$ & Pedicled TRAM & $7 / 78(9)$ & NR & NR \\
\hline & $\begin{array}{l}\text { Breast wound } \\
\text { infection }\end{array}$ & NRCS & Moderate & $2 y$ & AR with SIEA & $1 / 62(1.6)$ & NR & NR \\
\hline \multirow[t]{13}{*}{$\begin{array}{l}\text { Kulkarni, 2017, 28713853, } \\
\text { US \& Canada }\end{array}$} & $\begin{array}{l}\text { Breast wound } \\
\text { infection }\end{array}$ & NRCS & Moderate & $1 \mathrm{y}$ & DIEP & $14 / 365(3.8)$ & NR & NR \\
\hline & $\begin{array}{l}\text { Breast wound } \\
\text { infection }\end{array}$ & NRCS & Moderate & $1 \mathrm{y}$ & Free TRAM & $4 / 97(4.1)$ & NR & NR \\
\hline & $\begin{array}{l}\text { Breast wound } \\
\text { infection }\end{array}$ & NRCS & Moderate & $1 \mathrm{y}$ & Pedicled TRAM & $5 / 84(6)$ & NR & NR \\
\hline & $\begin{array}{l}\text { Breast wound } \\
\text { infection }\end{array}$ & NRCS & Moderate & $1 \mathrm{y}$ & LD & $6 / 73(8.2)$ & NR & NR \\
\hline & $\begin{array}{l}\text { Breast wound } \\
\text { infection }\end{array}$ & NRCS & Moderate & $2 y$ & DIEP & $27 / 390(6.9)$ & NR & NR \\
\hline & $\begin{array}{l}\text { Breast wound } \\
\text { infection }\end{array}$ & NRCS & Moderate & $2 y$ & Free TRAM & $5 / 95(5.3)$ & NR & NR \\
\hline & $\begin{array}{l}\text { Breast wound } \\
\text { infection }\end{array}$ & NRCS & Moderate & $2 y$ & Pedicled TRAM & $8 / 85(9.4)$ & NR & NR \\
\hline & $\begin{array}{l}\text { Breast wound } \\
\text { infection }\end{array}$ & NRCS & Moderate & $2 y$ & LD & $6 / 71(8.5)$ & NR & NR \\
\hline & $\begin{array}{l}\text { Breast wound } \\
\text { infection }\end{array}$ & NRCS & Moderate & $2 y$ & AR with SIEA & $8 / 65(12.3)$ & NR & NR \\
\hline & $\begin{array}{l}\text { Donor site wound } \\
\text { infection }\end{array}$ & NRCS & Moderate & $1 \mathrm{y}$ & DIEP & $12 / 365(3.3)$ & NR & NR \\
\hline & $\begin{array}{l}\text { Donor site wound } \\
\text { infection }\end{array}$ & NRCS & Moderate & $1 \mathrm{y}$ & Free TRAM & $2 / 97(2.1)$ & NR & NR \\
\hline & $\begin{array}{l}\text { Donor site wound } \\
\text { infection }\end{array}$ & NRCS & Moderate & $1 \mathrm{y}$ & Pedicled TRAM & $5 / 84(6)$ & NR & NR \\
\hline & $\begin{array}{l}\text { Donor site wound } \\
\text { infection }\end{array}$ & NRCS & Moderate & $1 \mathrm{y}$ & LD & $2 / 73(2.7)$ & NR & NR \\
\hline \multirow[t]{4}{*}{$\begin{array}{l}\text { Macadam, 2016, 26910656, } \\
\text { US, Canada, \& Japan }\end{array}$} & Any infection & NRCS & High & $\begin{array}{l}4.5-7.3 \\
y\end{array}$ & DIEP & $42 / 670(6.3)$ & NR & NR \\
\hline & Any infection & NRCS & High & $\begin{array}{l}4.5-7.3 \\
y\end{array}$ & Free TRAM & $14 / 144(9.7)$ & NR & NR \\
\hline & Any infection & NRCS & High & $\begin{array}{l}4.5-7.3 \\
y\end{array}$ & Pedicled TRAM & $\begin{array}{l}106 / 683 \\
(15.7)\end{array}$ & NR & NR \\
\hline & Any infection & NRCS & High & $\begin{array}{l}4.5-7.3 \\
y\end{array}$ & Muscle-sparing TRAM & $21 / 293(7.2)$ & NR & NR \\
\hline
\end{tabular}




\begin{tabular}{|c|c|c|c|c|c|c|c|c|}
\hline Study, Year, PMID, Country & $\begin{array}{l}\text { Outcome } \\
\text { Description }\end{array}$ & Design & $\begin{array}{l}\text { Overall } \\
\text { RoB }\end{array}$ & $\begin{array}{l}\text { Time } \\
\text { Point }\end{array}$ & Arm & $\mathrm{n} / \mathrm{N}(\%)$ & $\begin{array}{l}\text { Adjusted Odds } \\
\text { Ratio }(95 \% \mathrm{Cl})\end{array}$ & $\begin{array}{l}\mathbf{P} \\
\text { Value }\end{array}$ \\
\hline \multirow[t]{2}{*}{ Zoghbi, 2017, 28052051, US } & Wound infections & NRCS & High & $\begin{array}{l}\text { Post- } \\
\text { surgery }\end{array}$ & AR with TRAM & $\mathrm{NR} / 6137(\mathrm{NR})$ & $1.67(1.23,2.27)$ & 0.001 \\
\hline & Wound infections & NRCS & High & $\begin{array}{l}\text { Post- } \\
\text { surgery }\end{array}$ & DIEP & NR/9699 (NR) & Ref & Ref \\
\hline
\end{tabular}

Abbreviations: $\mathrm{AR}=$ autologous reconstruction, $\mathrm{CI}=$ confidence interval, $\mathrm{DIEP}=$ deep inferior epigastric perforator, $\mathrm{LD}=$ latissimus dorsi, $\mathrm{NR}=$ not reported, $\mathrm{NRCS}=$ nonrandomized comparative study, PMID $=$ PubMed identifier, $\mathrm{RCT}=$ randomized controlled trial, $\mathrm{Ref}=$ reference group, RoB $=$ risk of bias, $\mathrm{SIEA}=$ superficial inferior epigastric artery, TAP $=$ thoracodorsal artery perforator TRAM $=$ transverse rectus abdominis myocutaneous, $y=$ years.

Colors: Header rows are shaded orange. Rows for every alternate study are shaded blue. The colors do not add unique information.

* calculated

Table F-6.18. Full Evidence Table - Key Question 6: Comparison of flap types for AR - categorical outcomes (wound dehiscence)

\begin{tabular}{|c|c|c|c|c|c|c|c|c|}
\hline $\begin{array}{l}\text { Study, Year, PMID, } \\
\text { Country }\end{array}$ & Outcome Description & Design & $\begin{array}{l}\text { Overall } \\
\text { RoB }\end{array}$ & $\begin{array}{l}\text { Time } \\
\text { Point }\end{array}$ & Arm & $\mathrm{n} / \mathrm{N}(\%)$ & $\begin{array}{l}\text { Adjusted Odds } \\
\text { Ratio }(95 \% \mathrm{Cl})\end{array}$ & P Value \\
\hline \multirow{8}{*}{$\begin{array}{l}\text { Erdmann-Sager, 2018, } \\
\text { 29019862, US \& Canada }\end{array}$} & Donor site wound dehiscence & NRCS & Moderate & $2 y$ & DIEP & $37 / 355(10.4)$ & NR & NR \\
\hline & Donor site wound dehiscence & NRCS & Moderate & $2 y$ & Free TRAM & $3 / 92(3.3)$ & NR & NR \\
\hline & Donor site wound dehiscence & NRCS & Moderate & $2 y$ & Pedicled TRAM & $2 / 78(2.6)$ & NR & NR \\
\hline & Donor site wound dehiscence & NRCS & Moderate & $2 y$ & AR with SIEA & $17 / 62(27.4)$ & NR & NR \\
\hline & Breast wound dehiscence & NRCS & Moderate & $2 y$ & DIEP & $23 / 355(6.5)$ & NR & NR \\
\hline & Breast wound dehiscence & NRCS & Moderate & $2 y$ & Free TRAM & $3 / 92(3.3)$ & NR & NR \\
\hline & Breast wound dehiscence & NRCS & Moderate & $2 y$ & Pedicled TRAM & $2 / 78(2.6)$ & NR & NR \\
\hline & Breast wound dehiscence & NRCS & Moderate & $2 y$ & AR with SIEA & $4 / 62(6.5)$ & NR & NR \\
\hline \multirow[t]{2}{*}{ Israeli, 2014, 24572840, US } & $\begin{array}{l}\text { Wound problems/need for } \\
\text { negative pressure wound therapy }\end{array}$ & NRCS & High & $1.5 \mathrm{y}$ & AR with TRAM & $14 / 252(5.6)$ & NR & NR \\
\hline & $\begin{array}{l}\text { Wound problems/need for } \\
\text { negative pressure wound therapy }\end{array}$ & NRCS & High & $1.5 \mathrm{y}$ & LD & $2 / 302(1)$ & NR & NR \\
\hline \multirow{8}{*}{$\begin{array}{l}\text { Kulkarni, 2017, 28713853, } \\
\text { US \& Canada }\end{array}$} & Breast wound dehiscence & NRCS & Moderate & $1 \mathrm{y}$ & DIEP & $13 / 365(3.6)$ & NR & NR \\
\hline & Breast wound dehiscence & NRCS & Moderate & $1 \mathrm{y}$ & Free TRAM & $1 / 97(1)$ & NR & NR \\
\hline & Breast wound dehiscence & NRCS & Moderate & $1 \mathrm{y}$ & Pedicled TRAM & $1 / 84(1.2)$ & NR & NR \\
\hline & Breast wound dehiscence & NRCS & Moderate & $1 \mathrm{y}$ & LD & $1 / 73(1.4)$ & NR & NR \\
\hline & Donor site wound dehiscence & NRCS & Moderate & $1 \mathrm{y}$ & DIEP & $31 / 365(8.5)$ & NR & NR \\
\hline & Donor site wound dehiscence & NRCS & Moderate & $1 \mathrm{y}$ & Free TRAM & $3 / 97(3.1)$ & NR & NR \\
\hline & Donor site wound dehiscence & NRCS & Moderate & $1 \mathrm{y}$ & Pedicled TRAM & $1 / 84(1.2)$ & NR & NR \\
\hline & Donor site wound dehiscence & NRCS & Moderate & $1 \mathrm{y}$ & LD & $0 / 73(0)$ & NR & NR \\
\hline \multirow[t]{2}{*}{ Zoghbi, 2017, 28052051, US } & Wound dehiscence & NRCS & High & NR & DIEP & NR/9699 (NR) & Ref & Ref \\
\hline & Wound dehiscence & NRCS & High & NR & AR with TRAM & NR/6137 (NR) & 4.3 (NR) & $<0.00001$ \\
\hline
\end{tabular}

Abbreviations: $\mathrm{AR}=$ autologous reconstruction, $\mathrm{CI}=$ confidence interval, $\mathrm{DIEP}=$ deep inferior epigastric perforator, $\mathrm{LD}=$ latissimus dorsi, $\mathrm{NR}=$ not reported, $\mathrm{NRCS}=$ nonrandomized comparative study, PMID $=$ PubMed identifier, Ref $=$ reference group, $\mathrm{RoB}=$ risk of bias, $\mathrm{SIEA}=$ superficial inferior epigastric artery, TRAM $=$ transverse rectus abdominis myocutaneous, $\mathrm{y}=$ years.

Colors: Header rows are shaded orange. Rows for every alternate study are shaded blue. The colors do not add unique information. 
Table F-6.19. Full Evidence Table - Key Question 6: Comparison of flap types for AR - categorical outcomes (delayed healing)

\begin{tabular}{|c|c|c|c|c|c|c|c|}
\hline Study, Year, PMID, Country & Design & Overall RoB & Time Point & Arm & $\mathrm{n} / \mathrm{N}(\%)$ & Effect Size $(95 \% \mathrm{Cl})$ & P Value \\
\hline \multirow{4}{*}{$\begin{array}{l}\text { Macadam, 2016, 26910656, US, } \\
\text { Canada, \& Japan }\end{array}$} & NRCS & High & $4.5-7.3 \mathrm{y}$ & DIEP & $103 / 670(18.5)$ & NR & NR \\
\hline & NRCS & High & $4.5-7.3 y$ & Free TRAM & $26 / 144(22.4)$ & NR & NR \\
\hline & NRCS & High & $4.5-7.3 \mathrm{y}$ & Pedicled TRAM & $174 / 683(28.2)$ & NR & NR \\
\hline & NRCS & High & $4.5-7.3 y$ & Muscle-sparing TRAM & $51 / 293(26.7)$ & NR & NR \\
\hline
\end{tabular}

Abbreviations: $\mathrm{AR}=$ autologous reconstruction, $\mathrm{CI}=$ confidence interval, $\mathrm{DIEP}=$ deep inferior epigastric perforator, $\mathrm{NR}=$ not reported, $\mathrm{NRCS}=$ nonrandomized comparative

study, PMID = PubMed identifier, RoB = risk of bias, TRAM = transverse rectus abdominis myocutaneous, $\mathrm{y}=$ years.

Colors: Header rows are shaded orange. The color does not add unique information.

Table F-6.20. Full Evidence Table - Key Question 6: Comparison of flap types for AR - categorical outcomes (seroma)

\begin{tabular}{|c|c|c|c|c|c|c|c|c|}
\hline Study, Year, PMID, Country & $\begin{array}{l}\text { Outcome } \\
\text { Description }\end{array}$ & Design & $\begin{array}{l}\text { Overall } \\
\text { RoB }\end{array}$ & $\begin{array}{l}\text { Time } \\
\text { Point }\end{array}$ & Arm & $\mathrm{n} / \mathrm{N}(\%)$ & $\begin{array}{l}\text { Adjusted Odds } \\
\text { Ratio }(95 \% \mathrm{Cl})\end{array}$ & $\begin{array}{l}\mathbf{P} \\
\text { Value }\end{array}$ \\
\hline \multirow{2}{*}{$\begin{array}{l}\text { Rindom, 2019, 31515191, } \\
\text { Denmark }\end{array}$} & Seroma & RCT & Moderate & $1 \mathrm{y}$ & LD & $1 / 18(5.6)$ & Ref & Ref \\
\hline & Seroma & RCT & Moderate & $1 \mathrm{y}$ & TAP & $0 / 22(0)$ & $2.53(0.08,80.0)^{*}$ & $0.60^{*}$ \\
\hline \multirow{8}{*}{$\begin{array}{l}\text { Erdmann-Sager, 2018, } \\
\text { 29019862, US \& Canada }\end{array}$} & Donor site seroma & NRCS & Moderate & $2 y$ & DIEP & $25 / 355(7)$ & NR & NR \\
\hline & Donor site seroma & NRCS & Moderate & $2 y$ & Free TRAM & $3 / 92(3.3)$ & NR & NR \\
\hline & Donor site seroma & NRCS & Moderate & $2 y$ & Pedicled TRAM & $2 / 78(2.6)$ & NR & NR \\
\hline & Donor site seroma & NRCS & Moderate & $2 y$ & AR with SIEA & $19 / 62(30.7)$ & NR & NR \\
\hline & Breast seroma & NRCS & Moderate & $2 y$ & DIEP & $4 / 355(1.1)$ & NR & NR \\
\hline & Breast seroma & NRCS & Moderate & $2 y$ & Free TRAM & $0 / 92(0)$ & NR & NR \\
\hline & Breast seroma & NRCS & Moderate & $2 y$ & Pedicled TRAM & $2 / 78(2.6)$ & NR & NR \\
\hline & Breast seroma & NRCS & Moderate & $2 y$ & AR with SIEA & $1 / 62(1.6)$ & NR & NR \\
\hline \multirow[t]{2}{*}{ Israeli, 2014, 24572840, US } & Seroma/hematoma & NRCS & High & $1.5 \mathrm{y}$ & AR with TRAM & $13 / 252(5.2)$ & NR & NR \\
\hline & Seroma/hematoma & NRCS & High & $1.5 \mathrm{y}$ & LD & $19 / 302(6.3)$ & NR & NR \\
\hline \multirow{8}{*}{$\begin{array}{l}\text { Kulkarni, 2017, 28713853, US \& } \\
\text { Canada }\end{array}$} & Breast seroma & NRCS & Moderate & $1 \mathrm{y}$ & DIEP & $3 / 365(0.8)$ & NR & NR \\
\hline & Breast seroma & NRCS & Moderate & $1 \mathrm{y}$ & Free TRAM & $0 / 97(0)$ & NR & NR \\
\hline & Breast seroma & NRCS & Moderate & $1 \mathrm{y}$ & Pedicled TRAM & $2 / 84(2.4)$ & NR & NR \\
\hline & Breast seroma & NRCS & Moderate & $1 \mathrm{y}$ & LD & $2 / 73(2.7)$ & NR & NR \\
\hline & Donor site seroma & NRCS & Moderate & $1 \mathrm{y}$ & DIEP & $19 / 365(5.2)$ & NR & NR \\
\hline & Donor site seroma & NRCS & Moderate & $1 \mathrm{y}$ & Free TRAM & $2 / 97(2.1)$ & NR & NR \\
\hline & Donor site seroma & NRCS & Moderate & $1 \mathrm{y}$ & Pedicled TRAM & $0 / 84(0)$ & NR & NR \\
\hline & Donor site seroma & NRCS & Moderate & $1 \mathrm{y}$ & LD & $14 / 73(19.2)$ & NR & NR \\
\hline \multirow[t]{4}{*}{$\begin{array}{l}\text { Macadam, 2016, 26910656, US, } \\
\text { Canada, \& Japan }\end{array}$} & Seroma & NRCS & High & $\begin{array}{l}4.5- \\
7.3 y\end{array}$ & DIEP & $34 / 670(5.1)$ & NR & NR \\
\hline & Seroma & NRCS & High & $\begin{array}{l}4.5- \\
7.3 \mathrm{y}\end{array}$ & Free TRAM & $15 / 144(10.4)$ & NR & NR \\
\hline & Seroma & NRCS & High & $\begin{array}{l}4.5- \\
7.3 y\end{array}$ & Pedicled TRAM & $78 / 683(11.5)$ & NR & NR \\
\hline & Seroma & NRCS & High & $\begin{array}{l}4.5- \\
7.3 y\end{array}$ & Muscle-sparing TRAM & $20 / 293(6.8)$ & NR & NR \\
\hline
\end{tabular}

Abbreviations: $\mathrm{AR}=$ autologous reconstruction, $\mathrm{CI}=$ confidence interval, $\mathrm{DIEP}=$ deep inferior epigastric perforator, $\mathrm{LD}=$ latissimus dorsi, $\mathrm{NR}=$ not reported, $\mathrm{NRCS}=$

nonrandomized comparative study, PMID $=$ PubMed identifier, RCT $=$ randomized controlled trial, Ref $=$ reference group, RoB $=$ risk of bias, SIEA $=$ superficial inferior 
epigastric artery, TAP $=$ thoracodorsal artery perforator, TRAM $=$ transverse rectus abdominis myocutaneous, $\mathrm{y}=$ years.

Colors: Header rows are shaded orange. Rows for every alternate study are shaded blue. The colors do not add unique information.

* Calculated.

Table F-6.21. Full Evidence Table - Key Question 6: Comparison of flap types for AR - categorical outcomes (scarring)

\begin{tabular}{|c|c|c|c|c|c|c|c|c|}
\hline Study, Year, PMID, Country & Outcome Description & Design & $\begin{array}{l}\text { Overall } \\
\text { RoB }\end{array}$ & $\begin{array}{l}\text { Time } \\
\text { Point }\end{array}$ & Arm & $\mathrm{n} / \mathrm{N}(\%)$ & $\begin{array}{l}\text { Adjusted Odds } \\
\text { Ratio }(95 \% \mathrm{Cl})\end{array}$ & $\begin{array}{l}\mathbf{P} \\
\text { Value }\end{array}$ \\
\hline \multirow[t]{2}{*}{$\begin{array}{l}\text { Garbay, 1992, 1624727, } \\
\text { France }\end{array}$} & $\begin{array}{l}\text { Very visible or cheloid donor } \\
\text { site scarring }\end{array}$ & NRCS & High & $1.9 \mathrm{y}$ & AR with TRAM & $3 / 63(5)$ & NR & NR \\
\hline & $\begin{array}{l}\text { Very visible or cheloid donor } \\
\text { site scarring }\end{array}$ & NRCS & High & $1.9 \mathrm{y}$ & LD & NR/NR (24) & NR & NR \\
\hline \multirow[t]{8}{*}{$\begin{array}{l}\text { Erdmann-Sager, 2018, } \\
\text { 29019862, US \& Canada }\end{array}$} & $\begin{array}{l}\text { Donor site hypertrophic or } \\
\text { keloid scarring }\end{array}$ & NRCS & Moderate & $2 y$ & DIEP & $5 / 355(1.4)$ & NR & NR \\
\hline & $\begin{array}{l}\text { Donor site hypertrophic or } \\
\text { keloid scarring }\end{array}$ & NRCS & Moderate & $2 y$ & Free TRAM & $1 / 92(1.1)$ & NR & NR \\
\hline & $\begin{array}{l}\text { Donor site hypertrophic or } \\
\text { keloid scarring }\end{array}$ & NRCS & Moderate & $2 y$ & Pedicled TRAM & $1 / 78(1.3)$ & NR & NR \\
\hline & $\begin{array}{l}\text { Donor site hypertrophic or } \\
\text { keloid scarring }\end{array}$ & NRCS & Moderate & $2 y$ & AR with SIEA & $0 / 62(0)$ & NR & NR \\
\hline & $\begin{array}{l}\text { Breast hypertrophic or keloid } \\
\text { scarring }\end{array}$ & NRCS & Moderate & $2 y$ & DIEP & $8 / 355(2.3)$ & NR & NR \\
\hline & $\begin{array}{l}\text { Breast hypertrophic or keloid } \\
\text { scarring }\end{array}$ & NRCS & Moderate & $2 y$ & Free TRAM & $0 / 92(0)$ & NR & NR \\
\hline & $\begin{array}{l}\text { Breast hypertrophic or keloid } \\
\text { scarring }\end{array}$ & NRCS & Moderate & $2 y$ & Pedicled TRAM & $0 / 78(0)$ & NR & NR \\
\hline & $\begin{array}{l}\text { Breast hypertrophic or keloid } \\
\text { scarring }\end{array}$ & NRCS & Moderate & $2 y$ & AR with SIEA & $1 / 62(1.6)$ & NR & NR \\
\hline
\end{tabular}

Abbreviations: $\mathrm{AR}=$ autologous reconstruction, $\mathrm{CI}=$ confidence interval, $\mathrm{DIEP}=$ deep inferior epigastric perforator, $\mathrm{LD}=$ latissimus dorsi, $\mathrm{NR}=$ not reported, $\mathrm{NRCS}=$

nonrandomized comparative study, PMID $=$ PubMed identifier, RoB $=$ risk of bias, SIEA $=$ superficial inferior epigastric artery, TRAM $=$ transverse rectus abdominis

myocutaneous, $\mathrm{y}=$ years.

Colors: Header rows are shaded orange. Rows for every alternate study are shaded blue. The colors do not add unique information.

Table F-6.22. Full Evidence Table - Key Question 6: Comparison of flap types for AR - categorical outcomes (harms to area of flap harvest)

\begin{tabular}{|c|c|c|c|c|c|c|c|c|c|}
\hline $\begin{array}{l}\text { Study, Year, PMID, } \\
\text { Country }\end{array}$ & Outcome Description & Design & $\begin{array}{l}\text { Overall } \\
\text { RoB }\end{array}$ & $\begin{array}{l}\text { Time } \\
\text { Point }\end{array}$ & Arm & $\mathrm{n} / \mathrm{N}(\%)$ & Comparison & $\begin{array}{l}\text { Adjusted Odds } \\
\text { Ratio }(95 \% \mathrm{Cl})\end{array}$ & $\begin{array}{l}\mathbf{P} \\
\text { Value }\end{array}$ \\
\hline \multirow{4}{*}{$\begin{array}{l}\text { Carramaschi, 1989, } \\
2602589, \text { France }\end{array}$} & Abdominal hernia & NRCS & High & NR & AR with TRAM & $1 / 40(2.5)$ & NR & NR & NR \\
\hline & Abdominal hernia & NRCS & High & NR & LD & NR & NR & NR & NR \\
\hline & $\begin{array}{l}\text { Surgical abdominal } \\
\text { asymmetry }\end{array}$ & NRCS & High & NR & AR with TRAM & $7 / 40(17.5)$ & NR & NR & NR \\
\hline & $\begin{array}{l}\text { Surgical abdominal } \\
\text { asymmetry }\end{array}$ & NRCS & High & NR & LD & NR & NR & NR & NR \\
\hline
\end{tabular}




\begin{tabular}{|c|c|c|c|c|c|c|c|c|c|}
\hline $\begin{array}{l}\text { Study, Year, PMID, } \\
\text { Country }\end{array}$ & Outcome Description & Design & $\begin{array}{l}\text { Overall } \\
\text { RoB }\end{array}$ & $\begin{array}{l}\text { Time } \\
\text { Point }\end{array}$ & Arm & $\mathrm{n} / \mathrm{N}(\%)$ & Comparison & $\begin{array}{l}\text { Adjusted Odds } \\
\text { Ratio }(95 \% \mathrm{CI})\end{array}$ & $\begin{array}{l}P \\
\text { Value }\end{array}$ \\
\hline \multirow[t]{8}{*}{$\begin{array}{l}\text { Erdmann-Sager, 2018, } \\
\text { 29019862, US \& Canada }\end{array}$} & $\begin{array}{l}\text { Abdominal wall bulge, } \\
\text { laxity, or hernia }\end{array}$ & NRCS & Moderate & $2 y$ & DIEP & $6 / 355(1.7)$ & NR & NR & NR \\
\hline & $\begin{array}{l}\text { Abdominal wall bulge, } \\
\text { laxity, or hernia }\end{array}$ & NRCS & Moderate & $2 y$ & Free TRAM & $5 / 92(5.4)$ & NR & NR & NR \\
\hline & $\begin{array}{l}\text { Abdominal wall bulge, } \\
\text { laxity, or hernia }\end{array}$ & NRCS & Moderate & $2 y$ & Pedicled TRAM & $7 / 78(9)$ & NR & NR & NR \\
\hline & $\begin{array}{l}\text { Abdominal wall bulge, } \\
\text { laxity, or hernia }\end{array}$ & NRCS & Moderate & $2 y$ & AR with SIEA & $0 / 62(0)$ & NR & NR & NR \\
\hline & $\begin{array}{l}\text { Any donor site } \\
\text { complication }\end{array}$ & NRCS & Moderate & $2 y$ & DIEP & $99 / 355$ (27.9) & Ref & Ref & Ref \\
\hline & $\begin{array}{l}\text { Any donor site } \\
\text { complication }\end{array}$ & NRCS & Moderate & $2 y$ & Free TRAM & $14 / 92(15.2)$ & vs. DIEP & $\begin{array}{l}0.52(0.27 \\
1.02)\end{array}$ & 0.057 \\
\hline & $\begin{array}{l}\text { Any donor site } \\
\text { complication }\end{array}$ & NRCS & Moderate & $2 y$ & Pedicled TRAM & $14 / 78(18)$ & vs. DIEP & $\begin{array}{l}0.63(0.32 \\
1.24)\end{array}$ & 0.178 \\
\hline & $\begin{array}{l}\text { Any donor site } \\
\text { complication }\end{array}$ & NRCS & Moderate & $2 y$ & AR with SIEA & $33 / 62(53.2)$ & vs. DIEP & $\begin{array}{l}2.73(1.51 \\
4.96)\end{array}$ & 0.001 \\
\hline \multirow[t]{2}{*}{$\begin{array}{l}\text { Knox, 2016, 26267400, } \\
\text { Canada }\end{array}$} & $\begin{array}{l}\text { Abdominal bulge or } \\
\text { hernia }\end{array}$ & NRCS & High & $\begin{array}{l}1.7- \\
2.3 y\end{array}$ & DIEP & $4 / 130(3.1)$ & Ref & Ref & Ref \\
\hline & $\begin{array}{l}\text { Abdominal bulge or } \\
\text { hernia }\end{array}$ & NRCS & High & $\begin{array}{l}1.7- \\
2.3 \mathrm{y}\end{array}$ & AR with TRAM & $80 / 377(21.2)$ & vs. DIEP & $5.2(1.3,20.9)$ & 0.002 \\
\hline \multirow[t]{4}{*}{$\begin{array}{l}\text { Kulkarni, 2017, } \\
\text { 28713853, US \& Canada }\end{array}$} & $\begin{array}{l}\text { Abdominal wall bulge, } \\
\text { laxity, or hernia }\end{array}$ & NRCS & Moderate & $1 \mathrm{y}$ & DIEP & $6 / 365(1.6)$ & NR & NR & NR \\
\hline & $\begin{array}{l}\text { Abdominal wall bulge, } \\
\text { laxity, or hernia }\end{array}$ & NRCS & Moderate & $1 \mathrm{y}$ & Free TRAM & $3 / 97(3.1)$ & NR & NR & NR \\
\hline & $\begin{array}{l}\text { Abdominal wall bulge, } \\
\text { laxity, or hernia }\end{array}$ & NRCS & Moderate & $1 \mathrm{y}$ & Pedicled TRAM & $4 / 84(4.8)$ & NR & NR & NR \\
\hline & $\begin{array}{l}\text { Abdominal wall bulge, } \\
\text { laxity, or hernia }\end{array}$ & NRCS & Moderate & $1 \mathrm{y}$ & LD & $0 / 73(0)$ & NR & NR & NR \\
\hline \multirow{7}{*}{$\begin{array}{l}\text { Macadam, 2016, } \\
\text { 26910656, US, Canada, } \\
\text { \& Japan }\end{array}$} & Abdominal hernia & NRCS & High & $\begin{array}{l}4.5- \\
7.3 y\end{array}$ & DIEP & $13 / 670(1.9)$ & NR & NR & NR \\
\hline & Abdominal hernia & NRCS & High & $\begin{array}{l}4.5- \\
7.3 \mathrm{y}\end{array}$ & Free TRAM & $4 / 144(2.8)$ & NR & NR & NR \\
\hline & Abdominal hernia & NRCS & High & $\begin{array}{l}4.5- \\
7.3 y\end{array}$ & Pedicled TRAM & $46 / 683(6.7)$ & NR & NR & NR \\
\hline & Abdominal hernia & NRCS & High & $\begin{array}{l}4.5- \\
7.3 y\end{array}$ & Muscle-sparing TRAM & $4 / 293(4.8)$ & NR & NR & NR \\
\hline & Abdominal bulge & NRCS & High & $\begin{array}{l}4.5- \\
7.3 y\end{array}$ & DIEP & $18 / 670(2.7)$ & NR & NR & NR \\
\hline & Abdominal bulge & NRCS & High & $\begin{array}{l}4.5- \\
7.3 y\end{array}$ & Free TRAM & $5 / 144(3.5)$ & NR & NR & NR \\
\hline & Abdominal bulge & NRCS & High & $\begin{array}{l}4.5- \\
7.3 \mathrm{y}\end{array}$ & Pedicled TRAM & $74 / 683(10.9)$ & NR & NR & NR \\
\hline
\end{tabular}




\begin{tabular}{|c|c|c|c|c|c|c|c|c|c|}
\hline $\begin{array}{l}\text { Study, Year, PMID, } \\
\text { Country }\end{array}$ & Outcome Description & Design & $\begin{array}{l}\text { Overall } \\
\text { RoB }\end{array}$ & $\begin{array}{l}\text { Time } \\
\text { Point }\end{array}$ & Arm & n/N (\%) & Comparison & $\begin{array}{l}\text { Adjusted Odds } \\
\text { Ratio }(95 \% \mathrm{Cl})\end{array}$ & $\begin{array}{l}\mathbf{P} \\
\text { Value }\end{array}$ \\
\hline & Abdominal bulge & NRCS & High & $\begin{array}{l}4.5- \\
7.3 y\end{array}$ & Muscle-sparing TRAM & $15 / 293(5.1)$ & NR & NR & NR \\
\hline \multirow{3}{*}{$\begin{array}{l}\text { Mennie, 2015, } \\
\text { 25839173, UK }\end{array}$} & Hernia repair & NRCS & High & $3 y$ & DIEP & $63 / 5144(1.2)$ & Ref & Ref & Ref \\
\hline & Hernia repair & NRCS & High & $3 y$ & Free TRAM & $50 / 1963(2.6)$ & vs. DIEP & $\begin{array}{l}1.81(1.24 \\
2.64)\end{array}$ & NR \\
\hline & Hernia repair & NRCS & High & $3 y$ & Pedicled TRAM & $36 / 822(4.4)$ & vs. DIEP & $\begin{array}{l}2.89(1.91 \\
4.37)\end{array}$ & NR \\
\hline \multirow[t]{2}{*}{$\begin{array}{l}\text { Zhong, 2014, 24675183, } \\
\text { Canada }\end{array}$} & $\begin{array}{l}\text { Abdominal bulge or } \\
\text { hernia }\end{array}$ & NRCS & High & NR & DIEP & $15 / 244(6)$ & Ref & Ref & Ref \\
\hline & $\begin{array}{l}\text { Abdominal bulge or } \\
\text { hernia }\end{array}$ & NRCS & High & NR & AR with TRAM & $8 / 48(17)$ & vs. DIEP & $\begin{array}{l}2.73(1.01 \\
7.07)\end{array}$ & 0.04 \\
\hline
\end{tabular}

Abbreviations: $\mathrm{AR}=$ autologous reconstruction, $\mathrm{CI}=$ confidence interval, $\mathrm{DIEP}=$ deep inferior epigastric perforator, $\mathrm{LD}=$ latissimus dorsi, $\mathrm{NR}=$ not reported, $\mathrm{NRCS}=$

nonrandomized comparative study, PMID = PubMed identifier, Ref $=$ reference group, RoB $=$ risk of bias, SIEA = superficial inferior epigastric artery, TRAM = transverse rectus abdominis myocutaneous, $\mathrm{y}=$ years.

Colors: Header rows are shaded orange. Rows for every alternate study are shaded blue. The colors do not add unique information.

Table F-6.23. Full Evidence Table - Key Question 6: Comparison of flap types for AR - categorical outcomes (flap failure/loss)

\begin{tabular}{|c|c|c|c|c|c|c|c|c|c|}
\hline $\begin{array}{l}\text { Study, Year, PMID, } \\
\text { Country }\end{array}$ & $\begin{array}{l}\text { Outcome } \\
\text { Description }\end{array}$ & Design & $\begin{array}{l}\text { Overall } \\
\text { RoB }\end{array}$ & $\begin{array}{l}\text { Time } \\
\text { Point }\end{array}$ & Arm & $n / N(\%)$ & Comparison & $\begin{array}{l}\text { Adjusted Odds } \\
\text { Ratio }(95 \% \mathrm{Cl})\end{array}$ & $\begin{array}{l}\mathbf{P} \\
\text { Value }\end{array}$ \\
\hline \multirow[t]{4}{*}{$\begin{array}{l}\text { Erdmann-Sager, 2018, } \\
\text { 29019862, US \& Canada }\end{array}$} & $\begin{array}{l}\text { Flap } \\
\text { failure/loss }\end{array}$ & NRCS & Moderate & $2 y$ & DIEP & $4 / 355(1.1)$ & NR & NR & NR \\
\hline & $\begin{array}{l}\text { Flap } \\
\text { failure/loss }\end{array}$ & NRCS & Moderate & $2 y$ & Free TRAM & $2 / 92(2.17)$ & NR & NR & NR \\
\hline & $\begin{array}{l}\text { Flap } \\
\text { failure/loss }\end{array}$ & NRCS & Moderate & $2 y$ & Pedicled TRAM & $1 / 78(1.2)$ & NR & NR & NR \\
\hline & $\begin{array}{l}\text { Flap } \\
\text { failure/loss }\end{array}$ & NRCS & Moderate & $2 y$ & AR with SIEA & $0 / 62(0)$ & NR & NR & NR \\
\hline \multirow[t]{2}{*}{ Kroll, 2000,10987463, US } & $\begin{array}{l}\text { Partial flap } \\
\text { loss }\end{array}$ & NRCS & High & $3 \mathrm{mo}$ & DIEP & $5 / 31$ breasts $(16.1)$ & vs. TRAM & $6.74(1.83,24.7)$ & 0.004 \\
\hline & $\begin{array}{l}\text { Partial flap } \\
\text { loss }\end{array}$ & NRCS & High & $3 \mathrm{mo}$ & AR with TRAM & $6 / 279$ breasts $(2.2)$ & Ref & Ref & Ref \\
\hline \multirow[t]{5}{*}{$\begin{array}{l}\text { Kulkarni, 2017, 28713853, } \\
\text { US \& Canada }\end{array}$} & $\begin{array}{l}\text { Total flap } \\
\text { loss }\end{array}$ & NRCS & Moderate & $1 \mathrm{y}$ & DIEP & $5 / 365(1.4)$ & NR & NR & NR \\
\hline & $\begin{array}{l}\text { Total flap } \\
\text { loss }\end{array}$ & NRCS & Moderate & $1 \mathrm{y}$ & Free TRAM & $2 / 97(2.1)$ & NR & NR & NR \\
\hline & $\begin{array}{l}\text { Total flap } \\
\text { loss }\end{array}$ & NRCS & Moderate & $1 \mathrm{y}$ & Pedicled TRAM & $1 / 84(1.2)$ & NR & NR & NR \\
\hline & $\begin{array}{l}\text { Total flap } \\
\text { loss }\end{array}$ & NRCS & Moderate & $1 \mathrm{y}$ & LD & $0 / 73(0)$ & NR & NR & NR \\
\hline & $\begin{array}{l}\text { Reconstructi } \\
\text { ve failure }\end{array}$ & NRCS & Moderate & $1 \mathrm{y}$ & DIEP & $5 / 390(1.3)$ & NR & NR & NR \\
\hline
\end{tabular}




\begin{tabular}{|c|c|c|c|c|c|c|c|c|c|}
\hline $\begin{array}{l}\text { Study, Year, PMID, } \\
\text { Country }\end{array}$ & $\begin{array}{l}\text { Outcome } \\
\text { Description }\end{array}$ & Design & $\begin{array}{l}\text { Overall } \\
\text { RoB }\end{array}$ & $\begin{array}{l}\text { Time } \\
\text { Point }\end{array}$ & Arm & $\mathrm{n} / \mathrm{N}(\%)$ & Comparison & $\begin{array}{l}\text { Adjusted Odds } \\
\text { Ratio }(95 \% \mathrm{Cl})\end{array}$ & $\begin{array}{l}P \\
\text { Value }\end{array}$ \\
\hline & $\begin{array}{l}\text { Reconstructi } \\
\text { ve failure }\end{array}$ & NRCS & Moderate & $1 \mathrm{y}$ & Free TRAM & $2 / 95(2.1)$ & NR & NR & NR \\
\hline & $\begin{array}{l}\text { Reconstructi } \\
\text { ve failure }\end{array}$ & NRCS & Moderate & $1 \mathrm{y}$ & Pedicled TRAM & $1 / 85(1.2)$ & NR & NR & NR \\
\hline & $\begin{array}{l}\text { Reconstructi } \\
\text { ve failure }\end{array}$ & NRCS & Moderate & $1 \mathrm{y}$ & LD & $2 / 71(2.8)$ & NR & NR & NR \\
\hline & $\begin{array}{l}\text { Reconstructi } \\
\text { ve failure }\end{array}$ & NRCS & Moderate & $1 \mathrm{y}$ & AR with SIEA & $0 / 65(0)$ & NR & NR & NR \\
\hline \multirow{8}{*}{$\begin{array}{l}\text { Macadam, 2016, } \\
26910656, \text { US, Canada, \& } \\
\text { Japan }\end{array}$} & $\begin{array}{l}\text { Partial flap } \\
\text { loss }\end{array}$ & NRCS & High & $4.5-7.3 y$ & DIEP & $47 / 670(4)$ & NR & NR & NR \\
\hline & $\begin{array}{l}\text { Partial flap } \\
\text { loss }\end{array}$ & NRCS & High & $4.5-7.3 y$ & Free TRAM & $11 / 144(7.6)$ & NR & NR & NR \\
\hline & $\begin{array}{l}\text { Partial flap } \\
\text { loss }\end{array}$ & NRCS & High & $4.5-7.3 y$ & Pedicled TRAM & $60 / 683(8.9)$ & NR & NR & NR \\
\hline & $\begin{array}{l}\text { Partial flap } \\
\text { loss }\end{array}$ & NRCS & High & $4.5-7.3 y$ & Muscle-sparing TRAM & $14 / 293(4.8)$ & NR & NR & NR \\
\hline & $\begin{array}{l}\text { Total flap } \\
\text { loss }\end{array}$ & NRCS & High & $4.5-7.3 y$ & DIEP & $11 / 670(1.6)$ & NR & NR & NR \\
\hline & $\begin{array}{l}\text { Total flap } \\
\text { loss }\end{array}$ & NRCS & High & $4.5-7.3 y$ & Free TRAM & $3 / 144(2.1)$ & NR & NR & NR \\
\hline & $\begin{array}{l}\text { Total flap } \\
\text { loss }\end{array}$ & NRCS & High & $4.5-7.3 y$ & Pedicled TRAM & $8 / 683(1.2)$ & NR & NR & NR \\
\hline & $\begin{array}{l}\text { Total flap } \\
\text { loss }\end{array}$ & NRCS & High & $4.5-7.3 y$ & Muscle-sparing TRAM & $4 / 293(1.4)$ & NR & NR & NR \\
\hline \multirow[t]{3}{*}{$\begin{array}{l}\text { Massenburg, 2015, } \\
\text { 26487657, US }\end{array}$} & $\begin{array}{l}\text { Flap } \\
\text { failure/loss }\end{array}$ & NRCS & High & $2 y$ & Free TRAM & $56 / 2306(2.4)$ & vs. LD & $3.17(1.90,5.30)$ & $<0.001$ \\
\hline & $\begin{array}{l}\text { Flap } \\
\text { failure/loss }\end{array}$ & NRCS & High & $2 y$ & Pedicled TRAM & $67 / 2464(2.7)$ & vs. LD & $2.28(1.38,3.77)$ & 0.001 \\
\hline & $\begin{array}{l}\text { Flap } \\
\text { failure/loss }\end{array}$ & NRCS & High & $2 y$ & LD & $22 / 2085(1.1)$ & Ref & Ref & Ref \\
\hline
\end{tabular}

Abbreviations: $\mathrm{AR}=$ autologous reconstruction, $\mathrm{CI}=$ confidence interval, $\mathrm{DIEP}=$ deep inferior epigastric perforator, $\mathrm{LD}=$ latissimus dorsi, $\mathrm{mo}=$ months, $\mathrm{NR}=$ not reported, $\mathrm{NRCS}=$ nonrandomized comparative study, PMID $=$ PubMed identifier, Ref $=$ reference group, RoB $=$ risk of bias, SIEA $=$ superficial inferior epigastric artery, TRAM = transverse rectus abdominis myocutaneous, $\mathrm{y}=$ years.

Colors: Header rows are shaded orange. Rows for every alternate study are shaded blue. The colors do not add unique information.

Table F-6.24. Full Evidence Table - Key Question 6: Comparison of flap types for AR - categorical outcomes (hematoma/hemorrhage)

\begin{tabular}{|l|l|l|l|l|l|l|l|l|}
\hline Study, Year, PMID, Country & $\begin{array}{l}\text { Outcome } \\
\text { Description }\end{array}$ & Design & $\begin{array}{l}\text { Overall } \\
\text { RoB }\end{array}$ & $\begin{array}{l}\text { Time } \\
\text { Point }\end{array}$ & Arm & $\begin{array}{l}\text { n/N (\%) } \\
\text { Ratjusted Odds } \\
\text { Ratio (95\% Cl) }\end{array}$ & $\begin{array}{l}\text { P } \\
\text { Value }\end{array}$ \\
\hline Rindom, 2019, 31515191, Denmark & Hematoma & RCT & Moderate & 1 y & LD & $0 / 18(0)$ & Ref \\
\cline { 2 - 8 } & Hematoma & RCT & Moderate & 1 y & TAP & Ref \\
\hline & Donor site hematoma & NRCS & Moderate & 2 y & DIEP & $1 / 22(4.5)$ & $0.6(0.02,19.0)^{*}$ & $0.77^{*}$ \\
\hline
\end{tabular}




\begin{tabular}{|c|c|c|c|c|c|c|c|c|}
\hline Study, Year, PMID, Country & $\begin{array}{l}\text { Outcome } \\
\text { Description }\end{array}$ & Design & $\begin{array}{l}\text { Overall } \\
\text { RoB }\end{array}$ & $\begin{array}{l}\text { Time } \\
\text { Point }\end{array}$ & Arm & $\mathrm{n} / \mathrm{N}(\%)$ & $\begin{array}{l}\text { Adjusted Odds } \\
\text { Ratio }(95 \% \mathrm{Cl})\end{array}$ & $\begin{array}{l}\mathbf{P} \\
\text { Value }\end{array}$ \\
\hline \multirow{7}{*}{$\begin{array}{l}\text { Erdmann-Sager, 2018, } 29019862 \text {, } \\
\text { US \& Canada }\end{array}$} & Donor site hematoma & NRCS & Moderate & $2 y$ & Free TRAM & $0 / 92(0)$ & NR & NR \\
\hline & Donor site hematoma & NRCS & Moderate & $2 y$ & Pedicled TRAM & $0 / 78(0)$ & NR & NR \\
\hline & Donor site hematoma & NRCS & Moderate & $2 y$ & AR with SIEA & $4 / 62(6.5)$ & NR & NR \\
\hline & Breast hematoma & NRCS & Moderate & $2 y$ & DIEP & $25 / 355(7)$ & NR & NR \\
\hline & Breast hematoma & NRCS & Moderate & $2 y$ & Free TRAM & $5 / 92(5.4)$ & NR & NR \\
\hline & Breast hematoma & NRCS & Moderate & $2 y$ & Pedicled TRAM & $3 / 78(3.9)$ & NR & NR \\
\hline & Breast hematoma & NRCS & Moderate & $2 y$ & AR with SIEA & $7 / 62(11.3)$ & NR & NR \\
\hline \multirow{8}{*}{$\begin{array}{l}\text { Kulkarni, 2017, 28713853, US \& } \\
\text { Canada }\end{array}$} & Breast hematoma & NRCS & Moderate & $1 \mathrm{y}$ & DIEP & $22 / 365(6)$ & NR & NR \\
\hline & Breast hematoma & NRCS & Moderate & $1 \mathrm{y}$ & Free TRAM & $4 / 97(4.1)$ & NR & NR \\
\hline & Breast hematoma & NRCS & Moderate & $1 \mathrm{y}$ & Pedicled TRAM & $3 / 84(3.6)$ & NR & NR \\
\hline & Breast hematoma & NRCS & Moderate & $1 y$ & LD & $3 / 73(4.1)$ & NR & NR \\
\hline & Donor site hematoma & NRCS & Moderate & $1 \mathrm{y}$ & DIEP & $10 / 365(2.7)$ & NR & NR \\
\hline & Donor site hematoma & NRCS & Moderate & $1 \mathrm{y}$ & Free TRAM & $0 / 97(0)$ & NR & NR \\
\hline & Donor site hematoma & NRCS & Moderate & $1 \mathrm{y}$ & Pedicled TRAM & $0 / 84(0)$ & NR & NR \\
\hline & Donor site hematoma & NRCS & Moderate & $1 \mathrm{y}$ & LD & $0 / 73(0)$ & NR & NR \\
\hline \multirow[t]{4}{*}{$\begin{array}{l}\text { Macadam, 2016, 26910656, US, } \\
\text { Canada, \& Japan }\end{array}$} & $\begin{array}{l}\text { Hematoma requiring } \\
\text { surgery }\end{array}$ & NRCS & High & $\begin{array}{l}4.5- \\
7.3 \mathrm{y}\end{array}$ & DIEP & $56 / 670(8.4)$ & NR & NR \\
\hline & $\begin{array}{l}\text { Hematoma requiring } \\
\text { surgery }\end{array}$ & NRCS & High & $\begin{array}{l}4.5- \\
7.3 y\end{array}$ & Free TRAM & $5 / 144(3.5)$ & NR & NR \\
\hline & $\begin{array}{l}\text { Hematoma requiring } \\
\text { surgery }\end{array}$ & NRCS & High & $\begin{array}{l}4.5- \\
7.3 y\end{array}$ & Pedicled TRAM & $26 / 683(3.8)$ & NR & NR \\
\hline & $\begin{array}{l}\text { Hematoma requiring } \\
\text { surgery }\end{array}$ & NRCS & High & $\begin{array}{l}4.5- \\
7.3 y\end{array}$ & Muscle-sparing TRAM & $14 / 293(4.4)$ & NR & NR \\
\hline
\end{tabular}

Abbreviations: $\mathrm{AR}=$ autologous reconstruction, $\mathrm{CI}=$ confidence interval, $\mathrm{DIEP}=$ deep inferior epigastric perforator, $\mathrm{LD}=$ latissimus dorsi, $\mathrm{NR}=$ not reported, $\mathrm{NRCS}=$ nonrandomized comparative study, PMID $=$ PubMed identifier, $\mathrm{RCT}=$ randomized controlled trial, Ref $=$ reference group, RoB $=$ risk of bias, $\mathrm{SIEA}=$ superficial inferior epigastric artery, TAP $=$ thoracodorsal artery perforator, TRAM $=$ transverse rectus abdominis myocutaneous, $\mathrm{y}=$ years.

Colors: Header rows are shaded orange. Rows for every alternate study are shaded blue. The colors do not add unique information.

* calculated

Table F-6.25. Full Evidence Table - Key Question 6: Comparison of flap types for AR - categorical outcomes (composite/unspecified harms)

\begin{tabular}{|c|c|c|c|c|c|c|c|c|c|}
\hline $\begin{array}{l}\text { Study, Year, } \\
\text { PMID, } \\
\text { Country }\end{array}$ & Outcome Description & Design & $\begin{array}{l}\text { Overall } \\
\text { RoB }\end{array}$ & $\begin{array}{l}\text { Time } \\
\text { Point }\end{array}$ & Arm & $\mathrm{n} / \mathrm{N}(\%)$ & Comparison & $\begin{array}{l}\text { Adjusted Odds } \\
\text { Ratio }(95 \% \mathrm{Cl})\end{array}$ & $\begin{array}{l}\mathbf{P} \\
\text { Value }\end{array}$ \\
\hline \multirow{7}{*}{$\begin{array}{l}\text { Brorson } \\
2020 \mathrm{~b}, \\
32807615 \text {, } \\
\text { Sweden }\end{array}$} & Clavien-Dindo Grade I complications & $\mathrm{RCT}$ & High & $1 \mathrm{mo}$ & DIEP & $16 / 34(47)$ & Ref & Ref & Ref \\
\hline & Clavien-Dindo Grade I complications & RCT & High & $1 \mathrm{mo}$ & LD & $20 / 33(63)$ & VS. DIEP & $1.73(0.66,4.57)$ & 0.27 \\
\hline & Clavien-Dindo Grade II complications & RCT & High & $1 \mathrm{mo}$ & DIEP & $14 / 34(41)$ & Ref & Ref & Ref \\
\hline & Clavien-Dindo Grade II complications & RCT & High & $1 \mathrm{mo}$ & LD & $16 / 32(50)$ & vs. DIEP & $1.29(0.48,3.44)$ & 0.76 \\
\hline & Clavien-Dindo Grade IIla complications & RCT & High & $1 \mathrm{mo}$ & DIEP & $9 / 34(26)$ & Ref & Ref & Ref \\
\hline & Clavien-Dindo Grade IIla complications & RCT & High & $1 \mathrm{mo}$ & LD & $7 / 32(22)$ & vs. DIEP & $0.85(0.27,2.64)$ & 0.77 \\
\hline & Clavien-Dindo Grade IIIb complications & RCT & High & $1 \mathrm{mo}$ & DIEP & $11 / 34(32)$ & Ref & Ref & Ref \\
\hline
\end{tabular}




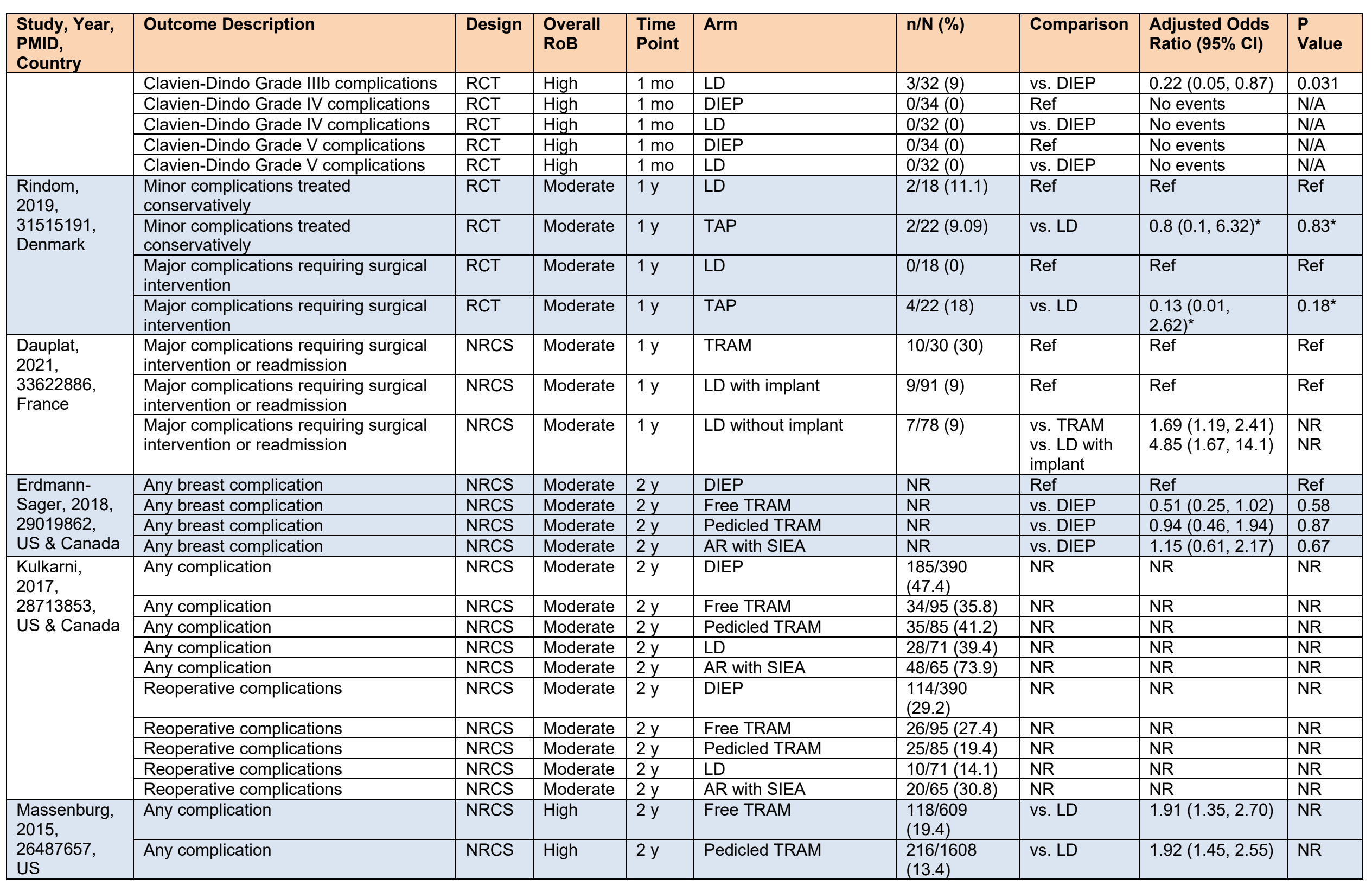

F-115 


\begin{tabular}{|c|c|c|c|c|c|c|c|c|c|}
\hline $\begin{array}{l}\text { Study, Year, } \\
\text { PMID, } \\
\text { Country }\end{array}$ & Outcome Description & Design & $\begin{array}{l}\text { Overall } \\
\text { RoB }\end{array}$ & $\begin{array}{l}\text { Time } \\
\text { Point }\end{array}$ & Arm & $\mathrm{n} / \mathrm{N}(\%)$ & Comparison & $\begin{array}{l}\text { Adjusted Odds } \\
\text { Ratio }(95 \% \mathrm{Cl})\end{array}$ & $\begin{array}{l}\text { P } \\
\text { Value }\end{array}$ \\
\hline & Any complication & NRCS & High & $2 y$ & LD & $77 / 1079(7.1)$ & Ref & Ref & Ref \\
\hline & $\begin{array}{l}\text { Superficial SSI, deep SSI, organ space } \\
\text { infection, or wound disruption/ } \\
\text { dehiscence }\end{array}$ & NRCS & High & $2 y$ & Free TRAM & $\begin{array}{l}143 / 2306 \\
(6.2)\end{array}$ & vs. LD & $1.46(1.00,2.12)$ & 0.046 \\
\hline & $\begin{array}{l}\text { Superficial SSI, deep SSI, organ space } \\
\text { infection, or wound disruption/ } \\
\text { dehiscence }\end{array}$ & NRCS & High & $2 y$ & Pedicled TRAM & $\begin{array}{l}199 / 2464 \\
(8.1)\end{array}$ & vs. LD & $1.80(1.29,2.51)$ & 0.001 \\
\hline & $\begin{array}{l}\text { Superficial SSI, deep SSI, organ space } \\
\text { infection, or wound disruption/ } \\
\text { dehiscence }\end{array}$ & NRCS & High & $2 y$ & LD & $90 / 2085(4.3)$ & Ref & Ref & Ref \\
\hline \multirow{2}{*}{$\begin{array}{l}\text { Woo, 2018, } \\
30360958, \\
\text { South Korea }\end{array}$} & Lymphedema & NRCS & High & NR & DIEP & $23 / 163(14.1)$ & NR & NR & NR \\
\hline & Lymphedema & NRCS & High & NR & LD & $19 / 44(43.2)$ & NR & NR & NR \\
\hline $\begin{array}{l}\text { Zhong, 2014, } \\
24675183, \\
\text { Canada }\end{array}$ & $\begin{array}{l}\text { Major breast complications included } \\
\text { total or partial flap loss, fat necrosis, } \\
\text { and breast hematoma }\end{array}$ & NRCS & High & NR & DIEP & $50 / 244(20.5)$ & Ref & Ref & Ref \\
\hline
\end{tabular}

Abbreviations: $\mathrm{AR}=$ autologous reconstruction, $\mathrm{CI}=$ confidence interval, $\mathrm{DIEP}=$ deep inferior epigastric perforator, $\mathrm{LD}=$ latissimus dorsi, $\mathrm{NR}=$ not reported, $\mathrm{NRCS}=$ nonrandomized comparative study, PMID $=$ PubMed identifier, $\mathrm{RCT}=$ randomized controlled trial, $\mathrm{Ref}=$ reference group, $\mathrm{RoB}=$ risk of bias, $\mathrm{SIEA}=$ superficial inferior epigastric artery, SSI $=$ surgical site infection, TAP $=$ thoracodorsal artery perforator, TRAM $=$ transverse rectus abdominis myocutaneous, $\mathrm{y}=\mathrm{years}$.

* calculated

Colors: Header rows are shaded orange. Rows for every alternate study are shaded blue. The colors do not add unique information. 


\section{Appendix G. Results: Evidence Profiles}

Table G-1. Key Question 1: IBR versus AR - full evidence profile

\begin{tabular}{|c|c|c|c|c|c|c|c|c|c|}
\hline $\begin{array}{l}\text { Outcome } \\
\text { Category }\end{array}$ & Outcome & $\begin{array}{l}\text { N Studies } \\
\text { (Patients) }\end{array}$ & RoB & Consistency & Precision & Directness & Other & SoE & Conclusions (Reason, if None) \\
\hline \multirow[t]{7}{*}{ Clinical } & General quality of life & $3(709)$ & Moderate & Consistent & Precise & Direct & None & Moderate & Comparable in both groups \\
\hline & Physical well-being & $6(5717)$ & Moderate & Consistent & Precise & Direct & None & Insufficient & None (Inconsistent results) \\
\hline & Psychosocial well-being & $5(2760)$ & Moderate & Consistent & Precise & Direct & None & Moderate & $\begin{array}{l}\text { Clinically comparable in both } \\
\text { groups: summary adjMD } 3.14 \\
(95 \% \mathrm{Cl} 1.26,5.02) ; 3 \text { studies }\end{array}$ \\
\hline & Sexual well-being & $4(3307)$ & Moderate & Inconsistent & Precise & Direct & None & Moderate & $\begin{array}{l}\text { Clinically significant better with } \\
\text { AR: summary adjMD } 5.83(95 \% \\
\text { Cl 3.44, 8.23); } 3 \text { studies }\end{array}$ \\
\hline & Patient satisfaction with breast & $7(4557)$ & Moderate & Consistent & Precise & Direct & None & Moderate & $\begin{array}{l}\text { Clinically significant better } \\
\text { satisfaction with breast with } \mathrm{AR} \text { : } \\
\text { summary adjMD } 8.08(95 \% \mathrm{Cl} \\
6.11,10.1) ; 3 \text { studies. Inconsistent } \\
\text { results regarding satisfaction with } \\
\text { nipples. }\end{array}$ \\
\hline & $\begin{array}{l}\text { Patient satisfaction with surgical } \\
\text { outcome }\end{array}$ & $5(1432)$ & Moderate & Inconsistent & Precise & Direct & None & Insufficient & None (Inconsistent results) \\
\hline & Mortality & $1(4061)$ & High & $\mathrm{N} / \mathrm{A}$ & Precise & Direct & Sparse & Insufficient & None (Sparse evidence) \\
\hline \multirow{12}{*}{$\begin{array}{l}\text { Surgical } \\
\text { complications }\end{array}$} & Unplanned repeat hospitalization & $3(50675)$ & High & Consistent & Precise & Direct & None & Moderate & Comparable in both groups \\
\hline & $\begin{array}{l}\text { Duration of unplanned repeat } \\
\text { hospitalization }\end{array}$ & $0(0)$ & N/A & $\mathrm{N} / \mathrm{A}$ & $\mathrm{N} / \mathrm{A}$ & $\mathrm{N} / \mathrm{A}$ & $\mathrm{N} / \mathrm{A}$ & $\mathrm{N} / \mathrm{A}$ & $\mathrm{N} / \mathrm{A}$ \\
\hline & $\begin{array}{l}\text { Unplanned repeat surgeries for } \\
\text { revision }\end{array}$ & $3(3138)$ & High & Inconsistent & Precise & Direct & None & Insufficient & None (Inconsistent results) \\
\hline & $\begin{array}{l}\text { Unplanned repeat surgeries for } \\
\text { complications }\end{array}$ & $3(14313)$ & High & Inconsistent & Precise & Direct & None & Insufficient & None (Inconsistent results) \\
\hline & Pain & $5(3173)$ & Moderate & Inconsistent & Precise & Direct & None & Insufficient & None (Inconsistent results) \\
\hline & Analgesic use & $1(90)$ & High & $\mathrm{N} / \mathrm{A}$ & Imprecise & Direct & Sparse & Insufficient & None (Sparse evidence) \\
\hline & Necrosis & $4(34742)$ & High & Inconsistent & Imprecise & Direct & None & Insufficient & None (Inconsistent results) \\
\hline & Animation deformity & $0(0)$ & $\mathrm{N} / \mathrm{A}$ & $\mathrm{N} / \mathrm{A}$ & $\mathrm{N} / \mathrm{A}$ & $\mathrm{N} / \mathrm{A}$ & $\mathrm{N} / \mathrm{A}$ & $\mathrm{N} / \mathrm{A}$ & $\mathrm{N} / \mathrm{A}$ \\
\hline & $\begin{array}{l}\text { Complications delaying other } \\
\text { cancer treatments }\end{array}$ & $0(0)$ & $\mathrm{N} / \mathrm{A}$ & $\mathrm{N} / \mathrm{A}$ & $\mathrm{N} / \mathrm{A}$ & $\mathrm{N} / \mathrm{A}$ & N/A & N/A & $\mathrm{N} / \mathrm{A}$ \\
\hline & Thromboembolic events & $2(34555)$ & High & Consistent & Precise & Direct & None & Moderate & $\begin{array}{l}\text { Increased risk of deep vein } \\
\text { thrombosis or pulmonary } \\
\text { embolism in AR group }\end{array}$ \\
\hline & $\begin{array}{l}\text { Infections (not explicitly implant- } \\
\text { related) }\end{array}$ & $4(17246)$ & Moderate & Inconsistent & Precise & Direct & None & Insufficient & None (Inconsistent results) \\
\hline & Seroma & $2(1300)$ & Moderate & Consistent & Unclear & Direct & None & Low & $\begin{array}{l}\text { Increased risk of breast seroma in } \\
\text { IBR group }\end{array}$ \\
\hline
\end{tabular}




\begin{tabular}{|c|c|c|c|c|c|c|c|c|c|}
\hline $\begin{array}{l}\text { Outcome } \\
\text { Category }\end{array}$ & Outcome & $\begin{array}{l}\text { N Studies } \\
\text { (Patients) }\end{array}$ & RoB & Consistency & Precision & Directness & Other & SoE & Conclusions (Reason, if None) \\
\hline & Reconstructive Failure & $5(21090)$ & Moderate & Consistent & Precise & Direct & None & Moderate & $\begin{array}{l}\text { Increased risk with IBR in the } \\
\text { long-term ( } 1.5 \text { to } 4 \text { years of } \\
\text { followup) }\end{array}$ \\
\hline
\end{tabular}

Abbreviations: adj = adjusted, $\mathrm{AR}=$ autologous reconstruction, $\mathrm{DIEP}=$ deep inferior epigastric perforator, $\mathrm{IBR}=$ implant-based reconstruction, $\mathrm{MD}=\mathrm{mean}$ difference, $\mathrm{N} / \mathrm{A}=$ not applicable, $\mathrm{NR}=$ not reported, $\mathrm{RoB}=$ risk of bias, $\mathrm{SIEA}=$ superficial inferior epigastric artery, $\mathrm{SoE}=$ strength of evidence, TRAM $=$ transverse rectus abdominis myocutaneous .

For continuous outcomes, clinical significance is based on published estimates of minimal clinically important differences (MCIDs), where available.

Colors: Header rows are shaded orange. The color does not add unique information. 
Table G-2. Key Question 2: Timing of chemotherapy and radiation therapy relative to IBR and AR - full evidence profile

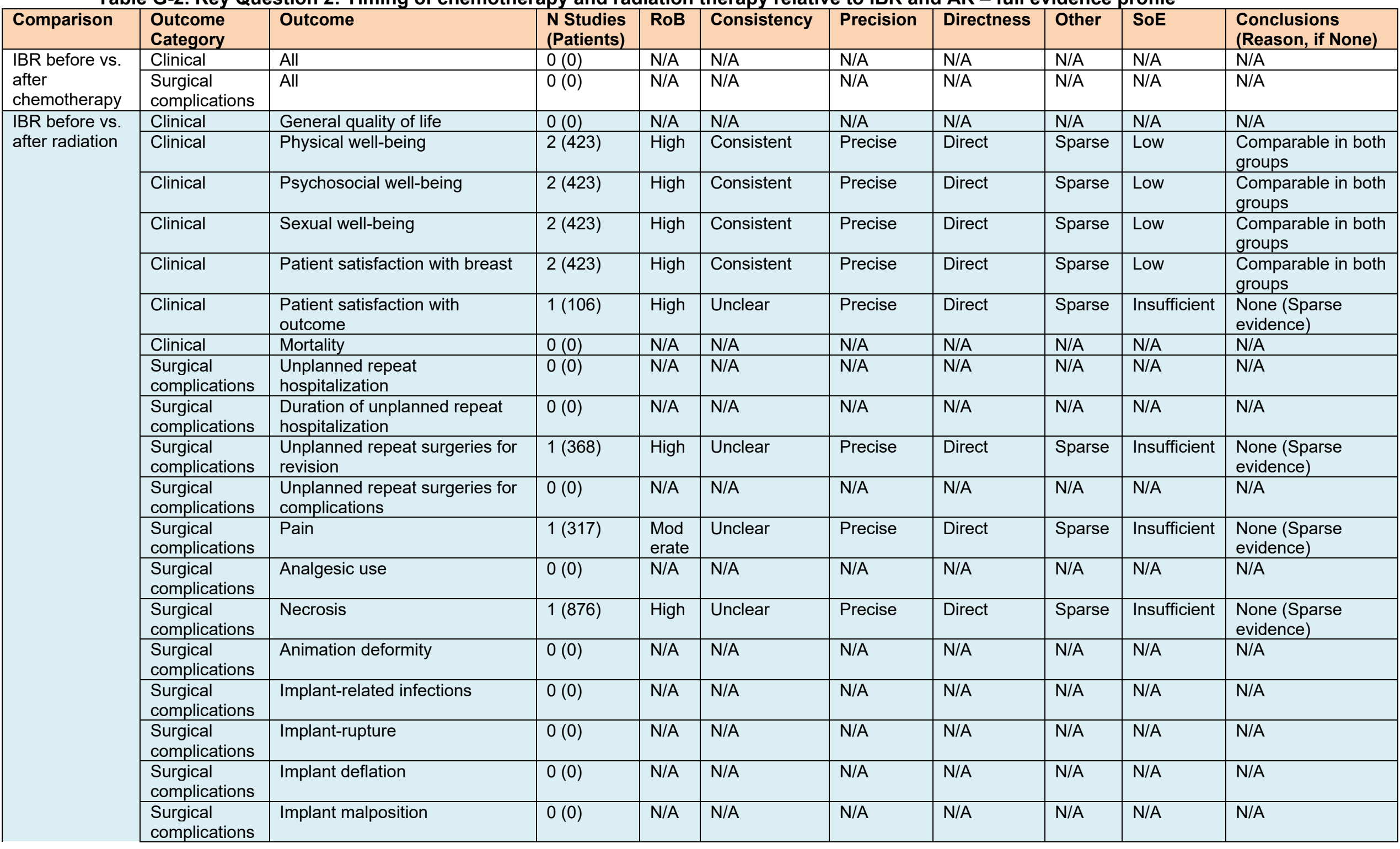




\begin{tabular}{|c|c|c|c|c|c|c|c|c|c|c|}
\hline Comparison & $\begin{array}{l}\text { Outcome } \\
\text { Category }\end{array}$ & Outcome & $\begin{array}{l}\text { N Studies } \\
\text { (Patients) }\end{array}$ & RoB & Consistency & Precision & Directness & Other & SoE & $\begin{array}{l}\text { Conclusions } \\
\text { (Reason, if None) }\end{array}$ \\
\hline & $\begin{array}{l}\text { Surgical } \\
\text { complications }\end{array}$ & $\begin{array}{l}\text { Implant failure/loss or need for } \\
\text { explant surgery }\end{array}$ & $4(2537)$ & High & Consistent & Precise & Direct & None & Moderate & $\begin{array}{l}\text { Comparable in both } \\
\text { groups: summary } \\
\text { adjOR } 0.87(95 \% \\
\text { Cl } 0.65,1.17)\end{array}$ \\
\hline & $\begin{array}{l}\text { Surgical } \\
\text { complications }\end{array}$ & $\begin{array}{l}\text { Complications delaying other } \\
\text { cancer treatments }\end{array}$ & $0(0)$ & $\mathrm{N} / \mathrm{A}$ & $\mathrm{N} / \mathrm{A}$ & $\mathrm{N} / \mathrm{A}$ & $\mathrm{N} / \mathrm{A}$ & $\mathrm{N} / \mathrm{A}$ & $\mathrm{N} / \mathrm{A}$ & $\mathrm{N} / \mathrm{A}$ \\
\hline & $\begin{array}{l}\text { Surgical } \\
\text { complications }\end{array}$ & Thromboembolic events & $0(0)$ & $\mathrm{N} / \mathrm{A}$ & N/A & $\mathrm{N} / \mathrm{A}$ & $\mathrm{N} / \mathrm{A}$ & $\mathrm{N} / \mathrm{A}$ & $\mathrm{N} / \mathrm{A}$ & N/A \\
\hline & $\begin{array}{l}\text { Surgical } \\
\text { complications }\end{array}$ & Seroma & $1(150)$ & $\begin{array}{l}\text { Mod } \\
\text { erate }\end{array}$ & Unclear & Unclear & Direct & . & Insufficient & $\begin{array}{l}\text { None (Sparse } \\
\text { evidence) }\end{array}$ \\
\hline & $\begin{array}{l}\text { Surgical } \\
\text { complications }\end{array}$ & Chronic conditions & $0(0)$ & N/A & $\mathrm{N} / \mathrm{A}$ & $\mathrm{N} / \mathrm{A}$ & $\mathrm{N} / \mathrm{A}$ & $\mathrm{N} / \mathrm{A}$ & $\mathrm{N} / \mathrm{A}$ & $\mathrm{N} / \mathrm{A}$ \\
\hline \multirow{2}{*}{$\begin{array}{l}\text { AR before vs. } \\
\text { after } \\
\text { chemotherapy }\end{array}$} & Clinical & All & $0(0)$ & $\mathrm{N} / \mathrm{A}$ & $\mathrm{N} / \mathrm{A}$ & $\mathrm{N} / \mathrm{A}$ & $\mathrm{N} / \mathrm{A}$ & $\mathrm{N} / \mathrm{A}$ & $\mathrm{N} / \mathrm{A}$ & $\mathrm{N} / \mathrm{A}$ \\
\hline & $\begin{array}{l}\text { Surgical } \\
\text { complications }\end{array}$ & All & $0(0)$ & $\mathrm{N} / \mathrm{A}$ & $\mathrm{N} / \mathrm{A}$ & $\mathrm{N} / \mathrm{A}$ & $\mathrm{N} / \mathrm{A}$ & $\mathrm{N} / \mathrm{A}$ & $\mathrm{N} / \mathrm{A}$ & $\mathrm{N} / \mathrm{A}$ \\
\hline \multirow{2}{*}{$\begin{array}{l}\text { IBR before vs. } \\
\text { after radiation }\end{array}$} & Clinical & All & $0(0)$ & $\mathrm{N} / \mathrm{A}$ & $\mathrm{N} / \mathrm{A}$ & $\mathrm{N} / \mathrm{A}$ & $\mathrm{N} / \mathrm{A}$ & $\mathrm{N} / \mathrm{A}$ & $\mathrm{N} / \mathrm{A}$ & $\mathrm{N} / \mathrm{A}$ \\
\hline & $\begin{array}{l}\text { Surgical } \\
\text { complications }\end{array}$ & All & $0(0)$ & $\mathrm{N} / \mathrm{A}$ & N/A & N/A & $\mathrm{N} / \mathrm{A}$ & $\mathrm{N} / \mathrm{A}$ & $\mathrm{N} / \mathrm{A}$ & $\mathrm{N} / \mathrm{A}$ \\
\hline
\end{tabular}

Abbreviations: adj = adjusted, $\mathrm{AR}=$ autologous reconstruction, $\mathrm{CI}=$ confidence interval, $\mathrm{IBR}=$ implant-based reconstruction, $\mathrm{OR}=\mathrm{odds}$ ratio, $\mathrm{RoB}=$ risk of bias, SoE $=$ strength of evidence.

Colors: Header rows are shaded orange. Rows for every alternate comparison are shaded blue. The colors do not add unique information. 
Table G-3. Key Question 3: Comparison of materials for IBR - full evidence profile

\begin{tabular}{|c|c|c|c|c|c|c|c|c|c|c|}
\hline Comparison & $\begin{array}{l}\text { Outcome } \\
\text { Category }\end{array}$ & Outcome & $\begin{array}{l}\text { N Studies } \\
\text { (Patients) }\end{array}$ & RoB & Consistency & Precision & Directness & Other & SoE & $\begin{array}{l}\text { Conclusions } \\
\text { (Reason, if None) }\end{array}$ \\
\hline \multirow[t]{20}{*}{$\begin{array}{l}\text { Silicone vs. } \\
\text { saline }\end{array}$} & Clinical & General quality of life & $1(139)$ & High & $\mathrm{N} / \mathrm{A}$ & Unclear & Direct & Sparse & Insufficient & $\begin{array}{l}\text { None (Sparse } \\
\text { evidence) }\end{array}$ \\
\hline & Clinical & Physical well-being & $1(142)$ & High & $\mathrm{N} / \mathrm{A}$ & Unclear & Direct & Sparse & Insufficient & $\begin{array}{l}\text { None (Sparse } \\
\text { evidence) }\end{array}$ \\
\hline & Clinical & Psychosocial well-being & $1(142)$ & High & $\mathrm{N} / \mathrm{A}$ & Unclear & Direct & Sparse & Insufficient & $\begin{array}{l}\text { None (Sparse } \\
\text { evidence) }\end{array}$ \\
\hline & Clinical & Sexual well-being & $1(137)$ & High & $\mathrm{N} / \mathrm{A}$ & Unclear & Direct & Sparse & Insufficient & $\begin{array}{l}\text { None (Sparse } \\
\text { evidence) }\end{array}$ \\
\hline & Clinical & Patient satisfaction with breast & $2(624)$ & High & Consistent & Unclear & Direct & None & Low & $\begin{array}{l}\text { Comparable in both } \\
\text { groups }\end{array}$ \\
\hline & Clinical & Patient satisfaction with outcome & $1(143)$ & High & $\mathrm{N} / \mathrm{A}$ & Unclear & Direct & Sparse & Insufficient & $\begin{array}{l}\text { None (Sparse } \\
\text { evidence) }\end{array}$ \\
\hline & Clinical & $\begin{array}{l}\text { Planned surgeries for } \\
\text { reconstruction }\end{array}$ & $0(0)$ & $\mathrm{N} / \mathrm{A}$ & $\mathrm{N} / \mathrm{A}$ & $\mathrm{N} / \mathrm{A}$ & $\mathrm{N} / \mathrm{A}$ & $\mathrm{N} / \mathrm{A}$ & $\mathrm{N} / \mathrm{A}$ & $\mathrm{N} / \mathrm{A}$ \\
\hline & Clinical & Mortality & $1(\mathrm{NR})$ & High & $\mathrm{N} / \mathrm{A}$ & Imprecise & Direct & Sparse & Insufficient & $\begin{array}{l}\text { None (Sparse } \\
\text { evidence) }\end{array}$ \\
\hline & $\begin{array}{l}\text { Surgical } \\
\text { complications }\end{array}$ & Unplanned repeat hospitalization & $0(0)$ & $\mathrm{N} / \mathrm{A}$ & $\mathrm{N} / \mathrm{A}$ & $\mathrm{N} / \mathrm{A}$ & $\mathrm{N} / \mathrm{A}$ & $\mathrm{N} / \mathrm{A}$ & $\mathrm{N} / \mathrm{A}$ & $\mathrm{N} / \mathrm{A}$ \\
\hline & $\begin{array}{l}\text { Surgical } \\
\text { complications }\end{array}$ & $\begin{array}{l}\text { Duration of unplanned repeat } \\
\text { hospitalization }\end{array}$ & $0(0)$ & $\mathrm{N} / \mathrm{A}$ & $\mathrm{N} / \mathrm{A}$ & $\mathrm{N} / \mathrm{A}$ & $\mathrm{N} / \mathrm{A}$ & $\mathrm{N} / \mathrm{A}$ & $\mathrm{N} / \mathrm{A}$ & $\mathrm{N} / \mathrm{A}$ \\
\hline & $\begin{array}{l}\text { Surgical } \\
\text { complications }\end{array}$ & $\begin{array}{l}\text { Unplanned repeat surgeries for } \\
\text { revision }\end{array}$ & $0(0)$ & $\mathrm{N} / \mathrm{A}$ & $\mathrm{N} / \mathrm{A}$ & $\mathrm{N} / \mathrm{A}$ & $\mathrm{N} / \mathrm{A}$ & $\mathrm{N} / \mathrm{A}$ & $\mathrm{N} / \mathrm{A}$ & $\mathrm{N} / \mathrm{A}$ \\
\hline & $\begin{array}{l}\text { Surgical } \\
\text { complications }\end{array}$ & $\begin{array}{l}\text { Unplanned repeat surgeries for } \\
\text { complications }\end{array}$ & $0(0)$ & $\mathrm{N} / \mathrm{A}$ & $\mathrm{N} / \mathrm{A}$ & $\mathrm{N} / \mathrm{A}$ & $\mathrm{N} / \mathrm{A}$ & N/A & $\mathrm{N} / \mathrm{A}$ & $\mathrm{N} / \mathrm{A}$ \\
\hline & $\begin{array}{l}\text { Surgical } \\
\text { complications }\end{array}$ & Pain & $0(0)$ & $\mathrm{N} / \mathrm{A}$ & $\mathrm{N} / \mathrm{A}$ & $\mathrm{N} / \mathrm{A}$ & $\mathrm{N} / \mathrm{A}$ & $\mathrm{N} / \mathrm{A}$ & $\mathrm{N} / \mathrm{A}$ & $\mathrm{N} / \mathrm{A}$ \\
\hline & $\begin{array}{l}\text { Surgical } \\
\text { complications }\end{array}$ & Analgesic use & $0(0)$ & $\mathrm{N} / \mathrm{A}$ & $\mathrm{N} / \mathrm{A}$ & $\mathrm{N} / \mathrm{A}$ & $\mathrm{N} / \mathrm{A}$ & $\mathrm{N} / \mathrm{A}$ & $\mathrm{N} / \mathrm{A}$ & $\mathrm{N} / \mathrm{A}$ \\
\hline & $\begin{array}{l}\text { Surgical } \\
\text { complications }\end{array}$ & Necrosis & $0(0)$ & $\mathrm{N} / \mathrm{A}$ & $\mathrm{N} / \mathrm{A}$ & $\mathrm{N} / \mathrm{A}$ & $\mathrm{N} / \mathrm{A}$ & $\mathrm{N} / \mathrm{A}$ & $\mathrm{N} / \mathrm{A}$ & $\mathrm{N} / \mathrm{A}$ \\
\hline & $\begin{array}{l}\text { Surgical } \\
\text { complications }\end{array}$ & Animation deformity & $0(0)$ & $\mathrm{N} / \mathrm{A}$ & $\mathrm{N} / \mathrm{A}$ & $\mathrm{N} / \mathrm{A}$ & $\mathrm{N} / \mathrm{A}$ & $\mathrm{N} / \mathrm{A}$ & $\mathrm{N} / \mathrm{A}$ & $\mathrm{N} / \mathrm{A}$ \\
\hline & $\begin{array}{l}\text { Surgical } \\
\text { complications }\end{array}$ & Implant-related infections & $0(0)$ & $\mathrm{N} / \mathrm{A}$ & $\mathrm{N} / \mathrm{A}$ & $\mathrm{N} / \mathrm{A}$ & $\mathrm{N} / \mathrm{A}$ & $\mathrm{N} / \mathrm{A}$ & $\mathrm{N} / \mathrm{A}$ & $\mathrm{N} / \mathrm{A}$ \\
\hline & $\begin{array}{l}\text { Surgical } \\
\text { complications }\end{array}$ & Implant-rupture & $0(0)$ & $\mathrm{N} / \mathrm{A}$ & $\mathrm{N} / \mathrm{A}$ & $\mathrm{N} / \mathrm{A}$ & $\mathrm{N} / \mathrm{A}$ & $\mathrm{N} / \mathrm{A}$ & $\mathrm{N} / \mathrm{A}$ & $\mathrm{N} / \mathrm{A}$ \\
\hline & $\begin{array}{l}\text { Surgical } \\
\text { complications }\end{array}$ & Implant deflation & $0(0)$ & $\mathrm{N} / \mathrm{A}$ & $\mathrm{N} / \mathrm{A}$ & $\mathrm{N} / \mathrm{A}$ & $\mathrm{N} / \mathrm{A}$ & N/A & $\mathrm{N} / \mathrm{A}$ & $\mathrm{N} / \mathrm{A}$ \\
\hline & $\begin{array}{l}\text { Surgical } \\
\text { complications }\end{array}$ & Implant malposition & $0(0)$ & $\mathrm{N} / \mathrm{A}$ & $\mathrm{N} / \mathrm{A}$ & $\mathrm{N} / \mathrm{A}$ & $\mathrm{N} / \mathrm{A}$ & $\mathrm{N} / \mathrm{A}$ & $\mathrm{N} / \mathrm{A}$ & N/A \\
\hline
\end{tabular}




\begin{tabular}{|c|c|c|c|c|c|c|c|c|c|c|}
\hline Comparison & $\begin{array}{l}\text { Outcome } \\
\text { Category }\end{array}$ & Outcome & $\begin{array}{l}\text { N Studies } \\
\text { (Patients) }\end{array}$ & RoB & Consistency & Precision & Directness & Other & SoE & $\begin{array}{l}\text { Conclusions } \\
\text { (Reason, if None) }\end{array}$ \\
\hline & $\begin{array}{l}\text { Surgical } \\
\text { complications }\end{array}$ & $\begin{array}{l}\text { Implant failure/loss or need for } \\
\text { explant surgery }\end{array}$ & $1(288)$ & High & $\mathrm{N} / \mathrm{A}$ & Precise & Direct & Sparse & Insufficient & $\begin{array}{l}\text { None (Sparse } \\
\text { evidence) }\end{array}$ \\
\hline & $\begin{array}{l}\text { Surgical } \\
\text { complications }\end{array}$ & Capsular contracture & $1(345)$ & High & $\mathrm{N} / \mathrm{A}$ & Unclear & Direct & Sparse & Insufficient & $\begin{array}{l}\text { None (Sparse } \\
\text { evidence) }\end{array}$ \\
\hline & $\begin{array}{l}\text { Surgical } \\
\text { complications }\end{array}$ & New neoplasms & $0(0)$ & $\mathrm{N} / \mathrm{A}$ & $\mathrm{N} / \mathrm{A}$ & $\mathrm{N} / \mathrm{A}$ & $\mathrm{N} / \mathrm{A}$ & $\mathrm{N} / \mathrm{A}$ & $\mathrm{N} / \mathrm{A}$ & $\mathrm{N} / \mathrm{A}$ \\
\hline & $\begin{array}{l}\text { Surgical } \\
\text { complications }\end{array}$ & $\begin{array}{l}\text { Complications delaying other } \\
\text { cancer treatments }\end{array}$ & $0(0)$ & N/A & $\mathrm{N} / \mathrm{A}$ & $\mathrm{N} / \mathrm{A}$ & $\mathrm{N} / \mathrm{A}$ & $\mathrm{N} / \mathrm{A}$ & $\mathrm{N} / \mathrm{A}$ & $\mathrm{N} / \mathrm{A}$ \\
\hline & $\begin{array}{l}\text { Surgical } \\
\text { complications }\end{array}$ & Thromboembolic events & $0(0)$ & $\mathrm{N} / \mathrm{A}$ & $\mathrm{N} / \mathrm{A}$ & $\mathrm{N} / \mathrm{A}$ & $\mathrm{N} / \mathrm{A}$ & $\mathrm{N} / \mathrm{A}$ & $\mathrm{N} / \mathrm{A}$ & $\mathrm{N} / \mathrm{A}$ \\
\hline & $\begin{array}{l}\text { Surgical } \\
\text { complications }\end{array}$ & Seroma & $0(0)$ & $\mathrm{N} / \mathrm{A}$ & $\mathrm{N} / \mathrm{A}$ & $\mathrm{N} / \mathrm{A}$ & N/A & $\mathrm{N} / \mathrm{A}$ & $\mathrm{N} / \mathrm{A}$ & $\mathrm{N} / \mathrm{A}$ \\
\hline & $\begin{array}{l}\text { Surgical } \\
\text { complications }\end{array}$ & Chronic conditions & $0(0)$ & $\mathrm{N} / \mathrm{A}$ & $\mathrm{N} / \mathrm{A}$ & $\mathrm{N} / \mathrm{A}$ & $\mathrm{N} / \mathrm{A}$ & $\mathrm{N} / \mathrm{A}$ & $\mathrm{N} / \mathrm{A}$ & $\mathrm{N} / \mathrm{A}$ \\
\hline \multirow{16}{*}{$\begin{array}{l}\text { Silicone vs. } \\
\text { double lumen }\end{array}$} & Clinical & General quality of life & $0(0)$ & $\mathrm{N} / \mathrm{A}$ & $\mathrm{N} / \mathrm{A}$ & $\mathrm{N} / \mathrm{A}$ & $\mathrm{N} / \mathrm{A}$ & $\mathrm{N} / \mathrm{A}$ & $\mathrm{N} / \mathrm{A}$ & $\mathrm{N} / \mathrm{A}$ \\
\hline & Clinical & Physical well-being & $0(0)$ & $\mathrm{N} / \mathrm{A}$ & $\mathrm{N} / \mathrm{A}$ & $\mathrm{N} / \mathrm{A}$ & $\mathrm{N} / \mathrm{A}$ & $\mathrm{N} / \mathrm{A}$ & $\mathrm{N} / \mathrm{A}$ & $\mathrm{N} / \mathrm{A}$ \\
\hline & Clinical & Psychosocial well-being & $0(0)$ & $\mathrm{N} / \mathrm{A}$ & $\mathrm{N} / \mathrm{A}$ & $\mathrm{N} / \mathrm{A}$ & $\mathrm{N} / \mathrm{A}$ & $\mathrm{N} / \mathrm{A}$ & $\mathrm{N} / \mathrm{A}$ & $\mathrm{N} / \mathrm{A}$ \\
\hline & Clinical & Sexual well-being & $0(0)$ & $\mathrm{N} / \mathrm{A}$ & $\mathrm{N} / \mathrm{A}$ & $\mathrm{N} / \mathrm{A}$ & $\mathrm{N} / \mathrm{A}$ & $\mathrm{N} / \mathrm{A}$ & $\mathrm{N} / \mathrm{A}$ & $\mathrm{N} / \mathrm{A}$ \\
\hline & Clinical & Patient satisfaction with breast & $0(0)$ & $\mathrm{N} / \mathrm{A}$ & $\mathrm{N} / \mathrm{A}$ & $\mathrm{N} / \mathrm{A}$ & $\mathrm{N} / \mathrm{A}$ & $\mathrm{N} / \mathrm{A}$ & $\mathrm{N} / \mathrm{A}$ & $\mathrm{N} / \mathrm{A}$ \\
\hline & Clinical & Patient satisfaction with outcome & $0(0)$ & $\mathrm{N} / \mathrm{A}$ & $\mathrm{N} / \mathrm{A}$ & $\mathrm{N} / \mathrm{A}$ & N/A & $\mathrm{N} / \mathrm{A}$ & $\mathrm{N} / \mathrm{A}$ & $\mathrm{N} / \mathrm{A}$ \\
\hline & Clinical & $\begin{array}{l}\text { Planned surgeries for } \\
\text { reconstruction }\end{array}$ & $0(0)$ & N/A & $\mathrm{N} / \mathrm{A}$ & $\mathrm{N} / \mathrm{A}$ & $\mathrm{N} / \mathrm{A}$ & $\mathrm{N} / \mathrm{A}$ & $\mathrm{N} / \mathrm{A}$ & $\mathrm{N} / \mathrm{A}$ \\
\hline & Clinical & Mortality & $1(\mathrm{NR})$ & High & Unclear & Imprecise & Direct & Sparse & Insufficient & $\begin{array}{l}\text { None (Sparse } \\
\text { evidence) }\end{array}$ \\
\hline & $\begin{array}{l}\text { Surgical } \\
\text { complications }\end{array}$ & Unplanned repeat hospitalization & $0(0)$ & $\mathrm{N} / \mathrm{A}$ & $\mathrm{N} / \mathrm{A}$ & $\mathrm{N} / \mathrm{A}$ & $\mathrm{N} / \mathrm{A}$ & $\mathrm{N} / \mathrm{A}$ & $\mathrm{N} / \mathrm{A}$ & $\mathrm{N} / \mathrm{A}$ \\
\hline & $\begin{array}{l}\text { Surgical } \\
\text { complications }\end{array}$ & $\begin{array}{l}\text { Duration of unplanned repeat } \\
\text { hospitalization }\end{array}$ & $0(0)$ & N/A & $\mathrm{N} / \mathrm{A}$ & N/A & N/A & $\mathrm{N} / \mathrm{A}$ & $\mathrm{N} / \mathrm{A}$ & $\mathrm{N} / \mathrm{A}$ \\
\hline & $\begin{array}{l}\text { Surgical } \\
\text { complications }\end{array}$ & $\begin{array}{l}\text { Unplanned repeat surgeries for } \\
\text { revision }\end{array}$ & $0(0)$ & $\mathrm{N} / \mathrm{A}$ & $\mathrm{N} / \mathrm{A}$ & $\mathrm{N} / \mathrm{A}$ & N/A & $\mathrm{N} / \mathrm{A}$ & $\mathrm{N} / \mathrm{A}$ & $\mathrm{N} / \mathrm{A}$ \\
\hline & $\begin{array}{l}\text { Surgical } \\
\text { complications }\end{array}$ & $\begin{array}{l}\text { Unplanned repeat surgeries for } \\
\text { complications }\end{array}$ & $0(0)$ & $\mathrm{N} / \mathrm{A}$ & $\mathrm{N} / \mathrm{A}$ & $\mathrm{N} / \mathrm{A}$ & $\mathrm{N} / \mathrm{A}$ & $\mathrm{N} / \mathrm{A}$ & $\mathrm{N} / \mathrm{A}$ & $\mathrm{N} / \mathrm{A}$ \\
\hline & $\begin{array}{l}\text { Surgical } \\
\text { complications }\end{array}$ & Pain & $0(0)$ & $\mathrm{N} / \mathrm{A}$ & $\mathrm{N} / \mathrm{A}$ & $\mathrm{N} / \mathrm{A}$ & $\mathrm{N} / \mathrm{A}$ & $\mathrm{N} / \mathrm{A}$ & $\mathrm{N} / \mathrm{A}$ & $\mathrm{N} / \mathrm{A}$ \\
\hline & $\begin{array}{l}\text { Surgical } \\
\text { complications }\end{array}$ & Analgesic use & $0(0)$ & $\mathrm{N} / \mathrm{A}$ & $\mathrm{N} / \mathrm{A}$ & $\mathrm{N} / \mathrm{A}$ & $\mathrm{N} / \mathrm{A}$ & $\mathrm{N} / \mathrm{A}$ & $\mathrm{N} / \mathrm{A}$ & $\mathrm{N} / \mathrm{A}$ \\
\hline & $\begin{array}{l}\text { Surgical } \\
\text { complications }\end{array}$ & Necrosis & $0(0)$ & N/A & $\mathrm{N} / \mathrm{A}$ & $\mathrm{N} / \mathrm{A}$ & $\mathrm{N} / \mathrm{A}$ & $\mathrm{N} / \mathrm{A}$ & $\mathrm{N} / \mathrm{A}$ & $\mathrm{N} / \mathrm{A}$ \\
\hline & $\begin{array}{l}\text { Surgical } \\
\text { complications }\end{array}$ & Animation deformity & $0(0)$ & $\mathrm{N} / \mathrm{A}$ & $\mathrm{N} / \mathrm{A}$ & $\mathrm{N} / \mathrm{A}$ & $\mathrm{N} / \mathrm{A}$ & $\mathrm{N} / \mathrm{A}$ & $\mathrm{N} / \mathrm{A}$ & $\mathrm{N} / \mathrm{A}$ \\
\hline
\end{tabular}




\begin{tabular}{|c|c|c|c|c|c|c|c|c|c|c|}
\hline Comparison & $\begin{array}{l}\text { Outcome } \\
\text { Category }\end{array}$ & Outcome & $\begin{array}{l}\text { N Studies } \\
\text { (Patients) }\end{array}$ & RoB & Consistency & Precision & Directness & Other & SoE & $\begin{array}{l}\text { Conclusions } \\
\text { (Reason, if None) }\end{array}$ \\
\hline & $\begin{array}{l}\text { Surgical } \\
\text { complications }\end{array}$ & Implant-related infections & $0(0)$ & $\mathrm{N} / \mathrm{A}$ & $\mathrm{N} / \mathrm{A}$ & $\mathrm{N} / \mathrm{A}$ & $\mathrm{N} / \mathrm{A}$ & $\mathrm{N} / \mathrm{A}$ & N/A & $\mathrm{N} / \mathrm{A}$ \\
\hline & $\begin{array}{l}\text { Surgical } \\
\text { complications }\end{array}$ & Implant-rupture & $0(0)$ & $\mathrm{N} / \mathrm{A}$ & $\mathrm{N} / \mathrm{A}$ & $\mathrm{N} / \mathrm{A}$ & $\mathrm{N} / \mathrm{A}$ & $\mathrm{N} / \mathrm{A}$ & $\mathrm{N} / \mathrm{A}$ & $\mathrm{N} / \mathrm{A}$ \\
\hline & $\begin{array}{l}\text { Surgical } \\
\text { complications }\end{array}$ & Implant deflation & $0(0)$ & $\mathrm{N} / \mathrm{A}$ & $\mathrm{N} / \mathrm{A}$ & $\mathrm{N} / \mathrm{A}$ & $\mathrm{N} / \mathrm{A}$ & $\mathrm{N} / \mathrm{A}$ & $\mathrm{N} / \mathrm{A}$ & $\mathrm{N} / \mathrm{A}$ \\
\hline & $\begin{array}{l}\text { Surgical } \\
\text { complications }\end{array}$ & Implant malposition & $0(0)$ & $\mathrm{N} / \mathrm{A}$ & $\mathrm{N} / \mathrm{A}$ & $\mathrm{N} / \mathrm{A}$ & $\mathrm{N} / \mathrm{A}$ & $\mathrm{N} / \mathrm{A}$ & $\mathrm{N} / \mathrm{A}$ & $\mathrm{N} / \mathrm{A}$ \\
\hline & $\begin{array}{l}\text { Surgical } \\
\text { complications }\end{array}$ & $\begin{array}{l}\text { Implant failure/loss/need for explant } \\
\text { surgery }\end{array}$ & $0(0)$ & $\mathrm{N} / \mathrm{A}$ & $\mathrm{N} / \mathrm{A}$ & $\mathrm{N} / \mathrm{A}$ & $\mathrm{N} / \mathrm{A}$ & $\mathrm{N} / \mathrm{A}$ & $\mathrm{N} / \mathrm{A}$ & $\mathrm{N} / \mathrm{A}$ \\
\hline & $\begin{array}{l}\text { Surgical } \\
\text { complications }\end{array}$ & Capsular contracture & $0(0)$ & $\mathrm{N} / \mathrm{A}$ & $\mathrm{N} / \mathrm{A}$ & $\mathrm{N} / \mathrm{A}$ & $\mathrm{N} / \mathrm{A}$ & $\mathrm{N} / \mathrm{A}$ & $\mathrm{N} / \mathrm{A}$ & $\mathrm{N} / \mathrm{A}$ \\
\hline & $\begin{array}{l}\text { Surgical } \\
\text { complications }\end{array}$ & New neoplasms & $0(0)$ & $\mathrm{N} / \mathrm{A}$ & $\mathrm{N} / \mathrm{A}$ & $\mathrm{N} / \mathrm{A}$ & $\mathrm{N} / \mathrm{A}$ & $\mathrm{N} / \mathrm{A}$ & $\mathrm{N} / \mathrm{A}$ & $\mathrm{N} / \mathrm{A}$ \\
\hline & $\begin{array}{l}\text { Surgical } \\
\text { complications }\end{array}$ & $\begin{array}{l}\text { Complications delaying other } \\
\text { cancer treatments }\end{array}$ & $0(0)$ & $\mathrm{N} / \mathrm{A}$ & $\mathrm{N} / \mathrm{A}$ & $\mathrm{N} / \mathrm{A}$ & $\mathrm{N} / \mathrm{A}$ & $\mathrm{N} / \mathrm{A}$ & $\mathrm{N} / \mathrm{A}$ & $\mathrm{N} / \mathrm{A}$ \\
\hline & $\begin{array}{l}\text { Surgical } \\
\text { complications }\end{array}$ & Thromboembolic events & $0(0)$ & $\mathrm{N} / \mathrm{A}$ & $\mathrm{N} / \mathrm{A}$ & $\mathrm{N} / \mathrm{A}$ & $\mathrm{N} / \mathrm{A}$ & $\mathrm{N} / \mathrm{A}$ & N/A & $\mathrm{N} / \mathrm{A}$ \\
\hline & $\begin{array}{l}\text { Surgical } \\
\text { complications }\end{array}$ & Seroma & $0(0)$ & $\mathrm{N} / \mathrm{A}$ & $\mathrm{N} / \mathrm{A}$ & $\mathrm{N} / \mathrm{A}$ & $\mathrm{N} / \mathrm{A}$ & $\mathrm{N} / \mathrm{A}$ & $\mathrm{N} / \mathrm{A}$ & $\mathrm{N} / \mathrm{A}$ \\
\hline & $\begin{array}{l}\text { Surgical } \\
\text { complications }\end{array}$ & Chronic conditions & $0(0)$ & $\mathrm{N} / \mathrm{A}$ & $\mathrm{N} / \mathrm{A}$ & $\mathrm{N} / \mathrm{A}$ & $\mathrm{N} / \mathrm{A}$ & $\mathrm{N} / \mathrm{A}$ & $\mathrm{N} / \mathrm{A}$ & $\mathrm{N} / \mathrm{A}$ \\
\hline \multirow{13}{*}{$\begin{array}{l}\text { Saline vs. } \\
\text { double lumen }\end{array}$} & Clinical & General quality of life & $0(0)$ & $\mathrm{N} / \mathrm{A}$ & $\mathrm{N} / \mathrm{A}$ & $\mathrm{N} / \mathrm{A}$ & $\mathrm{N} / \mathrm{A}$ & $\mathrm{N} / \mathrm{A}$ & $\mathrm{N} / \mathrm{A}$ & $\mathrm{N} / \mathrm{A}$ \\
\hline & Clinical & Physical well-being & $0(0)$ & $\mathrm{N} / \mathrm{A}$ & $\mathrm{N} / \mathrm{A}$ & $\mathrm{N} / \mathrm{A}$ & $\mathrm{N} / \mathrm{A}$ & $\mathrm{N} / \mathrm{A}$ & $\mathrm{N} / \mathrm{A}$ & $\mathrm{N} / \mathrm{A}$ \\
\hline & Clinical & Psychosocial well-being & $0(0)$ & $\mathrm{N} / \mathrm{A}$ & $\mathrm{N} / \mathrm{A}$ & $\mathrm{N} / \mathrm{A}$ & $\mathrm{N} / \mathrm{A}$ & $\mathrm{N} / \mathrm{A}$ & $\mathrm{N} / \mathrm{A}$ & $\mathrm{N} / \mathrm{A}$ \\
\hline & Clinical & Sexual well-being & $0(0)$ & $\mathrm{N} / \mathrm{A}$ & $\mathrm{N} / \mathrm{A}$ & $\mathrm{N} / \mathrm{A}$ & $\mathrm{N} / \mathrm{A}$ & $\mathrm{N} / \mathrm{A}$ & $\mathrm{N} / \mathrm{A}$ & $\mathrm{N} / \mathrm{A}$ \\
\hline & Clinical & Patient satisfaction with breast & $0(0)$ & $\mathrm{N} / \mathrm{A}$ & $\mathrm{N} / \mathrm{A}$ & $\mathrm{N} / \mathrm{A}$ & $\mathrm{N} / \mathrm{A}$ & $\mathrm{N} / \mathrm{A}$ & $\mathrm{N} / \mathrm{A}$ & $\mathrm{N} / \mathrm{A}$ \\
\hline & Clinical & Patient satisfaction with outcome & $0(0)$ & $\mathrm{N} / \mathrm{A}$ & $\mathrm{N} / \mathrm{A}$ & $\mathrm{N} / \mathrm{A}$ & $\mathrm{N} / \mathrm{A}$ & $\mathrm{N} / \mathrm{A}$ & $\mathrm{N} / \mathrm{A}$ & $\mathrm{N} / \mathrm{A}$ \\
\hline & Clinical & $\begin{array}{l}\text { Planned surgeries for } \\
\text { reconstruction }\end{array}$ & $0(0)$ & $\mathrm{N} / \mathrm{A}$ & $\mathrm{N} / \mathrm{A}$ & $\mathrm{N} / \mathrm{A}$ & $\mathrm{N} / \mathrm{A}$ & $\mathrm{N} / \mathrm{A}$ & $\mathrm{N} / \mathrm{A}$ & $\mathrm{N} / \mathrm{A}$ \\
\hline & Clinical & Mortality & $0(0)$ & $\mathrm{N} / \mathrm{A}$ & $\mathrm{N} / \mathrm{A}$ & $\mathrm{N} / \mathrm{A}$ & $\mathrm{N} / \mathrm{A}$ & $\mathrm{N} / \mathrm{A}$ & $\mathrm{N} / \mathrm{A}$ & $\mathrm{N} / \mathrm{A}$ \\
\hline & $\begin{array}{l}\text { Surgical } \\
\text { complications }\end{array}$ & Unplanned repeat hospitalization & $0(0)$ & $\mathrm{N} / \mathrm{A}$ & $\mathrm{N} / \mathrm{A}$ & $\mathrm{N} / \mathrm{A}$ & $\mathrm{N} / \mathrm{A}$ & $\mathrm{N} / \mathrm{A}$ & $\mathrm{N} / \mathrm{A}$ & $\mathrm{N} / \mathrm{A}$ \\
\hline & $\begin{array}{l}\text { Surgical } \\
\text { complications }\end{array}$ & $\begin{array}{l}\text { Duration of unplanned repeat } \\
\text { hospitalization }\end{array}$ & $0(0)$ & $\mathrm{N} / \mathrm{A}$ & $\mathrm{N} / \mathrm{A}$ & $\mathrm{N} / \mathrm{A}$ & $\mathrm{N} / \mathrm{A}$ & $\mathrm{N} / \mathrm{A}$ & $\mathrm{N} / \mathrm{A}$ & $\mathrm{N} / \mathrm{A}$ \\
\hline & $\begin{array}{l}\text { Surgical } \\
\text { complications }\end{array}$ & $\begin{array}{l}\text { Unplanned repeat surgeries for } \\
\text { revision }\end{array}$ & $0(0)$ & $\mathrm{N} / \mathrm{A}$ & $\mathrm{N} / \mathrm{A}$ & $\mathrm{N} / \mathrm{A}$ & $\mathrm{N} / \mathrm{A}$ & $\mathrm{N} / \mathrm{A}$ & $\mathrm{N} / \mathrm{A}$ & $\mathrm{N} / \mathrm{A}$ \\
\hline & $\begin{array}{l}\text { Surgical } \\
\text { complications }\end{array}$ & $\begin{array}{l}\text { Unplanned repeat surgeries for } \\
\text { complications }\end{array}$ & $0(0)$ & $\mathrm{N} / \mathrm{A}$ & $\mathrm{N} / \mathrm{A}$ & $\mathrm{N} / \mathrm{A}$ & $\mathrm{N} / \mathrm{A}$ & $\mathrm{N} / \mathrm{A}$ & $\mathrm{N} / \mathrm{A}$ & $\mathrm{N} / \mathrm{A}$ \\
\hline & $\begin{array}{l}\text { Surgical } \\
\text { complications }\end{array}$ & Pain & $0(0)$ & $\mathrm{N} / \mathrm{A}$ & $\mathrm{N} / \mathrm{A}$ & $\mathrm{N} / \mathrm{A}$ & $\mathrm{N} / \mathrm{A}$ & $\mathrm{N} / \mathrm{A}$ & $\mathrm{N} / \mathrm{A}$ & $\mathrm{N} / \mathrm{A}$ \\
\hline
\end{tabular}




\begin{tabular}{|c|c|c|c|c|c|c|c|c|c|c|}
\hline Comparison & $\begin{array}{l}\text { Outcome } \\
\text { Category }\end{array}$ & Outcome & $\begin{array}{l}\text { N Studies } \\
\text { (Patients) }\end{array}$ & RoB & Consistency & Precision & Directness & Other & SoE & $\begin{array}{l}\text { Conclusions } \\
\text { (Reason, if None) }\end{array}$ \\
\hline & $\begin{array}{l}\text { Surgical } \\
\text { complications }\end{array}$ & Analgesic use & $0(0)$ & $\mathrm{N} / \mathrm{A}$ & $\mathrm{N} / \mathrm{A}$ & $\mathrm{N} / \mathrm{A}$ & $\mathrm{N} / \mathrm{A}$ & $\mathrm{N} / \mathrm{A}$ & $\mathrm{N} / \mathrm{A}$ & N/A \\
\hline & $\begin{array}{l}\text { Surgical } \\
\text { complications }\end{array}$ & Necrosis & $0(0)$ & $\mathrm{N} / \mathrm{A}$ & $\mathrm{N} / \mathrm{A}$ & $\mathrm{N} / \mathrm{A}$ & $\mathrm{N} / \mathrm{A}$ & $\mathrm{N} / \mathrm{A}$ & N/A & $\mathrm{N} / \mathrm{A}$ \\
\hline & $\begin{array}{l}\text { Surgical } \\
\text { complications }\end{array}$ & Animation deformity & $0(0)$ & $\mathrm{N} / \mathrm{A}$ & $\mathrm{N} / \mathrm{A}$ & $\mathrm{N} / \mathrm{A}$ & $\mathrm{N} / \mathrm{A}$ & $\mathrm{N} / \mathrm{A}$ & $\mathrm{N} / \mathrm{A}$ & $\mathrm{N} / \mathrm{A}$ \\
\hline & $\begin{array}{l}\text { Surgical } \\
\text { complications }\end{array}$ & Implant-related infections & $0(0)$ & N/A & $\mathrm{N} / \mathrm{A}$ & $\mathrm{N} / \mathrm{A}$ & $\mathrm{N} / \mathrm{A}$ & $\mathrm{N} / \mathrm{A}$ & N/A & $\mathrm{N} / \mathrm{A}$ \\
\hline & $\begin{array}{l}\text { Surgical } \\
\text { complications }\end{array}$ & Implant-rupture & $0(0)$ & $\mathrm{N} / \mathrm{A}$ & $\mathrm{N} / \mathrm{A}$ & $\mathrm{N} / \mathrm{A}$ & $\mathrm{N} / \mathrm{A}$ & $\mathrm{N} / \mathrm{A}$ & N/A & $\mathrm{N} / \mathrm{A}$ \\
\hline & $\begin{array}{l}\text { Surgical } \\
\text { complications }\end{array}$ & Implant deflation & $0(0)$ & $\mathrm{N} / \mathrm{A}$ & $\mathrm{N} / \mathrm{A}$ & $\mathrm{N} / \mathrm{A}$ & $\mathrm{N} / \mathrm{A}$ & $\mathrm{N} / \mathrm{A}$ & $\mathrm{N} / \mathrm{A}$ & $\mathrm{N} / \mathrm{A}$ \\
\hline & $\begin{array}{l}\text { Surgical } \\
\text { complications }\end{array}$ & Implant malposition & $0(0)$ & $\mathrm{N} / \mathrm{A}$ & $\mathrm{N} / \mathrm{A}$ & $\mathrm{N} / \mathrm{A}$ & $\mathrm{N} / \mathrm{A}$ & $\mathrm{N} / \mathrm{A}$ & $\mathrm{N} / \mathrm{A}$ & $\mathrm{N} / \mathrm{A}$ \\
\hline & $\begin{array}{l}\text { Surgical } \\
\text { complications }\end{array}$ & $\begin{array}{l}\text { Implant failure/loss/need for explant } \\
\text { surgery }\end{array}$ & $0(0)$ & $\mathrm{N} / \mathrm{A}$ & $\mathrm{N} / \mathrm{A}$ & $\mathrm{N} / \mathrm{A}$ & $\mathrm{N} / \mathrm{A}$ & $\mathrm{N} / \mathrm{A}$ & N/A & $\mathrm{N} / \mathrm{A}$ \\
\hline & $\begin{array}{l}\text { Surgical } \\
\text { complications }\end{array}$ & Capsular contracture & $0(0)$ & $\mathrm{N} / \mathrm{A}$ & $\mathrm{N} / \mathrm{A}$ & $\mathrm{N} / \mathrm{A}$ & $\mathrm{N} / \mathrm{A}$ & $\mathrm{N} / \mathrm{A}$ & N/A & $\mathrm{N} / \mathrm{A}$ \\
\hline & $\begin{array}{l}\text { Surgical } \\
\text { complications }\end{array}$ & New neoplasms & $0(0)$ & $\mathrm{N} / \mathrm{A}$ & $\mathrm{N} / \mathrm{A}$ & $\mathrm{N} / \mathrm{A}$ & $\mathrm{N} / \mathrm{A}$ & $\mathrm{N} / \mathrm{A}$ & $\mathrm{N} / \mathrm{A}$ & $\mathrm{N} / \mathrm{A}$ \\
\hline & $\begin{array}{l}\text { Surgical } \\
\text { complications }\end{array}$ & $\begin{array}{l}\text { Complications delaying other } \\
\text { cancer treatments }\end{array}$ & $0(0)$ & $\mathrm{N} / \mathrm{A}$ & $\mathrm{N} / \mathrm{A}$ & $\mathrm{N} / \mathrm{A}$ & $\mathrm{N} / \mathrm{A}$ & $\mathrm{N} / \mathrm{A}$ & $\mathrm{N} / \mathrm{A}$ & $\mathrm{N} / \mathrm{A}$ \\
\hline & $\begin{array}{l}\text { Surgical } \\
\text { complications }\end{array}$ & Thromboembolic events & $0(0)$ & $\mathrm{N} / \mathrm{A}$ & $\mathrm{N} / \mathrm{A}$ & $\mathrm{N} / \mathrm{A}$ & $\mathrm{N} / \mathrm{A}$ & $\mathrm{N} / \mathrm{A}$ & $\mathrm{N} / \mathrm{A}$ & $\mathrm{N} / \mathrm{A}$ \\
\hline & $\begin{array}{l}\text { Surgical } \\
\text { complications }\end{array}$ & Seroma & $0(0)$ & $\mathrm{N} / \mathrm{A}$ & $\mathrm{N} / \mathrm{A}$ & $\mathrm{N} / \mathrm{A}$ & $\mathrm{N} / \mathrm{A}$ & $\mathrm{N} / \mathrm{A}$ & $\mathrm{N} / \mathrm{A}$ & $\mathrm{N} / \mathrm{A}$ \\
\hline & $\begin{array}{l}\text { Surgical } \\
\text { complications }\end{array}$ & Chronic conditions & $0(0)$ & $\mathrm{N} / \mathrm{A}$ & $\mathrm{N} / \mathrm{A}$ & $\mathrm{N} / \mathrm{A}$ & $\mathrm{N} / \mathrm{A}$ & $\mathrm{N} / \mathrm{A}$ & $\mathrm{N} / \mathrm{A}$ & $\mathrm{N} / \mathrm{A}$ \\
\hline
\end{tabular}

Abbreviations: $\mathrm{N} / \mathrm{A}=$ not applicable, $\mathrm{NR}=$ not reported, $\mathrm{RoB}=$ risk of bias, $\mathrm{SoE}=$ strength of evidence.

For continuous outcomes, clinical significance is based on published estimates of minimal clinically important differences (MCIDs), where available.

Colors: Header rows are shaded orange. Rows for every alternate comparison are shaded blue. The colors do not add unique information. 
Table G-4. Key Question 4: Comparison of anatomic planes of implant placement for IBR - full evidence profile

\begin{tabular}{|c|c|c|c|c|c|c|c|c|c|c|}
\hline Comparison & $\begin{array}{l}\text { Outcome } \\
\text { Category }\end{array}$ & Outcome & $\begin{array}{l}\text { N Studies } \\
\text { (Patients) }\end{array}$ & RoB & Consistency & Precision & Directness & Other & SoE & $\begin{array}{l}\text { Conclusions } \\
\text { (Reason, if } \\
\text { None) }\end{array}$ \\
\hline \multirow{22}{*}{$\begin{array}{l}\text { Prepectoral } \\
\text { versus total } \\
\text { submuscular }\end{array}$} & Clinical & General quality of life & $0(0)$ & $\mathrm{N} / \mathrm{A}$ & $\mathrm{N} / \mathrm{A}$ & $\mathrm{N} / \mathrm{A}$ & $\mathrm{N} / \mathrm{A}$ & $\mathrm{N} / \mathrm{A}$ & $\mathrm{N} / \mathrm{A}$ & $\mathrm{N} / \mathrm{A}$ \\
\hline & Clinical & Physical well-being & $1(84)$ & High & Unclear & Unclear & Direct & Sparse & Insufficient & $\begin{array}{l}\text { None (Sparse } \\
\text { evidence) }\end{array}$ \\
\hline & Clinical & Psychosocial well-being & $0(0)$ & $\mathrm{N} / \mathrm{A}$ & $\mathrm{N} / \mathrm{A}$ & $\mathrm{N} / \mathrm{A}$ & $\mathrm{N} / \mathrm{A}$ & $\mathrm{N} / \mathrm{A}$ & $\mathrm{N} / \mathrm{A}$ & $\mathrm{N} / \mathrm{A}$ \\
\hline & Clinical & Sexual well-being & $0(0)$ & $\mathrm{N} / \mathrm{A}$ & $\mathrm{N} / \mathrm{A}$ & $\mathrm{N} / \mathrm{A}$ & $\mathrm{N} / \mathrm{A}$ & $\mathrm{N} / \mathrm{A}$ & $\mathrm{N} / \mathrm{A}$ & $\mathrm{N} / \mathrm{A}$ \\
\hline & Clinical & Patient satisfaction with breast & $1(84)$ & High & Unclear & Unclear & Direct & Sparse & Insufficient & $\begin{array}{l}\text { None (Sparse } \\
\text { evidence) }\end{array}$ \\
\hline & Clinical & Patient satisfaction with outcome & $0(0)$ & $\mathrm{N} / \mathrm{A}$ & $\mathrm{N} / \mathrm{A}$ & $\mathrm{N} / \mathrm{A}$ & $\mathrm{N} / \mathrm{A}$ & $\mathrm{N} / \mathrm{A}$ & $\mathrm{N} / \mathrm{A}$ & $\mathrm{N} / \mathrm{A}$ \\
\hline & Clinical & Planned surgeries for reconstruction & $0(0)$ & $\mathrm{N} / \mathrm{A}$ & $\mathrm{N} / \mathrm{A}$ & $\mathrm{N} / \mathrm{A}$ & $\mathrm{N} / \mathrm{A}$ & $\mathrm{N} / \mathrm{A}$ & $\mathrm{N} / \mathrm{A}$ & $\mathrm{N} / \mathrm{A}$ \\
\hline & Clinical & Mortality & $0(0)$ & $\mathrm{N} / \mathrm{A}$ & $\mathrm{N} / \mathrm{A}$ & N/A & $\mathrm{N} / \mathrm{A}$ & $\mathrm{N} / \mathrm{A}$ & $\mathrm{N} / \mathrm{A}$ & $\mathrm{N} / \mathrm{A}$ \\
\hline & $\begin{array}{l}\text { Surgical } \\
\text { complications }\end{array}$ & $\begin{array}{l}\text { Unplanned repeat surgeries for } \\
\text { revision }\end{array}$ & $1(405)$ & High & $\mathrm{N} / \mathrm{A}$ & Precise & Direct & Sparse & Insufficient & $\begin{array}{l}\text { None (Sparse } \\
\text { evidence) }\end{array}$ \\
\hline & $\begin{array}{l}\text { Surgical } \\
\text { complications }\end{array}$ & Unplanned repeat hospitalization & $0(0)$ & $\mathrm{N} / \mathrm{A}$ & $\mathrm{N} / \mathrm{A}$ & $\mathrm{N} / \mathrm{A}$ & $\mathrm{N} / \mathrm{A}$ & $\mathrm{N} / \mathrm{A}$ & $\mathrm{N} / \mathrm{A}$ & $\mathrm{N} / \mathrm{A}$ \\
\hline & $\begin{array}{l}\text { Surgical } \\
\text { complications }\end{array}$ & $\begin{array}{l}\text { Duration of unplanned repeat } \\
\text { hospitalization }\end{array}$ & $0(0)$ & $\mathrm{N} / \mathrm{A}$ & $\mathrm{N} / \mathrm{A}$ & $\mathrm{N} / \mathrm{A}$ & $\mathrm{N} / \mathrm{A}$ & $\mathrm{N} / \mathrm{A}$ & $\mathrm{N} / \mathrm{A}$ & $\mathrm{N} / \mathrm{A}$ \\
\hline & $\begin{array}{l}\text { Surgical } \\
\text { complications }\end{array}$ & $\begin{array}{l}\text { Unplanned repeat surgeries for } \\
\text { revision }\end{array}$ & $0(0)$ & $\mathrm{N} / \mathrm{A}$ & $\mathrm{N} / \mathrm{A}$ & $\mathrm{N} / \mathrm{A}$ & N/A & $\mathrm{N} / \mathrm{A}$ & $\mathrm{N} / \mathrm{A}$ & N/A \\
\hline & $\begin{array}{l}\text { Surgical } \\
\text { complications }\end{array}$ & $\begin{array}{l}\text { Unplanned repeat surgeries for } \\
\text { complications }\end{array}$ & $0(0)$ & $\mathrm{N} / \mathrm{A}$ & $\mathrm{N} / \mathrm{A}$ & $\mathrm{N} / \mathrm{A}$ & $\mathrm{N} / \mathrm{A}$ & $\mathrm{N} / \mathrm{A}$ & $\mathrm{N} / \mathrm{A}$ & $\mathrm{N} / \mathrm{A}$ \\
\hline & $\begin{array}{l}\text { Surgical } \\
\text { complications }\end{array}$ & Pain & $2(230)$ & High & Inconsistent & Precise & Direct & $\mathrm{N} / \mathrm{A}$ & Insufficient & $\begin{array}{l}\text { None } \\
\text { (Inconsistent } \\
\text { results) }\end{array}$ \\
\hline & $\begin{array}{l}\text { Surgical } \\
\text { complications }\end{array}$ & Analgesic use & $1(146)$ & High & $\mathrm{N} / \mathrm{A}$ & Precise & Direct & Sparse & Insufficient & $\begin{array}{l}\text { None (Sparse } \\
\text { evidence) }\end{array}$ \\
\hline & $\begin{array}{l}\text { Surgical } \\
\text { complications }\end{array}$ & Necrosis & $1(256)$ & High & Unclear & Imprecise & Direct & Sparse & Insufficient & $\begin{array}{l}\text { None (Sparse } \\
\text { evidence) }\end{array}$ \\
\hline & $\begin{array}{l}\text { Surgical } \\
\text { complications }\end{array}$ & Animation deformity & $0(0)$ & $\mathrm{N} / \mathrm{A}$ & $\mathrm{N} / \mathrm{A}$ & $\mathrm{N} / \mathrm{A}$ & N/A & $\mathrm{N} / \mathrm{A}$ & $\mathrm{N} / \mathrm{A}$ & $\mathrm{N} / \mathrm{A}$ \\
\hline & $\begin{array}{l}\text { Surgical } \\
\text { complications }\end{array}$ & Implant-related infections & $0(0)$ & $\mathrm{N} / \mathrm{A}$ & $\mathrm{N} / \mathrm{A}$ & $\mathrm{N} / \mathrm{A}$ & $\mathrm{N} / \mathrm{A}$ & $\mathrm{N} / \mathrm{A}$ & $\mathrm{N} / \mathrm{A}$ & $\mathrm{N} / \mathrm{A}$ \\
\hline & $\begin{array}{l}\text { Surgical } \\
\text { complications }\end{array}$ & Implant-rupture & $0(0)$ & $\mathrm{N} / \mathrm{A}$ & $\mathrm{N} / \mathrm{A}$ & $\mathrm{N} / \mathrm{A}$ & $\mathrm{N} / \mathrm{A}$ & $\mathrm{N} / \mathrm{A}$ & $\mathrm{N} / \mathrm{A}$ & $\mathrm{N} / \mathrm{A}$ \\
\hline & $\begin{array}{l}\text { Surgical } \\
\text { complications }\end{array}$ & Implant deflation & $0(0)$ & $\mathrm{N} / \mathrm{A}$ & $\mathrm{N} / \mathrm{A}$ & $\mathrm{N} / \mathrm{A}$ & $\mathrm{N} / \mathrm{A}$ & $\mathrm{N} / \mathrm{A}$ & $\mathrm{N} / \mathrm{A}$ & $\mathrm{N} / \mathrm{A}$ \\
\hline & $\begin{array}{l}\text { Surgical } \\
\text { complications }\end{array}$ & Implant malposition & $0(0)$ & $\mathrm{N} / \mathrm{A}$ & $\mathrm{N} / \mathrm{A}$ & $\mathrm{N} / \mathrm{A}$ & $\mathrm{N} / \mathrm{A}$ & $\mathrm{N} / \mathrm{A}$ & $\mathrm{N} / \mathrm{A}$ & $\mathrm{N} / \mathrm{A}$ \\
\hline & $\begin{array}{l}\text { Surgical } \\
\text { complications }\end{array}$ & $\begin{array}{l}\text { Implant failure/loss or need for } \\
\text { explant surgery }\end{array}$ & $1(256)$ & High & Unclear & Imprecise & Direct & Sparse & Insufficient & $\begin{array}{l}\text { None (Sparse } \\
\text { evidence) }\end{array}$ \\
\hline
\end{tabular}




\begin{tabular}{|c|c|c|c|c|c|c|c|c|c|c|}
\hline Comparison & $\begin{array}{l}\text { Outcome } \\
\text { Category }\end{array}$ & Outcome & $\begin{array}{l}\text { N Studies } \\
\text { (Patients) }\end{array}$ & RoB & Consistency & Precision & Directness & Other & SoE & $\begin{array}{l}\text { Conclusions } \\
\text { (Reason, if } \\
\text { None) }\end{array}$ \\
\hline & $\begin{array}{l}\text { Surgical } \\
\text { complications }\end{array}$ & Capsular contracture & $1(256)$ & High & Unclear & Imprecise & Direct & Sparse & Insufficient & $\begin{array}{l}\text { None (Sparse } \\
\text { evidence) }\end{array}$ \\
\hline & $\begin{array}{l}\text { Surgical } \\
\text { complications }\end{array}$ & New neoplasms & $0(0)$ & $\mathrm{N} / \mathrm{A}$ & $\mathrm{N} / \mathrm{A}$ & $\mathrm{N} / \mathrm{A}$ & $\mathrm{N} / \mathrm{A}$ & $\mathrm{N} / \mathrm{A}$ & $\mathrm{N} / \mathrm{A}$ & $\mathrm{N} / \mathrm{A}$ \\
\hline & $\begin{array}{l}\text { Surgical } \\
\text { complications }\end{array}$ & $\begin{array}{l}\text { Complications delaying other cancer } \\
\text { treatments }\end{array}$ & $0(0)$ & $\mathrm{N} / \mathrm{A}$ & $\mathrm{N} / \mathrm{A}$ & $\mathrm{N} / \mathrm{A}$ & $\mathrm{N} / \mathrm{A}$ & $\mathrm{N} / \mathrm{A}$ & $\mathrm{N} / \mathrm{A}$ & $\mathrm{N} / \mathrm{A}$ \\
\hline & $\begin{array}{l}\text { Surgical } \\
\text { complications }\end{array}$ & Thromboembolic events & $0(0)$ & $\mathrm{N} / \mathrm{A}$ & $\mathrm{N} / \mathrm{A}$ & $\mathrm{N} / \mathrm{A}$ & $\mathrm{N} / \mathrm{A}$ & $\mathrm{N} / \mathrm{A}$ & $\mathrm{N} / \mathrm{A}$ & $\mathrm{N} / \mathrm{A}$ \\
\hline & $\begin{array}{l}\text { Surgical } \\
\text { complications }\end{array}$ & $\begin{array}{l}\text { Infections (not explicitly implant- } \\
\text { related) }\end{array}$ & $2(542)$ & High & Direct & Precise & Direct & $\mathrm{N} / \mathrm{A}$ & Low & $\begin{array}{l}\text { Comparable } \\
\text { risk }\end{array}$ \\
\hline & $\begin{array}{l}\text { Surgical } \\
\text { complications }\end{array}$ & Seroma & $1(256)$ & High & Unclear & Imprecise & Direct & Sparse & Insufficient & $\begin{array}{l}\text { None (Sparse } \\
\text { evidence) }\end{array}$ \\
\hline & $\begin{array}{l}\text { Surgical } \\
\text { complications }\end{array}$ & Chronic conditions & $0(0)$ & $\mathrm{N} / \mathrm{A}$ & $\mathrm{N} / \mathrm{A}$ & $\mathrm{N} / \mathrm{A}$ & $\mathrm{N} / \mathrm{A}$ & $\mathrm{N} / \mathrm{A}$ & $\mathrm{N} / \mathrm{A}$ & N/A \\
\hline \multirow{16}{*}{$\begin{array}{l}\text { Prepectoral } \\
\text { versus } \\
\text { partial } \\
\text { submuscular }\end{array}$} & Clinical & General quality of life & $0(0)$ & $\mathrm{N} / \mathrm{A}$ & $\mathrm{N} / \mathrm{A}$ & $\mathrm{N} / \mathrm{A}$ & $\mathrm{N} / \mathrm{A}$ & $\mathrm{N} / \mathrm{A}$ & $\mathrm{N} / \mathrm{A}$ & $\mathrm{N} / \mathrm{A}$ \\
\hline & Clinical & Physical well-being & $1(34)$ & $\begin{array}{l}\text { Mod } \\
\text { erate }\end{array}$ & $\mathrm{N} / \mathrm{A}$ & Precise & Direct & Sparse & Insufficient & $\begin{array}{l}\text { None (Sparse } \\
\text { evidence) }\end{array}$ \\
\hline & Clinical & Psychosocial well-being & $1(34)$ & $\begin{array}{l}\text { Mod } \\
\text { erate }\end{array}$ & $\mathrm{N} / \mathrm{A}$ & Precise & Direct & Sparse & Insufficient & $\begin{array}{l}\text { None (Sparse } \\
\text { evidence) }\end{array}$ \\
\hline & Clinical & Sexual well-being & $0(0)$ & $\mathrm{N} / \mathrm{A}$ & $\mathrm{N} / \mathrm{A}$ & $\mathrm{N} / \mathrm{A}$ & $\mathrm{N} / \mathrm{A}$ & $\mathrm{N} / \mathrm{A}$ & $\mathrm{N} / \mathrm{A}$ & $\mathrm{N} / \mathrm{A}$ \\
\hline & Clinical & Patient satisfaction with breast & $0(0)$ & $\mathrm{N} / \mathrm{A}$ & $\mathrm{N} / \mathrm{A}$ & $\mathrm{N} / \mathrm{A}$ & $\mathrm{N} / \mathrm{A}$ & $\mathrm{N} / \mathrm{A}$ & N/A & $\mathrm{N} / \mathrm{A}$ \\
\hline & Clinical & Patient satisfaction with outcome & $0(0)$ & $\mathrm{N} / \mathrm{A}$ & $\mathrm{N} / \mathrm{A}$ & $\mathrm{N} / \mathrm{A}$ & $\mathrm{N} / \mathrm{A}$ & $\mathrm{N} / \mathrm{A}$ & $\mathrm{N} / \mathrm{A}$ & $\mathrm{N} / \mathrm{A}$ \\
\hline & Clinical & Planned surgeries for reconstruction & $0(0)$ & $\mathrm{N} / \mathrm{A}$ & $\mathrm{N} / \mathrm{A}$ & $\mathrm{N} / \mathrm{A}$ & $\mathrm{N} / \mathrm{A}$ & $\mathrm{N} / \mathrm{A}$ & $\mathrm{N} / \mathrm{A}$ & $\mathrm{N} / \mathrm{A}$ \\
\hline & Clinical & Mortality & $0(0)$ & $\mathrm{N} / \mathrm{A}$ & $\mathrm{N} / \mathrm{A}$ & $\mathrm{N} / \mathrm{A}$ & $\mathrm{N} / \mathrm{A}$ & $\mathrm{N} / \mathrm{A}$ & $\mathrm{N} / \mathrm{A}$ & $\mathrm{N} / \mathrm{A}$ \\
\hline & $\begin{array}{l}\text { Surgical } \\
\text { complications }\end{array}$ & Unplanned repeat hospitalization & $0(0)$ & $\mathrm{N} / \mathrm{A}$ & $\mathrm{N} / \mathrm{A}$ & $\mathrm{N} / \mathrm{A}$ & $\mathrm{N} / \mathrm{A}$ & $\mathrm{N} / \mathrm{A}$ & $\mathrm{N} / \mathrm{A}$ & $\mathrm{N} / \mathrm{A}$ \\
\hline & $\begin{array}{l}\text { Surgical } \\
\text { complications }\end{array}$ & $\begin{array}{l}\text { Duration of unplanned repeat } \\
\text { hospitalization }\end{array}$ & $0(0)$ & $\mathrm{N} / \mathrm{A}$ & $\mathrm{N} / \mathrm{A}$ & $\mathrm{N} / \mathrm{A}$ & $\mathrm{N} / \mathrm{A}$ & $\mathrm{N} / \mathrm{A}$ & $\mathrm{N} / \mathrm{A}$ & $\mathrm{N} / \mathrm{A}$ \\
\hline & $\begin{array}{l}\text { Surgical } \\
\text { complications }\end{array}$ & $\begin{array}{l}\text { Unplanned repeat surgeries for } \\
\text { revision }\end{array}$ & $0(0)$ & $\mathrm{N} / \mathrm{A}$ & $\mathrm{N} / \mathrm{A}$ & $\mathrm{N} / \mathrm{A}$ & $\mathrm{N} / \mathrm{A}$ & $\mathrm{N} / \mathrm{A}$ & $\mathrm{N} / \mathrm{A}$ & $\mathrm{N} / \mathrm{A}$ \\
\hline & $\begin{array}{l}\text { Surgical } \\
\text { complications }\end{array}$ & $\begin{array}{l}\text { Unplanned repeat surgeries for } \\
\text { complications }\end{array}$ & $0(0)$ & $\mathrm{N} / \mathrm{A}$ & $\mathrm{N} / \mathrm{A}$ & $\mathrm{N} / \mathrm{A}$ & $\mathrm{N} / \mathrm{A}$ & $N / A$ & $\mathrm{~N} / \mathrm{A}$ & $\mathrm{N} / \mathrm{A}$ \\
\hline & $\begin{array}{l}\text { Surgical } \\
\text { complications }\end{array}$ & Pain & $1(167)$ & $\begin{array}{l}\text { Mod } \\
\text { erate }\end{array}$ & $\mathrm{N} / \mathrm{A}$ & Precise & Direct & Sparse & Insufficient & $\begin{array}{l}\text { None (Sparse } \\
\text { evidence) }\end{array}$ \\
\hline & $\begin{array}{l}\text { Surgical } \\
\text { complications }\end{array}$ & Analgesic use & $0(0)$ & $\mathrm{N} / \mathrm{A}$ & $\mathrm{N} / \mathrm{A}$ & $\mathrm{N} / \mathrm{A}$ & $\mathrm{N} / \mathrm{A}$ & $\mathrm{N} / \mathrm{A}$ & $\mathrm{N} / \mathrm{A}$ & $\mathrm{N} / \mathrm{A}$ \\
\hline & $\begin{array}{l}\text { Surgical } \\
\text { complications }\end{array}$ & Necrosis & $0(0)$ & $\mathrm{N} / \mathrm{A}$ & $\mathrm{N} / \mathrm{A}$ & $\mathrm{N} / \mathrm{A}$ & $\mathrm{N} / \mathrm{A}$ & $\mathrm{N} / \mathrm{A}$ & $\mathrm{N} / \mathrm{A}$ & $\mathrm{N} / \mathrm{A}$ \\
\hline & $\begin{array}{l}\text { Surgical } \\
\text { complications }\end{array}$ & Animation deformity & $0(0)$ & $\mathrm{N} / \mathrm{A}$ & $\mathrm{N} / \mathrm{A}$ & $\mathrm{N} / \mathrm{A}$ & $\mathrm{N} / \mathrm{A}$ & $\mathrm{N} / \mathrm{A}$ & $\mathrm{N} / \mathrm{A}$ & $\mathrm{N} / \mathrm{A}$ \\
\hline
\end{tabular}




\begin{tabular}{|c|c|c|c|c|c|c|c|c|c|c|}
\hline Comparison & $\begin{array}{l}\text { Outcome } \\
\text { Category }\end{array}$ & Outcome & $\begin{array}{l}\text { N Studies } \\
\text { (Patients) }\end{array}$ & RoB & Consistency & Precision & Directness & Other & SoE & $\begin{array}{l}\text { Conclusions } \\
\text { (Reason, if } \\
\text { None) }\end{array}$ \\
\hline & $\begin{array}{l}\text { Surgical } \\
\text { complications }\end{array}$ & Implant-related infections & $0(0)$ & $\mathrm{N} / \mathrm{A}$ & $\mathrm{N} / \mathrm{A}$ & $\mathrm{N} / \mathrm{A}$ & $\mathrm{N} / \mathrm{A}$ & $\mathrm{N} / \mathrm{A}$ & $\mathrm{N} / \mathrm{A}$ & $\mathrm{N} / \mathrm{A}$ \\
\hline & $\begin{array}{l}\text { Surgical } \\
\text { complications }\end{array}$ & Implant-rupture & $0(0)$ & $\mathrm{N} / \mathrm{A}$ & $\mathrm{N} / \mathrm{A}$ & $\mathrm{N} / \mathrm{A}$ & $\mathrm{N} / \mathrm{A}$ & $\mathrm{N} / \mathrm{A}$ & $\mathrm{N} / \mathrm{A}$ & $\mathrm{N} / \mathrm{A}$ \\
\hline & $\begin{array}{l}\text { Surgical } \\
\text { complications }\end{array}$ & Implant deflation & $0(0)$ & $\mathrm{N} / \mathrm{A}$ & $\mathrm{N} / \mathrm{A}$ & $\mathrm{N} / \mathrm{A}$ & $\mathrm{N} / \mathrm{A}$ & $\mathrm{N} / \mathrm{A}$ & $\mathrm{N} / \mathrm{A}$ & $\mathrm{N} / \mathrm{A}$ \\
\hline & $\begin{array}{l}\text { Surgical } \\
\text { complications }\end{array}$ & Implant malposition & $0(0)$ & $\mathrm{N} / \mathrm{A}$ & $\mathrm{N} / \mathrm{A}$ & $\mathrm{N} / \mathrm{A}$ & $\mathrm{N} / \mathrm{A}$ & $\mathrm{N} / \mathrm{A}$ & $\mathrm{N} / \mathrm{A}$ & $\mathrm{N} / \mathrm{A}$ \\
\hline & $\begin{array}{l}\text { Surgical } \\
\text { complications }\end{array}$ & $\begin{array}{l}\text { Implant failure/loss or need for } \\
\text { explant surgery }\end{array}$ & $0(0)$ & $\mathrm{N} / \mathrm{A}$ & $\mathrm{N} / \mathrm{A}$ & $\mathrm{N} / \mathrm{A}$ & $\mathrm{N} / \mathrm{A}$ & $\mathrm{N} / \mathrm{A}$ & $\mathrm{N} / \mathrm{A}$ & $\mathrm{N} / \mathrm{A}$ \\
\hline & $\begin{array}{l}\text { Surgical } \\
\text { complications }\end{array}$ & Capsular contracture & $1(34)$ & $\begin{array}{l}\text { Mod } \\
\text { erate }\end{array}$ & $\mathrm{N} / \mathrm{A}$ & Imprecise & Direct & Sparse & Insufficient & $\begin{array}{l}\text { None (Sparse } \\
\text { evidence) }\end{array}$ \\
\hline & $\begin{array}{l}\text { Surgical } \\
\text { complications }\end{array}$ & New neoplasms & $0(0)$ & $\mathrm{N} / \mathrm{A}$ & $\mathrm{N} / \mathrm{A}$ & $\mathrm{N} / \mathrm{A}$ & $\mathrm{N} / \mathrm{A}$ & $\mathrm{N} / \mathrm{A}$ & $\mathrm{N} / \mathrm{A}$ & $\mathrm{N} / \mathrm{A}$ \\
\hline & $\begin{array}{l}\text { Surgical } \\
\text { complications }\end{array}$ & $\begin{array}{l}\text { Complications delaying other cancer } \\
\text { treatments }\end{array}$ & $0(0)$ & $\mathrm{N} / \mathrm{A}$ & $\mathrm{N} / \mathrm{A}$ & $\mathrm{N} / \mathrm{A}$ & $\mathrm{N} / \mathrm{A}$ & $\mathrm{N} / \mathrm{A}$ & $\mathrm{N} / \mathrm{A}$ & $\mathrm{N} / \mathrm{A}$ \\
\hline & $\begin{array}{l}\text { Surgical } \\
\text { complications }\end{array}$ & Thromboembolic events & $0(0)$ & $\mathrm{N} / \mathrm{A}$ & $\mathrm{N} / \mathrm{A}$ & $\mathrm{N} / \mathrm{A}$ & $\mathrm{N} / \mathrm{A}$ & $\mathrm{N} / \mathrm{A}$ & $\mathrm{N} / \mathrm{A}$ & $\mathrm{N} / \mathrm{A}$ \\
\hline & $\begin{array}{l}\text { Surgical } \\
\text { complications }\end{array}$ & $\begin{array}{l}\text { Infections (not explicitly implant- } \\
\text { related) }\end{array}$ & $0(0)$ & $\mathrm{N} / \mathrm{A}$ & $\mathrm{N} / \mathrm{A}$ & $\mathrm{N} / \mathrm{A}$ & $\mathrm{N} / \mathrm{A}$ & $\mathrm{N} / \mathrm{A}$ & $\mathrm{N} / \mathrm{A}$ & $\mathrm{N} / \mathrm{A}$ \\
\hline & $\begin{array}{l}\text { Surgical } \\
\text { complications }\end{array}$ & Seroma & $1(34)$ & $\begin{array}{l}\text { Mod } \\
\text { erate }\end{array}$ & $\mathrm{N} / \mathrm{A}$ & Imprecise & Direct & Sparse & Insufficient & $\begin{array}{l}\text { None (Sparse } \\
\text { evidence) }\end{array}$ \\
\hline & $\begin{array}{l}\text { Surgical } \\
\text { complications }\end{array}$ & Chronic conditions & $0(0)$ & $\mathrm{N} / \mathrm{A}$ & $\mathrm{N} / \mathrm{A}$ & $\mathrm{N} / \mathrm{A}$ & $\mathrm{N} / \mathrm{A}$ & $\mathrm{N} / \mathrm{A}$ & $\mathrm{N} / \mathrm{A}$ & $\mathrm{N} / \mathrm{A}$ \\
\hline \multirow{12}{*}{$\begin{array}{l}\text { Total versus } \\
\text { partial } \\
\text { submuscular }\end{array}$} & Clinical & General quality of life & $0(0)$ & $\mathrm{N} / \mathrm{A}$ & $\mathrm{N} / \mathrm{A}$ & $\mathrm{N} / \mathrm{A}$ & $\mathrm{N} / \mathrm{A}$ & $\mathrm{N} / \mathrm{A}$ & $\mathrm{N} / \mathrm{A}$ & $\mathrm{N} / \mathrm{A}$ \\
\hline & Clinical & Physical well-being & $0(0)$ & $\mathrm{N} / \mathrm{A}$ & $\mathrm{N} / \mathrm{A}$ & $\mathrm{N} / \mathrm{A}$ & $\mathrm{N} / \mathrm{A}$ & $\mathrm{N} / \mathrm{A}$ & $\mathrm{N} / \mathrm{A}$ & $\mathrm{N} / \mathrm{A}$ \\
\hline & Clinical & Psychosocial well-being & $0(0)$ & $\mathrm{N} / \mathrm{A}$ & $\mathrm{N} / \mathrm{A}$ & $\mathrm{N} / \mathrm{A}$ & $\mathrm{N} / \mathrm{A}$ & $\mathrm{N} / \mathrm{A}$ & $\mathrm{N} / \mathrm{A}$ & $\mathrm{N} / \mathrm{A}$ \\
\hline & Clinical & Sexual well-being & $0(0)$ & $\mathrm{N} / \mathrm{A}$ & $\mathrm{N} / \mathrm{A}$ & $\mathrm{N} / \mathrm{A}$ & $\mathrm{N} / \mathrm{A}$ & $\mathrm{N} / \mathrm{A}$ & $\mathrm{N} / \mathrm{A}$ & $\mathrm{N} / \mathrm{A}$ \\
\hline & Clinical & Patient satisfaction with breast & $0(0)$ & $\mathrm{N} / \mathrm{A}$ & $\mathrm{N} / \mathrm{A}$ & $\mathrm{N} / \mathrm{A}$ & $\mathrm{N} / \mathrm{A}$ & $\mathrm{N} / \mathrm{A}$ & $\mathrm{N} / \mathrm{A}$ & $\mathrm{N} / \mathrm{A}$ \\
\hline & Clinical & Patient satisfaction with outcome & $0(0)$ & $\mathrm{N} / \mathrm{A}$ & $\mathrm{N} / \mathrm{A}$ & $\mathrm{N} / \mathrm{A}$ & $\mathrm{N} / \mathrm{A}$ & $\mathrm{N} / \mathrm{A}$ & $\mathrm{N} / \mathrm{A}$ & $\mathrm{N} / \mathrm{A}$ \\
\hline & Clinical & Planned surgeries for reconstruction & $0(0)$ & $\mathrm{N} / \mathrm{A}$ & $\mathrm{N} / \mathrm{A}$ & $\mathrm{N} / \mathrm{A}$ & $\mathrm{N} / \mathrm{A}$ & $\mathrm{N} / \mathrm{A}$ & $\mathrm{N} / \mathrm{A}$ & $\mathrm{N} / \mathrm{A}$ \\
\hline & Clinical & Mortality & $0(0)$ & $\mathrm{N} / \mathrm{A}$ & $\mathrm{N} / \mathrm{A}$ & $\mathrm{N} / \mathrm{A}$ & $\mathrm{N} / \mathrm{A}$ & $\mathrm{N} / \mathrm{A}$ & $\mathrm{N} / \mathrm{A}$ & $\mathrm{N} / \mathrm{A}$ \\
\hline & $\begin{array}{l}\text { Surgical } \\
\text { complications }\end{array}$ & Unplanned repeat hospitalization & $0(0)$ & $\mathrm{N} / \mathrm{A}$ & $\mathrm{N} / \mathrm{A}$ & $\mathrm{N} / \mathrm{A}$ & $\mathrm{N} / \mathrm{A}$ & $\mathrm{N} / \mathrm{A}$ & $\mathrm{N} / \mathrm{A}$ & $\mathrm{N} / \mathrm{A}$ \\
\hline & $\begin{array}{l}\text { Surgical } \\
\text { complications }\end{array}$ & $\begin{array}{l}\text { Duration of unplanned repeat } \\
\text { hospitalization }\end{array}$ & $0(0)$ & $\mathrm{N} / \mathrm{A}$ & $\mathrm{N} / \mathrm{A}$ & $\mathrm{N} / \mathrm{A}$ & $\mathrm{N} / \mathrm{A}$ & $\mathrm{N} / \mathrm{A}$ & $\mathrm{N} / \mathrm{A}$ & $\mathrm{N} / \mathrm{A}$ \\
\hline & $\begin{array}{l}\text { Surgical } \\
\text { complications }\end{array}$ & $\begin{array}{l}\text { Unplanned repeat surgeries for } \\
\text { revision }\end{array}$ & $0(0)$ & $\mathrm{N} / \mathrm{A}$ & $\mathrm{N} / \mathrm{A}$ & $\mathrm{N} / \mathrm{A}$ & $\mathrm{N} / \mathrm{A}$ & $\mathrm{N} / \mathrm{A}$ & $\mathrm{N} / \mathrm{A}$ & $\mathrm{N} / \mathrm{A}$ \\
\hline & $\begin{array}{l}\text { Surgical } \\
\text { complications }\end{array}$ & $\begin{array}{l}\text { Unplanned repeat surgeries for } \\
\text { complications }\end{array}$ & $0(0)$ & $\mathrm{N} / \mathrm{A}$ & $\mathrm{N} / \mathrm{A}$ & $\mathrm{N} / \mathrm{A}$ & $\mathrm{N} / \mathrm{A}$ & $\mathrm{N} / \mathrm{A}$ & $\mathrm{N} / \mathrm{A}$ & $\mathrm{N} / \mathrm{A}$ \\
\hline
\end{tabular}




\begin{tabular}{|c|c|c|c|c|c|c|c|c|c|c|}
\hline Comparison & $\begin{array}{l}\text { Outcome } \\
\text { Category }\end{array}$ & Outcome & $\begin{array}{l}\text { N Studies } \\
\text { (Patients) }\end{array}$ & RoB & Consistency & Precision & Directness & Other & SoE & $\begin{array}{l}\text { Conclusions } \\
\text { (Reason, if } \\
\text { None) }\end{array}$ \\
\hline & $\begin{array}{l}\text { Surgical } \\
\text { complications }\end{array}$ & Pain & $0(0)$ & $\mathrm{N} / \mathrm{A}$ & $\mathrm{N} / \mathrm{A}$ & $\mathrm{N} / \mathrm{A}$ & $\mathrm{N} / \mathrm{A}$ & $\mathrm{N} / \mathrm{A}$ & $\mathrm{N} / \mathrm{A}$ & $\mathrm{N} / \mathrm{A}$ \\
\hline & $\begin{array}{l}\text { Surgical } \\
\text { complications }\end{array}$ & Analgesic use & $0(0)$ & $\mathrm{N} / \mathrm{A}$ & $\mathrm{N} / \mathrm{A}$ & $\mathrm{N} / \mathrm{A}$ & $\mathrm{N} / \mathrm{A}$ & $\mathrm{N} / \mathrm{A}$ & $\mathrm{N} / \mathrm{A}$ & $\mathrm{N} / \mathrm{A}$ \\
\hline & $\begin{array}{l}\text { Surgical } \\
\text { complications }\end{array}$ & Necrosis & $0(0)$ & $\mathrm{N} / \mathrm{A}$ & $\mathrm{N} / \mathrm{A}$ & $\mathrm{N} / \mathrm{A}$ & N/A & $\mathrm{N} / \mathrm{A}$ & $\mathrm{N} / \mathrm{A}$ & N/A \\
\hline & $\begin{array}{l}\text { Surgical } \\
\text { complications }\end{array}$ & Animation deformity & $0(0)$ & $\mathrm{N} / \mathrm{A}$ & $\mathrm{N} / \mathrm{A}$ & $\mathrm{N} / \mathrm{A}$ & $\mathrm{N} / \mathrm{A}$ & $\mathrm{N} / \mathrm{A}$ & $\mathrm{N} / \mathrm{A}$ & $\mathrm{N} / \mathrm{A}$ \\
\hline & $\begin{array}{l}\text { Surgical } \\
\text { complications }\end{array}$ & Implant-related infections & $0(0)$ & $\mathrm{N} / \mathrm{A}$ & $\mathrm{N} / \mathrm{A}$ & $\mathrm{N} / \mathrm{A}$ & $\mathrm{N} / \mathrm{A}$ & $\mathrm{N} / \mathrm{A}$ & $\mathrm{N} / \mathrm{A}$ & $\mathrm{N} / \mathrm{A}$ \\
\hline & $\begin{array}{l}\text { Surgical } \\
\text { complications }\end{array}$ & Implant-rupture & $0(0)$ & $\mathrm{N} / \mathrm{A}$ & $\mathrm{N} / \mathrm{A}$ & $\mathrm{N} / \mathrm{A}$ & $\mathrm{N} / \mathrm{A}$ & $\mathrm{N} / \mathrm{A}$ & $\mathrm{N} / \mathrm{A}$ & $\mathrm{N} / \mathrm{A}$ \\
\hline & $\begin{array}{l}\text { Surgical } \\
\text { complications }\end{array}$ & Implant deflation & $0(0)$ & $\mathrm{N} / \mathrm{A}$ & $\mathrm{N} / \mathrm{A}$ & $\mathrm{N} / \mathrm{A}$ & $\mathrm{N} / \mathrm{A}$ & $\mathrm{N} / \mathrm{A}$ & $\mathrm{N} / \mathrm{A}$ & $\mathrm{N} / \mathrm{A}$ \\
\hline & $\begin{array}{l}\text { Surgical } \\
\text { complications }\end{array}$ & Implant malposition & $0(0)$ & $\mathrm{N} / \mathrm{A}$ & $\mathrm{N} / \mathrm{A}$ & $\mathrm{N} / \mathrm{A}$ & $\mathrm{N} / \mathrm{A}$ & $\mathrm{N} / \mathrm{A}$ & $\mathrm{N} / \mathrm{A}$ & $\mathrm{N} / \mathrm{A}$ \\
\hline & $\begin{array}{l}\text { Surgical } \\
\text { complications }\end{array}$ & $\begin{array}{l}\text { Implant failure/loss or need for } \\
\text { explant surgery }\end{array}$ & $0(0)$ & $\mathrm{N} / \mathrm{A}$ & $\mathrm{N} / \mathrm{A}$ & $\mathrm{N} / \mathrm{A}$ & $\mathrm{N} / \mathrm{A}$ & $\mathrm{N} / \mathrm{A}$ & $\mathrm{N} / \mathrm{A}$ & $\mathrm{N} / \mathrm{A}$ \\
\hline & $\begin{array}{l}\text { Surgical } \\
\text { complications }\end{array}$ & Capsular contracture & $0(0)$ & $\mathrm{N} / \mathrm{A}$ & $\mathrm{N} / \mathrm{A}$ & $\mathrm{N} / \mathrm{A}$ & $\mathrm{N} / \mathrm{A}$ & $\mathrm{N} / \mathrm{A}$ & $\mathrm{N} / \mathrm{A}$ & $\mathrm{N} / \mathrm{A}$ \\
\hline & $\begin{array}{l}\text { Surgical } \\
\text { complications }\end{array}$ & New neoplasms & $0(0)$ & $\mathrm{N} / \mathrm{A}$ & $\mathrm{N} / \mathrm{A}$ & $\mathrm{N} / \mathrm{A}$ & N/A & $\mathrm{N} / \mathrm{A}$ & $\mathrm{N} / \mathrm{A}$ & $\mathrm{N} / \mathrm{A}$ \\
\hline & $\begin{array}{l}\text { Surgical } \\
\text { complications }\end{array}$ & $\begin{array}{l}\text { Complications delaying other cancer } \\
\text { treatments }\end{array}$ & $0(0)$ & $\mathrm{N} / \mathrm{A}$ & $\mathrm{N} / \mathrm{A}$ & $\mathrm{N} / \mathrm{A}$ & $\mathrm{N} / \mathrm{A}$ & $\mathrm{N} / \mathrm{A}$ & $\mathrm{N} / \mathrm{A}$ & $\mathrm{N} / \mathrm{A}$ \\
\hline & $\begin{array}{l}\text { Surgical } \\
\text { complications }\end{array}$ & Thromboembolic events & $0(0)$ & $\mathrm{N} / \mathrm{A}$ & $\mathrm{N} / \mathrm{A}$ & $\mathrm{N} / \mathrm{A}$ & N/A & $\mathrm{N} / \mathrm{A}$ & $\mathrm{N} / \mathrm{A}$ & $\mathrm{N} / \mathrm{A}$ \\
\hline & $\begin{array}{l}\text { Surgical } \\
\text { complications }\end{array}$ & $\begin{array}{l}\text { Infections (not explicitly implant- } \\
\text { related) }\end{array}$ & $0(0)$ & $\mathrm{N} / \mathrm{A}$ & $\mathrm{N} / \mathrm{A}$ & $\mathrm{N} / \mathrm{A}$ & $\mathrm{N} / \mathrm{A}$ & $\mathrm{N} / \mathrm{A}$ & $\mathrm{N} / \mathrm{A}$ & $\mathrm{N} / \mathrm{A}$ \\
\hline & $\begin{array}{l}\text { Surgical } \\
\text { complications }\end{array}$ & Seroma & $0(0)$ & $\mathrm{N} / \mathrm{A}$ & $\mathrm{N} / \mathrm{A}$ & $\mathrm{N} / \mathrm{A}$ & $\mathrm{N} / \mathrm{A}$ & $\mathrm{N} / \mathrm{A}$ & $\mathrm{N} / \mathrm{A}$ & $\mathrm{N} / \mathrm{A}$ \\
\hline & $\begin{array}{l}\text { Surgical } \\
\text { complications }\end{array}$ & Chronic conditions & $0(0)$ & $\mathrm{N} / \mathrm{A}$ & $\mathrm{N} / \mathrm{A}$ & $\mathrm{N} / \mathrm{A}$ & $\mathrm{N} / \mathrm{A}$ & $\mathrm{N} / \mathrm{A}$ & $\mathrm{N} / \mathrm{A}$ & $\mathrm{N} / \mathrm{A}$ \\
\hline
\end{tabular}

Abbreviations: $\mathrm{N} / \mathrm{A}=$ not applicable, $\mathrm{RoB}=$ risk of bias, $\mathrm{SoE}=$ strength of evidence.

Colors: Header rows are shaded orange. Rows for every alternate comparison are shaded blue. The colors do not add unique information. 
Table G-5. Key Question 5: Use versus nonuse of human ADMs during IBR - full evidence profile

\begin{tabular}{|c|c|c|c|c|c|c|c|c|c|}
\hline $\begin{array}{l}\text { Outcome } \\
\text { Category }\end{array}$ & Outcome & $\begin{array}{l}\text { N Studies } \\
\text { (Patients) }\end{array}$ & RoB & $\begin{array}{l}\text { Consistenc } \\
y\end{array}$ & Precision & Directness & Other & SoE & $\begin{array}{l}\text { Conclusions } \\
\text { (Reason, if None) }\end{array}$ \\
\hline Clinical & General quality of life & $0(0)$ & $\mathrm{N} / \mathrm{A}$ & $\mathrm{N} / \mathrm{A}$ & $\mathrm{N} / \mathrm{A}$ & $\mathrm{N} / \mathrm{A}$ & $\mathrm{N} / \mathrm{A}$ & $\mathrm{N} / \mathrm{A}$ & N/A \\
\hline Clinical & Physical well-being & $3(1604)$ & Moderate & Inconsistent & Precise & Direct & None & Insufficient & $\begin{array}{l}\text { None (Inconsistent } \\
\text { results) }\end{array}$ \\
\hline Clinical & Psychosocial well-being & $2(1535)$ & Moderate & Inconsistent & Precise & Direct & Low & Insufficient & $\begin{array}{l}\text { None (Inconsistent } \\
\text { results) }\end{array}$ \\
\hline Clinical & Sexual well-being & $1(1451)$ & Moderate & $\mathrm{N} / \mathrm{A}$ & Precise & Direct & Sparse & Insufficient & $\begin{array}{l}\text { None (Sparse } \\
\text { evidence) }\end{array}$ \\
\hline Clinical & Patient satisfaction with breast & $2(1535)$ & Moderate & Inconsistent & Precise & Direct & None & Insufficient & $\begin{array}{l}\text { None (Inconsistent } \\
\text { results) }\end{array}$ \\
\hline Clinical & Patient satisfaction with outcome & $0(0)$ & $\mathrm{N} / \mathrm{A}$ & $\mathrm{N} / \mathrm{A}$ & $\mathrm{N} / \mathrm{A}$ & $\mathrm{N} / \mathrm{A}$ & $\mathrm{N} / \mathrm{A}$ & $\mathrm{N} / \mathrm{A}$ & $\mathrm{N} / \mathrm{A}$ \\
\hline Clinical & $\begin{array}{l}\text { Planned surgeries for } \\
\text { reconstruction }\end{array}$ & $0(0)$ & $\mathrm{N} / \mathrm{A}$ & $\mathrm{N} / \mathrm{A}$ & $\mathrm{N} / \mathrm{A}$ & $\mathrm{N} / \mathrm{A}$ & $\mathrm{N} / \mathrm{A}$ & $\mathrm{N} / \mathrm{A}$ & $\mathrm{N} / \mathrm{A}$ \\
\hline Clinical & Mortality & $1(36)$ & High & $\mathrm{N} / \mathrm{A}$ & $\mathrm{N} / \mathrm{A}$ & Direct & Sparse & Insufficient & $\begin{array}{l}\text { None (Sparse } \\
\text { evidence) }\end{array}$ \\
\hline $\begin{array}{l}\text { Surgical } \\
\text { complications }\end{array}$ & Unplanned repeat hospitalization & $0(0)$ & $\mathrm{N} / \mathrm{A}$ & $\mathrm{N} / \mathrm{A}$ & $\mathrm{N} / \mathrm{A}$ & $\mathrm{N} / \mathrm{A}$ & $\mathrm{N} / \mathrm{A}$ & $\mathrm{N} / \mathrm{A}$ & $\mathrm{N} / \mathrm{A}$ \\
\hline $\begin{array}{l}\text { Surgical } \\
\text { complications }\end{array}$ & $\begin{array}{l}\text { Duration of unplanned repeat } \\
\text { hospitalization }\end{array}$ & $0(0)$ & $\mathrm{N} / \mathrm{A}$ & $\mathrm{N} / \mathrm{A}$ & $\mathrm{N} / \mathrm{A}$ & $\mathrm{N} / \mathrm{A}$ & $\mathrm{N} / \mathrm{A}$ & $\mathrm{N} / \mathrm{A}$ & $\mathrm{N} / \mathrm{A}$ \\
\hline $\begin{array}{l}\text { Surgical } \\
\text { complications }\end{array}$ & $\begin{array}{l}\text { Unplanned repeat surgeries for } \\
\text { revision }\end{array}$ & $3(20808)$ & High & Consistent & Precise & Direct & Moderate & None & $\begin{array}{l}\text { Comparable risks in } \\
\text { both groups }\end{array}$ \\
\hline $\begin{array}{l}\text { Surgical } \\
\text { complications }\end{array}$ & $\begin{array}{l}\text { Unplanned repeat surgeries for } \\
\text { complications }\end{array}$ & $1(128)$ & High & $\mathrm{N} / \mathrm{A}$ & Unclear & Direct & Sparse & Insufficient & $\begin{array}{l}\text { None (Sparse } \\
\text { evidence) }\end{array}$ \\
\hline $\begin{array}{l}\text { Surgical } \\
\text { complications }\end{array}$ & Pain & $2(153)$ & Moderate & Inconsistent & Unclear & Direct & None & Insufficient & $\begin{array}{l}\text { None (Inconsistent } \\
\text { results) }\end{array}$ \\
\hline $\begin{array}{l}\text { Surgical } \\
\text { complications }\end{array}$ & Analgesic use & $1(68)$ & Moderate & $\mathrm{N} / \mathrm{A}$ & Precise & Direct & Sparse & Insufficient & $\begin{array}{l}\text { None (Sparse } \\
\text { evidence) }\end{array}$ \\
\hline $\begin{array}{l}\text { Surgical } \\
\text { complications }\end{array}$ & Necrosis & $7(2101)$ & High & Consistent & Precise & Direct & None & Low & $\begin{array}{l}\text { Comparable risks in } \\
\text { both groups: } \\
\text { summary adjOR } 0.89 \\
(95 \% \mathrm{Cl} 0.63,1.25) ; 4 \\
\text { studies }\end{array}$ \\
\hline $\begin{array}{l}\text { Surgical } \\
\text { complications }\end{array}$ & Animation deformity & $0(0)$ & $\mathrm{N} / \mathrm{A}$ & $\mathrm{N} / \mathrm{A}$ & $\mathrm{N} / \mathrm{A}$ & $\mathrm{N} / \mathrm{A}$ & $\mathrm{N} / \mathrm{A}$ & $\mathrm{N} / \mathrm{A}$ & $\mathrm{N} / \mathrm{A}$ \\
\hline $\begin{array}{l}\text { Surgical } \\
\text { complications }\end{array}$ & Implant-related infections & $0(0)$ & $\mathrm{N} / \mathrm{A}$ & $\mathrm{N} / \mathrm{A}$ & $\mathrm{N} / \mathrm{A}$ & $\mathrm{N} / \mathrm{A}$ & $\mathrm{N} / \mathrm{A}$ & $\mathrm{N} / \mathrm{A}$ & $\mathrm{N} / \mathrm{A}$ \\
\hline $\begin{array}{l}\text { Surgical } \\
\text { complications }\end{array}$ & Implant-rupture & $1(1451)$ & Moderate & $\mathrm{N} / \mathrm{A}$ & Unclear & Direct & Sparse & Insufficient & $\begin{array}{l}\text { None (Sparse } \\
\text { evidence) }\end{array}$ \\
\hline $\begin{array}{l}\text { Surgical } \\
\text { complications }\end{array}$ & Implant deflation & $0(0)$ & $\mathrm{N} / \mathrm{A}$ & $\mathrm{N} / \mathrm{A}$ & $\mathrm{N} / \mathrm{A}$ & $\mathrm{N} / \mathrm{A}$ & $\mathrm{N} / \mathrm{A}$ & $\mathrm{N} / \mathrm{A}$ & $\mathrm{N} / \mathrm{A}$ \\
\hline $\begin{array}{l}\text { Surgical } \\
\text { complications }\end{array}$ & Implant malposition & $2(1654)$ & Moderate & Inconsistent & Unclear & Direct & Sparse & Insufficient & $\begin{array}{l}\text { None (Inconsistent } \\
\text { results) }\end{array}$ \\
\hline
\end{tabular}




\begin{tabular}{|c|c|c|c|c|c|c|c|c|c|}
\hline $\begin{array}{l}\text { Outcome } \\
\text { Category }\end{array}$ & Outcome & $\begin{array}{l}\text { N Studies } \\
\text { (Patients) }\end{array}$ & RoB & $\begin{array}{l}\text { Consistenc } \\
\text { y }\end{array}$ & Precision & Directness & Other & SoE & $\begin{array}{l}\text { Conclusions } \\
\text { (Reason, if None) }\end{array}$ \\
\hline $\begin{array}{l}\text { Surgical } \\
\text { complications }\end{array}$ & $\begin{array}{l}\text { Implant failure/loss/need for } \\
\text { explant surgery }\end{array}$ & $10(38983)$ & Moderate & Consistent & Precise & Direct & None & Moderate & $\begin{array}{l}\text { Higher risk with ADM: } \\
\text { summary adjOR } 1.28 \\
(95 \% \mathrm{Cl} 0.97,1.70) ; 6 \\
\text { studies }\end{array}$ \\
\hline $\begin{array}{l}\text { Surgical } \\
\text { complications }\end{array}$ & Capsular contracture & $4(3485)$ & High & Inconsistent & Precise & Direct & None & Insufficient & $\begin{array}{l}\text { None (Inconsistent } \\
\text { results) }\end{array}$ \\
\hline $\begin{array}{l}\text { Surgical } \\
\text { complications }\end{array}$ & New neoplasms & $0(0)$ & N/A & N/A & $\mathrm{N} / \mathrm{A}$ & $\mathrm{N} / \mathrm{A}$ & N/A & N/A & $\mathrm{N} / \mathrm{A}$ \\
\hline $\begin{array}{l}\text { Surgical } \\
\text { complications }\end{array}$ & $\begin{array}{l}\text { Complications delaying other } \\
\text { cancer treatments }\end{array}$ & $0(0)$ & $\mathrm{N} / \mathrm{A}$ & $\mathrm{N} / \mathrm{A}$ & $\mathrm{N} / \mathrm{A}$ & $\mathrm{N} / \mathrm{A}$ & $\mathrm{N} / \mathrm{A}$ & $\mathrm{N} / \mathrm{A}$ & $\mathrm{N} / \mathrm{A}$ \\
\hline $\begin{array}{l}\text { Surgical } \\
\text { complications }\end{array}$ & Thromboembolic events & $1(18997)$ & Moderate & $\mathrm{N} / \mathrm{A}$ & Unclear & Direct & Sparse & Insufficient & $\begin{array}{l}\text { None (Sparse } \\
\text { evidence) }\end{array}$ \\
\hline $\begin{array}{l}\text { Surgical } \\
\text { complications }\end{array}$ & $\begin{array}{l}\text { Infections (not explicitly implant- } \\
\text { related) }\end{array}$ & $13(25228)$ & Moderate & Inconsistent & Precise & Direct & None & Low & $\begin{array}{l}\text { Higher risk with ADM: } \\
\text { summary adjOR } 1.56 \\
(95 \% \mathrm{Cl} 0.96,2.53) ; 7 \\
\text { studies }\end{array}$ \\
\hline $\begin{array}{l}\text { Surgical } \\
\text { complications }\end{array}$ & Wound dehiscence & $4(21798)$ & Moderate & Inconsistent & Unclear & Direct & None & Insufficient & $\begin{array}{l}\text { None (Inconsistent } \\
\text { results) }\end{array}$ \\
\hline $\begin{array}{l}\text { Surgical } \\
\text { complications }\end{array}$ & Delayed healing & $1(398)$ & High & $\mathrm{N} / \mathrm{A}$ & Imprecise & Direct & Sparse & Insufficient & $\begin{array}{l}\text { None (Sparse } \\
\text { evidence) }\end{array}$ \\
\hline $\begin{array}{l}\text { Surgical } \\
\text { complications }\end{array}$ & Seroma & $6(3575)$ & Moderate & Consistent & Precise & Direct & None & Moderate & $\begin{array}{l}\text { Comparable risks in } \\
\text { both groups: } \\
\text { summary adjOR } 1.52 \\
(95 \% \mathrm{Cl} 0.62,3.71) ; 4 \\
\text { studies }\end{array}$ \\
\hline
\end{tabular}

Abbreviations: adj = adjusted, $\mathrm{ADM}=$ acellular dermal matrix, $\mathrm{CI}=$ confidence interval, $\mathrm{N} / \mathrm{A}=$ not applicable, $\mathrm{OR}=$ odds ratio, $\mathrm{RoB}=$ risk of bias, $\mathrm{SoE}=\mathrm{strength}$ of evidence.

Colors: Header rows are shaded orange. The color does not add unique information. 
Table G-6. Key Question 6: Comparison of flap types for AR - full evidence profile

\begin{tabular}{|c|c|c|c|c|c|c|c|c|c|}
\hline Comparison & $\begin{array}{l}\text { Outcome } \\
\text { Category }\end{array}$ & Outcome & $\begin{array}{l}\text { N Studies } \\
\text { (Patients) }\end{array}$ & RoB & Consistency & Precision & Directness & SoE & $\begin{array}{l}\text { Conclusions } \\
\text { (Reason, if None) }\end{array}$ \\
\hline \multirow{20}{*}{$\begin{array}{l}\text { TRAM vs. } \\
\text { DIEP }\end{array}$} & Clinical & General quality of life & $0(0)$ & $\mathrm{N} / \mathrm{A}$ & $\mathrm{N} / \mathrm{A}$ & $\mathrm{N} / \mathrm{A}$ & $\mathrm{N} / \mathrm{A}$ & $\mathrm{N} / \mathrm{A}$ & $\mathrm{N} / \mathrm{A}$ \\
\hline & Clinical & Physical well-being & $1(\mathrm{NR})$ & Moderate & $\mathrm{N} / \mathrm{A}$ & Precise & Direct & Insufficient & $\begin{array}{l}\text { None (Sparse } \\
\text { evidence) }\end{array}$ \\
\hline & Clinical & Psychosocial well-being & $1(\mathrm{NR})$ & Moderate & $\mathrm{N} / \mathrm{A}$ & Precise & Direct & Insufficient & $\begin{array}{l}\text { None (Sparse } \\
\text { evidence) }\end{array}$ \\
\hline & Clinical & Sexual well-being & 1 (NR) & Moderate & $\mathrm{N} / \mathrm{A}$ & Precise & Direct & Insufficient & $\begin{array}{l}\text { None (Sparse } \\
\text { evidence) }\end{array}$ \\
\hline & Clinical & Patient satisfaction with breast & $2(\mathrm{NR})$ & Moderate & Consistent & Precise & Direct & Low & $\begin{array}{l}\text { Comparable in } \\
\text { both groups }\end{array}$ \\
\hline & Clinical & Patient satisfaction with outcome & $1(260)$ & High & $\mathrm{N} / \mathrm{A}$ & Imprecise & Direct & Insufficient & $\begin{array}{l}\text { None (Sparse } \\
\text { evidence) }\end{array}$ \\
\hline & Clinical & Planned surgeries for reconstruction & $0(0)$ & $\mathrm{N} / \mathrm{A}$ & $\mathrm{N} / \mathrm{A}$ & $\mathrm{N} / \mathrm{A}$ & $\mathrm{N} / \mathrm{A}$ & $\mathrm{N} / \mathrm{A}$ & $\mathrm{N} / \mathrm{A}$ \\
\hline & Clinical & Duration of initial hospitalization & $1(15836)$ & High & $\mathrm{N} / \mathrm{A}$ & Unclear & Direct & Insufficient & $\begin{array}{l}\text { None (Sparse } \\
\text { evidence) }\end{array}$ \\
\hline & Clinical & Mortality & $0(0)$ & $\mathrm{N} / \mathrm{A}$ & $\mathrm{N} / \mathrm{A}$ & $\mathrm{N} / \mathrm{A}$ & $\mathrm{N} / \mathrm{A}$ & $\mathrm{N} / \mathrm{A}$ & $\mathrm{N} / \mathrm{A}$ \\
\hline & $\begin{array}{l}\text { Surgical } \\
\text { complications }\end{array}$ & Unplanned repeat hospitalization & $0(0)$ & $\mathrm{N} / \mathrm{A}$ & $\mathrm{N} / \mathrm{A}$ & $\mathrm{N} / \mathrm{A}$ & $\mathrm{N} / \mathrm{A}$ & $\mathrm{N} / \mathrm{A}$ & $\mathrm{N} / \mathrm{A}$ \\
\hline & $\begin{array}{l}\text { Surgical } \\
\text { complications }\end{array}$ & Duration of unplanned repeat hospitalization & $0(0)$ & $\mathrm{N} / \mathrm{A}$ & $\mathrm{N} / \mathrm{A}$ & $\mathrm{N} / \mathrm{A}$ & $\mathrm{N} / \mathrm{A}$ & $\mathrm{N} / \mathrm{A}$ & $\mathrm{N} / \mathrm{A}$ \\
\hline & $\begin{array}{l}\text { Surgical } \\
\text { complications }\end{array}$ & Unplanned repeat surgeries for revision & $0(0)$ & $\mathrm{N} / \mathrm{A}$ & $\mathrm{N} / \mathrm{A}$ & $\mathrm{N} / \mathrm{A}$ & $\mathrm{N} / \mathrm{A}$ & $\mathrm{N} / \mathrm{A}$ & $\mathrm{N} / \mathrm{A}$ \\
\hline & $\begin{array}{l}\text { Surgical } \\
\text { complications }\end{array}$ & Unplanned repeat surgeries for complications & $0(0)$ & $\mathrm{N} / \mathrm{A}$ & $\mathrm{N} / \mathrm{A}$ & $\mathrm{N} / \mathrm{A}$ & $\mathrm{N} / \mathrm{A}$ & $\mathrm{N} / \mathrm{A}$ & $\mathrm{N} / \mathrm{A}$ \\
\hline & $\begin{array}{l}\text { Surgical } \\
\text { complications }\end{array}$ & Pain & $0(0)$ & N/A & $\mathrm{N} / \mathrm{A}$ & $\mathrm{N} / \mathrm{A}$ & $\mathrm{N} / \mathrm{A}$ & $\mathrm{N} / \mathrm{A}$ & $\mathrm{N} / \mathrm{A}$ \\
\hline & $\begin{array}{l}\text { Surgical } \\
\text { complications }\end{array}$ & Analgesic use & $0(0)$ & N/A & $\mathrm{N} / \mathrm{A}$ & $\mathrm{N} / \mathrm{A}$ & $\mathrm{N} / \mathrm{A}$ & $\mathrm{N} / \mathrm{A}$ & $\mathrm{N} / \mathrm{A}$ \\
\hline & $\begin{array}{l}\text { Surgical } \\
\text { complications }\end{array}$ & Necrosis & $2(959)$ & High & Consistent & Unclear & Direct & Low & $\begin{array}{l}\text { Comparable in } \\
\text { both groups }\end{array}$ \\
\hline & $\begin{array}{l}\text { Surgical } \\
\text { complications }\end{array}$ & Harms to area of flap harvest & $4(9253)$ & High & Consistent & Precise & Direct & Moderate & $\begin{array}{l}\text { Increased risk of } \\
\text { abdominal } \\
\text { bulge/hernia and } \\
\text { abdominal hernia } \\
\text { repair }\end{array}$ \\
\hline & $\begin{array}{l}\text { Surgical } \\
\text { complications }\end{array}$ & Complications delaying other cancer treatments & $0(0)$ & N/A & $\mathrm{N} / \mathrm{A}$ & $\mathrm{N} / \mathrm{A}$ & $\mathrm{N} / \mathrm{A}$ & $\mathrm{N} / \mathrm{A}$ & N/A \\
\hline & $\begin{array}{l}\text { Surgical } \\
\text { complications }\end{array}$ & Thromboembolic events & $0(0)$ & $\mathrm{N} / \mathrm{A}$ & $\mathrm{N} / \mathrm{A}$ & $\mathrm{N} / \mathrm{A}$ & $\mathrm{N} / \mathrm{A}$ & $\mathrm{N} / \mathrm{A}$ & $\mathrm{N} / \mathrm{A}$ \\
\hline & $\begin{array}{l}\text { Surgical } \\
\text { complications }\end{array}$ & Infections & $1(15836)$ & High & $\mathrm{N} / \mathrm{A}$ & Unclear & Direct & Insufficient & $\begin{array}{l}\text { None (Sparse } \\
\text { evidence) }\end{array}$ \\
\hline
\end{tabular}




\begin{tabular}{|c|c|c|c|c|c|c|c|c|c|}
\hline Comparison & $\begin{array}{l}\text { Outcome } \\
\text { Category }\end{array}$ & Outcome & $\begin{array}{l}\text { N Studies } \\
\text { (Patients) }\end{array}$ & RoB & Consistency & Precision & Directness & SoE & $\begin{array}{l}\text { Conclusions } \\
\text { (Reason, if None) }\end{array}$ \\
\hline & $\begin{array}{l}\text { Surgical } \\
\text { complications }\end{array}$ & Wound dehiscence & $1(15836)$ & High & $\mathrm{N} / \mathrm{A}$ & Unclear & Direct & Insufficient & $\begin{array}{l}\text { None (Sparse } \\
\text { evidence) }\end{array}$ \\
\hline & $\begin{array}{l}\text { Surgical } \\
\text { complications }\end{array}$ & Delayed healing & $0(0)$ & $\mathrm{N} / \mathrm{A}$ & $\mathrm{N} / \mathrm{A}$ & $\mathrm{N} / \mathrm{A}$ & $\mathrm{N} / \mathrm{A}$ & $\mathrm{N} / \mathrm{A}$ & $\mathrm{N} / \mathrm{A}$ \\
\hline & $\begin{array}{l}\text { Surgical } \\
\text { complications }\end{array}$ & Seroma & $0(0)$ & $\mathrm{N} / \mathrm{A}$ & $\mathrm{N} / \mathrm{A}$ & $\mathrm{N} / \mathrm{A}$ & $\mathrm{N} / \mathrm{A}$ & $\mathrm{N} / \mathrm{A}$ & N/A \\
\hline \multirow{20}{*}{$\begin{array}{l}\text { SIEA vs. } \\
\text { DIEP }\end{array}$} & Clinical & General quality of life & $0(0)$ & $\mathrm{N} / \mathrm{A}$ & $\mathrm{N} / \mathrm{A}$ & $\mathrm{N} / \mathrm{A}$ & $\mathrm{N} / \mathrm{A}$ & $\mathrm{N} / \mathrm{A}$ & $\mathrm{N} / \mathrm{A}$ \\
\hline & Clinical & Physical well-being & $1(\mathrm{NR})$ & Moderate & N/A & Precise & Direct & Insufficient & $\begin{array}{l}\text { None (Sparse } \\
\text { evidence) }\end{array}$ \\
\hline & Clinical & Psychosocial well-being & $1(\mathrm{NR})$ & Moderate & $\mathrm{N} / \mathrm{A}$ & Precise & Direct & Insufficient & $\begin{array}{l}\text { None (Sparse } \\
\text { evidence) }\end{array}$ \\
\hline & Clinical & Sexual well-being & 1 (NR) & Moderate & N/A & Precise & Direct & Insufficient & $\begin{array}{l}\text { None (Sparse } \\
\text { evidence) }\end{array}$ \\
\hline & Clinical & Patient satisfaction with breast & $1(\mathrm{NR})$ & Moderate & $\mathrm{N} / \mathrm{A}$ & Precise & Direct & Insufficient & $\begin{array}{l}\text { None (Sparse } \\
\text { evidence) }\end{array}$ \\
\hline & Clinical & Patient satisfaction with outcome & $0(0)$ & $\mathrm{N} / \mathrm{A}$ & $\mathrm{N} / \mathrm{A}$ & $\mathrm{N} / \mathrm{A}$ & $\mathrm{N} / \mathrm{A}$ & $\mathrm{N} / \mathrm{A}$ & N/A \\
\hline & Clinical & Planned surgeries for reconstruction & $0(0)$ & $\mathrm{N} / \mathrm{A}$ & $\mathrm{N} / \mathrm{A}$ & $\mathrm{N} / \mathrm{A}$ & $\mathrm{N} / \mathrm{A}$ & $\mathrm{N} / \mathrm{A}$ & $\mathrm{N} / \mathrm{A}$ \\
\hline & Clinical & Duration of initial hospitalization & $0(0)$ & $\mathrm{N} / \mathrm{A}$ & $\mathrm{N} / \mathrm{A}$ & $\mathrm{N} / \mathrm{A}$ & $\mathrm{N} / \mathrm{A}$ & $\mathrm{N} / \mathrm{A}$ & $\mathrm{N} / \mathrm{A}$ \\
\hline & Clinical & Mortality & $0(0)$ & $\mathrm{N} / \mathrm{A}$ & $\mathrm{N} / \mathrm{A}$ & $\mathrm{N} / \mathrm{A}$ & $\mathrm{N} / \mathrm{A}$ & $\mathrm{N} / \mathrm{A}$ & $\mathrm{N} / \mathrm{A}$ \\
\hline & $\begin{array}{l}\text { Surgical } \\
\text { complications }\end{array}$ & Unplanned repeat hospitalization & $0(0)$ & $\mathrm{N} / \mathrm{A}$ & $\mathrm{N} / \mathrm{A}$ & $\mathrm{N} / \mathrm{A}$ & $\mathrm{N} / \mathrm{A}$ & $\mathrm{N} / \mathrm{A}$ & $\mathrm{N} / \mathrm{A}$ \\
\hline & $\begin{array}{l}\text { Surgical } \\
\text { complications }\end{array}$ & Duration of unplanned repeat hospitalization & $0(0)$ & $\mathrm{N} / \mathrm{A}$ & $\mathrm{N} / \mathrm{A}$ & $\mathrm{N} / \mathrm{A}$ & $\mathrm{N} / \mathrm{A}$ & $\mathrm{N} / \mathrm{A}$ & $\mathrm{N} / \mathrm{A}$ \\
\hline & $\begin{array}{l}\text { Surgical } \\
\text { complications }\end{array}$ & Unplanned repeat surgeries for revision & $0(0)$ & $\mathrm{N} / \mathrm{A}$ & $\mathrm{N} / \mathrm{A}$ & $\mathrm{N} / \mathrm{A}$ & $\mathrm{N} / \mathrm{A}$ & $\mathrm{N} / \mathrm{A}$ & N/A \\
\hline & $\begin{array}{l}\text { Surgical } \\
\text { complications }\end{array}$ & Unplanned repeat surgeries for complications & $0(0)$ & $\mathrm{N} / \mathrm{A}$ & $\mathrm{N} / \mathrm{A}$ & $\mathrm{N} / \mathrm{A}$ & $\mathrm{N} / \mathrm{A}$ & $\mathrm{N} / \mathrm{A}$ & $\mathrm{N} / \mathrm{A}$ \\
\hline & $\begin{array}{l}\text { Surgical } \\
\text { complications }\end{array}$ & Pain & $0(0)$ & $\mathrm{N} / \mathrm{A}$ & $\mathrm{N} / \mathrm{A}$ & $\mathrm{N} / \mathrm{A}$ & $\mathrm{N} / \mathrm{A}$ & $\mathrm{N} / \mathrm{A}$ & N/A \\
\hline & $\begin{array}{l}\text { Surgical } \\
\text { complications }\end{array}$ & Analgesic use & $0(0)$ & $\mathrm{N} / \mathrm{A}$ & $\mathrm{N} / \mathrm{A}$ & $\mathrm{N} / \mathrm{A}$ & $\mathrm{N} / \mathrm{A}$ & $\mathrm{N} / \mathrm{A}$ & N/A \\
\hline & $\begin{array}{l}\text { Surgical } \\
\text { complications }\end{array}$ & Necrosis & $0(0)$ & $\mathrm{N} / \mathrm{A}$ & $\mathrm{N} / \mathrm{A}$ & $\mathrm{N} / \mathrm{A}$ & N/A & $\mathrm{N} / \mathrm{A}$ & N/A \\
\hline & $\begin{array}{l}\text { Surgical } \\
\text { complications }\end{array}$ & Harms to area of flap harvest & $1(417)$ & Moderate & N/A & Precise & Direct & Insufficient & $\begin{array}{l}\text { None (Sparse } \\
\text { evidence) }\end{array}$ \\
\hline & $\begin{array}{l}\text { Surgical } \\
\text { complications }\end{array}$ & Complications delaying other cancer treatments & $0(0)$ & $\mathrm{N} / \mathrm{A}$ & $\mathrm{N} / \mathrm{A}$ & $\mathrm{N} / \mathrm{A}$ & $\mathrm{N} / \mathrm{A}$ & $\mathrm{N} / \mathrm{A}$ & N/A \\
\hline & $\begin{array}{l}\text { Surgical } \\
\text { complications }\end{array}$ & Thromboembolic events & $0(0)$ & $\mathrm{N} / \mathrm{A}$ & $\mathrm{N} / \mathrm{A}$ & $\mathrm{N} / \mathrm{A}$ & $\mathrm{N} / \mathrm{A}$ & $\mathrm{N} / \mathrm{A}$ & N/A \\
\hline & $\begin{array}{l}\text { Surgical } \\
\text { complications }\end{array}$ & Infections & $0(0)$ & $\mathrm{N} / \mathrm{A}$ & $\mathrm{N} / \mathrm{A}$ & $\mathrm{N} / \mathrm{A}$ & $\mathrm{N} / \mathrm{A}$ & $\mathrm{N} / \mathrm{A}$ & N/A \\
\hline
\end{tabular}




\begin{tabular}{|c|c|c|c|c|c|c|c|c|c|}
\hline Comparison & $\begin{array}{l}\text { Outcome } \\
\text { Category }\end{array}$ & Outcome & $\begin{array}{l}\text { N Studies } \\
\text { (Patients) }\end{array}$ & RoB & Consistency & Precision & Directness & SoE & $\begin{array}{l}\text { Conclusions } \\
\text { (Reason, if None) }\end{array}$ \\
\hline & $\begin{array}{l}\text { Surgical } \\
\text { complications }\end{array}$ & Wound dehiscence & $0(0)$ & $\mathrm{N} / \mathrm{A}$ & $\mathrm{N} / \mathrm{A}$ & $\mathrm{N} / \mathrm{A}$ & $\mathrm{N} / \mathrm{A}$ & $\mathrm{N} / \mathrm{A}$ & N/A \\
\hline & $\begin{array}{l}\text { Surgical } \\
\text { complications }\end{array}$ & Delayed healing & $0(0)$ & $\mathrm{N} / \mathrm{A}$ & $\mathrm{N} / \mathrm{A}$ & $\mathrm{N} / \mathrm{A}$ & $\mathrm{N} / \mathrm{A}$ & $\mathrm{N} / \mathrm{A}$ & N/A \\
\hline & $\begin{array}{l}\text { Surgical } \\
\text { complications }\end{array}$ & Seroma & $0(0)$ & $\mathrm{N} / \mathrm{A}$ & $\mathrm{N} / \mathrm{A}$ & $\mathrm{N} / \mathrm{A}$ & $\mathrm{N} / \mathrm{A}$ & $\mathrm{N} / \mathrm{A}$ & $\mathrm{N} / \mathrm{A}$ \\
\hline \multirow[t]{21}{*}{ TAP vs. LD } & Clinical & General quality of life & $0(0)$ & $\mathrm{N} / \mathrm{A}$ & $\mathrm{N} / \mathrm{A}$ & $\mathrm{N} / \mathrm{A}$ & $\mathrm{N} / \mathrm{A}$ & $\mathrm{N} / \mathrm{A}$ & $\mathrm{N} / \mathrm{A}$ \\
\hline & Clinical & Physical well-being & $1(40)$ & Moderate & N/A & Imprecise & Direct & Insufficient & $\begin{array}{l}\text { None (Sparse } \\
\text { evidence) }\end{array}$ \\
\hline & Clinical & Psychosocial well-being & $0(0)$ & $\mathrm{N} / \mathrm{A}$ & $\mathrm{N} / \mathrm{A}$ & $\mathrm{N} / \mathrm{A}$ & $\mathrm{N} / \mathrm{A}$ & $\mathrm{N} / \mathrm{A}$ & $\mathrm{N} / \mathrm{A}$ \\
\hline & Clinical & Sexual well-being & $0(0)$ & $\mathrm{N} / \mathrm{A}$ & $\mathrm{N} / \mathrm{A}$ & $\mathrm{N} / \mathrm{A}$ & $\mathrm{N} / \mathrm{A}$ & $\mathrm{N} / \mathrm{A}$ & N/A \\
\hline & Clinical & Patient satisfaction with breast & $0(0)$ & $\mathrm{N} / \mathrm{A}$ & $\mathrm{N} / \mathrm{A}$ & $\mathrm{N} / \mathrm{A}$ & $\mathrm{N} / \mathrm{A}$ & $\mathrm{N} / \mathrm{A}$ & $\mathrm{N} / \mathrm{A}$ \\
\hline & Clinical & Patient satisfaction with outcome & $0(0)$ & $\mathrm{N} / \mathrm{A}$ & $\mathrm{N} / \mathrm{A}$ & $\mathrm{N} / \mathrm{A}$ & $\mathrm{N} / \mathrm{A}$ & $\mathrm{N} / \mathrm{A}$ & $\mathrm{N} / \mathrm{A}$ \\
\hline & Clinical & Planned surgeries for reconstruction & $0(0)$ & $\mathrm{N} / \mathrm{A}$ & $\mathrm{N} / \mathrm{A}$ & $\mathrm{N} / \mathrm{A}$ & $\mathrm{N} / \mathrm{A}$ & $\mathrm{N} / \mathrm{A}$ & $\mathrm{N} / \mathrm{A}$ \\
\hline & Clinical & Duration of initial hospitalization & $1(40)$ & Moderate & $\mathrm{N} / \mathrm{A}$ & Precise & Direct & Insufficient & $\begin{array}{l}\text { None (Sparse } \\
\text { evidence) }\end{array}$ \\
\hline & Clinical & Mortality & $0(0)$ & $\mathrm{N} / \mathrm{A}$ & $\mathrm{N} / \mathrm{A}$ & $\mathrm{N} / \mathrm{A}$ & $\mathrm{N} / \mathrm{A}$ & $\mathrm{N} / \mathrm{A}$ & N/A \\
\hline & $\begin{array}{l}\text { Surgical } \\
\text { complications }\end{array}$ & Unplanned repeat hospitalization & $0(0)$ & $\mathrm{N} / \mathrm{A}$ & $\mathrm{N} / \mathrm{A}$ & $\mathrm{N} / \mathrm{A}$ & $\mathrm{N} / \mathrm{A}$ & $\mathrm{N} / \mathrm{A}$ & $\mathrm{N} / \mathrm{A}$ \\
\hline & $\begin{array}{l}\text { Surgical } \\
\text { complications }\end{array}$ & Duration of unplanned repeat hospitalization & $0(0)$ & $\mathrm{N} / \mathrm{A}$ & $\mathrm{N} / \mathrm{A}$ & $\mathrm{N} / \mathrm{A}$ & $\mathrm{N} / \mathrm{A}$ & $\mathrm{N} / \mathrm{A}$ & N/A \\
\hline & $\begin{array}{l}\text { Surgical } \\
\text { complications }\end{array}$ & Unplanned repeat surgeries for revision & $0(0)$ & $\mathrm{N} / \mathrm{A}$ & $\mathrm{N} / \mathrm{A}$ & $\mathrm{N} / \mathrm{A}$ & $\mathrm{N} / \mathrm{A}$ & $\mathrm{N} / \mathrm{A}$ & $\mathrm{N} / \mathrm{A}$ \\
\hline & $\begin{array}{l}\text { Surgical } \\
\text { complications }\end{array}$ & Unplanned repeat surgeries for complications & $0(0)$ & $\mathrm{N} / \mathrm{A}$ & $\mathrm{N} / \mathrm{A}$ & $\mathrm{N} / \mathrm{A}$ & $\mathrm{N} / \mathrm{A}$ & $\mathrm{N} / \mathrm{A}$ & $\mathrm{N} / \mathrm{A}$ \\
\hline & $\begin{array}{l}\text { Surgical } \\
\text { complications }\end{array}$ & Pain & $1(40)$ & Moderate & $\mathrm{N} / \mathrm{A}$ & Imprecise & Direct & Insufficient & $\begin{array}{l}\text { None (Sparse } \\
\text { evidence) }\end{array}$ \\
\hline & $\begin{array}{l}\text { Surgical } \\
\text { complications }\end{array}$ & Analgesic use & $0(0)$ & $\mathrm{N} / \mathrm{A}$ & $\mathrm{N} / \mathrm{A}$ & $\mathrm{N} / \mathrm{A}$ & $\mathrm{N} / \mathrm{A}$ & $\mathrm{N} / \mathrm{A}$ & N/A \\
\hline & $\begin{array}{l}\text { Surgical } \\
\text { complications }\end{array}$ & Necrosis & $1(40)$ & Moderate & $\mathrm{N} / \mathrm{A}$ & Imprecise & Direct & Insufficient & $\begin{array}{l}\text { None (Sparse } \\
\text { evidence) }\end{array}$ \\
\hline & $\begin{array}{l}\text { Surgical } \\
\text { complications }\end{array}$ & Harms to area of flap harvest & $0(0)$ & $\mathrm{N} / \mathrm{A}$ & $\mathrm{N} / \mathrm{A}$ & $\mathrm{N} / \mathrm{A}$ & $\mathrm{N} / \mathrm{A}$ & $\mathrm{N} / \mathrm{A}$ & $\mathrm{N} / \mathrm{A}$ \\
\hline & $\begin{array}{l}\text { Surgical } \\
\text { complications }\end{array}$ & Complications delaying other cancer treatments & $0(0)$ & $\mathrm{N} / \mathrm{A}$ & $\mathrm{N} / \mathrm{A}$ & $\mathrm{N} / \mathrm{A}$ & $\mathrm{N} / \mathrm{A}$ & $\mathrm{N} / \mathrm{A}$ & $\mathrm{N} / \mathrm{A}$ \\
\hline & $\begin{array}{l}\text { Surgical } \\
\text { complications }\end{array}$ & Thromboembolic events & $0(0)$ & $\mathrm{N} / \mathrm{A}$ & $\mathrm{N} / \mathrm{A}$ & $\mathrm{N} / \mathrm{A}$ & $\mathrm{N} / \mathrm{A}$ & $\mathrm{N} / \mathrm{A}$ & N/A \\
\hline & $\begin{array}{l}\text { Surgical } \\
\text { complications }\end{array}$ & Infections & $1(40)$ & Moderate & $\mathrm{N} / \mathrm{A}$ & Imprecise & Direct & Insufficient & $\begin{array}{l}\text { None (Sparse } \\
\text { evidence) }\end{array}$ \\
\hline & $\begin{array}{l}\text { Surgical } \\
\text { complications }\end{array}$ & Wound dehiscence & $0(0)$ & $\mathrm{N} / \mathrm{A}$ & $\mathrm{N} / \mathrm{A}$ & $\mathrm{N} / \mathrm{A}$ & $\mathrm{N} / \mathrm{A}$ & $\mathrm{N} / \mathrm{A}$ & $\mathrm{N} / \mathrm{A}$ \\
\hline
\end{tabular}




\begin{tabular}{|c|c|c|c|c|c|c|c|c|c|}
\hline Comparison & $\begin{array}{l}\text { Outcome } \\
\text { Category }\end{array}$ & Outcome & \begin{tabular}{|l|} 
N Studies \\
(Patients)
\end{tabular} & RoB & Consistency & Precision & Directness & SoE & $\begin{array}{l}\text { Conclusions } \\
\text { (Reason, if None) }\end{array}$ \\
\hline & $\begin{array}{l}\text { Surgical } \\
\text { complications }\end{array}$ & Delayed healing & $0(0)$ & $\mathrm{N} / \mathrm{A}$ & $\mathrm{N} / \mathrm{A}$ & $\mathrm{N} / \mathrm{A}$ & $\mathrm{N} / \mathrm{A}$ & $\mathrm{N} / \mathrm{A}$ & $\mathrm{N} / \mathrm{A}$ \\
\hline & $\begin{array}{l}\text { Surgical } \\
\text { complications }\end{array}$ & Seroma & $1(40)$ & Moderate & $\mathrm{N} / \mathrm{A}$ & Imprecise & Direct & Insufficient & $\begin{array}{l}\text { None (Sparse } \\
\text { evidence) }\end{array}$ \\
\hline \multirow[t]{21}{*}{ TRAM vs. LD } & Clinical & General quality of life & $0(0)$ & $\mathrm{N} / \mathrm{A}$ & $\mathrm{N} / \mathrm{A}$ & $\mathrm{N} / \mathrm{A}$ & $\mathrm{N} / \mathrm{A}$ & $\mathrm{N} / \mathrm{A}$ & $\mathrm{N} / \mathrm{A}$ \\
\hline & Clinical & Physical well-being & $0(0)$ & $\mathrm{N} / \mathrm{A}$ & $\mathrm{N} / \mathrm{A}$ & $\mathrm{N} / \mathrm{A}$ & $\mathrm{N} / \mathrm{A}$ & $\mathrm{N} / \mathrm{A}$ & $\mathrm{N} / \mathrm{A}$ \\
\hline & Clinical & Psychosocial well-being & $0(0)$ & $\mathrm{N} / \mathrm{A}$ & $\mathrm{N} / \mathrm{A}$ & $\mathrm{N} / \mathrm{A}$ & $\mathrm{N} / \mathrm{A}$ & $\mathrm{N} / \mathrm{A}$ & $\mathrm{N} / \mathrm{A}$ \\
\hline & Clinical & Sexual well-being & $0(0)$ & $\mathrm{N} / \mathrm{A}$ & $\mathrm{N} / \mathrm{A}$ & N/A & N/A & $\mathrm{N} / \mathrm{A}$ & N/A \\
\hline & Clinical & Patient satisfaction with breast & $1(49)$ & High & $\mathrm{N} / \mathrm{A}$ & Unclear & Direct & Insufficient & $\begin{array}{l}\text { None (Sparse } \\
\text { evidence) }\end{array}$ \\
\hline & Clinical & Patient satisfaction with outcome & $1(255)$ & High & $\mathrm{N} / \mathrm{A}$ & Imprecise & Direct & Insufficient & $\begin{array}{l}\text { None (Sparse } \\
\text { evidence) }\end{array}$ \\
\hline & Clinical & Planned surgeries for reconstruction & $0(0)$ & $\mathrm{N} / \mathrm{A}$ & $\mathrm{N} / \mathrm{A}$ & $\mathrm{N} / \mathrm{A}$ & $\mathrm{N} / \mathrm{A}$ & $\mathrm{N} / \mathrm{A}$ & $\mathrm{N} / \mathrm{A}$ \\
\hline & Clinical & Duration of initial hospitalization & $0(0)$ & $\mathrm{N} / \mathrm{A}$ & N/A & $\mathrm{N} / \mathrm{A}$ & N/A & $\mathrm{N} / \mathrm{A}$ & N/A \\
\hline & Clinical & Mortality & $1(59)$ & High & $\mathrm{N} / \mathrm{A}$ & Imprecise & Direct & Insufficient & $\begin{array}{l}\text { None (Sparse } \\
\text { evidence) }\end{array}$ \\
\hline & $\begin{array}{l}\text { Surgical } \\
\text { complications }\end{array}$ & Unplanned repeat hospitalization & $0(0)$ & $\mathrm{N} / \mathrm{A}$ & $\mathrm{N} / \mathrm{A}$ & $\mathrm{N} / \mathrm{A}$ & $\mathrm{N} / \mathrm{A}$ & $\mathrm{N} / \mathrm{A}$ & N/A \\
\hline & $\begin{array}{l}\text { Surgical } \\
\text { complications }\end{array}$ & Duration of unplanned repeat hospitalization & $0(0)$ & $\mathrm{N} / \mathrm{A}$ & $\mathrm{N} / \mathrm{A}$ & $\mathrm{N} / \mathrm{A}$ & $\mathrm{N} / \mathrm{A}$ & $\mathrm{N} / \mathrm{A}$ & $\mathrm{N} / \mathrm{A}$ \\
\hline & $\begin{array}{l}\text { Surgical } \\
\text { complications }\end{array}$ & Unplanned repeat surgeries for revision & $1(3296)$ & High & $\mathrm{N} / \mathrm{A}$ & Precise & Direct & Insufficient & $\begin{array}{l}\text { None (Sparse } \\
\text { evidence) }\end{array}$ \\
\hline & $\begin{array}{l}\text { Surgical } \\
\text { complications }\end{array}$ & Unplanned repeat surgeries for complications & $0(0)$ & $\mathrm{N} / \mathrm{A}$ & $\mathrm{N} / \mathrm{A}$ & $\mathrm{N} / \mathrm{A}$ & $\mathrm{N} / \mathrm{A}$ & $\mathrm{N} / \mathrm{A}$ & $\mathrm{N} / \mathrm{A}$ \\
\hline & $\begin{array}{l}\text { Surgical } \\
\text { complications }\end{array}$ & Pain & $0(0)$ & $\mathrm{N} / \mathrm{A}$ & $\mathrm{N} / \mathrm{A}$ & $\mathrm{N} / \mathrm{A}$ & $\mathrm{N} / \mathrm{A}$ & $\mathrm{N} / \mathrm{A}$ & $\mathrm{N} / \mathrm{A}$ \\
\hline & $\begin{array}{l}\text { Surgical } \\
\text { complications }\end{array}$ & Analgesic use & $0(0)$ & $\mathrm{N} / \mathrm{A}$ & $\mathrm{N} / \mathrm{A}$ & $\mathrm{N} / \mathrm{A}$ & $\mathrm{N} / \mathrm{A}$ & $\mathrm{N} / \mathrm{A}$ & $\mathrm{N} / \mathrm{A}$ \\
\hline & $\begin{array}{l}\text { Surgical } \\
\text { complications }\end{array}$ & Necrosis & $0(0)$ & $\mathrm{N} / \mathrm{A}$ & $\mathrm{N} / \mathrm{A}$ & $\mathrm{N} / \mathrm{A}$ & $\mathrm{N} / \mathrm{A}$ & $\mathrm{N} / \mathrm{A}$ & $\mathrm{N} / \mathrm{A}$ \\
\hline & $\begin{array}{l}\text { Surgical } \\
\text { complications }\end{array}$ & Harms to area of flap harvest & $0(0)$ & $\mathrm{N} / \mathrm{A}$ & $\mathrm{N} / \mathrm{A}$ & $\mathrm{N} / \mathrm{A}$ & $\mathrm{N} / \mathrm{A}$ & $\mathrm{N} / \mathrm{A}$ & $\mathrm{N} / \mathrm{A}$ \\
\hline & $\begin{array}{l}\text { Surgical } \\
\text { complications }\end{array}$ & Complications delaying other cancer treatments & $0(0)$ & $\mathrm{N} / \mathrm{A}$ & $\mathrm{N} / \mathrm{A}$ & $\mathrm{N} / \mathrm{A}$ & $\mathrm{N} / \mathrm{A}$ & $\mathrm{N} / \mathrm{A}$ & $\mathrm{N} / \mathrm{A}$ \\
\hline & $\begin{array}{l}\text { Surgical } \\
\text { complications }\end{array}$ & Thromboembolic events & $0(0)$ & $\mathrm{N} / \mathrm{A}$ & $\mathrm{N} / \mathrm{A}$ & $\mathrm{N} / \mathrm{A}$ & $\mathrm{N} / \mathrm{A}$ & $\mathrm{N} / \mathrm{A}$ & $\mathrm{N} / \mathrm{A}$ \\
\hline & $\begin{array}{l}\text { Surgical } \\
\text { complications }\end{array}$ & Infections & $0(0)$ & $\mathrm{N} / \mathrm{A}$ & $\mathrm{N} / \mathrm{A}$ & $\mathrm{N} / \mathrm{A}$ & $\mathrm{N} / \mathrm{A}$ & $\mathrm{N} / \mathrm{A}$ & $\mathrm{N} / \mathrm{A}$ \\
\hline & $\begin{array}{l}\text { Surgical } \\
\text { complications }\end{array}$ & Wound dehiscence & $0(0)$ & $\mathrm{N} / \mathrm{A}$ & $\mathrm{N} / \mathrm{A}$ & $\mathrm{N} / \mathrm{A}$ & $\mathrm{N} / \mathrm{A}$ & $\mathrm{N} / \mathrm{A}$ & $\mathrm{N} / \mathrm{A}$ \\
\hline
\end{tabular}




\begin{tabular}{|c|c|c|c|c|c|c|c|c|c|}
\hline Comparison & $\begin{array}{l}\text { Outcome } \\
\text { Category }\end{array}$ & Outcome & $\begin{array}{l}\text { N Studies } \\
\text { (Patients) }\end{array}$ & RoB & Consistency & Precision & Directness & SoE & $\begin{array}{l}\text { Conclusions } \\
\text { (Reason, if None) }\end{array}$ \\
\hline & $\begin{array}{l}\text { Surgical } \\
\text { complications }\end{array}$ & Delayed healing & $0(0)$ & $\mathrm{N} / \mathrm{A}$ & $\mathrm{N} / \mathrm{A}$ & $\mathrm{N} / \mathrm{A}$ & $\mathrm{N} / \mathrm{A}$ & $\mathrm{N} / \mathrm{A}$ & N/A \\
\hline & $\begin{array}{l}\text { Surgical } \\
\text { complications }\end{array}$ & Seroma & $0(0)$ & $\mathrm{N} / \mathrm{A}$ & $\mathrm{N} / \mathrm{A}$ & $\mathrm{N} / \mathrm{A}$ & $\mathrm{N} / \mathrm{A}$ & $\mathrm{N} / \mathrm{A}$ & $\mathrm{N} / \mathrm{A}$ \\
\hline \multirow[t]{22}{*}{ DIEP vs. LD } & Clinical & General quality of life & $0(0)$ & $\mathrm{N} / \mathrm{A}$ & $\mathrm{N} / \mathrm{A}$ & $\mathrm{N} / \mathrm{A}$ & $\mathrm{N} / \mathrm{A}$ & $\mathrm{N} / \mathrm{A}$ & $\mathrm{N} / \mathrm{A}$ \\
\hline & Clinical & Physical well-being & $0(0)$ & $\mathrm{N} / \mathrm{A}$ & $\mathrm{N} / \mathrm{A}$ & $\mathrm{N} / \mathrm{A}$ & $\mathrm{N} / \mathrm{A}$ & $\mathrm{N} / \mathrm{A}$ & $\mathrm{N} / \mathrm{A}$ \\
\hline & Clinical & Psychosocial well-being & $0(0)$ & $\mathrm{N} / \mathrm{A}$ & $\mathrm{N} / \mathrm{A}$ & $\mathrm{N} / \mathrm{A}$ & $\mathrm{N} / \mathrm{A}$ & $\mathrm{N} / \mathrm{A}$ & $\mathrm{N} / \mathrm{A}$ \\
\hline & Clinical & Sexual well-being & $0(0)$ & $\mathrm{N} / \mathrm{A}$ & $\mathrm{N} / \mathrm{A}$ & $\mathrm{N} / \mathrm{A}$ & $\mathrm{N} / \mathrm{A}$ & $\mathrm{N} / \mathrm{A}$ & $\mathrm{N} / \mathrm{A}$ \\
\hline & Clinical & Patient satisfaction with breast & $1(229)$ & High & $\mathrm{N} / \mathrm{A}$ & Imprecise & Direct & Insufficient & $\begin{array}{l}\text { None (Sparse } \\
\text { evidence) }\end{array}$ \\
\hline & Clinical & Patient satisfaction with outcome & $1(229)$ & High & $\mathrm{N} / \mathrm{A}$ & Imprecise & Direct & Insufficient & $\begin{array}{l}\text { None (Sparse } \\
\text { evidence) }\end{array}$ \\
\hline & Clinical & Planned surgeries for reconstruction & $0(0)$ & $\mathrm{N} / \mathrm{A}$ & $\mathrm{N} / \mathrm{A}$ & $\mathrm{N} / \mathrm{A}$ & $\mathrm{N} / \mathrm{A}$ & $\mathrm{N} / \mathrm{A}$ & $\mathrm{N} / \mathrm{A}$ \\
\hline & Clinical & Duration of initial hospitalization & $0(0)$ & $\mathrm{N} / \mathrm{A}$ & $\mathrm{N} / \mathrm{A}$ & $\mathrm{N} / \mathrm{A}$ & $\mathrm{N} / \mathrm{A}$ & $\mathrm{N} / \mathrm{A}$ & $\mathrm{N} / \mathrm{A}$ \\
\hline & Clinical & Mortality & $0(0)$ & $\mathrm{N} / \mathrm{A}$ & $\mathrm{N} / \mathrm{A}$ & $\mathrm{N} / \mathrm{A}$ & $\mathrm{N} / \mathrm{A}$ & $\mathrm{N} / \mathrm{A}$ & $\mathrm{N} / \mathrm{A}$ \\
\hline & $\begin{array}{l}\text { Surgical } \\
\text { complications }\end{array}$ & Unplanned repeat hospitalization & $0(0)$ & $\mathrm{N} / \mathrm{A}$ & $\mathrm{N} / \mathrm{A}$ & $\mathrm{N} / \mathrm{A}$ & $\mathrm{N} / \mathrm{A}$ & N/A & N/A \\
\hline & $\begin{array}{l}\text { Surgical } \\
\text { complications }\end{array}$ & Duration of unplanned repeat hospitalization & $0(0)$ & $\mathrm{N} / \mathrm{A}$ & $\mathrm{N} / \mathrm{A}$ & $\mathrm{N} / \mathrm{A}$ & $\mathrm{N} / \mathrm{A}$ & $\mathrm{N} / \mathrm{A}$ & $\mathrm{N} / \mathrm{A}$ \\
\hline & $\begin{array}{l}\text { Surgical } \\
\text { complications }\end{array}$ & Unplanned repeat surgeries for revision & $0(0)$ & $\mathrm{N} / \mathrm{A}$ & $\mathrm{N} / \mathrm{A}$ & $\mathrm{N} / \mathrm{A}$ & $\mathrm{N} / \mathrm{A}$ & $\mathrm{N} / \mathrm{A}$ & $\mathrm{N} / \mathrm{A}$ \\
\hline & $\begin{array}{l}\text { Surgical } \\
\text { complications }\end{array}$ & Unplanned repeat surgeries for complications & $0(0)$ & $\mathrm{N} / \mathrm{A}$ & $\mathrm{N} / \mathrm{A}$ & $\mathrm{N} / \mathrm{A}$ & $\mathrm{N} / \mathrm{A}$ & $\mathrm{N} / \mathrm{A}$ & $\mathrm{N} / \mathrm{A}$ \\
\hline & $\begin{array}{l}\text { Surgical } \\
\text { complications }\end{array}$ & Pain & $0(0)$ & $\mathrm{N} / \mathrm{A}$ & $\mathrm{N} / \mathrm{A}$ & $\mathrm{N} / \mathrm{A}$ & $\mathrm{N} / \mathrm{A}$ & $\mathrm{N} / \mathrm{A}$ & $\mathrm{N} / \mathrm{A}$ \\
\hline & $\begin{array}{l}\text { Surgical } \\
\text { complications }\end{array}$ & Analgesic use & $0(0)$ & $\mathrm{N} / \mathrm{A}$ & $\mathrm{N} / \mathrm{A}$ & $\mathrm{N} / \mathrm{A}$ & $\mathrm{N} / \mathrm{A}$ & $\mathrm{N} / \mathrm{A}$ & $\mathrm{N} / \mathrm{A}$ \\
\hline & $\begin{array}{l}\text { Surgical } \\
\text { complications }\end{array}$ & Necrosis & $0(0)$ & $\mathrm{N} / \mathrm{A}$ & $\mathrm{N} / \mathrm{A}$ & $\mathrm{N} / \mathrm{A}$ & $\mathrm{N} / \mathrm{A}$ & $\mathrm{N} / \mathrm{A}$ & $\mathrm{N} / \mathrm{A}$ \\
\hline & $\begin{array}{l}\text { Surgical } \\
\text { complications }\end{array}$ & Harms to area of flap harvest & $0(0)$ & $\mathrm{N} / \mathrm{A}$ & $\mathrm{N} / \mathrm{A}$ & $\mathrm{N} / \mathrm{A}$ & $\mathrm{N} / \mathrm{A}$ & $\mathrm{N} / \mathrm{A}$ & $\mathrm{N} / \mathrm{A}$ \\
\hline & $\begin{array}{l}\text { Surgical } \\
\text { complications }\end{array}$ & Complications delaying other cancer treatments & $0(0)$ & $\mathrm{N} / \mathrm{A}$ & $\mathrm{N} / \mathrm{A}$ & $\mathrm{N} / \mathrm{A}$ & $\mathrm{N} / \mathrm{A}$ & $\mathrm{N} / \mathrm{A}$ & $\mathrm{N} / \mathrm{A}$ \\
\hline & $\begin{array}{l}\text { Surgical } \\
\text { complications }\end{array}$ & Thromboembolic events & $1(56)$ & High & $\mathrm{N} / \mathrm{A}$ & Imprecise & Direct & Insufficient & $\begin{array}{l}\text { None (Sparse } \\
\text { evidence) }\end{array}$ \\
\hline & $\begin{array}{l}\text { Surgical } \\
\text { complications }\end{array}$ & Infections & $0(0)$ & $\mathrm{N} / \mathrm{A}$ & $\mathrm{N} / \mathrm{A}$ & $\mathrm{N} / \mathrm{A}$ & $\mathrm{N} / \mathrm{A}$ & $\mathrm{N} / \mathrm{A}$ & N/A \\
\hline & $\begin{array}{l}\text { Surgical } \\
\text { complications }\end{array}$ & Wound dehiscence & $0(0)$ & $\mathrm{N} / \mathrm{A}$ & $\mathrm{N} / \mathrm{A}$ & $\mathrm{N} / \mathrm{A}$ & $\mathrm{N} / \mathrm{A}$ & $\mathrm{N} / \mathrm{A}$ & $\mathrm{N} / \mathrm{A}$ \\
\hline & $\begin{array}{l}\text { Surgical } \\
\text { complications }\end{array}$ & Delayed healing & $0(0)$ & $\mathrm{N} / \mathrm{A}$ & $\mathrm{N} / \mathrm{A}$ & $\mathrm{N} / \mathrm{A}$ & $\mathrm{N} / \mathrm{A}$ & $\mathrm{N} / \mathrm{A}$ & $\mathrm{N} / \mathrm{A}$ \\
\hline
\end{tabular}




\begin{tabular}{|c|c|c|c|c|c|c|c|c|c|}
\hline Comparison & $\begin{array}{l}\text { Outcome } \\
\text { Category }\end{array}$ & Outcome & $\begin{array}{l}\text { N Studies } \\
\text { (Patients) }\end{array}$ & RoB & Consistency & Precision & Directness & SoE & $\begin{array}{l}\text { Conclusions } \\
\text { (Reason, if None) }\end{array}$ \\
\hline & $\begin{array}{l}\text { Surgical } \\
\text { complications }\end{array}$ & Seroma & $0(0)$ & $\mathrm{N} / \mathrm{A}$ & N/A & $\mathrm{N} / \mathrm{A}$ & $\mathrm{N} / \mathrm{A}$ & $\mathrm{N} / \mathrm{A}$ & $\mathrm{N} / \mathrm{A}$ \\
\hline \multirow{23}{*}{$\begin{array}{l}\text { TRAM vs. } \\
\text { LTD }\end{array}$} & Clinical & General quality of life & $0(0)$ & $\mathrm{N} / \mathrm{A}$ & N/A & $\mathrm{N} / \mathrm{A}$ & $\mathrm{N} / \mathrm{A}$ & $\mathrm{N} / \mathrm{A}$ & $\mathrm{N} / \mathrm{A}$ \\
\hline & Clinical & Physical well-being & $0(0)$ & $\mathrm{N} / \mathrm{A}$ & N/A & $\mathrm{N} / \mathrm{A}$ & $\mathrm{N} / \mathrm{A}$ & $\mathrm{N} / \mathrm{A}$ & $\mathrm{N} / \mathrm{A}$ \\
\hline & Clinical & Psychosocial well-being & $0(0)$ & $\mathrm{N} / \mathrm{A}$ & N/A & N/A & N/A & N/A & N/A \\
\hline & Clinical & Sexual well-being & $0(0)$ & $\mathrm{N} / \mathrm{A}$ & $\mathrm{N} / \mathrm{A}$ & $\mathrm{N} / \mathrm{A}$ & $\mathrm{N} / \mathrm{A}$ & $\mathrm{N} / \mathrm{A}$ & $\mathrm{N} / \mathrm{A}$ \\
\hline & Clinical & Patient satisfaction with breast & $1(38)$ & High & $\mathrm{N} / \mathrm{A}$ & Unclear & Direct & Insufficient & $\begin{array}{l}\text { None (Sparse } \\
\text { evidence) }\end{array}$ \\
\hline & Clinical & Patient satisfaction with outcome & $0(0)$ & $\mathrm{N} / \mathrm{A}$ & N/A & $\mathrm{N} / \mathrm{A}$ & N/A & $\mathrm{N} / \mathrm{A}$ & N/A \\
\hline & Clinical & Planned surgeries for reconstruction & $0(0)$ & $\mathrm{N} / \mathrm{A}$ & N/A & N/A & $\mathrm{N} / \mathrm{A}$ & N/A & N/A \\
\hline & Clinical & Duration of initial hospitalization & $0(0)$ & $\mathrm{N} / \mathrm{A}$ & N/A & $\mathrm{N} / \mathrm{A}$ & $\mathrm{N} / \mathrm{A}$ & $\mathrm{N} / \mathrm{A}$ & $\mathrm{N} / \mathrm{A}$ \\
\hline & Clinical & Mortality & $1(45)$ & High & N/A & Imprecise & Direct & Insufficient & $\begin{array}{l}\text { None (Sparse } \\
\text { evidence) }\end{array}$ \\
\hline & $\begin{array}{l}\text { Surgical } \\
\text { complications }\end{array}$ & Unplanned repeat hospitalization & $0(0)$ & $\mathrm{N} / \mathrm{A}$ & $\mathrm{N} / \mathrm{A}$ & $\mathrm{N} / \mathrm{A}$ & $\mathrm{N} / \mathrm{A}$ & $\mathrm{N} / \mathrm{A}$ & $\mathrm{N} / \mathrm{A}$ \\
\hline & $\begin{array}{l}\text { Surgical } \\
\text { complications }\end{array}$ & Duration of unplanned repeat hospitalization & $0(0)$ & $\mathrm{N} / \mathrm{A}$ & N/A & N/A & $\mathrm{N} / \mathrm{A}$ & $\mathrm{N} / \mathrm{A}$ & $\mathrm{N} / \mathrm{A}$ \\
\hline & $\begin{array}{l}\text { Surgical } \\
\text { complications }\end{array}$ & Unplanned repeat surgeries for revision & $0(0)$ & $\mathrm{N} / \mathrm{A}$ & $\mathrm{N} / \mathrm{A}$ & $\mathrm{N} / \mathrm{A}$ & $\mathrm{N} / \mathrm{A}$ & $\mathrm{N} / \mathrm{A}$ & $\mathrm{N} / \mathrm{A}$ \\
\hline & $\begin{array}{l}\text { Surgical } \\
\text { complications }\end{array}$ & Unplanned repeat surgeries for complications & $0(0)$ & $\mathrm{N} / \mathrm{A}$ & $\mathrm{N} / \mathrm{A}$ & N/A & $\mathrm{N} / \mathrm{A}$ & $\mathrm{N} / \mathrm{A}$ & $\mathrm{N} / \mathrm{A}$ \\
\hline & $\begin{array}{l}\text { Surgical } \\
\text { complications }\end{array}$ & Pain & $0(0)$ & $\mathrm{N} / \mathrm{A}$ & $\mathrm{N} / \mathrm{A}$ & $\mathrm{N} / \mathrm{A}$ & $\mathrm{N} / \mathrm{A}$ & $\mathrm{N} / \mathrm{A}$ & $\mathrm{N} / \mathrm{A}$ \\
\hline & $\begin{array}{l}\text { Surgical } \\
\text { complications }\end{array}$ & Analgesic use & $0(0)$ & $\mathrm{N} / \mathrm{A}$ & $\mathrm{N} / \mathrm{A}$ & $\mathrm{N} / \mathrm{A}$ & $\mathrm{N} / \mathrm{A}$ & $\mathrm{N} / \mathrm{A}$ & $\mathrm{N} / \mathrm{A}$ \\
\hline & $\begin{array}{l}\text { Surgical } \\
\text { complications }\end{array}$ & Necrosis & $0(0)$ & N/A & $\mathrm{N} / \mathrm{A}$ & $\mathrm{N} / \mathrm{A}$ & $\mathrm{N} / \mathrm{A}$ & $\mathrm{N} / \mathrm{A}$ & $\mathrm{N} / \mathrm{A}$ \\
\hline & $\begin{array}{l}\text { Surgical } \\
\text { complications }\end{array}$ & Harms to area of flap harvest & $0(0)$ & $\mathrm{N} / \mathrm{A}$ & $\mathrm{N} / \mathrm{A}$ & $\mathrm{N} / \mathrm{A}$ & $\mathrm{N} / \mathrm{A}$ & $\mathrm{N} / \mathrm{A}$ & $\mathrm{N} / \mathrm{A}$ \\
\hline & $\begin{array}{l}\text { Surgical } \\
\text { complications }\end{array}$ & Complications delaying other cancer treatments & $0(0)$ & $\mathrm{N} / \mathrm{A}$ & N/A & $\mathrm{N} / \mathrm{A}$ & $\mathrm{N} / \mathrm{A}$ & $\mathrm{N} / \mathrm{A}$ & $\mathrm{N} / \mathrm{A}$ \\
\hline & $\begin{array}{l}\text { Surgical } \\
\text { complications }\end{array}$ & Thromboembolic events & $0(0)$ & $\mathrm{N} / \mathrm{A}$ & N/A & $\mathrm{N} / \mathrm{A}$ & $\mathrm{N} / \mathrm{A}$ & $\mathrm{N} / \mathrm{A}$ & $\mathrm{N} / \mathrm{A}$ \\
\hline & $\begin{array}{l}\text { Surgical } \\
\text { complications }\end{array}$ & Infections & $0(0)$ & $\mathrm{N} / \mathrm{A}$ & $\mathrm{N} / \mathrm{A}$ & $\mathrm{N} / \mathrm{A}$ & $\mathrm{N} / \mathrm{A}$ & $\mathrm{N} / \mathrm{A}$ & $\mathrm{N} / \mathrm{A}$ \\
\hline & $\begin{array}{l}\text { Surgical } \\
\text { complications }\end{array}$ & Wound dehiscence & $0(0)$ & $\mathrm{N} / \mathrm{A}$ & N/A & N/A & $\mathrm{N} / \mathrm{A}$ & $\mathrm{N} / \mathrm{A}$ & $\mathrm{N} / \mathrm{A}$ \\
\hline & $\begin{array}{l}\text { Surgical } \\
\text { complications }\end{array}$ & Delayed healing & $0(0)$ & $\mathrm{N} / \mathrm{A}$ & $\mathrm{N} / \mathrm{A}$ & $\mathrm{N} / \mathrm{A}$ & $\mathrm{N} / \mathrm{A}$ & $\mathrm{N} / \mathrm{A}$ & $\mathrm{N} / \mathrm{A}$ \\
\hline & $\begin{array}{l}\text { Surgical } \\
\text { complications }\end{array}$ & Seroma & $0(0)$ & $\mathrm{N} / \mathrm{A}$ & $\mathrm{N} / \mathrm{A}$ & $\mathrm{N} / \mathrm{A}$ & $\mathrm{N} / \mathrm{A}$ & $\mathrm{N} / \mathrm{A}$ & $\mathrm{N} / \mathrm{A}$ \\
\hline
\end{tabular}




\begin{tabular}{|c|c|c|c|c|c|c|c|c|c|}
\hline Comparison & $\begin{array}{l}\text { Outcome } \\
\text { Category }\end{array}$ & Outcome & $\begin{array}{l}\text { N Studies } \\
\text { (Patients) }\end{array}$ & RoB & Consistency & Precision & Directness & SoE & $\begin{array}{l}\text { Conclusions } \\
\text { (Reason, if None) }\end{array}$ \\
\hline \multirow[t]{23}{*}{ LD vs. LTD } & Clinical & General quality of life & $0(0)$ & $\mathrm{N} / \mathrm{A}$ & $\mathrm{N} / \mathrm{A}$ & $\mathrm{N} / \mathrm{A}$ & $\mathrm{N} / \mathrm{A}$ & $\mathrm{N} / \mathrm{A}$ & $\mathrm{N} / \mathrm{A}$ \\
\hline & Clinical & Physical well-being & $0(0)$ & $\mathrm{N} / \mathrm{A}$ & $\mathrm{N} / \mathrm{A}$ & $\mathrm{N} / \mathrm{A}$ & $\mathrm{N} / \mathrm{A}$ & $\mathrm{N} / \mathrm{A}$ & $\mathrm{N} / \mathrm{A}$ \\
\hline & Clinical & Psychosocial well-being & $0(0)$ & $\mathrm{N} / \mathrm{A}$ & $\mathrm{N} / \mathrm{A}$ & $\mathrm{N} / \mathrm{A}$ & $\mathrm{N} / \mathrm{A}$ & $\mathrm{N} / \mathrm{A}$ & $\mathrm{N} / \mathrm{A}$ \\
\hline & Clinical & Sexual well-being & $0(0)$ & $\mathrm{N} / \mathrm{A}$ & $\mathrm{N} / \mathrm{A}$ & $\mathrm{N} / \mathrm{A}$ & $\mathrm{N} / \mathrm{A}$ & $\mathrm{N} / \mathrm{A}$ & $\mathrm{N} / \mathrm{A}$ \\
\hline & Clinical & Patient satisfaction with breast & $1(35)$ & High & $\mathrm{N} / \mathrm{A}$ & Unclear & Direct & Insufficient & $\begin{array}{l}\text { None (Sparse } \\
\text { evidence) }\end{array}$ \\
\hline & Clinical & Patient satisfaction with outcome & $0(0)$ & $\mathrm{N} / \mathrm{A}$ & $\mathrm{N} / \mathrm{A}$ & $\mathrm{N} / \mathrm{A}$ & $\mathrm{N} / \mathrm{A}$ & $\mathrm{N} / \mathrm{A}$ & $\mathrm{N} / \mathrm{A}$ \\
\hline & Clinical & Planned surgeries for reconstruction & $0(0)$ & $\mathrm{N} / \mathrm{A}$ & $\mathrm{N} / \mathrm{A}$ & $\mathrm{N} / \mathrm{A}$ & $\mathrm{N} / \mathrm{A}$ & $\mathrm{N} / \mathrm{A}$ & $\mathrm{N} / \mathrm{A}$ \\
\hline & Clinical & Duration of initial hospitalization & $0(0)$ & $\mathrm{N} / \mathrm{A}$ & $\mathrm{N} / \mathrm{A}$ & $\mathrm{N} / \mathrm{A}$ & $\mathrm{N} / \mathrm{A}$ & $\mathrm{N} / \mathrm{A}$ & $\mathrm{N} / \mathrm{A}$ \\
\hline & Clinical & Mortality & $1(46)$ & High & $\mathrm{N} / \mathrm{A}$ & Imprecise & Direct & Insufficient & $\begin{array}{l}\text { None (Sparse } \\
\text { evidence) }\end{array}$ \\
\hline & $\begin{array}{l}\text { Surgical } \\
\text { complications }\end{array}$ & Unplanned repeat hospitalization & $0(0)$ & $\mathrm{N} / \mathrm{A}$ & $\mathrm{N} / \mathrm{A}$ & $\mathrm{N} / \mathrm{A}$ & $\mathrm{N} / \mathrm{A}$ & $\mathrm{N} / \mathrm{A}$ & N/A \\
\hline & $\begin{array}{l}\text { Surgical } \\
\text { complications }\end{array}$ & Duration of unplanned repeat hospitalization & $0(0)$ & $\mathrm{N} / \mathrm{A}$ & $\mathrm{N} / \mathrm{A}$ & $\mathrm{N} / \mathrm{A}$ & $\mathrm{N} / \mathrm{A}$ & $\mathrm{N} / \mathrm{A}$ & $\mathrm{N} / \mathrm{A}$ \\
\hline & $\begin{array}{l}\text { Surgical } \\
\text { complications }\end{array}$ & Unplanned repeat surgeries for revision & $0(0)$ & $\mathrm{N} / \mathrm{A}$ & $\mathrm{N} / \mathrm{A}$ & $\mathrm{N} / \mathrm{A}$ & $\mathrm{N} / \mathrm{A}$ & $\mathrm{N} / \mathrm{A}$ & $\mathrm{N} / \mathrm{A}$ \\
\hline & $\begin{array}{l}\text { Surgical } \\
\text { complications }\end{array}$ & Unplanned repeat surgeries for complications & $0(0)$ & $\mathrm{N} / \mathrm{A}$ & $\mathrm{N} / \mathrm{A}$ & $\mathrm{N} / \mathrm{A}$ & $\mathrm{N} / \mathrm{A}$ & $\mathrm{N} / \mathrm{A}$ & $\mathrm{N} / \mathrm{A}$ \\
\hline & $\begin{array}{l}\text { Surgical } \\
\text { complications }\end{array}$ & Pain & $0(0)$ & $\mathrm{N} / \mathrm{A}$ & $\mathrm{N} / \mathrm{A}$ & $\mathrm{N} / \mathrm{A}$ & $\mathrm{N} / \mathrm{A}$ & $\mathrm{N} / \mathrm{A}$ & $\mathrm{N} / \mathrm{A}$ \\
\hline & $\begin{array}{l}\text { Surgical } \\
\text { complications }\end{array}$ & Analgesic use & $0(0)$ & $\mathrm{N} / \mathrm{A}$ & N/A & $\mathrm{N} / \mathrm{A}$ & $\mathrm{N} / \mathrm{A}$ & $\mathrm{N} / \mathrm{A}$ & $\mathrm{N} / \mathrm{A}$ \\
\hline & $\begin{array}{l}\text { Surgical } \\
\text { complications }\end{array}$ & Necrosis & $0(0)$ & $\mathrm{N} / \mathrm{A}$ & $\mathrm{N} / \mathrm{A}$ & $\mathrm{N} / \mathrm{A}$ & $\mathrm{N} / \mathrm{A}$ & $\mathrm{N} / \mathrm{A}$ & $\mathrm{N} / \mathrm{A}$ \\
\hline & $\begin{array}{l}\text { Surgical } \\
\text { complications }\end{array}$ & Harms to area of flap harvest & $0(0)$ & $\mathrm{N} / \mathrm{A}$ & $\mathrm{N} / \mathrm{A}$ & $\mathrm{N} / \mathrm{A}$ & $\mathrm{N} / \mathrm{A}$ & $\mathrm{N} / \mathrm{A}$ & $\mathrm{N} / \mathrm{A}$ \\
\hline & $\begin{array}{l}\text { Surgical } \\
\text { complications }\end{array}$ & Complications delaying other cancer treatments & $0(0)$ & $\mathrm{N} / \mathrm{A}$ & $\mathrm{N} / \mathrm{A}$ & $\mathrm{N} / \mathrm{A}$ & $\mathrm{N} / \mathrm{A}$ & $\mathrm{N} / \mathrm{A}$ & $\mathrm{N} / \mathrm{A}$ \\
\hline & $\begin{array}{l}\text { Surgical } \\
\text { complications }\end{array}$ & Thromboembolic events & $0(0)$ & $\mathrm{N} / \mathrm{A}$ & $\mathrm{N} / \mathrm{A}$ & $\mathrm{N} / \mathrm{A}$ & $\mathrm{N} / \mathrm{A}$ & $\mathrm{N} / \mathrm{A}$ & $\mathrm{N} / \mathrm{A}$ \\
\hline & $\begin{array}{l}\text { Surgical } \\
\text { complications }\end{array}$ & Infections & $0(0)$ & $\mathrm{N} / \mathrm{A}$ & N/A & $\mathrm{N} / \mathrm{A}$ & $\mathrm{N} / \mathrm{A}$ & $\mathrm{N} / \mathrm{A}$ & $\mathrm{N} / \mathrm{A}$ \\
\hline & $\begin{array}{l}\text { Surgical } \\
\text { complications }\end{array}$ & Wound dehiscence & $0(0)$ & $\mathrm{N} / \mathrm{A}$ & $\mathrm{N} / \mathrm{A}$ & $\mathrm{N} / \mathrm{A}$ & $\mathrm{N} / \mathrm{A}$ & $\mathrm{N} / \mathrm{A}$ & $\mathrm{N} / \mathrm{A}$ \\
\hline & $\begin{array}{l}\text { Surgical } \\
\text { complications }\end{array}$ & Delayed healing & $0(0)$ & $\mathrm{N} / \mathrm{A}$ & $\mathrm{N} / \mathrm{A}$ & $\mathrm{N} / \mathrm{A}$ & $\mathrm{N} / \mathrm{A}$ & $\mathrm{N} / \mathrm{A}$ & $\mathrm{N} / \mathrm{A}$ \\
\hline & $\begin{array}{l}\text { Surgical } \\
\text { complications }\end{array}$ & Seroma & $0(0)$ & $\mathrm{N} / \mathrm{A}$ & $\mathrm{N} / \mathrm{A}$ & $\mathrm{N} / \mathrm{A}$ & $\mathrm{N} / \mathrm{A}$ & $\mathrm{N} / \mathrm{A}$ & $\mathrm{N} / \mathrm{A}$ \\
\hline
\end{tabular}

Abbreviations: adj $=$ adjusted, $\mathrm{AR}=$ autologous reconstruction, $\mathrm{CI}=$ confidence interval, $\mathrm{DIEP}=$ deep inferior epigastric perforator, $\mathrm{LD}=$ latissimus dorsi, $\mathrm{LTD}=$ lateral

thoracodorsal flap, $\mathrm{OR}=$ odds ratio, N/A = not applicable, RoB $=$ risk of bias, SIEA = superficial inferior epigastric artery, SoE $=$ strength of evidence, TAP $=$ thoracodorsal artery perforator, TRAM $=$ transverse rectus abdominis myocutaneous. 
Colors: Header rows are shaded orange. Rows for every alternate comparison are shaded blue. The colors do not add unique information. 


\section{Appendix H. Appendix References}

1. Abedi N, Ho AL, Knox A, et al. Predictors of Mastectomy Flap Necrosis in Patients Undergoing Immediate Breast Reconstruction: A Review of 718 Patients. Ann Plast Surg. 2016 Jun;76(6):629-34. doi: 10.1097/sap.0000000000000262. PMID: 25003437.

2. Acosta R, Smit JM, Audolfsson T, et al. A clinical review of 9 years of free perforator flap breast reconstructions: an analysis of 675 flaps and the influence of new techniques on clinical practice. J Reconstr Microsurg. 2011 Feb;27(2):91-8. doi: 10.1055/s-00301267835. PMID: 21046538.

3. Albornoz CR, Cordeiro PG, Hishon L, et al. A nationwide analysis of the relationship between hospital volume and outcome for autologous breast reconstruction. Plast Reconstr Surg. 2013 Aug;132(2):192e-200e. doi: 10.1097/PRS.0b013e31829586c1. PMID: 23897346.

4. Andree C, Munder BI, Seidenstuecker K, et al. Skin-sparing mastectomy and immediate reconstruction with DIEP flap after breast-conserving therapy. Med Sci Monit. 2012 Dec;18(12):Cr716-20. doi: 10.12659/msm.883598. PMID: 23197233.

5. Antony AK, McCarthy C, Disa JJ, et al. Bilateral implant breast reconstruction: outcomes, predictors, and matched cohort analysis in 730 2-stage breast reconstructions over 10 years. Ann Plast Surg. 2014;72(6):625-30. doi: 10.1097/SAP.0b013e31826af03e. PMID: 24135689.

6. Atisha D, Alderman AK, Lowery JC, et al. Prospective analysis of long-term psychosocial outcomes in breast reconstruction: two-year postoperative results from the Michigan Breast
Reconstruction Outcomes Study. Ann Surg. 2008 Jun;247(6):1019-28. doi: 10.1097/SLA.0b013e3181728a5c. PMID: 18520230.

7. Avila A, Bartholomew AJ, Sosin M, et al. Acute Postoperative Complications in Prepectoral versus Subpectoral Reconstruction following NippleSparing Mastectomy. Plast Reconstr Surg. 2020 Dec;146(6):715e-20e. doi: 10.1097/prs.0000000000007326. PMID: 33234947.

8. Banuelos J, Taslakian EN, Nguyen MT, et al. The American College of Surgeons National Quality Improvement Program Incompletely Captures Implant-Based Breast Reconstruction Complications. Ann Plast Surg. 2020 Mar;84(3):271-5. doi: 10.1097/sap.0000000000002051. PMID: 31663932.

9. Baumann DP, Lin HY, Chevray PM. Perforator number predicts fat necrosis in a prospective analysis of breast reconstruction with free TRAM, DIEP, and SIEA flaps. Plast Reconstr Surg. 2010 May;125(5):1335-41. doi: 10.1097/PRS.0b013e3181d4fb4a. PMID: 20440154.

10. Bennett KG, Qi J, Kim HM, et al. Comparison of 2-Year Complication Rates Among Common Techniques for Postmastectomy Breast Reconstruction. JAMA Surg. 2018 Oct 1;153(10):901-8. doi: 10.1001/jamasurg.2018.1687. PMID: 29926077.

11. Beugels J, Bod L, van Kuijk SMJ, et al. Complications following immediate compared to delayed deep inferior epigastric artery perforator flap breast reconstructions. Breast Cancer Res Treat. 2018 Jun;169(2):349-57. doi: 10.1007/s10549-018-4695-0. PMID: 29399731. 
12. Beugels J, Kool M, Hoekstra LT, et al. Quality of Life of Patients After Immediate or Delayed Autologous Breast Reconstruction: A Multicenter Study. Ann Plast Surg. 2018

Nov;81(5):523-7. doi: 10.1097/sap.0000000000001618. PMID: 30247195.

13. Bozzuto LM, Bartholomew AJ, Tung S, et al. Decreased postoperative pain and opioid use following prepectoral versus subpectoral breast reconstruction after mastectomy: A retrospective cohort study: Pain after pre- versus subpectoral reconstruction. J Plast Reconstr Aesthet Surg. 2020 Dec 13. doi:

10.1016/j.bjps.2020.12.009. PMID: 33451949.

14. Brandberg Y, Malm M, Blomqvist L. A prospective and randomized study, "SVEA," comparing effects of three methods for delayed breast reconstruction on quality of life, patient-defined problem areas of life, and cosmetic result. Plast Reconstr Surg. 2000 Jan;105(1):66-74; discussion 5-6. doi: 10.1097/00006534200001000-00011. PMID: 10626972.

15. Brandberg Y, Malm M, Rutqvist LE, et al. A prospective randomised study (named SVEA) of three methods of delayed breast reconstruction. Study design, patients' preoperative problems and expectations. Scand J Plast Reconstr Surg Hand Surg. 1999 Jun;33(2):209-16. doi: 10.1080/02844319950159479. PMID: 10450579.

16. Brooke S, Mesa J, Uluer M, et al. Complications in tissue expander breast reconstruction: a comparison of AlloDerm, DermaMatrix, and FlexHD acellular inferior pole dermal slings. Ann Plast Surg. 2012 Oct;69(4):347-9. doi: 10.1097/SAP.0b013e31824b3d97. PMID: 22868313.
17. Brooks S, Djohan R, Tendulkar R, et al. Risk factors for complications of radiation therapy on tissue expander breast reconstructions. Breast J. 2012 Jan-Feb;18(1):28-34. doi: 10.1111/j.1524-4741.2011.01182.x. PMID: 22098451.

18. Brorson F, Thorarinsson A, Kölby L, et al. Early complications in delayed breast reconstruction: A prospective, randomized study comparing different reconstructive methods in radiated and non-radiated patients. Eur J Surg Oncol. 2020 Dec;46(12):2208-17. doi: 10.1016/j.ejso.2020.07.010. PMID: 32807615 .

19. Carramaschi F, Bricout N, Servant JM, et al. [Breast reconstruction after mastectomy. Critical analysis of 240 cases]. AMB Rev Assoc Med Bras. 1989 Jan-Feb;35(1):15-9. PMID: 2602589.

20. Cattelani L, Polotto S, Arcuri MF, et al. One-Step Prepectoral Breast Reconstruction With Dermal MatrixCovered Implant Compared to Submuscular Implantation: Functional and Cost Evaluation. Clin Breast Cancer. 2018 Aug;18(4):e703-e11. doi: 10.1016/j.clbc.2017.11.015. PMID: 29275104.

21. Chang DW, Wang B, Robb GL, et al. Effect of obesity on flap and donor-site complications in free transverse rectus abdominis myocutaneous flap breast reconstruction. Plast Reconstr Surg. 2000 Apr;105(5):1640-8. doi: 10.1097/00006534-200004050-00007. PMID: 10809092.

22. Chang EI, Chang EI, Soto-Miranda MA, et al. Demystifying the use of internal mammary vessels as recipient vessels in free flap breast reconstruction. Plast Reconstr Surg. 2013 Oct;132(4):763-8. doi: 
10.1097/PRS.0b013e31829fe321. PMID: 24076668.

23. Chang EI, Chang EI, Soto-Miranda MA, et al. Comprehensive Evaluation of Risk Factors and Management of Impending Flap Loss in 2138 Breast Free Flaps. Ann Plast Surg. 2016 Jan;77(1):67-71. doi: 10.1097/sap.0000000000000263. PMID: 25003429.

24. Chang EI, Chang EI, Soto-Miranda MA, et al. Comprehensive analysis of donor-site morbidity in abdominally based free flap breast reconstruction. Plast Reconstr Surg. 2013 Dec;132(6):1383-91. doi: 10.1097/PRS.0b013e3182a805a3. PMID: 24005365.

25. Chang EI, Vaca L, DaLio AL, et al. Assessment of advanced age as a risk factor in microvascular breast reconstruction. Ann Plast Surg. 2011 Sep;67(3):255-9. doi: 10.1097/SAP.0b013e3181f9b20c. PMID: 21407063.

26. Chen AD, Chi D, Wu WW, et al. The Influence of Connective Tissue Disease in Breast Reconstruction: A National Database Analysis. Ann Plast Surg. 2018 Apr;80(4 Suppl 4):S182-s8. doi: $10.1097 /$ sap.0000000000001387. PMID: 29596085.

27. Chen CF, Lin SF, Hung CF, et al. Risk of infection is associated more with drain duration than daily drainage volume in prosthesis-based breast reconstruction: A cohort study. Medicine (Baltimore). 2016 Dec;95(49):e5605. doi: 10.1097/md.0000000000005605. PMID: 27930584.

28. Chen Y, Chen J, Chen J, et al. [Current trends of breast reconstruction after mastectomy for breast cancer patients in China: a survey report]. Zhonghua
Zhong Liu Za Zhi. 2014

Nov;36(11):851-7. PMID: 25620484.

29. Chetta MD, Aliu O, Zhong L, et al. Reconstruction of the Irradiated Breast: A National Claims-Based Assessment of Postoperative Morbidity. Plast Reconstr Surg. 2017 Apr;139(4):78392. doi:

10.1097/prs.0000000000003168. PMID: 28002254.

30. Chun YS, Verma K, Rosen H, et al. Implant-based breast reconstruction using acellular dermal matrix and the risk of postoperative complications. Plast Reconstr Surg. 2010 Feb;125(2):429-36. doi: 10.1097/PRS.0b013e3181c82d90. PMID: 20124828.

31. Clarke-Pearson EM, Lin AM, Hertl C, et al. Revisions in Implant-Based Breast Reconstruction: How Does Direct-toImplant Measure Up? Plast Reconstr Surg. 2016 Jun;137(6):1690-9. doi: 10.1097/prs.0000000000002173. PMID: 27219225.

32. Cleveland EC, Fischer JP, Nelson JA, et al. Optimizing the fascial closure: an analysis of 1261 abdominally based free flap reconstructions. Ann Plast Surg. 2013 Sep;71(3):255-60. doi: 10.1097/SAP.0b013e318286380e. PMID: 23945529.

33. Cohen WA, Ballard TN, Hamill JB, et al. Understanding and Optimizing the Patient Experience in Breast Reconstruction. Ann Plast Surg. 2016 Aug;77(2):237-41. doi: 10.1097/sap.0000000000000550. PMID: 26101986.

34. Collier W, Scheefer Van Boerum M, Kim J, et al. Are 30-Day Outcomes Enough? Late Infectious Readmissions following Prosthetic-Based Breast Reconstruction. Plast Reconstr Surg. 2019 Sep;144(3):360e-8e. doi: 
10.1097/prs.0000000000005903.

PMID: 31461001.

35. Cordeiro PG, Albornoz CR, McCormick B, et al. The impact of postmastectomy radiotherapy on twostage implant breast reconstruction: an analysis of long-term surgical outcomes, aesthetic results, and satisfaction over 13 years. Plast Reconstr Surg. 2014 Oct;134(4):58895. doi:

$10.1097 /$ prs.0000000000000523. PMID: 25357021.

36. Cordeiro PG, Albornoz CR, McCormick B, et al. What Is the Optimum Timing of Postmastectomy Radiotherapy in Two-Stage Prosthetic Reconstruction: Radiation to the Tissue Expander or Permanent Implant? Plast Reconstr Surg. 2015 Jun;135(6):150917. doi: $10.1097 /$ prs.0000000000001278. PMID: 25742523.

37. Cordeiro PG, McCarthy CM. A single surgeon's 12-year experience with tissue expander/implant breast reconstruction: part I. A prospective analysis of early complications. Plast Reconstr Surg. 2006 Sep 15;118(4):825-31. doi: 10.1097/01.prs.0000232362.82402.e8. PMID: 16980842.

38. Cordeiro PG, McGuire P, Murphy DK. Natrelle 410 Extra-Full Projection Silicone Breast Implants: 2-Year Results from Two Prospective Studies. Plast Reconstr Surg. 2015 Oct;136(4):638-46. doi: 10.1097 /prs.0000000000001636. PMID: 26090764.

39. Cordeiro PG, Snell L, Heerdt A, et al. Immediate tissue expander/implast breast reconstruction after salvage mastectomy for cancer recurrence following lumpectomy/irradiation. Plast Reconstr Surg. 2012 Feb;129(2):341-
50. doi:

10.1097/PRS.0b013e318205f203.

PMID: 22286416.

40. Coroneos CJ, Selber JC, Offodile AC, 2nd, et al. US FDA Breast Implant Postapproval Studies: Long-term Outcomes in 99,993 Patients. Ann Surg. 2019 Jan;269(1):30-6. doi: 10.1097/sla.0000000000002990. PMID: 30222598.

41. Craig ES, Clemens MW, Koshy JC, et al. Outcomes of Acellular Dermal Matrix for Immediate Tissue Expander Reconstruction with Radiotherapy: A Retrospective Cohort Study. Aesthet Surg J. 2019 Feb 15;39(3):279-88. doi: 10.1093/asj/sjy127. PMID: 29800083.

42. Daly LT, Doval AF, Lin SJ, et al. Role of CTA in Women with Abdominal Scars Undergoing DIEP Breast Reconstruction: Review of 1,187 Flaps. J Reconstr Microsurg. 2020 May;36(4):294-300. doi: 10.1055/s0039-1701040. PMID: 31994156.

43. Dauplat J, Thivat E, Rouanet P, et al. Risk Factors Associated With Complications After Unilateral Immediate Breast Reconstruction: A French Prospective Multicenter Study. In Vivo. 2021 Mar-Apr;35(2):937-45. doi: 10.21873/invivo.12334. PMID: 33622886.

44. Davila AA, Seth AK, Wang E, et al. Human Acellular Dermis versus Submuscular Tissue Expander Breast Reconstruction: A Multivariate Analysis of Short-Term Complications. Arch Plast Surg. 2013 Jan;40(1):19-27. doi: 10.5999/aps.2013.40.1.19. PMID: 23362476.

45. de Araujo TB, Jue Xu M, Susarla SM, et al. Impact of Prior Unilateral Chest Wall Radiotherapy on Outcomes in Bilateral Breast Reconstruction. Plast Reconstr Surg. 2016 Oct;138(4):575e80e. doi: 
10.1097/prs.0000000000002532.

PMID: 27673527.

46. Eltahir Y, Werners LL, Dreise MM, et al. Which breast is the best? Successful autologous or alloplastic breast reconstruction: patient-reported qualityof-life outcomes. Plast Reconstr Surg.

2015 Jan;135(1):43-50. doi:

$10.1097 /$ prs.0000000000000804.

PMID: 25539295.

47. Enajat M, Rozen WM, Whitaker IS, et al. A single center comparison of one versus two venous anastomoses in 564 consecutive DIEP flaps: investigating the effect on venous congestion and flap survival. Microsurgery. 2010;30(3):185-91. doi: 10.1002/micr.20712. PMID: 19790180.

48. Erdmann-Sager J, Wilkins EG, Pusic AL, et al. Complications and PatientReported Outcomes after Abdominally Based Breast Reconstruction: Results of the Mastectomy Reconstruction Outcomes Consortium Study. Plast Reconstr Surg. 2018 Feb;141(2):27181. doi:

10.1097/prs.0000000000004016. PMID: 29019862.

49. Eriksson M, Anveden L, Celebioglu F, et al. Radiotherapy in implant-based immediate breast reconstruction: risk factors, surgical outcomes, and patientreported outcome measures in a large Swedish multicenter cohort. Breast Cancer Res Treat. 2013 Dec;142(3):591-601. doi: 10.1007/s10549-013-2770-0. PMID: 24258257.

50. Fischer JP, Fox JP, Nelson JA, et al. A Longitudinal Assessment of Outcomes and Healthcare Resource Utilization After Immediate Breast ReconstructionComparing Implant- and Autologousbased Breast Reconstruction. Ann Surg. 2015 Oct;262(4):692-9. doi: 10.1097/sla.0000000000001457.

PMID: 26366550.

51. Fischer JP, Nelson JA, Cleveland E, et al. Breast reconstruction modality outcome study: a comparison of expander/implants and free flaps in select patients. Plast Reconstr Surg. 2013 May;131(5):928-34. doi:

10.1097/PRS.0b013e3182865977. PMID: 23629074.

52. Fischer JP, Wes AM, Nelson JA, et al. Propensity-matched, longitudinal outcomes analysis of complications and cost: comparing abdominal free flaps and implant-based breast reconstruction. J Am Coll Surg. 2014 Aug;219(2):303-12. doi: 10.1016/j.jamcollsurg.2014.02.028. PMID: 24916480.

53. Fitzgerald O'Connor E, Rozen WM, Chowdhry M, et al. The microvascular anastomotic coupler for venous anastomoses in free flap breast reconstruction improves outcomes. Gland Surg. 2016 Apr;5(2):88-92. doi: 10.3978/j.issn.2227-684X.2015.05.14. PMID: 27047776.

54. Gabriel A, Sigalove S, Storm-Dickerson TL, et al. Dual-Plane versus Prepectoral Breast Reconstruction in High-Body Mass Index Patients. Plast Reconstr Surg. 2020 Jun;145(6):1357-65. doi: 10.1097/prs.0000000000006840. PMID: 32195862.

55. Ganesh Kumar N, Berlin NL, Kim HM, et al. Development of an evidencebased approach to the use of acellular dermal matrix in immediate expanderimplant-based breast reconstruction. J Plast Reconstr Aesthet Surg. 2021 Jan;74(1):30-40. doi: 10.1016/j.bjps.2020.10.005. PMID: 33172826.

56. Garbay JR, Rietjens M, Petit JY. [Esthetic results of breast reconstruction after amputation for cancer. 323 cases]. 
J Gynecol Obstet Biol Reprod (Paris). 1992;21(4):405-12. PMID: 1624727.

57. Gart MS, Smetona JT, Hanwright PJ, et al. Autologous options for postmastectomy breast reconstruction: a comparison of outcomes based on the American College of Surgeons National Surgical Quality Improvement Program. J Am Coll Surg. 2013 Feb;216(2):229-38. doi: 10.1016/j.jamcollsurg.2012.11.003. PMID: 23211118.

58. Garvey PB, Villa MT, Rozanski AT, et al. The advantages of free abdominalbased flaps over implants for breast reconstruction in obese patients. Plast Reconstr Surg. 2012 Nov;130(5):9911000. doi:

10.1097/PRS.0b013e318267efc5. PMID: 23096600.

59. Gfrerer L, Mattos D, Mastroianni M, et al. Assessment of patient factors, surgeons, and surgeon teams in immediate implant-based breast reconstruction outcomes. Plast Reconstr Surg. 2015 Feb;135(2):245e-52e. doi: $10.1097 /$ prs.0000000000000912. PMID: 25626807.

60. Gill PS, Hunt JP, Guerra AB, et al. A 10-year retrospective review of 758 DIEP flaps for breast reconstruction. Plast Reconstr Surg. 2004 Apr 1;113(4):1153-60. doi: 10.1097/01.prs.0000110328.47206.50. PMID: 15083015.

61. Goodwin SJ, McCarthy CM, Pusic AL, et al. Complications in smokers after postmastectomy tissue expander/implant breast reconstruction. Ann Plast Surg. 2005 Jul;55(1):16-9; discussion 9-20. doi: 10.1097/01.sap.0000168282.81348.b3. PMID: 15985785.

62. Ha JH, Hong KY, Lee HB, et al. Oncologic outcomes after immediate breast reconstruction following mastectomy: comparison of implant and flap using propensity score matching. BMC Cancer. 2020 Jan 30;20(1):78. doi: 10.1186/s12885-020-6568-2. PMID: 32000718.

63. Haddock NT, Cho MJ, Teotia SS. Comparative Analysis of Single versus Stacked Free Flap Breast Reconstruction: A Single-Center Experience. Plast Reconstr Surg. 2019 Sep;144(3):369e-77e. doi: 10.1097/prs.0000000000005906. PMID: 31461004.

64. Haddock NT, Culver AJ, Teotia SS. Abdominal weakness, bulge, or hernia after DIEP flaps: An algorithm of management, prevention, and surgical repair with classification. J Plast Reconstr Aesthet Surg. 2020 Dec 26. doi: 10.1016/j.bjps.2020.12.044. PMID: 33487570 .

65. Hamdi M, Andrades P, Thiessen F, et al. Is a second free flap still an option in a failed free flap breast reconstruction? Plast Reconstr Surg. 2010 Aug;126(2):375-84. doi: 10.1097/PRS.0b013e3181de22f3. PMID: 20679823.

66. Hamdi M, Casaer B, Andrades P, et al. Salvage (tertiary) breast reconstruction after implant failure. J Plast Reconstr Aesthet Surg. 2011 Mar;64(3):353-9. doi: 10.1016/j.bjps.2010.05.019. PMID: 20576480.

67. Hangge PT, Jogerst K, Mohsen A, et al. Making an informed choice: Which breast reconstruction type has the lowest complication rate? Am J Surg. 2019 Dec;218(6):1040-5. doi: 10.1016/j.amjsurg.2019.09.033. PMID: 31606126.

68. Hansen N, Espino S, Blough JT, et al. Evaluating Mastectomy Skin Flap Necrosis in the Extended Breast Reconstruction Risk Assessment Score for 1-Year Prediction of Prosthetic 
Reconstruction Outcomes. J Am Coll

Surg. 2018 Jul;227(1):96-104. doi:

10.1016/j.jamcollsurg.2018.05.003.

PMID: 29778821.

69. Hembd AS, Yan J, Zhu H, et al. Intraoperative Assessment of DIEP Flap Breast Reconstruction Using Indocyanine Green Angiography: Reduction of Fat Necrosis, Resection Volumes, and Postoperative

Surveillance. Plast Reconstr Surg. 2020

Jul;146(1):1e-10e. doi: 10.1097/prs.0000000000006888. PMID: 32590635.

70. Heo JW, Park SO, Jin US. Donor-site morbidities in 615 patients after breast reconstruction using a free musclesparing type I transverse rectus abdominis myocutaneous flap: a single surgeon experience. J Plast Surg Hand Surg. 2018 Dec;52(6):325-32. doi: 10.1080/2000656x.2018.1493389. PMID: 30039735.

71. Hirsch EM, Seth AK, Kim JY, et al. Analysis of risk factors for complications in expander/implant breast reconstruction by stage of reconstruction. Plast Reconstr Surg. 2014 Nov;134(5):692e-9e. doi: $10.1097 /$ prs.0000000000000607. PMID: 25347643.

72. Hunsicker LM, Ashikari AY, Berry C, et al. Short-Term Complications Associated With Acellular Dermal Matrix-Assisted Direct-to-Implant Breast Reconstruction. Ann Plast Surg. 2017 Jan;78(1):35-40. doi: 10.1097/sap.0000000000000742. PMID: 26849284.

73. Huo J, Smith BD, Giordano SH, et al. A comparison of patient-centered economic and clinical outcomes of post-mastectomy breast reconstruction between obese and non-obese patients. Breast. 2016 Dec;30:118-24. doi: 10.1016/j.breast.2016.09.004. PMID: 27697676.

74. Ibrahim AM, Shuster M, Koolen PG, et al. Analysis of the National Surgical Quality Improvement Program database in 19,100 patients undergoing implantbased breast reconstruction: complication rates with acellular dermal matrix. Plast Reconstr Surg. 2013 Nov;132(5):1057-66. doi: 10.1097/PRS.0b013e3182a3beec. PMID: 24165587.

75. Israeli R, Funk S, Reaven NL. Comparative analysis of 18-month outcomes and costs of breast reconstruction flap procedures. Plast Reconstr Surg. 2014 Mar;133(3):471-9. doi: $10.1097 /$ prs.0000000000000064. PMID: 24572840.

76. Jiang YZ, Liu YR, Yu KD, et al. Immediate postmastectomy breast reconstruction showed limited advantage in patient survival after stratifying by family income. PLoS One. 2013;8(12):e82807. doi: 10.1371/journal.pone.0082807. PMID: 24349366.

77. Jo T, Hur J, Min K, et al. Immediate breast reconstruction after salvage mastectomy: Case control outcome comparisons of DIEP flap and DTI reconstruction. J Plast Reconstr Aesthet Surg. 2020 Dec 9. doi: 10.1016/j.bjps.2020.11.034. PMID: 33386262.

78. Kanuri A, Liu AS, Guo L. Whom should we SPY? A cost analysis of laser-assisted indocyanine green angiography in prevention of mastectomy skin flap necrosis during prosthesis-based breast reconstruction. Plast Reconstr Surg. 2014 Apr;133(4):448e-54e. doi: 10.1097/prs.0000000000000025. PMID: 24675199. 
79. Kato H, Nakagami G, Iwahira Y, et al. Risk factors and risk scoring tool for infection during tissue expansion in tissue expander and implant breast reconstruction. Breast J. 2013 NovDec;19(6):618-26. doi: 10.1111/tbj.12175. PMID: 24011080.

80. Kim EK, Eom JS, Ahn SH, et al. Evolution of the pedicled TRAM flap: a prospective study of 500 consecutive cases by a single surgeon in Asian patients. Ann Plast Surg. 2009 Oct;63(4):378-82. doi: 10.1097/SAP.0b013e3181951708. PMID: 19745709.

81. Kim EK, Eom JS, Ahn SH, et al. The efficacy of prophylactic low-molecularweight heparin to prevent pulmonary thromboembolism in immediate breast reconstruction using the TRAM flap. Plast Reconstr Surg. 2009 Jan;123(1):912. doi: 10.1097/PRS.0b013e3181904be7. PMID: 19116509.

82. Kim HJ, Park EH, Lim WS, et al. Nipple areola skin-sparing mastectomy with immediate transverse rectus abdominis musculocutaneous flap reconstruction is an oncologically safe procedure: a single center study. Ann Surg. 2010 Mar;251(3):493-8. doi: 10.1097/SLA.0b013e3181c5dc4e. PMID: 20134317.

83. Kim JH, Hong SE. A Comparative Analysis between Subpectoral versus Prepectoral Single Stage Direct-toImplant Breast Reconstruction. Medicina (Kaunas). 2020 Oct 13;56(10). doi: 10.3390/medicina56100537. PMID: 33066236.

84. Knox AD, Ho AL, Leung L, et al. Comparison of Outcomes following Autologous Breast Reconstruction Using the DIEP and Pedicled TRAM Flaps: A 12-Year Clinical Retrospective
Study and Literature Review. Plast Reconstr Surg. 2016 Jul;138(1):16-28. doi: $10.1097 /$ prs.0000000000001747. PMID: 26267400.

85. Kouwenberg CAE, de Ligt KM, Kranenburg LW, et al. Long-Term Health-Related Quality of Life after Four Common Surgical Treatment Options for Breast Cancer and the Effect of Complications: A Retrospective Patient-Reported Survey among 1871 Patients. Plast Reconstr Surg. 2020 Jul;146(1):1-13. doi: 10.1097/prs.0000000000006887. PMID: 32590633.

86. Kouwenberg CAE, Kranenburg LW, Visser MS, et al. "The validity of the EQ-5D-5L in measuring quality of life benefits of breast reconstruction". J Plast Reconstr Aesthet Surg. 2019 Jan;72(1):52-61. doi: 10.1016/j.bjps.2018.08.023. PMID: 30270015.

87. Kraenzlin F, Darrach H, Khavanin N, et al. Tissue Expander-Based Breast Reconstruction in the Prepectoral Versus Subpectoral Plane: An Analysis of Short-Term Outcomes. Ann Plast Surg. 2021 Jan;86(1):19-23. doi: 10.1097/sap.0000000000002415. PMID: 32568752.

88. Kroll SS. Fat necrosis in free transverse rectus abdominis myocutaneous and deep inferior epigastric perforator flaps. Plast Reconstr Surg. 2000

Sep;106(3):576-83. doi: 10.1097/00006534-200009030-00008. PMID: 10987463.

89. Kulkarni AR, Pusic AL, Hamill JB, et al. Factors Associated with Acute Postoperative Pain Following Breast Reconstruction. JPRAS Open. 2017 Mar;11:1-13. doi: 10.1016/j.jpra.2016.08.005. PMID: 28713853. 
90. Langer S, Munder B, Seidenstuecker K, et al. Development of a surgical algorithm and optimized management of complications - based on a review of 706 abdominal free flaps for breast reconstruction. Med Sci Monit. 2010 Nov;16(11):Cr518-22. PMID: 20980954.

91. Lantieri L, Hivelin M, Benjoar MD, et al. [Setting of a breast autologous microsurgical reconstructive surgery evolution in 20 years and review of 1138 cases]. Ann Chir Plast Esthet. 2015 Dec;60(6):484-9. doi: 10.1016/j.anplas.2015.06.009. PMID: 26238173.

92. Laporta R, Sorotos M, Longo B, et al. Breast Reconstruction in Elderly Patients: Risk Factors, Clinical Outcomes, and Aesthetic Results. J Reconstr Microsurg. 2017 May;33(4):257-67. doi: 10.1055/s0036-1597822. PMID: 28061518.

93. Law TY, Moeller E, Hubbard ZS, et al. Preoperative Hypoglycemia and Hyperglycemia Are Related to Postoperative Infection Rates in Implant-Based Breast Reconstruction. J Surg Res. 2018 Dec;232:437-41. doi: 10.1016/j.jss.2018.06.068. PMID: 30463754.

94. Le GM, O'Malley CD, Glaser SL, et al. Breast implants following mastectomy in women with early-stage breast cancer: prevalence and impact on survival. Breast Cancer Res. 2005;7(2):R184-93. doi: 10.1186/bcr974. PMID: 15743498.

95. Lee JS, Park E, Lee JH, et al. A prospective comparison study of early functional outcomes after implantbased breast reconstruction: subpectoral versus prepectoral technique. Ann Palliat Med. 2021 Mar;10(3):2520-9. doi: 10.21037/apm-20-1550. PMID: 33691448 .
96. Lee KT, Bae J, Jeon BJ, et al. Adjuvant Chemotherapy in Two-Stage Tissue Expander/Implant Breast Reconstruction: Does it Affect Final Outcomes? Ann Surg Oncol. 2021 Apr;28(4):2191-8. doi: 10.1245/s10434-020-09177-z. PMID: 32974692.

97. Lee KT, Bang SI, Pyon JK, et al. Method of breast reconstruction and the development of lymphoedema. Br J Surg. 2017 Feb;104(3):230-7. doi: 10.1002/bjs.10397. PMID: 27861758.

98. Lee KT, Kim S, Jeon BJ, et al. Association of the Implant Surface Texture Used in Reconstruction With Breast Cancer Recurrence. JAMA Surg. 2020 Dec 1;155(12):1132-40. doi: 10.1001/jamasurg.2020.4124. PMID: 33026424.

99. Lee KT, Lee H, Jeon BJ, et al. Impact of overweight/obesity on the development of hematoma following tissue expander-based breast reconstruction. J Plast Reconstr Aesthet Surg. 2020;74(2):307-15.

100. Lee KT, Pyon JK, Bang SI, et al. Does the reconstruction method influence development of mastectomy flap complications in nipple-sparing mastectomy? J Plast Reconstr Aesthet Surg. 2013 Nov;66(11):1543-50. doi: 10.1016/j.bjps.2013.06.032. PMID: 23834909.

101. Lei C, Xu L, Xu F, et al. Patient satisfaction in one-stage immediate breast reconstruction after mastectomy: A multi-center comparative patient evaluation of prosthesis, LDMF, and TRAM techniques. Medicine (Baltimore). 2020 May 29;99(22):e19991. doi: 10.1097/md.0000000000019991. PMID: 32481367.

102. Liao EC, Taghinia AH, Nguyen LP, et al. Incidence of hematoma complication 
with heparin venous thrombosis prophylaxis after TRAM flap breast reconstruction. Plast Reconstr Surg. 2008 Apr;121(4):1101-7. doi: 10.1097/01.prs.0000302454.43201.83. PMID: 18349626.

103. Liu AS, Kao HK, Reish RG, et al. Postoperative complications in prosthesis-based breast reconstruction using acellular dermal matrix. Plast Reconstr Surg. 2011 May;127(5):175562. doi: 10.1097/PRS.0b013e31820cf233. PMID: 21228744.

104. Liu C, Momeni A, Zhuang Y, et al. Outcome analysis of expander/implant versus microsurgical abdominal flap breast reconstruction: a critical study of 254 cases. Ann Surg Oncol. 2014 Jun;21(6):2074-82. doi: 10.1245/s10434-014-3521-0. PMID: 24558063.

105. Lovecchio F, Jordan SW, Lim S, et al. Risk Factors for Complications Differ Between Stages of Tissue-Expander Breast Reconstruction. Ann Plast Surg. 2015 Sep;75(3):275-80. doi: 10.1097/sap.0000000000000109. PMID: 24691330.

106. Macadam SA, Ho AL, Cook EF, Jr., et al. Patient satisfaction and healthrelated quality of life following breast reconstruction: patient-reported outcomes among saline and silicone implant recipients. Plast Reconstr Surg. 2010 Mar;125(3):761-71. doi: 10.1097/PRS.0b013e3181cb5cf8. PMID: 20009795.

107. Macadam SA, Zhong T, Weichman K, et al. Quality of Life and PatientReported Outcomes in Breast Cancer Survivors: A Multicenter Comparison of Four Abdominally Based Autologous Reconstruction Methods. Plast Reconstr Surg. 2016 Mar;137(3):758-71. doi: 10.1097/01.prs.0000479932.11170.8f. PMID: 26910656.

108. Mak JC, Kwong A. Complications in Post-mastectomy Immediate Breast Reconstruction: A Ten-year Analysis of Outcomes. Clin Breast Cancer. 2020 Oct;20(5):402-7. doi: 10.1016/j.clbc.2019.12.002. PMID: 32665188.

109. Masoomi H, Blumenauer BJ, Blakkolb $\mathrm{CL}$, et al. Predictors of blood transfusion in autologous breast reconstruction surgery: A retrospective study using the nationwide inpatient sample database. J Plast Reconstr Aesthet Surg. 2019 Oct;72(10):161622. doi: 10.1016/j.bjps.2019.06.012. PMID: 31331721.

110. Masoomi H, Paydar KZ, Wirth GA, et al. Predictive risk factors of venous thromboembolism in autologous breast reconstruction surgery. Ann Plast Surg. 2014 Jan;72(1):30-3. doi: 10.1097/sap.0000000000000003. PMID: 24317244.

111. Massenburg BB, Sanati-Mehrizy P, Ingargiola MJ, et al. Flap Failure and Wound Complications in Autologous Breast Reconstruction: A National Perspective. Aesthetic Plast Surg. 2015 Dec;39(6):902-9. doi: 10.1007/s00266015-0575-8. PMID: 26487657.

112. McCarthy CM, Klassen AF, Cano SJ, et al. Patient satisfaction with postmastectomy breast reconstruction: a comparison of saline and silicone implants. Cancer. 2010 Dec 15;116(24):5584-91. doi: 10.1002/cncr.25552. PMID: 21136577.

113. McCarthy CM, Lee CN, Halvorson EG, et al. The use of acellular dermal matrices in two-stage expander/implant reconstruction: a multicenter, blinded, randomized controlled trial. Plast Reconstr Surg. 2012 Nov;130(5 Suppl 2):57s-66s. doi: 
10.1097/PRS.0b013e31825f05b4. PMID: 23096987.

114. McCarthy CM, Mehrara BJ, Long T, et al. Chest and upper body morbidity following immediate postmastectomy breast reconstruction. Ann Surg Oncol. 2014 Jan;21(1):107-12. doi: 10.1245/s10434-013-3231-z. PMID: 24201740.

115. McCarthy CM, Mehrara BJ, Riedel E, et al. Predicting complications following expander/implant breast reconstruction: an outcomes analysis based on preoperative clinical risk. Plast Reconstr Surg. 2008 Jun;121(6):1886-92. doi: 10.1097/PRS.0b013e31817151c4. PMID: 18520873.

116. Mehrara BJ, Santoro TD, Arcilla E, et al. Complications after microvascular breast reconstruction: experience with 1195 flaps. Plast Reconstr Surg. 2006 Oct;118(5):1100-9; discussion 10-1. doi:

10.1097/01.prs.0000236898.87398.d6. PMID: 17016173.

117. Mennie JC, Mohanna PN, O'Donoghue JM, et al. Donor-Site Hernia Repair in Abdominal Flap Breast Reconstruction: A Population-Based Cohort Study of 7929 Patients. Plast Reconstr Surg. 2015 Jul;136(1):1-9. doi: $10.1097 /$ prs. 0000000000001398 . PMID: 25839173.

118. Merchant SJ, Goldstein L, Kruper LL. Patterns and Trends in Immediate Postmastectomy Reconstruction in California: Complications and Unscheduled Readmissions. Plast Reconstr Surg. 2015 Jul;136(1):10e-9e. doi: 10.1097/prs.0000000000001326. PMID: 26111325.

119. Mioton LM, Smetona JT, Hanwright $\mathrm{PJ}$, et al. Comparing thirty-day outcomes in prosthetic and autologous breast reconstruction: a multivariate analysis of 13,082 patients? J Plast

Reconstr Aesthet Surg. 2013

Jul;66(7):917-25. doi:

10.1016/j.bjps.2013.03.009. PMID: 23562485.

120. Mirzabeigi MN, Nelson JA, Fischer JP, et al. Tamoxifen (selective estrogenreceptor modulators) and aromatase inhibitors as potential perioperative thrombotic risk factors in free flap breast reconstruction. Plast Reconstr Surg. 2015 Apr;135(4):670e-9e. doi: 10.1097/prs.0000000000001127. PMID: 25811579.

121. Mlodinow AS, Ver Halen JP, Lim S, et al. Predictors of readmission after breast reconstruction: a multiinstitutional analysis of 5012 patients. Ann Plast Surg. 2013 Oct;71(4):335-41. doi: 10.1097/SAP.0b013e3182a0df25. PMID: 24025652.

122. Momeni A, Fox JP. Venous Thromboembolism After Surgical Treatment of Breast Cancer. Ann Plast Surg. 2018 Feb;80(2):188-92. doi: 10.1097/sap.0000000000001249. PMID: 29095189.

123. Munder B, Andree C, Witzel C, et al. The DIEP Flap as Well-established Method of Choice for Autologous Breast Reconstruction with a Low Complication Rate - Retrospective Single-centre 10-Year Experience. Geburtshilfe Frauenheilkd. 2020 Jun;80(6):628-38. doi: 10.1055/a-11162102. PMID: 32565553.

124. Naoum GE, Oladeru OT, Niemierko A, et al. Optimal breast reconstruction type for patients treated with neoadjuvant chemotherapy, mastectomy followed by radiation therapy. Breast Cancer Res Treat. 2020 Aug;183(1):127-36. doi: 10.1007/s10549-020-05747-7. PMID: 32607638 . 
125. Naoum GE, Salama L, Niemierko A, et al. Single Stage Direct-to-Implant Breast Reconstruction Has Lower Complication Rates Than Tissue Expander and Implant and Comparable Rates to Autologous Reconstruction in Patients Receiving Postmastectomy Radiation. Int J Radiat Oncol Biol Phys. 2020 Mar 1;106(3):514-24. doi: 10.1016/j.ijrobp.2019.11.008. PMID: 31756414.

126. Nasser JS, Huetteman HE, Chung TT, et al. Unplanned Emergency Department Visits within 30 Days of Mastectomy and Breast Reconstruction. Plast Reconstr Surg. 2018 Dec;142(6):1411-20. doi: 10.1097/prs.0000000000004970. PMID: 30204678.

127. Nealon KP, Sobti N, Gadd M, et al. Assessing the additional surgical risk of contralateral prophylactic mastectomy and immediate breast implant reconstruction. Breast Cancer Res Treat. 2020 Jan;179(2):255-65. doi: 10.1007/s10549-019-05460-0. PMID: 31605310.

128. Nealon KP, Weitzman RE, Sobti N, et al. Prepectoral Direct-to-Implant Breast Reconstruction: Safety Outcome Endpoints and Delineation of Risk Factors. Plast Reconstr Surg. 2020 Feb 5. doi: 10.1097/prs.0000000000006721. PMID: 32032345.

129. Nelson JA, Allen RJ, Jr., Polanco T, et al. Long-term Patient-reported Outcomes Following Postmastectomy Breast Reconstruction: An 8-year Examination of 3268 Patients. Ann Surg. 2019 Sep;270(3):473-83. doi: 10.1097/sla.0000000000003467. PMID: 31356276.

130. Nelson JA, Fischer JP, Grover R, et al. The impact of anemia on microsurgical breast reconstruction complications and outcomes. Microsurgery. 2014
May;34(4):261-70. doi: 10.1002/micr.22202. PMID: 24436188.

131. Nelson JA, Stransky CA, Fischer JP, et al. Reexamining free flap breast reconstruction in the community and university setting: is there a difference? Ann Plast Surg. 2014 Dec;73 Suppl

2:S171-4. doi: 10.1097/sap.0000000000000204. PMID: 25046665.

132. Nelson JA, Voineskos SH, Qi J, et al. Elective Revisions after Breast Reconstruction: Results from the Mastectomy Reconstruction Outcomes Consortium. Plast Reconstr Surg. 2019 Dec;144(6):1280-90. doi: 10.1097/prs.0000000000006225. PMID: 31764633.

133. O'Neill AC, Barandun M, Cha J, et al. Restrictive use of perioperative blood transfusion does not increase complication rates in microvascular breast reconstruction. J Plast Reconstr Aesthet Surg. 2016 Aug;69(8):1092-6. doi: 10.1016/j.bjps.2016.04.021. PMID: 27236504.

134. O'Neill AC, Sebastiampillai S, Zhong $\mathrm{T}$, et al. Increasing body mass index increases complications but not failure rates in microvascular breast reconstruction: A retrospective cohort study. J Plast Reconstr Aesthet Surg. 2019 Sep;72(9):1518-24. doi: 10.1016/j.bjps.2019.05.013. PMID: 31196805.

135. Ozgur I, Kurul S, Bademler S, et al. Comparison of subpectoral versus dualplane implant based immediate breast reconstruction after nipple-areola sparing mastectomy. Ann Chir Plast Esthet. 2020 Nov 19. doi: 10.1016/j.anplas.2020.10.002. PMID: 33223365.

136. Palve JS, Luukkaala TH, Kääriäinen MT. Predictive risk factors of complications in different breast 
reconstruction methods. Breast Cancer

Res Treat. 2020 Jul;182(2):345-54. doi: 10.1007/s10549-020-05705-3. PMID: 32468337.

137. Pannucci CJ, Antony AK, Wilkins EG. The impact of acellular dermal matrix on tissue expander/implant loss in breast reconstruction: an analysis of the tracking outcomes and operations in plastic surgery database. Plast Reconstr Surg. 2013 Jul;132(1):1-10. doi: 10.1097/PRS.0b013e318290f917. PMID: 23508050.

138. Parikh RP, Brown GM, Sharma K, et al. Immediate Implant-Based Breast Reconstruction with Acellular Dermal Matrix: A Comparison of Sterile and Aseptic AlloDerm in 2039 Consecutive Cases. Plast Reconstr Surg. 2018 Dec;142(6):1401-9. doi: 10.1097/prs.0000000000004968. PMID: 30204676.

139. Park JW, Lee KT, Jeon BJ, et al. Effects of Antihypertensive Drugs on Outcomes of Breast Reconstruction. Ann Surg Oncol. 2019 Jun;26(6):17129. doi: 10.1245/s10434-019-07293-z. PMID: 30863940.

140. Peled AW, Foster RD, Garwood ER, et al. The effects of acellular dermal matrix in expander-implant breast reconstruction after total skin-sparing mastectomy: results of a prospective practice improvement study. Plast Reconstr Surg. 2012 Jun;129(6):901e8e. doi: 10.1097/PRS.0b013e31824ec447. PMID: 22634688.

141. Phan R, Rozen WM, Chowdhry M, et al. Risk factors and timing of postoperative hematomas following microvascular breast reconstruction: A prospective cohort study. Microsurgery. 2020 Feb;40(2):99-103. doi: 10.1002/micr.30473. PMID: 31124177.
142. Polanco TO, Shamsunder MG, Hicks MEV, et al. Goal-directed fluid therapy in autologous breast reconstruction results in less fluid and more vasopressor administration without outcome compromise. J Plast Reconstr Aesthet Surg. 2021 Feb 4. doi: 10.1016/j.bjps.2021.01.017. PMID: 33745850 .

143. Potter S, Conroy EJ, Cutress RI, et al. Short-term safety outcomes of mastectomy and immediate implantbased breast reconstruction with and without mesh (iBRA): a multicentre, prospective cohort study. Lancet Oncol. 2019 Feb;20(2):254-66. doi: 10.1016/s1470-2045(18)30781-2. PMID: 30639093.

144. Prantl L, Moellhoff N, von Fritschen $\mathrm{U}$, et al. Immediate versus secondary DIEP flap breast reconstruction: a multicenter outcome study. Arch Gynecol Obstet. 2020 Dec;302(6):1451-9. doi: 10.1007/s00404-020-05779-w. PMID: 32895743.

145. Pusic AL, Matros E, Fine N, et al. Patient-Reported Outcomes 1 Year After Immediate Breast Reconstruction: Results of the Mastectomy Reconstruction Outcomes Consortium Study. J Clin Oncol. 2017 Aug 1;35(22):2499-506. doi: 10.1200/jco.2016.69.9561. PMID: 28346808.

146. Qin Q, Tan Q, Lian B, et al. Postoperative outcomes of breast reconstruction after mastectomy: A retrospective study. Medicine (Baltimore). 2018 Feb;97(5):e9766. doi: 10.1097/md.0000000000009766. PMID: 29384865.

147. Qureshi AA, Broderick KP, Belz J, et al. Uneventful versus Successful Reconstruction and Outcome Pathways in Implant-Based Breast Reconstruction 
with Acellular Dermal Matrices. Plast

Reconstr Surg. 2016 Aug;138(2):173e-

83e. doi:

10.1097/prs.0000000000002402.

PMID: 27465177.

148. Ricci JA, Kamali P, Becherer BE, et al. Umbilical necrosis rates after abdominal-based microsurgical breast reconstruction. J Surg Res. 2017

Jul;215:257-63. doi:

10.1016/j.jss.2017.04.010. PMID: 28688657.

149. Rindom MB, Gunnarsson GL, Lautrup $\mathrm{MD}$, et al. Shoulder-related donor site morbidity after delayed breast reconstruction with pedicled flaps from the back: An open label randomized controlled clinical trial. J Plast Reconstr Aesthet Surg. 2019 Dec;72(12):1942-9. doi: 10.1016/j.bjps.2019.07.027. PMID: 31515191.

150. Rogoff H, Marquez JE, Ghosh K, et al. Clinical Outcomes After Hematoma Development: A Study of 627 Tissue Expander Breast Reconstructions. Ann Plast Surg. 2020 Jul;85(S1 Suppl

1):S63-s7. doi: 10.1097/sap.0000000000002343. PMID: 32243320.

151. Roth RS, Lowery JC, Davis J, et al. Persistent pain following postmastectomy breast reconstruction: long-term effects of type and timing of surgery. Ann Plast Surg. 2007

Apr;58(4):371-6. doi: 10.1097/01.sap.0000239810.38137.84. PMID: 17413877.

152. Roth RS, Qi J, Hamill JB, et al. Is chronic postsurgical pain surgeryinduced? A study of persistent postoperative pain following breast reconstruction. Breast. 2018

Feb;37:119-25. doi: 10.1016/j.breast.2017.11.001. PMID: 29145033.
153. Roy M, Sebastiampillai S, Zhong T, et al. Synergistic Interaction Increases Complication Rates following Microvascular Breast Reconstruction. Plast Reconstr Surg. 2019 Jul;144(1):1e-8e. doi: 10.1097/prs.0000000000005695. PMID: 31246793.

154. Rubio GA, McGee CS, Thaller SR. Autologous breast reconstruction surgery outcomes in patients with autoimmune connective tissue disease. J Plast Reconstr Aesthet Surg. 2019 May;72(5):848-62. doi: 10.1016/j.bjps.2018.12.038. PMID: 30665841.

155. Safran T, Al-Halabi B, Viezel-Mathieu A, et al. Direct-to-Implant, Prepectoral Breast Reconstruction: A SingleSurgeon Experience with 201 Consecutive Patients. Plast Reconstr Surg. 2020 Apr;145(4):686e-96e. doi: 10.1097/prs.0000000000006654. PMID: 32221195.

156. Salibian AA, Frey JD, Bekisz JM, et al. Ischemic Complications after Nipple-sparing Mastectomy: Predictors of Reconstructive Failure in Implantbased Reconstruction and Implications for Decision-making. Plast Reconstr Surg Glob Open. 2019 May;7(5):e2280. doi: 10.1097/gox.0000000000002280. PMID: 31333984.

157. Santosa KB, Chen X, Qi J, et al. Postmastectomy Radiation Therapy and Two-Stage Implant-Based Breast Reconstruction: Is There a Better Time to Irradiate? Plast Reconstr Surg. 2016 Oct;138(4):761-9. doi: 10.1097/prs.0000000000002534. PMID: 27673513.

158. Santosa KB, Qi J, Kim HM, et al. Long-term Patient-Reported Outcomes in Postmastectomy Breast

Reconstruction. JAMA Surg. 2018 Oct 1;153(10):891-9. doi: 
10.1001/jamasurg.2018.1677. PMID: 29926096.

159. Seidenstuecker $K$, van Waes $C$, Munder BI, et al. DIEAP flap for safe definitive autologous breast reconstruction. Breast. 2016 Apr;26:5966. doi: 10.1016/j.breast.2015.12.005. PMID: 27017243.

160. Selber JC, Bergey M, Sonnad SS, et al. Free flap breast reconstruction in advanced age: is it safe? Plast Reconstr Surg. 2009 Oct;124(4):1015-22. doi: 10.1097/PRS.0b013e3181b453fd. PMID: 19935283.

161. Seth AK, Hirsch EM, Fine NA, et al. Additive risk of tumescent technique in patients undergoing mastectomy with immediate reconstruction. Ann Surg Oncol. 2011 Oct;18(11):3041-6. doi: 10.1245/s10434-011-1913-y. PMID: 21947584.

162. Seth AK, Hirsch EM, Fine NA, et al. Utility of acellular dermis-assisted breast reconstruction in the setting of radiation: a comparative analysis. Plast Reconstr Surg. 2012 Oct;130(4):750-8. doi: 10.1097/PRS.0b013e318262f009. PMID: 23018687.

163. Seth AK, Hirsch EM, Kim JY, et al. Two surgeons, one patient: the impact of surgeon-surgeon familiarity on patient outcomes following mastectomy with immediate reconstruction. Breast. 2013 Oct;22(5):914-8. doi: 10.1016/j.breast.2013.04.013. PMID: 23673077.

164. Seth AK, Hirsch EM, Kim JY, et al. Hematoma after mastectomy with immediate reconstruction: an analysis of risk factors in 883 patients. Ann Plast Surg. 2013 Jul;71(1):20-3. doi: 10.1097/SAP.0b013e318243355f. PMID: 22791066.

165. Seth AK, Hirsch EM, Kim JY, et al. Long-term outcomes following fat grafting in prosthetic breast reconstruction: a comparative analysis. Plast Reconstr Surg. 2012

Nov;130(5):984-90. doi: 10.1097/PRS.0b013e318267d34d. PMID: 22777039.

166. Seth AK, Silver HR, Hirsch EM, et al. Comparison of Delayed and Immediate Tissue Expander Breast Reconstruction in the Setting of Postmastectomy Radiation Therapy. Ann Plast Surg. 2015 Nov;75(5):503-7. doi: 10.1097/sap.0000000000000191. PMID: 25180955.

167. Sewart E, Turner NL, Conroy EJ, et al. Patient-reported outcomes of immediate implant-based breast reconstruction with and without biological or synthetic mesh. BJS Open. 2021 Jan 8;5(1). doi: 10.1093/bjsopen/zraa063. PMID: 33609398.

168. Shaikh IA, Thomas H, Vidyadharan R, et al. Post mastectomy immediate breast reconstruction 13 years experience in a single centre. Indian J Surg Oncol. 2010 Sep;1(3):250-5. doi: 10.1007/s13193011-0051-0. PMID: 22693373.

169. Shammas RL, Ren Y, Thomas SM, et al. The Association of Overall Annual Hospital Volume and Perioperative Outcomes following Free Flap Breast Reconstruction. Plast Reconstr Surg. 2021 Feb 1;147(2):196e-206e. doi: 10.1097/prs.0000000000007549. PMID: 33565821.

170. Shiraishi M, Sowa Y, Fujikawa K, et al. Factors associated with chronic pain following breast reconstruction in Japanese women. J Plast Surg Hand Surg. 2020 Oct;54(5):317-22. doi: 10.1080/2000656x.2020.1780246. PMID: 32589082.

171. Simon P, Barrou J, Cohen M, et al. Types of Mastectomies and Immediate Reconstructions for Ipsilateral Breast Local Recurrences. Front Oncol. 2020;10:567298. doi: 
10.3389/fonc.2020.567298. PMID: 33363007.

172. Singh D, Slavin BR, Holton T. Comparing Surgical Site Occurrences in 1 versus 2-stage Breast Reconstruction via Federated EMR Network. Plast Reconstr Surg Glob Open. 2021 Jan;9(1):e3385. doi: $10.1097 /$ gox.0000000000003385. PMID: 33564597.

173. Singh N, Reaven NL, Funk SE. Immediate 1-stage vs. tissue expander postmastectomy implant breast reconstructions: a retrospective realworld comparison over 18 months. J Plast Reconstr Aesthet Surg. 2012 Jul;65(7):917-23. doi: 10.1016/j.bjps.2011.12.040. PMID: 22342636.

174. Sobti N, Ji E, Brown RL, et al. Evaluation of Acellular Dermal Matrix Efficacy in Prosthesis-Based Breast Reconstruction. Plast Reconstr Surg. 2018 Mar;141(3):541-9. doi: $10.1097 /$ prs.0000000000004109. PMID: 29481386.

175. Song D, Slater K, Papsdorf M, et al. Autologous Breast Reconstruction in Women Older Than 65 Years Versus Women Younger Than 65 Years: A Multi-Center Analysis. Ann Plast Surg. 2016 Feb;76(2):155-63. doi: 10.1097/sap.0000000000000527. PMID: 26637165.

176. Sorkin M, Qi J, Kim HM, et al. Acellular Dermal Matrix in Immediate Expander/Implant Breast Reconstruction: A Multicenter Assessment of Risks and Benefits. Plast Reconstr Surg. 2017 Dec;140(6):1091100. doi: $10.1097 /$ prs. 0000000000003842 . PMID: 28806288.

177. Stein MJ, Chung A, Arnaout A, et al. Complication rates of acellular dermal matrix in immediate breast reconstruction with radiation: A singleinstitution retrospective comparison study. J Plast Reconstr Aesthet Surg. 2020 Dec;73(12):2156-63. doi: 10.1016/j.bjps.2020.05.065. PMID: 32561384.

178. Tallroth L, Velander P, Klasson S. A short-term comparison of expander prosthesis and DIEP flap in breast reconstructions: A prospective randomized study. J Plast Reconstr Aesthet Surg. 2020 Nov 12. doi: 10.1016/j.bjps.2020.10.104. PMID: 33436336.

179. Taylor EM, Wilkins EG, Pusic AL, et al. Impact of Unilateral versus Bilateral Breast Reconstruction on Procedure Choices and Outcomes. Plast Reconstr Surg. 2019 Jun;143(6):1159e-68e. doi: 10.1097/prs.0000000000005602. PMID: 31136472.

180. Tong WM, Baumann DP, Villa MT, et al. Obese Women Experience Fewer Complications after Oncoplastic Breast Repair following Partial Mastectomy Than after Immediate Total Breast Reconstruction. Plast Reconstr Surg. 2016 Mar; 137(3):777-91. doi: 10.1097/01.prs.0000479939.69211.19. PMID: 26910658.

181. Tran BNN, Ruan QZ, Cohen JB, et al. Does Hormone Therapy Use Increase Perioperative Complications in Abdominally Based Microsurgical Breast Reconstruction? Plast Reconstr Surg. 2018 Jun;141(6):805e-13e. doi: 10.1097/prs.0000000000004359. PMID: 29794694.

182. Vardanian AJ, Clayton JL, Roostaeian $\mathrm{J}$, et al. Comparison of implant-based immediate breast reconstruction with and without acellular dermal matrix. Plast Reconstr Surg. 2011 Nov;128(5):403e-10e. doi: 10.1097/PRS.0b013e31822b6637. PMID: 22030500. 
183. Vargas CR, Koolen PG, Ho OA, et al. Preoperative CT-angiography in autologous breast reconstruction. Microsurgery. 2016 Nov;36(8):623-7. doi: 10.1002/micr.30065. PMID: 27159778.

184. Warren DK, Nickel KB, Hostler CJ, et al. Surgeon choice in the use of postdischarge antibiotics for prophylaxis following mastectomy with and without breast reconstruction. Infect Control Hosp Epidemiol. 2021 Apr;42(4):467-70. doi: 10.1017/ice.2020.462. PMID: 33040748 .

185. Watterson PA, Bostwick J, 3rd, Hester TR, Jr., et al. TRAM flap anatomy correlated with a 10 -year clinical experience with 556 patients. Plast Reconstr Surg. 1995 Jun;95(7):118594. doi: 10.1097/00006534-19950600000007. PMID: 7761505.

186. Weichman KE, Clavin NW, Miller $\mathrm{HC}$, et al. Does the use of biopatch devices at drain sites reduce perioperative infectious complications in patients undergoing immediate tissue expander breast reconstruction? Plast Reconstr Surg. 2015 Jan;135(1):9e-17e. doi: 10.1097/prs.0000000000000810. PMID: 25539357.

187. Weichman KE, Hamill JB, Kim HM, et al. Understanding the recovery phase of breast reconstructions: Patientreported outcomes correlated to the type and timing of reconstruction. $\mathrm{J}$ Plast Reconstr Aesthet Surg. 2015 Oct;68(10):1370-8. doi: 10.1016/j.bjps.2015.05.039. PMID: 26165633.

188. Weichman KE, Wilson SC, Weinstein AL, et al. The use of acellular dermal matrix in immediate two-stage tissue expander breast reconstruction. Plast Reconstr Surg. 2012 May;129(5):104958. doi:
10.1097/PRS.0b013e31824a2acb.

PMID: 22544088.

189. Wendel J. Use of Dermal Matrix in Breast Reconstruction. ClinicalTrials.gov; 2013.

190. Wilkins EG, Hamill JB, Kim HM, et al. Complications in Postmastectomy Breast Reconstruction: One-year Outcomes of the Mastectomy Reconstruction Outcomes Consortium (MROC) Study. Ann Surg. 2018 Jan;267(1):164-70. doi: 10.1097/sla.0000000000002033. PMID: 27906762.

191. Williams JK, Bostwick J, 3rd, Bried JT, et al. TRAM flap breast reconstruction after radiation treatment. Ann Surg. 1995 Jun;221(6):756-64; discussion 64-6. doi: 10.1097/00000658-199506000-00014. PMID: 7794079.

192. Woo KJ, Lee KT, Mun GH, et al. Effect of breast reconstruction modality on the development of postmastectomy shoulder morbidity. J Plast Reconstr Aesthet Surg. 2018 Dec;71(12):1761-7. doi: 10.1016/j.bjps.2018.07.033. PMID: 30360958.

193. Woo KJ, Park JW, Mun GH, et al. Does the Use of Acellular Dermal Matrix Increase Postoperative Complications of the First-Stage Reconstruction of Immediate ExpanderImplant Breast Reconstruction: A Matched Cohort Study. Ann Plast Surg. 2017 Oct;79(4):341-5. doi: 10.1097/sap.0000000000001067. PMID: 28509694.

194. Wu ZY, Han HH, Kim HJ, et al. A propensity score-matched comparison of recurrence outcomes after immediate implant vs autologous flap reconstruction in patients receiving neoadjuvant chemotherapy for breast cancer. Breast Cancer Res Treat. 2021 
Mar 19. doi: 10.1007/s10549-021-

06114-w. PMID: 33740204.

195. Xu F, Sun H, Zhang C, et al.

Comparison of surgical complication between immediate implant and autologous breast reconstruction after mastectomy: A multicenter study of 426 cases. J Surg Oncol. 2018 Nov;118(6):953-8. doi: 10.1002/jso.25238. PMID: 30261115.

196. Yoo H, Kim BH, Kim HH, et al. Local recurrence of breast cancer in reconstructed breasts using TRAM flap after skin-sparing mastectomy: clinical and imaging features. Eur Radiol. 2014 Sep;24(9):2220-6. doi: 10.1007/s00330014-3214-x. PMID: 24852813.

197. Yoon AP, Qi J, Kim HM, et al. Patient-Reported Outcomes after Irradiation of Tissue Expander versus Permanent Implant in Breast Reconstruction: A Multicenter Prospective Study. Plast Reconstr Surg. 2020 May;145(5):917e-26e. doi: $10.1097 /$ prs.0000000000006724. PMID: 32332528.

198. Yueh JH, Slavin SA, Adesiyun T, et al. Patient satisfaction in postmastectomy breast reconstruction: a comparative evaluation of DIEP, TRAM, latissimus flap, and implant techniques. Plast Reconstr Surg. 2010 Jun;125(6):158595. doi: 10.1097/PRS.0b013e3181cb6351. PMID: 20517080.
199. Yueh JH, Slavin SA, Bar-Meir ED, et al. Impact of regional referral centers for microsurgical breast reconstruction: the New England perforator flap program experience. J Am Coll Surg. 2009 Feb;208(2):246-54. doi: 10.1016/j.jamcollsurg.2008.10.033. PMID: 19228537.

200. Zhang L, Jin K, Wang X, et al. The Impact of Radiotherapy on Reoperation Rates in Patients Undergoing Mastectomy and Breast Reconstruction. Ann Surg Oncol. 2019 Apr;26(4):9618. doi: 10.1245/s10434-018-07135-4. PMID: 30675702.

201. Zhong T, Novak CB, Bagher S, et al. Using propensity score analysis to compare major complications between DIEP and free muscle-sparing TRAM flap breast reconstructions. Plast Reconstr Surg. 2014 Apr;133(4):77482. doi:

10.1097/prs.0000000000000024. PMID: 24675183.

202. Zoghbi Y, Gerth DJ, Tashiro J, et al. Deep Inferior Epigastric Perforator Versus Free Transverse Rectus Abdominis Myocutaneous Flap: Complications and Resource Utilization. Ann Plast Surg. 2017

May;78(5):516-20. doi: 10.1097/sap.0000000000000936. PMID: 28052051. 